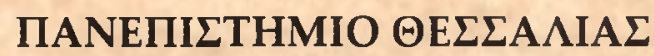

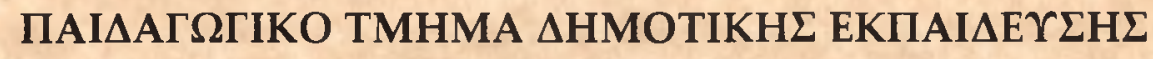

\title{
$\underline{\Delta \mathrm{I} \Delta \mathrm{AKTOPIKH} \triangle \mathrm{IATPIBH}}$
}

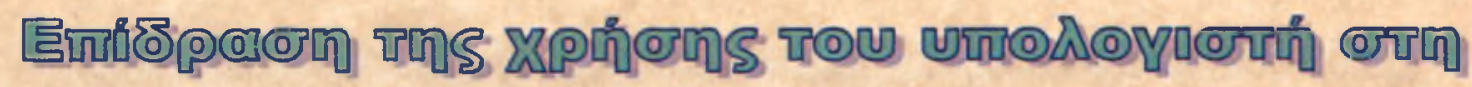

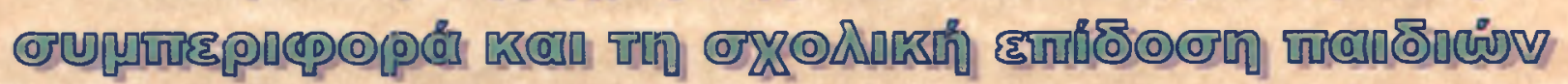

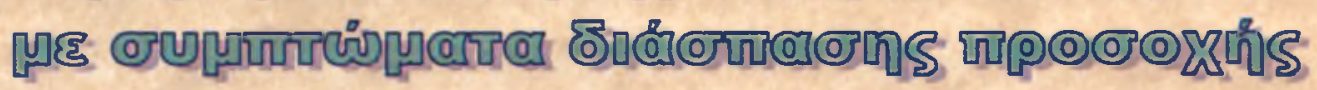

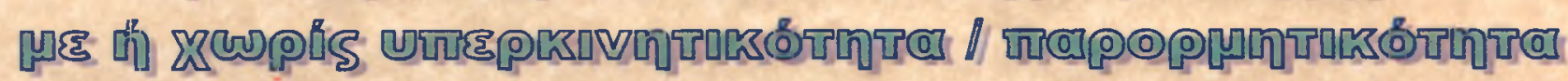

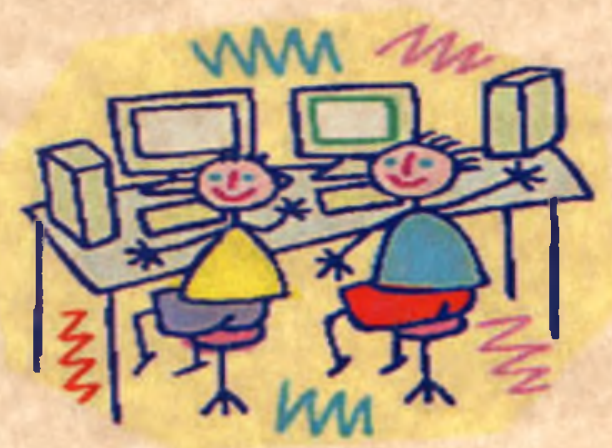

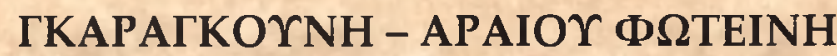

TPIME $\Lambda$ ЕП ЕПТРОПН:

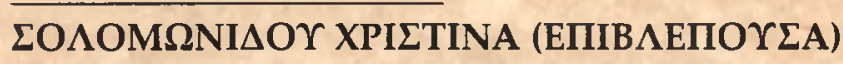

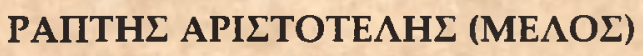

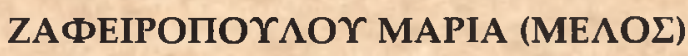

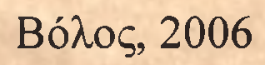




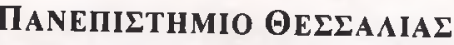
YПHPEEIA BIBAIOQHKHЕ \& ПАНPOФOPHЕHЕ ЕIАIKH ГYААОГН «ГКРІZА ВIBAIOГРАФІА»

Apı̈. Eıб.: 4924/1

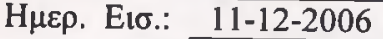

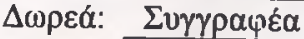

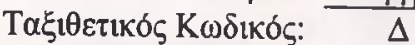

\begin{tabular}{l}
\hline 37.334 \\
\hline$\Gamma \mathrm{KA}$ \\
\hline
\end{tabular}




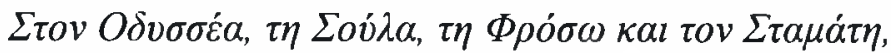
$\mu \varepsilon \dot{\alpha} \lambda \eta \mu o v \tau \eta v \alpha \gamma \alpha \dot{\pi} \eta \ldots$

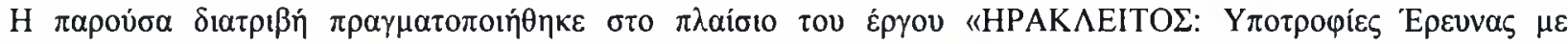

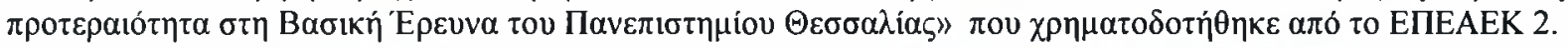

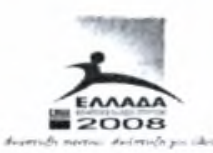

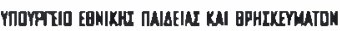 ELS:KH YIHPEEIA AIAXEPPISHI EILAEK




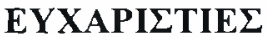

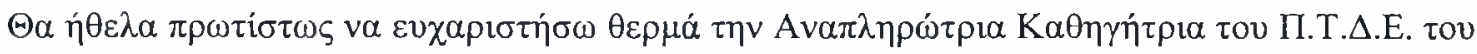

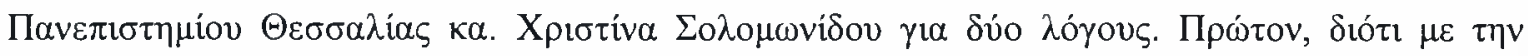

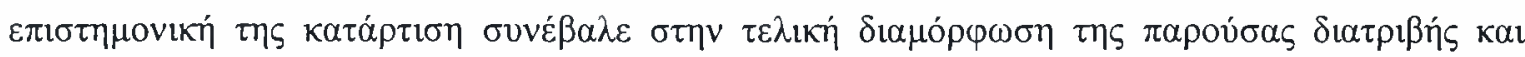

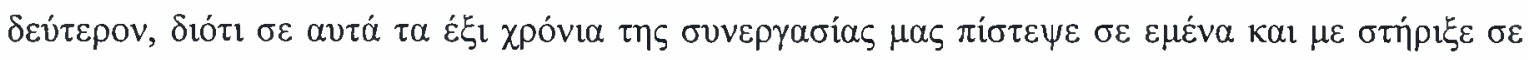

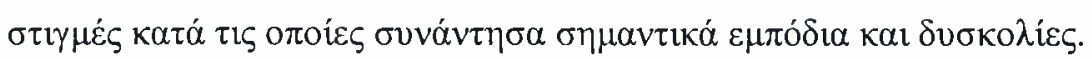

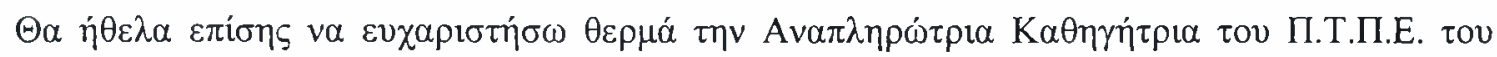

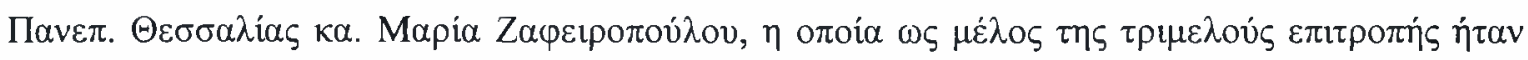



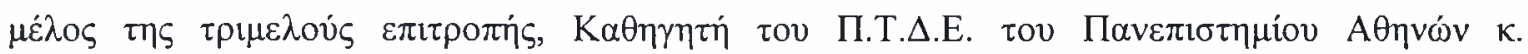

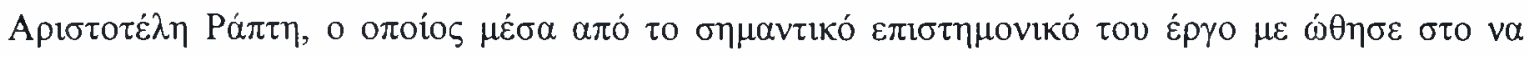

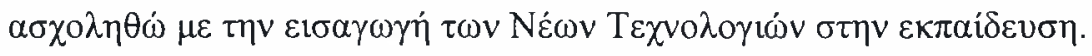

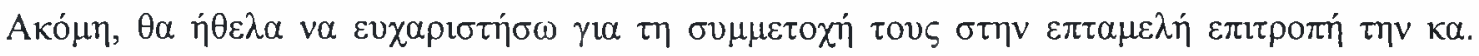

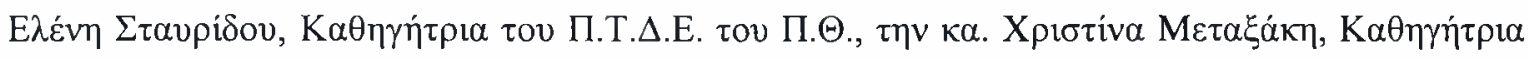

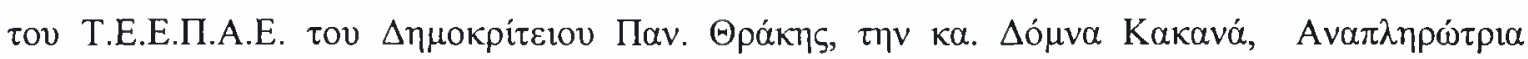

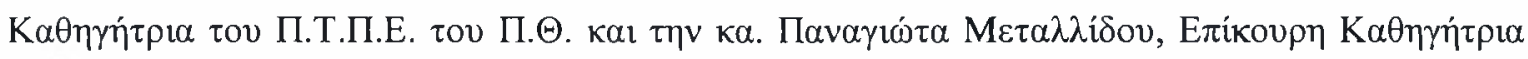

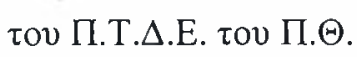

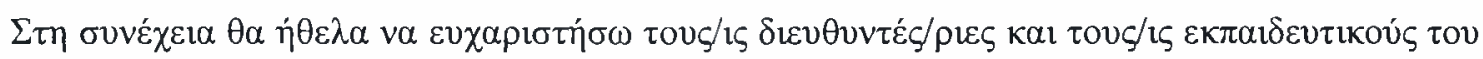

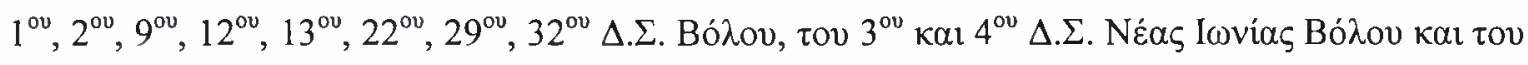

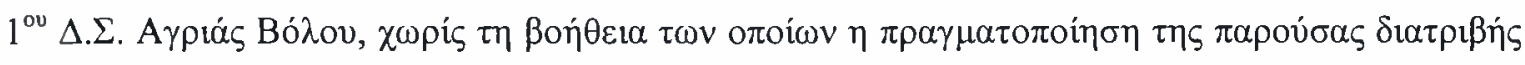

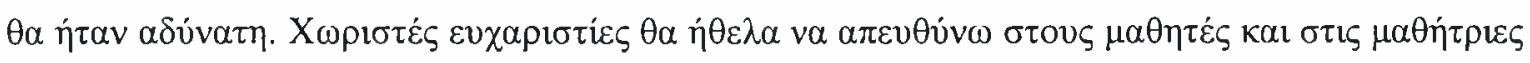

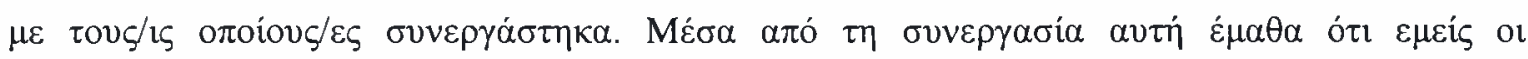

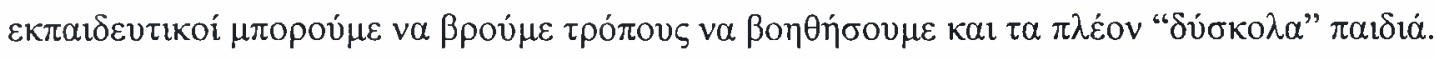

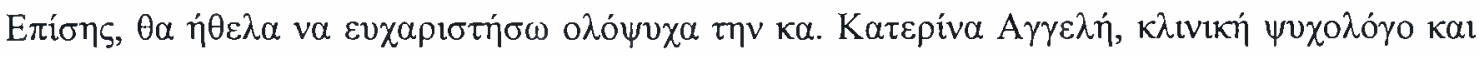

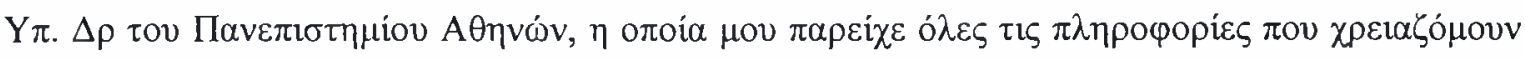

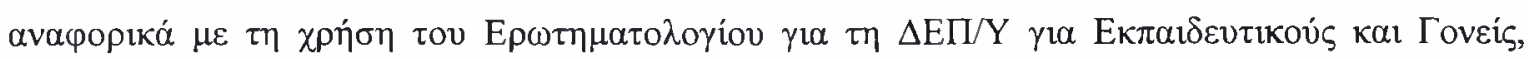

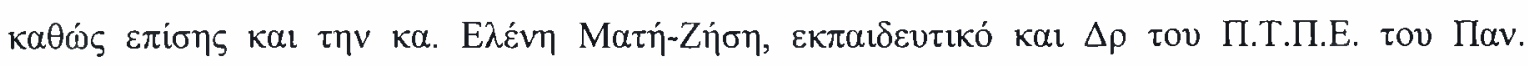

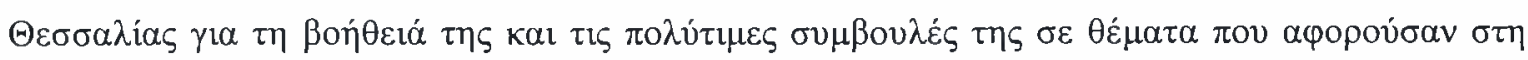

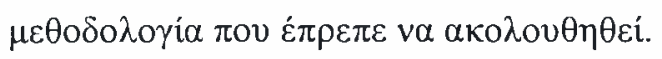

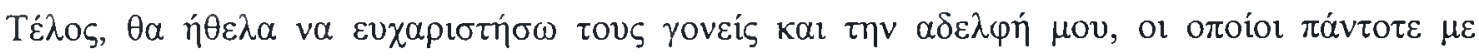

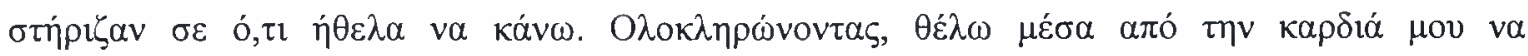

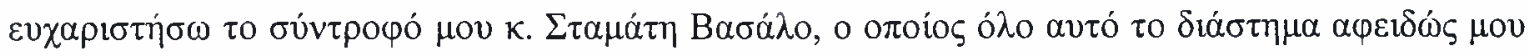

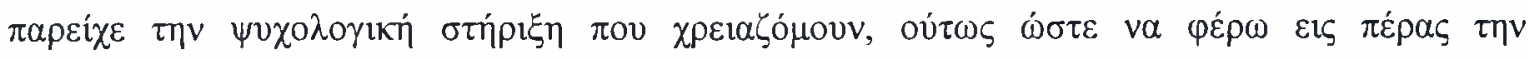

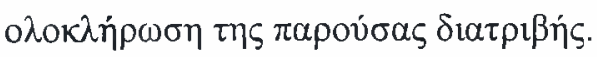




\begin{tabular}{|c|c|}
\hline$\Pi E P I A H \Psi H$ & 8 \\
\hline$S U M M A R Y$ & 9 \\
\hline$E I \Sigma A \Gamma \Omega \Gamma H$ & 10 \\
\hline ПРДТО МЕРО - ӨЕ & 15 \\
\hline 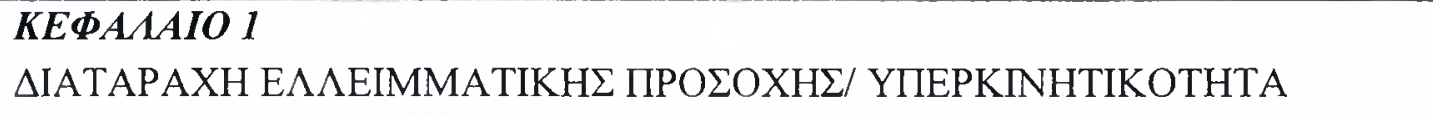 & 16 \\
\hline 1.1 Eı $\sigma \alpha \gamma \omega \gamma \eta \dot{~}$ & 16 \\
\hline 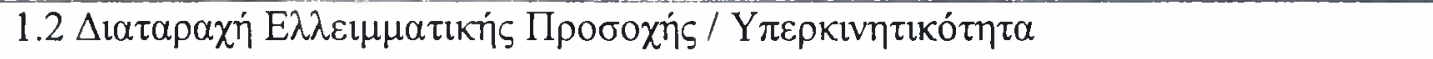 & 16 \\
\hline 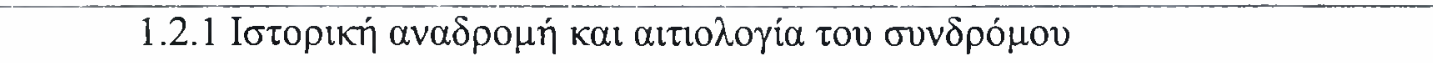 & 18 \\
\hline 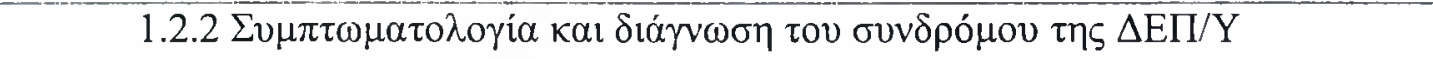 & 24 \\
\hline 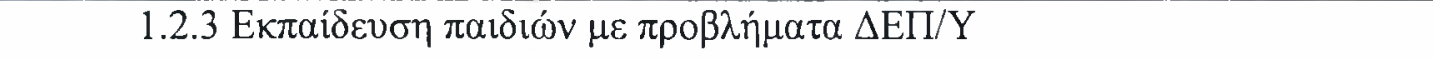 & 27 \\
\hline 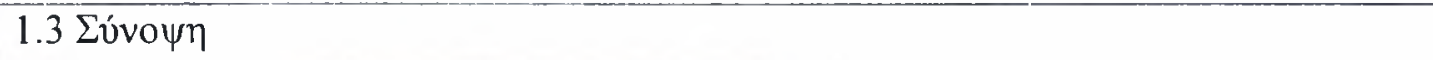 & 30 \\
\hline 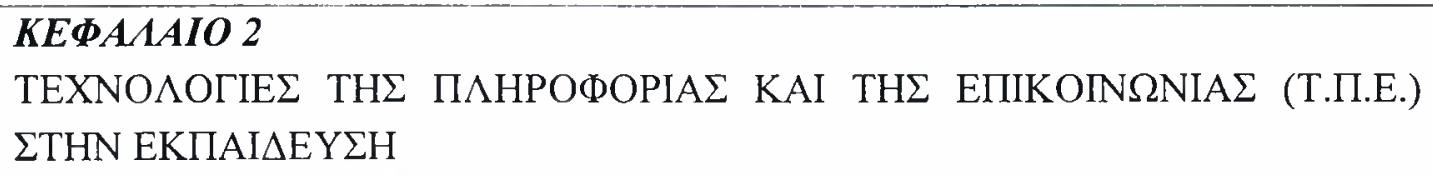 & 31 \\
\hline 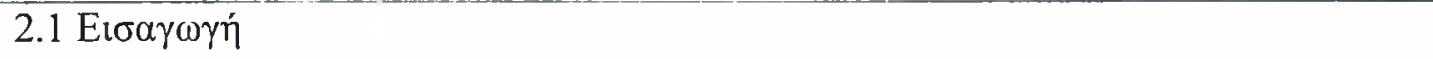 & 31 \\
\hline 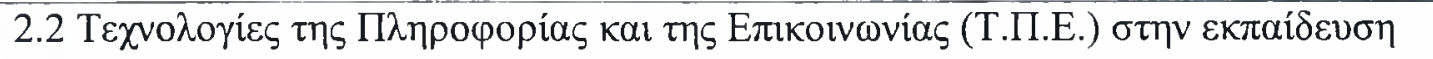 & 31 \\
\hline 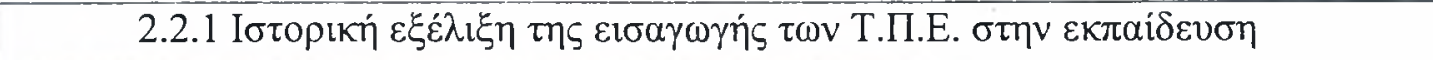 & 31 \\
\hline 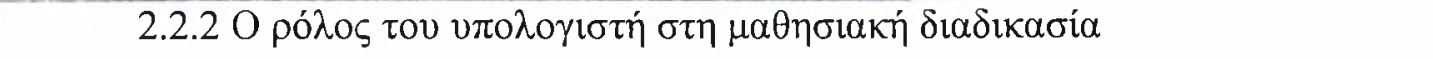 & 33 \\
\hline 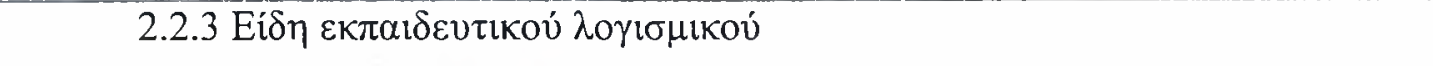 & 38 \\
\hline 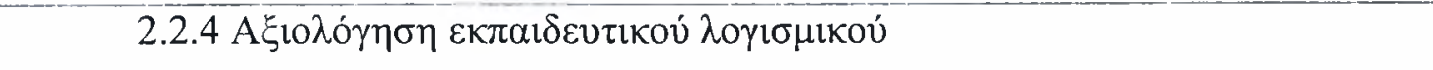 & 41 \\
\hline 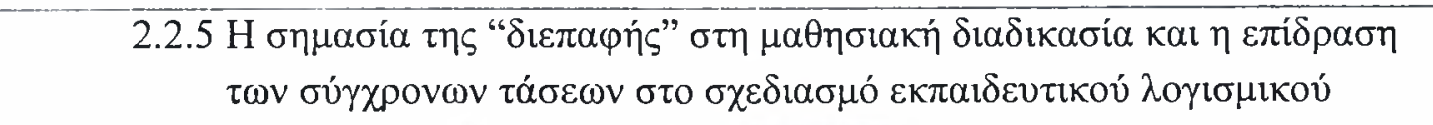 & 42 \\
\hline 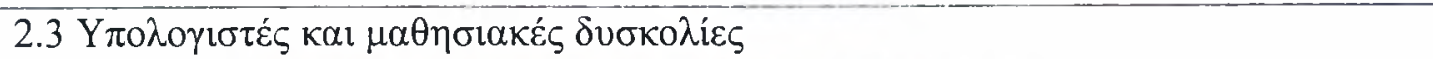 & 44 \\
\hline 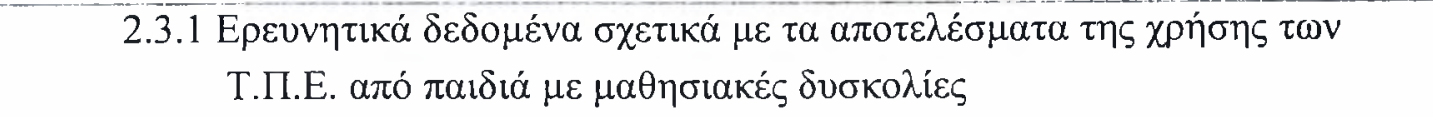 & 48 \\
\hline 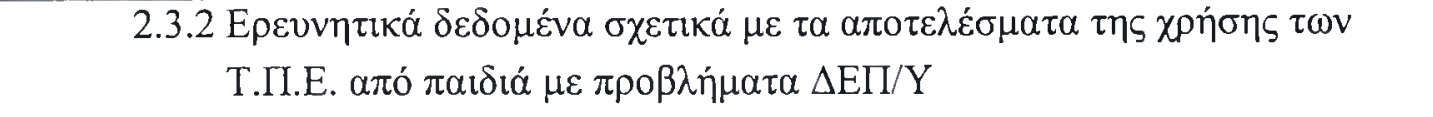 & 50 \\
\hline 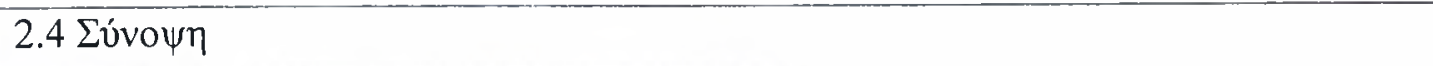 & 55 \\
\hline
\end{tabular}




\begin{tabular}{|c|c|}
\hline$\triangle E Y T E P O ~ M E P O \Sigma-H E P E Y N A$ & 57 \\
\hline 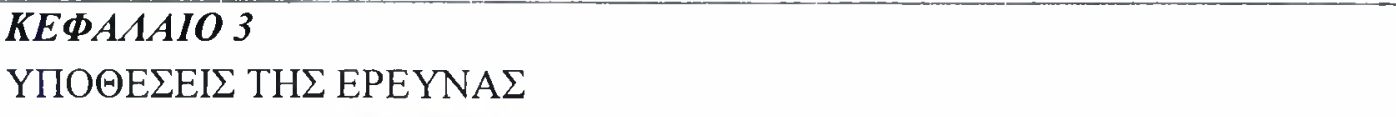 & 58 \\
\hline 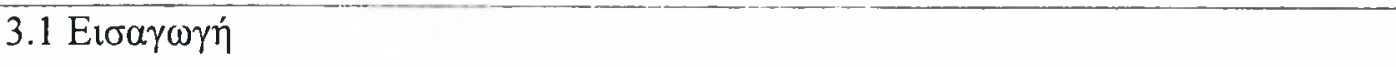 & 58 \\
\hline 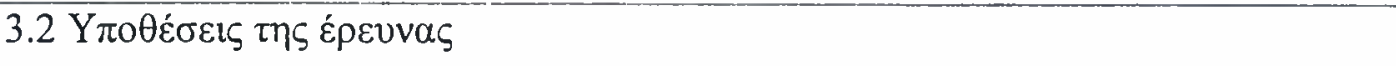 & 58 \\
\hline 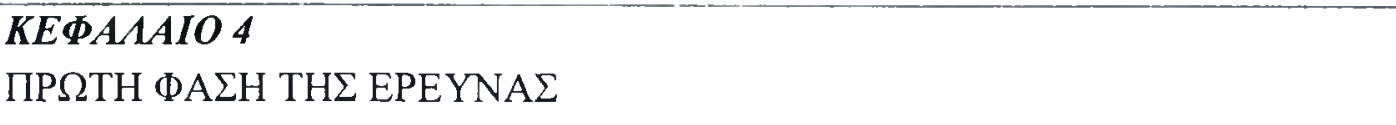 & 60 \\
\hline 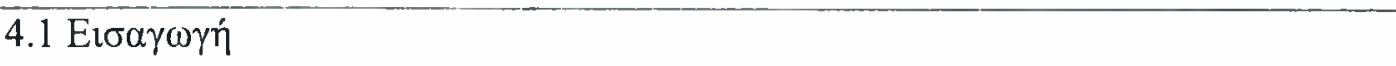 & 60 \\
\hline 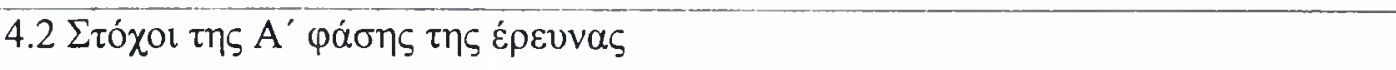 & 60 \\
\hline 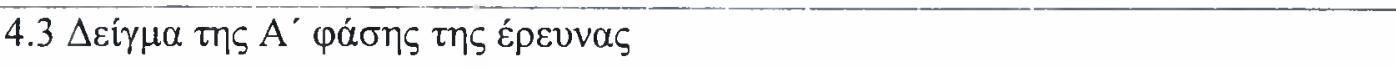 & 61 \\
\hline 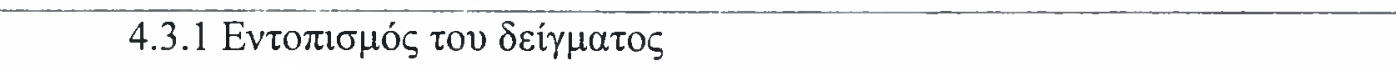 & 61 \\
\hline 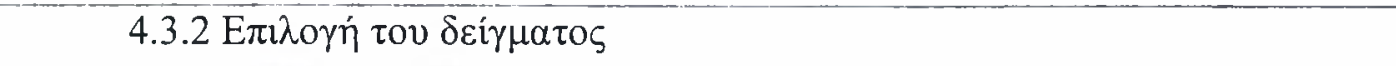 & 62 \\
\hline 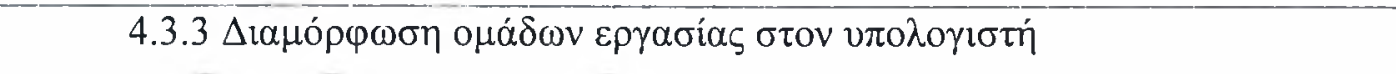 & 63 \\
\hline 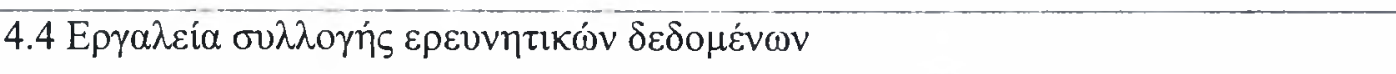 & 63 \\
\hline 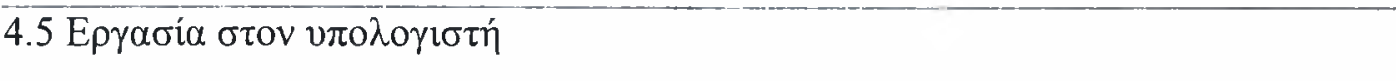 & 64 \\
\hline 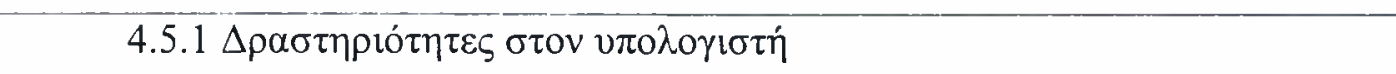 & 65 \\
\hline 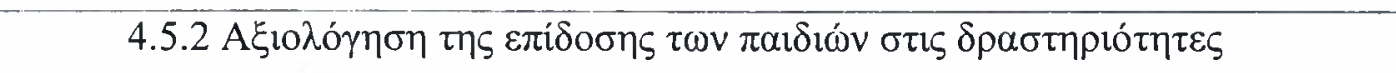 & 78 \\
\hline 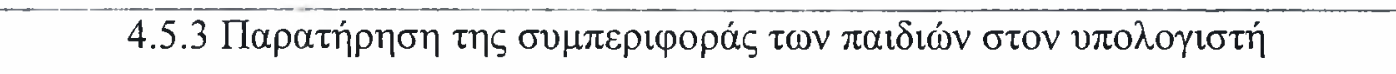 & 79 \\
\hline 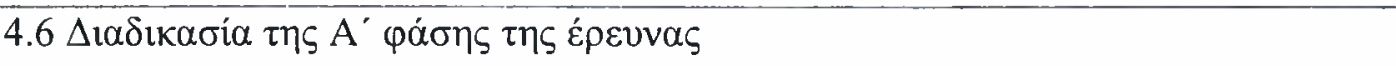 & 81 \\
\hline 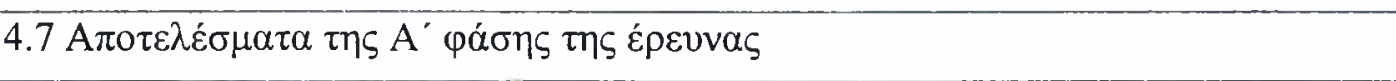 & 83 \\
\hline 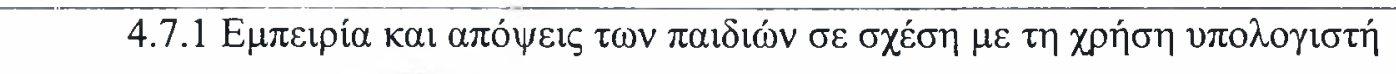 & 83 \\
\hline 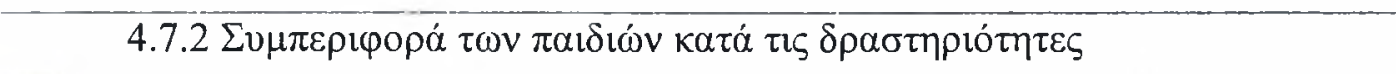 & 84 \\
\hline 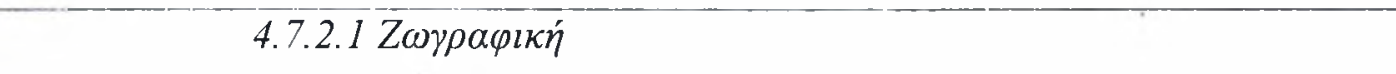 & 85 \\
\hline 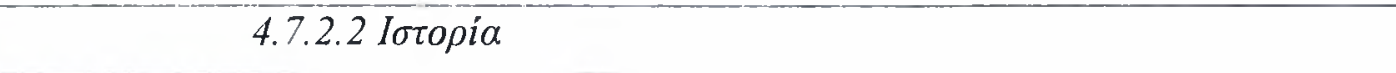 & 92 \\
\hline 4.7.2.3 $\Phi v \sigma i k \eta ́$ & 96 \\
\hline 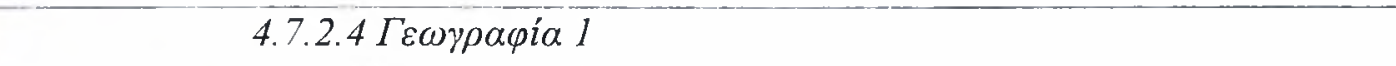 & 100 \\
\hline $4.7 .2 .5 \Gamma \varepsilon \omega \gamma \rho \alpha \varphi i \alpha 2$ & 108 \\
\hline 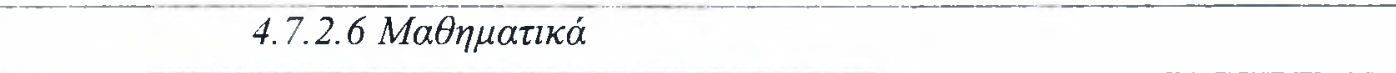 & 114 \\
\hline 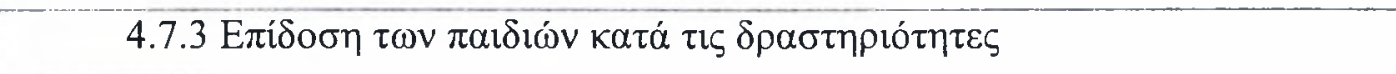 & 122 \\
\hline 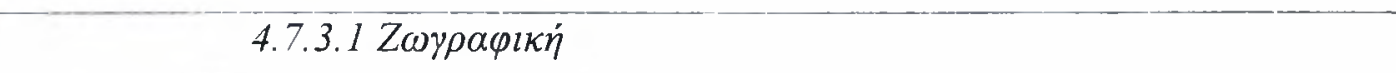 & 122 \\
\hline 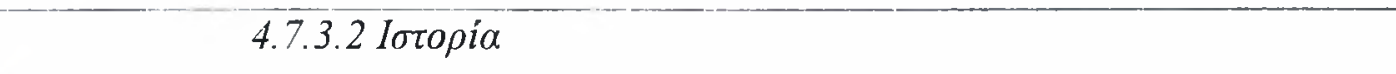 & 124 \\
\hline 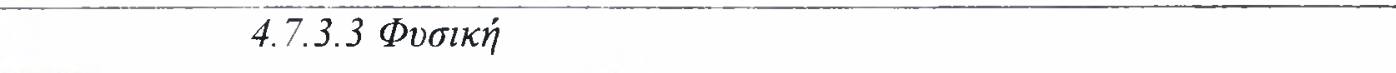 & 125 \\
\hline 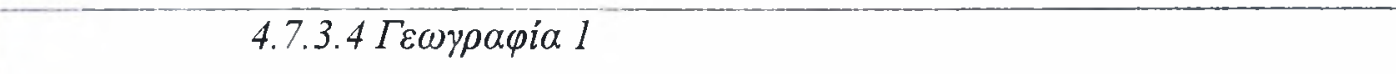 & 126 \\
\hline $4.7 .3 .5 \Gamma \varepsilon \omega \gamma \rho \alpha \varphi i \alpha 2$ & 127 \\
\hline 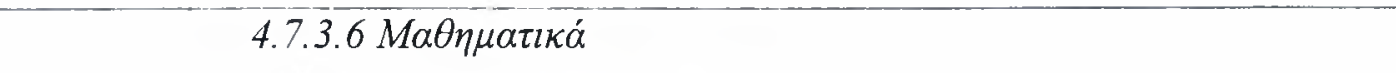 & 128 \\
\hline 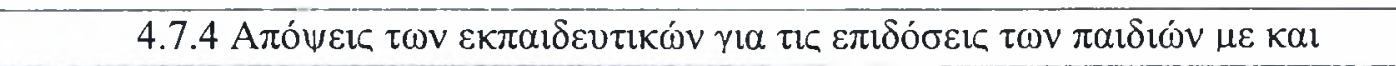 & 130 \\
\hline
\end{tabular}




\begin{tabular}{|c|c|}
\hline 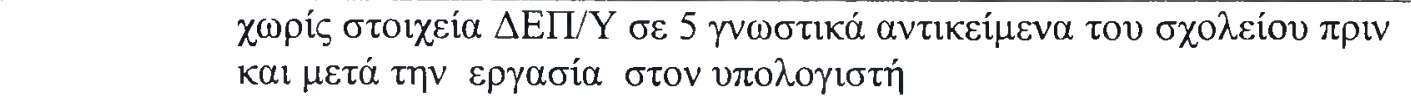 & \\
\hline 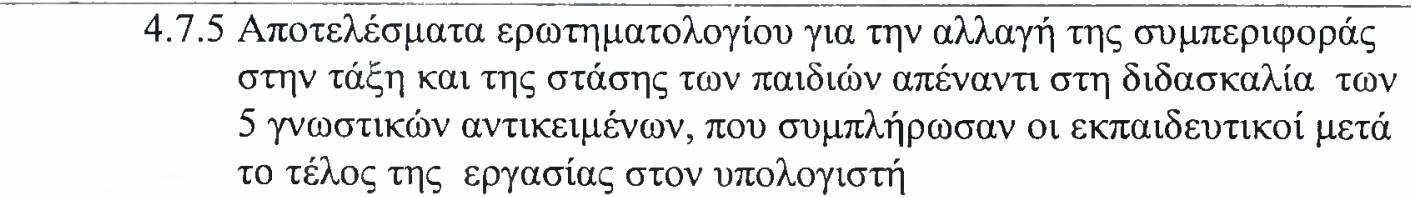 & 131 \\
\hline 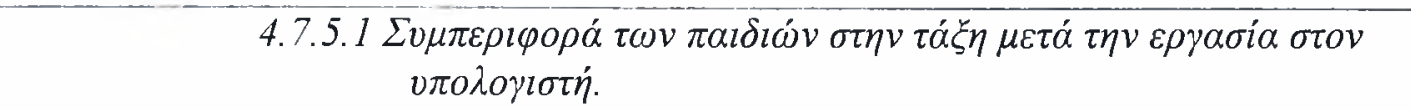 & 132 \\
\hline 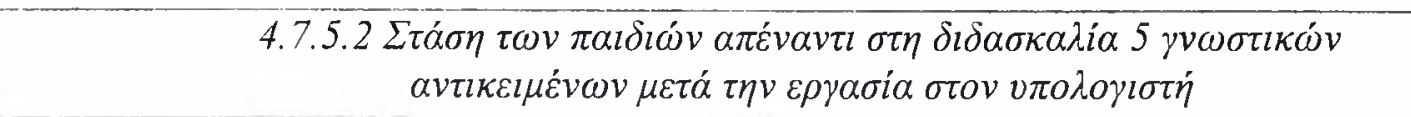 & 132 \\
\hline 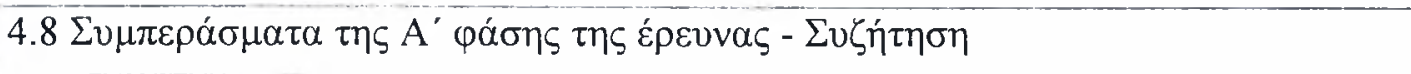 & 135 \\
\hline 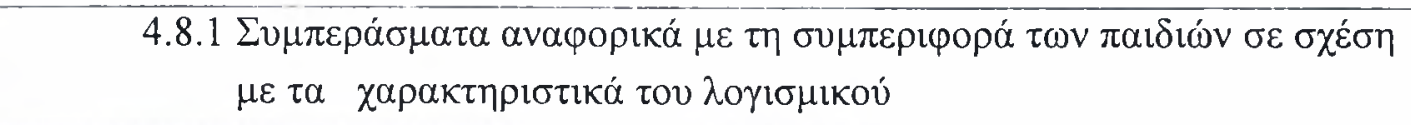 & 135 \\
\hline 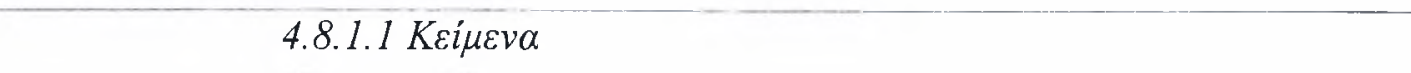 & 135 \\
\hline $4.8 .1 .2 A \varphi \eta \gamma \eta \dot{\sigma} \sigma \varepsilon l \varsigma$ & 137 \\
\hline 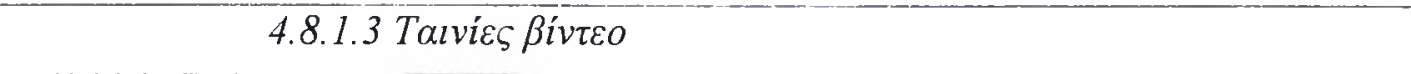 & 138 \\
\hline 4.8.1.4 Elкóves & 139 \\
\hline 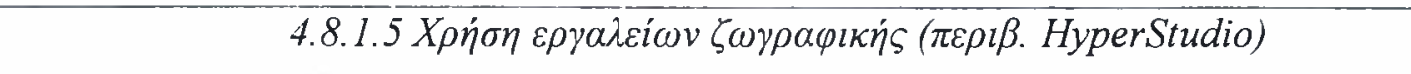 & 139 \\
\hline 4.8. $1.6 \Pi \lambda o \eta \gamma \eta \eta \sigma \eta \sigma \tau o \Delta \imath \alpha \delta i \kappa \tau v o$ & 140 \\
\hline 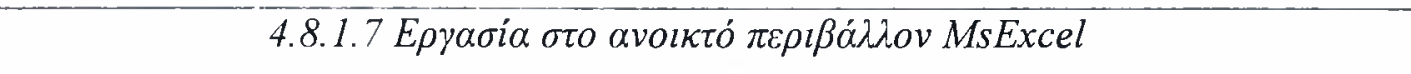 & 140 \\
\hline 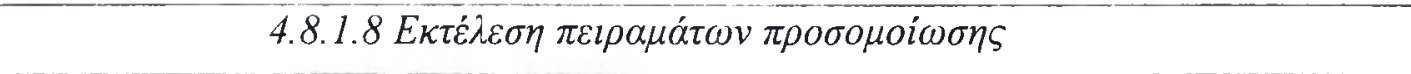 & 141 \\
\hline 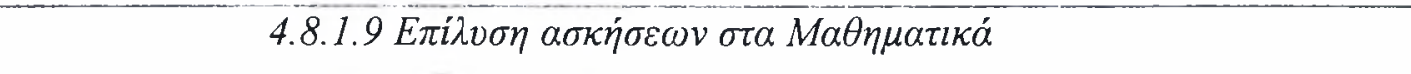 & 141 \\
\hline 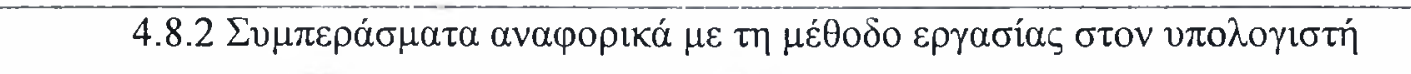 & 142 \\
\hline 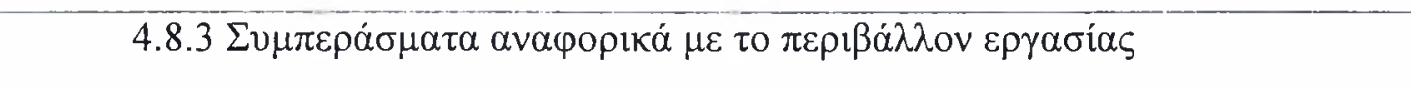 & 143 \\
\hline 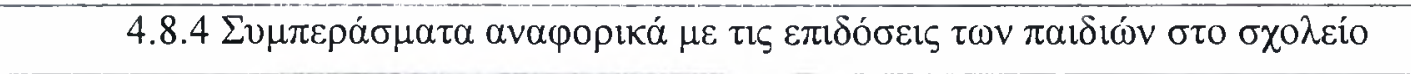 & 143 \\
\hline 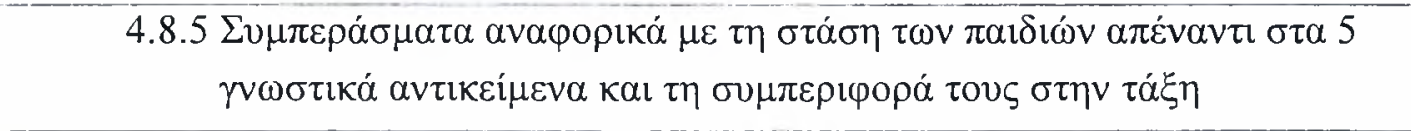 & 144 \\
\hline 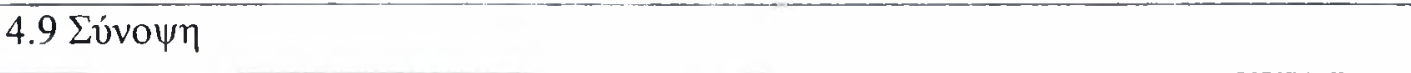 & 144 \\
\hline $\begin{array}{l}\boldsymbol{K} \boldsymbol{E \Phi} \boldsymbol{A \boldsymbol { A } A I O} \mathbf{5} \\
\triangle \mathrm{EYTEPH} \mathrm{ФА \Sigma Н} \mathrm{TH \Sigma} \mathrm{EPEYNA \Sigma}\end{array}$ & 146 \\
\hline 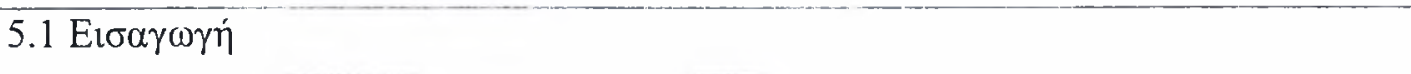 & 146 \\
\hline 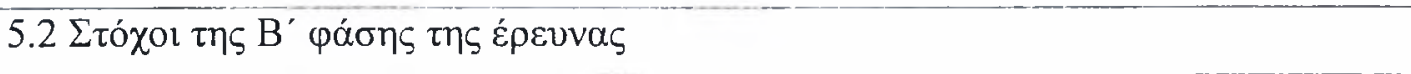 & 146 \\
\hline 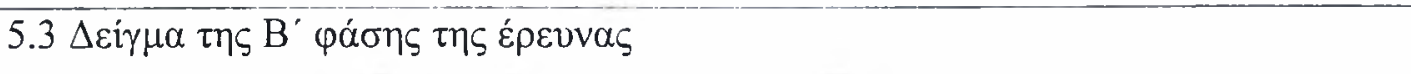 & 148 \\
\hline 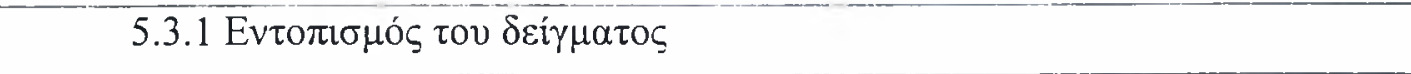 & 148 \\
\hline 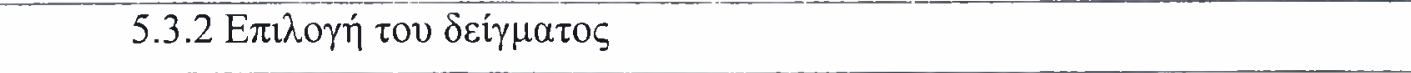 & 148 \\
\hline 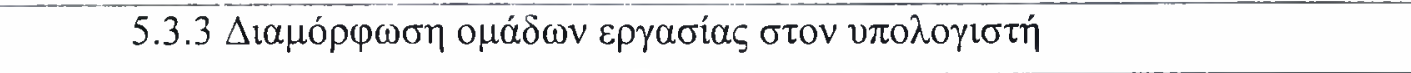 & 149 \\
\hline 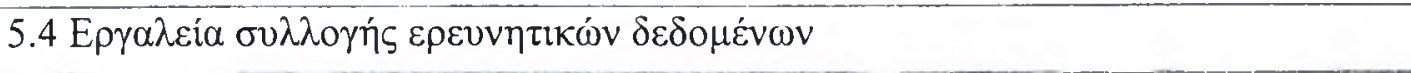 & 150 \\
\hline
\end{tabular}




\begin{tabular}{|c|c|}
\hline 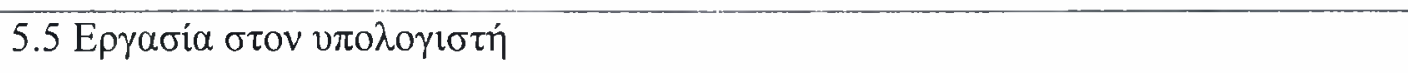 & 151 \\
\hline 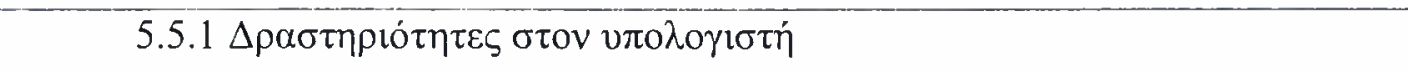 & 152 \\
\hline 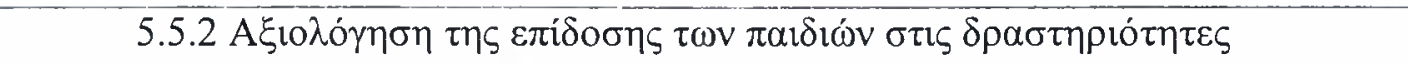 & 159 \\
\hline 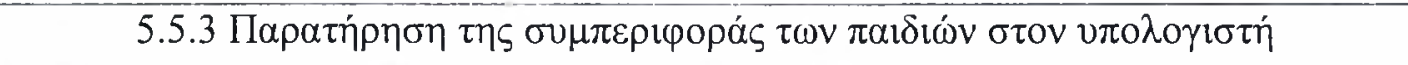 & 160 \\
\hline 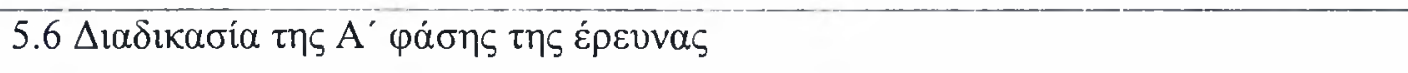 & 160 \\
\hline 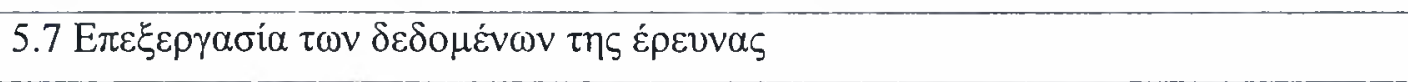 & 161 \\
\hline 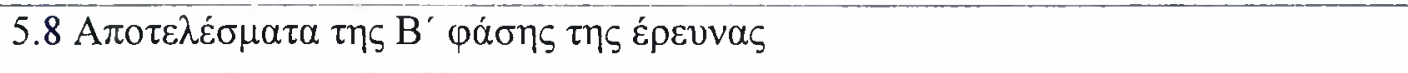 & 162 \\
\hline 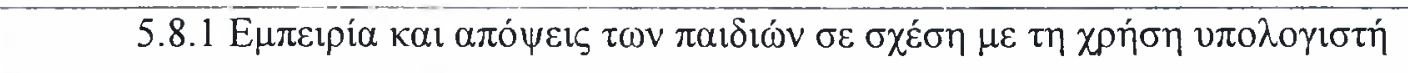 & 162 \\
\hline 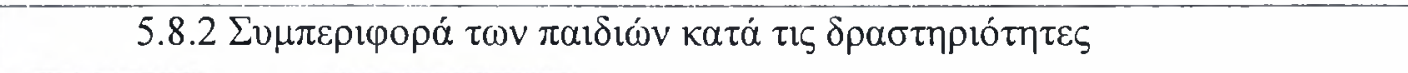 & 163 \\
\hline 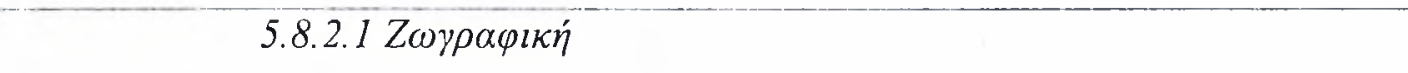 & 163 \\
\hline 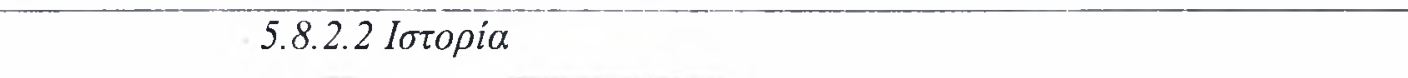 & 167 \\
\hline 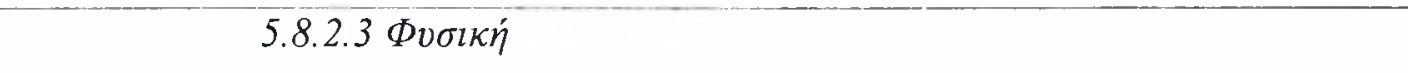 & 170 \\
\hline 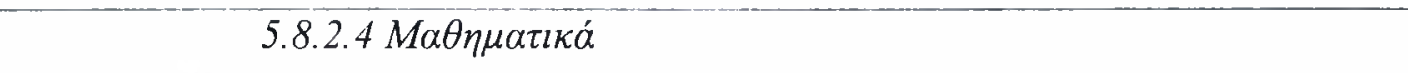 & 175 \\
\hline 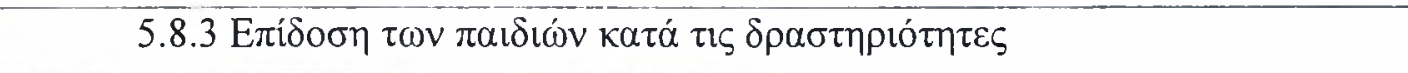 & 182 \\
\hline 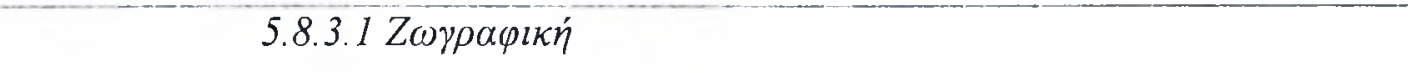 & 182 \\
\hline 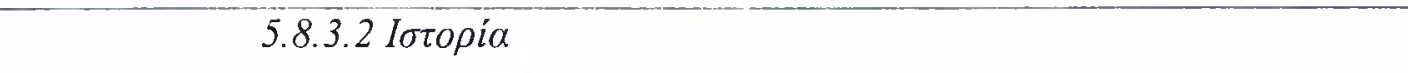 & 183 \\
\hline $5.8 .3 .3 \Phi v \sigma i k \eta$ & 185 \\
\hline 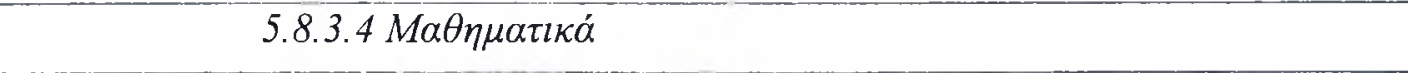 & 186 \\
\hline 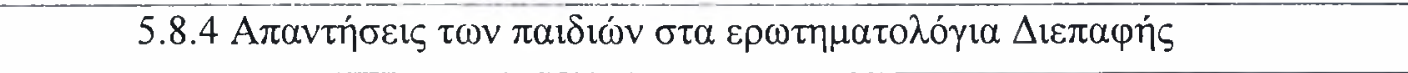 & 188 \\
\hline 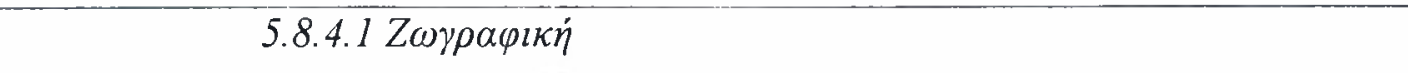 & 189 \\
\hline 5.8.4.2 Iбtopía & 193 \\
\hline $5.8 . \overline{4.3} \Phi v \sigma l \kappa \dot{n}$ & 198 \\
\hline 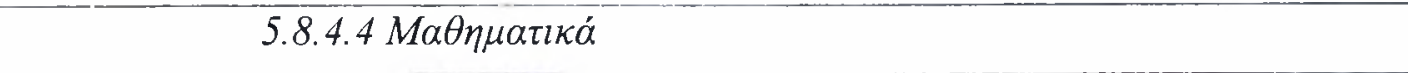 & 202 \\
\hline 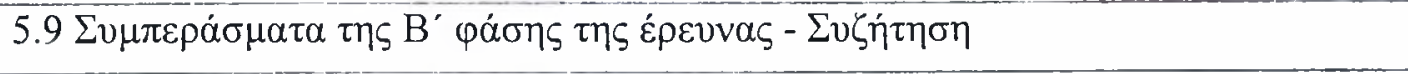 & 209 \\
\hline 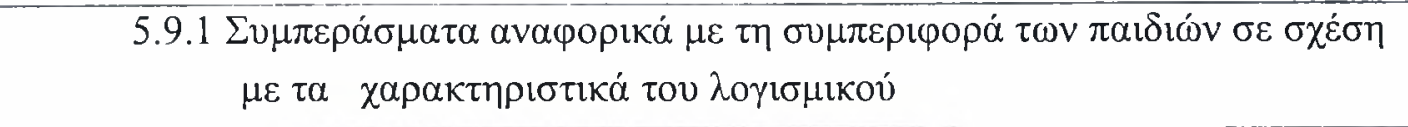 & 209 \\
\hline 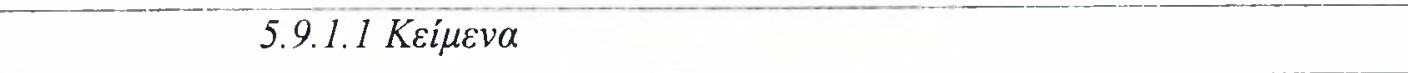 & 209 \\
\hline 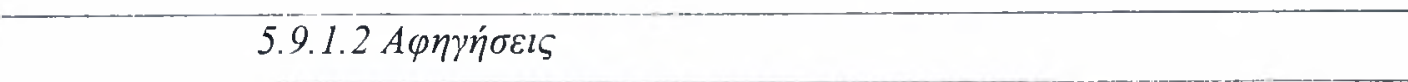 & 212 \\
\hline 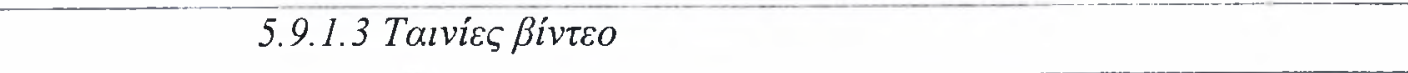 & 214 \\
\hline 5.9.1.4 Eıkóveৎ & 215 \\
\hline 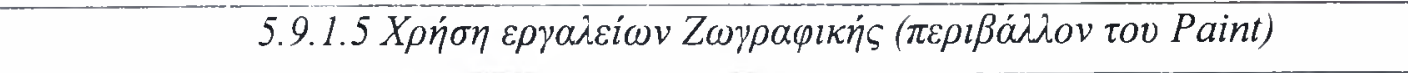 & 215 \\
\hline 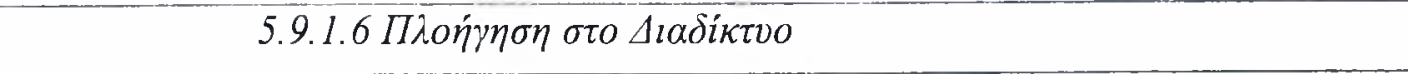 & 216 \\
\hline 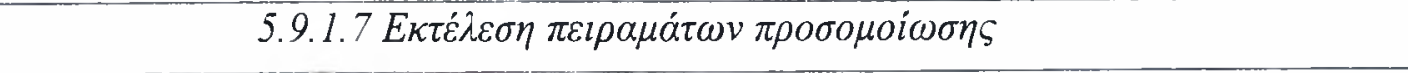 & 216 \\
\hline 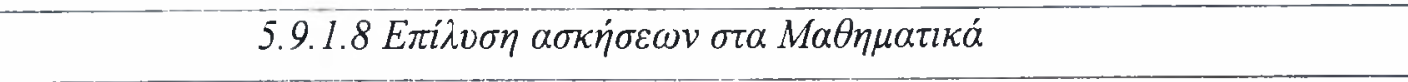 & 216 \\
\hline 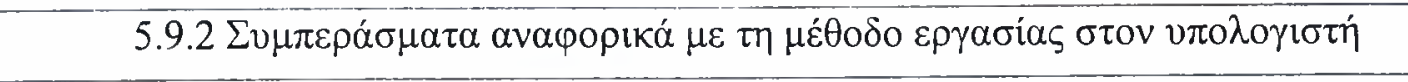 & 218 \\
\hline 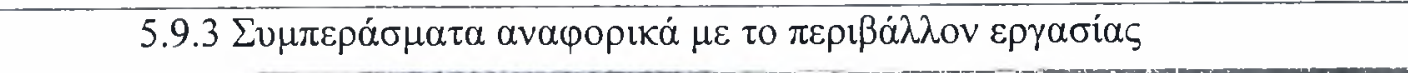 & 219 \\
\hline
\end{tabular}




\begin{tabular}{|c|c|}
\hline 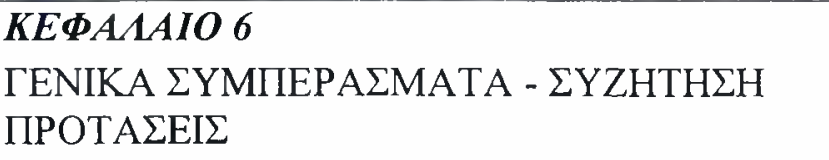 & 220 \\
\hline 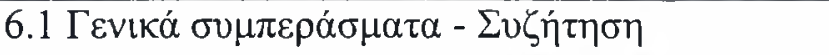 & 220 \\
\hline 6.1.1 Y Үó $\theta \varepsilon \sigma \eta \pi \rho \omega ́ \tau \eta$ & 222 \\
\hline 6.1.2 Yสó $\theta \varepsilon \sigma \eta ~ \delta \varepsilon v ́ \tau \varepsilon \rho \eta$ & 225 \\
\hline 6.1.3 Y & 226 \\
\hline 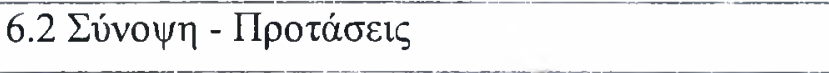 & 227 \\
\hline ВIBAIOГРAФIA & 230 \\
\hline ПАРАРТНMA $1-A^{\prime} \Phi A \Sigma H$ TH $\Sigma$ EPEYNA $\Sigma$ & 243 \\
\hline ПАPAPTHMA $2-B^{\prime} \Phi A \Sigma H T H \Sigma E P E Y N A \Sigma$ & 269 \\
\hline
\end{tabular}




\section{ПЕРІАНЧН}

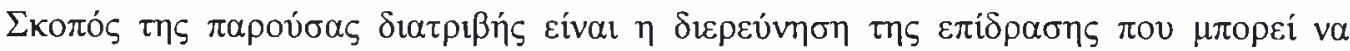

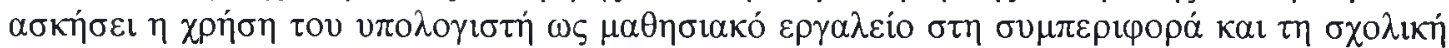

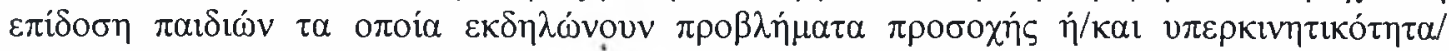

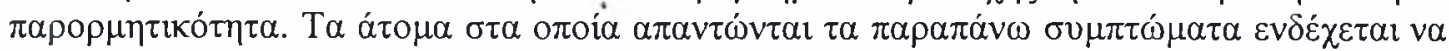

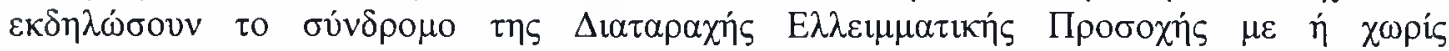

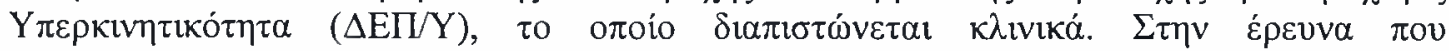

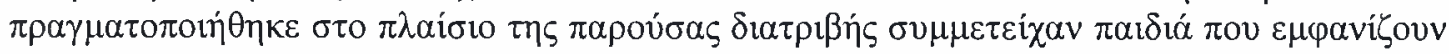

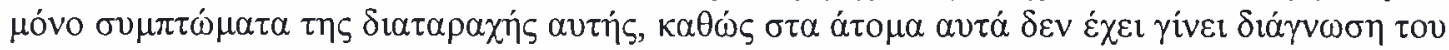
$\sigma v v \delta \rho{ }^{\prime} \mu \mathrm{ov} \Delta \mathrm{E \Pi /Y}$.

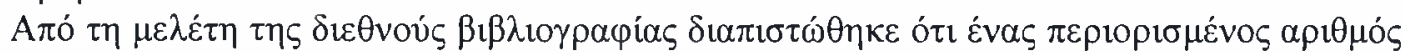

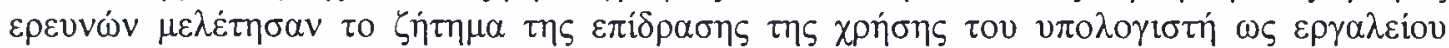

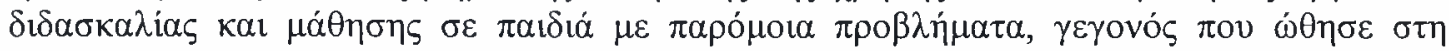

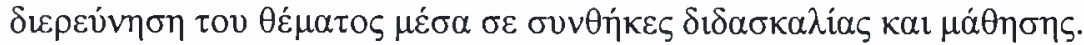

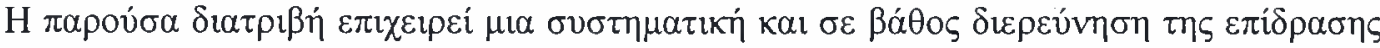

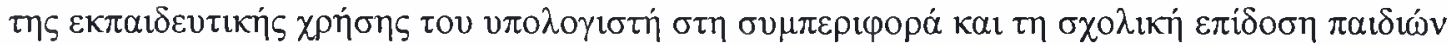

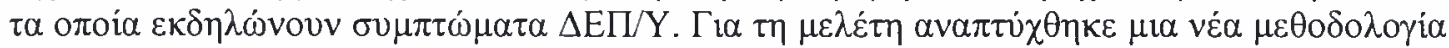

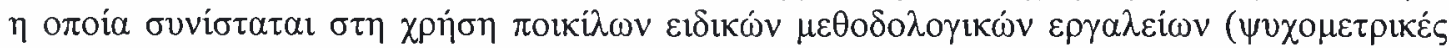

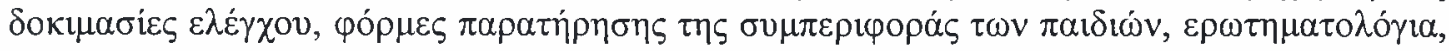
К.á.).

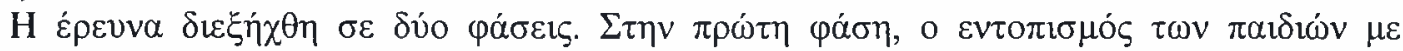

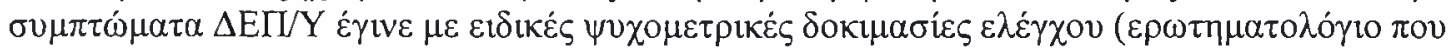

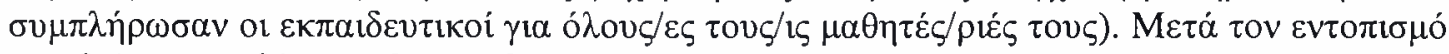

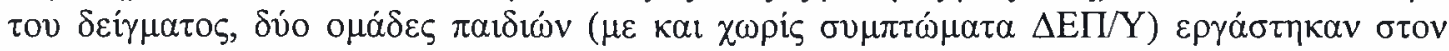

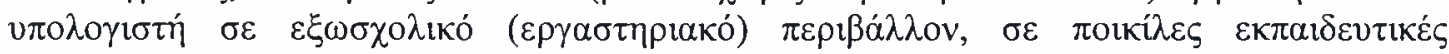

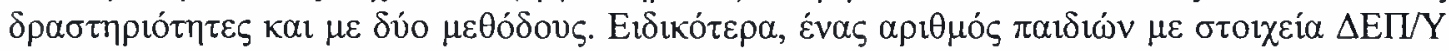

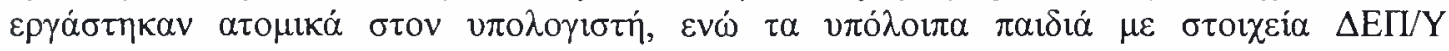

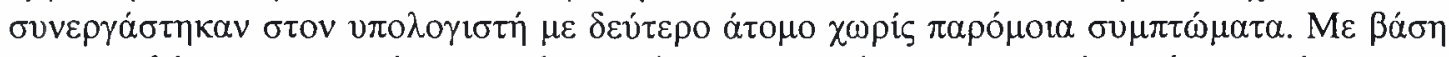

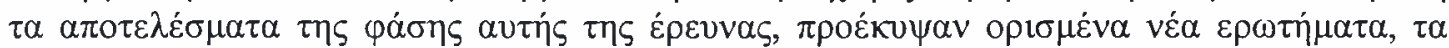

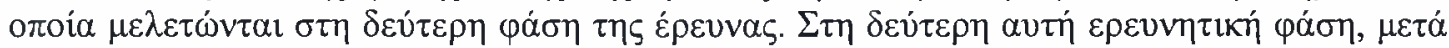

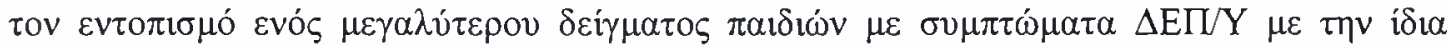

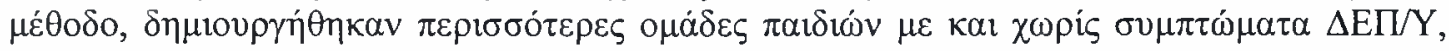

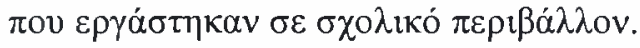

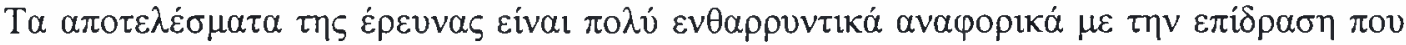

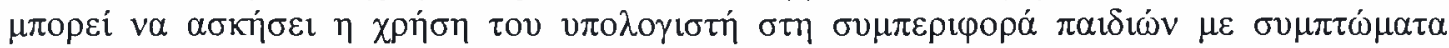

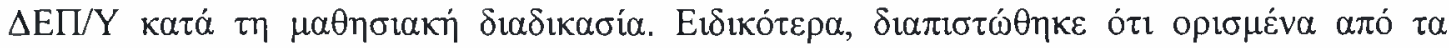

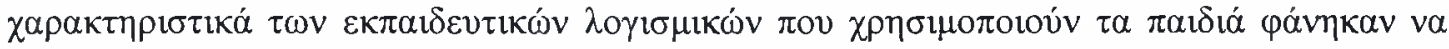

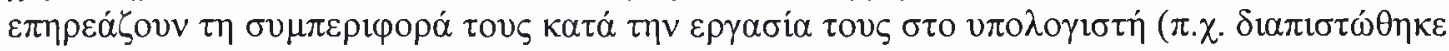

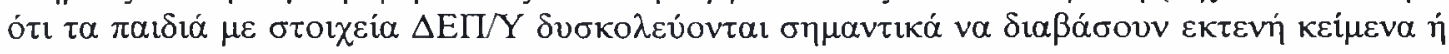

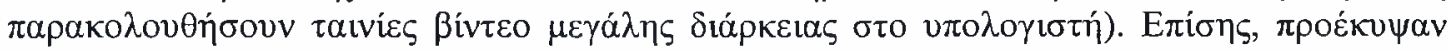

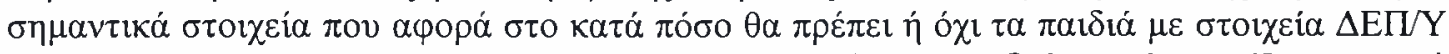

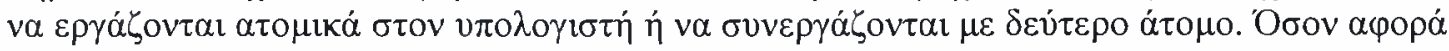

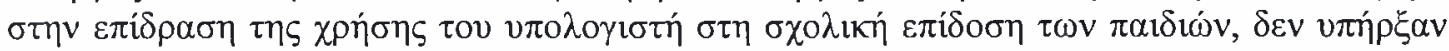

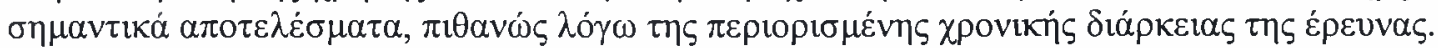

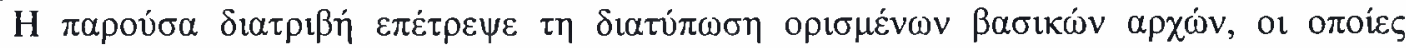

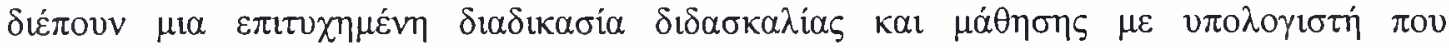

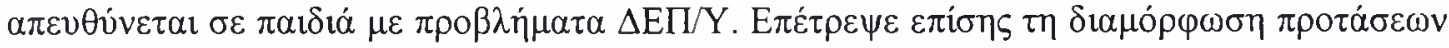

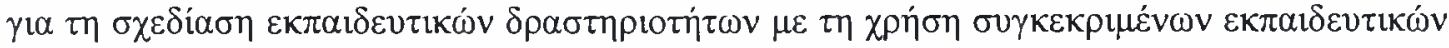

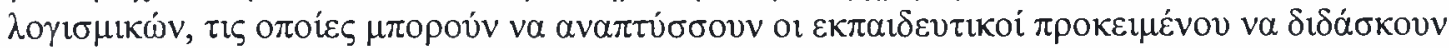

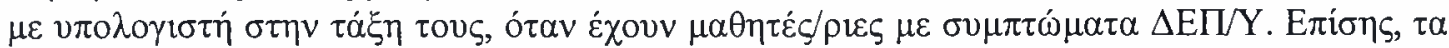

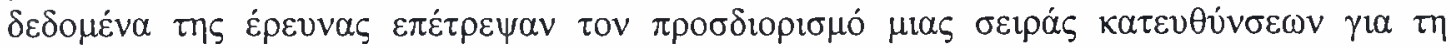

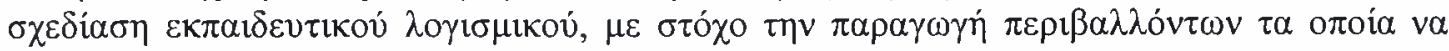

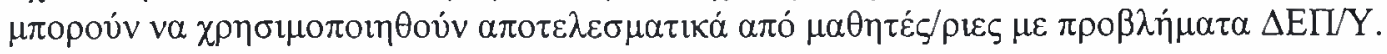




\section{SUMMARY}

The aim of the present dissertation is to investigate the effects computer use on the behavior and academic achievement of students with attention and hyperactivity/impulsivity problems. Those students are likely to exhibit the Attention Deficit Hyperactivity Disorder (ADHD) syndrome, which is a syndrome that is clinically diagnosed. Yet, in the present study only students that manifest ADHD symptoms participated, since they did not previously receive clinical diagnosis.

The review of the international literature has showed that only a limited number of research studies had investigated the effect of using the computer as a teaching and learning tool in students with ADHD problems. Thus, a need to study the above subject in real learning conditions arose.

The present study aims to achieve a systematic and profound assessment of the effect of the educational use of the computer on the behavior and academic achievement of students with attention and hyperactivity/impulsivity symptoms. To accomplish this aim, we designed a methodology based on the use of various research tools (psychometric assessment, observation sheets for measuring students' behavior, various questionnaires, etc.).

The study was conducted in two stages. At the first stage of the study, after the location of the subject with the use of a psychometric test (a questionnaire filled in by teachers regarding their students' behavior), two groups of students (with and without ADHD symptoms) worked in a laboratory (outside school) in a series of learning activities and in two different working methods (individual and collaborative). According to the results of the first stage of the study, the students' behavior while working on the computer could be affected by the characteristics of the software environment and by the working method they use. Based on the results of the first stage of the study, new questions came up, which have been investigated in the second stage of the study. In this second stage, after the location of a wider sample of students with ADHD symptoms by using the same method, a greater number of working groups of students with and without ADHD problems were formed. Those student groups worked in fewer learning activities in school settings, so as to assess the effect of computer use within the school environment.

The results of the study are very encouraging regarding the effect of computer use on the behavior of students with ADHD problems during the learning process. More precisely, it was found that specific software characteristics could affect the behavior of students with ADHD symptoms (e.g. it was found that students with $\mathrm{ADHD}$ symptoms show great difficulty in reading long texts or watching long videos on the computer). Furthermore, it was found that students with ADHD symptoms performed better while they working alone on the computer, than when they collaborated with another student without ADHD symptoms. On the other hand, the computer use did not seem to have affected the students' academic achievement, probably because of the short duration of the teaching and learning process with the use of the computer.

The present study allowed the formulation of some basic guidelines, which should exist in a successful learning process with the use of Information and Communication Technologies (ICT), designed for students with ADHD problems. Also the results allowed the formulation of propositions for the design of educational activities with the use of specific educational software that teachers could develop in order to use the computer in teaching students with ADHD symptoms. Furthermore, a series of guidelines are formulated for the design of educational software environments, which could be effective when used by students with ADHD problems. 


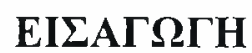

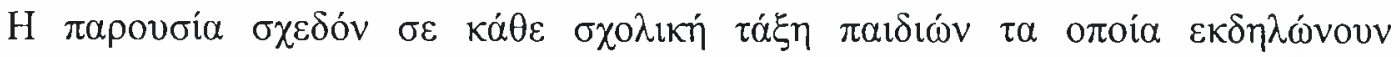

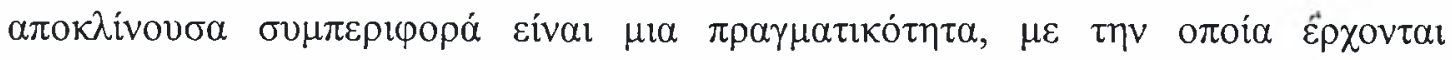

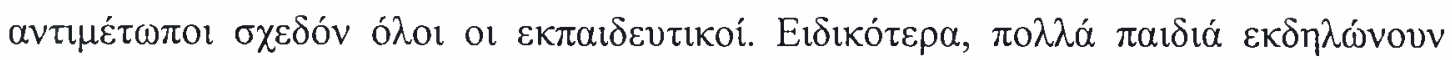

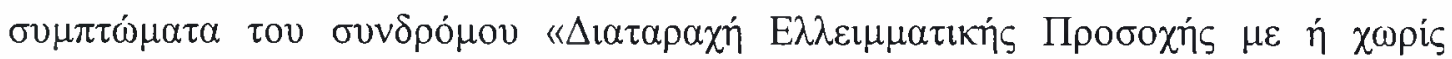

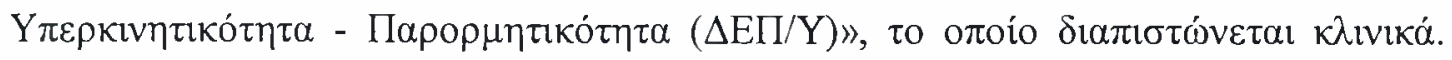

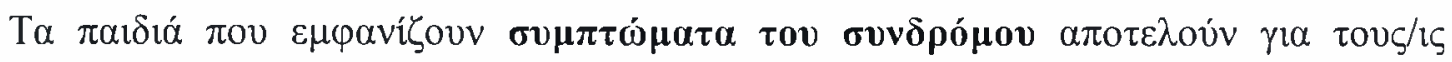

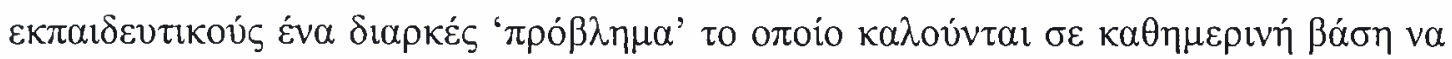

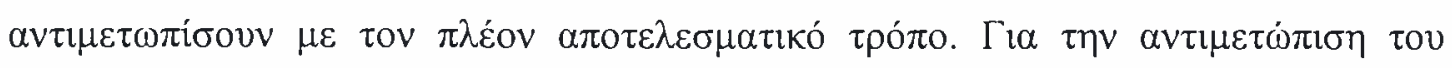

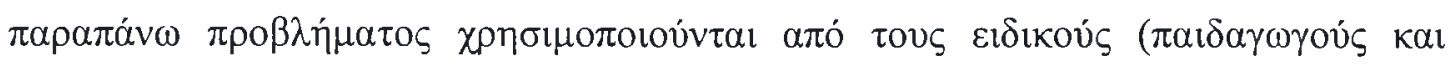

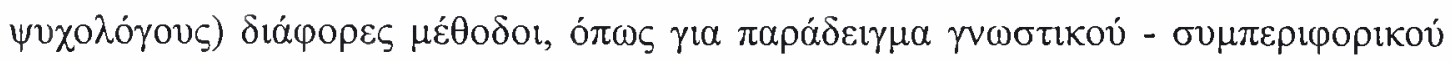

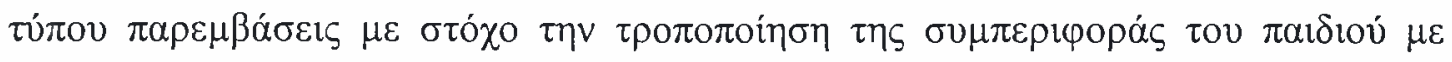

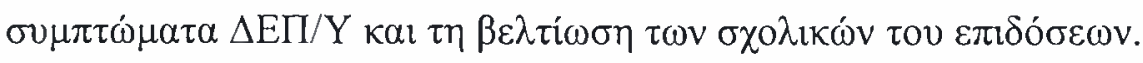

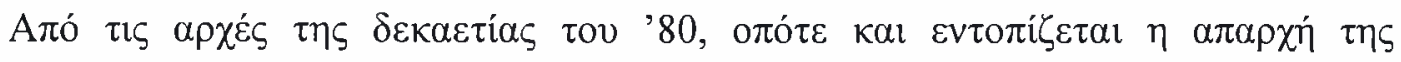

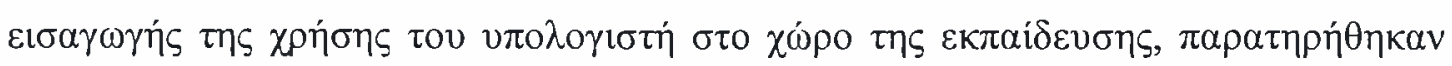

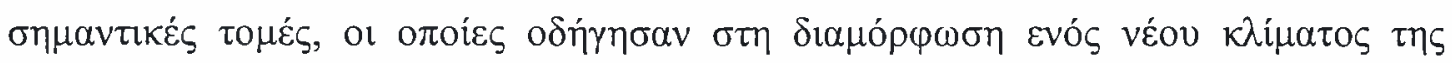

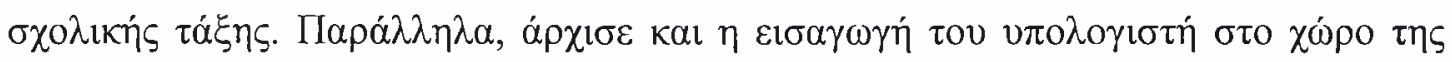

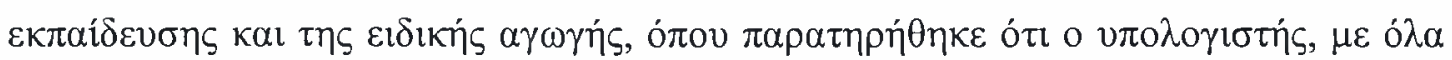

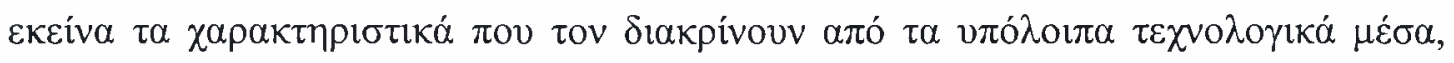

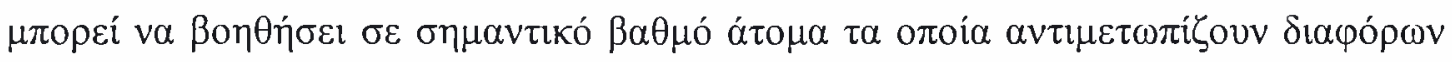

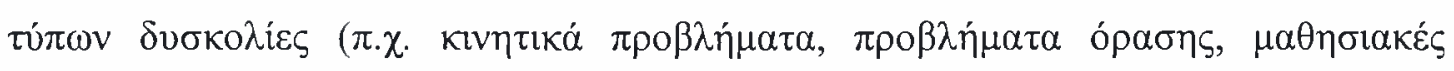

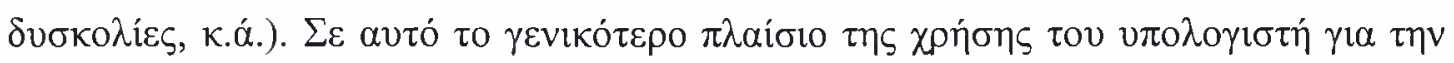

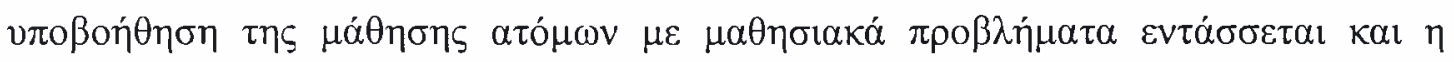

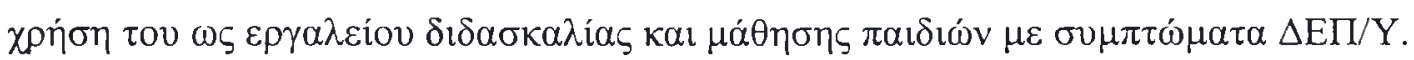

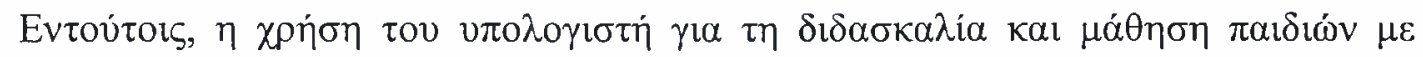

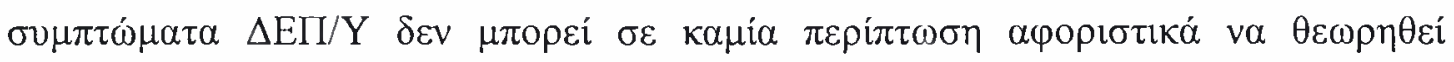

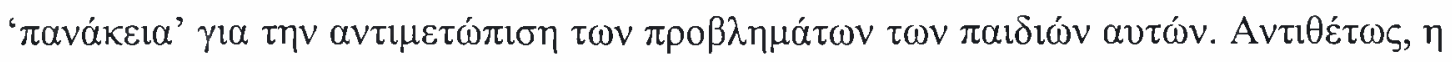

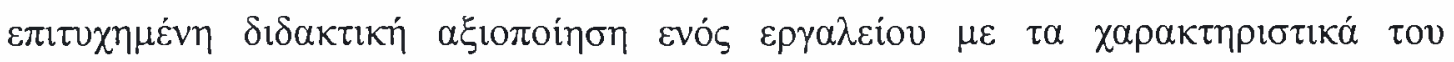

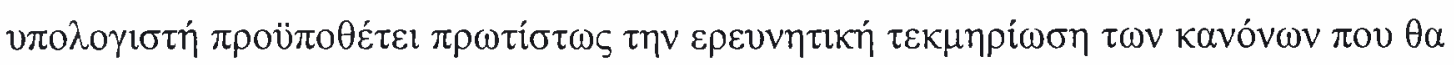

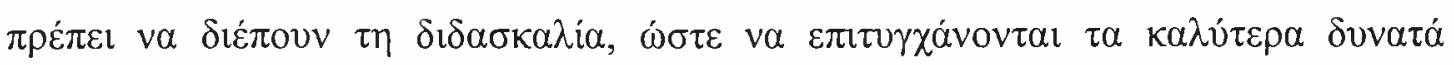

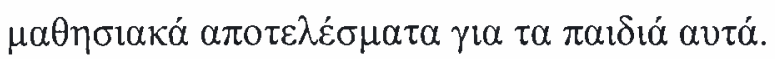




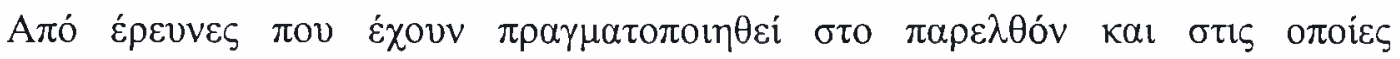

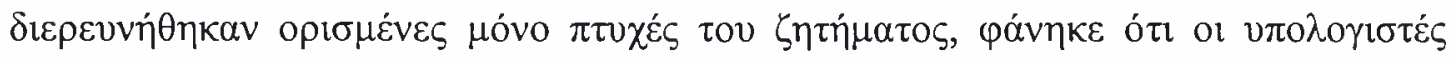

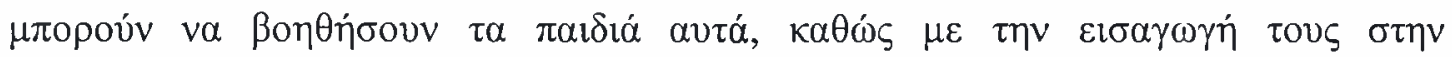

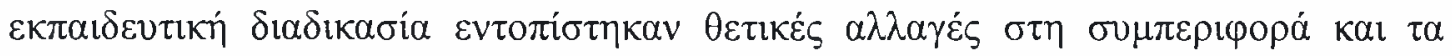

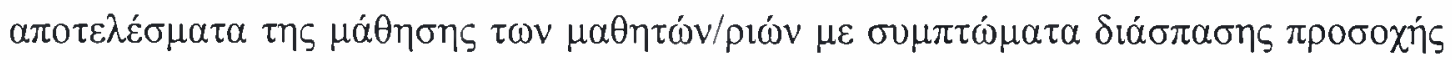

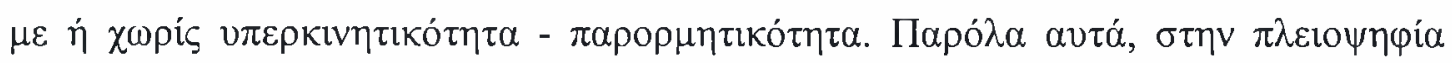

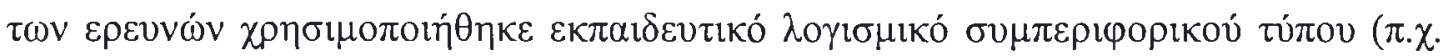

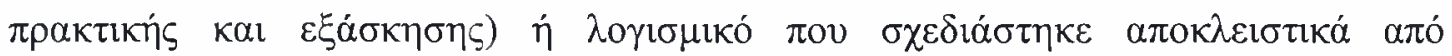

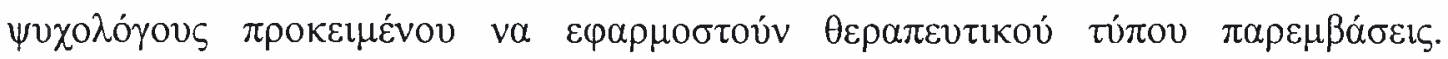

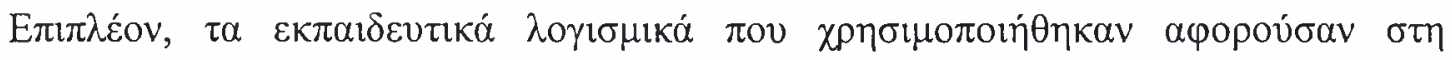

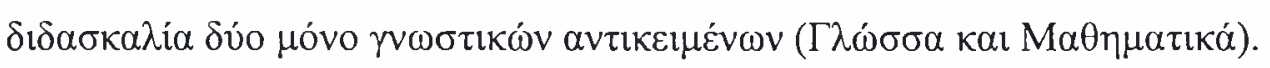

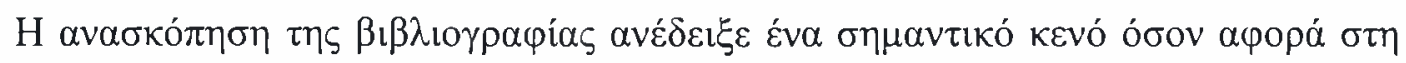

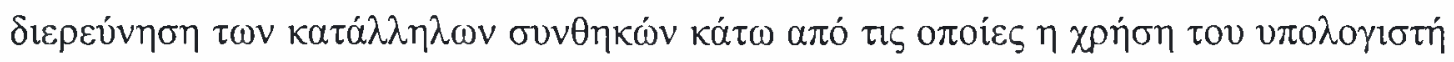

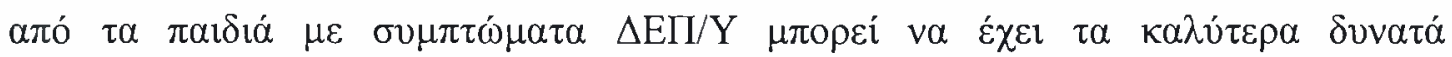

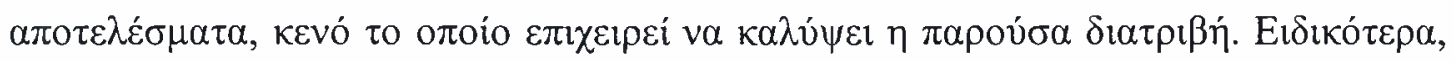

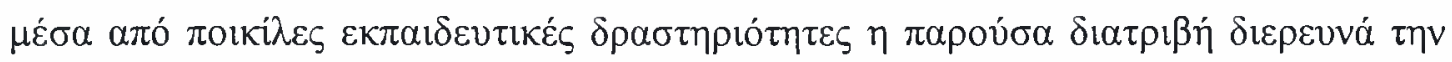

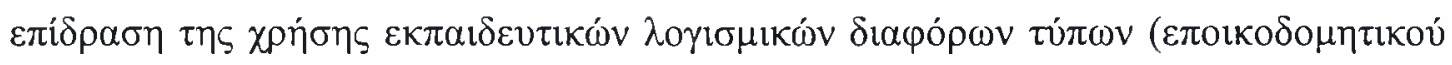

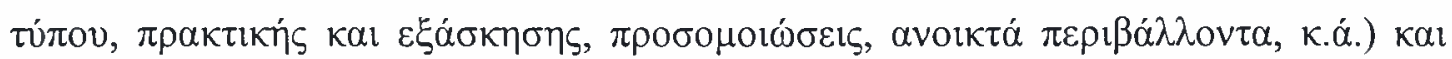

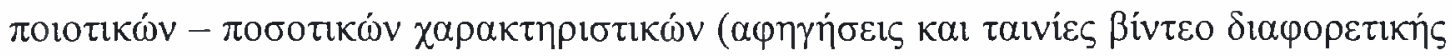

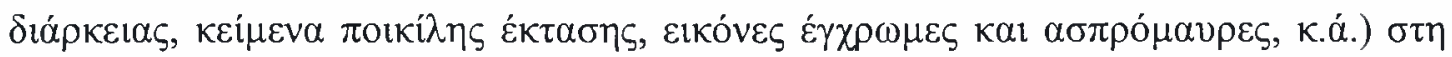

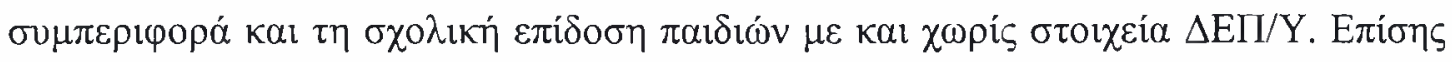

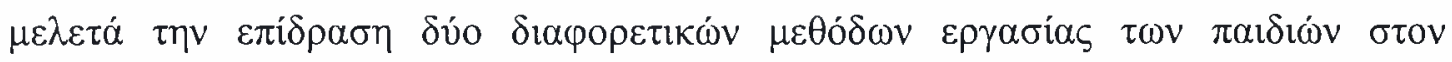

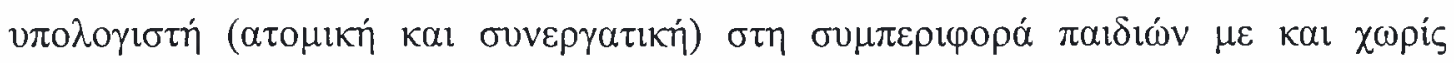

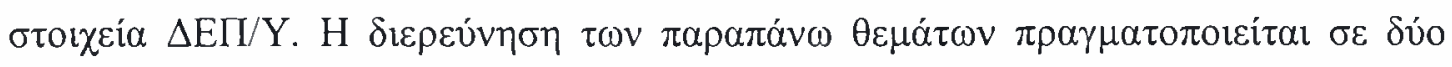

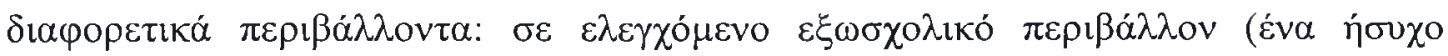

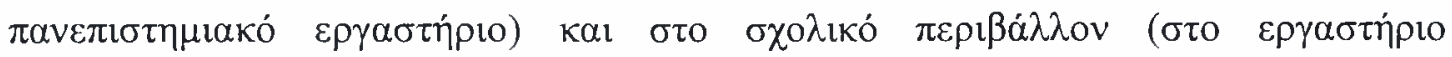

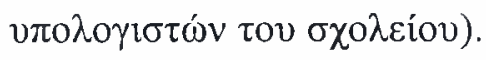

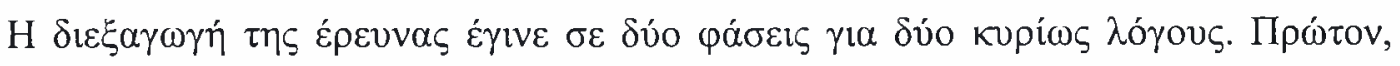

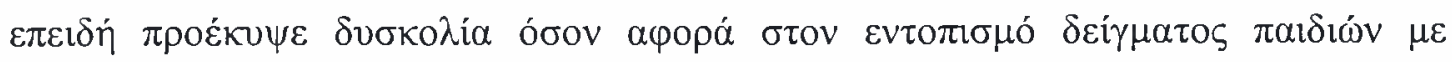

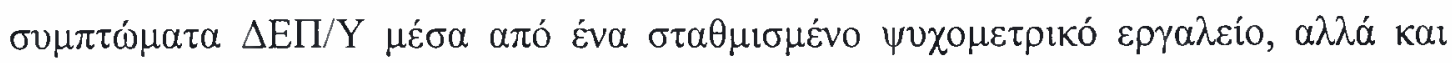

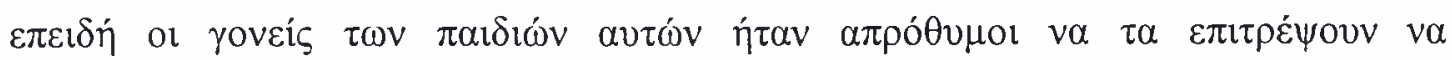

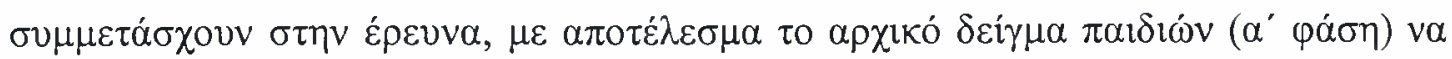

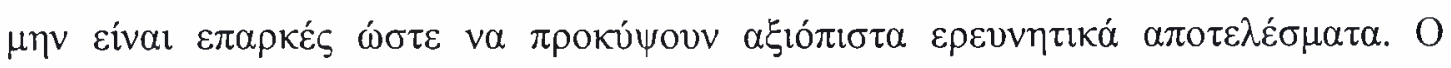

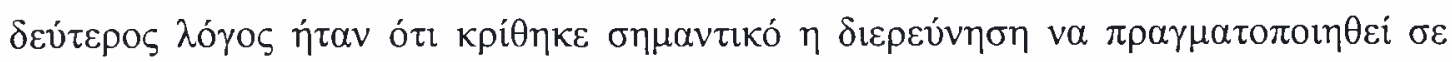




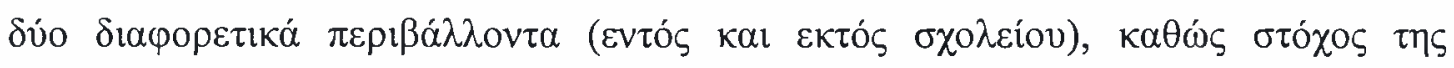

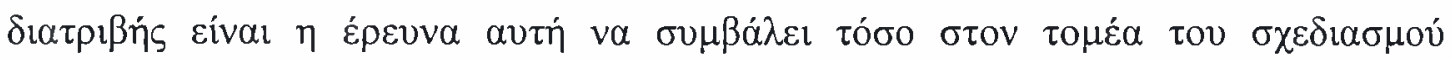

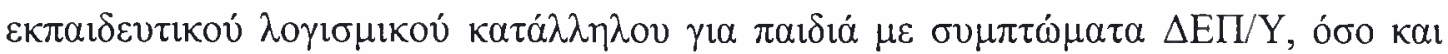

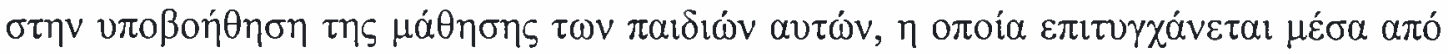

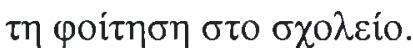

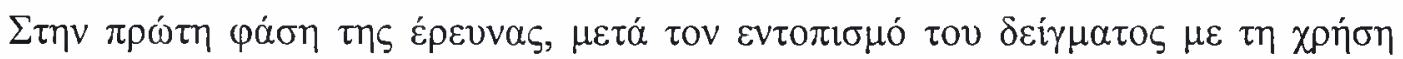

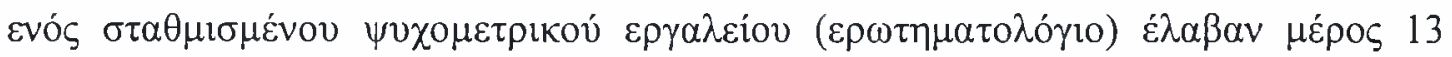

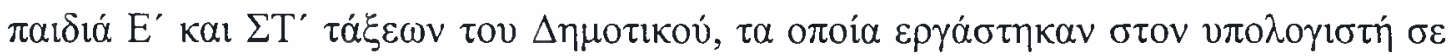

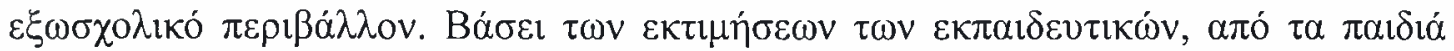

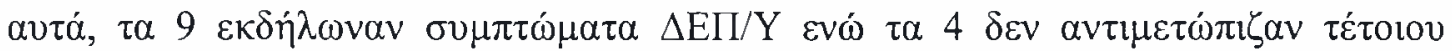

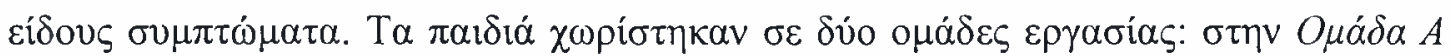

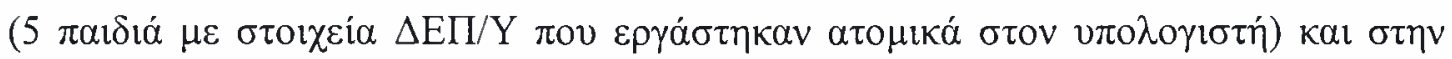

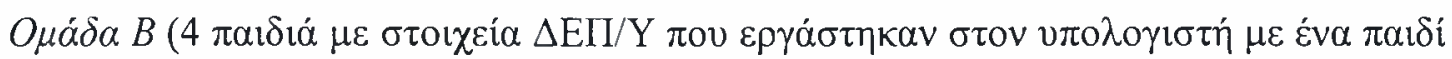

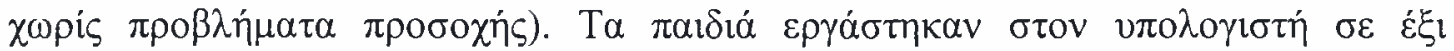

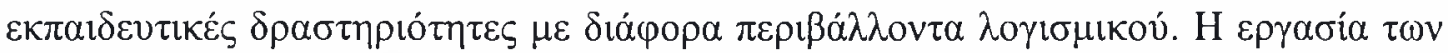

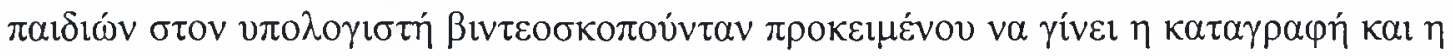

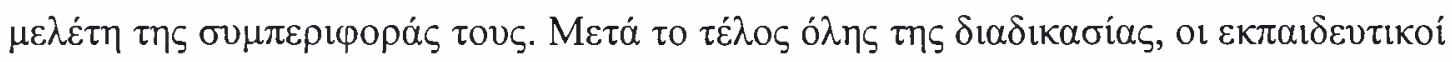

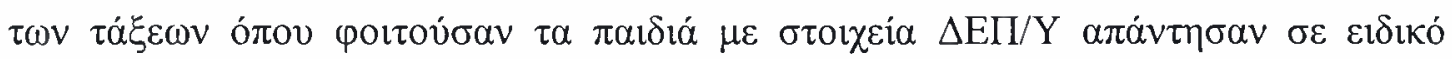

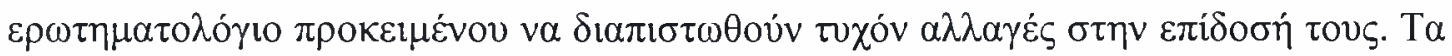

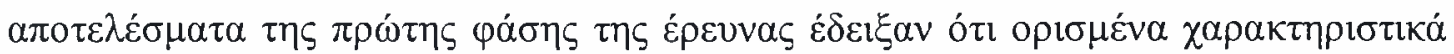

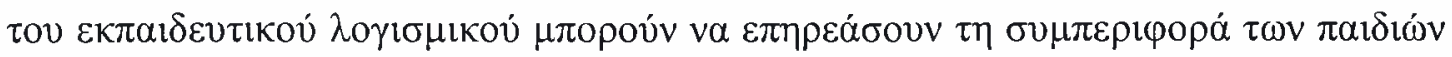

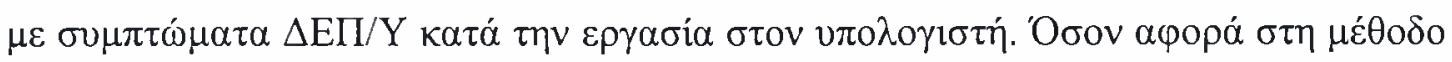

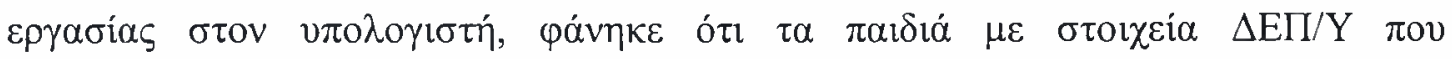

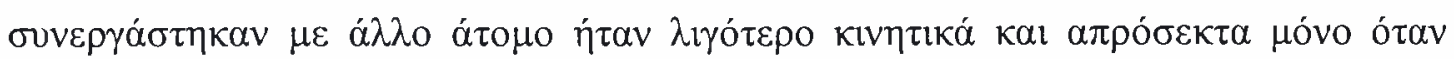

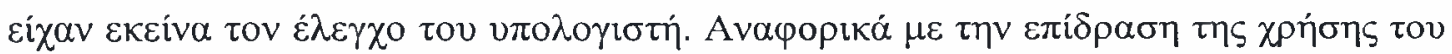

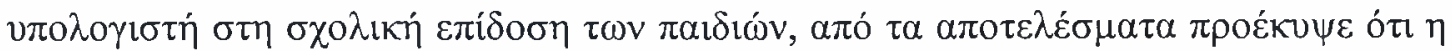

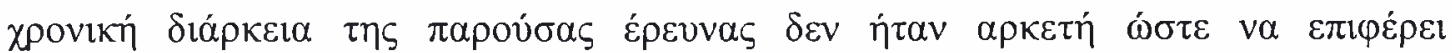

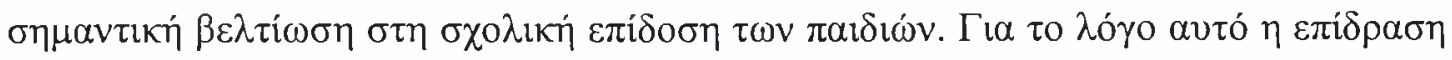

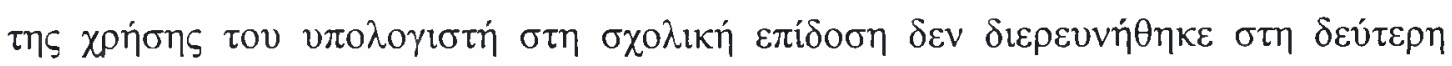

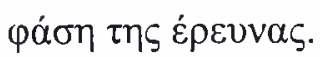

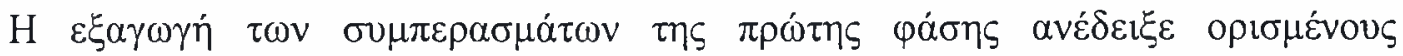

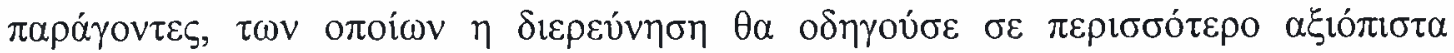

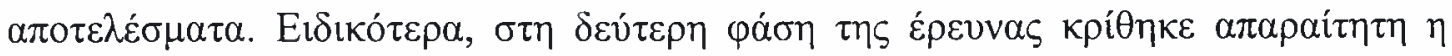

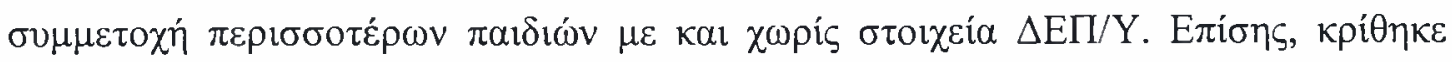




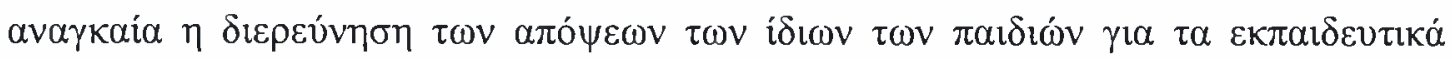

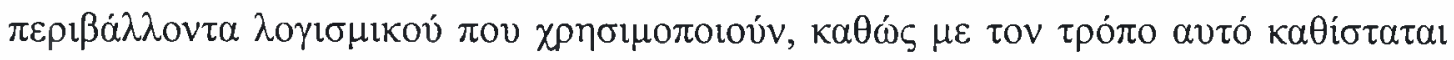

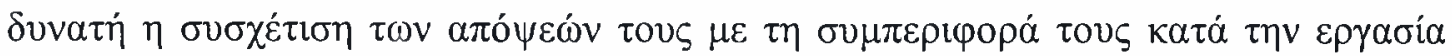

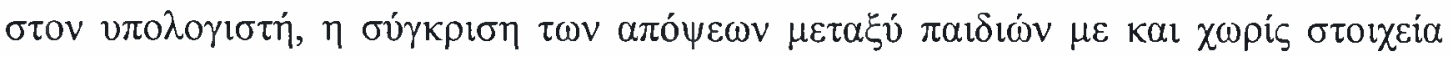

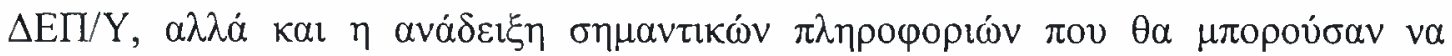

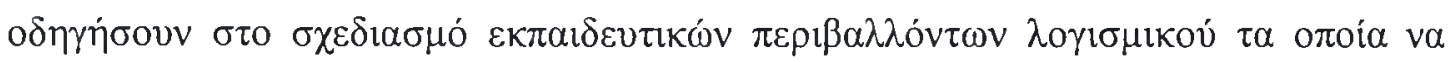

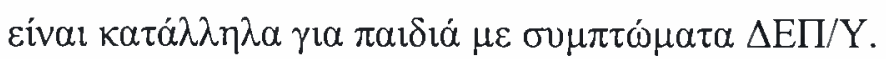

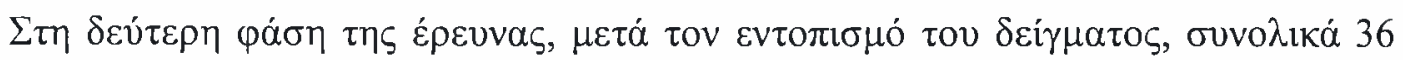

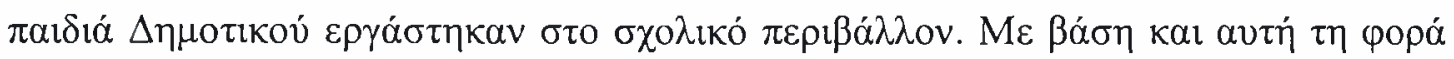

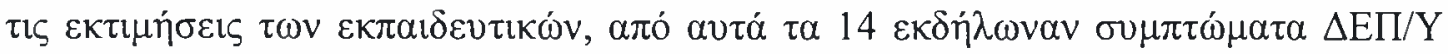

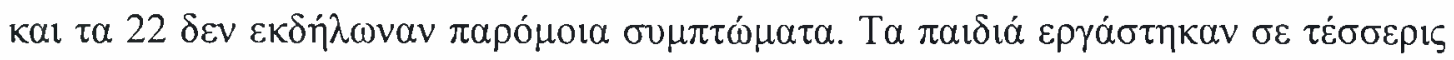

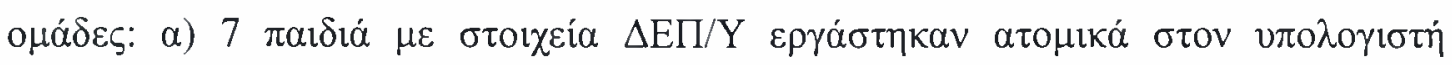

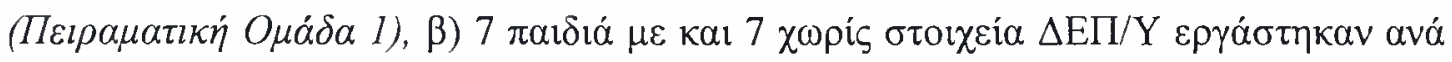

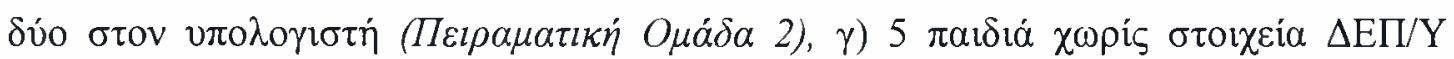

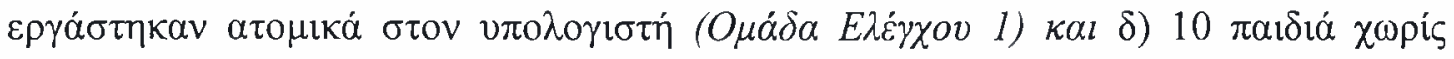

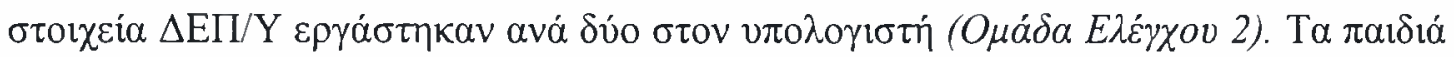

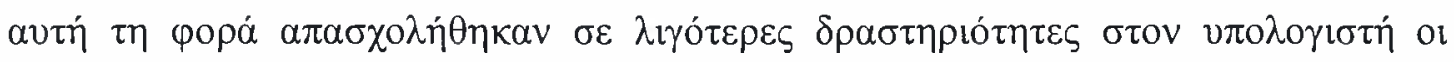

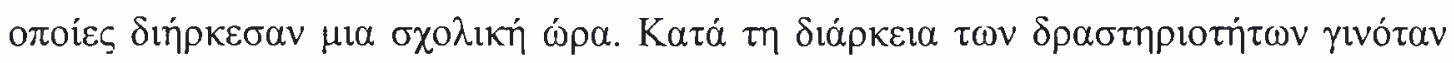

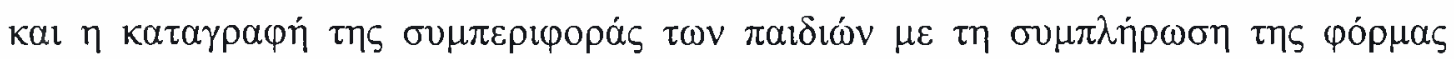

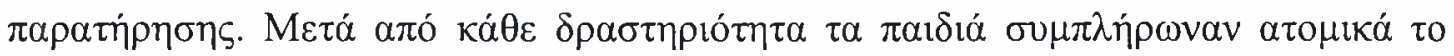

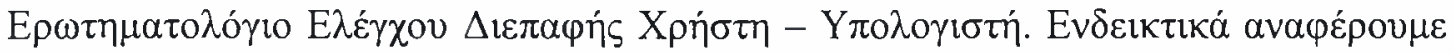

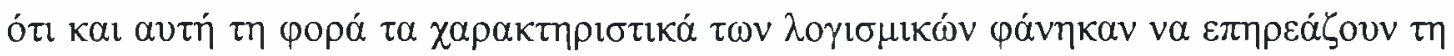

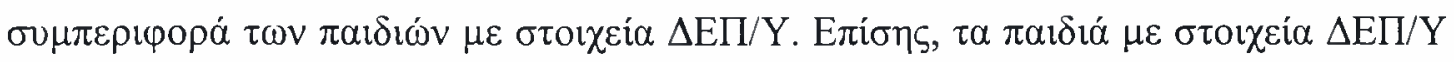

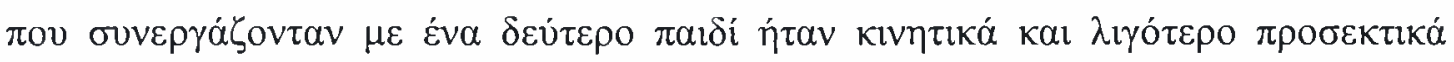

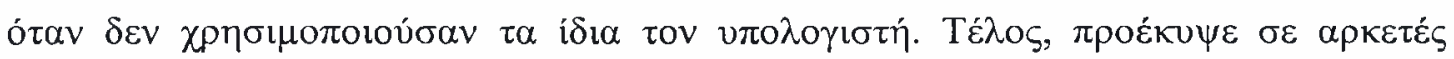

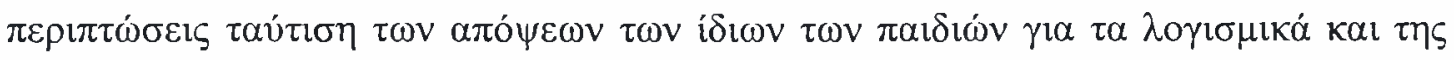

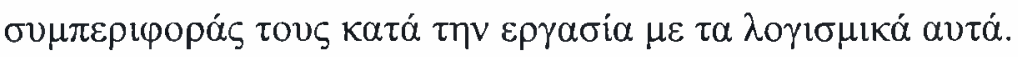

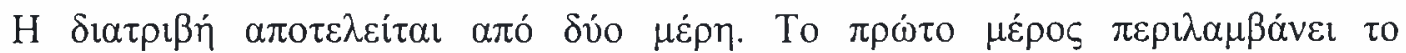

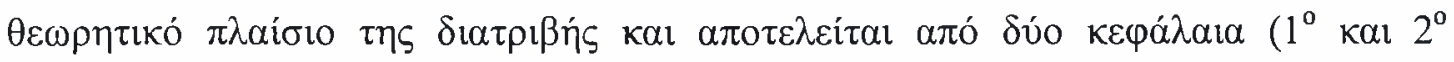

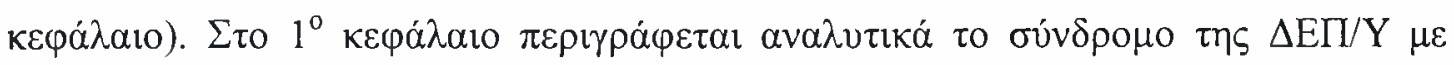

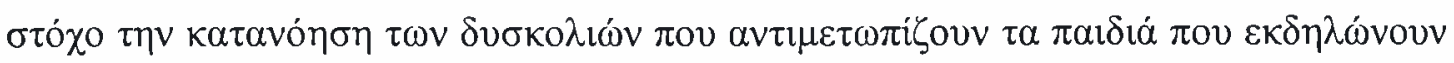

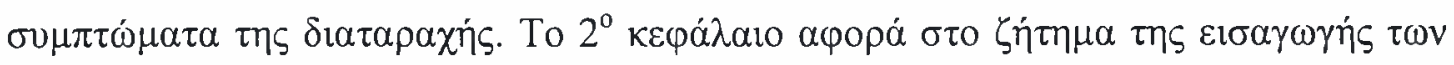

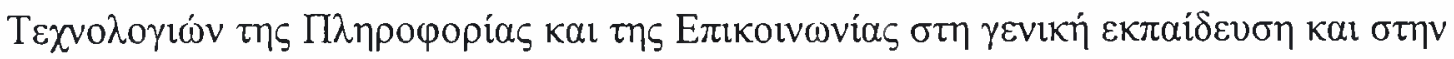

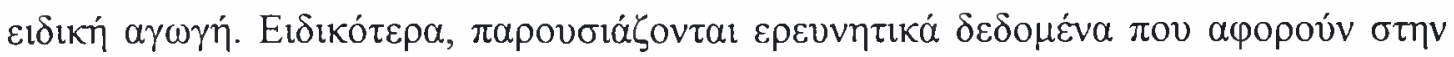




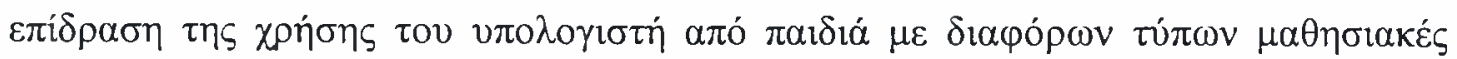

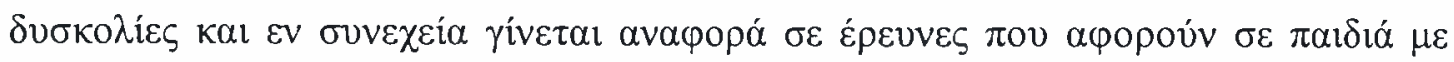

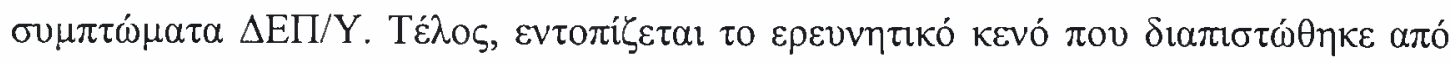

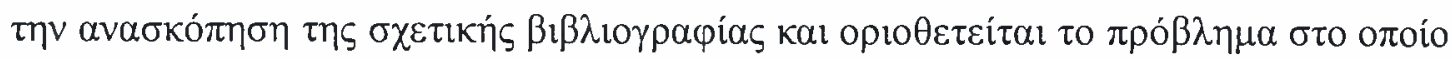

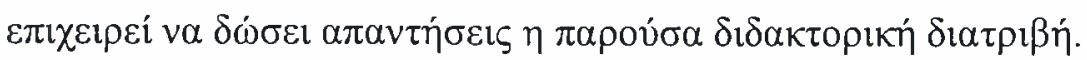

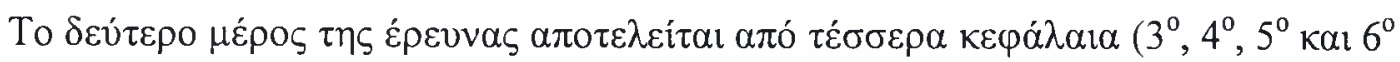

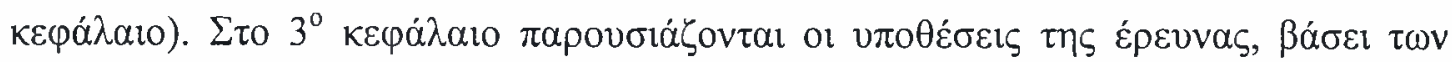

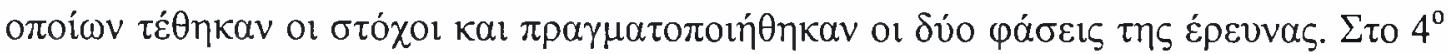

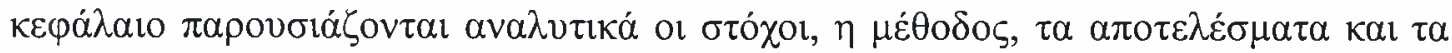

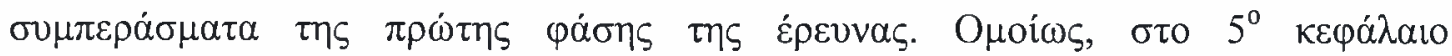

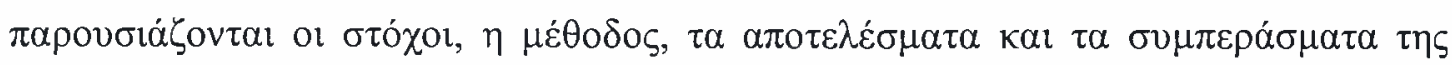

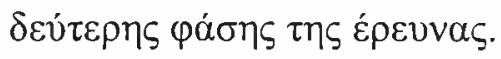

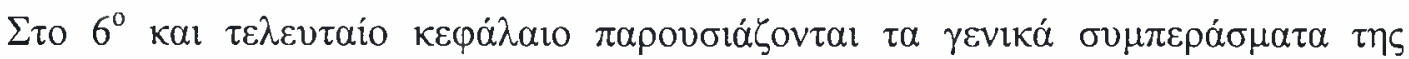

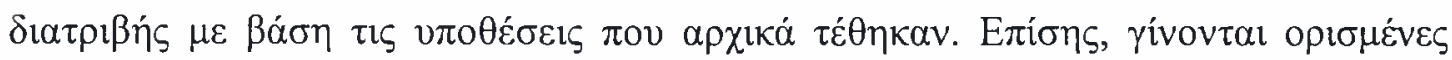

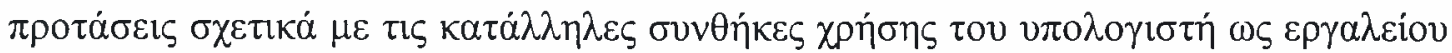

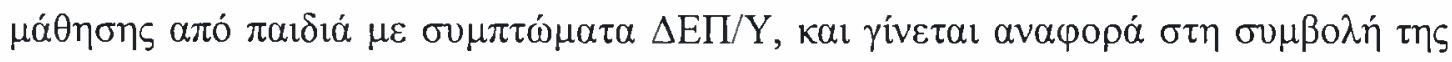

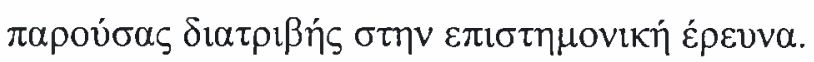

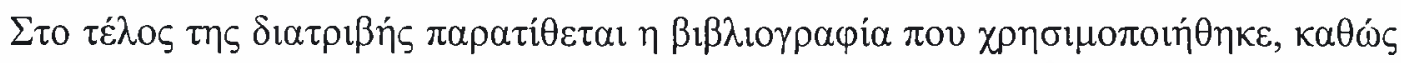

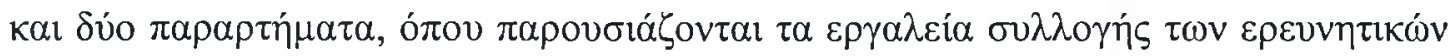
$\delta \varepsilon \delta \circ \mu \varepsilon ́ v \omega v$.

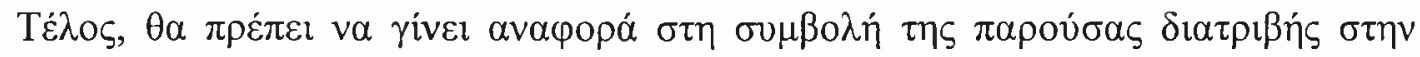

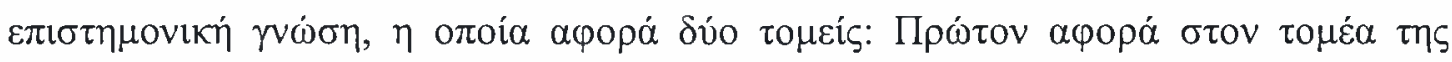

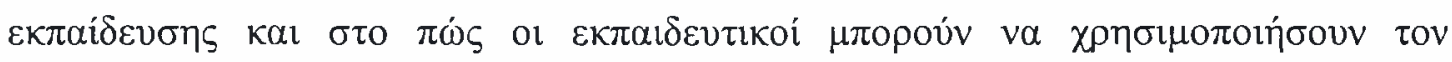

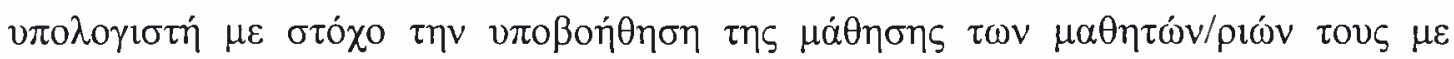

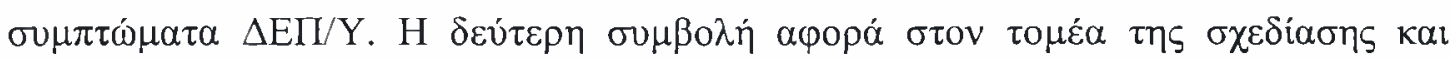

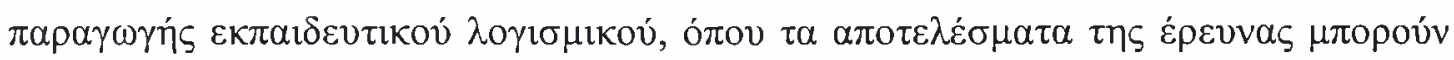

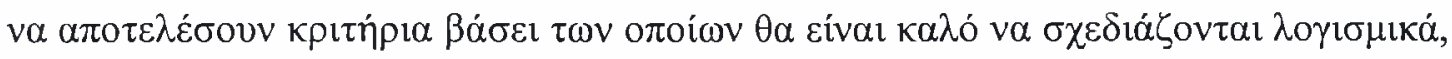

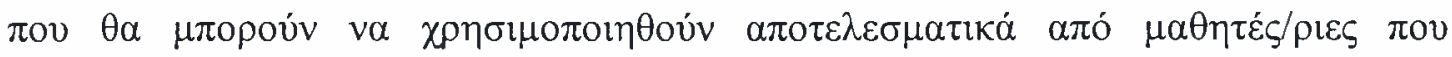

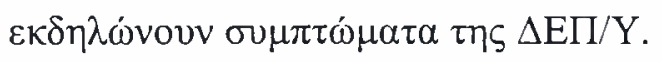




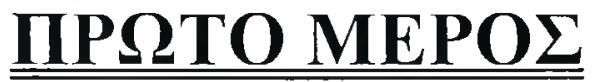

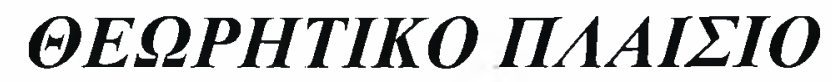




\section{$\underline{\underline{\text { IP } \Omega T O ~ K E \Phi A \Lambda A I O ~}}$}

\section{$\triangle$ IATAPAXH E $\Lambda$ \ЕIMMATIKH}

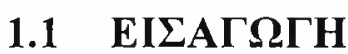

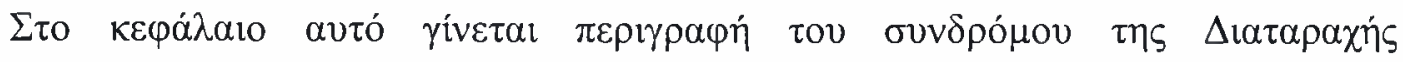

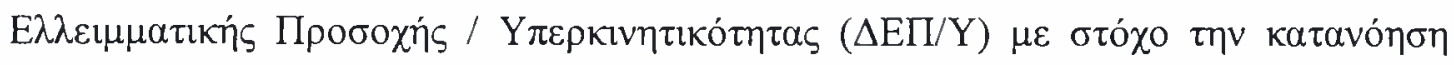

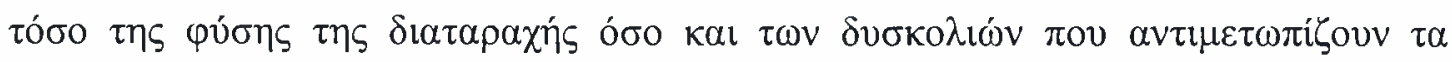

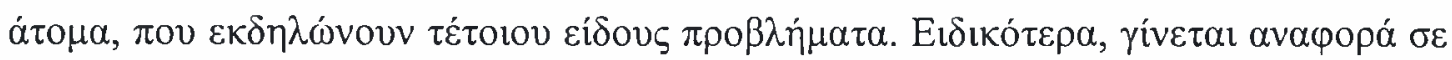

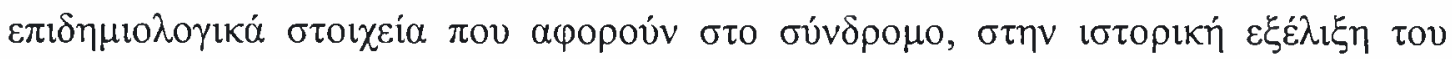

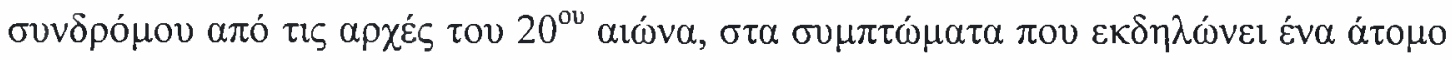

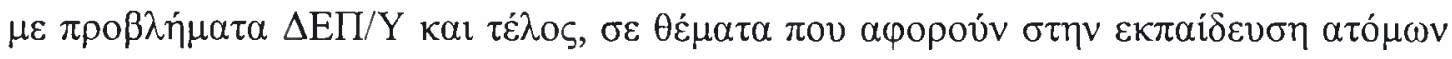
$\mu \varepsilon \sigma v \mu \pi \tau \omega \dot{\mu} \mu \alpha \alpha \Delta \mathrm{E} \Pi / \mathrm{Y}$.

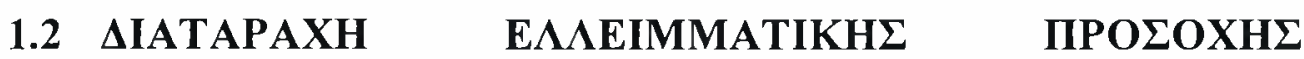 YПEPKINHTIKOTHTA $(\Delta E \Pi / Y)$}

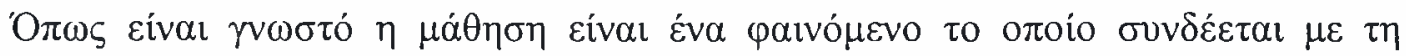

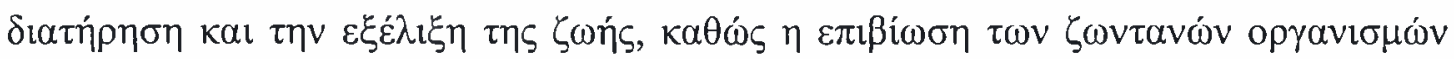

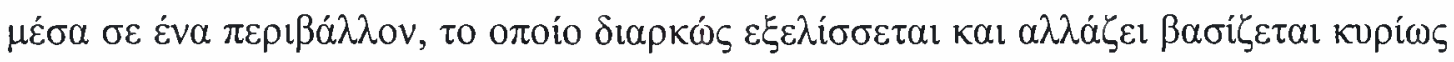

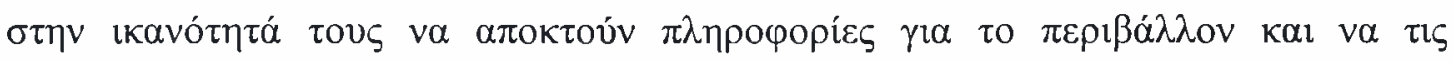

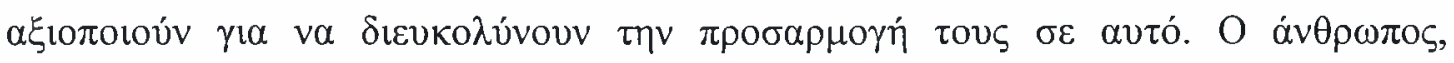

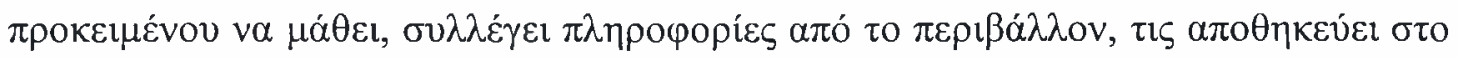

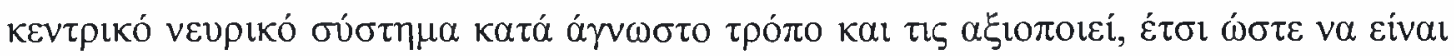

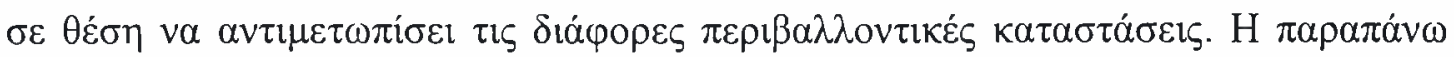

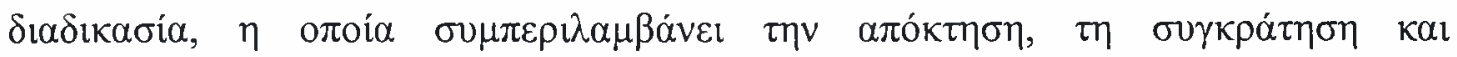

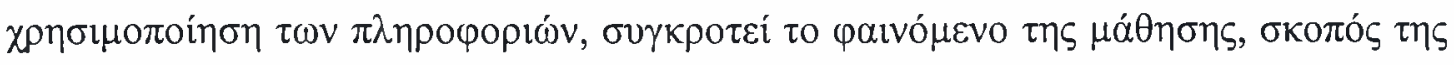

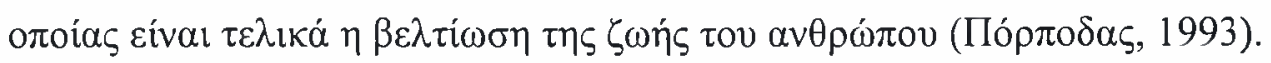




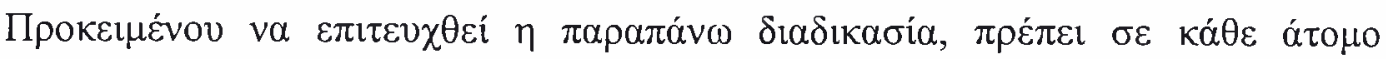

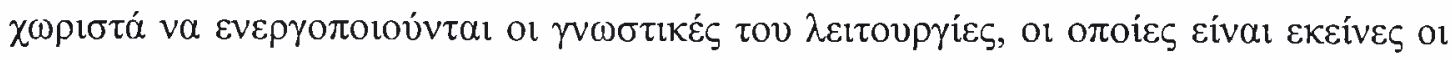

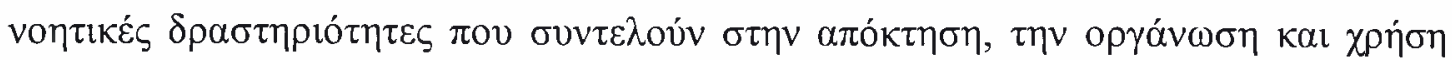

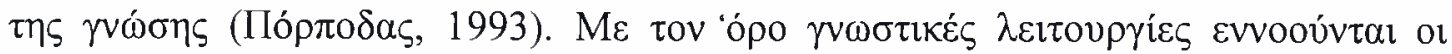

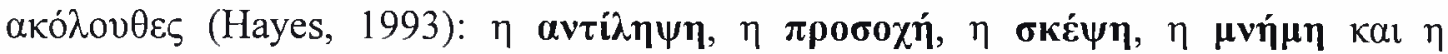

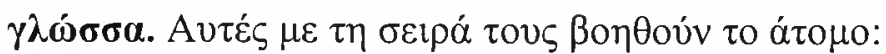

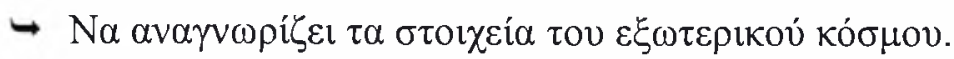

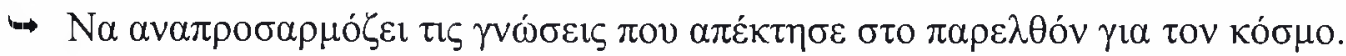

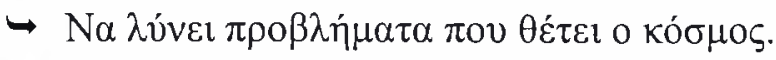

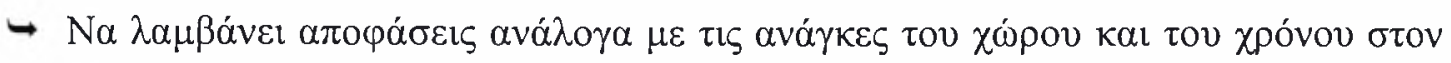

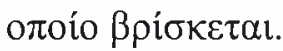

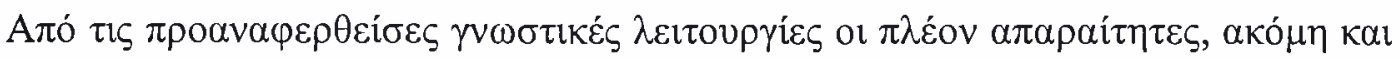

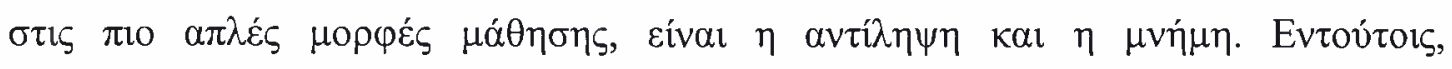

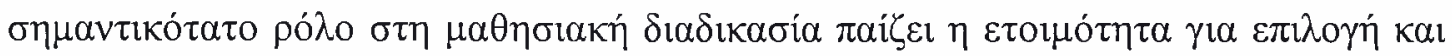

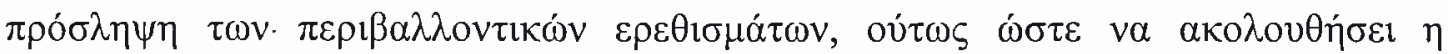

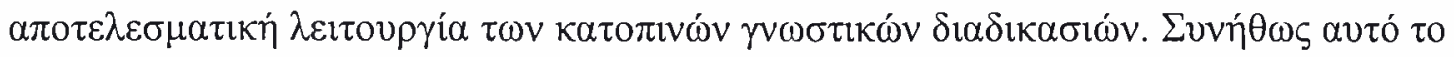

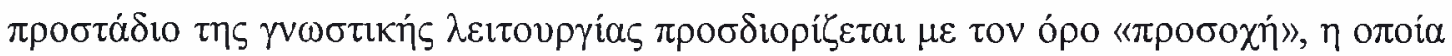

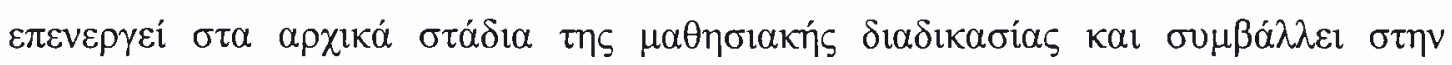

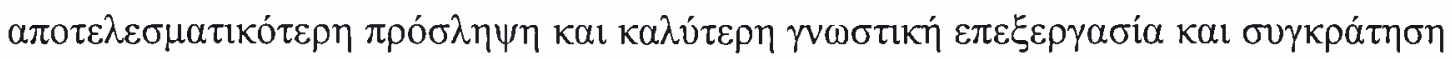

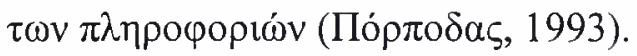

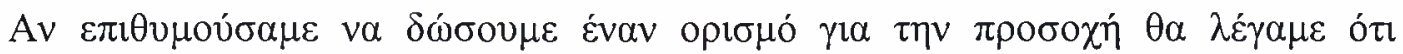

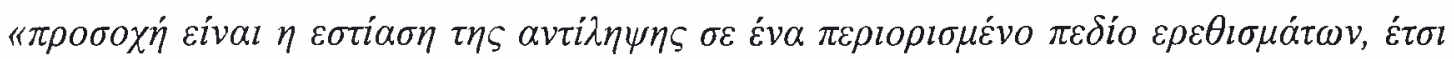

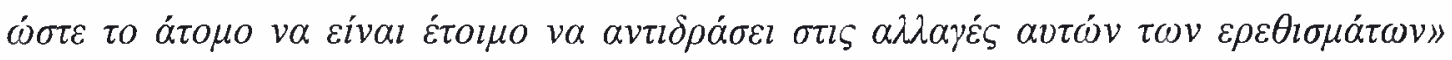

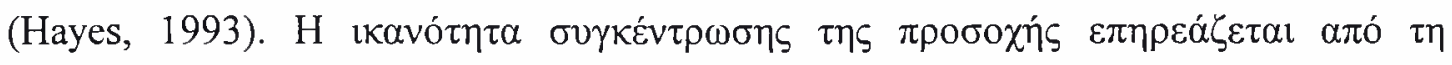

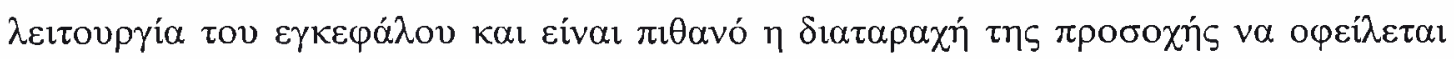

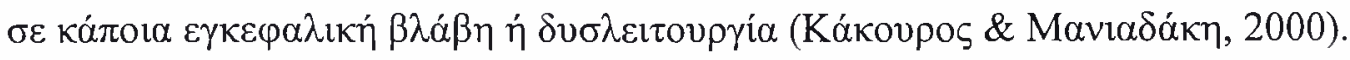

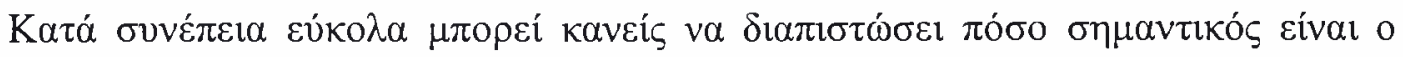

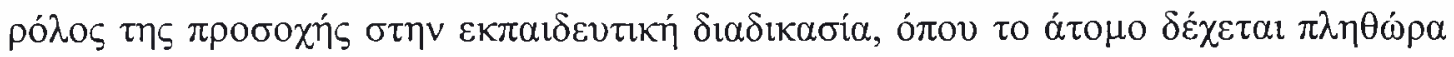

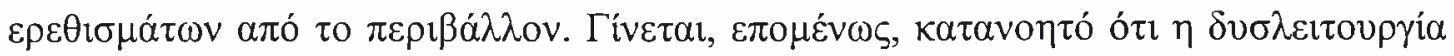

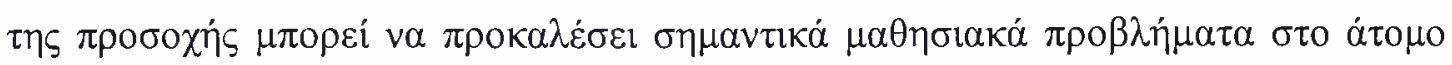

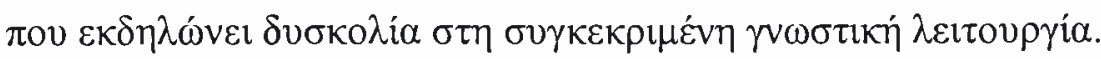

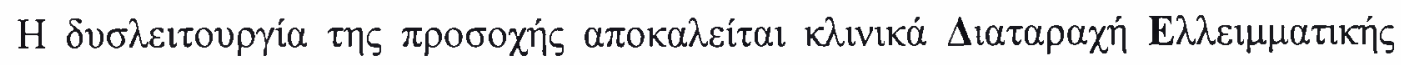

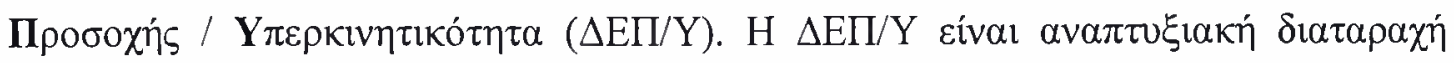

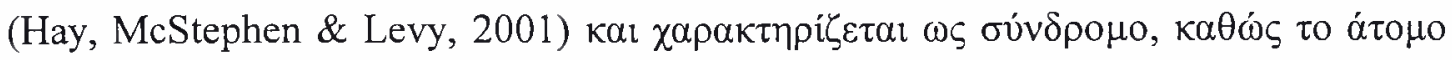




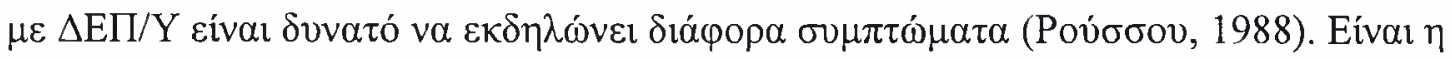

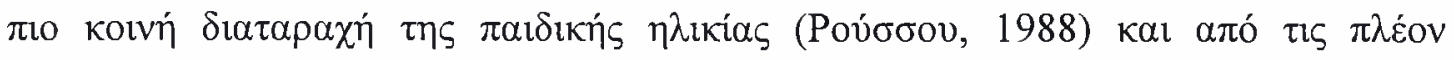

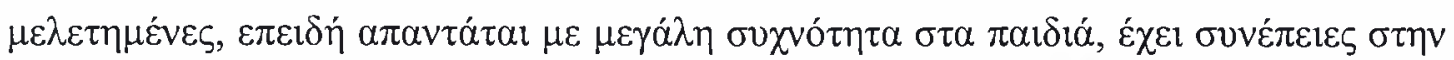

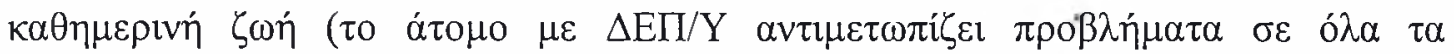

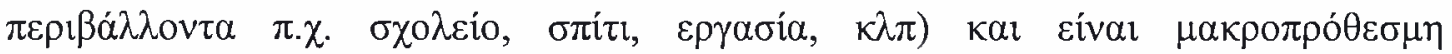

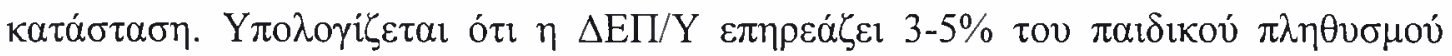
(American Psychiatric Association, 1994).

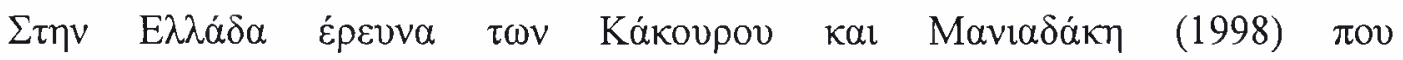

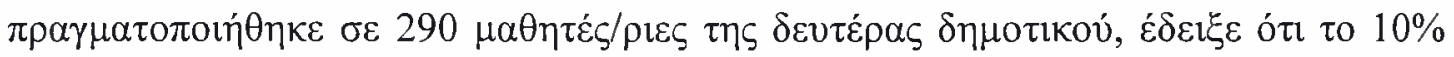

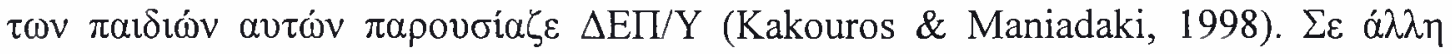

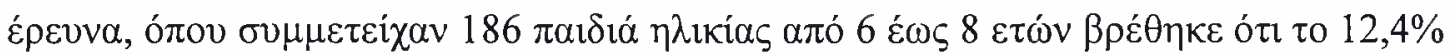

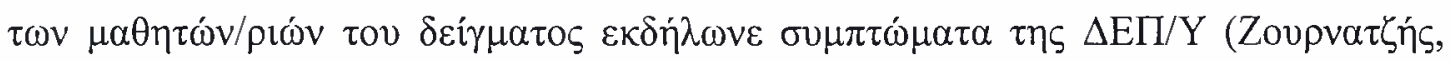

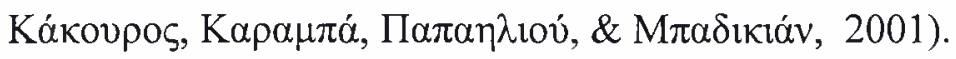

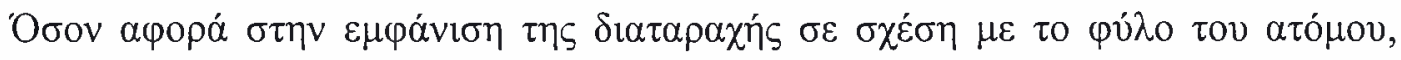

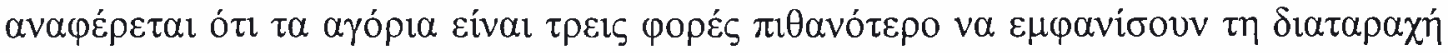

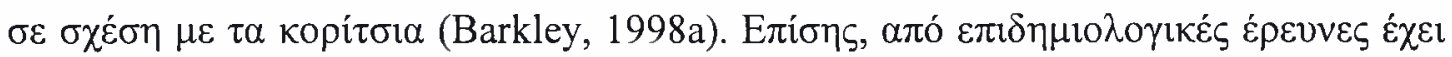

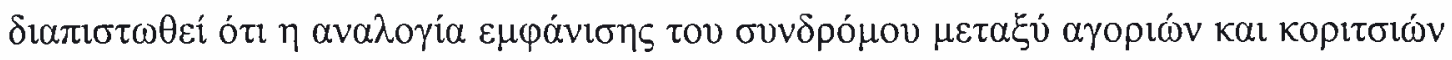

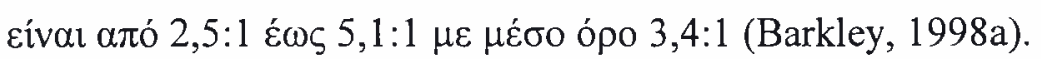

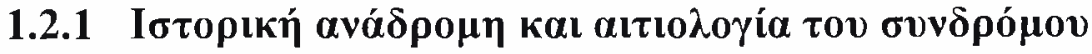

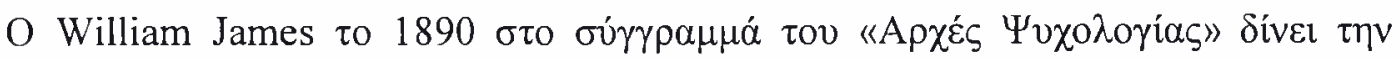

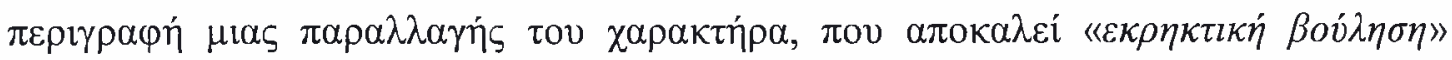

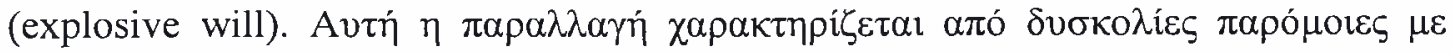

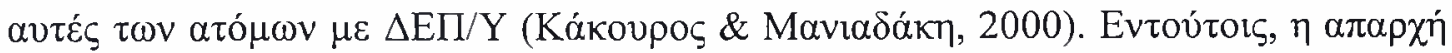

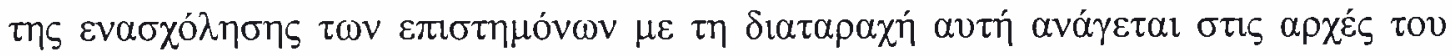

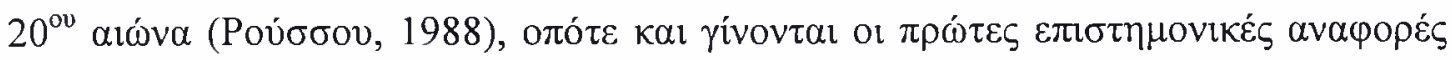

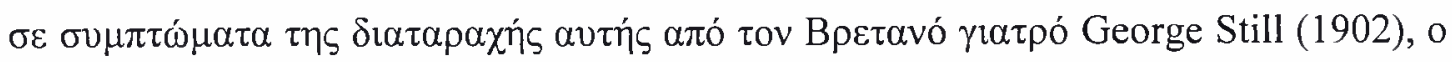

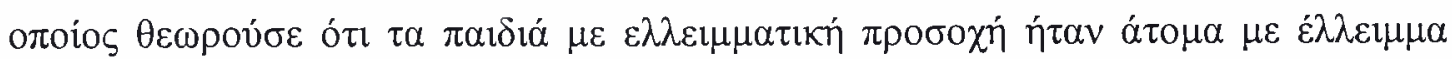

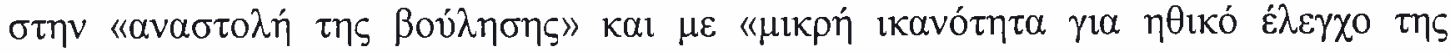

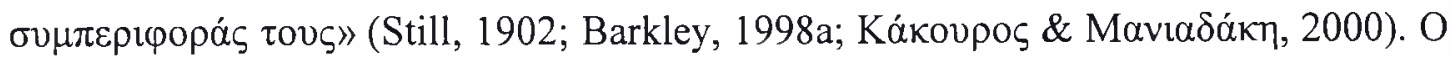

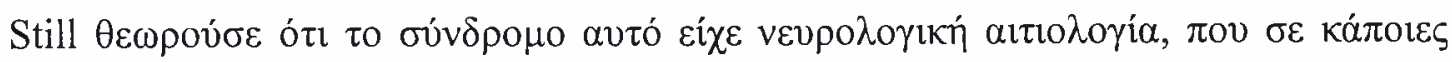




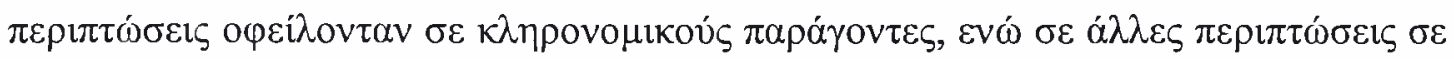

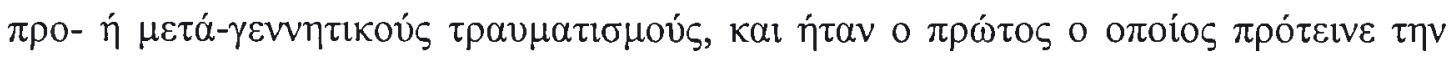

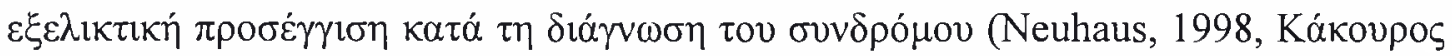

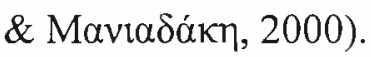

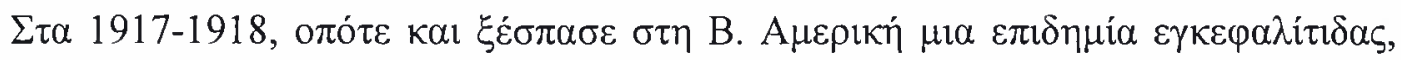

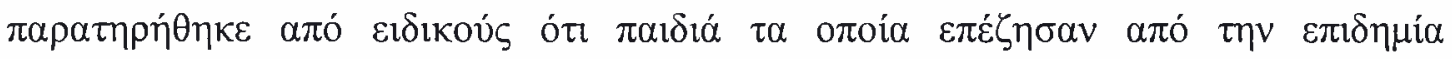

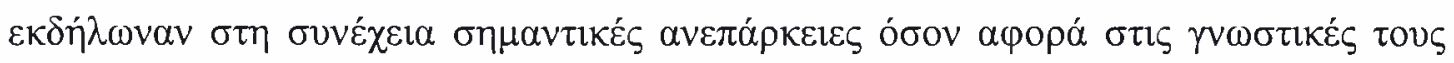

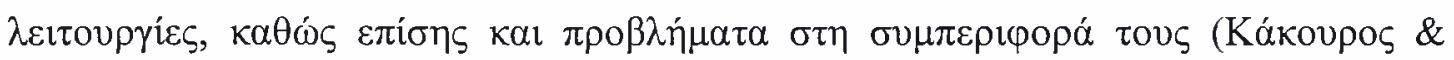

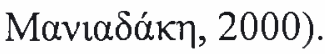

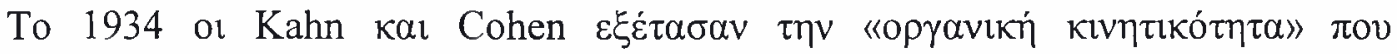

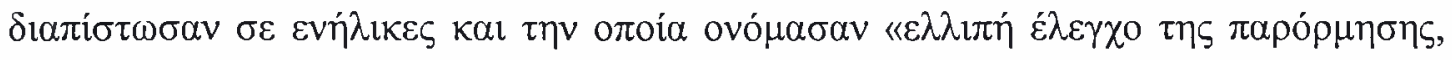

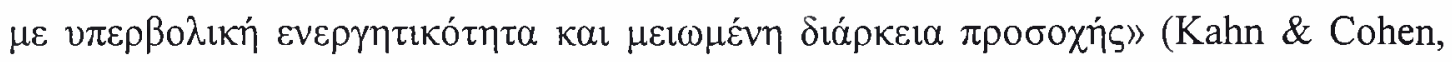

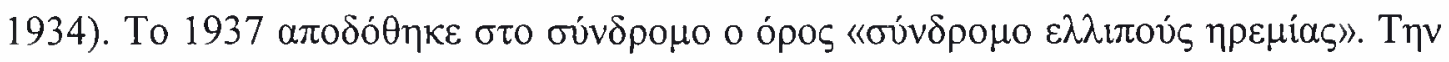

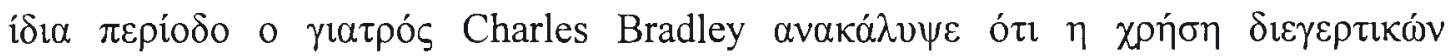

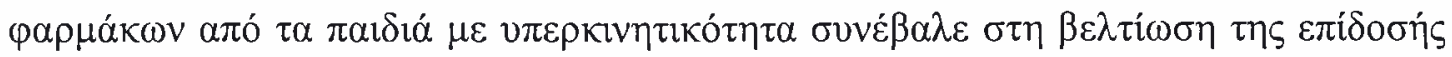

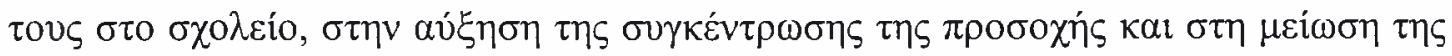

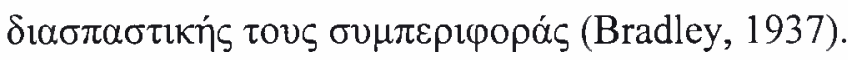

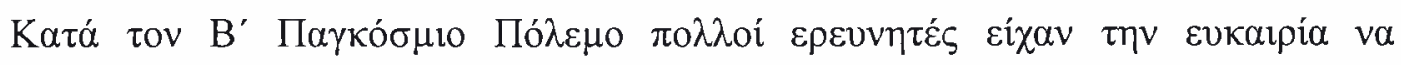

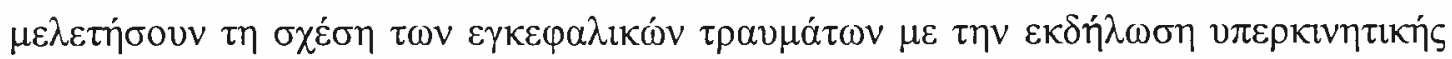

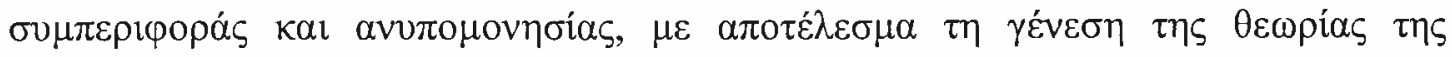

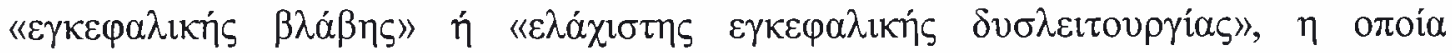

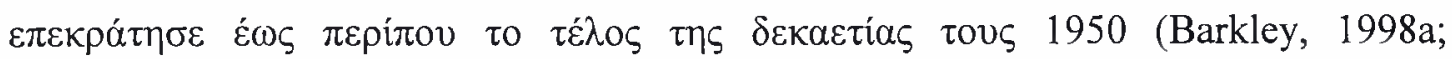

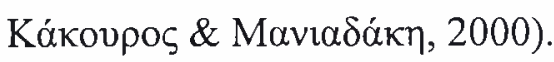

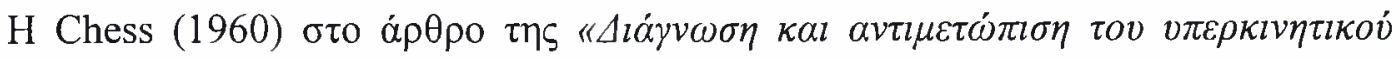

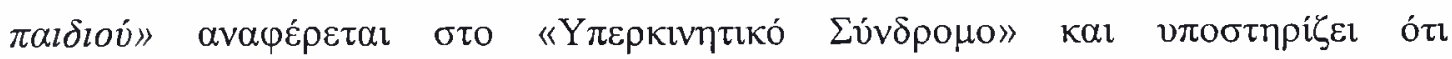

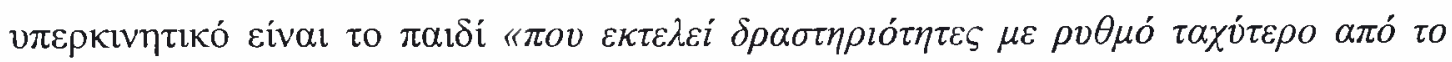

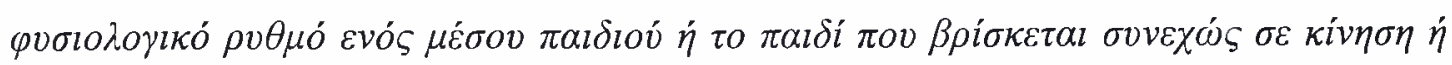

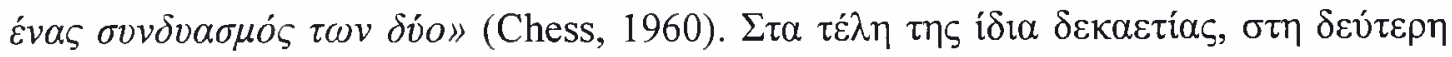

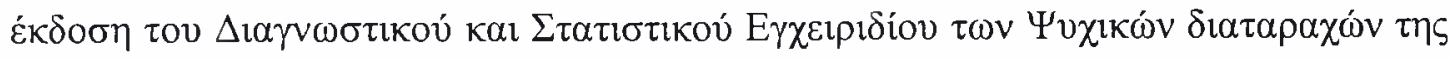

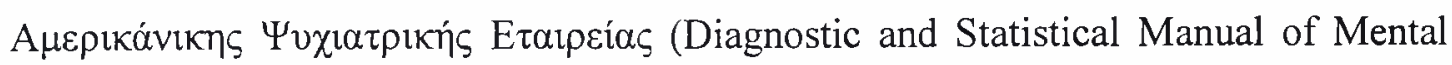
Disorders-II $\eta \dot{~ D S M-I I) ~(A m e r i c a n ~ P s y c h i a t r i c ~ A s s o c i a t i o n, ~ 1968) ~} \alpha \pi \circ \delta i \delta \varepsilon \tau \alpha \iota ~ \sigma \tau o$

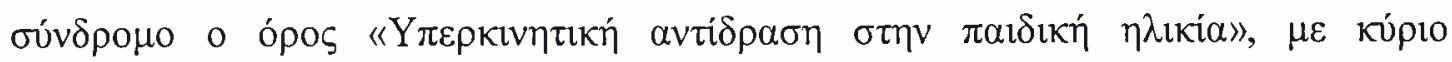

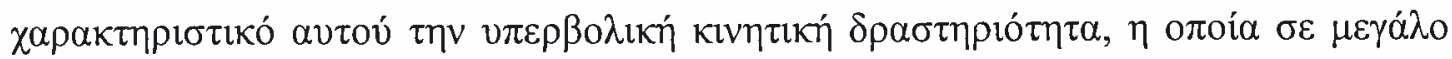

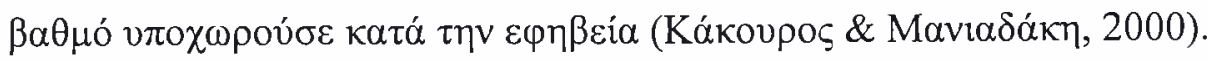




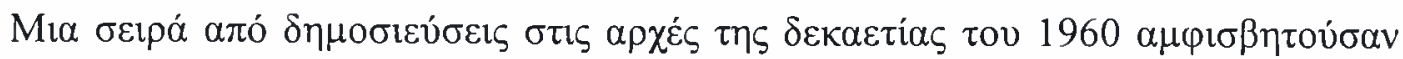

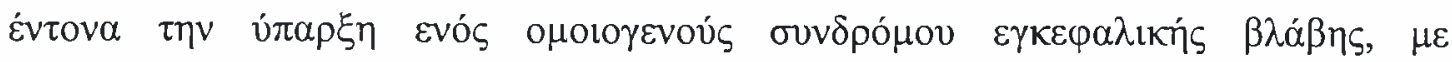

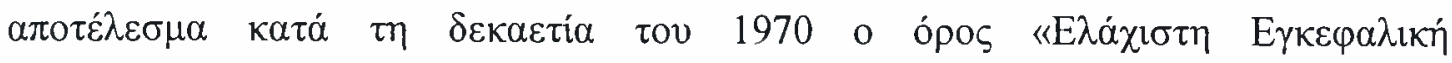

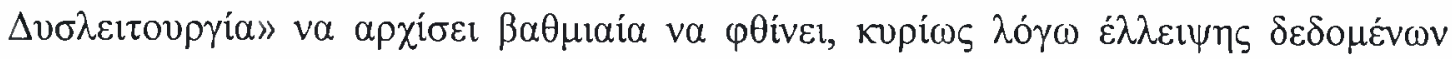

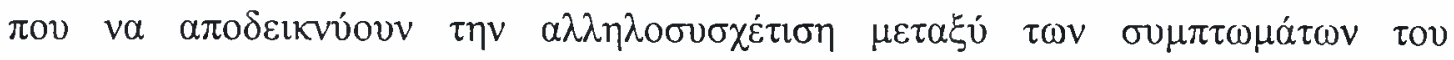

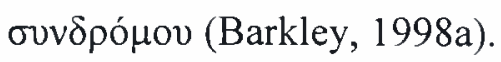

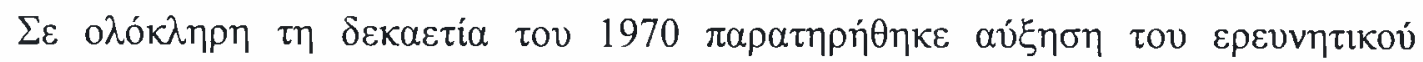

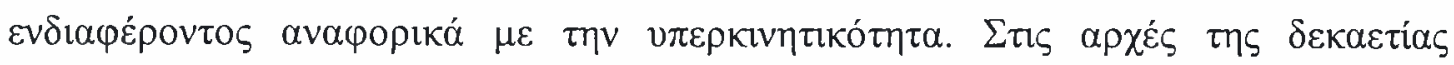

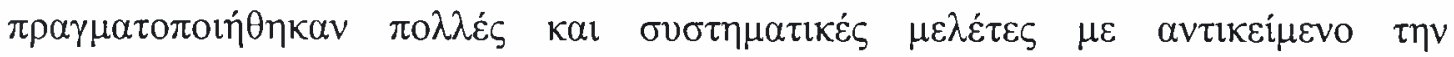

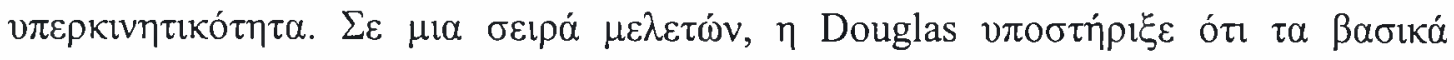

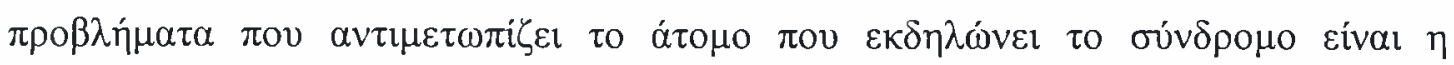

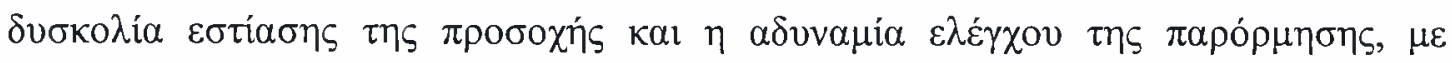

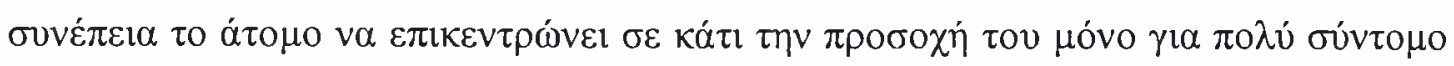

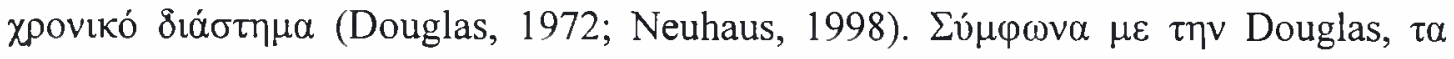

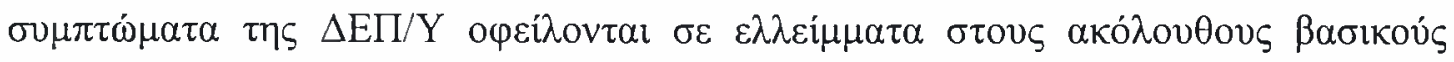

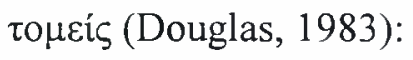

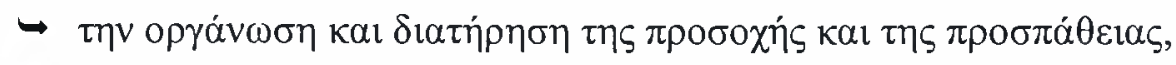

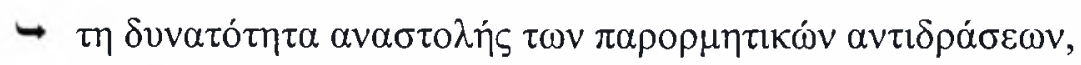

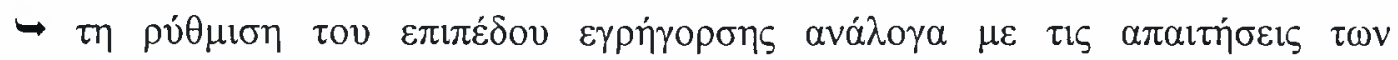
$\pi \varepsilon \rho \iota \sigma \tau \alpha \dot{\sigma \varepsilon} \omega v, \kappa \alpha \imath$

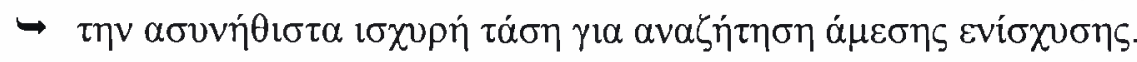

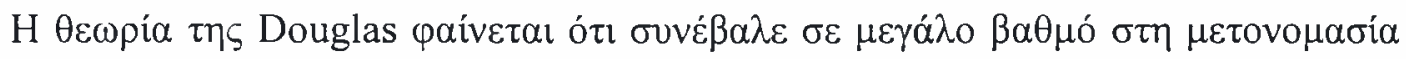

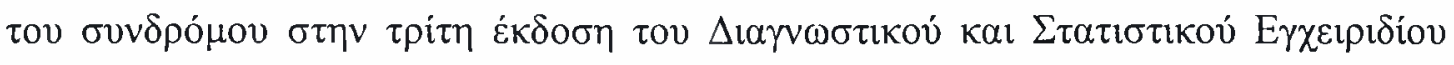

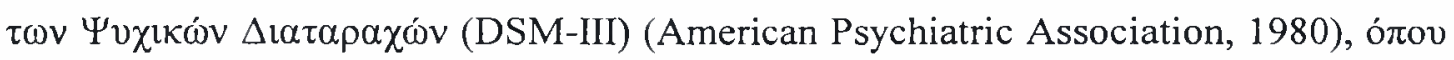

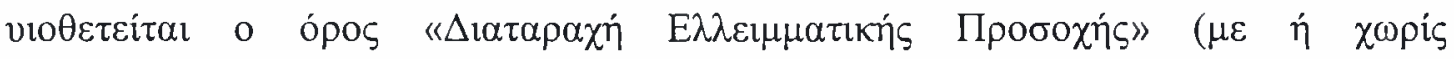
Y

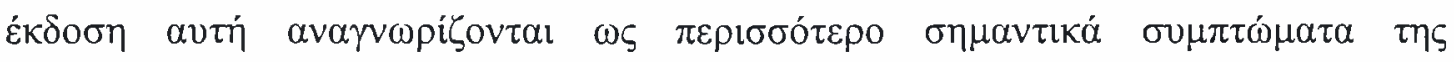

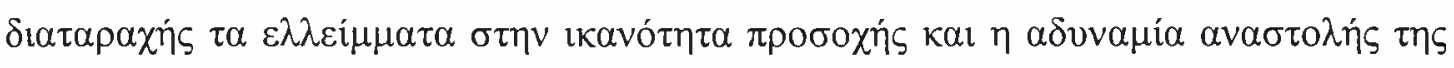

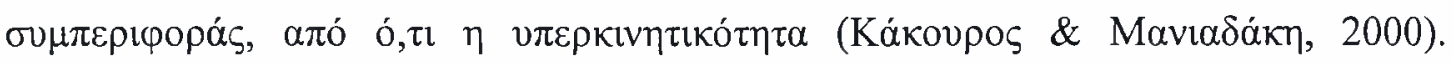

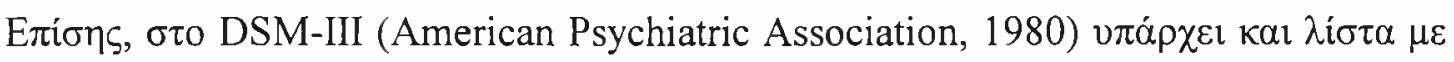

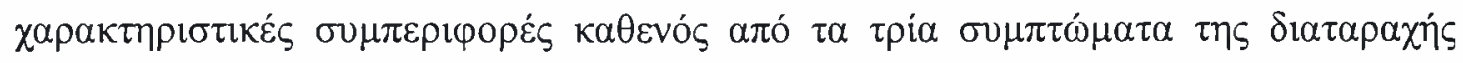

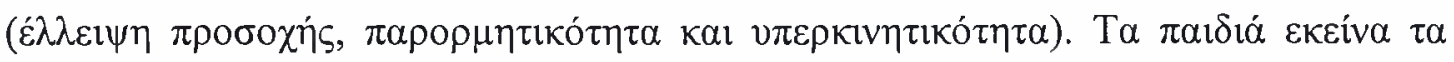

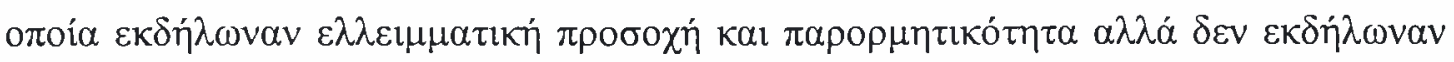




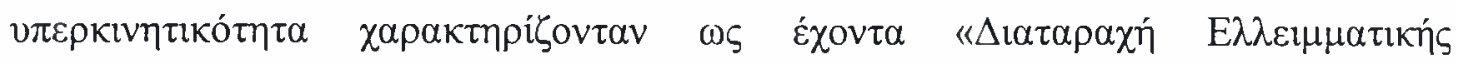

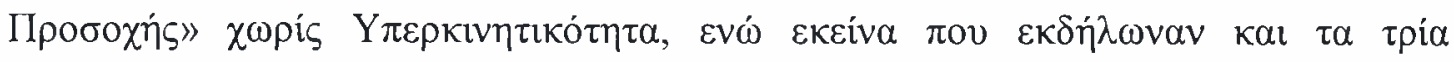

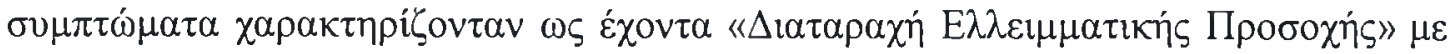

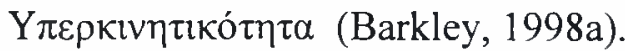

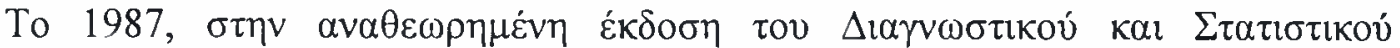

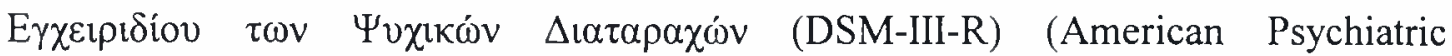

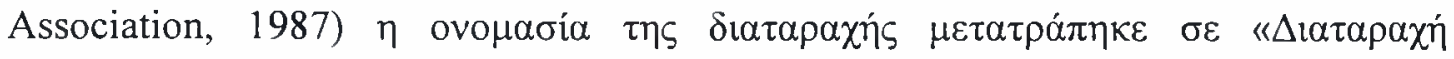

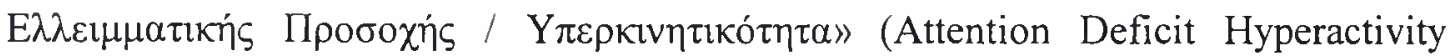

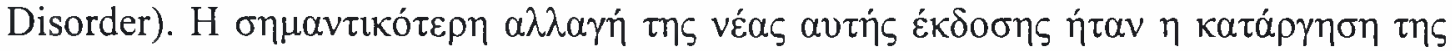

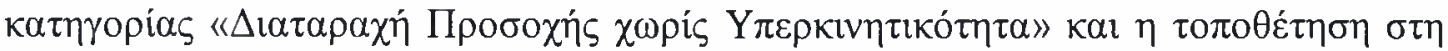

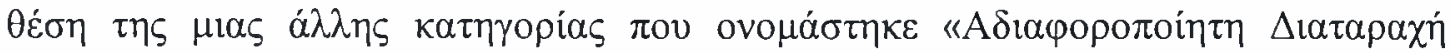

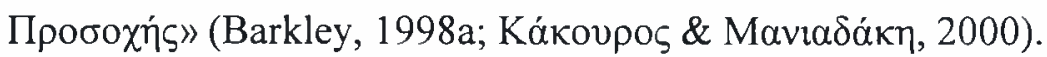

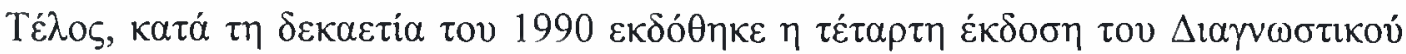

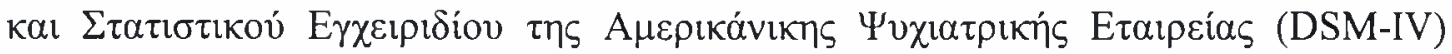

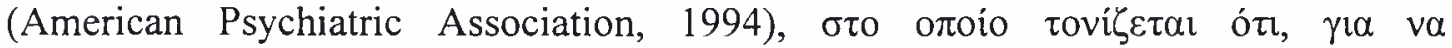

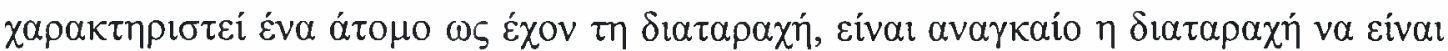

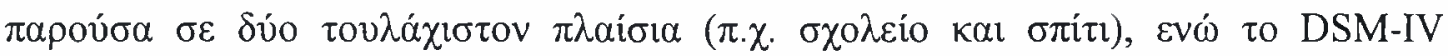

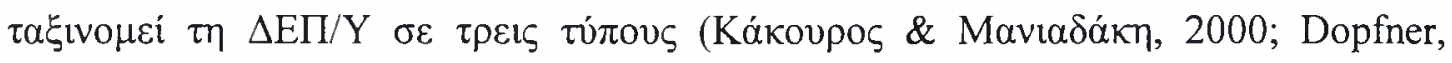
2002):

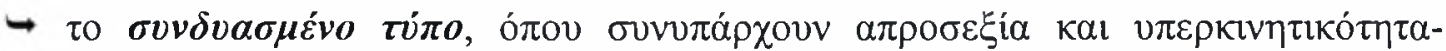
$\pi \alpha \rho \circ \rho \mu \eta \tau \kappa \kappa ́ \tau \eta \tau \alpha$,

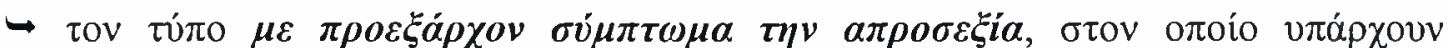

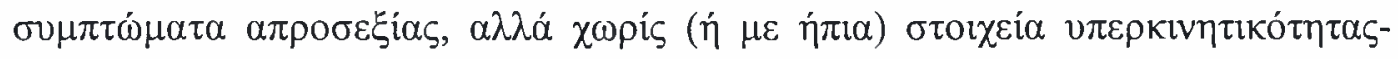

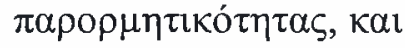

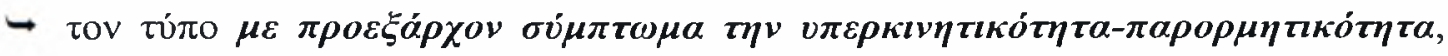

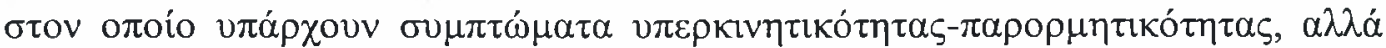

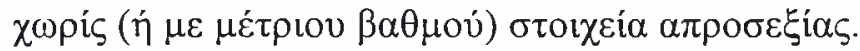

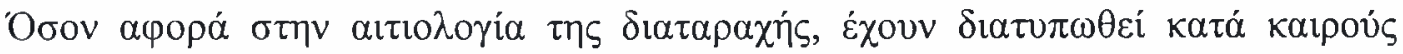
$\delta$ lá

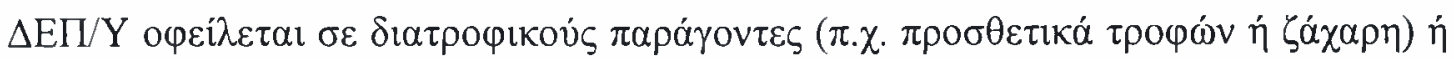

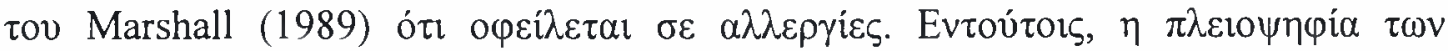

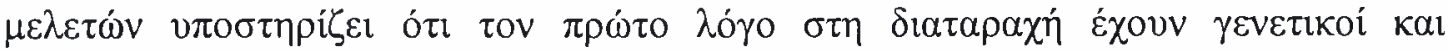

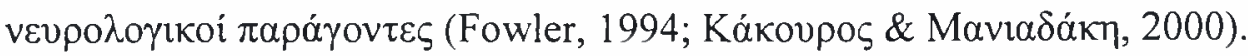




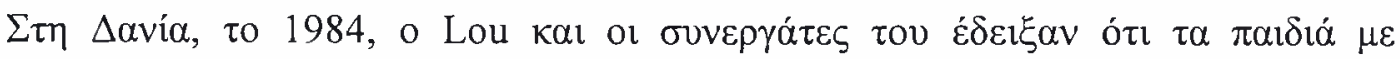

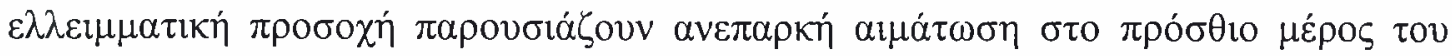

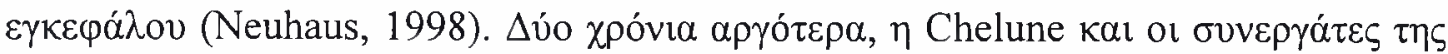

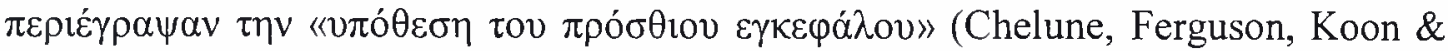

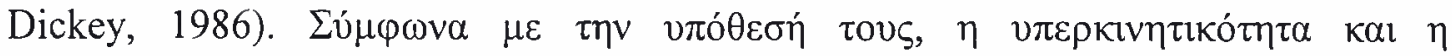

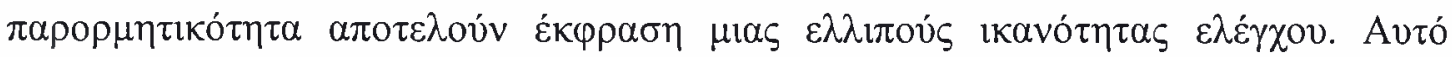

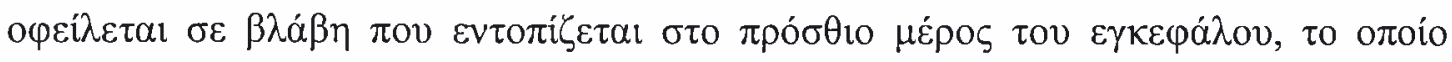

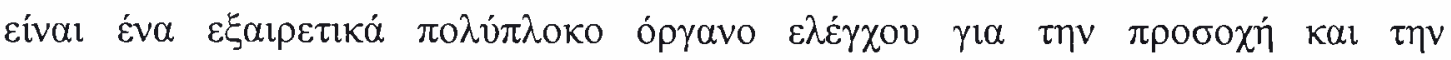

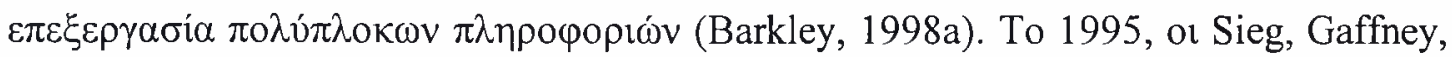

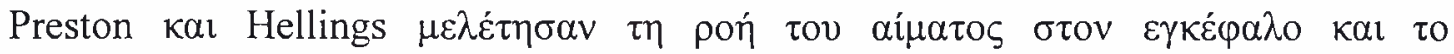

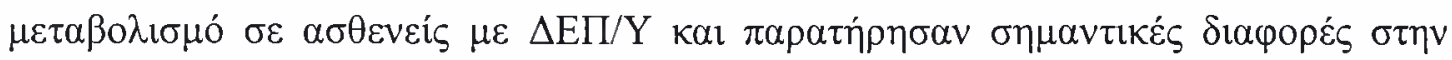

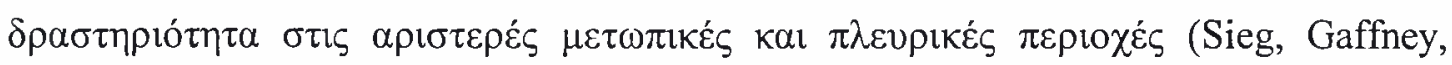

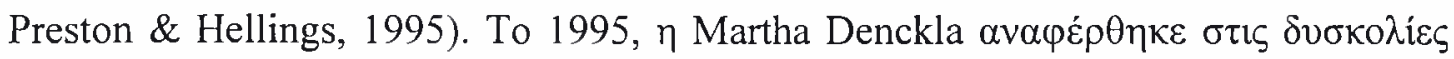

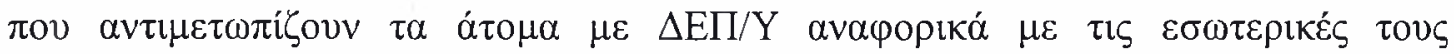

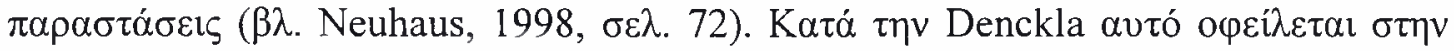

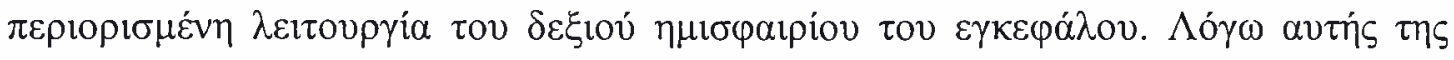

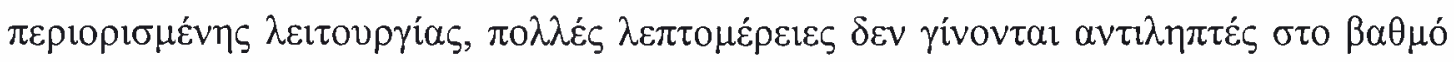

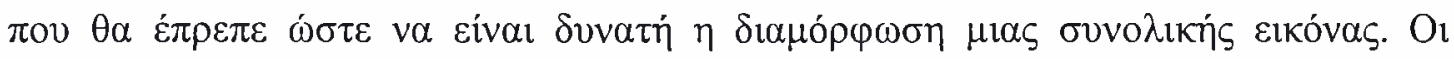

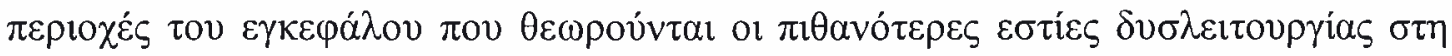

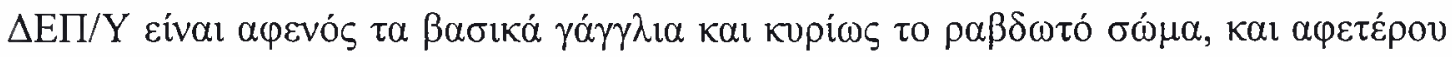

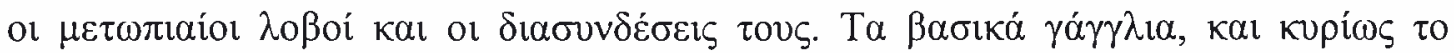

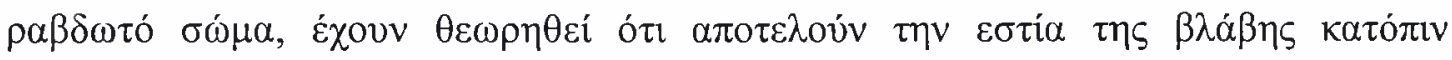

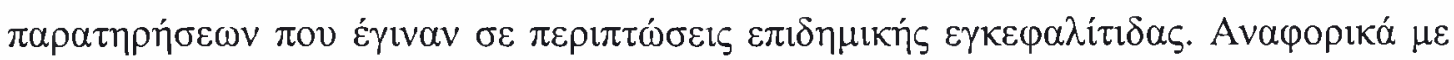

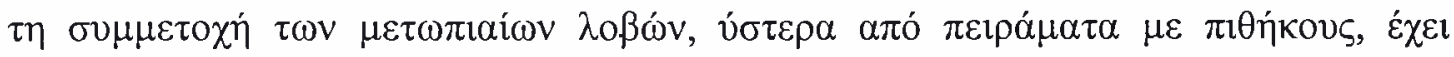

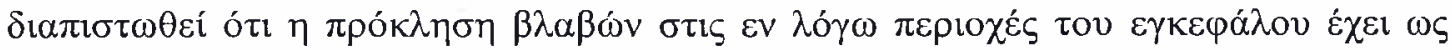

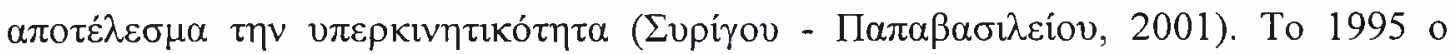

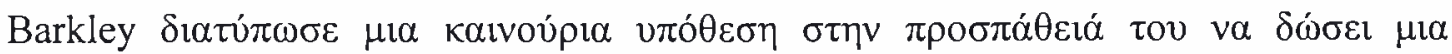

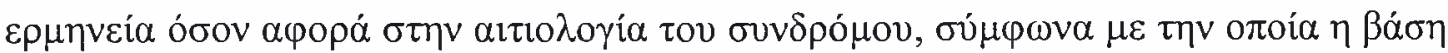

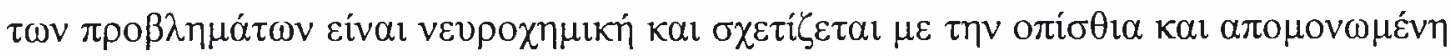

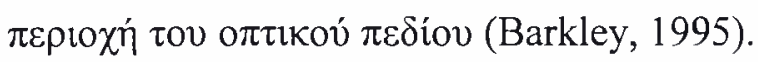

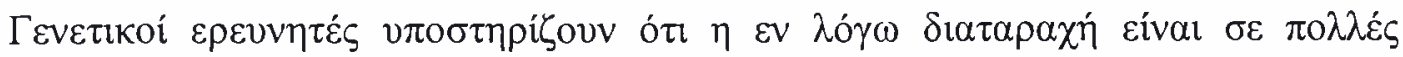

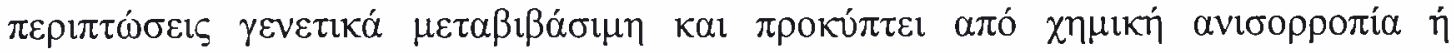

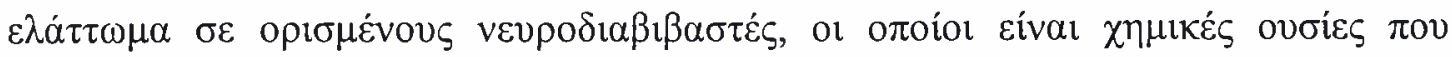

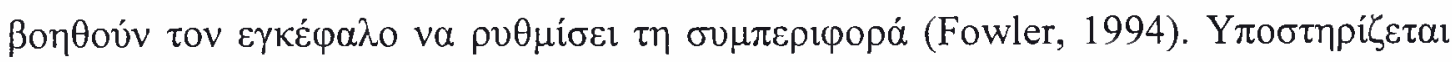




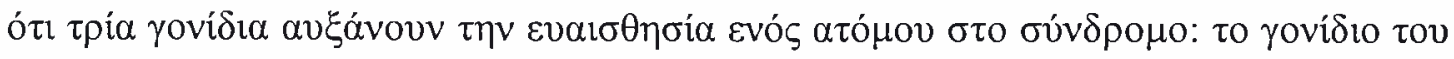

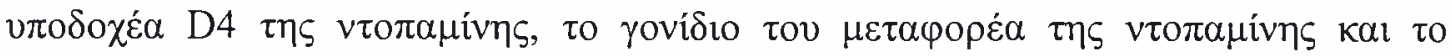

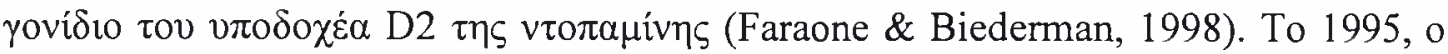

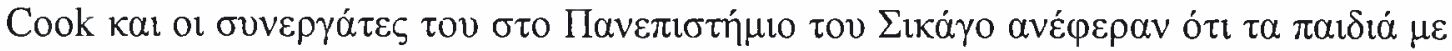

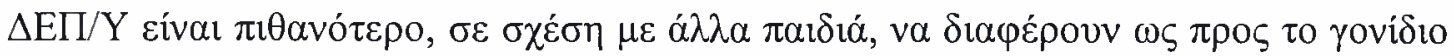

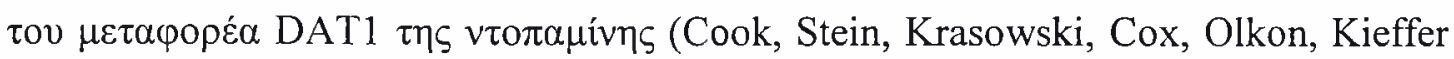

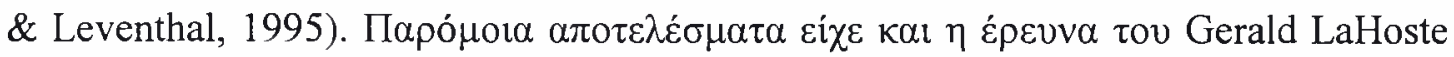

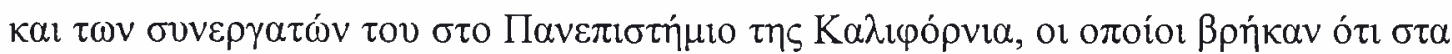

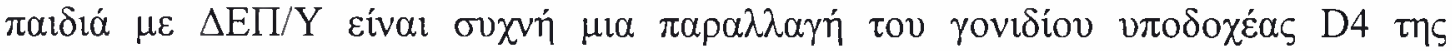

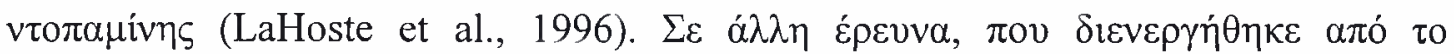

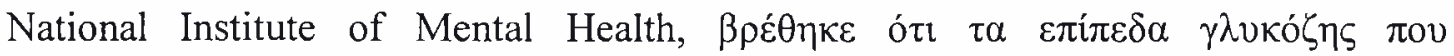

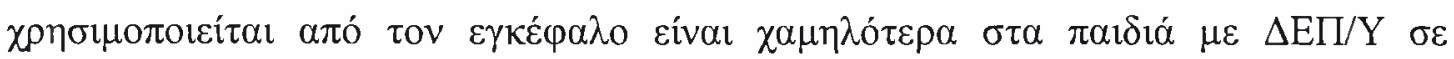

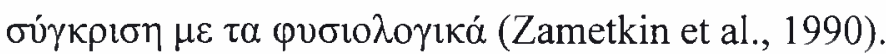

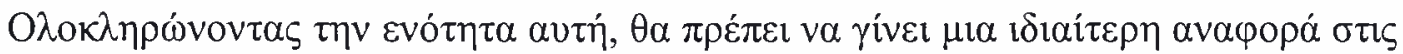

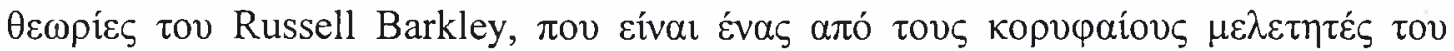

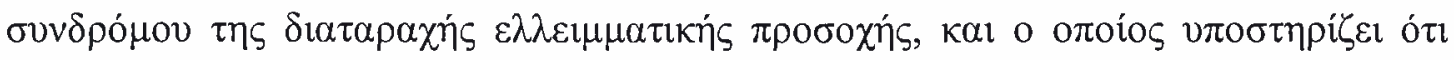

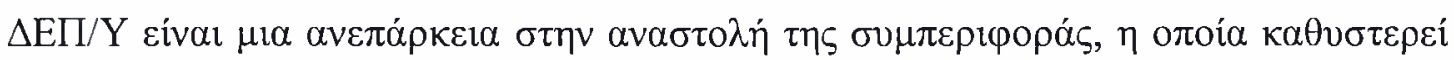

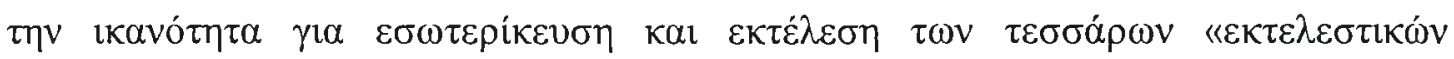

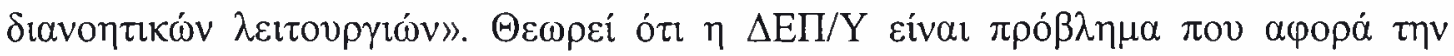

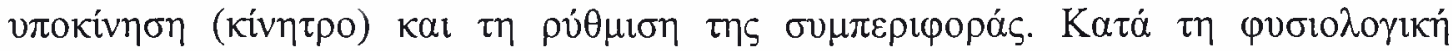

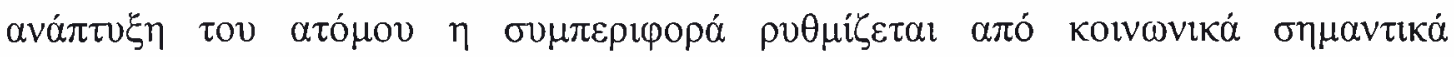

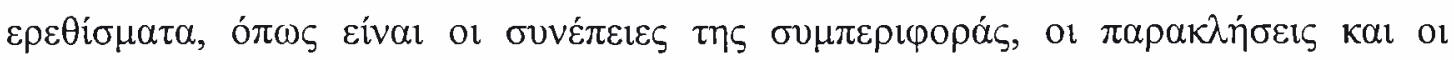

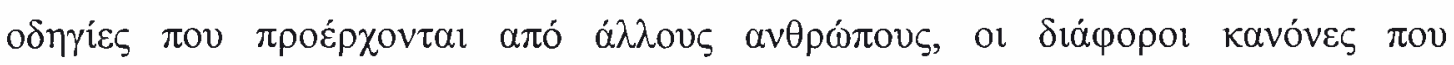

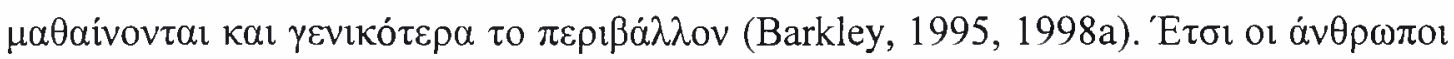

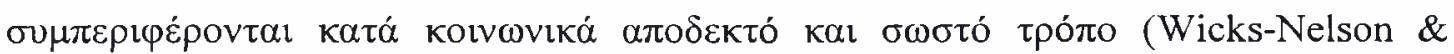

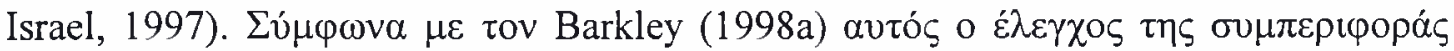

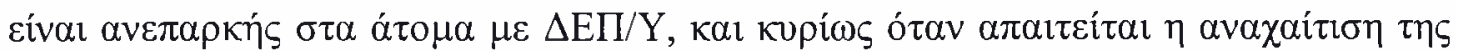

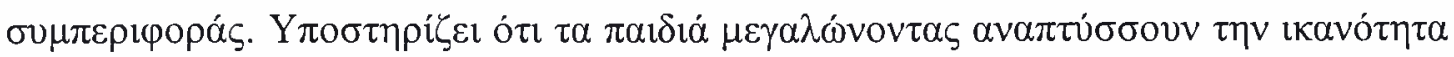

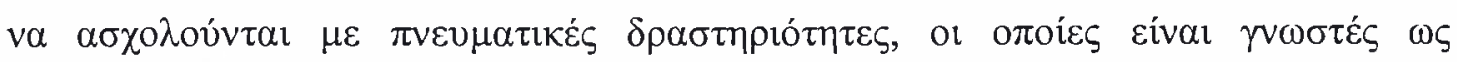

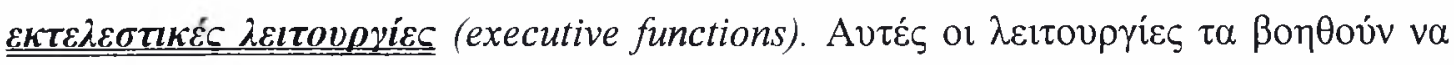

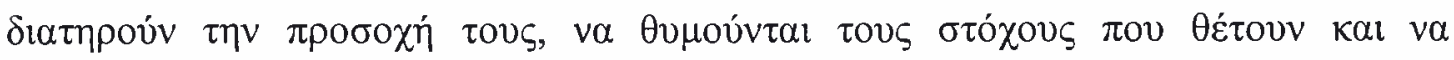

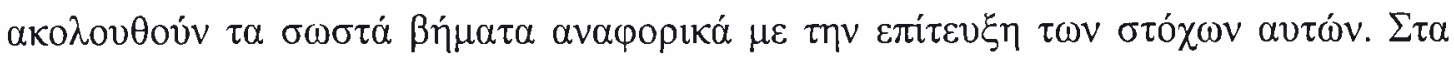

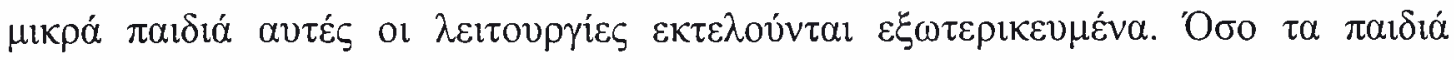

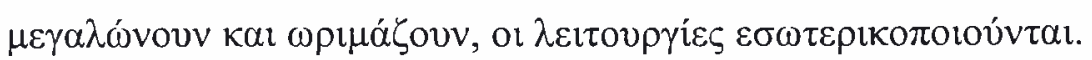




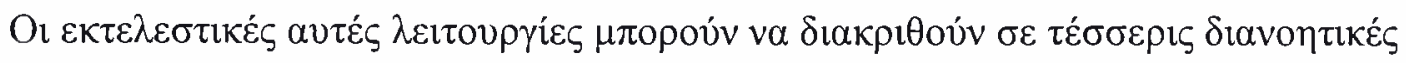

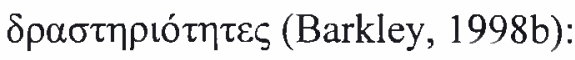

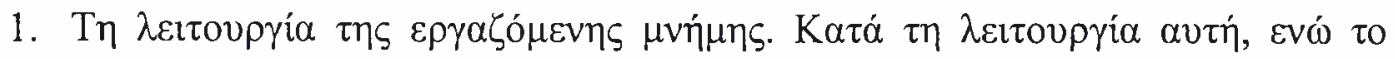

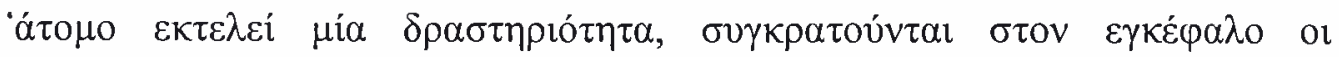

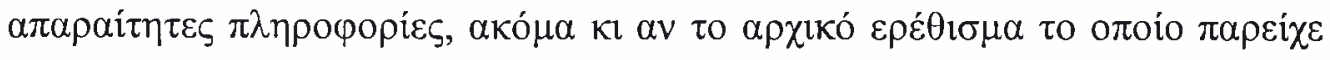

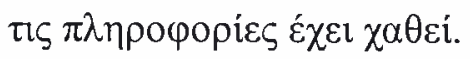

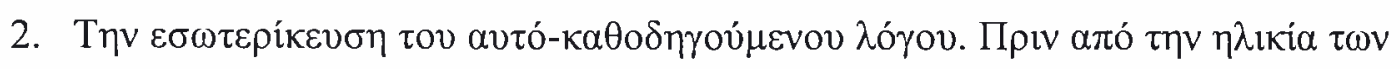

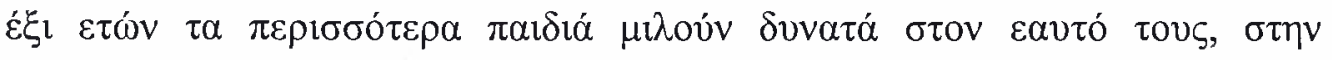

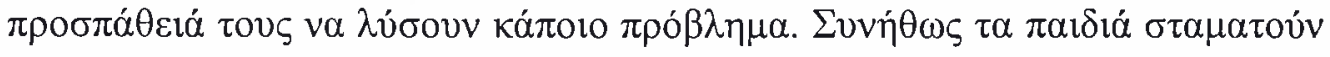

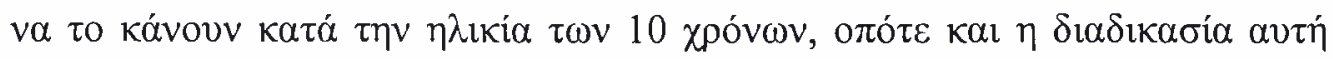

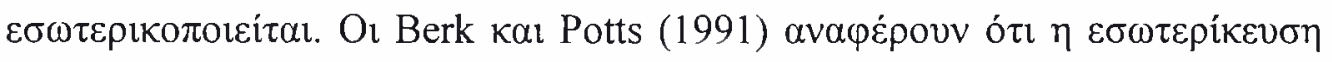

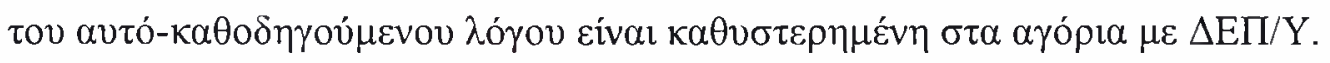

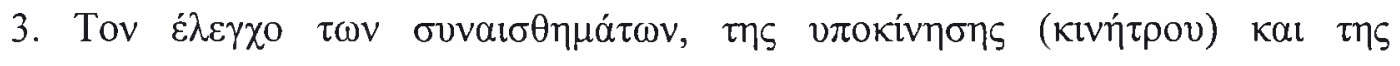

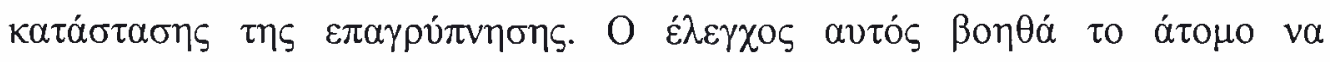

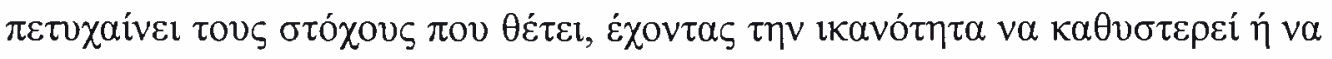

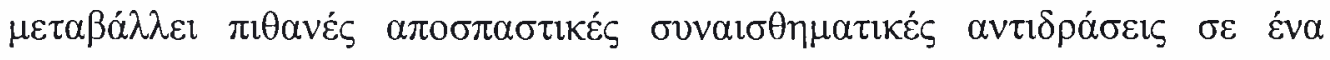

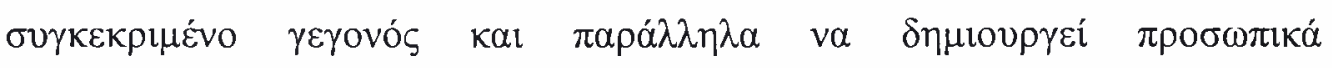

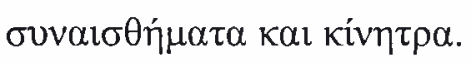

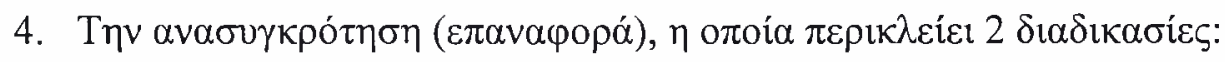

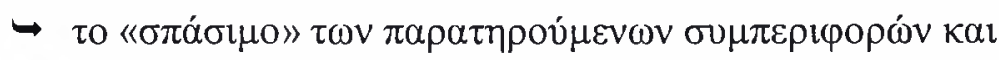

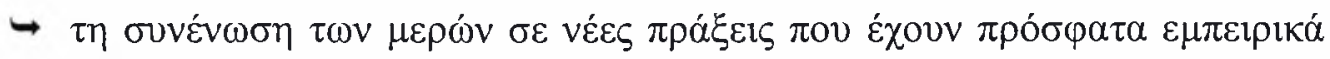

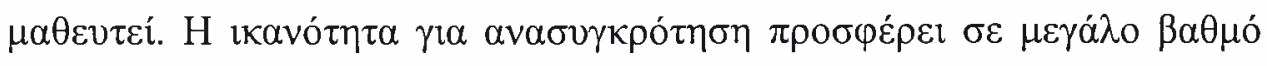

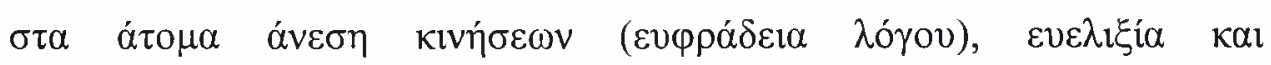

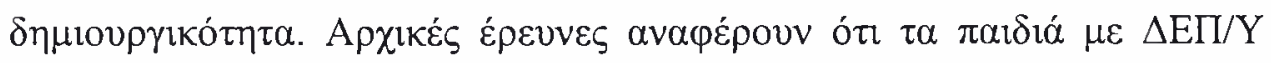

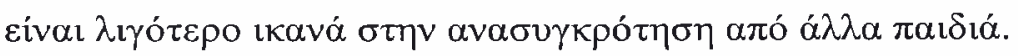

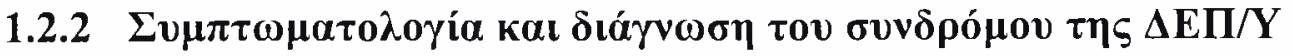

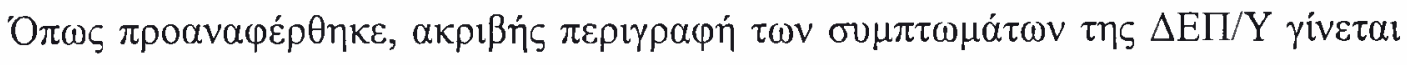

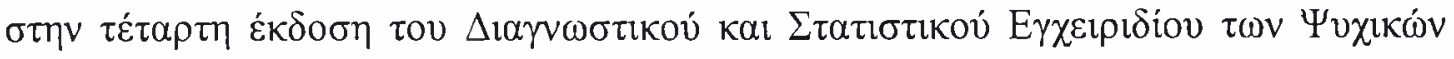

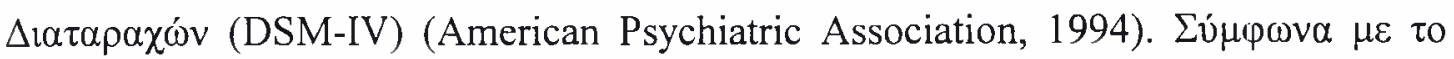

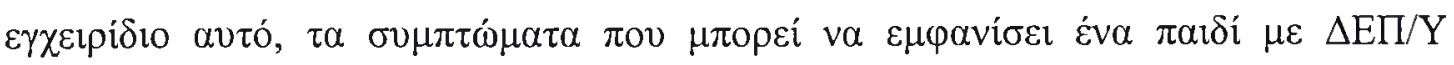




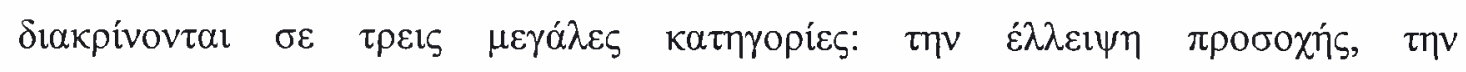

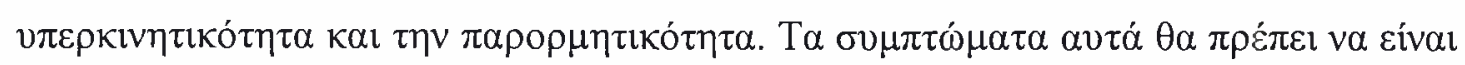

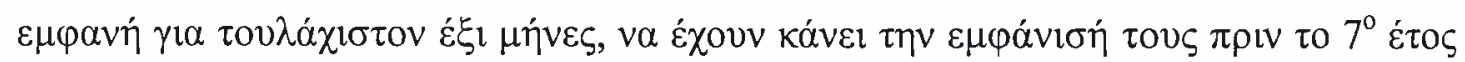

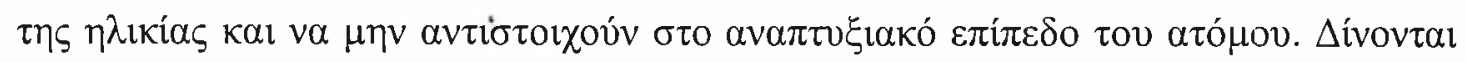

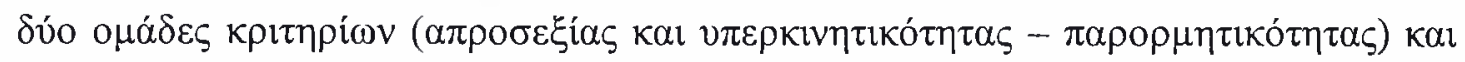

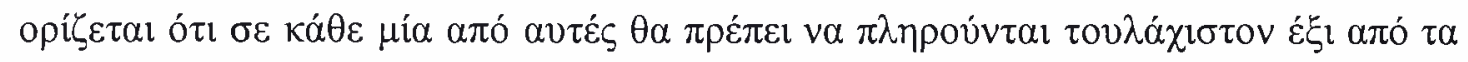

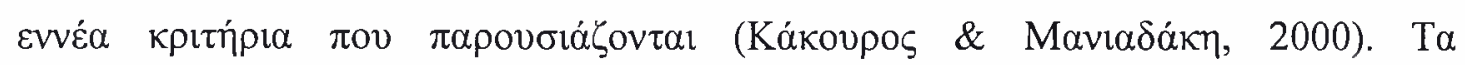

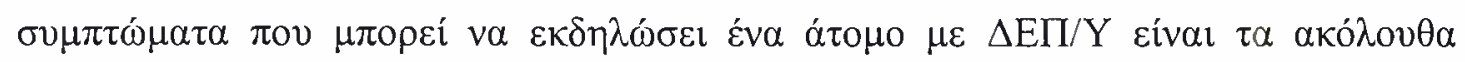

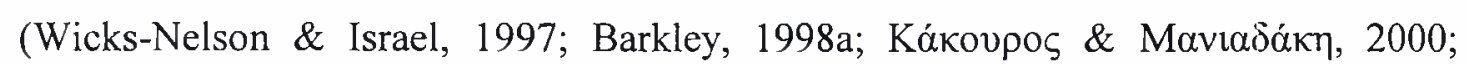
Dopfner, 2002):

\section{A. A}

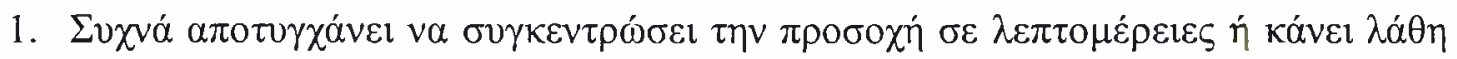

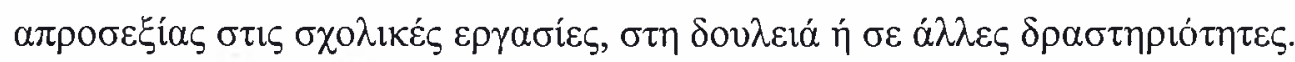

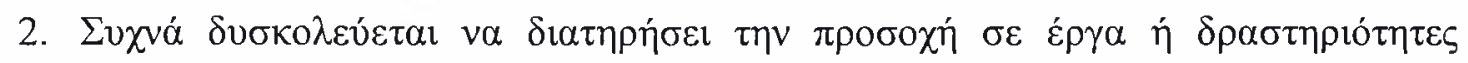
$\pi \alpha \imath \chi v i \delta ı v ́$.

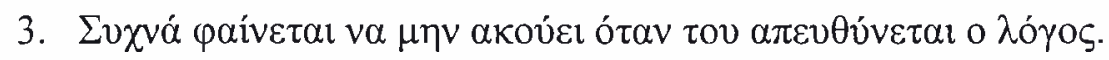

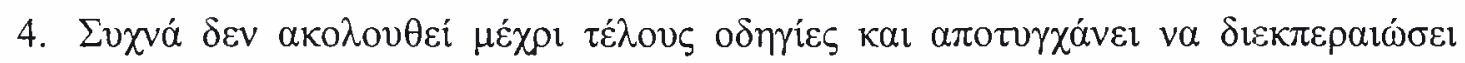

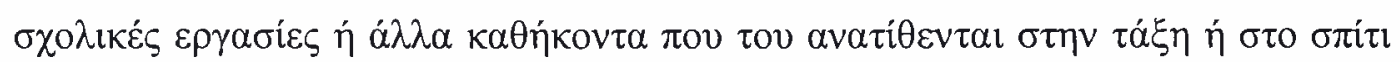

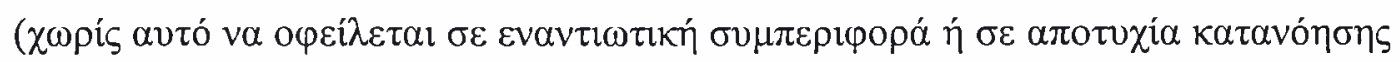

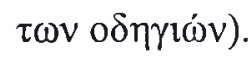

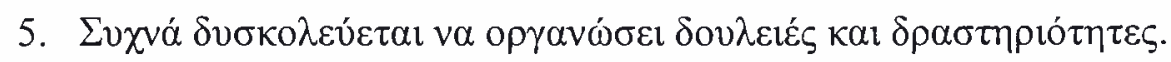

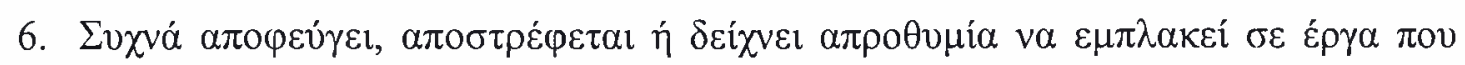

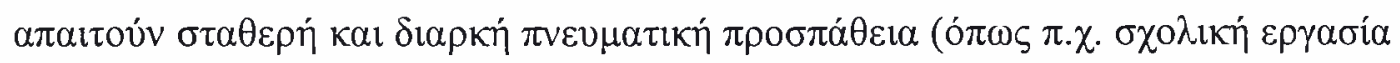

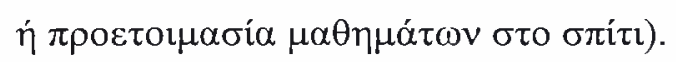

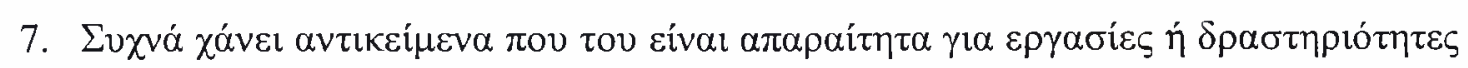

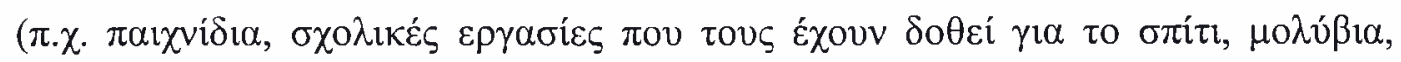
$\beta \mathrm{\imath} \beta \lambda i \alpha, \kappa . \alpha ́ \alpha$.

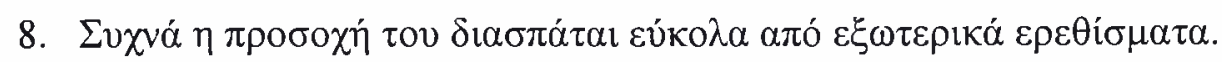

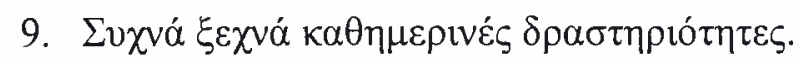




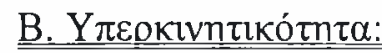

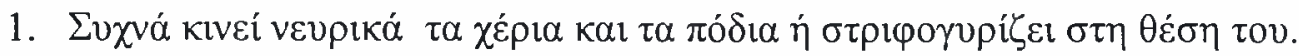

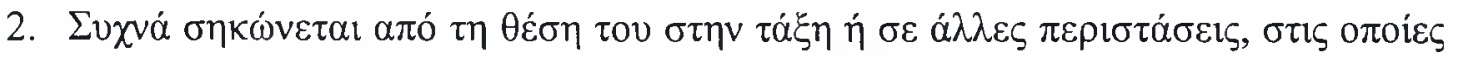

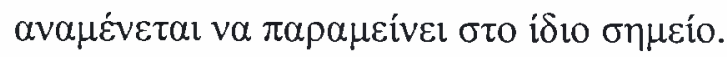

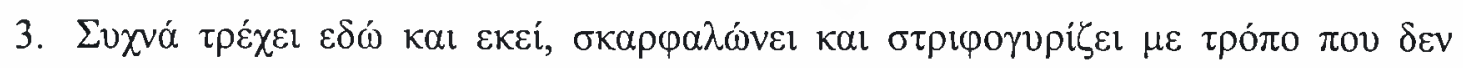

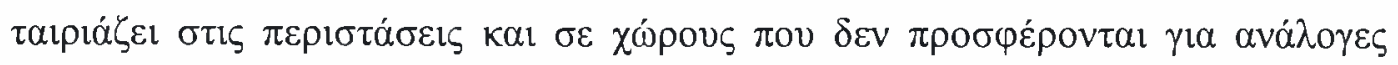
$\delta \rho \alpha \sigma \tau \eta \rho$ เó $\tau \varepsilon \zeta$.

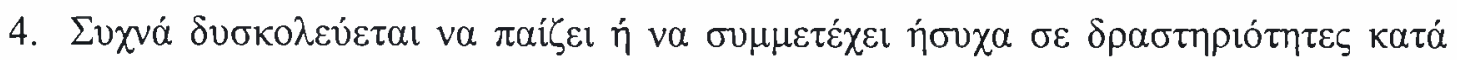

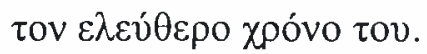

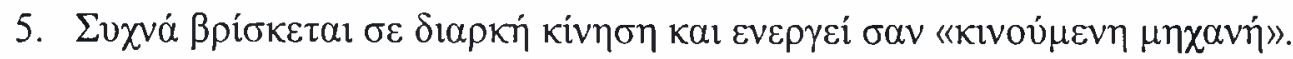

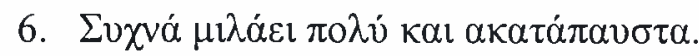

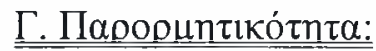

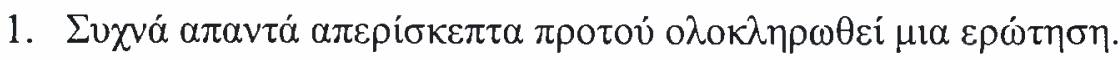

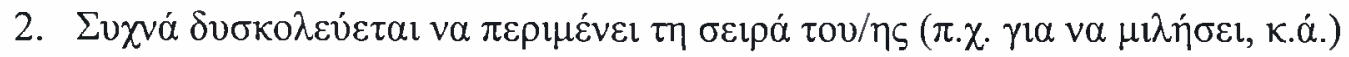

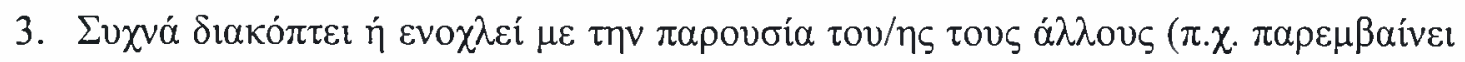

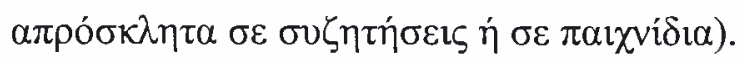

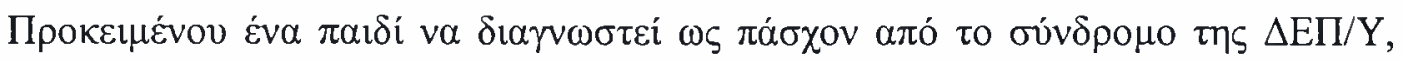

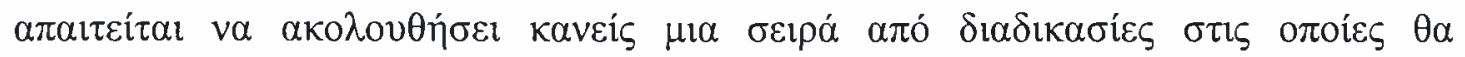

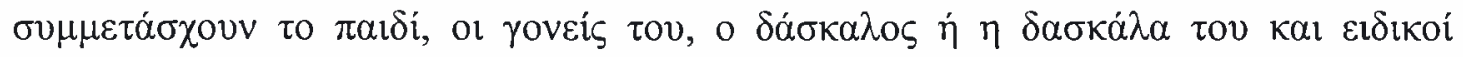

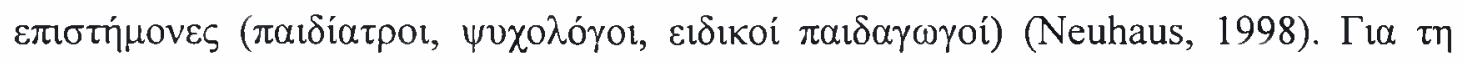

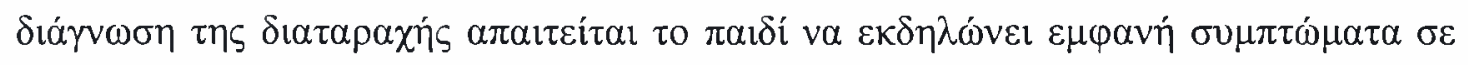

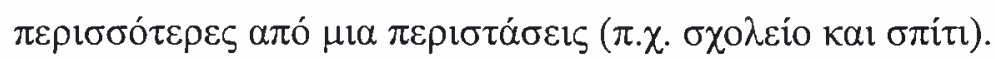

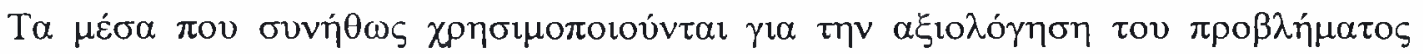

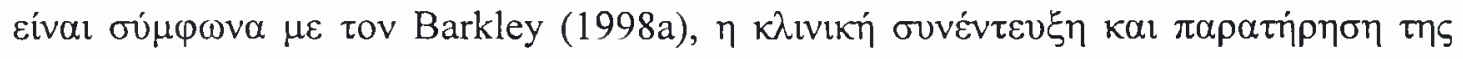

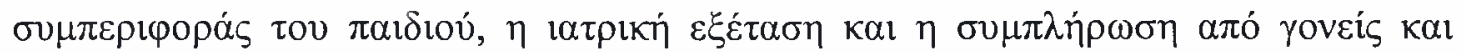

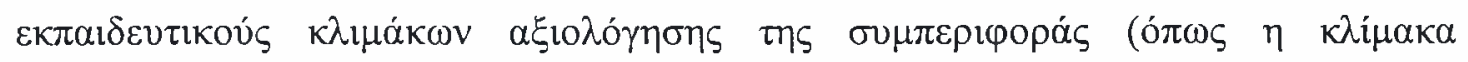

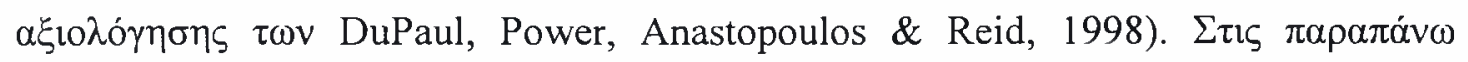

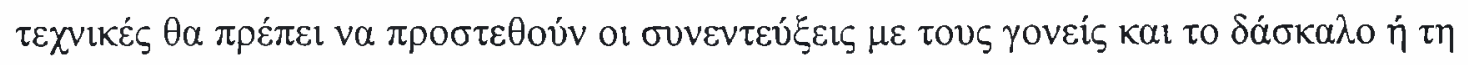

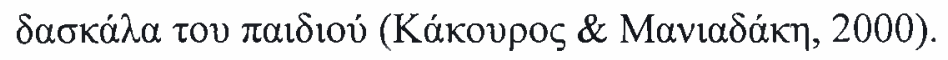




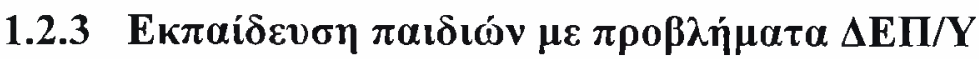

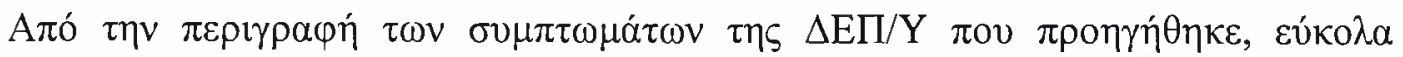

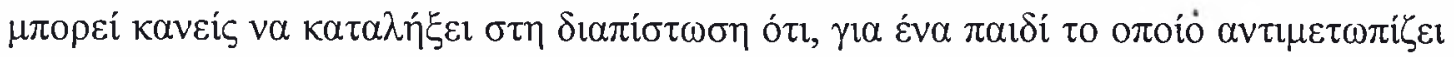

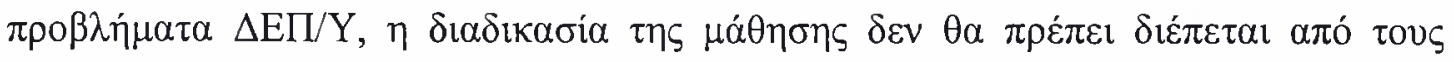

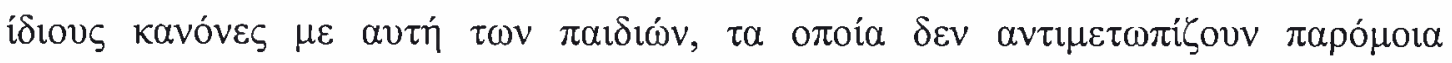

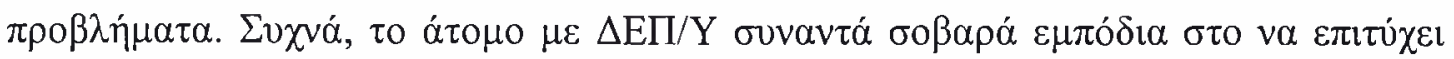

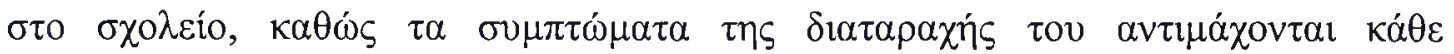

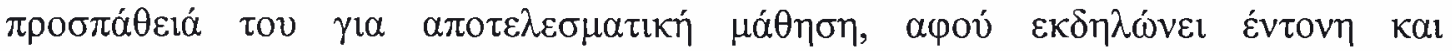

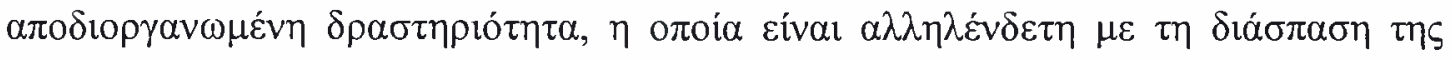

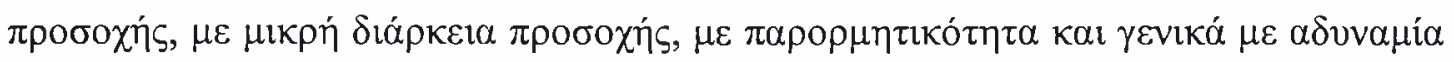

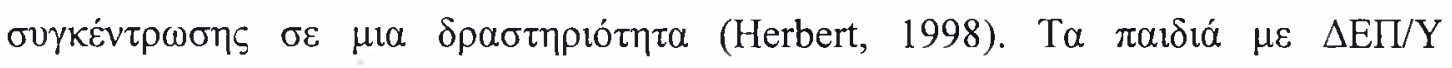

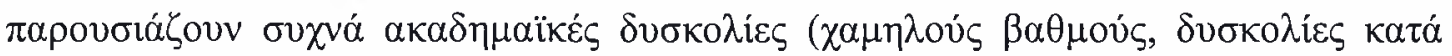

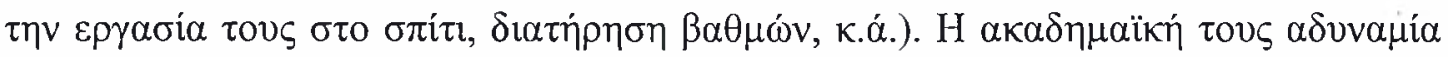

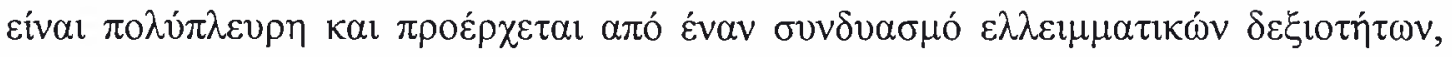

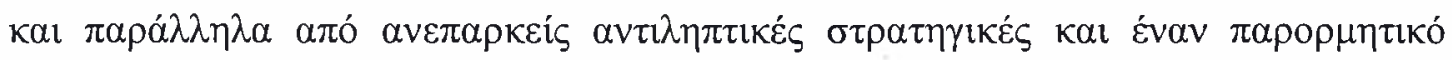

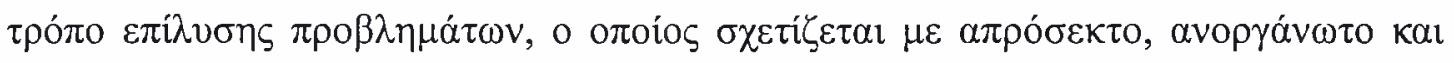

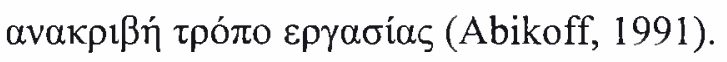

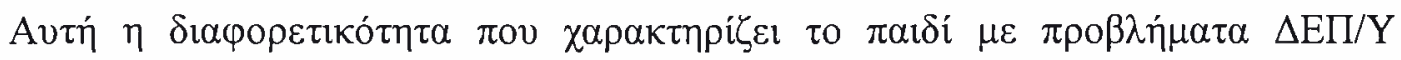

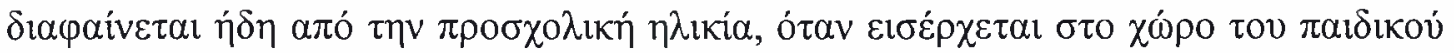

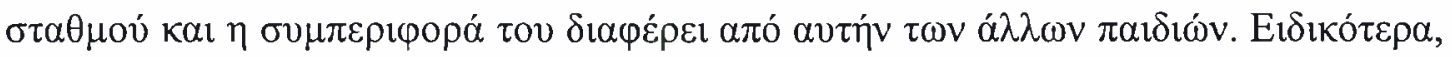

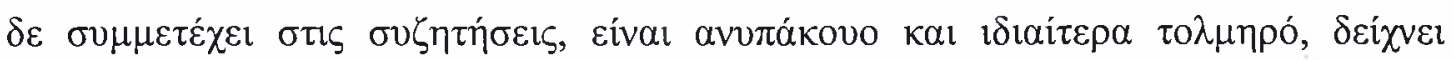

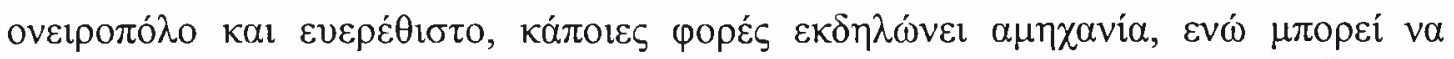

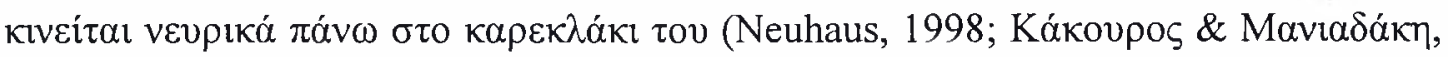
2000).

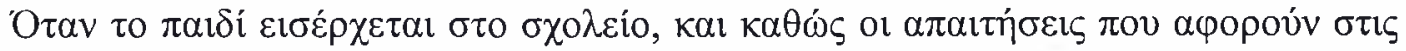

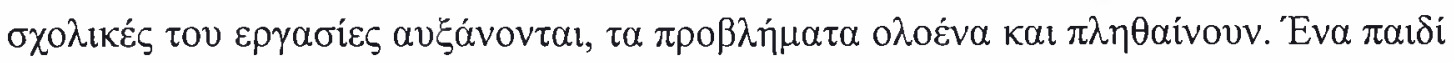

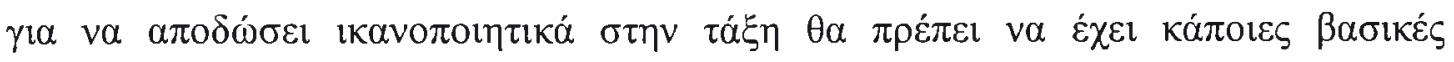

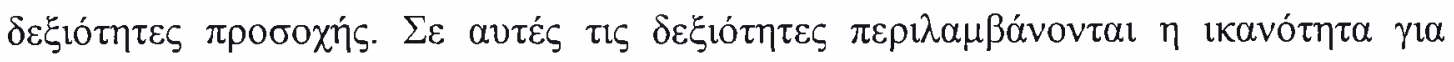

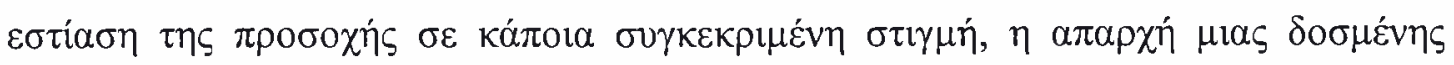

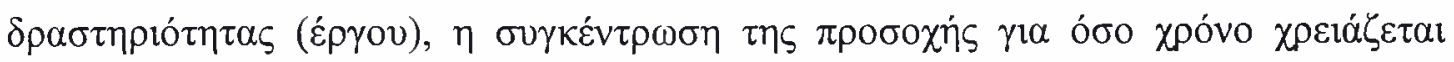

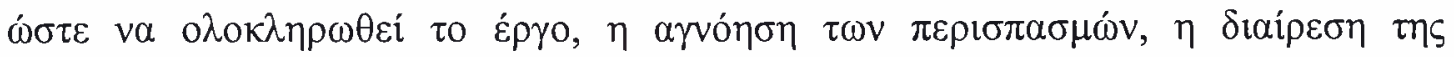

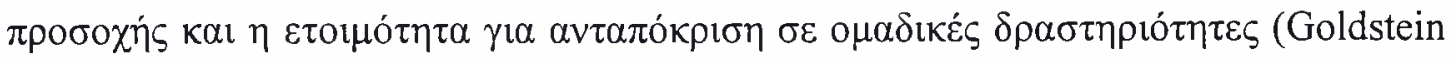




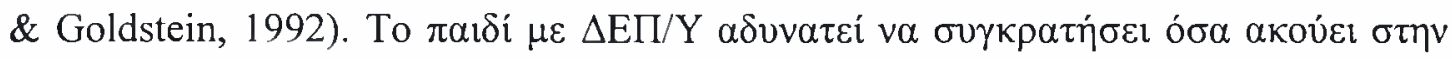

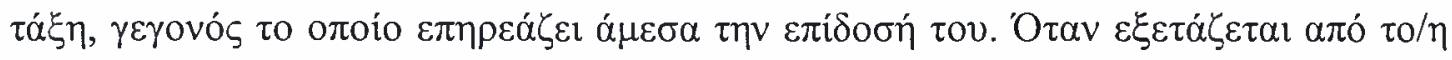

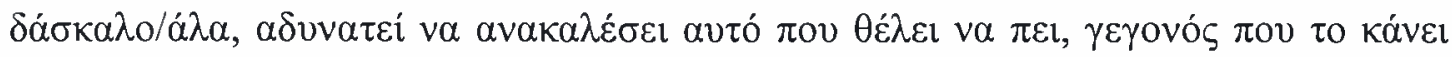

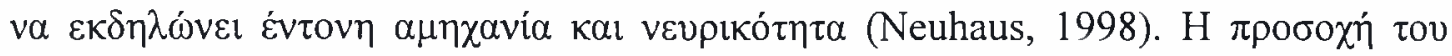

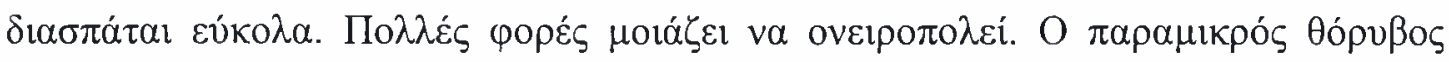

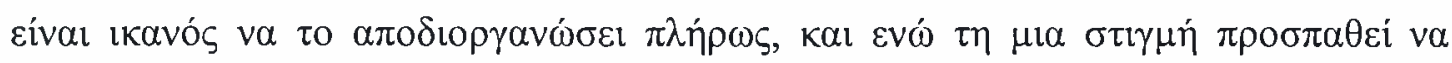

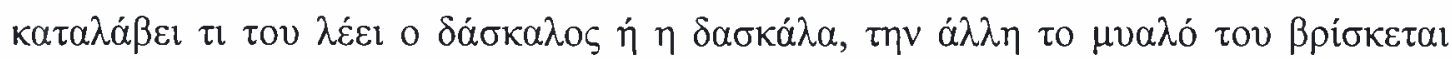
$\alpha \lambda \lambda o v$.

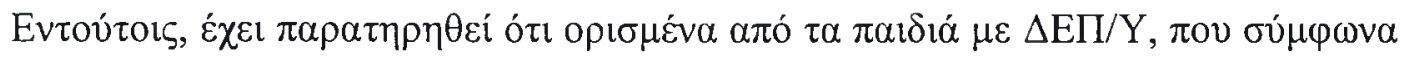

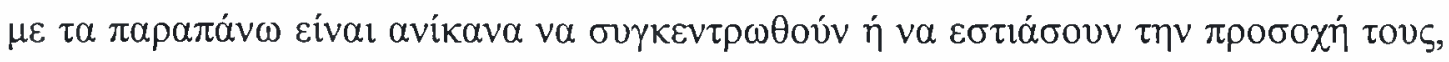

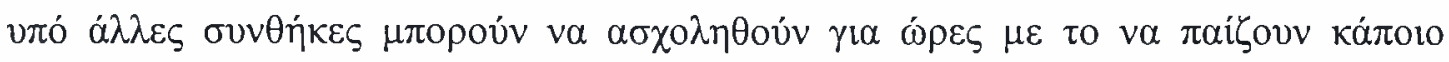

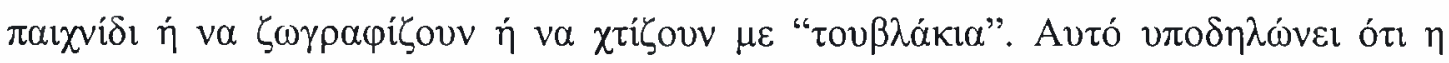

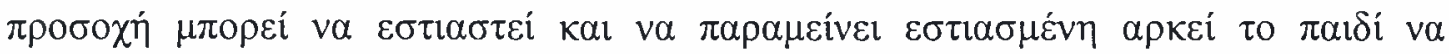

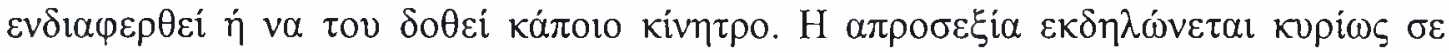

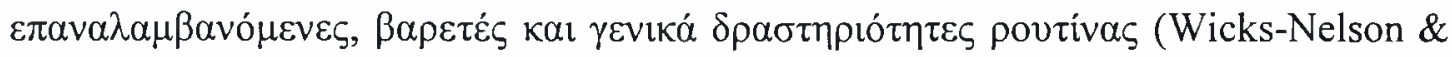

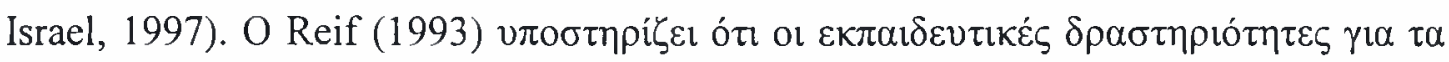

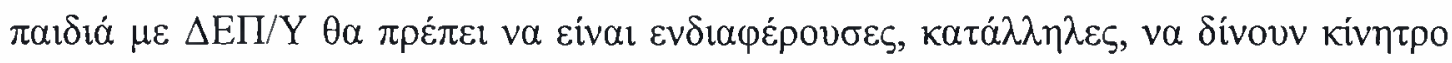

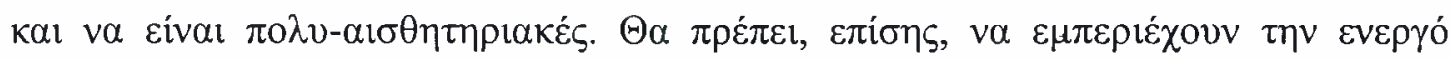

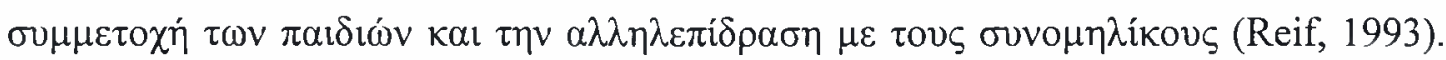

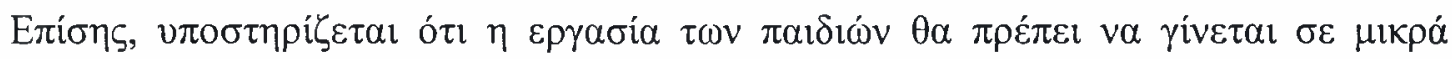

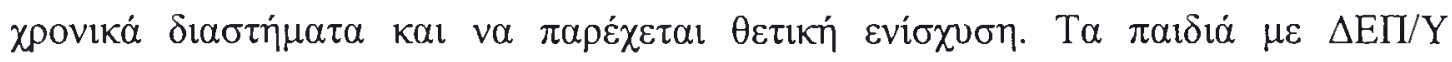

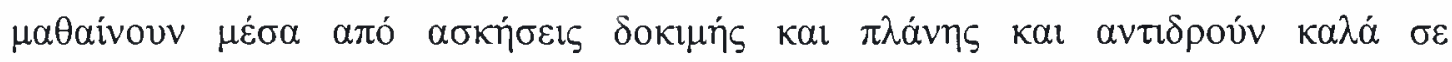

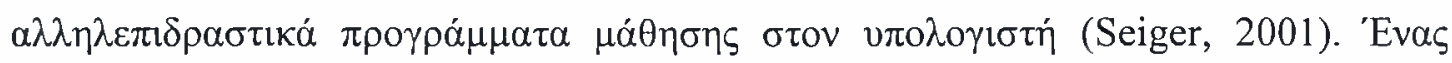

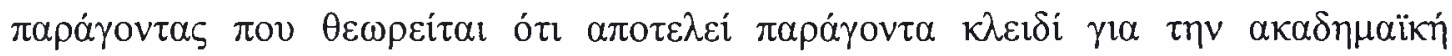

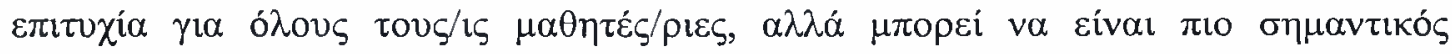

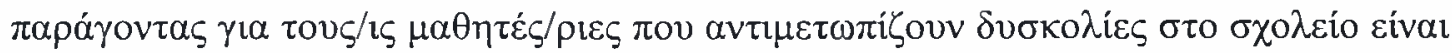

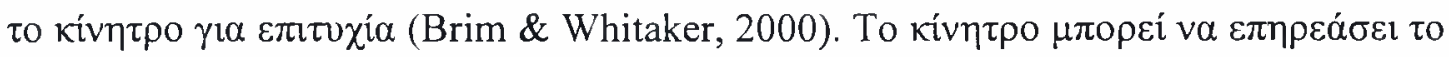

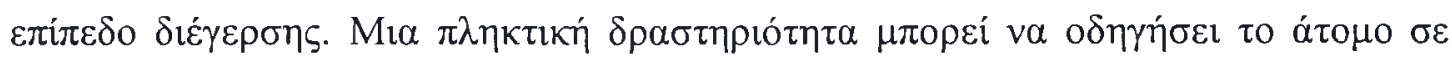

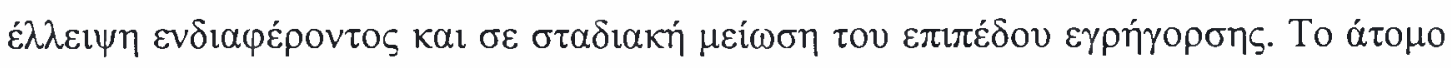

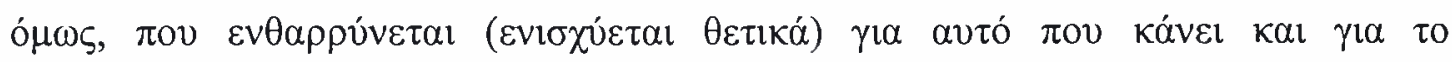

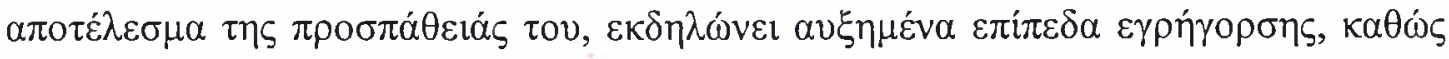

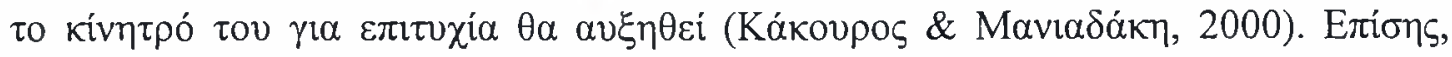

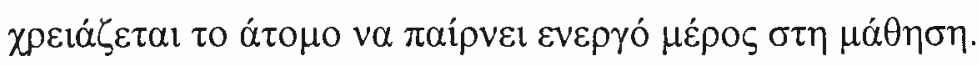




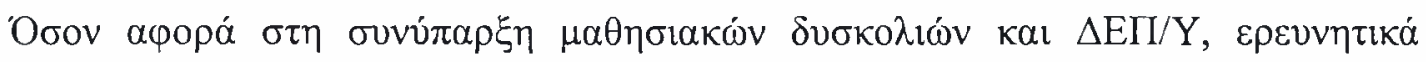

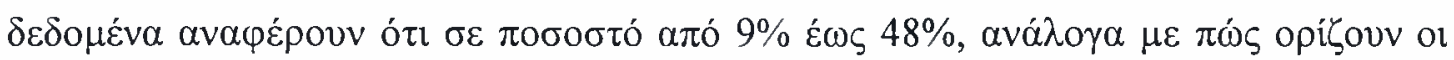

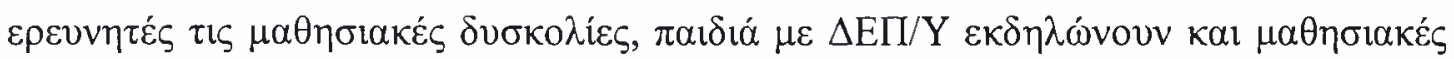

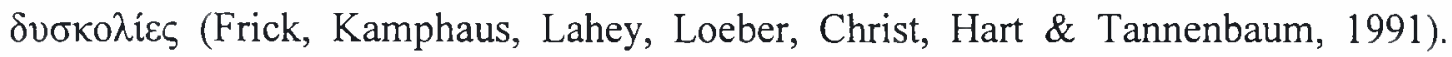

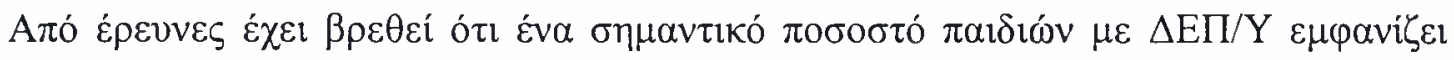

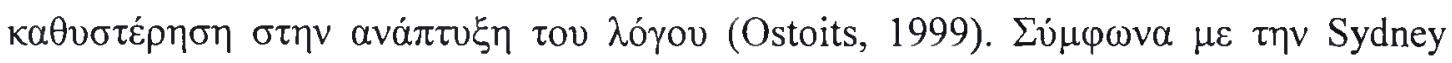

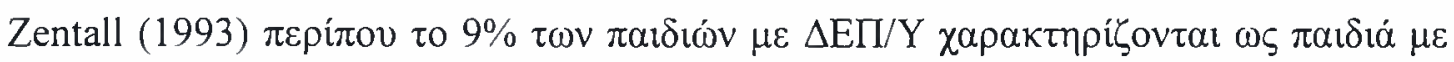

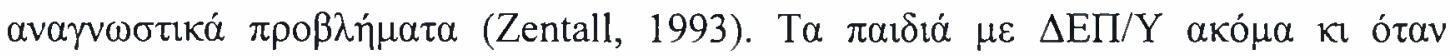

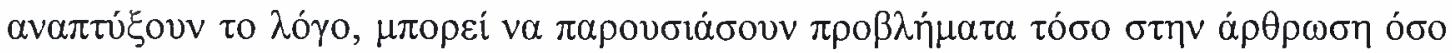

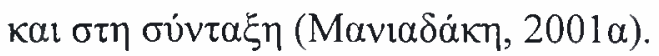

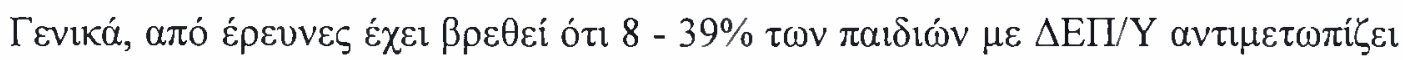

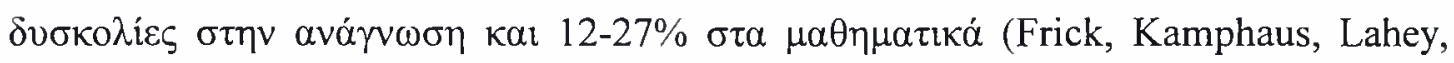

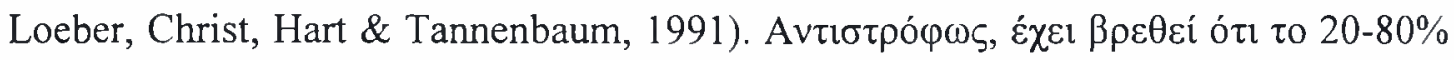

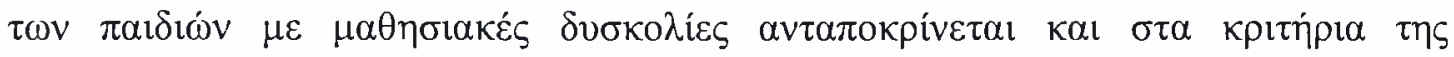

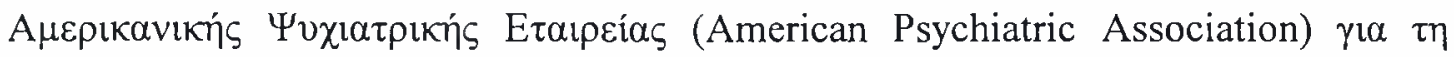
$\Delta \mathrm{E \Pi /Y} \mathrm{(Ká \kappa oupos} \mathrm{\&} \mathrm{Mavı \alpha \delta \alpha ́ \kappa \eta ,} \mathrm{2000,} \mathrm{\sigma \varepsilon \lambda .} \mathrm{104).}$

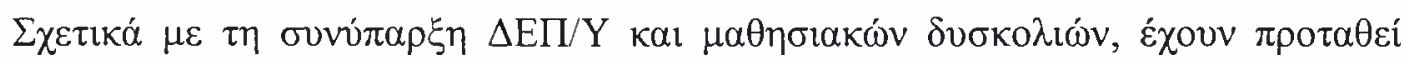

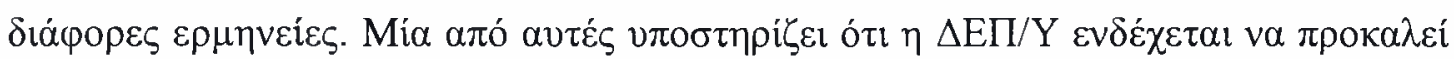

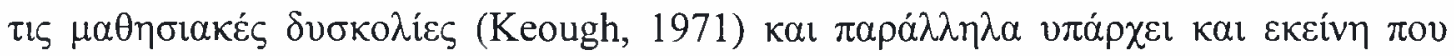

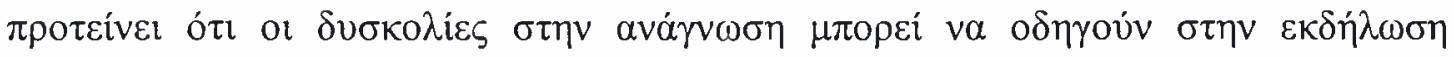

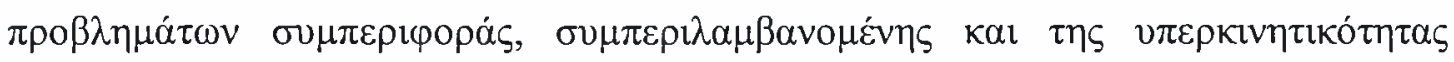
(Cunningham \& Barkley, 1978). П'́)

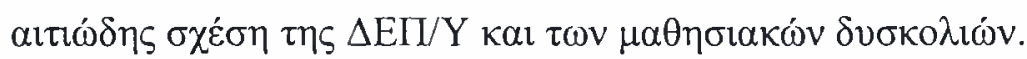

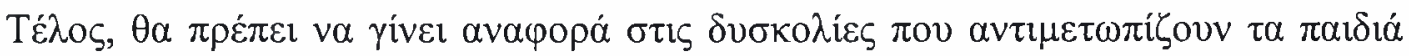

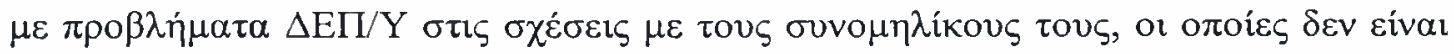

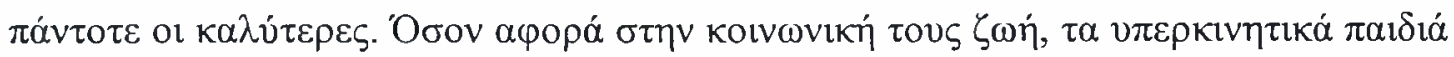

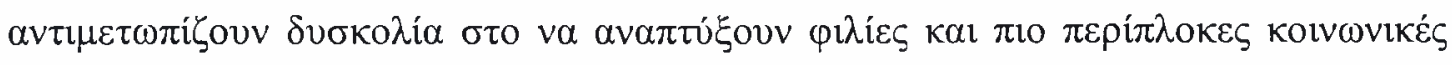

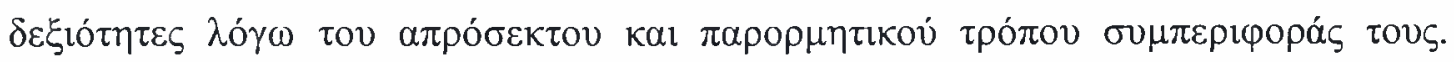

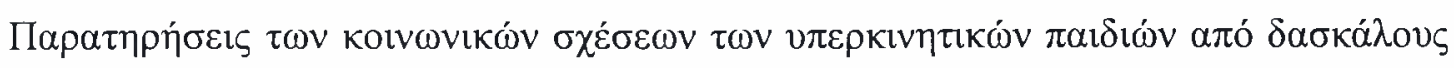

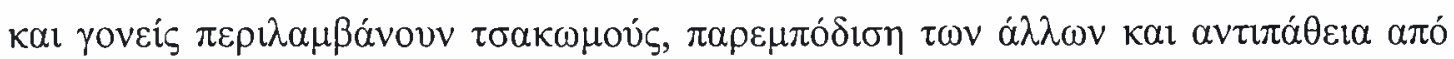

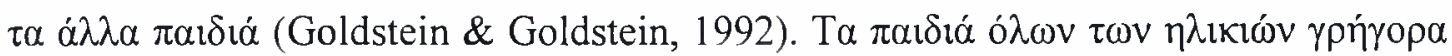

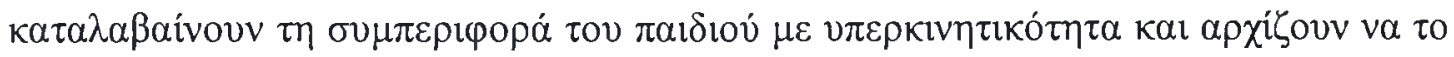

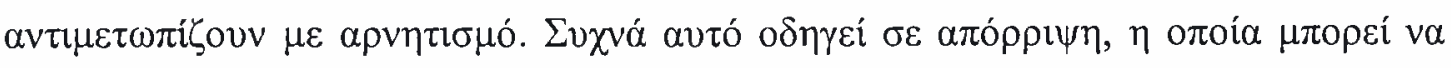

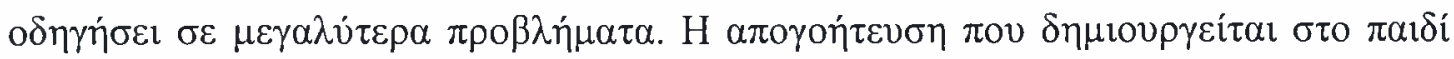




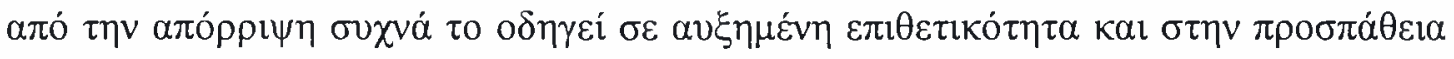

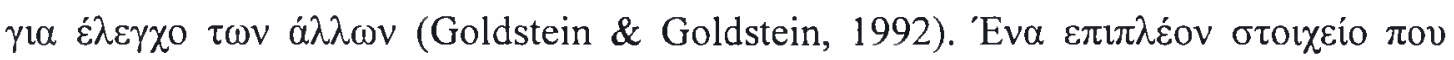

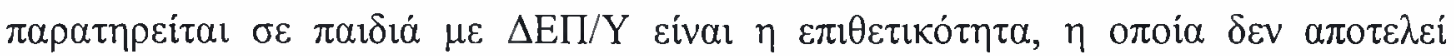

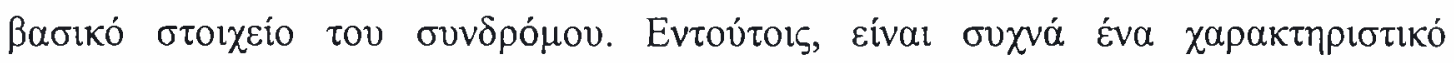

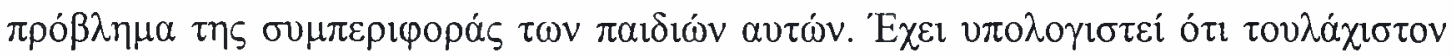

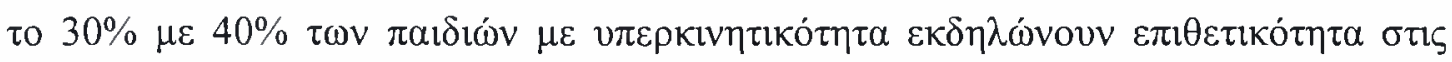

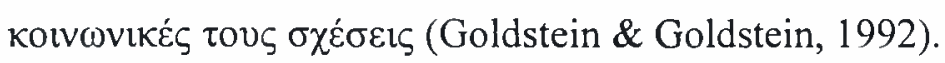

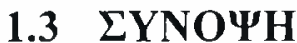

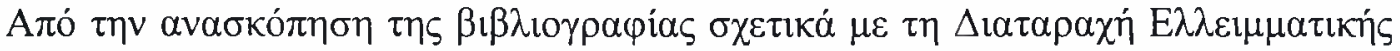

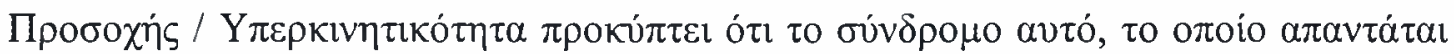

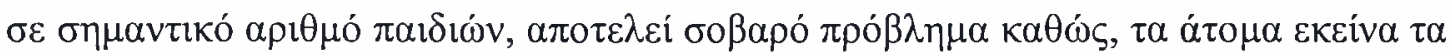

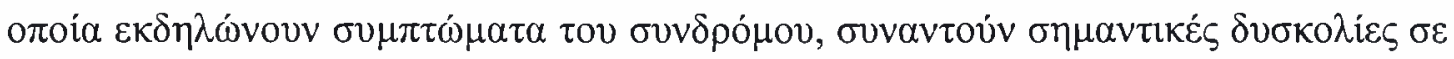

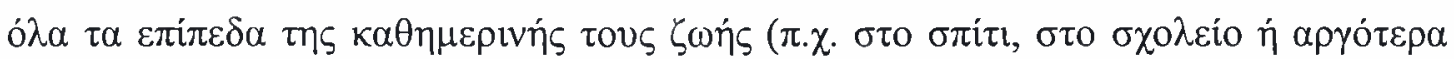

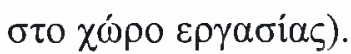

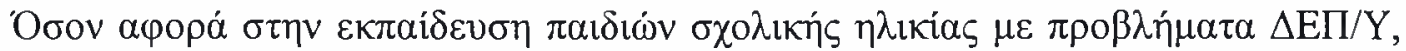

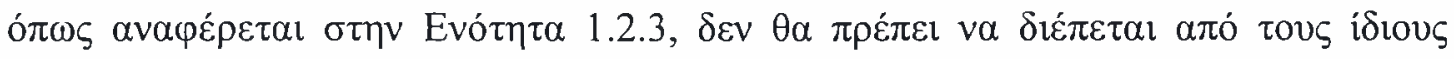

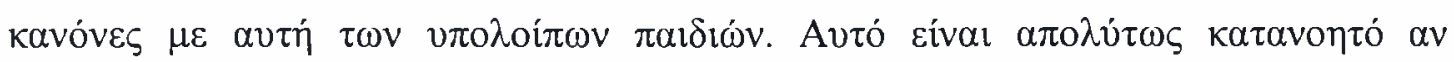

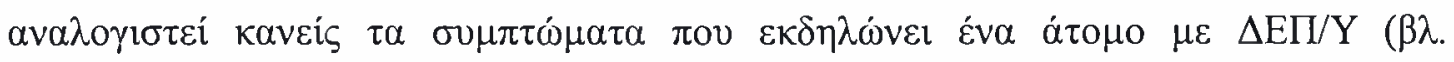

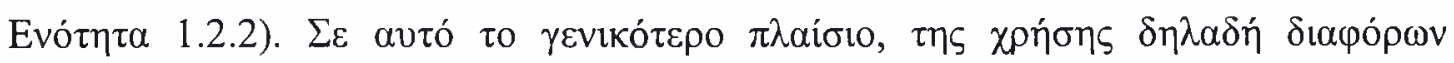

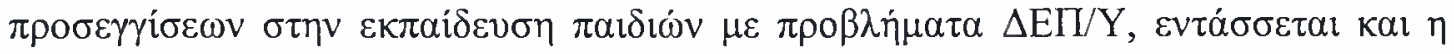

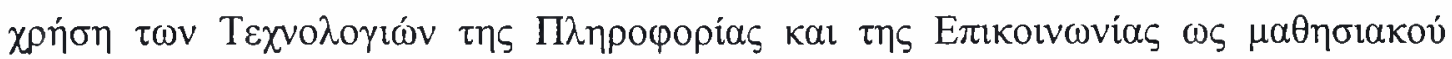

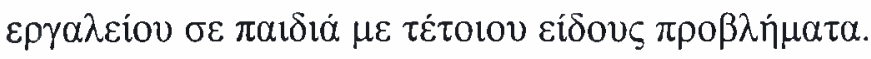




\section{$\triangle \mathrm{EYTEPO} \mathrm{KEФA \Lambda AIO}$}

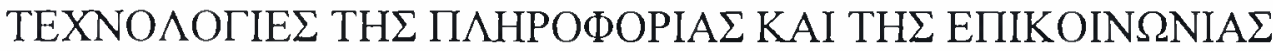 (T.П.E.) $\Sigma T H N$ EKПAI $\triangle E Y \Sigma H$}

\section{$2.1 \quad \mathrm{EI} \Sigma \mathrm{A} \Gamma \Omega \Gamma \mathrm{H}$}

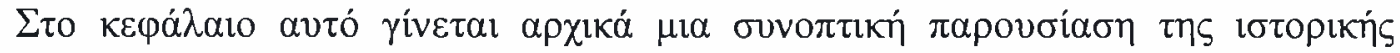

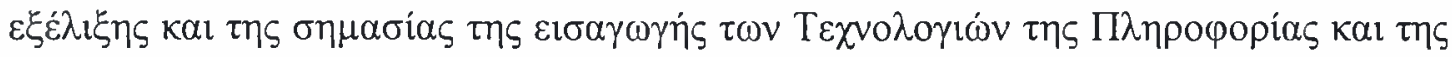

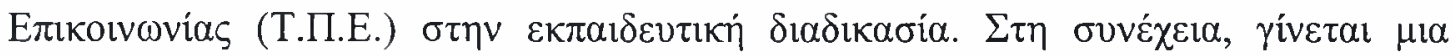

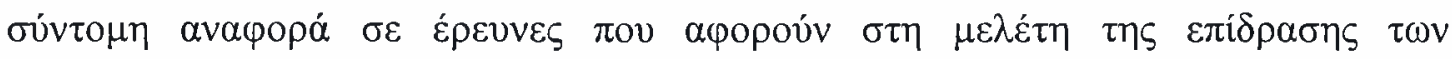

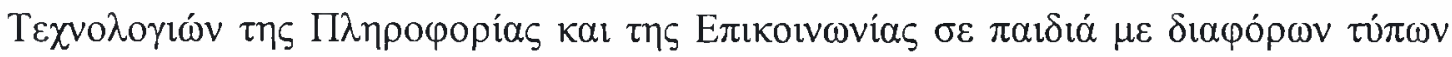

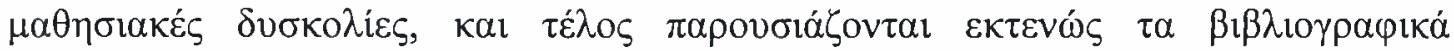

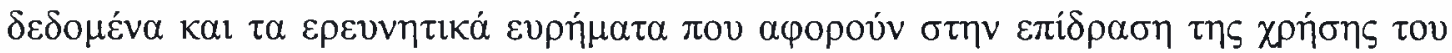

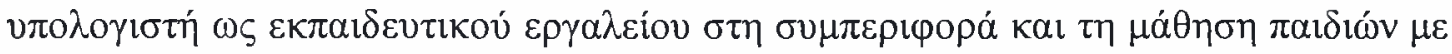
$\pi \rho \circ \beta \lambda \eta \dot{\mu \alpha} \alpha \alpha \mathrm{E \Pi /Y}$.

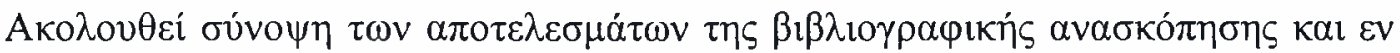

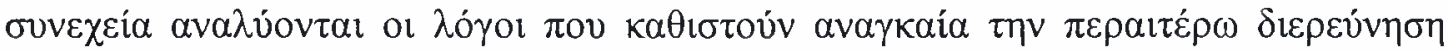

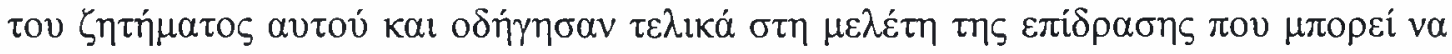

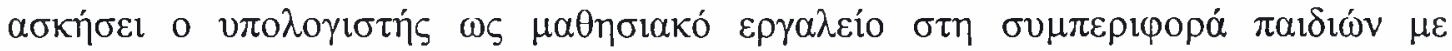
$о \mu \pi \tau \dot{\omega} \mu \alpha \tau \alpha \Delta \mathrm{E} \Pi / \mathrm{Y}$.

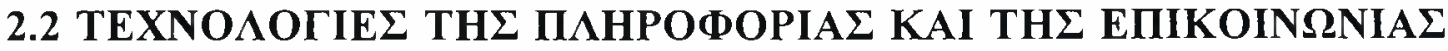

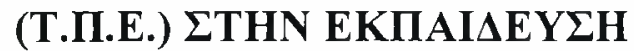

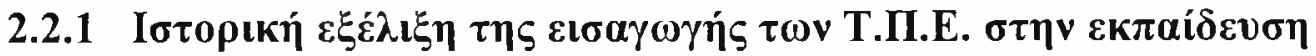

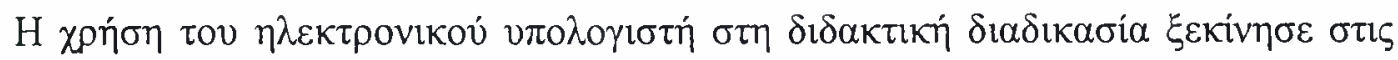

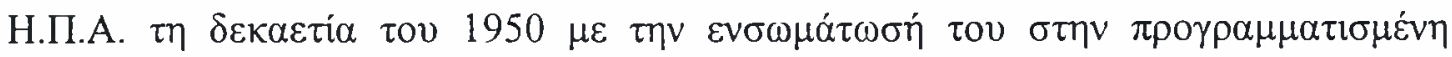




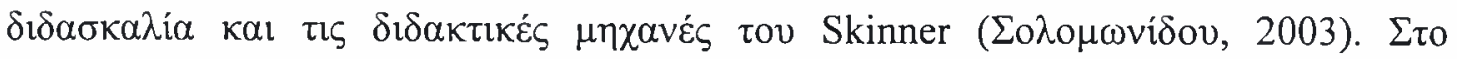

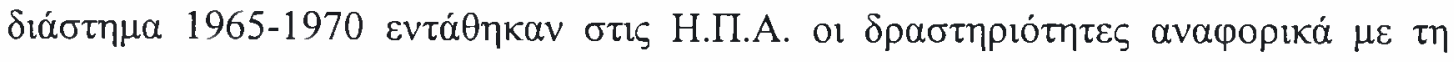

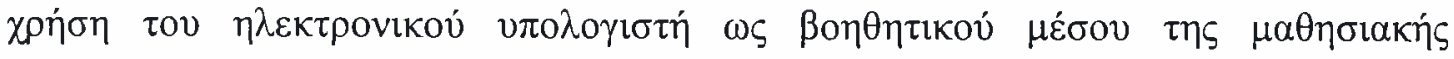

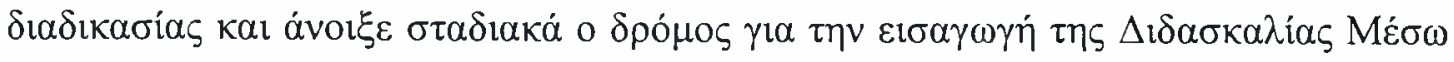

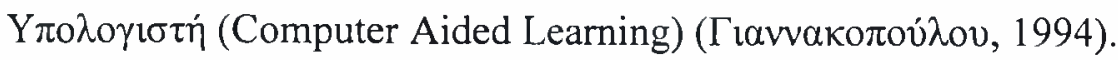

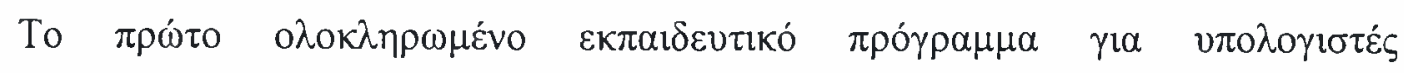

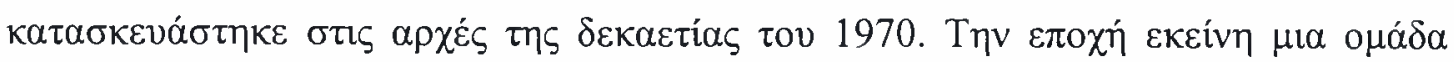

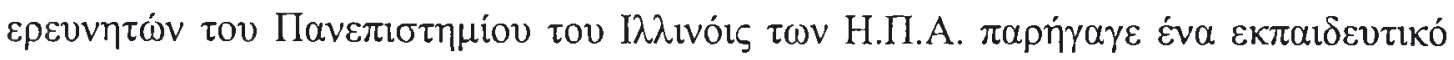

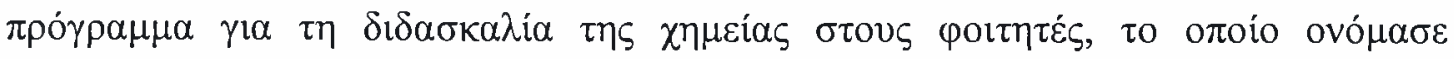

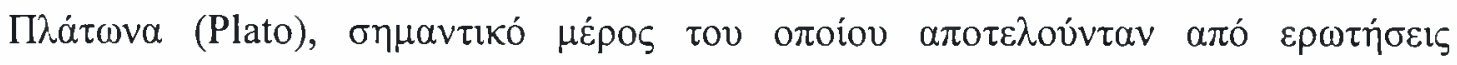

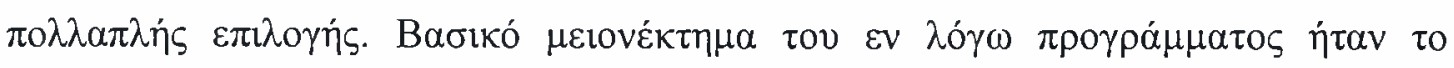

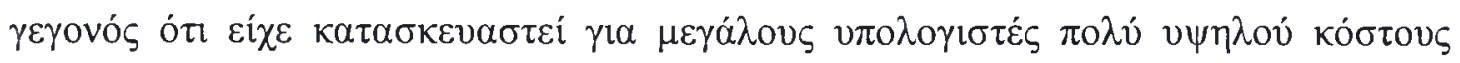

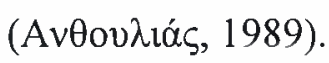

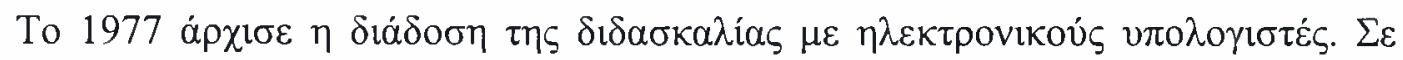

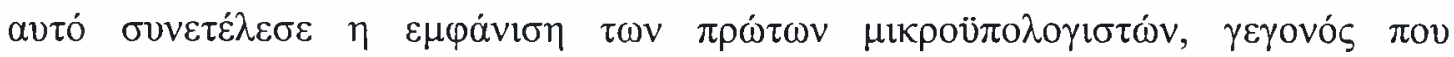

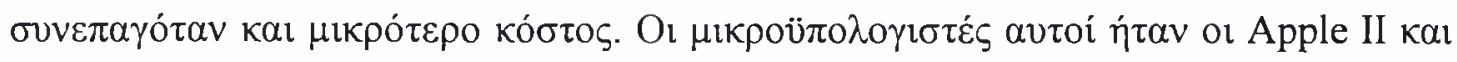

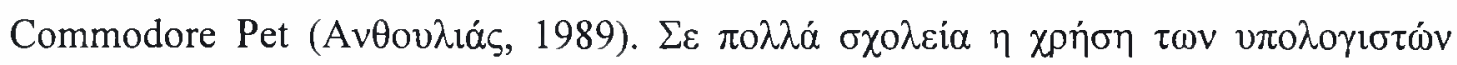

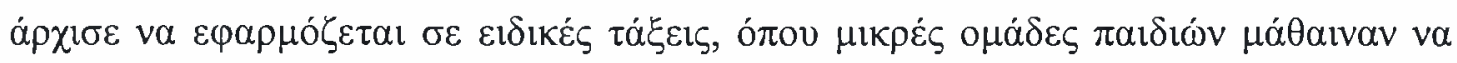

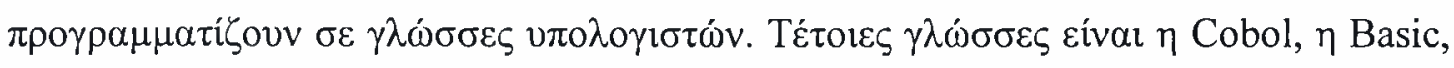
$\eta$ Fortran, $\eta$ Logo (Collins, Hammond \& Wellington, 1997).

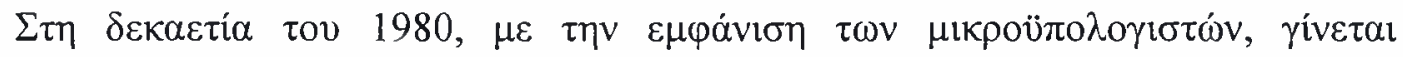

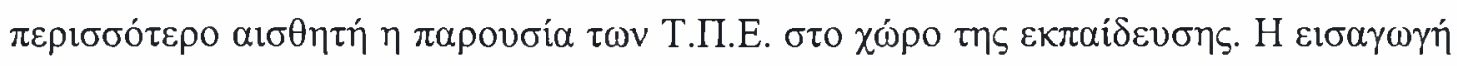

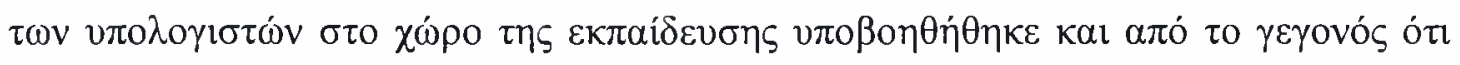

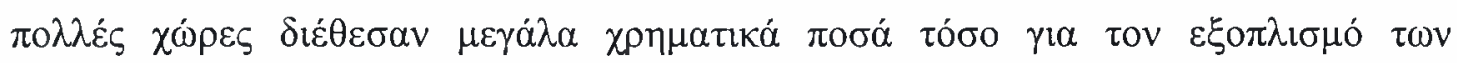

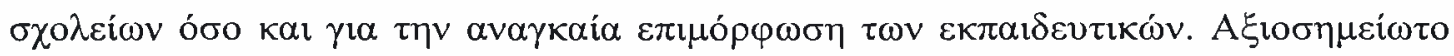

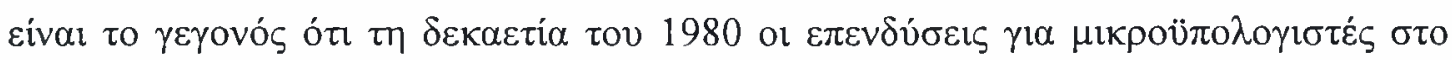

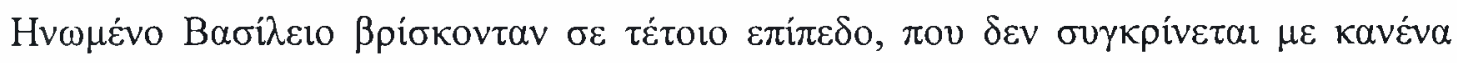

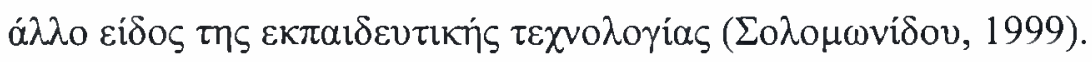

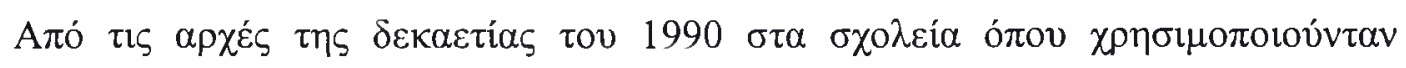

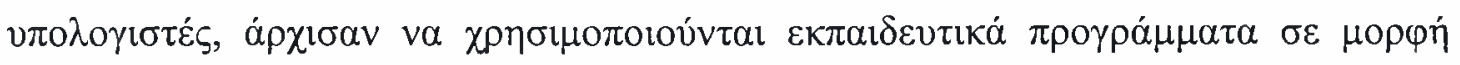

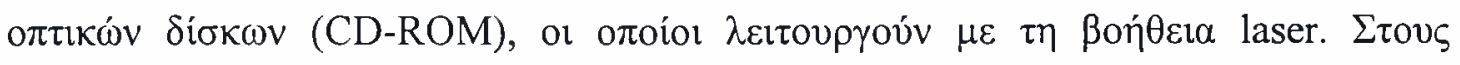

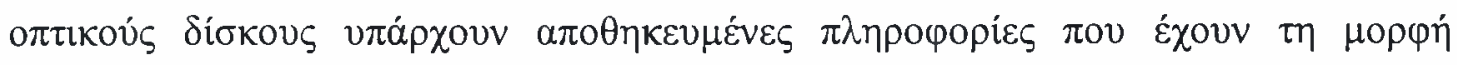

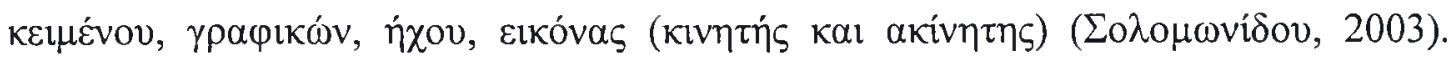

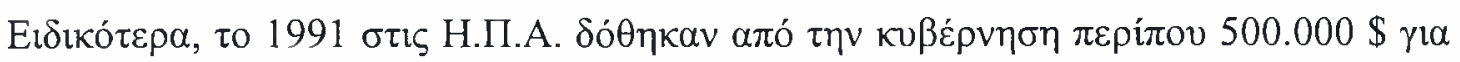




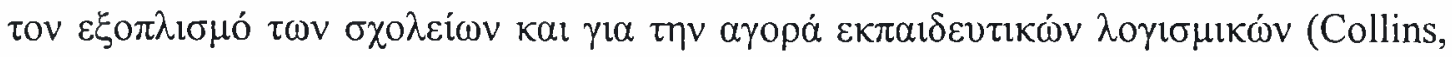

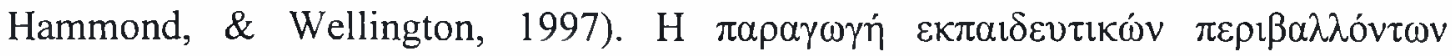

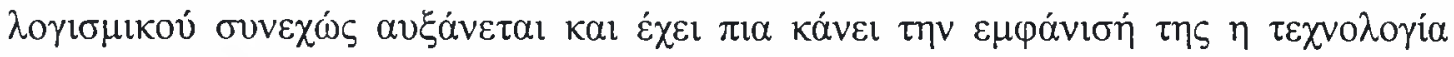

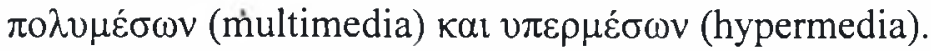

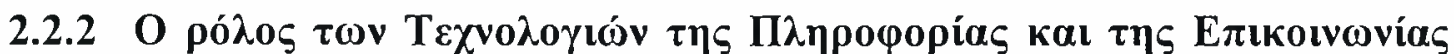

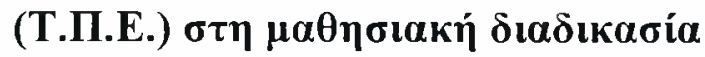

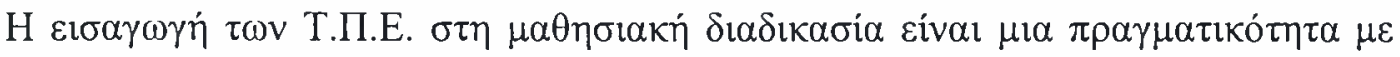
$\tau \eta \eta$ o

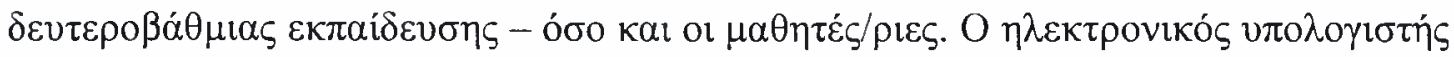

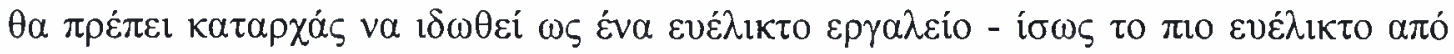

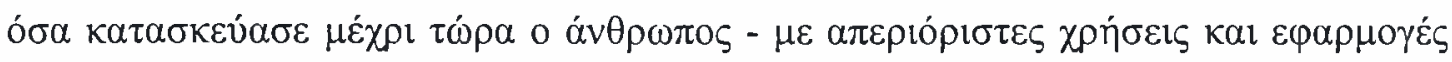

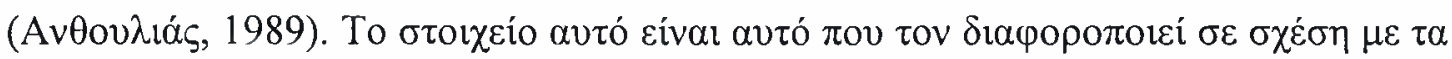

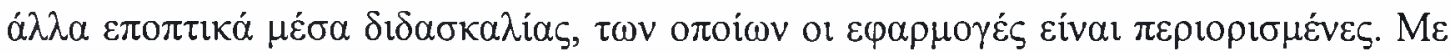

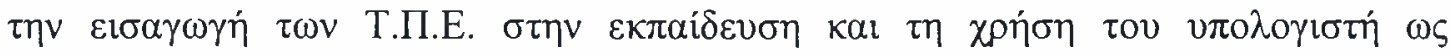

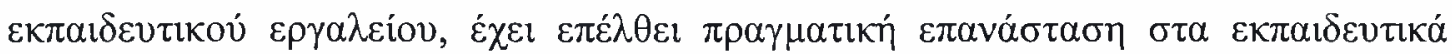
$\pi \rho \alpha ́ \gamma \mu \alpha \tau \alpha$.

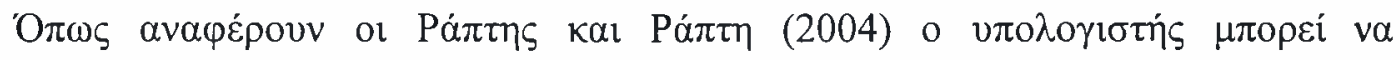

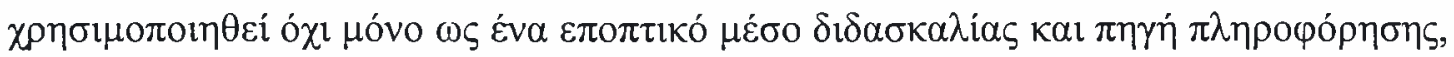

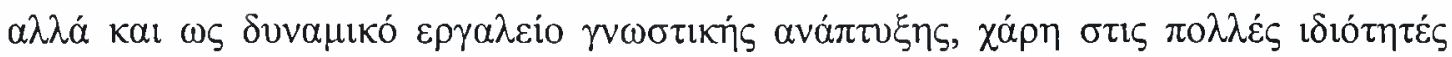

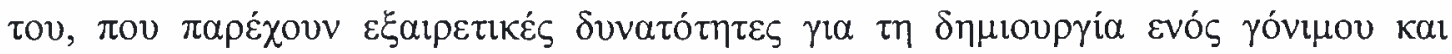

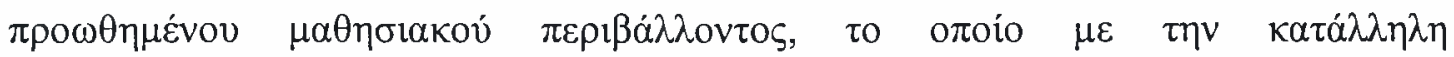

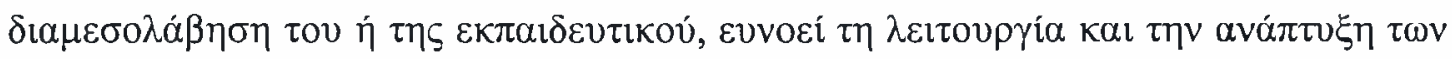

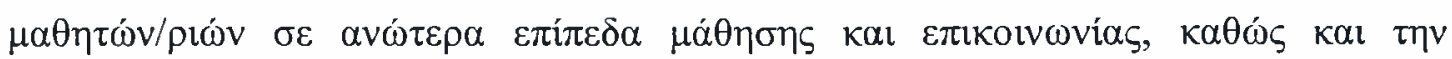

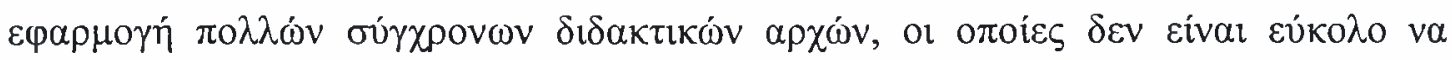

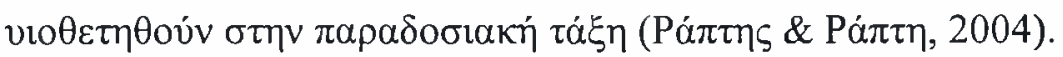

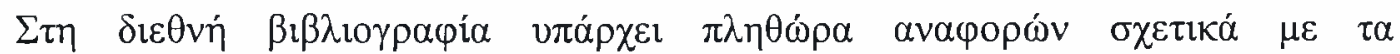

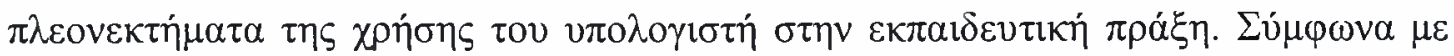

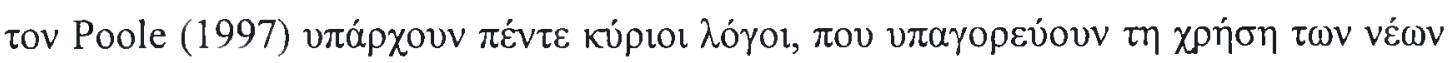

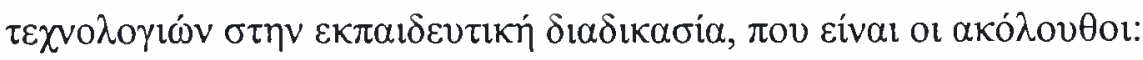

$\Rightarrow \eta v \pi \circ \beta \circ \eta \dot{\theta} \eta \sigma \eta \tau \eta \varsigma \mu \alpha \dot{\theta} \eta \sigma \eta \varsigma$, 


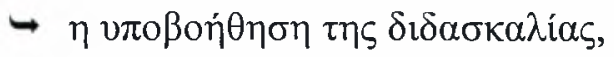

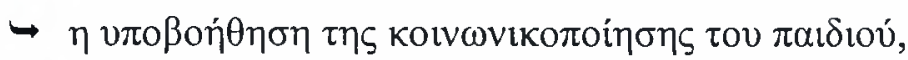

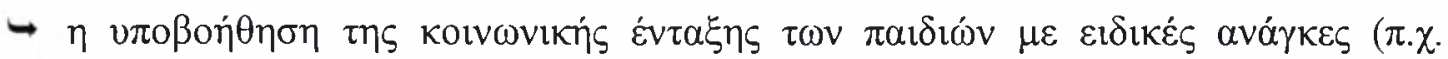

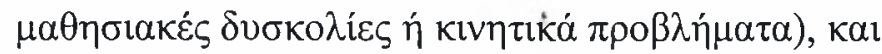

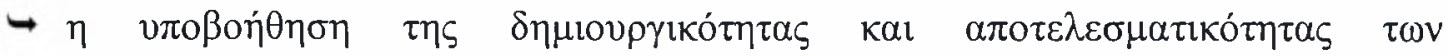
$\varepsilon \kappa \pi \alpha \iota \delta \varepsilon v \tau \kappa \kappa \dot{v}$.

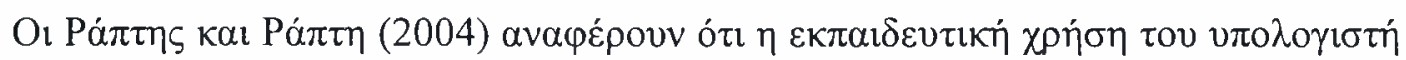

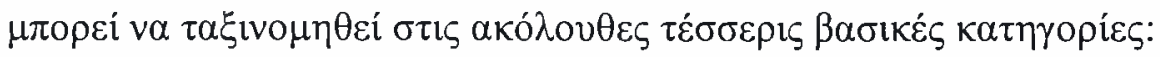

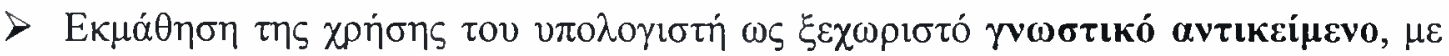

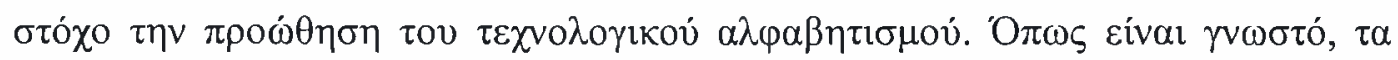

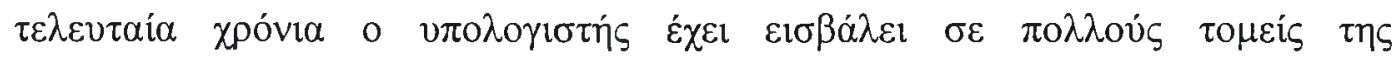

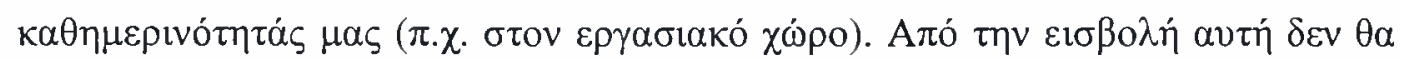

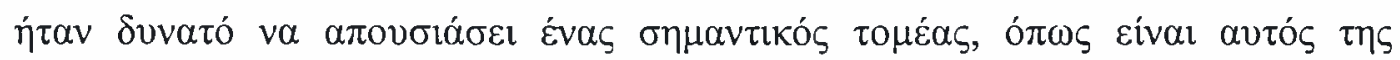

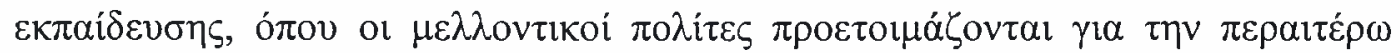

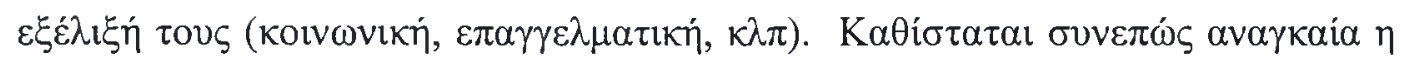

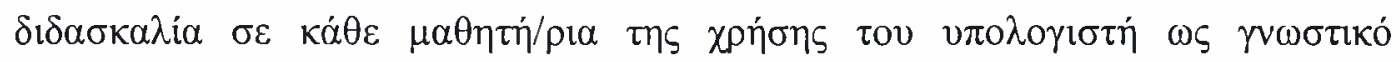

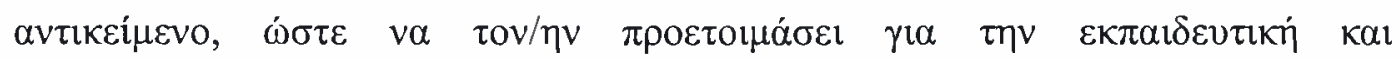

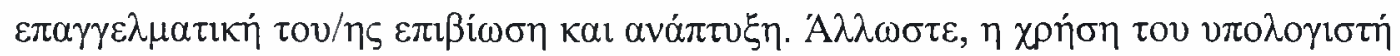

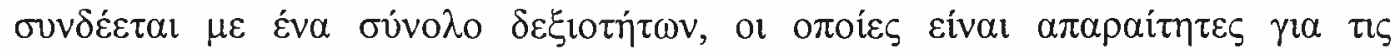

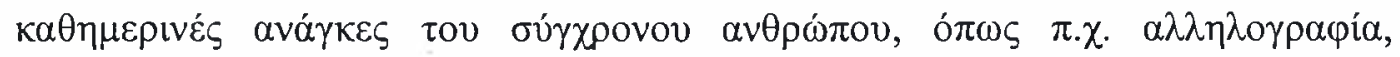

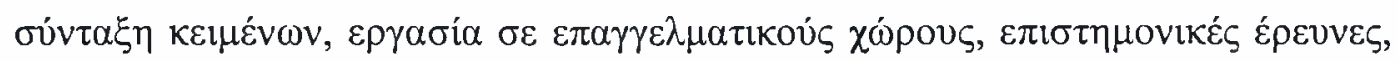

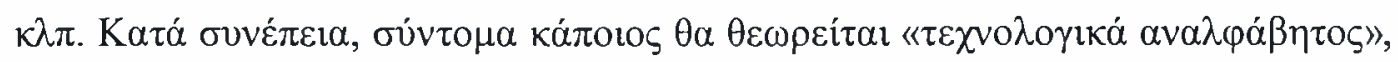

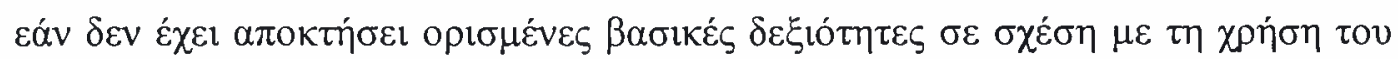

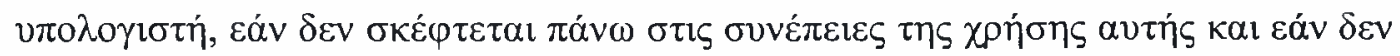

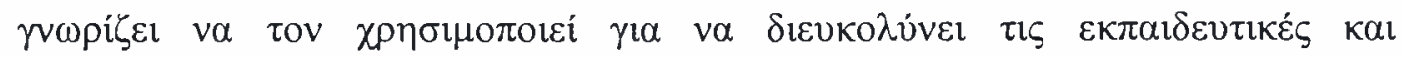
$\varepsilon \pi \alpha \gamma \gamma \varepsilon \lambda \mu \alpha \tau$ «

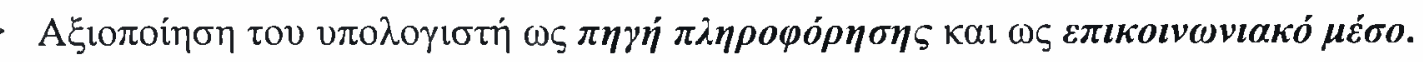

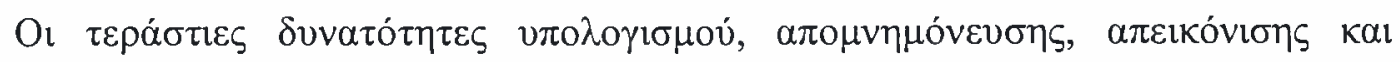

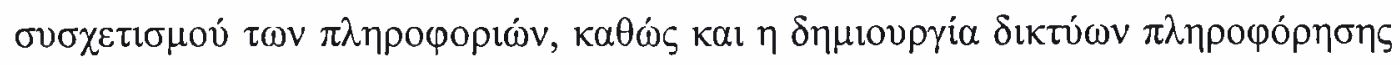

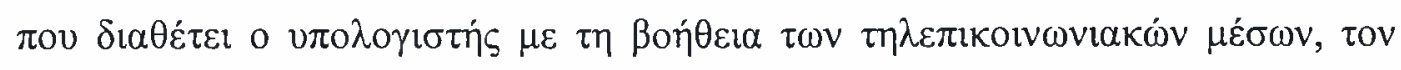

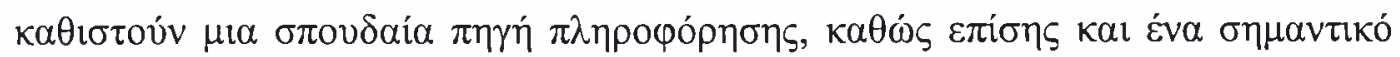

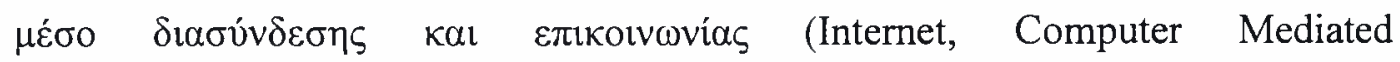

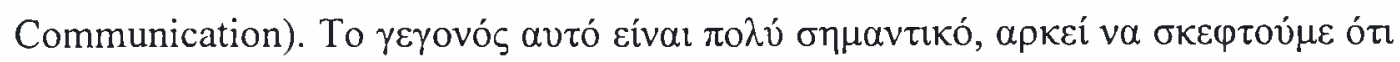




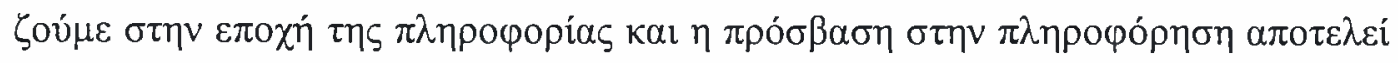

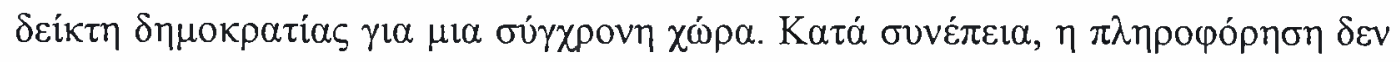

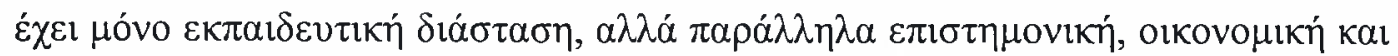

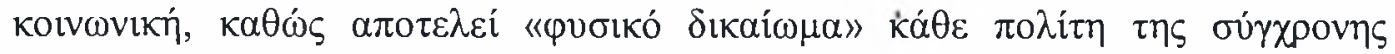

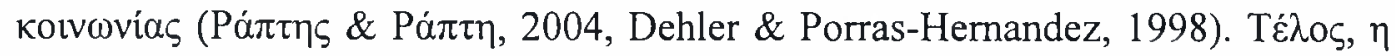

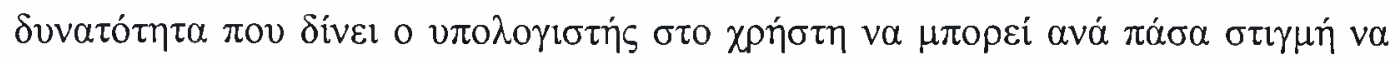

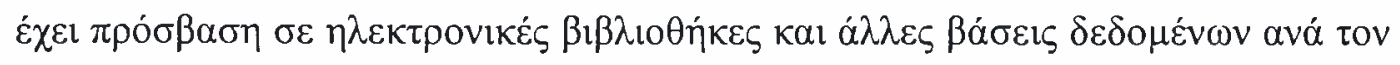

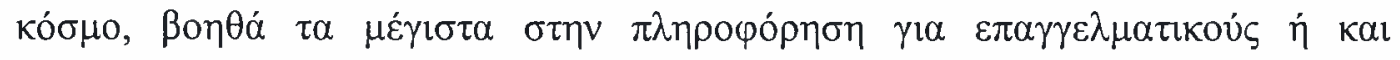

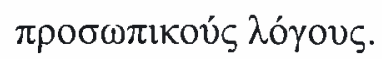

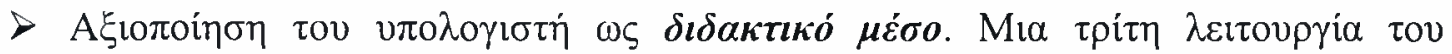

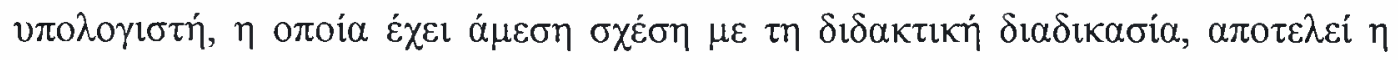

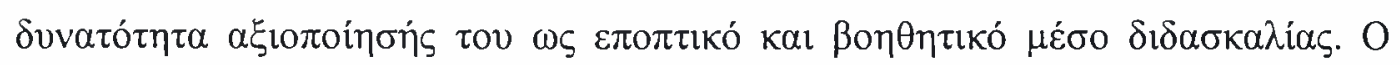

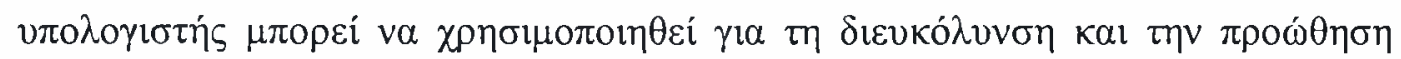

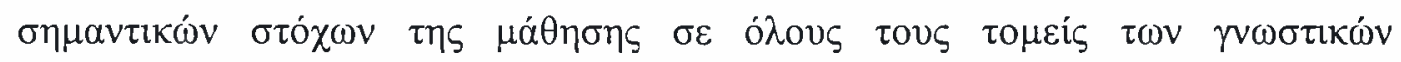

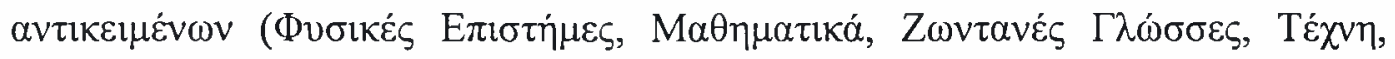

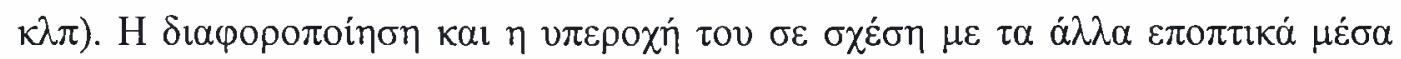

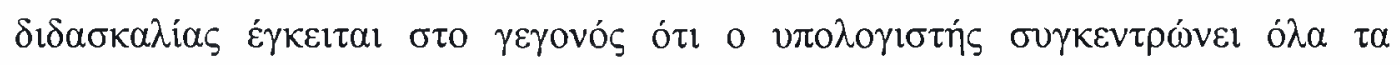

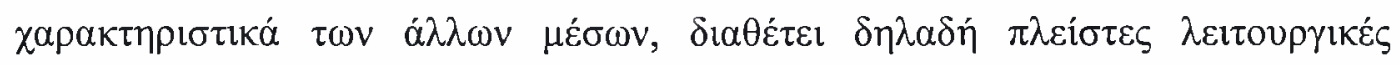

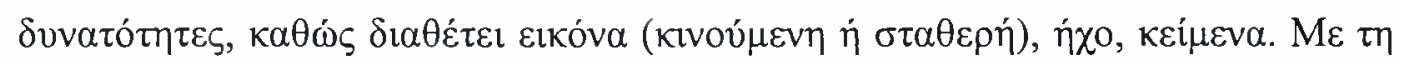

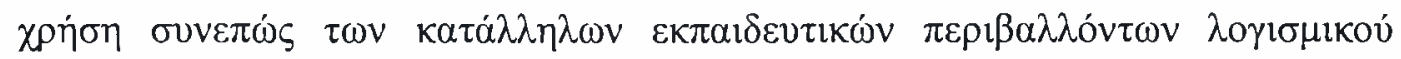

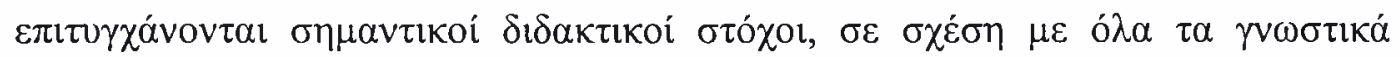

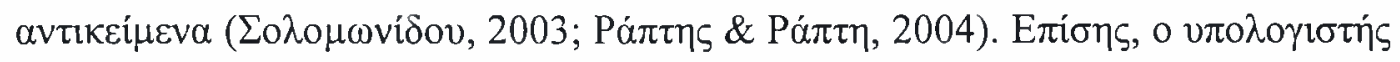

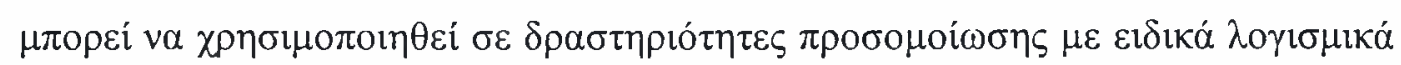

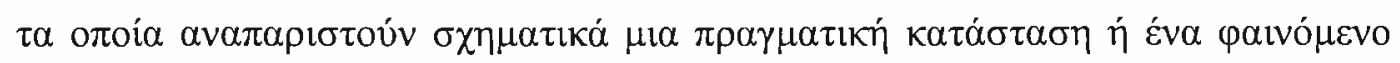

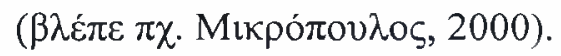

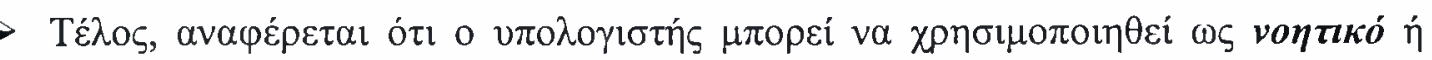

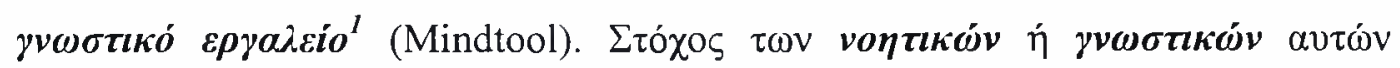

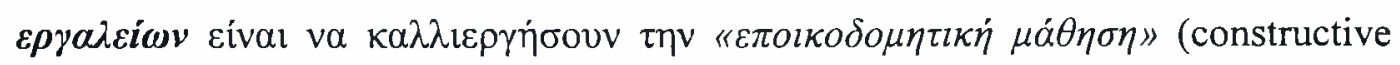

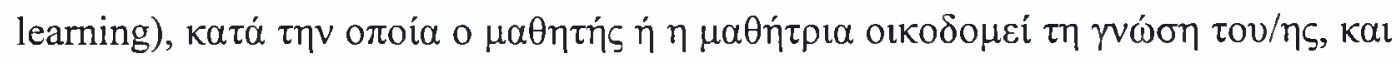

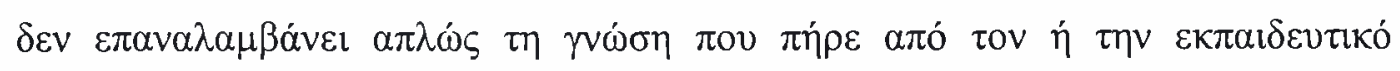

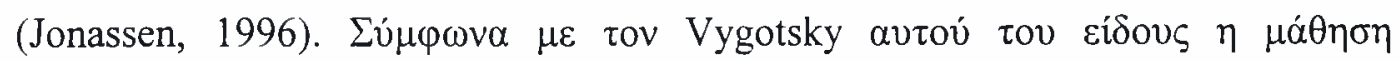

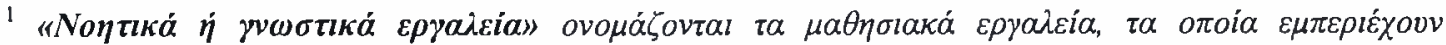

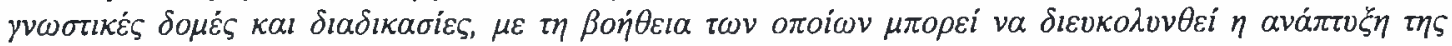

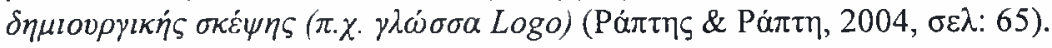




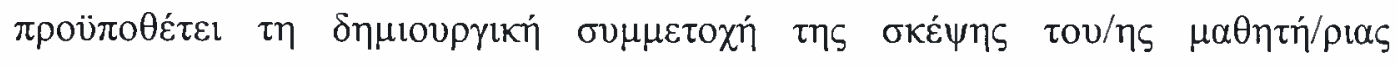

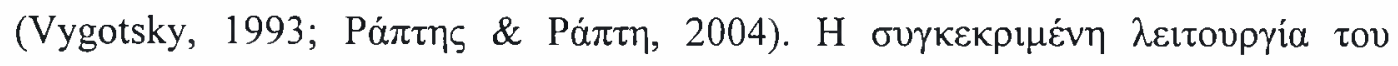

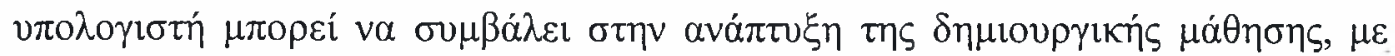

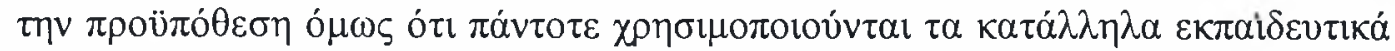

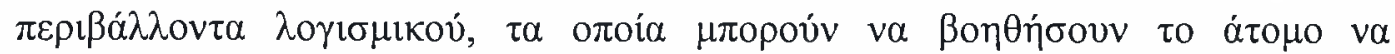

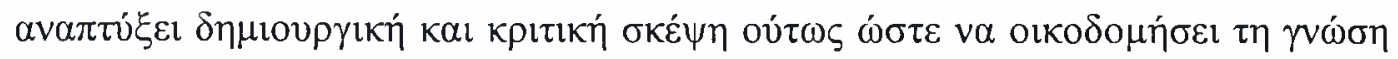

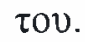

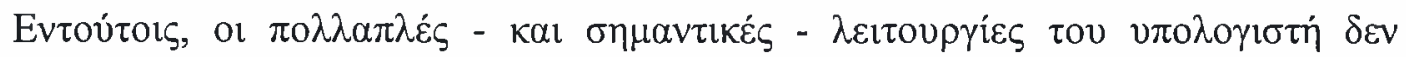

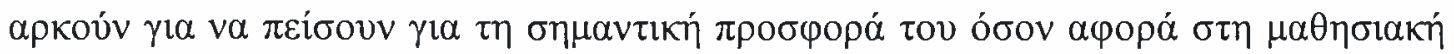

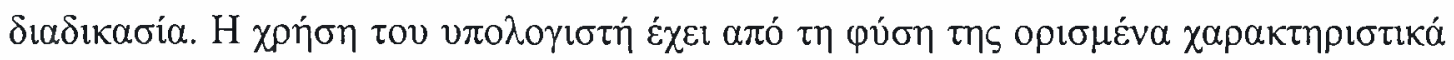

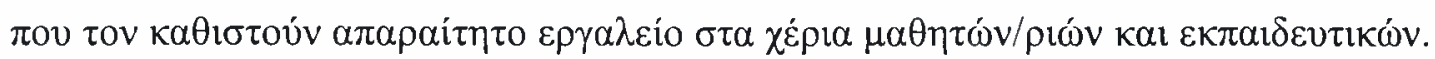

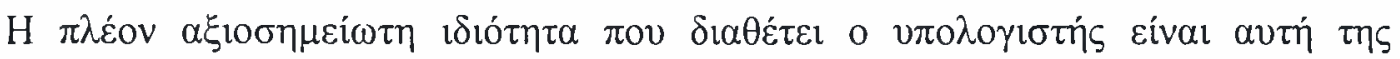

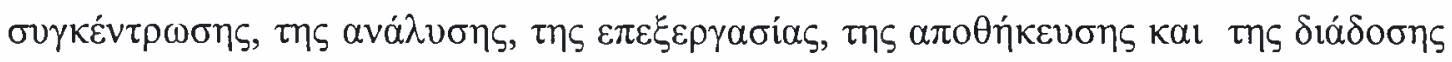

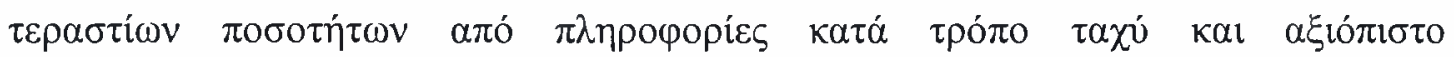

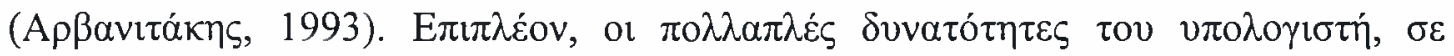

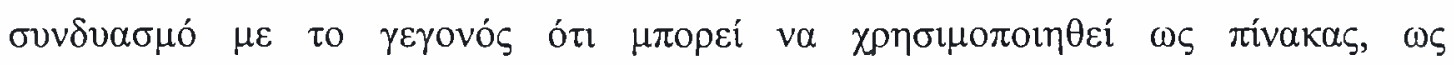

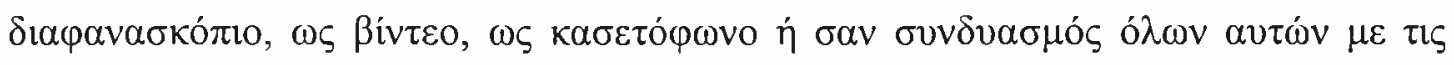

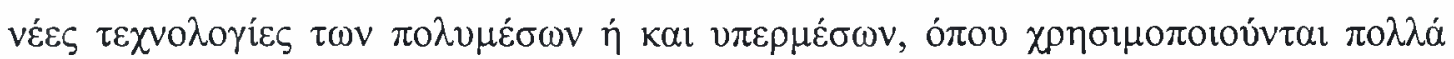

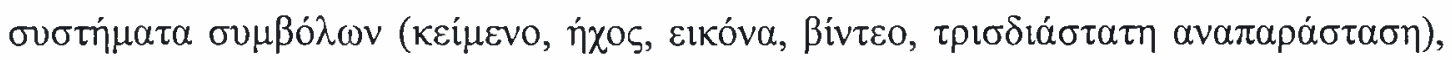

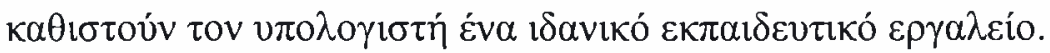

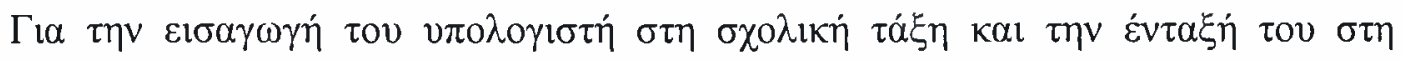

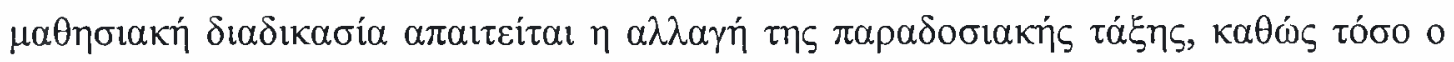

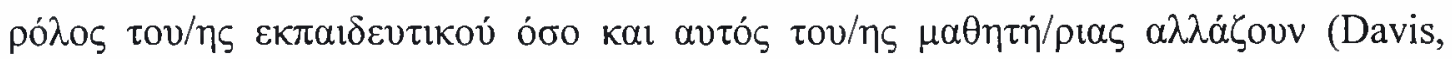

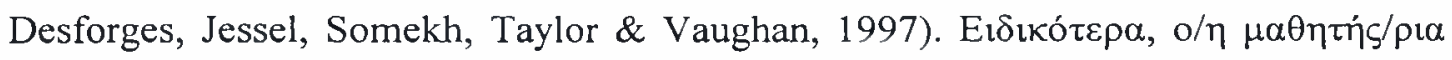

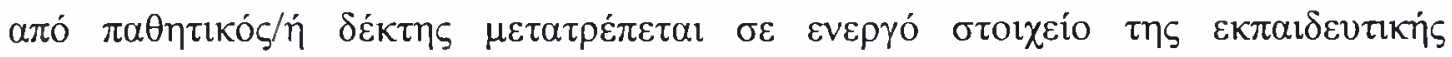

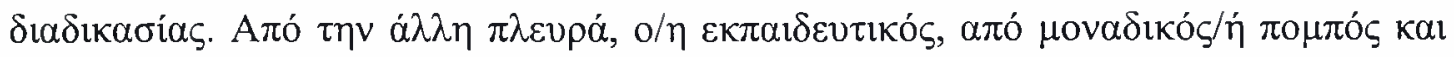

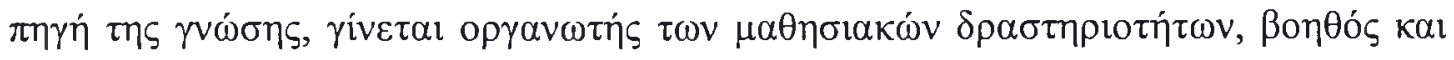

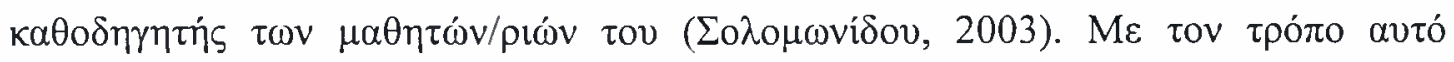

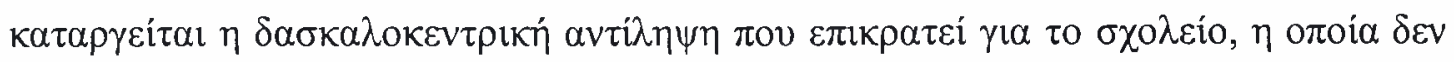

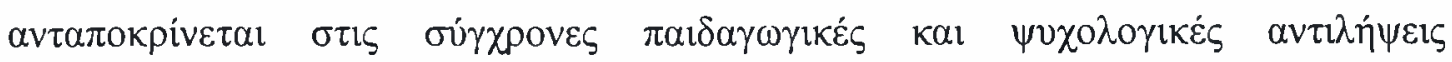

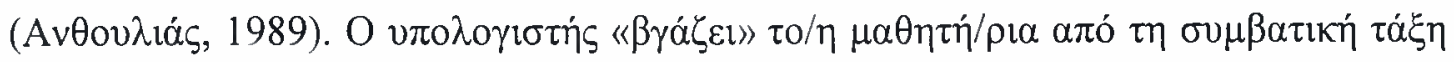

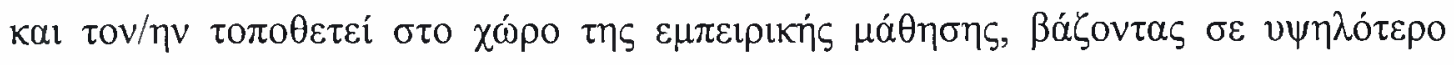

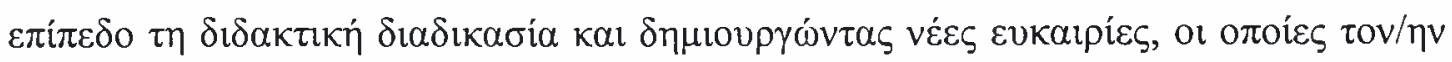




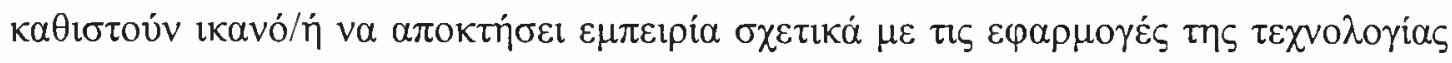
(Dehler \& Porras-Hernandez, 1998).

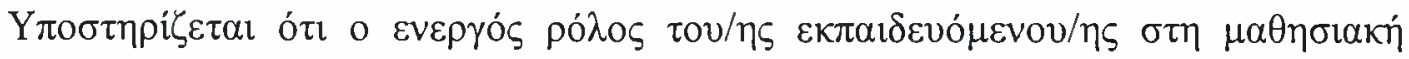

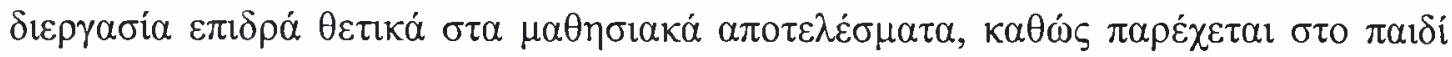

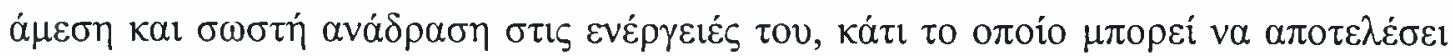

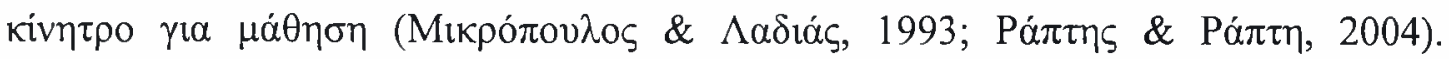

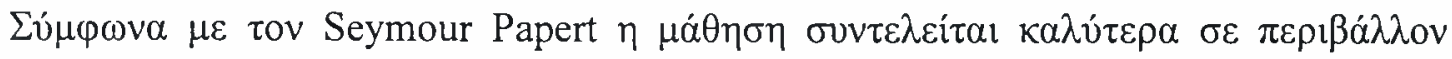

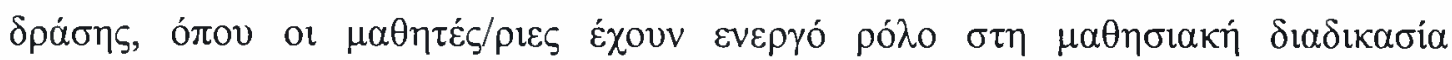

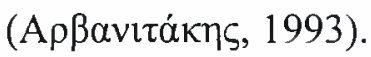

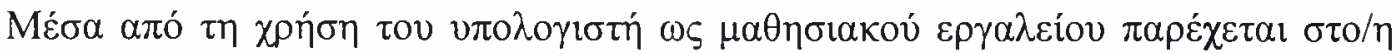

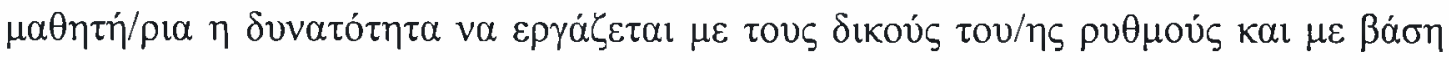

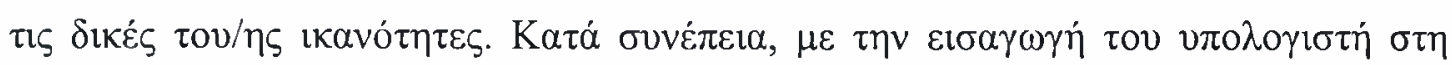

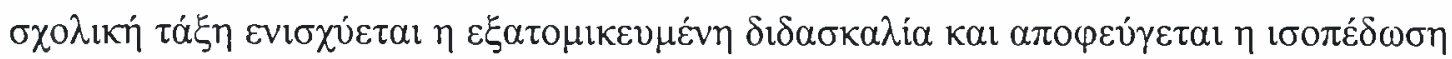

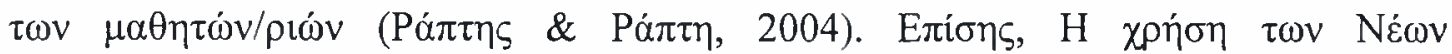

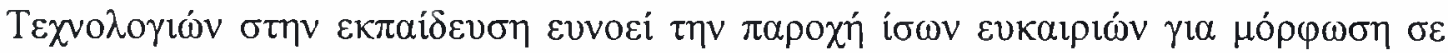

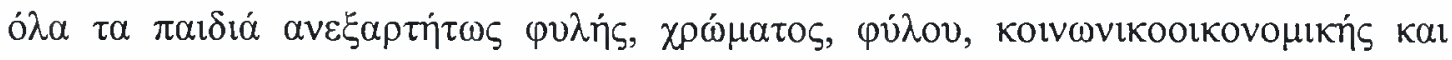

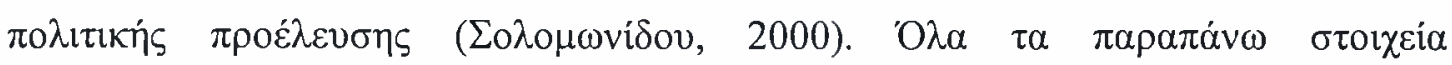

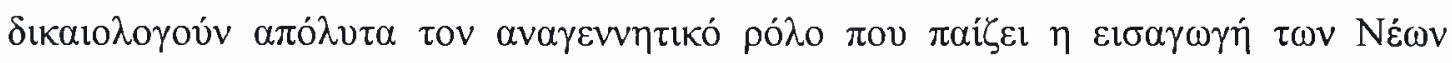

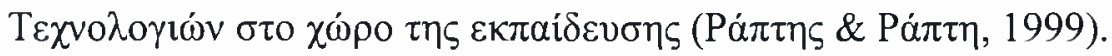

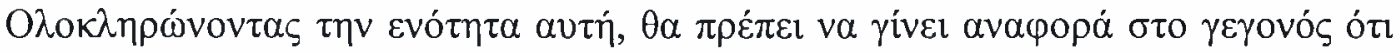

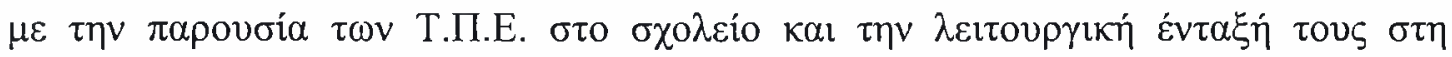

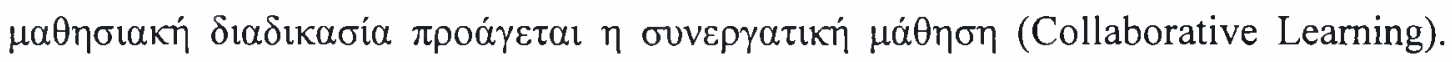

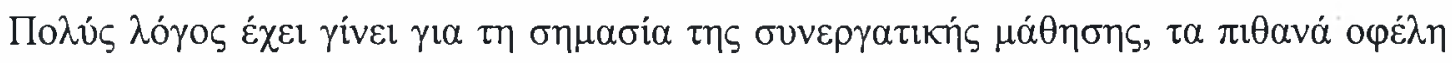

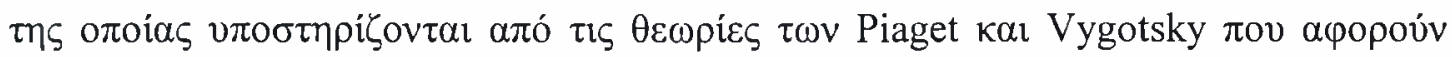

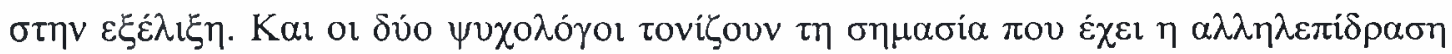

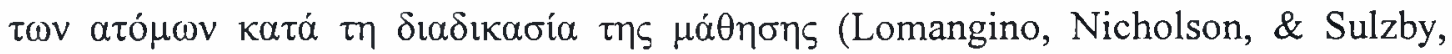
1999).

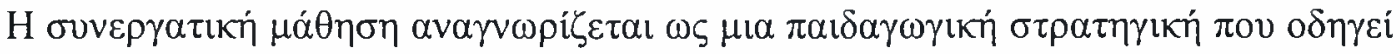

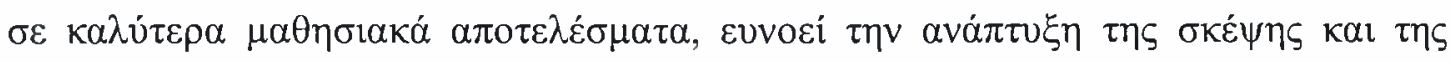

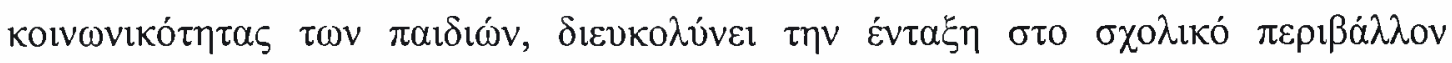

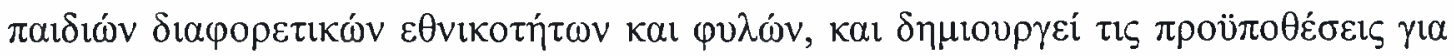

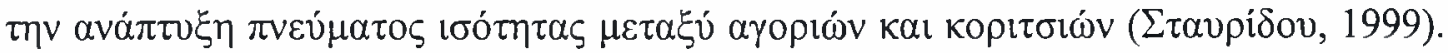

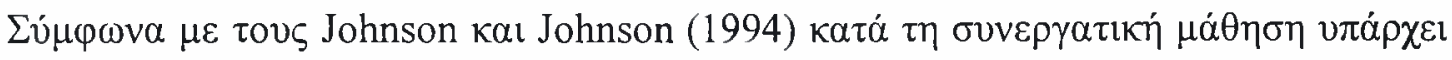

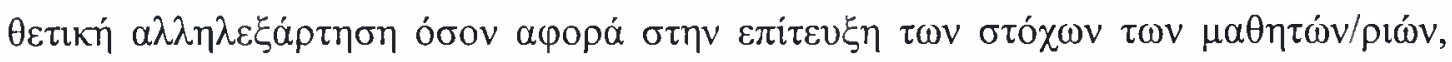




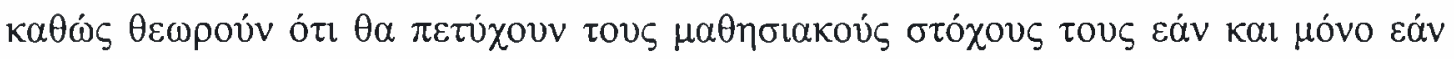

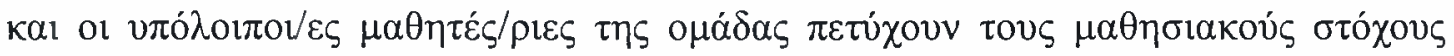

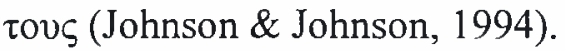

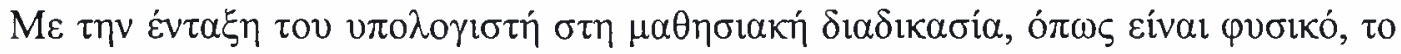

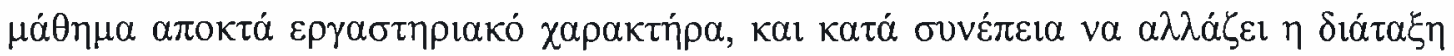

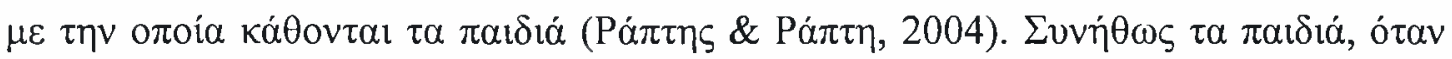

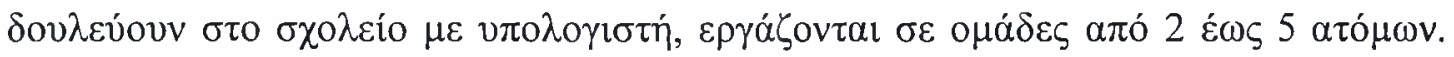

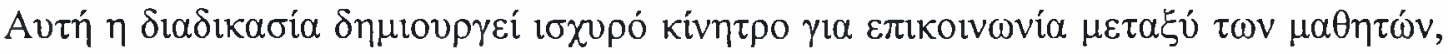

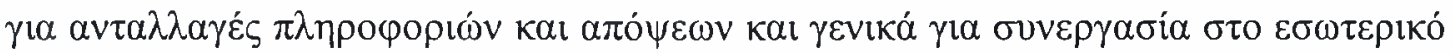

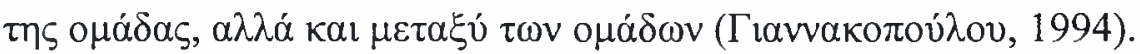

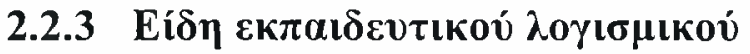

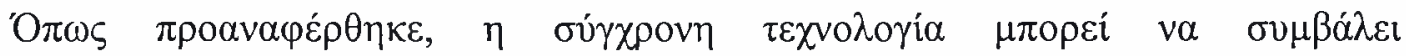

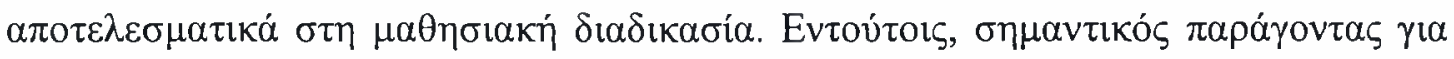

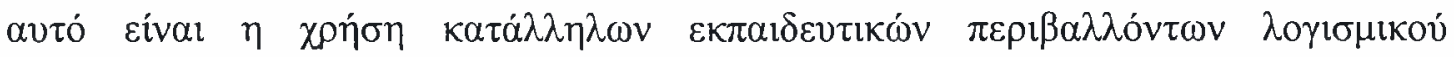

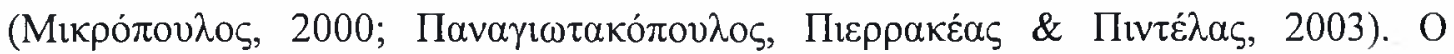

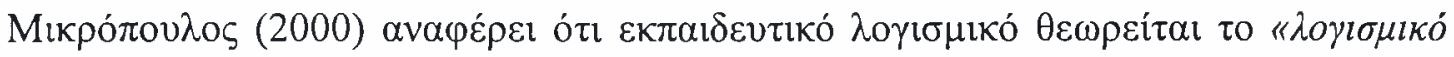

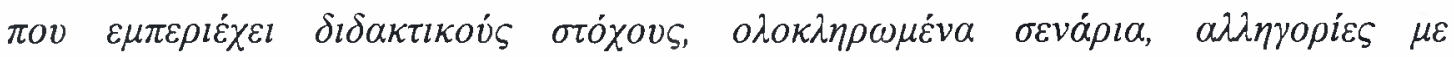

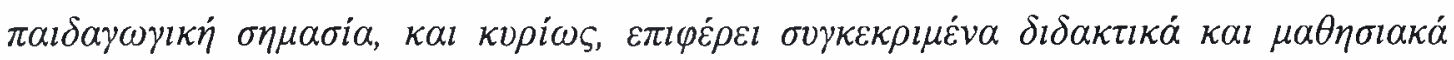

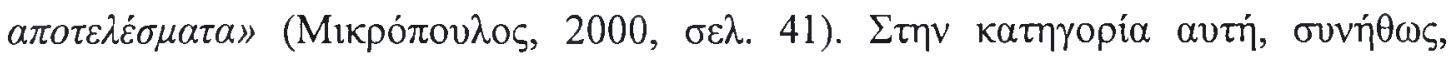

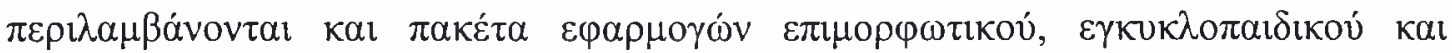

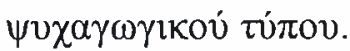

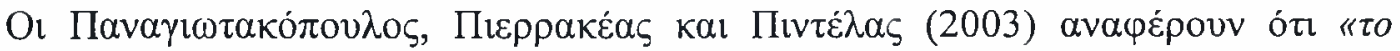

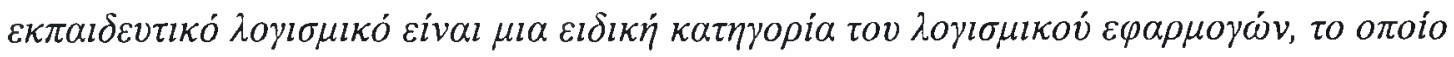

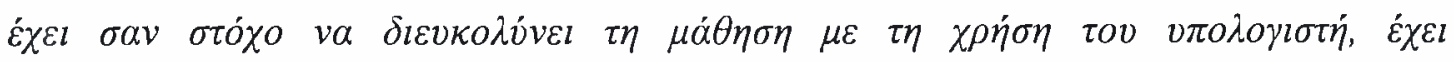

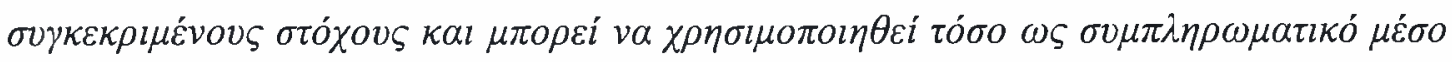

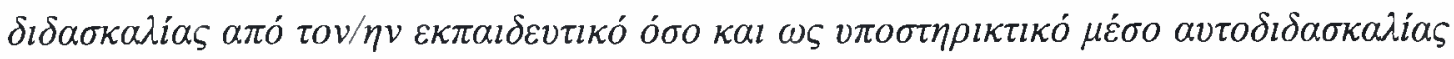

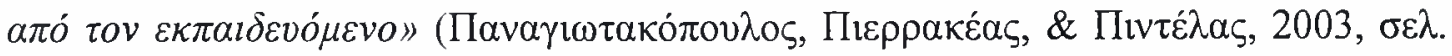
21).

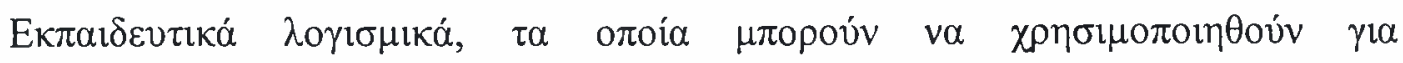

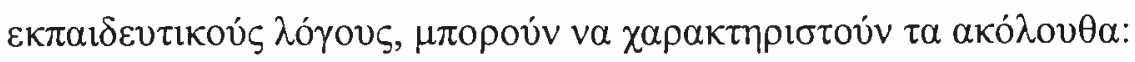




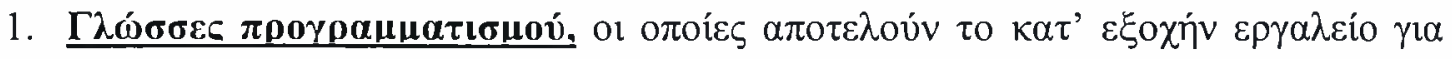

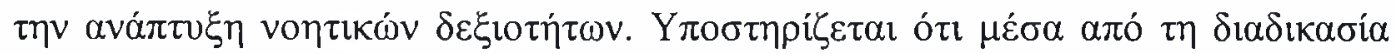

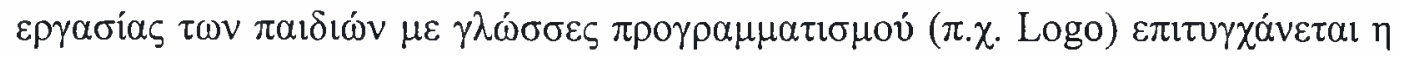

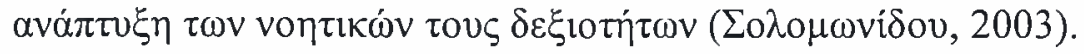

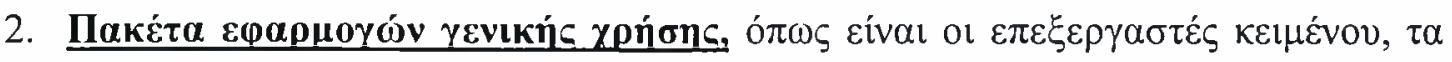

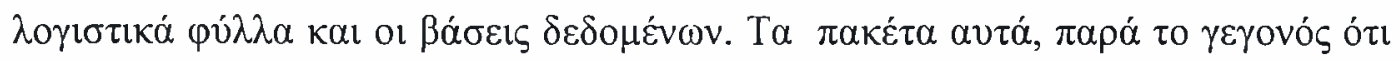

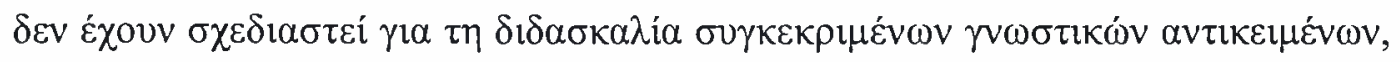

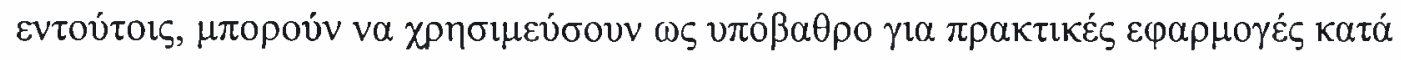

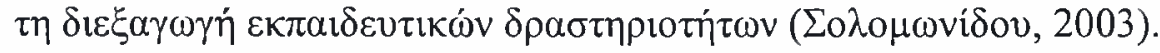

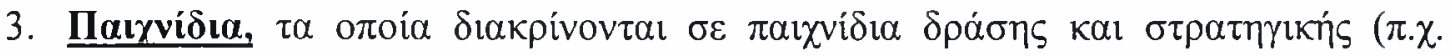

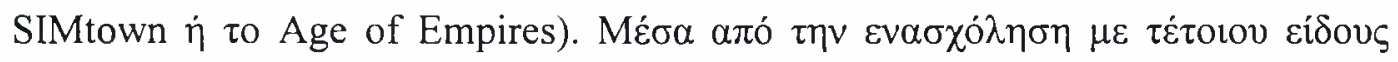

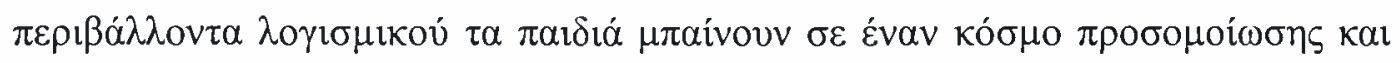

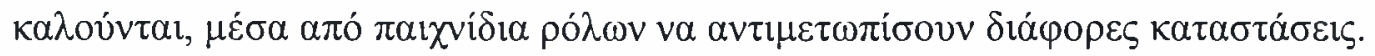

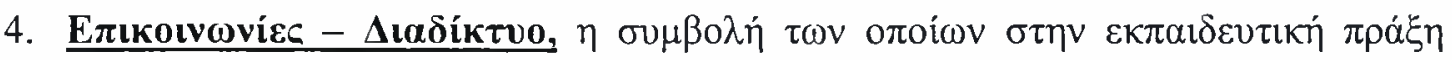

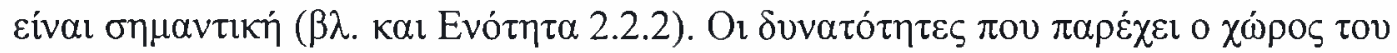

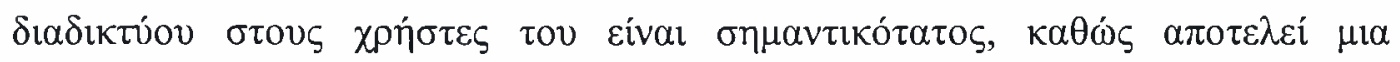

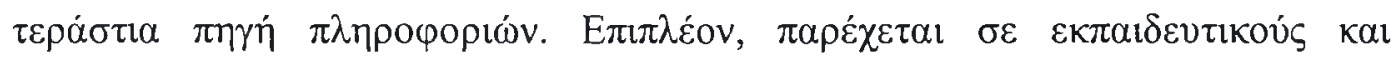

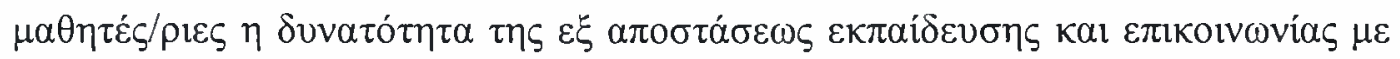

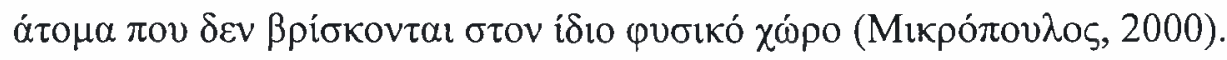

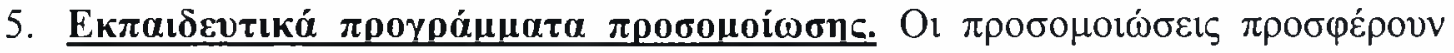

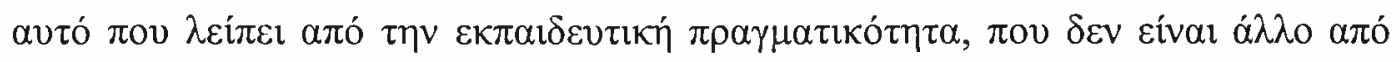

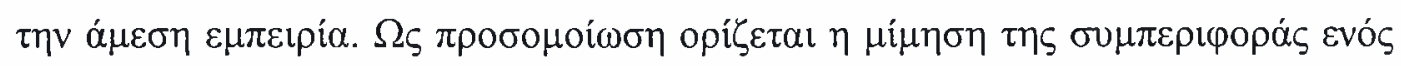

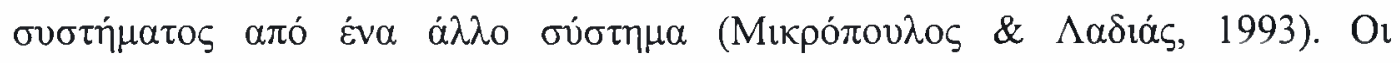

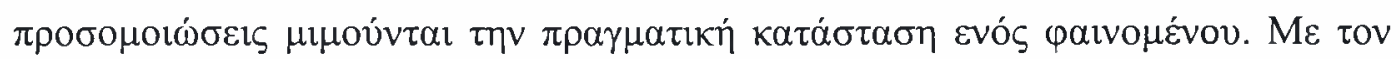

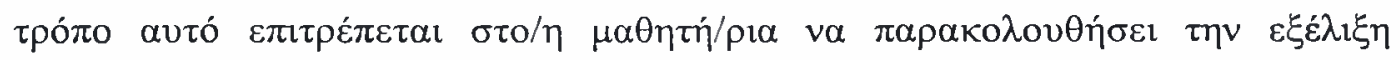

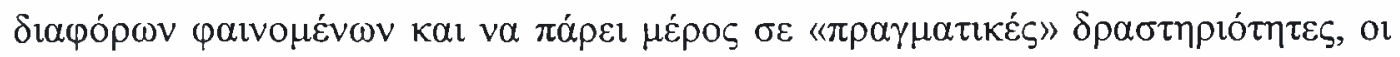

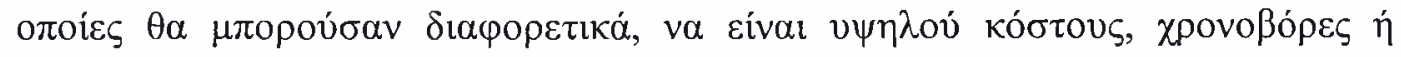

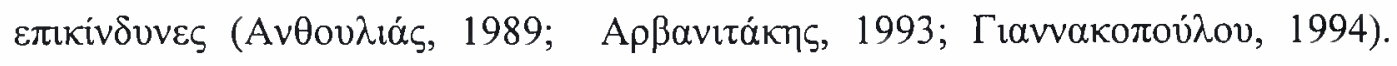

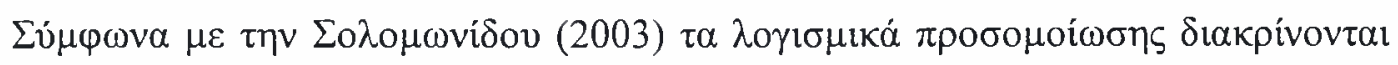

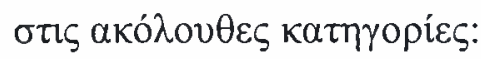

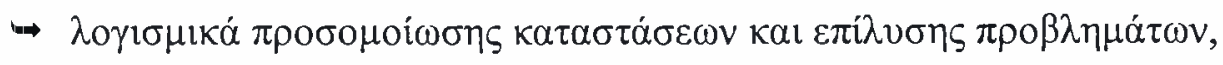

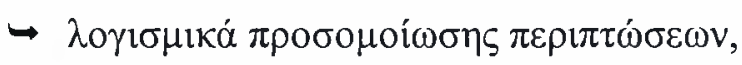

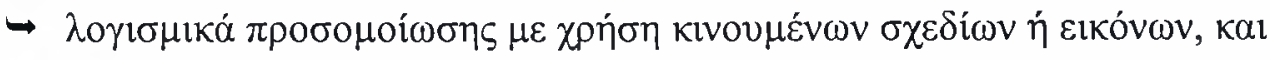

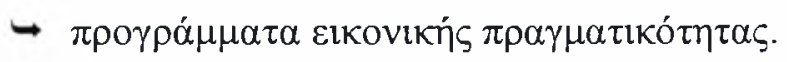




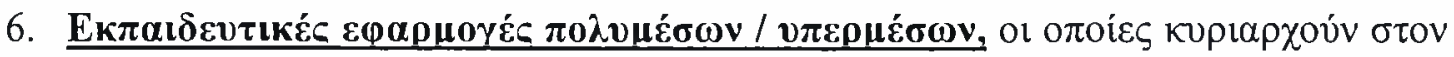

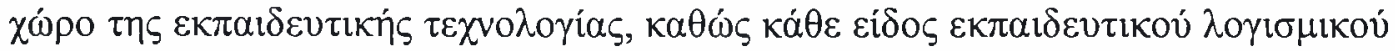

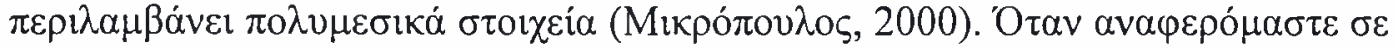

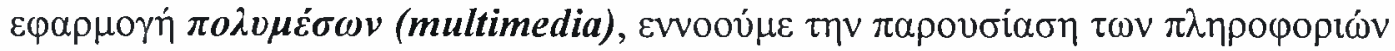

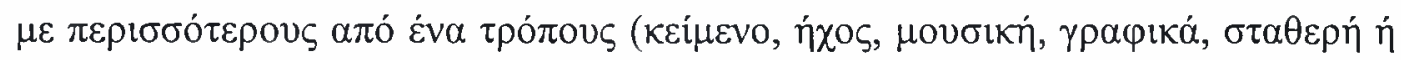

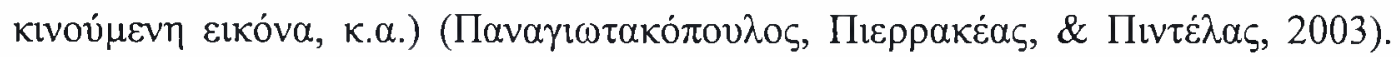

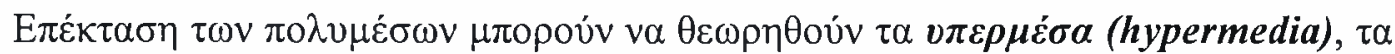

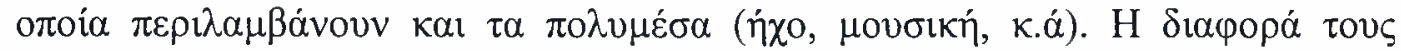

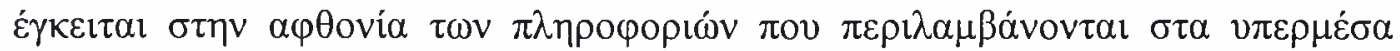

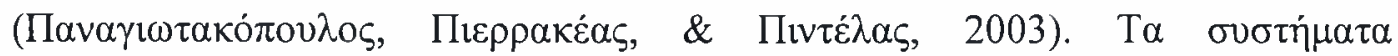

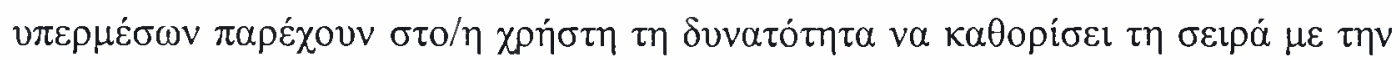

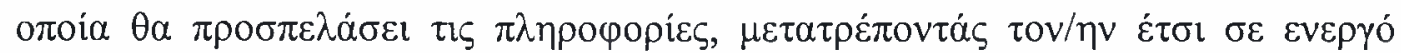

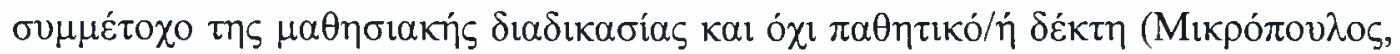

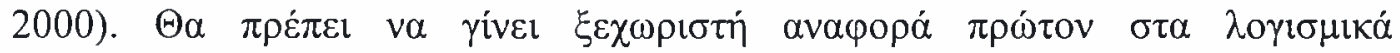

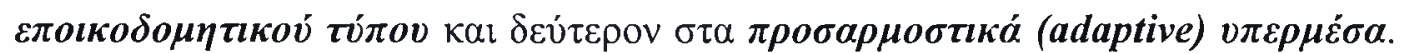

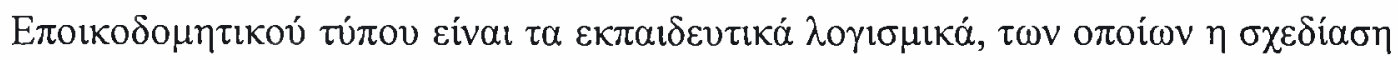

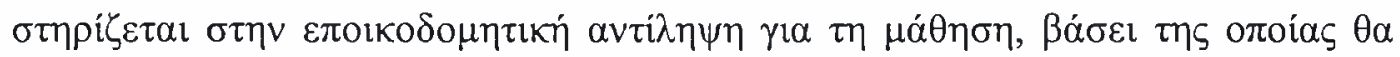

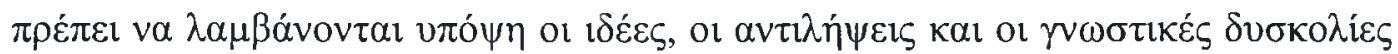

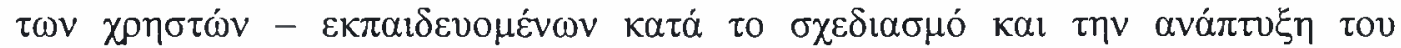

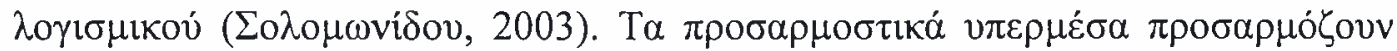

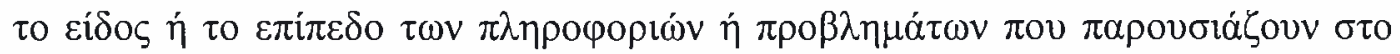

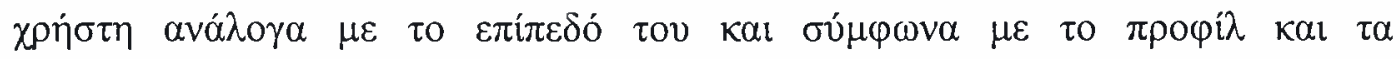

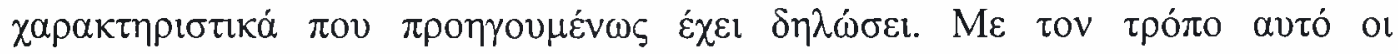

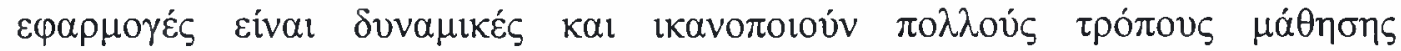

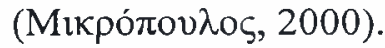

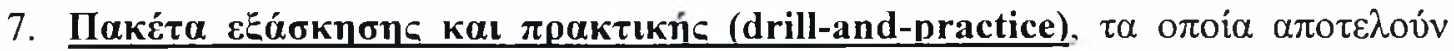

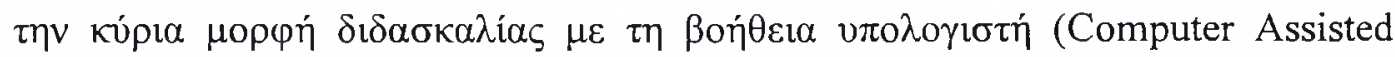

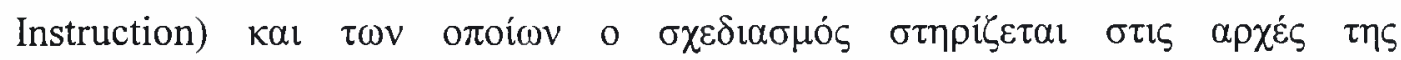

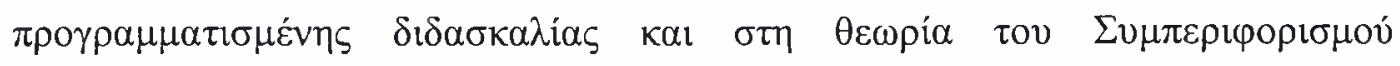

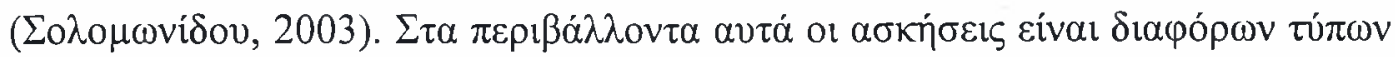

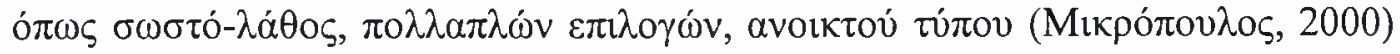

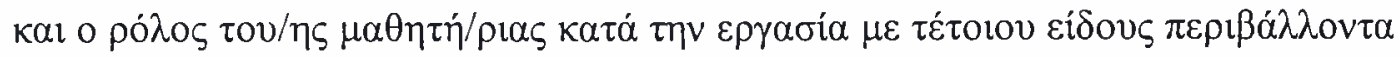

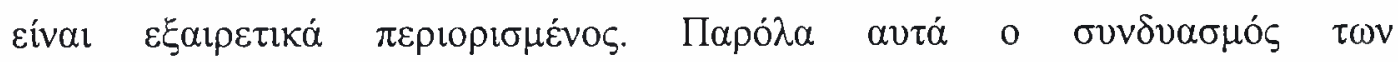




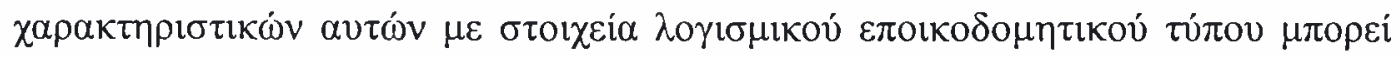

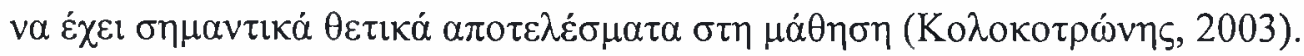

\subsection{4 А}

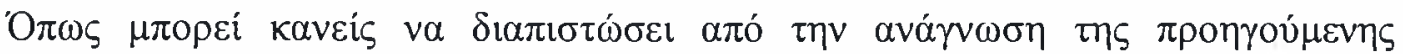

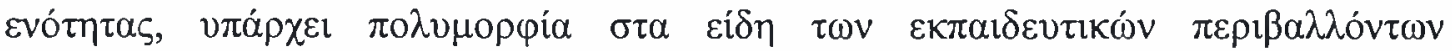

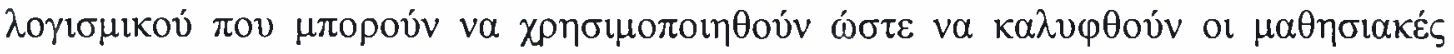

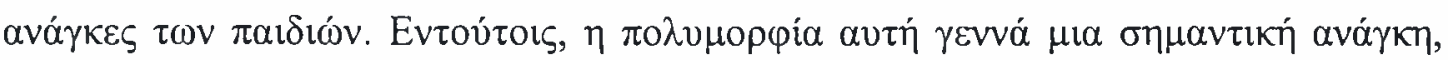

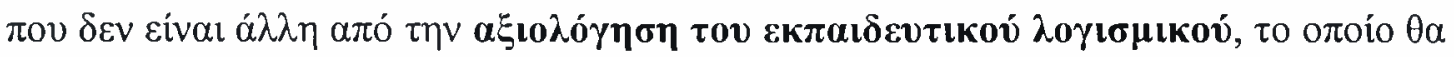

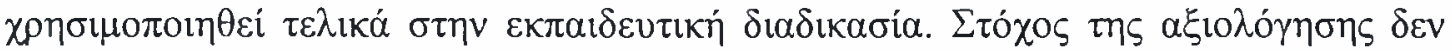

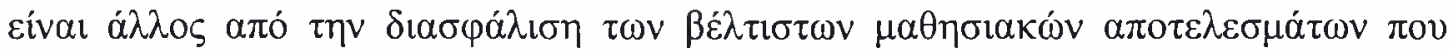

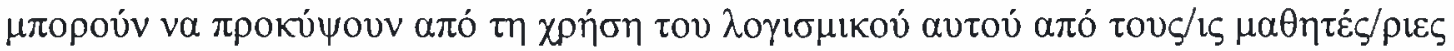

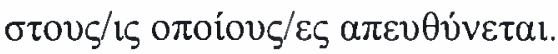

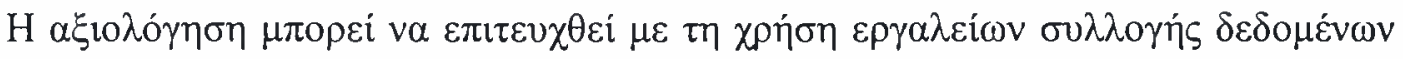

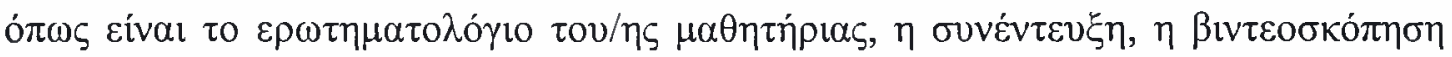

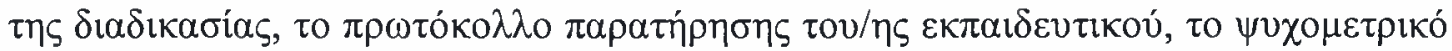

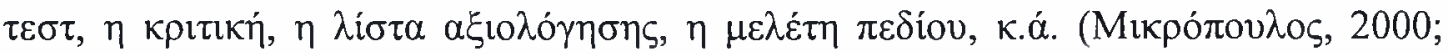

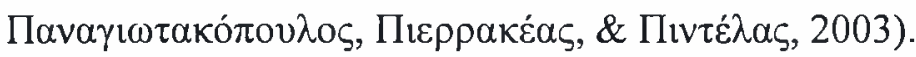

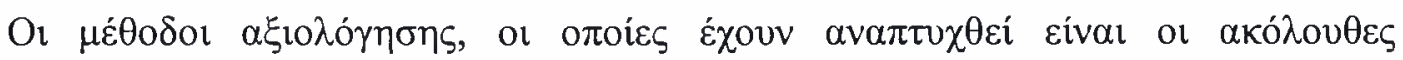

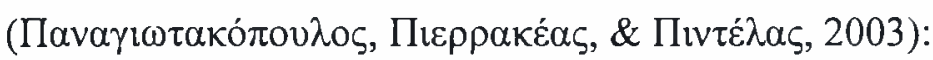

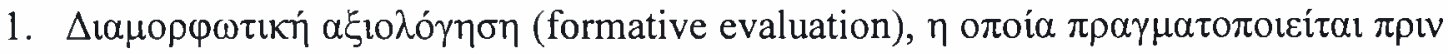

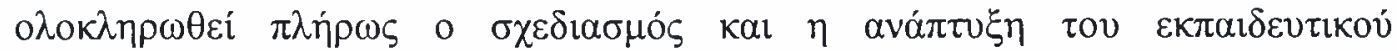

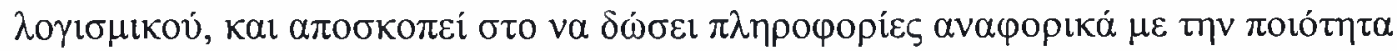

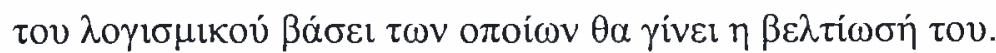

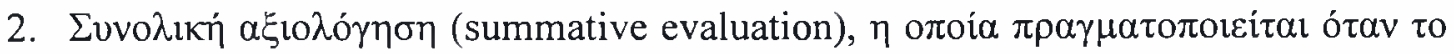

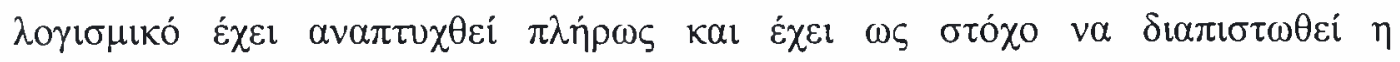
$\alpha \pi \circ \tau \varepsilon \lambda \varepsilon \sigma \mu \alpha \tau$ otóxous.

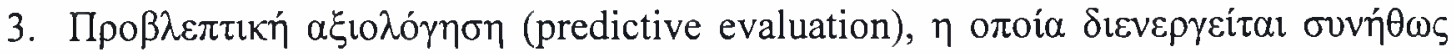

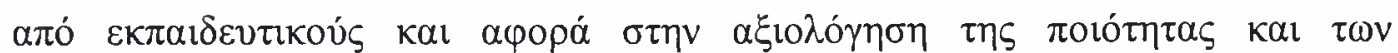




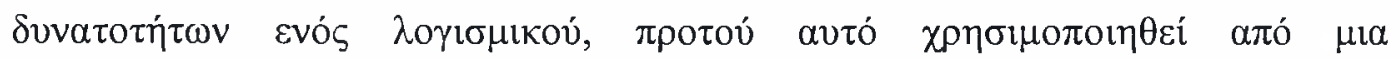
$\sigma \vartheta \gamma \kappa \varepsilon \kappa \rho \mu \varepsilon \dot{v} \eta\rceil \mu \alpha ́ \delta \alpha-\sigma \tau o ́ \chi 0$.

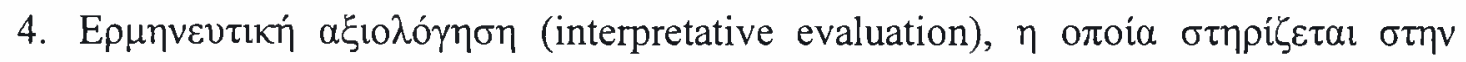

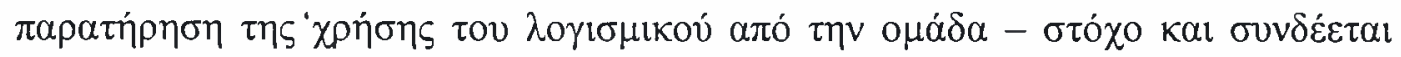

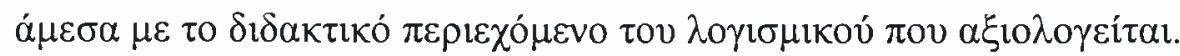

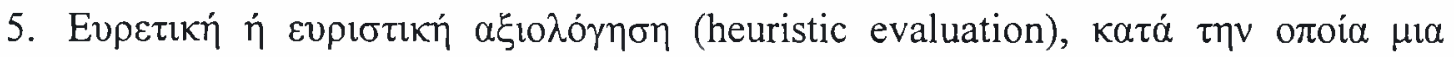

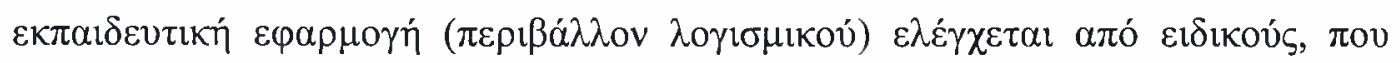

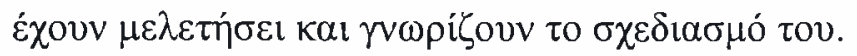

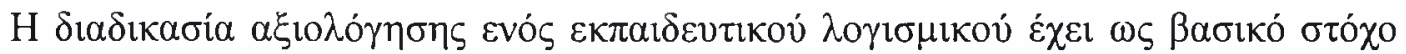
$\tau \eta \beta \varepsilon \lambda \tau i \omega \sigma \eta:$

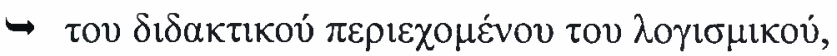

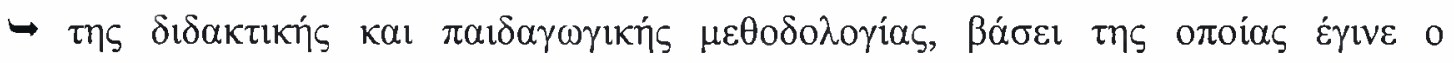

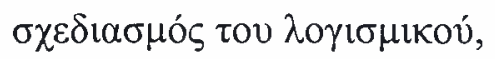

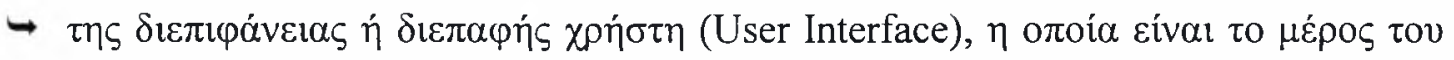

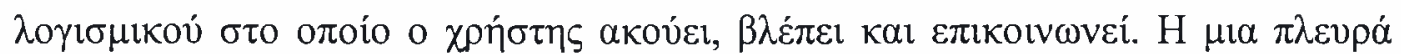

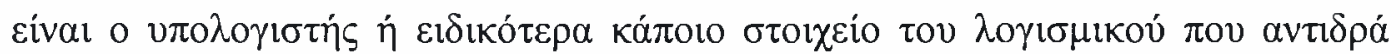

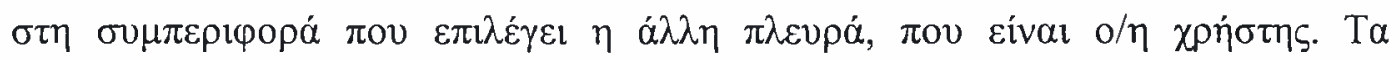

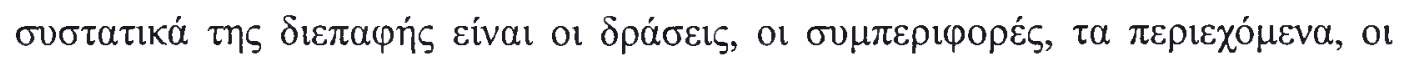

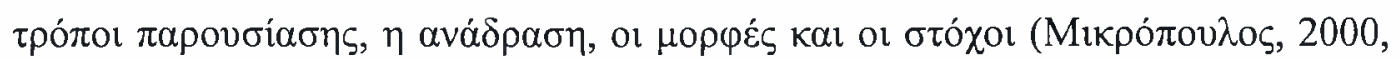

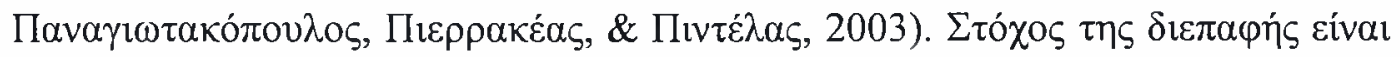

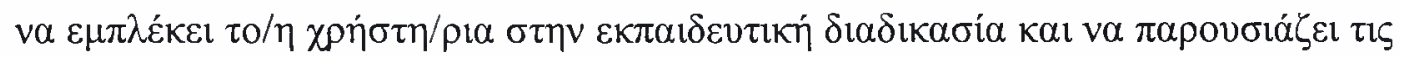

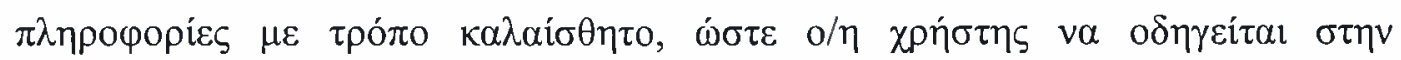

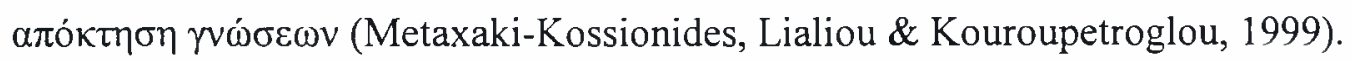

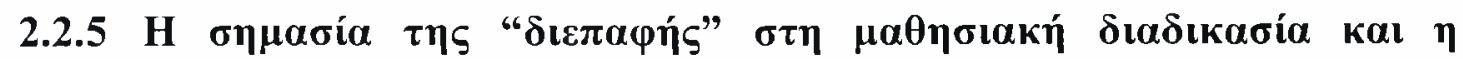

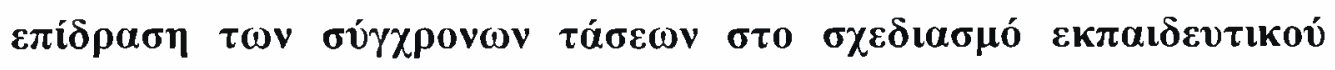 $\lambda о \gamma \iota \sigma \mu \iota \kappa о v ́$}

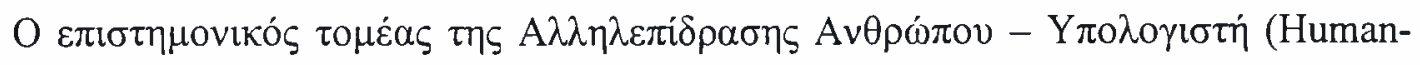

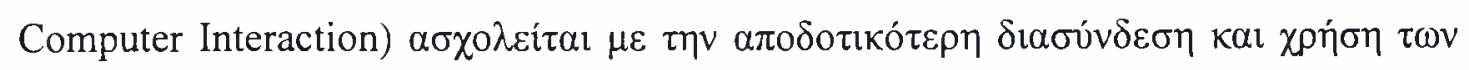

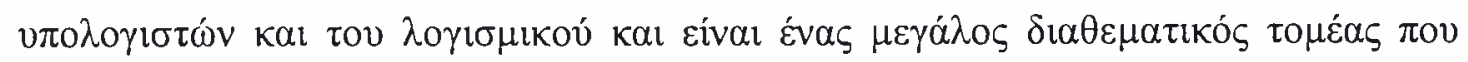

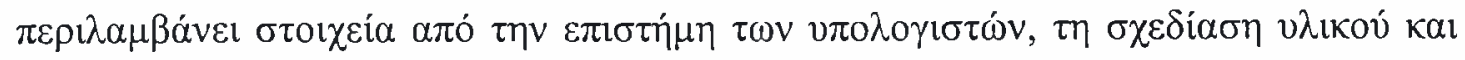




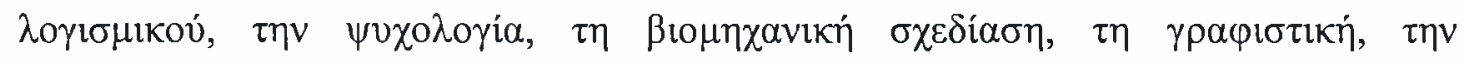

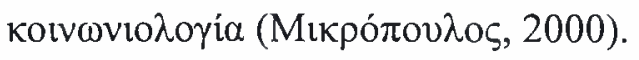

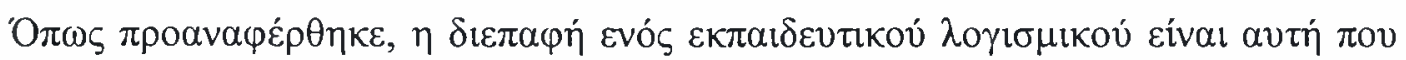

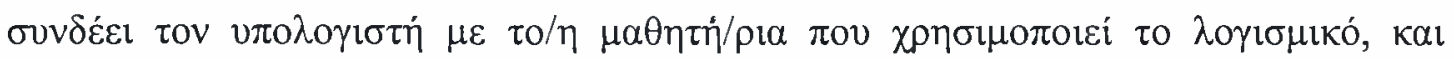

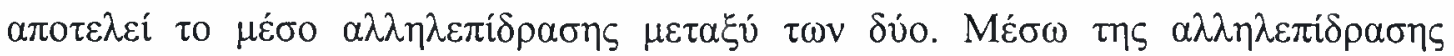

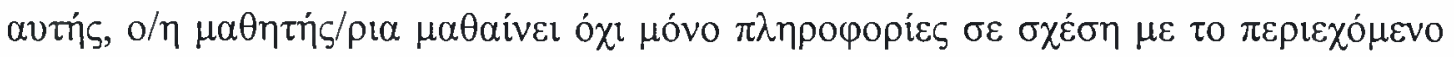

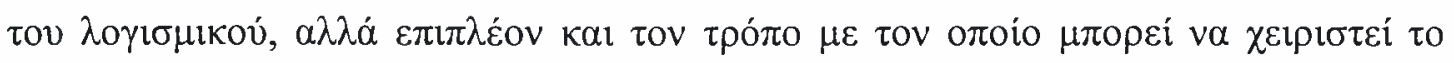

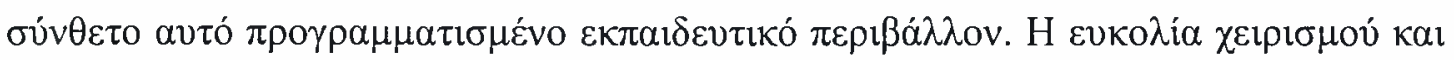

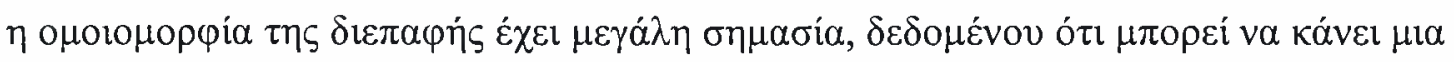

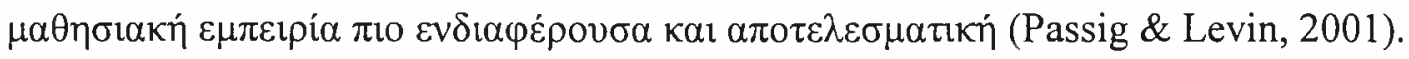

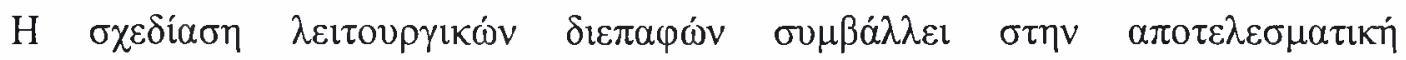

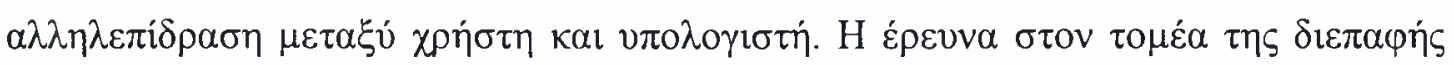

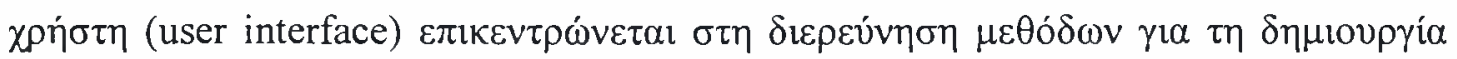

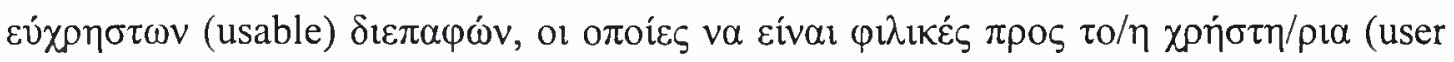

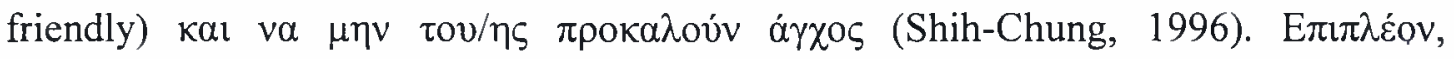

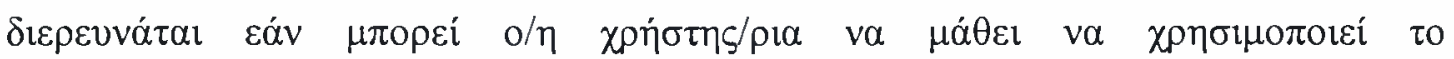

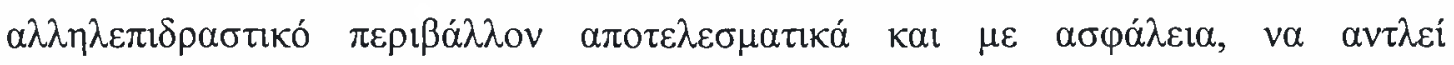

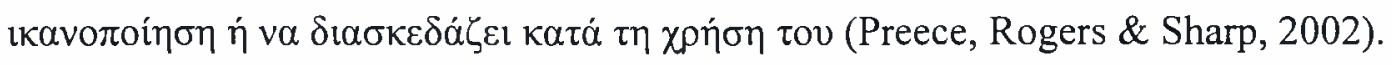

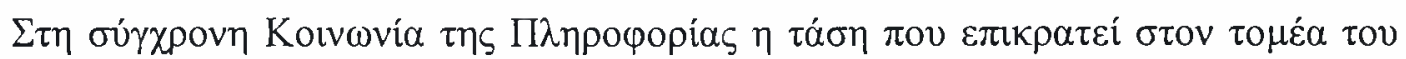

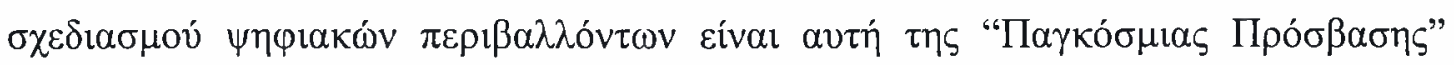

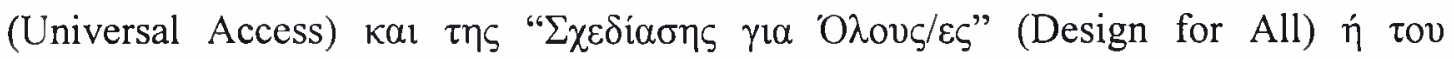

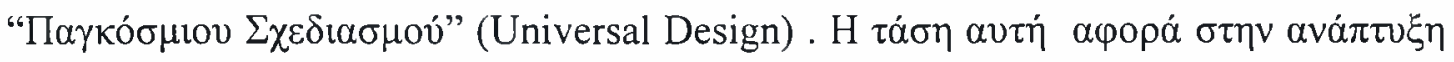

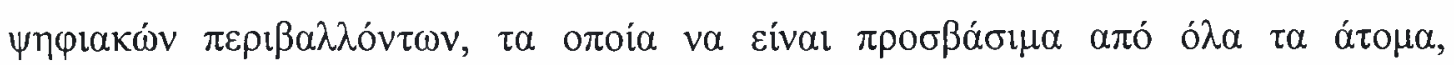

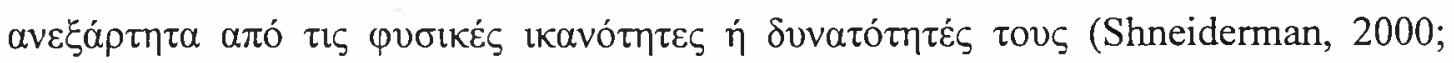
Preece, Rogers \& Sharp, 2002; Keates, Clarkson \& Robinson, 2002; Stephanidis,

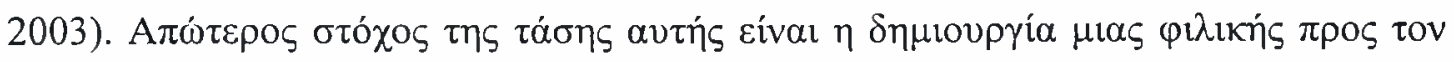

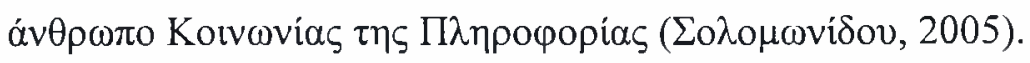

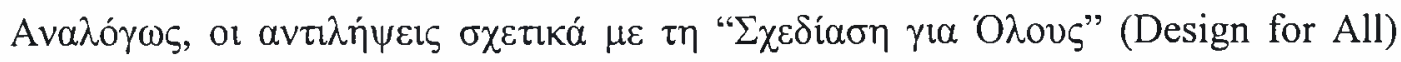

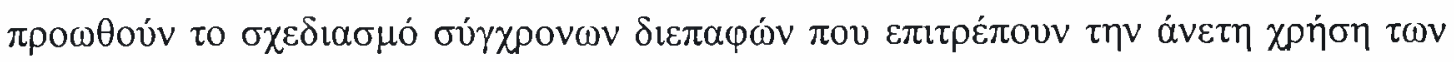

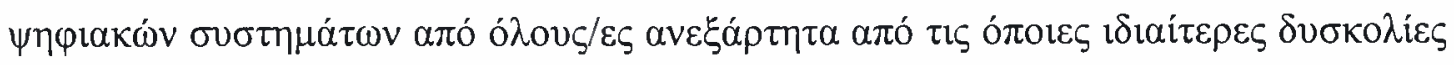

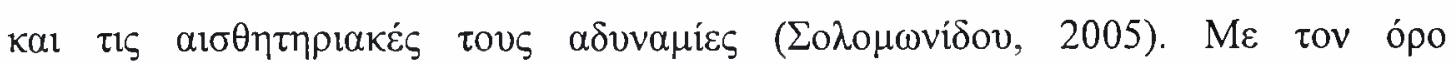

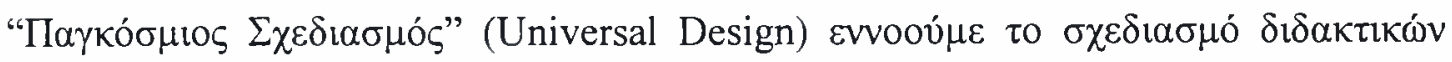

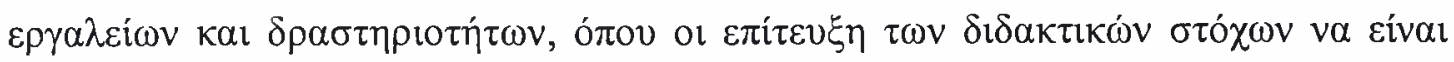

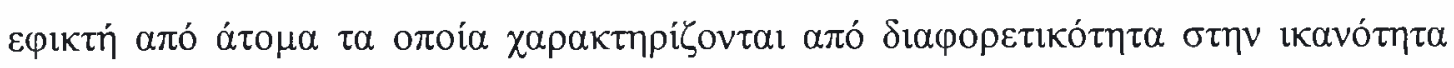




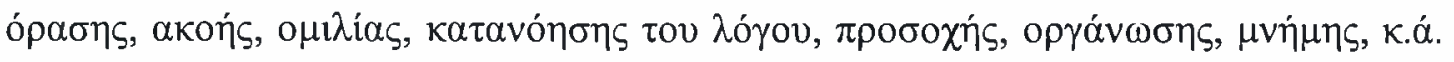
(Bauder \& Simmons, 2003).

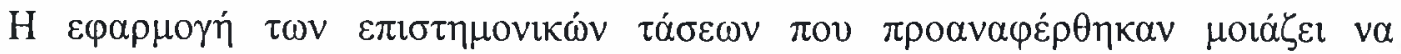

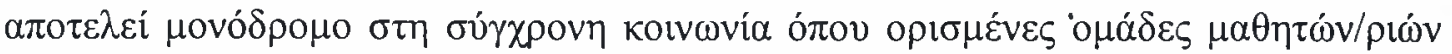

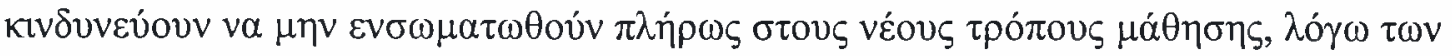

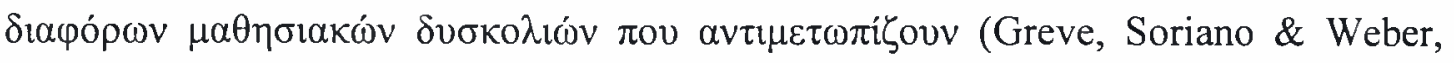

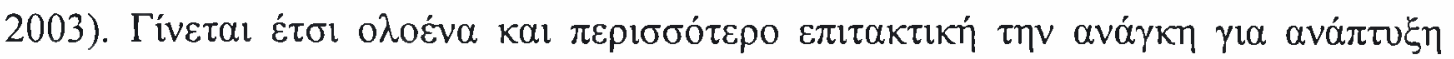

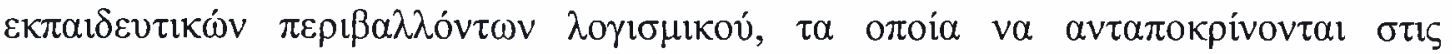

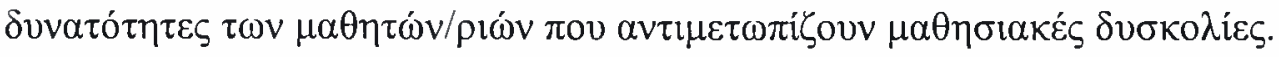

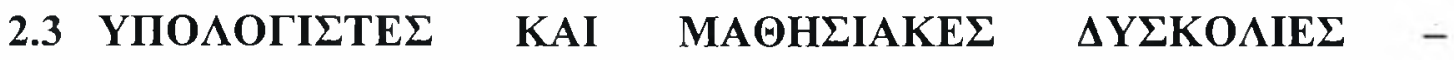 EPEYNHTIKA $\triangle$ EDOMENA}

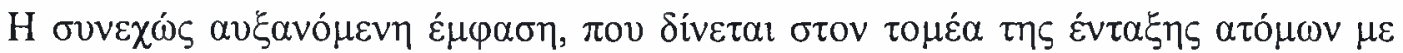

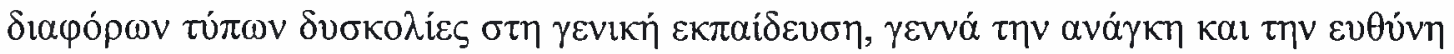

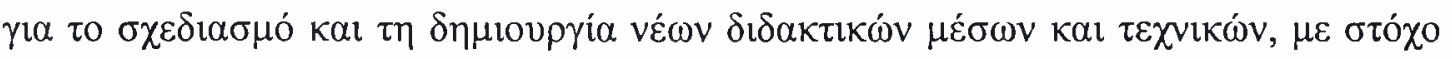

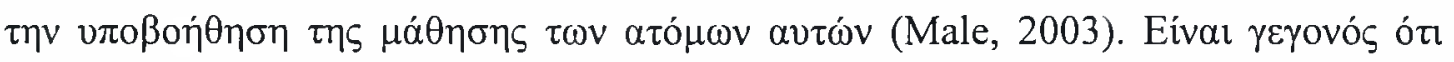

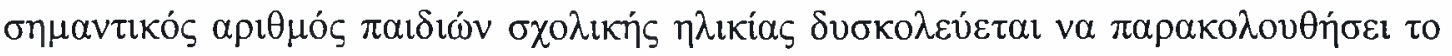

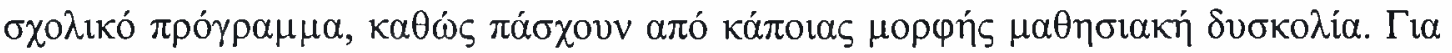

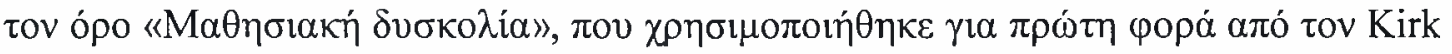

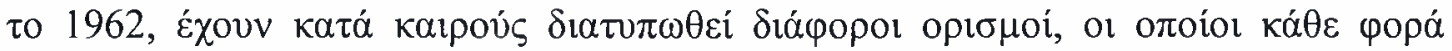

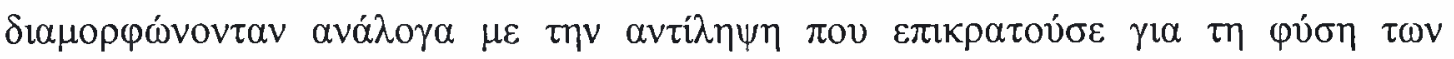

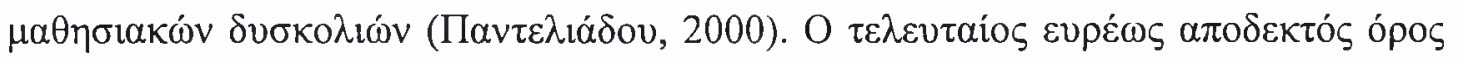

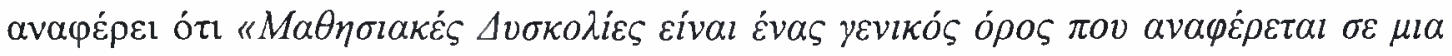

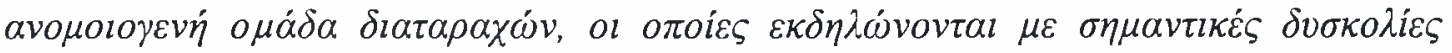

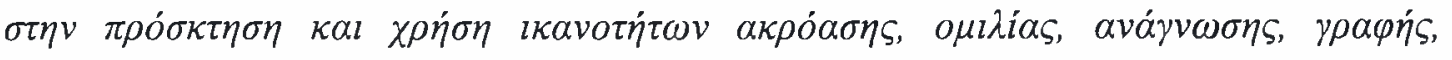

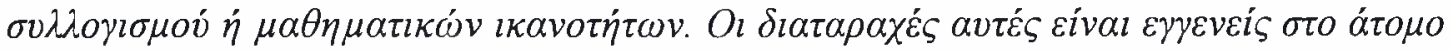

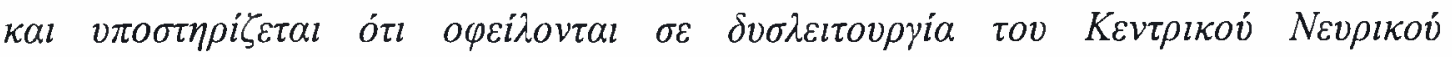

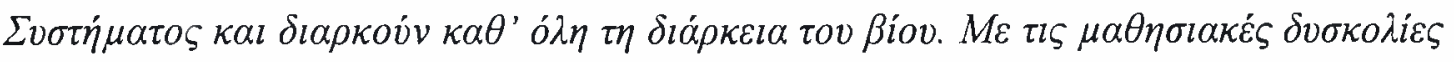

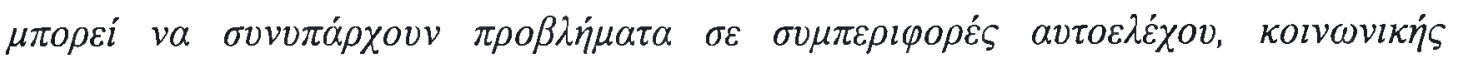

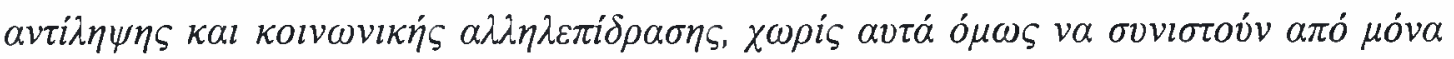

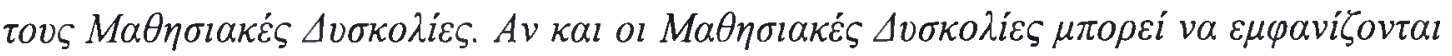




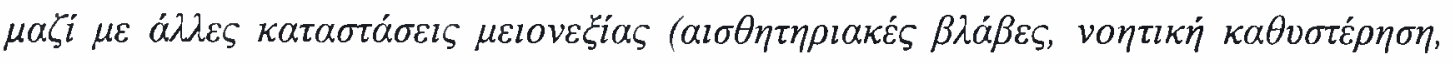

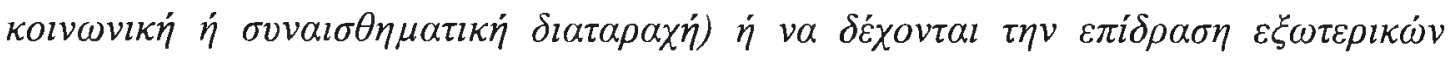

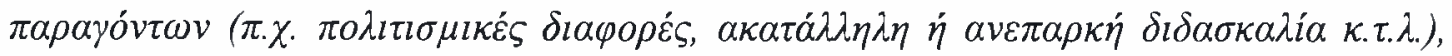

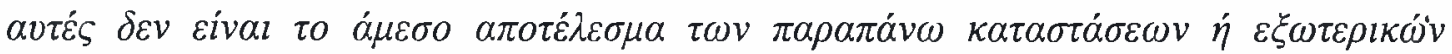

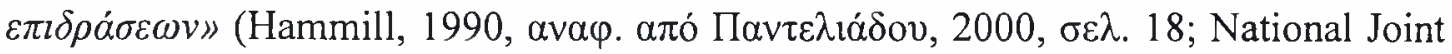

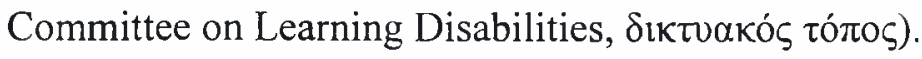

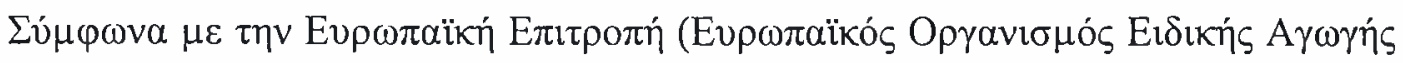

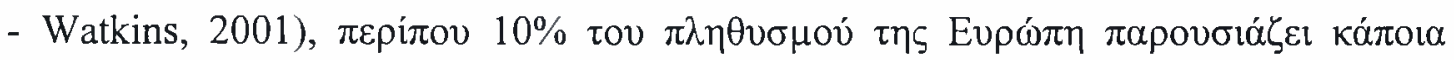

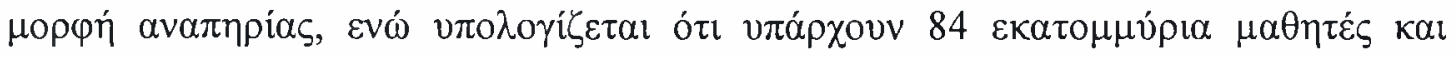

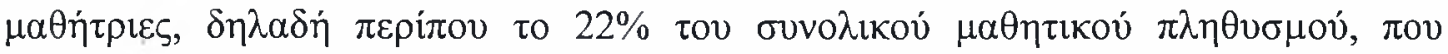

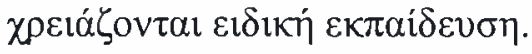

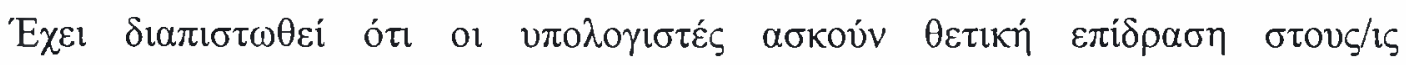

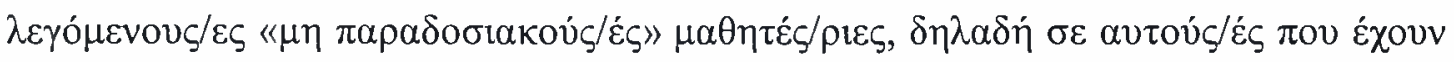

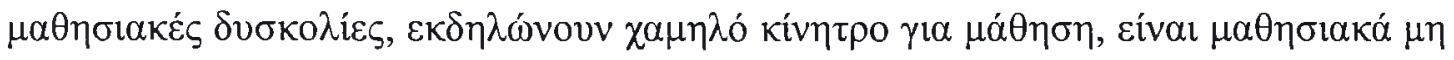

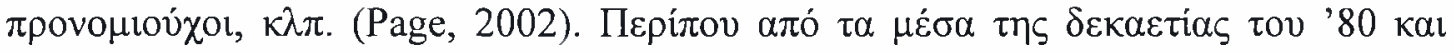

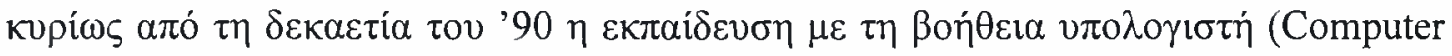

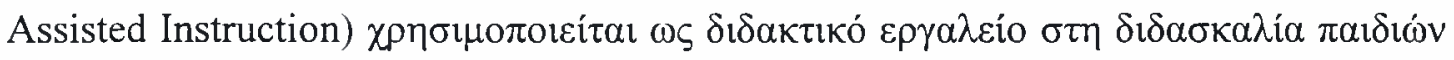

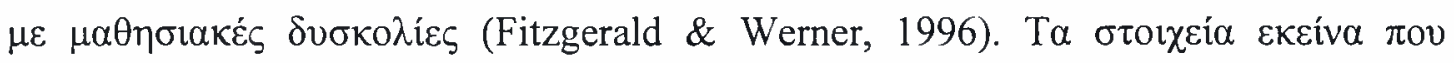

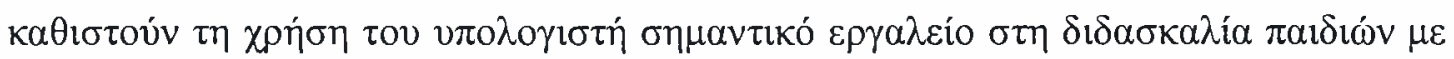

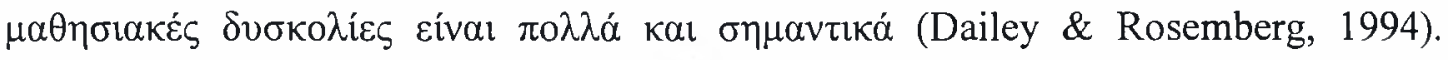

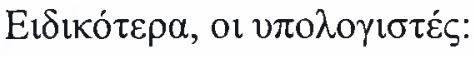

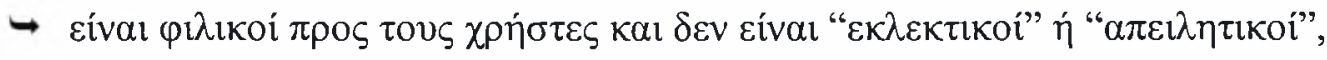

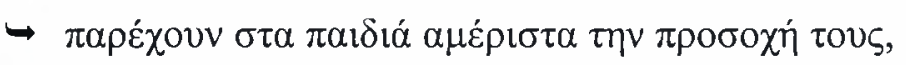

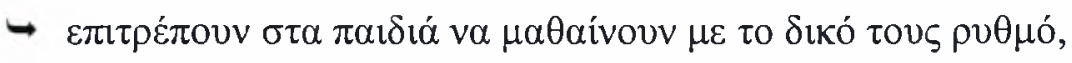

$\rightarrow \pi \alpha \rho \varepsilon ́ \chi 0 v v \varepsilon v i \sigma \chi v \sigma \eta, \delta เ \rho \rho \theta \omega \tau \iota \kappa \eta ́ ~ \alpha v \alpha ́ \delta \rho \alpha \sigma \eta ~ \kappa \alpha \iota ~ \alpha ́ \mu \varepsilon \sigma \eta ~ \varepsilon \pi ı \beta \rho \alpha \beta \varepsilon v \sigma \eta$,

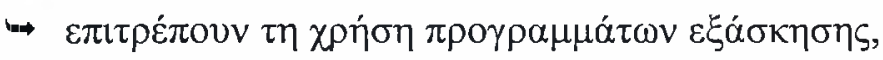

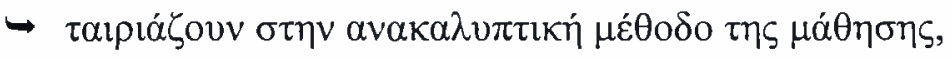

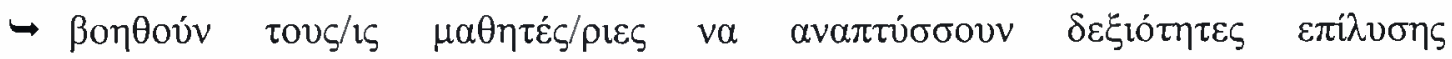
$\pi \rho \circ \beta \lambda \eta \mu \alpha ́ \tau \omega v$,

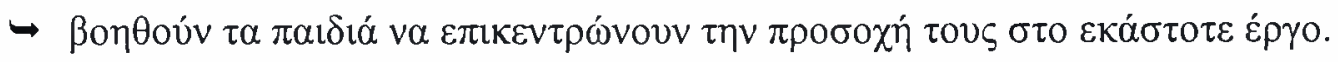

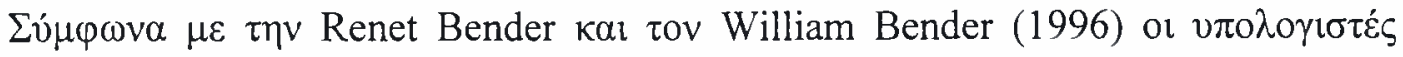

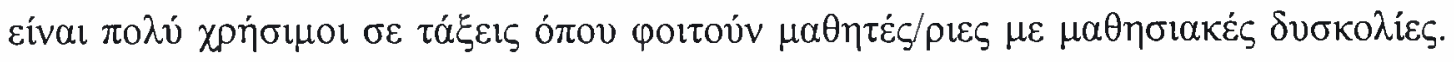

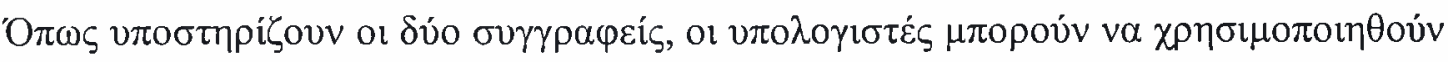




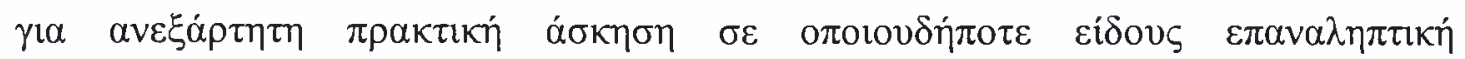

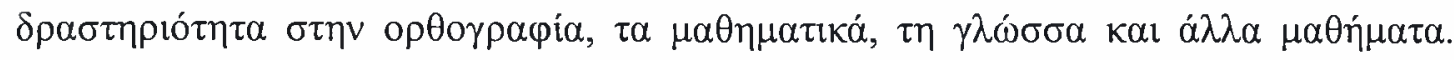

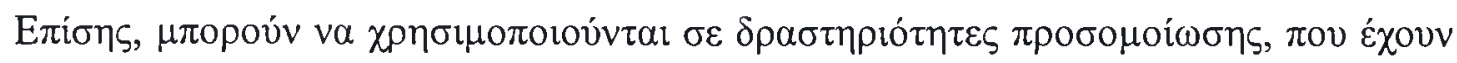

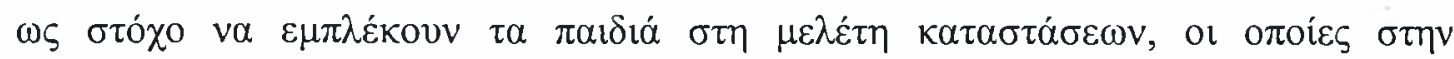

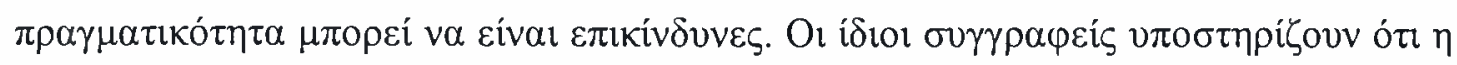

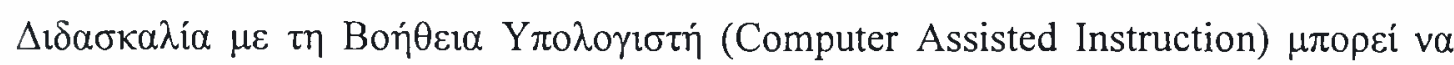

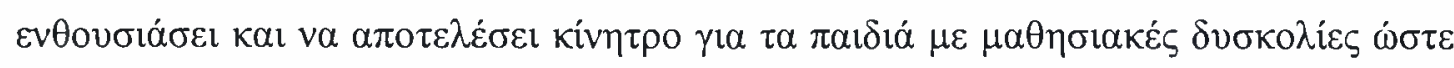

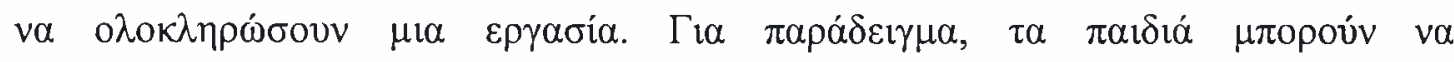

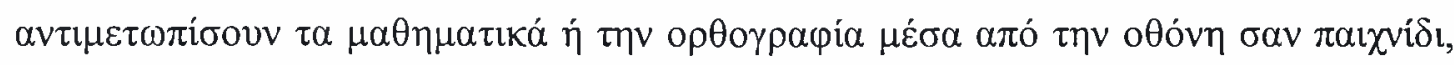

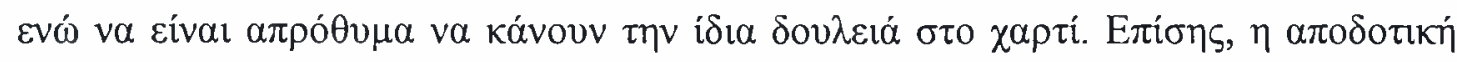

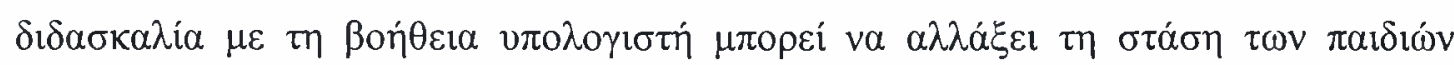

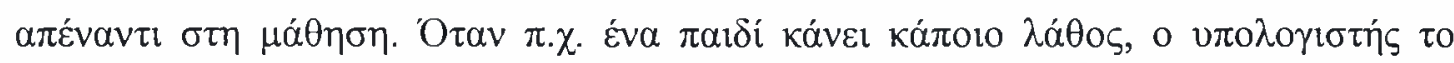

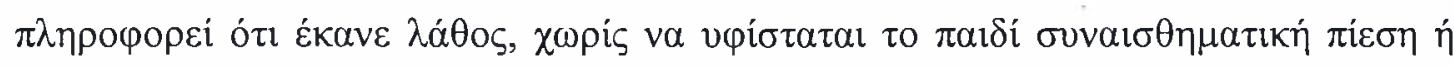

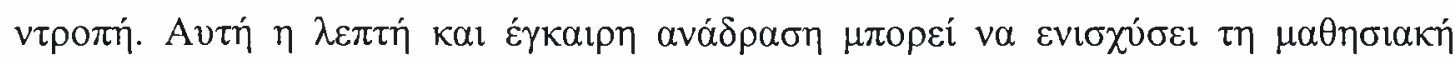

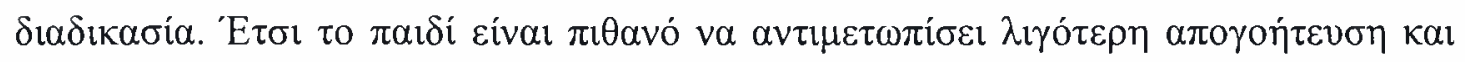

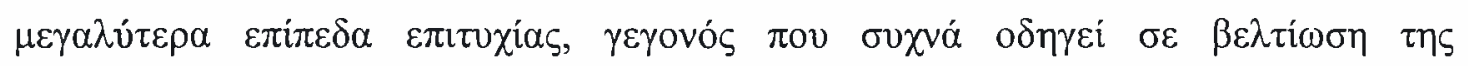

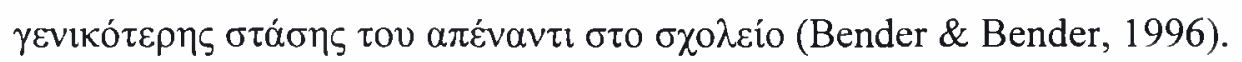

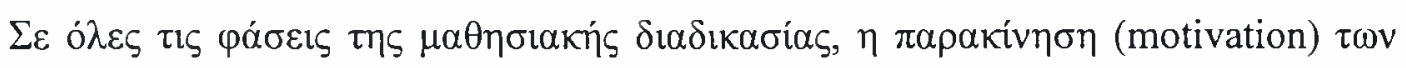

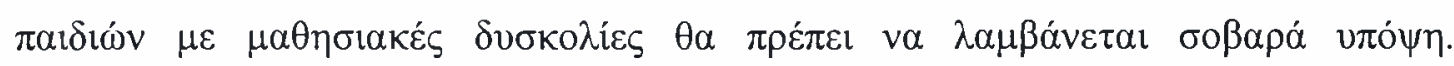

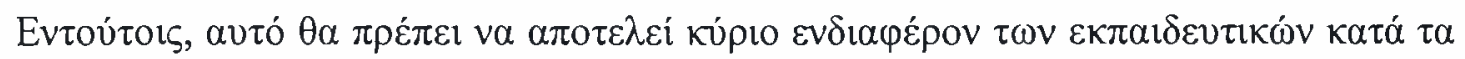

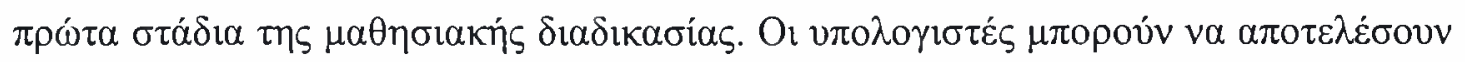

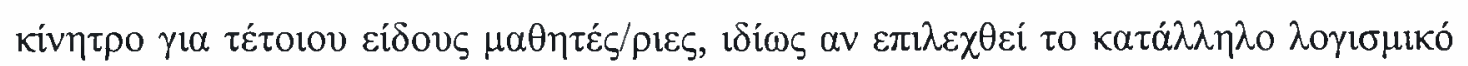

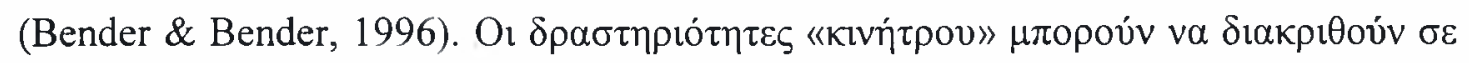

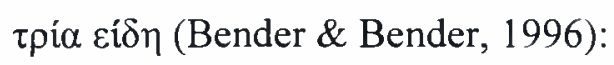

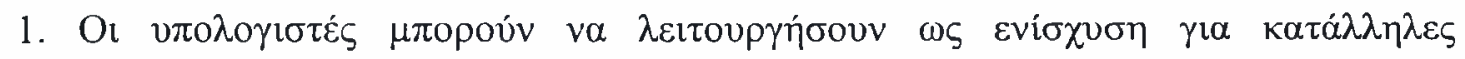

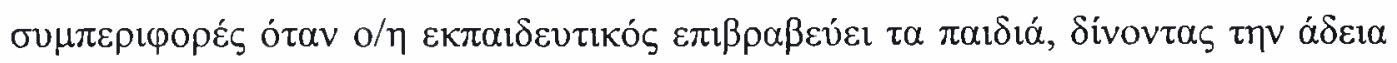

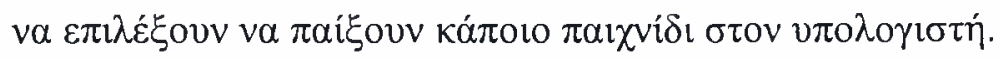

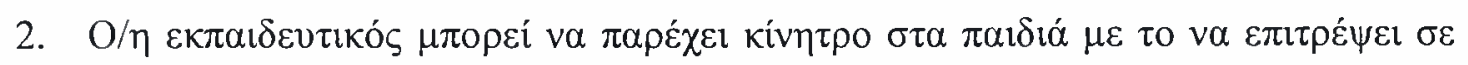

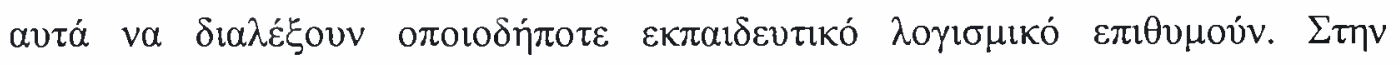

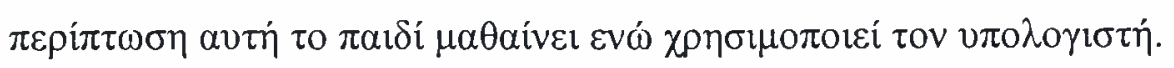

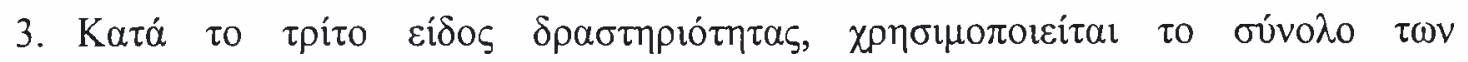

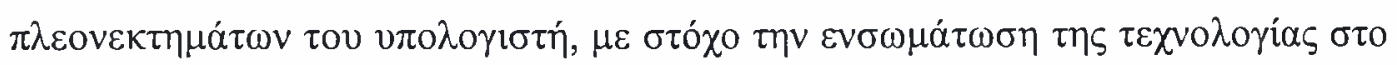
$\sigma \chi 0 \lambda \imath \kappa o ́ ~ \pi \rho o ́ \gamma \rho \alpha \mu \mu \alpha$. 


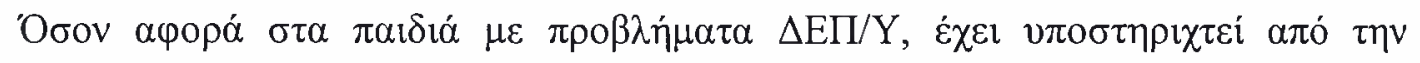

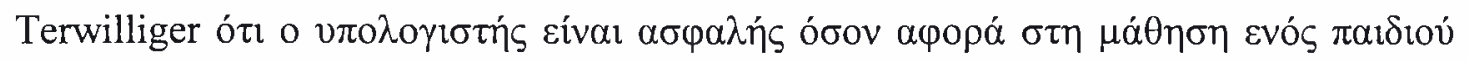

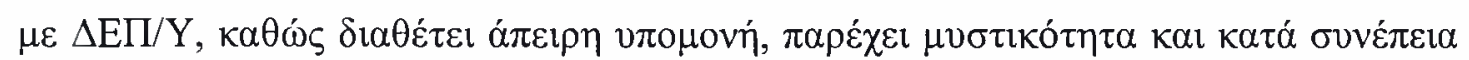

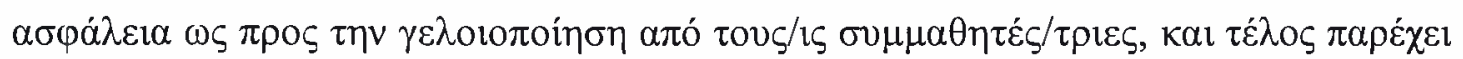

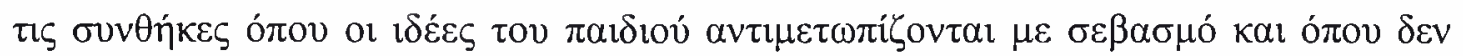

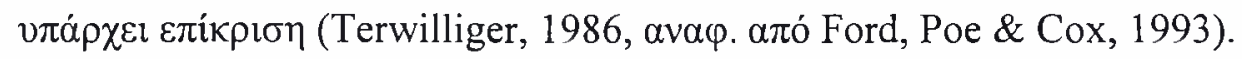

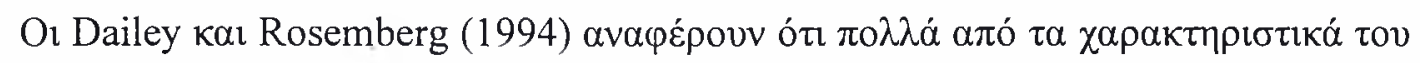

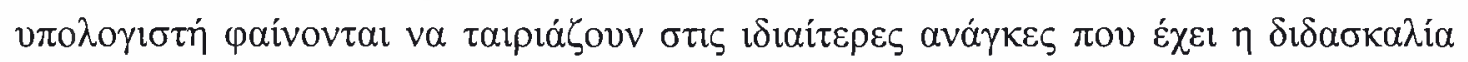

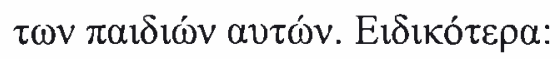

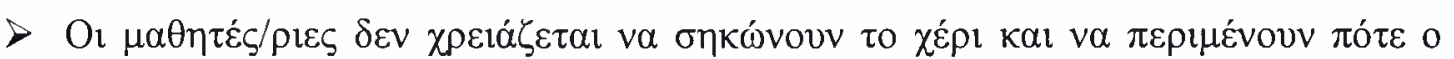

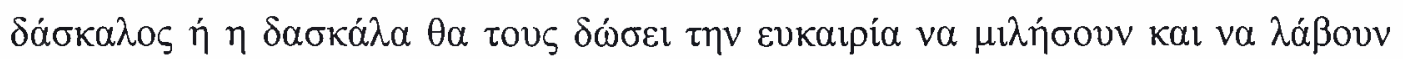

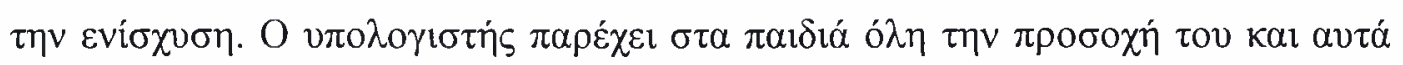

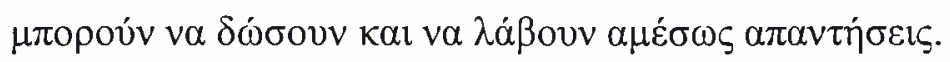

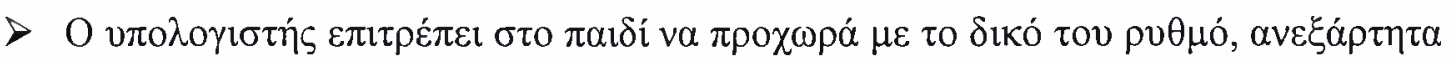

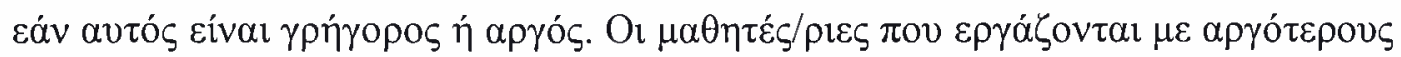

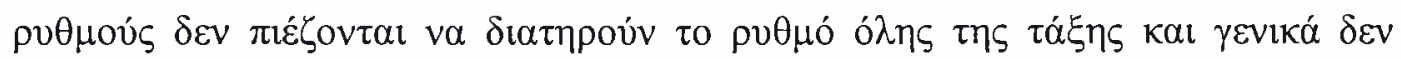

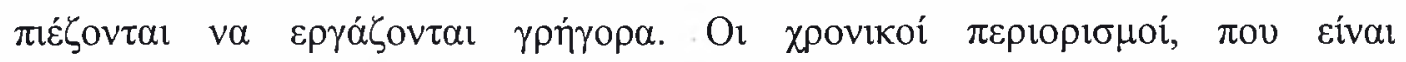

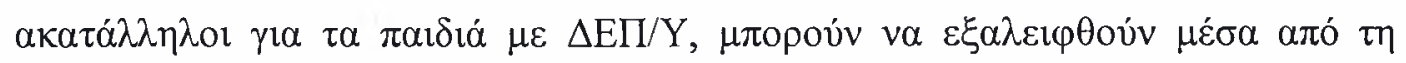

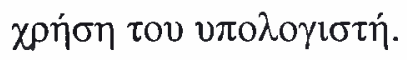

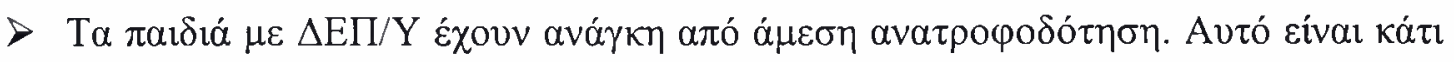

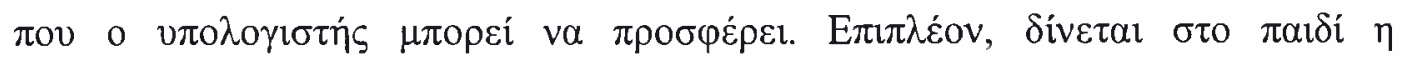

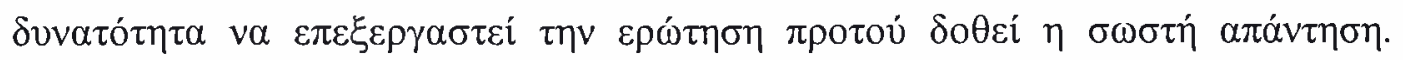

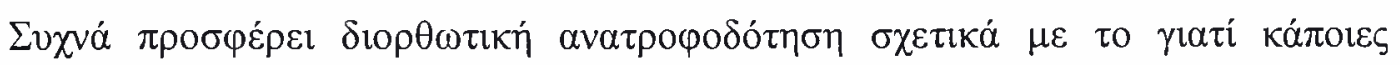

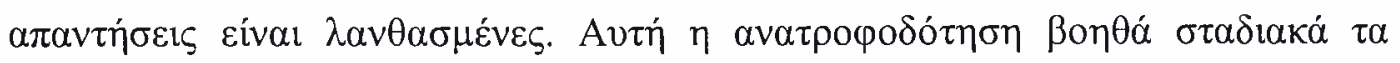

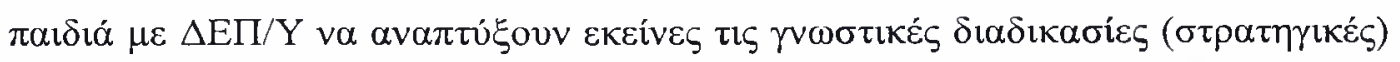

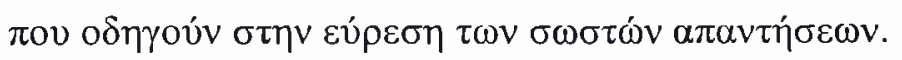

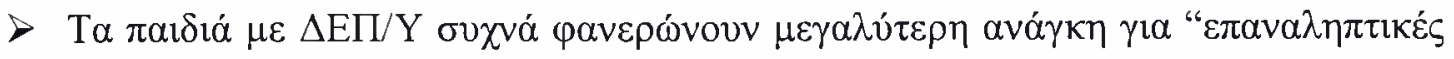

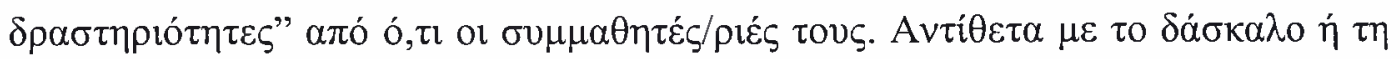

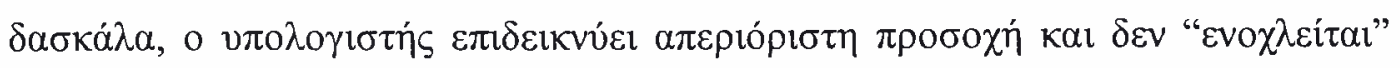

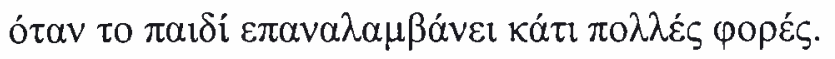

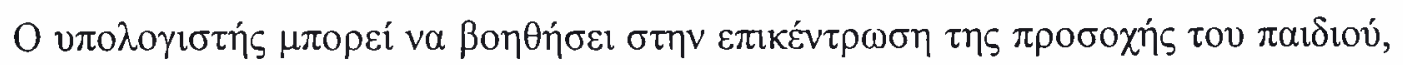

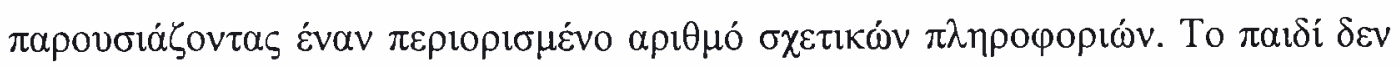

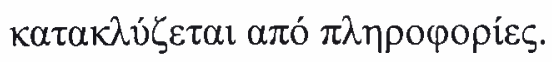




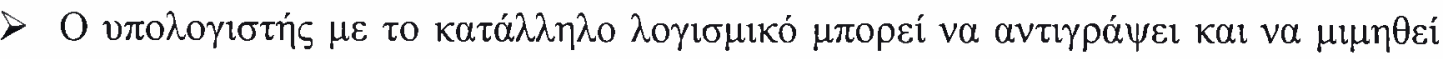

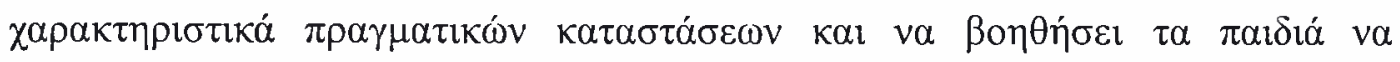

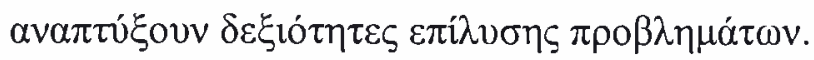

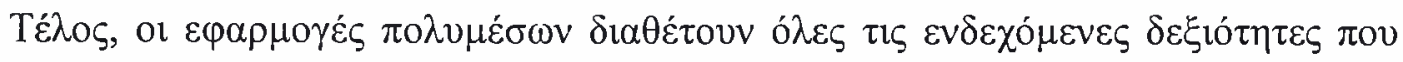

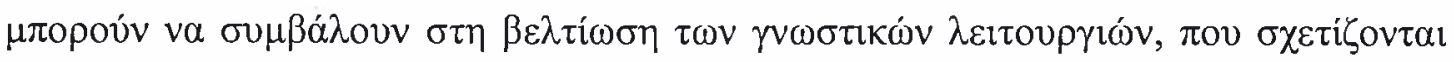

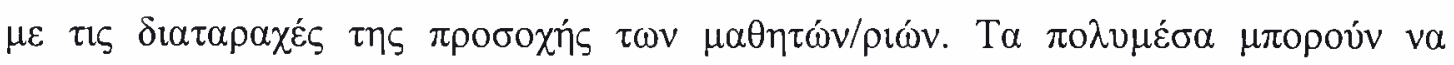

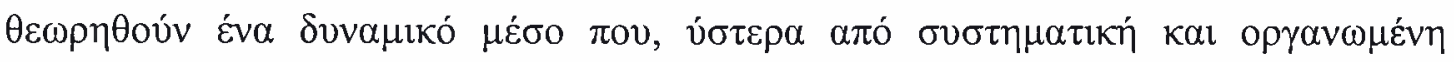

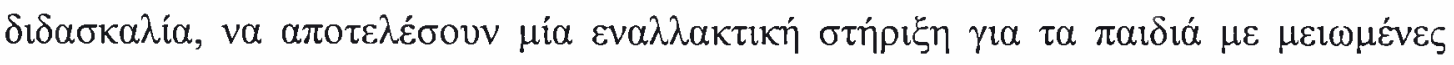

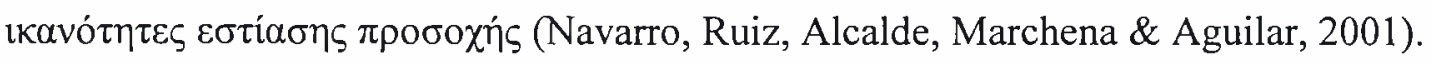

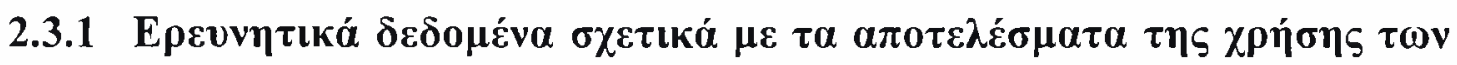

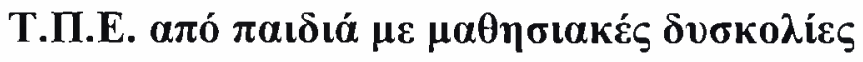

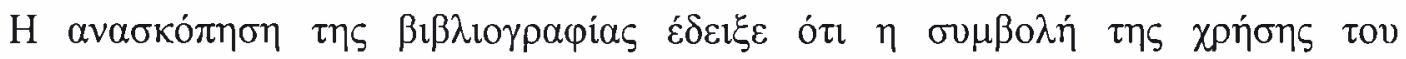

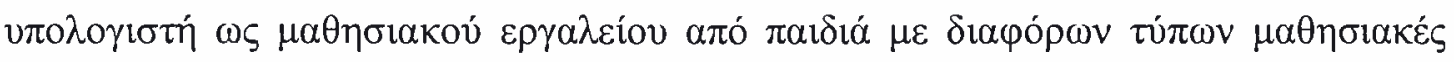

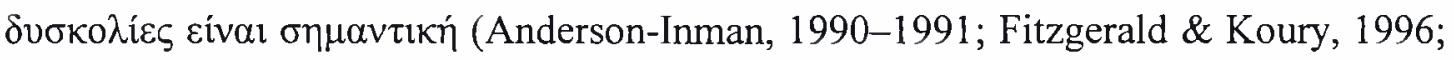
Maccini, McNaughton \& Ruhl, 1999; Hall, Hughes \& Filbert, 2000; McArthur,

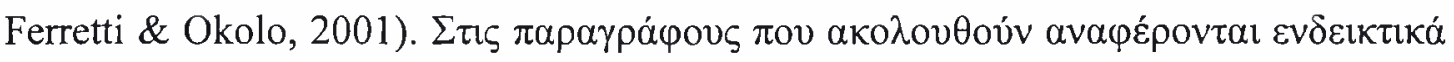

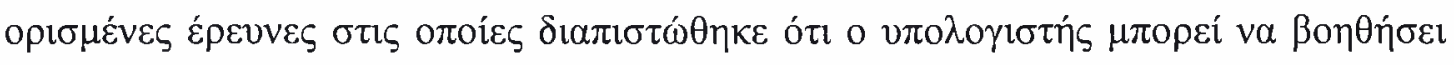

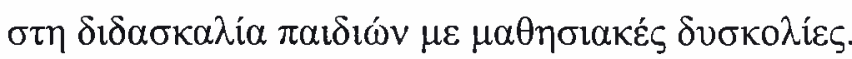

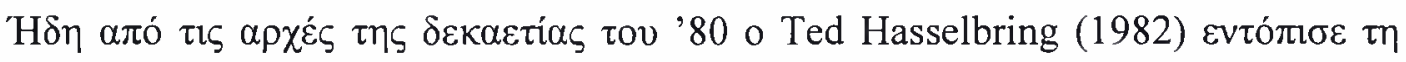

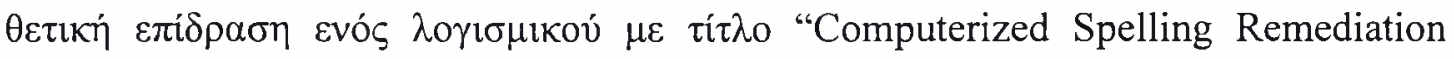

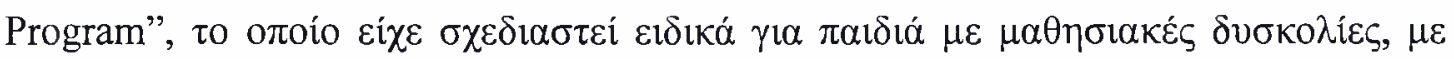

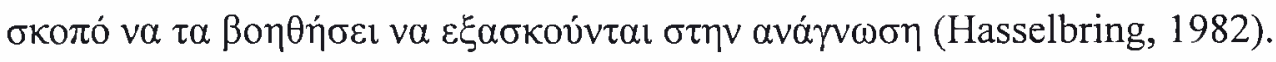

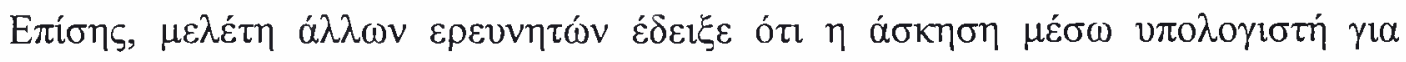

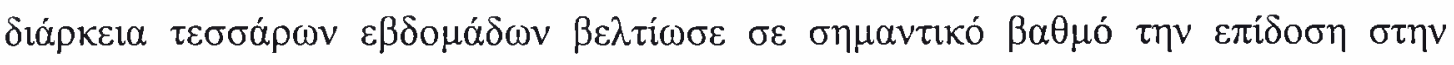

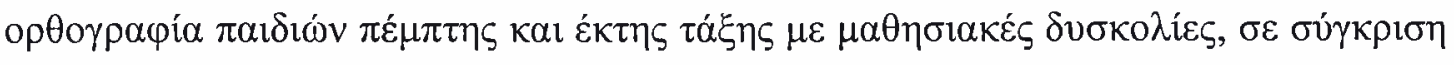

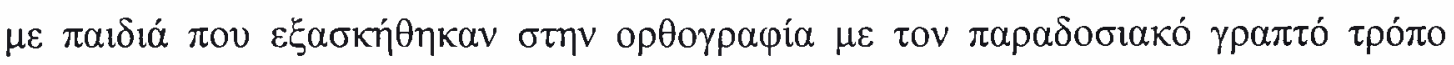
(McArthur, Haynes, Malouf \& Harris, 1987).

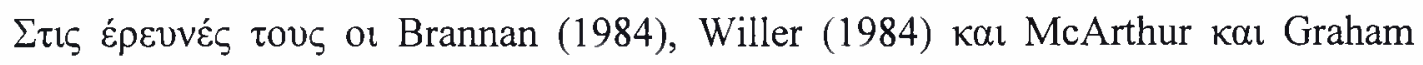

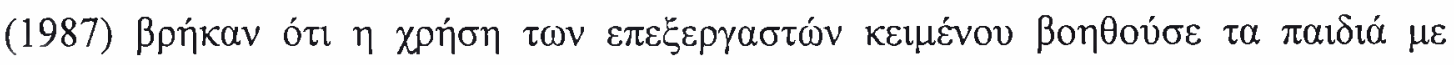




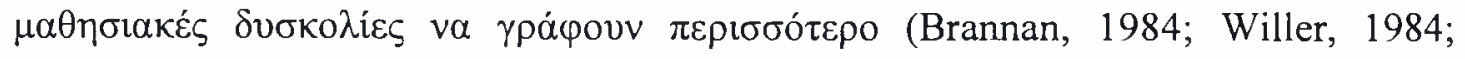
McArthur \& Graham, 1987).

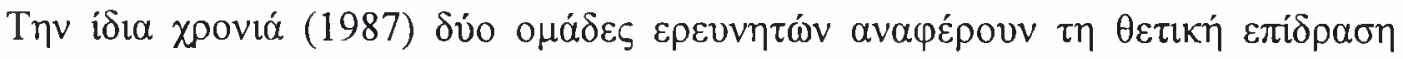

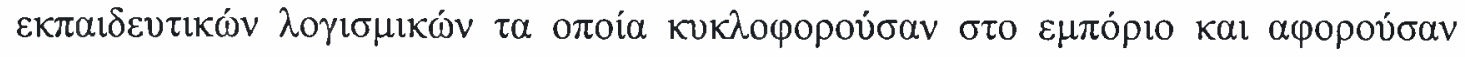

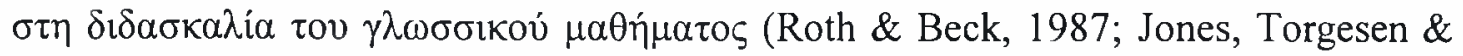

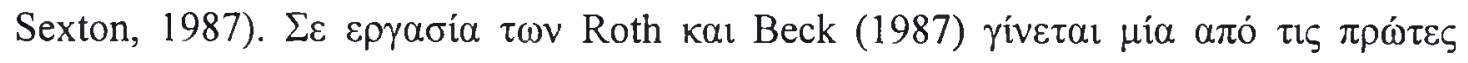

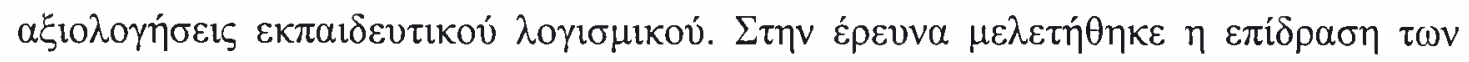

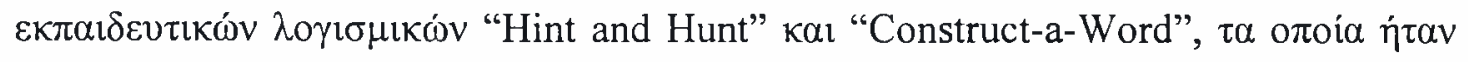

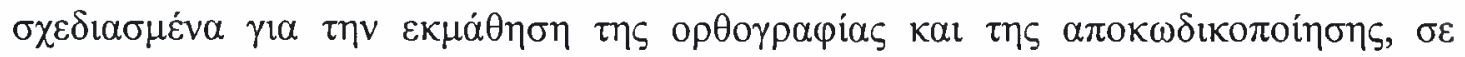

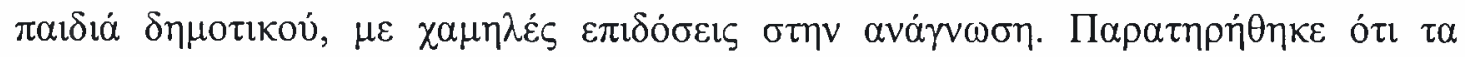

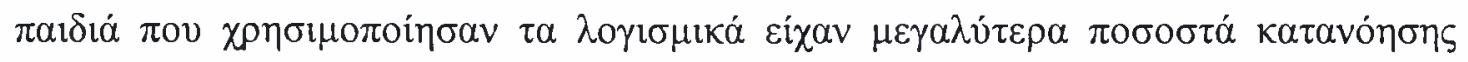

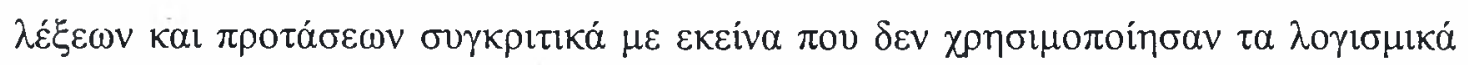

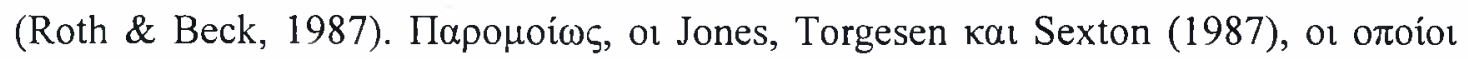

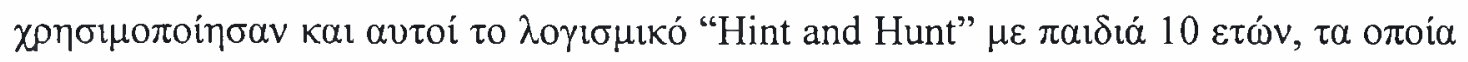

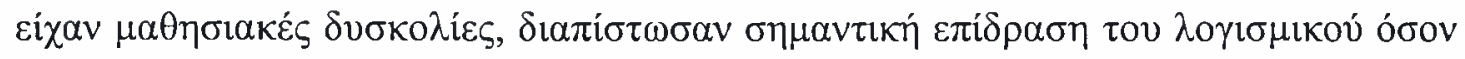

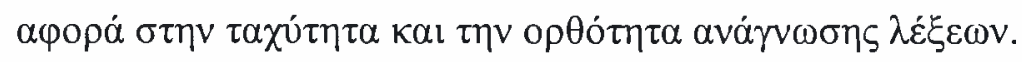

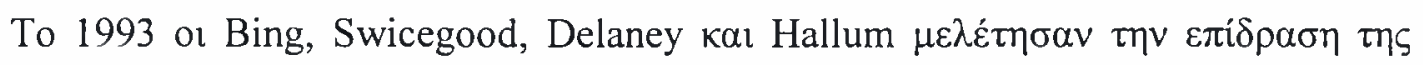

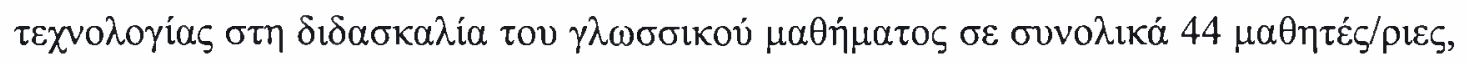

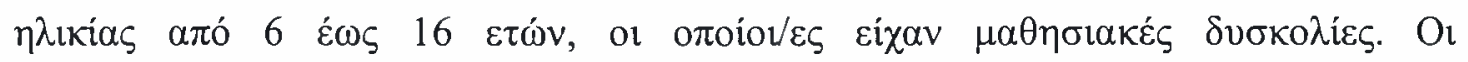

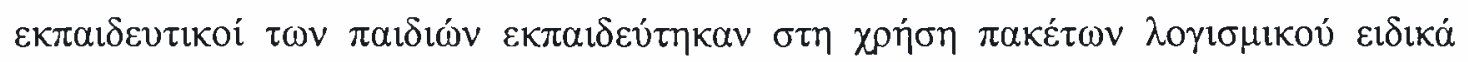

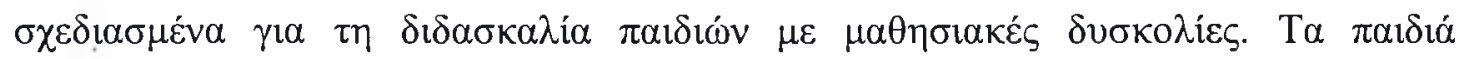

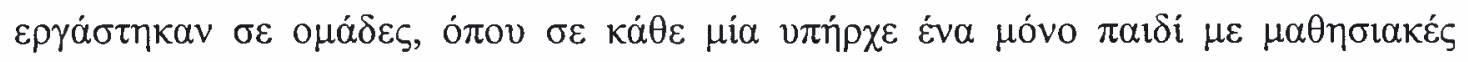

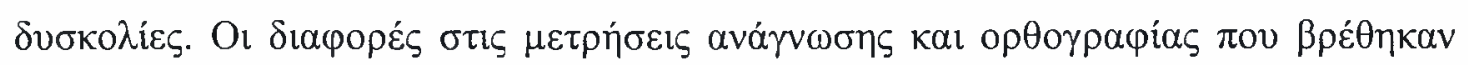

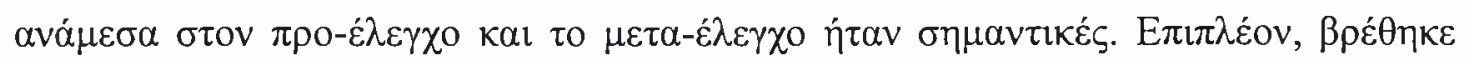

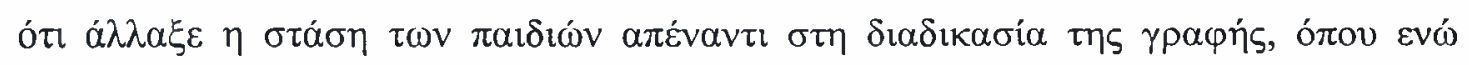

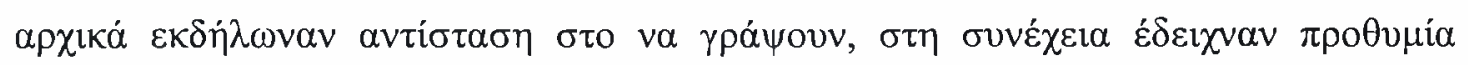

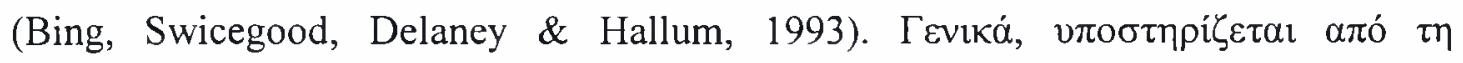

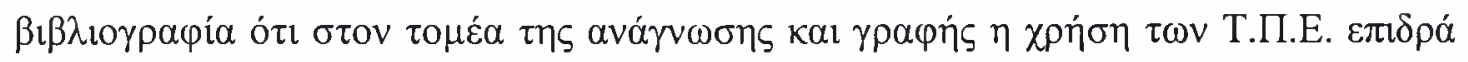

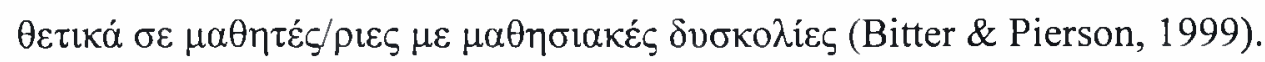

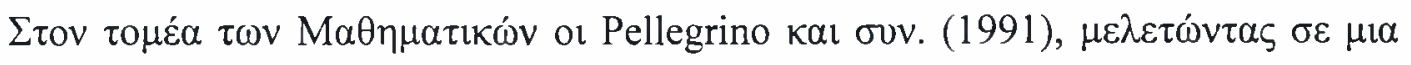

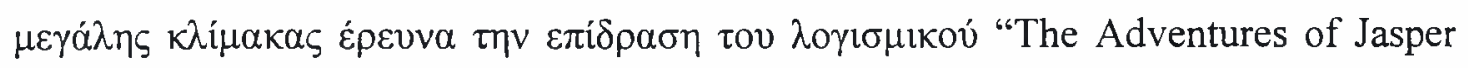

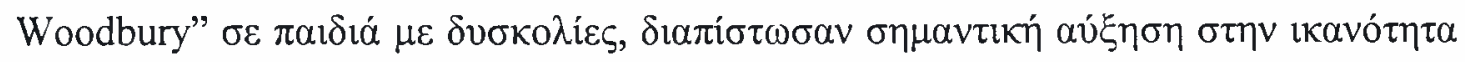

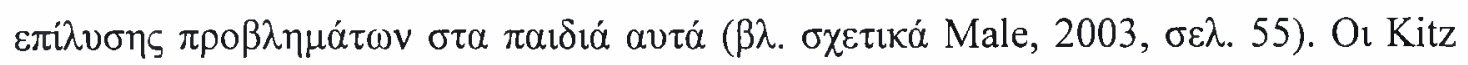

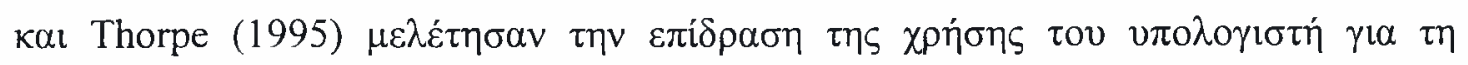




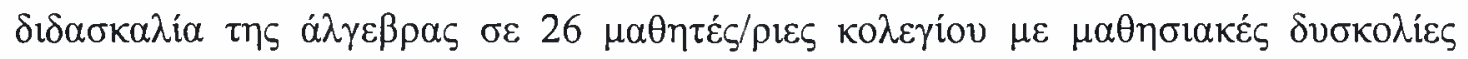

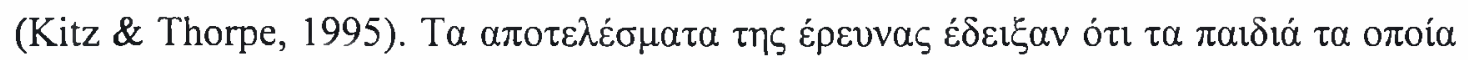

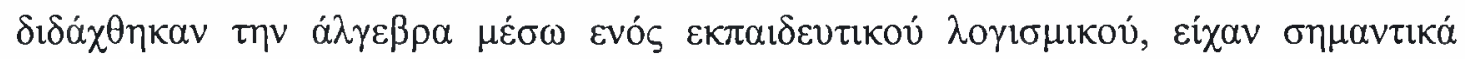

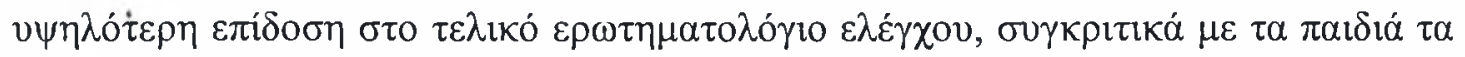

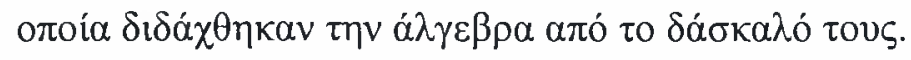

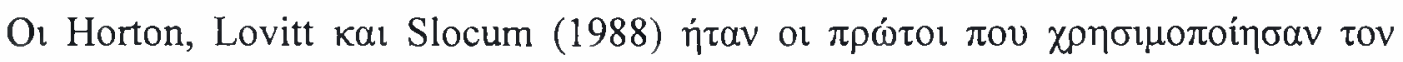

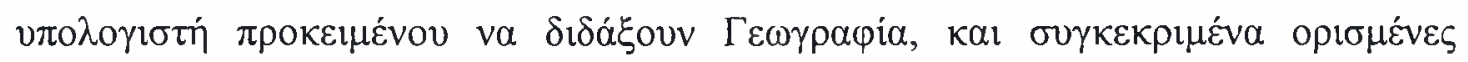

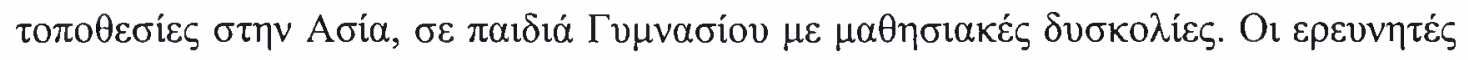

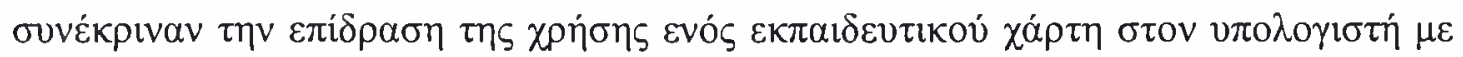

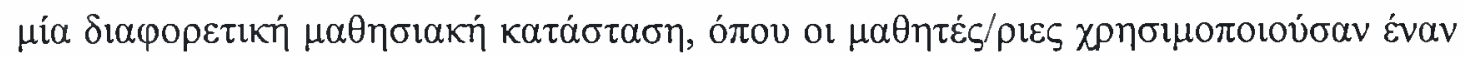

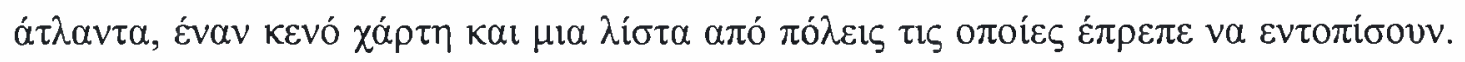

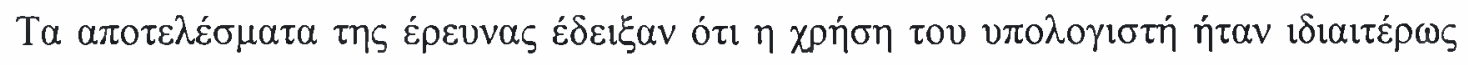

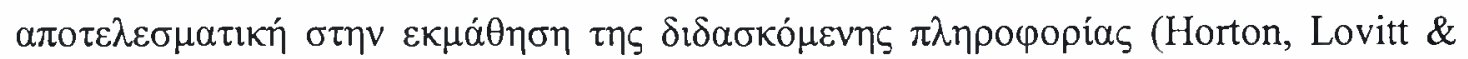
Slocum, 1988).

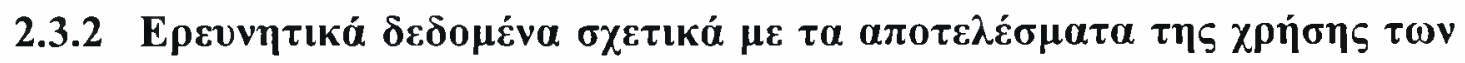

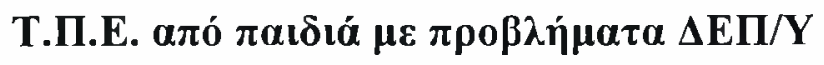

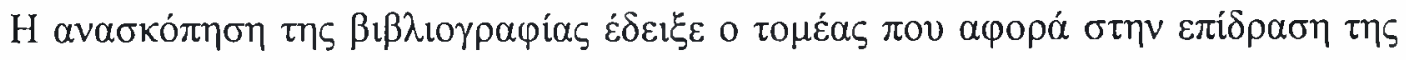

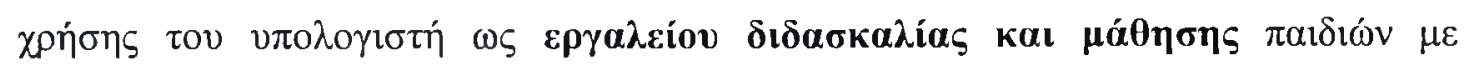

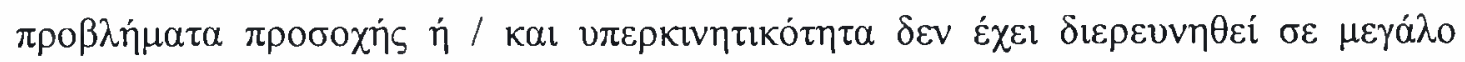

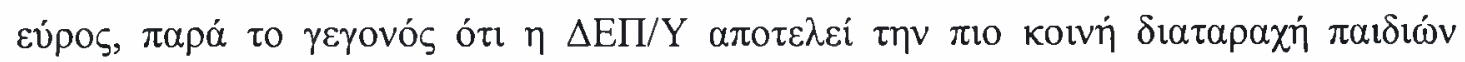

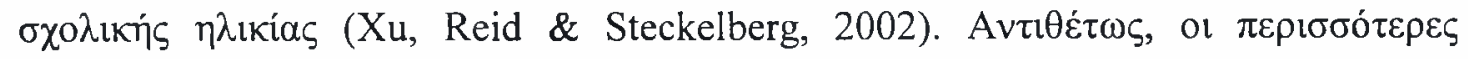

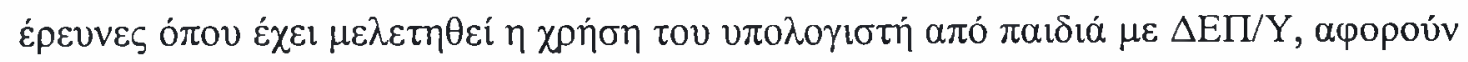

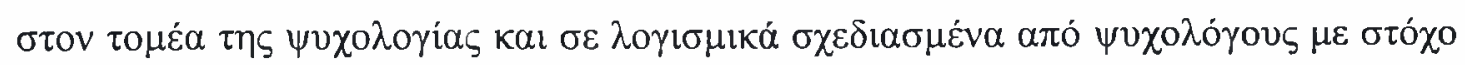

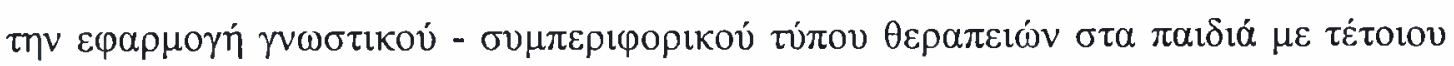

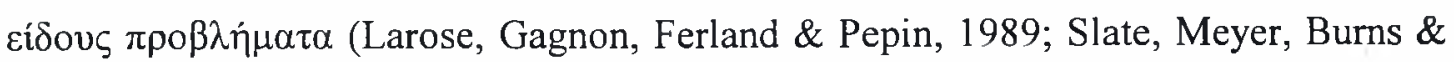
Montgomery, 1998; Navarro, Ruiz, Alcalde, Marchena \& Aguilar, 2001).

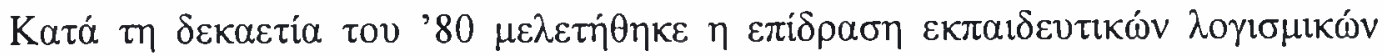

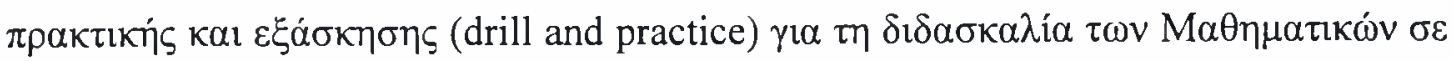

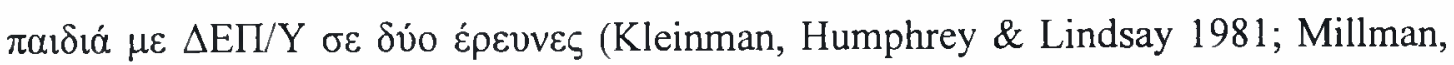

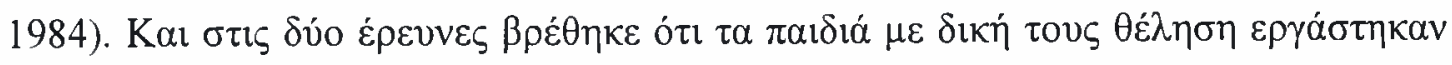




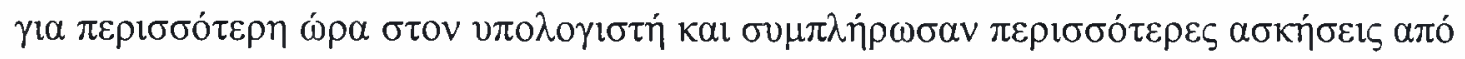

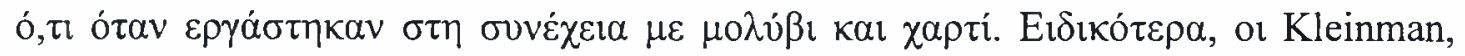

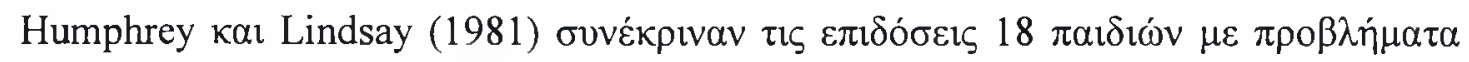

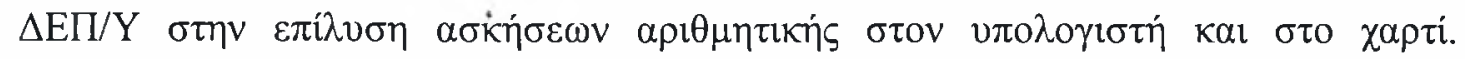

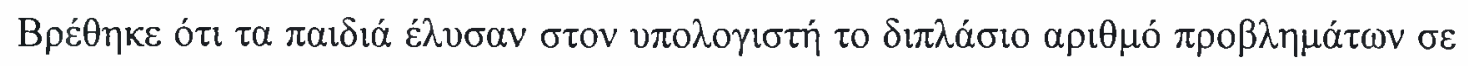

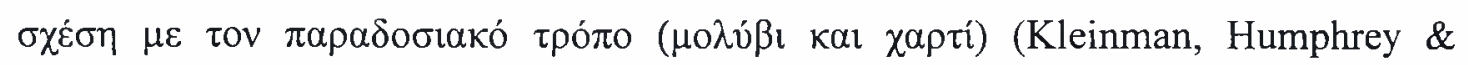
Lindsay, 1981).

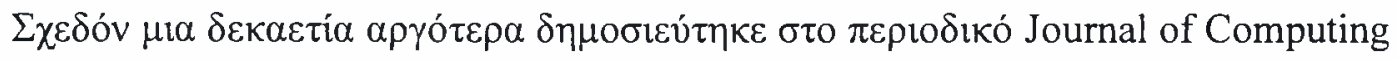

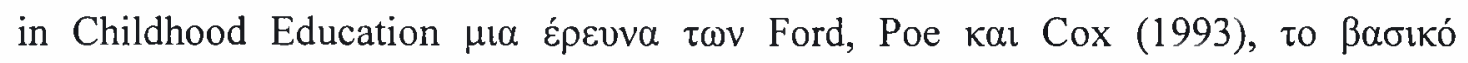

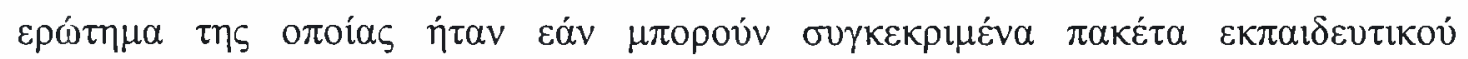

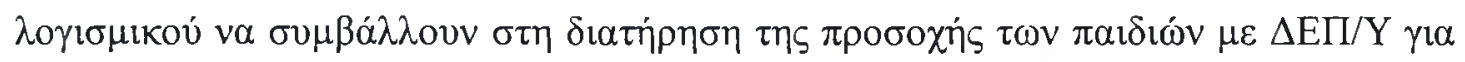

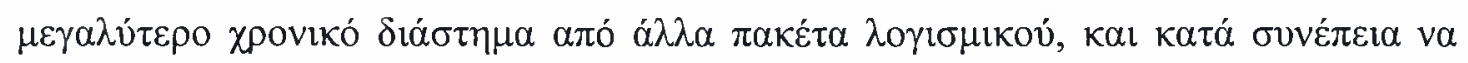

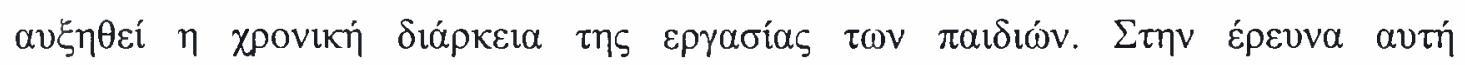

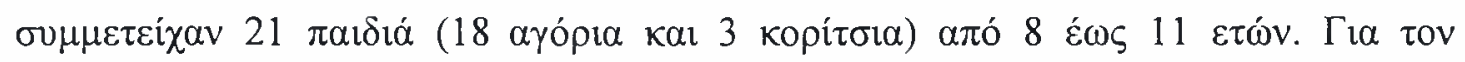

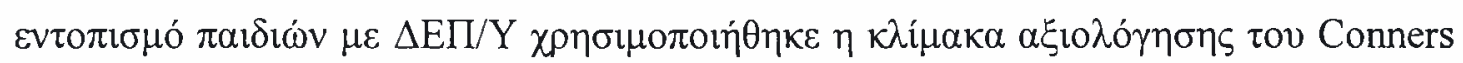

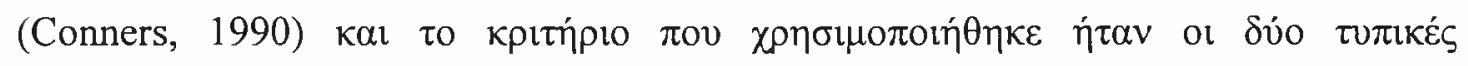

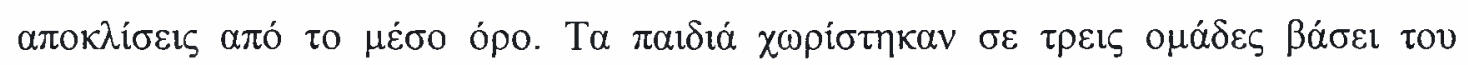

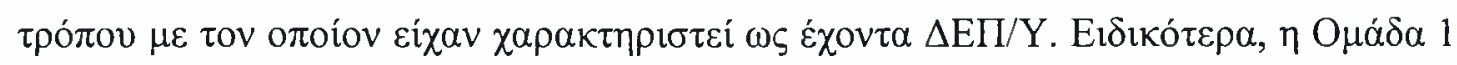

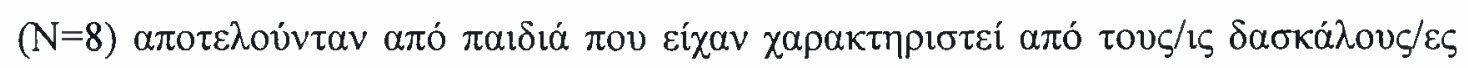

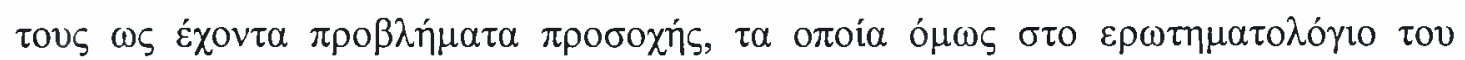

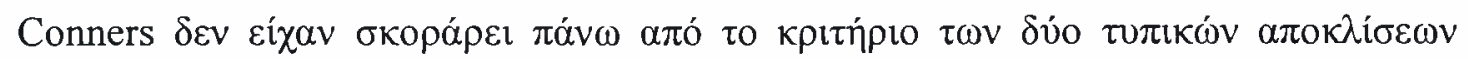

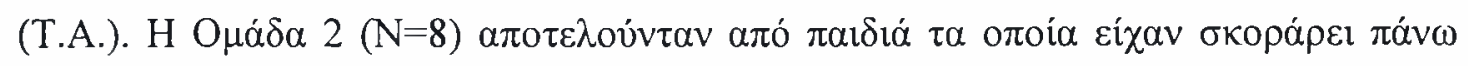

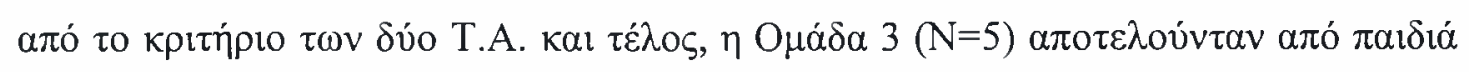

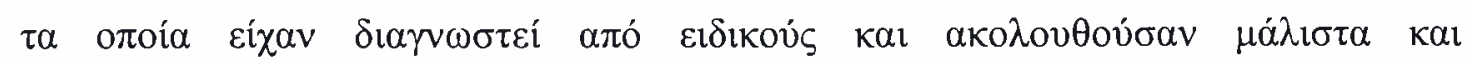

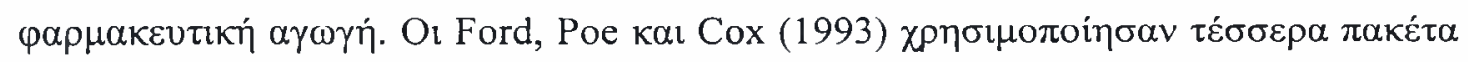

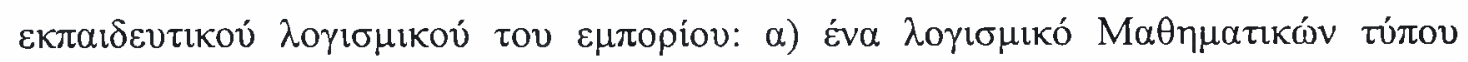

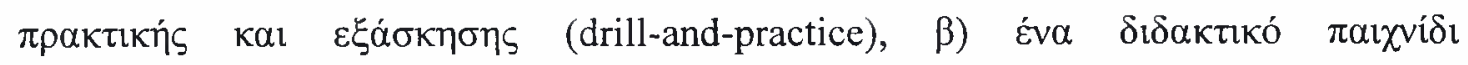

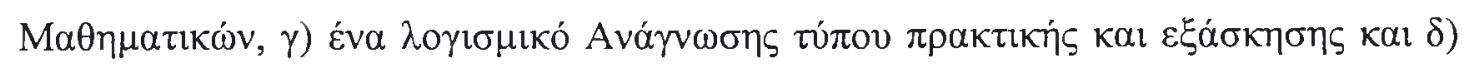

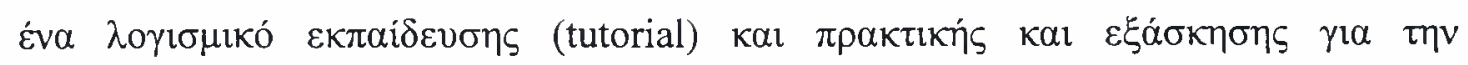

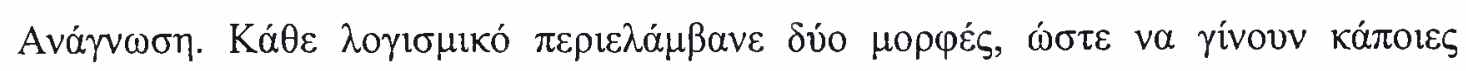

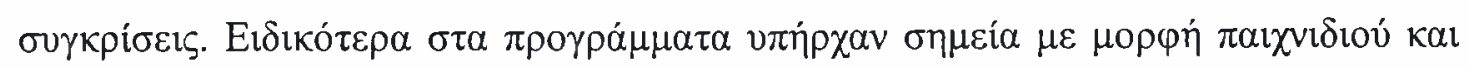

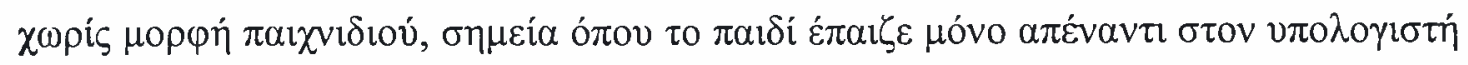

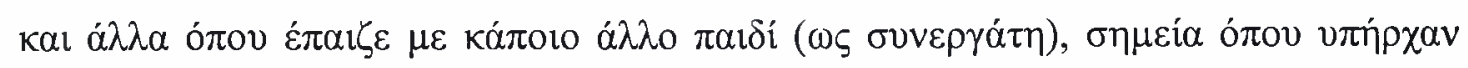

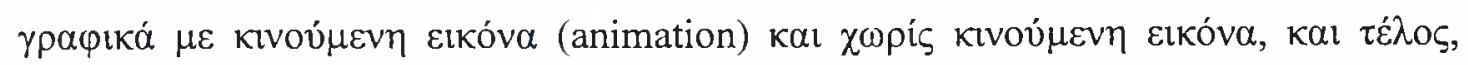




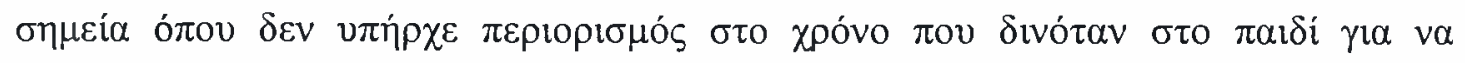

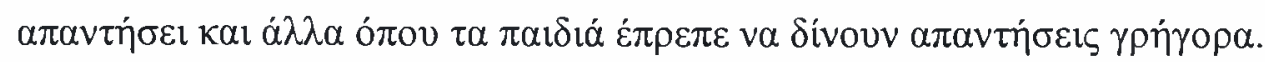

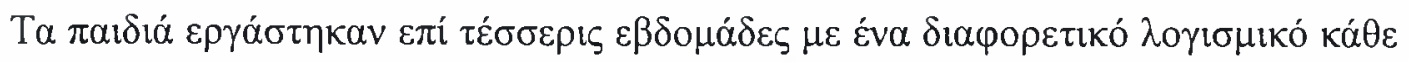

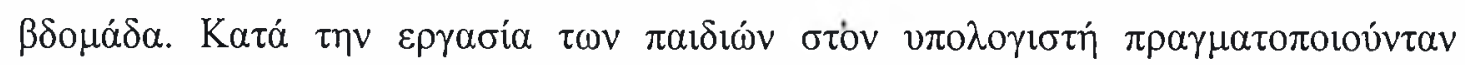

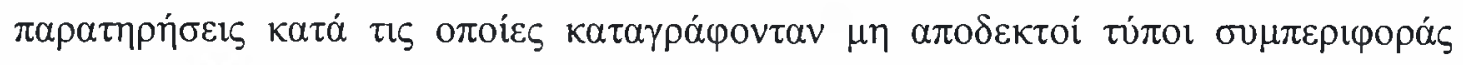

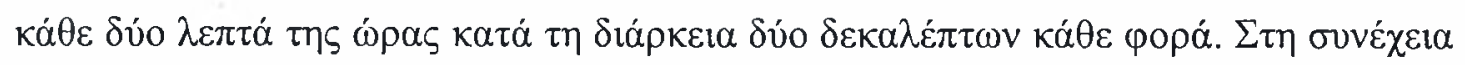

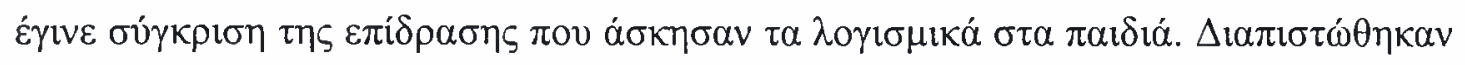
$\tau \alpha \alpha \kappa o ́ \lambda o v \theta \alpha$ :

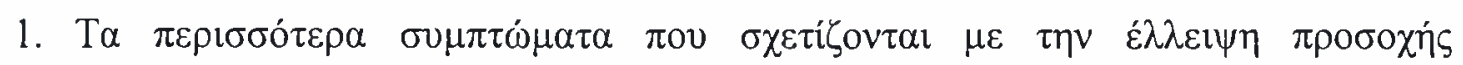

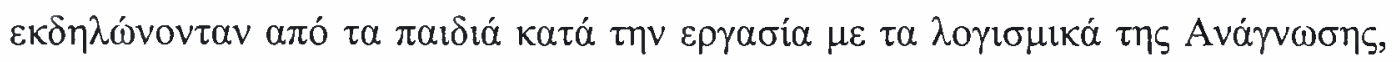

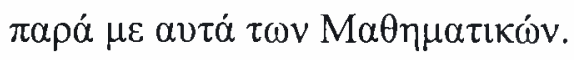

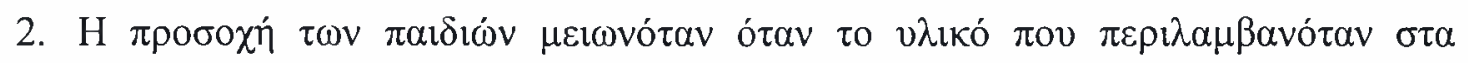

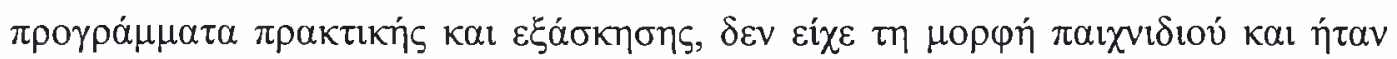

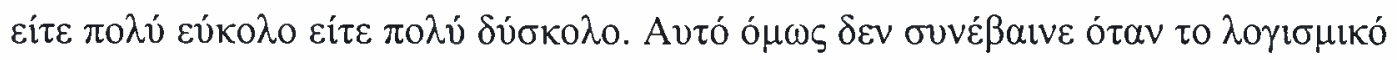

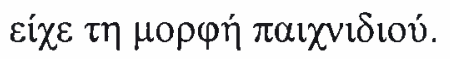

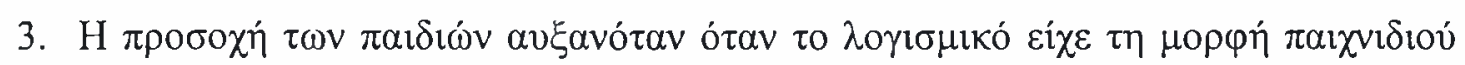

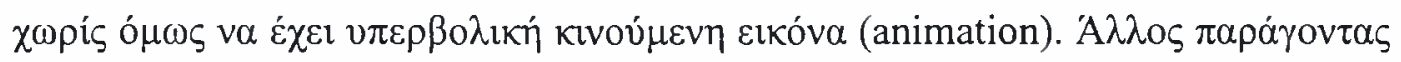

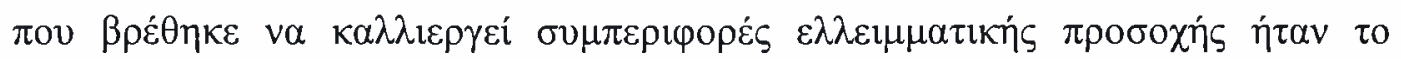

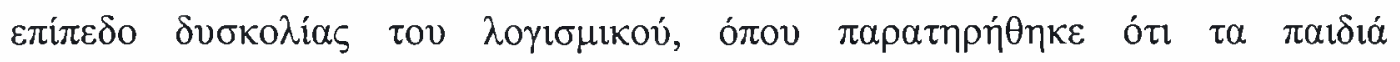

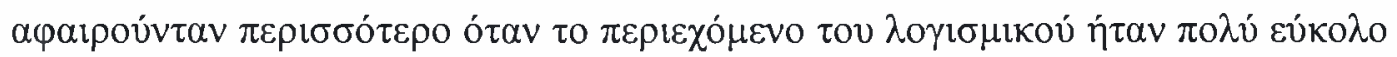

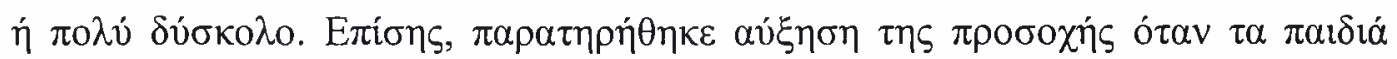

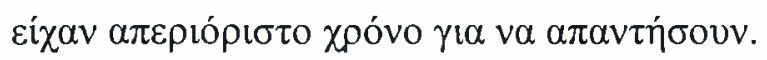

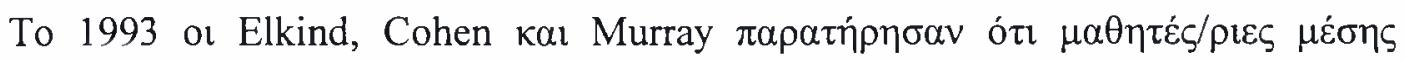

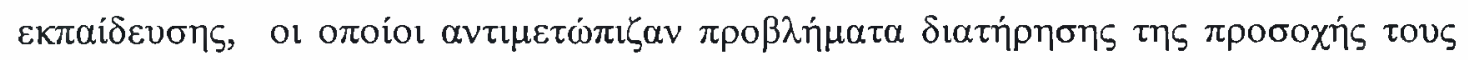

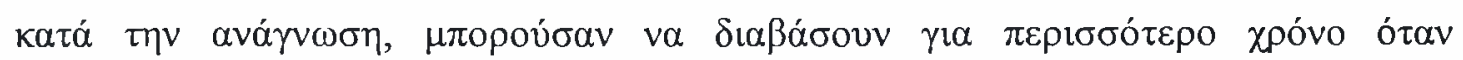

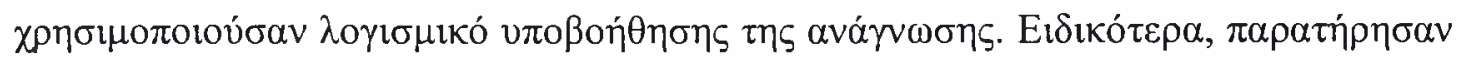

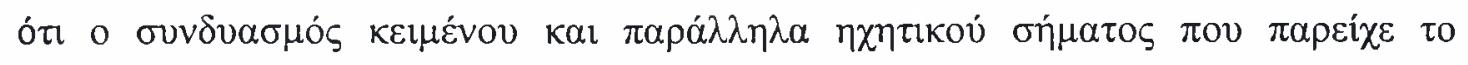

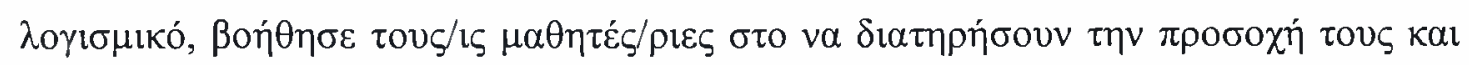

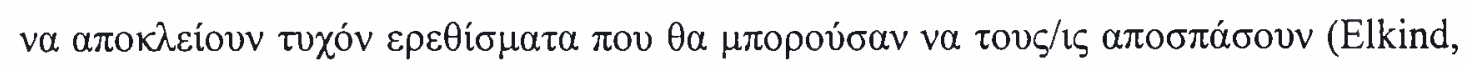
Cohen \& Murray, 1993).

To $1997 \delta \eta \mu о \sigma \imath \varepsilon \dot{\tau \eta \kappa \varepsilon ~ \varepsilon ́ \rho \varepsilon u v a ~ \tau \omega v ~ C a r e y ~ \kappa a \imath ~ S a l e, ~ \sigma \tau \eta v ~ o \pi o i ́ a ~ o l ~ \varepsilon \rho \varepsilon u v \eta \tau \varepsilon ́ \varsigma ~}$

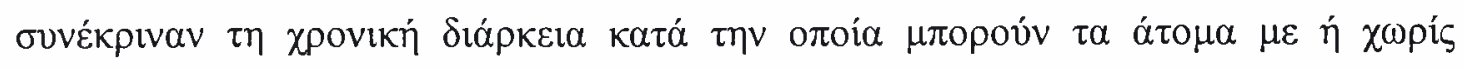

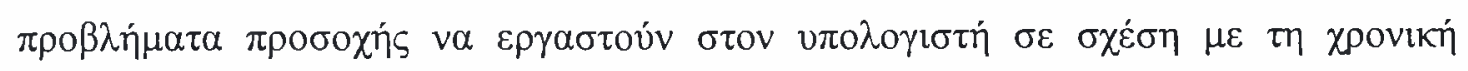




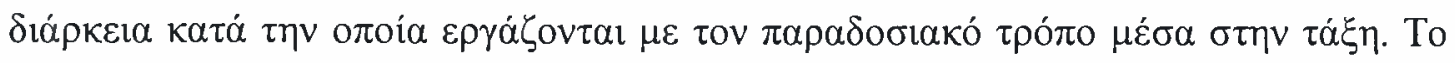

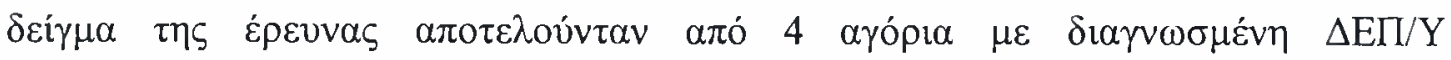

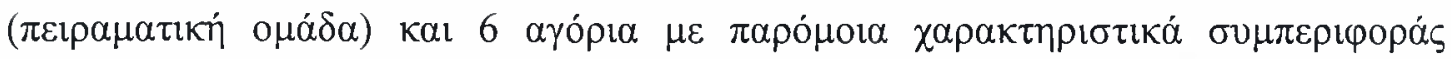

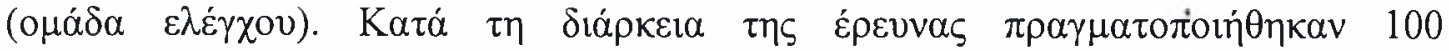

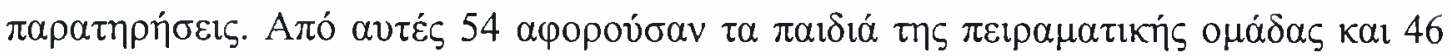

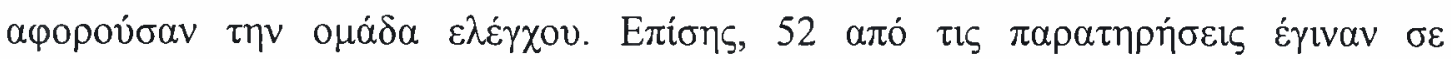

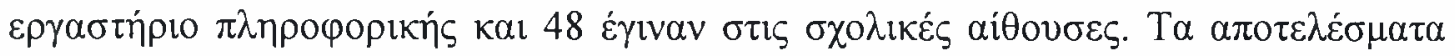

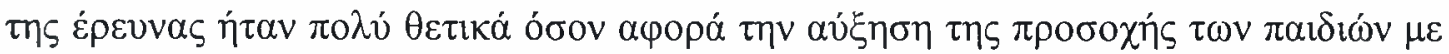

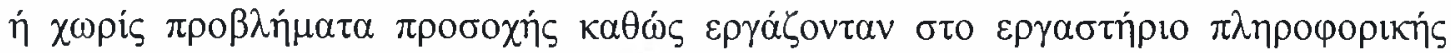
(Carey \& Sale, 1997).

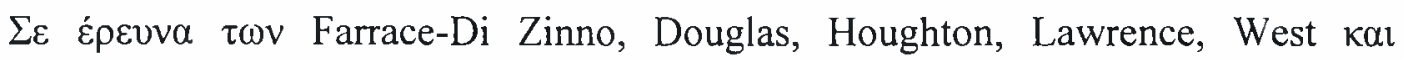

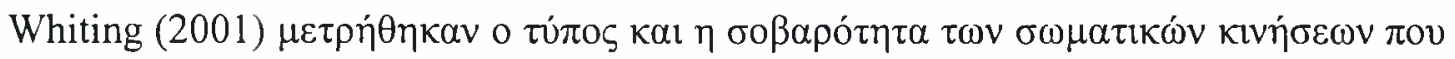

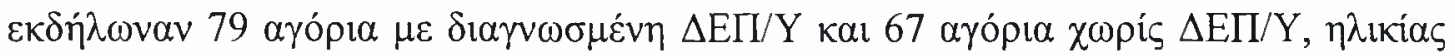

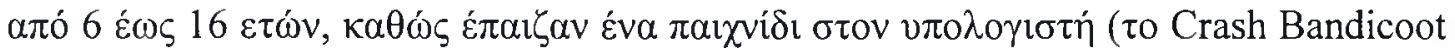

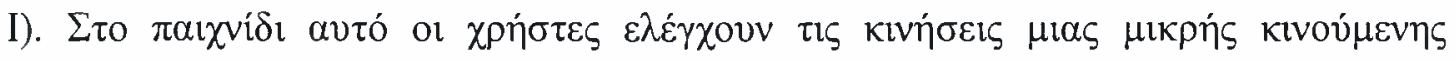

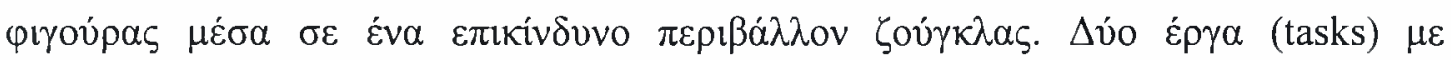

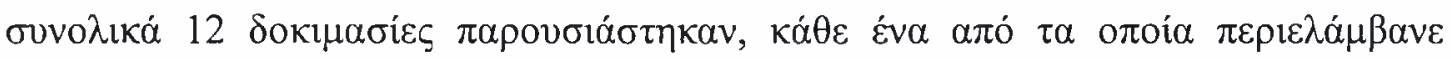

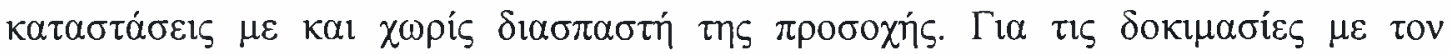

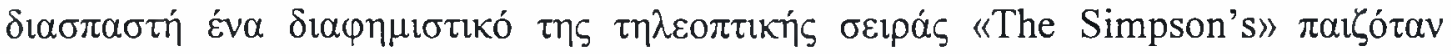

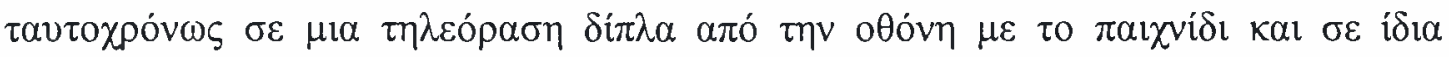

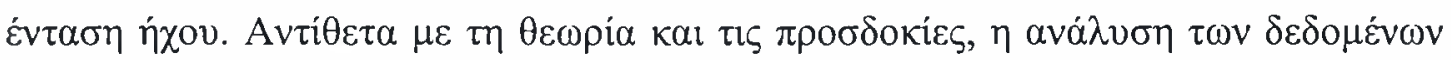

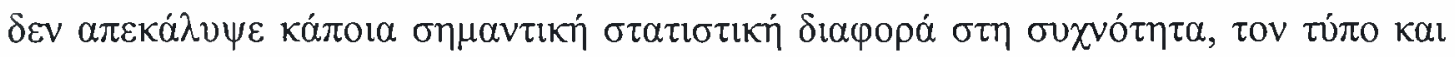

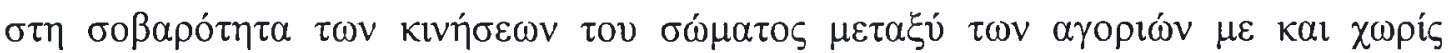

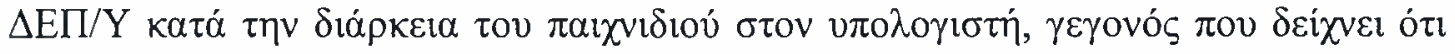

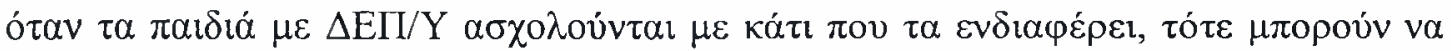

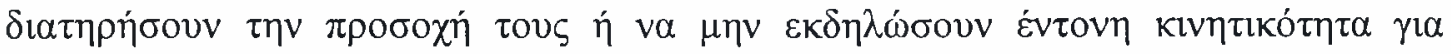

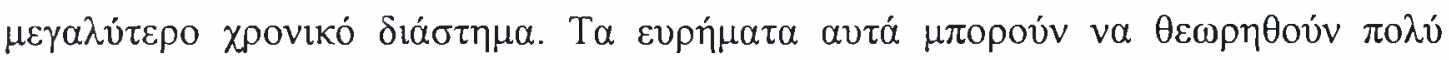

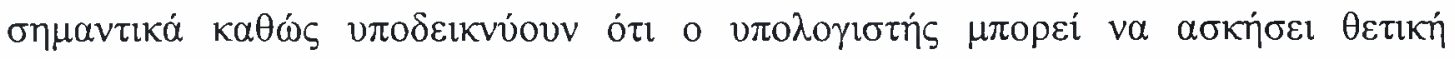

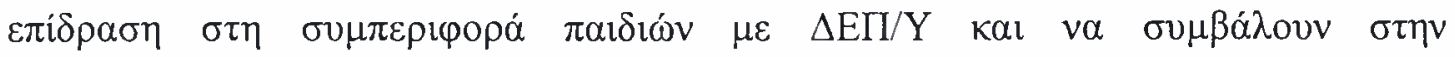
$\alpha \pi \circ \tau \varepsilon \lambda \varepsilon \sigma \mu \alpha \tau \iota \kappa \eta ́ ~ \varepsilon \kappa \pi \alpha i ́ \delta \varepsilon v \sigma \eta ́ ~ \tau o v \varsigma$ (Farrace-Di Zinno, Douglas, Houghton, Lawrence, West \& Whiting, 2001).

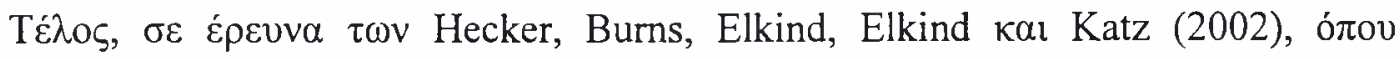

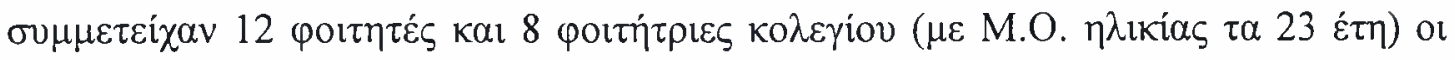

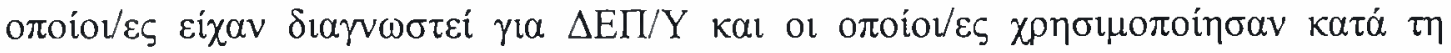




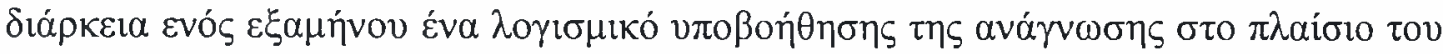

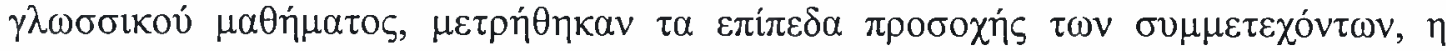

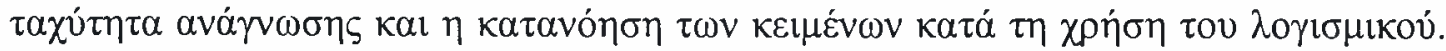

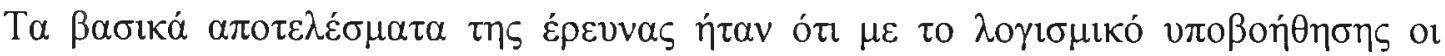

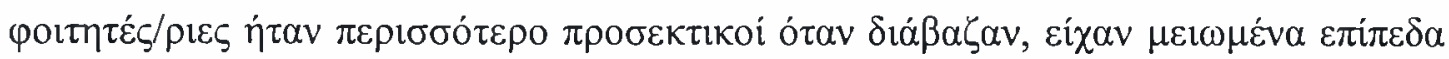

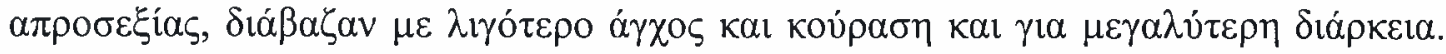

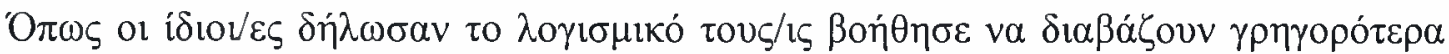

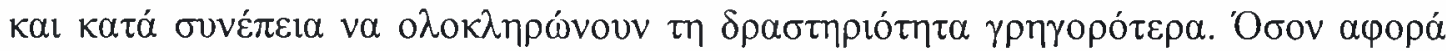

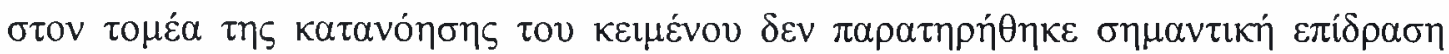

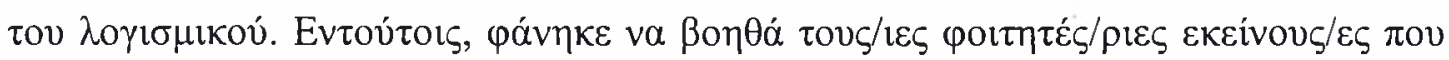

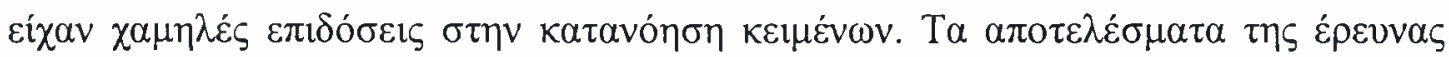

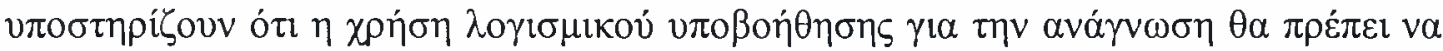

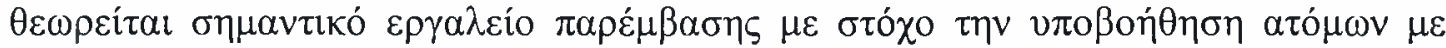
$\delta 1 \alpha \tau \alpha \rho \alpha \chi \varepsilon ́ \varsigma \tau \eta \varsigma \pi \rho \circ \sigma o \chi \eta ́ \varsigma$ (Hecker, Burns, Elkind, Elkind \& Katz, 2002).

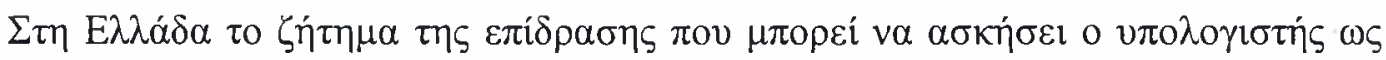

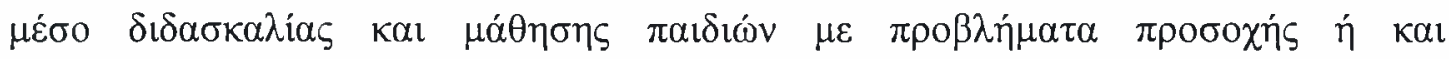

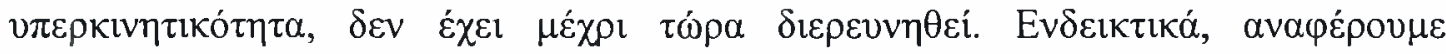

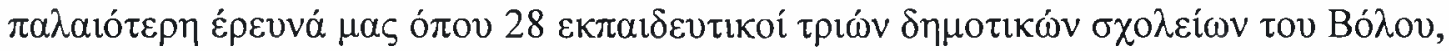

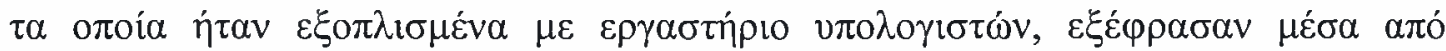

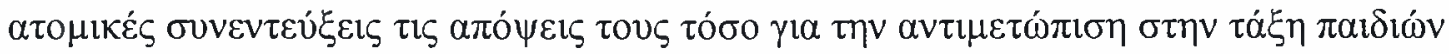

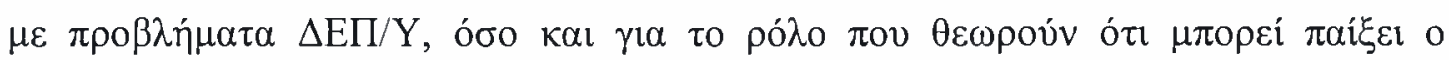

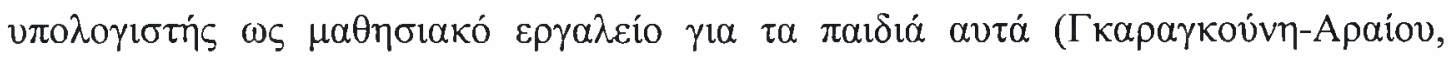

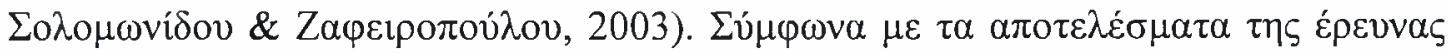

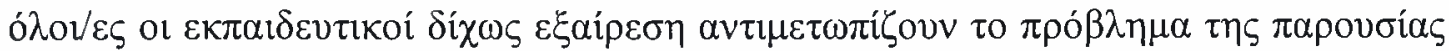

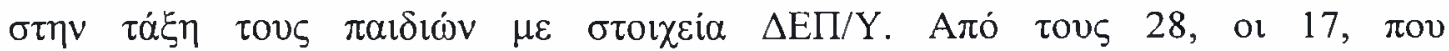

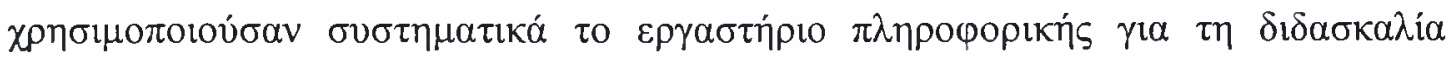

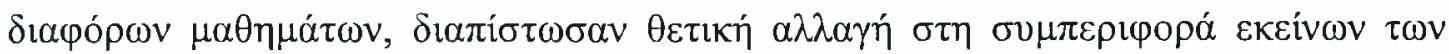

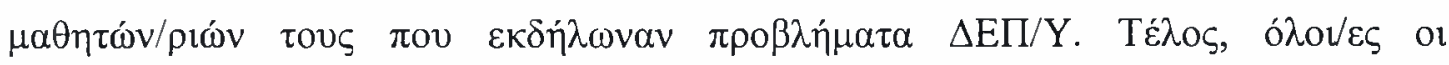

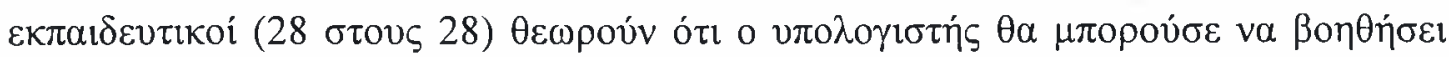

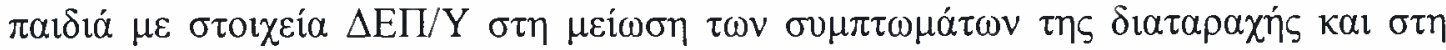

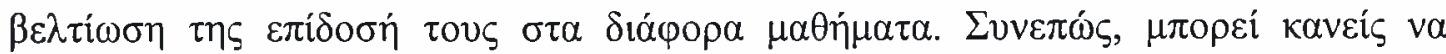

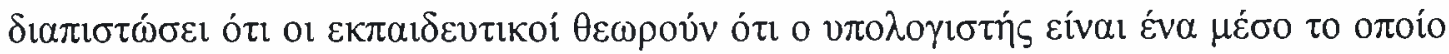

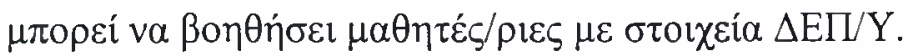




\section{$2.4 \Sigma Y N O \Psi H$}

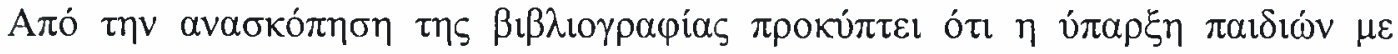

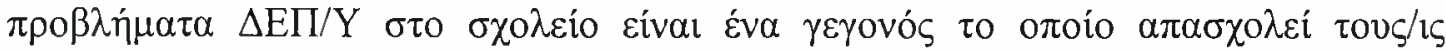

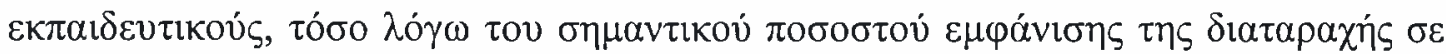

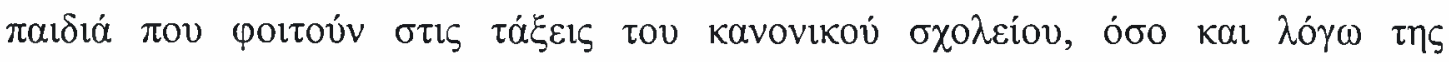

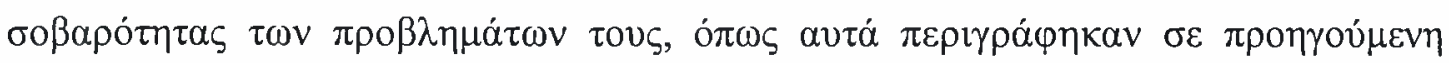

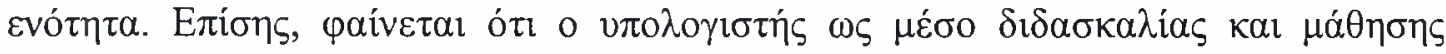

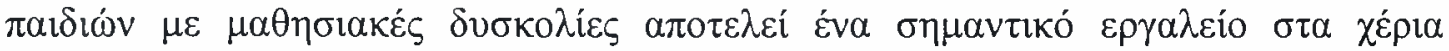

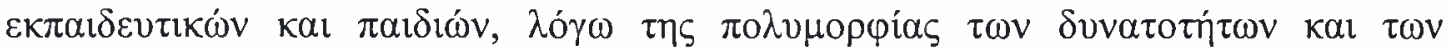

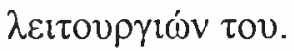

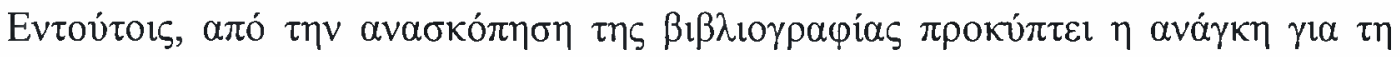

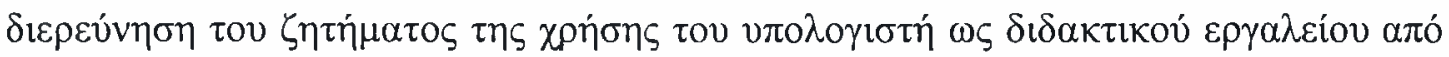

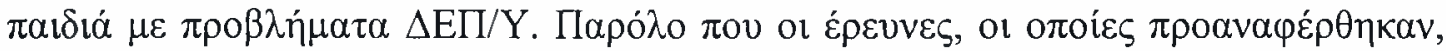

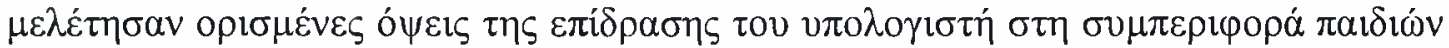

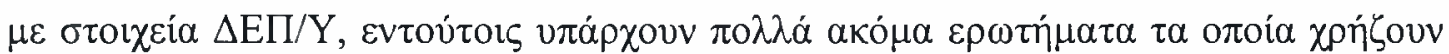

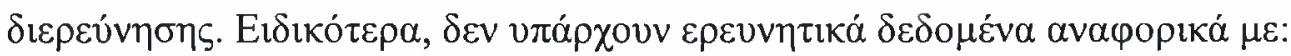

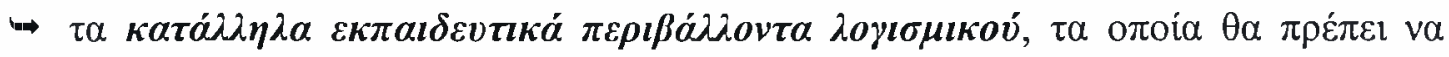

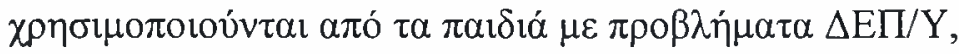

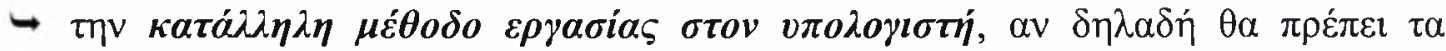

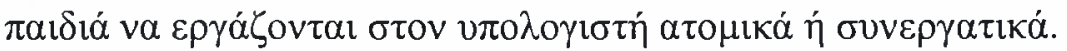

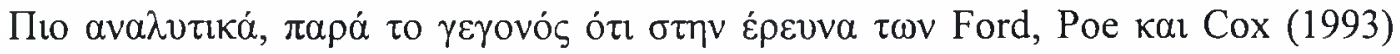

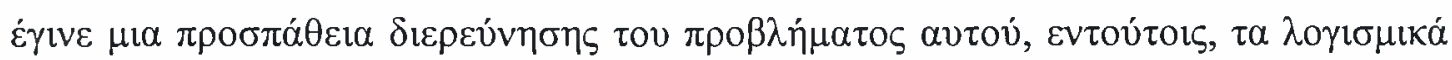

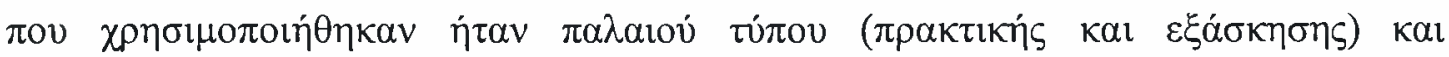

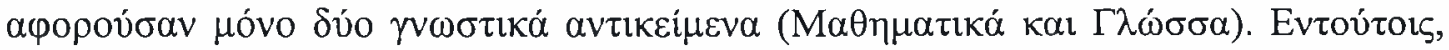

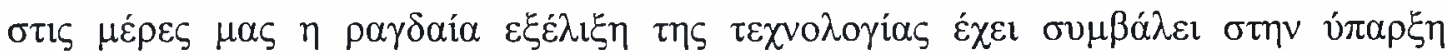

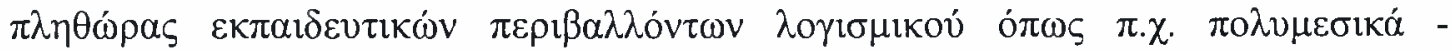

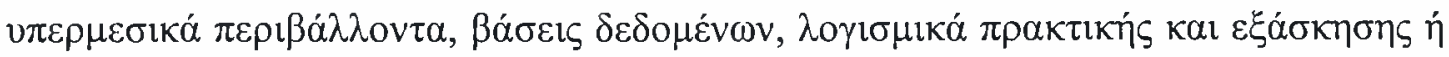

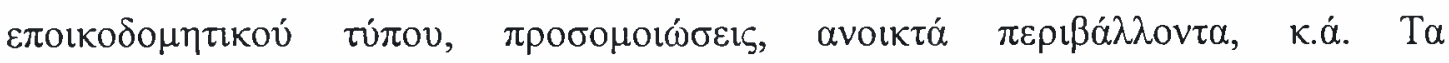

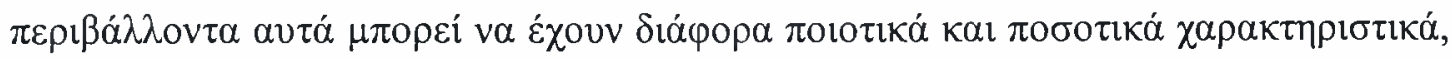

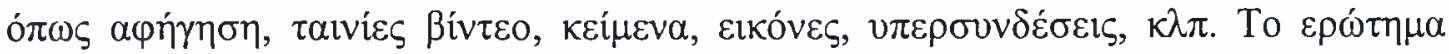

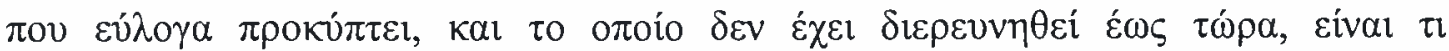

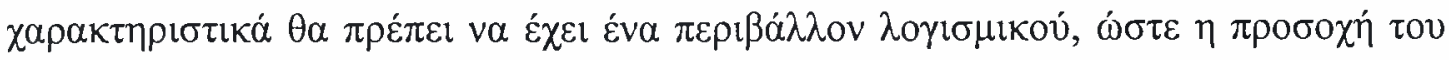




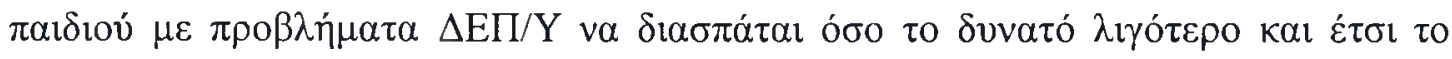

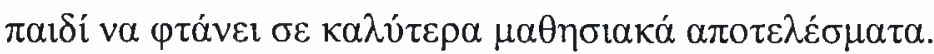

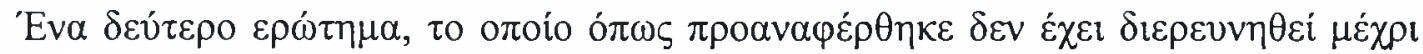

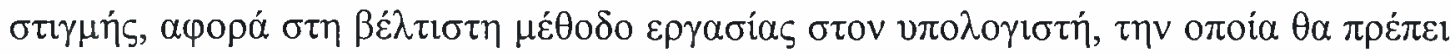

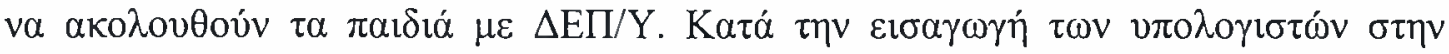

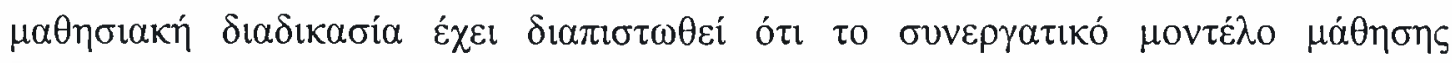

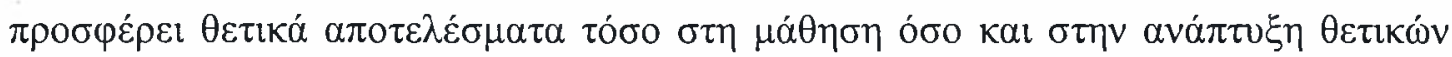

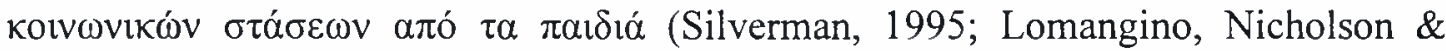

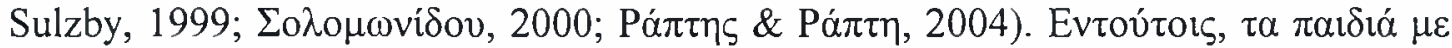

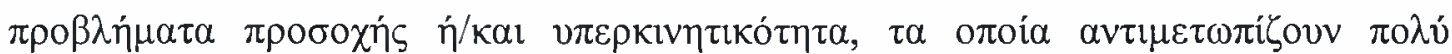

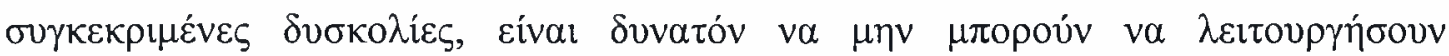

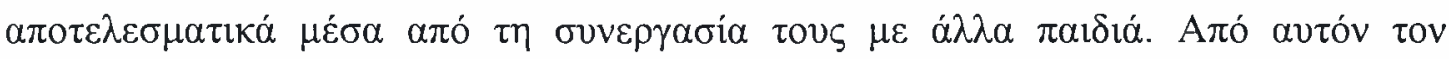

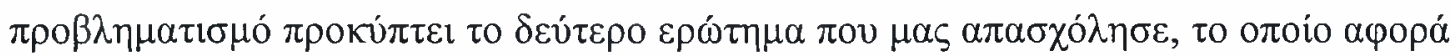

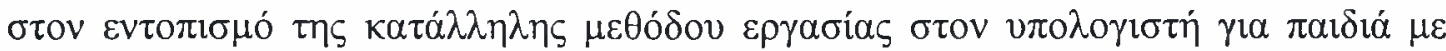
$\Delta \mathrm{E} \Pi / \mathrm{Y}$.

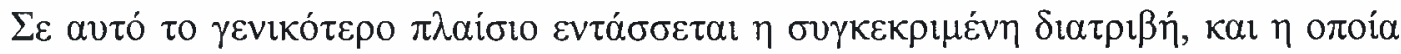

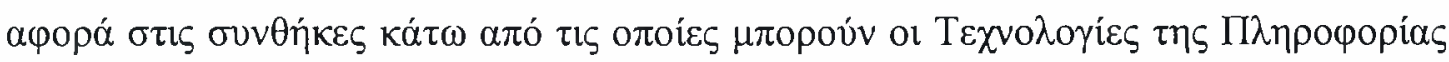

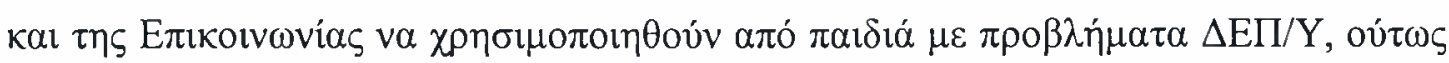

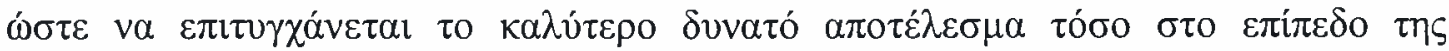

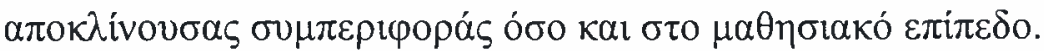




\title{
$\triangle \mathrm{EYTEPO} \mathrm{MEPO \Sigma}$
}

\author{
H EPEYNA
}




\section{TPITO KEФA $\triangle$ AIO}

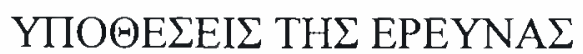

\section{$3.1 \mathrm{EI \Sigma AГ \Omega ГН}$}

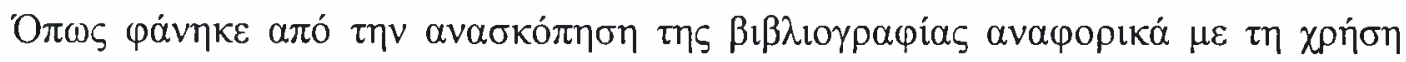

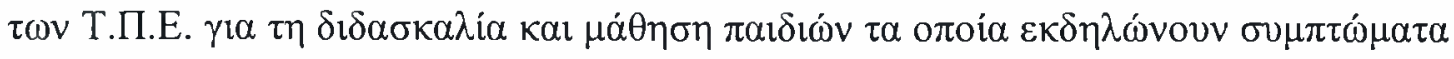

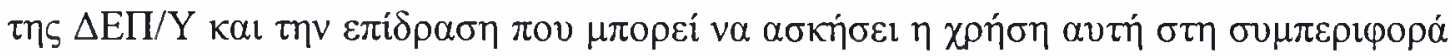

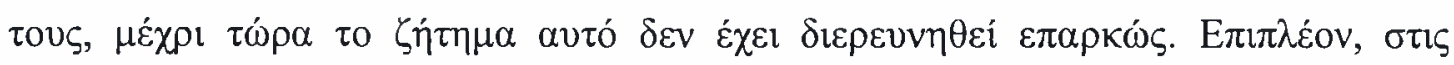

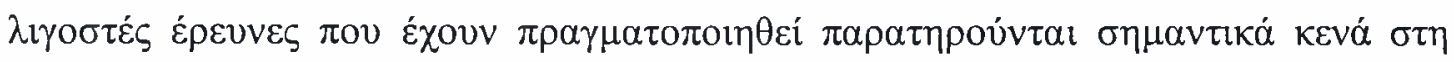

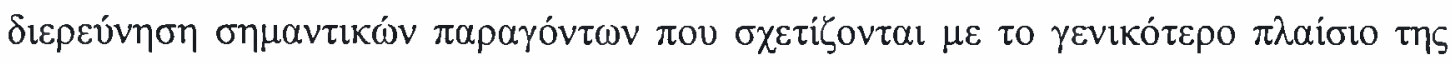

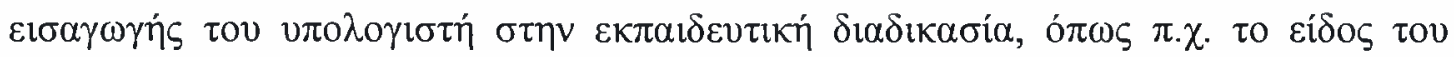

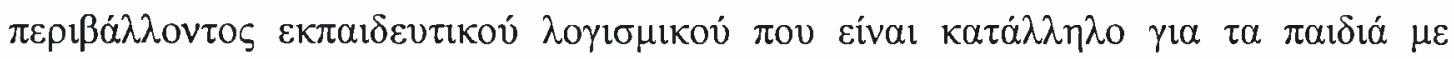

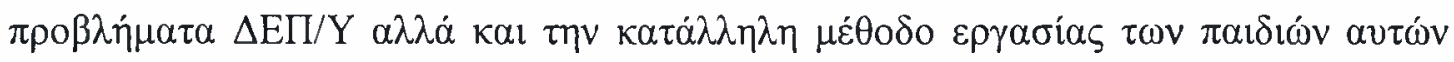

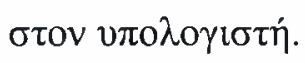

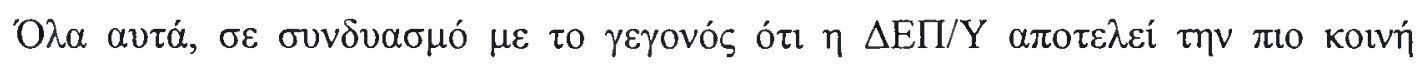

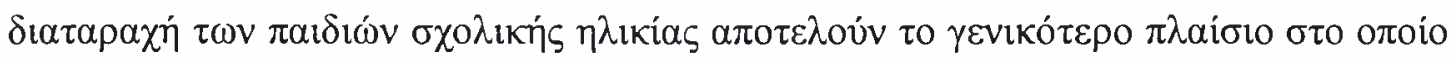

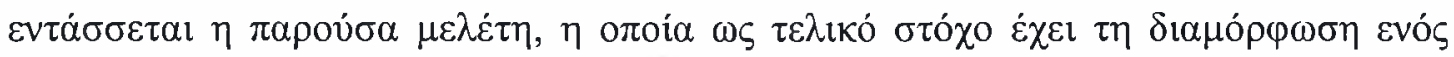

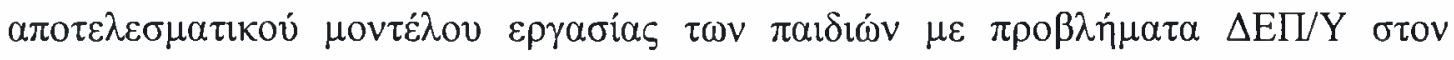

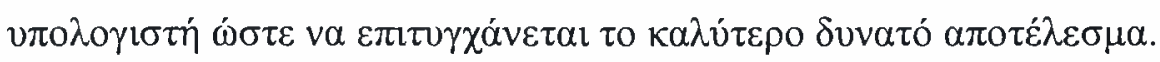

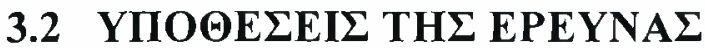

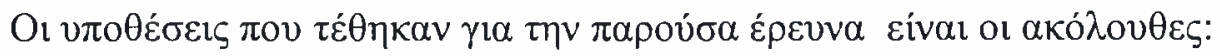

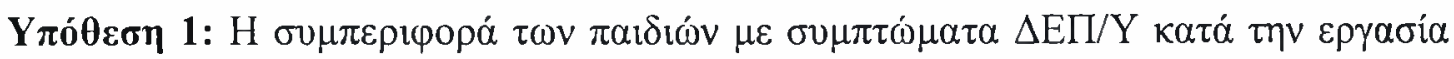

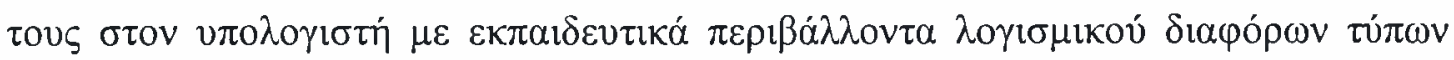

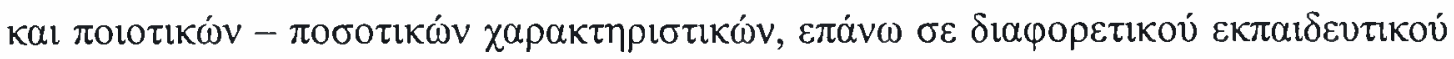

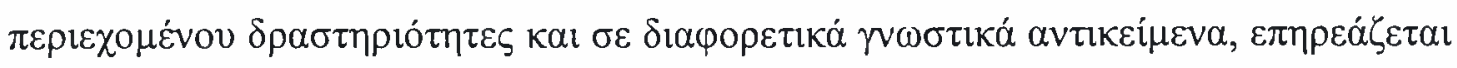

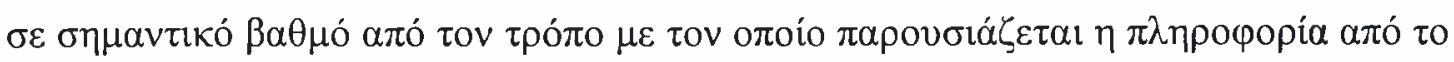




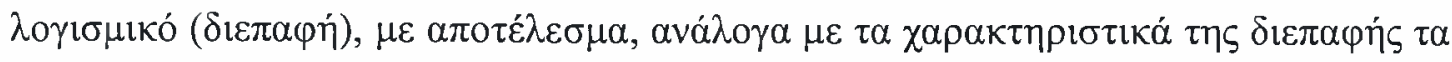

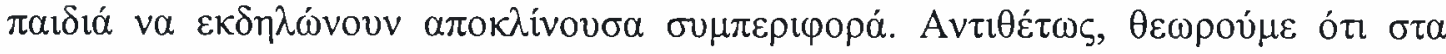

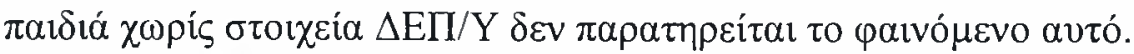

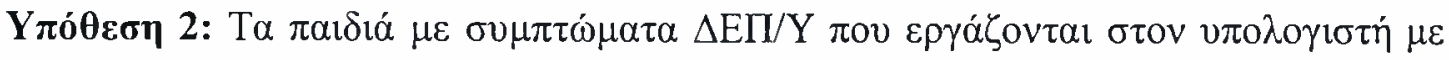

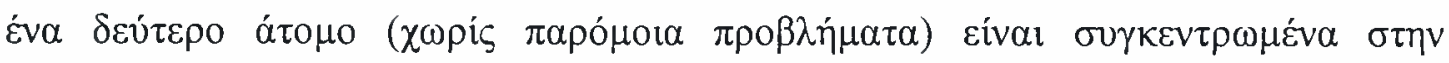

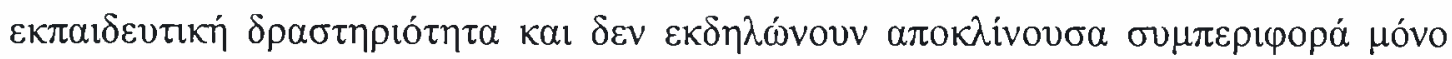

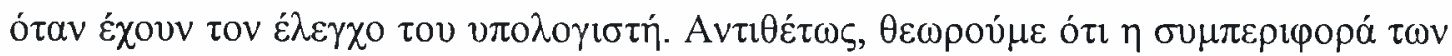

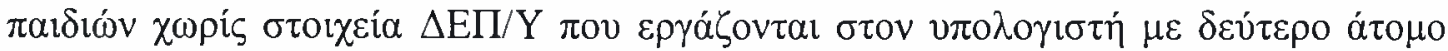

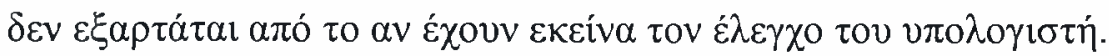

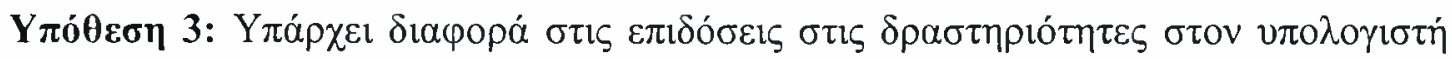

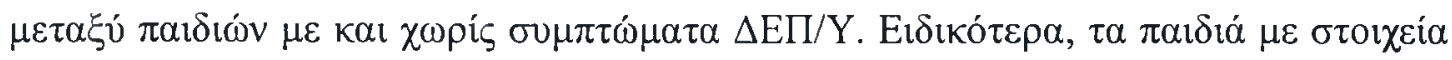

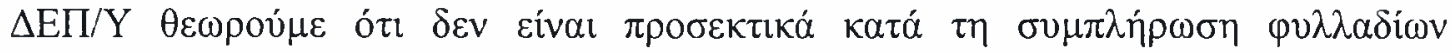

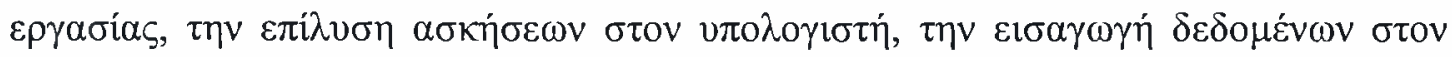

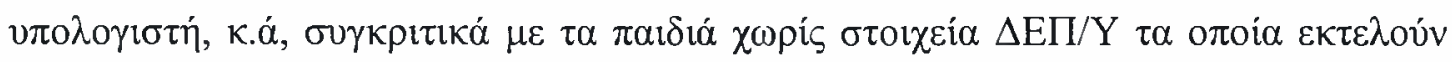

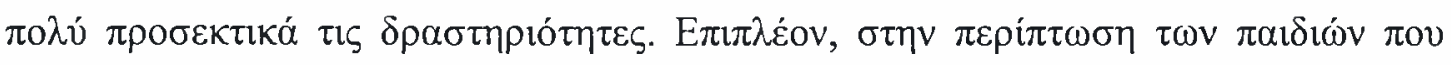

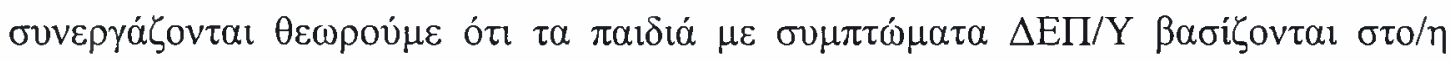

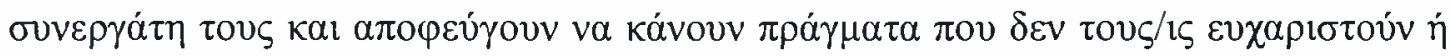

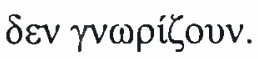

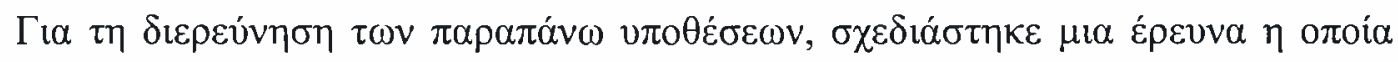

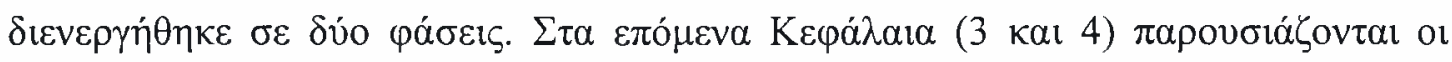

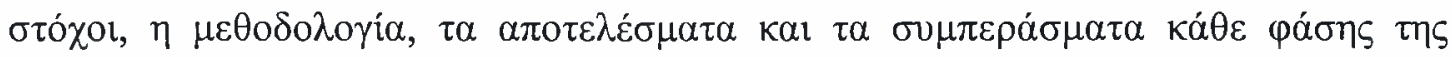

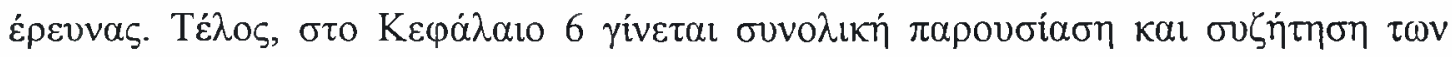

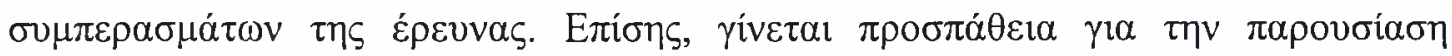

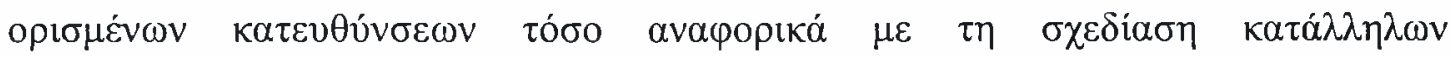

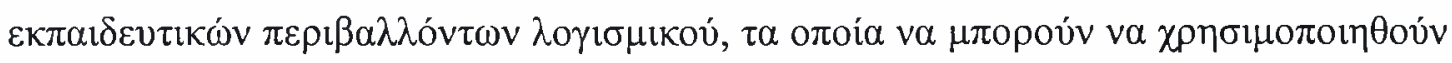

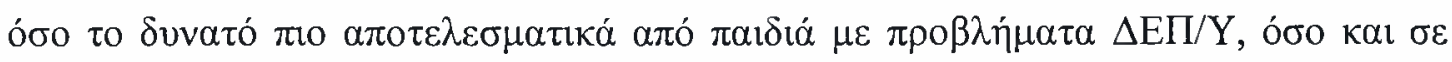

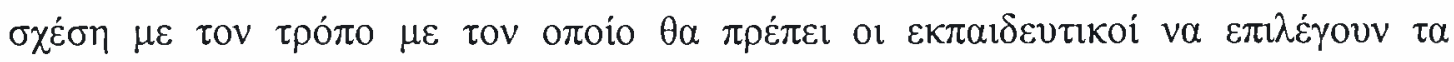

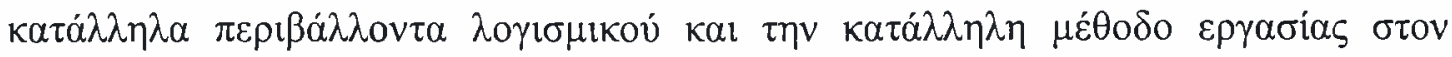

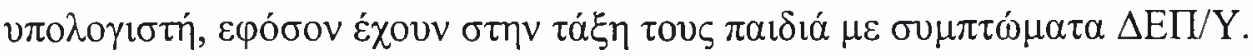




\section{TETAPTO KEФA $\triangle A I O$}

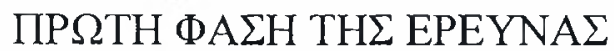

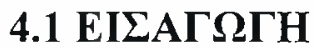

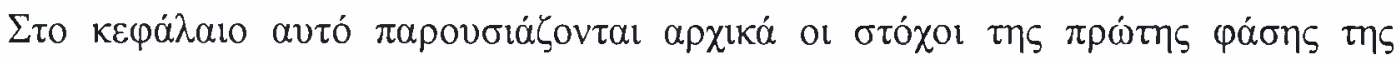

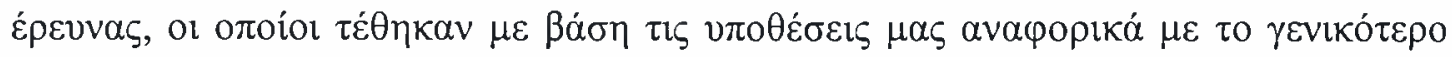

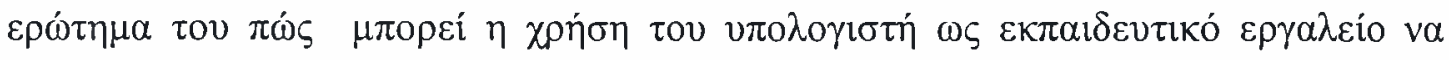

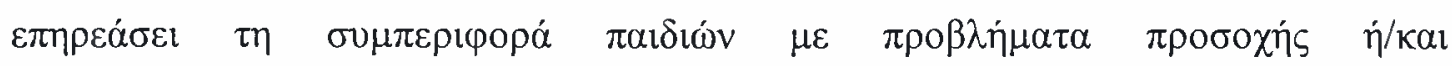

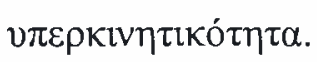

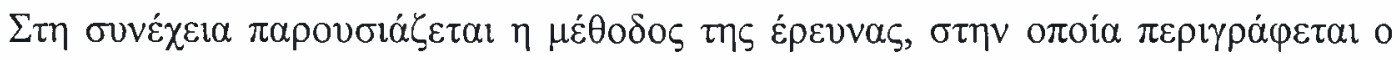

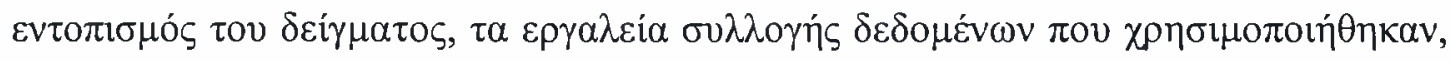

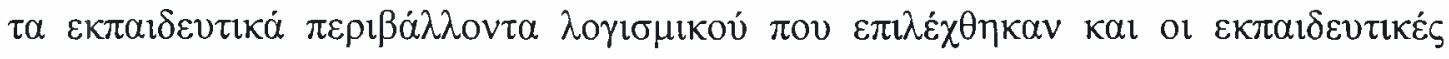

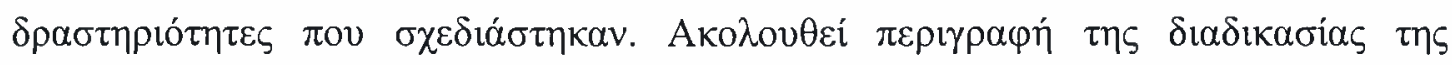

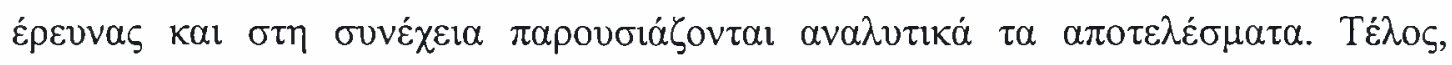

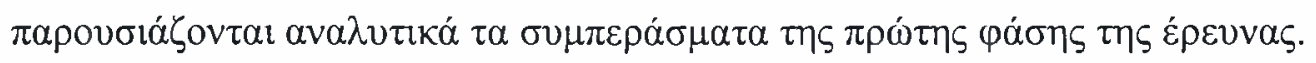

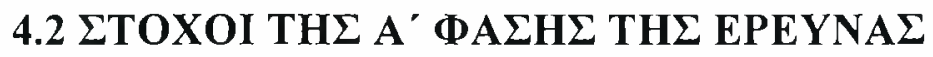

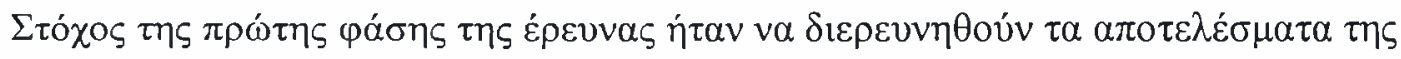

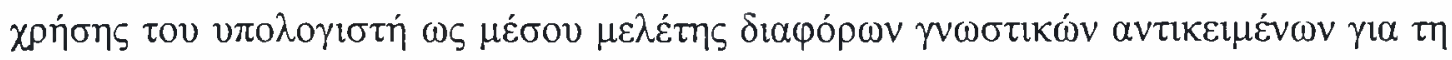

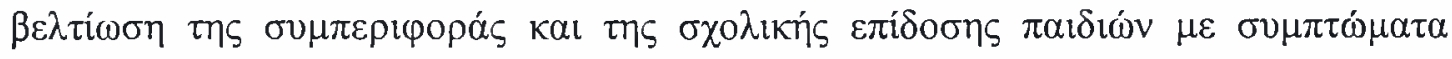

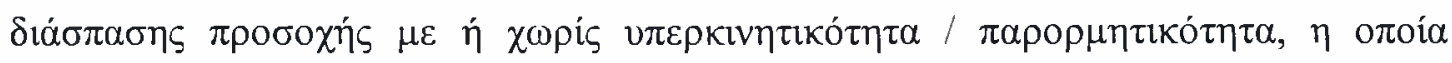

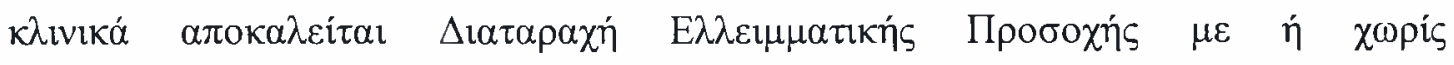

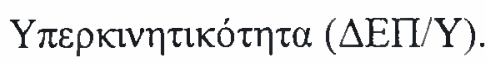

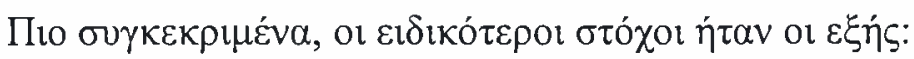

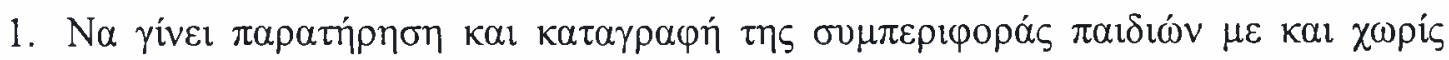

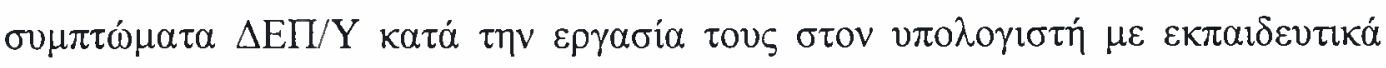

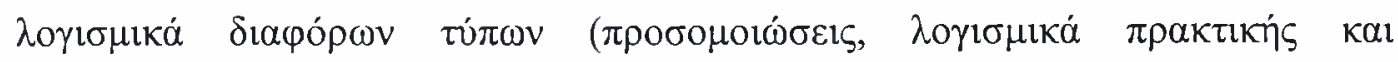




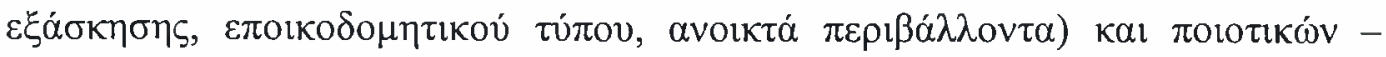

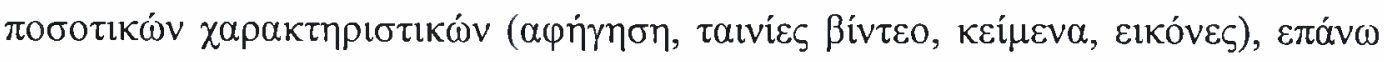

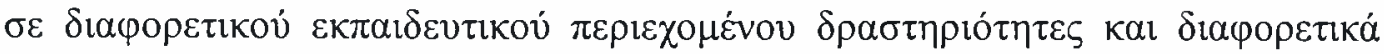

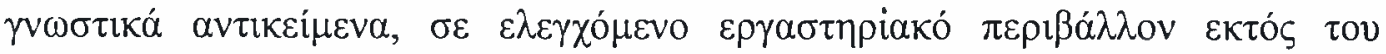

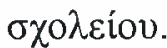

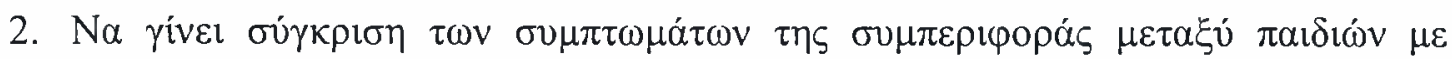

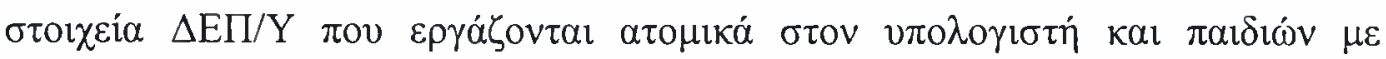

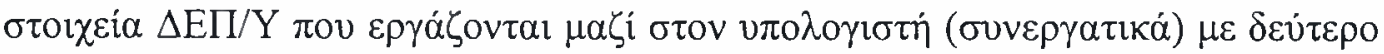

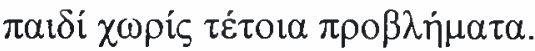

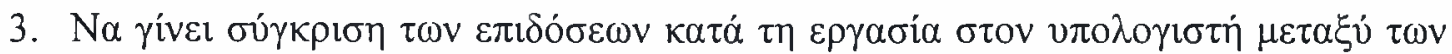

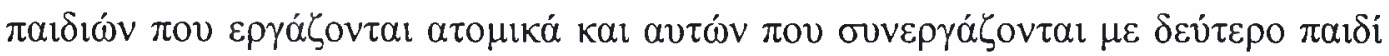

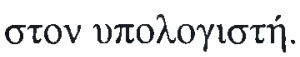

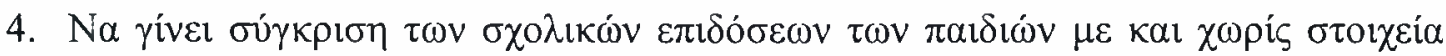

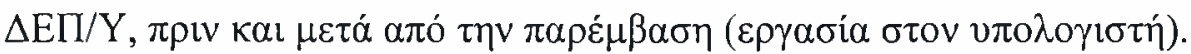

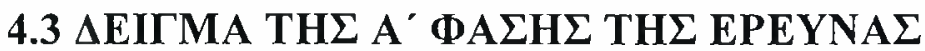

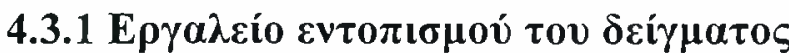

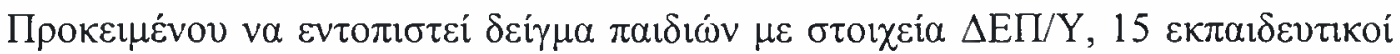

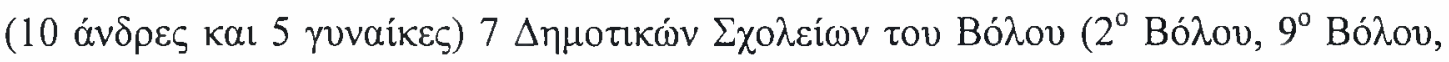

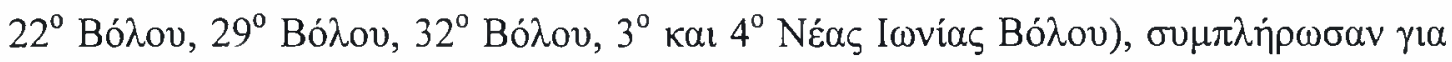

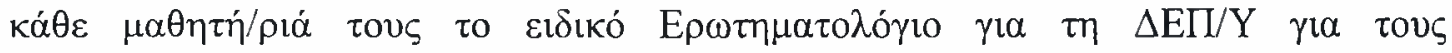

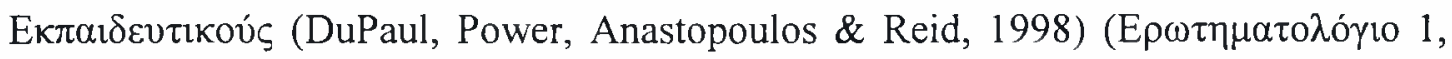

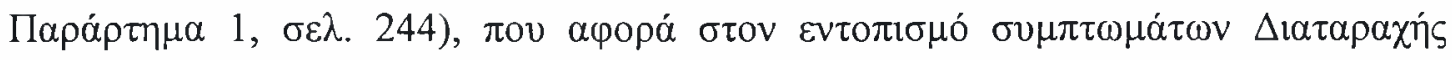

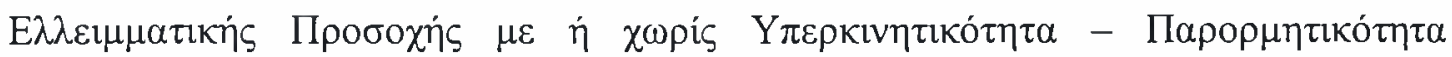

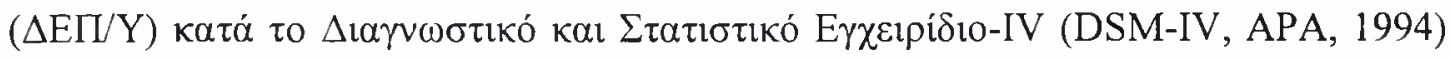

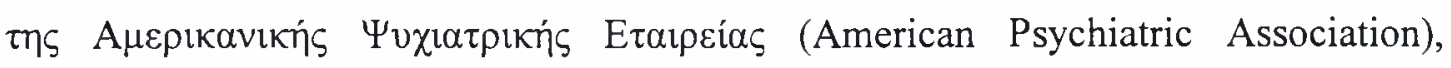

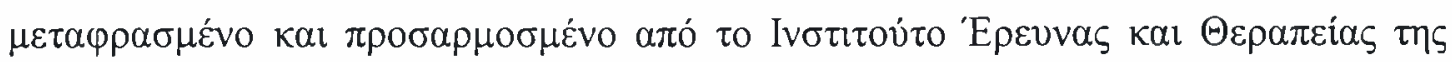

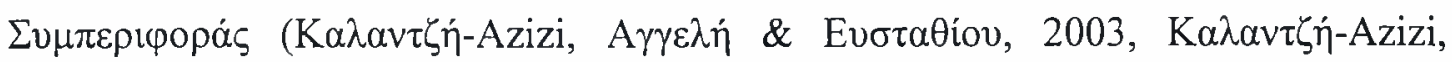

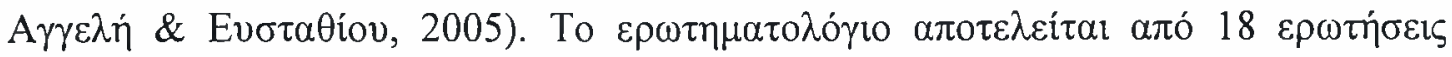

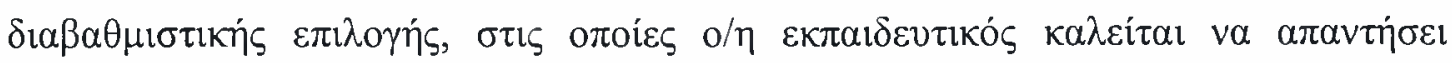




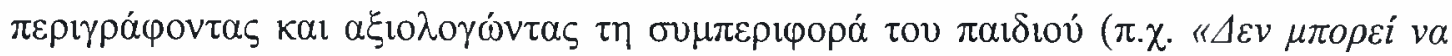

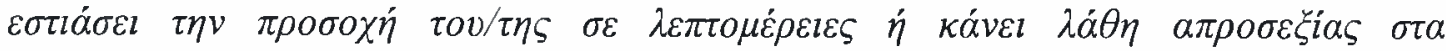

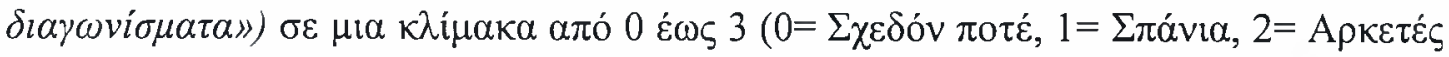

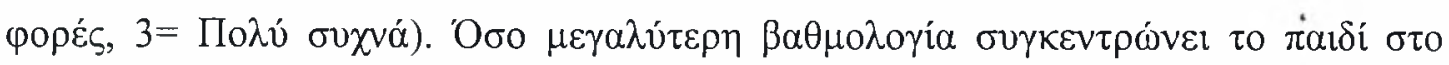

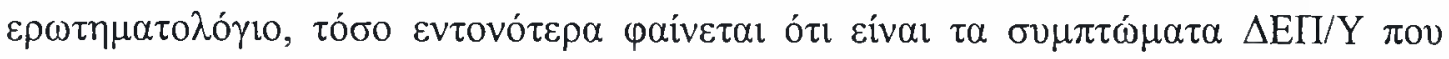

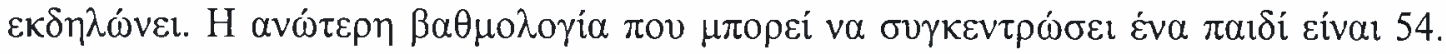

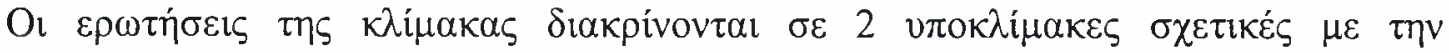

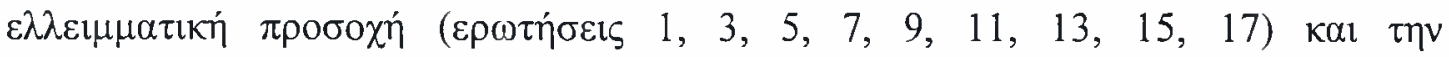

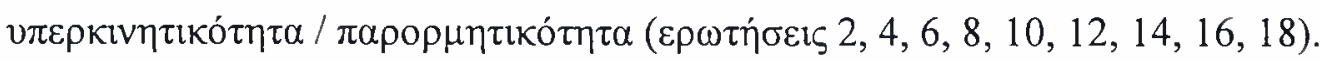

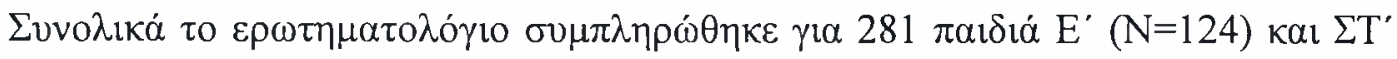

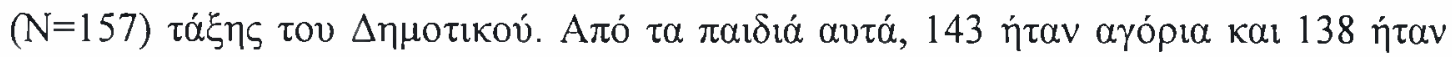

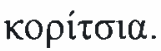

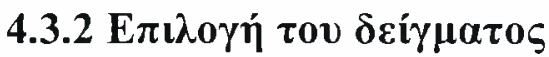

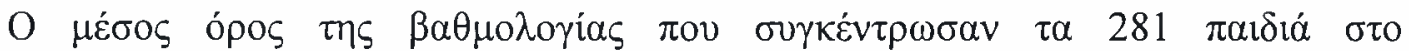

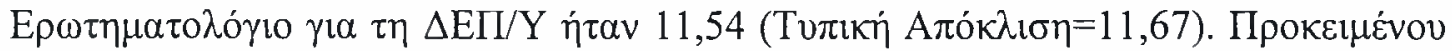

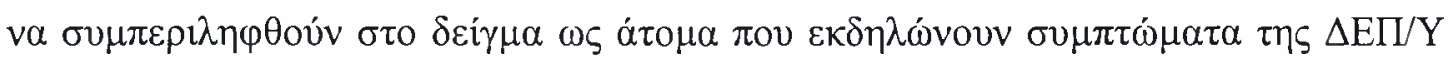

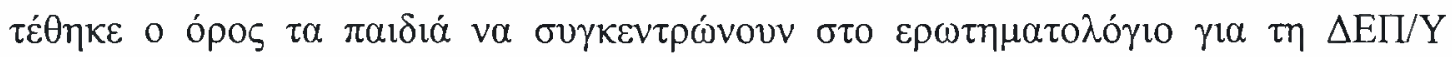

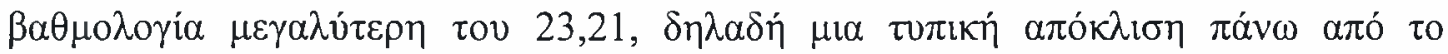

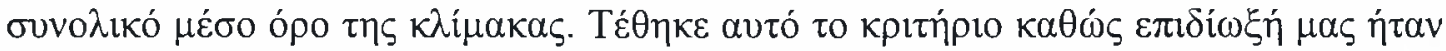

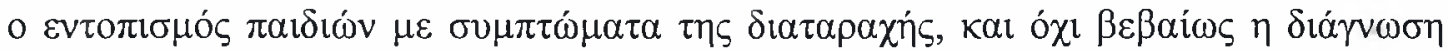

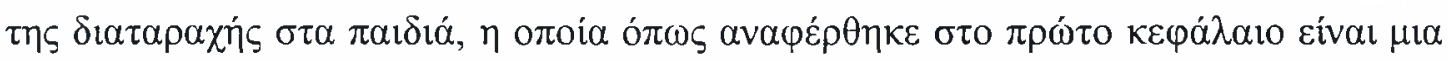
$\pi \circ \lambda$ ú $\pi \lambda \circ \kappa \eta \delta$ i $\alpha \delta 1 \kappa \alpha \sigma i \alpha$.

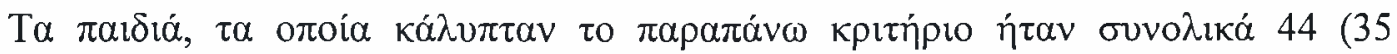

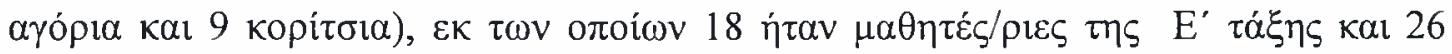

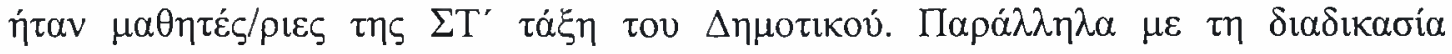

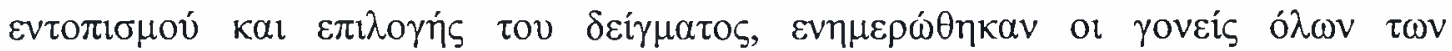

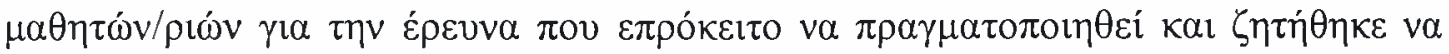

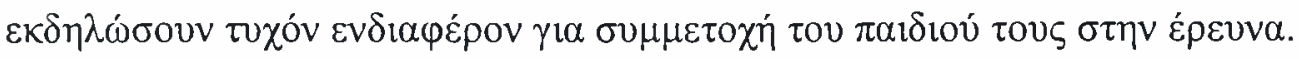

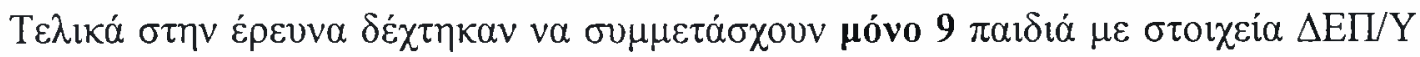

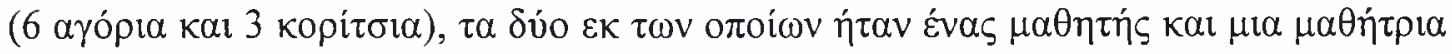

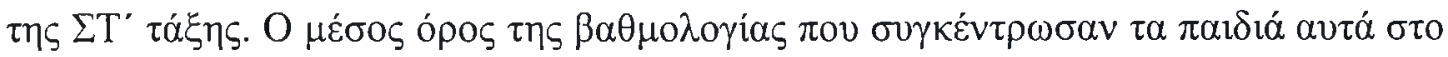


Е

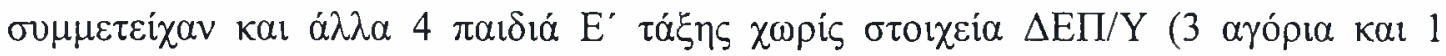

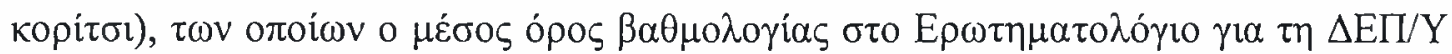

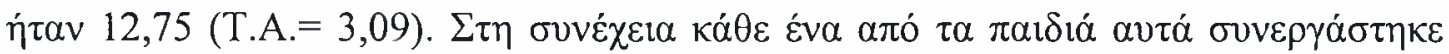

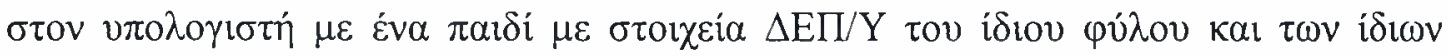

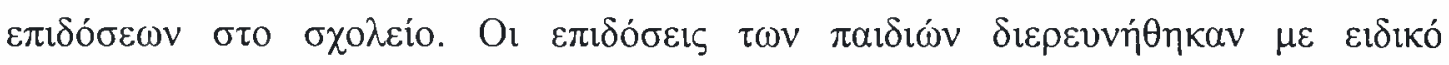

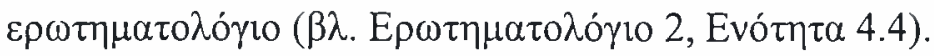

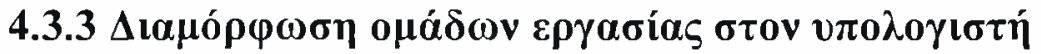

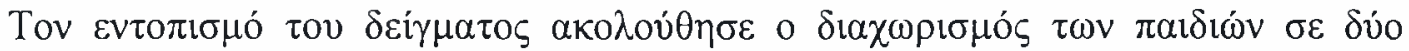

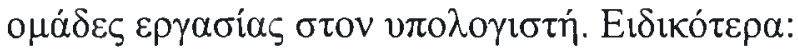

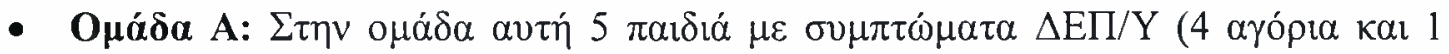

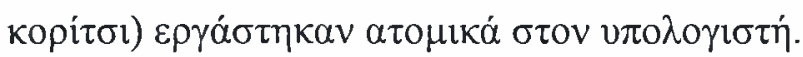

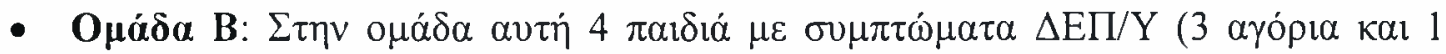

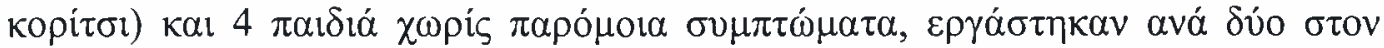

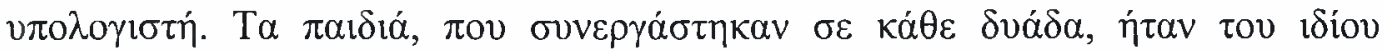

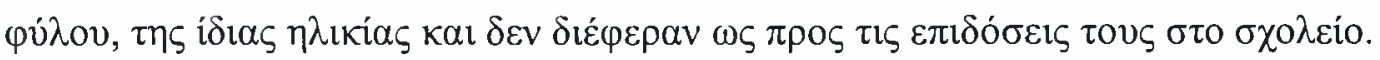

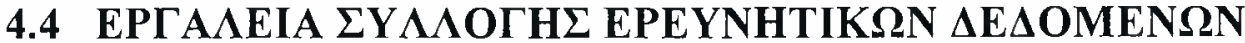

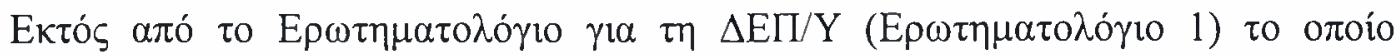

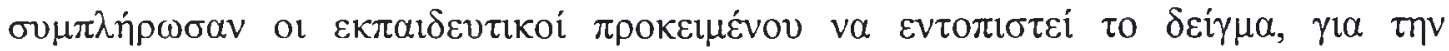

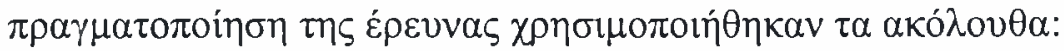

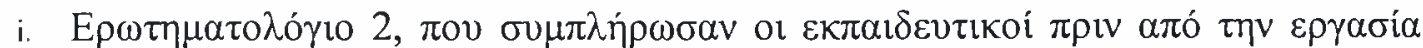

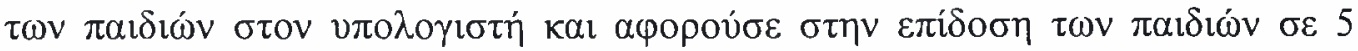

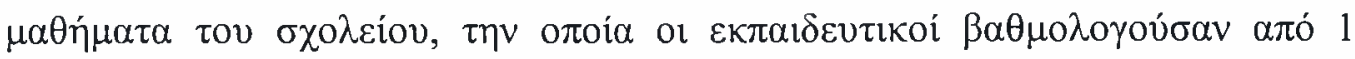

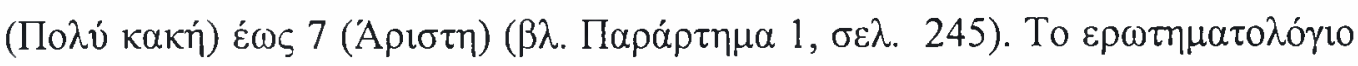

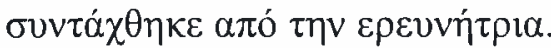

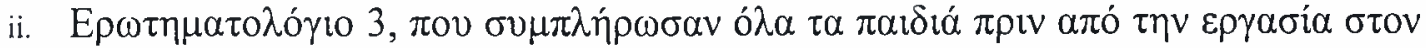

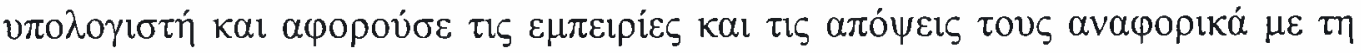




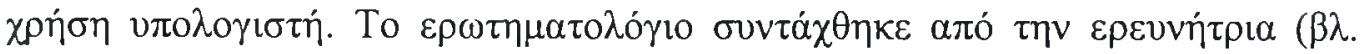

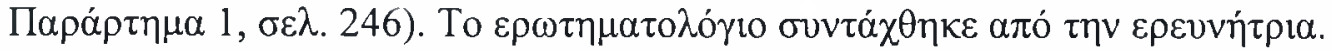

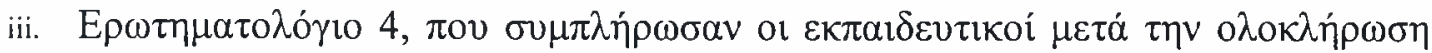

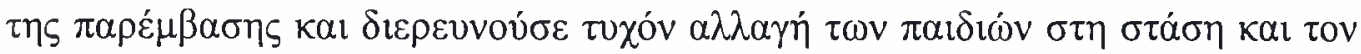

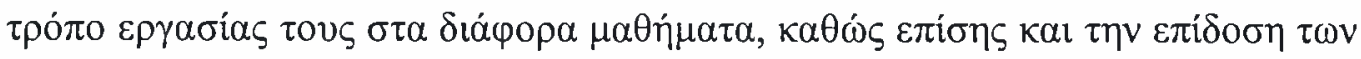

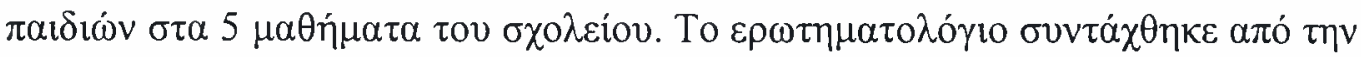

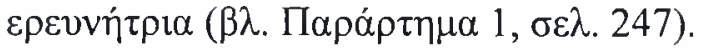

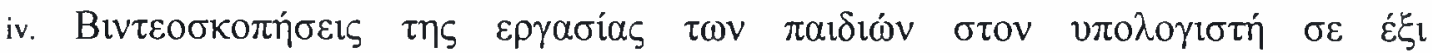

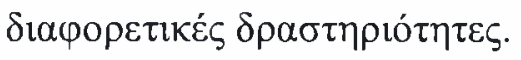

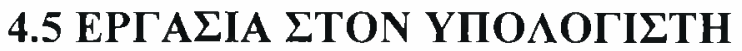

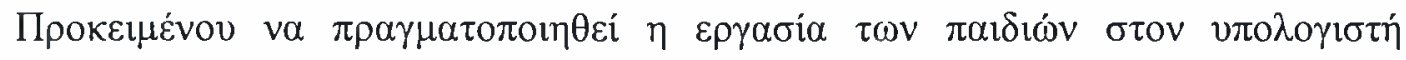

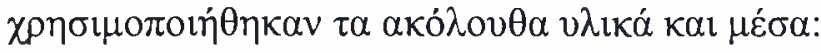

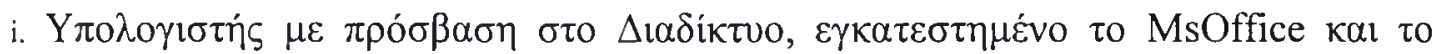

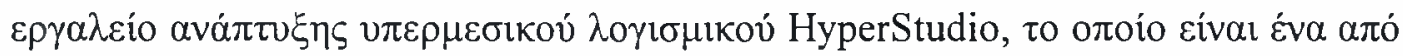

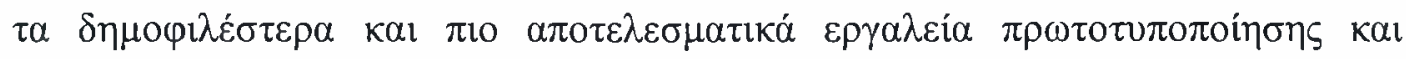

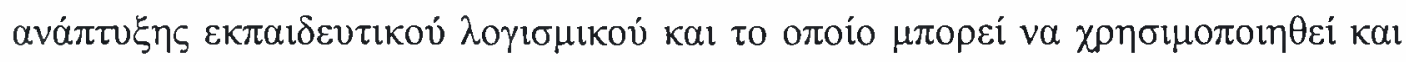

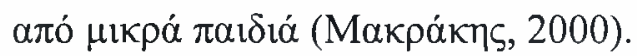

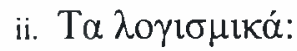

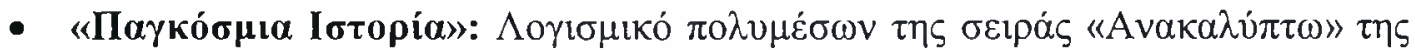

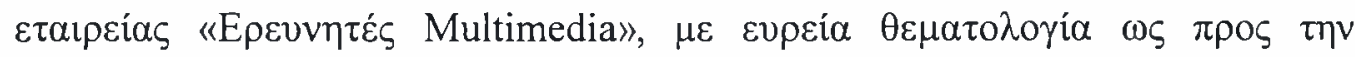

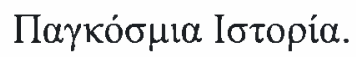

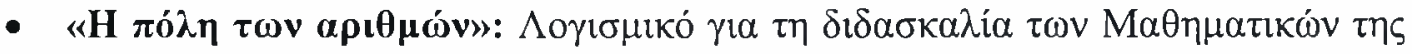

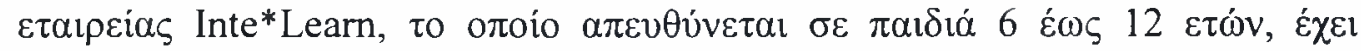

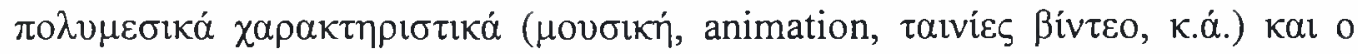

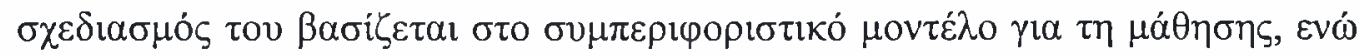

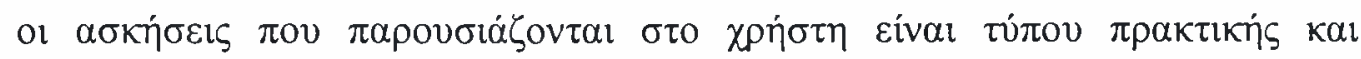
$\varepsilon \xi \dot{\alpha} \sigma \kappa \eta \sigma \eta \varsigma$ (drill-and-practice).

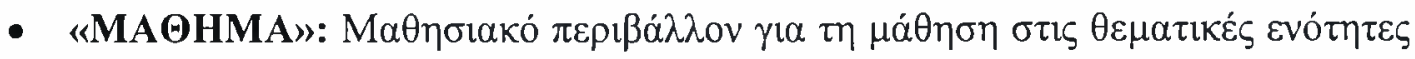

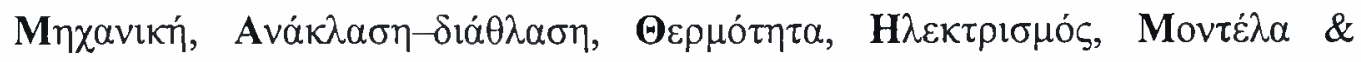

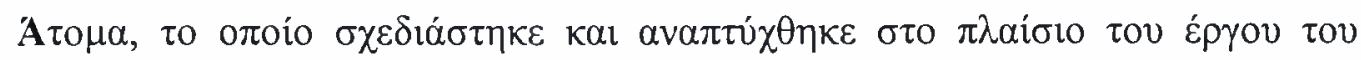




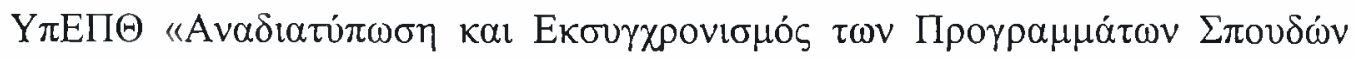

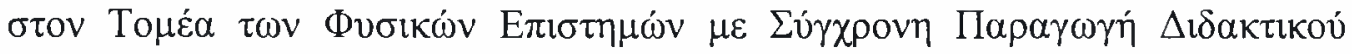

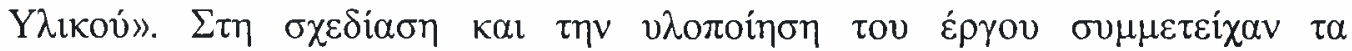

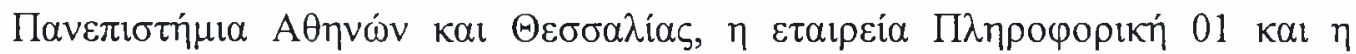

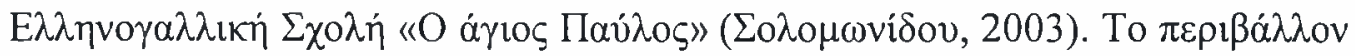

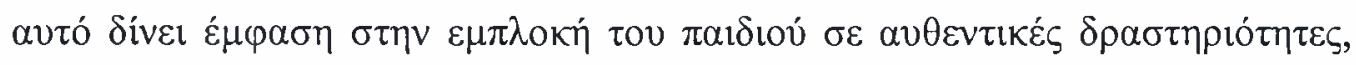

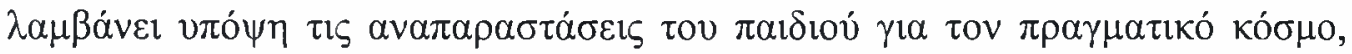

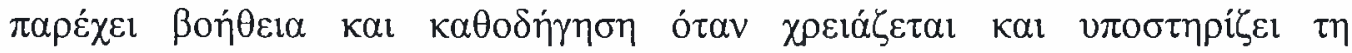

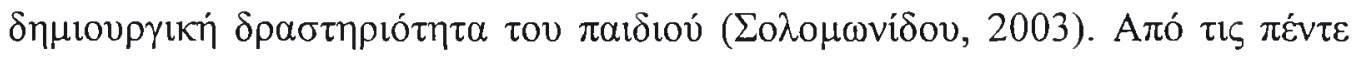

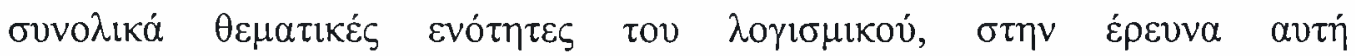

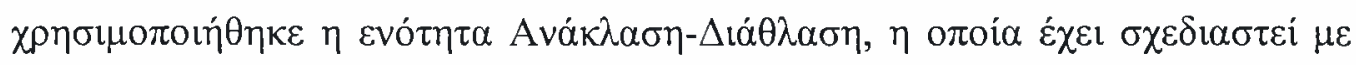

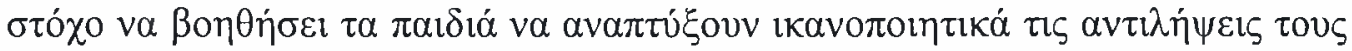

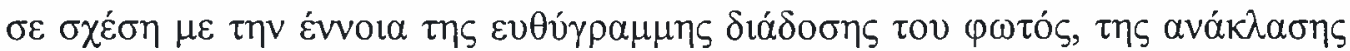

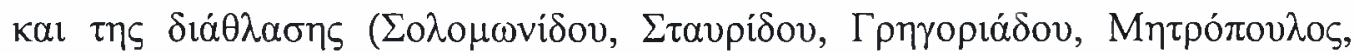

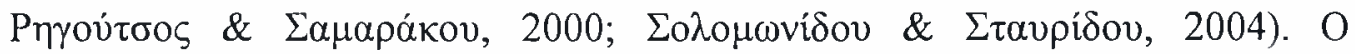

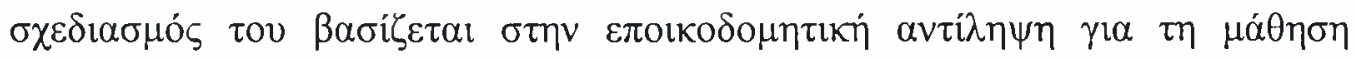

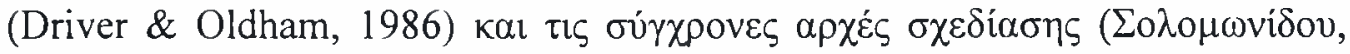
2003).

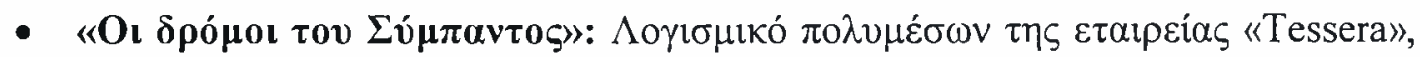

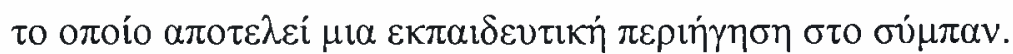

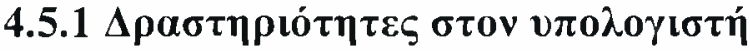

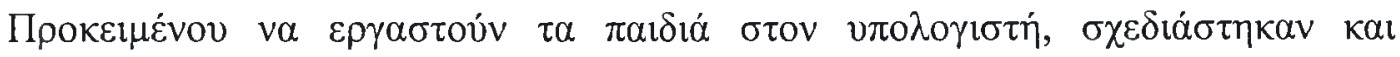

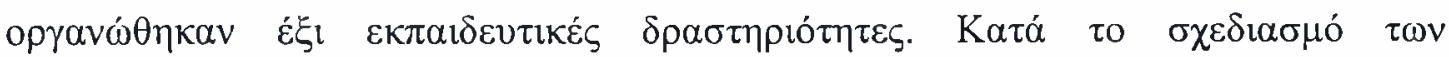

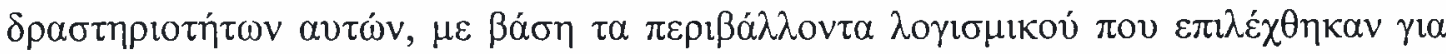

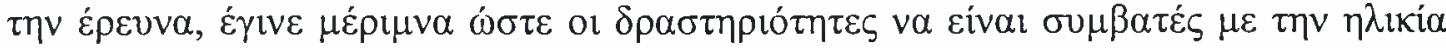

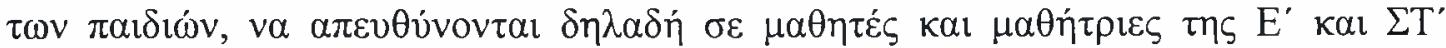

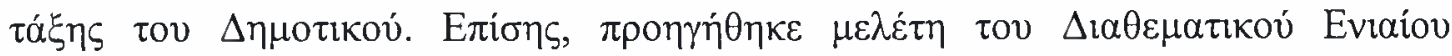

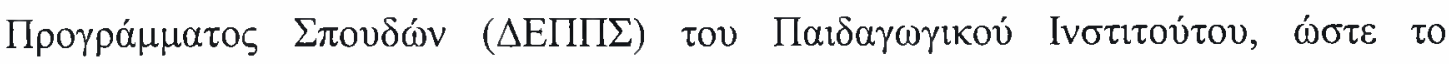

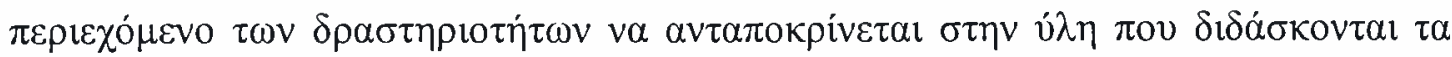

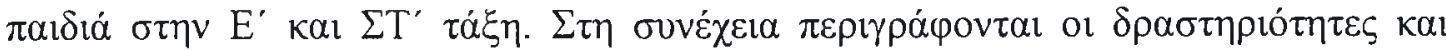




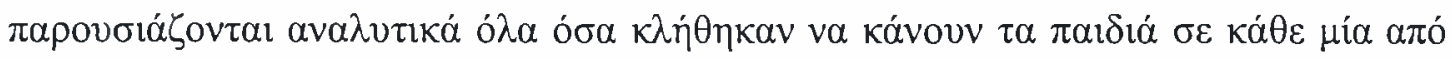

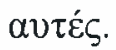

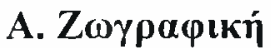

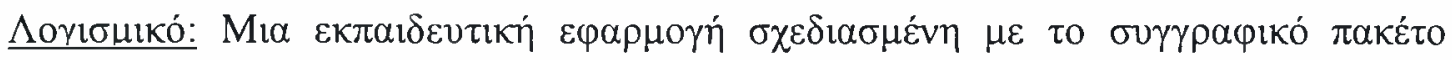

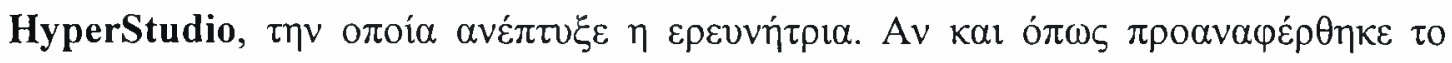

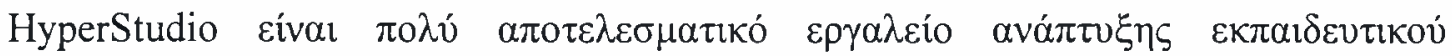

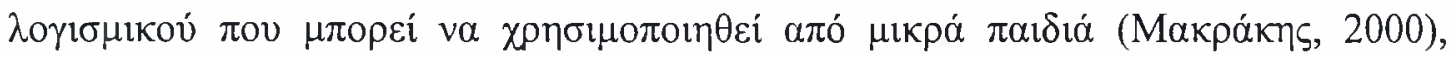

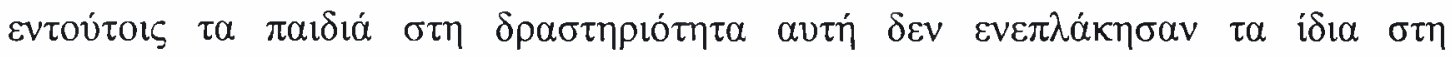

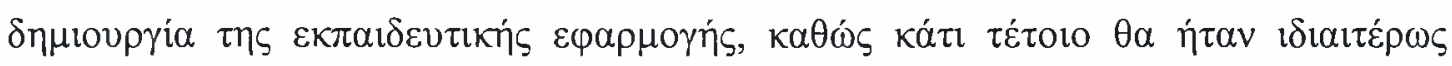

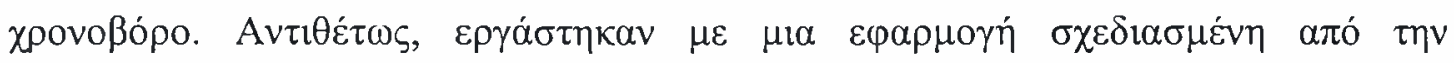

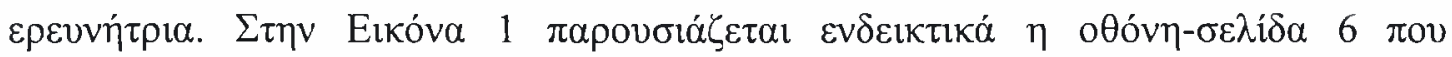

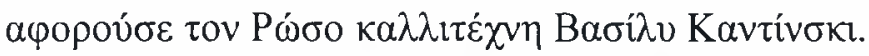

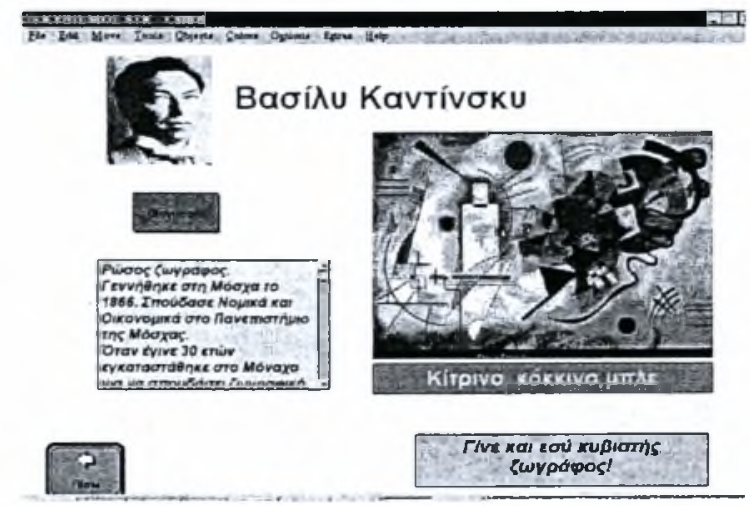

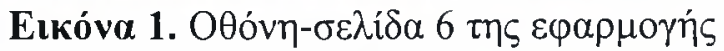

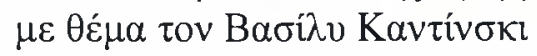

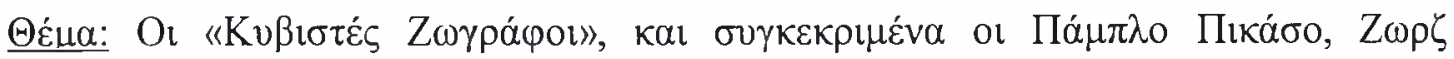

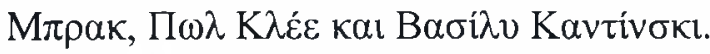

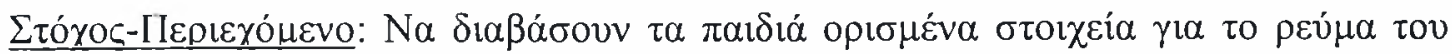

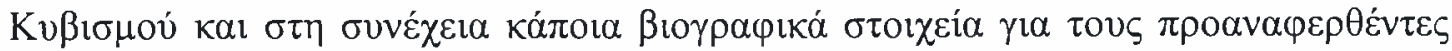

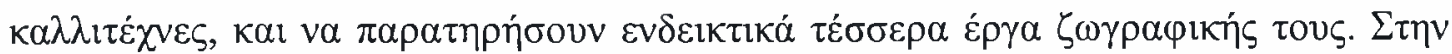

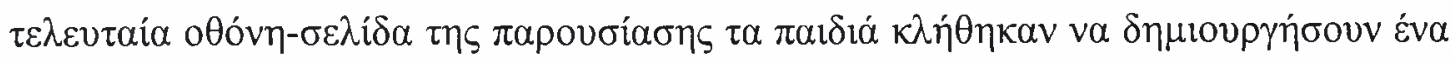

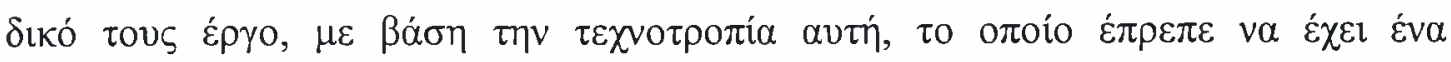

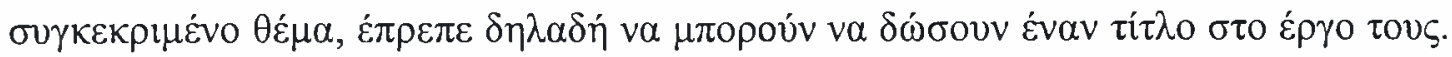

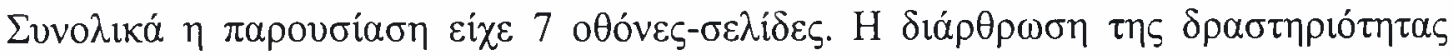

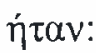




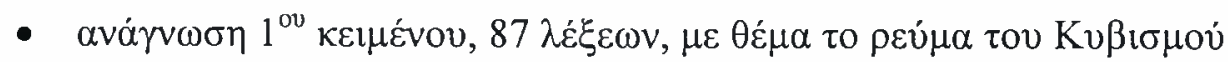

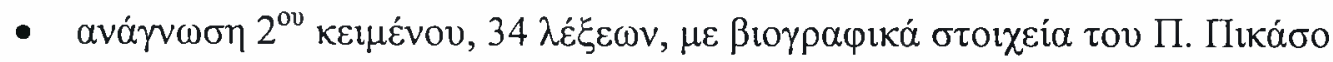

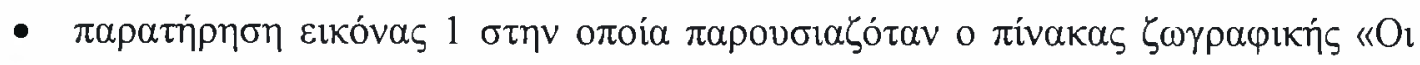

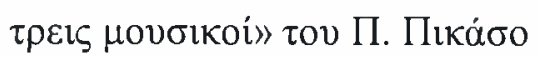

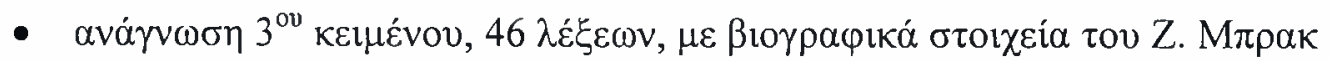

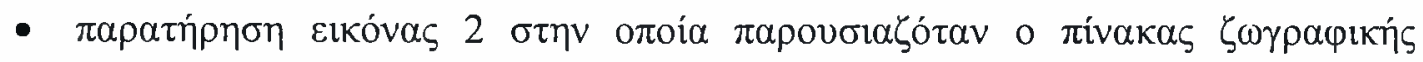

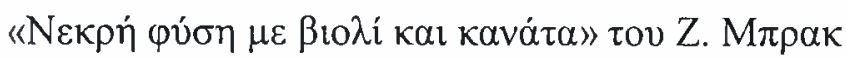

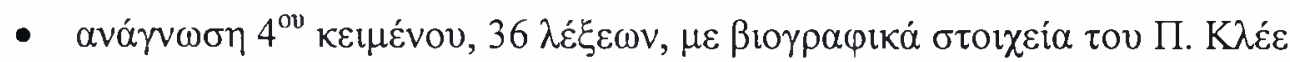

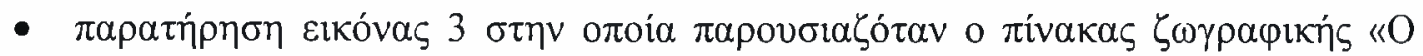

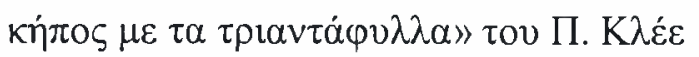

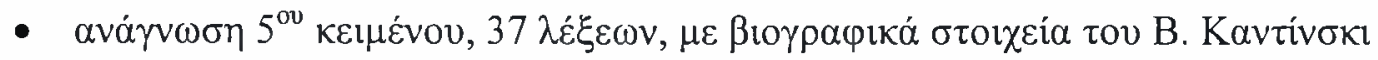

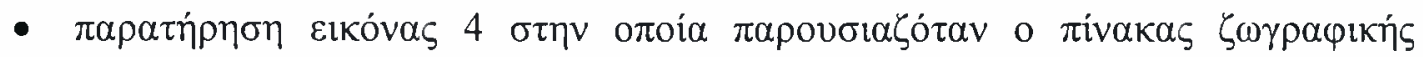

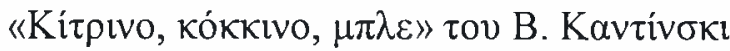

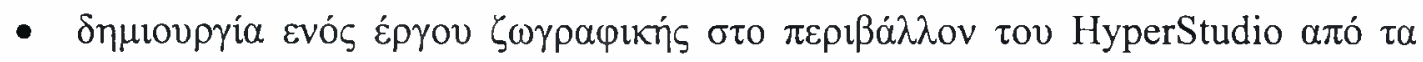

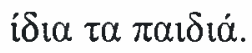

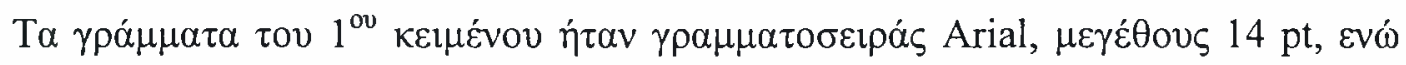

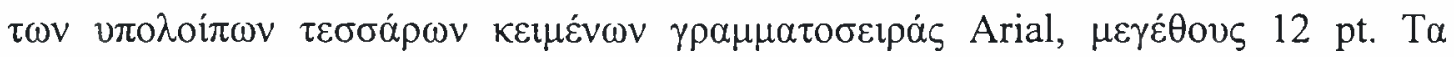

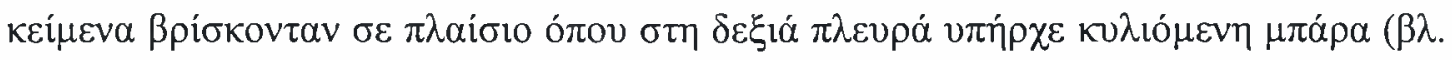

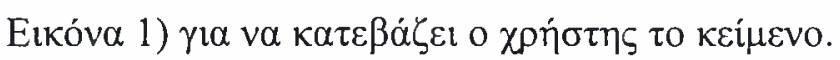

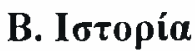

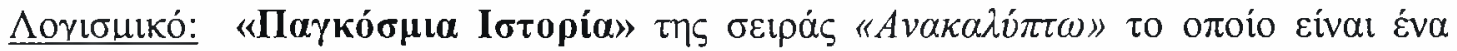

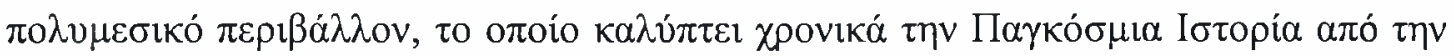

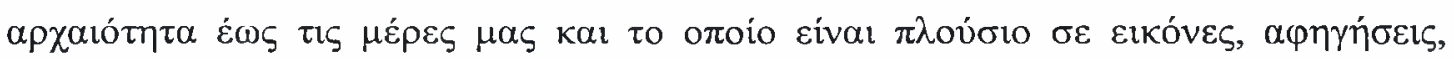

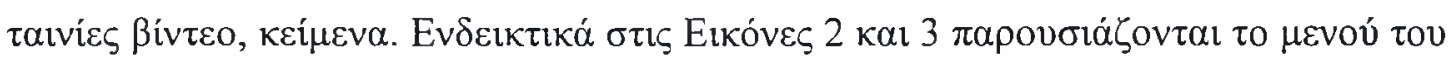

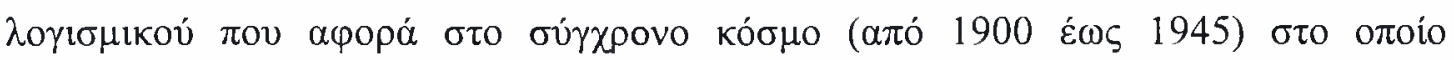

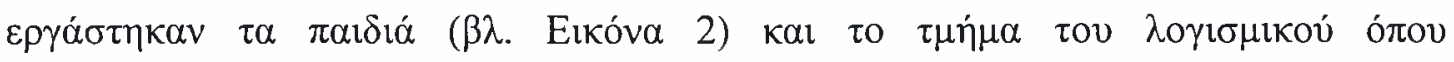

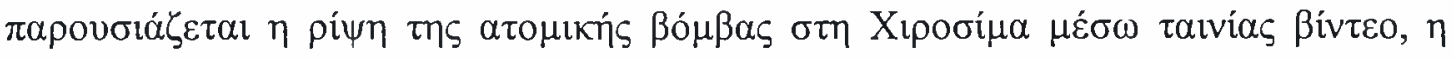

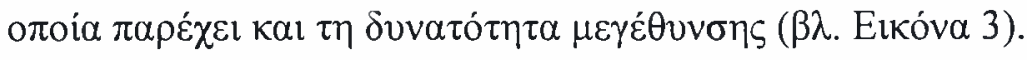




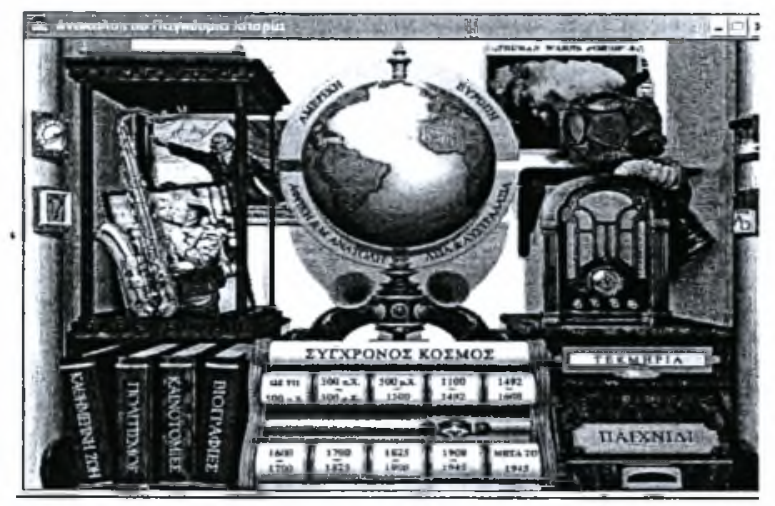

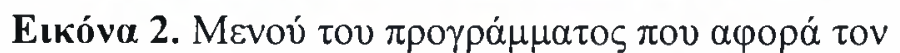

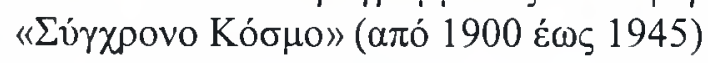

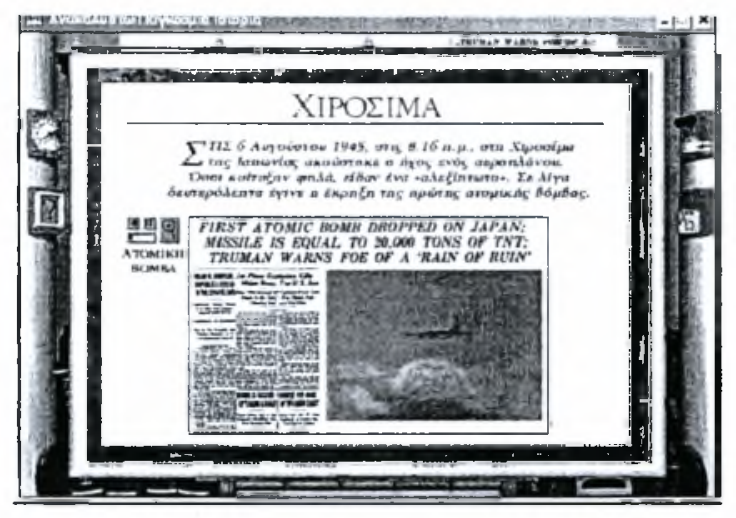

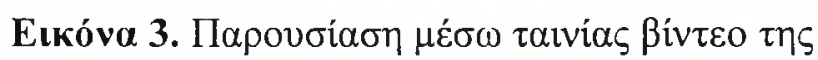

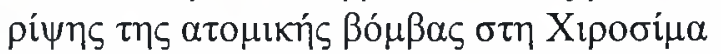

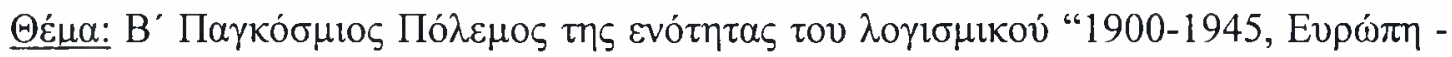

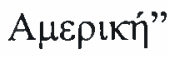

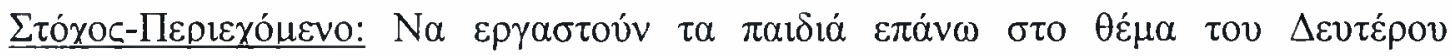

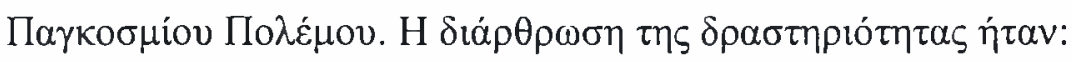

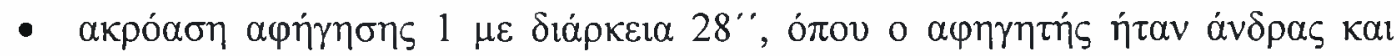

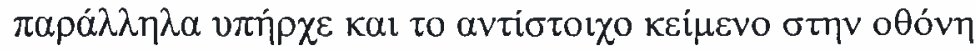

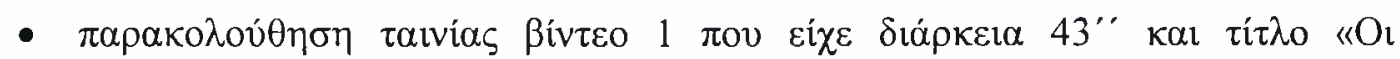
$\delta i \alpha \delta \eta \lambda \dot{\omega} \sigma \varepsilon l \varsigma \tau \omega \vee N \alpha \zeta \zeta\rangle$

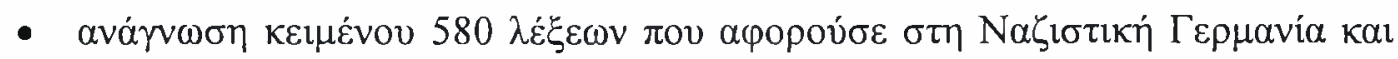

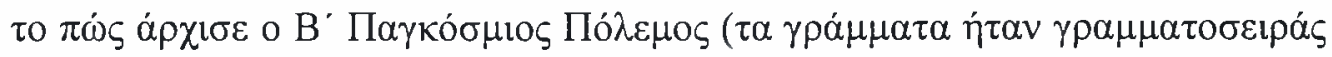

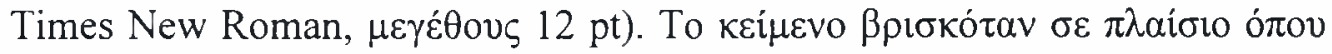

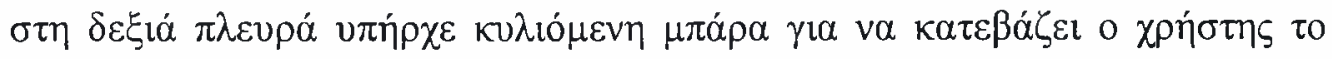
квífevo. 


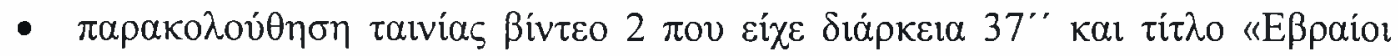

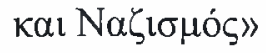

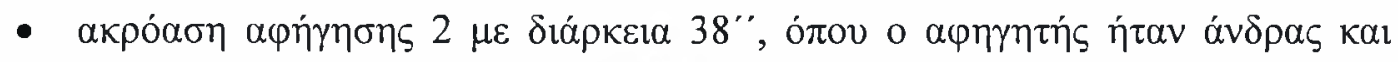

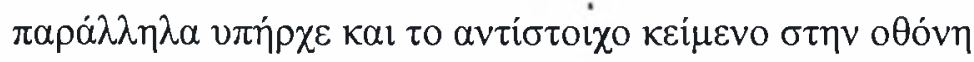

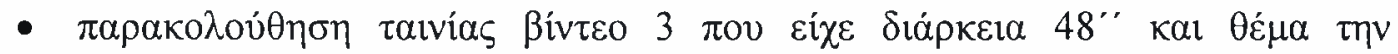

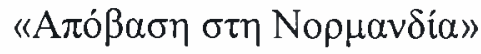

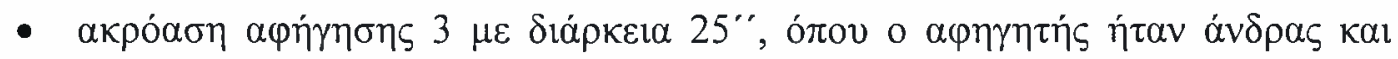

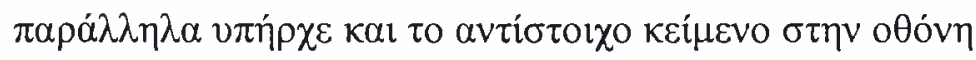

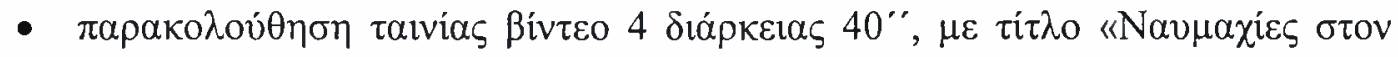

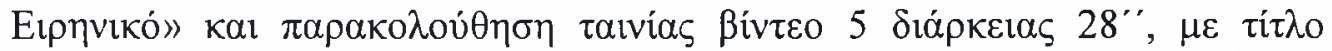

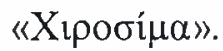

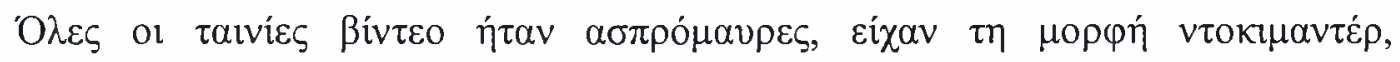

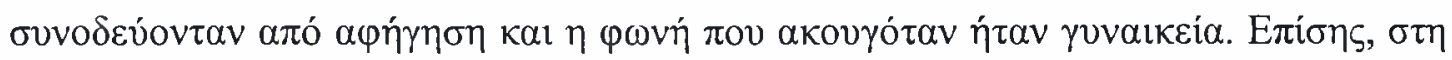

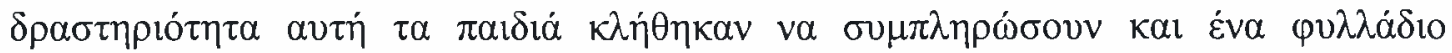

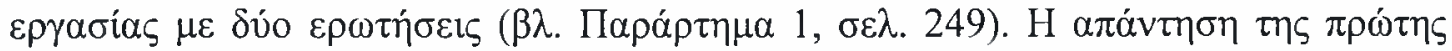

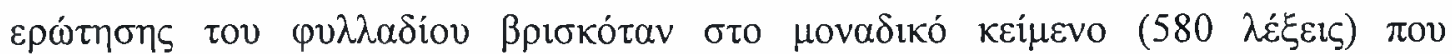

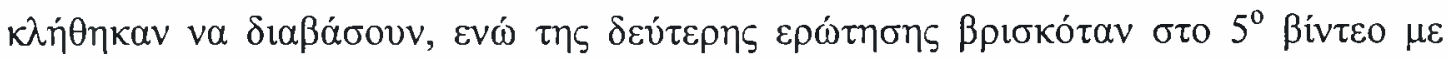

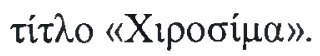

\section{Г. Фибเки́}

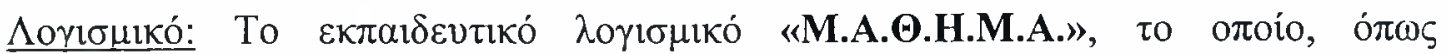

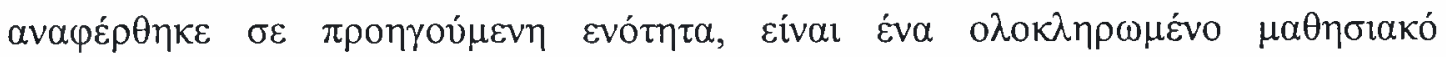

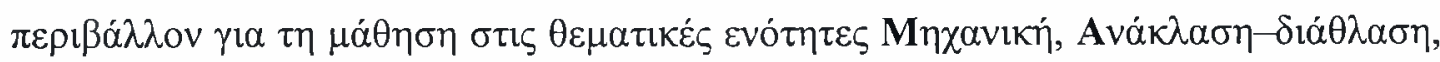

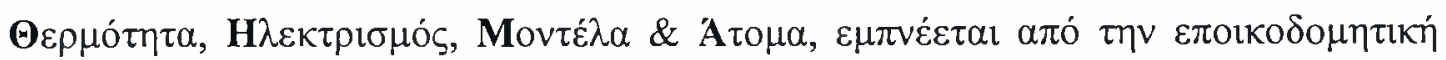

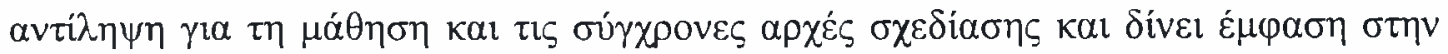

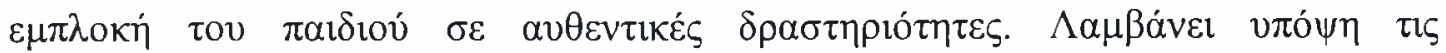

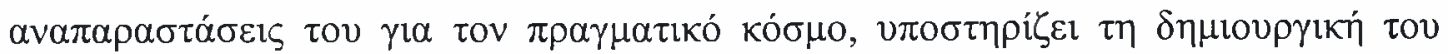

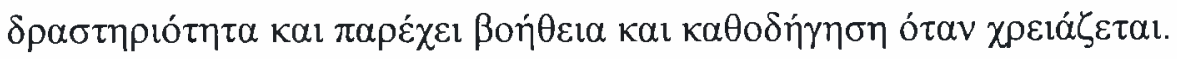

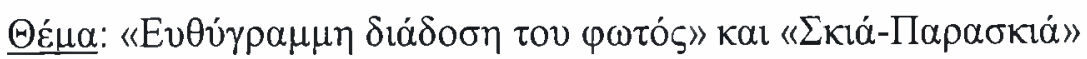

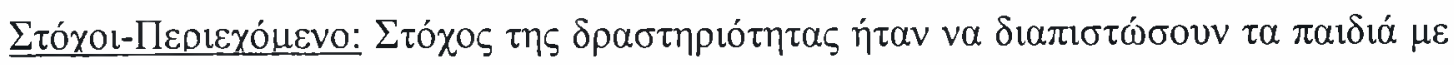

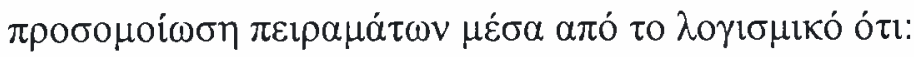

1. To $\varphi \omega \varsigma \delta 1 \alpha \delta i \delta \varepsilon \tau \alpha \iota \varepsilon v \theta \dot{\gamma} \gamma \rho \alpha \mu \mu \alpha$. 


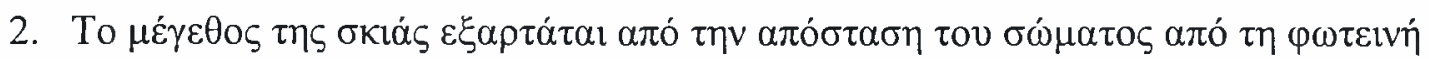
$\pi \eta \gamma \eta \dot{~}$

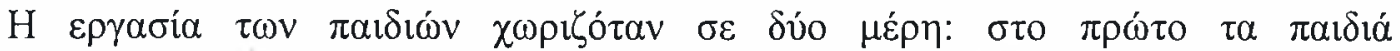

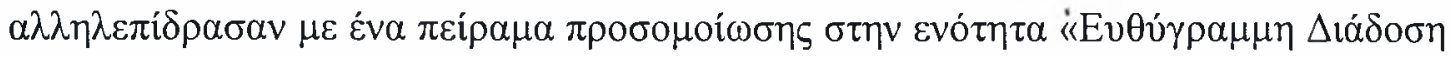

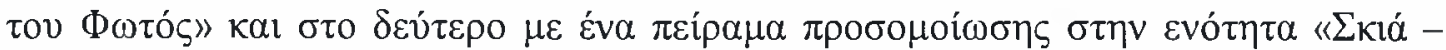

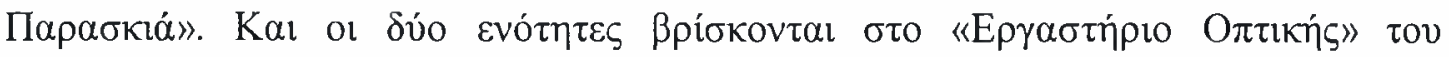

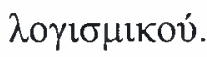

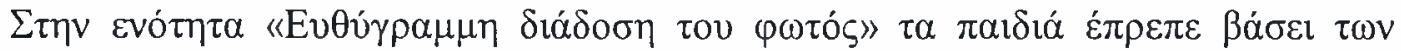

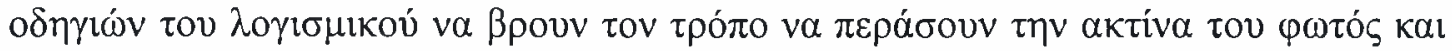

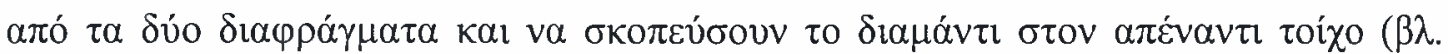

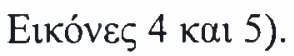

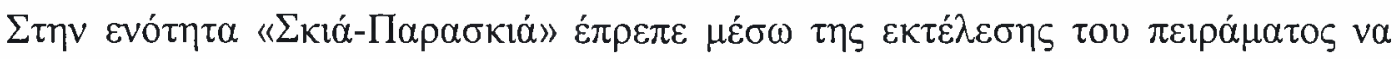

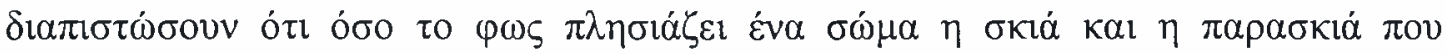

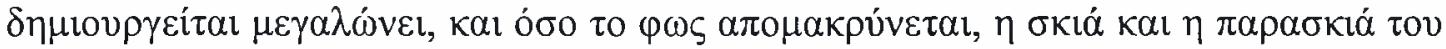

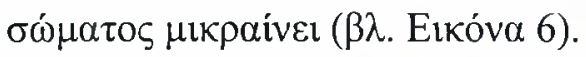

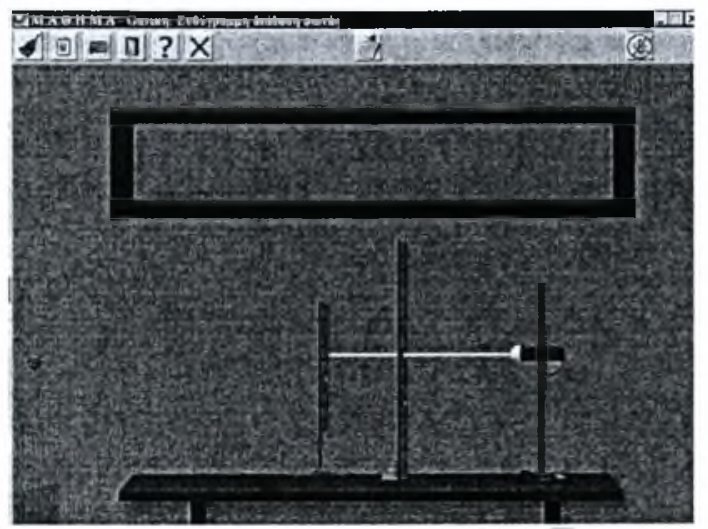

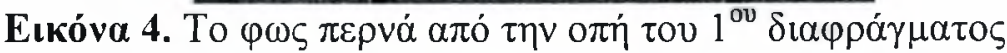

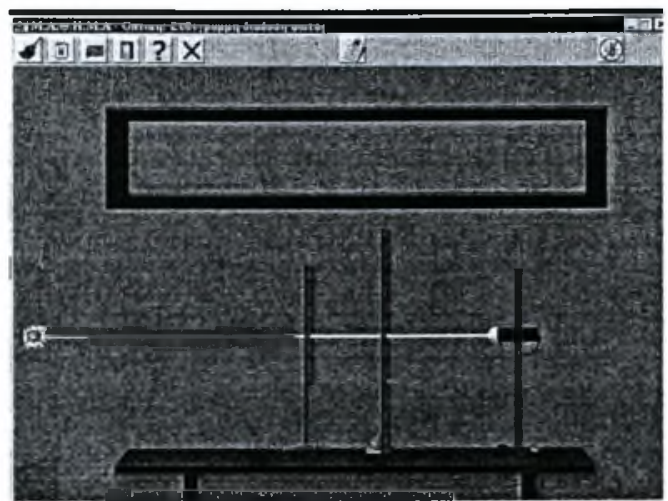

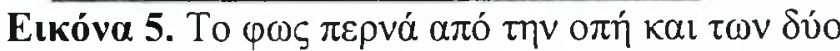

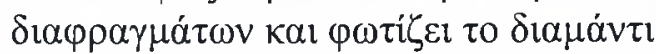




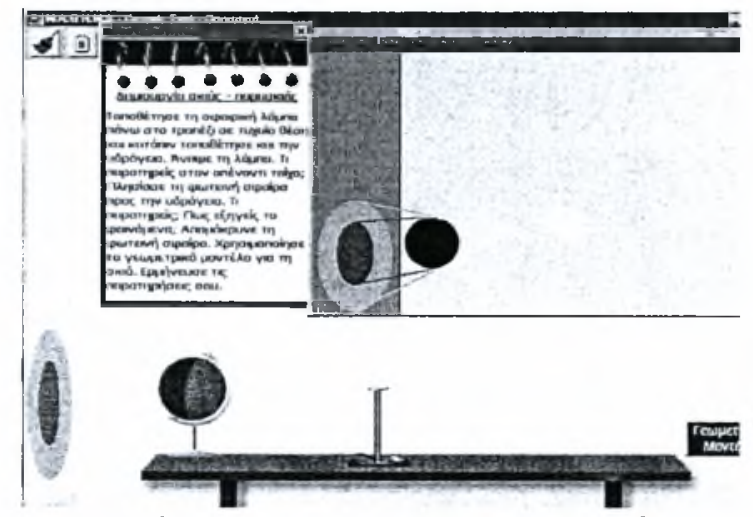

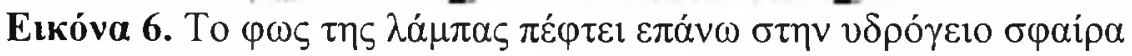

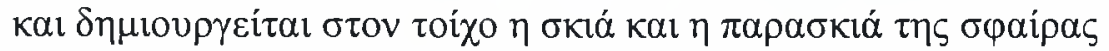

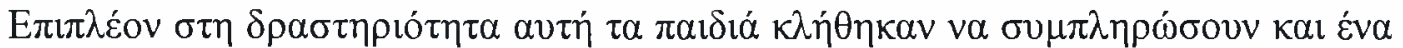

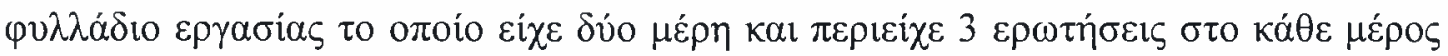

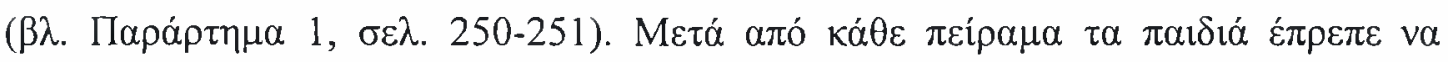

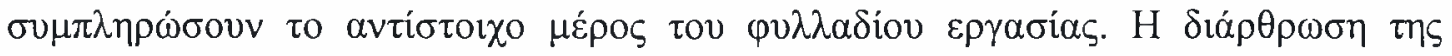

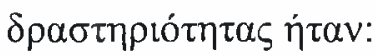

$A^{\prime \prime \mu \varepsilon ́ \rho o s: ~}$

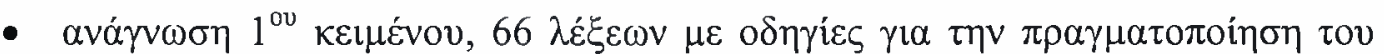

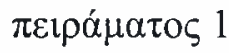

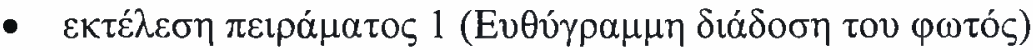

- $\sigma u \mu \pi \lambda \eta \dot{\eta} \rho \omega \sigma \eta \mathrm{A}^{\prime} \mu \varepsilon \dot{\rho} \rho v_{\varsigma} \varphi v \lambda \lambda \alpha \delta$ íov $\varepsilon \rho \gamma \alpha \sigma i \alpha \varsigma$

$B^{\prime \prime \mu} \boldsymbol{\varepsilon} \rho \circ \varsigma^{\prime}$

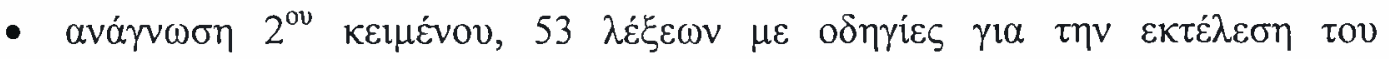

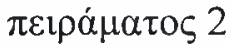

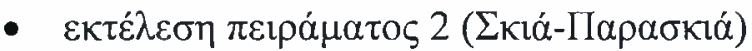

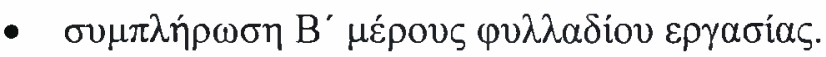

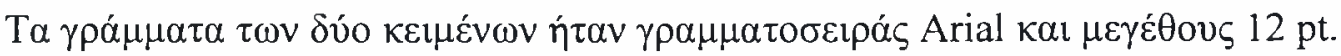

\section{$\Delta . \Gamma \varepsilon \omega \gamma \rho \alpha \varphi \alpha_{\alpha} 1$}

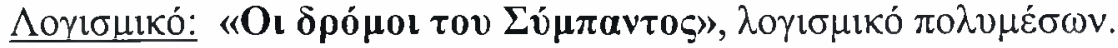

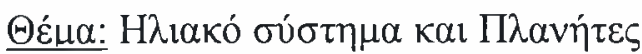

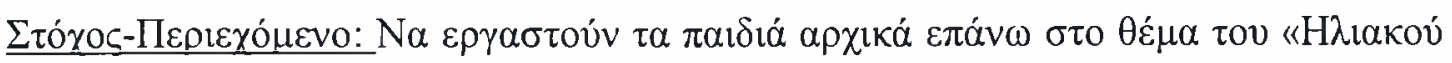

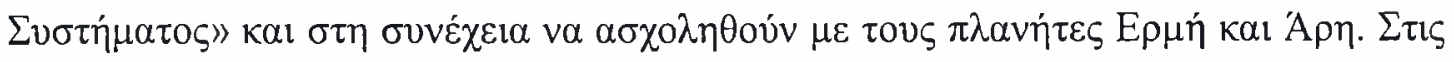




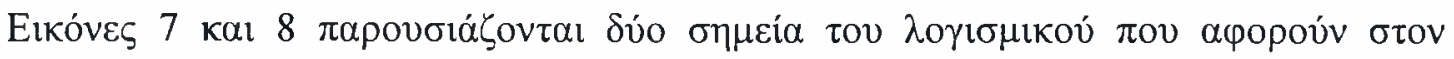

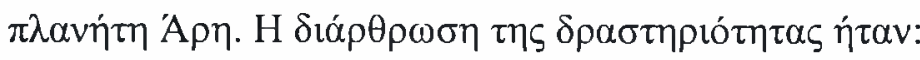

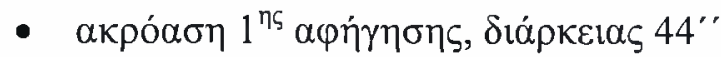

- $\alpha \kappa \rho o ́ \alpha \sigma \eta 2^{\eta \varsigma} \alpha \varphi \eta \dot{\gamma \eta \sigma \eta \zeta, ~ \delta i \alpha ́ \rho \kappa \varepsilon i \alpha \varsigma ~ 36 " ~}$

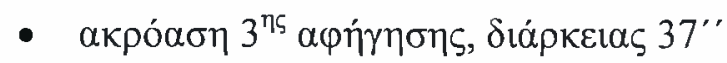

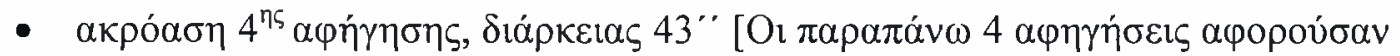

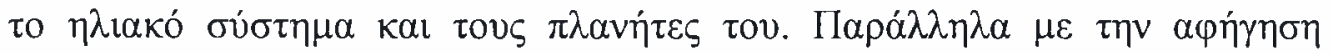

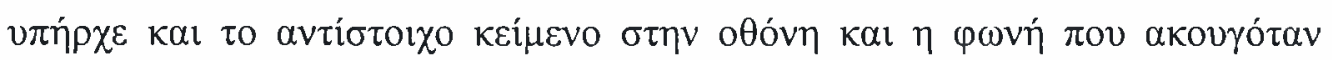

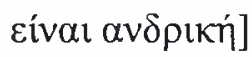

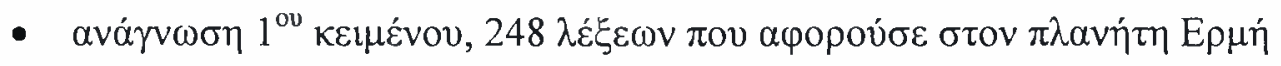

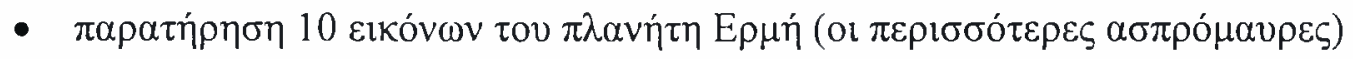

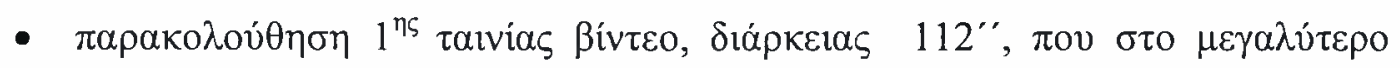

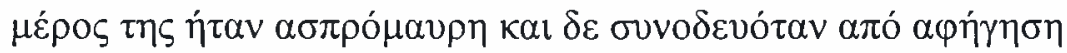

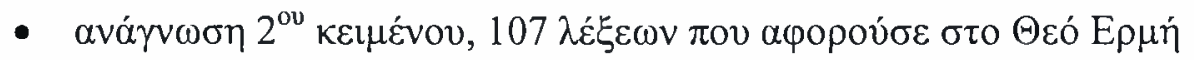

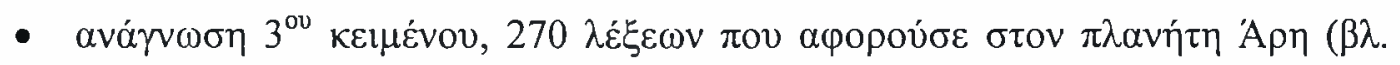
Eıкóva 7)

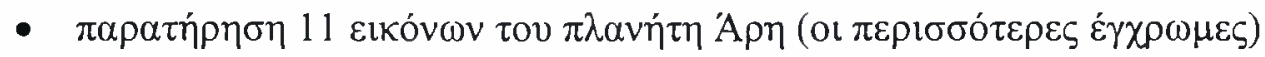

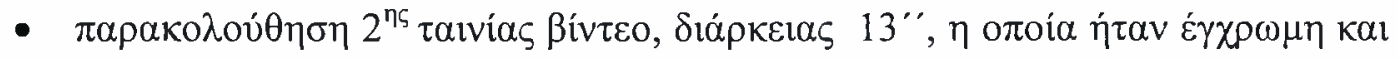

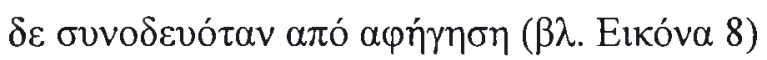

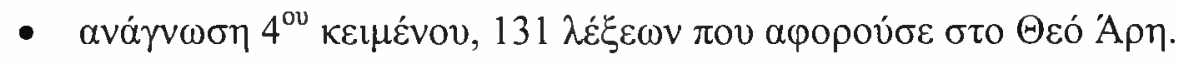

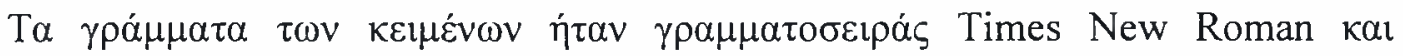

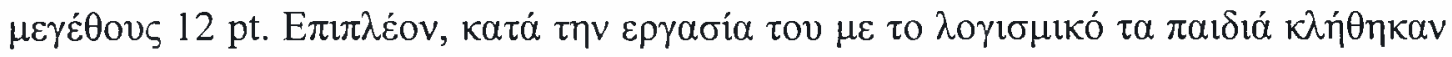

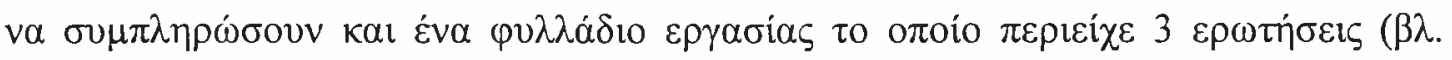

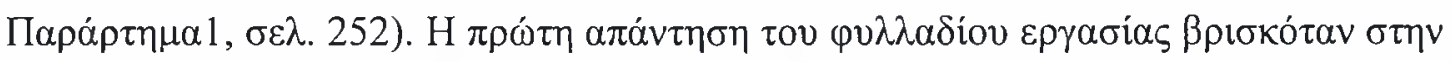

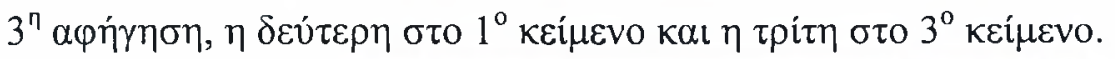

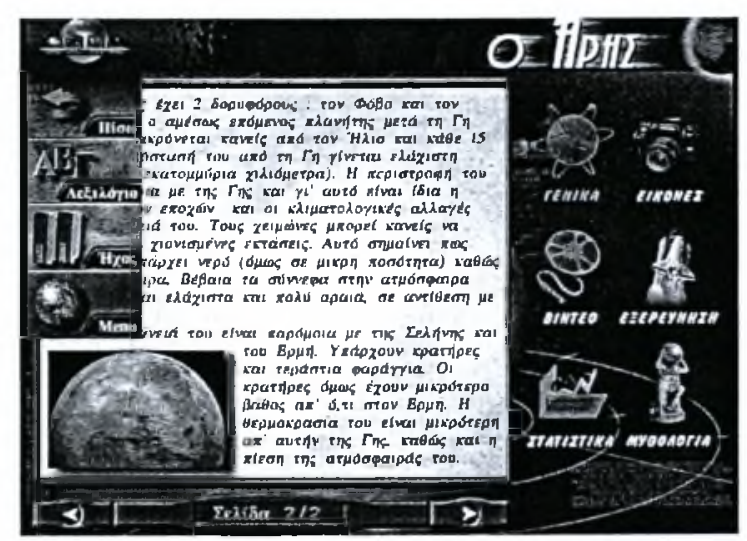

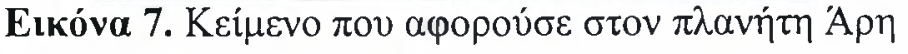




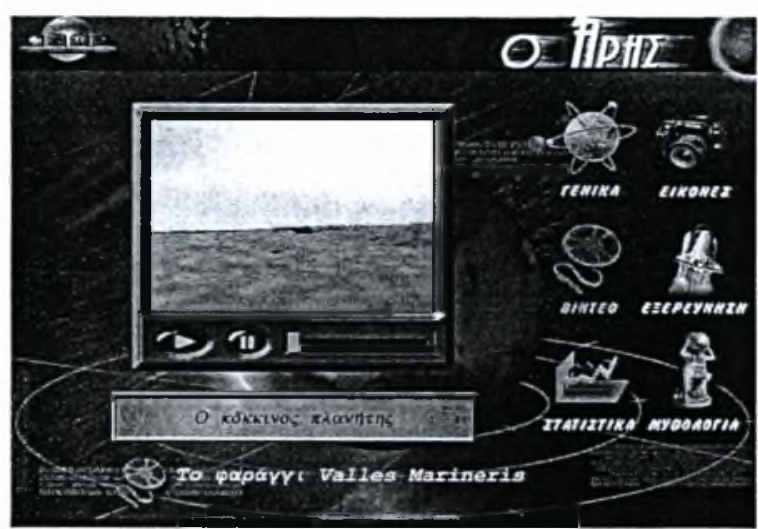

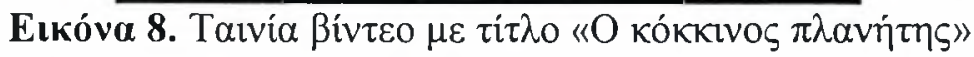

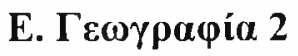

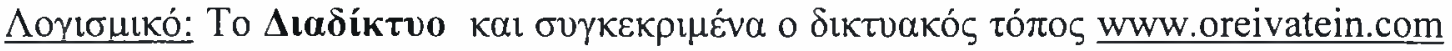
kal to MsExcel

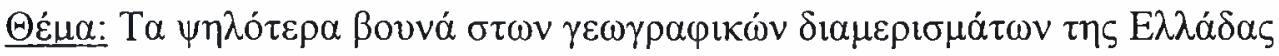

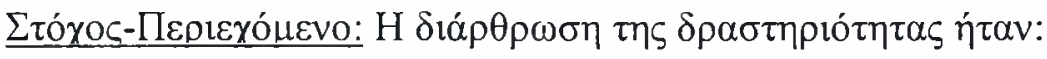

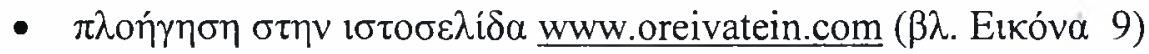

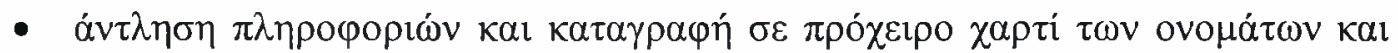

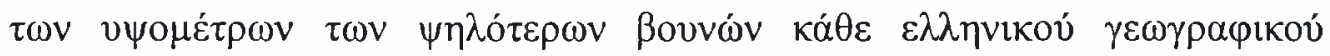
$\delta 1 \alpha \mu \varepsilon \rho i \sigma \mu \alpha \tau o \varsigma$

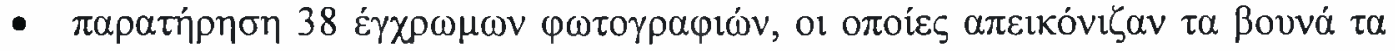

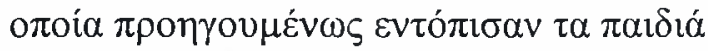

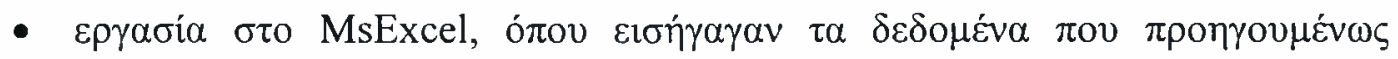

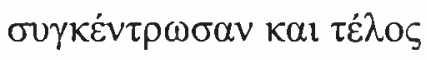

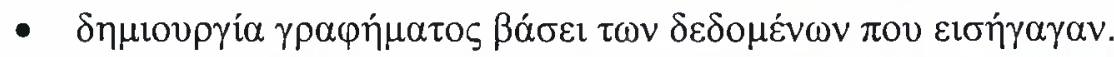

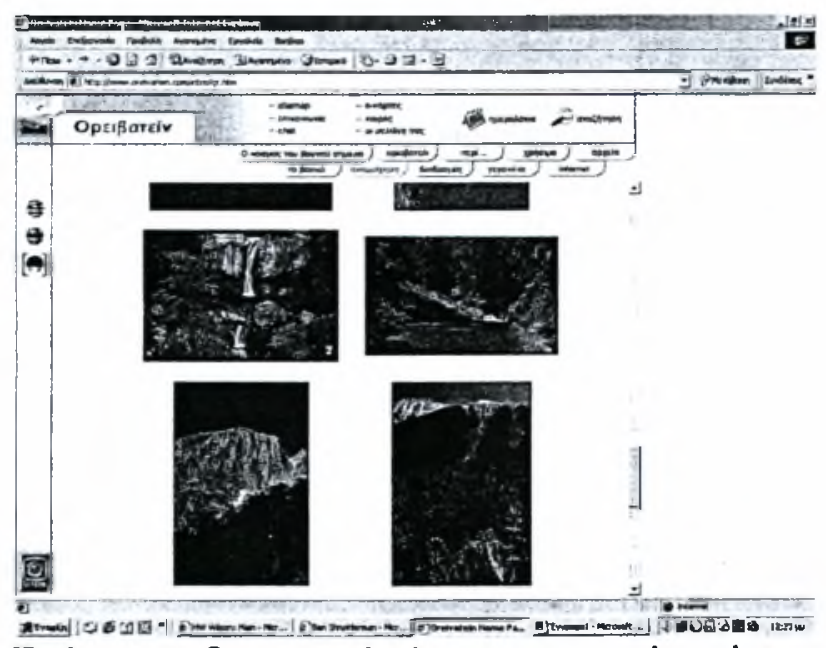

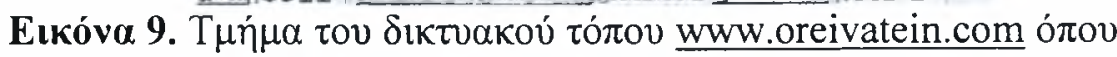

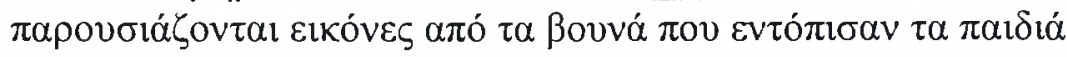




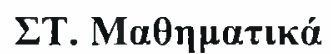

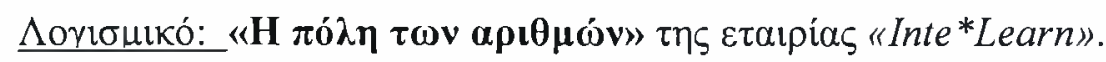

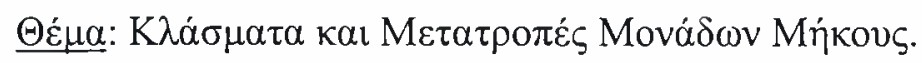

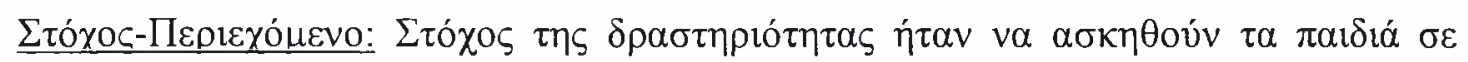

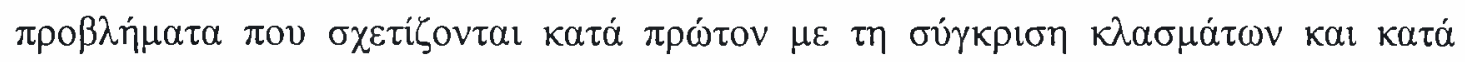

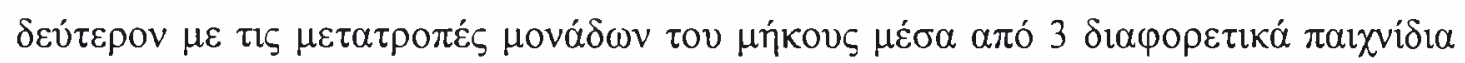

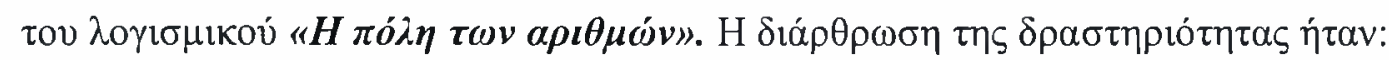

\section{$A^{\prime} \mu \varepsilon \dot{\rho o \varsigma:}$}

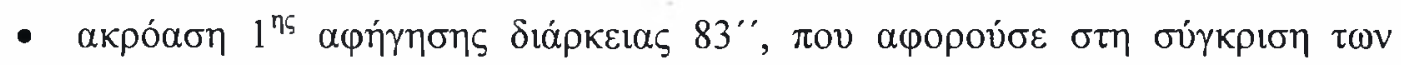
$\kappa \lambda \alpha \sigma \mu \alpha \dot{\tau} \omega \omega$

- $\varepsilon \pi i ́ \lambda v \sigma \eta 1^{\eta \varsigma} \alpha \dot{\sigma} \sigma \kappa \sigma \eta \varsigma$

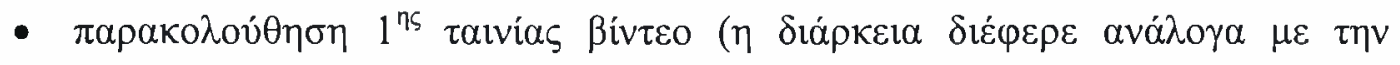
$\varepsilon \pi \imath \lambda \circ \gamma \eta \dot{~ \tau o v ~ \kappa a ́ ~} \theta \varepsilon \pi \alpha \iota \delta$ เov́)

- $\varepsilon \pi i \lambda v \sigma \eta 2^{\eta \varsigma} \alpha ́ \sigma \kappa \eta \sigma \eta \zeta$

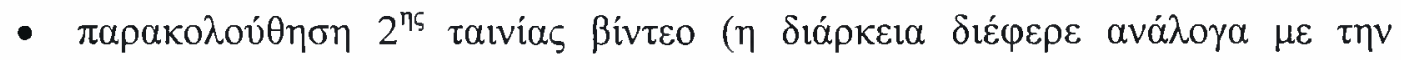
$\varepsilon \pi \imath \lambda \circ \gamma \eta \dot{~ \tau o v ~ \kappa a ́ \theta \varepsilon ~} \pi \alpha 1 \delta ı$ ).

\section{$B^{\prime \prime \mu \varepsilon ́ \rho o \varsigma: ~}$}

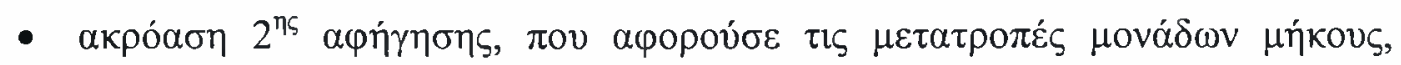

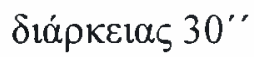

- $\varepsilon \pi i \lambda v \sigma \eta 3^{75} \alpha \sigma \kappa \eta \sigma \eta \varsigma$

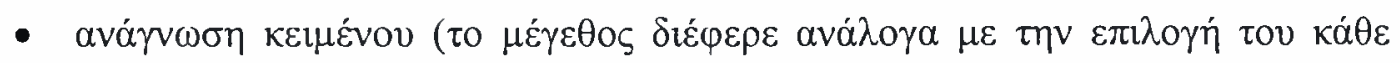

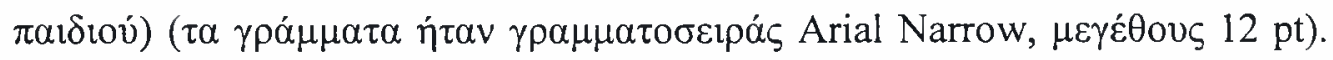

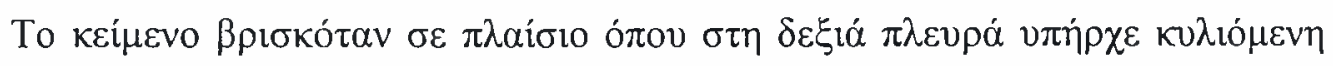

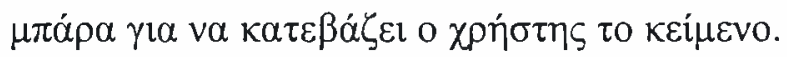

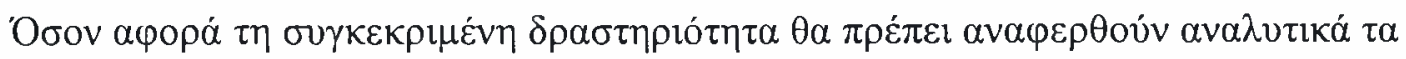
$\alpha \kappa o ́ \lambda o v \theta \alpha$ :

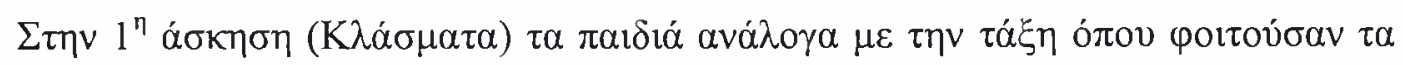

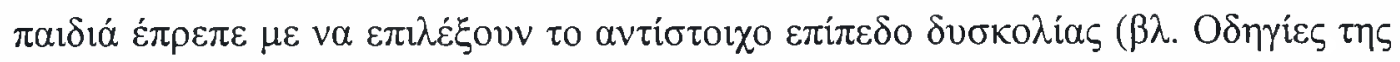

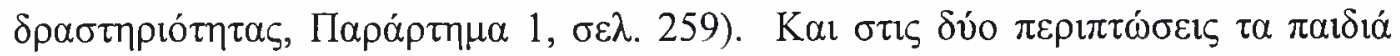

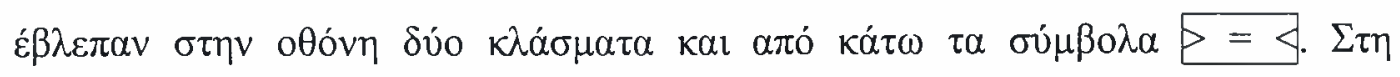

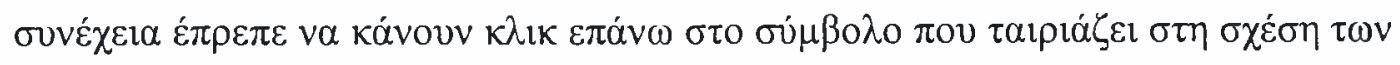




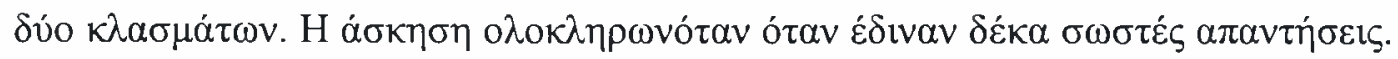

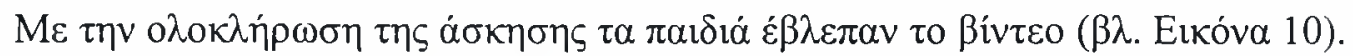

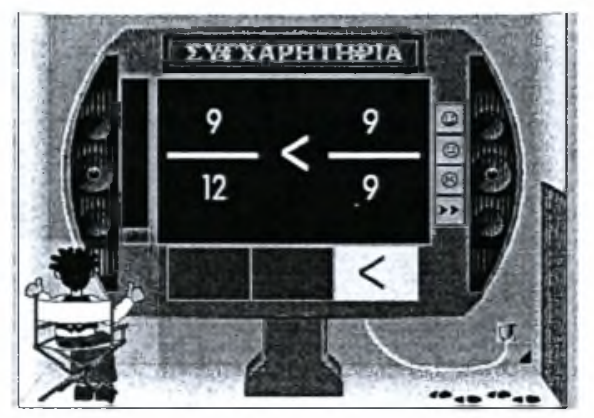

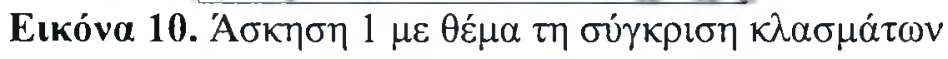

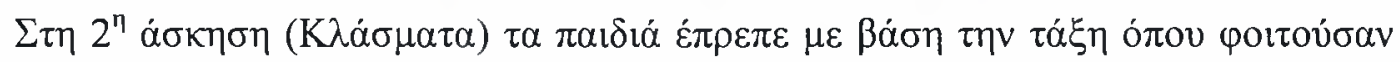

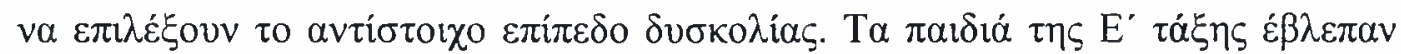

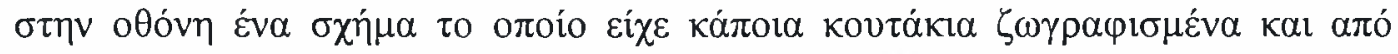

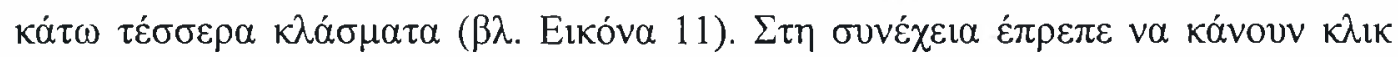

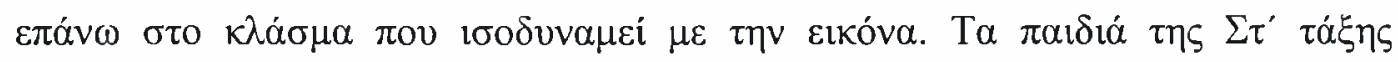

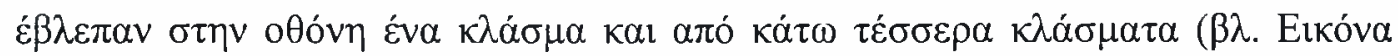

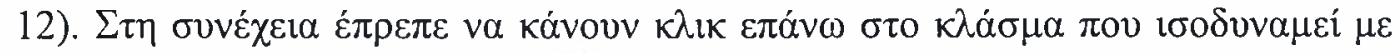

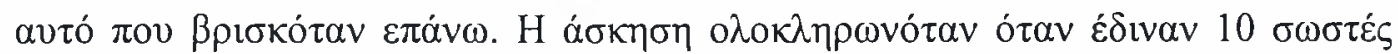

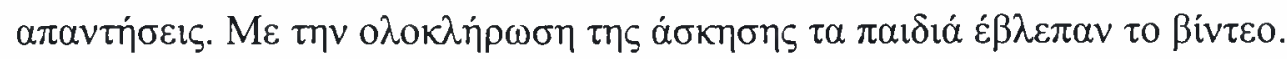

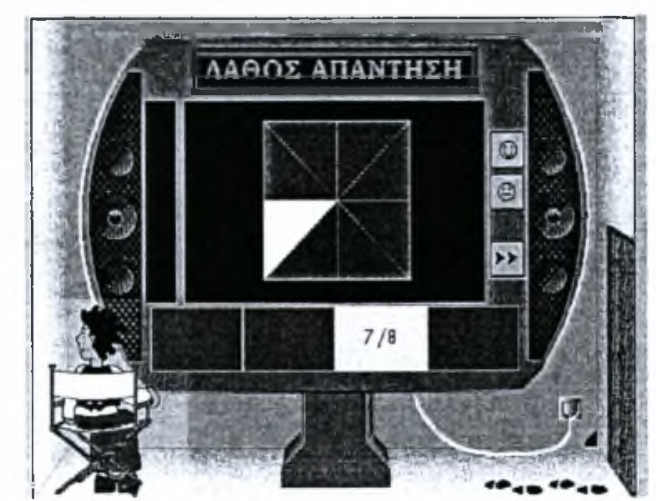

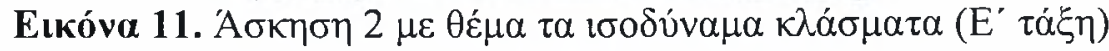




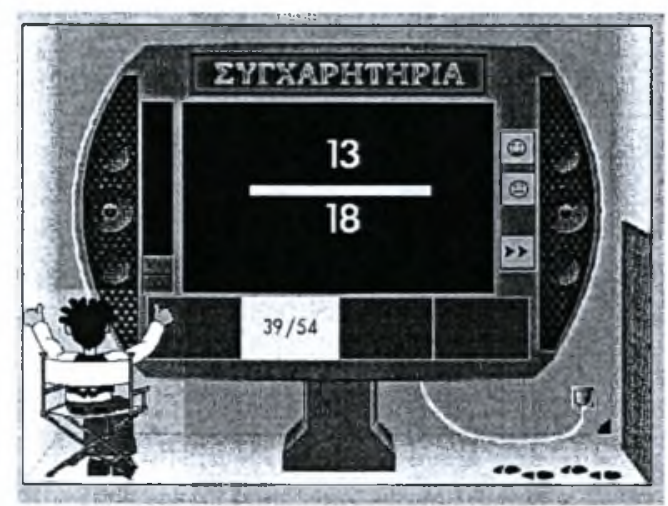

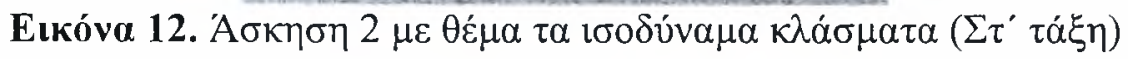

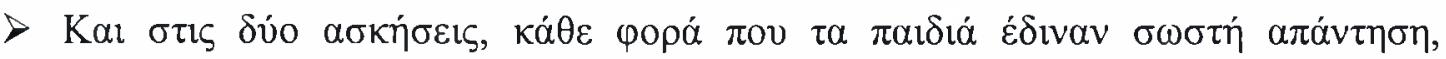

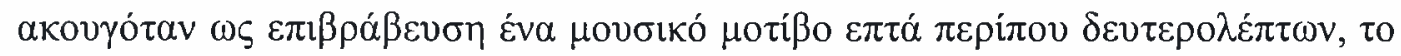

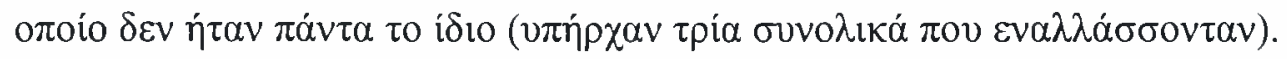

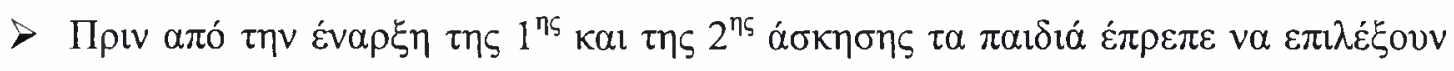

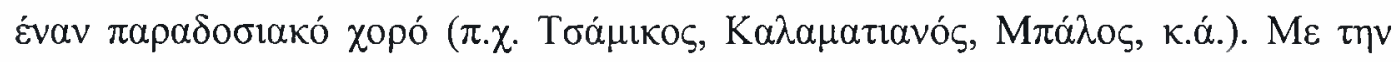

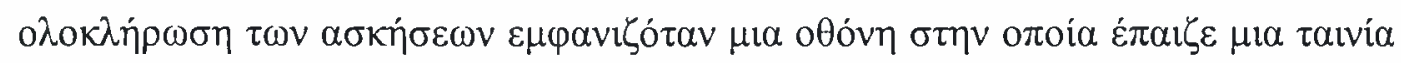

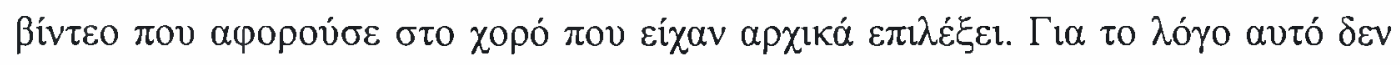

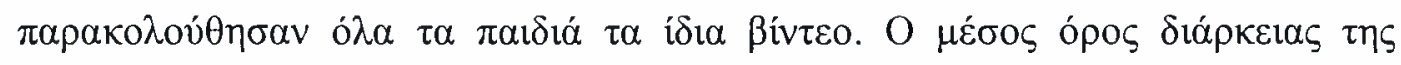

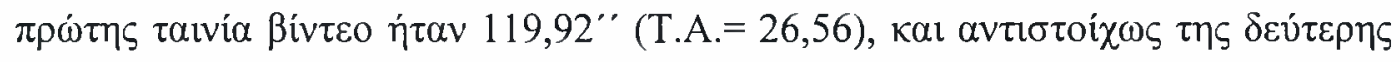

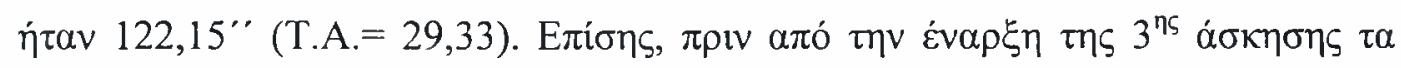

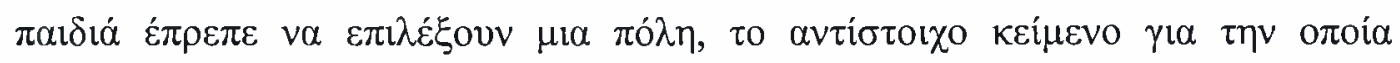

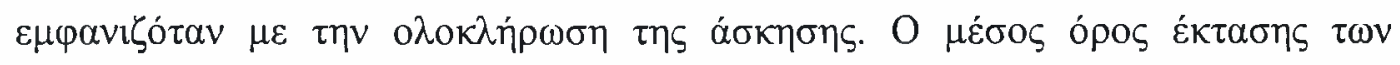

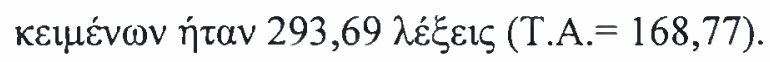

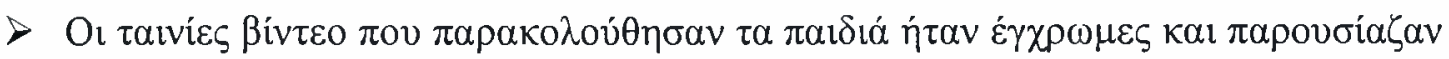

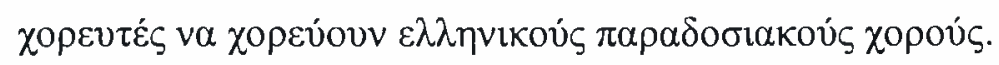

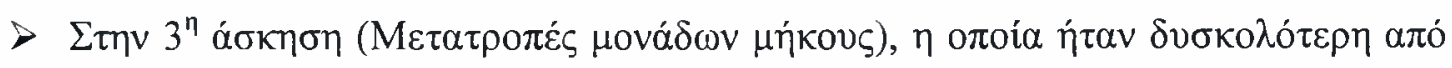

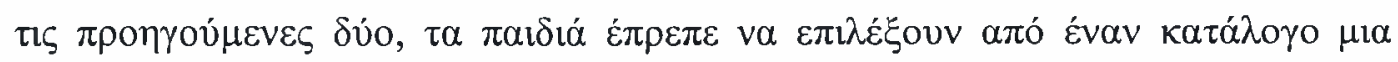

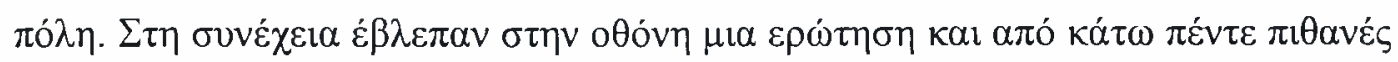

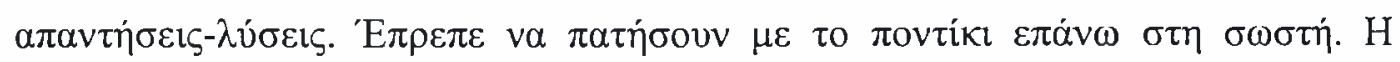

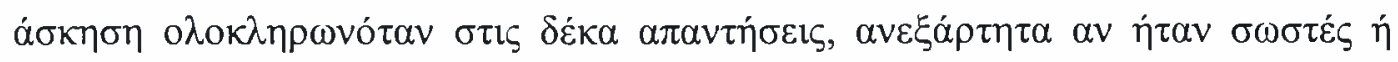

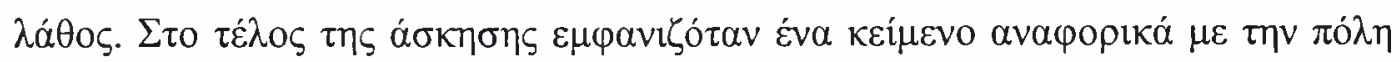

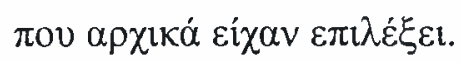




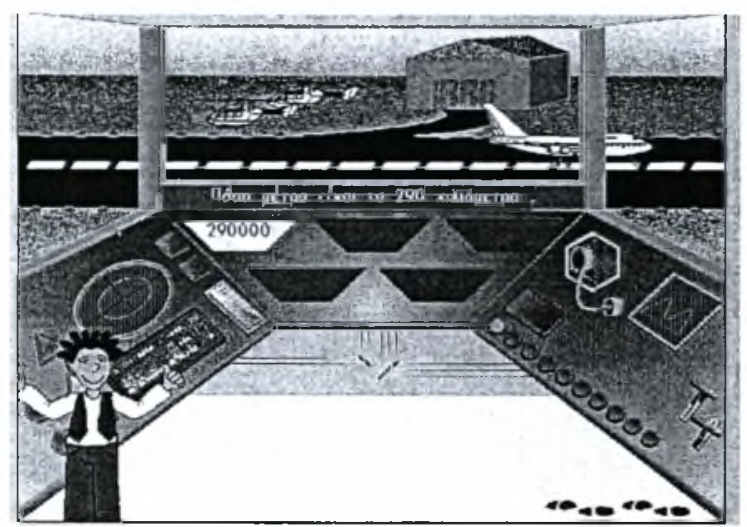

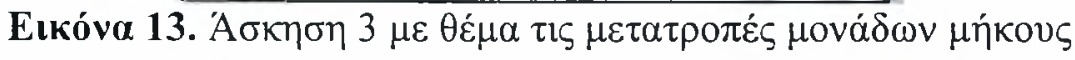

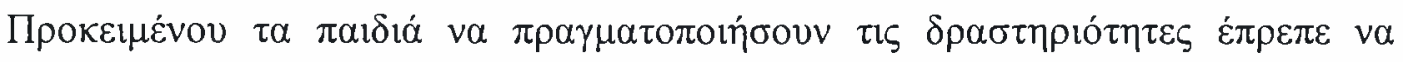

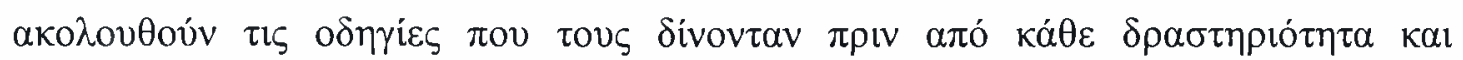

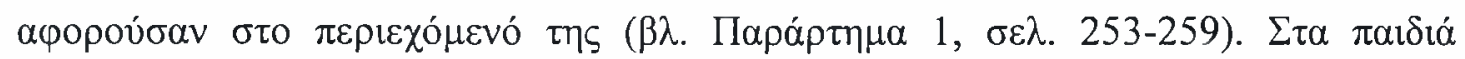

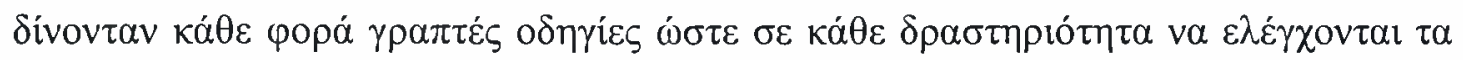
$\alpha \kappa o ́ \lambda o v \theta \alpha$ :

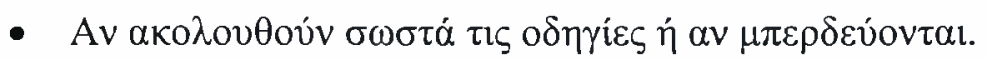

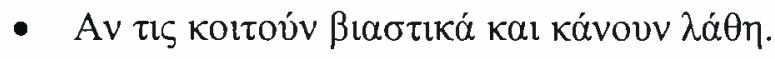

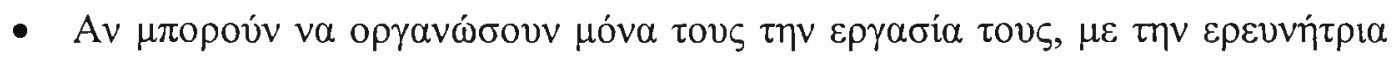

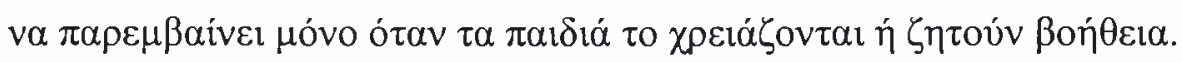

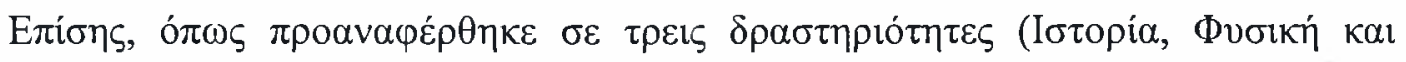

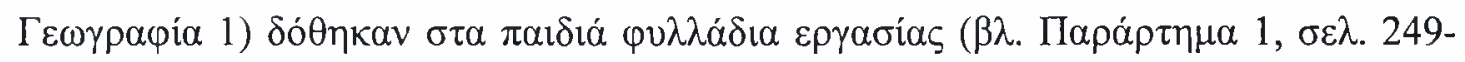

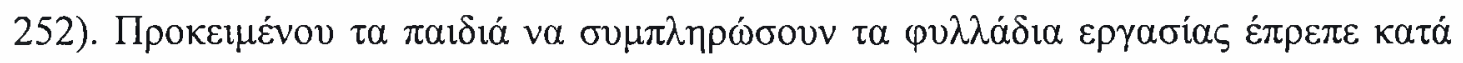

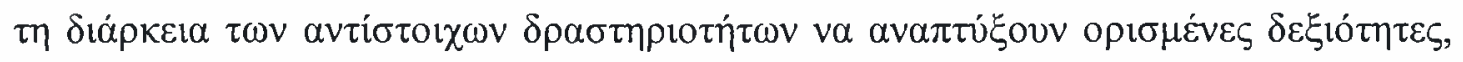

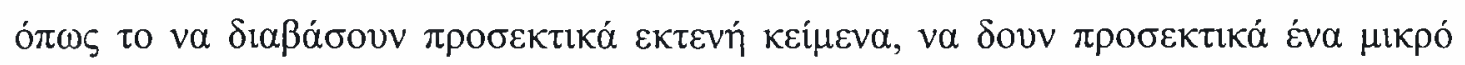

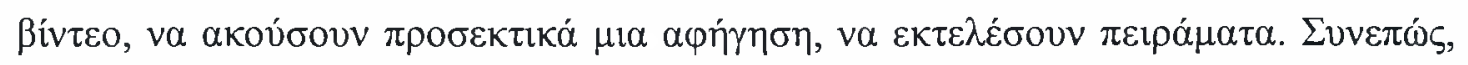

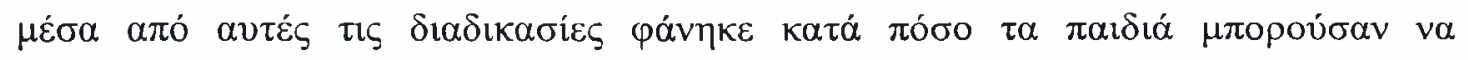

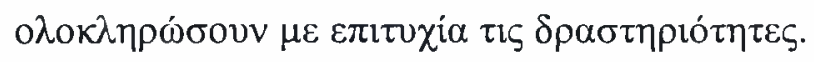

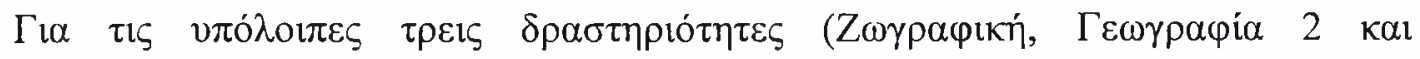

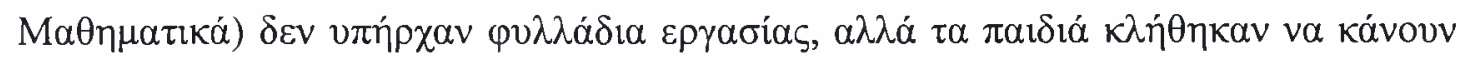
$\tau \alpha \alpha \kappa o ́ \lambda o v \theta \alpha$ :

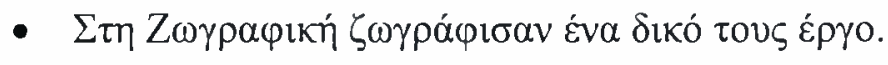

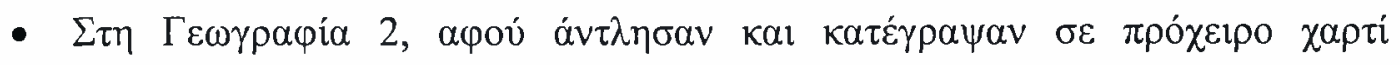

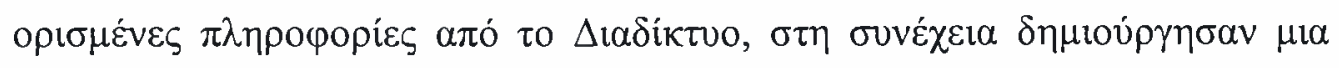

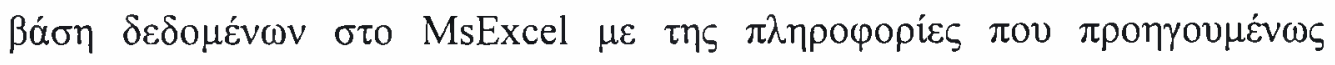
$\sigma u \gamma \kappa \varepsilon ́ v \tau \rho \omega \sigma \alpha \nu$. 


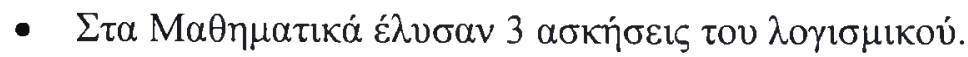

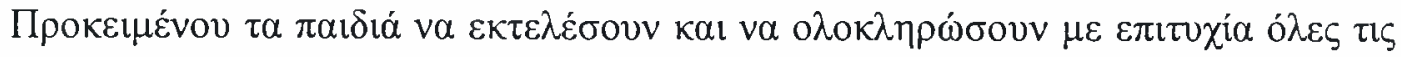

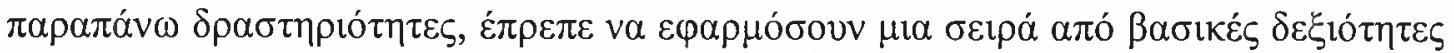
ó $\pi \omega \varsigma$ :

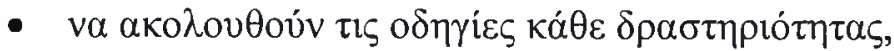

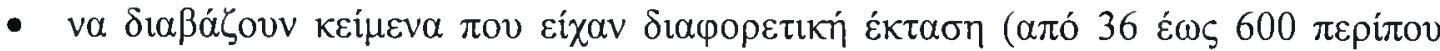

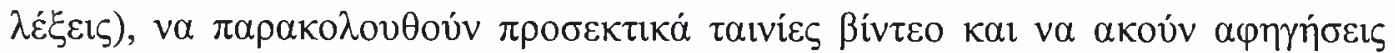

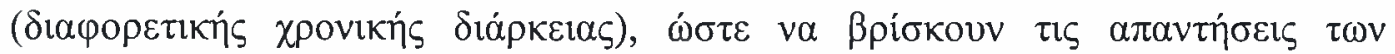

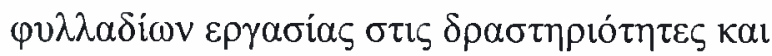

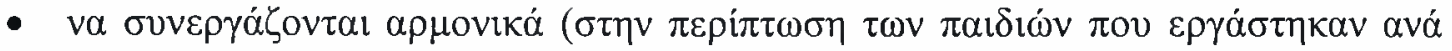
Súo).

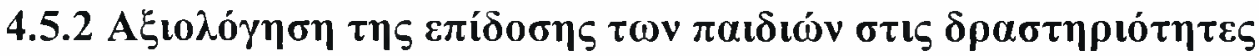

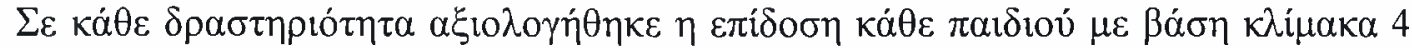

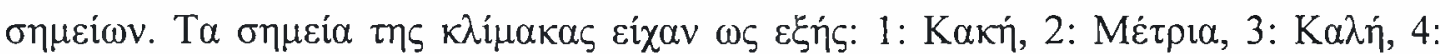

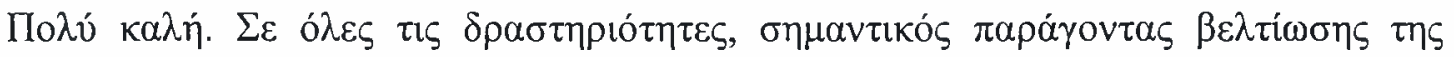

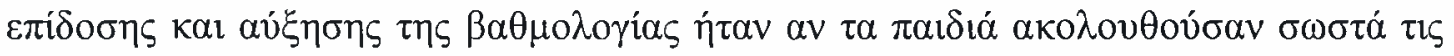

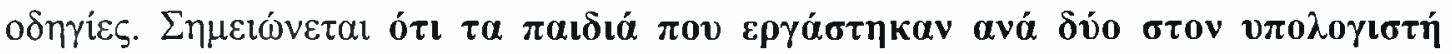

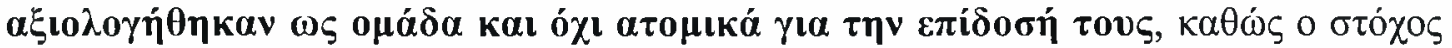

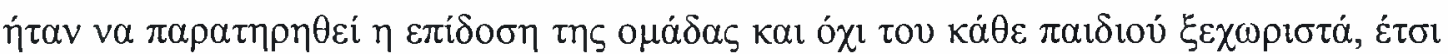

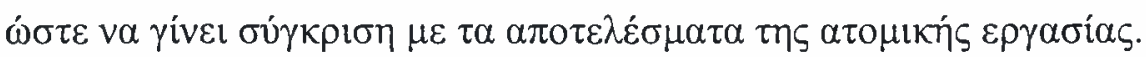

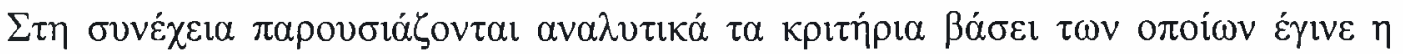

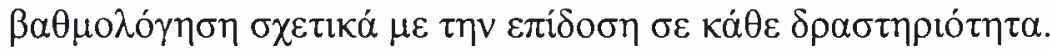

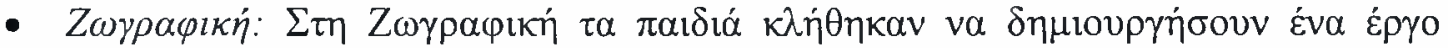

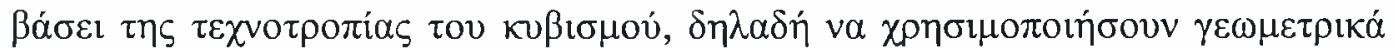

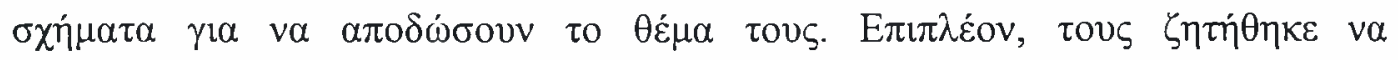

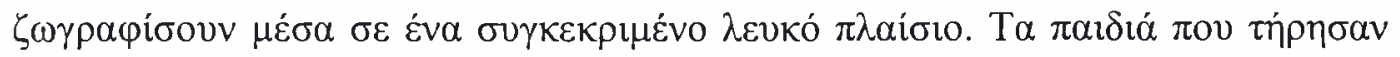

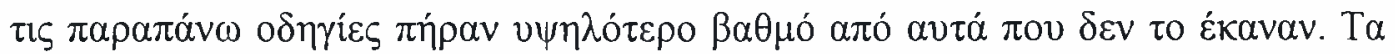

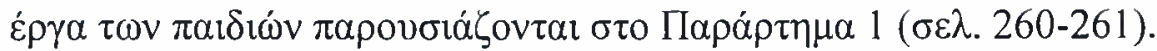

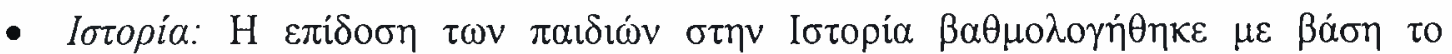

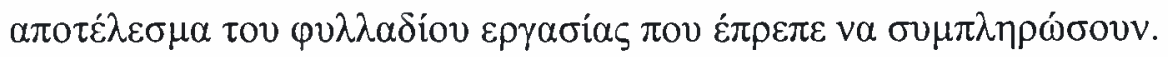




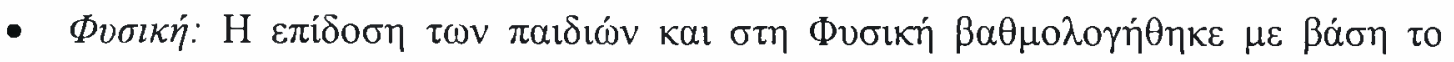

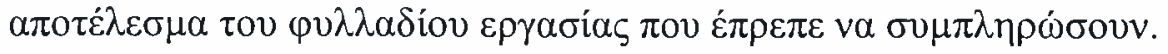

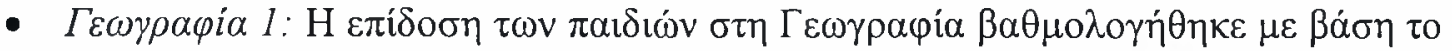

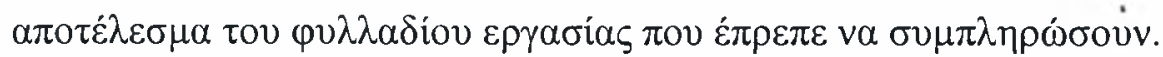

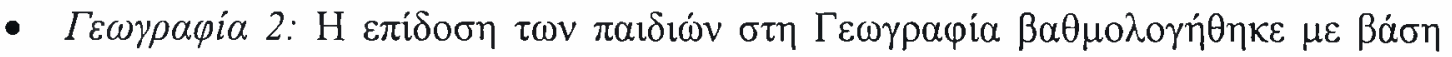

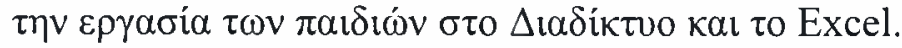

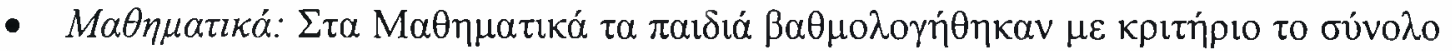

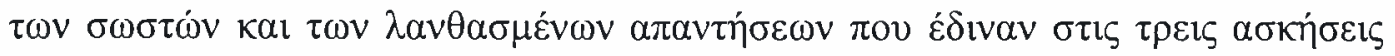

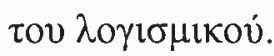

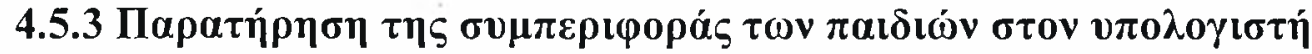

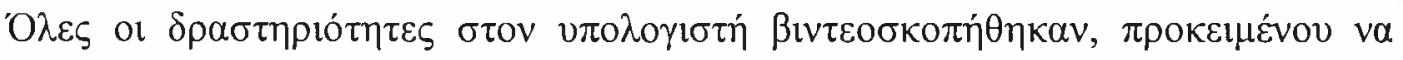

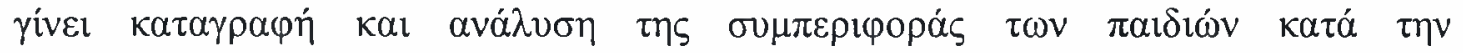

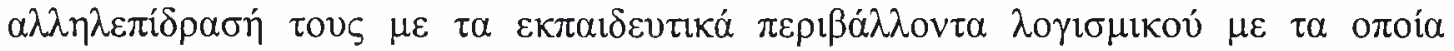

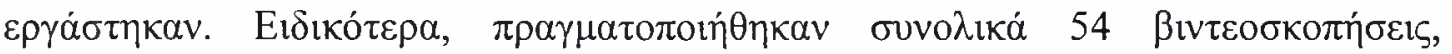

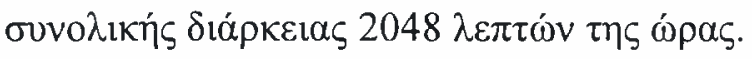

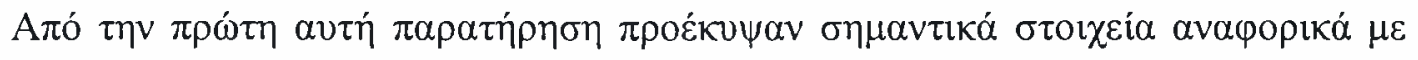

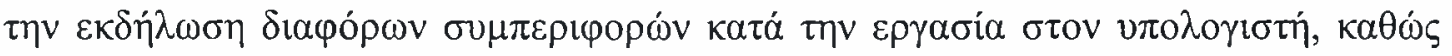

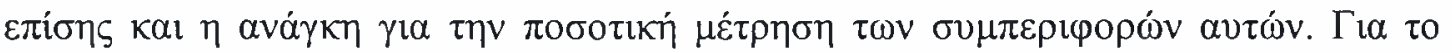

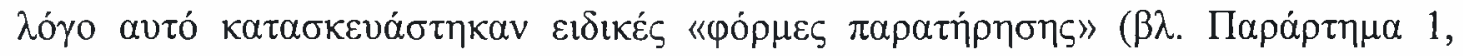

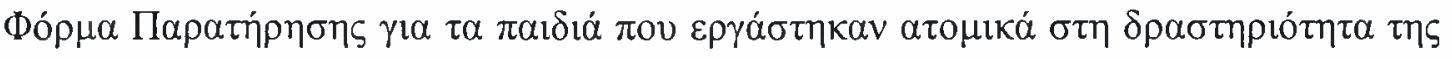
Фvбוкท́

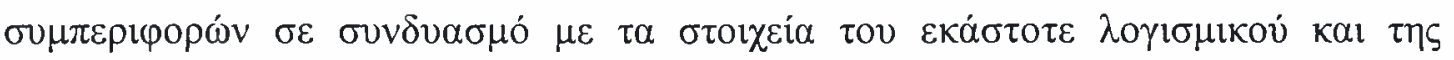

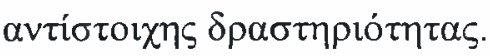

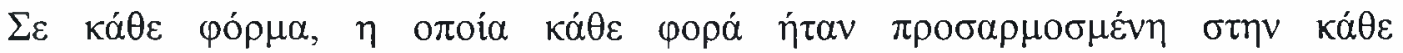

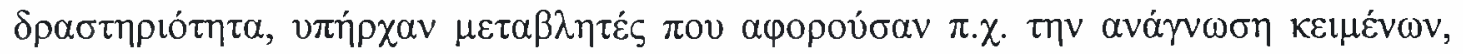

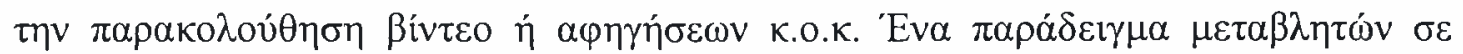

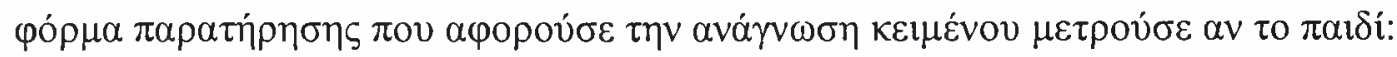

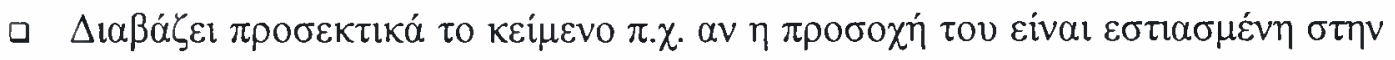

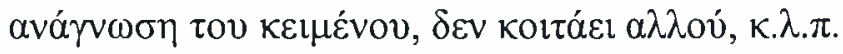




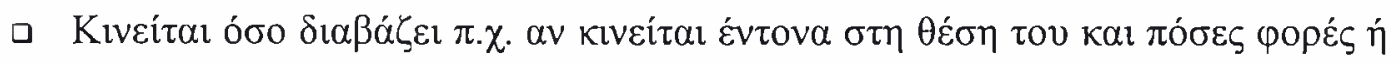

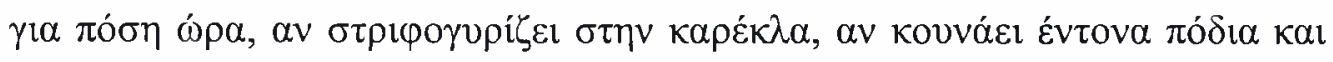
$\chi \varepsilon \dot{\rho} \rho t \alpha, \kappa . \alpha ́$.

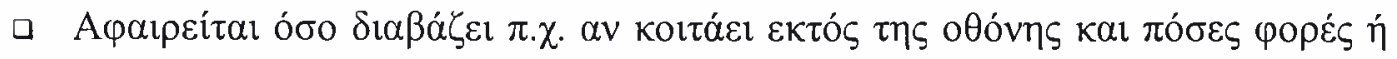

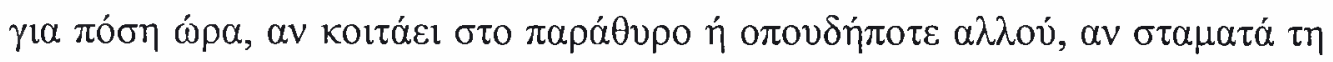

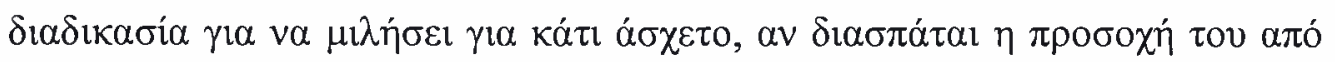

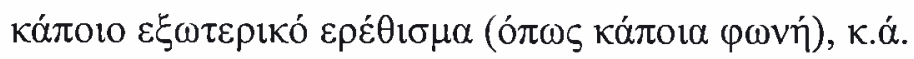

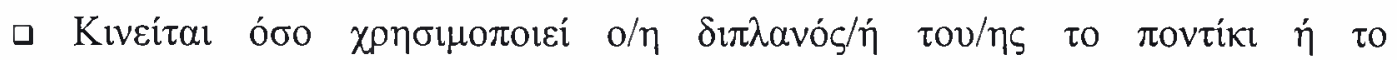
$\pi \lambda \eta \kappa \tau \rho \circ \lambda o ́ \gamma ı$.

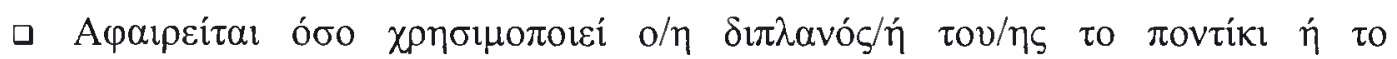
$\pi \lambda \eta \kappa \tau \rho \circ \lambda o ́ \gamma 10$.

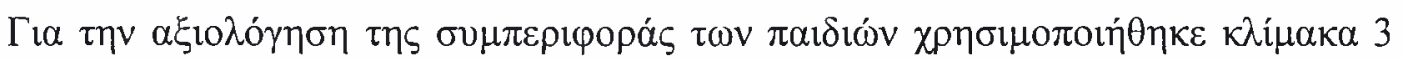

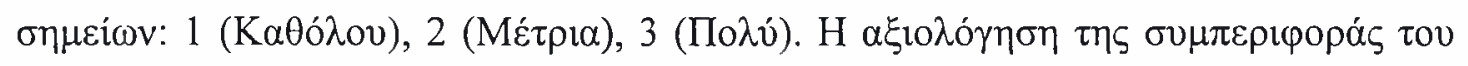

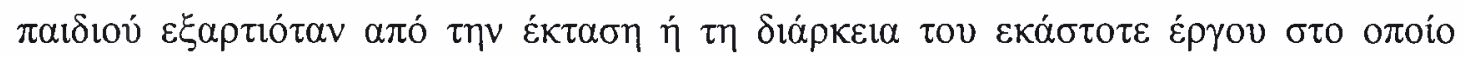

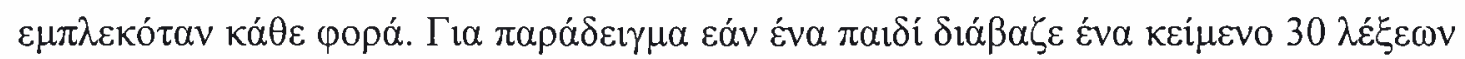

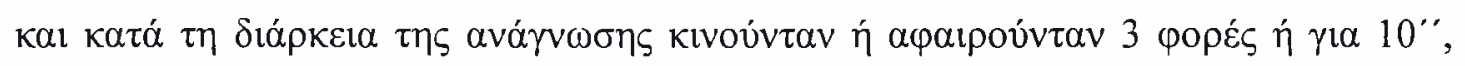

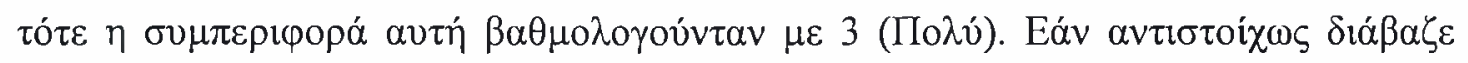

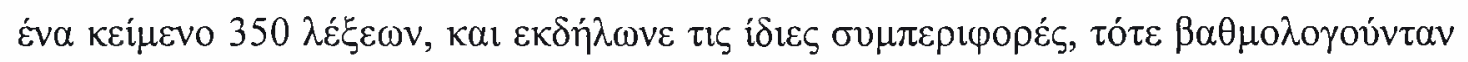

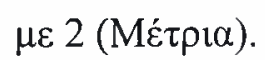

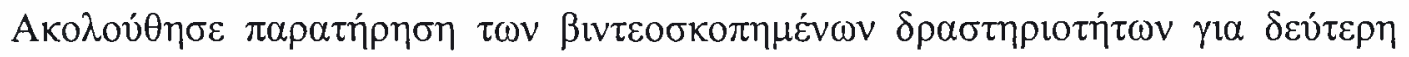

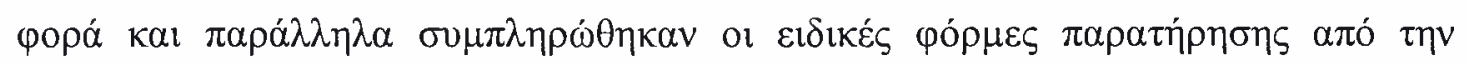

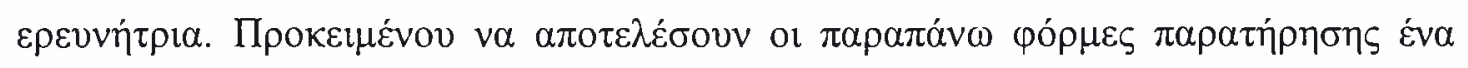

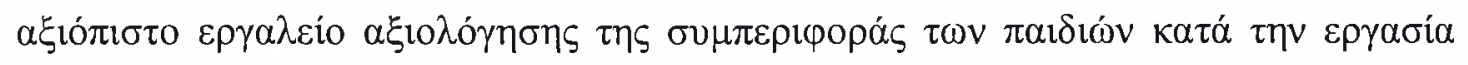

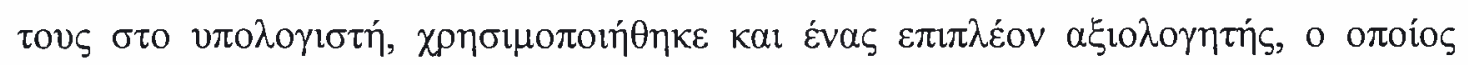

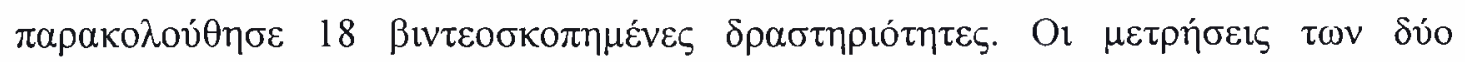

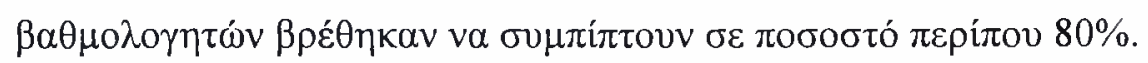

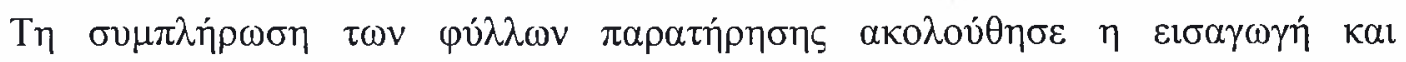

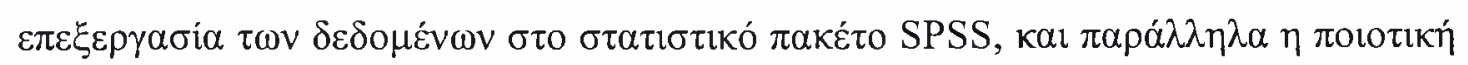

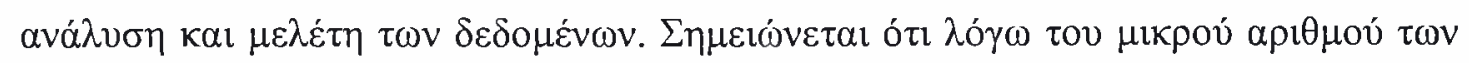

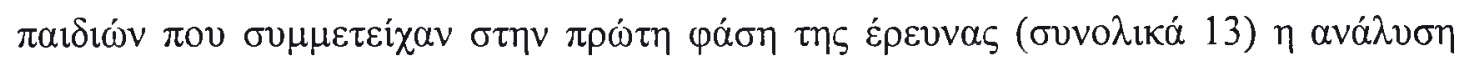

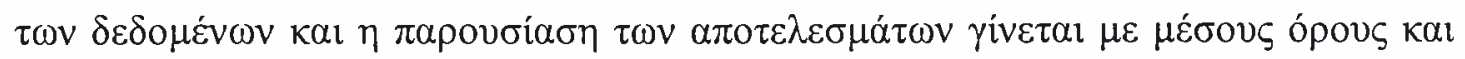

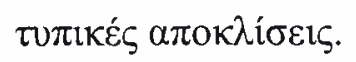




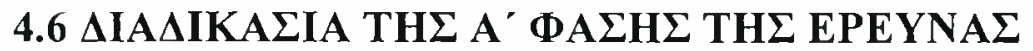

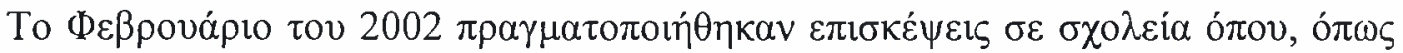

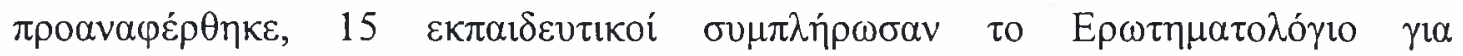

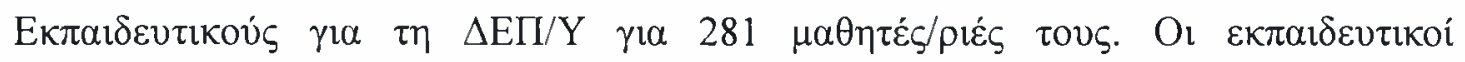

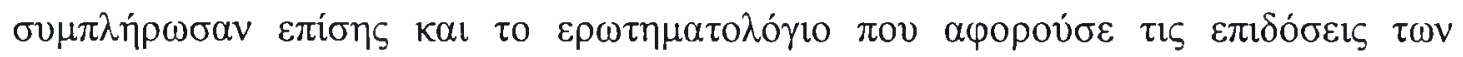

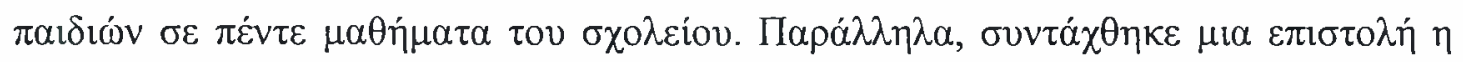

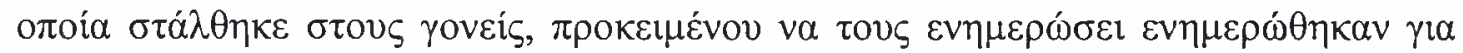

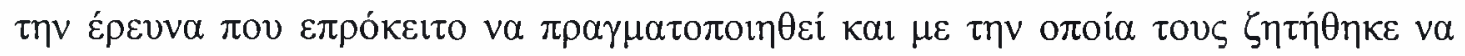

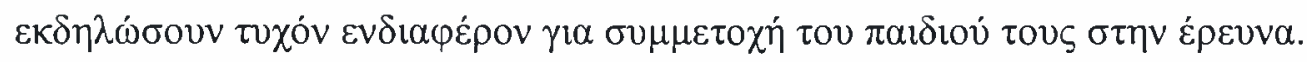

A

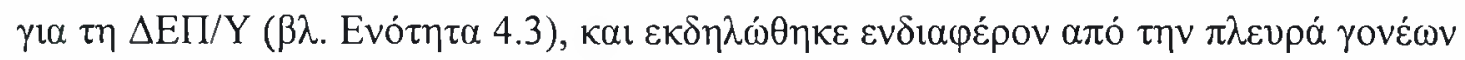

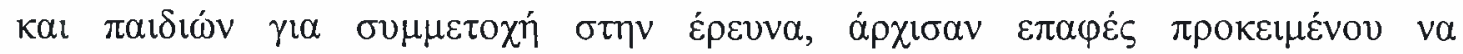

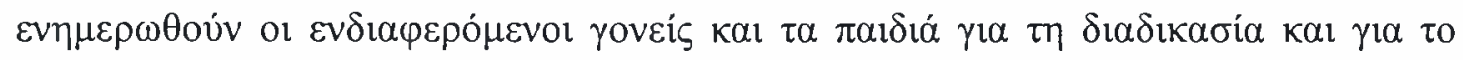

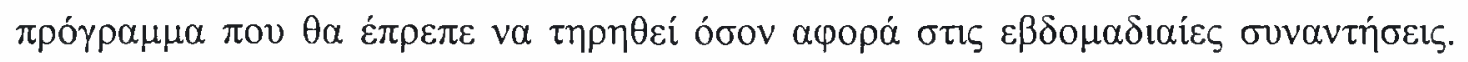

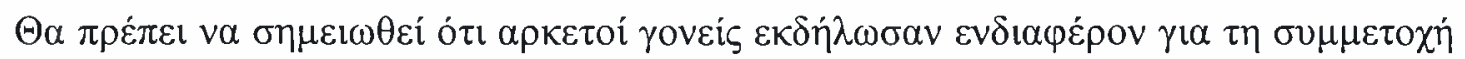

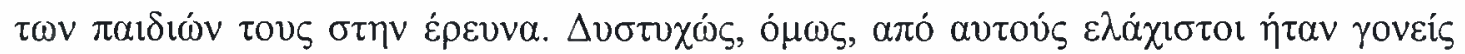
$\pi \alpha \iota \delta\llcorner\dot{v} \mu \varepsilon \sigma v \mu \pi \tau \omega \dot{\mu} \mu \tau \alpha \Delta \mathrm{E} \Pi / \mathrm{Y}$.

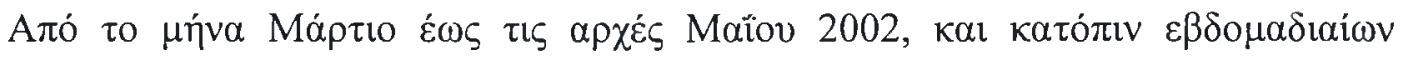

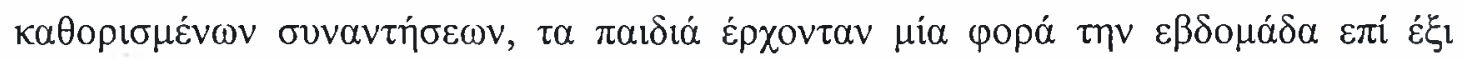

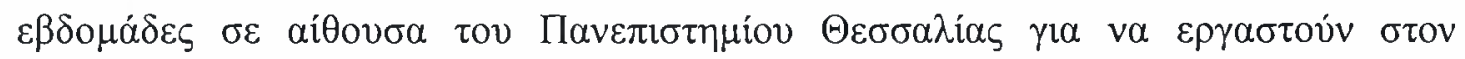

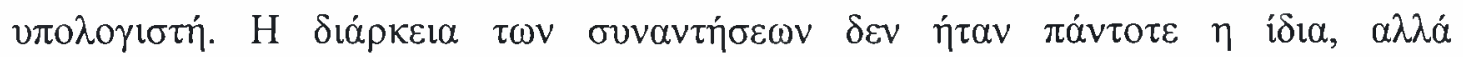

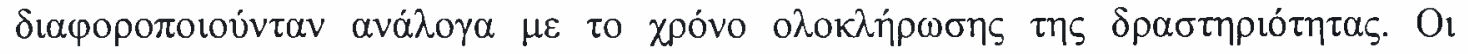

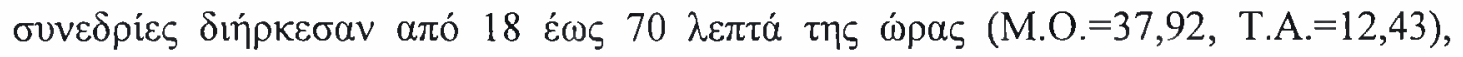

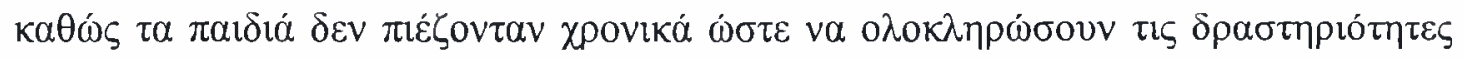

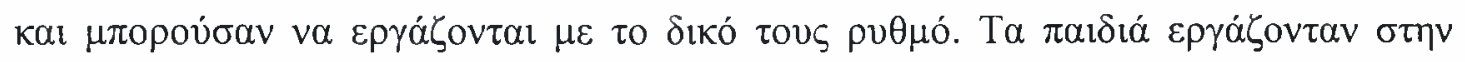

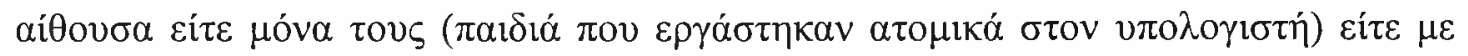

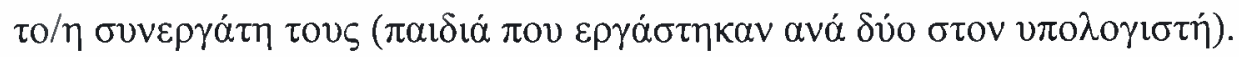

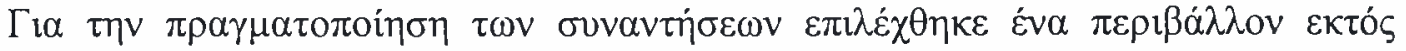

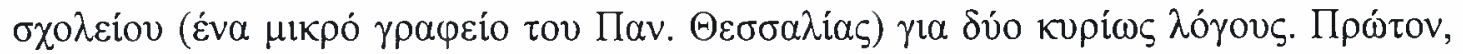

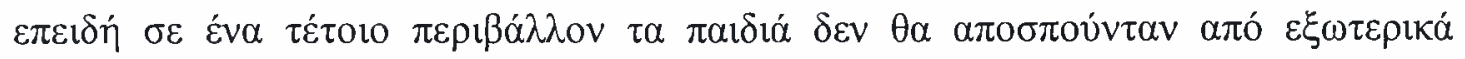

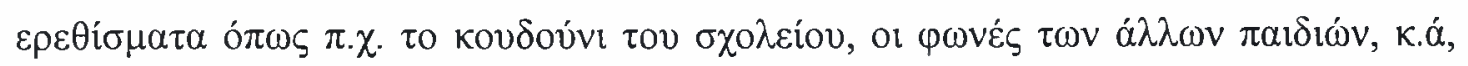




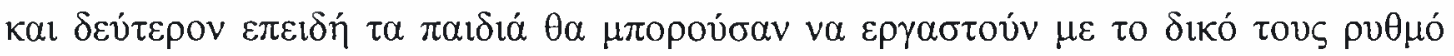

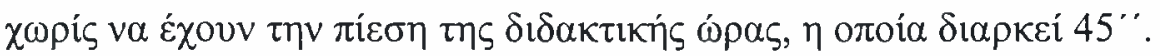

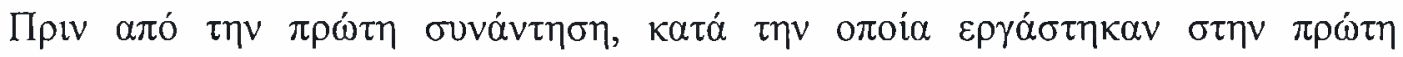

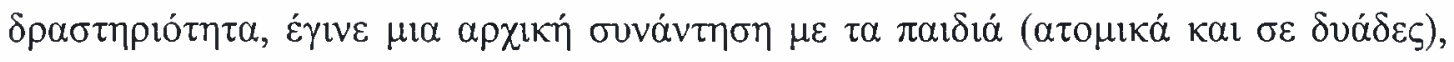

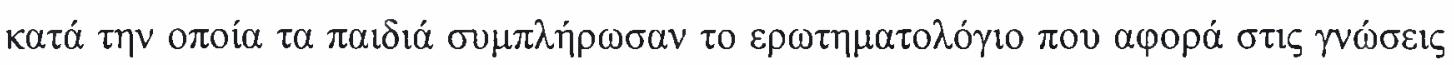

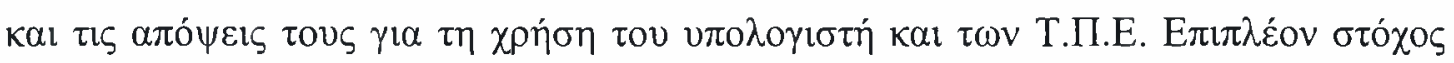

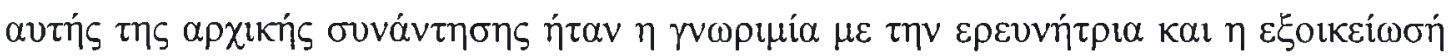

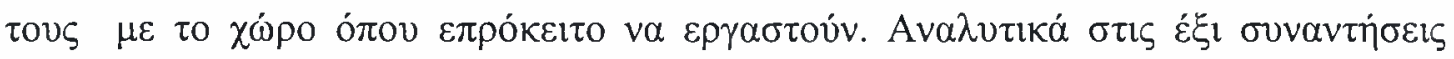

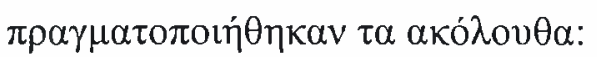

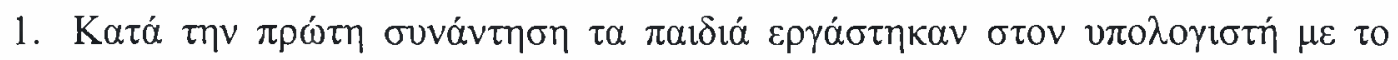

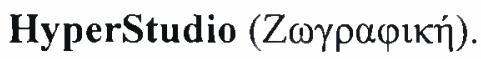

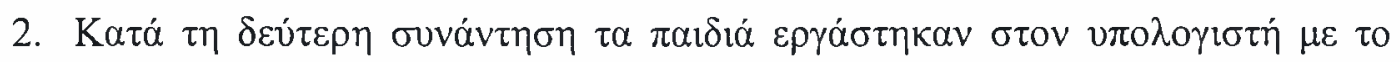

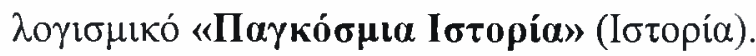

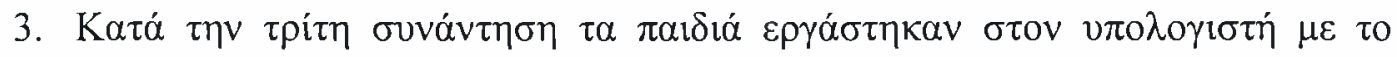
$\lambda$ о

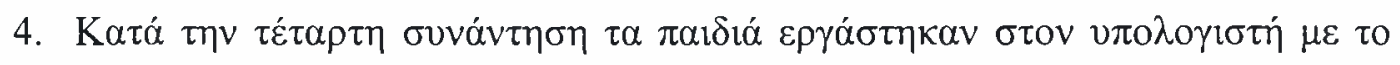

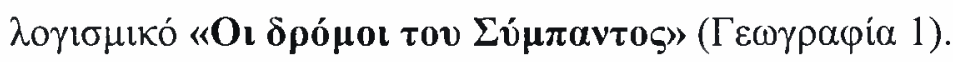

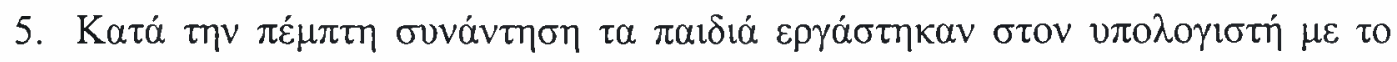

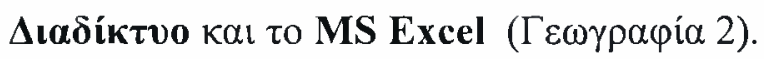

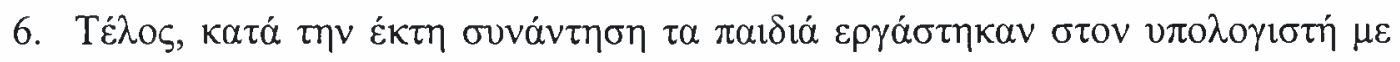

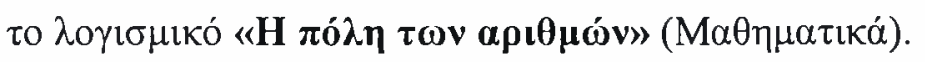

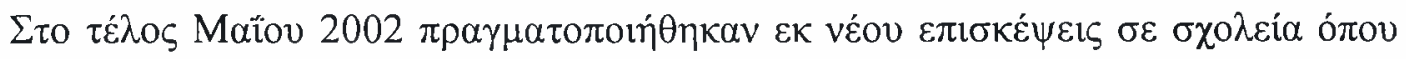

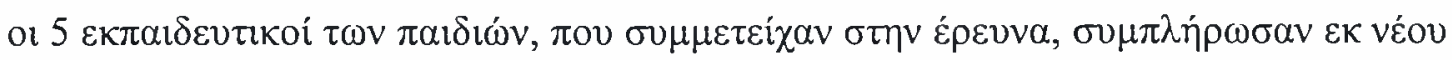

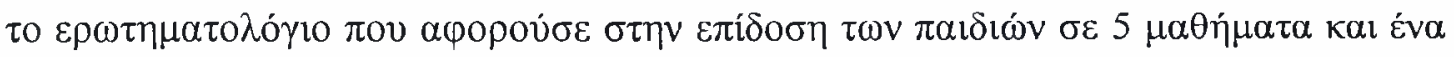

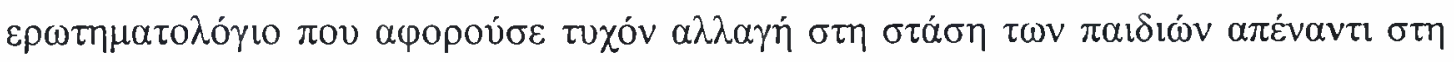

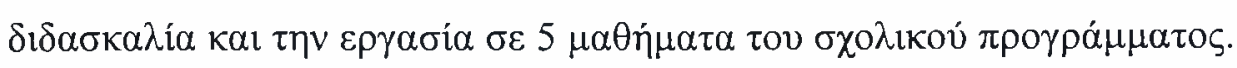




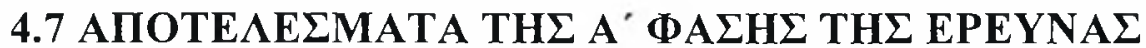

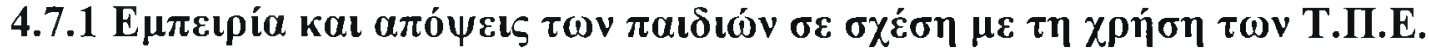

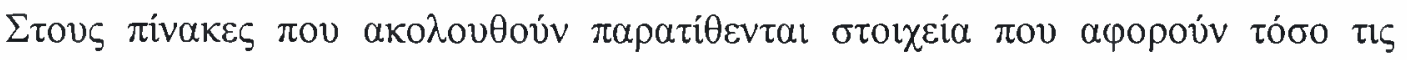

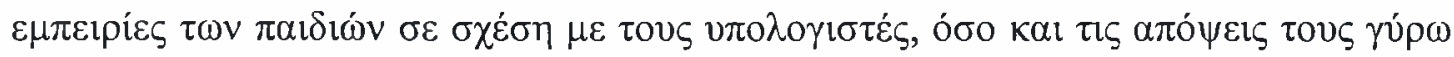

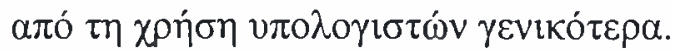

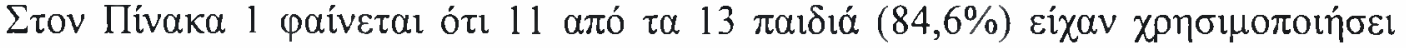

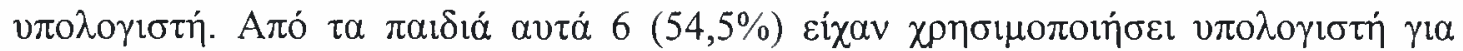

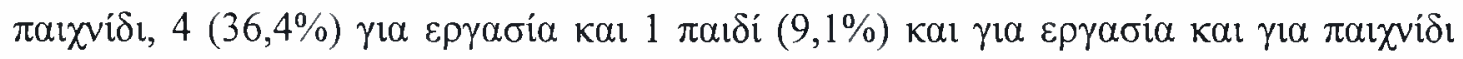

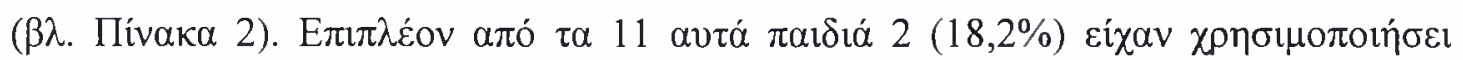

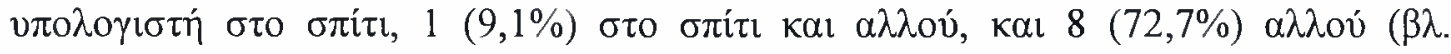
Пívaka 3).

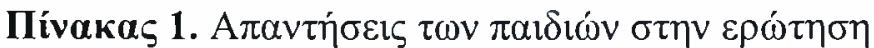

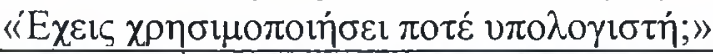

\begin{tabular}{|c|c|}
\hline$A \pi \alpha \nu \tau \tilde{j} \sigma \varepsilon l \varsigma$ & $\begin{array}{c}A \rho \imath \theta \mu o ́ \varsigma \\
N(=13)\end{array}$ \\
\hline$N a l$ & 11 \\
\hline$O \chi \imath$ & 2 \\
\hline
\end{tabular}

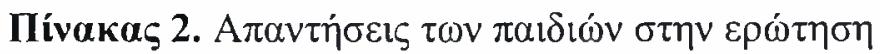

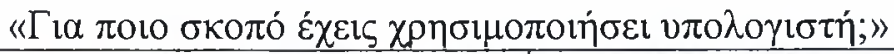

\begin{tabular}{|c|c|}
\hline 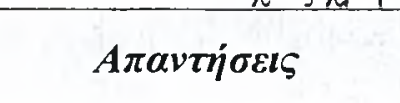 & $\begin{array}{c}A \rho \imath \theta \mu o ́ \varsigma \pi \alpha \iota \delta \imath{ }^{\prime} v \\
N(=11)\end{array}$ \\
\hline 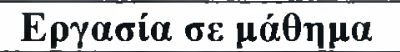 & 4 \\
\hline$\Pi \alpha \iota \chi v i ́ \delta ı$ & 6 \\
\hline 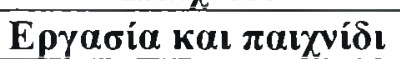 & 1 \\
\hline
\end{tabular}

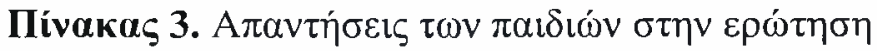

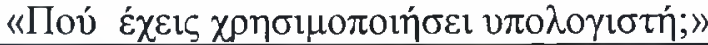

\begin{tabular}{|c|c|}
\hline 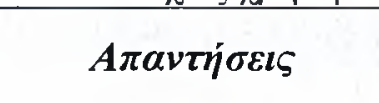 & $\begin{array}{c}A \rho \imath \theta \mu o ́ \varsigma \pi \alpha \iota \delta \imath \omega ́ v \\
N(=11)\end{array}$ \\
\hline$\Sigma \tau 0 \sigma \pi i ́ \tau \imath$ & 2 \\
\hline 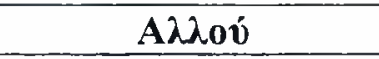 & 8 \\
\hline 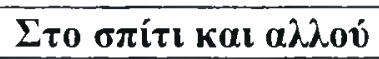 & 1 \\
\hline
\end{tabular}

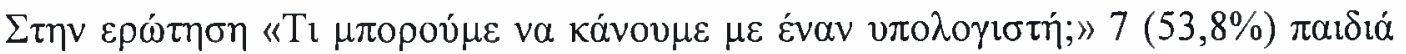

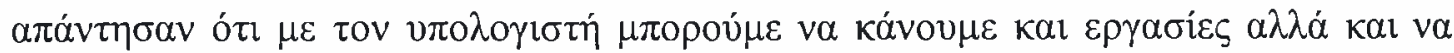

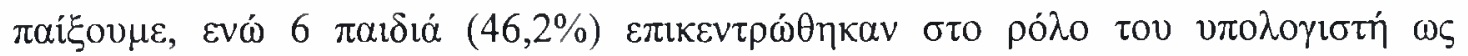




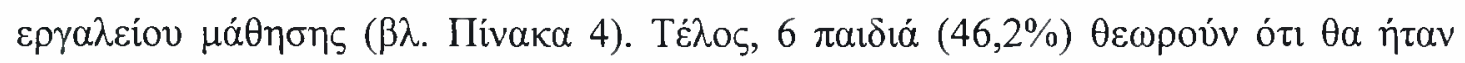

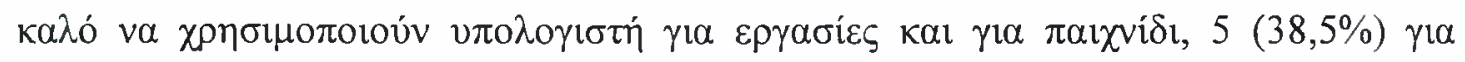

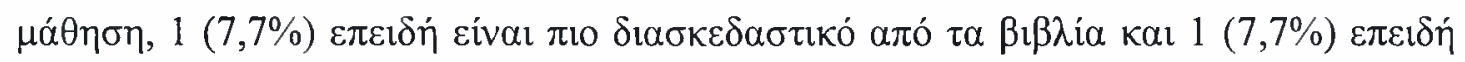

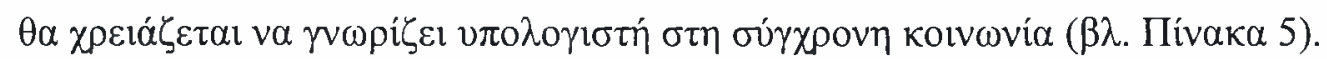

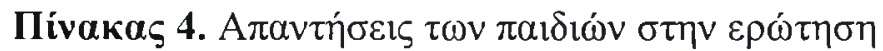

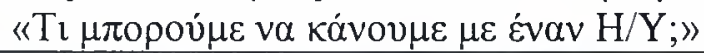

\begin{tabular}{|c|c|}
\hline 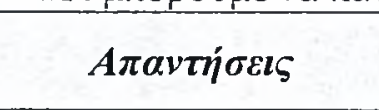 & $\begin{array}{c}A \rho \imath \theta \mu o ́ \varsigma \pi a \imath \delta \imath \omega ́ v \\
N(=13)\end{array}$ \\
\hline 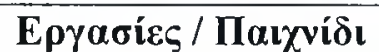 & 7 \\
\hline 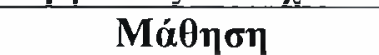 & 6 \\
\hline
\end{tabular}

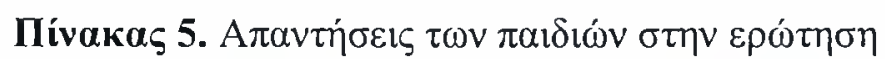

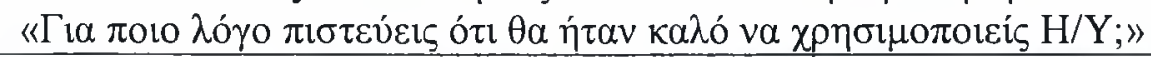

\begin{tabular}{|c|c|}
\hline 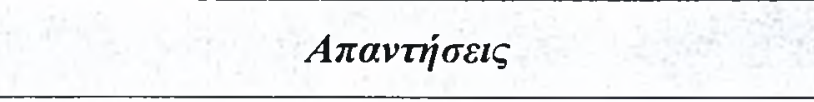 & $\begin{array}{c}A \rho \imath \theta \mu o ́ \varsigma \pi a \imath \delta \iota \omega ́ v \\
N(=13)\end{array}$ \\
\hline 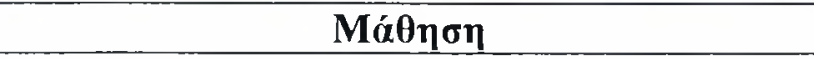 & 5 \\
\hline 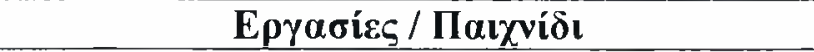 & 6 \\
\hline 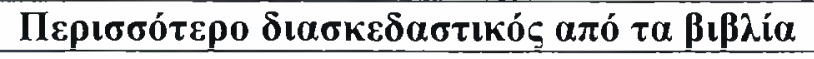 & 1 \\
\hline 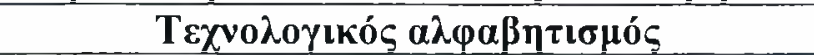 & 1 \\
\hline
\end{tabular}

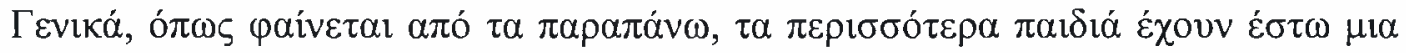

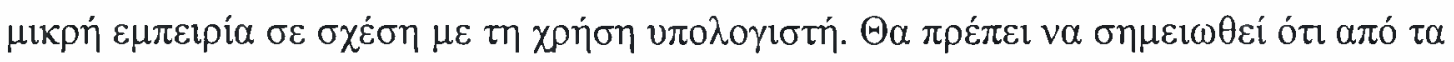

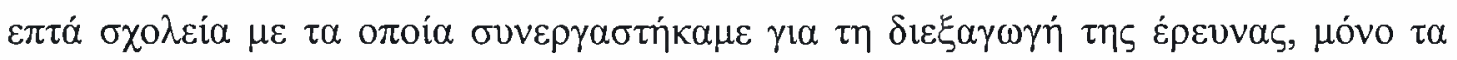

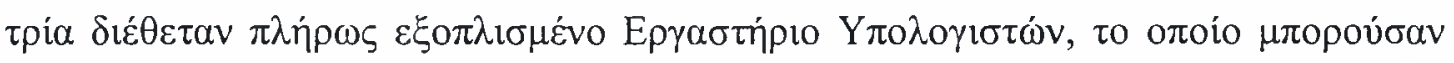

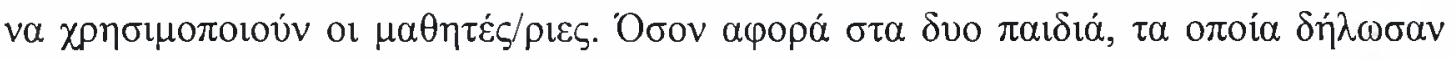

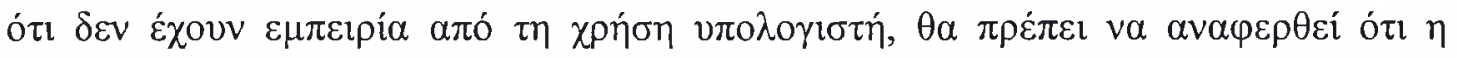

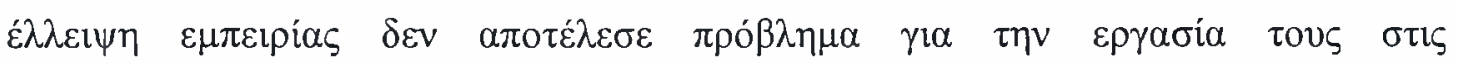

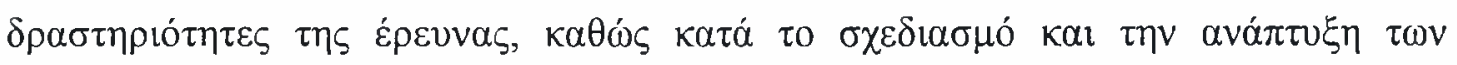

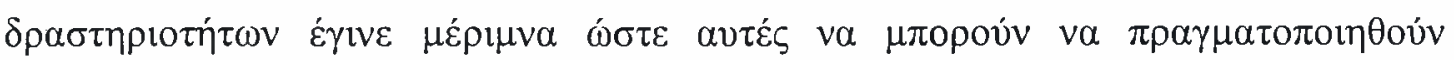

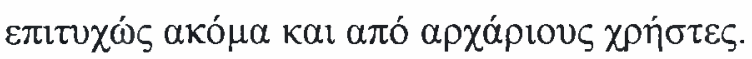

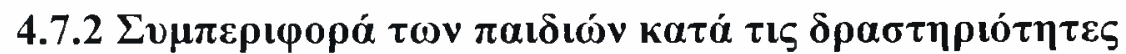

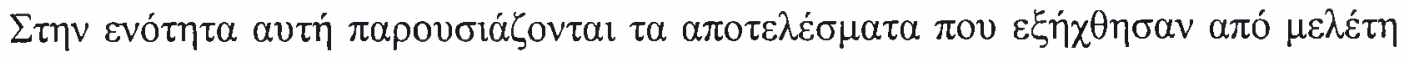

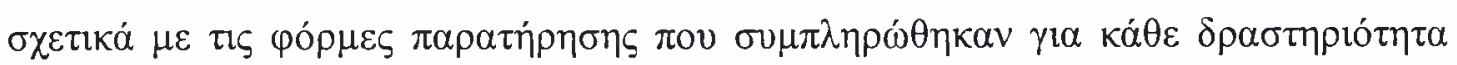

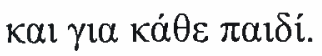




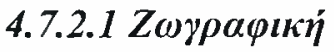

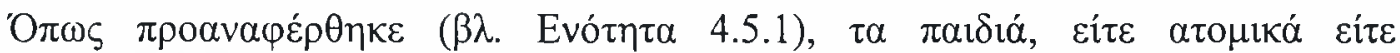

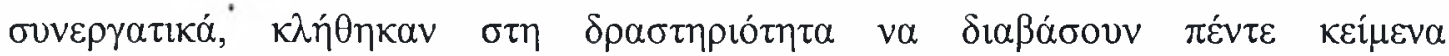

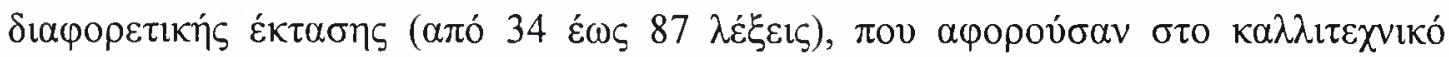

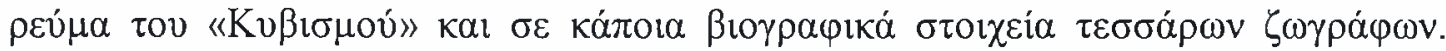

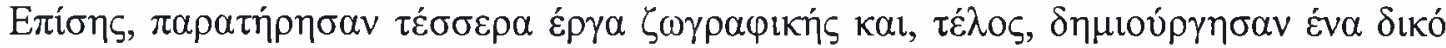

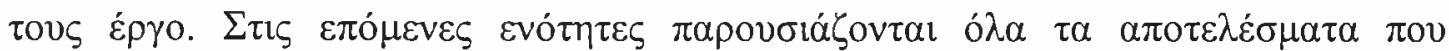

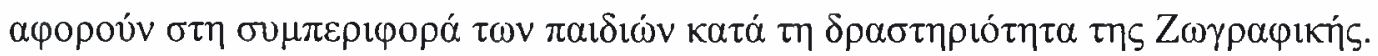

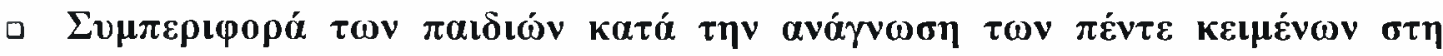

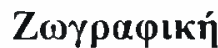

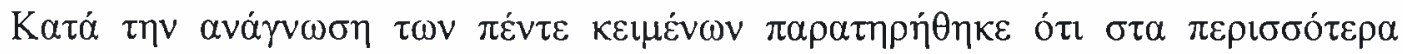

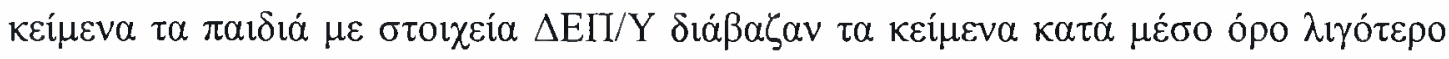

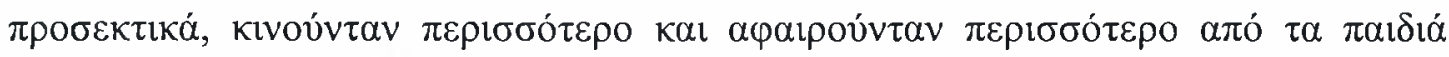

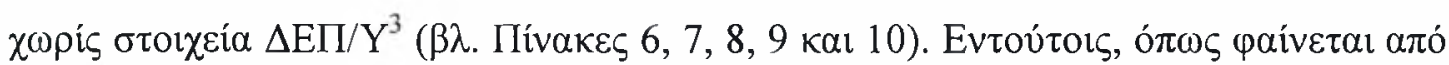

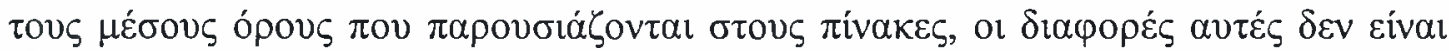
$\pi \circ \lambda v \dot{u} \mu \varepsilon \gamma \alpha \dot{\lambda} \varepsilon \varsigma$.

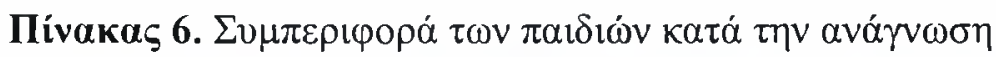

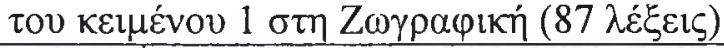

\begin{tabular}{|c|c|c|c|c|}
\hline \multirow{3}{*}{ 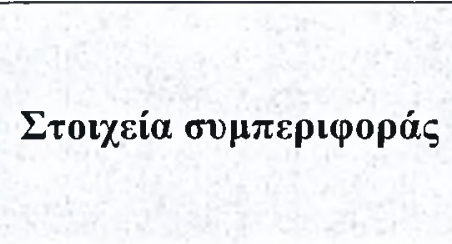 } & \multicolumn{4}{|c|}{ 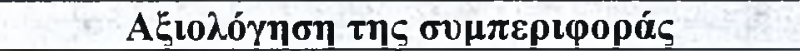 } \\
\hline & \multicolumn{2}{|c|}{ 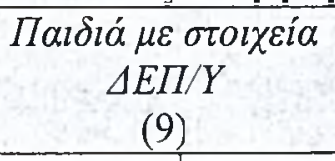 } & \multicolumn{2}{|c|}{ 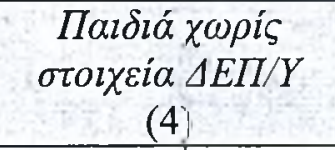 } \\
\hline & M.O. & T.A. & M.O. & T.A. \\
\hline 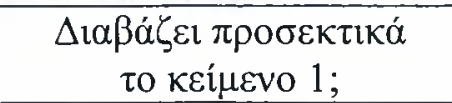 & 2,56 & 0,73 & 2,75 & 0,50 \\
\hline 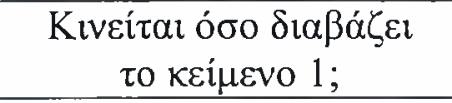 & 1,33 & 0,50 & 1,25 & 0,50 \\
\hline 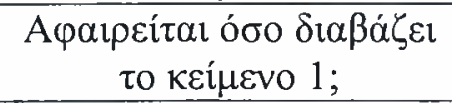 & 1,33 & 0,50 & 1,00 & 0,00 \\
\hline
\end{tabular}

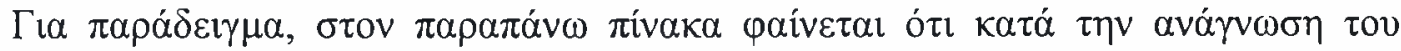

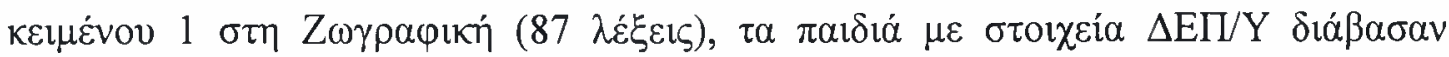

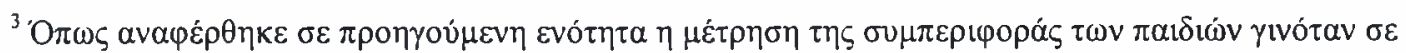

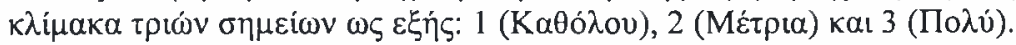




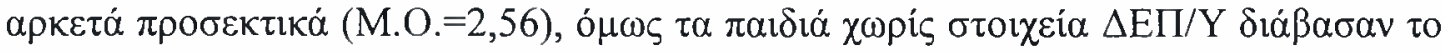

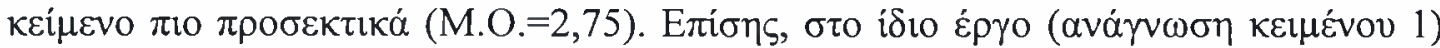

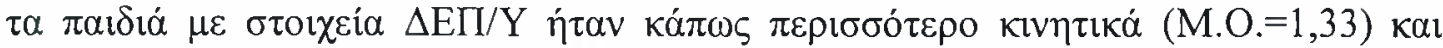

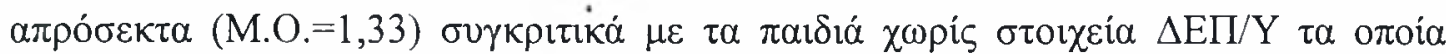

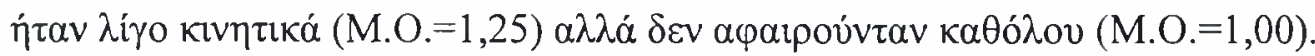

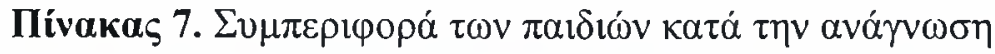

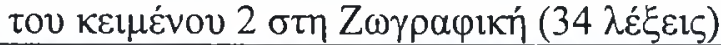

\begin{tabular}{|c|c|c|c|c|}
\hline \multirow{3}{*}{ 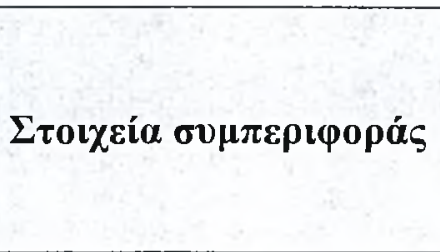 } & \multicolumn{4}{|c|}{ 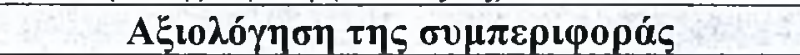 } \\
\hline & \multicolumn{2}{|c|}{ 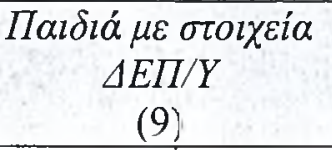 } & \multicolumn{2}{|c|}{ 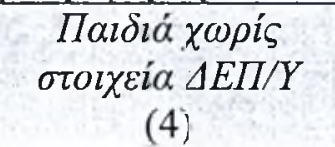 } \\
\hline & M.O. & T.A. & M.O. & T.A. \\
\hline 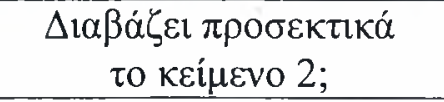 & 2,67 & 0,50 & 3,00 & 0,00 \\
\hline 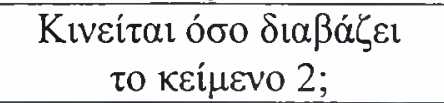 & 1,22 & 0,67 & 1,00 & 0,00 \\
\hline 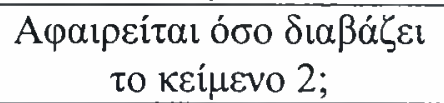 & 1,22 & 0,44 & 1,00 & 0,00 \\
\hline
\end{tabular}

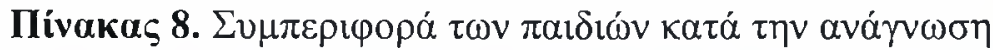

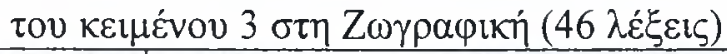

\begin{tabular}{|c|c|c|c|c|}
\hline \multirow{3}{*}{ 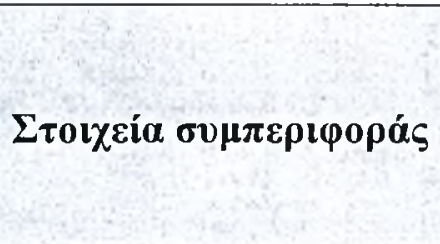 } & \multicolumn{4}{|c|}{ 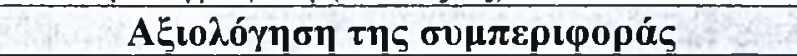 } \\
\hline & \multicolumn{2}{|c|}{ 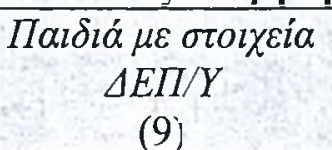 } & \multicolumn{2}{|c|}{ 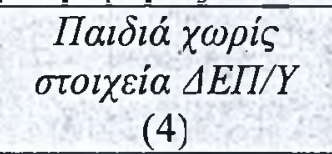 } \\
\hline & M.O. & T.A. & M.O. & T.A. \\
\hline 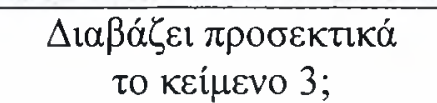 & 2,44 & 0,73 & 2,75 & 0,50 \\
\hline 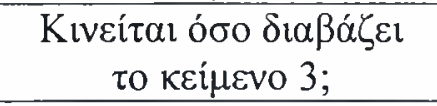 & 1,44 & 0,53 & 1,00 & 0,00 \\
\hline 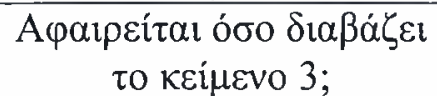 & 1,11 & 0,33 & 1,00 & 0,00 \\
\hline
\end{tabular}

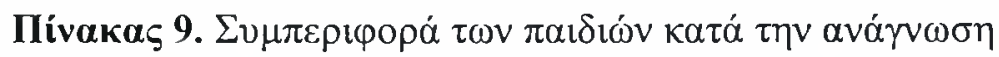

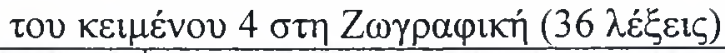

\begin{tabular}{|c|c|c|c|c|}
\hline \multirow{3}{*}{ 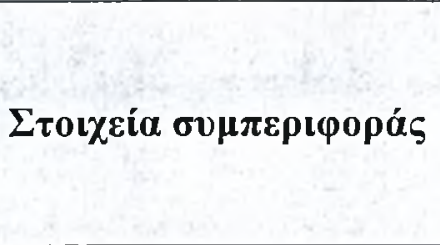 } & \multicolumn{4}{|c|}{ 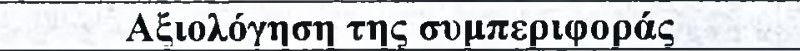 } \\
\hline & \multicolumn{2}{|c|}{ 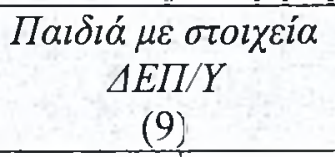 } & \multicolumn{2}{|c|}{ 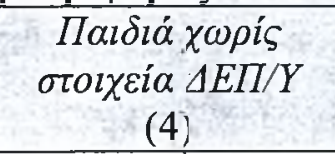 } \\
\hline & M.O. & T.A. & M.O. & T.A. \\
\hline 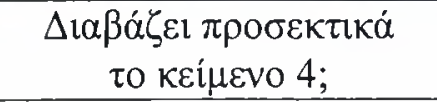 & 2,56 & 0,73 & 2,75 & 0,50 \\
\hline 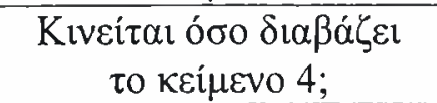 & 1,44 & 0,73 & 1,00 & 0,00 \\
\hline 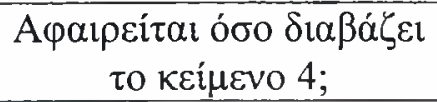 & 1,44 & 0,53 & 1,25 & 0,50 \\
\hline
\end{tabular}




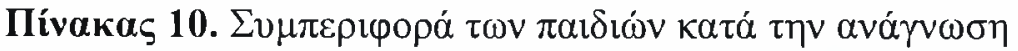

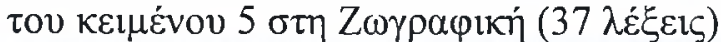

\begin{tabular}{|c|c|c|c|c|}
\hline \multirow{3}{*}{ 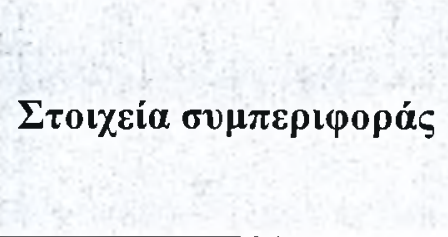 } & \multicolumn{4}{|c|}{ 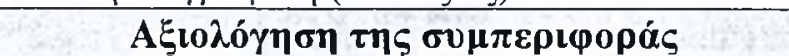 } \\
\hline & \multicolumn{2}{|c|}{$\begin{array}{c}\Pi a l \delta l \alpha \dot{\alpha} \mu \varepsilon \sigma \tau o l \chi \varepsilon i \alpha \\
\Delta E \Pi / Y \\
(9)\end{array}$} & \multicolumn{2}{|c|}{ 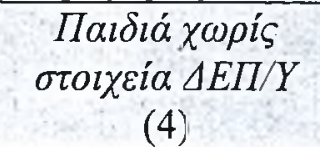 } \\
\hline & M.O. & T.A. & M.O. & T.A. \\
\hline 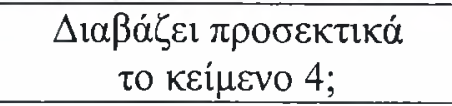 & 2,78 & 0,44 & 3,00 & 0,00 \\
\hline 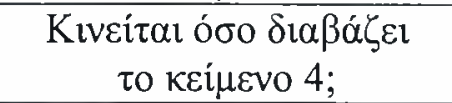 & 1,22 & 0,44 & 1,00 & 0,00 \\
\hline 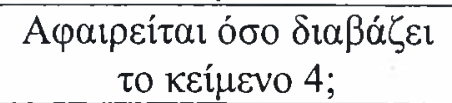 & 1,00 & 0,00 & 1,00 & 0,00 \\
\hline
\end{tabular}

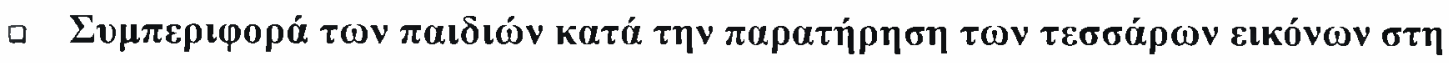

\section{Z}

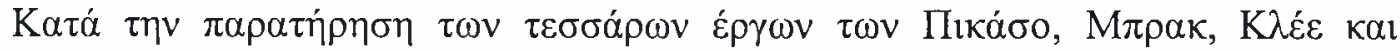

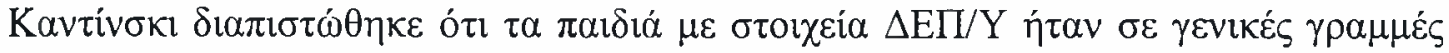

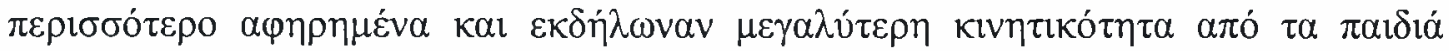

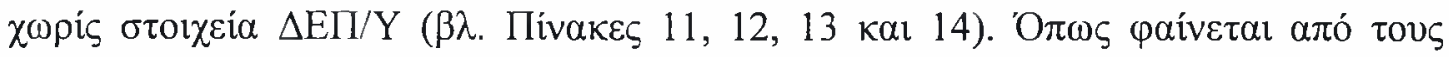

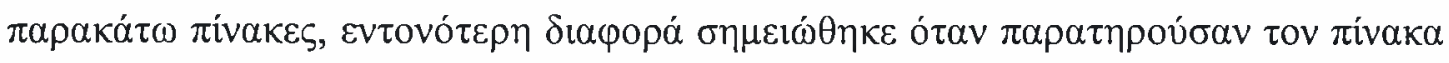

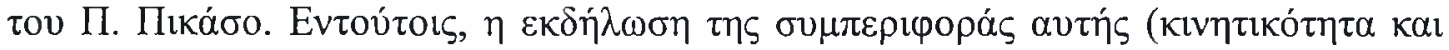

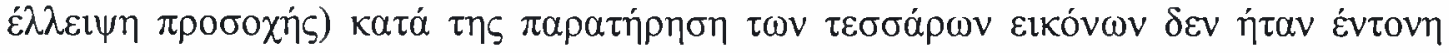

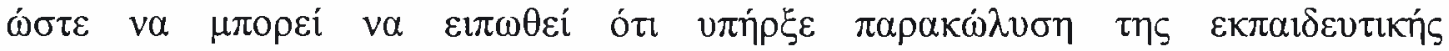

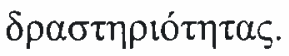

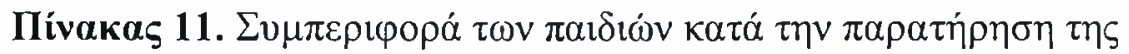

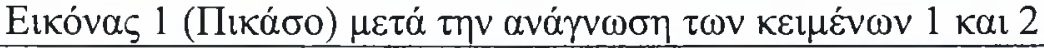

\begin{tabular}{|c|c|c|c|c|}
\hline \multirow{3}{*}{$\Sigma \tau о \iota \chi \varepsilon i ́ \alpha \sigma \nu \mu \pi \varepsilon \rho \iota \varphi о \rho \alpha ́ s$} & \multicolumn{4}{|c|}{ 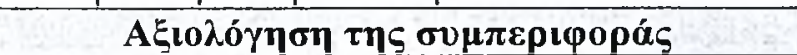 } \\
\hline & \multicolumn{2}{|c|}{ 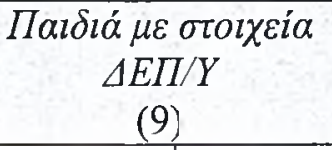 } & \multicolumn{2}{|c|}{ 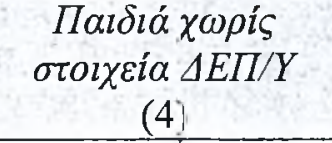 } \\
\hline & M.O. & T.A. & M.O. & T.A. \\
\hline 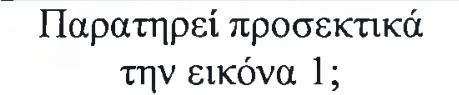 & 2,00 & 0,71 & 2,25 & 0,50 \\
\hline 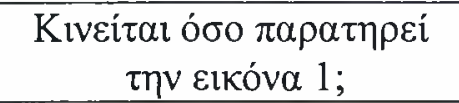 & 1,56 & 0,53 & 1,00 & 0,00 \\
\hline 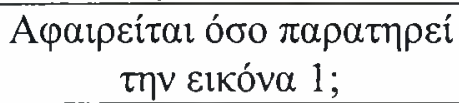 & 1,44 & 0,53 & 1,00 & 0,00 \\
\hline
\end{tabular}




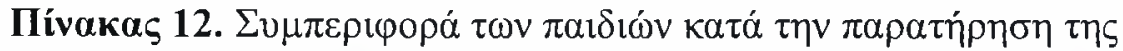

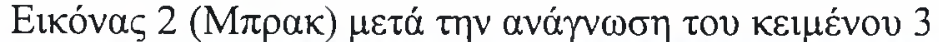

\begin{tabular}{|c|c|c|c|c|}
\hline \multirow{3}{*}{ 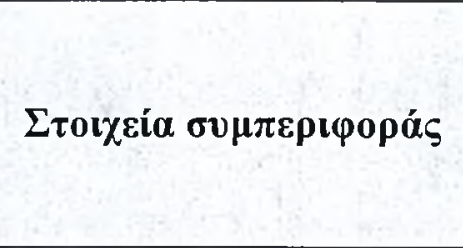 } & \multicolumn{4}{|c|}{ 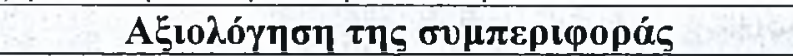 } \\
\hline & \multicolumn{2}{|c|}{ 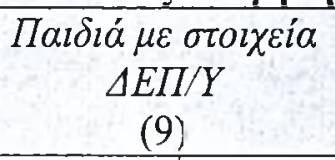 } & \multicolumn{2}{|c|}{ 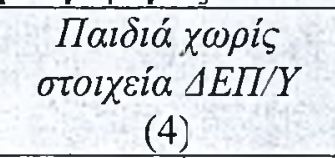 } \\
\hline & M.O. & T.A. & M.O. & T.A. \\
\hline 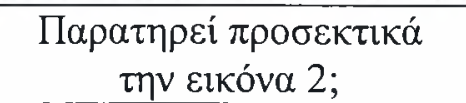 & 2,44 & 0,53 & 2,75 & 0,50 \\
\hline 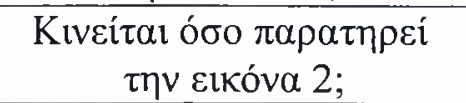 & 1,11 & 0,33 & 1,00 & 0,00 \\
\hline 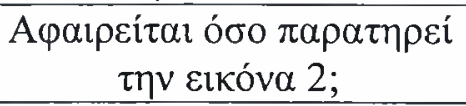 & 1,11 & 0,33 & 1,25 & 0,50 \\
\hline
\end{tabular}

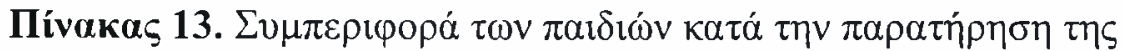

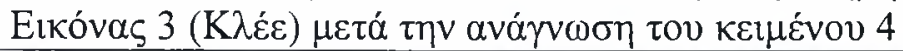

\begin{tabular}{|c|c|c|c|c|}
\hline \multirow{3}{*}{ 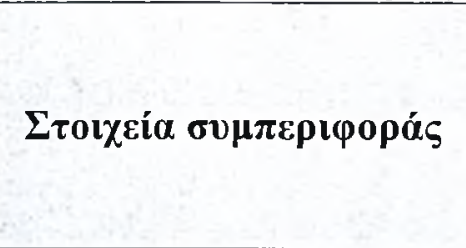 } & \multicolumn{4}{|c|}{ 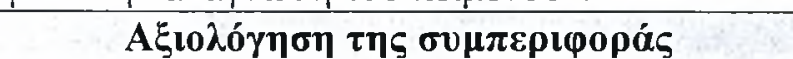 } \\
\hline & \multicolumn{2}{|c|}{ 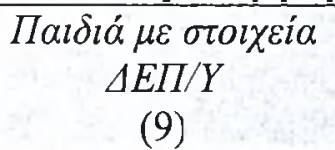 } & \multicolumn{2}{|c|}{ 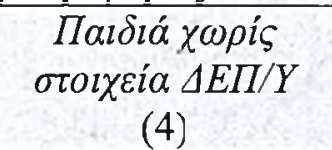 } \\
\hline & M.O. & T.A. & M.O. & T.A. \\
\hline 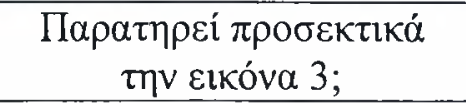 & 2,00 & 0,60 & 2,11 & 0,50 \\
\hline 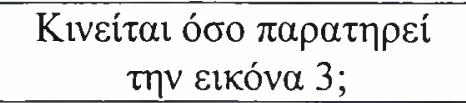 & 1,44 & 0,53 & 1,00 & 0,00 \\
\hline 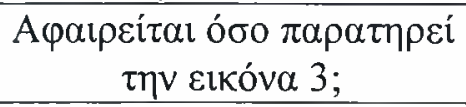 & 1,33 & 0,50 & 1,25 & 0,50 \\
\hline
\end{tabular}

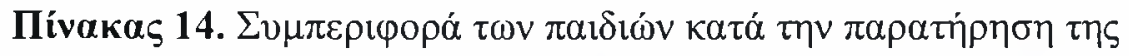

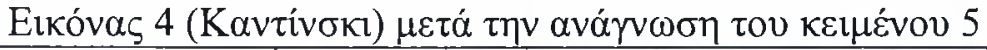

\begin{tabular}{|c|c|c|c|c|}
\hline \multirow{3}{*}{ 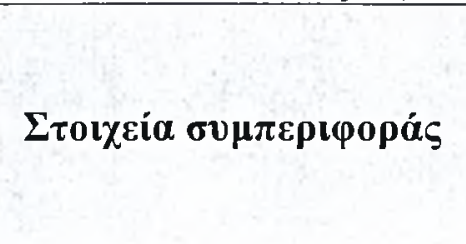 } & \multicolumn{4}{|c|}{ 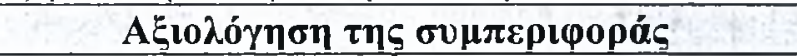 } \\
\hline & \multicolumn{2}{|c|}{ 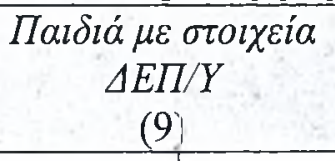 } & \multicolumn{2}{|c|}{ 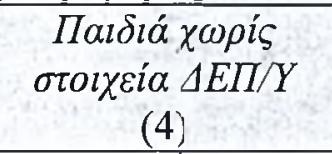 } \\
\hline & M.O. & T.A. & M.O. & T.A. \\
\hline 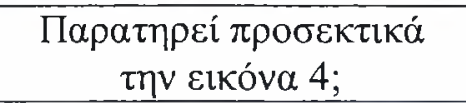 & 2,22 & 0,67 & 2,50 & 0,58 \\
\hline 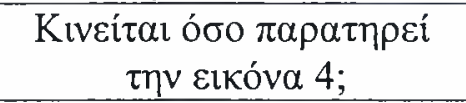 & 1,44 & 0,73 & 1,25 & 0,50 \\
\hline 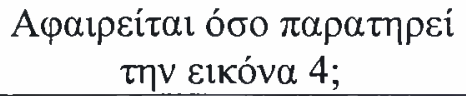 & 1,33 & 0,71 & 1,25 & 0,50 \\
\hline
\end{tabular}

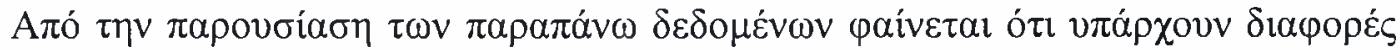

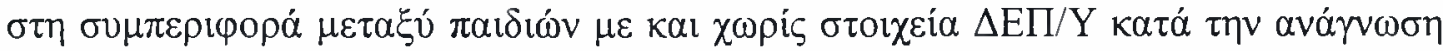

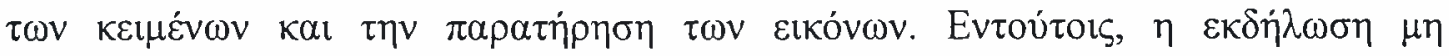

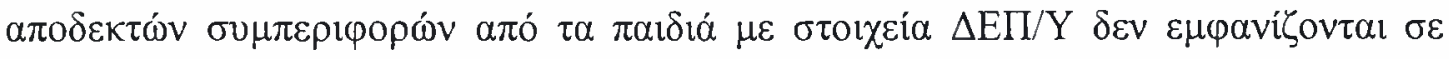




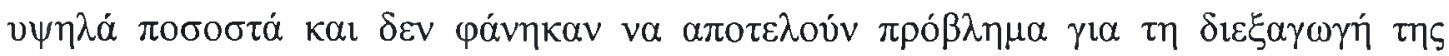

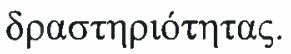

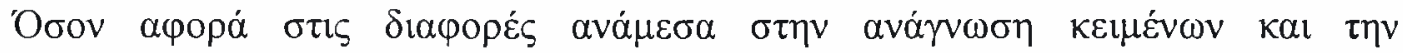

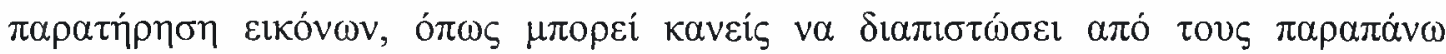

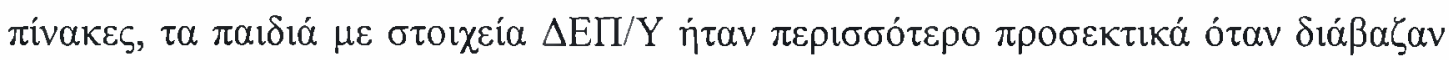

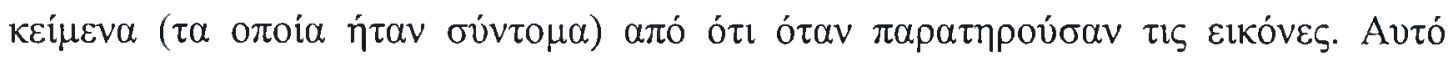

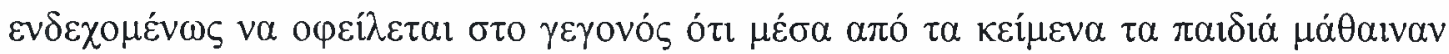

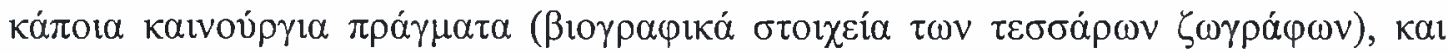

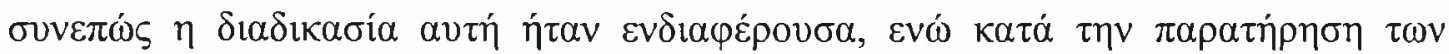

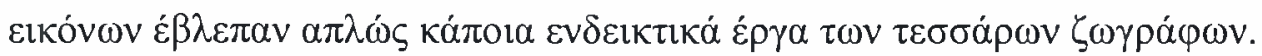

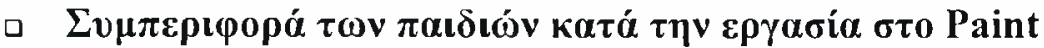

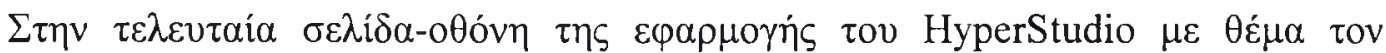

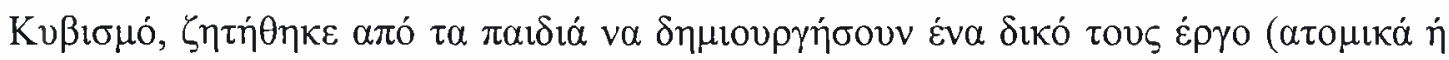

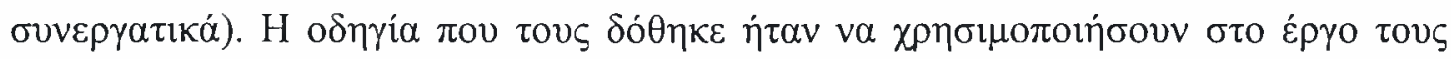

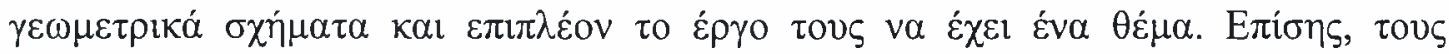

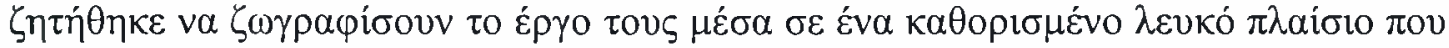

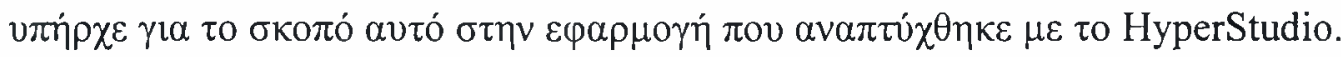

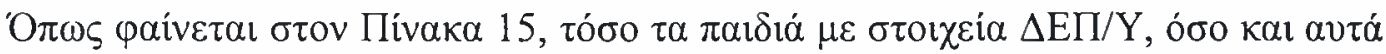

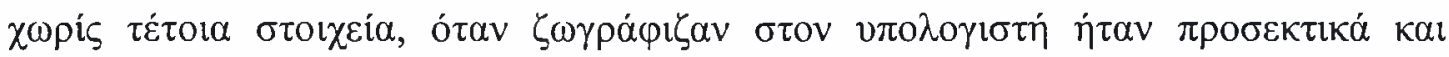

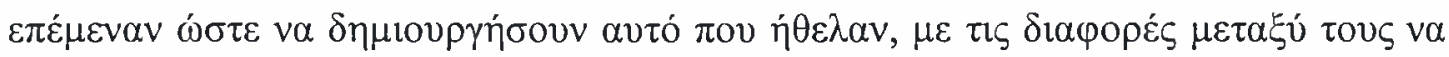

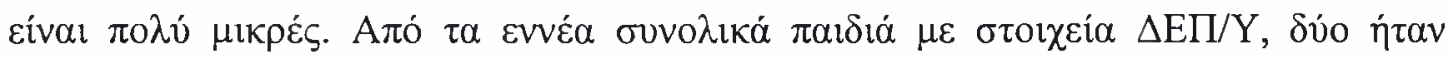

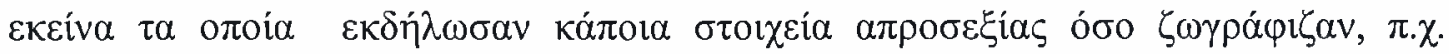

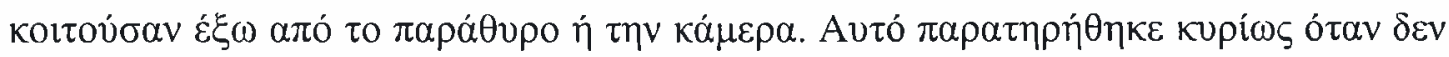

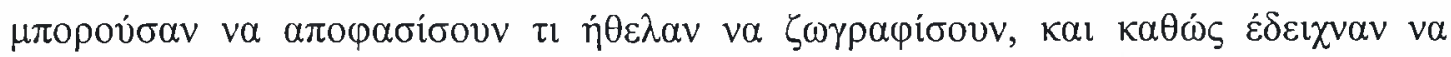

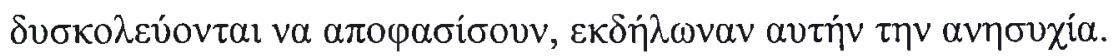




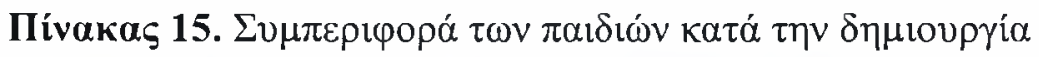

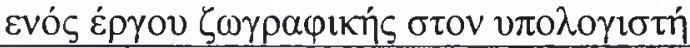

\begin{tabular}{|c|c|c|c|c|}
\hline \multirow{3}{*}{ 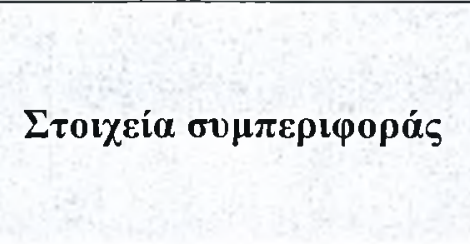 } & \multicolumn{4}{|c|}{ 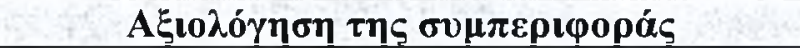 } \\
\hline & \multicolumn{2}{|c|}{ 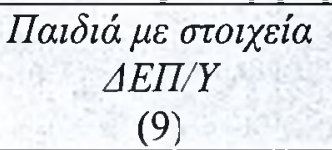 } & \multicolumn{2}{|c|}{ 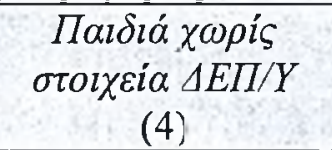 } \\
\hline & M.O. & T.A. & M.O. & T.A. \\
\hline 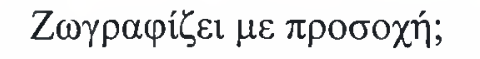 & 2,89 & 0,33 & 3,00 & 0,00 \\
\hline 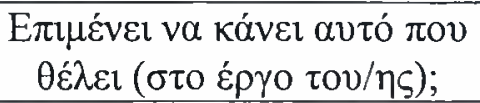 & 2,78 & 0,44 & 2,75 & 0,50 \\
\hline 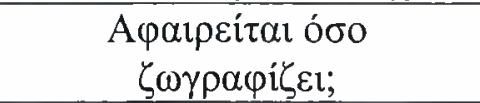 & 1,33 & 0,71 & 1,00 & 0,00 \\
\hline
\end{tabular}

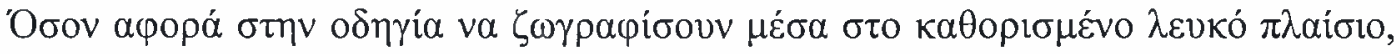

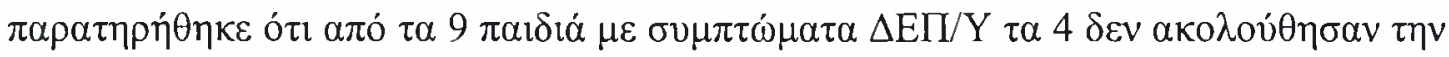

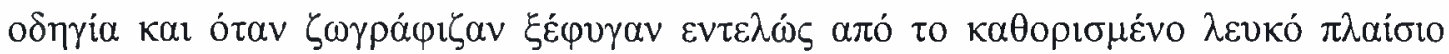

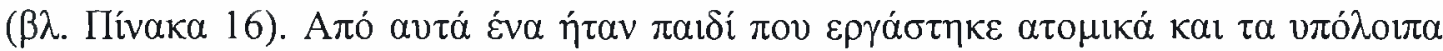

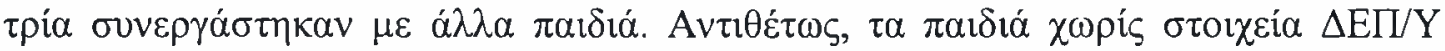

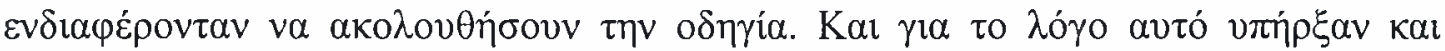

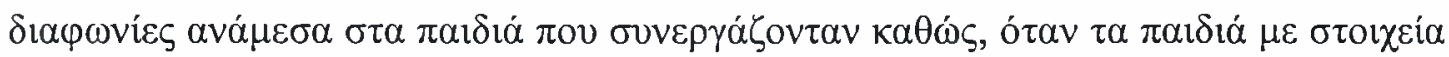

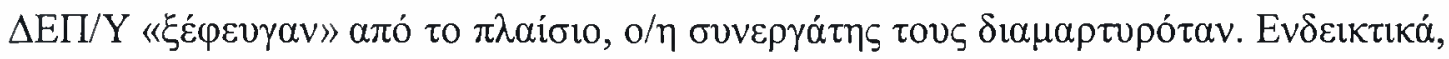

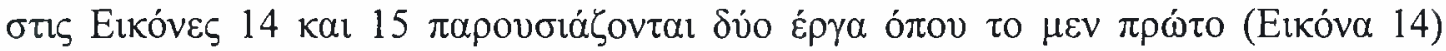

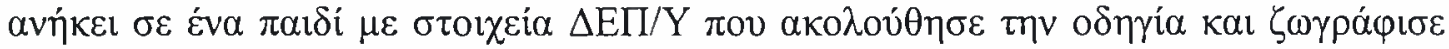

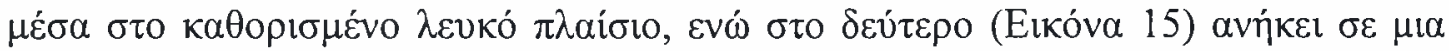

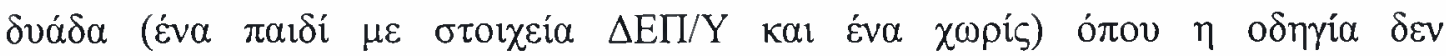

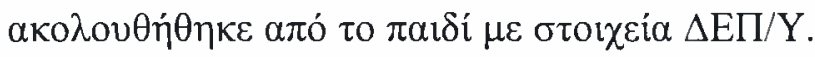

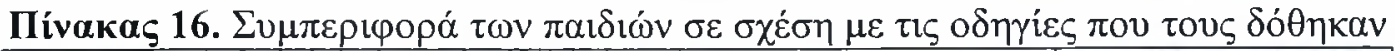

\begin{tabular}{|c|c|c|}
\hline 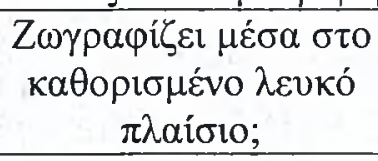 & $\begin{array}{c}\prod \alpha \imath \delta \imath \alpha \dot{\alpha} \mu \varepsilon \sigma \tau o l \chi \varepsilon i \alpha \Delta E \Pi / Y \\
(\mathrm{~N}=9)\end{array}$ & 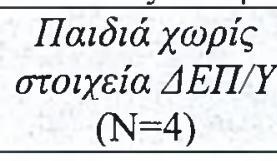 \\
\hline NAI & 5 & 4 \\
\hline OXI & 4 & \\
\hline
\end{tabular}




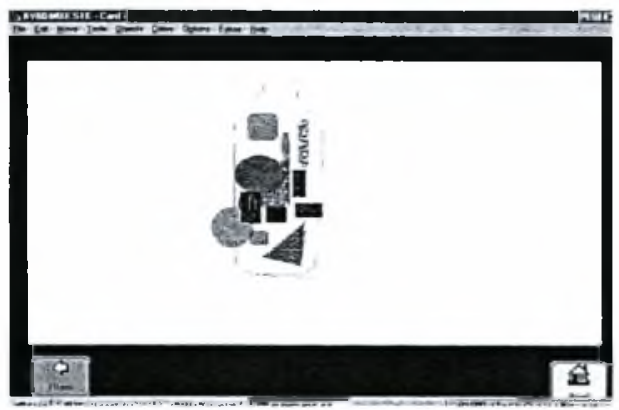

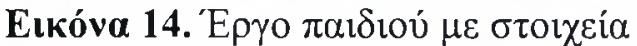

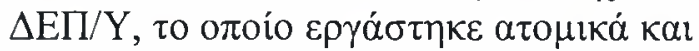
$\alpha \kappa o \lambda \circ u ́ \theta \eta \sigma \varepsilon \tau \eta \vee ~ o \delta \eta \gamma i ́ \alpha$

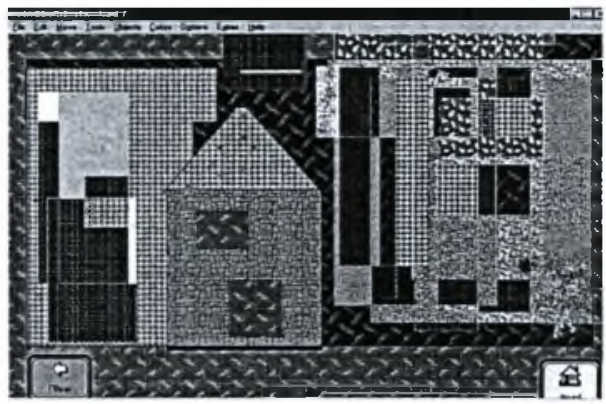

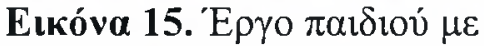

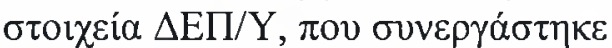
$\mu \varepsilon \dot{\alpha} \lambda \lambda \mathrm{o} \pi \alpha \mathrm{l} \delta$ í $\kappa \alpha \mathrm{\iota} \delta \varepsilon v \alpha \kappa \circ \lambda \circ u ́ \theta \eta \sigma \varepsilon$

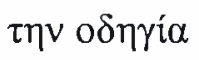

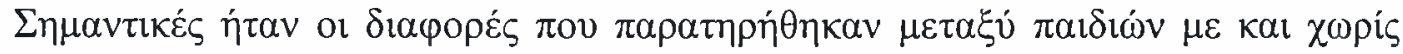

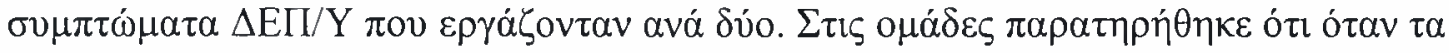

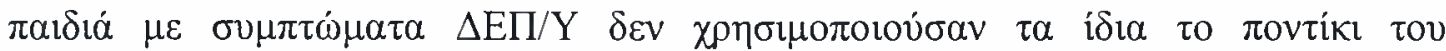

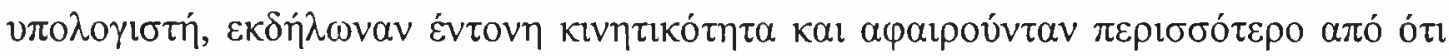

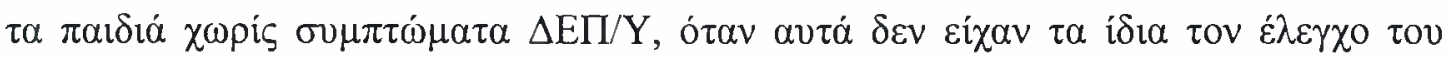

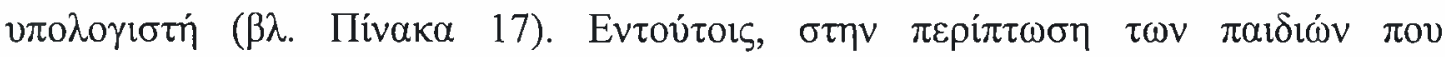

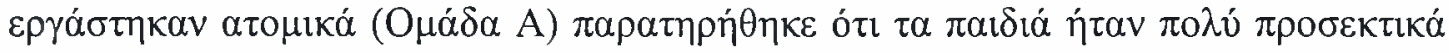

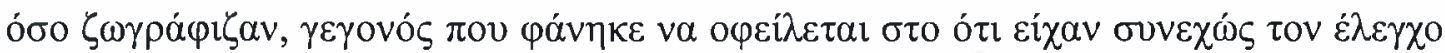

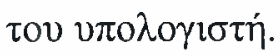

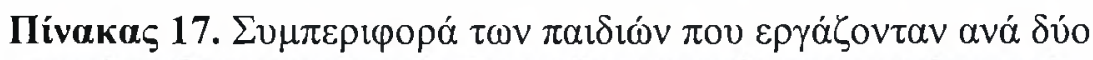

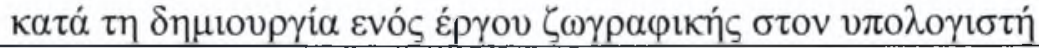

\begin{tabular}{|c|c|c|c|c|}
\hline \multirow{3}{*}{$\Sigma \tau 0 \iota \chi \varepsilon i ́ \alpha \sigma u \mu \pi \varepsilon \rho \iota \varphi 0 \rho \alpha ́ \varsigma$} & \multicolumn{4}{|c|}{ 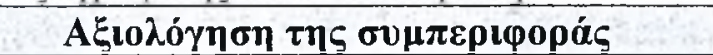 } \\
\hline & \multicolumn{2}{|c|}{ 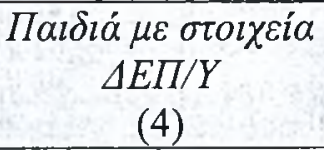 } & \multicolumn{2}{|c|}{ 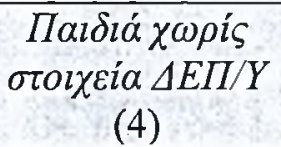 } \\
\hline & M.O. & T.A. & M.O. & T.A. \\
\hline 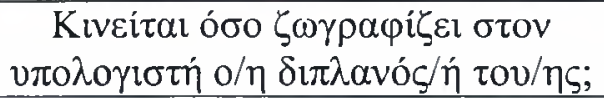 & 3,00 & 0,00 & 1,75 & 0,50 \\
\hline 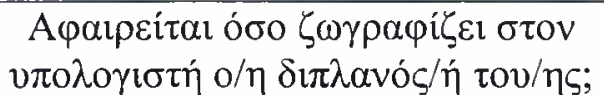 & 2,50 & 0,58 & 1,50 & 0,58 \\
\hline
\end{tabular}

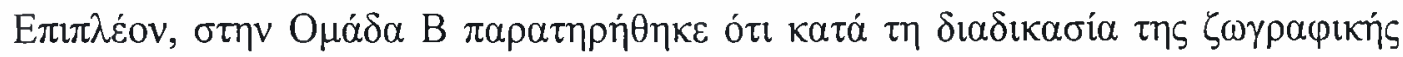

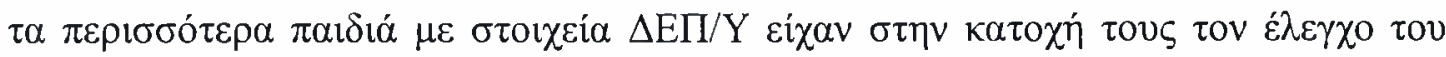

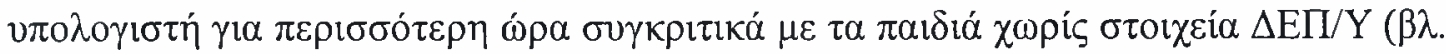

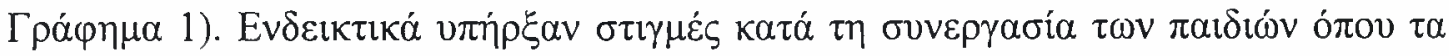

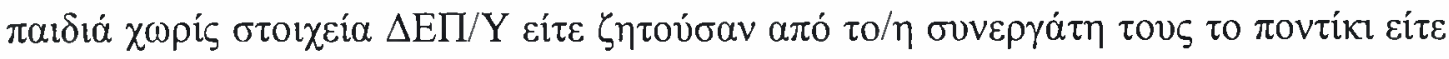




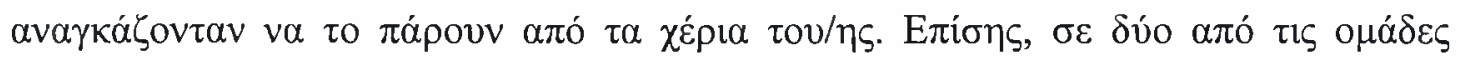

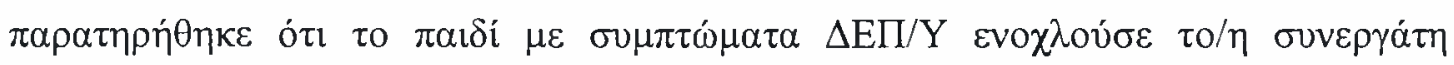

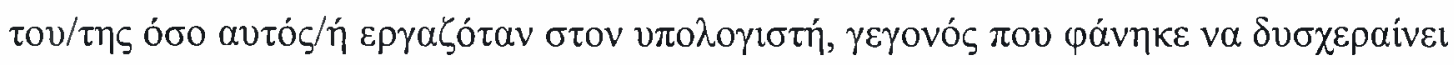

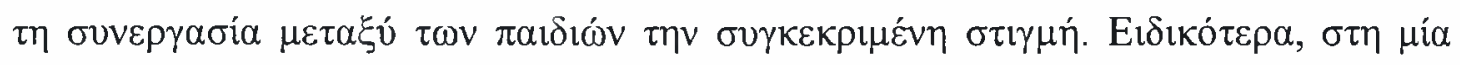

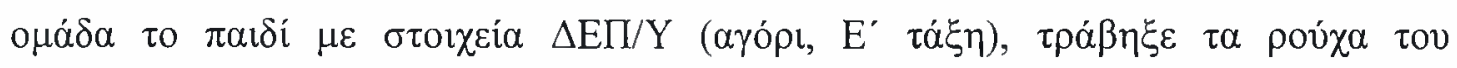

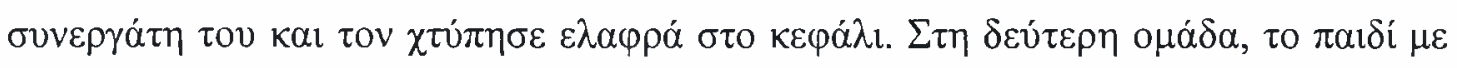

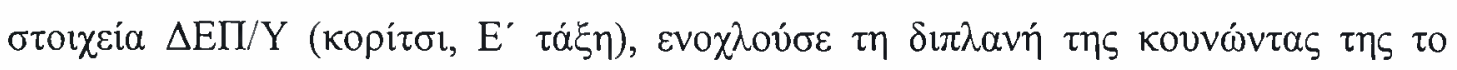

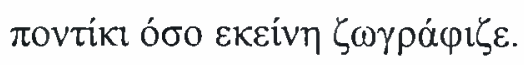

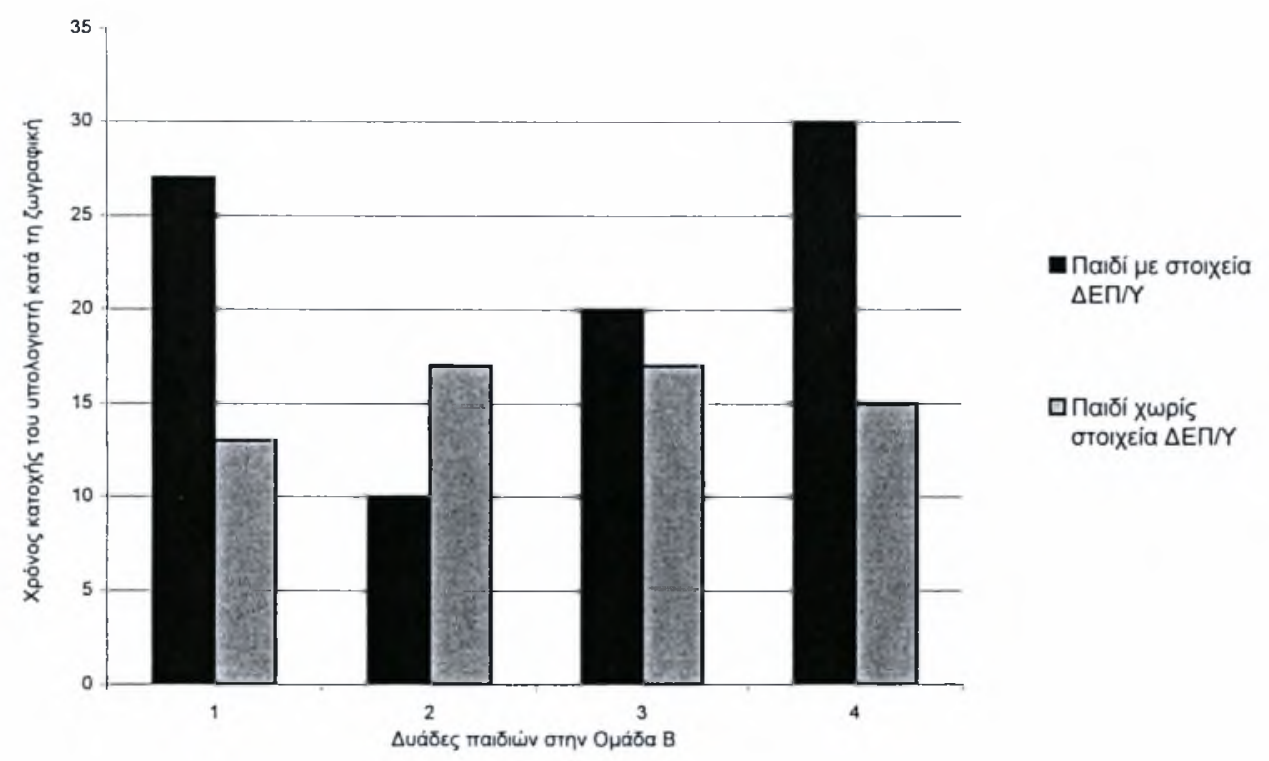

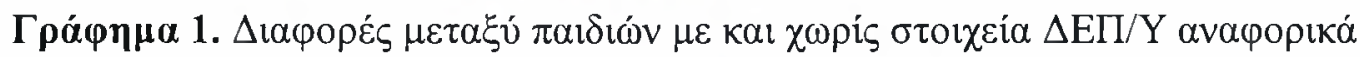

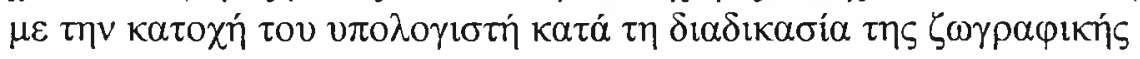

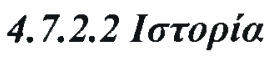

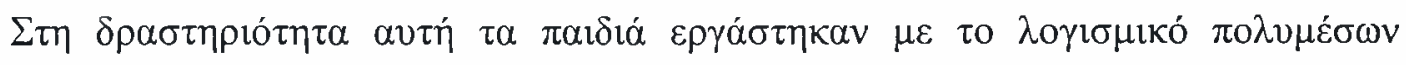

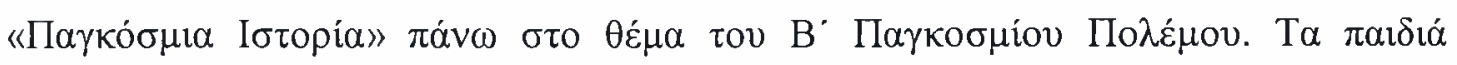

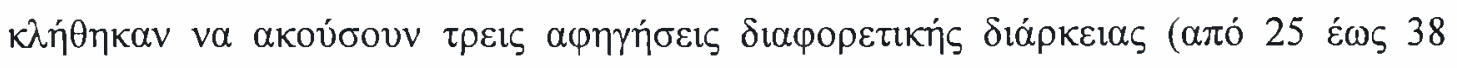

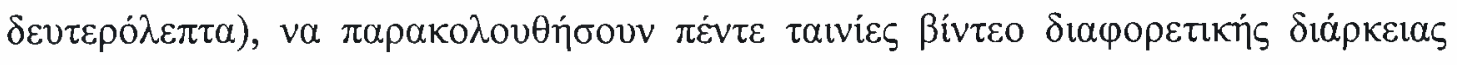

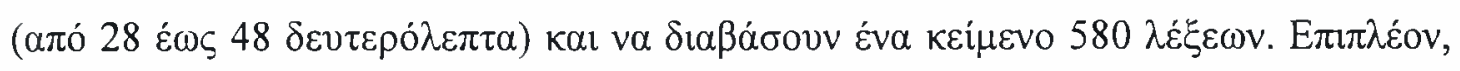

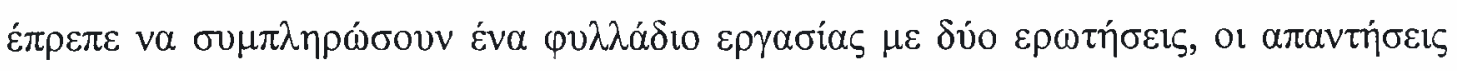

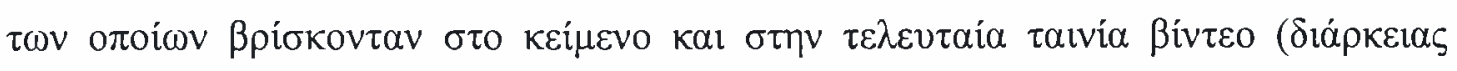

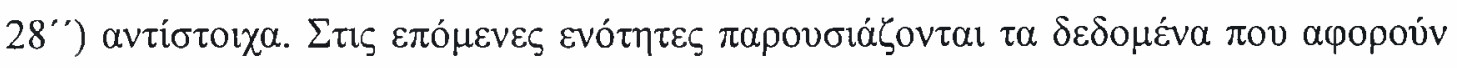

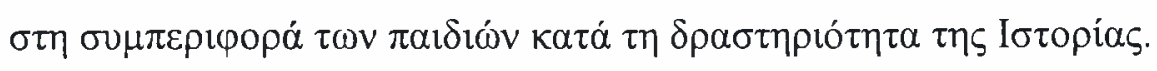




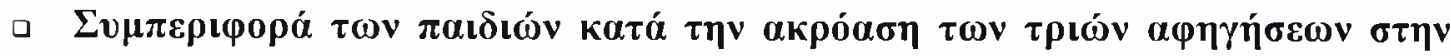

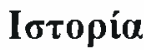

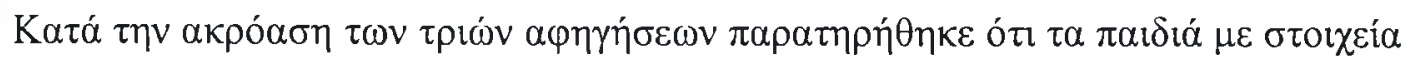

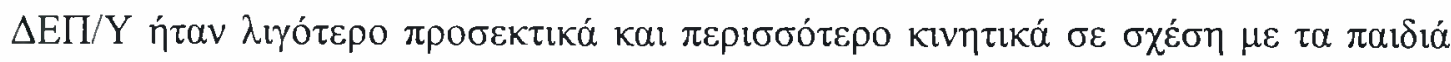

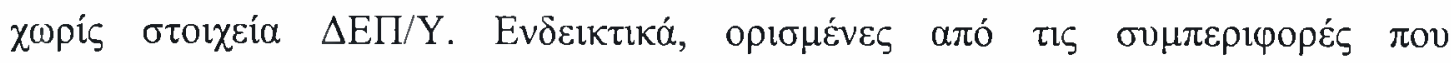

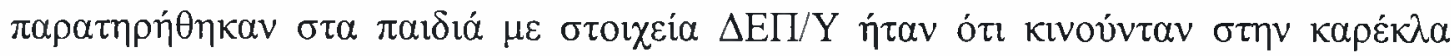

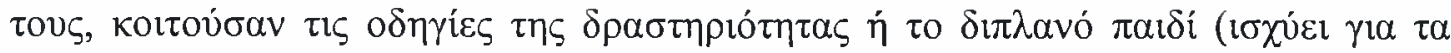

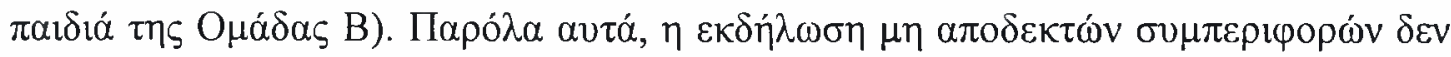

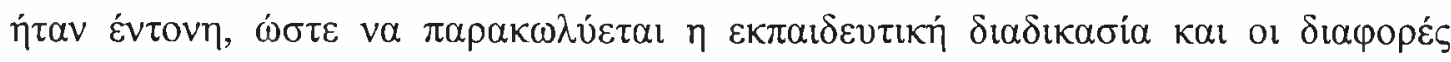

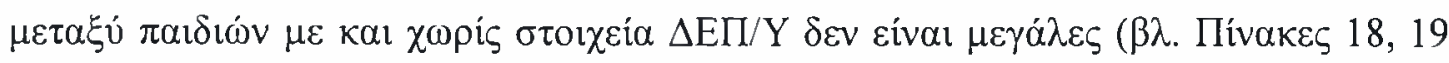
ка 20).

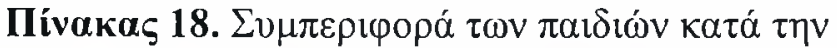
$\alpha \kappa \rho o ́ \alpha \sigma \eta \tau\rceil \zeta \alpha \varphi \eta ́ \gamma \eta \sigma \eta \zeta ~ 1 \sigma \tau \eta v \operatorname{I} \sigma \tau o \rho i \alpha\left(28^{\prime \prime}\right)$

\begin{tabular}{|c|c|c|c|c|}
\hline \multirow{3}{*}{ 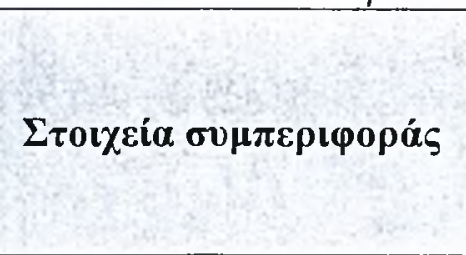 } & \multicolumn{4}{|c|}{ 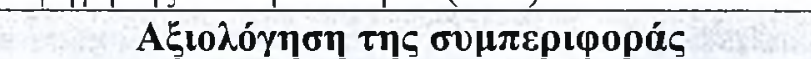 } \\
\hline & \multicolumn{2}{|c|}{ 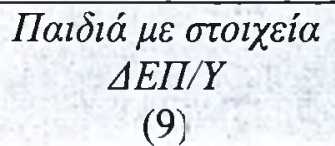 } & \multicolumn{2}{|c|}{ 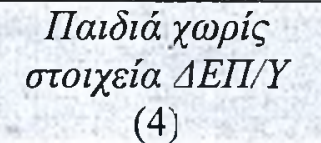 } \\
\hline & M.O. & T.A. & M.O. & T.A. \\
\hline 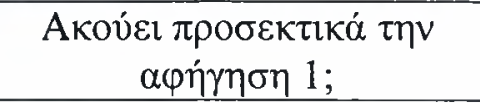 & 2,56 & 0,53 & 3,00 & 0,00 \\
\hline 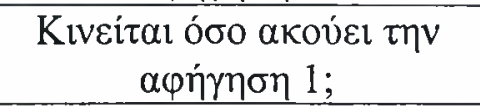 & 1,67 & 0,71 & 1,50 & 0,58 \\
\hline 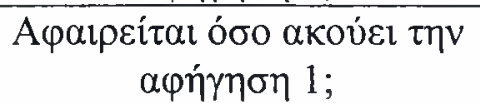 & 1,33 & 0,50 & 1,25 & 0,50 \\
\hline
\end{tabular}

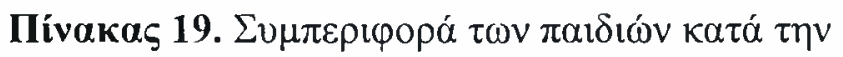

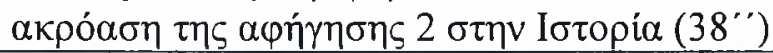

\begin{tabular}{|c|c|c|c|c|}
\hline \multirow{3}{*}{ 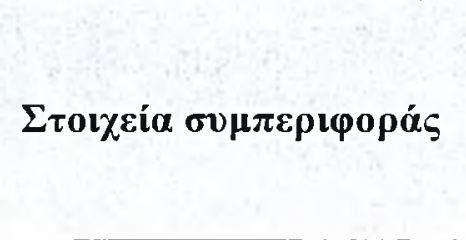 } & \multicolumn{4}{|c|}{ 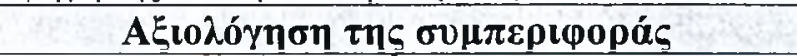 } \\
\hline & \multicolumn{2}{|c|}{ 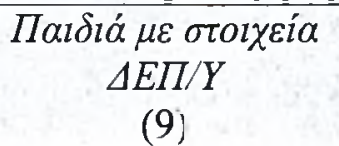 } & \multicolumn{2}{|c|}{ 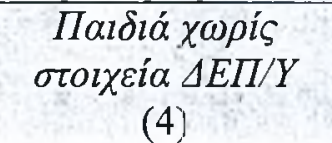 } \\
\hline & M.O. & T.A. & M.O. & T.A. \\
\hline 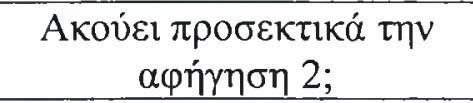 & 2,56 & 0,53 & 2,75 & 0,50 \\
\hline 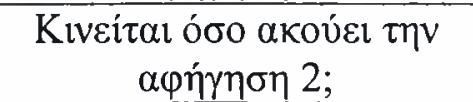 & 1,44 & 0,73 & 1,25 & 0,50 \\
\hline 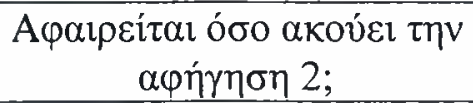 & 1,44 & 0,53 & 1,25 & 0,50 \\
\hline
\end{tabular}




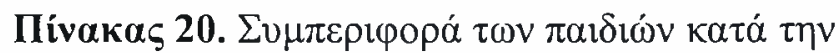

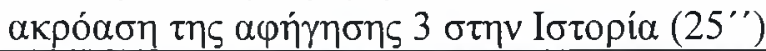

\begin{tabular}{|c|c|c|c|c|}
\hline \multirow{3}{*}{$\Sigma \tau 0 \iota \chi \varepsilon i ́ \alpha \sigma v \mu \pi \varepsilon \rho \iota \varphi 0 \rho \alpha ́ \varsigma$} & \multicolumn{4}{|c|}{ 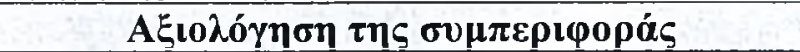 } \\
\hline & \multicolumn{2}{|c|}{ 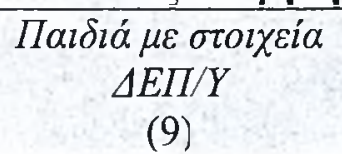 } & \multicolumn{2}{|c|}{ 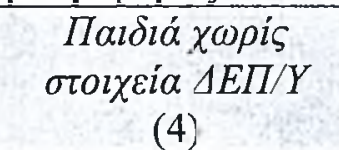 } \\
\hline & M.O. & T.A. & M.O. & T.A. \\
\hline 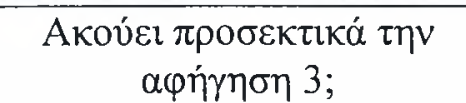 & 2,00 & 0,71 & 2,50 & 0,58 \\
\hline 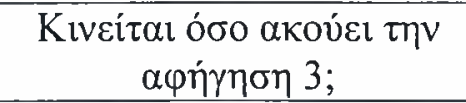 & 2,00 & 0,71 & 1,50 & 0,58 \\
\hline 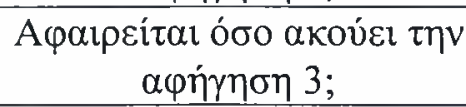 & 1,67 & 0,71 & 1,25 & 0,50 \\
\hline
\end{tabular}

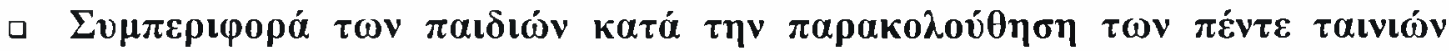

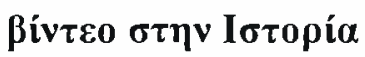

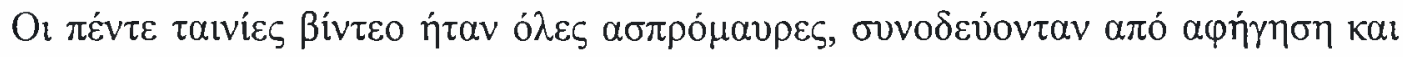

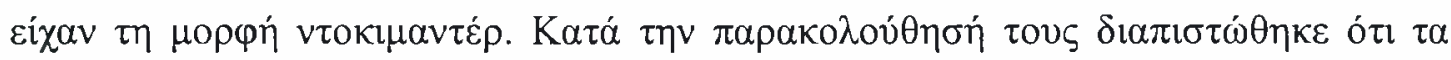

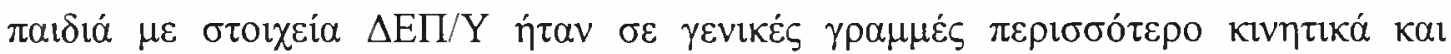

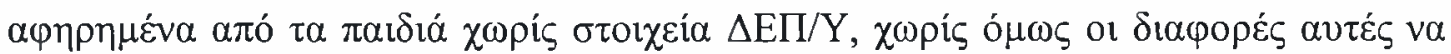

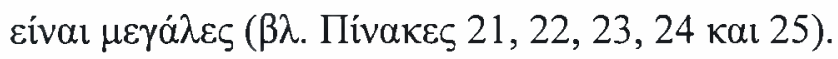

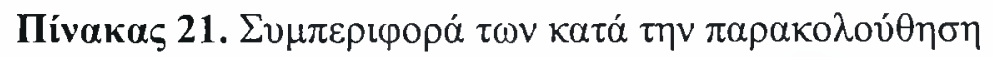

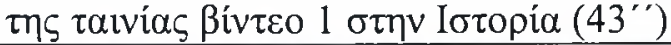

\begin{tabular}{|c|c|c|c|c|}
\hline \multirow{3}{*}{ 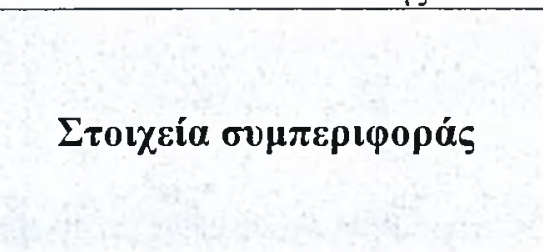 } & \multicolumn{4}{|c|}{ 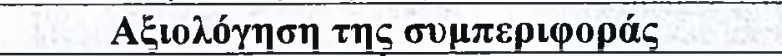 } \\
\hline & \multicolumn{2}{|c|}{ 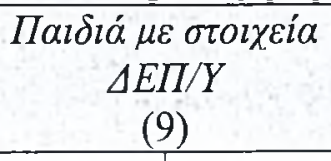 } & \multicolumn{2}{|c|}{ 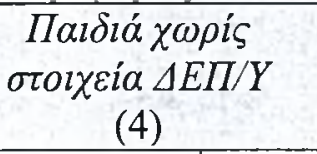 } \\
\hline & M.O. & T.A. & M.O. & T.A. \\
\hline 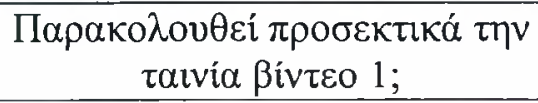 & 2,44 & 0,53 & 2,75 & 0,50 \\
\hline 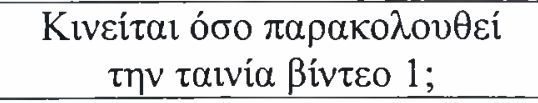 & 1,33 & 0,50 & 1,25 & 0,50 \\
\hline 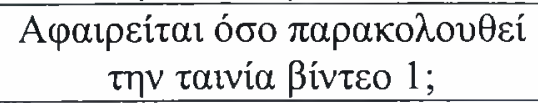 & 1,67 & 0,50 & 1,25 & 0,50 \\
\hline
\end{tabular}

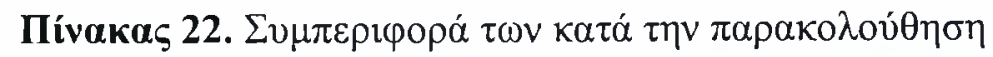

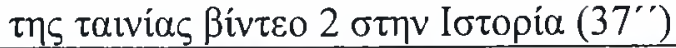

\begin{tabular}{|c|c|c|c|c|}
\hline \multirow{3}{*}{ 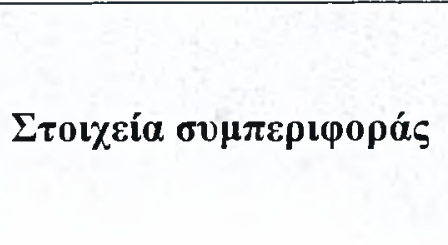 } & \multicolumn{4}{|c|}{ 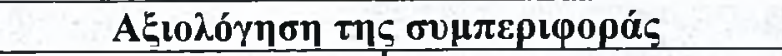 } \\
\hline & $\Pi \alpha \imath \delta l c$ & 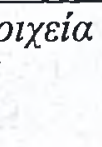 & $\begin{array}{r}\Pi \alpha \downarrow \delta \\
\sigma \tau o\llcorner\chi \varepsilon\end{array}$ & \\
\hline & M.O. & T.A. & M.O. & T.A. \\
\hline
\end{tabular}




\begin{tabular}{|c|c|c|c|c|}
\hline 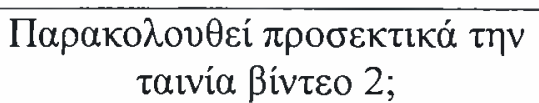 & 2,89 & 0,33 & 2,75 & 0,50 \\
\hline 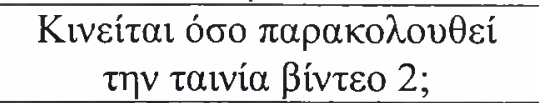 & 1,44 & 0,53 & 1,00 & 0,00 \\
\hline 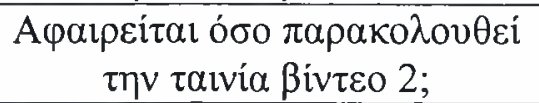 & $1,0 \dot{0}$ & 0,00 & 1,25 & 0,50 \\
\hline
\end{tabular}

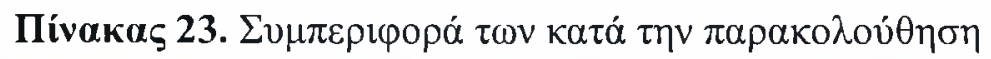

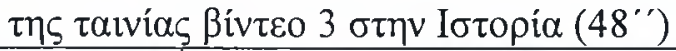

\begin{tabular}{|c|c|c|c|c|}
\hline \multirow{3}{*}{ 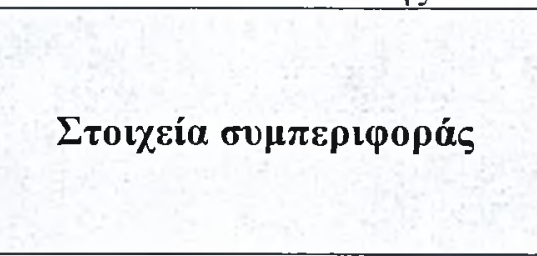 } & \multicolumn{4}{|c|}{ 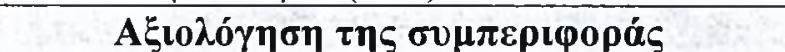 } \\
\hline & \multicolumn{2}{|c|}{$\begin{array}{c}\Pi \alpha \iota \delta \imath \alpha \dot{\alpha} \mu \sigma \tau o \imath \chi \varepsilon i ́ a \\
\Delta E \Pi / Y \\
(9)\end{array}$} & \multicolumn{2}{|c|}{ 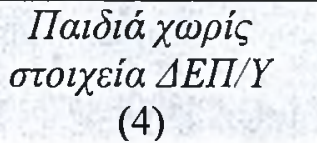 } \\
\hline & M.O. & T.A. & M.O. & T.A. \\
\hline 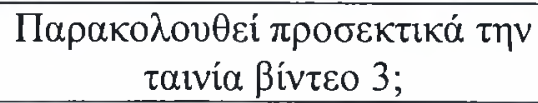 & 2,56 & 0,53 & 2,75 & 0,50 \\
\hline 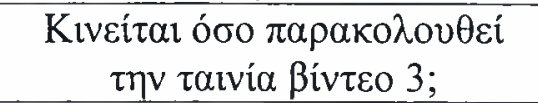 & 1,56 & 0,53 & 1,25 & 0,50 \\
\hline 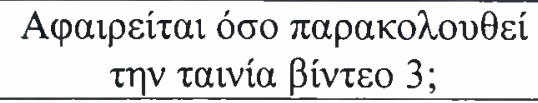 & 1,44 & 0,53 & 1,25 & 0,50 \\
\hline
\end{tabular}

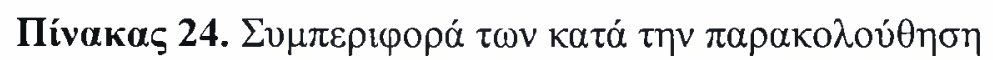

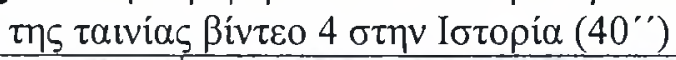

\begin{tabular}{|c|c|c|c|c|}
\hline \multirow{3}{*}{ 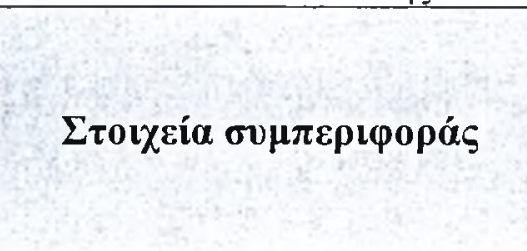 } & \multicolumn{4}{|c|}{ 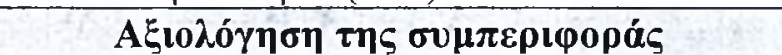 } \\
\hline & \multicolumn{2}{|c|}{ 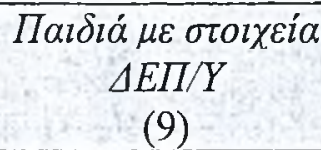 } & \multicolumn{2}{|c|}{ 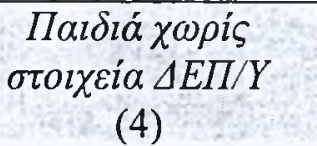 } \\
\hline & M.O. & T.A. & M.O. & T.A. \\
\hline 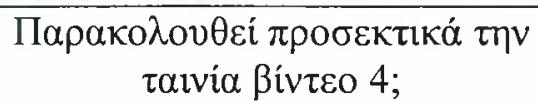 & 2,67 & 0,50 & 3,00 & 0,00 \\
\hline 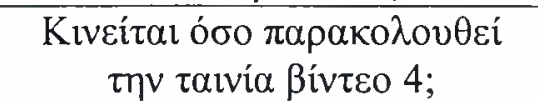 & 1,44 & 0,53 & 1,25 & 0,50 \\
\hline 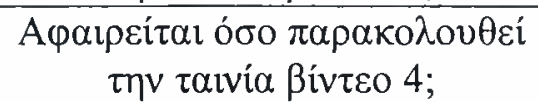 & 1,22 & 0,44 & 1,00 & 0,00 \\
\hline
\end{tabular}

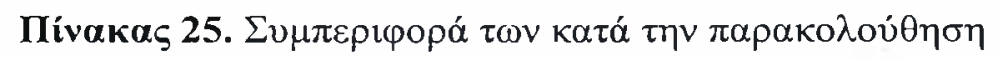

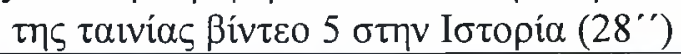

\begin{tabular}{|c|c|c|c|c|}
\hline \multirow{3}{*}{ 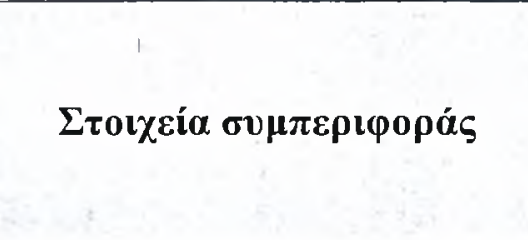 } & \multicolumn{4}{|c|}{ 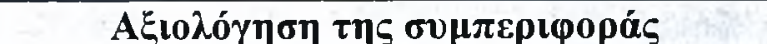 } \\
\hline & \multicolumn{2}{|c|}{ 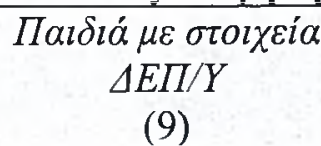 } & \multicolumn{2}{|c|}{$\begin{array}{c}\Pi \alpha \imath \delta \imath \alpha \dot{\alpha} \chi \omega \rho i \zeta \\
\sigma \tau o \imath \chi \varepsilon i ́ \alpha \Delta E \Pi / Y \\
\text { (4) }\end{array}$} \\
\hline & M.O. & T.A. & M.O. & T.A. \\
\hline 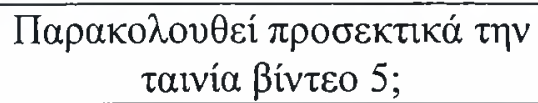 & 2,44 & 0,53 & 2,75 & 0,50 \\
\hline 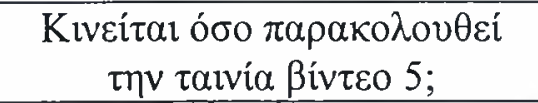 & 1,33 & 0,50 & 1,00 & 0,00 \\
\hline 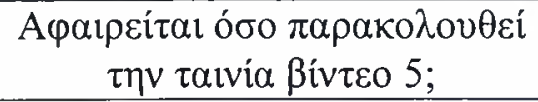 & 1,22 & 0,44 & 1,00 & 0,00 \\
\hline
\end{tabular}




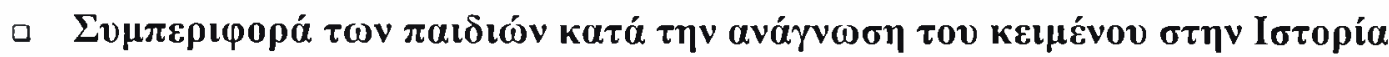

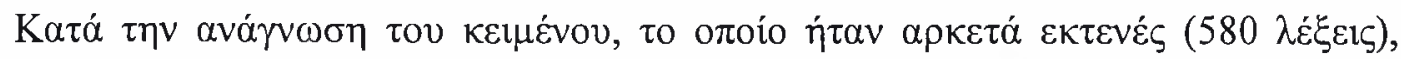

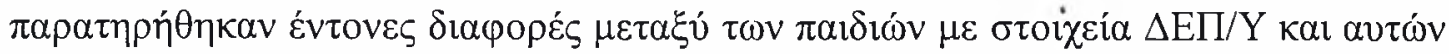

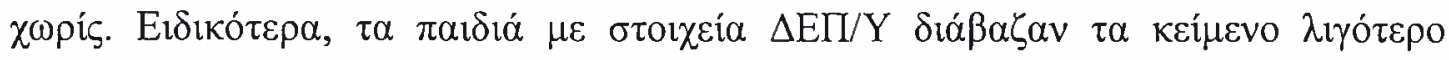

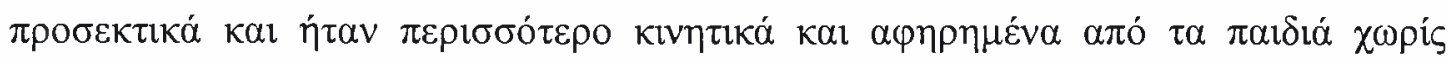

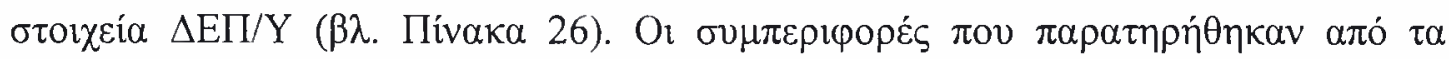

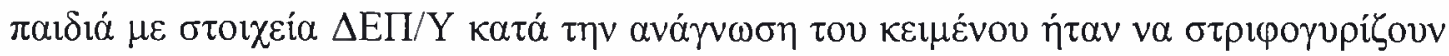

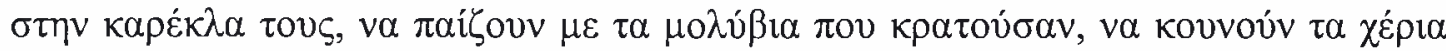

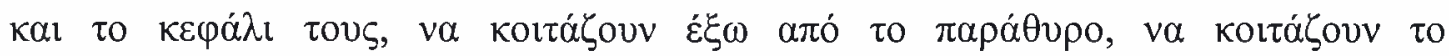

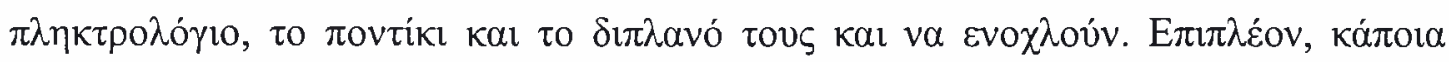

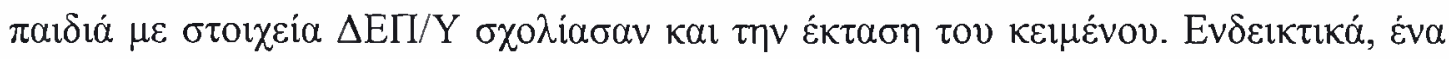

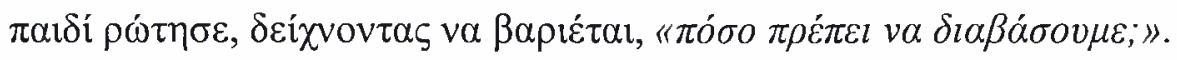

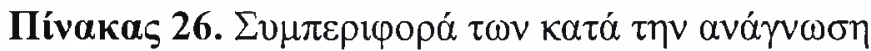

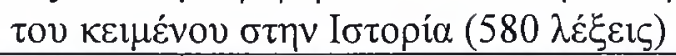

\begin{tabular}{|c|c|c|c|c|}
\hline \multirow{3}{*}{ 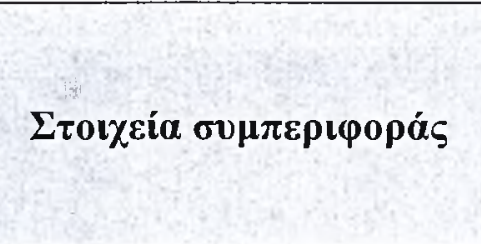 } & \multicolumn{4}{|c|}{ 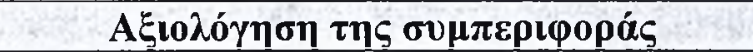 } \\
\hline & \multicolumn{2}{|c|}{ 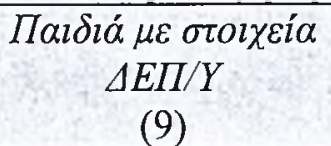 } & \multicolumn{2}{|c|}{ 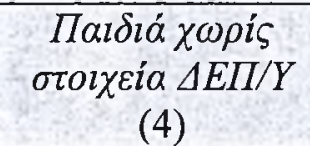 } \\
\hline & M.O. & T.A. & M.O. & T.A. \\
\hline 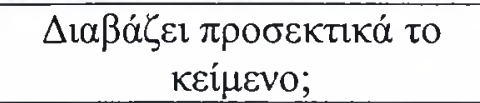 & 1,89 & 0,60 & 2,25 & 0,96 \\
\hline 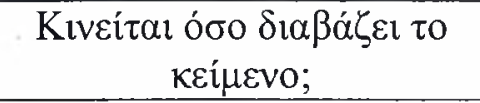 & 2,56 & 0,73 & 1,25 & 0,50 \\
\hline 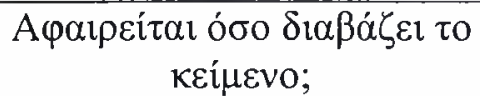 & 2,11 & 0,78 & 1,25 & 0,50 \\
\hline
\end{tabular}

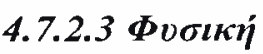

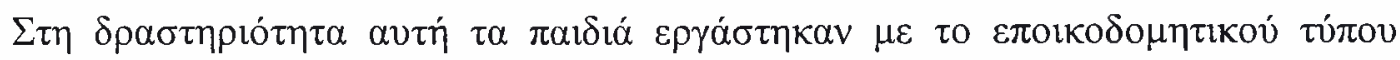
$\lambda$

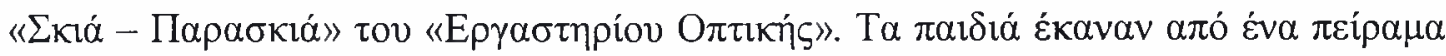

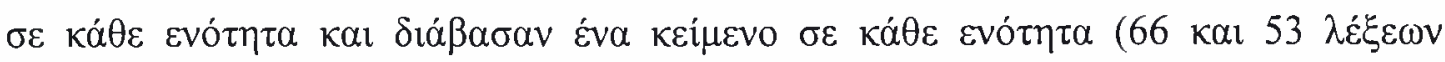

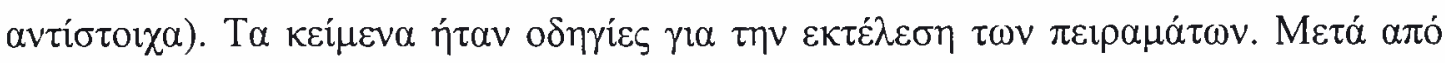

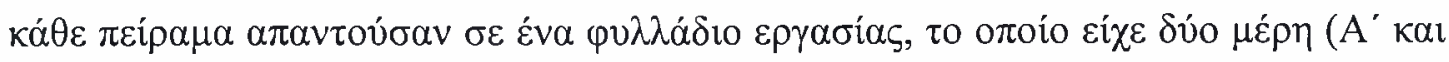

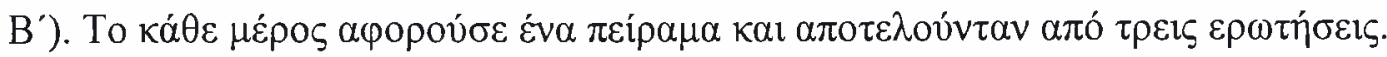




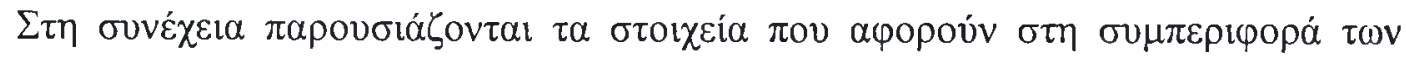

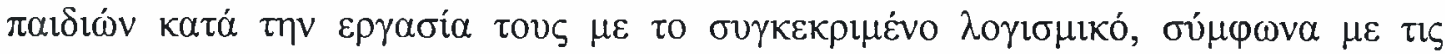

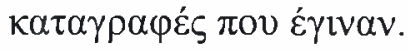

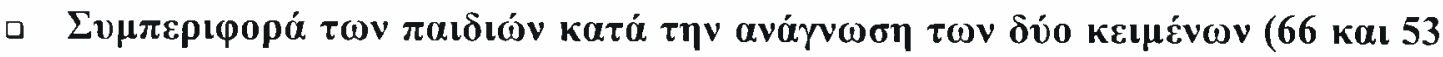

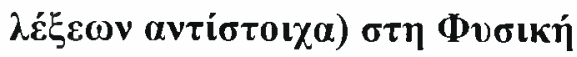

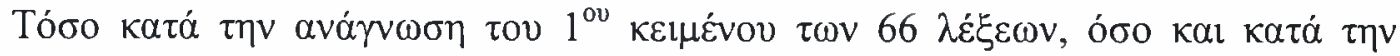

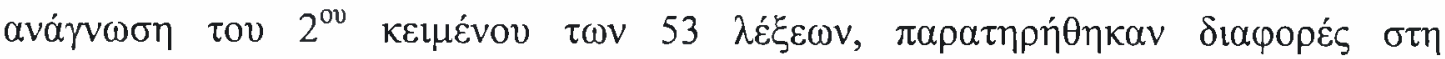

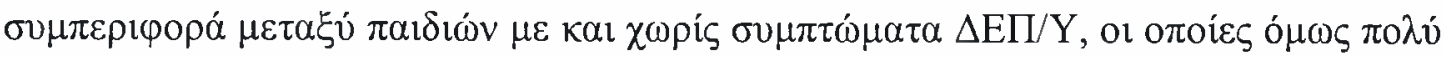

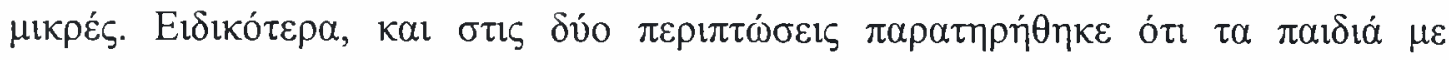

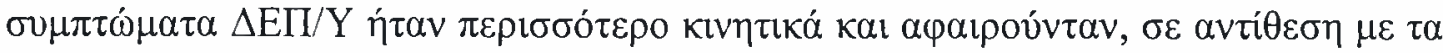

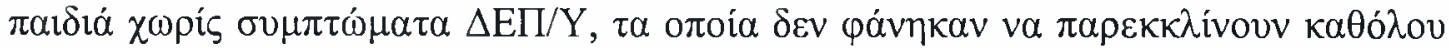

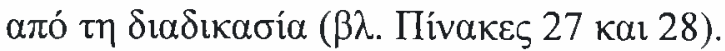

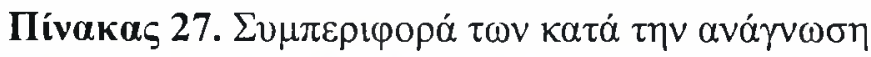

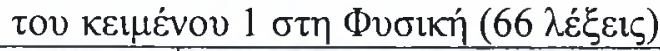

\begin{tabular}{|c|c|c|c|c|}
\hline \multirow{3}{*}{ 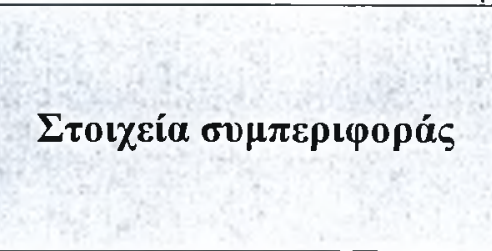 } & \multicolumn{4}{|c|}{ 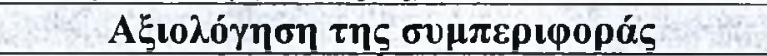 } \\
\hline & \multicolumn{2}{|c|}{$\begin{array}{c}\Pi \alpha \iota \delta ı \alpha \text { } \mu \varepsilon \sigma \tau o \iota \chi \varepsilon i \alpha \\
\Delta E \Pi / Y \\
(9)\end{array}$} & \multicolumn{2}{|c|}{ 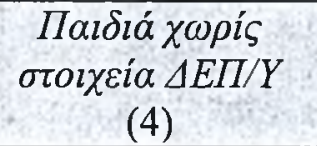 } \\
\hline & M.O. & T.A. & M.O. & T.A. \\
\hline 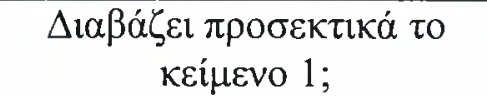 & 2,67 & 0,50 & 3,00 & 0,00 \\
\hline 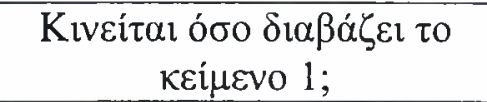 & 1,33 & 0,50 & 1,00 & 0,00 \\
\hline 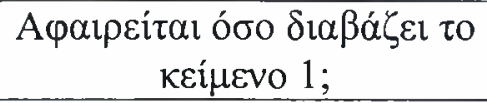 & 1,22 & 0,44 & 1,00 & 0,00 \\
\hline
\end{tabular}

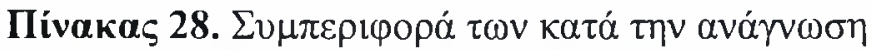

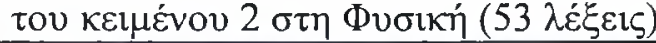

\begin{tabular}{|c|c|c|c|c|}
\hline \multirow{3}{*}{ 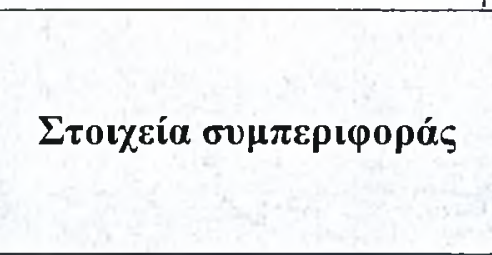 } & \multicolumn{4}{|c|}{ 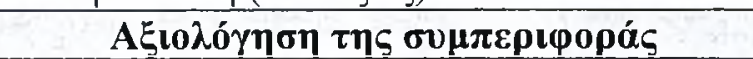 } \\
\hline & \multicolumn{2}{|c|}{$\begin{array}{c}\Pi \alpha \imath \delta \imath \alpha \mu \varepsilon \sigma \tau o \imath \chi \varepsilon i \alpha \\
\Delta E \Pi / Y \\
(9)\end{array}$} & \multicolumn{2}{|c|}{ 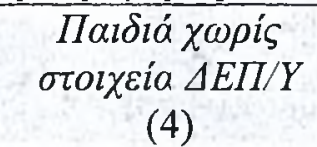 } \\
\hline & M.O. & T.A. & M.O. & T.A. \\
\hline 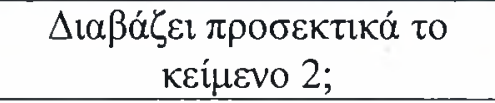 & 2,78 & 0,44 & 3,00 & 0,00 \\
\hline 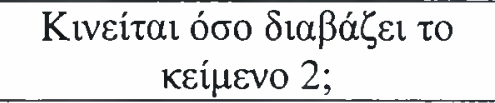 & 1,33 & 0,50 & 1,00 & 0,00 \\
\hline 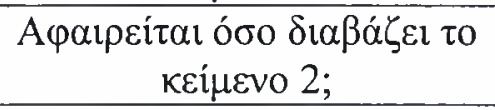 & 1,22 & 0,44 & 1,00 & 0,00 \\
\hline
\end{tabular}




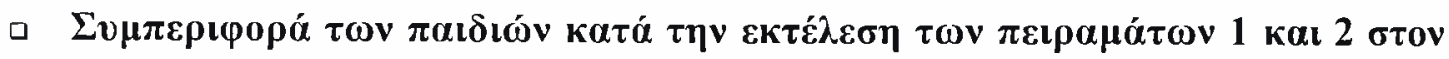 vлодо}

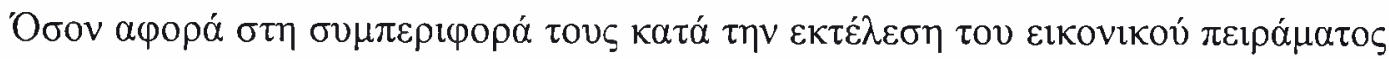

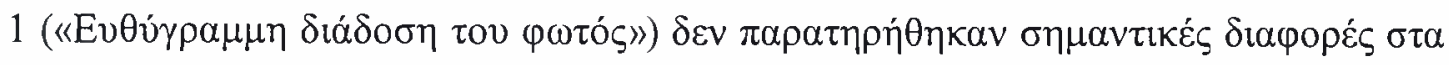

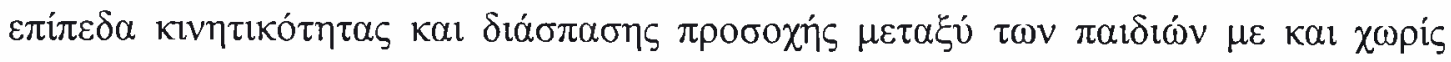

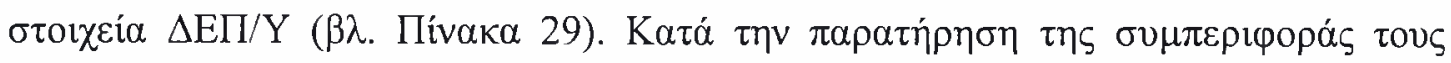

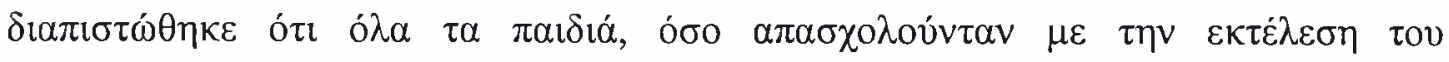

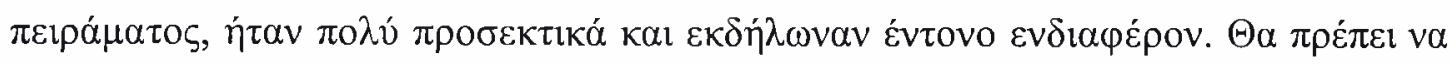

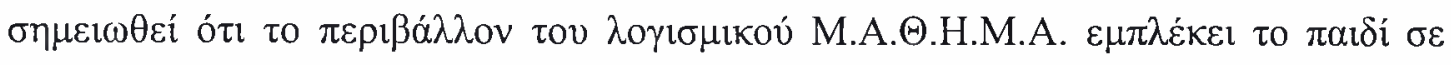

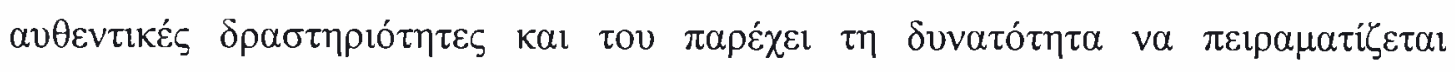

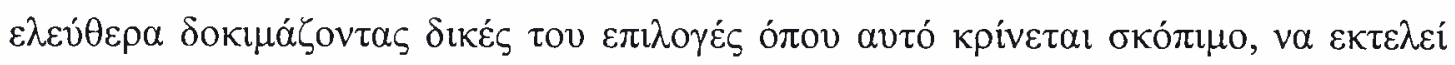

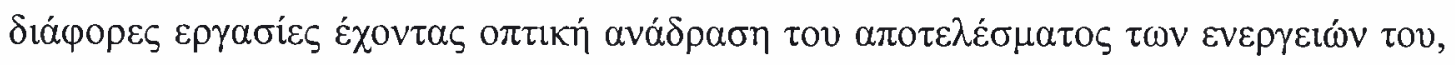

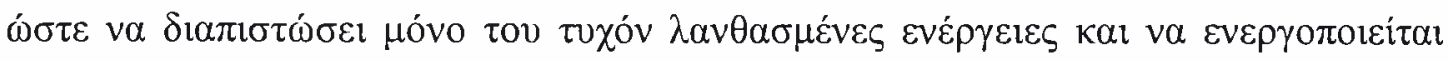

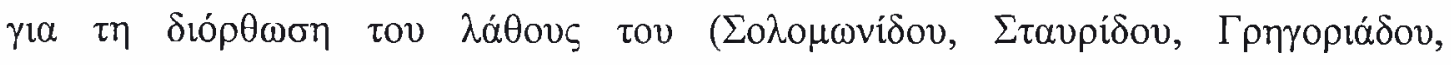

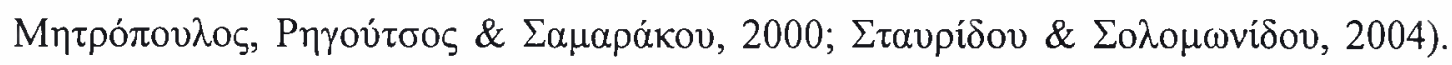

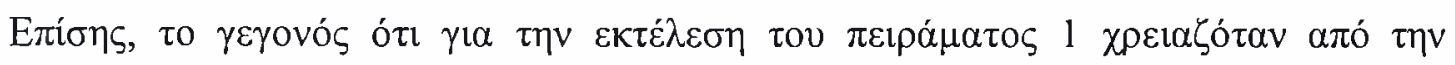

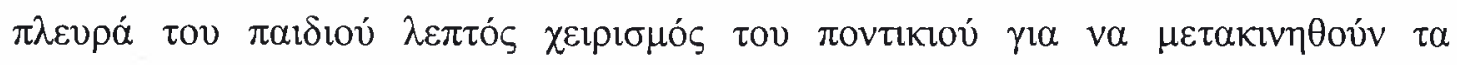

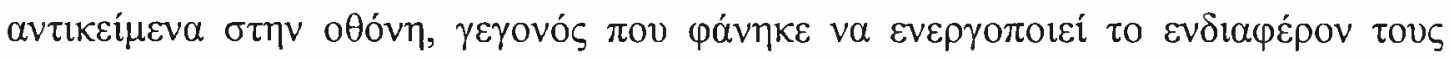

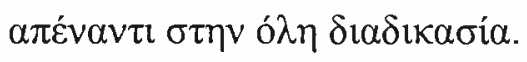

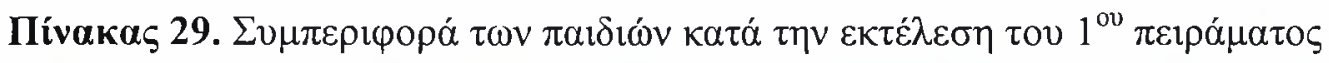

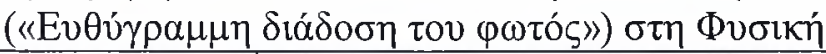

\begin{tabular}{|c|c|c|c|c|}
\hline \multirow{3}{*}{ 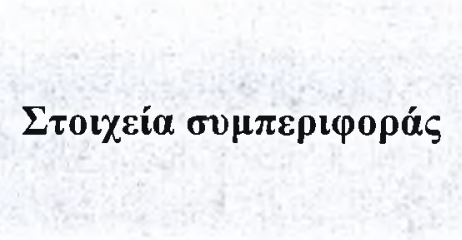 } & \multicolumn{4}{|c|}{ 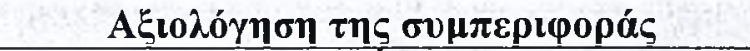 } \\
\hline & \multicolumn{2}{|c|}{ 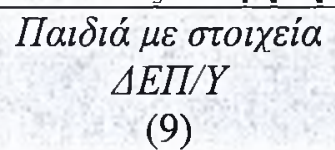 } & \multicolumn{2}{|c|}{ 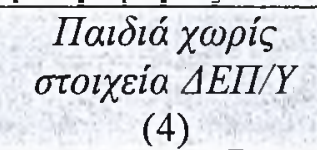 } \\
\hline & M.O. & T.A. & M.O. & T.A. \\
\hline 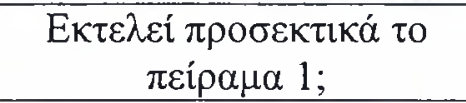 & 3,00 & 0,00 & 3,00 & 0,00 \\
\hline 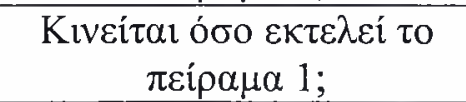 & 1,11 & 0,33 & 1,00 & 0,00 \\
\hline 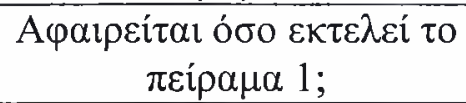 & 1,00 & 0,00 & 1,00 & 0,00 \\
\hline
\end{tabular}

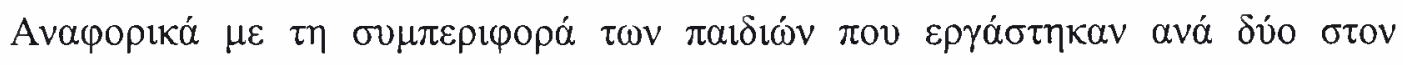

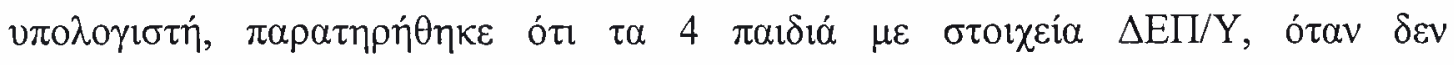

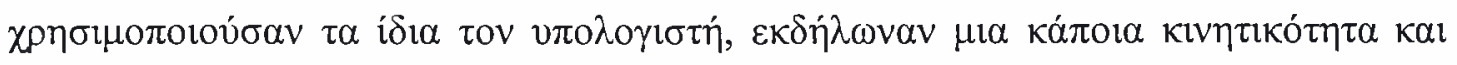




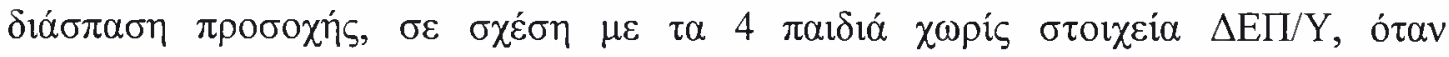

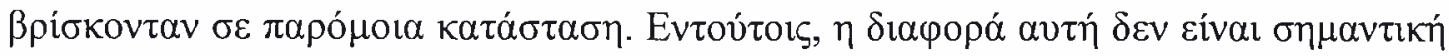

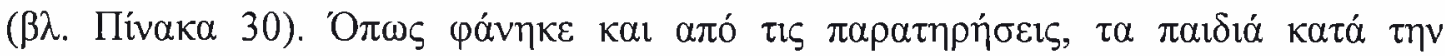

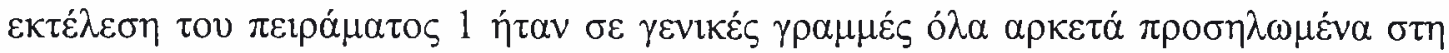

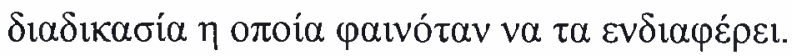

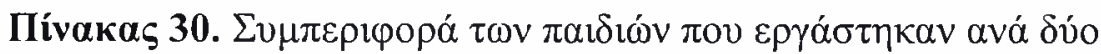

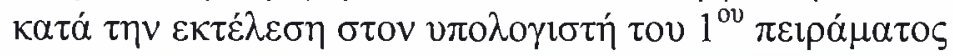

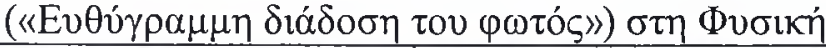

\begin{tabular}{|c|c|c|c|c|}
\hline \multirow{3}{*}{ 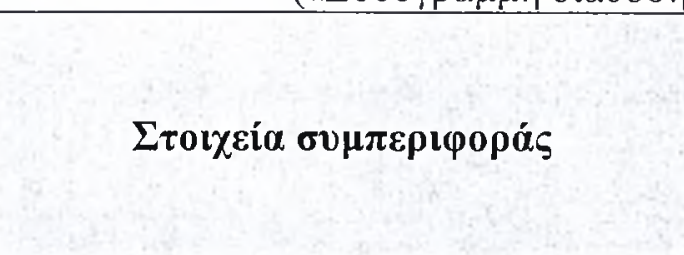 } & \multicolumn{4}{|c|}{ 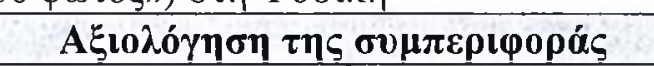 } \\
\hline & \multicolumn{2}{|c|}{ 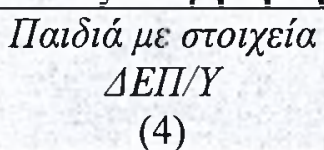 } & \multicolumn{2}{|c|}{ 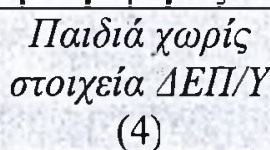 } \\
\hline & M.O. & T.A. & M.O. & T.A. \\
\hline 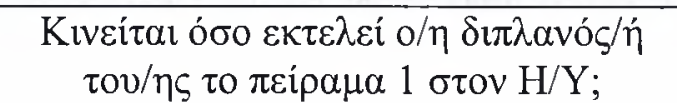 & 1,25 & 0,50 & 1,00 & 0,00 \\
\hline 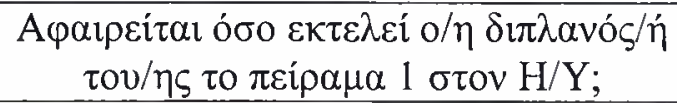 & 1,25 & 0,50 & 1,00 & 0,00 \\
\hline
\end{tabular}

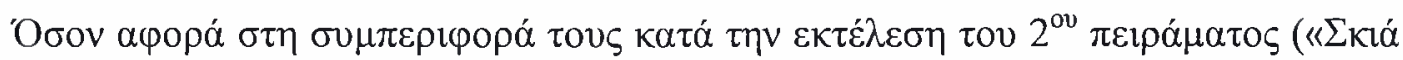

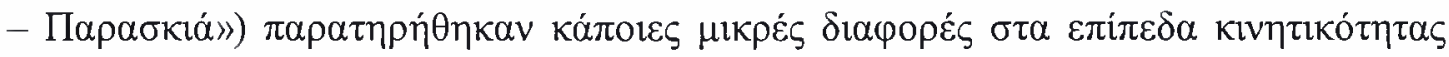

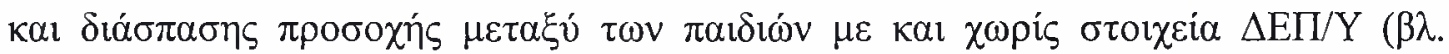

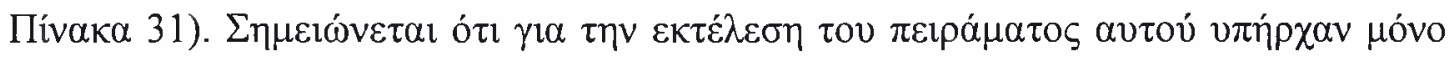

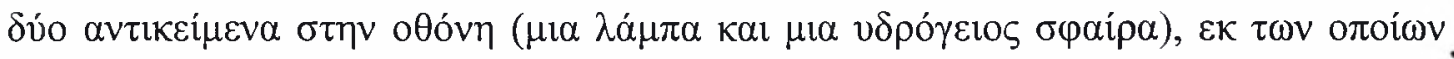

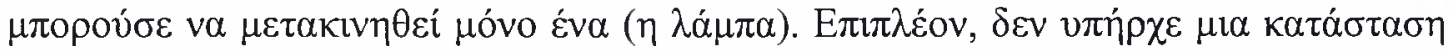

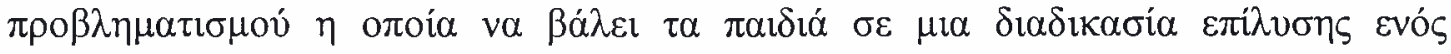

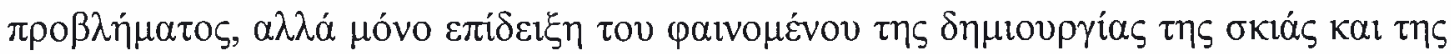

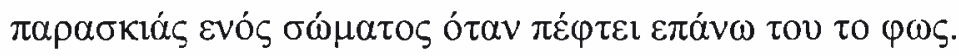

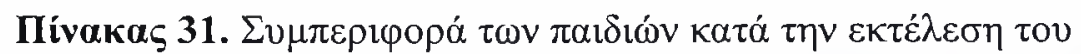

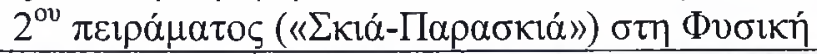

\begin{tabular}{|c|c|c|c|c|}
\hline \multirow{3}{*}{ 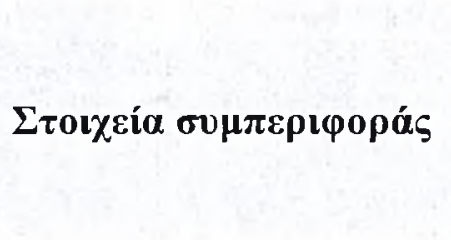 } & \multicolumn{4}{|c|}{ 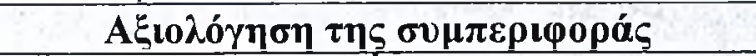 } \\
\hline & \multicolumn{2}{|c|}{$\begin{array}{c}\Pi \alpha \iota \delta \imath \dot{\alpha} \mu \varepsilon \sigma \tau o l \chi \varepsilon i \alpha \\
\Delta E \Pi / Y \\
\text { (9) }\end{array}$} & \multicolumn{2}{|c|}{ 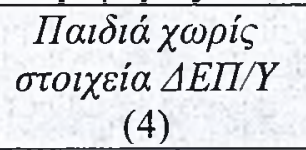 } \\
\hline & M.O. & T.A. & M.O. & T.A. \\
\hline 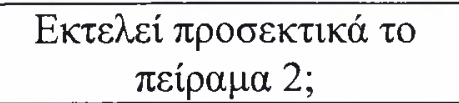 & 2,67 & 0,50 & 3,00 & 0,00 \\
\hline 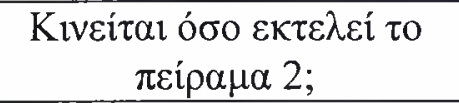 & 1,33 & 0,50 & 1,00 & 0,00 \\
\hline 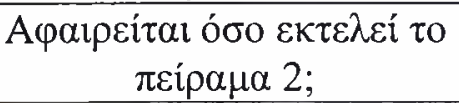 & 1,22 & 0,44 & 1,00 & 0,00 \\
\hline
\end{tabular}




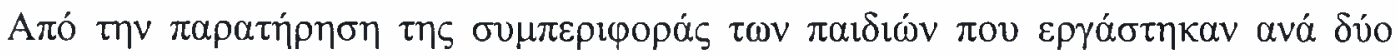

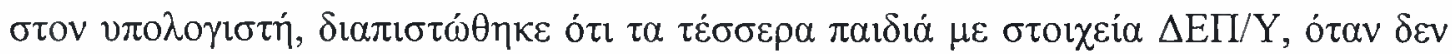

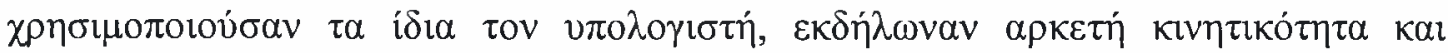

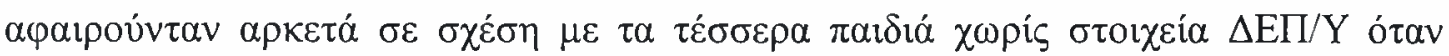

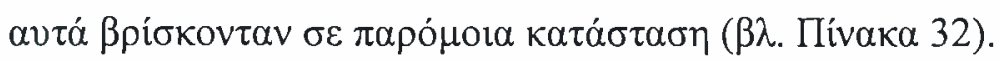

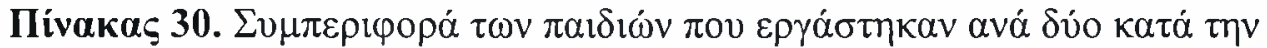

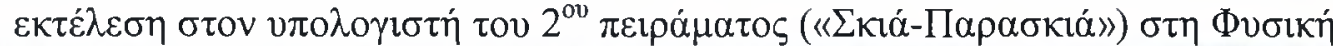

\begin{tabular}{|c|c|c|c|c|}
\hline \multirow{3}{*}{ 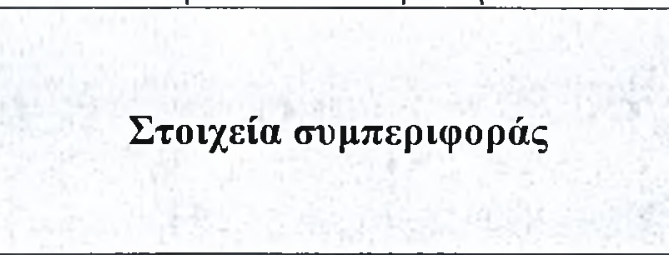 } & \multicolumn{4}{|c|}{ 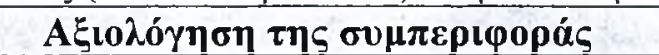 } \\
\hline & \multicolumn{2}{|c|}{ 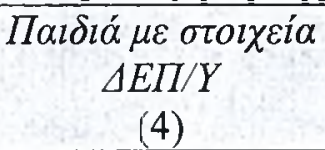 } & \multicolumn{2}{|c|}{ 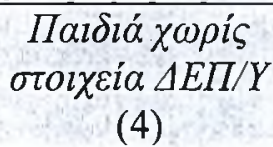 } \\
\hline & M.O. & T.A. & M.O. & T.A. \\
\hline 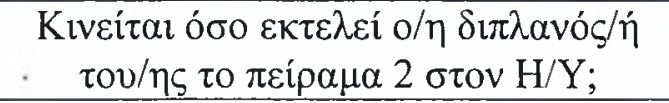 & 1,75 & 0,50 & 1,00 & 0,00 \\
\hline 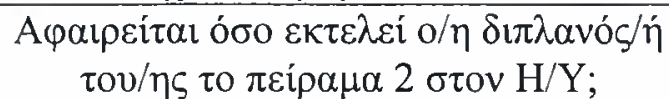 & 1,50 & 0,58 & 1,00 & 0,00 \\
\hline
\end{tabular}

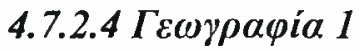

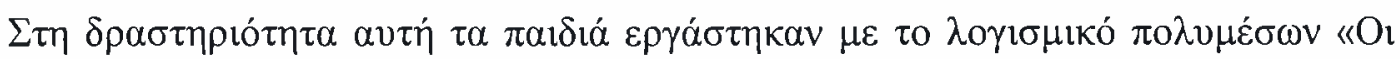

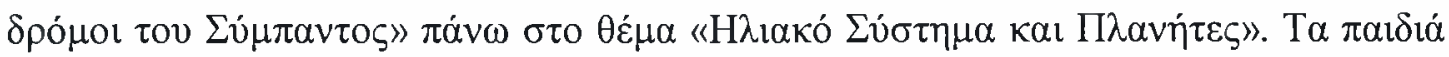

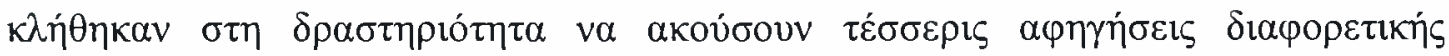

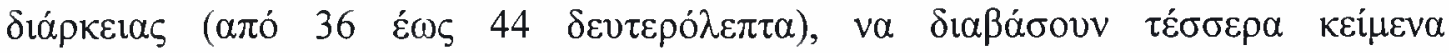

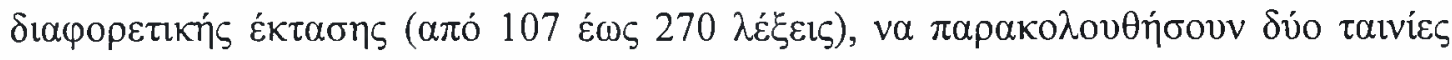

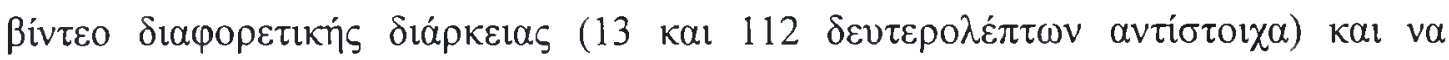

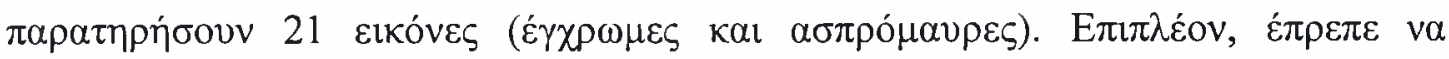

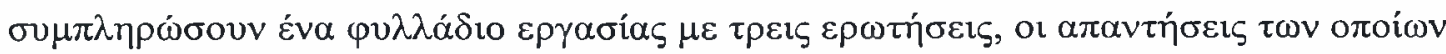

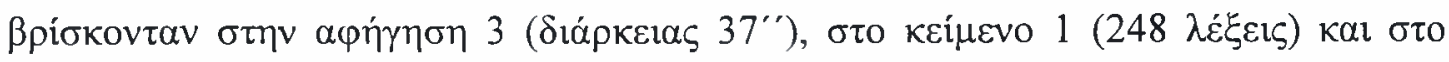

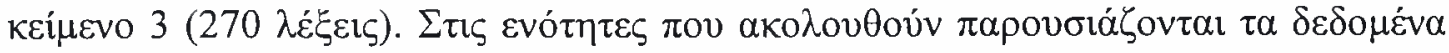

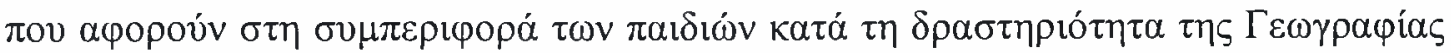
1.

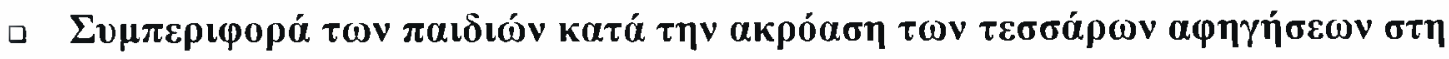

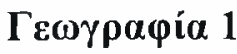

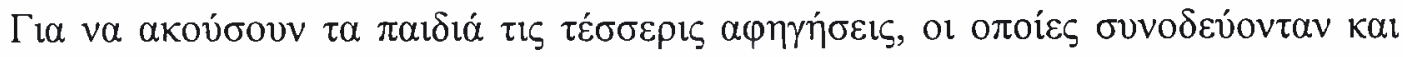

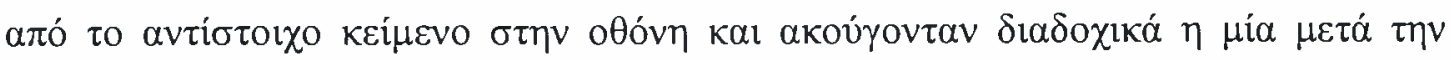




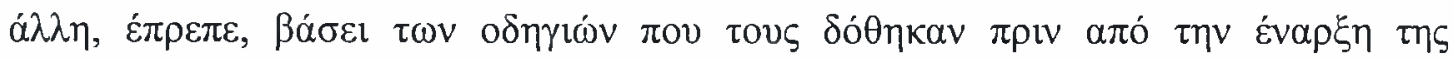

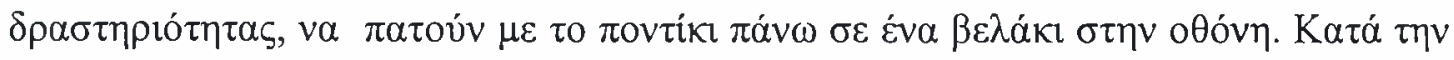

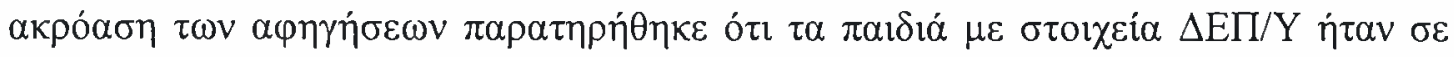

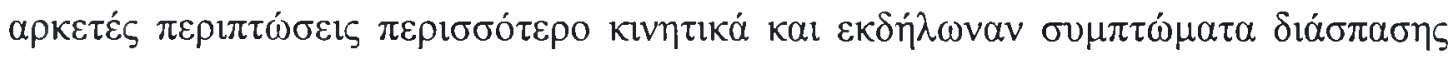

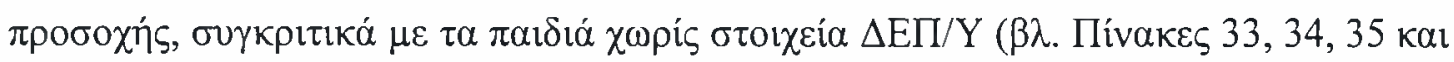
36).

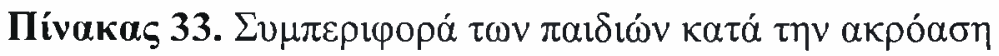

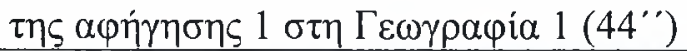

\begin{tabular}{|c|c|c|c|c|}
\hline \multirow{3}{*}{ 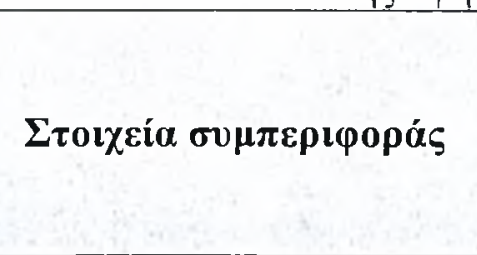 } & \multicolumn{4}{|c|}{ 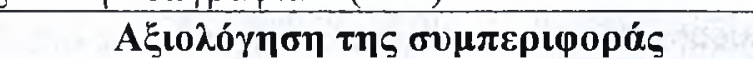 } \\
\hline & \multicolumn{2}{|c|}{$\begin{array}{c}\text { Паı } \delta \imath \alpha \dot{\alpha} \mu \varepsilon \sigma \tau o l \chi \varepsilon i \alpha \\
\Delta E \Pi / Y \\
(9)\end{array}$} & \multicolumn{2}{|c|}{ 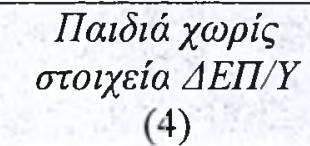 } \\
\hline & M.O. & T.A. & M.O. & T.A. \\
\hline 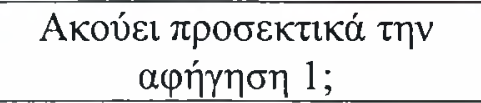 & 2,56 & 0,53 & 3,00 & 0,00 \\
\hline 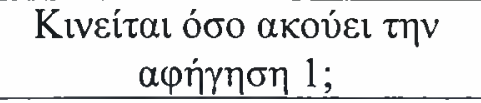 & 1,67 & 0,71 & 1,25 & 0,50 \\
\hline 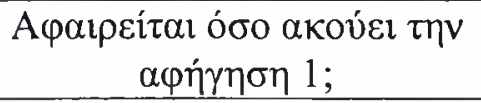 & 1,44 & 0,53 & 1,00 & 0,00 \\
\hline
\end{tabular}

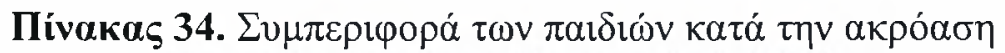
$\tau \eta \varsigma \alpha \varphi \eta \dot{\gamma \eta \sigma \eta \varsigma} 2 \sigma \tau \eta \Gamma \varepsilon \omega \gamma \rho \alpha \varphi \dot{\alpha} \alpha 1$ (36")

\begin{tabular}{|c|c|c|c|c|}
\hline \multirow{3}{*}{ 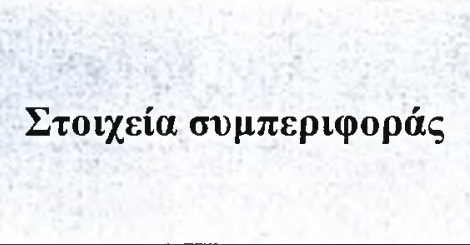 } & \multicolumn{4}{|c|}{ 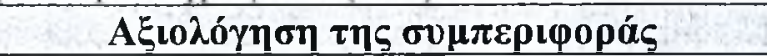 } \\
\hline & \multicolumn{2}{|c|}{$\begin{array}{c}\Pi \alpha \imath \delta \imath \dot{\alpha} \mu \varepsilon \sigma \tau o \imath \chi \varepsilon i \alpha \\
\Delta E \Pi / Y \\
(9)\end{array}$} & \multicolumn{2}{|c|}{ 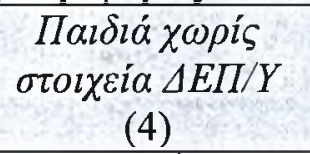 } \\
\hline & M.O. & T.A. & M.O. & T.A. \\
\hline 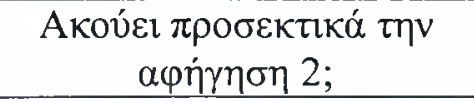 & 2,44 & 0,53 & 3,00 & 0,00 \\
\hline 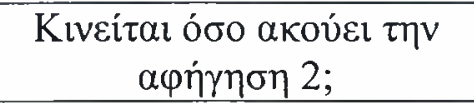 & 1,67 & 0,71 & 1,25 & 0,50 \\
\hline 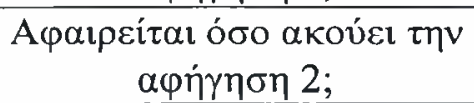 & 1,22 & 0,44 & 1,00 & 0,00 \\
\hline
\end{tabular}

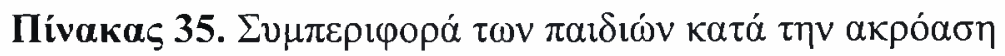

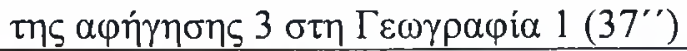

\begin{tabular}{|c|c|c|c|c|}
\hline \multirow{3}{*}{$\Sigma \tau 0 \iota \chi \varepsilon i ́ \alpha \sigma v \mu \pi \varepsilon \rho \iota \varphi 0 \rho \alpha ́ \varsigma$} & \multicolumn{4}{|c|}{ 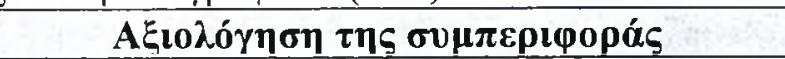 } \\
\hline & \multicolumn{2}{|c|}{$\begin{array}{c}\text { Пaıbı́́ } \mu \varepsilon \sigma \tau o \imath \chi \varepsilon i ́ a \\
\Delta E \Pi / Y \\
(9)\end{array}$} & \multicolumn{2}{|c|}{ 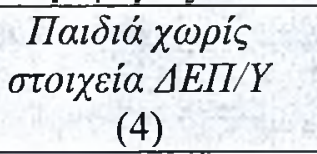 } \\
\hline & M.O. & T.A. & M.O. & T.A. \\
\hline 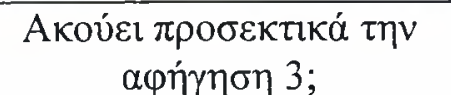 & 2,11 & 0,78 & 2,75 & 0,50 \\
\hline 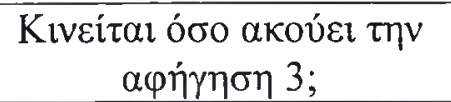 & 1,89 & 0,60 & 1,50 & 0,58 \\
\hline
\end{tabular}




\begin{tabular}{|c|c|c|c|c|}
\hline 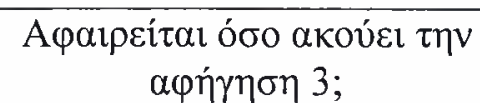 & 2,11 & 1,17 & 1,25 & 0,50 \\
\hline
\end{tabular}

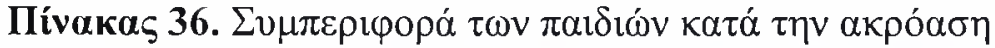

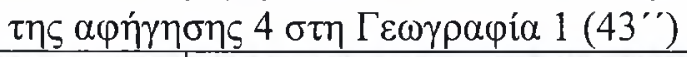

\begin{tabular}{|c|c|c|c|c|}
\hline \multirow{3}{*}{ 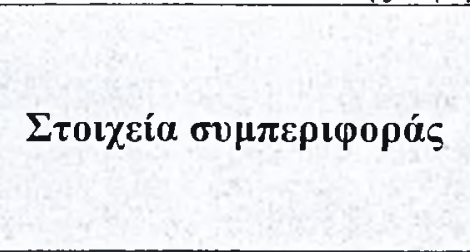 } & \multicolumn{4}{|c|}{ 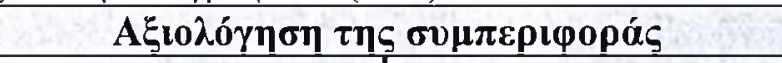 } \\
\hline & \multicolumn{2}{|c|}{ 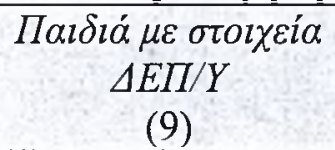 } & \multicolumn{2}{|c|}{ 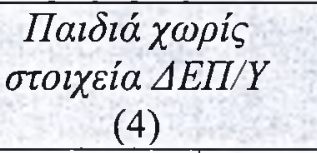 } \\
\hline & M.O. & T.A. & M.O. & T.A. \\
\hline 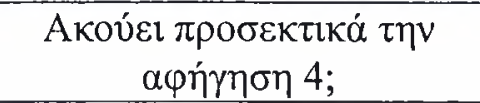 & 2,11 & 0,78 & 2,50 & 0,58 \\
\hline 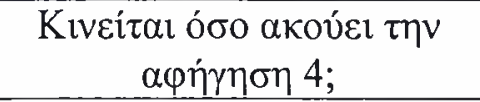 & 2,11 & 0,78 & 1,50 & 0,58 \\
\hline 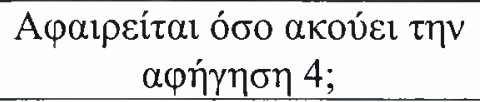 & 1,89 & 0,93 & 1,50 & 0,58 \\
\hline
\end{tabular}

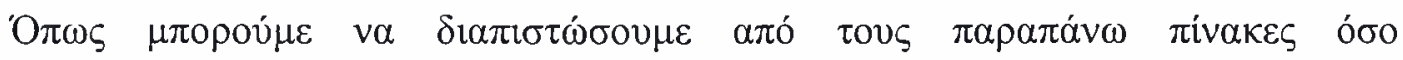

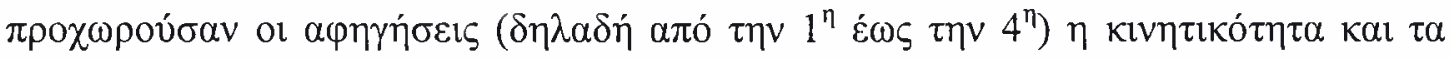

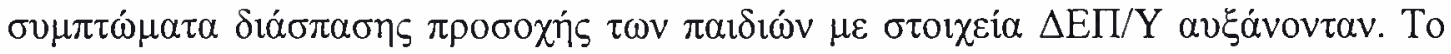

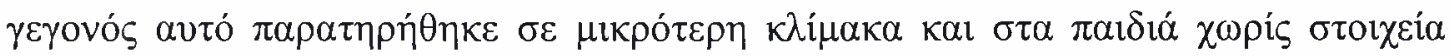

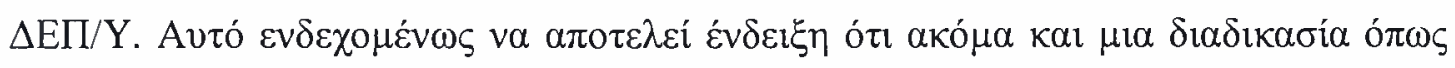

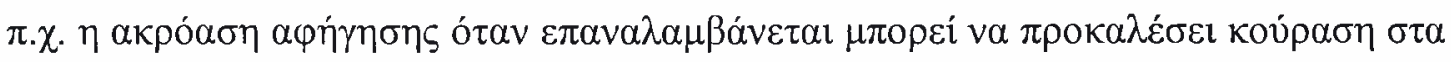

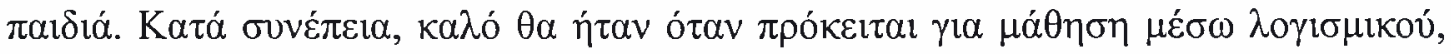

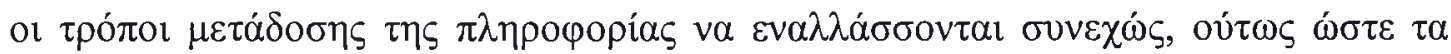

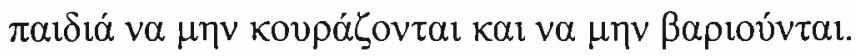

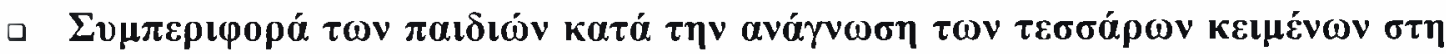 Гесүрафía 1}

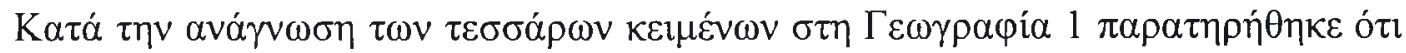

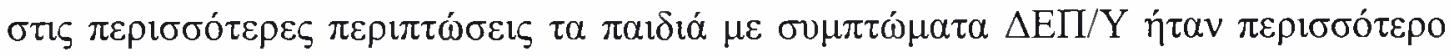

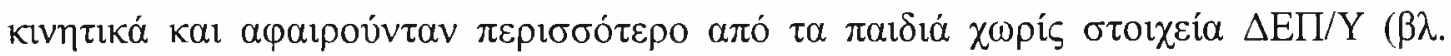

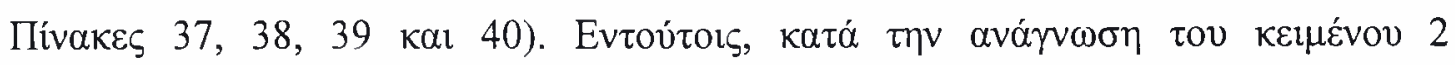

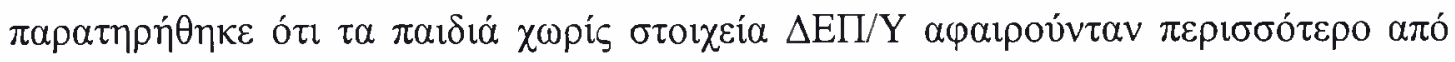

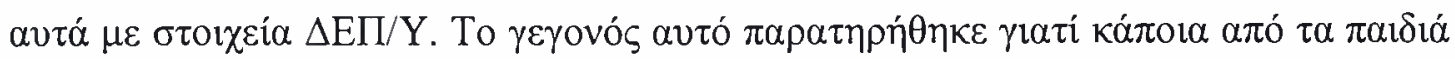

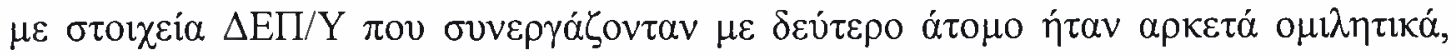

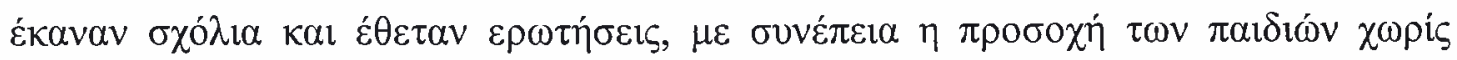




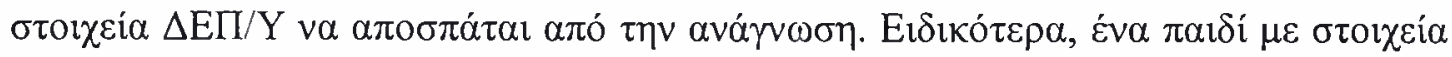

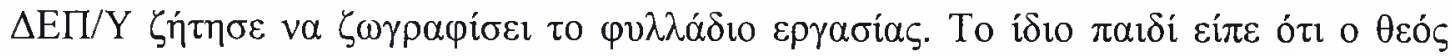

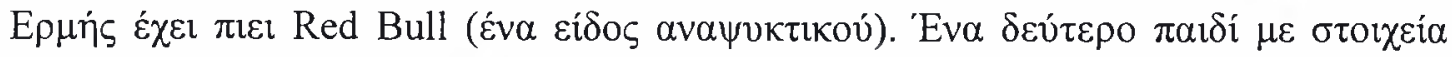

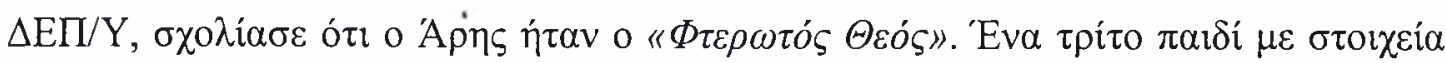

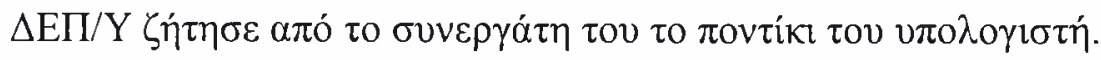

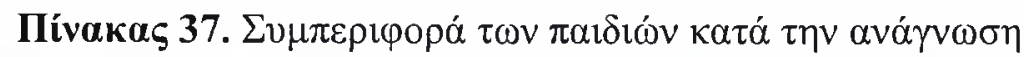

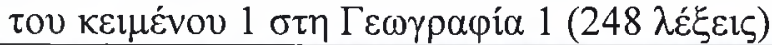

\begin{tabular}{|c|c|c|c|c|}
\hline \multirow{3}{*}{$\Sigma \tau 0 \iota \chi \varepsilon i ́ \alpha \sigma v \mu \pi \varepsilon \rho ı \varphi 0 \rho \alpha ́ \varsigma$} & \multicolumn{4}{|c|}{ 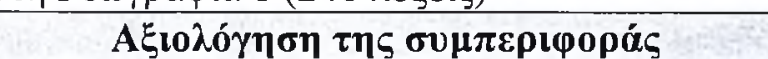 } \\
\hline & \multicolumn{2}{|c|}{ 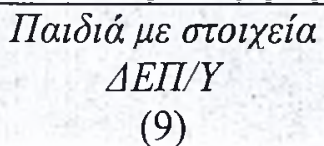 } & \multicolumn{2}{|c|}{ 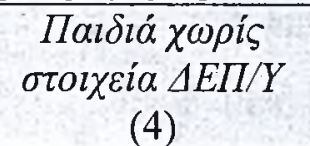 } \\
\hline & M.O. & T.A. & M.O. & T.A. \\
\hline 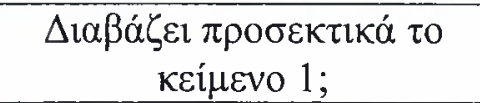 & 2,11 & 0,60 & 2,50 & 0,58 \\
\hline 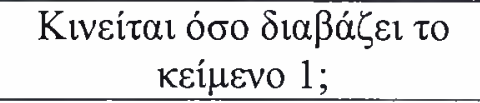 & 2,11 & 0,93 & 1,50 & 0,58 \\
\hline 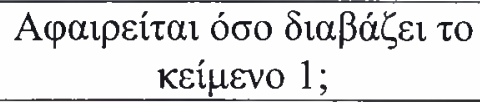 & 1,67 & 0,87 & 1,25 & 0,50 \\
\hline
\end{tabular}

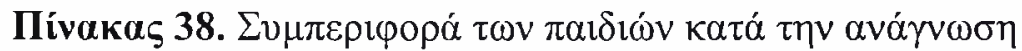

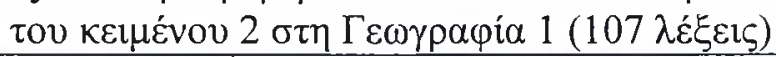

\begin{tabular}{|c|c|c|c|c|}
\hline \multirow{3}{*}{ 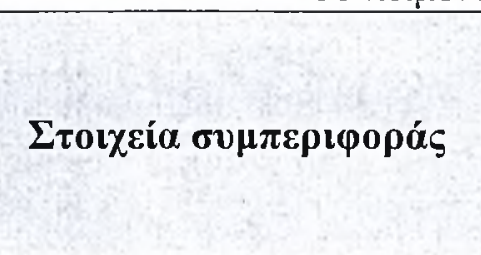 } & \multicolumn{4}{|c|}{ 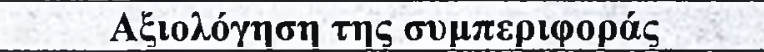 } \\
\hline & \multicolumn{2}{|c|}{ 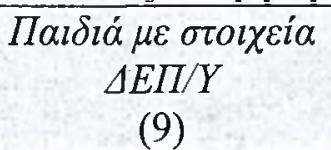 } & \multicolumn{2}{|c|}{ 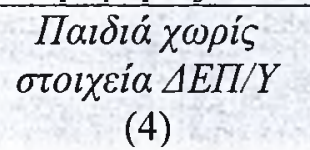 } \\
\hline & M.O. & T.A. & M.O. & T.A. \\
\hline 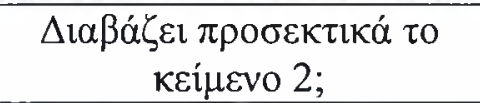 & 2,33 & 0,71 & 2,50 & 0,58 \\
\hline 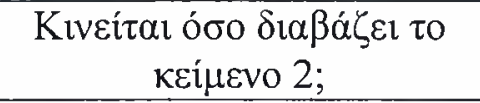 & 1,89 & 0,78 & 1,25 & 0,50 \\
\hline 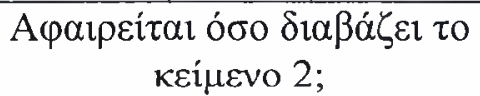 & 1,33 & 0,71 & 1,50 & 0,58 \\
\hline
\end{tabular}

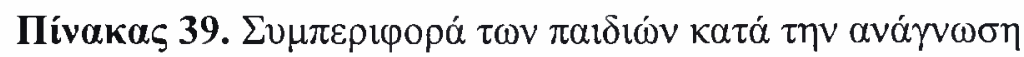

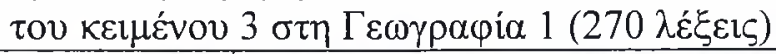

\begin{tabular}{|c|c|c|c|c|}
\hline \multirow{3}{*}{ 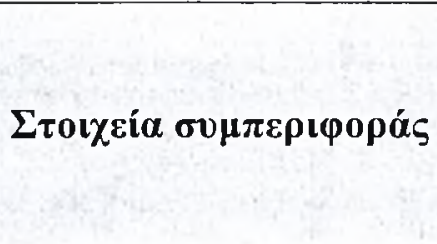 } & \multicolumn{4}{|c|}{ 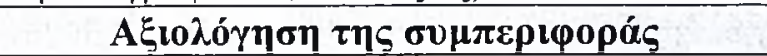 } \\
\hline & \multicolumn{2}{|c|}{ 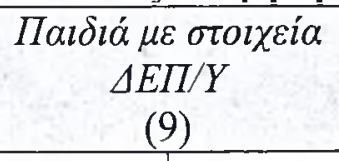 } & \multicolumn{2}{|c|}{ 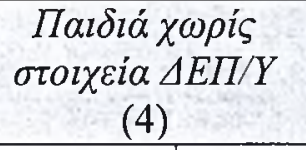 } \\
\hline & M.O. & T.A. & M.O. & T.A. \\
\hline 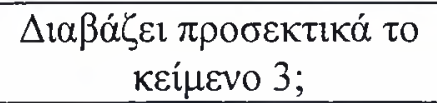 & 2,11 & 0,60 & 2,50 & 0,58 \\
\hline 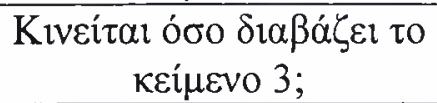 & 2,11 & 0,93 & 1,50 & 0,58 \\
\hline
\end{tabular}




\begin{tabular}{|c|c|c|c|c|}
\hline 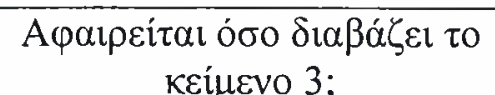 & 1,67 & 0,71 & 1,50 & 0,5 \\
\hline
\end{tabular}

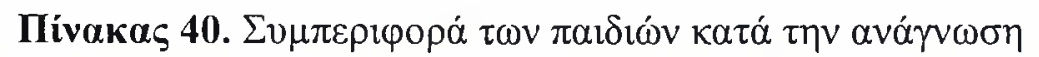

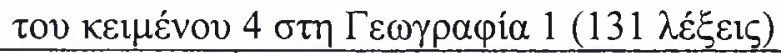

\begin{tabular}{|c|c|c|c|c|}
\hline \multirow{3}{*}{$\Sigma \tau 0 เ \chi \varepsilon \dot{\alpha} \alpha \sigma u \mu \pi \varepsilon \rho \iota \varphi о \rho \alpha \varsigma \varsigma$} & \multicolumn{4}{|c|}{ 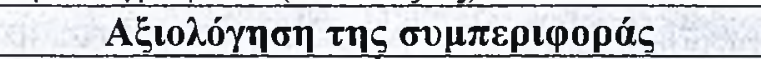 } \\
\hline & \multicolumn{2}{|c|}{$\begin{array}{c}\Pi \alpha ı \delta \imath \dot{\alpha} \mu \varepsilon \sigma \tau o l \chi \varepsilon i \alpha \\
\Delta E \Pi / Y \\
(9)\end{array}$} & \multicolumn{2}{|c|}{ 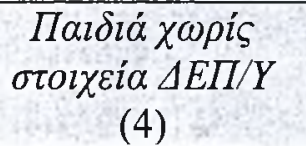 } \\
\hline & M.O. & T.A. & M.O. & T.A. \\
\hline 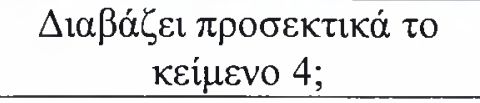 & 2,22 & 0,67 & 2,50 & 1,00 \\
\hline 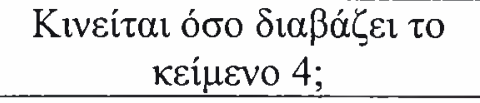 & 2,00 & 0,71 & 1,25 & 0,50 \\
\hline 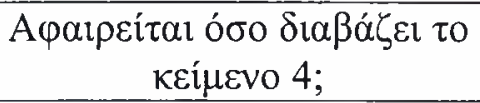 & 1,56 & 0,73 & 1,25 & 0,50 \\
\hline
\end{tabular}

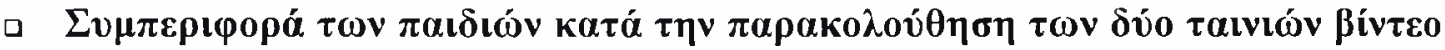 $\sigma \tau \eta \Gamma \varepsilon \omega \gamma \rho \alpha \varphi i ́ \alpha 1$}

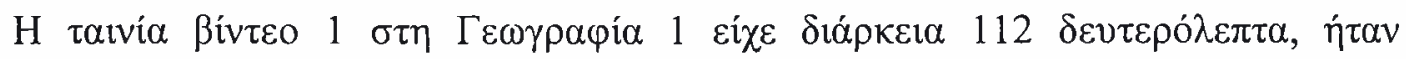

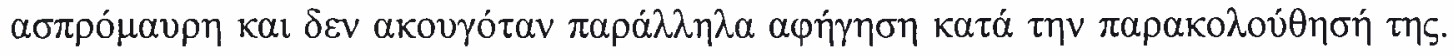

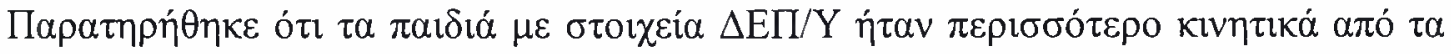

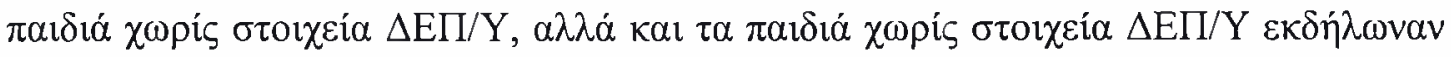

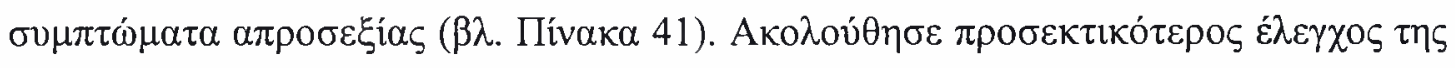

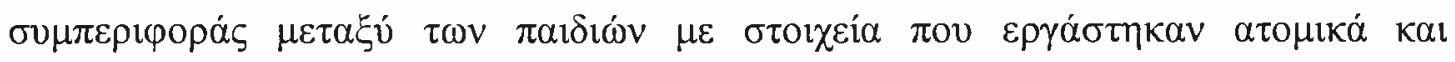

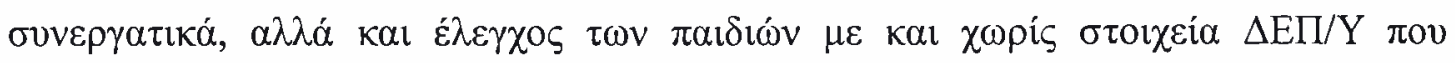

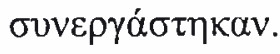

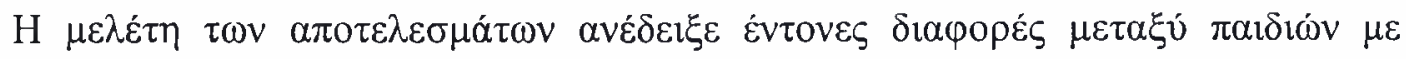

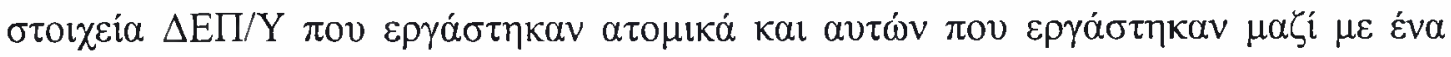

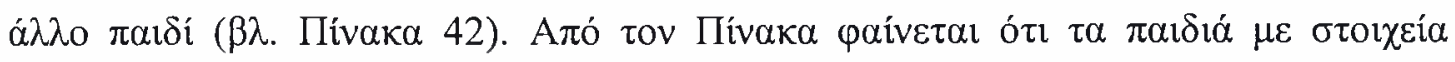

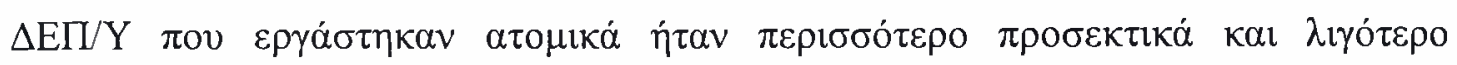

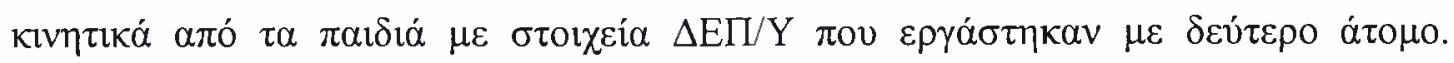

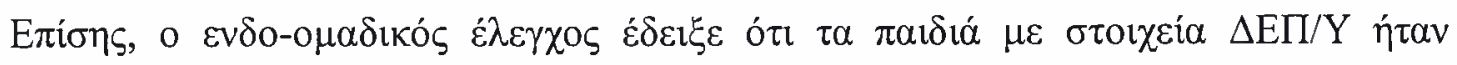

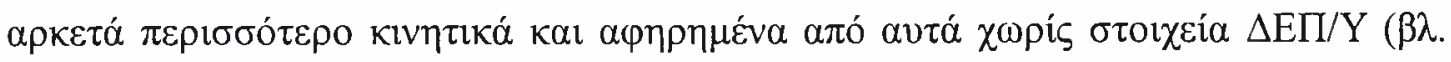
Пiv $\alpha \kappa \alpha 43)$. 


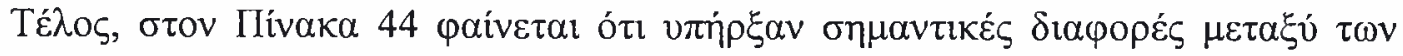

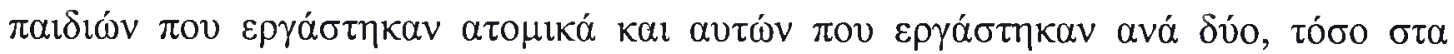

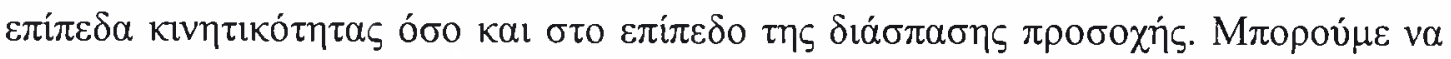

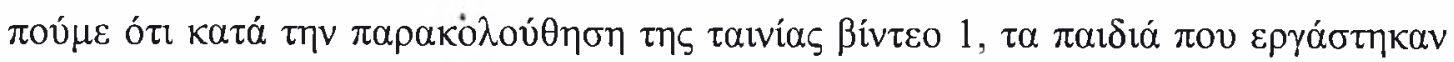

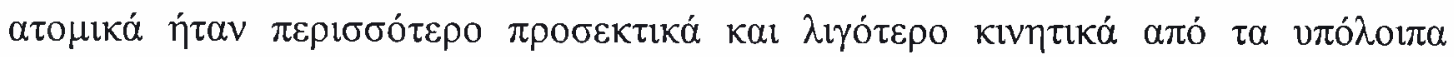
$\pi \alpha 1 \delta$ เó.

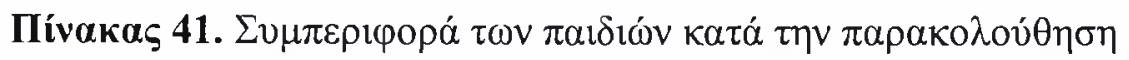

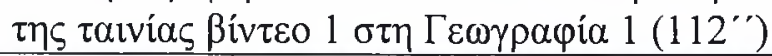

\begin{tabular}{|c|c|c|c|c|}
\hline \multirow{3}{*}{ 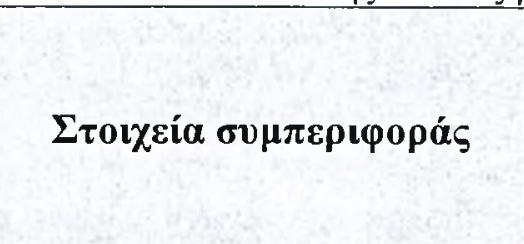 } & \multicolumn{4}{|c|}{ 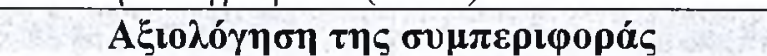 } \\
\hline & \multicolumn{2}{|c|}{ 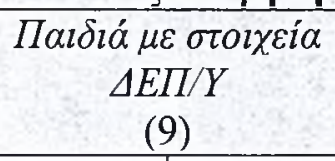 } & \multicolumn{2}{|c|}{ 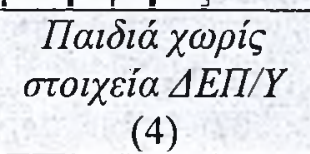 } \\
\hline & M.O. & T.A. & M.O. & T.A. \\
\hline 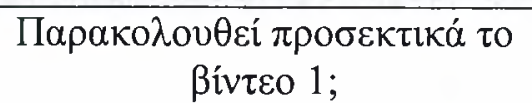 & 2,44 & 0,73 & 2,00 & 0,82 \\
\hline 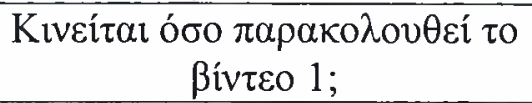 & 1,78 & 0,67 & 1,50 & 0,58 \\
\hline 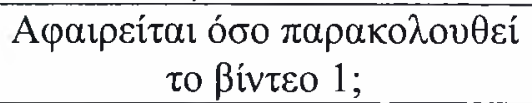 & 1,44 & 0,53 & 1,50 & 0,58 \\
\hline
\end{tabular}

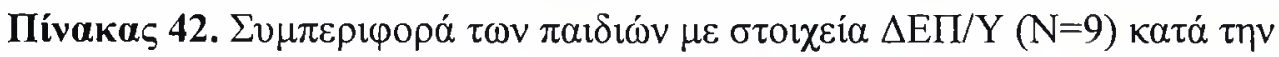

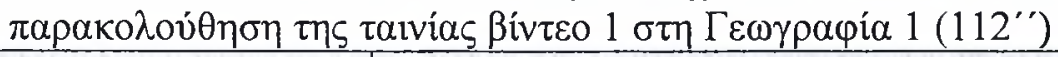

\begin{tabular}{|c|c|c|c|c|}
\hline \multirow{3}{*}{ 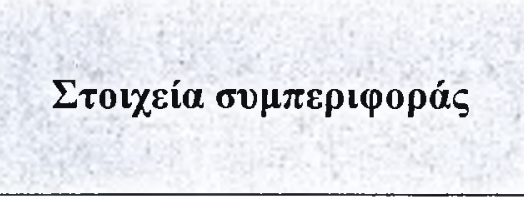 } & \multicolumn{4}{|c|}{ 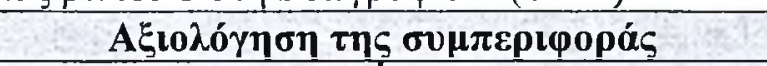 } \\
\hline & \multicolumn{2}{|c|}{ 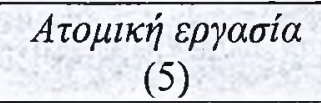 } & \multicolumn{2}{|c|}{ 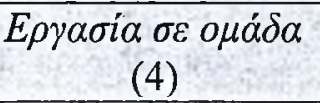 } \\
\hline & M.O. & T.A. & M.O. & T.A. \\
\hline 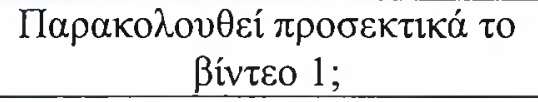 & 3,00 & 0,00 & 1,75 & 0,50 \\
\hline 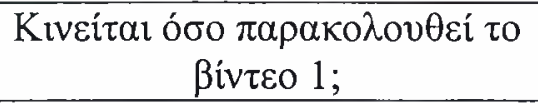 & 1,40 & 0,55 & 2,25 & 0,50 \\
\hline 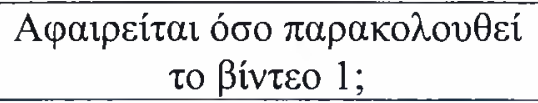 & 1,00 & 0,00 & 2,00 & 0,58 \\
\hline
\end{tabular}

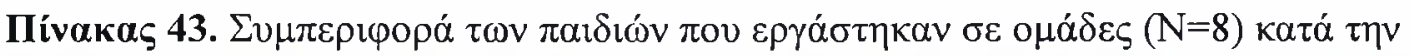

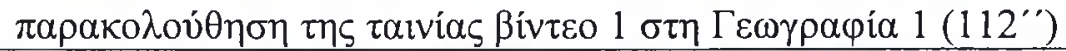

\begin{tabular}{|c|c|c|c|c|}
\hline \multirow{3}{*}{ 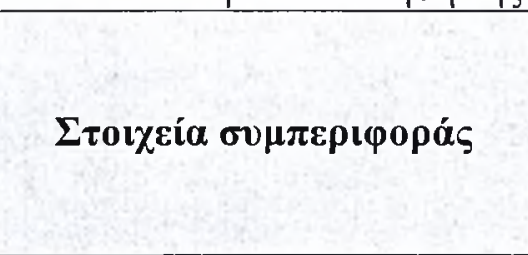 } & \multicolumn{4}{|c|}{ 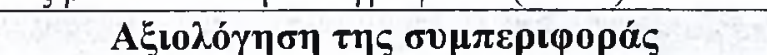 } \\
\hline & \multicolumn{2}{|c|}{ 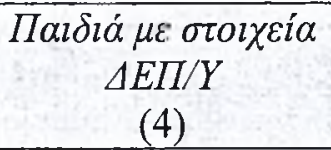 } & \multicolumn{2}{|c|}{ 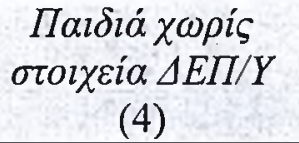 } \\
\hline & M.O. & T.A. & M.O. & T.A. \\
\hline 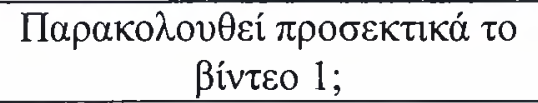 & 1,75 & 0,50 & 2,00 & 0,82 \\
\hline 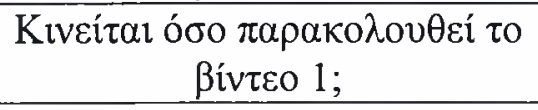 & 2,25 & 0,50 & 1,50 & 0,58 \\
\hline
\end{tabular}




\begin{tabular}{|c|c|c|c|c|}
\hline 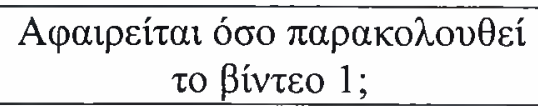 & 2,00 & 0,00 & 1,50 & 0,58 \\
\hline
\end{tabular}

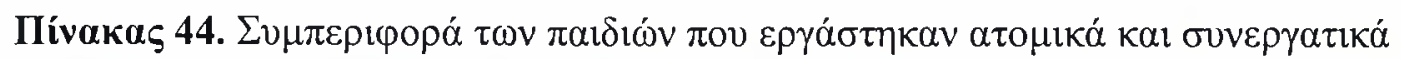

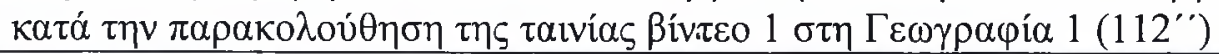

\begin{tabular}{|c|c|c|c|c|}
\hline \multirow{3}{*}{ 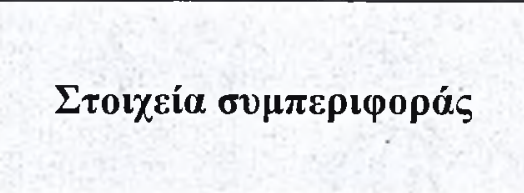 } & \multicolumn{4}{|c|}{ 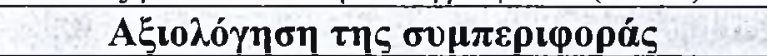 } \\
\hline & \multicolumn{2}{|c|}{$\begin{array}{c}A \tau o \mu \imath \kappa \eta ́ \\
(5)\end{array}$} & \multicolumn{2}{|c|}{ 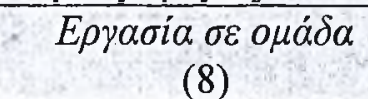 } \\
\hline & M.O. & T.A. & M.O. & T.A. \\
\hline 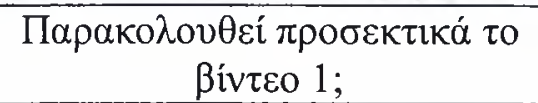 & 3,00 & 0,00 & 1,88 & 0,64 \\
\hline 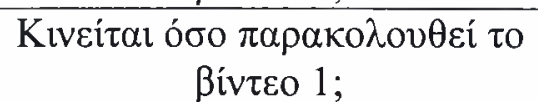 & 1,40 & 0,55 & 2,88 & 0,64 \\
\hline 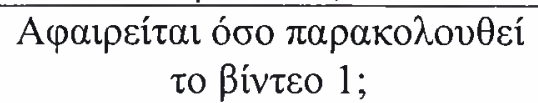 & 1,00 & 0,00 & 1,75 & 0,46 \\
\hline
\end{tabular}

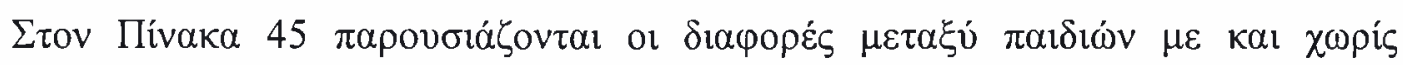

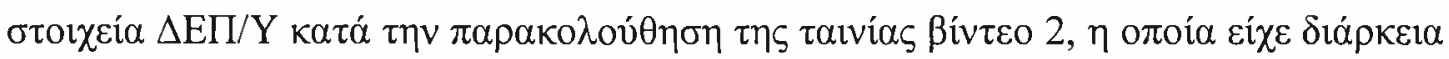

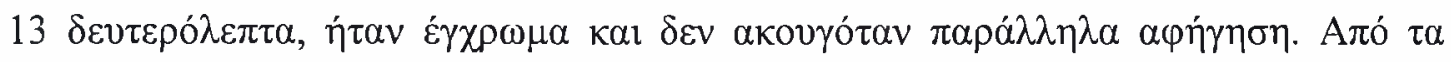

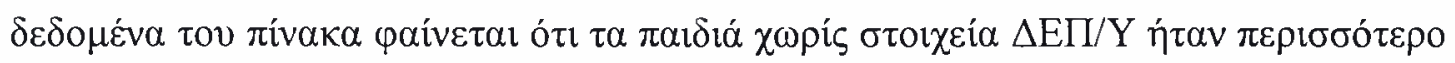

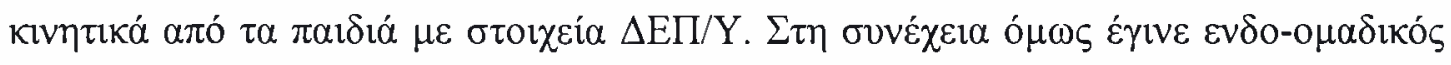

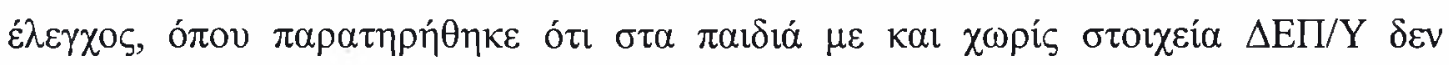

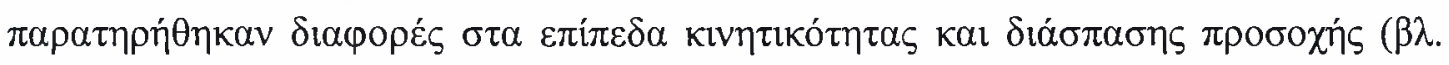

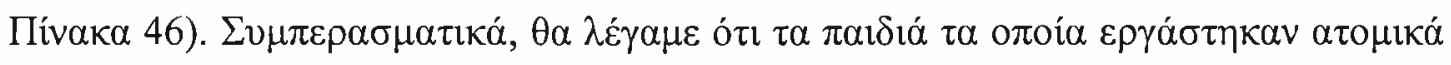

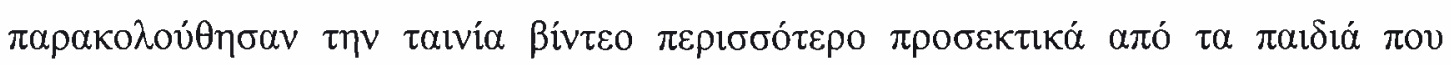

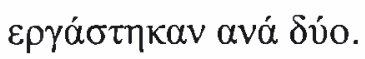

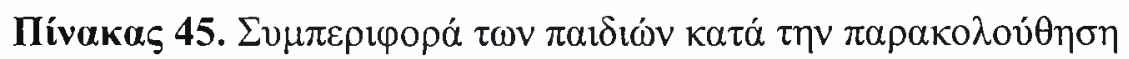

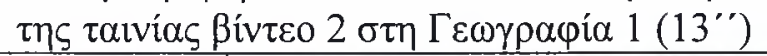

\begin{tabular}{|c|c|c|c|c|}
\hline \multirow{3}{*}{$\Sigma_{\tau \circ \chi \chi \varepsilon i ́ \alpha} \sigma 0 \mu \pi \varepsilon \rho \iota \varphi \rho \alpha \varsigma \zeta$} & \multicolumn{4}{|c|}{ 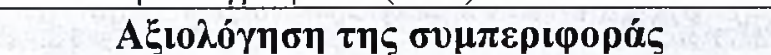 } \\
\hline & \multicolumn{2}{|c|}{ 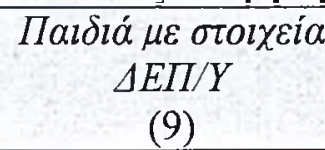 } & \multicolumn{2}{|c|}{ 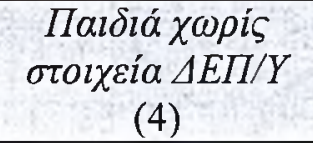 } \\
\hline & M.O. & T.A. & M.O. & T.A. \\
\hline 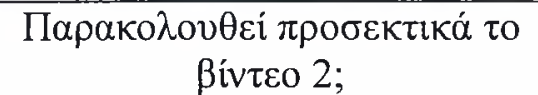 & 3,00 & 0,00 & 3,00 & 0,00 \\
\hline 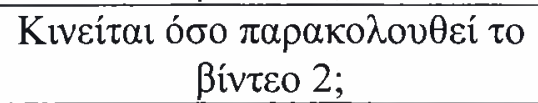 & 1,22 & 0,44 & 1,50 & 0,58 \\
\hline 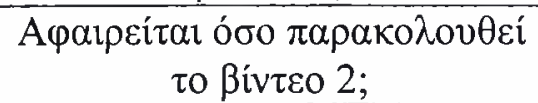 & 1,00 & 0,00 & 1,00 & 0,00 \\
\hline
\end{tabular}




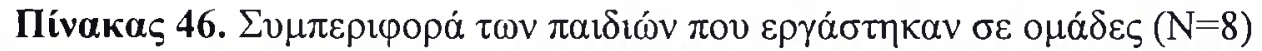

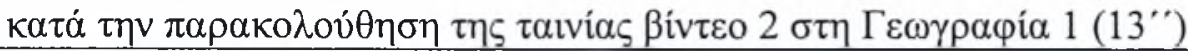

\begin{tabular}{|c|c|c|c|c|}
\hline \multirow{3}{*}{ 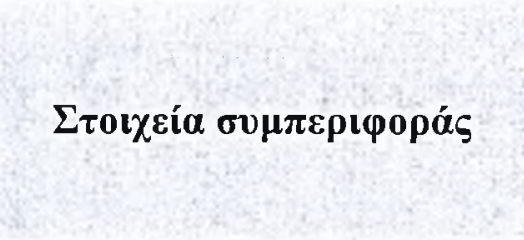 } & \multicolumn{4}{|c|}{ 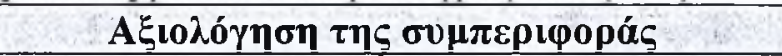 } \\
\hline & \multicolumn{2}{|c|}{$\begin{array}{c}\Pi \alpha \iota \delta \imath \alpha \dot{\alpha} \mu \sigma \tau o \imath \chi \varepsilon i \alpha \\
\Delta E \Pi / Y \\
\text { (4) }\end{array}$} & \multicolumn{2}{|c|}{ 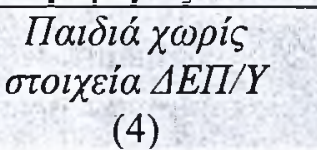 } \\
\hline & M.O. & T.A. & M.O. & T.A. \\
\hline 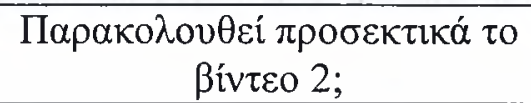 & 3,00 & 0,00 & 3,00 & 0,00 \\
\hline 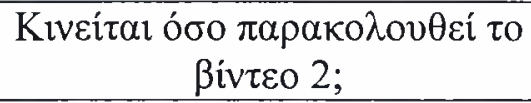 & 1,50 & 0,58 & 1,50 & 0,58 \\
\hline 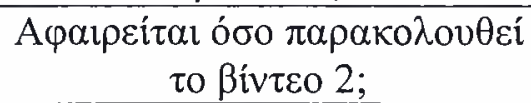 & 1,00 & 0,00 & 1,00 & 0,00 \\
\hline
\end{tabular}

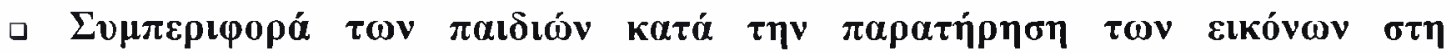
$\Gamma \varepsilon \omega \gamma \rho \alpha \varphi i ́ \alpha 1$

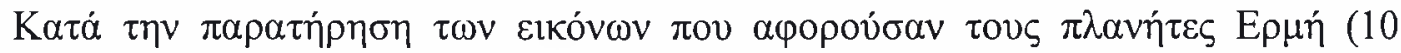

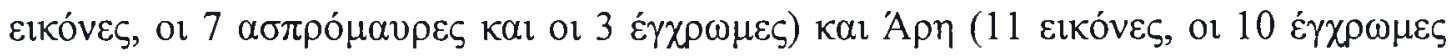

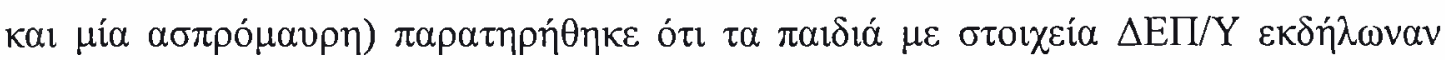

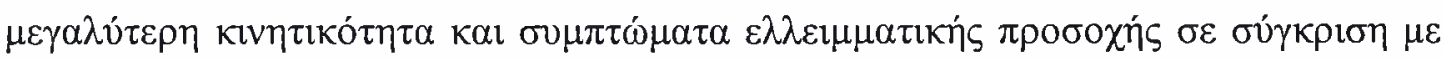

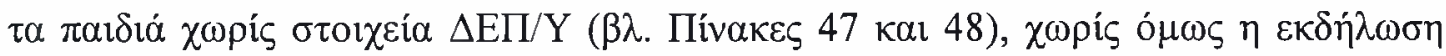

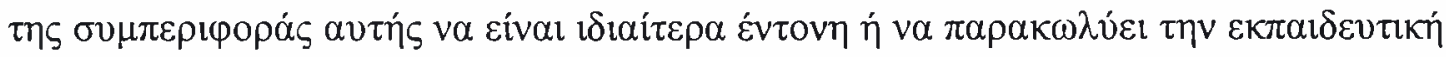
$\delta \rho \alpha \sigma \tau \eta \rho ์ \tau \eta \tau \alpha$.

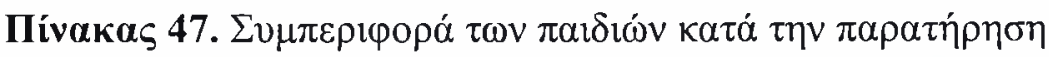

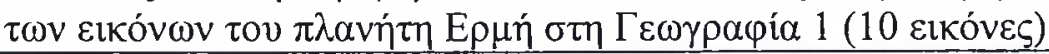

\begin{tabular}{|c|c|c|c|c|}
\hline \multirow{3}{*}{ 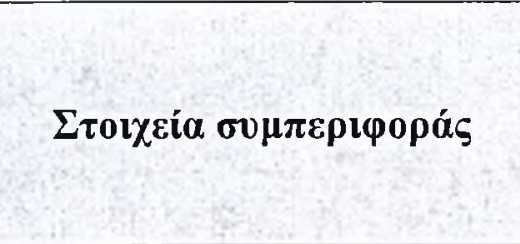 } & \multicolumn{4}{|c|}{ 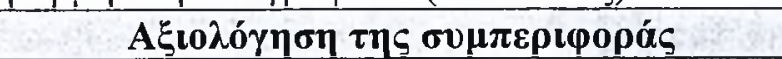 } \\
\hline & \multicolumn{2}{|c|}{$\begin{array}{c}\Pi \alpha \_\delta l \alpha \dot{\alpha} \mu \varepsilon \sigma \tau o \iota \chi \varepsilon i \alpha a \\
\Delta E \Pi / Y \\
(9)\end{array}$} & \multicolumn{2}{|c|}{ 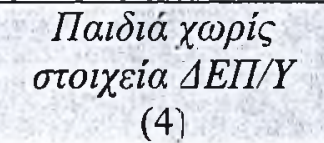 } \\
\hline & M.O. & T.A. & M.O. & T.A. \\
\hline 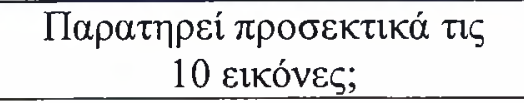 & 2,67 & 0,50 & 2,75 & 0,50 \\
\hline 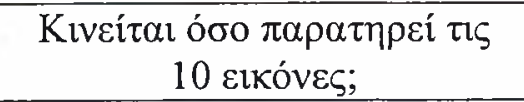 & 1,56 & 0,88 & 1,50 & 0,58 \\
\hline 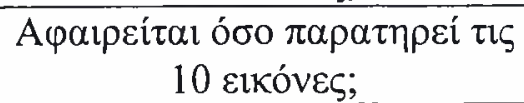 & 1,22 & 0,44 & 1,00 & 0,00 \\
\hline
\end{tabular}




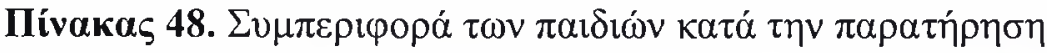

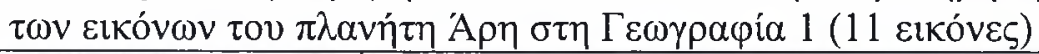

\begin{tabular}{|c|c|c|c|c|}
\hline \multirow{3}{*}{ 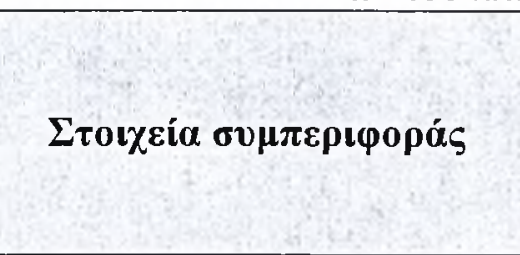 } & \multicolumn{4}{|c|}{ 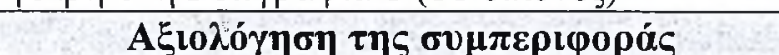 } \\
\hline & \multicolumn{2}{|c|}{ 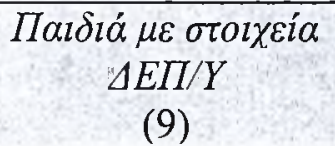 } & \multicolumn{2}{|c|}{ 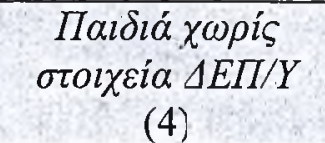 } \\
\hline & M.O. & T.A. & M.O. & T.A. \\
\hline 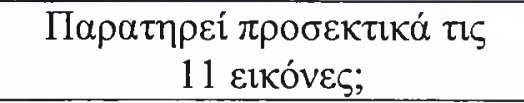 & 2,56 & 0,53 & 3,00 & 0,00 \\
\hline 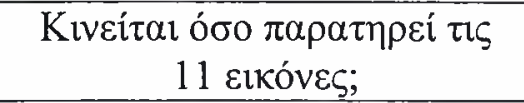 & 1,56 & 0,73 & 1,25 & 0,50 \\
\hline 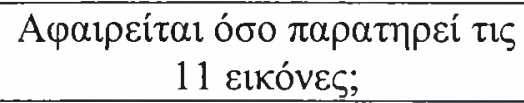 & 1,33 & 0,50 & 1,00 & 0,00 \\
\hline
\end{tabular}

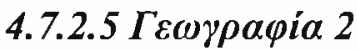

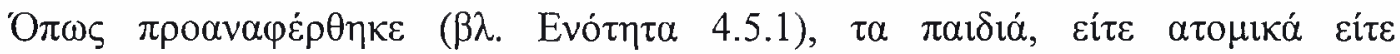

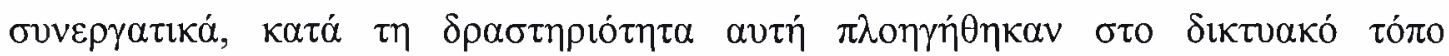

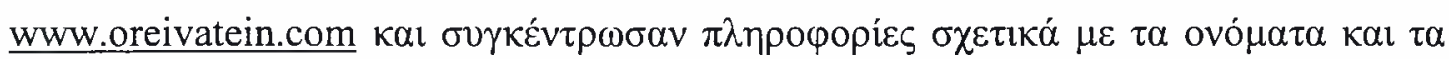

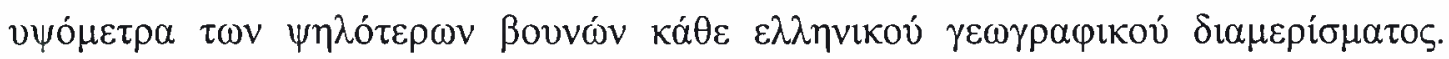

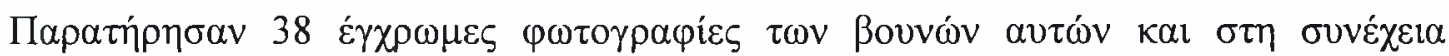

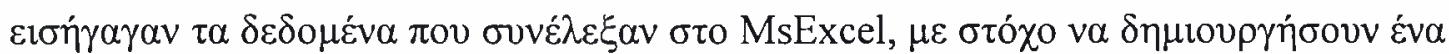

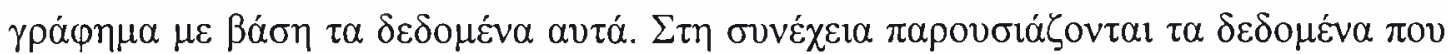

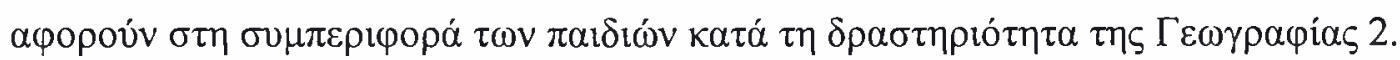

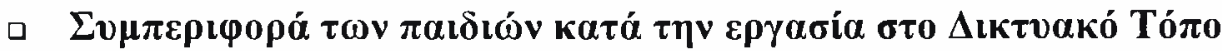

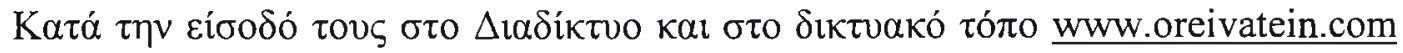

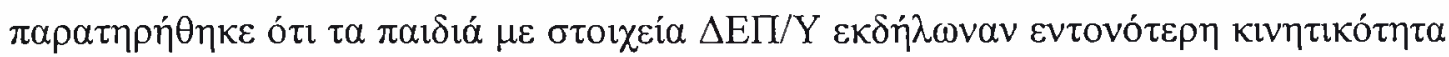

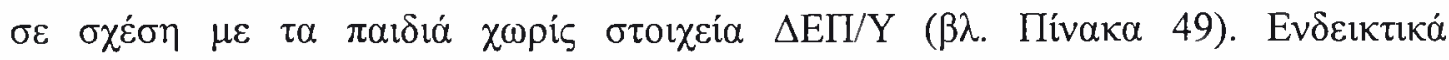

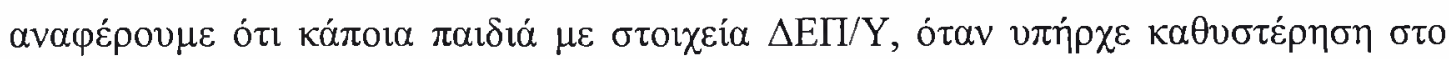

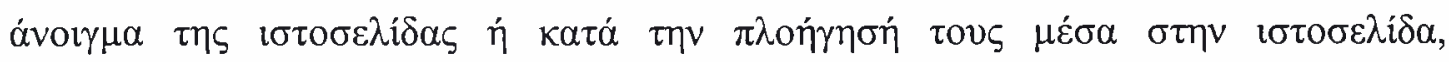

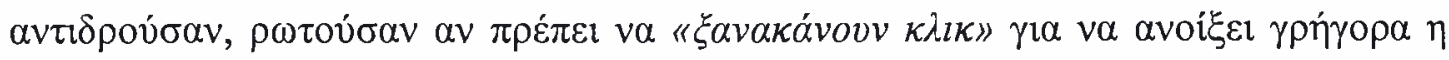

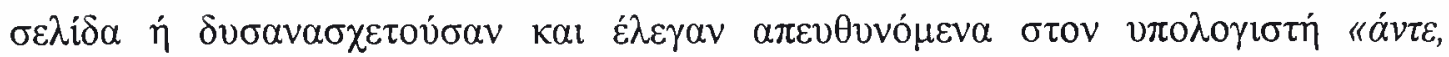
$\tau \varepsilon \lambda \varepsilon i \omega v \varepsilon ! »$. 


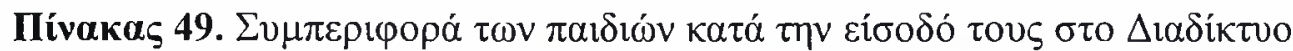

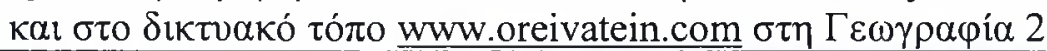

\begin{tabular}{|c|c|c|c|c|}
\hline \multirow{3}{*}{ 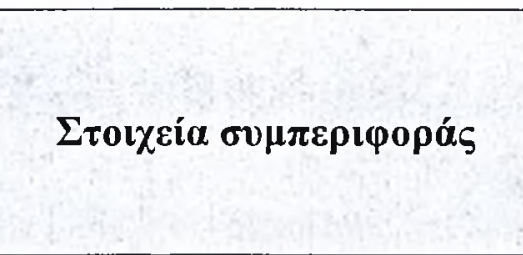 } & \multicolumn{4}{|c|}{ 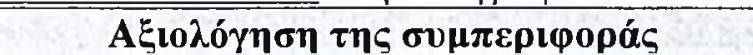 } \\
\hline & \multicolumn{2}{|c|}{$\begin{array}{c}\Pi \alpha \iota \delta \imath \dot{\alpha} \mu \varepsilon \sigma \tau o \imath \chi \varepsilon i \alpha \\
\Delta E \Pi / Y \\
(9)\end{array}$} & \multicolumn{2}{|c|}{ 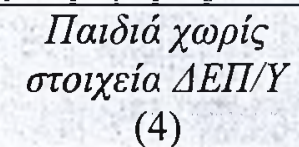 } \\
\hline & M.O. & T.A. & M.O. & T.A. \\
\hline 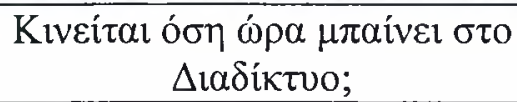 & 1,22 & 0,44 & 1,00 & 0,00 \\
\hline 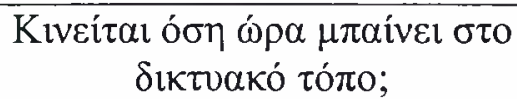 & 1,44 & 0,53 & 1,00 & 0,00 \\
\hline
\end{tabular}

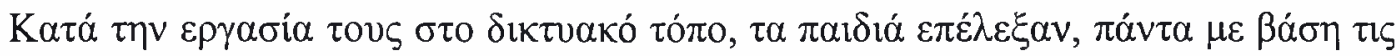

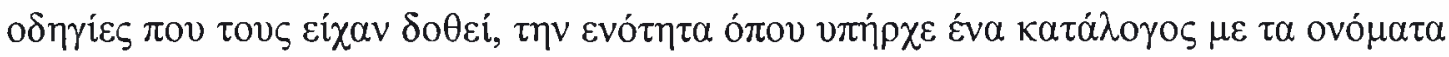

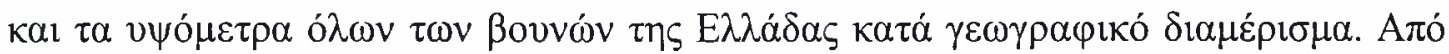

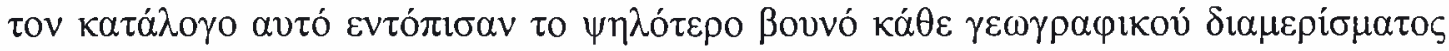

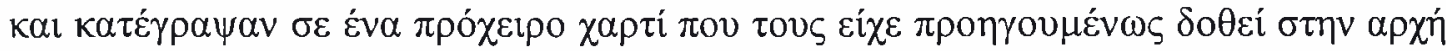

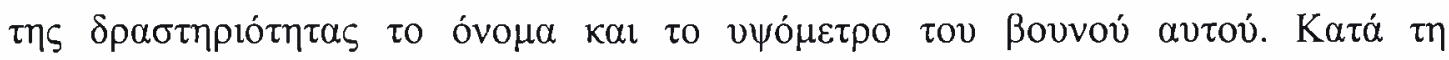

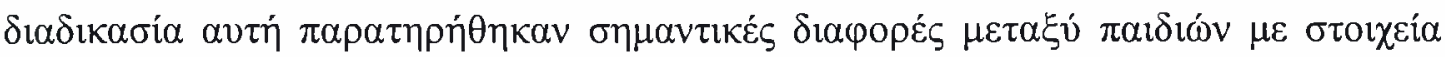

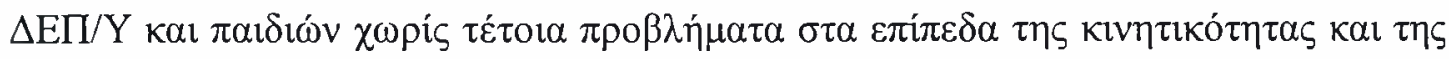

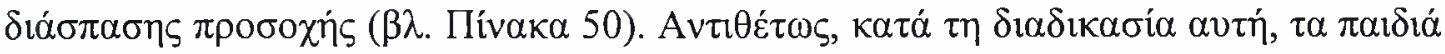

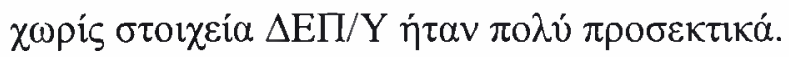

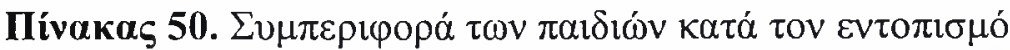

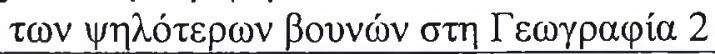

\begin{tabular}{|c|c|c|c|c|}
\hline \multirow{3}{*}{ 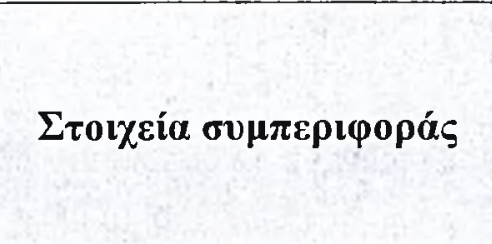 } & \multicolumn{4}{|c|}{ 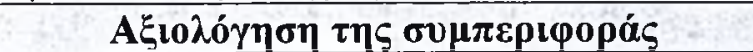 } \\
\hline & \multicolumn{2}{|c|}{ 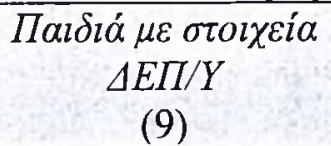 } & \multicolumn{2}{|c|}{ 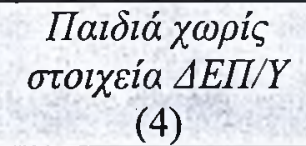 } \\
\hline & M.O. & T.A. & M.O. & T.A. \\
\hline 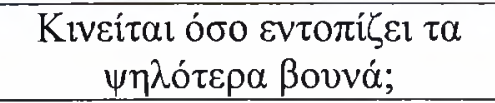 & 1,56 & 0,73 & 1,00 & 0,00 \\
\hline 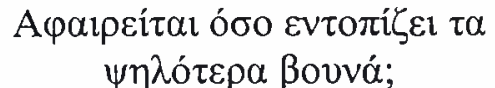 & 1,44 & 0,53 & 1,00 & 0,00 \\
\hline
\end{tabular}

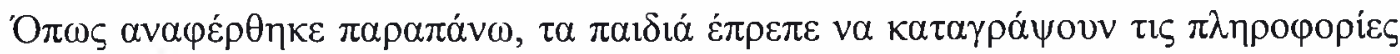

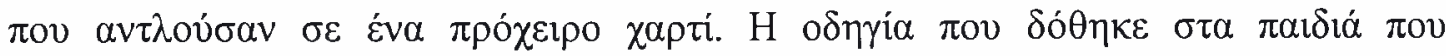

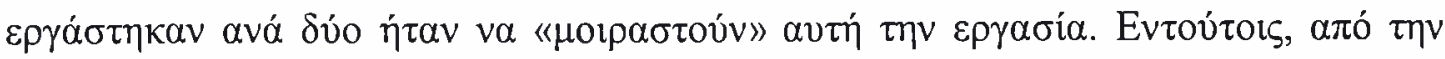

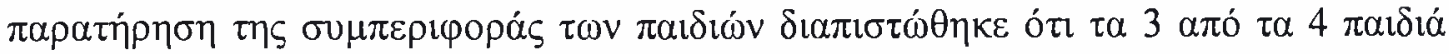

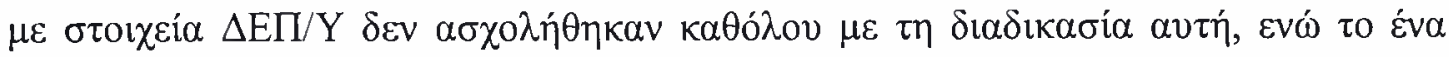

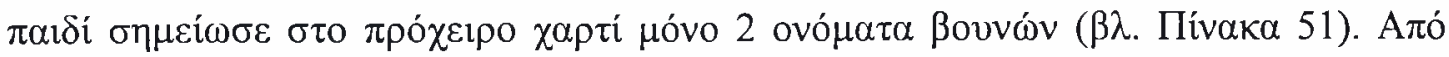




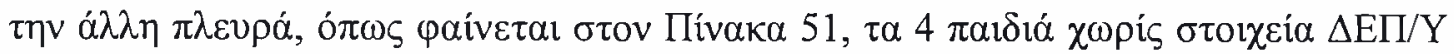

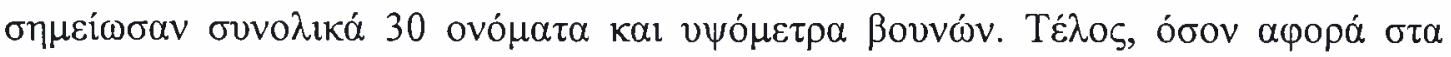

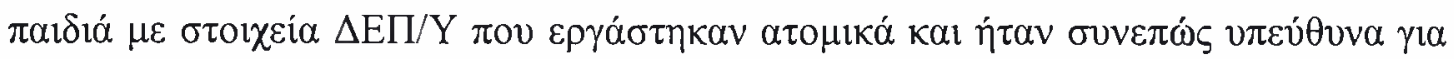

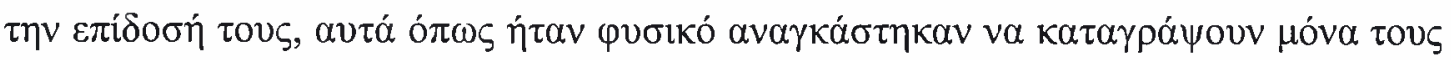

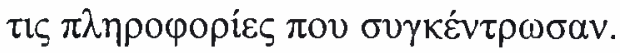

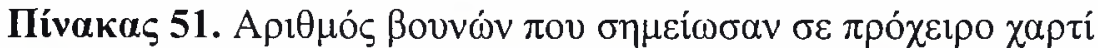

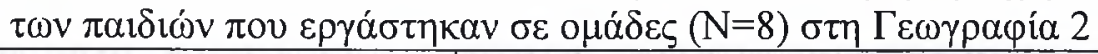

\begin{tabular}{|c|c|c|}
\hline \multirow[b]{2}{*}{ 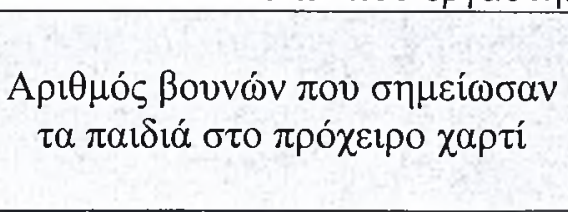 } & \multicolumn{2}{|c|}{ 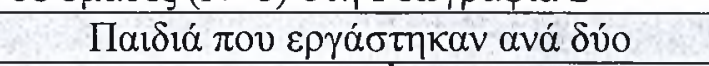 } \\
\hline & 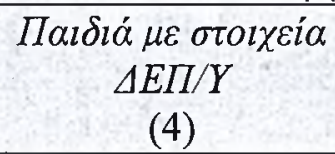 & 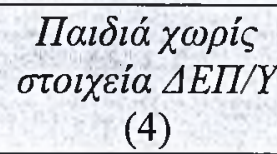 \\
\hline 0 & 3 & \\
\hline 2 & 1 & \\
\hline 6 & & 1 \\
\hline 8 & & 3 \\
\hline 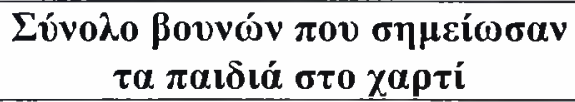 & 2 & 30 \\
\hline
\end{tabular}

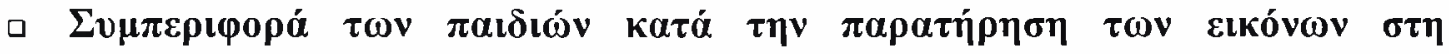

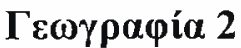

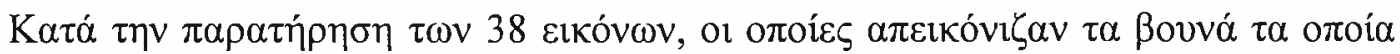

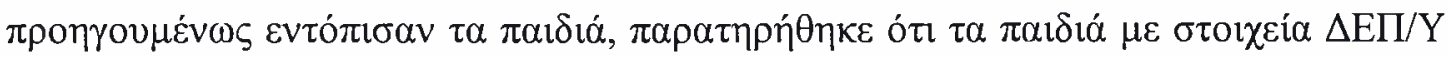

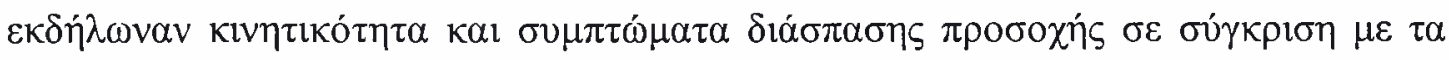

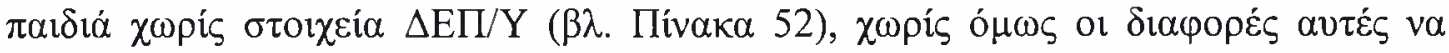

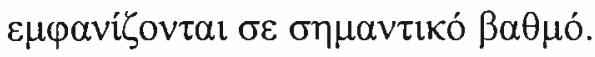

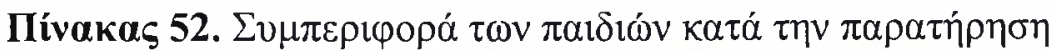

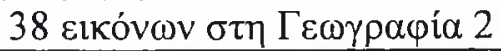

\begin{tabular}{|c|c|c|c|c|}
\hline \multirow{3}{*}{ 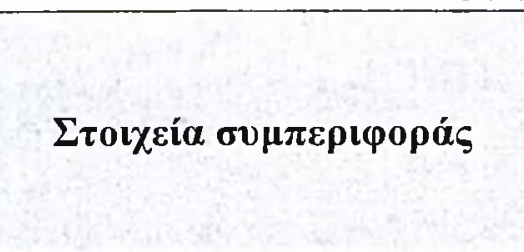 } & \multicolumn{4}{|c|}{ 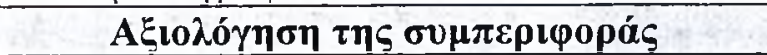 } \\
\hline & \multicolumn{2}{|c|}{ 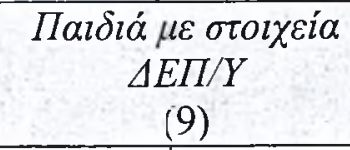 } & \multicolumn{2}{|c|}{ 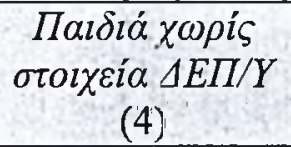 } \\
\hline & M.O. & T.A. & M.O. & T.A. \\
\hline 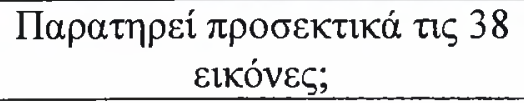 & 2,56 & 0,53 & 2,75 & 0,50 \\
\hline 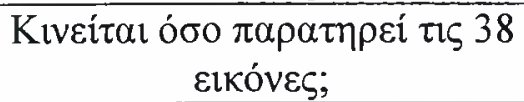 & 1,67 & 0,50 & 1,25 & 0,50 \\
\hline 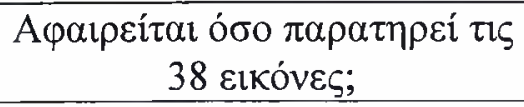 & 1,33 & 0,50 & 1,00 & 0,00 \\
\hline
\end{tabular}




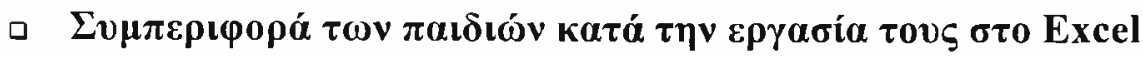

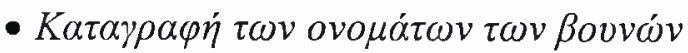

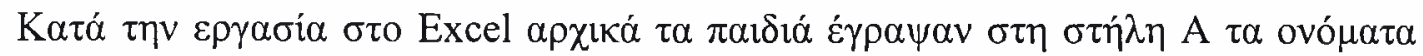

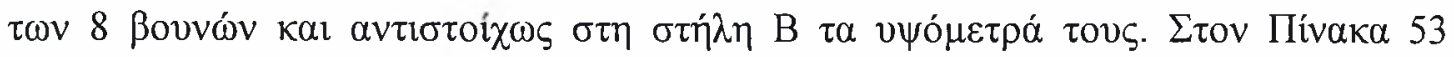

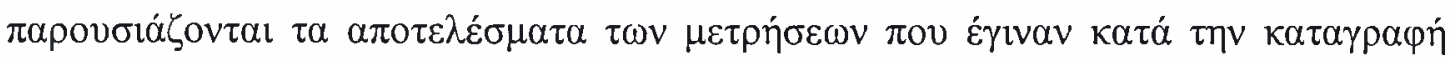

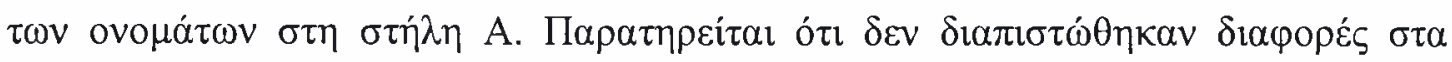

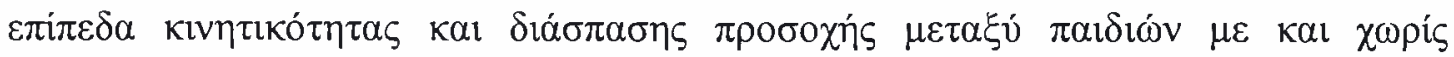

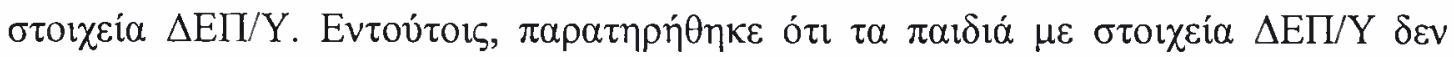

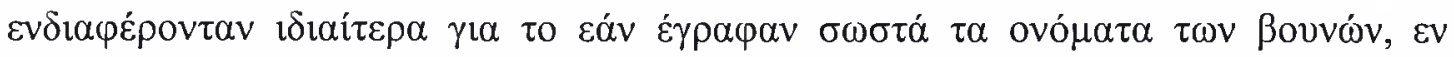

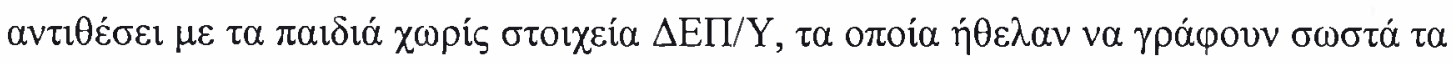
ovó $\mu \alpha \tau \alpha \tau \omega v \beta$ ßovvóv $\sigma \tau$ Excel.

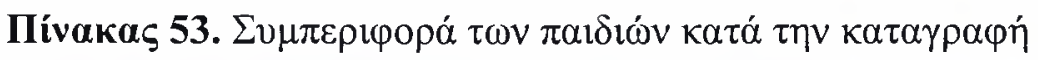

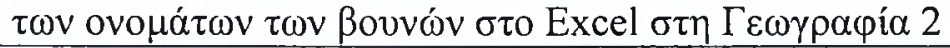

\begin{tabular}{|c|c|c|c|c|}
\hline \multirow{3}{*}{$\Sigma \tau 0\llcorner\chi \varepsilon i ́ \alpha \sigma v \mu \pi \varepsilon \rho \iota \varphi о \rho \alpha ́ \varsigma$} & \multicolumn{4}{|c|}{ 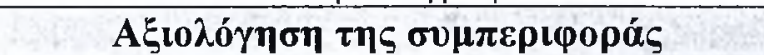 } \\
\hline & \multicolumn{2}{|c|}{ 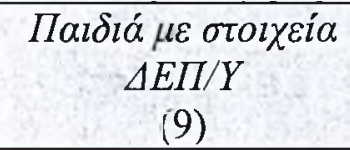 } & \multicolumn{2}{|c|}{ 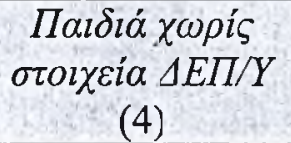 } \\
\hline & M.O. & T.A. & M.O. & T.A. \\
\hline 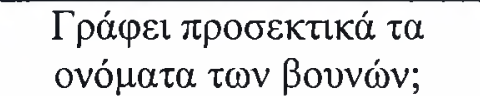 & 2,56 & 0,53 & 2,75 & 0,50 \\
\hline 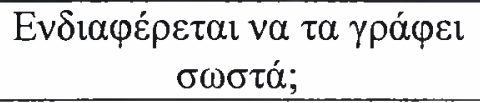 & 1,78 & 0,67 & 2,50 & 0,58 \\
\hline 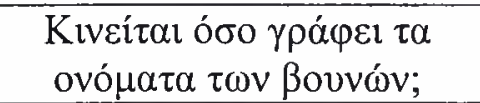 & 1,00 & 0,00 & 1,00 & 0,00 \\
\hline 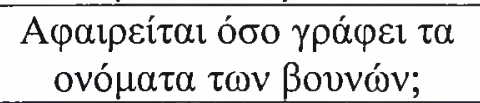 & 1,00 & 0,00 & 1,00 & 0,00 \\
\hline
\end{tabular}

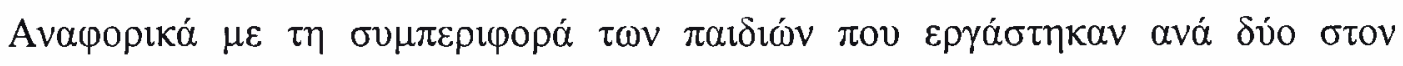

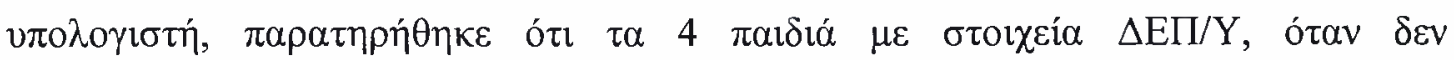

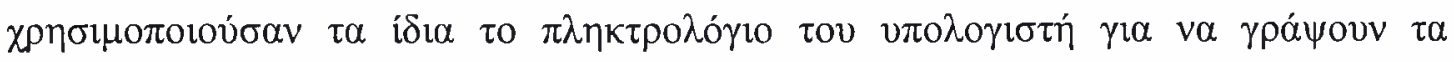

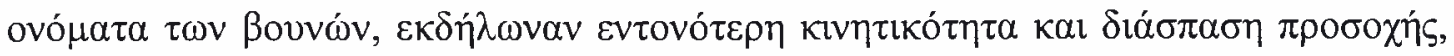

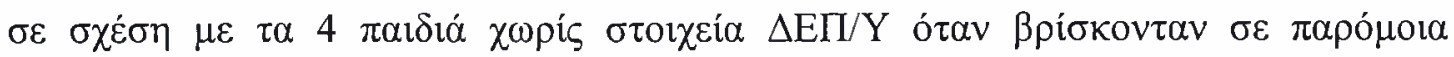

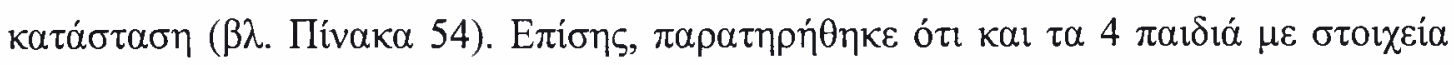

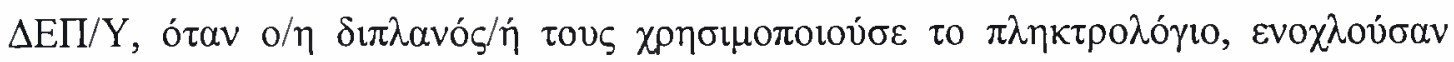

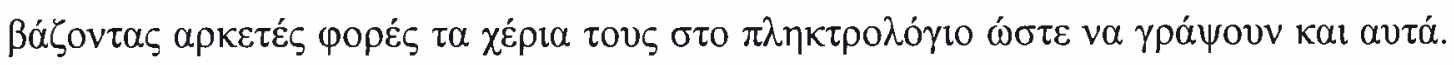

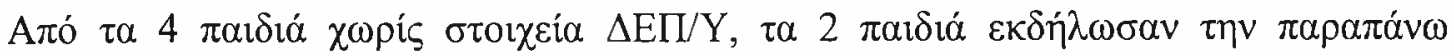




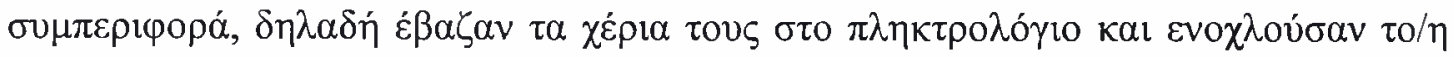

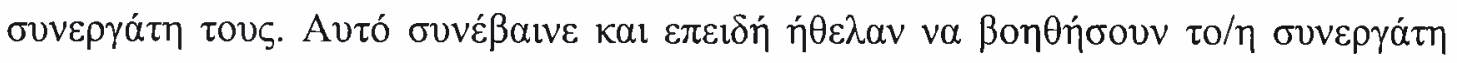

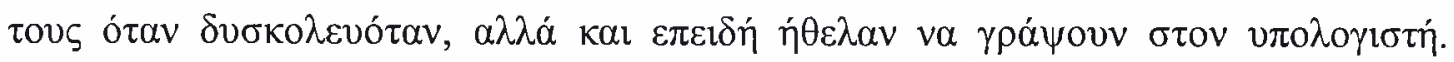

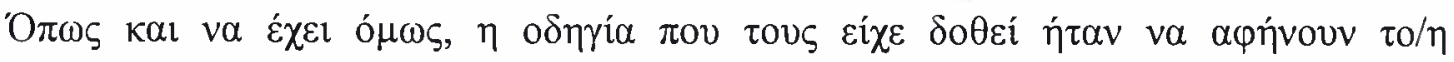

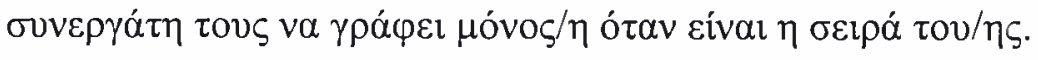

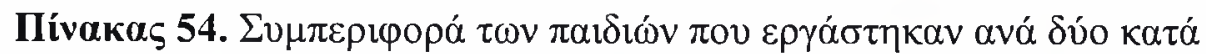

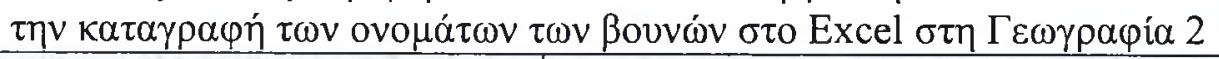

\begin{tabular}{|c|c|c|c|c|}
\hline \multirow{3}{*}{ 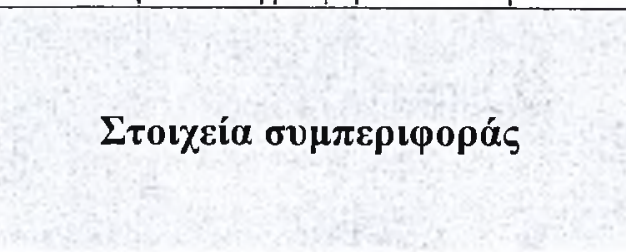 } & \multicolumn{4}{|c|}{ 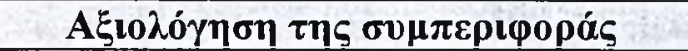 } \\
\hline & \multicolumn{2}{|c|}{$\begin{array}{c}\Pi \alpha \_\delta l \alpha \dot{\alpha} \mu \sigma \tau o l \chi \varepsilon i ́ \alpha \\
\Delta E \Pi / Y \\
\text { (4) }\end{array}$} & \multicolumn{2}{|c|}{ 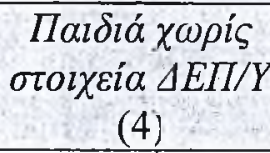 } \\
\hline & M.O. & T.A. & M.O. & T.A. \\
\hline 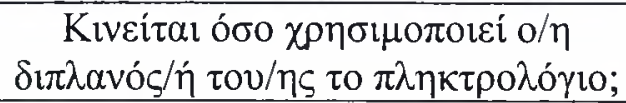 & 2,50 & 0,58 & 1,25 & 0,50 \\
\hline 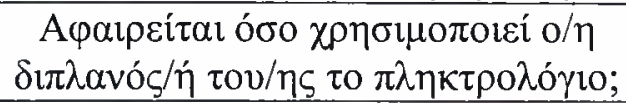 & 2,00 & 0,00 & 1,25 & 0,50 \\
\hline
\end{tabular}

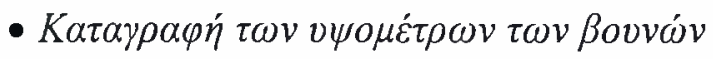

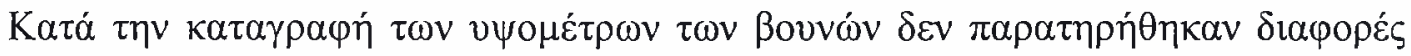

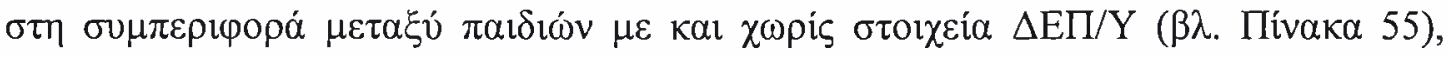

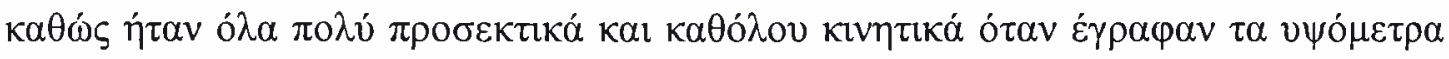

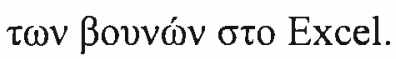

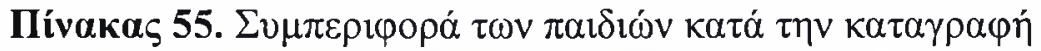

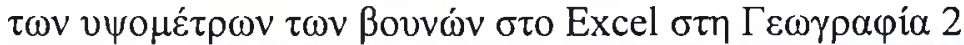

\begin{tabular}{|c|c|c|c|c|}
\hline \multirow{3}{*}{ 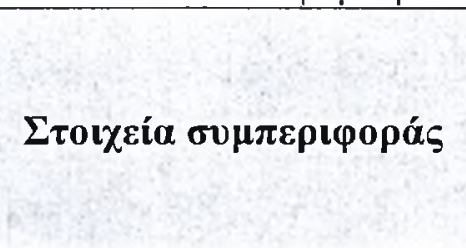 } & \multicolumn{4}{|c|}{ 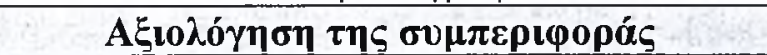 } \\
\hline & \multicolumn{2}{|c|}{ 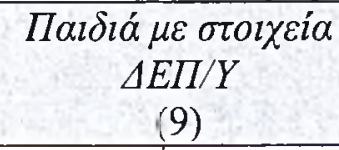 } & \multicolumn{2}{|c|}{ 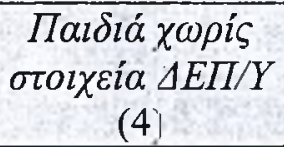 } \\
\hline & M.O. & T.A. & M.O. & T.A. \\
\hline 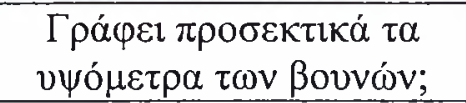 & 2,78 & 0,44 & 2,75 & 0,50 \\
\hline 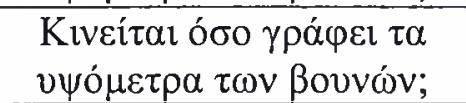 & 1,00 & 0,00 & 1,00 & 0,00 \\
\hline 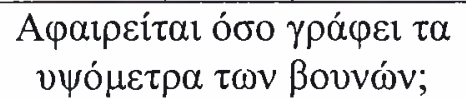 & 1,00 & 0,00 & 1,00 & 0,00 \\
\hline
\end{tabular}

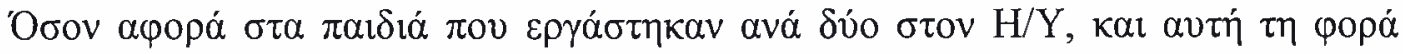

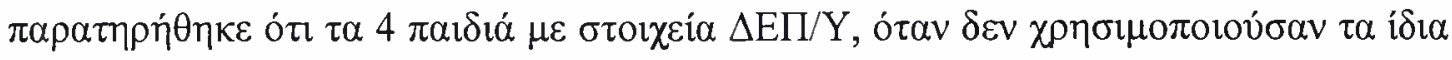

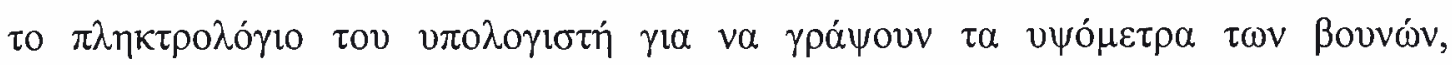




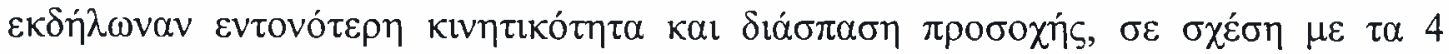

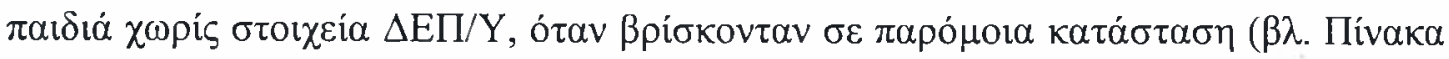

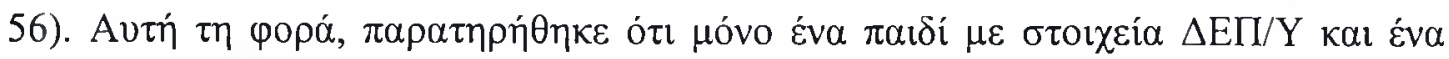

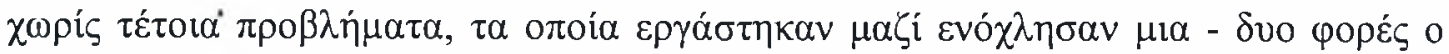

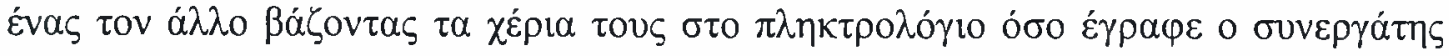

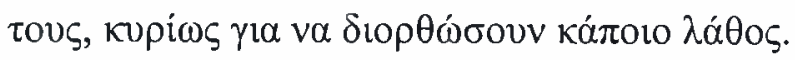

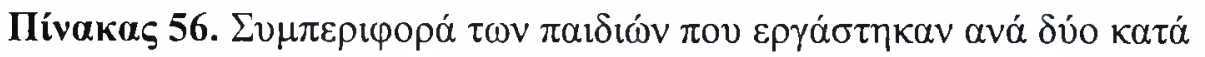

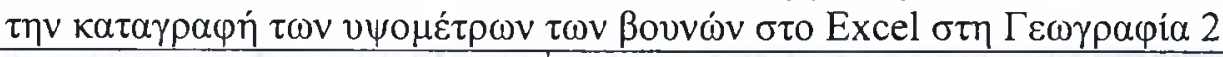

\begin{tabular}{|c|c|c|c|c|}
\hline \multirow{3}{*}{ 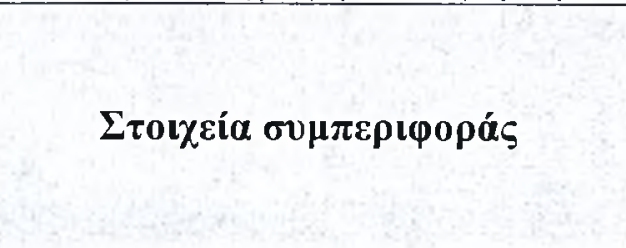 } & \multicolumn{4}{|c|}{ 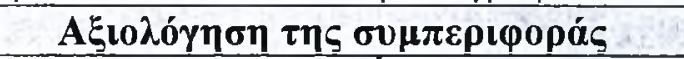 } \\
\hline & \multicolumn{2}{|c|}{ 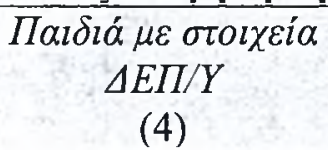 } & \multicolumn{2}{|c|}{ 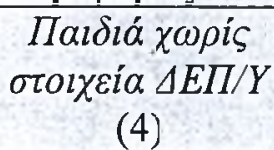 } \\
\hline & M.O. & T.A. & M.O. & T.A. \\
\hline 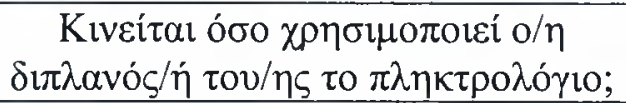 & 2,25 & 0,50 & 1,00 & 0,00 \\
\hline 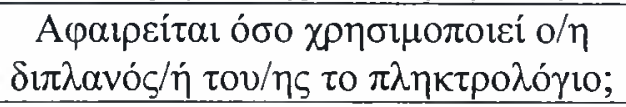 & 1,75 & 0,50 & 1,00 & 0,00 \\
\hline
\end{tabular}

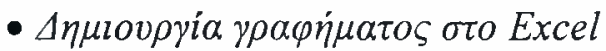

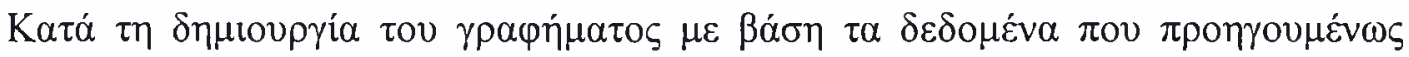

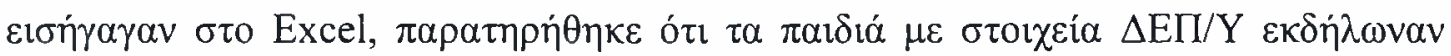

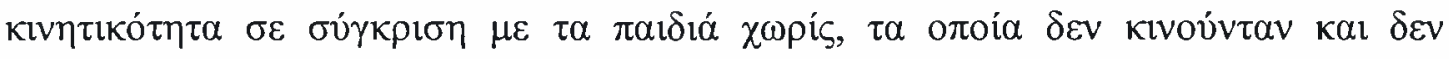

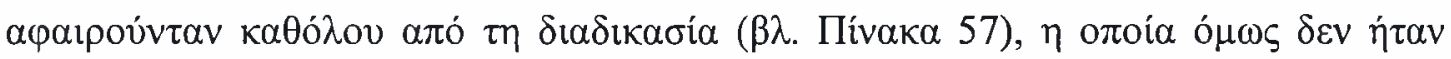

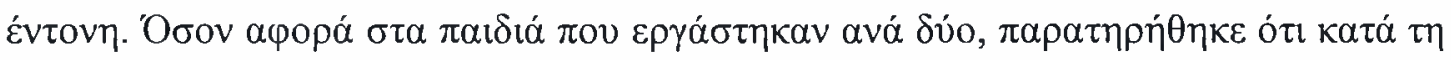

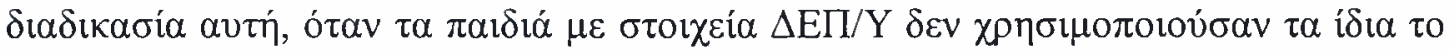

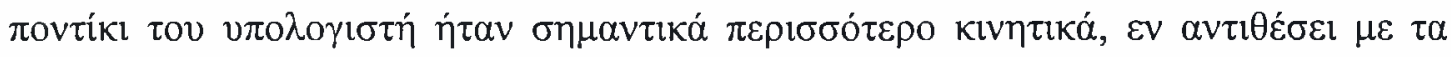

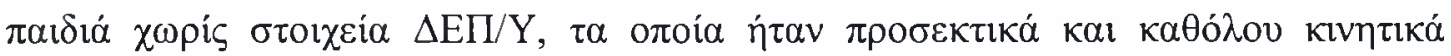

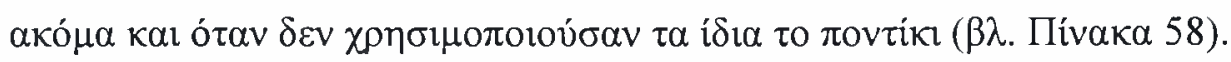

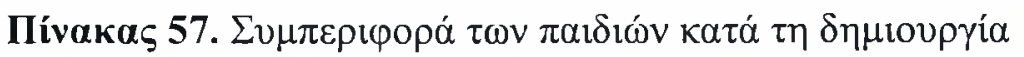

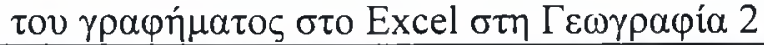

\begin{tabular}{|c|c|c|c|c|}
\hline \multirow{3}{*}{ 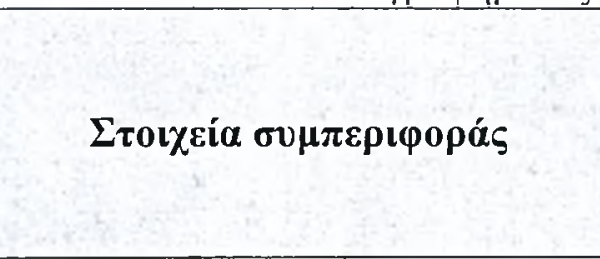 } & \multicolumn{4}{|c|}{ A } \\
\hline & \multicolumn{2}{|c|}{ 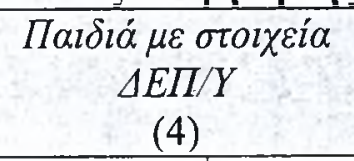 } & \multicolumn{2}{|c|}{ 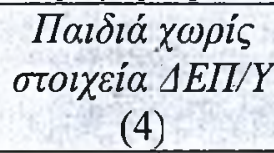 } \\
\hline & M.O. & T.A. & M.O. & T.A. \\
\hline 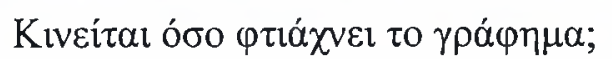 & 1,22 & 0,44 & 1,00 & 0,00 \\
\hline
\end{tabular}




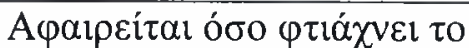
$\gamma \rho \alpha ́ \varphi \eta \mu \alpha$; 1,11 0,33 1,00 0,00

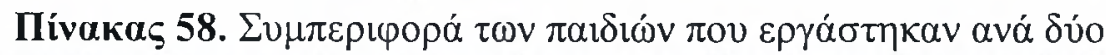

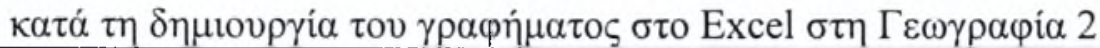

\begin{tabular}{|c|c|c|c|c|}
\hline \multirow{3}{*}{ 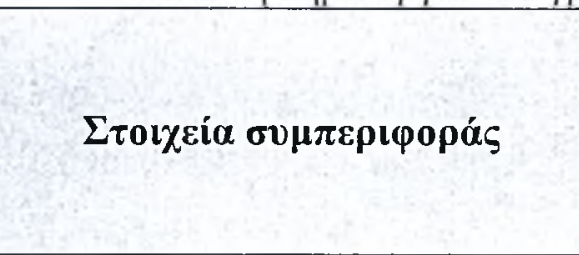 } & \multicolumn{4}{|c|}{ 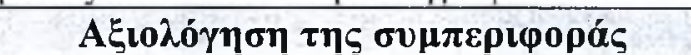 } \\
\hline & \multicolumn{2}{|c|}{ 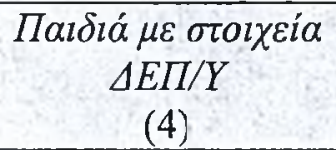 } & \multicolumn{2}{|c|}{ 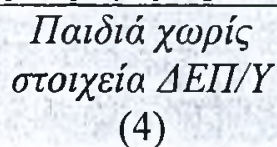 } \\
\hline & M.O. & T.A. & M.O. & T.A. \\
\hline 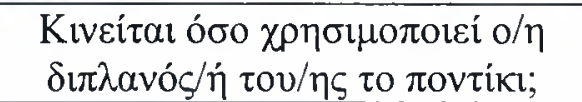 & 2,25 & 0,50 & 1,00 & 0,00 \\
\hline 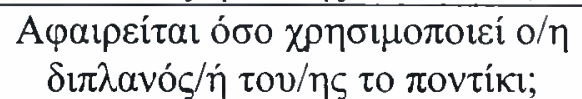 & 1,25 & 0,50 & 1,00 & 0,00 \\
\hline
\end{tabular}

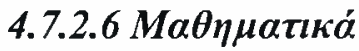

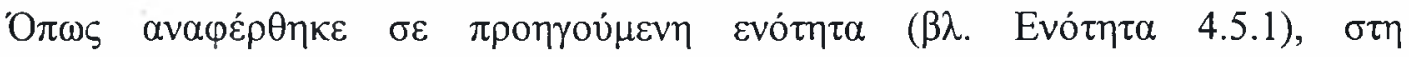

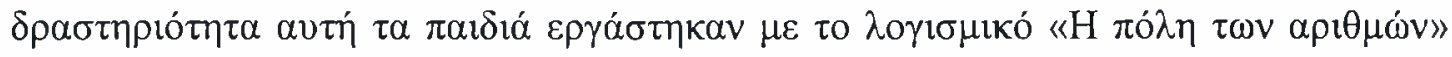

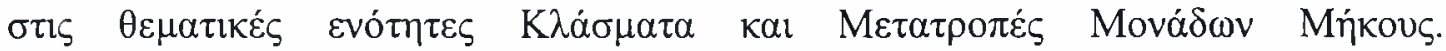

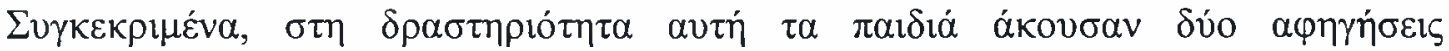

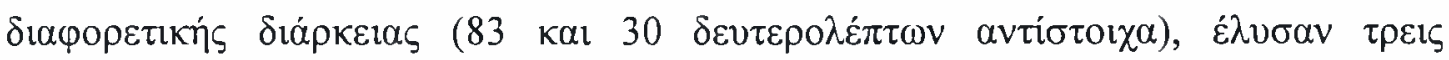

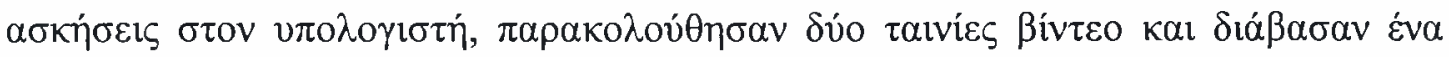

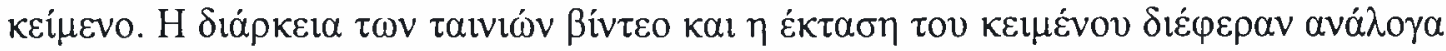

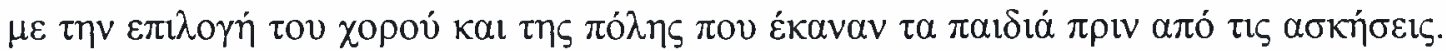

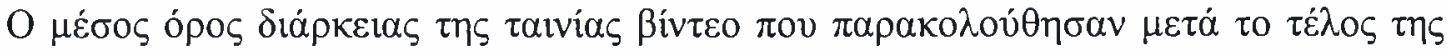

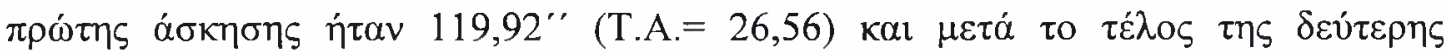

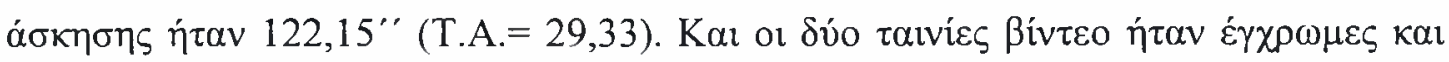

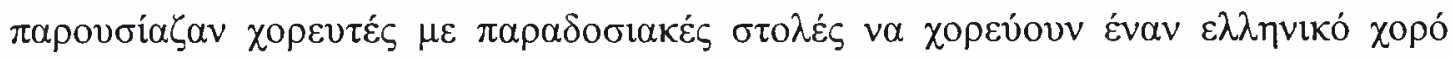

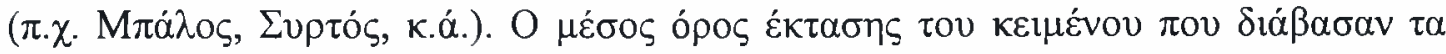

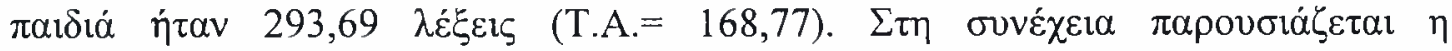

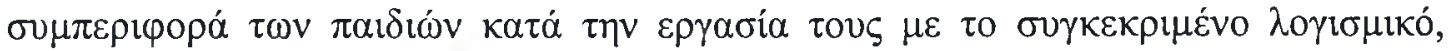

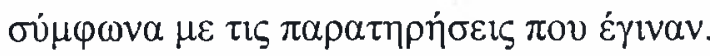




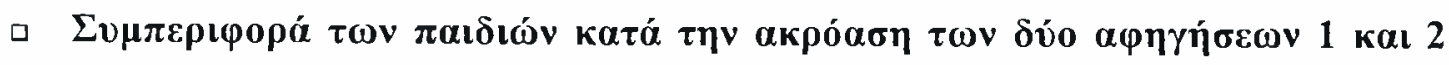
(83 каı $30 \delta \varepsilon v \tau \varepsilon \rho 0 \lambda \varepsilon ́ \pi \tau \omega v \alpha v \tau i ́ \sigma \tau 0 \iota \chi \alpha)$

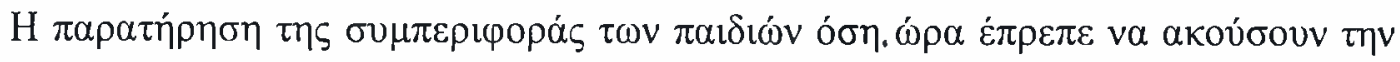

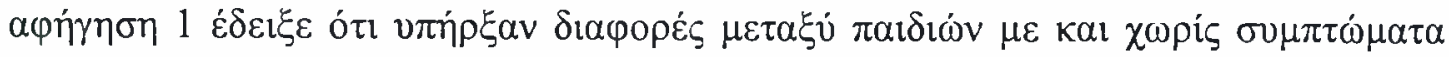

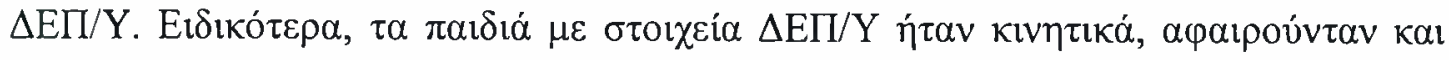

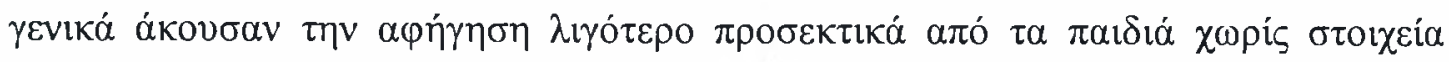

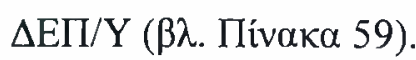

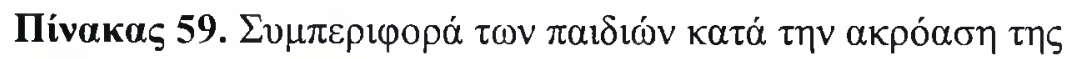

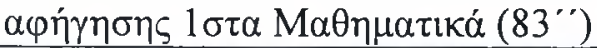

\begin{tabular}{|c|c|c|c|c|}
\hline \multirow{3}{*}{ 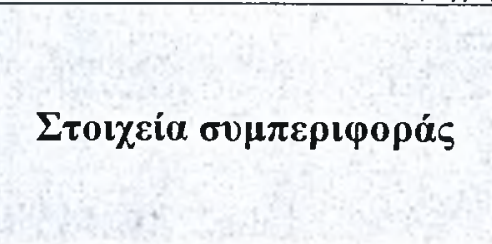 } & \multicolumn{4}{|c|}{ 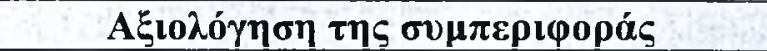 } \\
\hline & \multicolumn{2}{|c|}{ 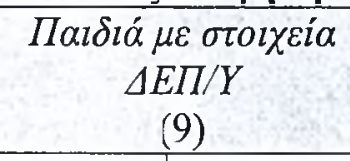 } & \multicolumn{2}{|c|}{ 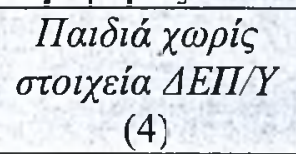 } \\
\hline & M.O. & T.A. & M.O. & T.A. \\
\hline 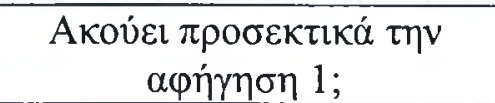 & 2,00 & 0,71 & 2,75 & 0,50 \\
\hline 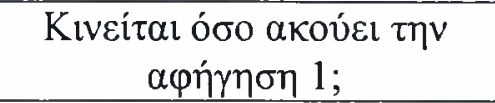 & 1,67 & 0,50 & 1,00 & 0,00 \\
\hline 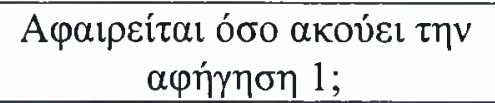 & 1,89 & 0,60 & 1,25 & 0,50 \\
\hline
\end{tabular}

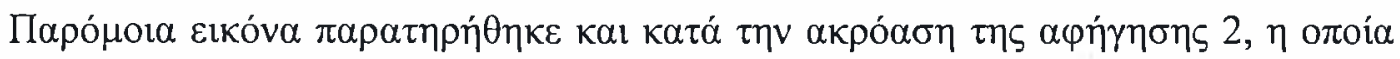

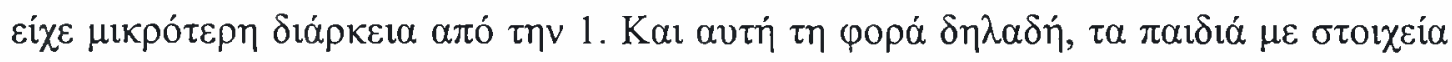

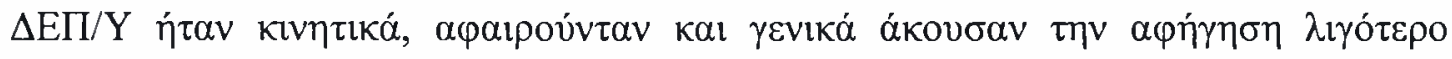

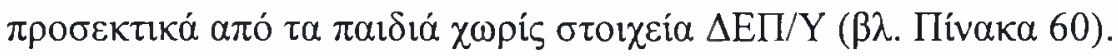

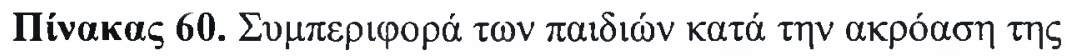

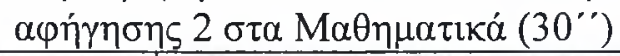

\begin{tabular}{|c|c|c|c|c|}
\hline \multirow{3}{*}{ 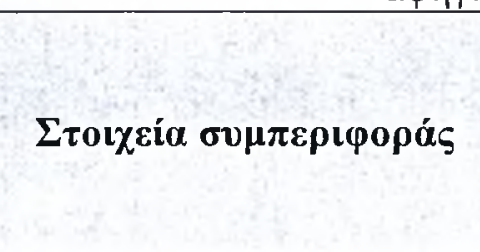 } & \multicolumn{4}{|c|}{ 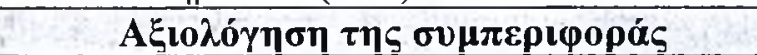 } \\
\hline & \multicolumn{2}{|c|}{$\begin{array}{c}\Pi \alpha \iota \delta l \dot{\alpha} \mu \varepsilon \sigma \tau o l \chi \varepsilon i \alpha \\
\Delta E \Pi / Y \\
(9)\end{array}$} & \multicolumn{2}{|c|}{ 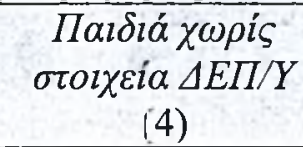 } \\
\hline & M.O. & T.A. & M.O. & T.A. \\
\hline 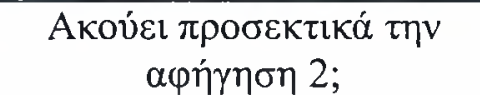 & 2,11 & 0,93 & 2,75 & 0,50 \\
\hline 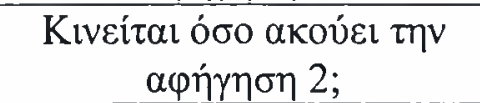 & 1,56 & 0,53 & 1,00 & 0,00 \\
\hline 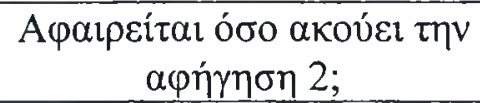 & 1,89 & 1,05 & 1,25 & 0,50 \\
\hline
\end{tabular}




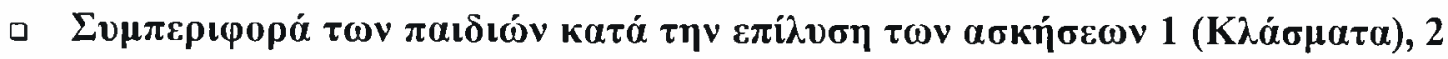

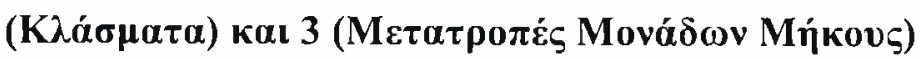

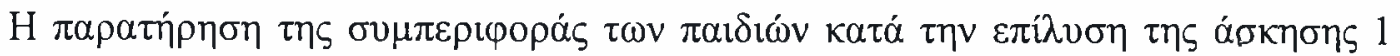

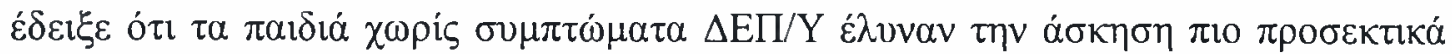

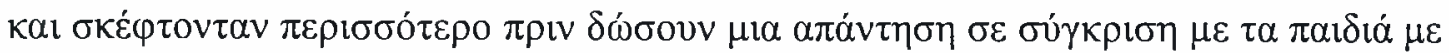

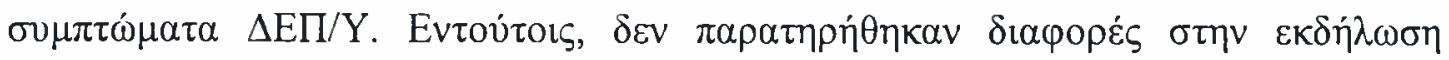

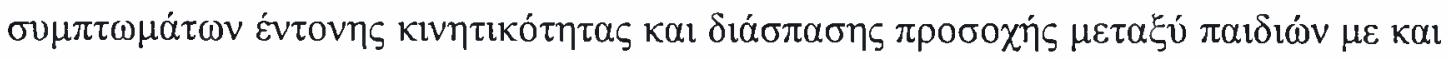

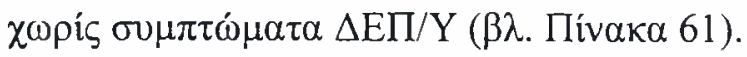

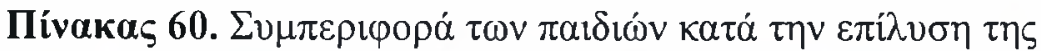
$\dot{\alpha} \sigma \kappa \eta \sigma \eta \varsigma \mathrm{I} \sigma \tau \alpha \mathrm{M} \alpha \theta \eta \mu \alpha \tau \iota \kappa \dot{~}$

\begin{tabular}{|c|c|c|c|c|}
\hline \multirow{3}{*}{ 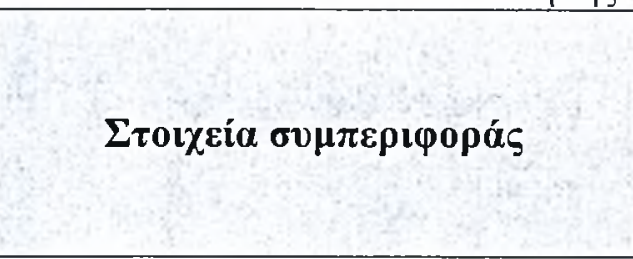 } & \multicolumn{4}{|c|}{ 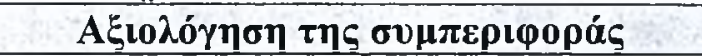 } \\
\hline & \multicolumn{2}{|c|}{ 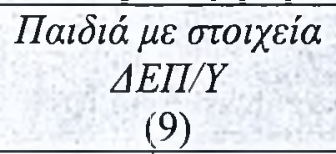 } & \multicolumn{2}{|c|}{ 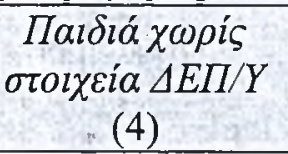 } \\
\hline & M.O. & T.A. & M.O. & T.A. \\
\hline 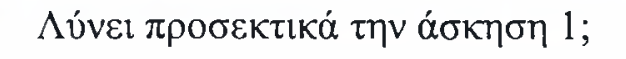 & 2,22 & 0,44 & 2,75 & 0,50 \\
\hline 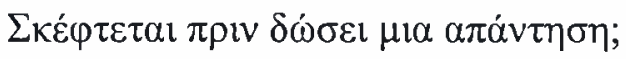 & 2,11 & 0,60 & 2,75 & 0,50 \\
\hline 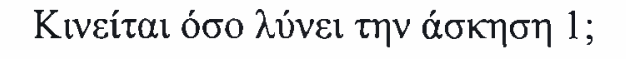 & 1,00 & 0,00 & 1,00 & 0,00 \\
\hline 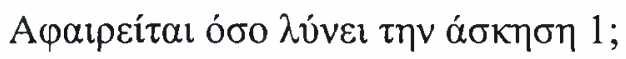 & 1,00 & 0,00 & 1,00 & 0,00 \\
\hline
\end{tabular}

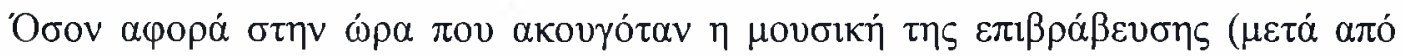

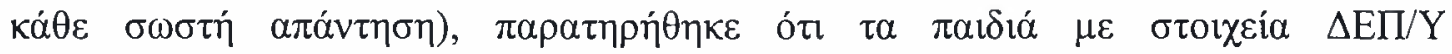

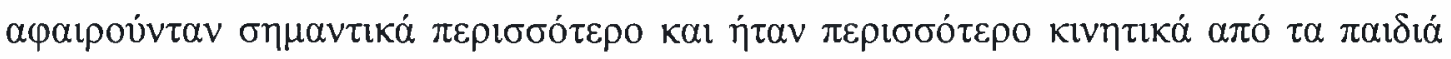
$\chi \omega$ pís $\Delta \mathrm{E \Pi /Y} \mathrm{( \beta \lambda .} \mathrm{Пív \alpha \kappa \alpha} \mathrm{62).}$

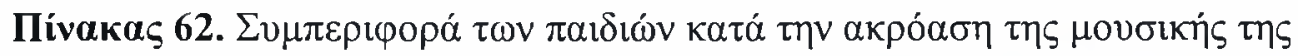

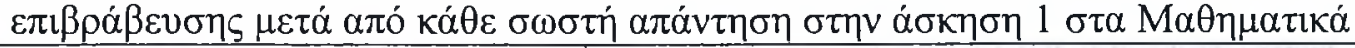

\begin{tabular}{|c|c|c|c|c|}
\hline \multirow{3}{*}{ 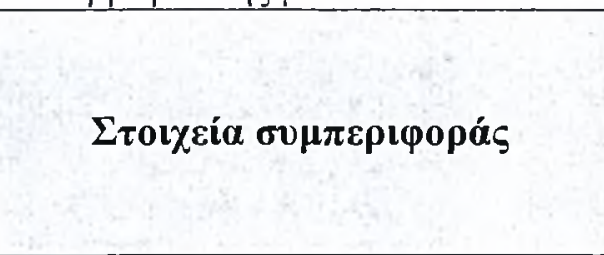 } & \multicolumn{4}{|c|}{ 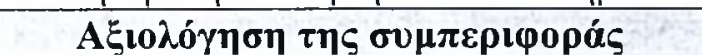 } \\
\hline & \multicolumn{2}{|c|}{$\begin{array}{c}\Pi \alpha \iota \delta \imath \dot{\alpha} \mu \varepsilon \sigma \tau o \imath \chi \varepsilon i \alpha \\
\Delta E \Pi / Y \\
(9)\end{array}$} & \multicolumn{2}{|c|}{ 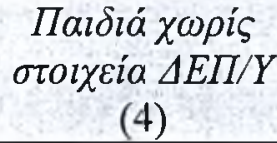 } \\
\hline & M.O. & T.A. & M.O. & T.A. \\
\hline 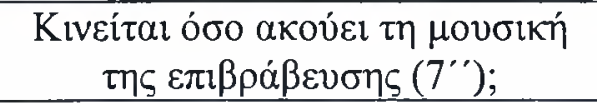 & 1,89 & 0,78 & 1,50 & 0,58 \\
\hline 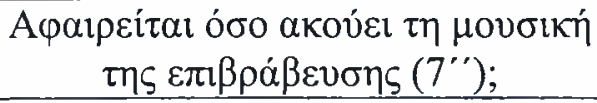 & 1,67 & 0,71 & 1,00 & 0,00 \\
\hline
\end{tabular}




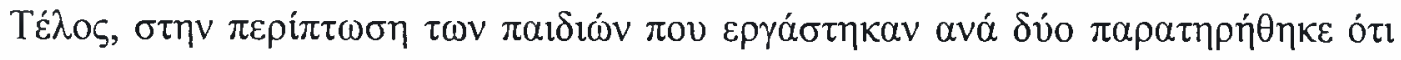

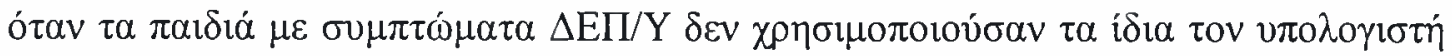

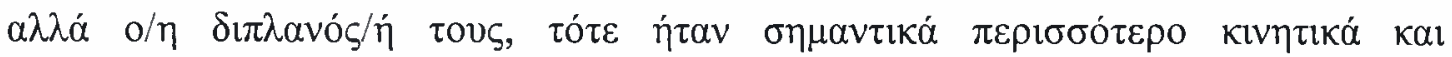

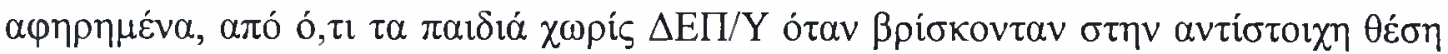
( $\beta \lambda$. Пiv $\alpha \kappa \alpha 63)$.

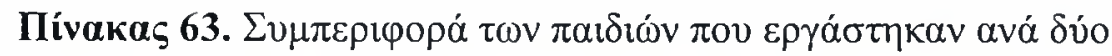

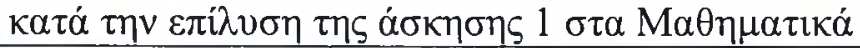

\begin{tabular}{|c|c|c|c|c|}
\hline \multirow{3}{*}{ 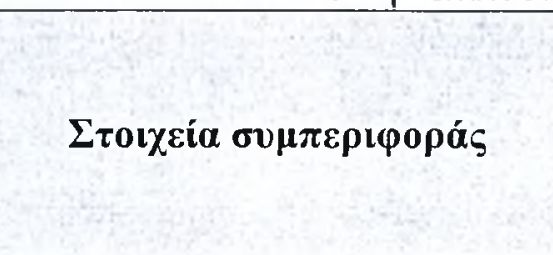 } & \multicolumn{4}{|c|}{ 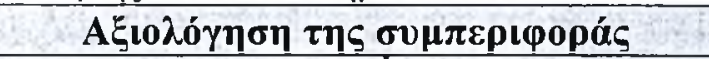 } \\
\hline & \multicolumn{2}{|c|}{ 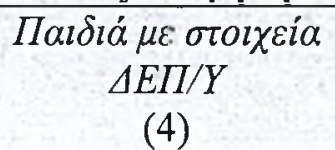 } & \multicolumn{2}{|c|}{ 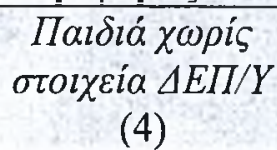 } \\
\hline & M.O. & T.A. & M.O. & T.A. \\
\hline 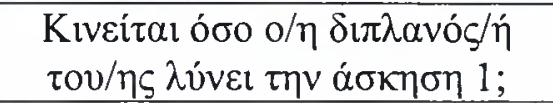 & 2,25 & 0,50 & 1,00 & 0,00 \\
\hline 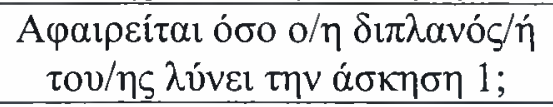 & 2,00 & 0,00 & 1,00 & 0,00 \\
\hline
\end{tabular}

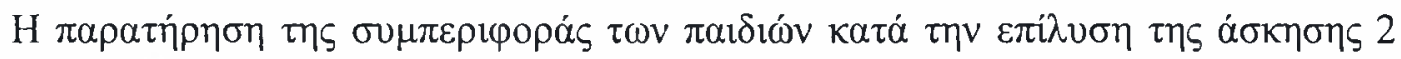

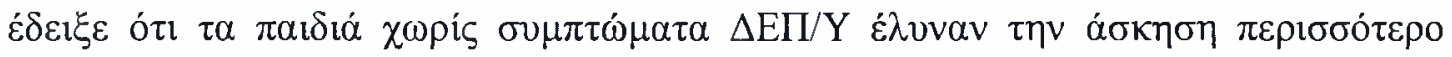

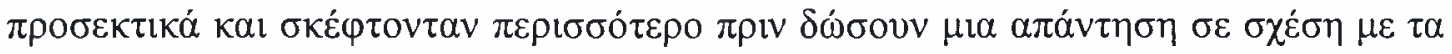

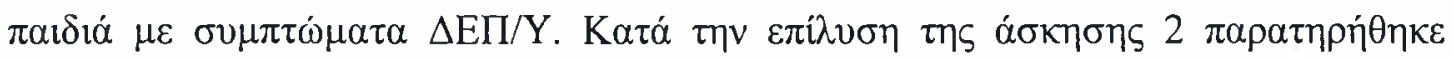

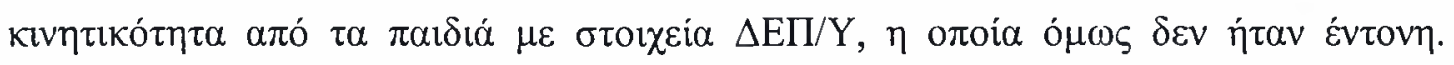

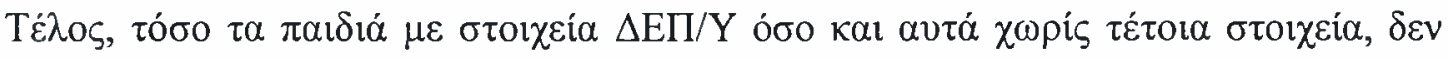

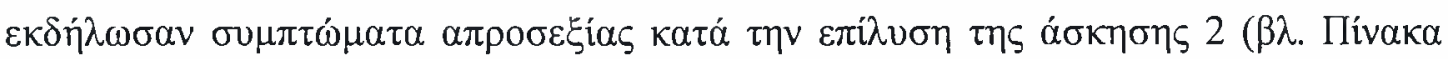
64).

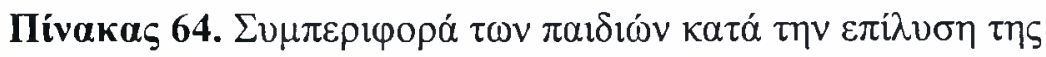

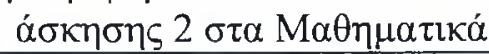

\begin{tabular}{|c|c|c|c|c|}
\hline \multirow{3}{*}{ 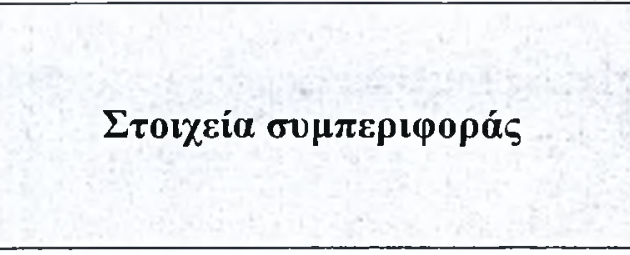 } & \multicolumn{4}{|c|}{ 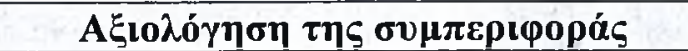 } \\
\hline & \multicolumn{2}{|c|}{ 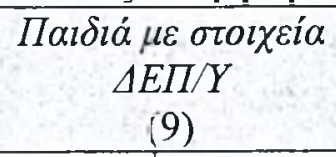 } & \multicolumn{2}{|c|}{ 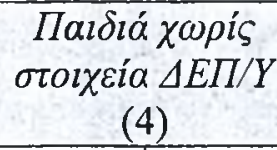 } \\
\hline & M.O. & T.A. & M.O. & T.A. \\
\hline 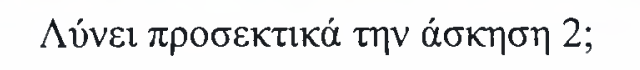 & 2,56 & 0,53 & 3,00 & 0,00 \\
\hline 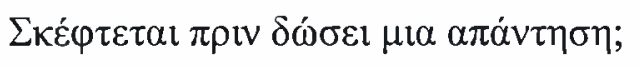 & 2,56 & 0,53 & 3,00 & 0,00 \\
\hline 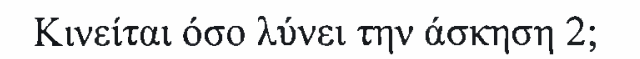 & 1,33 & 0,50 & 1,00 & 0,00 \\
\hline
\end{tabular}




\begin{tabular}{|c|c|c|c|c|}
\hline A & 1,00 & 0,00 & 1,00 & 0,00 \\
\hline
\end{tabular}

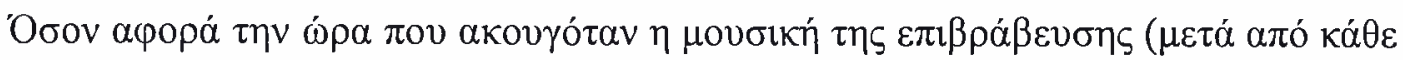

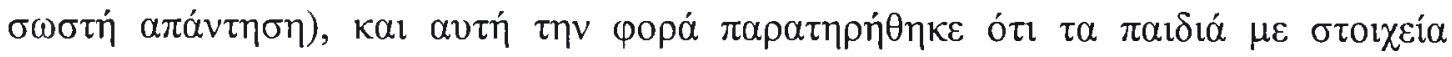

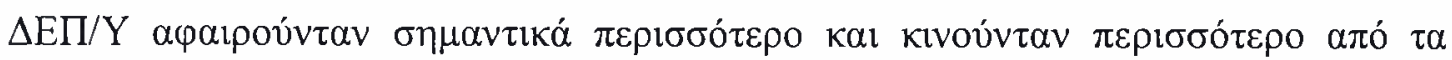

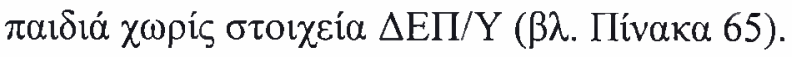

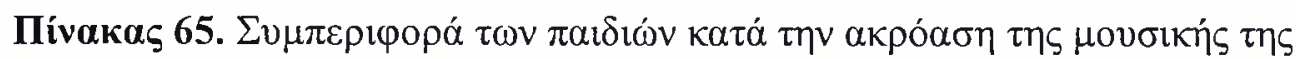

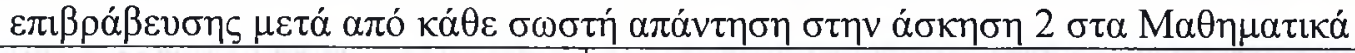

\begin{tabular}{|c|c|c|c|c|}
\hline \multirow{3}{*}{ 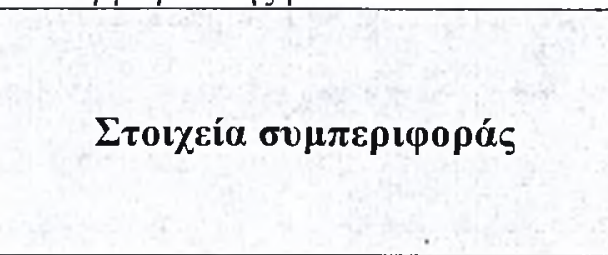 } & \multicolumn{4}{|c|}{ 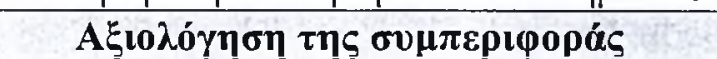 } \\
\hline & \multicolumn{2}{|c|}{ 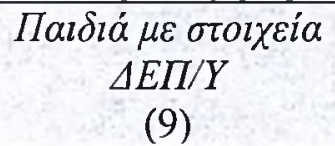 } & \multicolumn{2}{|c|}{ 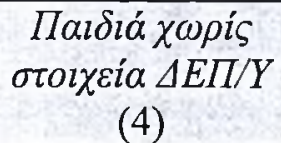 } \\
\hline & M.O. & T.A. & M.O. & T.A. \\
\hline 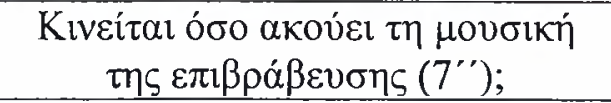 & 1,78 & 0,67 & 1,50 & 0,58 \\
\hline 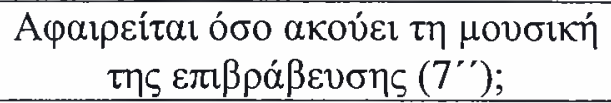 & 1,44 & 0,53 & 1,00 & 0,00 \\
\hline
\end{tabular}

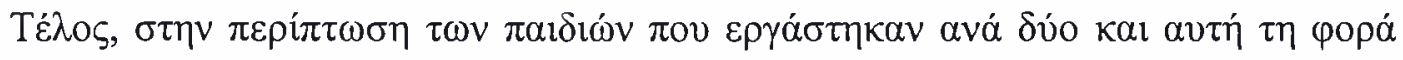

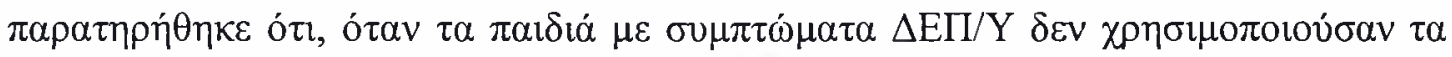

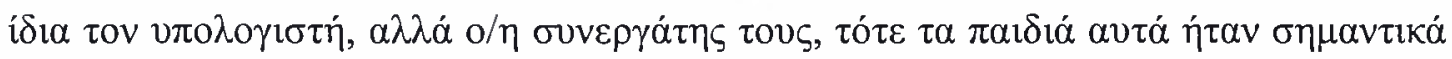

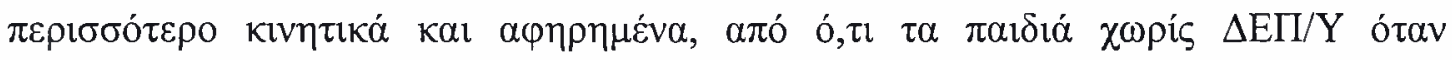

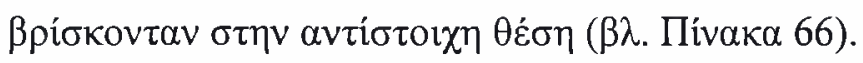

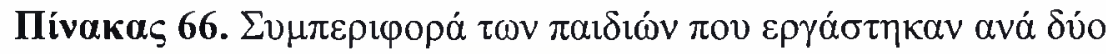

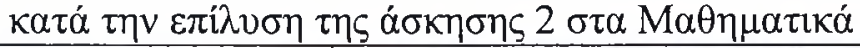

\begin{tabular}{|c|c|c|c|c|}
\hline \multirow{3}{*}{ 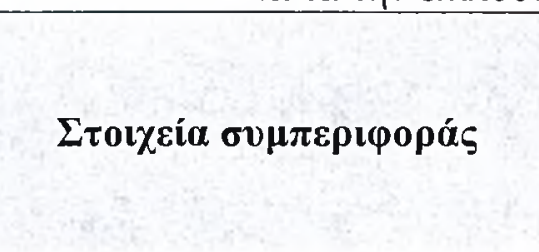 } & \multicolumn{4}{|c|}{ 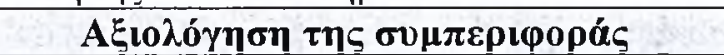 } \\
\hline & \multicolumn{2}{|c|}{ 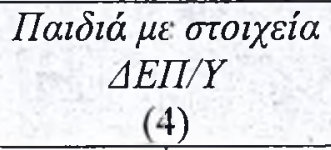 } & \multicolumn{2}{|c|}{ 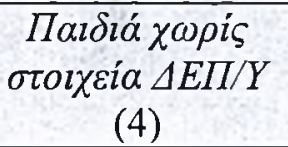 } \\
\hline & M.O. & T.A. & M.O. & T.A. \\
\hline 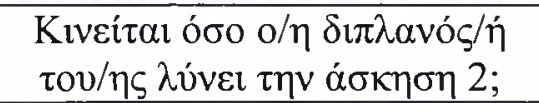 & 2,25 & 0,50 & 1,00 & 0,00 \\
\hline 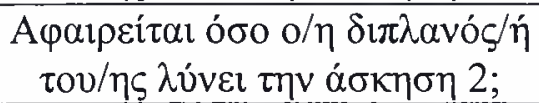 & 1,75 & 0,50 & 1,00 & 0,00 \\
\hline
\end{tabular}

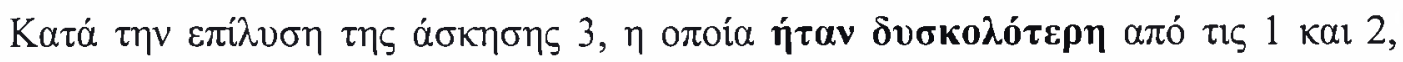

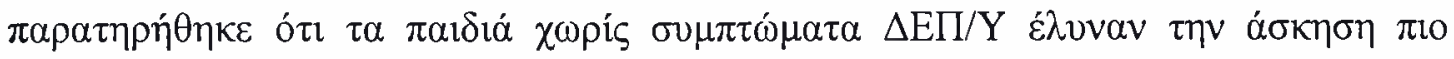

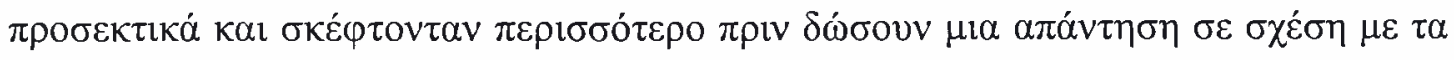




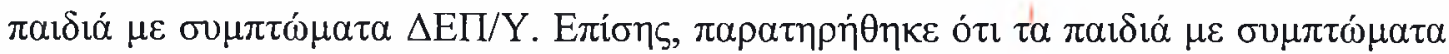

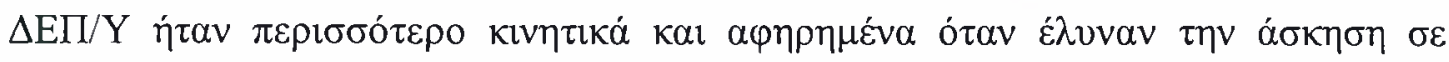

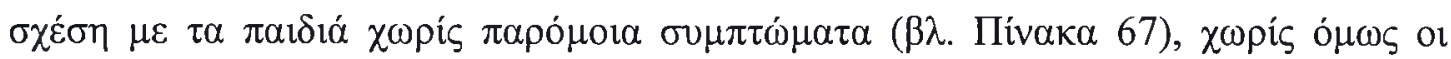

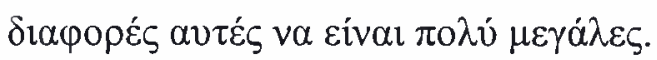

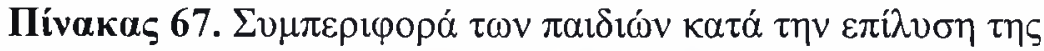
$\alpha \dot{\sigma} \sigma \eta \sigma \eta \varsigma 3 \sigma \tau \alpha \mathrm{M} \alpha \theta \eta \mu \alpha \tau \iota \kappa \alpha ́$

\begin{tabular}{|c|c|c|c|c|}
\hline \multirow{3}{*}{$\Sigma \tau 0\llcorner\chi \varepsilon i ́ \alpha \sigma v \mu \pi \varepsilon \rho\llcorner\varphi о \rho \alpha ́ \varsigma$} & \multicolumn{4}{|c|}{ 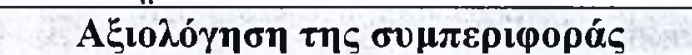 } \\
\hline & \multicolumn{2}{|c|}{ 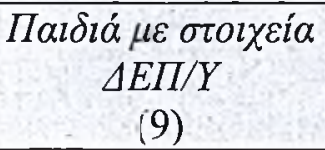 } & \multicolumn{2}{|c|}{ 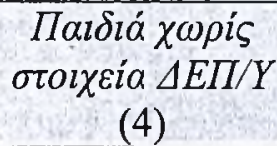 } \\
\hline & M.O. & T.A. & M.O. & T.A. \\
\hline 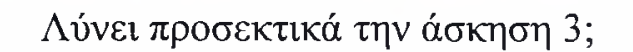 & 2,22 & 0,67 & 3,00 & 0,00 \\
\hline 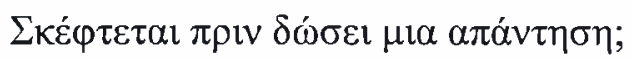 & 2,33 & 0,50 & 3,00 & 0,00 \\
\hline 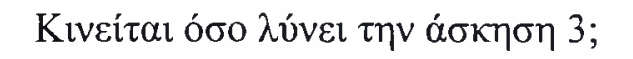 & 1,22 & 0,44 & 1,00 & 0,00 \\
\hline 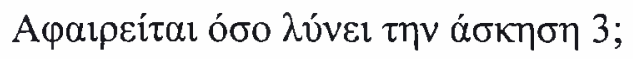 & 1,11 & 0,33 & 1,00 & 0,00 \\
\hline
\end{tabular}

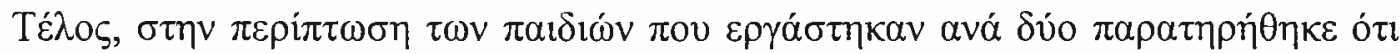

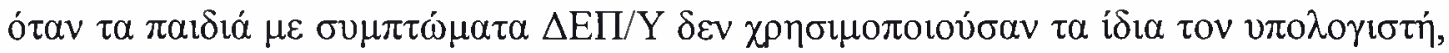

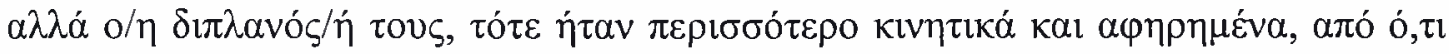

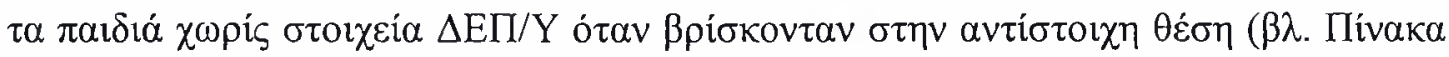

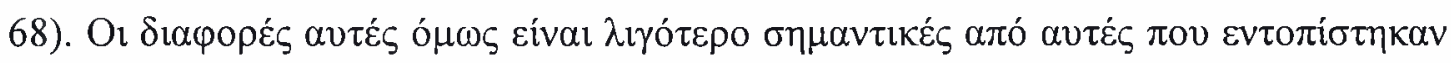

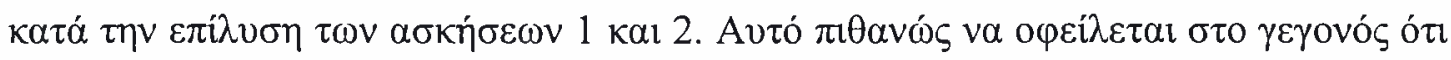

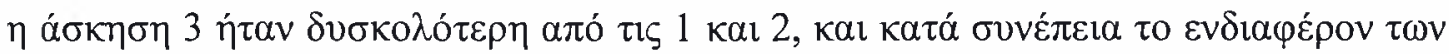

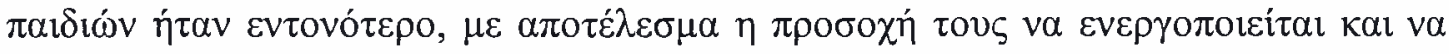

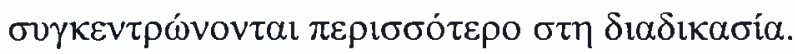

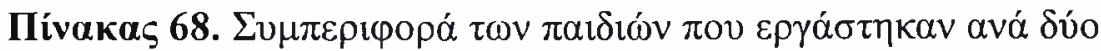

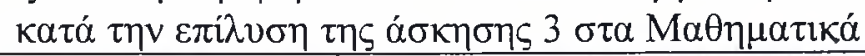

\begin{tabular}{|c|c|c|c|c|}
\hline \multirow{3}{*}{ 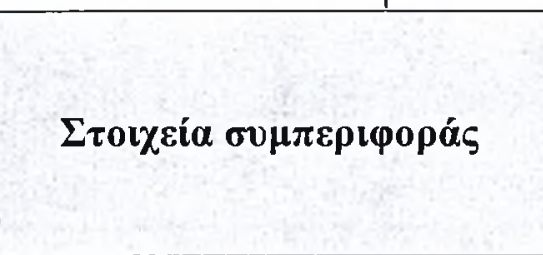 } & \multicolumn{4}{|c|}{ 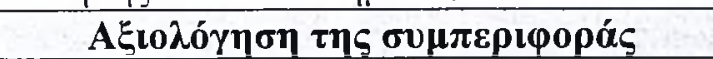 } \\
\hline & \multicolumn{2}{|c|}{ 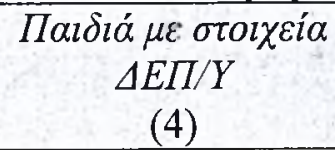 } & \multicolumn{2}{|c|}{ 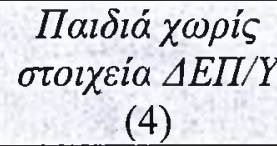 } \\
\hline & M.O. & T.A. & M.O. & T.A. \\
\hline 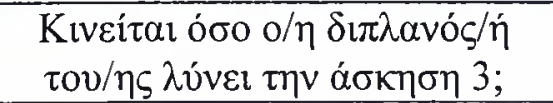 & 1,75 & 0,50 & 1,00 & 0,00 \\
\hline 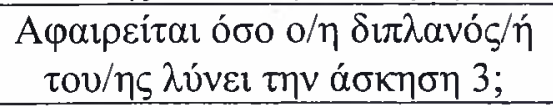 & 1,50 & 0,58 & 1,00 & 0,00 \\
\hline
\end{tabular}




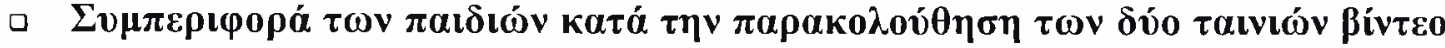 $\sigma \tau \alpha \operatorname{M\alpha \theta \eta \mu \alpha \tau \iota \kappa ó~}$}

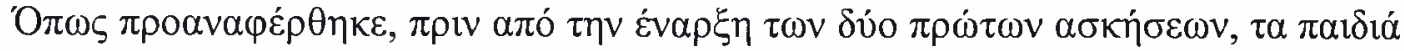

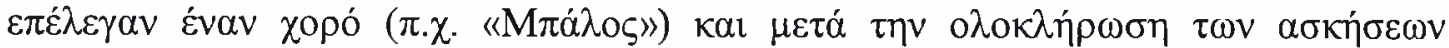

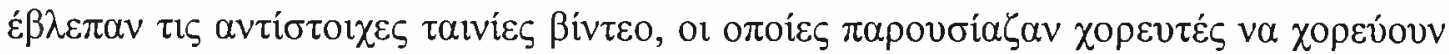

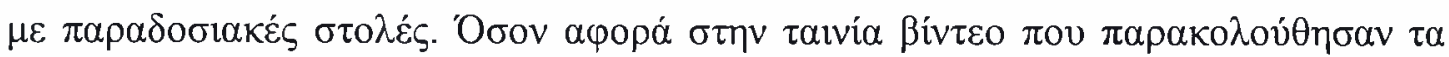

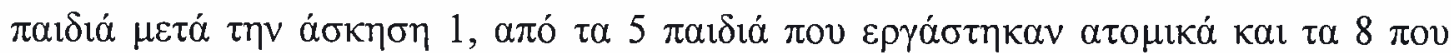

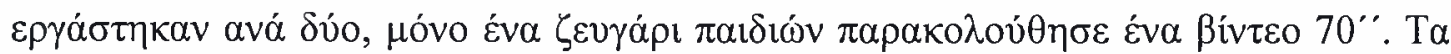

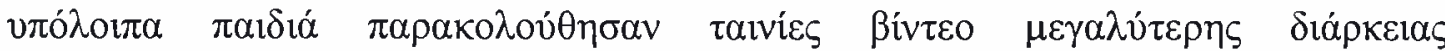

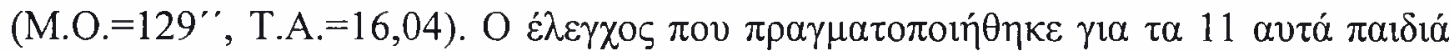

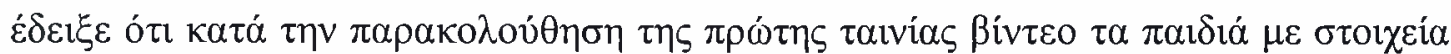

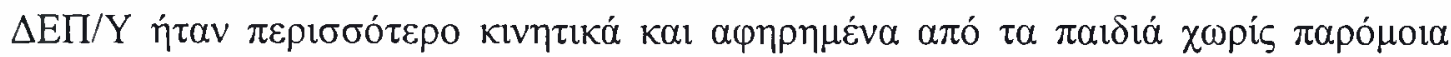

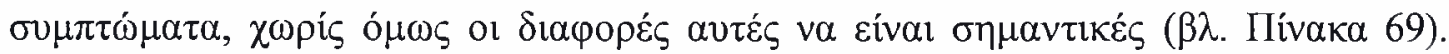

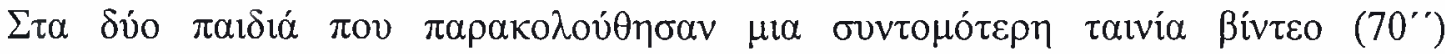

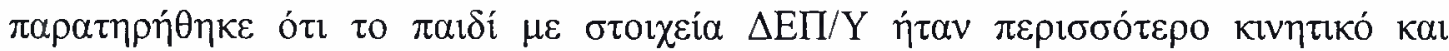

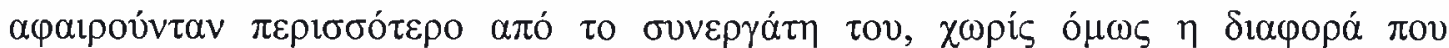

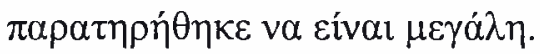

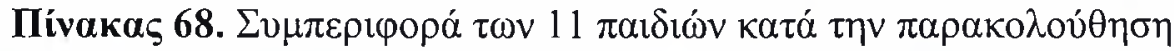

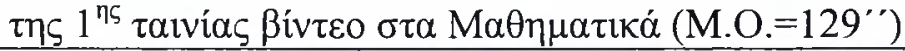

\begin{tabular}{|c|c|c|c|c|}
\hline \multirow{3}{*}{ 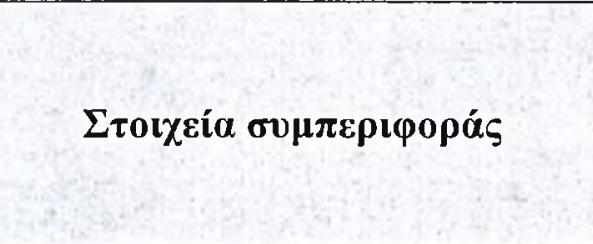 } & \multicolumn{4}{|c|}{ 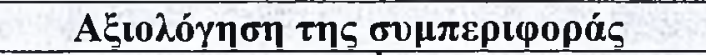 } \\
\hline & \multicolumn{2}{|c|}{ 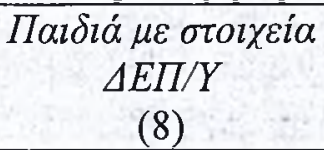 } & \multicolumn{2}{|c|}{ 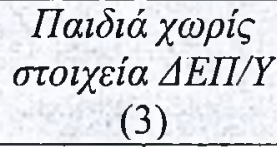 } \\
\hline & M.O. & T.A. & M.O. & T.A. \\
\hline 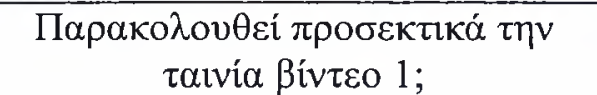 & 2,38 & 0,74 & 2,67 & 0,58 \\
\hline 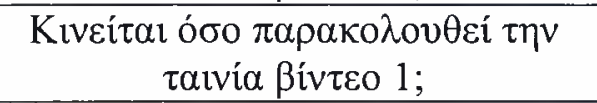 & 1,88 & 0,83 & 1,33 & 0,58 \\
\hline 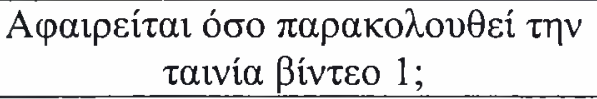 & 1,50 & 0,76 & 1,33 & 0,58 \\
\hline
\end{tabular}

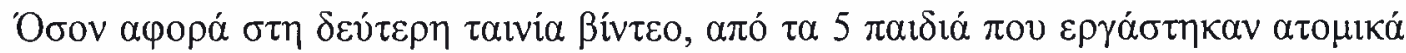

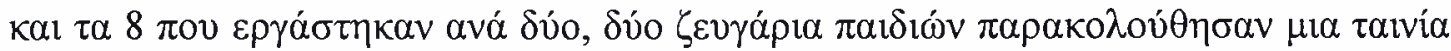

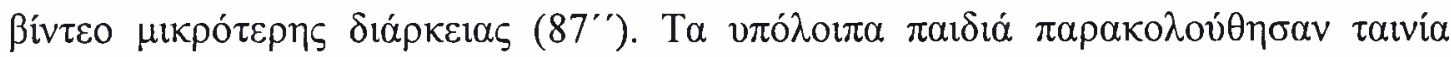

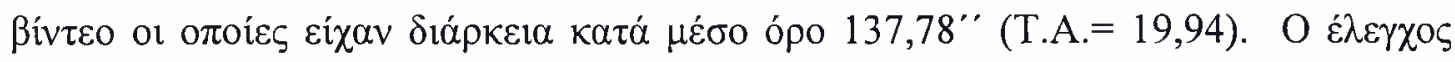

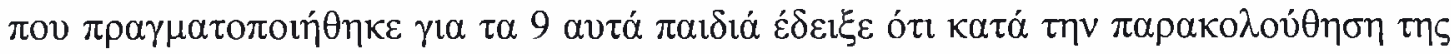




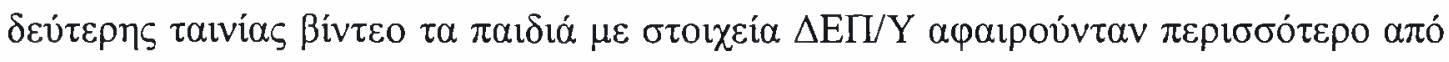

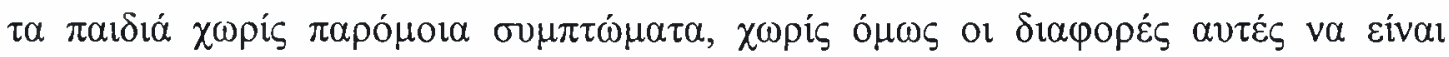

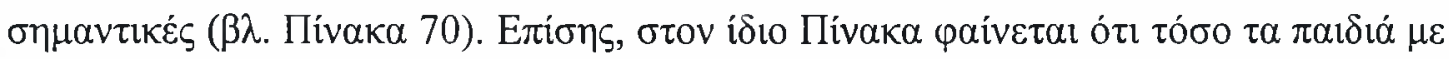

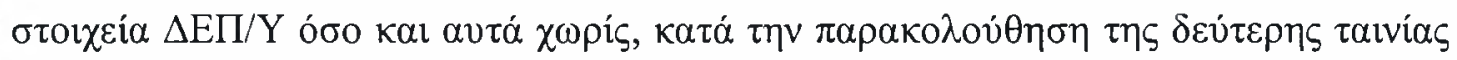

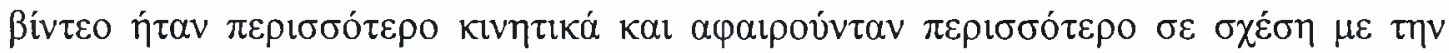

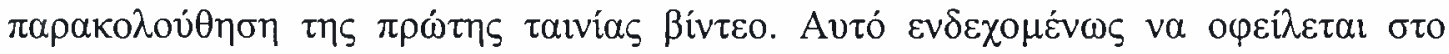

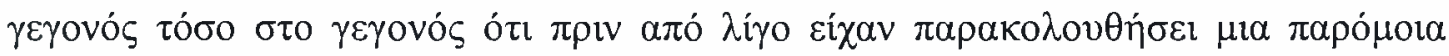

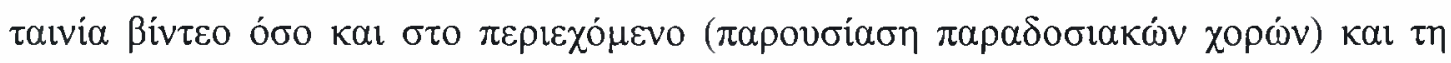

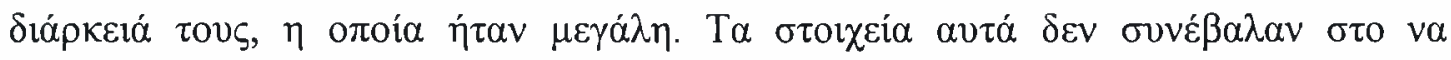

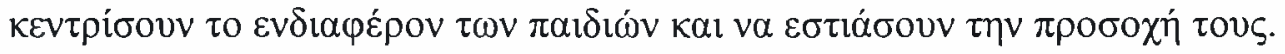

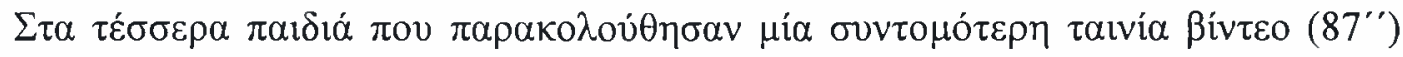

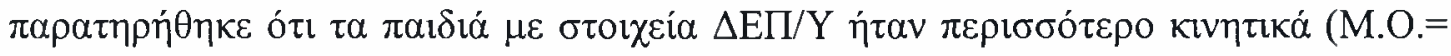

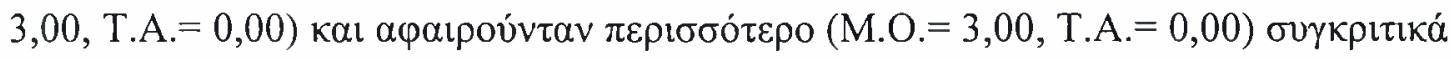

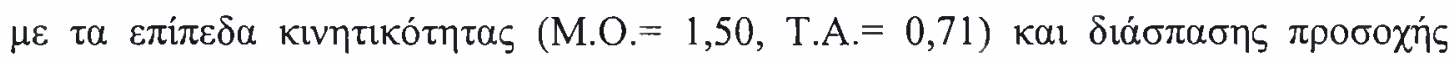

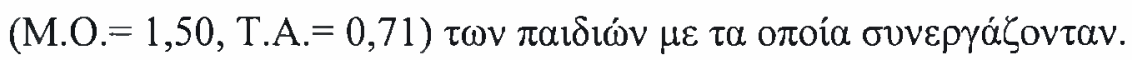

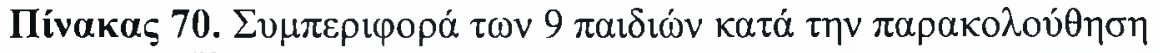

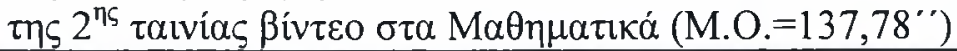

\begin{tabular}{|c|c|c|c|c|}
\hline \multirow{3}{*}{ 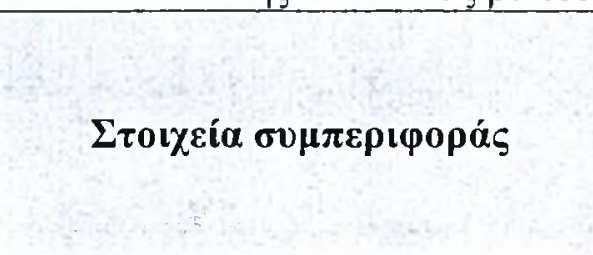 } & \multicolumn{4}{|c|}{ 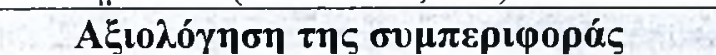 } \\
\hline & \multicolumn{2}{|c|}{ 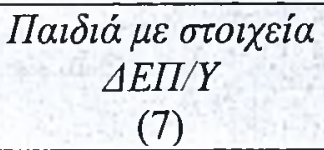 } & \multicolumn{2}{|c|}{ 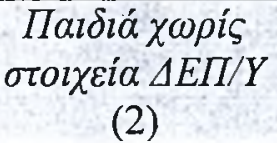 } \\
\hline & M.O. & T.A. & M.O. & T.A. \\
\hline 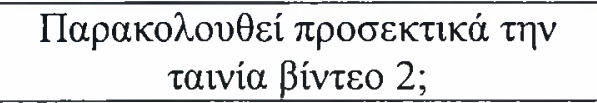 & 2,29 & 0,76 & 2,50 & 0,71 \\
\hline 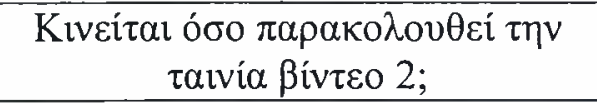 & 2,00 & 0,82 & 2,00 & 0,00 \\
\hline 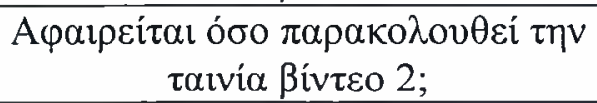 & 2,00 & 1,00 & 1,50 & 0,71 \\
\hline
\end{tabular}

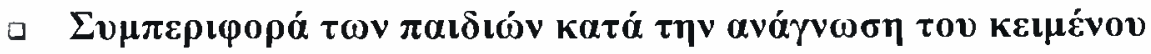

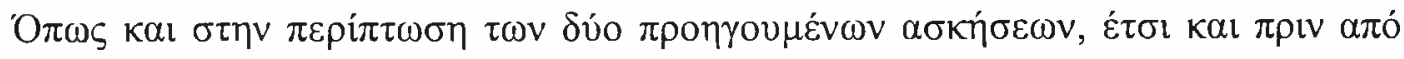

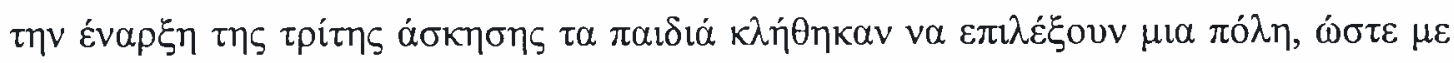

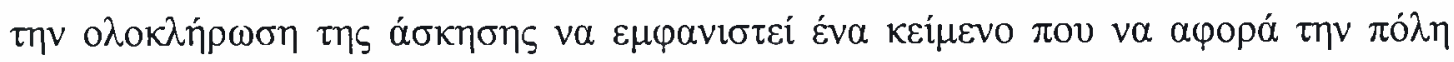

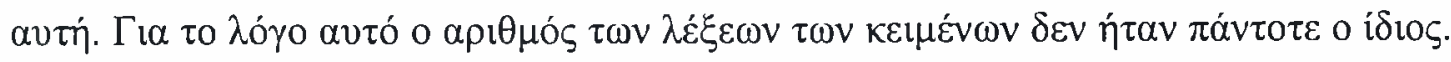

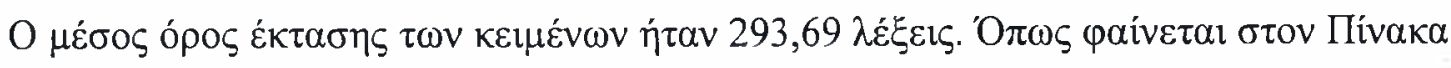

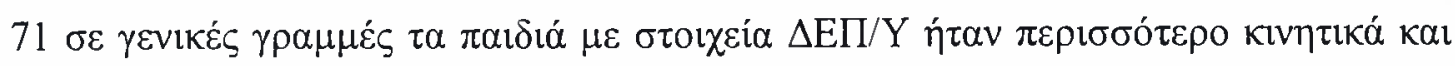




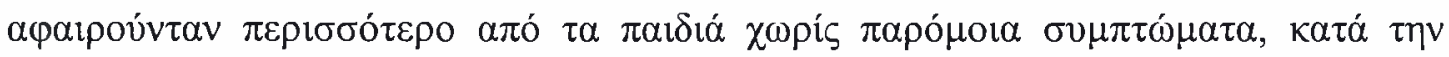

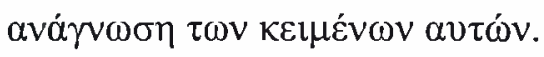

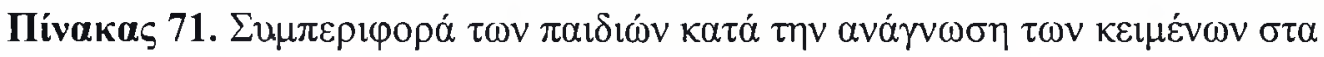

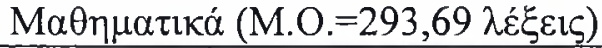

\begin{tabular}{|c|c|c|c|c|}
\hline \multirow{3}{*}{ 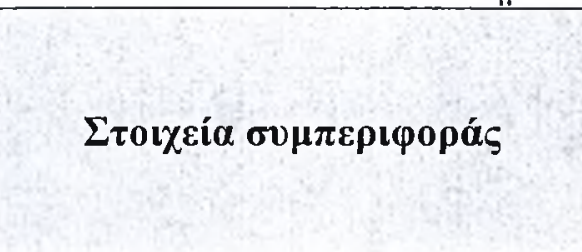 } & \multicolumn{4}{|c|}{ 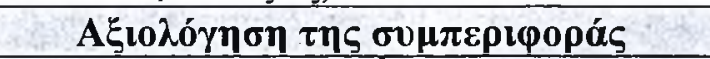 } \\
\hline & \multicolumn{2}{|c|}{ 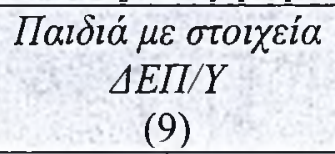 } & \multicolumn{2}{|c|}{ 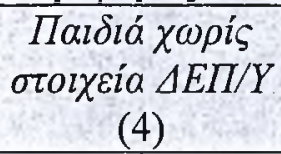 } \\
\hline & M.O. & T.A. & M.O. & T.A. \\
\hline 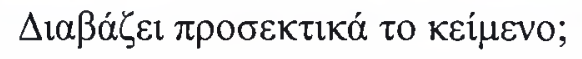 & 2,22 & 0,83 & 2,25 & 0,96 \\
\hline 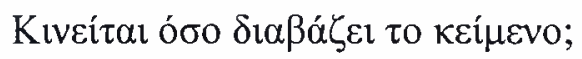 & 1,78 & 0,83 & 1,25 & 0,50 \\
\hline 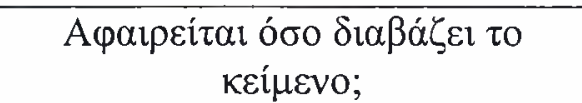 & 1,56 & 0,88 & 1,25 & 0,50 \\
\hline
\end{tabular}

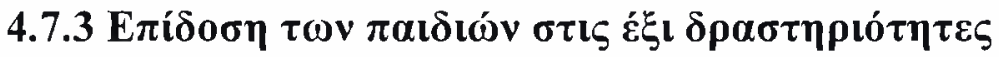

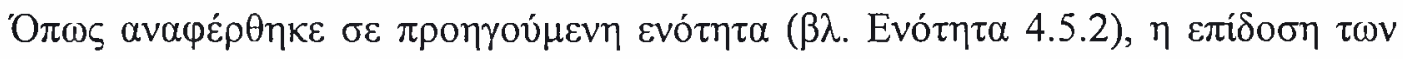

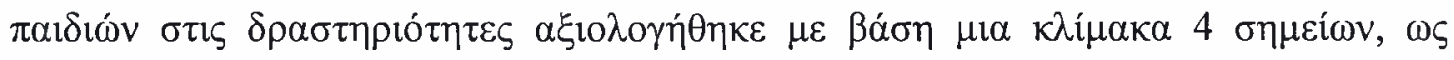

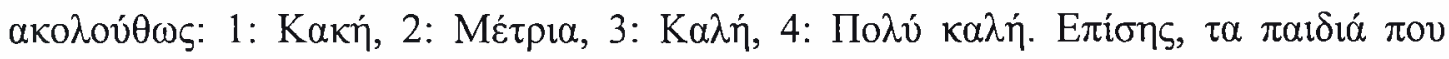

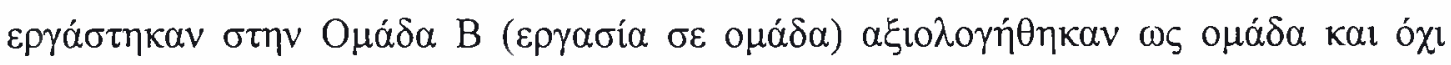

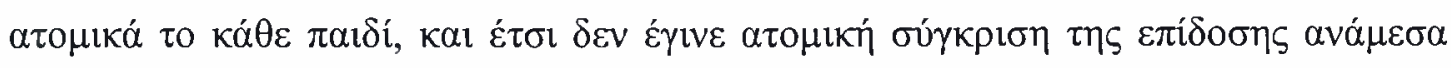

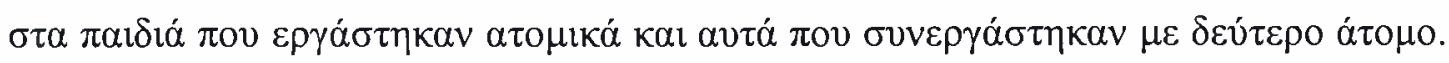

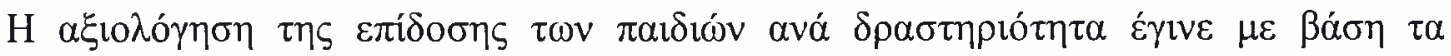

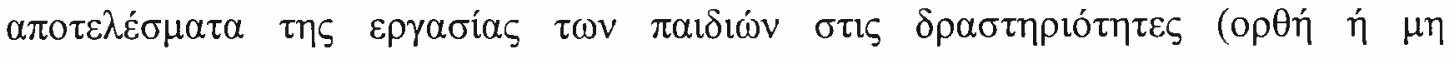

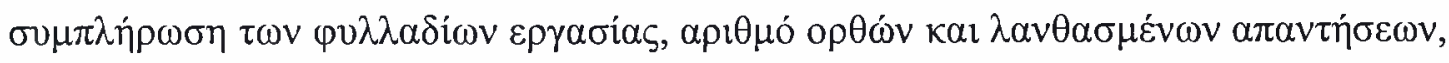

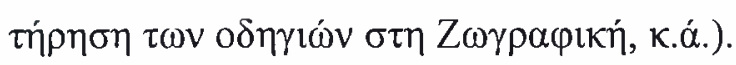

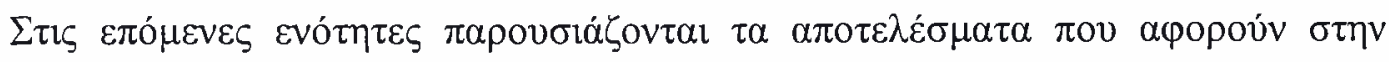

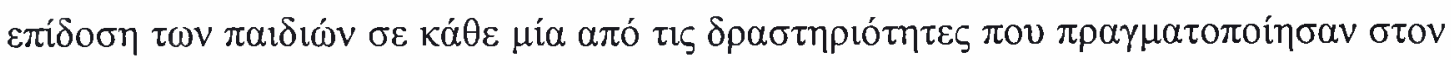

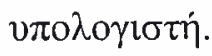

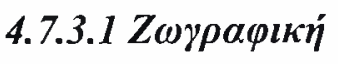

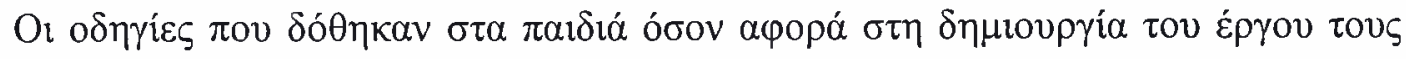

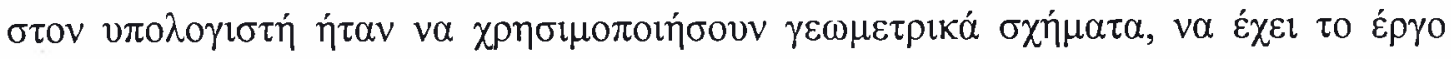




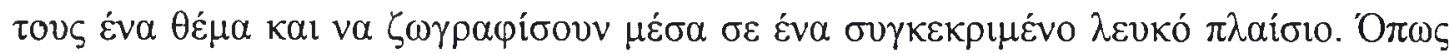

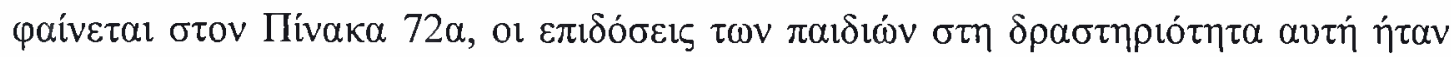

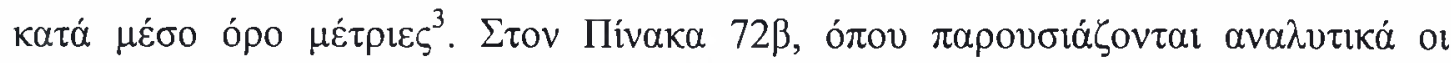

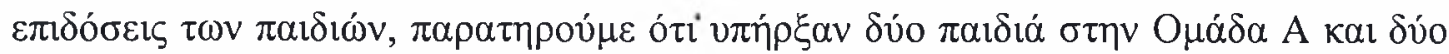

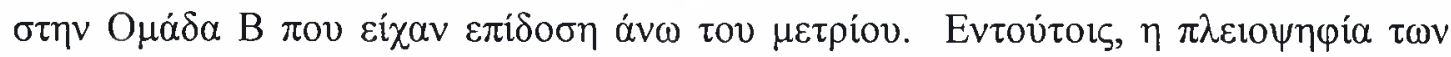

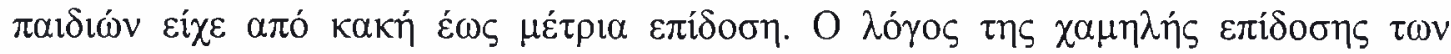

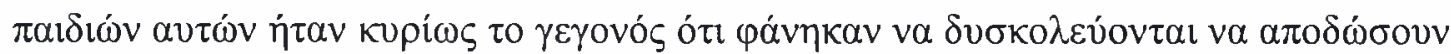

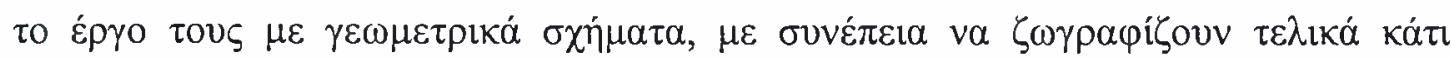

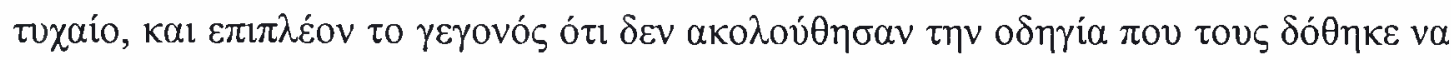

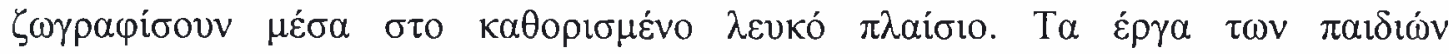

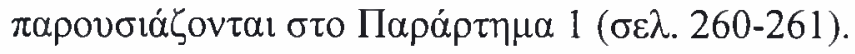

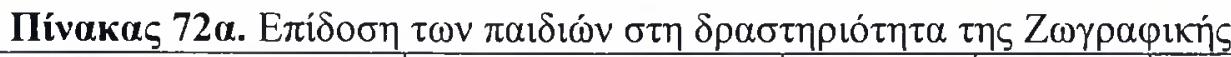

\begin{tabular}{|c|c|c|c|c|}
\hline & 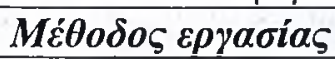 & $N(=13)$ & M.O. & T.A. \\
\hline \multirow{2}{*}{ 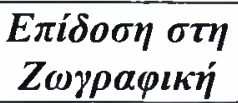 } & ATOMIKH & 5 & 2,20 & 0,84 \\
\hline & OMA $\triangle \mathrm{IKH}$ & 8 & 2,00 & 1,21 \\
\hline
\end{tabular}

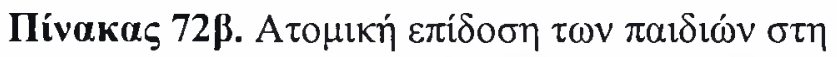

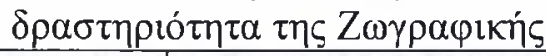

\begin{tabular}{|c|c|c|}
\hline 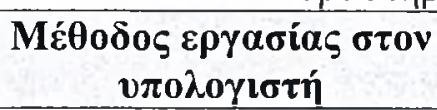 & 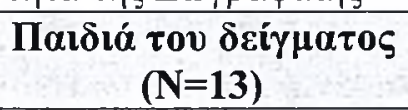 & 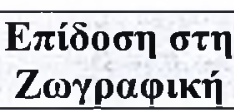 \\
\hline \multirow{5}{*}{ 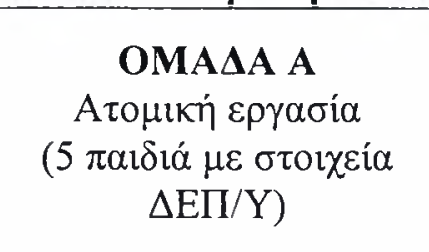 } & I. $A$. & 3 \\
\hline & P.H. & 2 \\
\hline & П.M. & 3 \\
\hline & $\Theta . \Sigma$ & 1 \\
\hline & T.П. & 2 \\
\hline \multirow{4}{*}{ 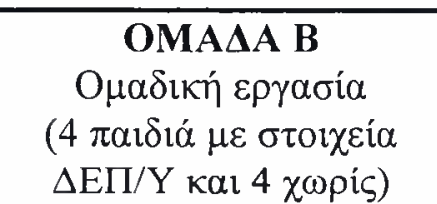 } & Z. $\Theta .-M . A$ & 1 \\
\hline & Г.Г. - Ф.П. & 2 \\
\hline & $Z . \Gamma .-\Sigma . \Lambda$ & 1 \\
\hline & B. $\Sigma .-$ Г.I. & 4 \\
\hline
\end{tabular}

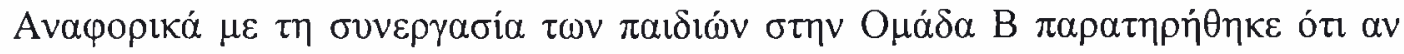

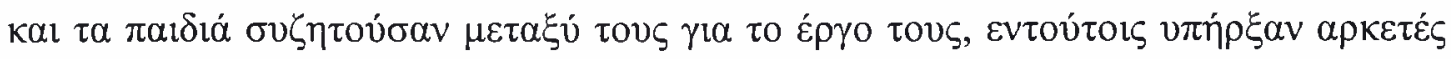

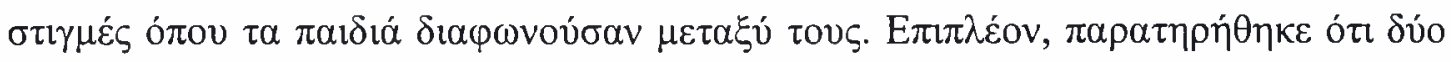

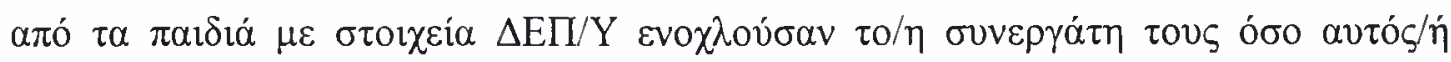

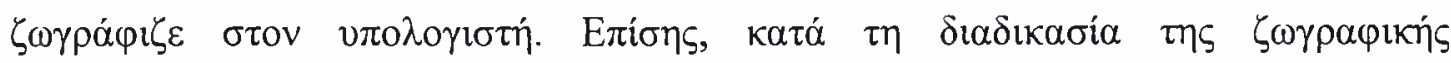

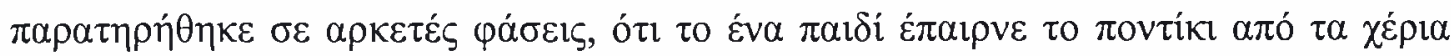

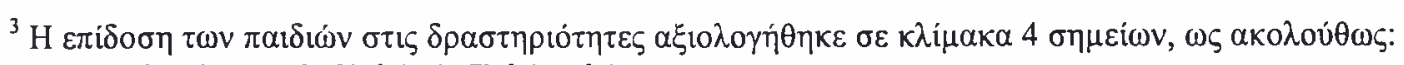

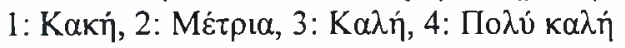




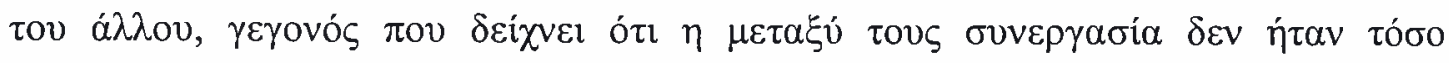

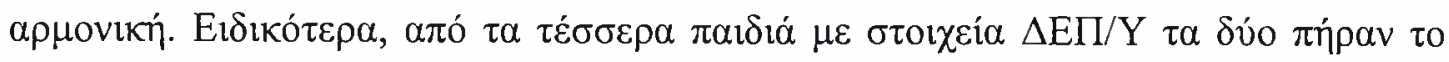

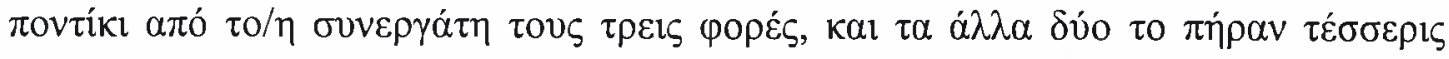

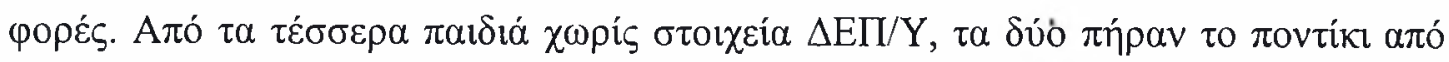

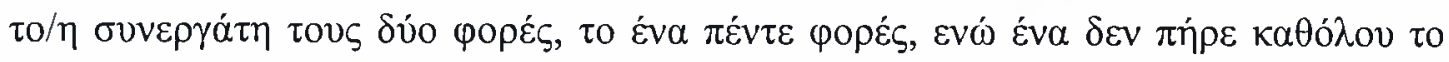

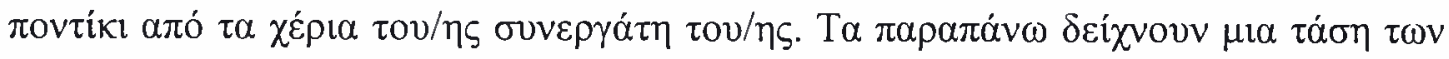

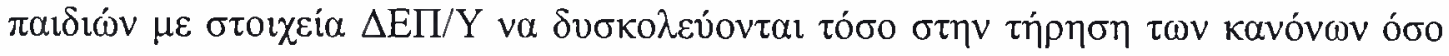

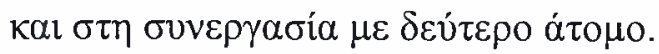

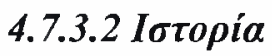

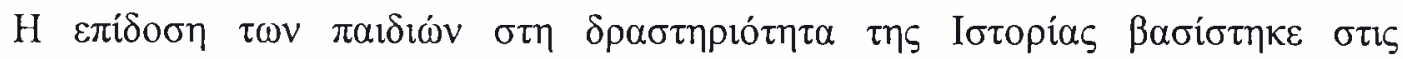

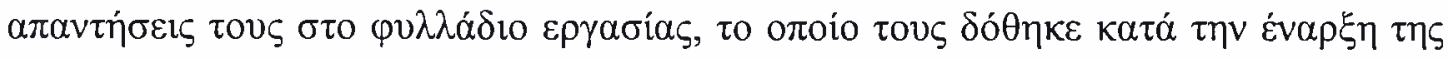

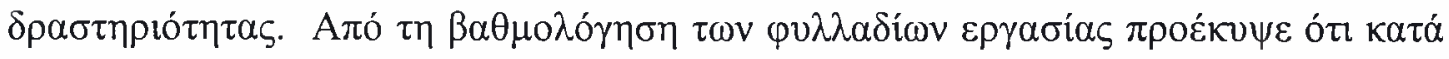

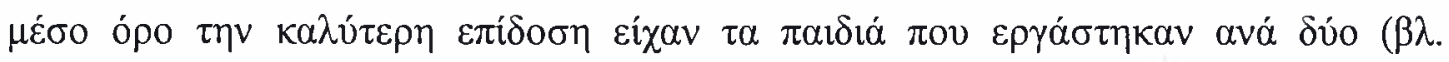

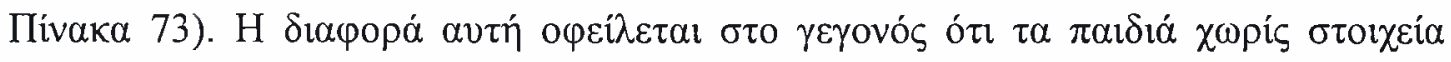

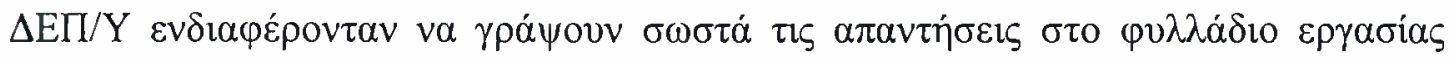

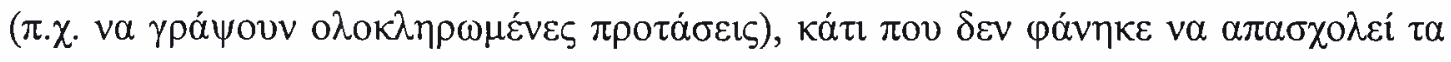

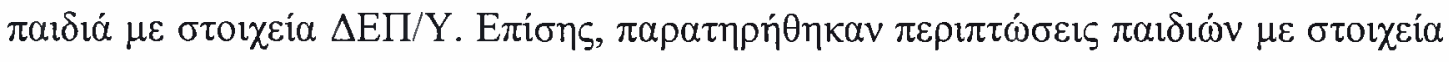

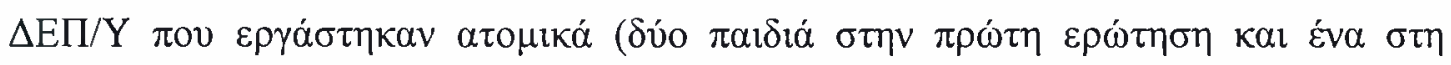

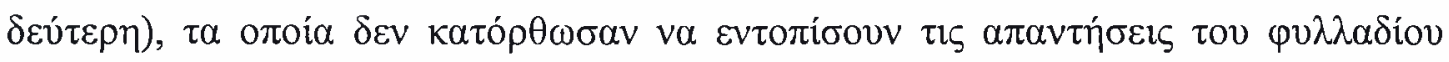

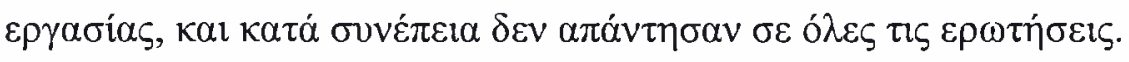

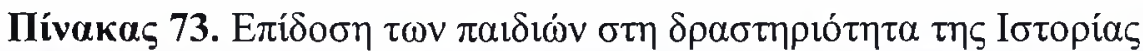

\begin{tabular}{|c|c|c|c|c|}
\hline & 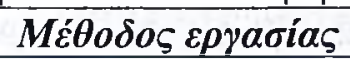 & $N(=13)$ & M.O. & T.A. \\
\hline \multirow{2}{*}{ 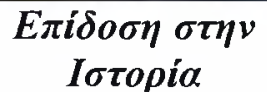 } & ATOMIKH & 5 & 2,20 & 0,84 \\
\hline & OMA $\triangle \mathrm{IKH}$ & 8 & 3,00 & 1,07 \\
\hline
\end{tabular}

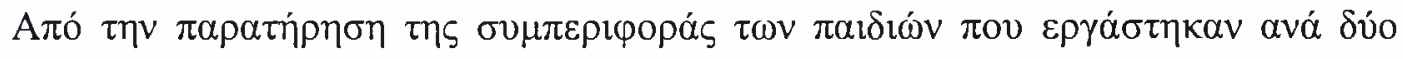

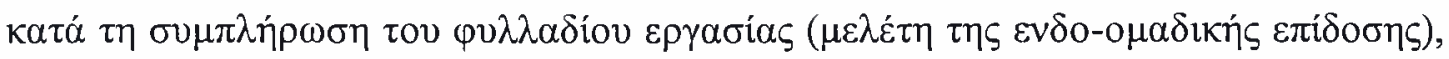
$\delta เ \alpha \pi \imath \tau \tau \omega ́ \theta \eta \kappa \alpha \nu \tau \alpha \alpha \kappa o ́ \lambda o v \theta \alpha$ :

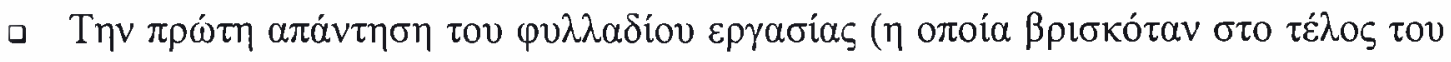

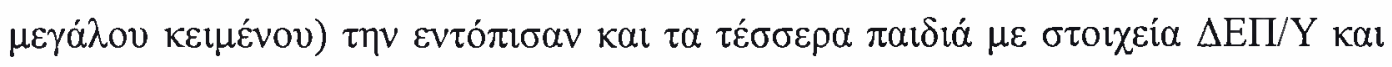

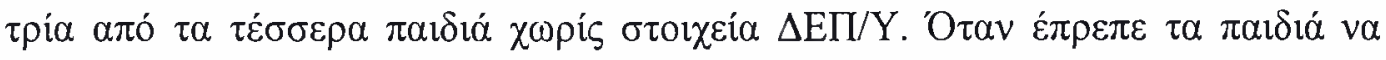

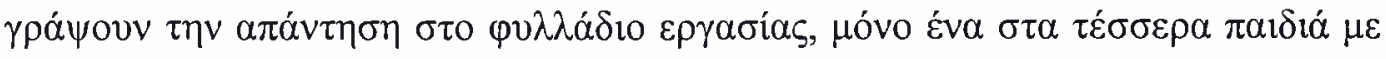




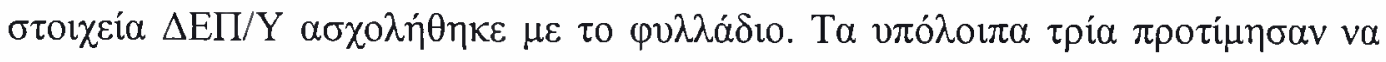

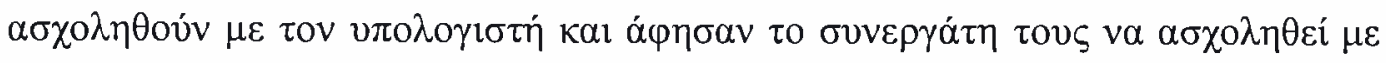

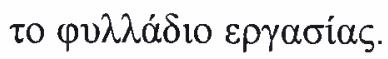

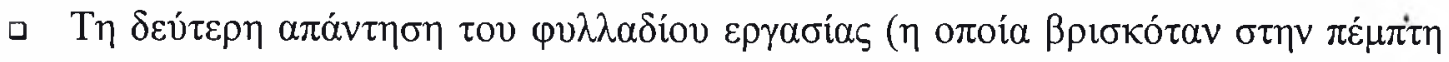

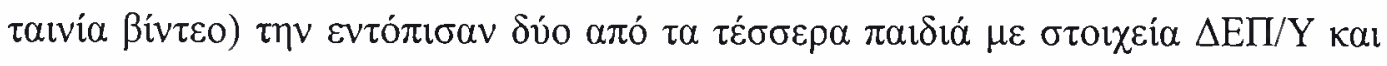

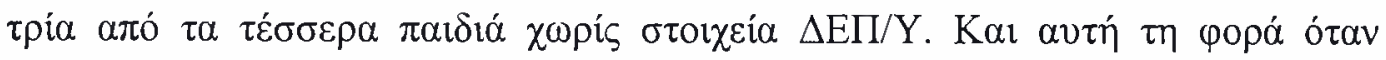

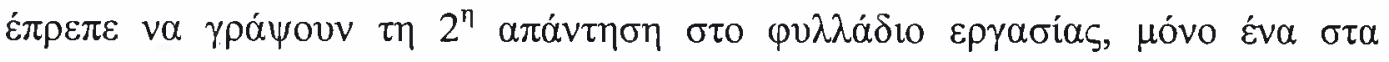

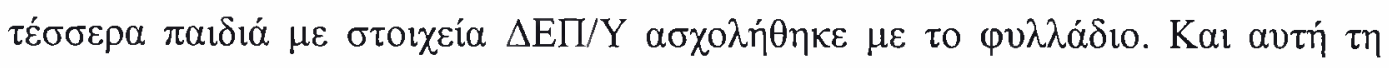

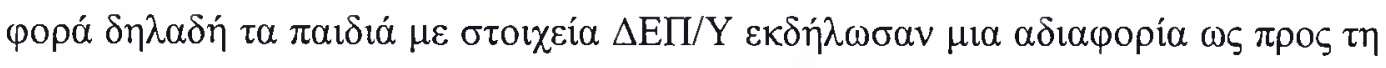

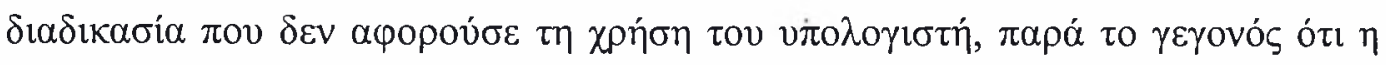

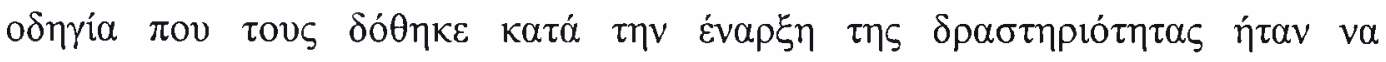

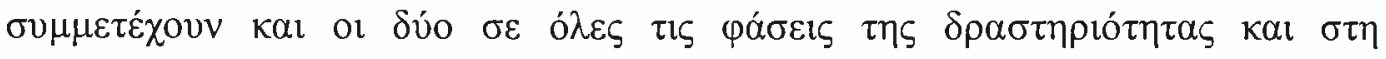

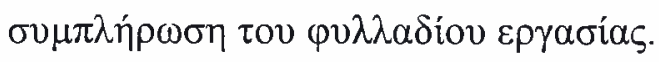

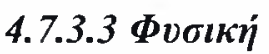

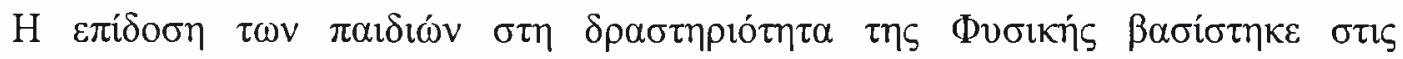

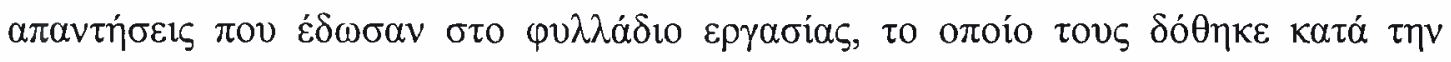

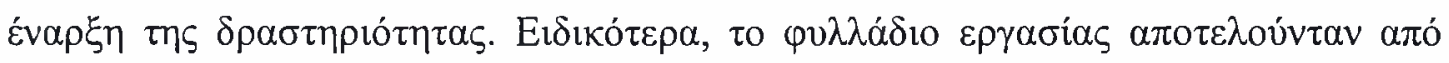

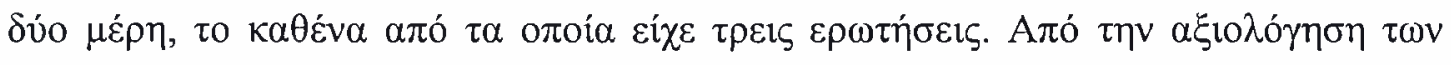

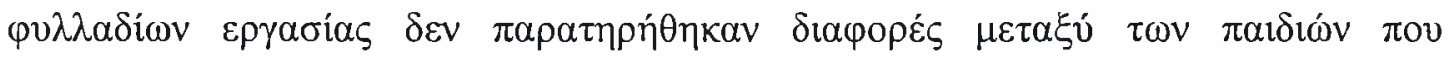

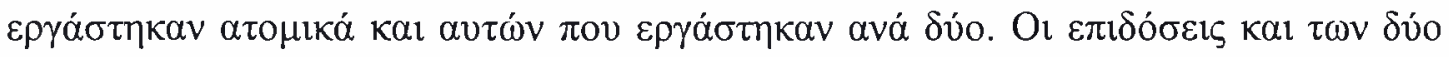

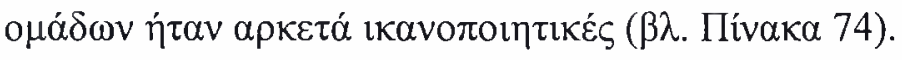

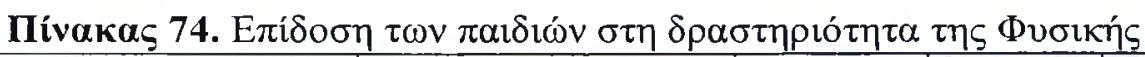

\begin{tabular}{|c|c|c|c|c|}
\hline & 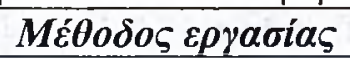 & $N(=13)$ & M.O. & T. A. \\
\hline \multirow{2}{*}{ 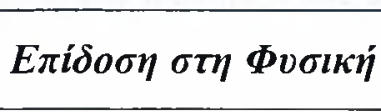 } & ATOMIKH & 5 & 3,00 & 0,71 \\
\hline & OMA $\triangle \mathrm{IKH}$ & 8 & 3,00 & 0,76 \\
\hline
\end{tabular}

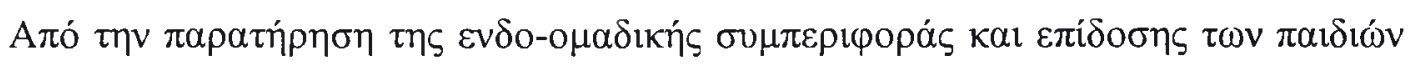

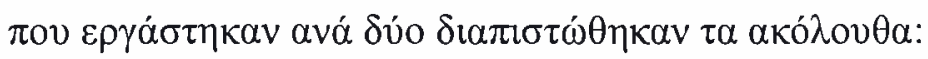

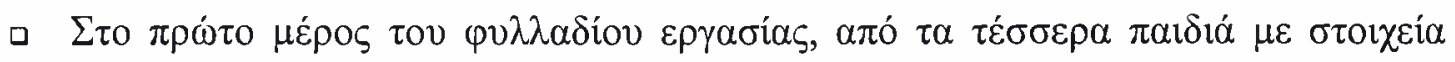

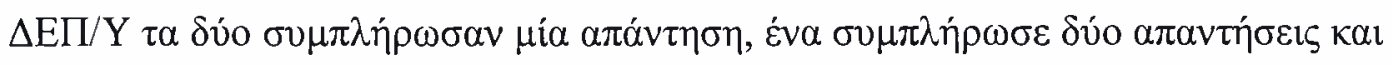

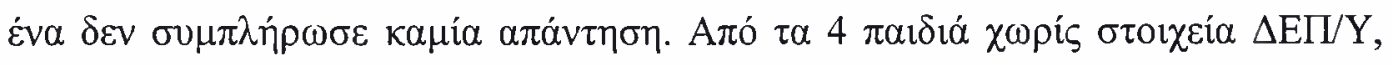




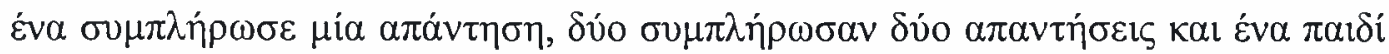

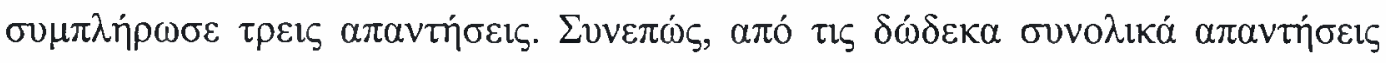

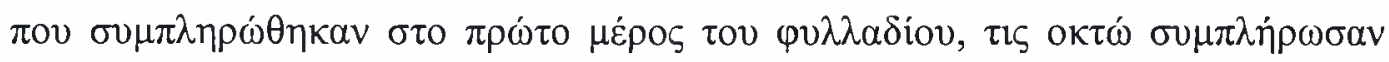

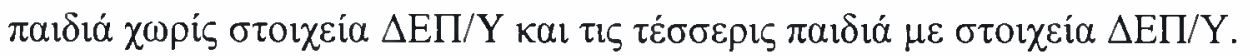

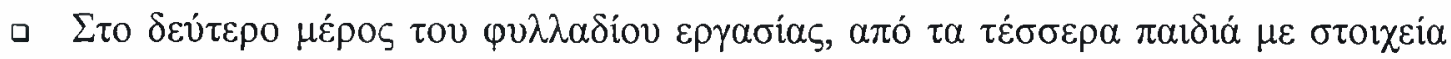

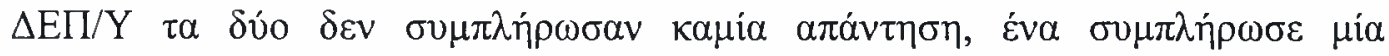

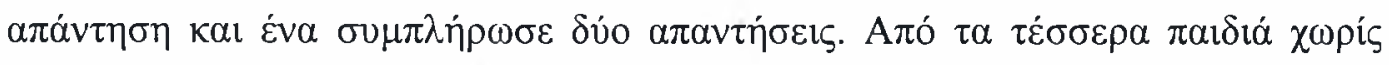

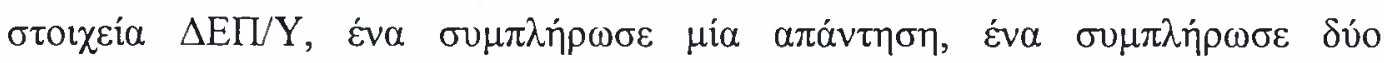

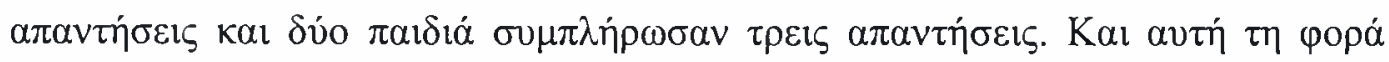

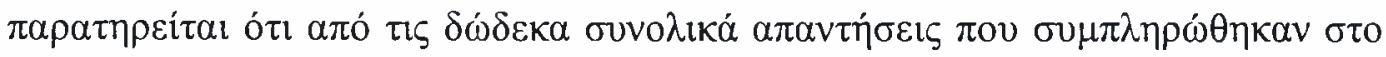

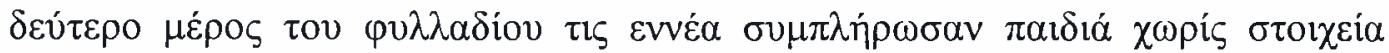

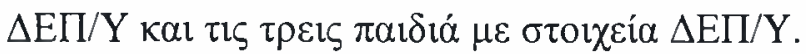

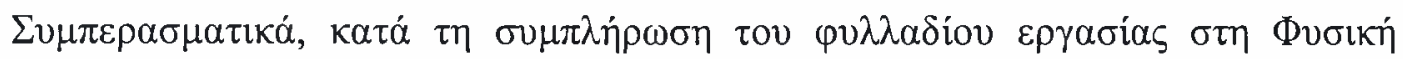

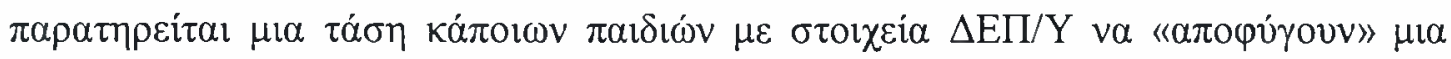

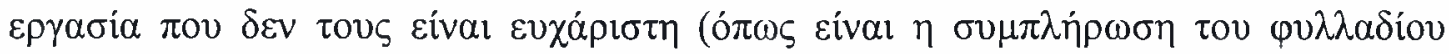
$\varepsilon \rho \gamma a \sigma i a \varsigma)$.

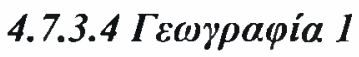

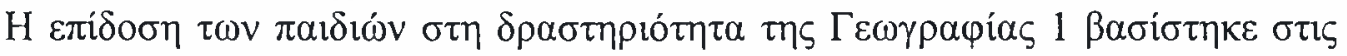

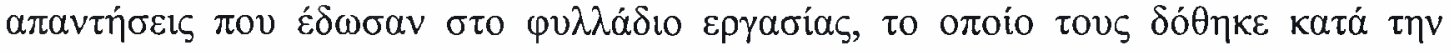

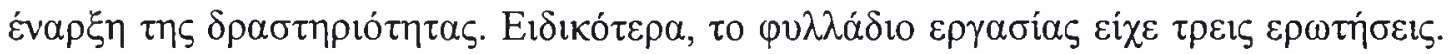

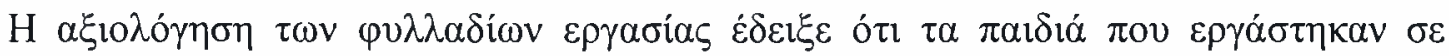

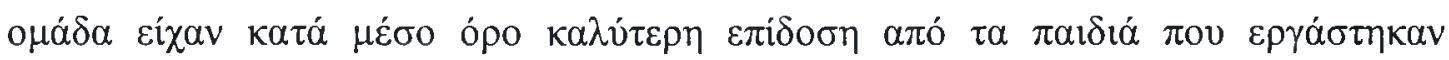

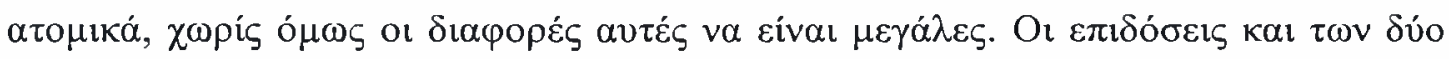

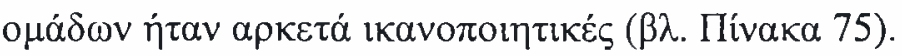

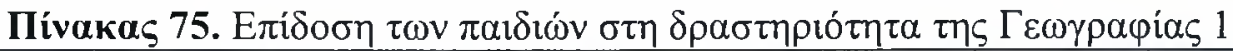

\begin{tabular}{|c|c|c|c|c|}
\hline & 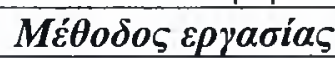 & $N(=13)$ & M.O. & $T . A$. \\
\hline \multirow{2}{*}{ 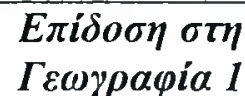 } & ATOMIKH & 5 & 3,20 & 1,30 \\
\hline & OMA $\triangle \mathrm{IKH}$ & 8 & 3,50 & 0,93 \\
\hline
\end{tabular}

A

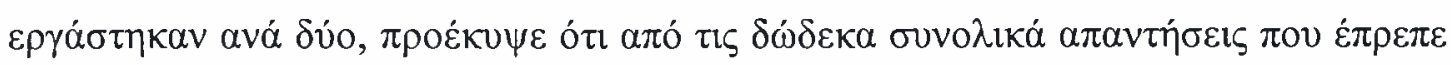




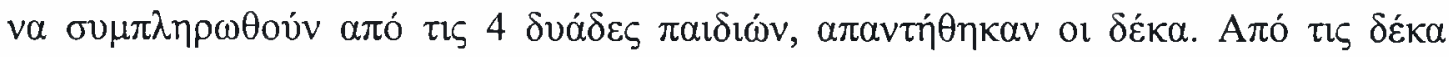

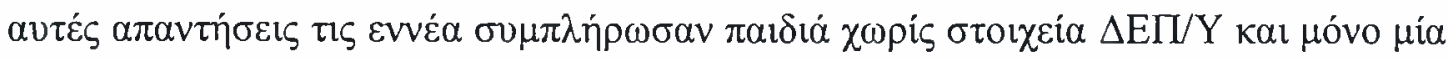

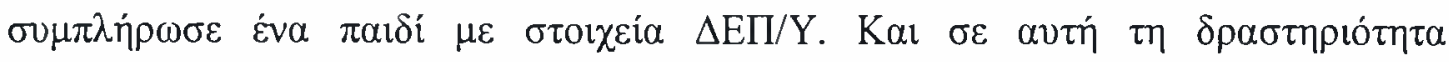

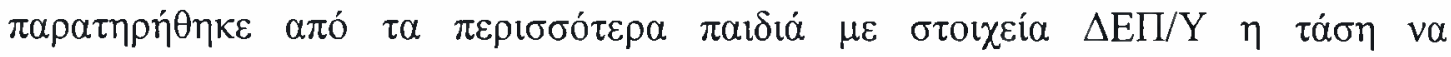

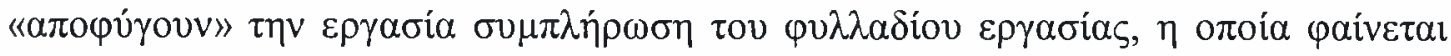

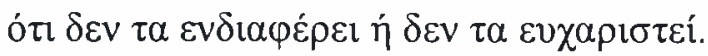

\subsubsection{5 Гвєурафiа 2}

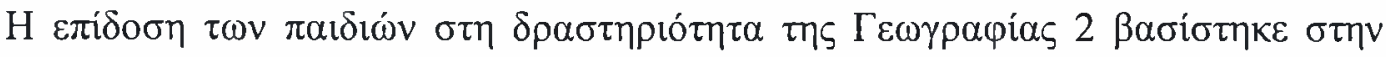

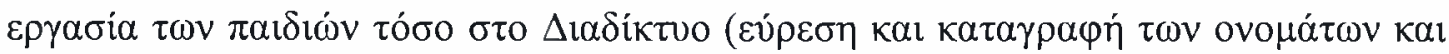

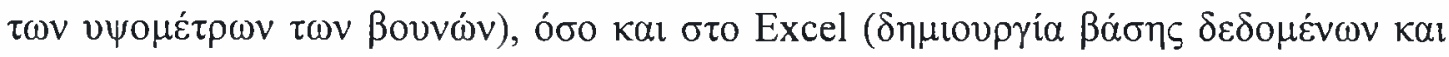

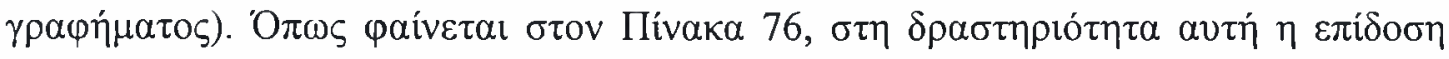

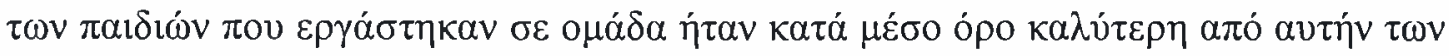

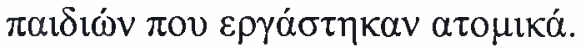

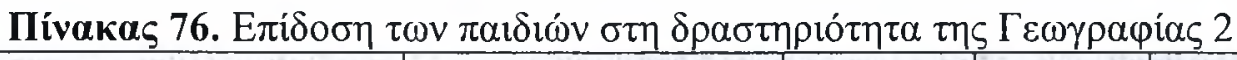

\begin{tabular}{|c|c|c|c|c|}
\hline & 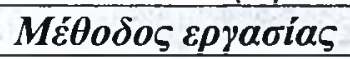 & $N(=13)$ & M.O. & T.A. \\
\hline \multirow{2}{*}{ 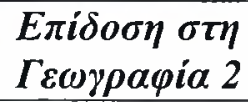 } & ATOMIKH & 5 & 2,60 & 0,55 \\
\hline & OMA $\triangle \mathrm{IKH}$ & 8 & 3,25 & 0,46 \\
\hline
\end{tabular}

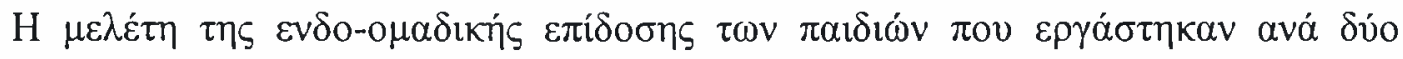
$\varepsilon \delta \varepsilon 1 \xi \varepsilon \tau \alpha \alpha \kappa o ́ \lambda o v \theta \alpha$ :

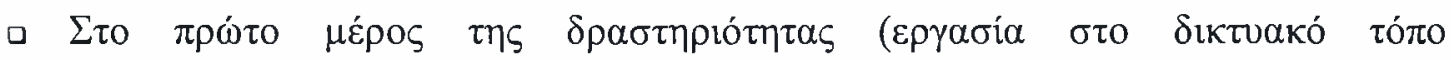

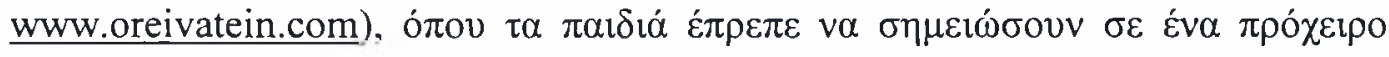

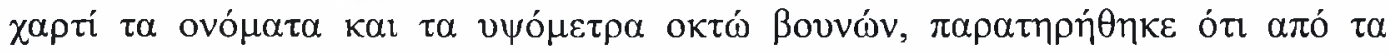

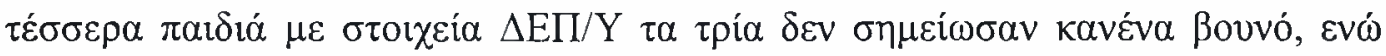

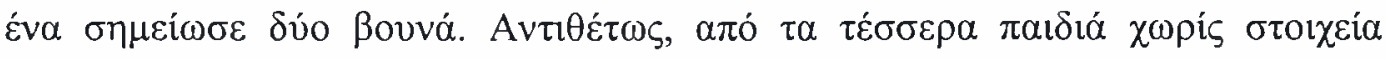

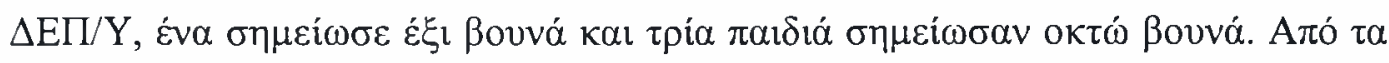

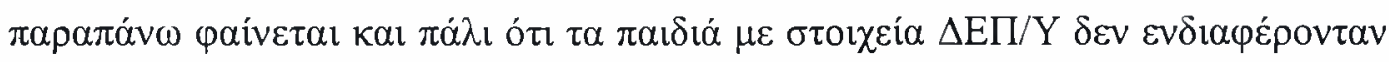

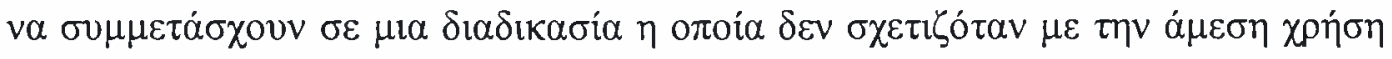

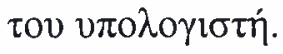

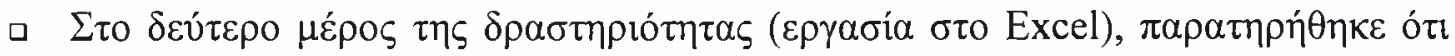

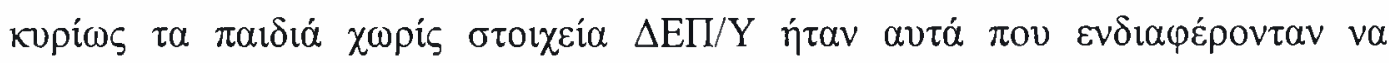




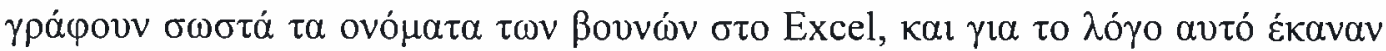

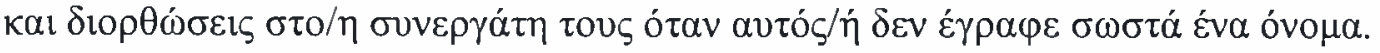

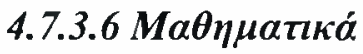

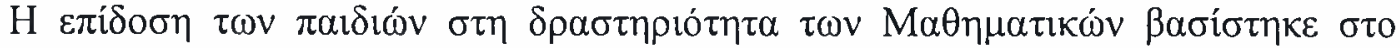

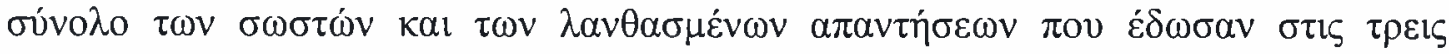

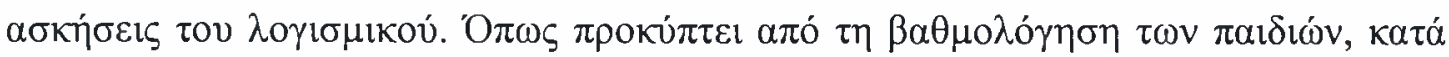

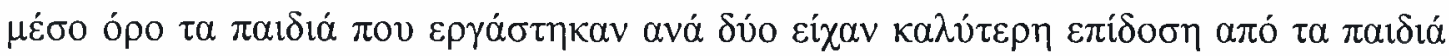

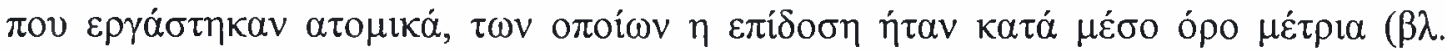
Пívaka 77).

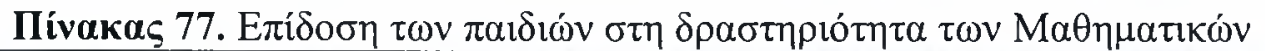

\begin{tabular}{|c|c|c|c|c|}
\hline & 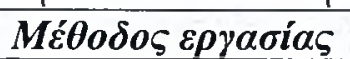 & $N(=13)$ & M.O. & T.A. \\
\hline \multirow{2}{*}{ 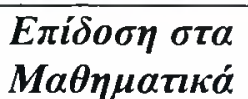 } & ATOMIKH & 5 & 2,20 & 1,10 \\
\hline & OMA $\triangle I K H$ & 8 & 3,00 & 0,00 \\
\hline
\end{tabular}

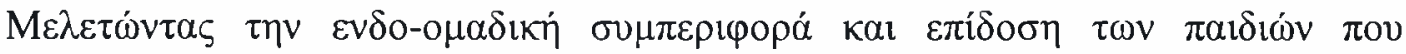

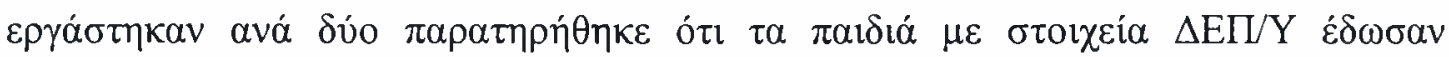

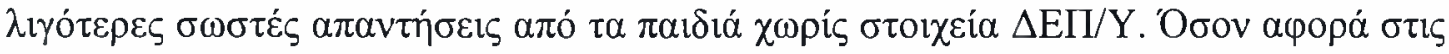

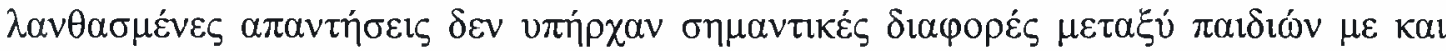

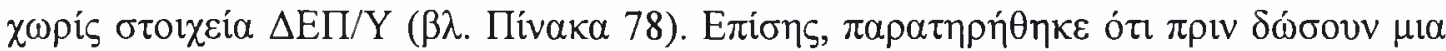

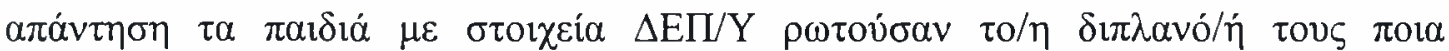

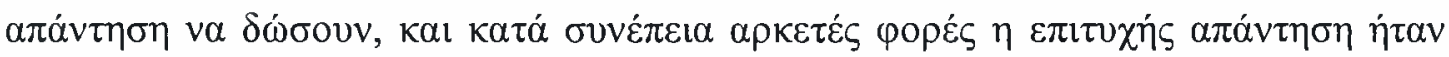

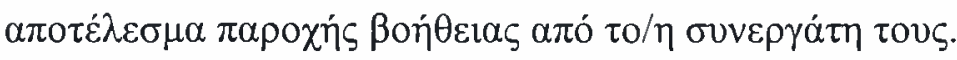

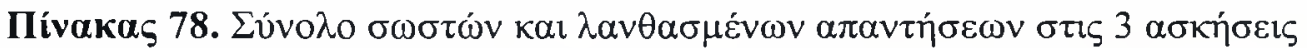

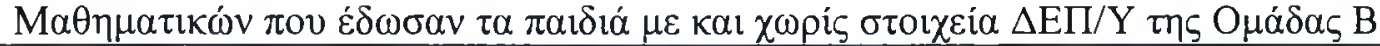

\begin{tabular}{|c|c|c|c|c|}
\hline & 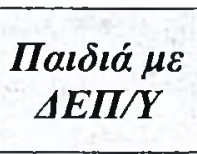 & $N(=8)$ & 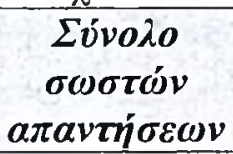 & $\begin{array}{c}\Sigma v ́ v o \lambda o \lambda \dot{\alpha} \theta 0 \varsigma \\
\alpha \pi \alpha v \tau \dot{\sigma} \sigma \varepsilon \omega v\end{array}$ \\
\hline \multirow{2}{*}{ 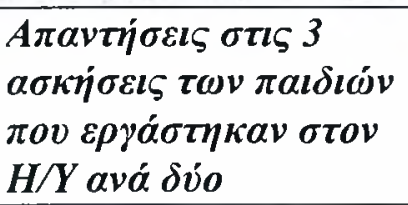 } & $\mathrm{NAI}$ & 4 & 52 & 12 \\
\hline & $\mathrm{OXI}$ & 4 & 58 & 10 \\
\hline
\end{tabular}

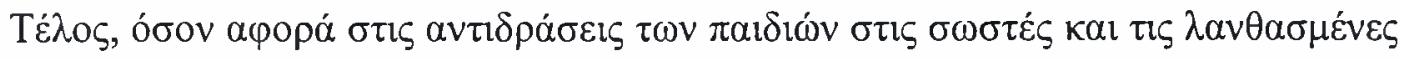

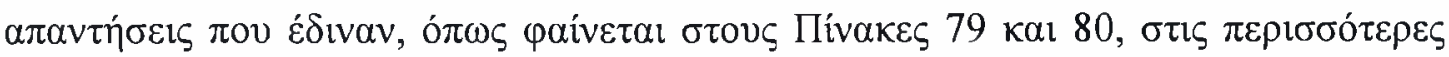




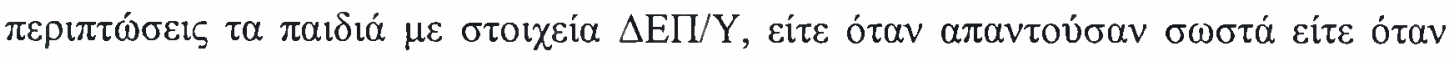

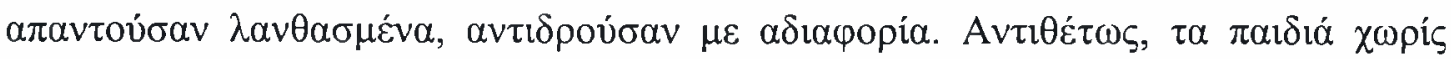

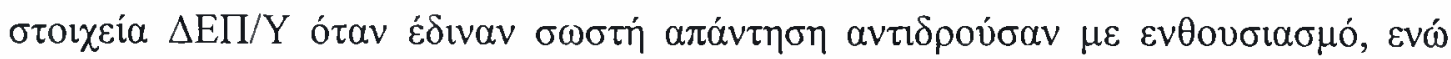

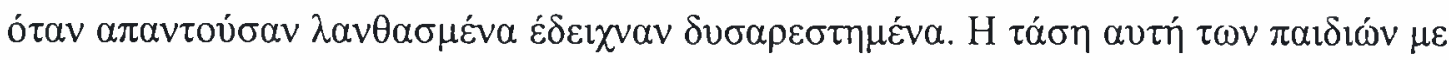

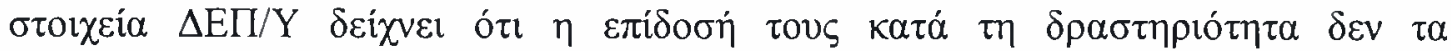

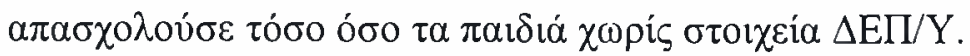

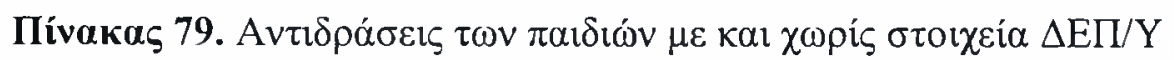

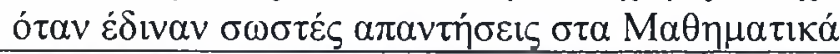

\begin{tabular}{|c|c|c|c|}
\hline & $\begin{array}{c}A v \tau i \delta \rho \alpha \sigma \eta \tau \omega v \pi \alpha \iota \delta \iota \omega ́ v \sigma \tau \iota \varsigma \sigma \omega \sigma \tau \varepsilon \dot{\zeta} \\
a \pi \alpha v \tau \dot{j} \sigma \varepsilon ı \varsigma\end{array}$ & 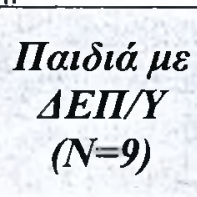 & 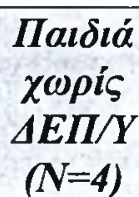 \\
\hline \multirow{3}{*}{$A \sigma \kappa \eta \sigma \eta 1$} & 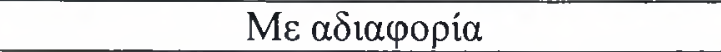 & 4 & \\
\hline & 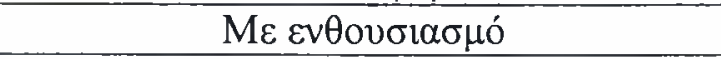 & 2 & 3 \\
\hline & 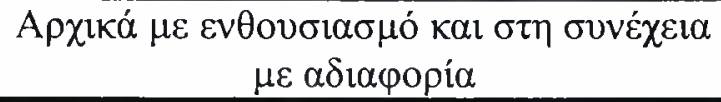 & 3 & 1 \\
\hline \multirow{3}{*}{ 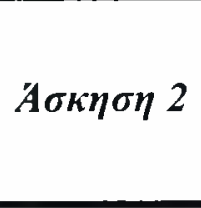 } & 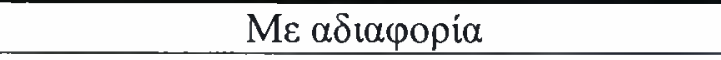 & 7 & \\
\hline & 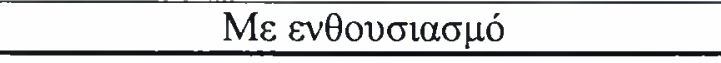 & 2 & 3 \\
\hline & 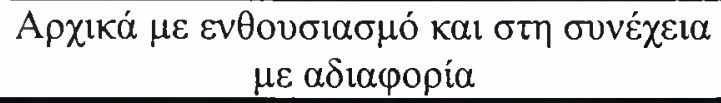 & & 1 \\
\hline \multirow{3}{*}{ 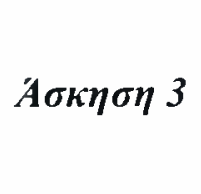 } & Mع $\alpha \delta 1 \alpha \varphi \circ \rho i \alpha$ & 6 & \\
\hline & 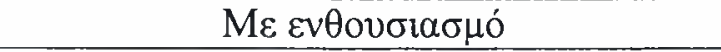 & 3 & 4 \\
\hline & 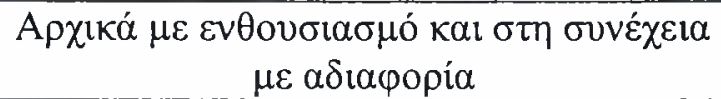 & & \\
\hline
\end{tabular}

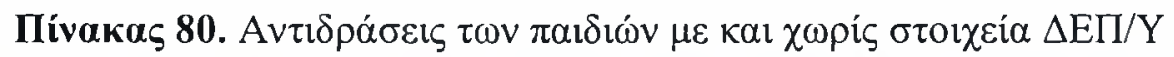

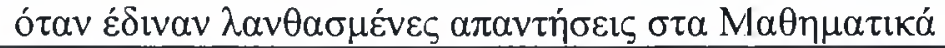

\begin{tabular}{|c|c|c|c|}
\hline & 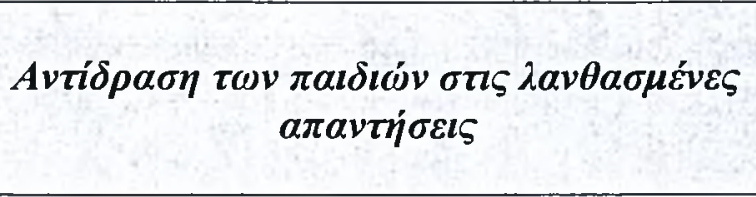 & 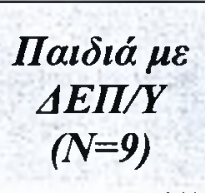 & 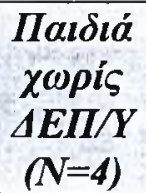 \\
\hline \multirow{3}{*}{ 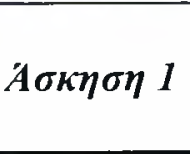 } & 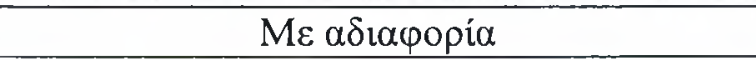 & 5 & \\
\hline & $\mathrm{M \varepsilon} \delta v \sigma \alpha \rho \varepsilon ́ \sigma \kappa \varepsilon 1 \alpha$ & & 1 \\
\hline & $\Delta \varepsilon v \varepsilon \dot{\varepsilon} \delta \omega \sigma \alpha \nu \lambda \alpha \dot{\alpha} \theta \circ \varsigma \alpha \pi \alpha \nu \tau \eta \dot{\sigma} \sigma \varepsilon l \varsigma$ & 4 & 3 \\
\hline \multirow{3}{*}{ 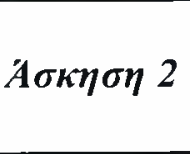 } & Mع $\alpha \delta 1 \alpha \varphi \circ \rho^{\prime} \alpha$ & 1 & \\
\hline & $\mathrm{M \varepsilon} \delta v \sigma \alpha \rho \varepsilon \dot{\sigma} \sigma \varepsilon \varepsilon l \alpha$ & 1 & \\
\hline & $\Delta \varepsilon v \varepsilon \dot{\varepsilon} \delta \sigma \alpha \alpha \nu \lambda \dot{\alpha} \theta 0 \varsigma \alpha \pi \alpha v \tau \eta \dot{\sigma} \sigma \varepsilon ı \zeta$ & 7 & 4 \\
\hline \multirow{3}{*}{$A \sigma \kappa \eta \sigma \eta 3$} & 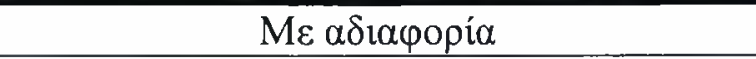 & 6 & \\
\hline & 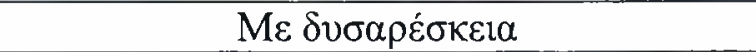 & 3 & 4 \\
\hline & 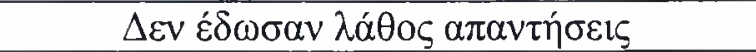 & & \\
\hline
\end{tabular}




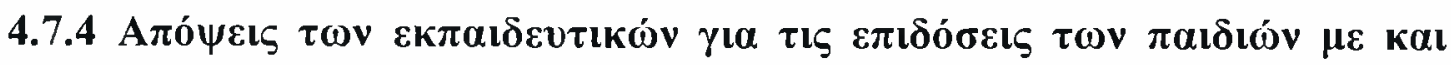

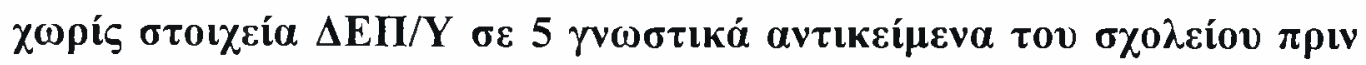

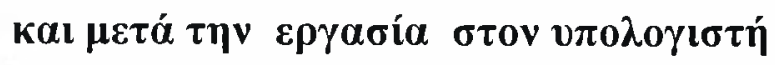

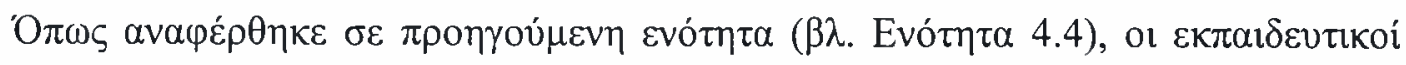

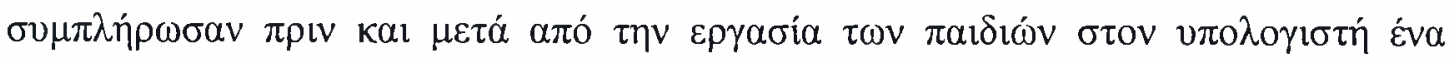

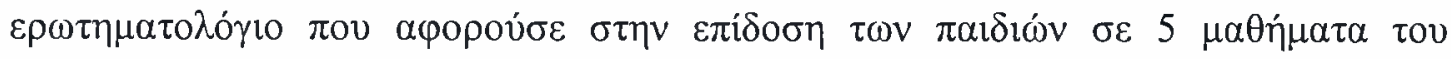

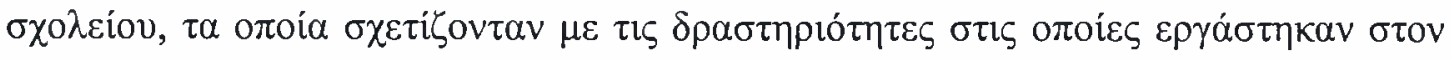

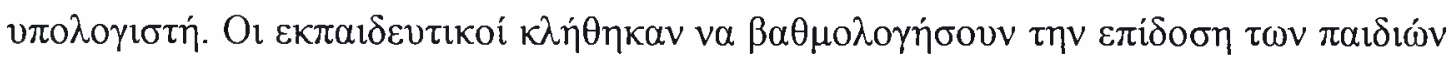

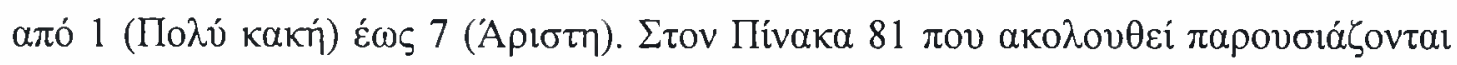

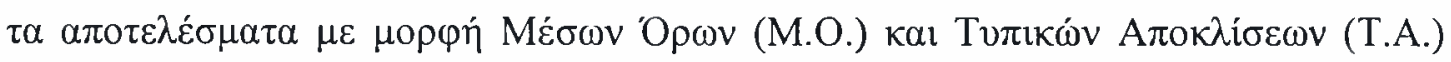

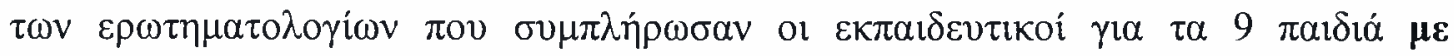

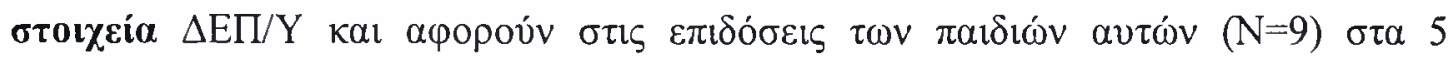

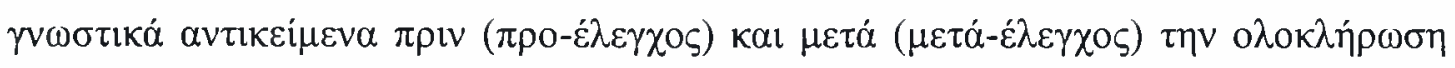
$\tau\rceil \varsigma \pi \alpha \rho \varepsilon ́ \mu \beta \alpha \sigma \eta \varsigma$.

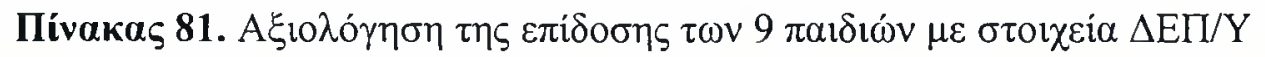

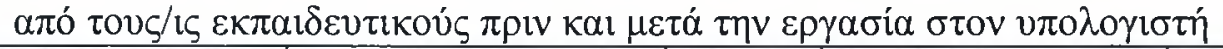

\begin{tabular}{|c|c|c|c|c|}
\hline 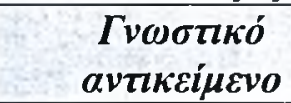 & $\begin{array}{c}\Pi \rho o-\varepsilon \lambda \varepsilon \gamma \chi \chi \varsigma \varsigma \\
\text { M.O. }\end{array}$ & T.A. & 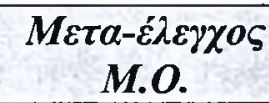 & T.A. \\
\hline 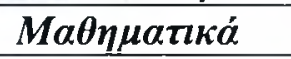 & 4,44 & 0,88 & 4,44 & 1,01 \\
\hline Iotopía & 4,44 & 1,24 & 4,78 & 1,39 \\
\hline 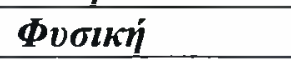 & 5,00 & 0,87 & 5,00 & 1,00 \\
\hline 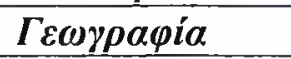 & 4,11 & 1,45 & 4,33 & 1,32 \\
\hline$K a \lambda \lambda \iota \tau \varepsilon \chi v ı \kappa a ́$ & 5,22 & 1,48 & 5,00 & 1,41 \\
\hline
\end{tabular}

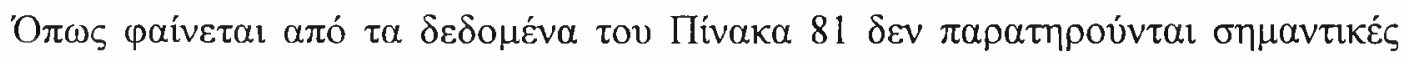

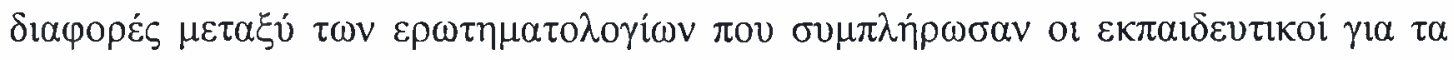

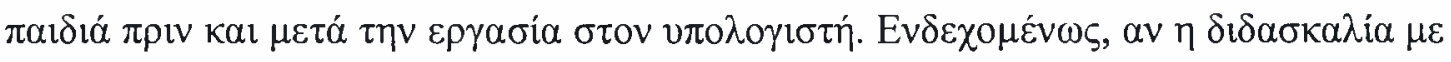

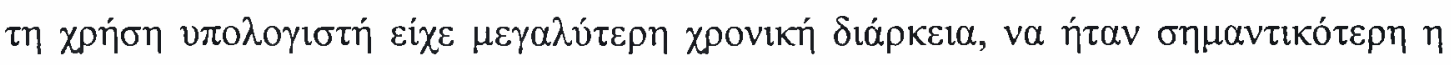

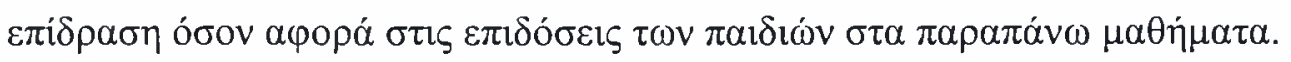

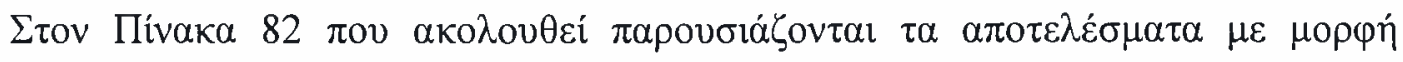

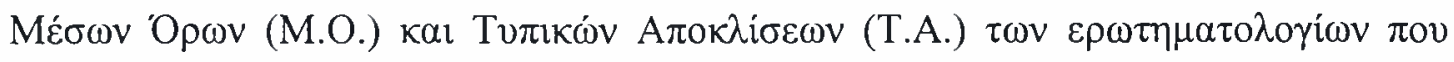

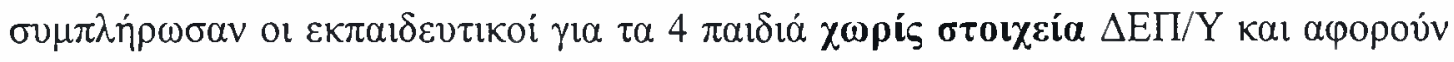

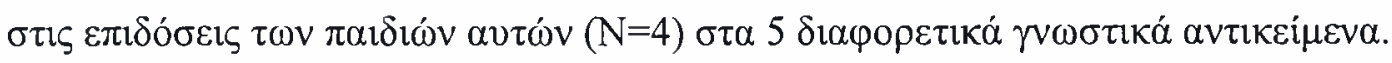




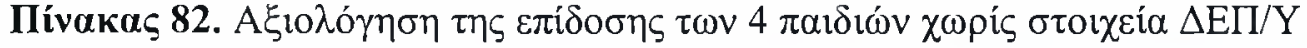

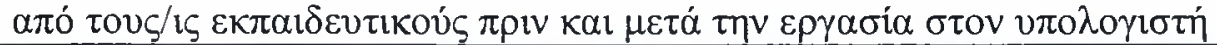

\begin{tabular}{|c|c|c|c|c|}
\hline 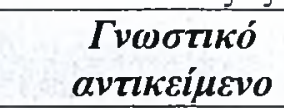 & 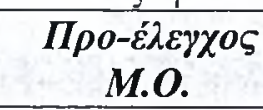 & T. A. & 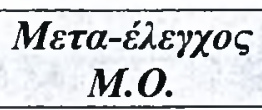 & T.A. \\
\hline 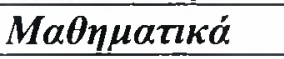 & 5,25 & 0,50 & 5,75 & 0,50 \\
\hline Iotopía & 5,00 & 0,82 & 5,25 & 0,96 \\
\hline 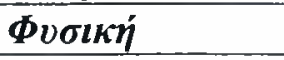 & 5,00 & 0,00 & 5,75 & 0,50 \\
\hline 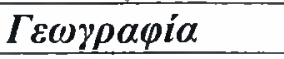 & 4,50 & 0,58 & 4,75 & 1,26 \\
\hline 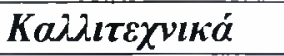 & 4,00 & 1,15 & 5,75 & 0,96 \\
\hline
\end{tabular}

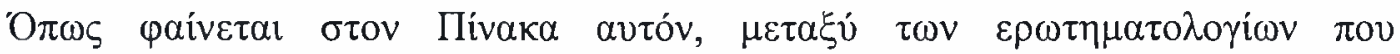

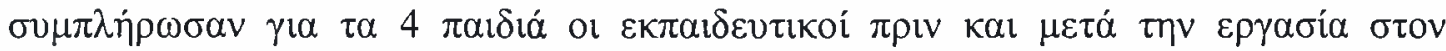

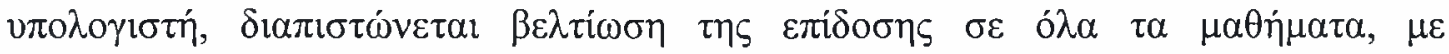

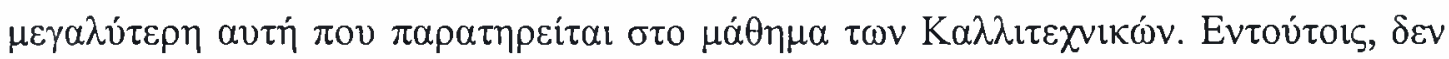

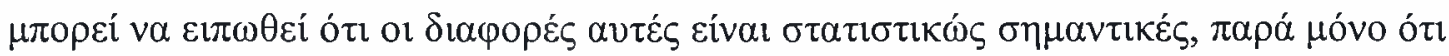

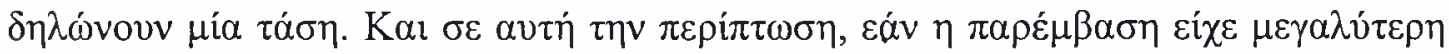

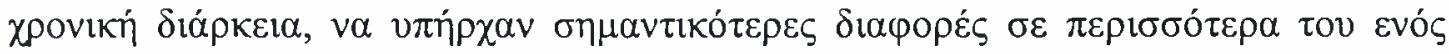
$\mu \alpha \theta \dot{\eta} \mu \alpha \tau \alpha$.

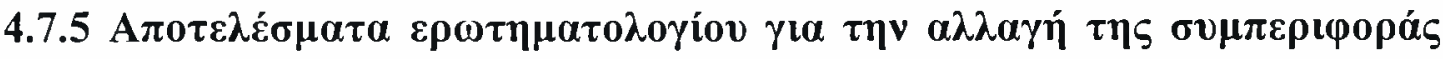

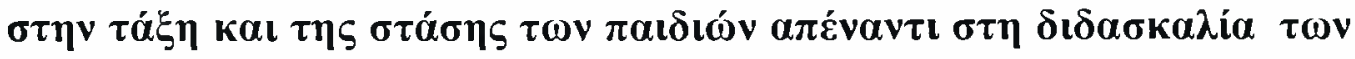

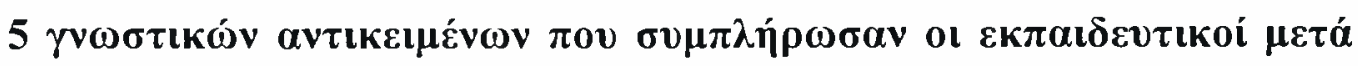

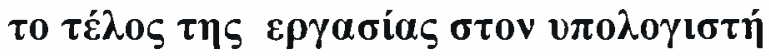

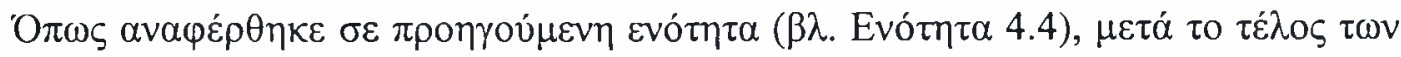

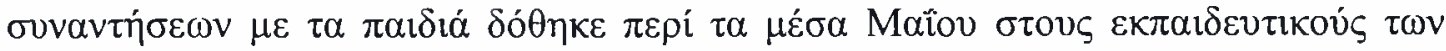

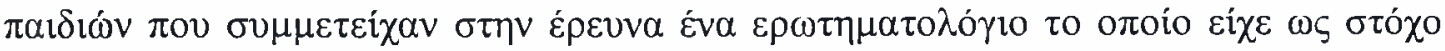

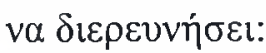

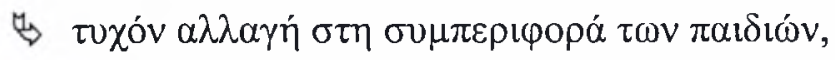

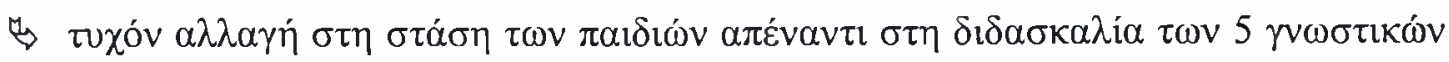

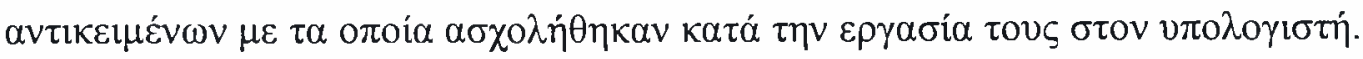

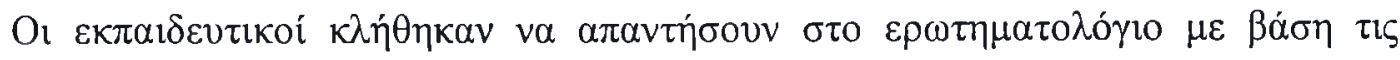

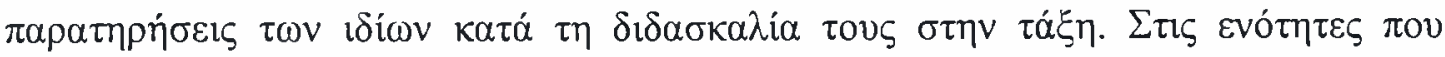

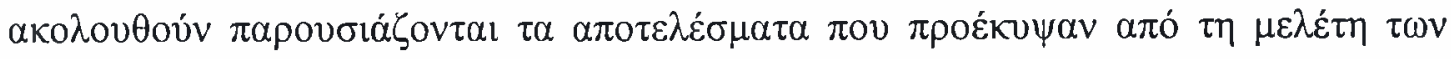

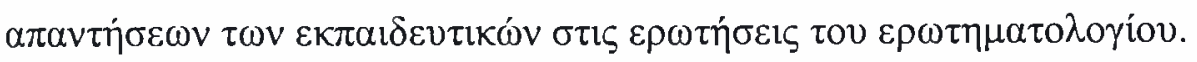




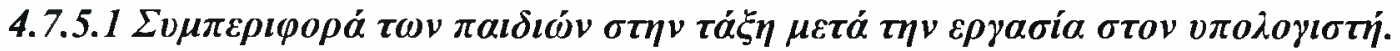

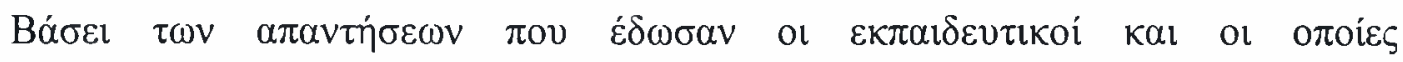

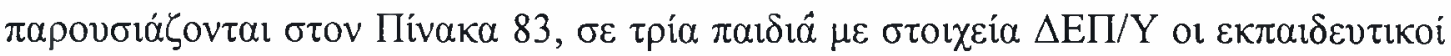

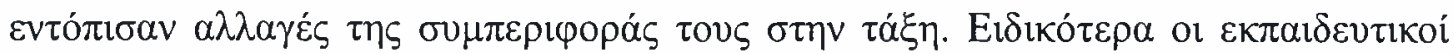

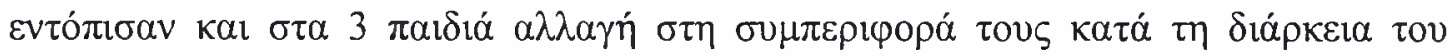

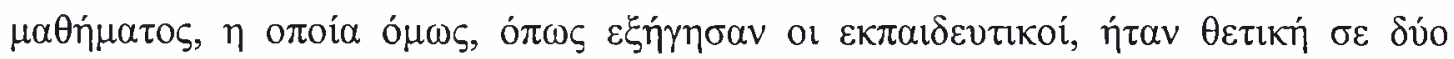

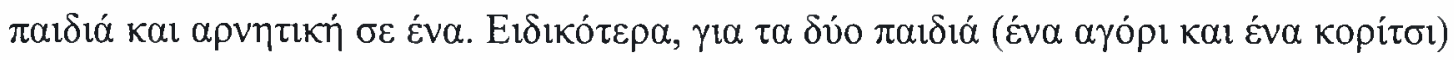

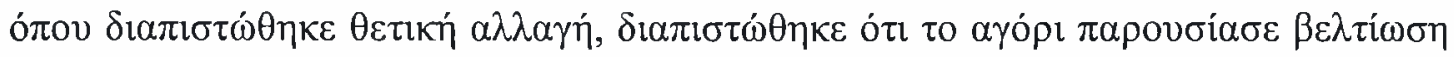

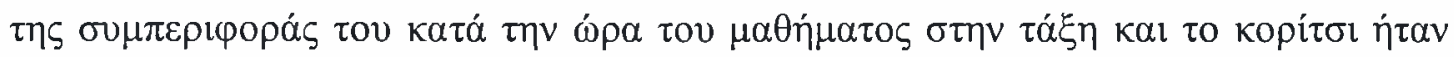

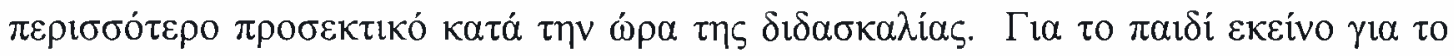

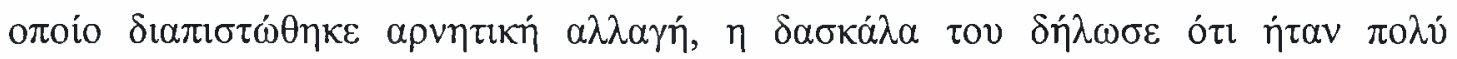
$\alpha \pi \rho \sigma_{\sigma \varepsilon \kappa \tau o \zeta}^{\sigma \tau \eta \eta} \nu \tau \dot{\alpha} \xi \eta$.

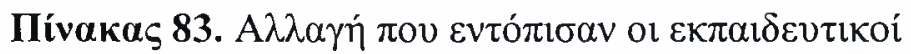

\begin{tabular}{|c|c|c|}
\hline 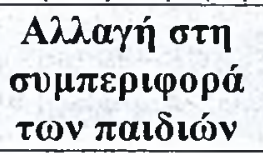 & 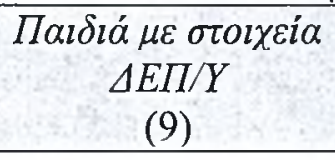 & 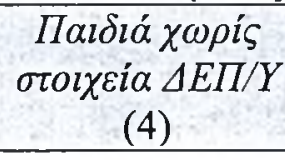 \\
\hline $\mathrm{N} \alpha \mathbf{l}$ & 3 & 0 \\
\hline $\mathrm{O} \chi \mathrm{l}$ & 6 & 4 \\
\hline
\end{tabular}

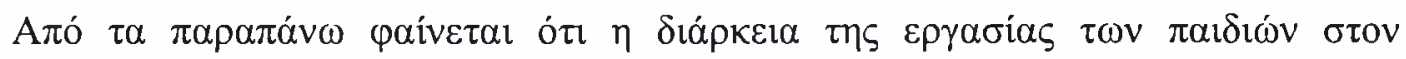

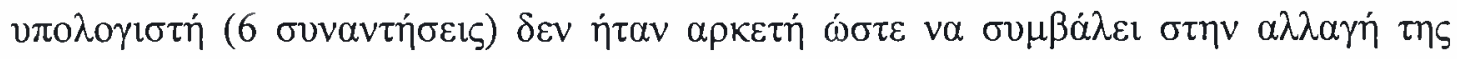

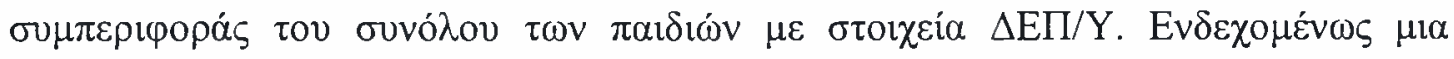

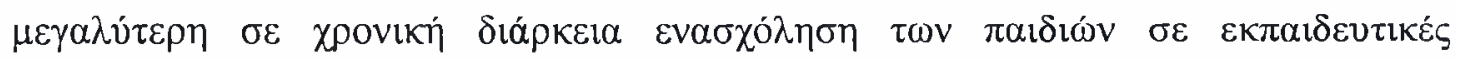

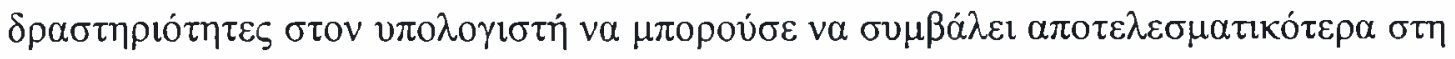

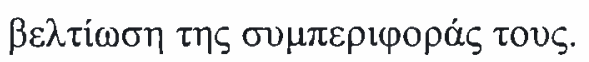

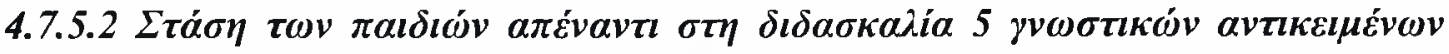

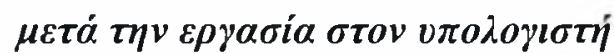

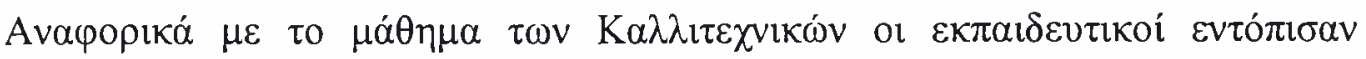

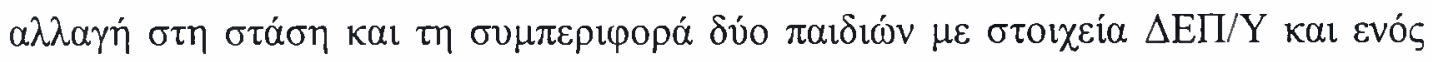




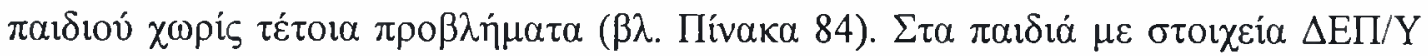

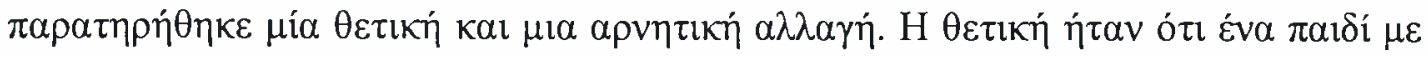

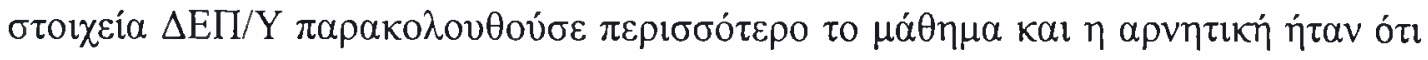

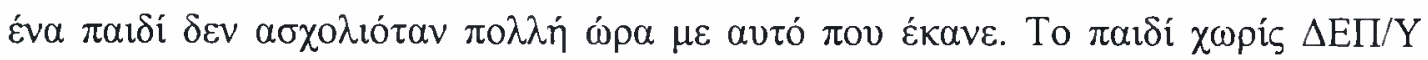

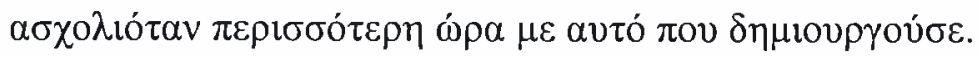

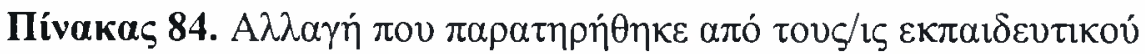

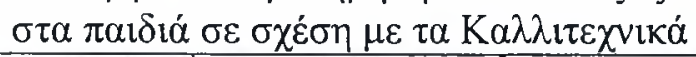

\begin{tabular}{|c|c|c|}
\hline 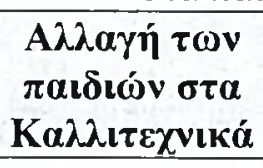 & $\begin{array}{c}\text { Пaldió } \mu \varepsilon \\
\sigma \tau o l \chi \varepsilon i ́ \alpha \Delta E \Pi / Y \\
(9)\end{array}$ & 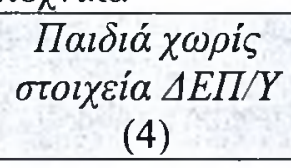 \\
\hline $\mathrm{Nal}$ & 2 & 1 \\
\hline${ }^{\prime} \mathrm{O} \chi \mathrm{l}$ & 7 & 3 \\
\hline
\end{tabular}

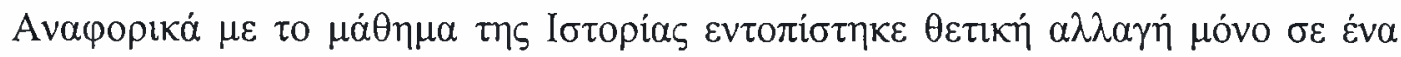

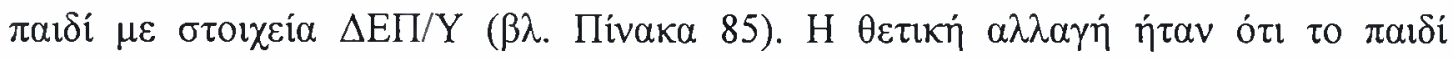

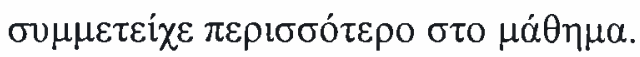

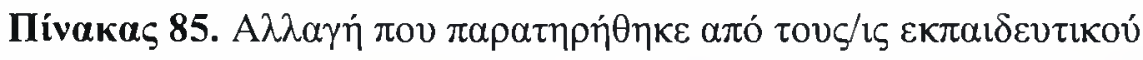

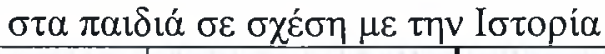

\begin{tabular}{|c|c|c|}
\hline 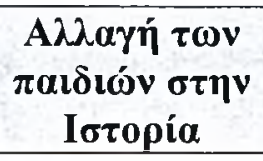 & $\begin{array}{c}\text { Пaldió } \mu \varepsilon \\
\sigma \tau o l \chi \varepsilon i \alpha \Delta E \Pi / Y \\
(9)\end{array}$ & $\begin{array}{c}\text { Пaıdiá } \chi \omega \rho i \zeta \\
\sigma \tau o \imath \chi \varepsilon i \alpha \Delta E \Pi / Y \\
\text { (4) }\end{array}$ \\
\hline $\mathrm{Nal}$ & 1 & 0 \\
\hline${ }^{\circ} \mathrm{O} \chi \mathrm{l}$ & 8 & 4 \\
\hline
\end{tabular}

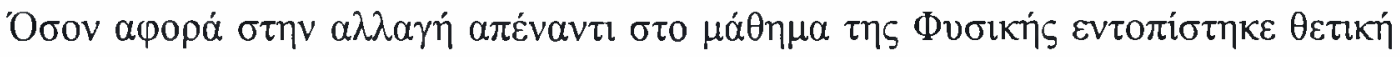

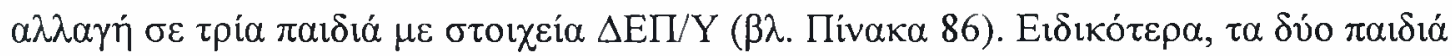

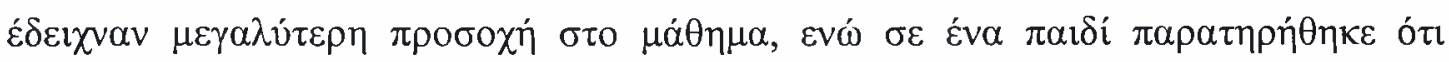

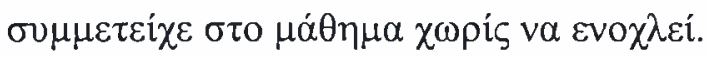

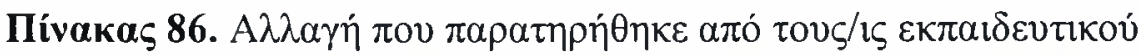
$\sigma \tau \alpha \pi \alpha 1 \delta 1 \alpha \dot{\alpha} \sigma \varepsilon \sigma \chi \varepsilon \dot{\varepsilon} \sigma \eta \mu \varepsilon \tau \eta$

\begin{tabular}{|c|c|c|}
\hline 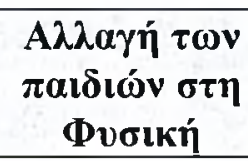 & 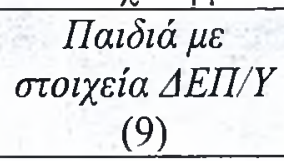 & 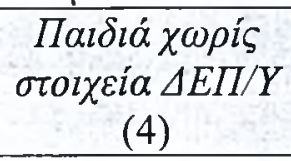 \\
\hline $\mathrm{Nal}$ & 3 & 0 \\
\hline
\end{tabular}




\begin{tabular}{|c|c|c|}
\hline $\mathrm{O} \chi \imath$ & 6 & 4 \\
\hline
\end{tabular}

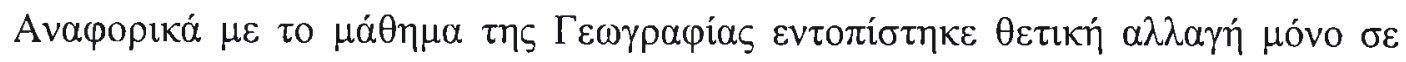

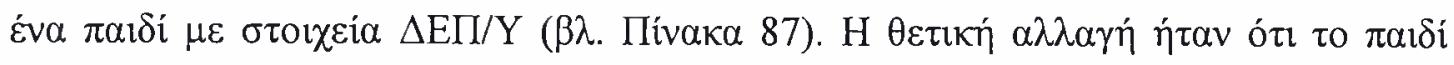

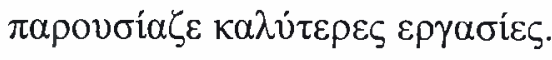

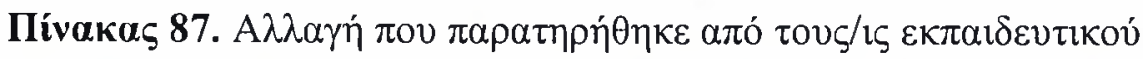
$\sigma \tau \alpha \pi \alpha i \delta i \alpha \dot{\alpha} \sigma \varepsilon \chi \varepsilon \dot{\varepsilon} \sigma \eta \mu \tau \tau \Gamma \varepsilon \omega \gamma \rho \alpha \varphi i \alpha$

\begin{tabular}{|c|c|c|}
\hline 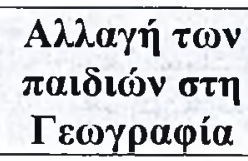 & $\begin{array}{c}\text { Пaldió } \mu \varepsilon \\
\sigma \tau o l \chi \varepsilon i ́ \alpha, \Delta E \Pi / Y \\
(9)\end{array}$ & $\begin{array}{c}\Pi \alpha \imath \delta \imath \dot{\alpha} \chi \omega \rho i \varsigma \\
\sigma \tau o \imath \chi \varepsilon i \alpha \Delta E \Pi / Y \\
(4)\end{array}$ \\
\hline $\mathrm{Nal}$ & 1 & 0 \\
\hline $\mathrm{O} \chi \mathrm{l}$ & 8 & 4 \\
\hline
\end{tabular}

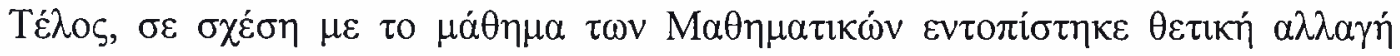

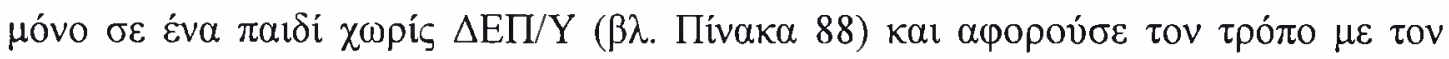

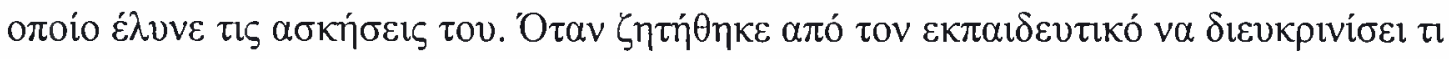

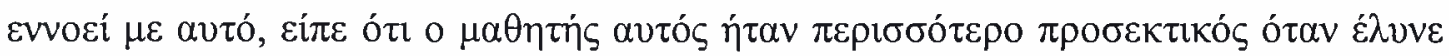
$\alpha \sigma \kappa \eta \dot{\sigma \varepsilon ı \varsigma ~ M \alpha \theta \eta \mu \alpha \tau ı \kappa \omega ́ v . ~}$

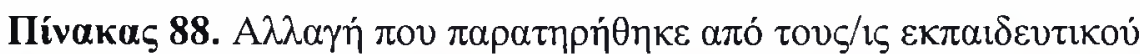

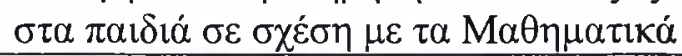

\begin{tabular}{|c|c|c|}
\hline 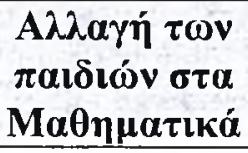 & 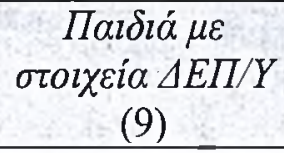 & 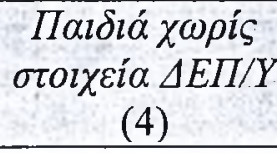 \\
\hline $\mathrm{N} \alpha \mathrm{l}$ & 0 & 1 \\
\hline${ }^{\circ} \mathrm{O} \chi \mathrm{l}$ & 9 & 3 \\
\hline
\end{tabular}

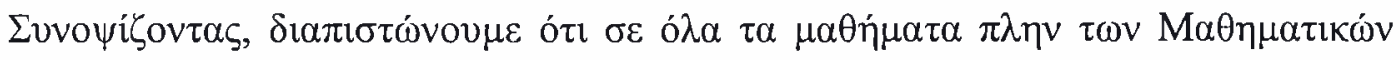

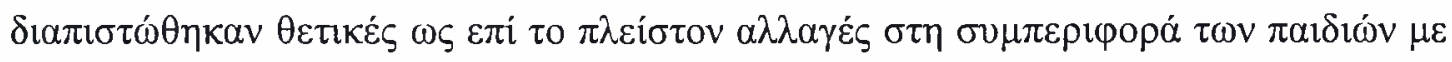

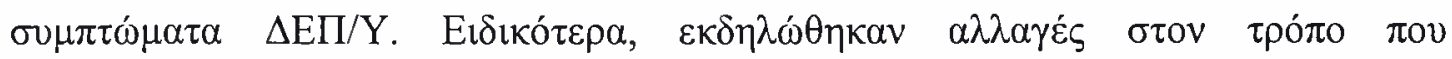

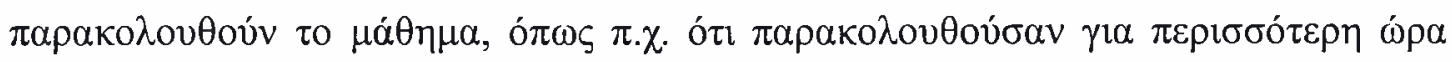

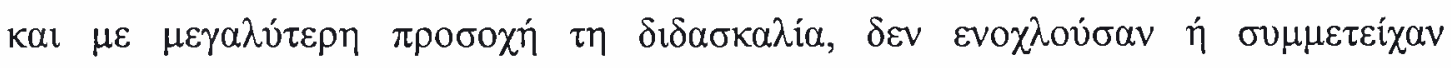

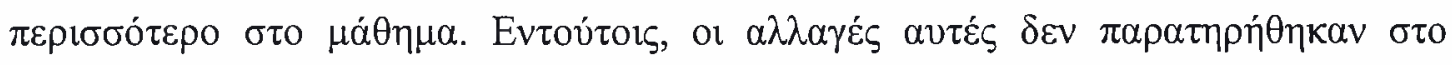

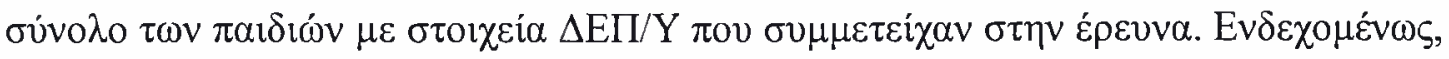




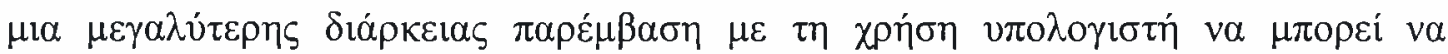

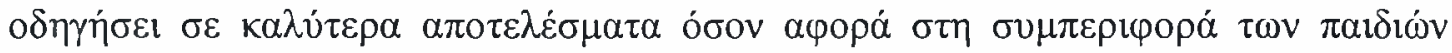

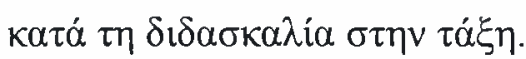

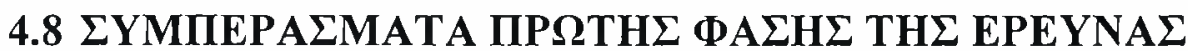

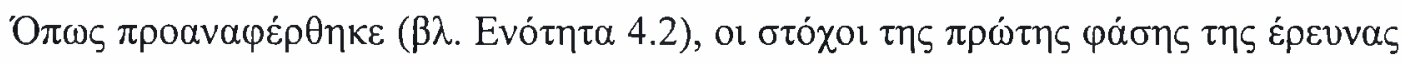

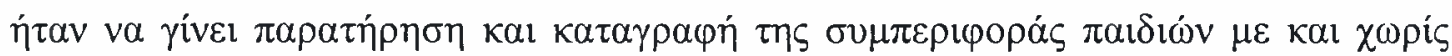

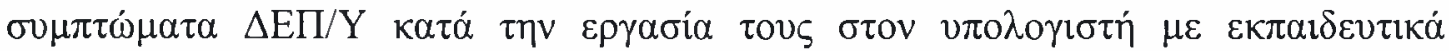

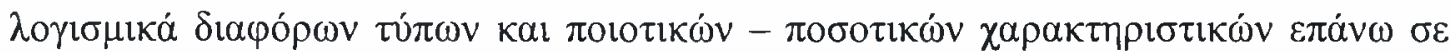

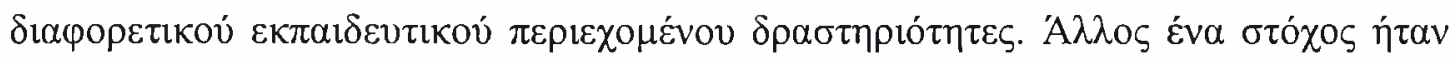

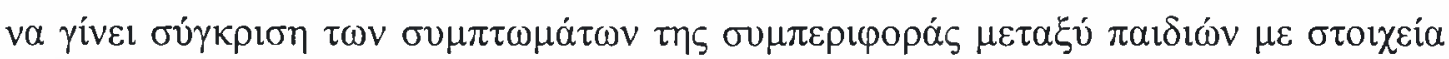

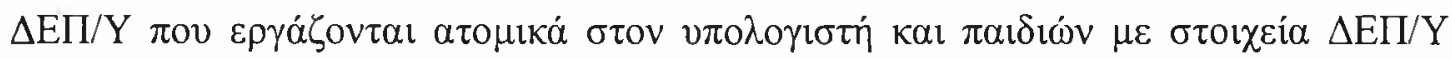

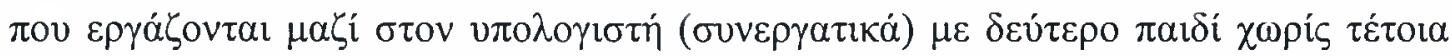

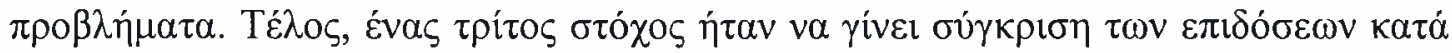

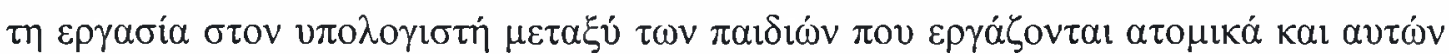

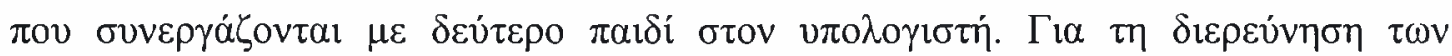

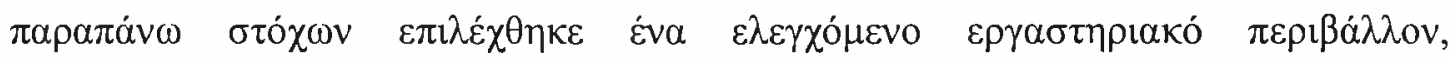

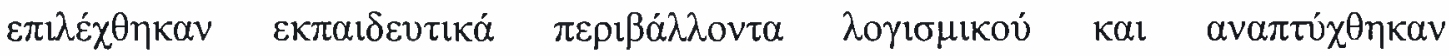

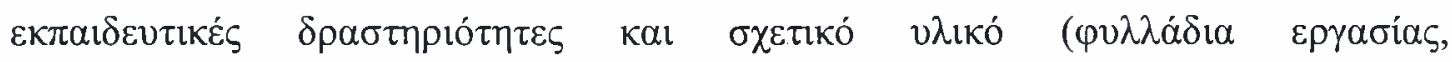
$\left.\varepsilon \rho \omega \tau \eta \mu \alpha \tau \lambda^{\prime} \operatorname{\gamma }_{\llcorner} \alpha\right)$

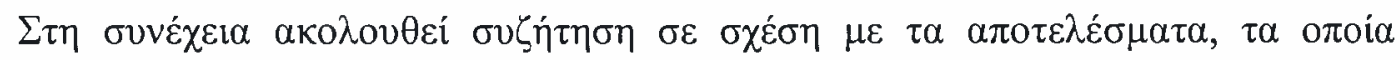

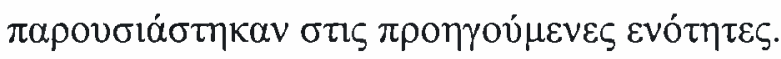

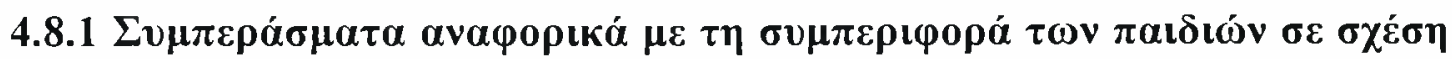

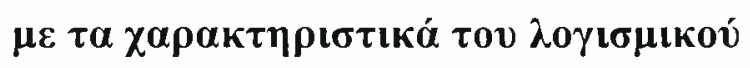

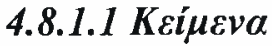

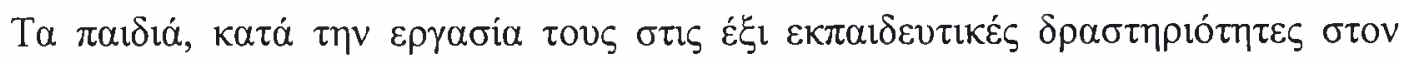

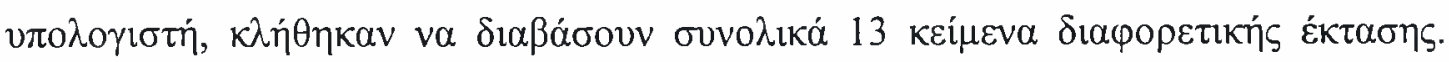

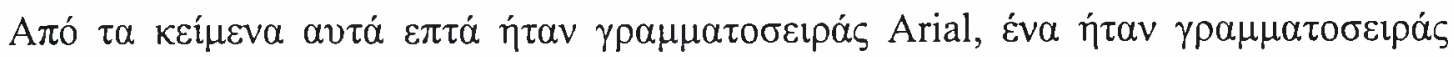




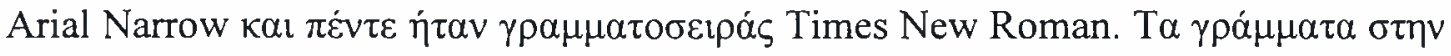

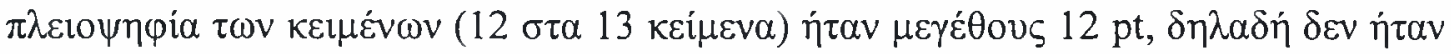

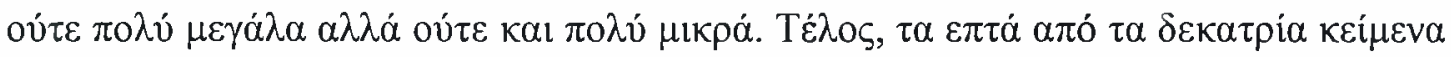

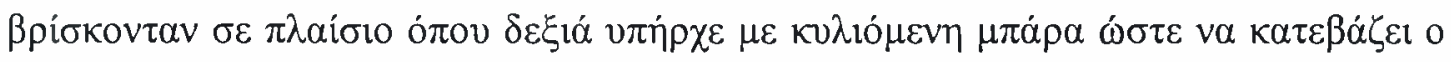

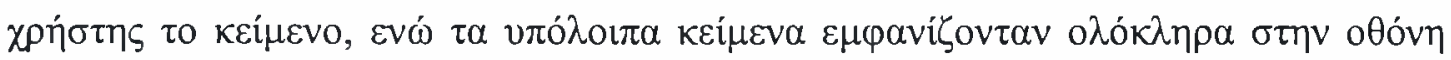

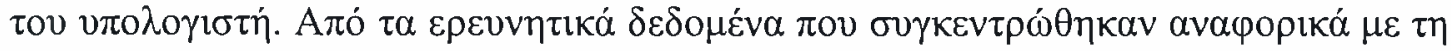

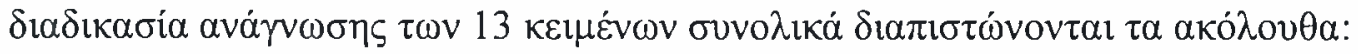

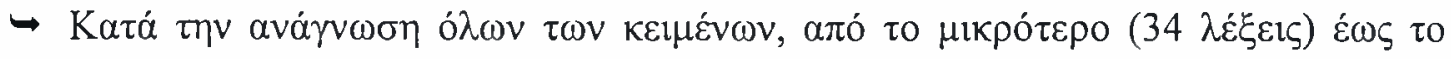

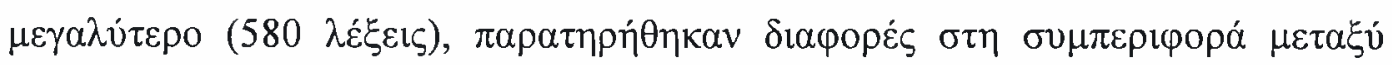

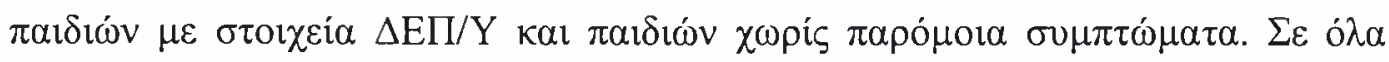

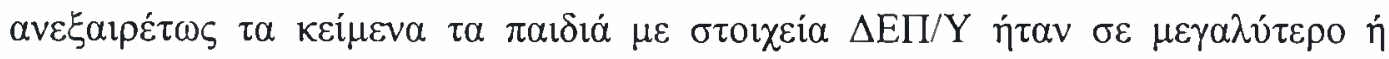

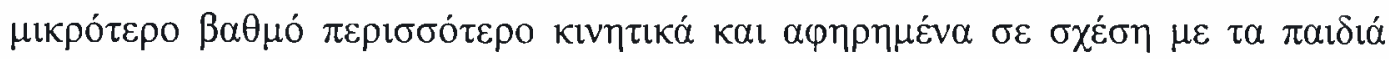

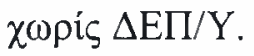

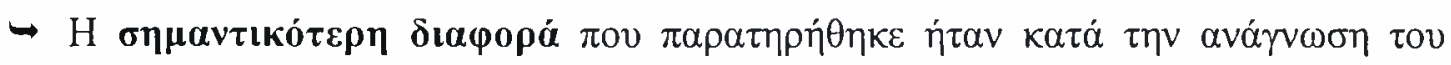

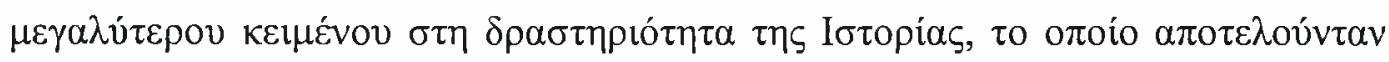

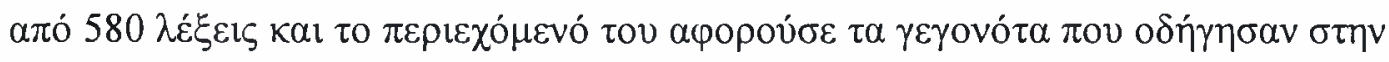

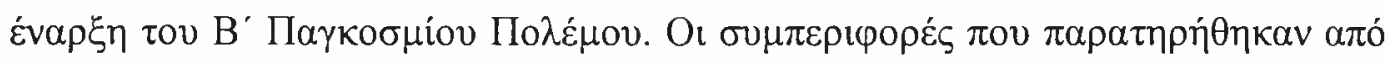

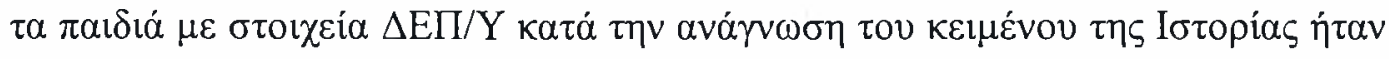

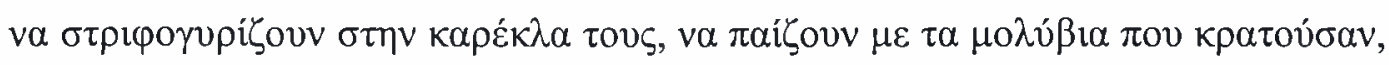

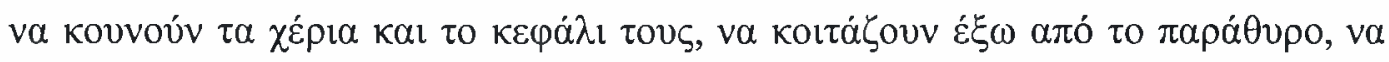

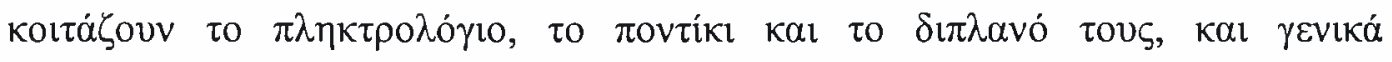

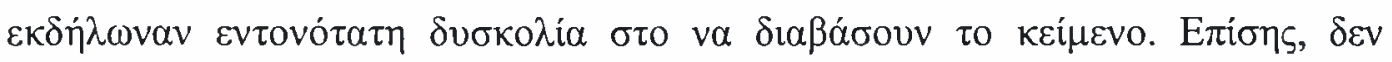

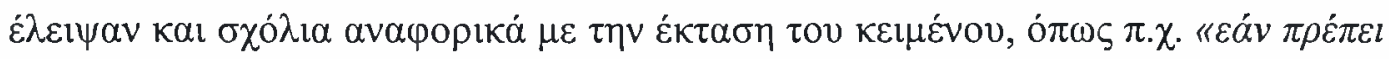

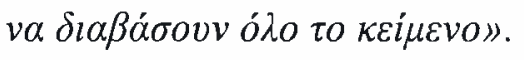

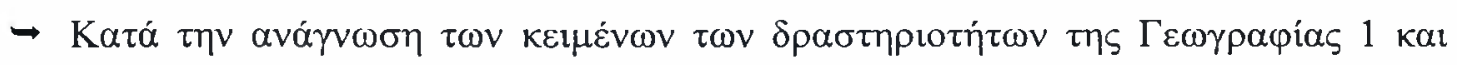

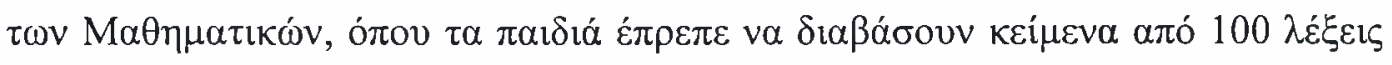

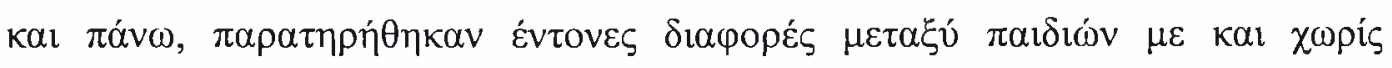

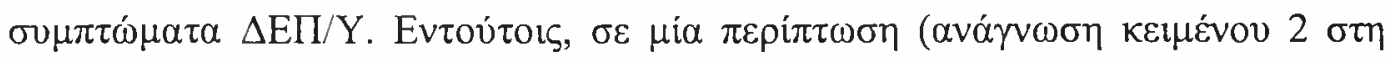

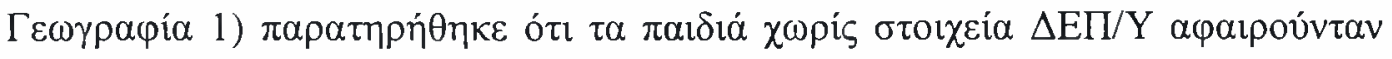

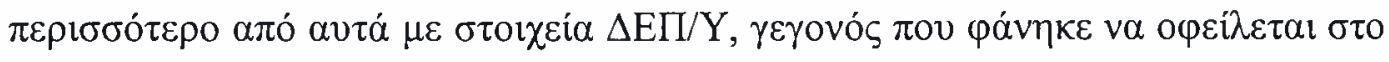

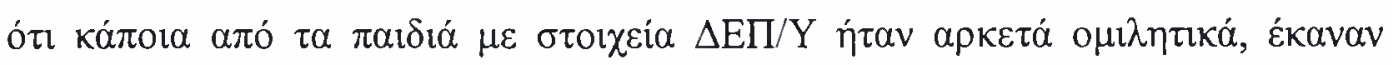

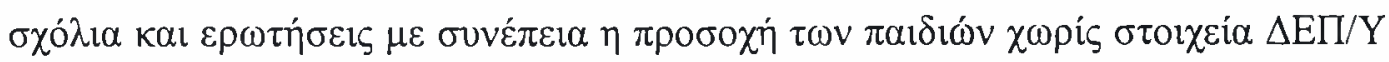

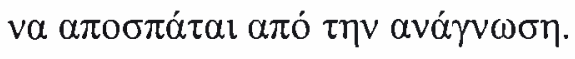




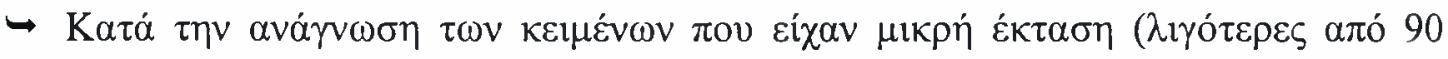

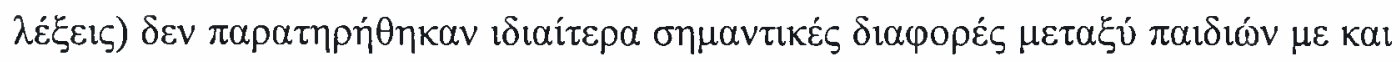

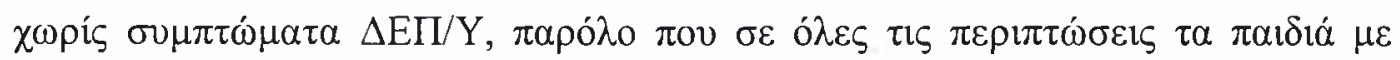

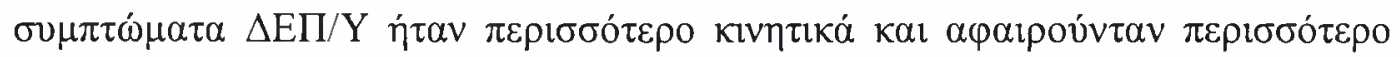

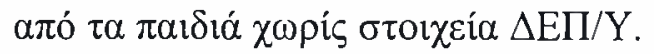

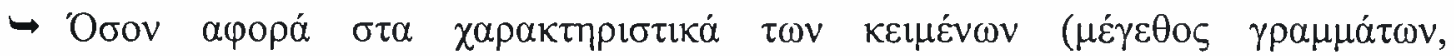

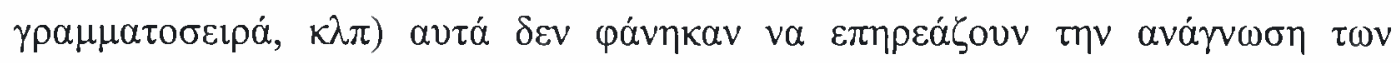

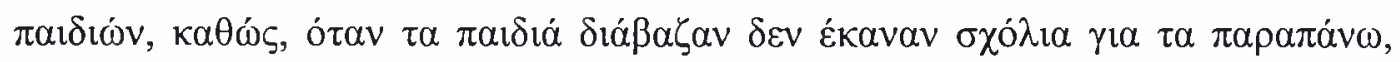

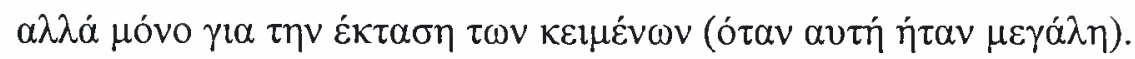

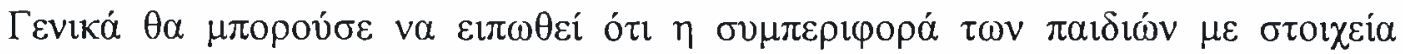

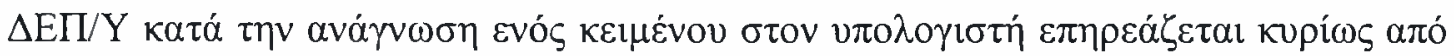

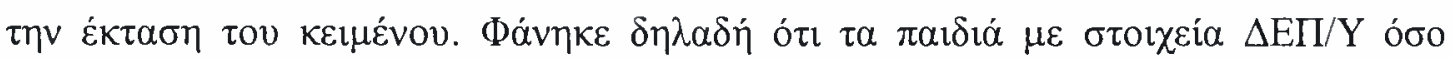

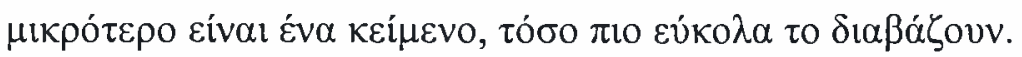

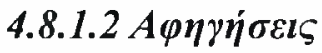

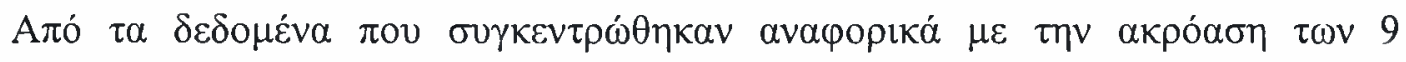

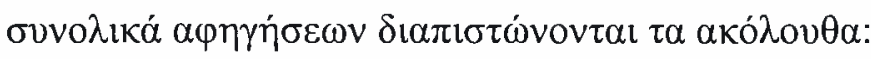

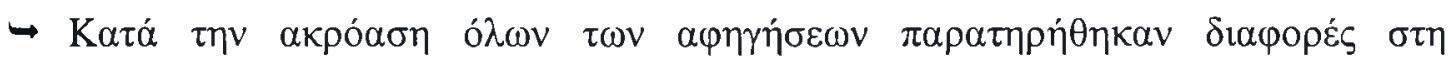

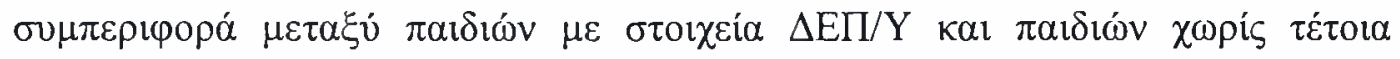

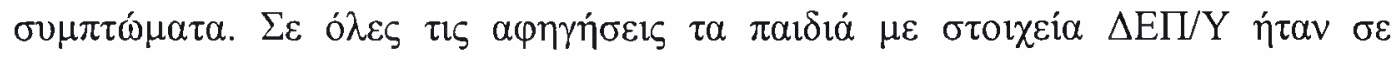

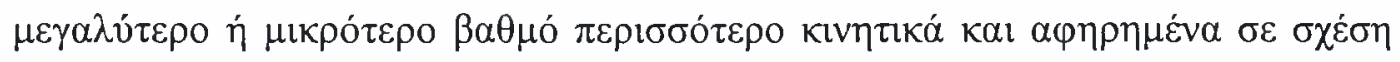

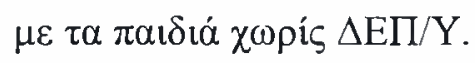

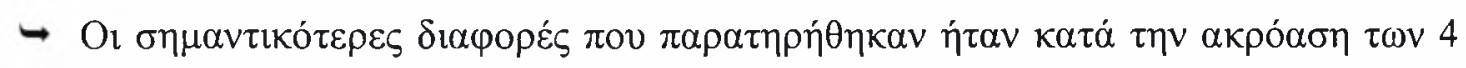

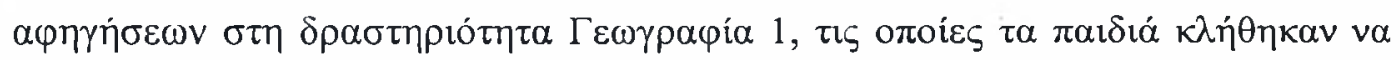

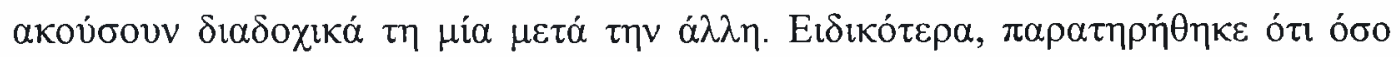

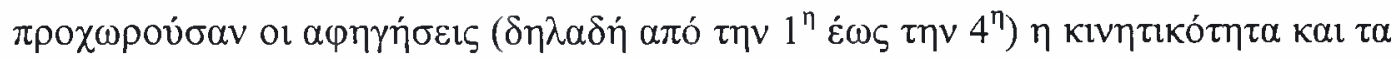

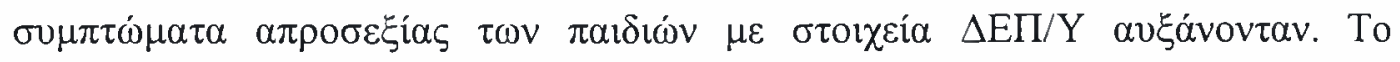

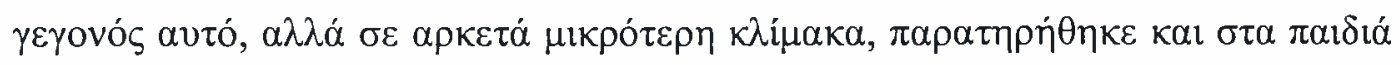

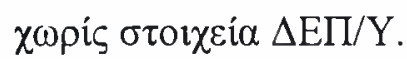

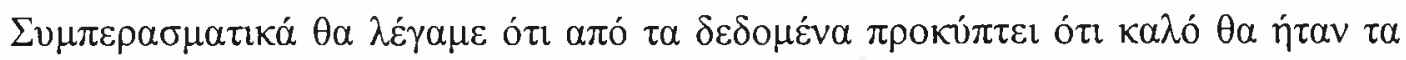

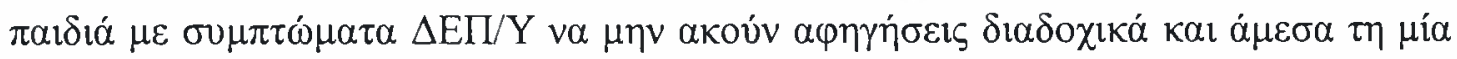

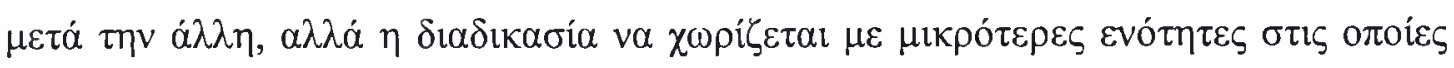

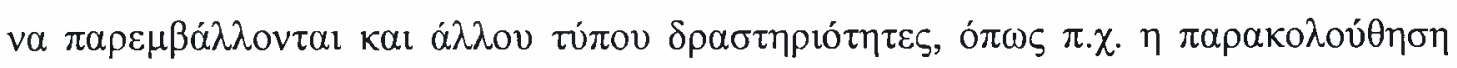




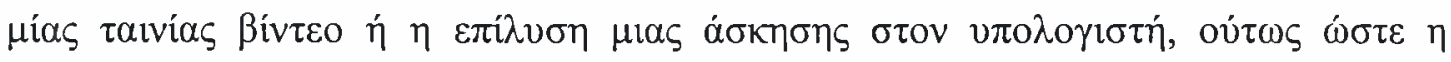

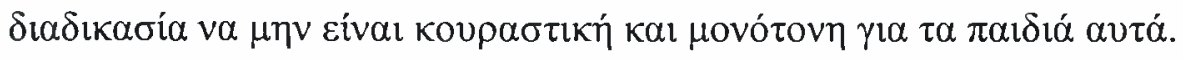

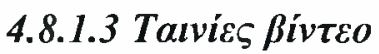

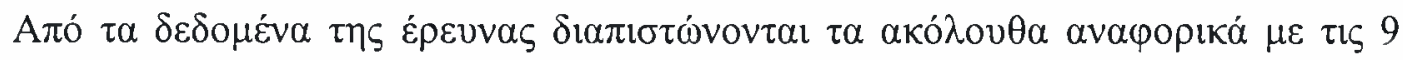

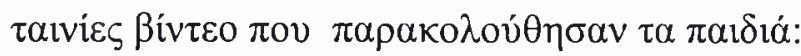

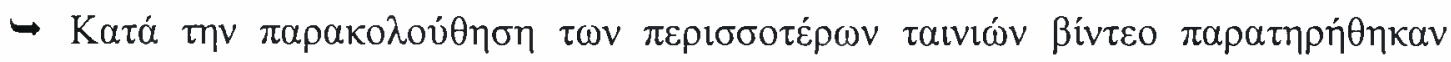

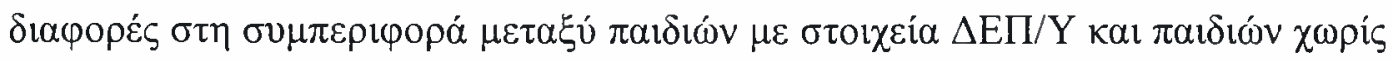

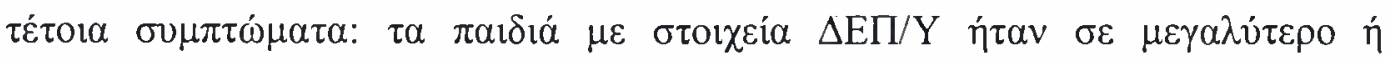

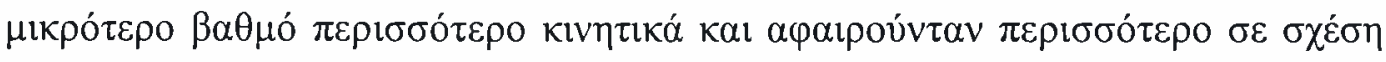
$\mu \varepsilon \tau \alpha \pi \alpha$ เíá $\chi \omega \rho i \varsigma \varsigma \Delta \Pi / Y$.

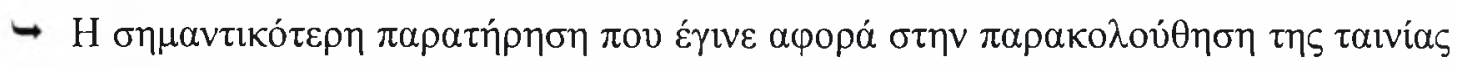

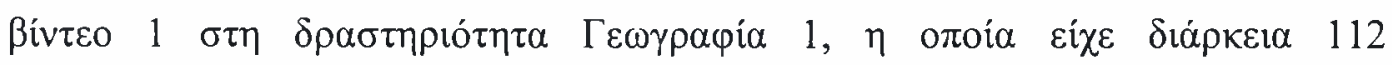

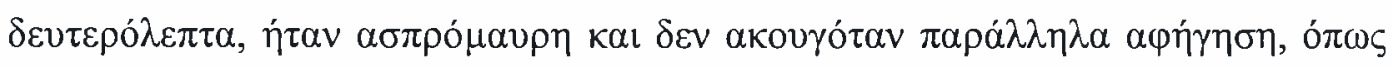

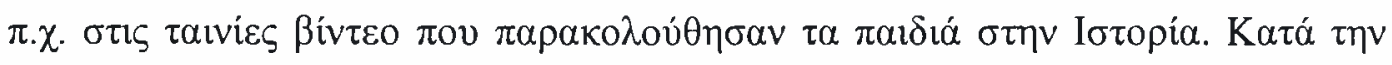

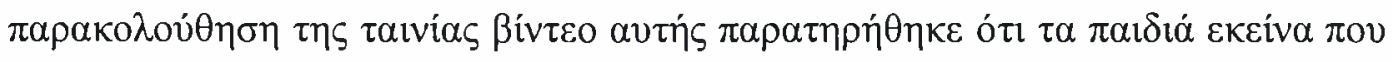

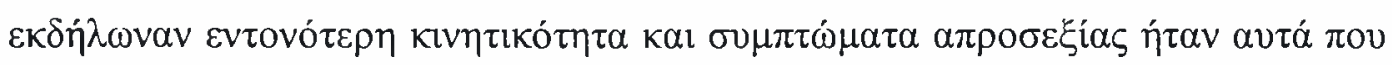

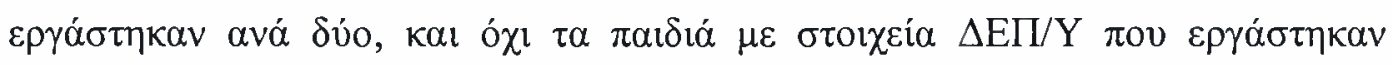

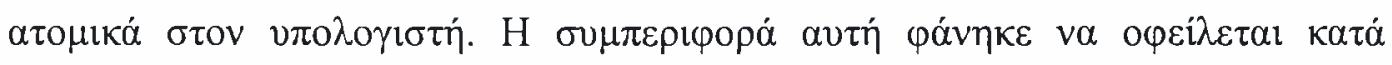

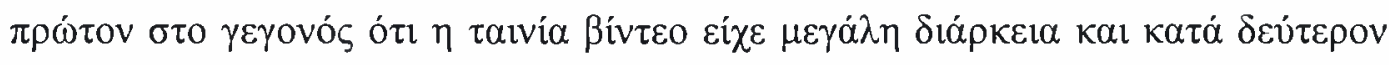

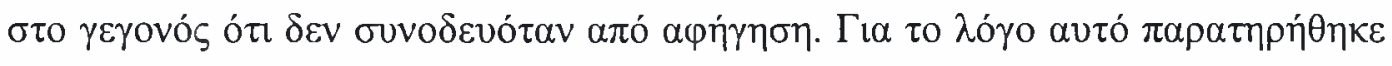

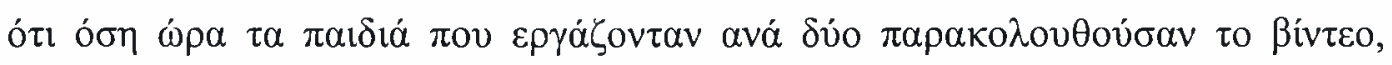

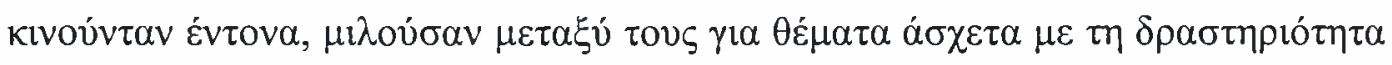

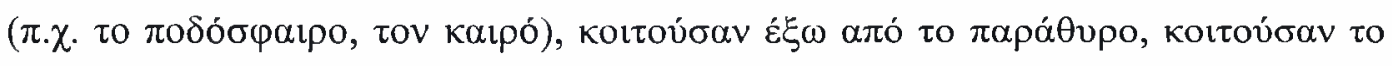

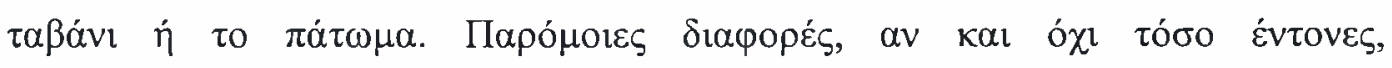

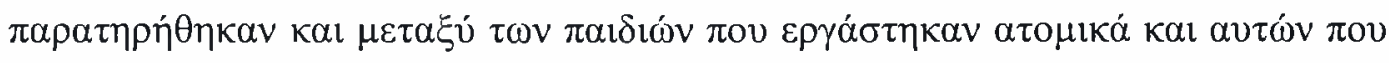

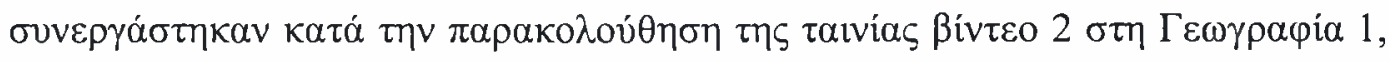

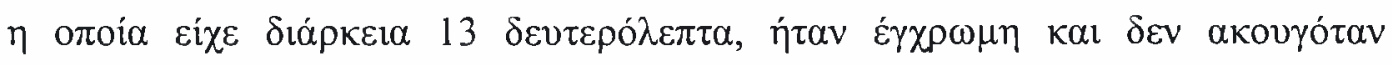

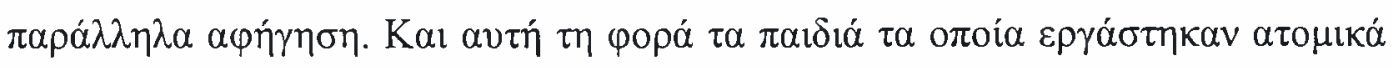

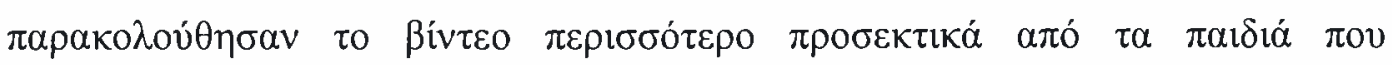

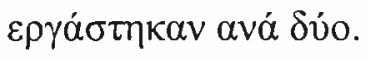

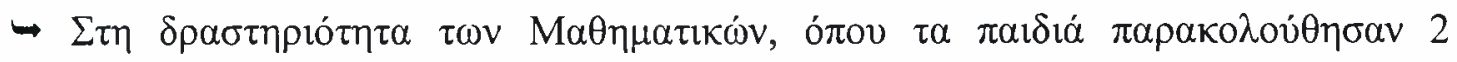

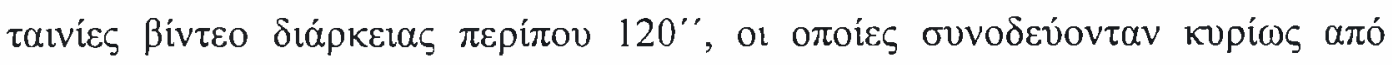




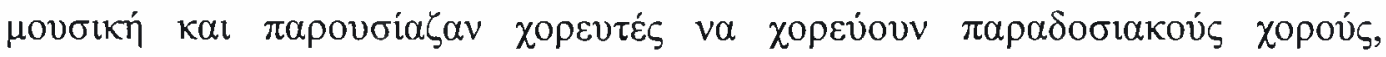

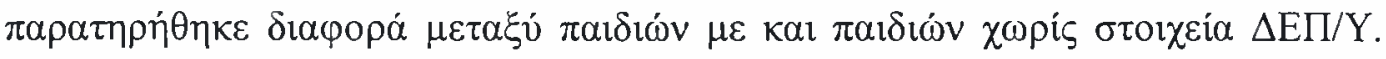

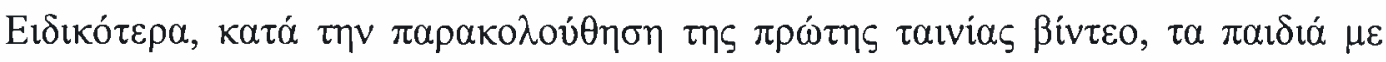

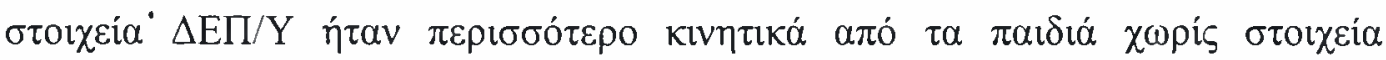

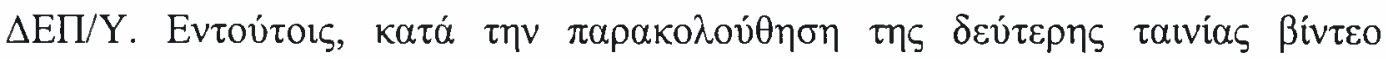

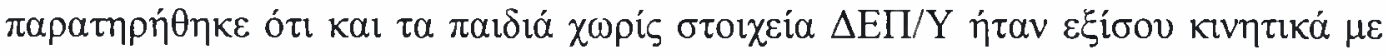

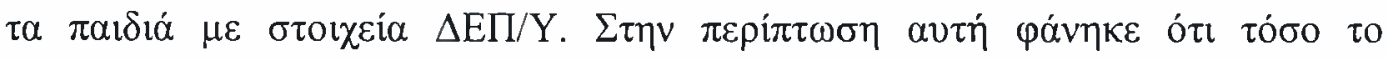

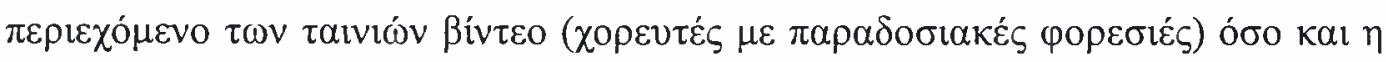

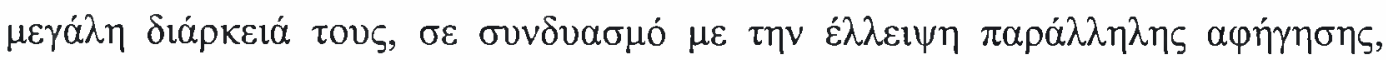

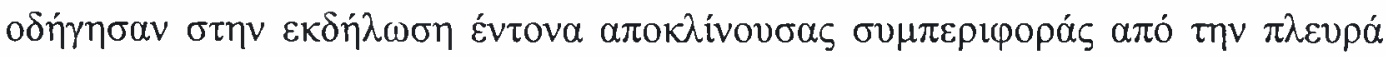

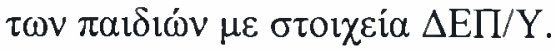

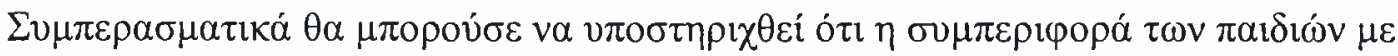

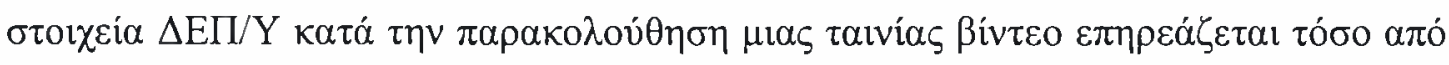

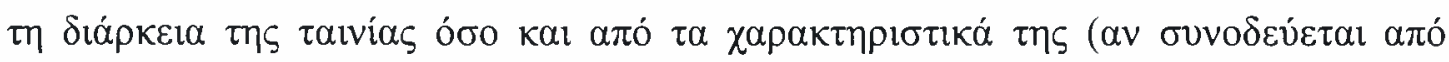

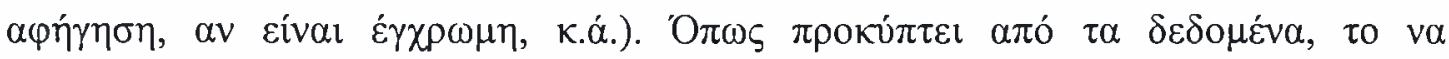

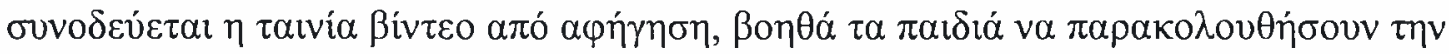

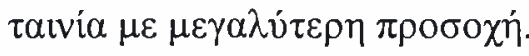

\subsubsection{Eıкóveৎ}

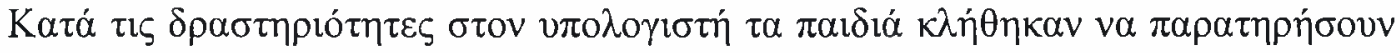

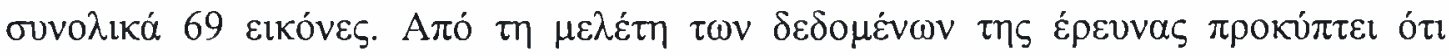

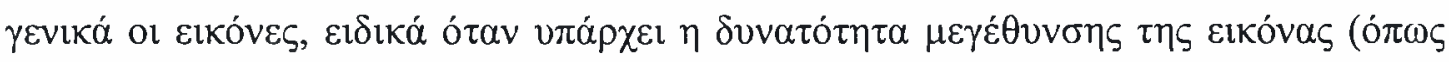

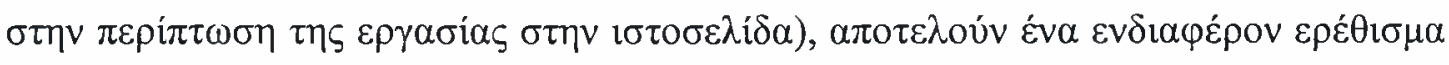

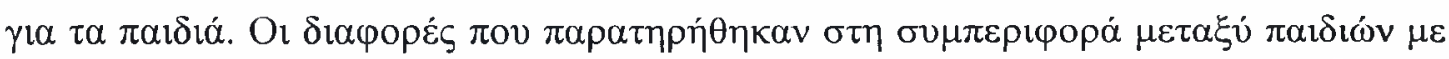

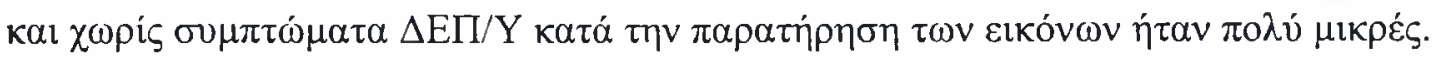

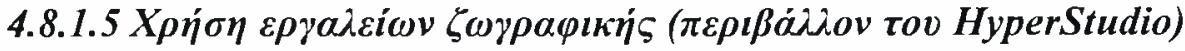

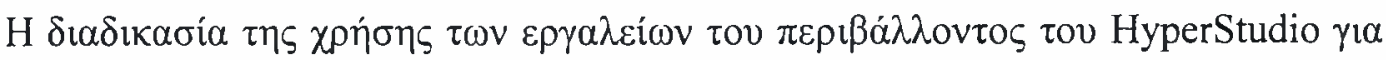

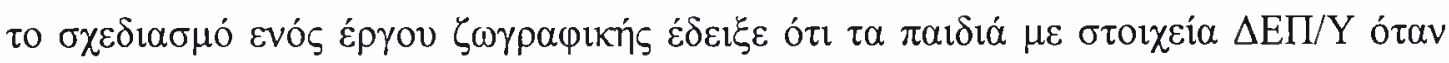

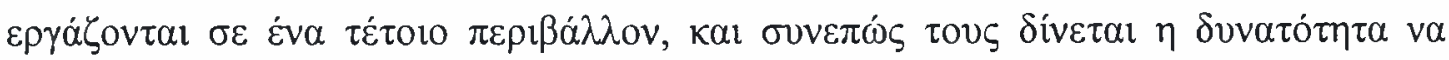

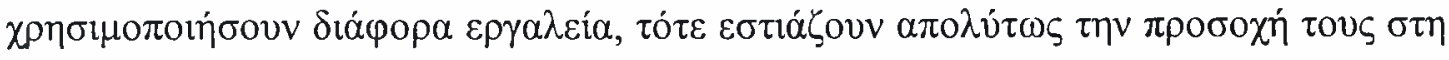

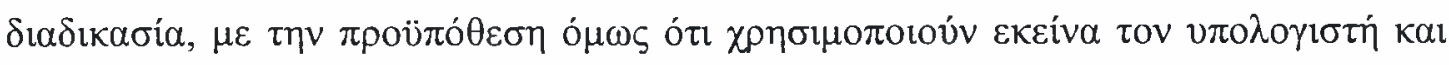




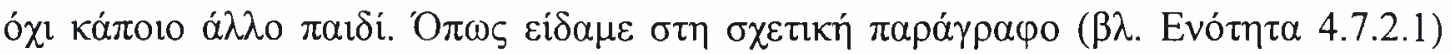

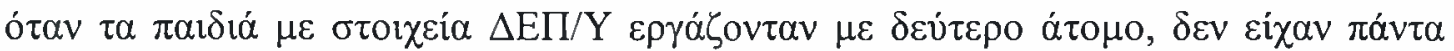

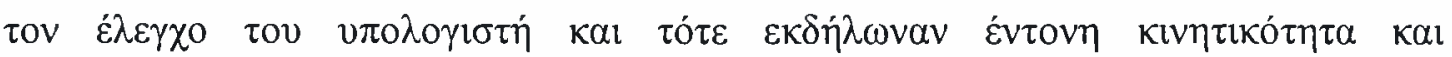

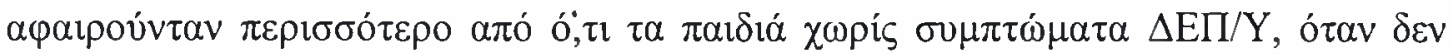

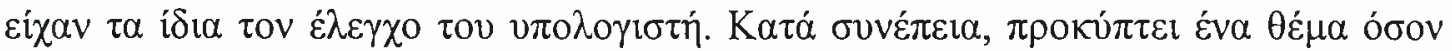

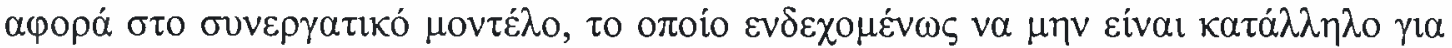

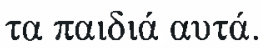

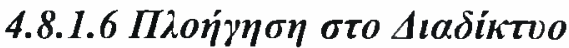

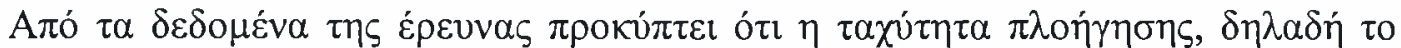

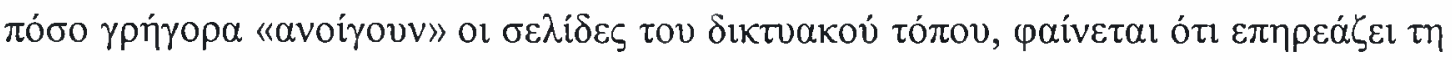

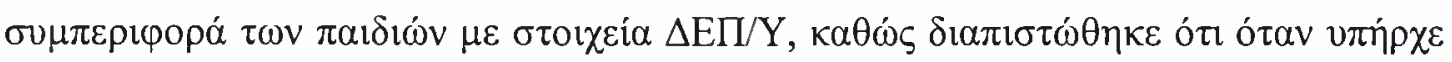

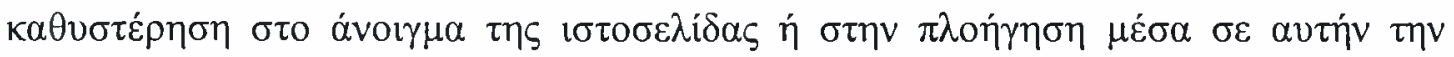

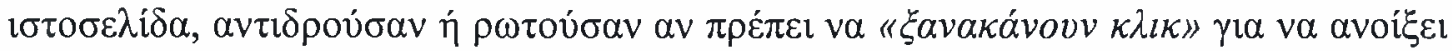

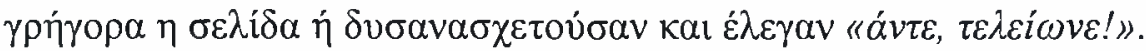

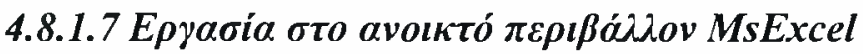

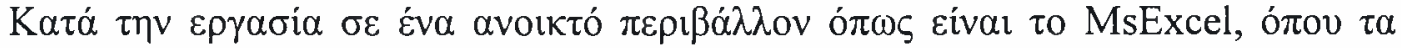

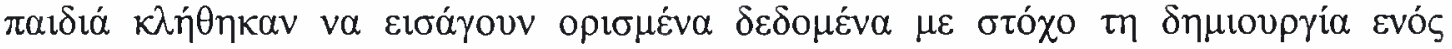

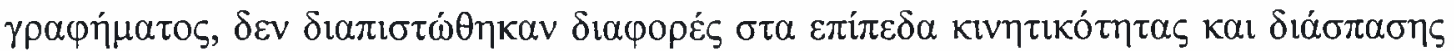

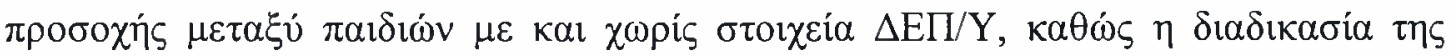

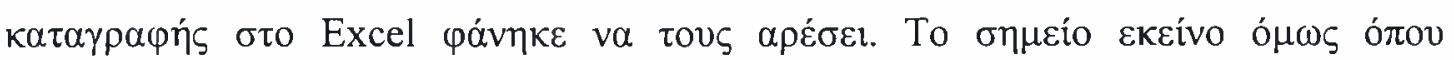

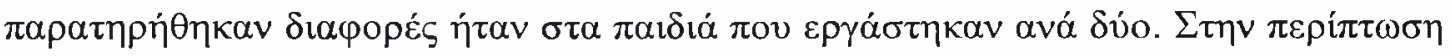

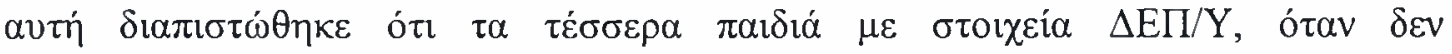

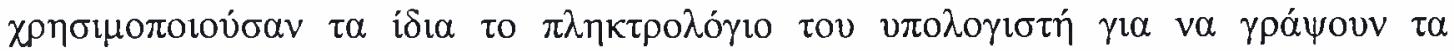

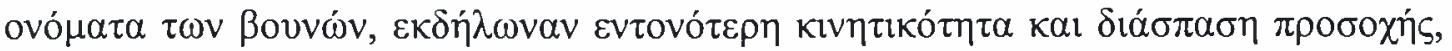

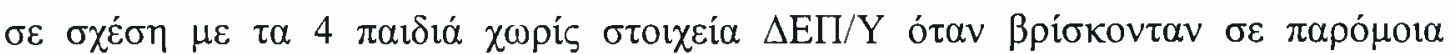

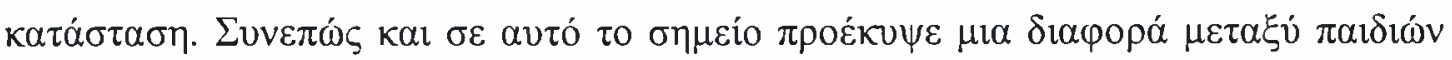

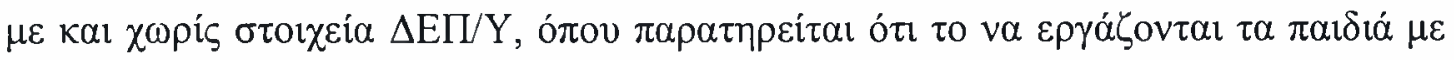

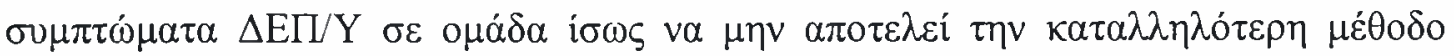
$\delta ı \delta \alpha \sigma \kappa \alpha \lambda i ́ \alpha \varsigma \kappa \alpha \iota \mu \alpha ́ \theta \eta \sigma \eta \varsigma$. 


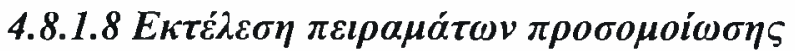

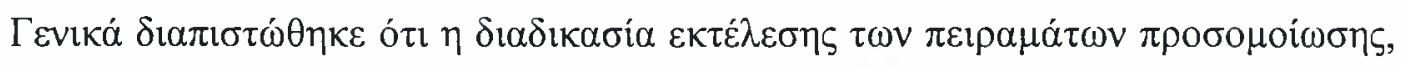

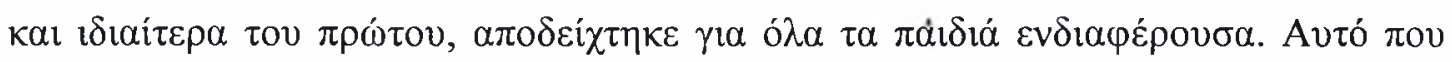

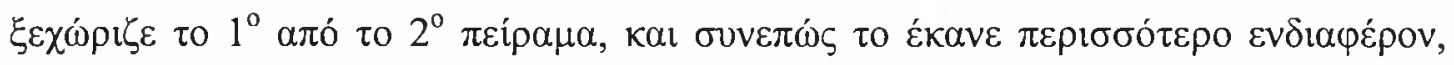

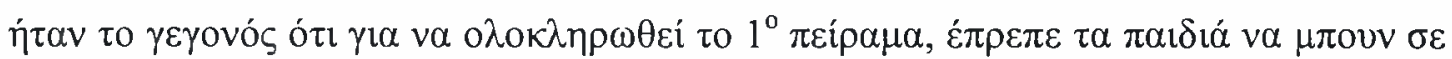

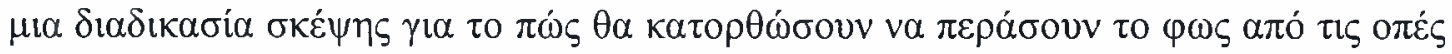

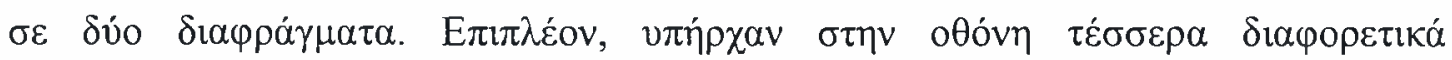

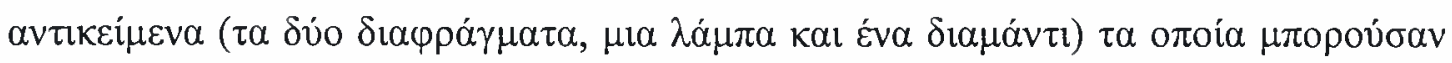

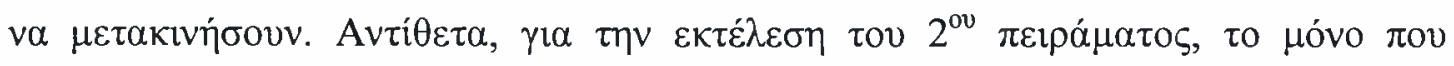

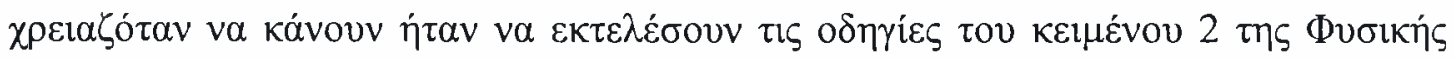

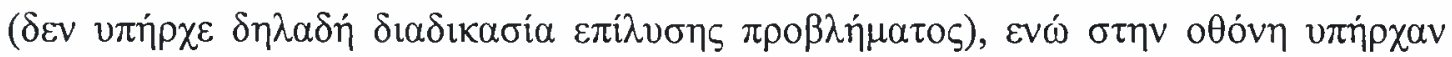

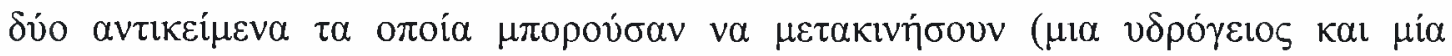

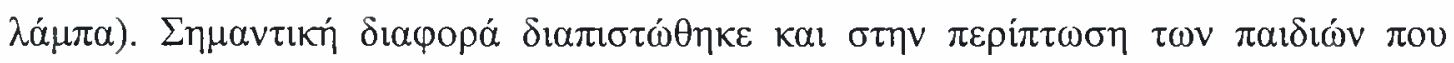

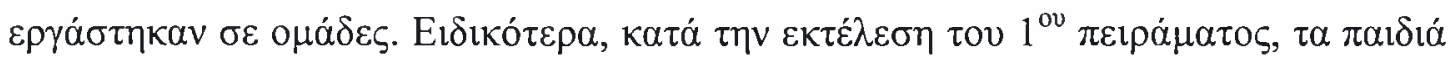

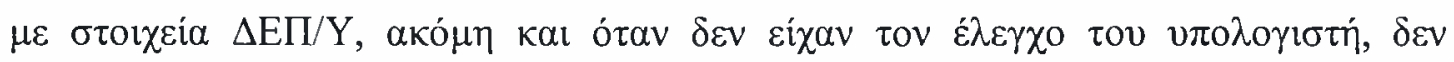

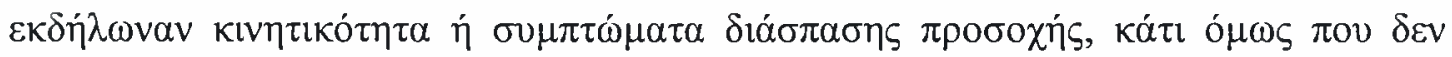

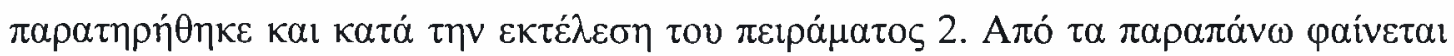

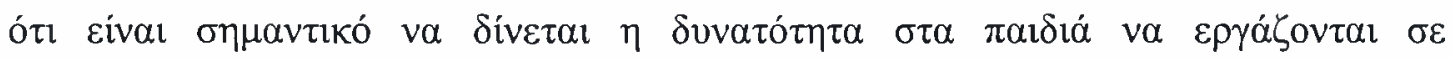

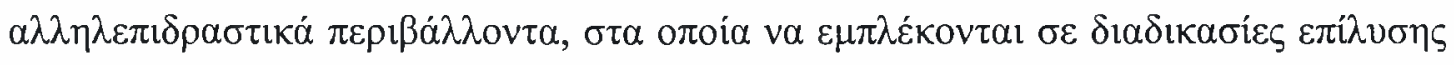

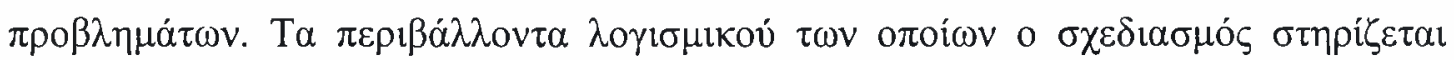

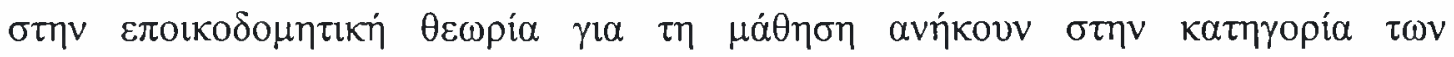

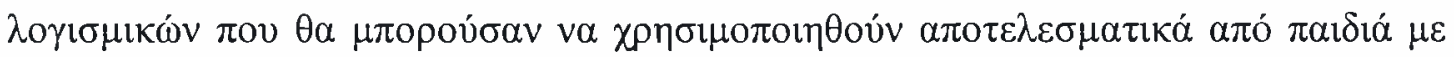
$\pi \rho \circ \beta \lambda \eta \dot{\mu} \mu \tau \alpha \Delta \mathrm{E} \Pi / \mathrm{Y}$.

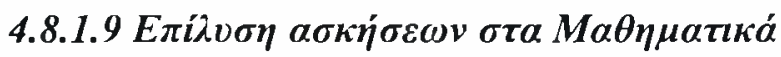

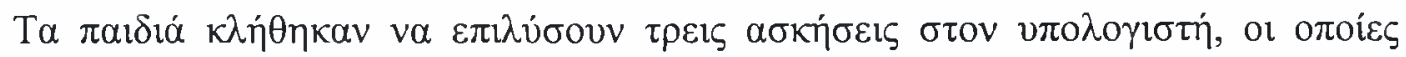

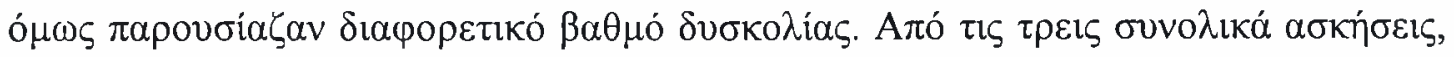

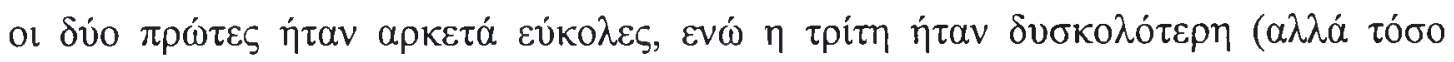

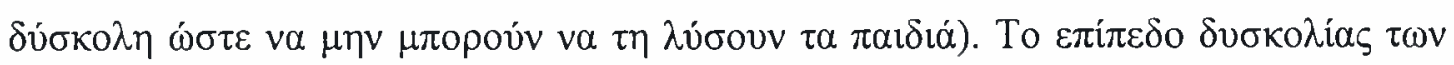

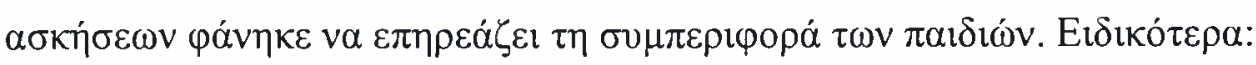

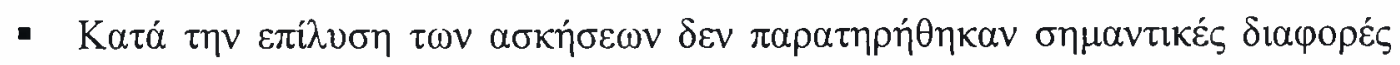

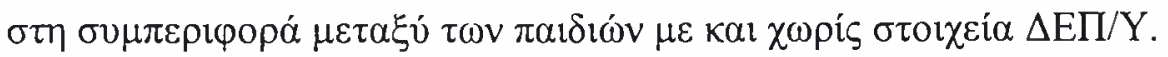




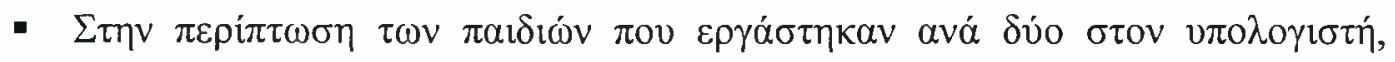

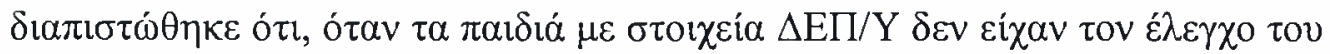

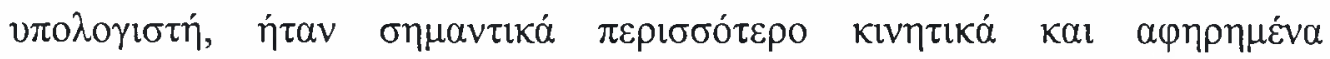

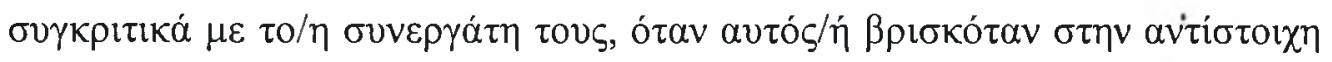

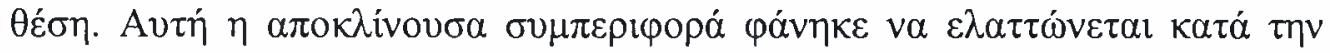

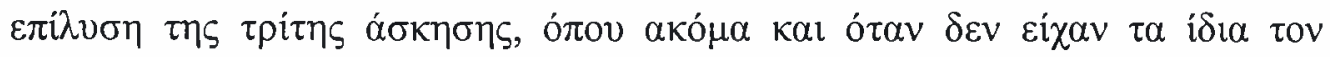

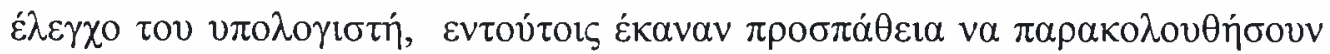

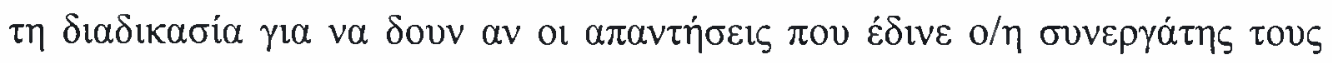

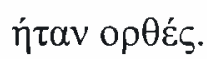

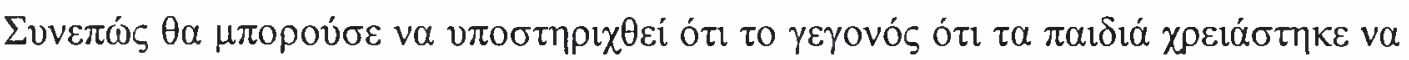

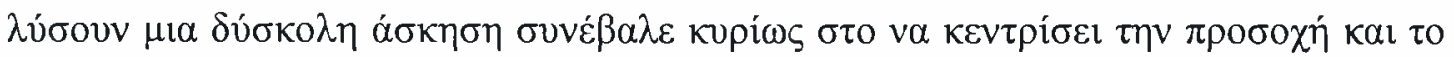

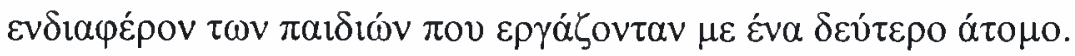

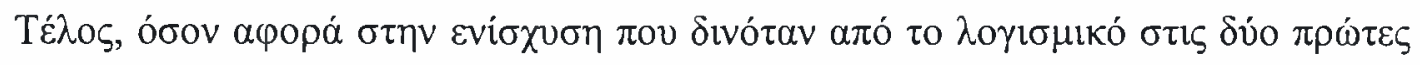

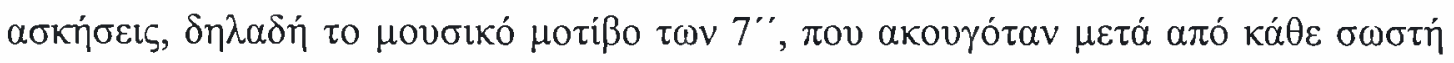

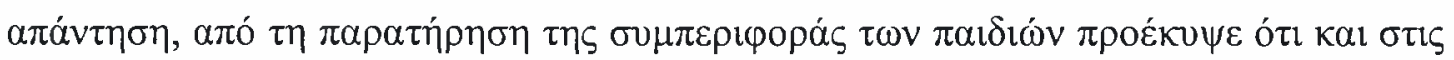

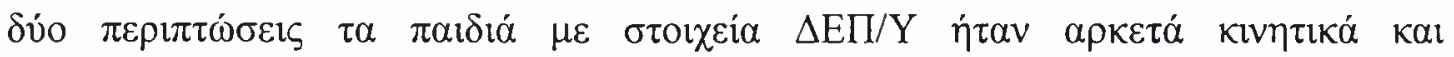

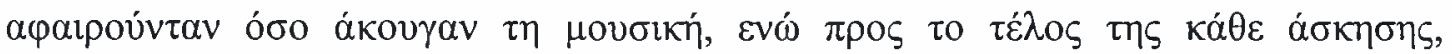

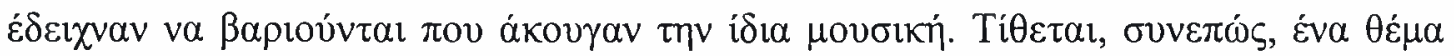

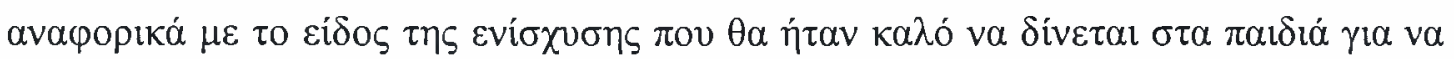

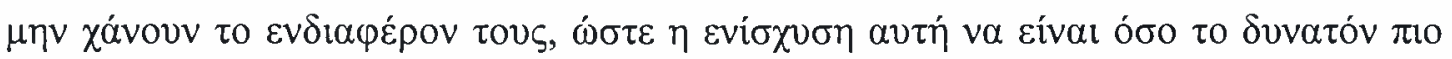

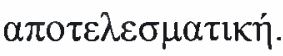

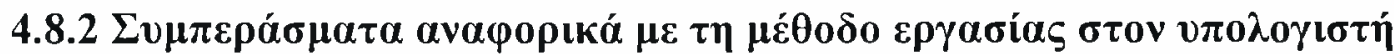

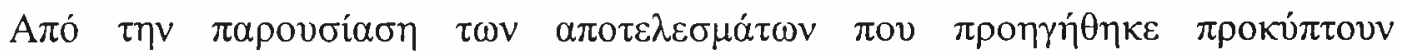

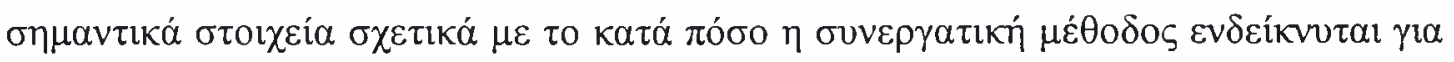

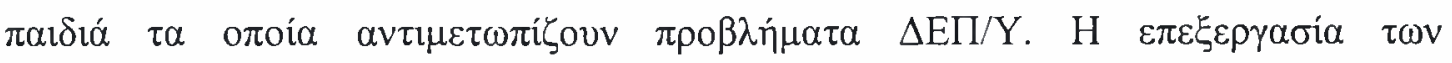

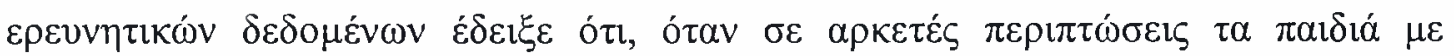

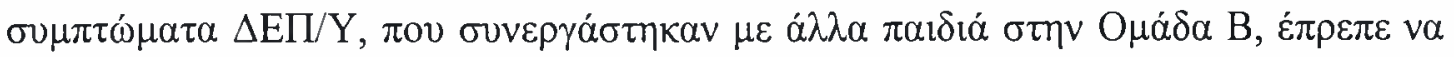

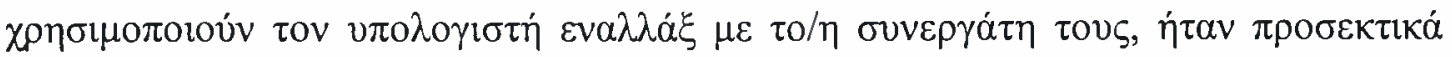

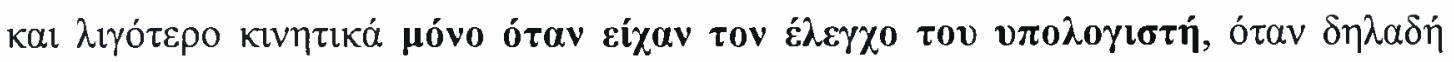

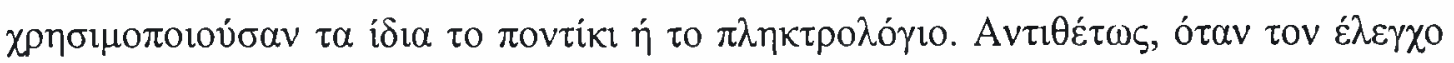

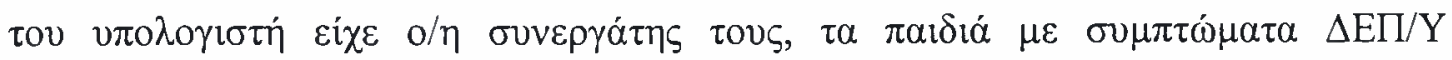




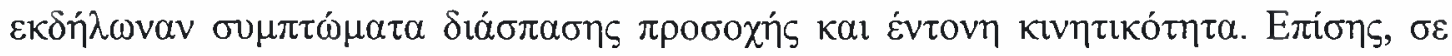

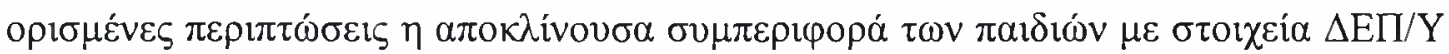

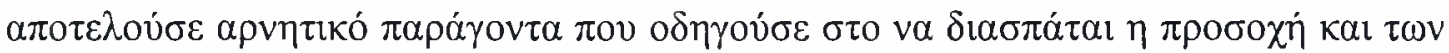

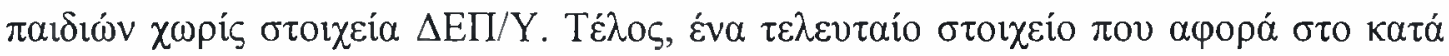

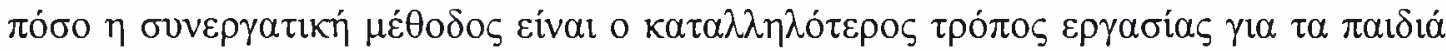

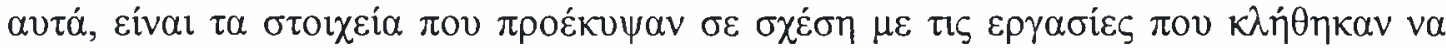

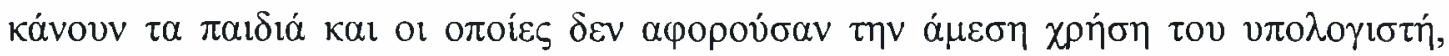

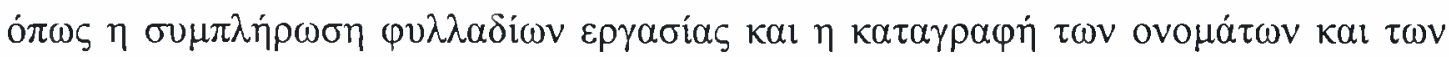

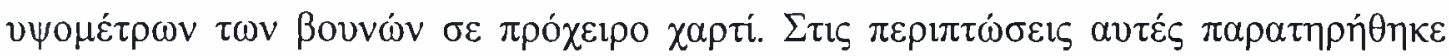

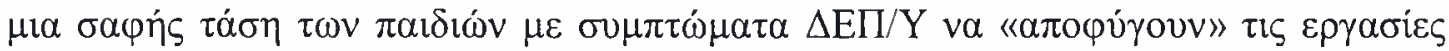

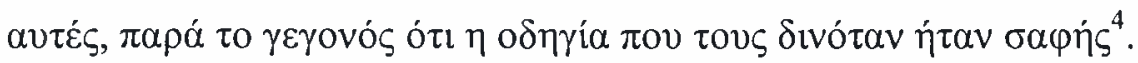

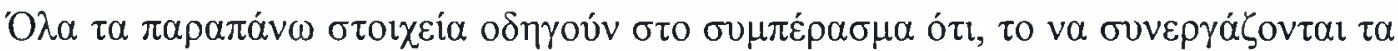

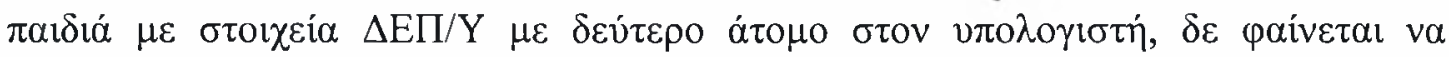

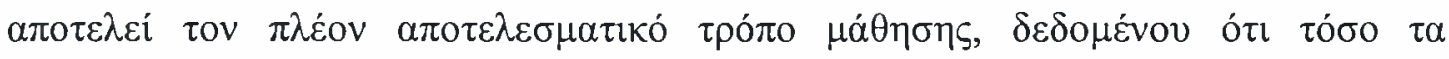

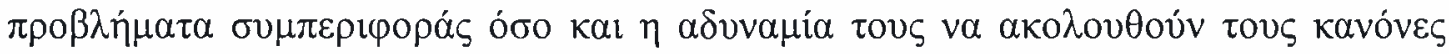

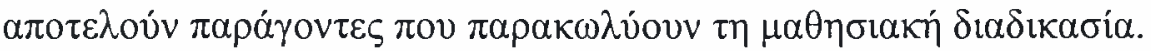

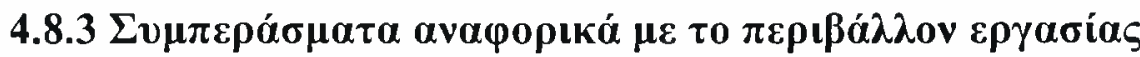

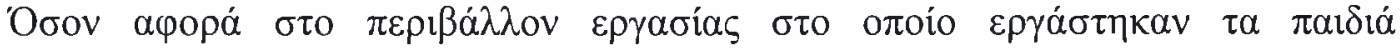

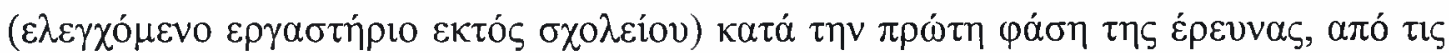

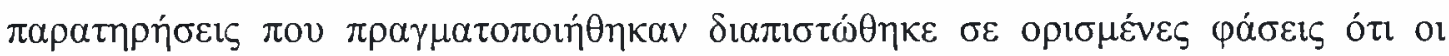

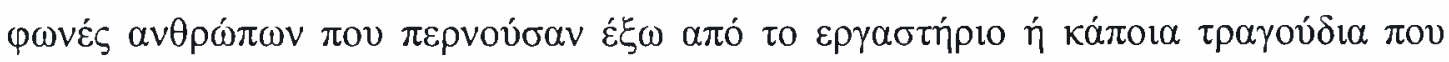

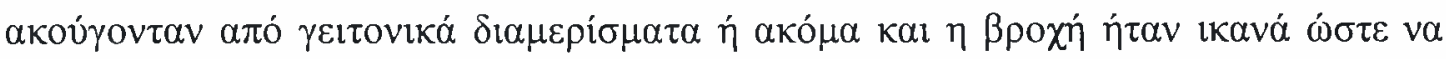

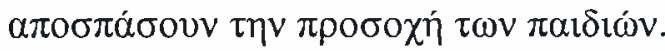

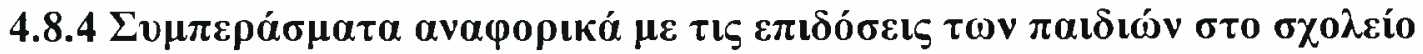

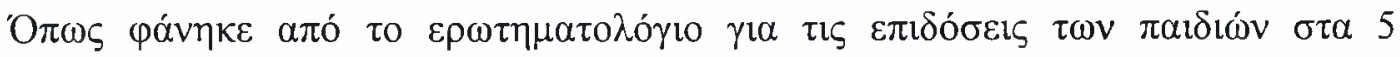

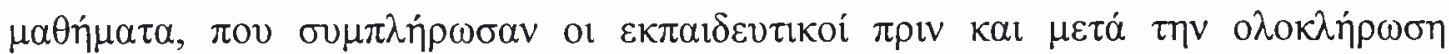

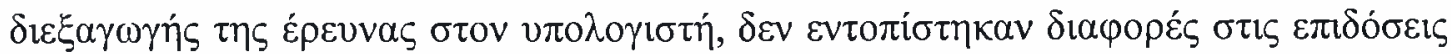

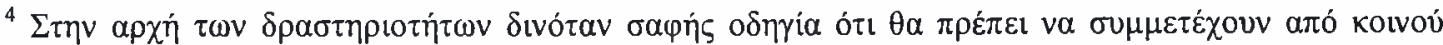

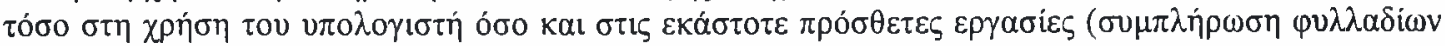
$\varepsilon \rho \gamma \alpha \sigma i \alpha \zeta, \kappa . \alpha ́)$.
} 


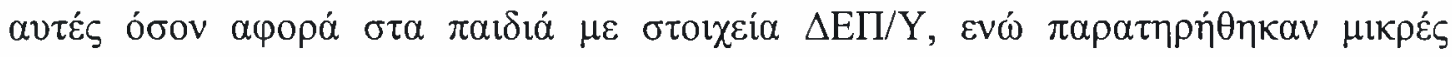

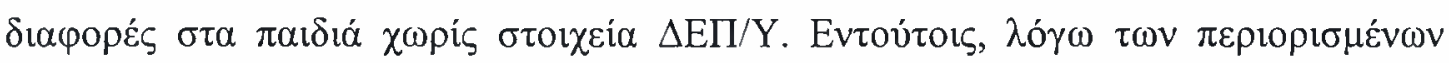

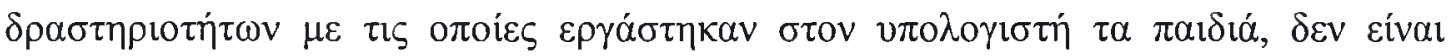

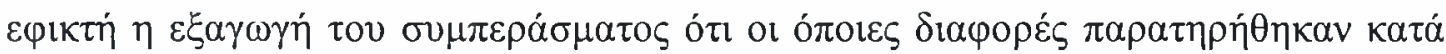

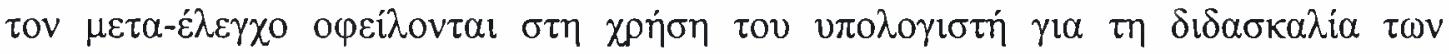

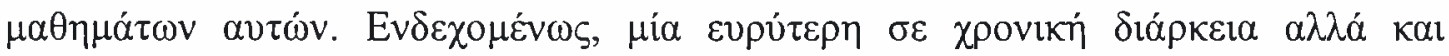

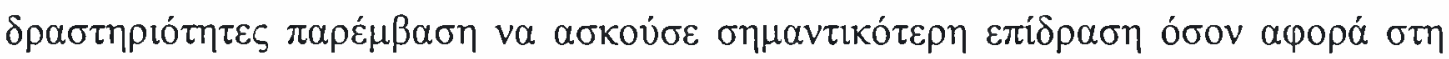

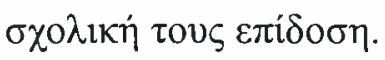

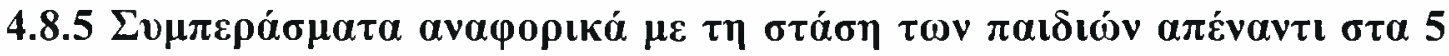

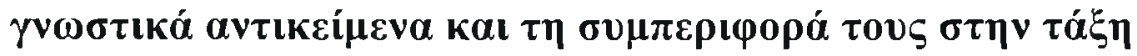

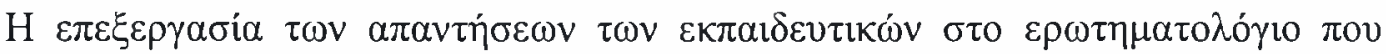

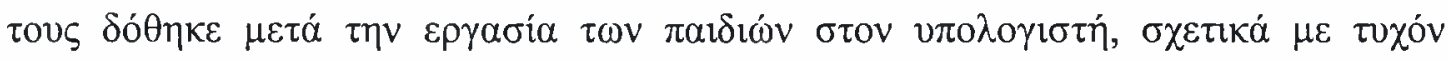

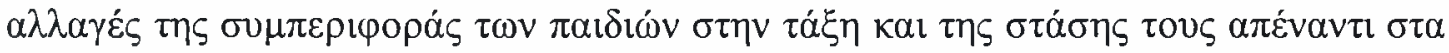

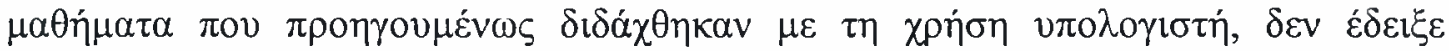

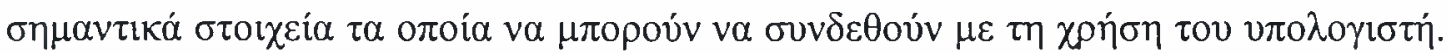

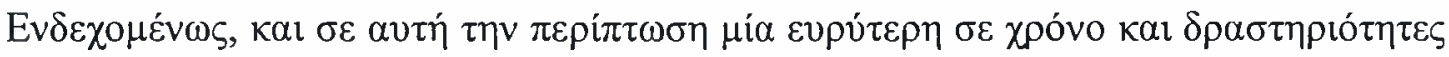

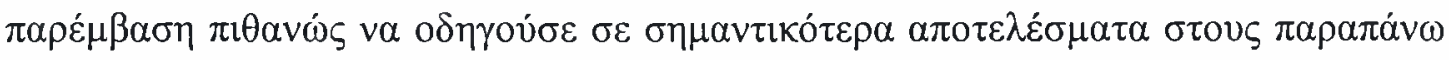

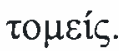

\section{$4.9 \Sigma Y N O \Psi H$}

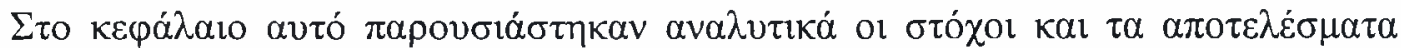

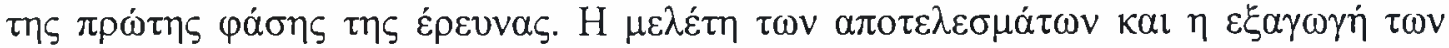

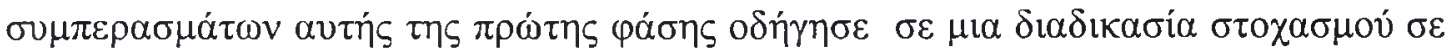

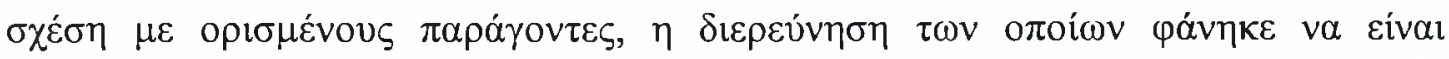

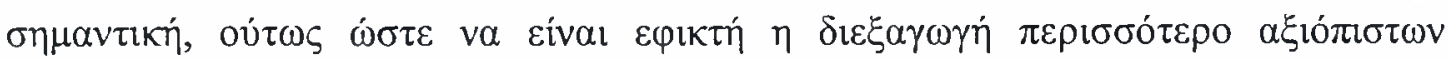

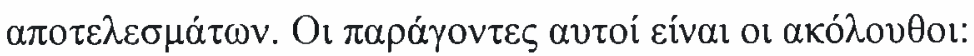

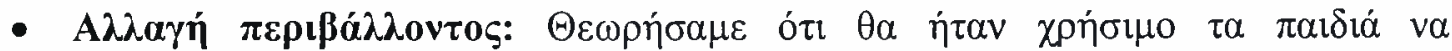

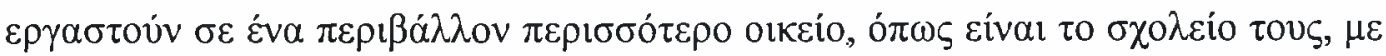

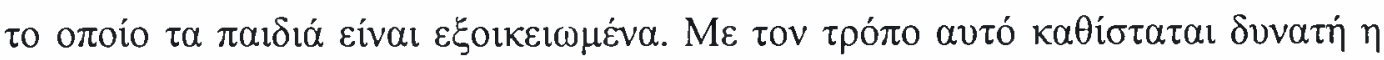




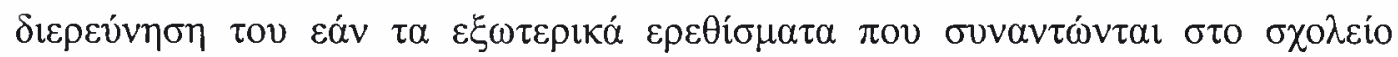

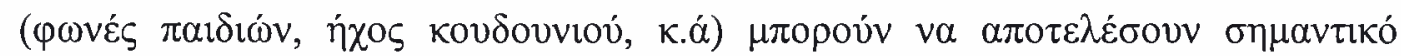

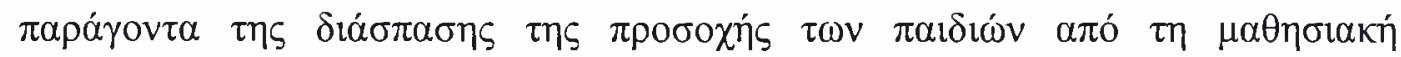

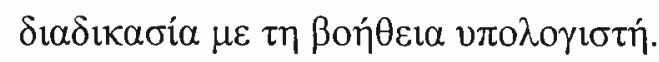

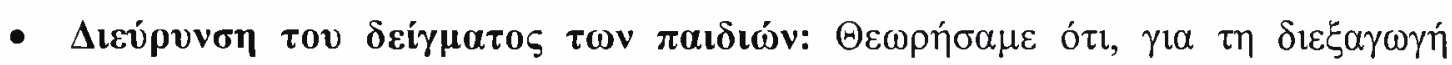

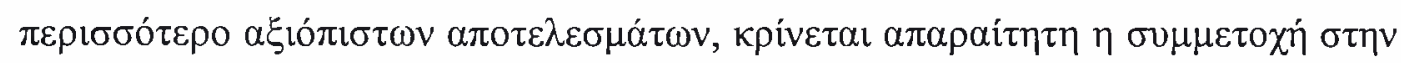

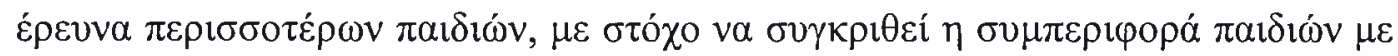

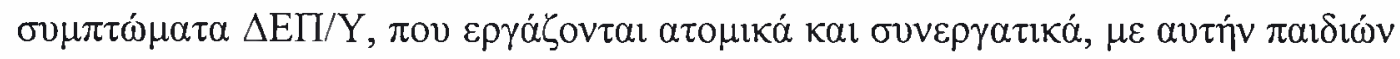

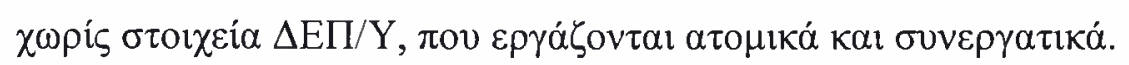

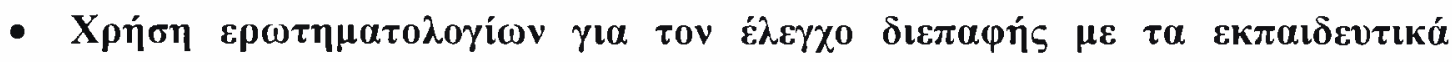

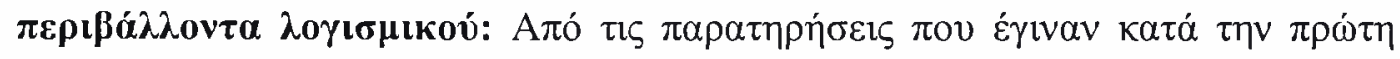

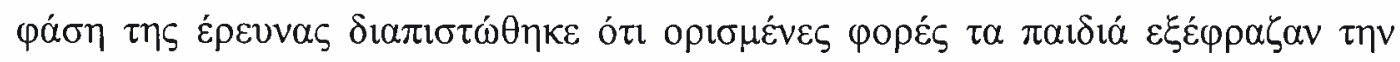

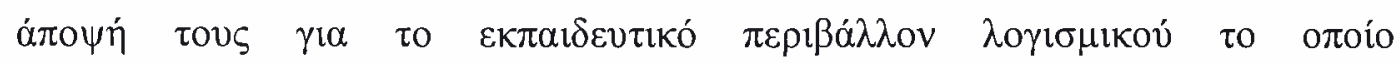
$\chi \rho \eta \sigma \mu о$ о

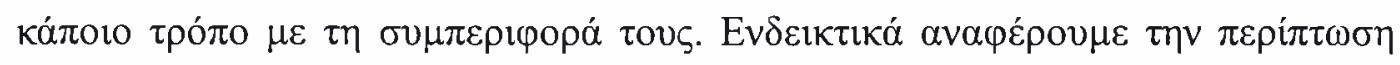

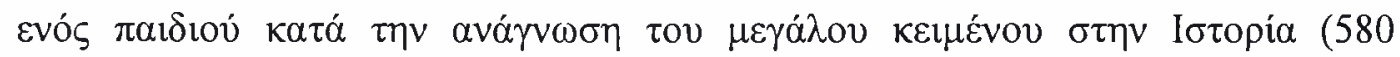

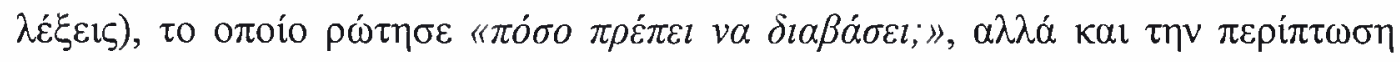

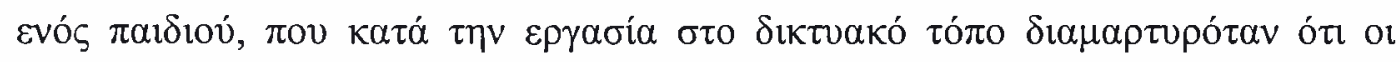

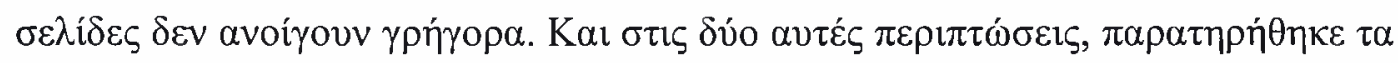

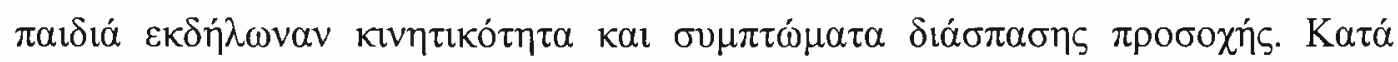

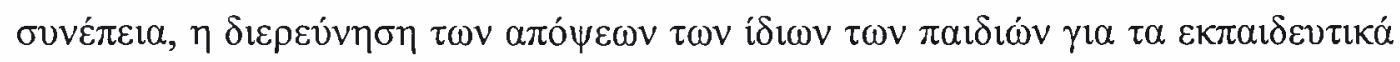

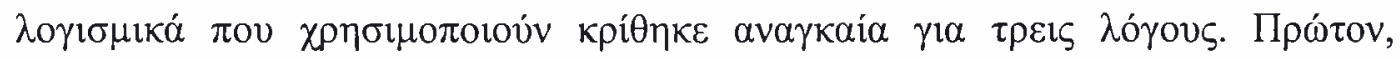

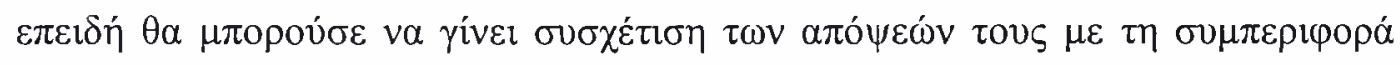

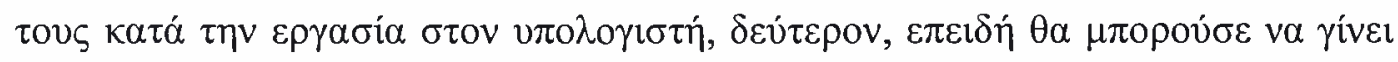

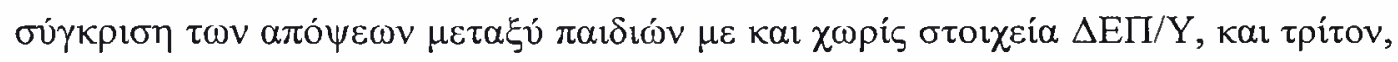

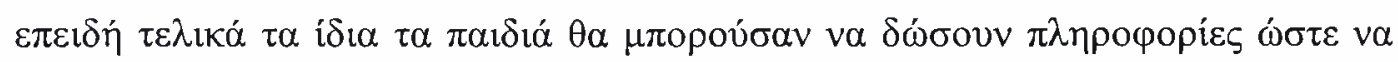

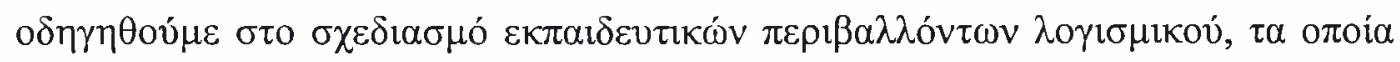

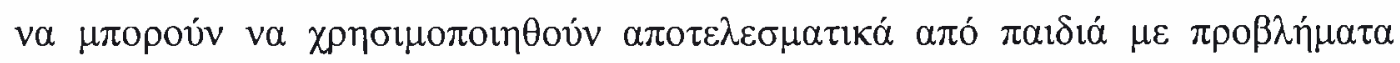

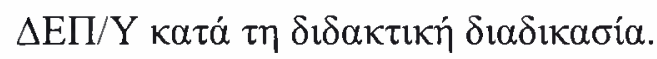




\section{ПЕМПТО КЕФА $\Lambda$ AIO}

\section{$\triangle$ EYTEPH $\Phi$ A $\Sigma H$ TH $\Sigma$ EPEYNA $\Sigma$}

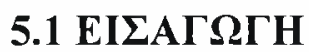

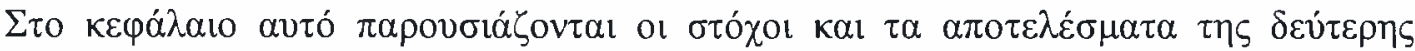

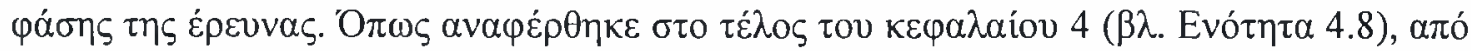

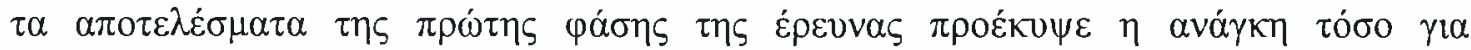

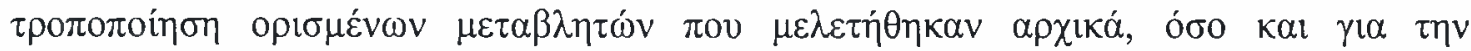

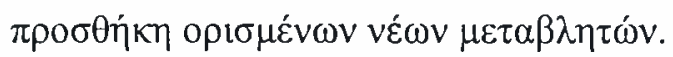

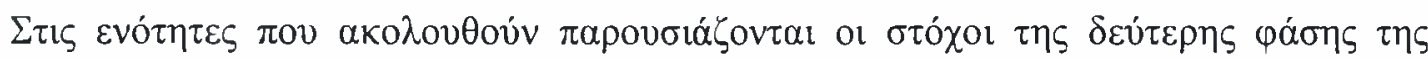

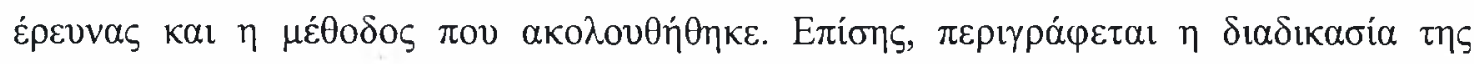

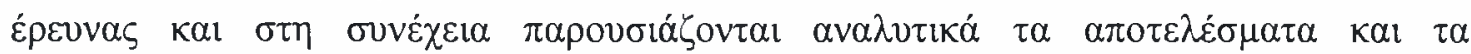

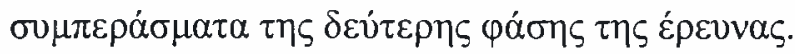

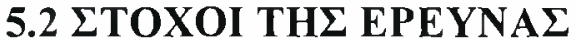

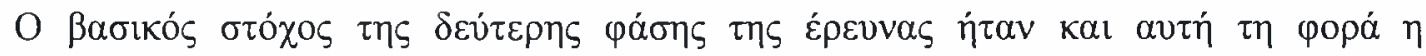

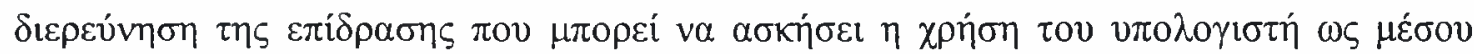

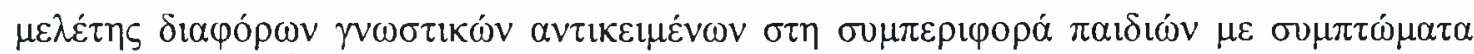

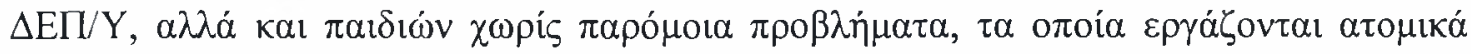

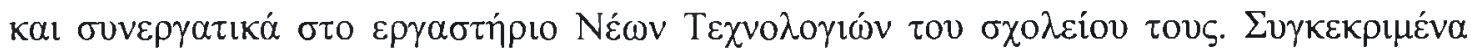

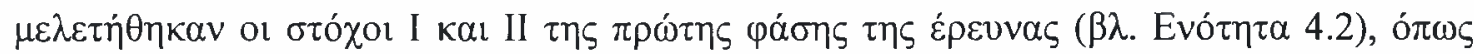

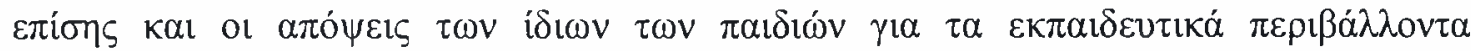

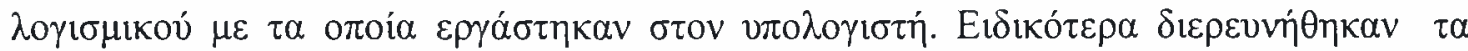
$\alpha \kappa o ́ \lambda o v \theta \alpha$ : 


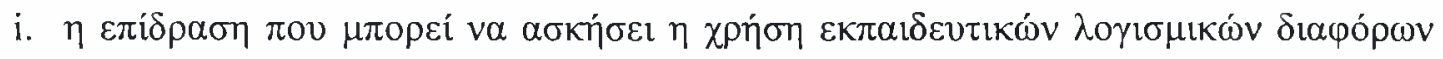

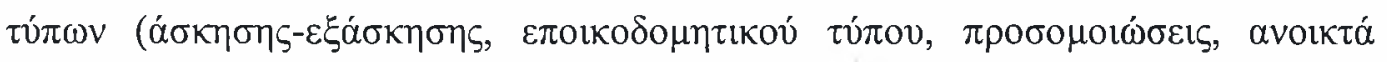

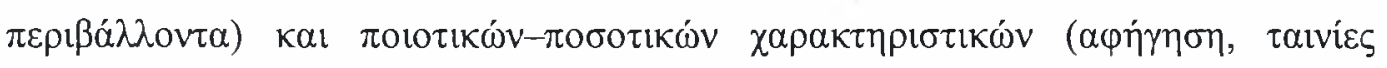

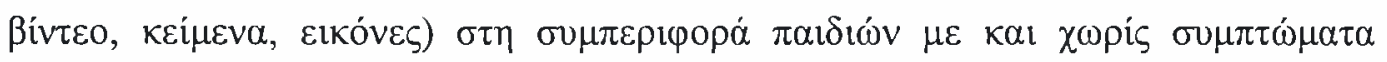
$\Delta \mathrm{E} \Pi / \mathrm{Y}$,

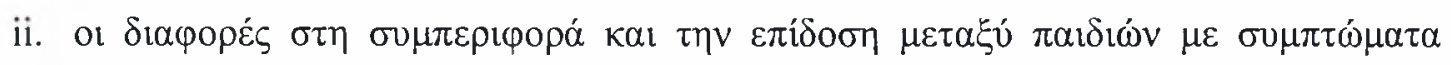

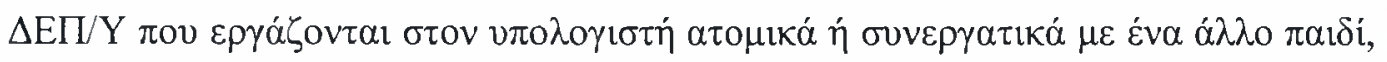

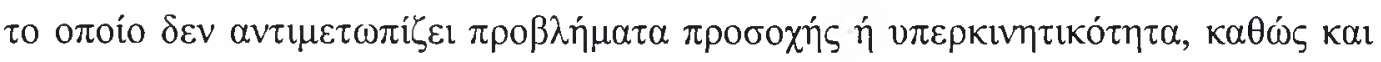

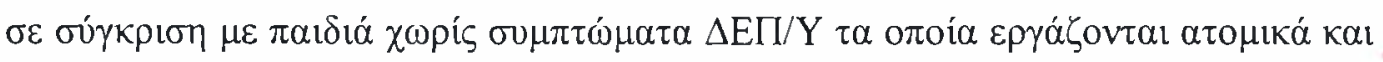

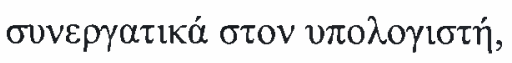

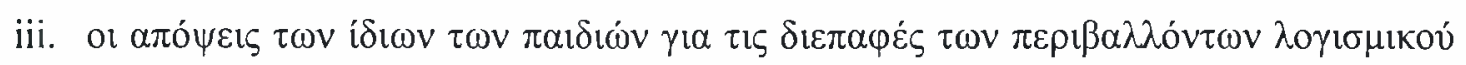

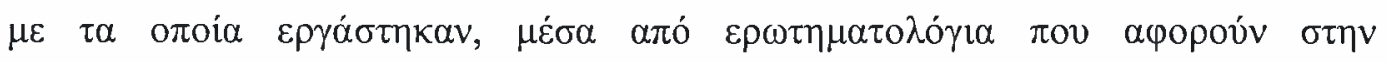

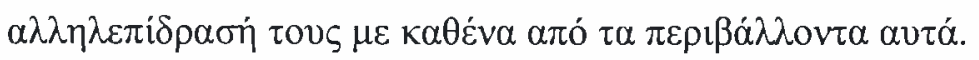

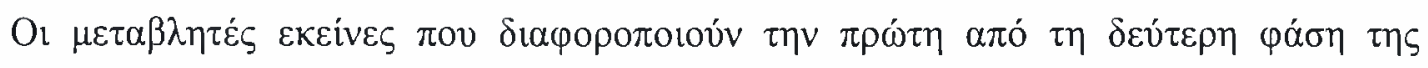
Épevvas gíval:

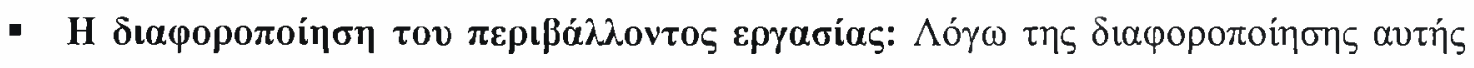

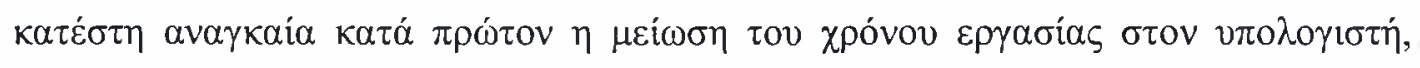

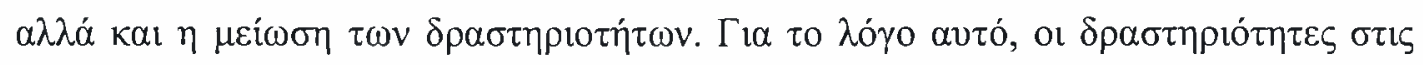

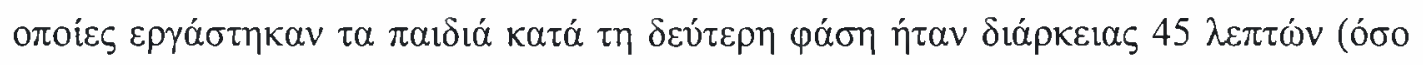

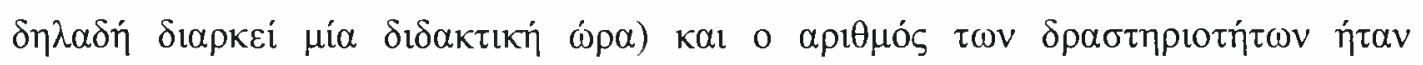

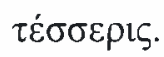

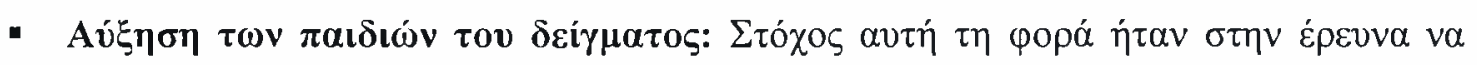

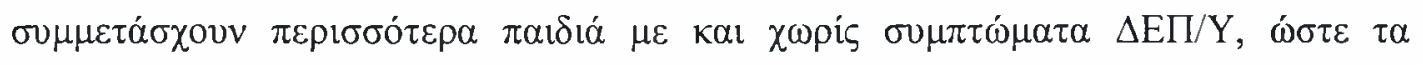

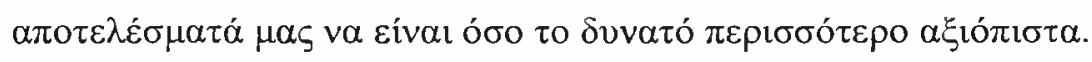

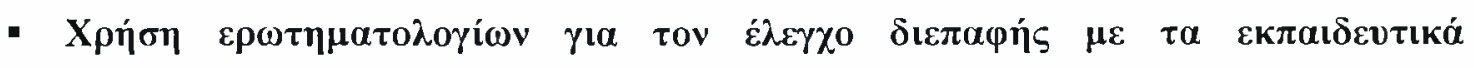

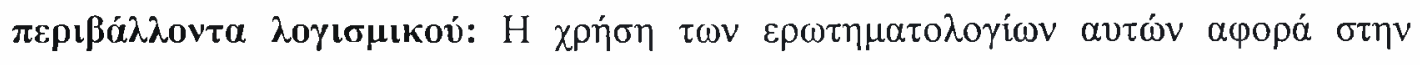

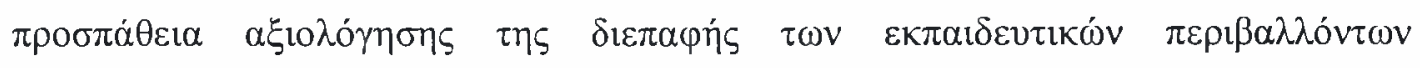

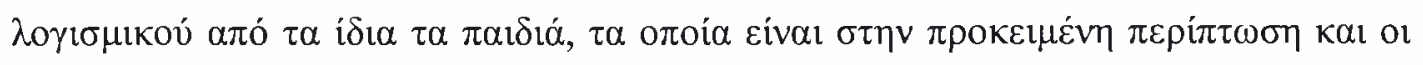

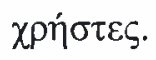

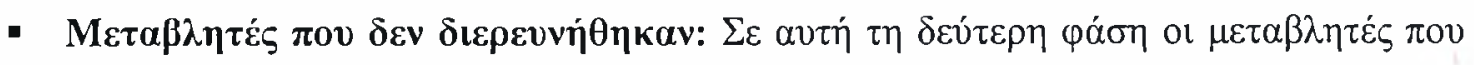

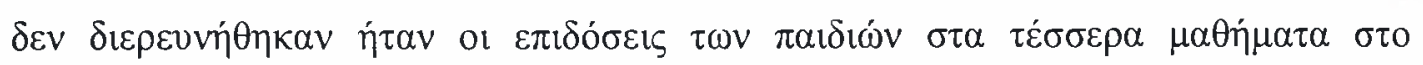




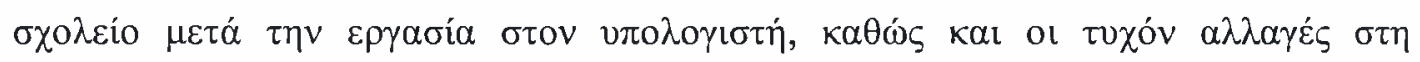

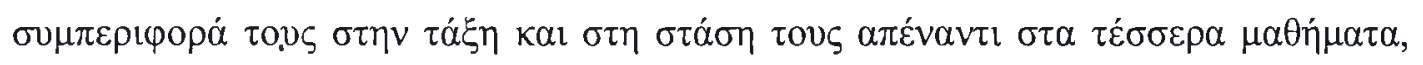

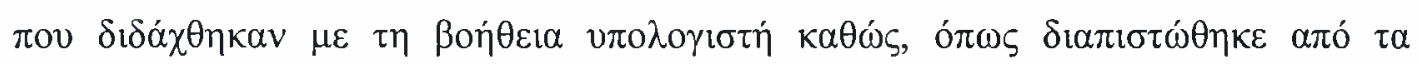

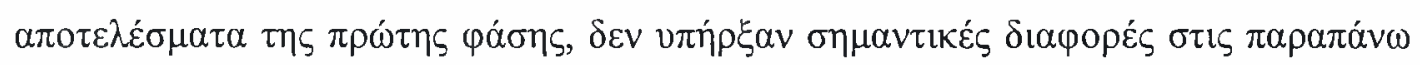

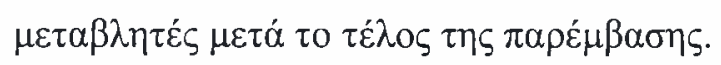

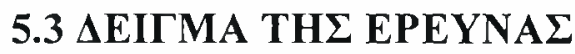

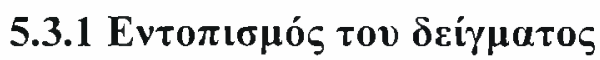

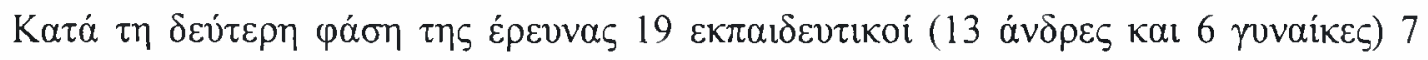

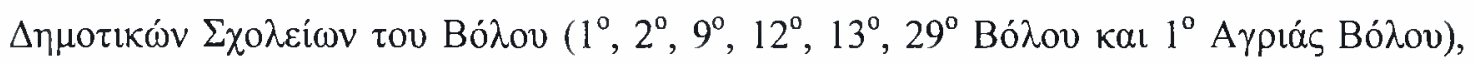

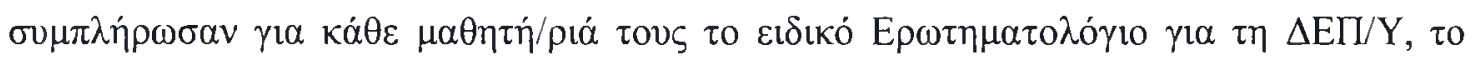

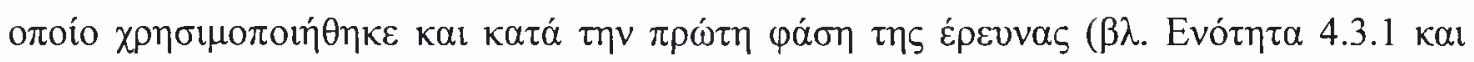

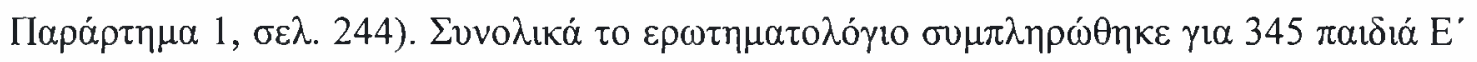

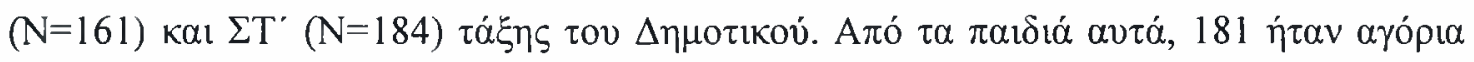

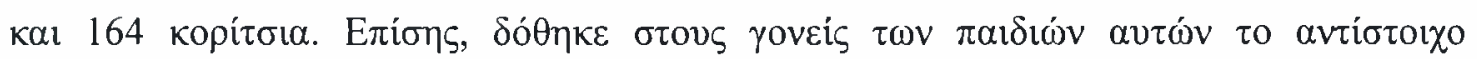

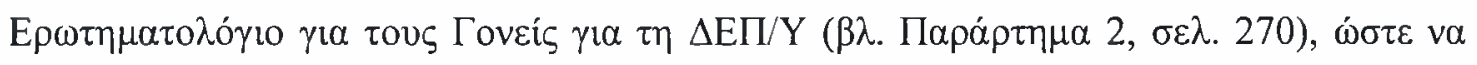

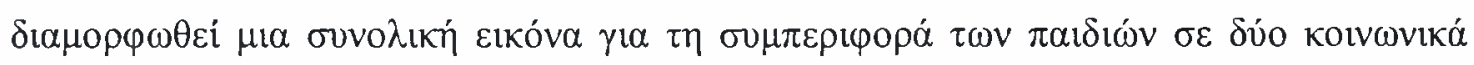

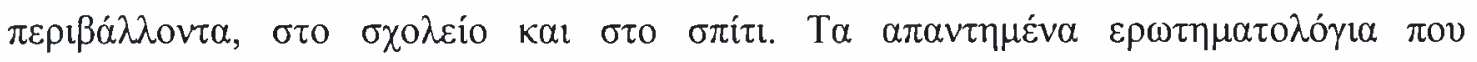

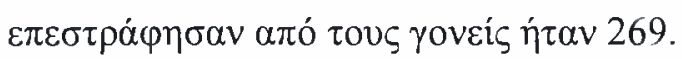

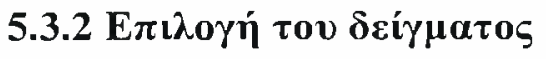

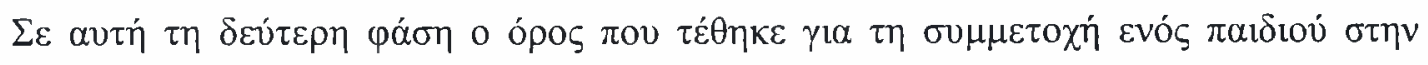

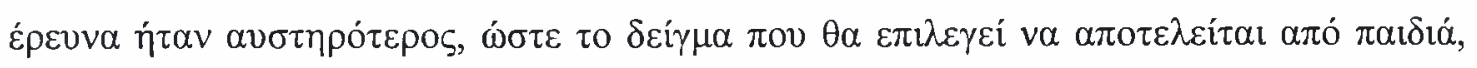

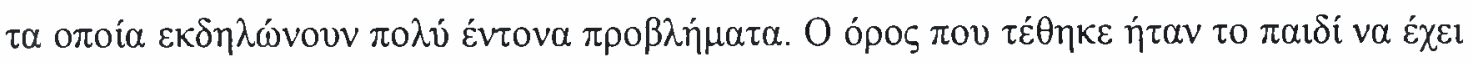

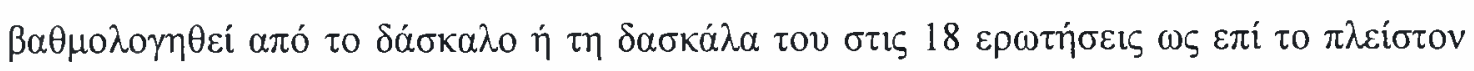

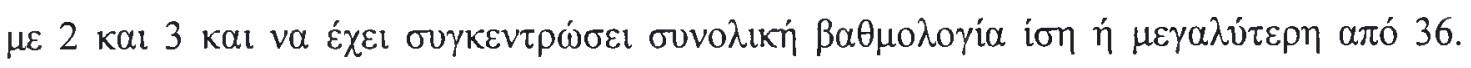

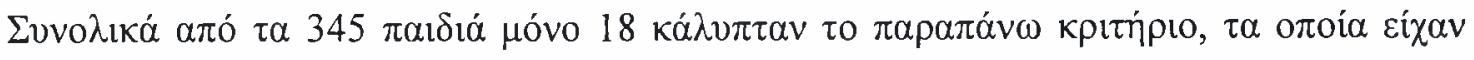




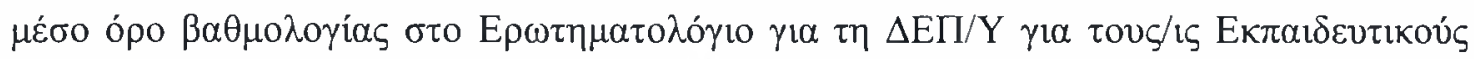

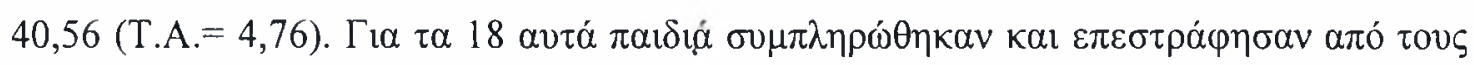

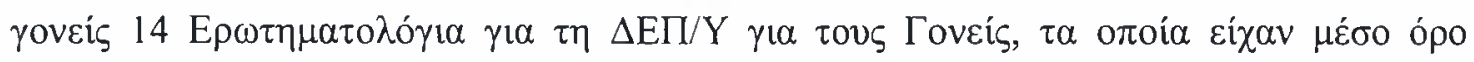

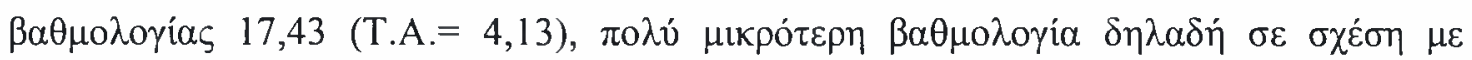

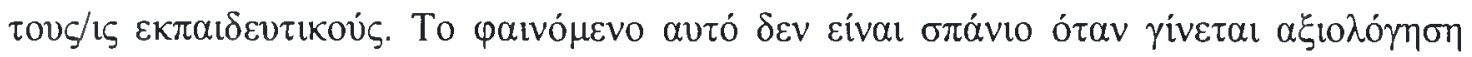

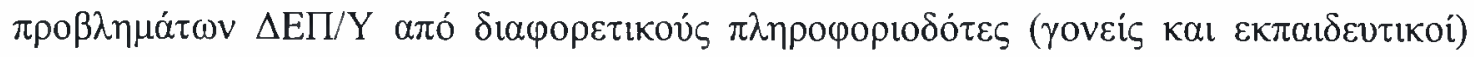
(Mitsis, McKay, Schulz, Newcorn \& Halperin, 2000).

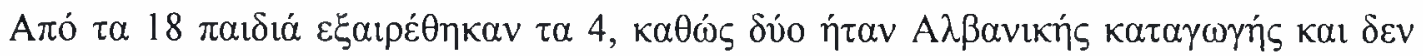

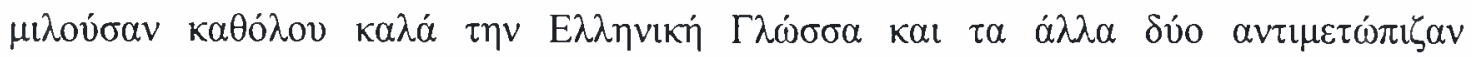

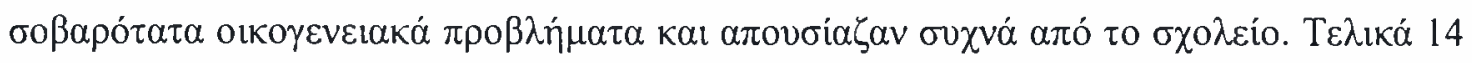

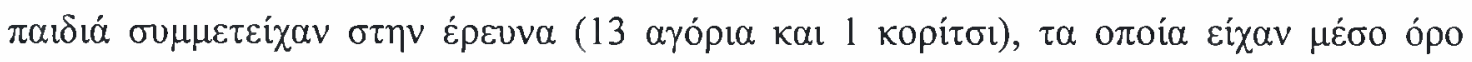

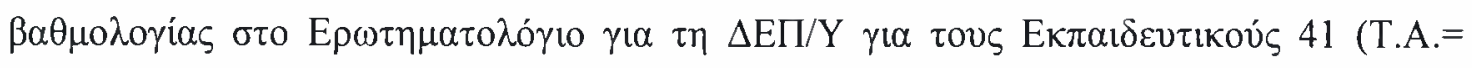

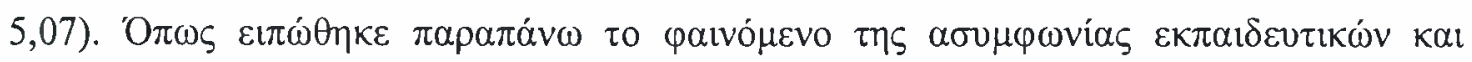

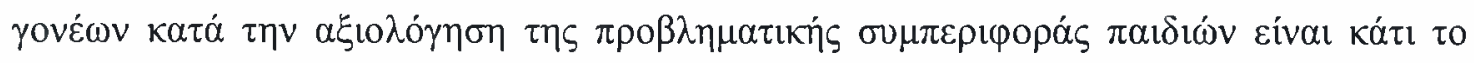

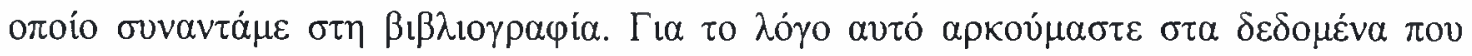

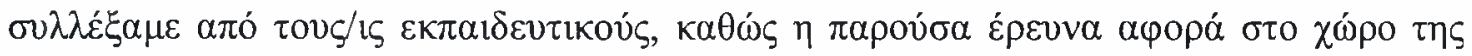

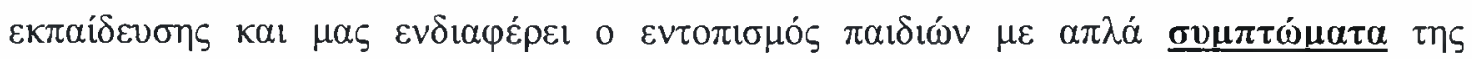
$\delta i \alpha \tau \alpha \rho \alpha \chi \dot{\eta} \zeta$.

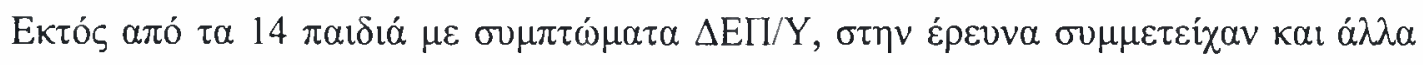

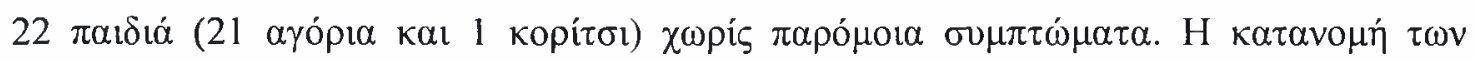

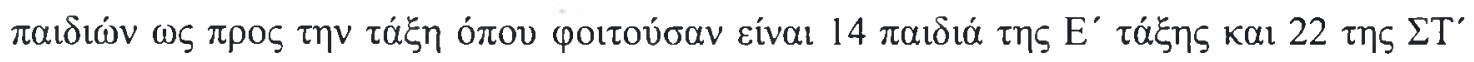

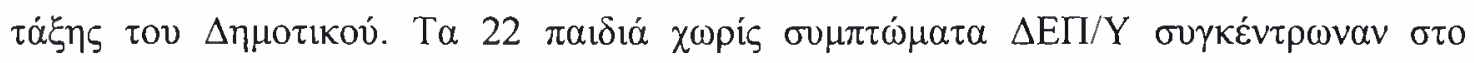

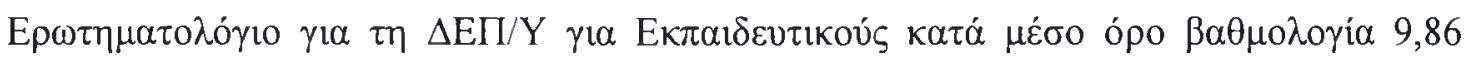
$(\mathrm{T} . \mathrm{A} .=7,66)$.

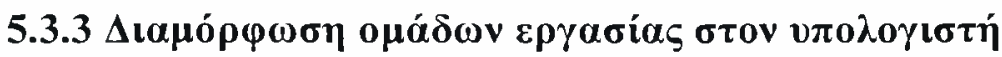

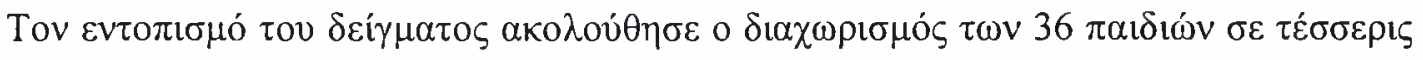

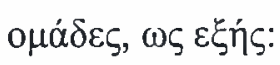

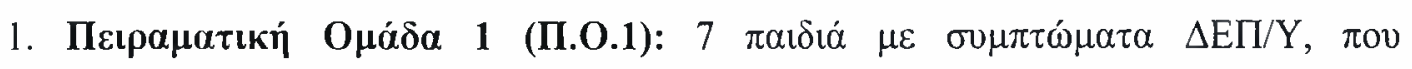

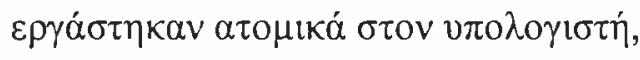




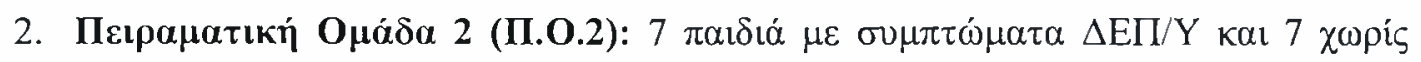

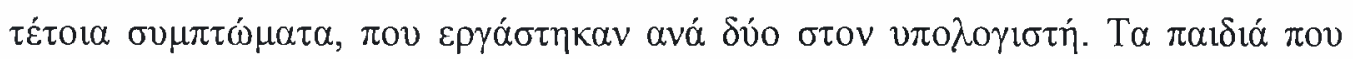

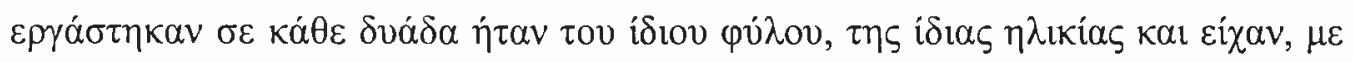

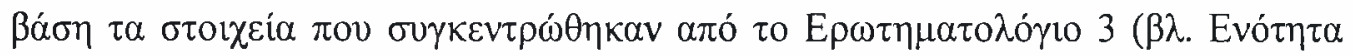

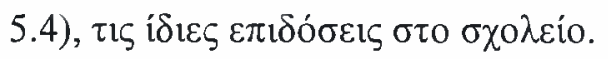

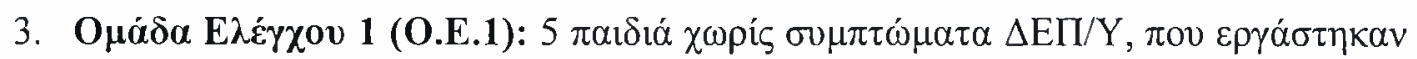

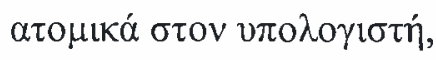

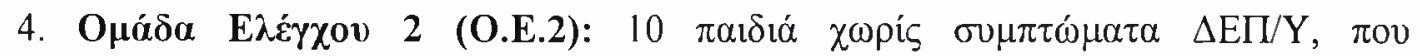

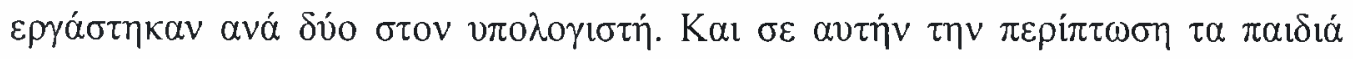

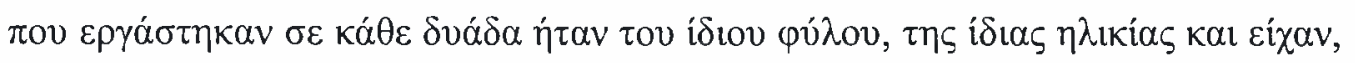

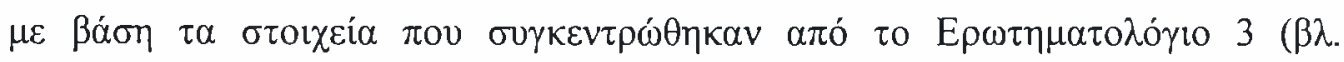

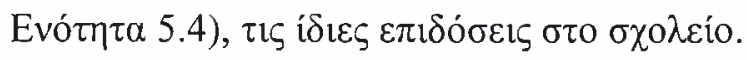

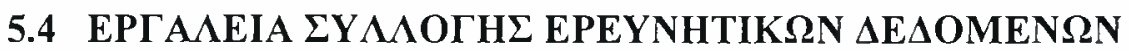

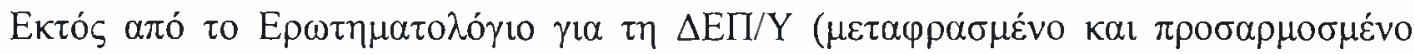

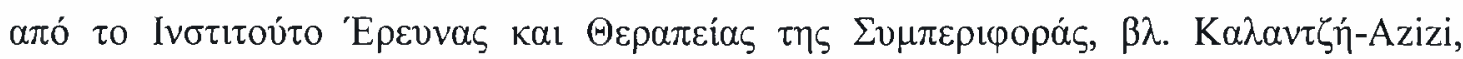

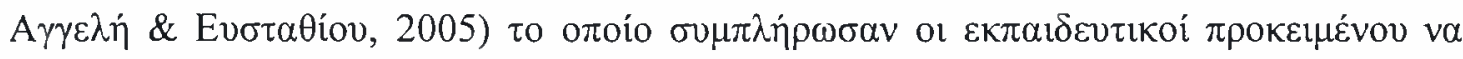

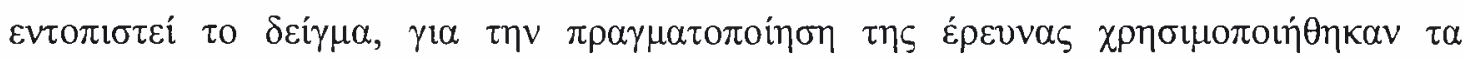
$\alpha \kappa o ́ \lambda o v \theta \alpha$ :

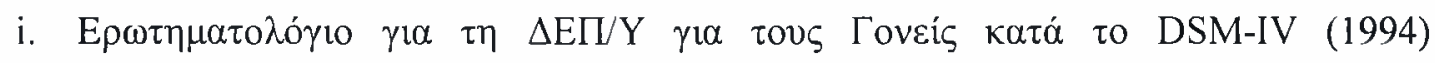

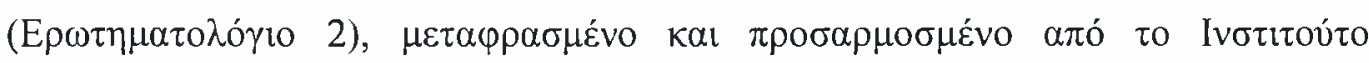

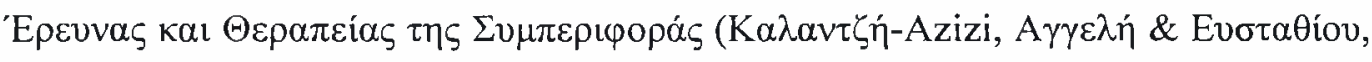

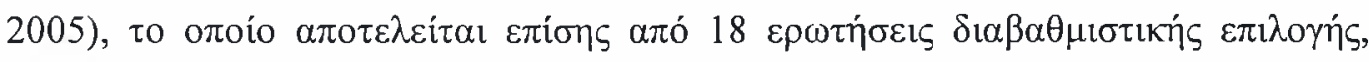

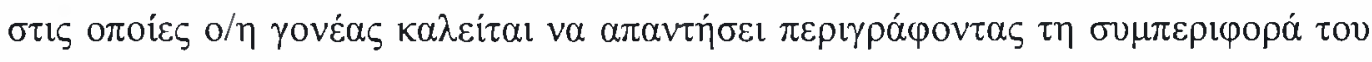

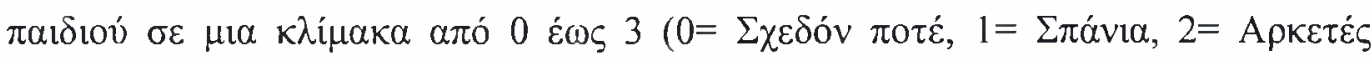

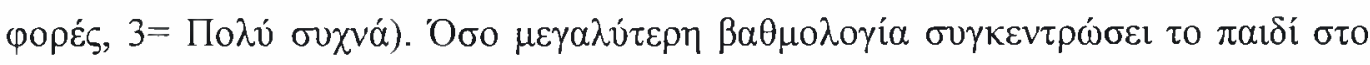

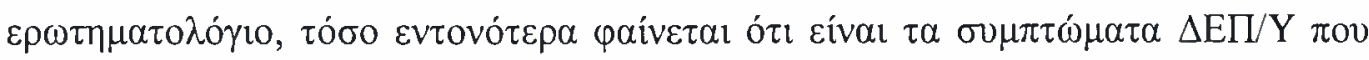

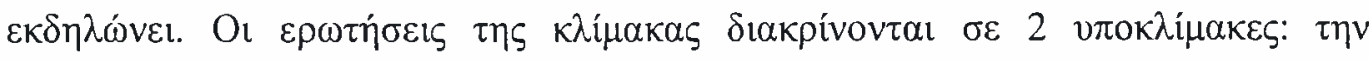

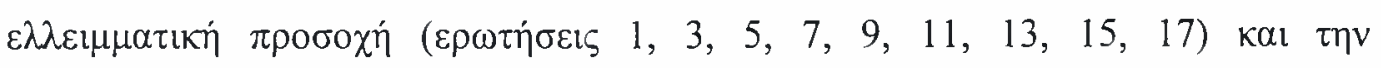




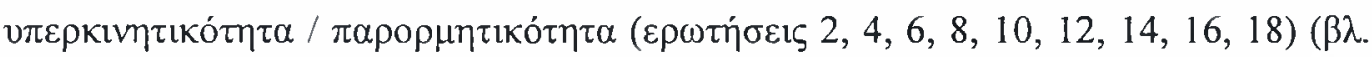

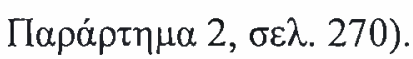

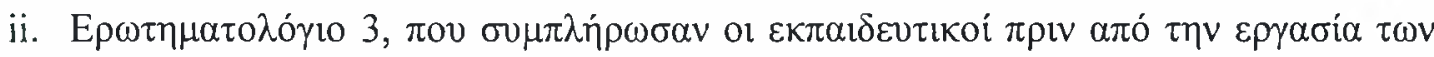

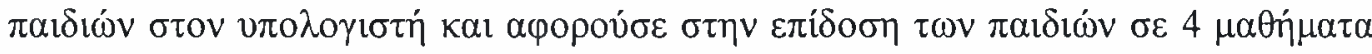

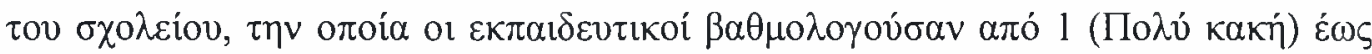

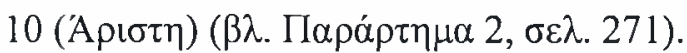

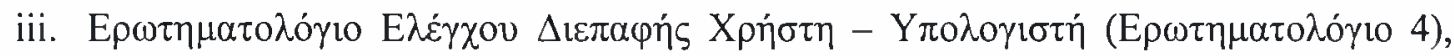

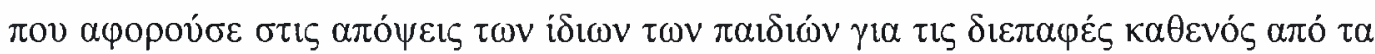

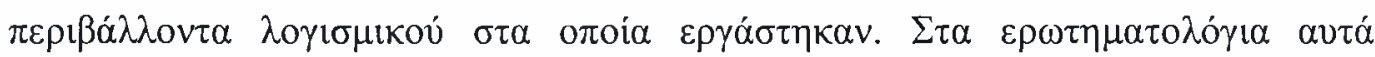

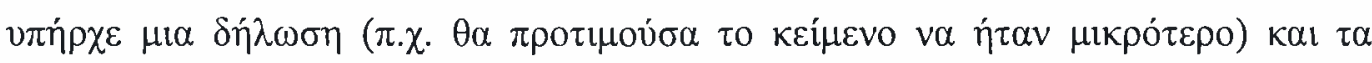

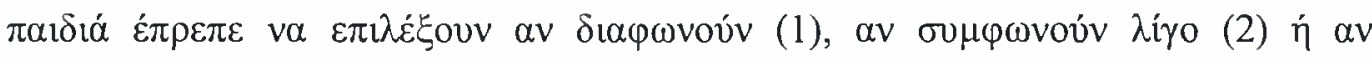

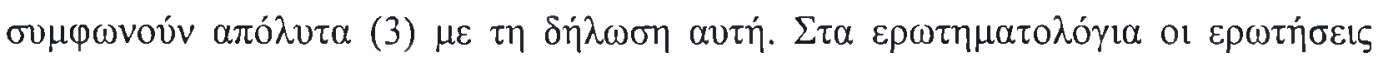

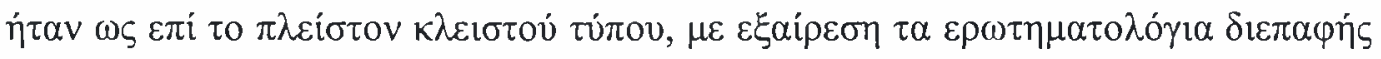

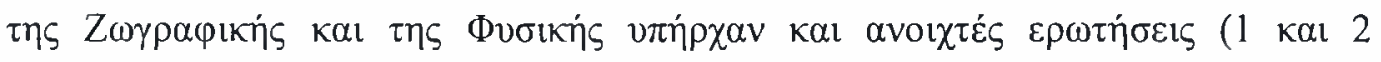

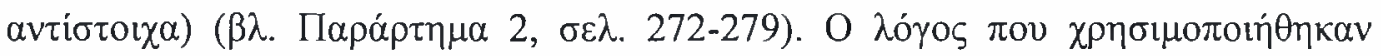

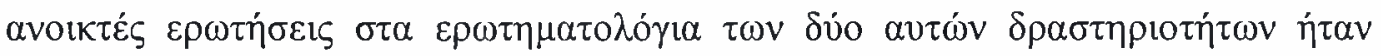

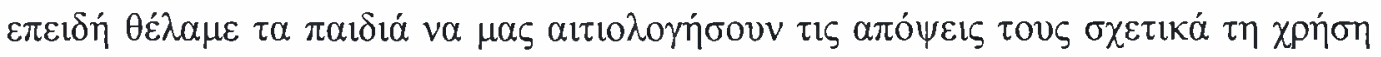

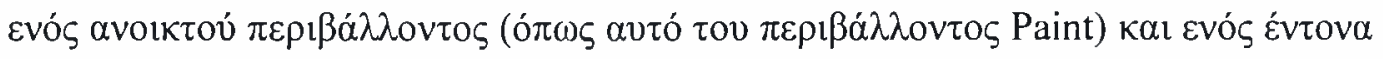

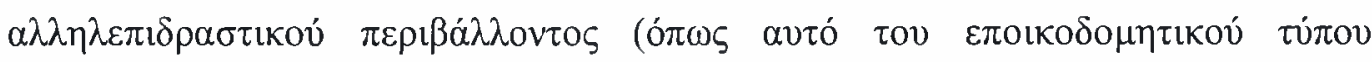

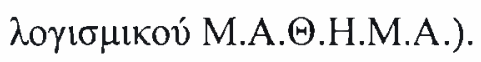

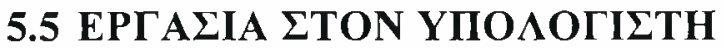

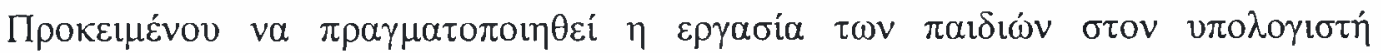

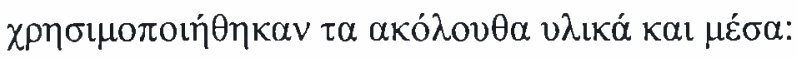

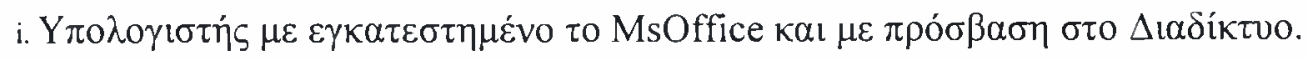

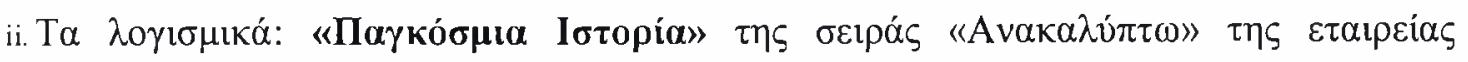

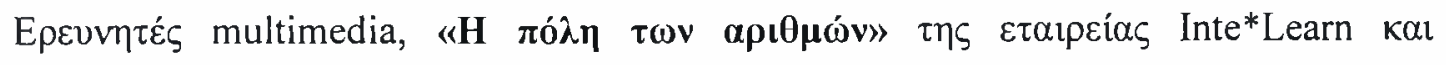

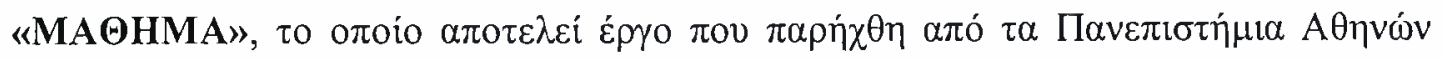




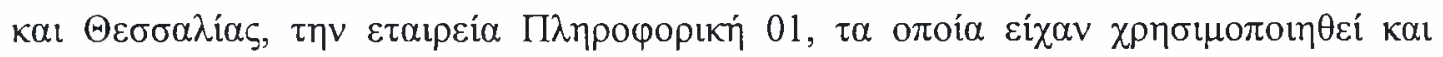

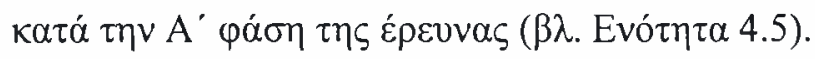

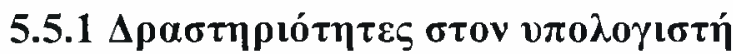

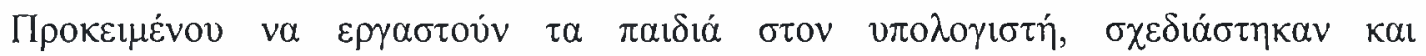

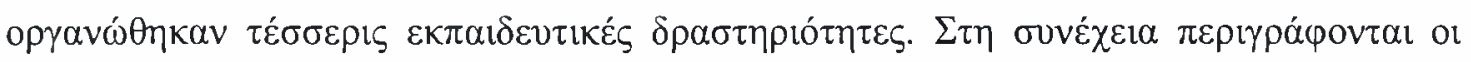

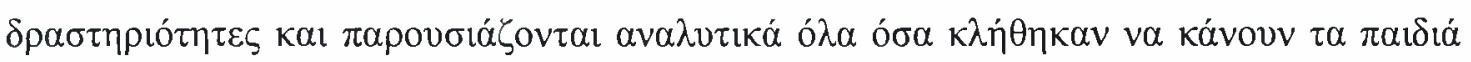

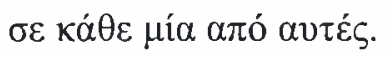

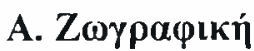

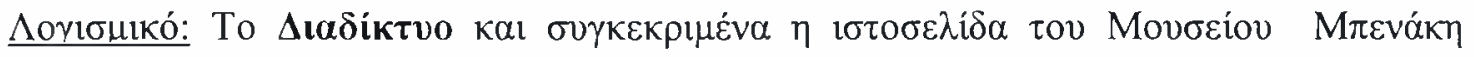

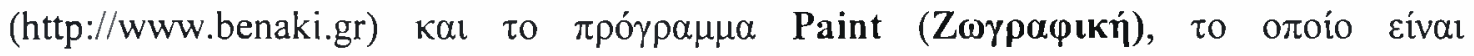
$\varepsilon \gamma \kappa \alpha \tau \varepsilon \sigma \tau \eta \mu \varepsilon ́ v o \sigma \tau \alpha$ Windows.

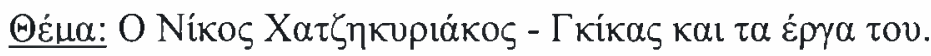

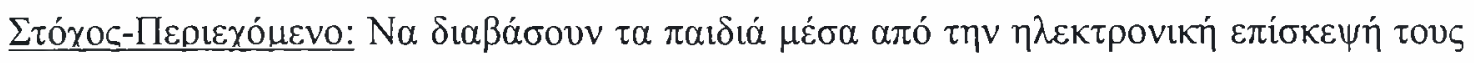

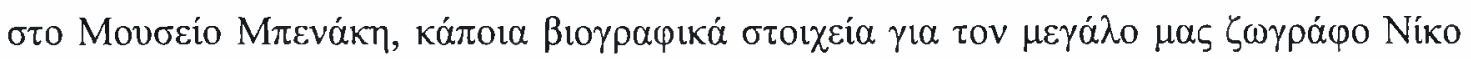

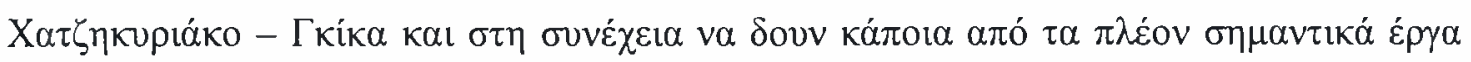

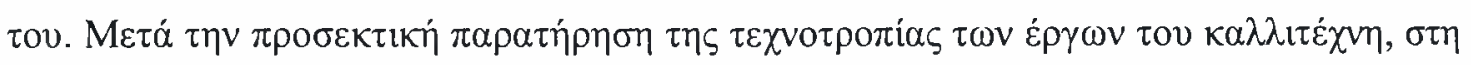

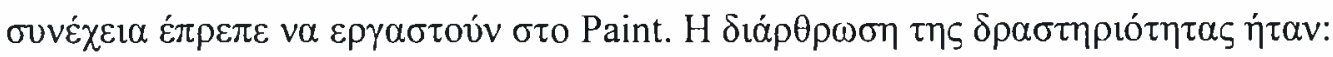

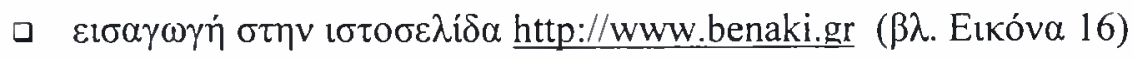

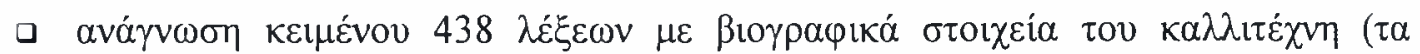

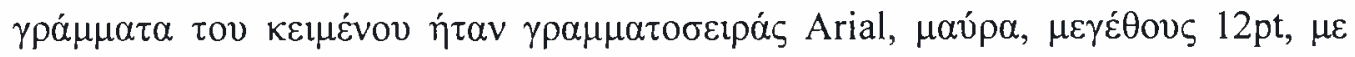

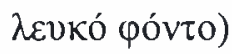

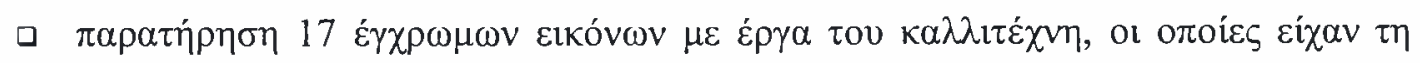

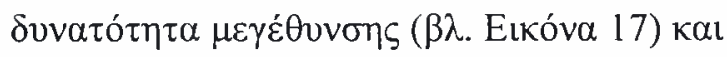

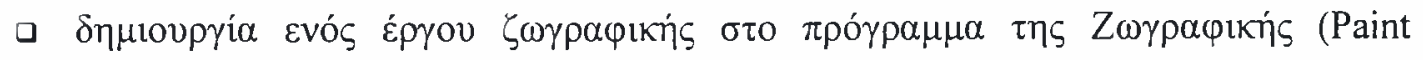
application). 


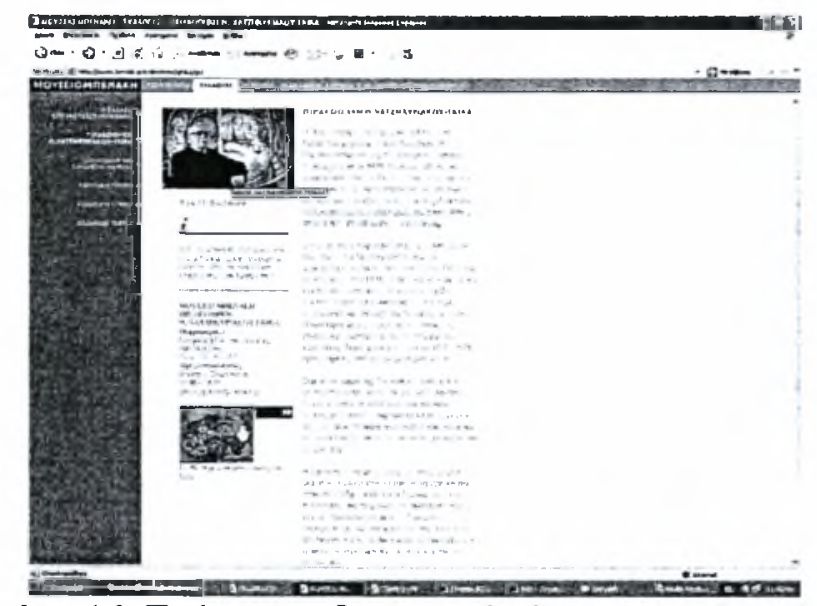

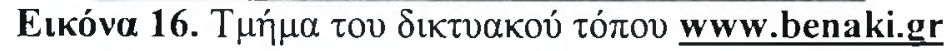

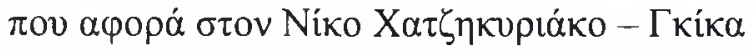

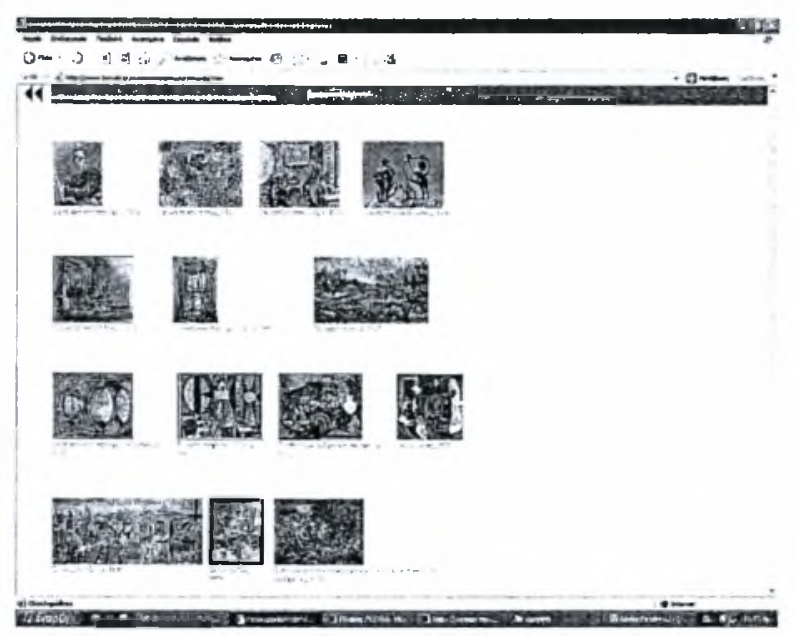

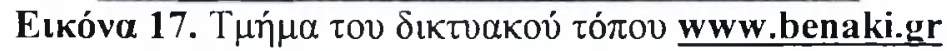

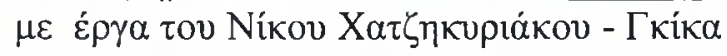

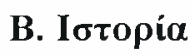

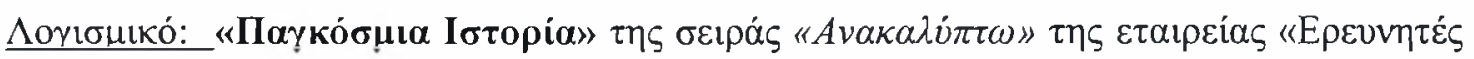

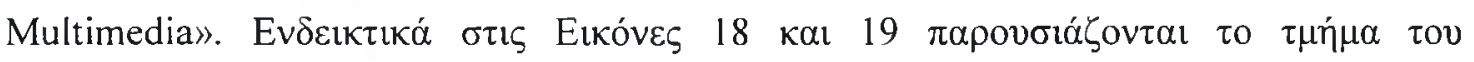

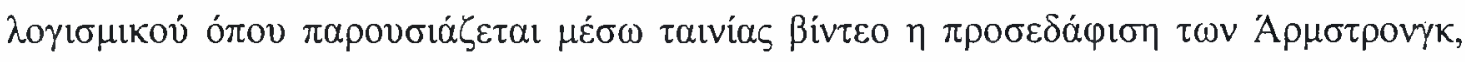

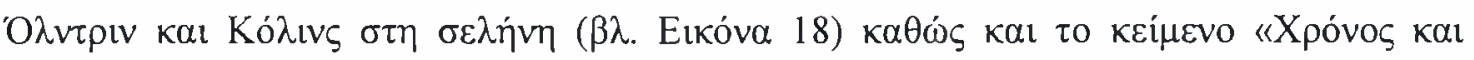

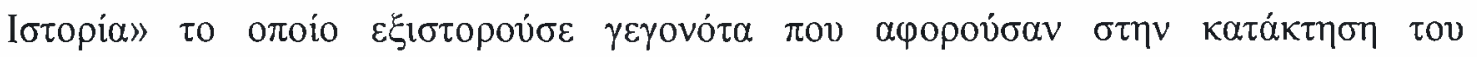

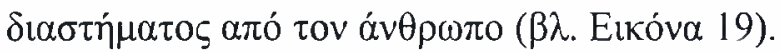




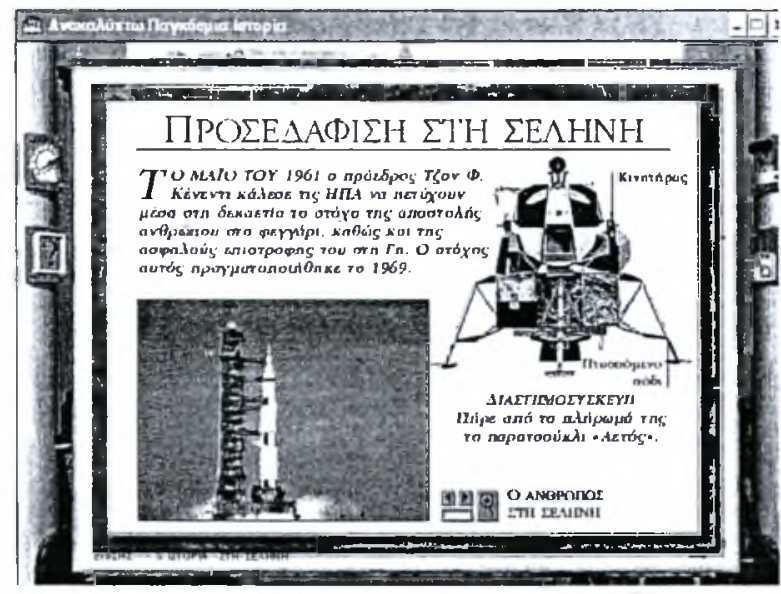

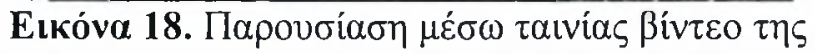

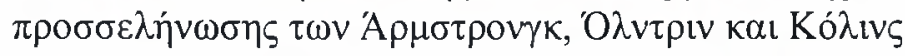

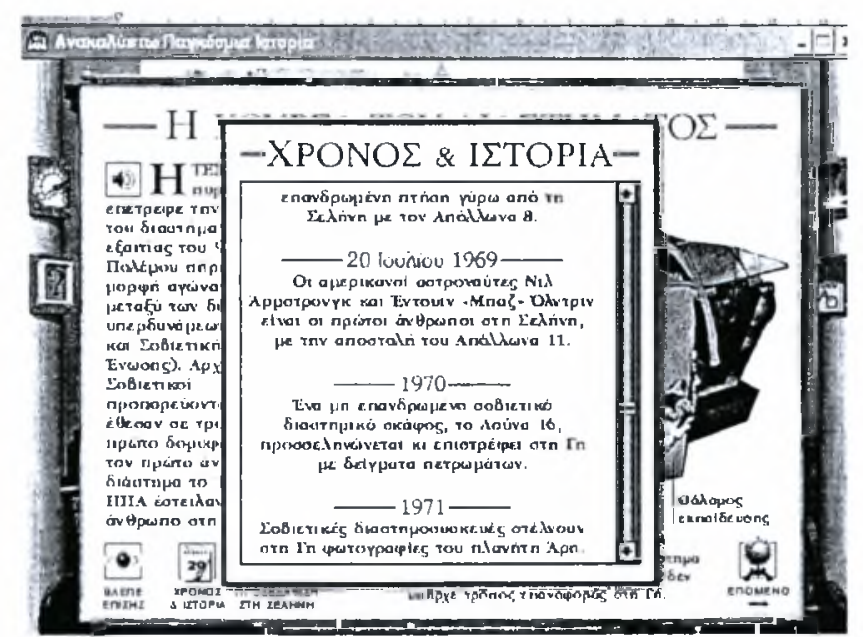

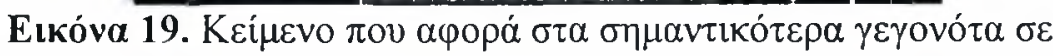

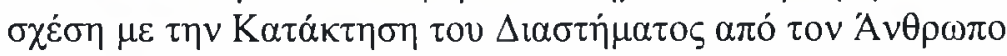

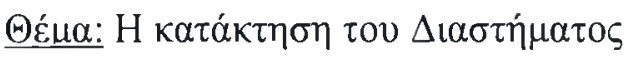

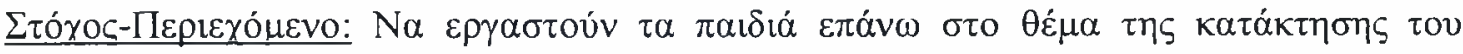

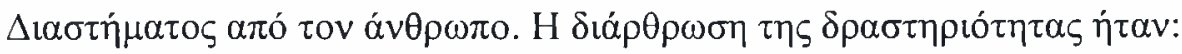

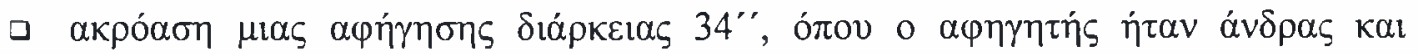

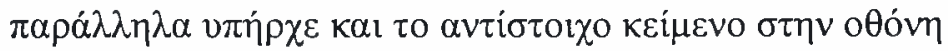

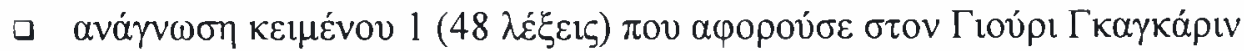

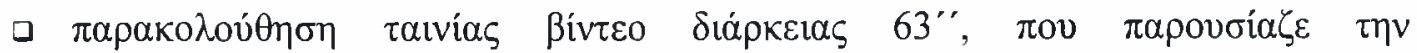

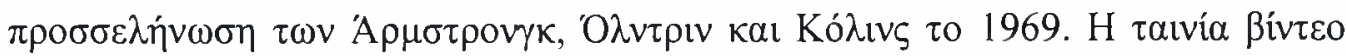




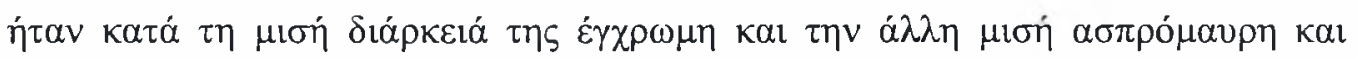

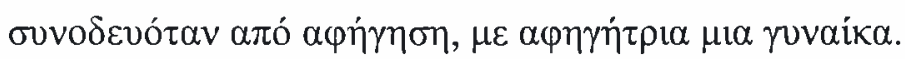

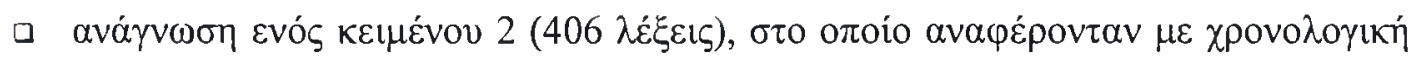

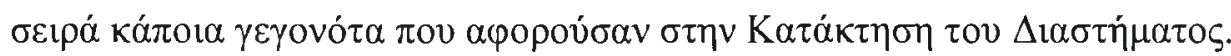

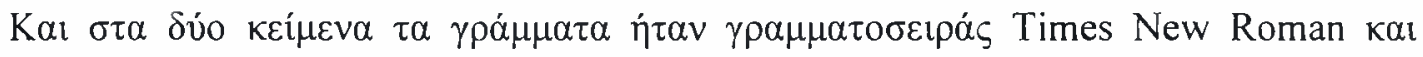

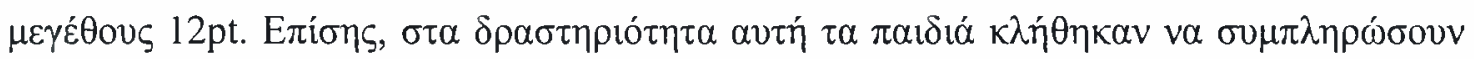

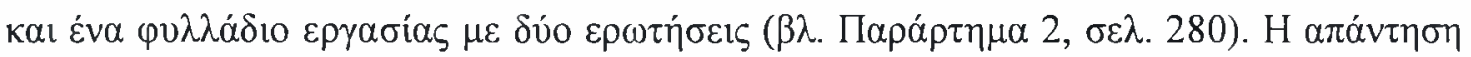

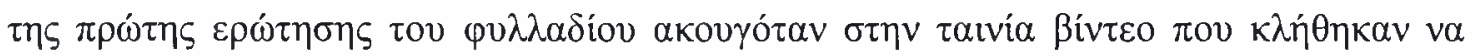

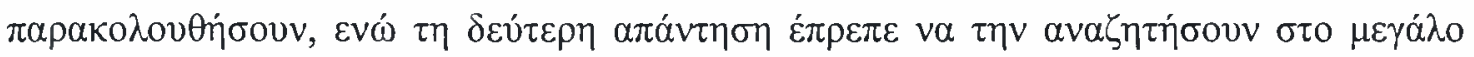

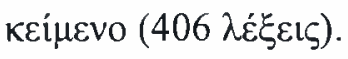

\section{Г. Фибเки́}

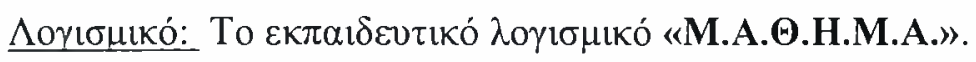

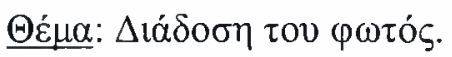

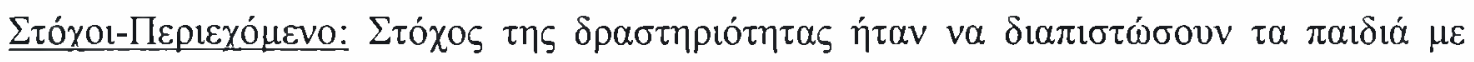

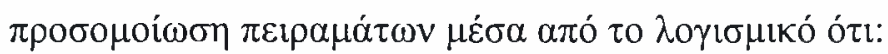

i. To $\varphi \omega \varsigma \delta \iota \alpha \delta i ́ \delta \varepsilon \tau \alpha \iota ~ \varepsilon v \theta \dot{\gamma} \rho \alpha \mu \mu \alpha$.

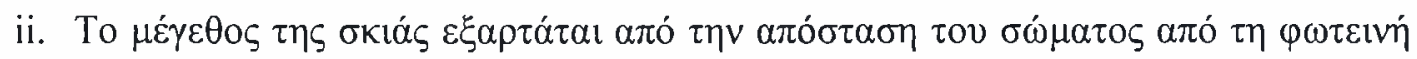
$\pi \eta \gamma \eta \dot{~}$

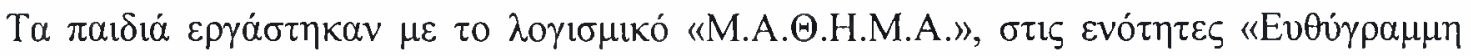

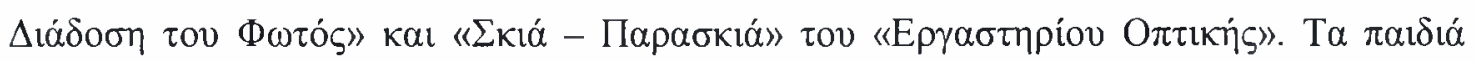

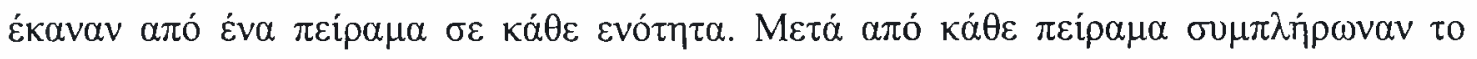

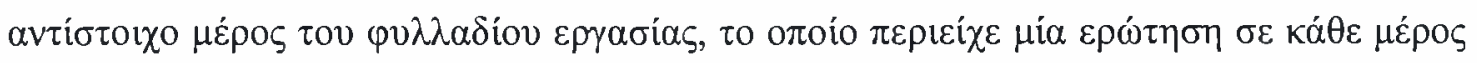

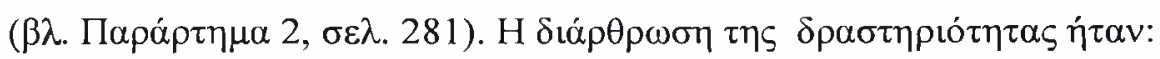

\section{$A^{\prime \prime \mu \varepsilon ́ \rho o s: ~}$}

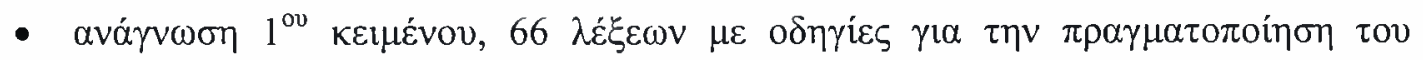
$\pi \varepsilon เ \rho \dot{\mu} \mu \alpha \tau \sigma \varsigma 1$

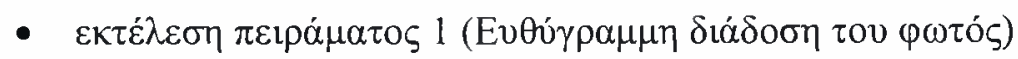

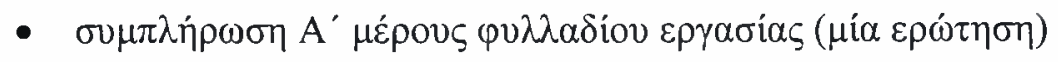

\section{$B^{\prime} \mu \varepsilon ́ \rho o \varsigma:$}

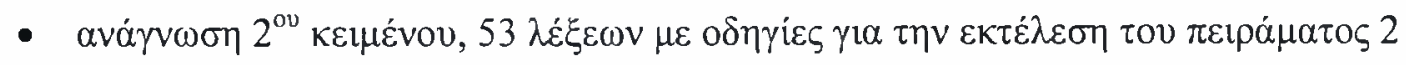




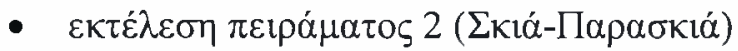

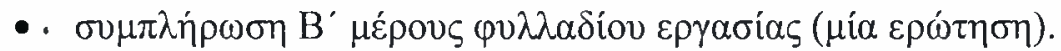

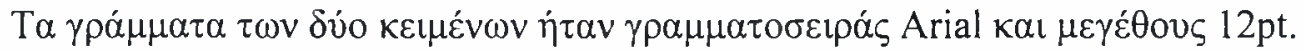

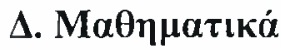

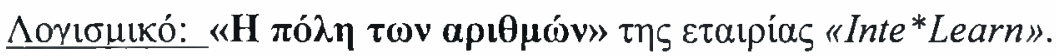

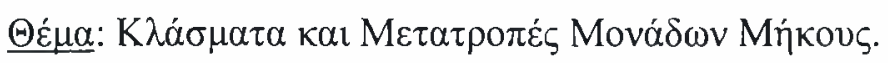

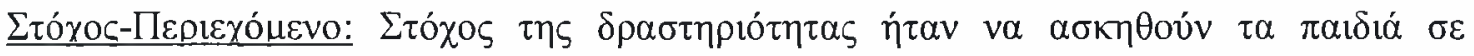

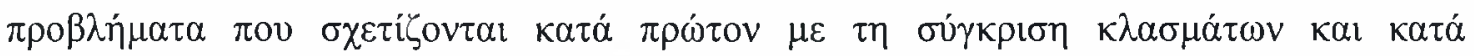

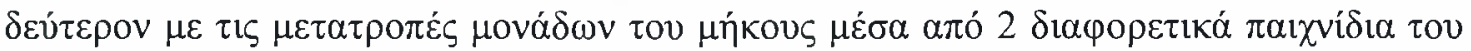

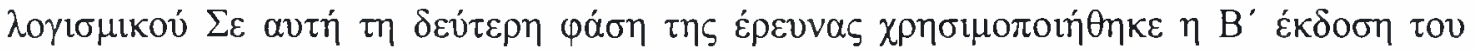

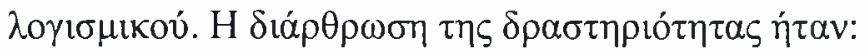

\section{$A^{\prime} \mu \varepsilon \dot{\rho} о \varsigma:$}

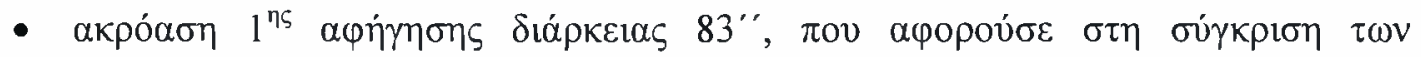
$\kappa \lambda \alpha \sigma \mu \alpha \dot{\tau} \tau \omega \nu$

- $\varepsilon \pi i ́ \lambda v \sigma \eta 1^{\mathrm{ns}} \alpha \dot{\sigma \kappa \kappa \eta \sigma \eta \zeta}$

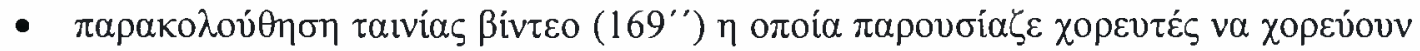

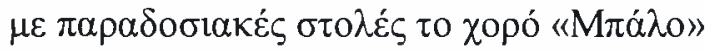

\section{$B^{\prime} \mu \varepsilon ́ p o s:$}

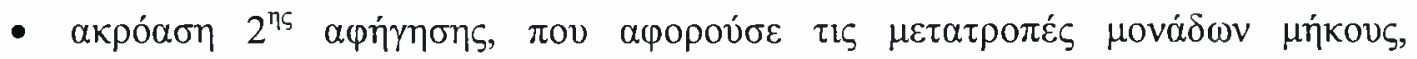

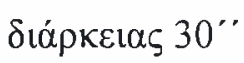

- $\varepsilon \pi i ́ \lambda v \sigma \eta 3^{\eta \varsigma} \alpha \dot{\alpha} \sigma \eta \sigma \eta \varsigma$

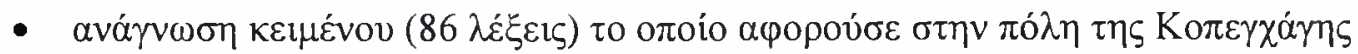

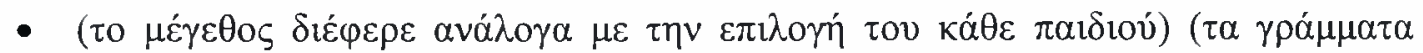

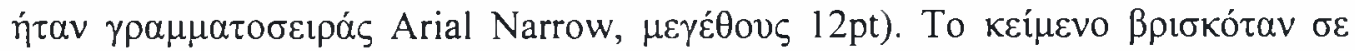

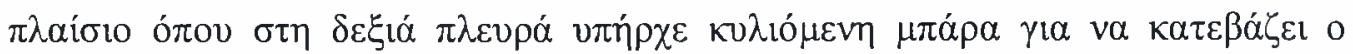

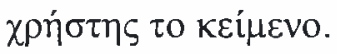

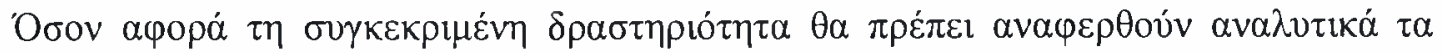
$\alpha \kappa o ́ \lambda \circ v \theta \alpha$ :

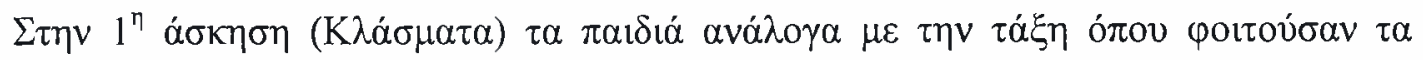

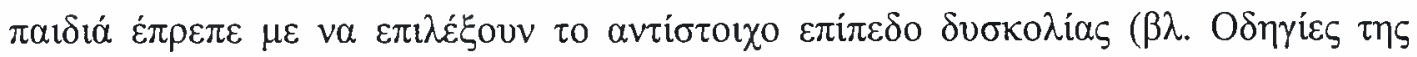




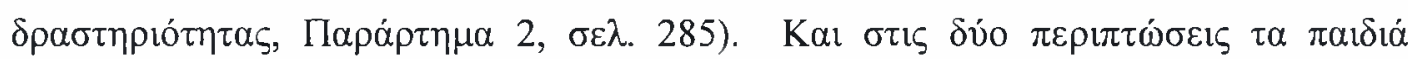

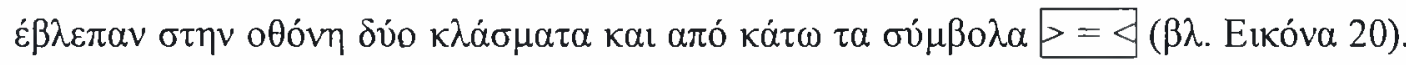

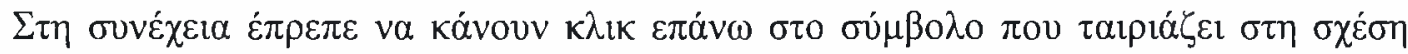

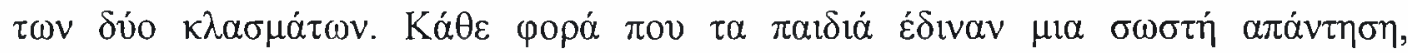

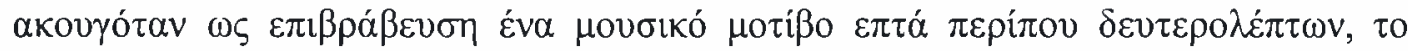

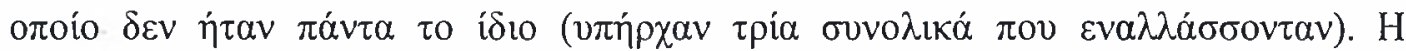

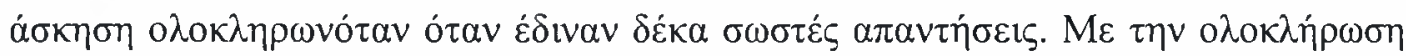

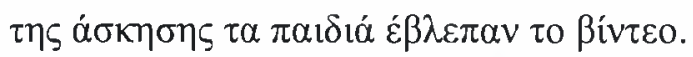

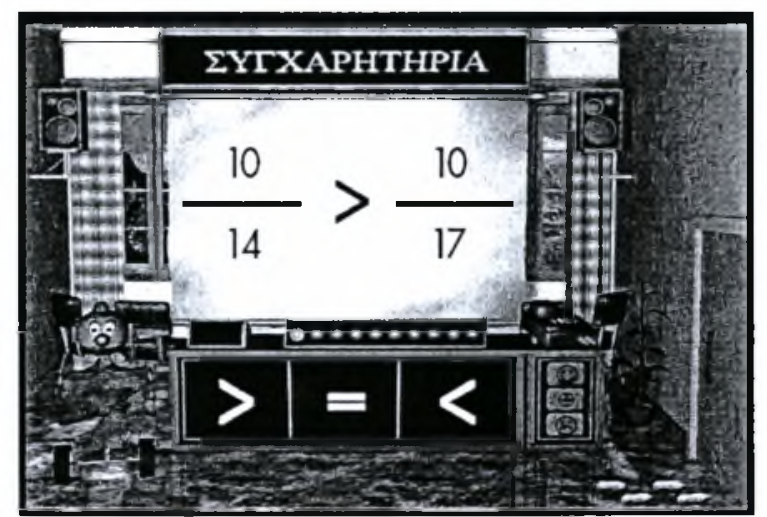

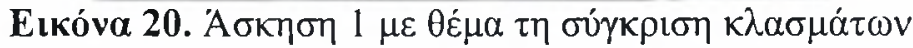

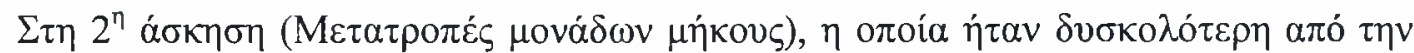

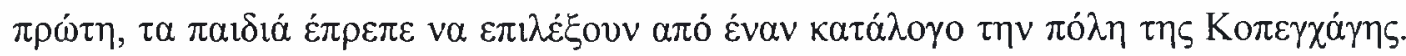

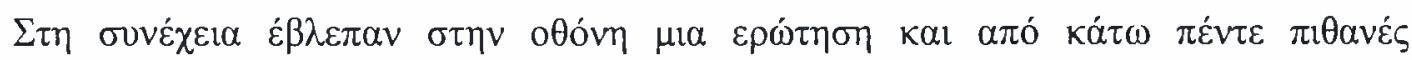

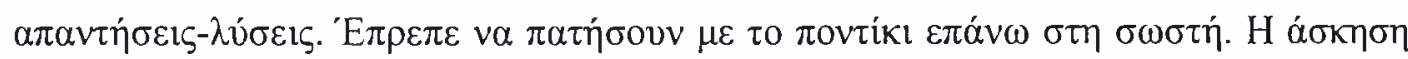

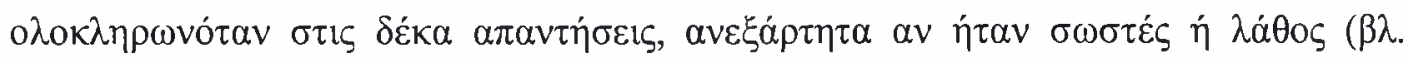

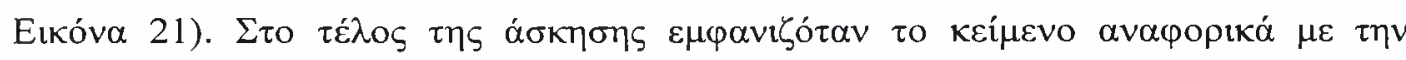

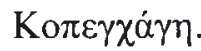




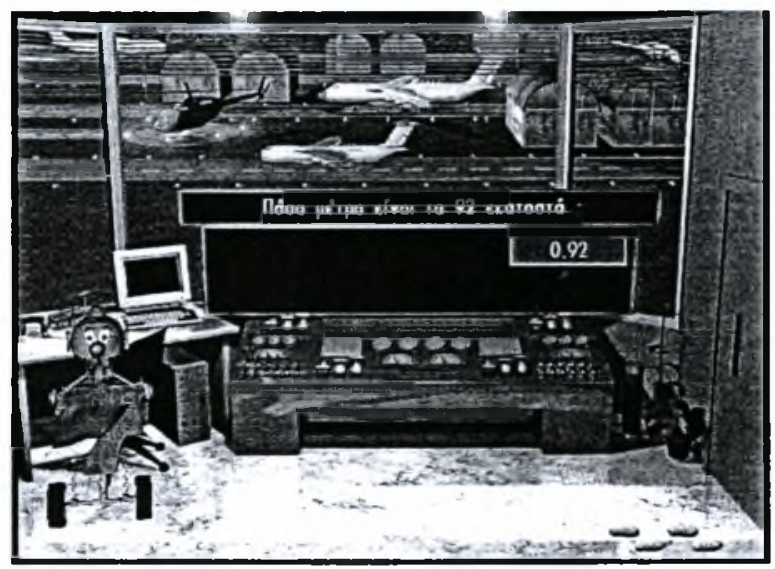

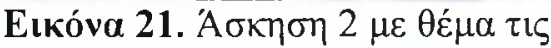

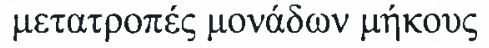

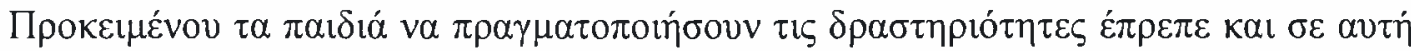

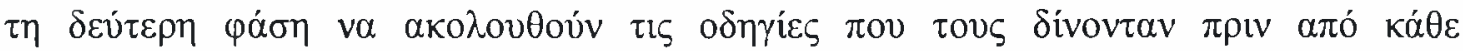

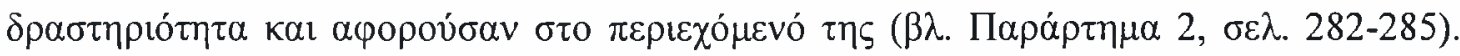

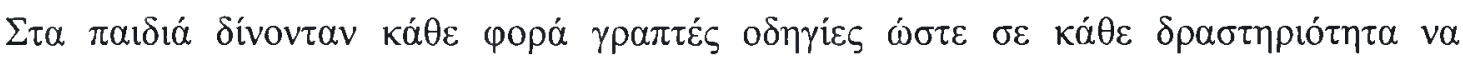
$\varepsilon \lambda \dot{\varepsilon} \gamma \chi 0 v \tau \alpha \mathrm{l} \tau \alpha \alpha \kappa o ́ \lambda o v \theta \alpha$ :

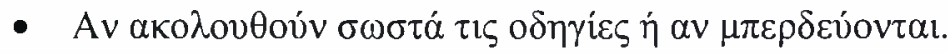

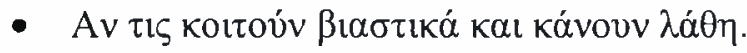

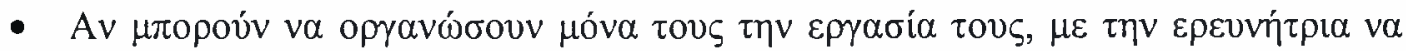

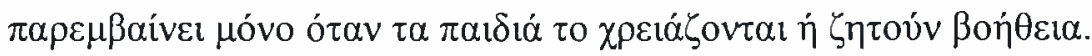

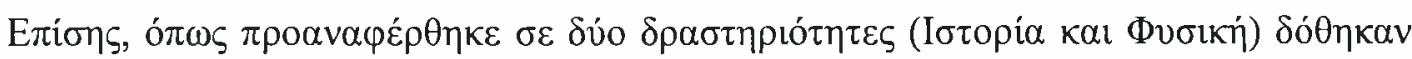

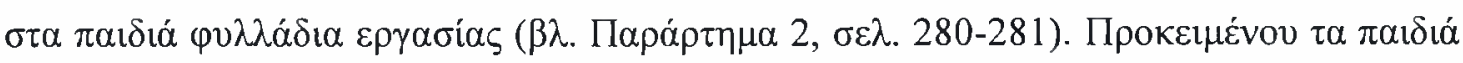

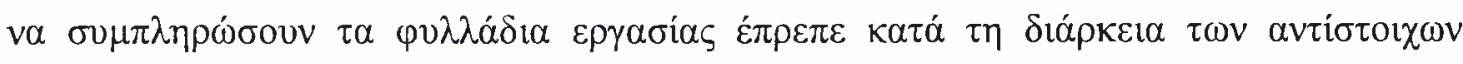

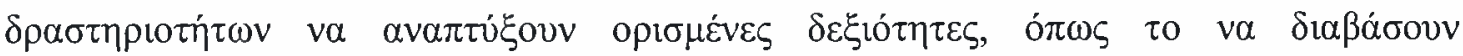

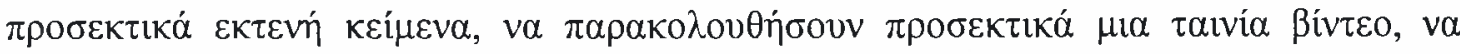

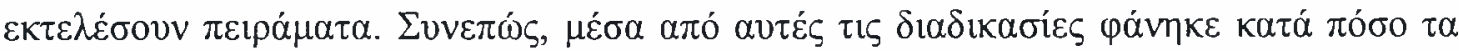

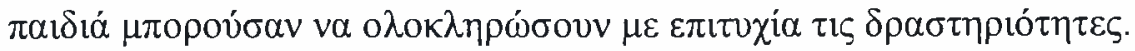

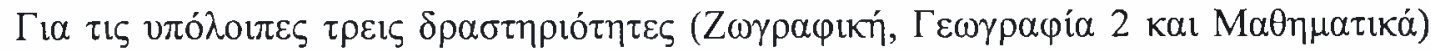

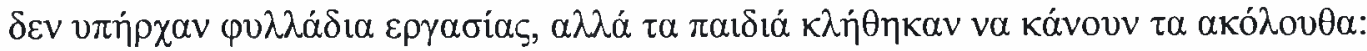

- $\quad \Sigma i \eta Z$ Z

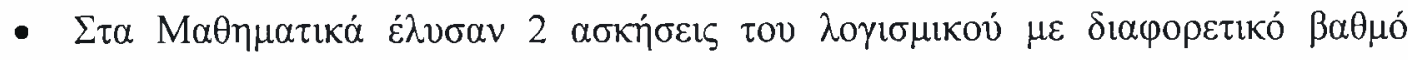
$\delta v \sigma \kappa o \lambda i ́ \alpha \varsigma \eta \kappa a ́ \theta \varepsilon \mu i ́ \alpha$. 


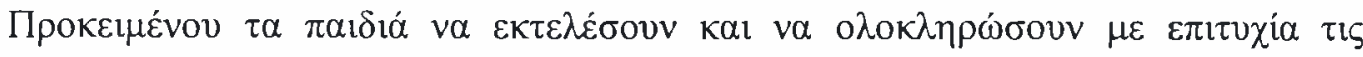

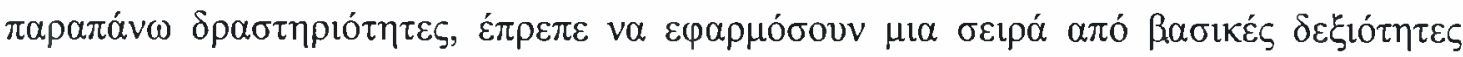
ó $\pi \omega \varsigma$ :

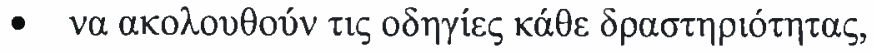

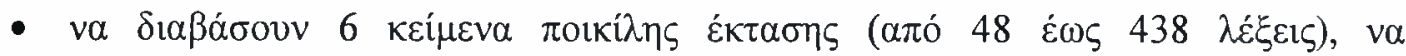

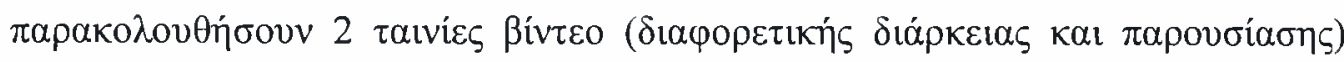

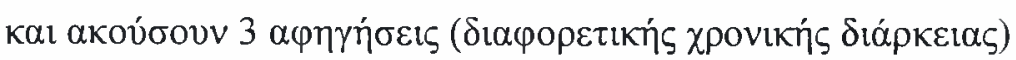

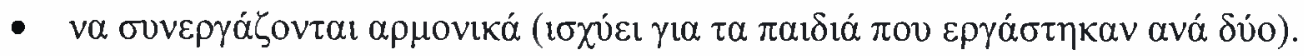

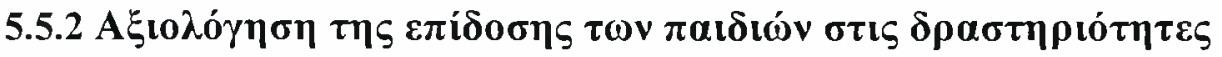

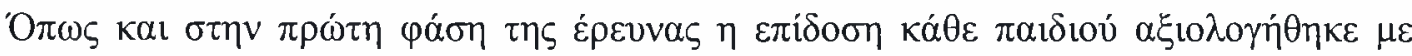

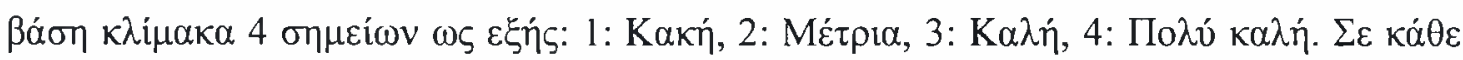

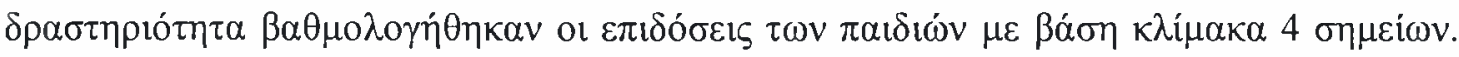

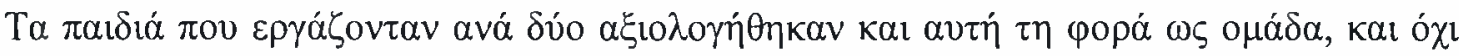

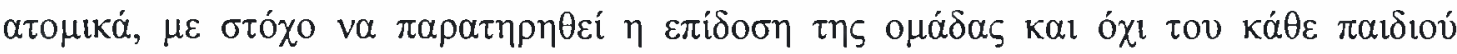

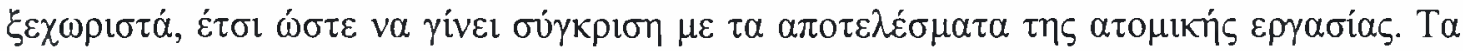

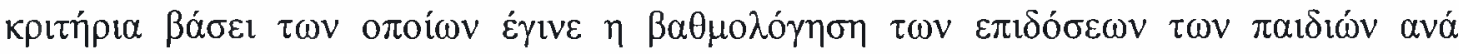

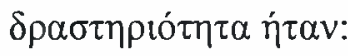

Z

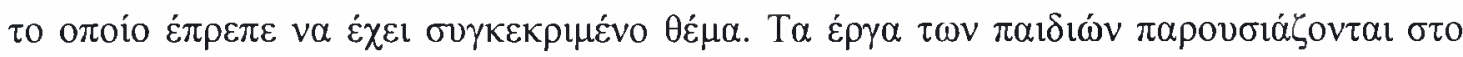

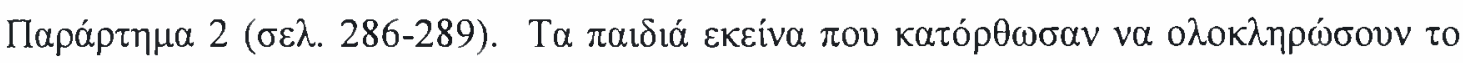

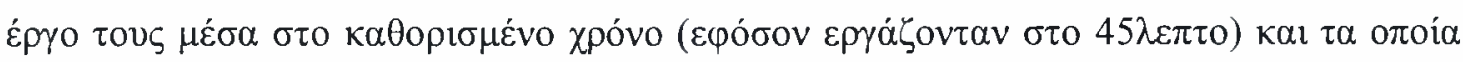

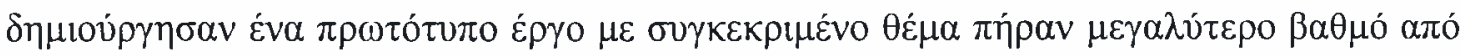

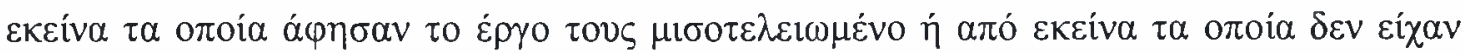

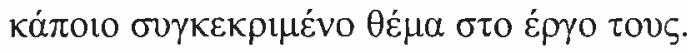

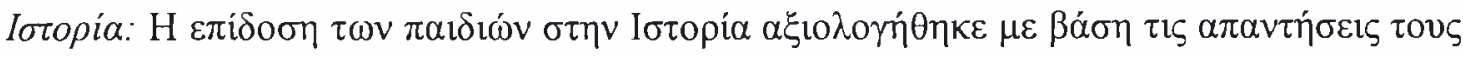

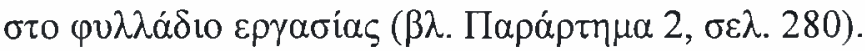

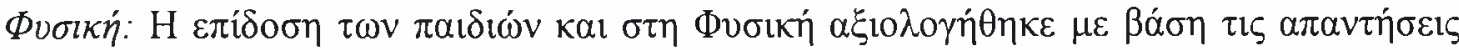

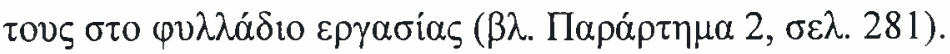




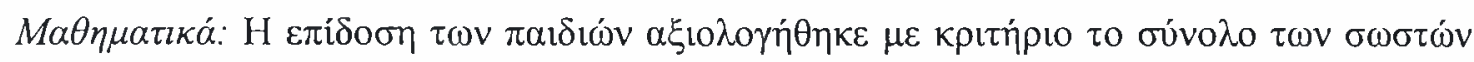

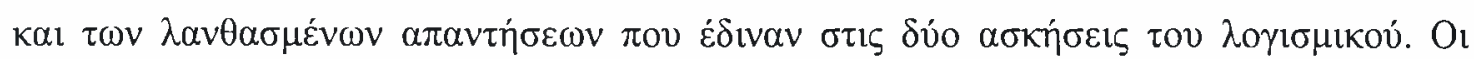

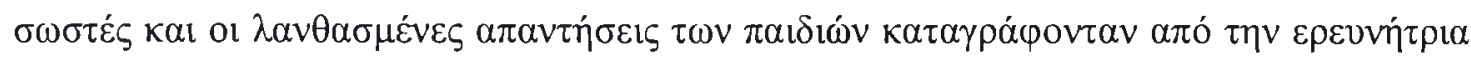

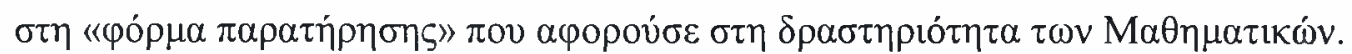

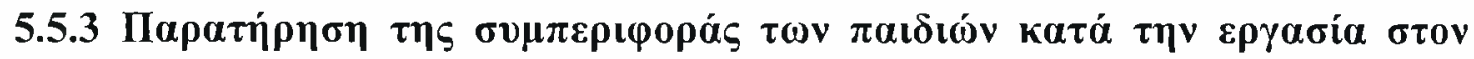

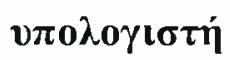

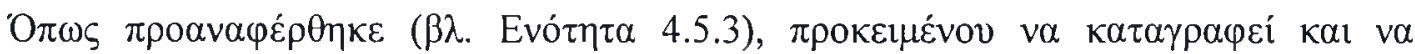

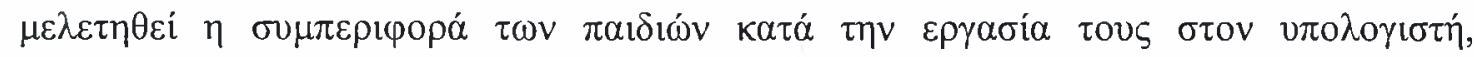

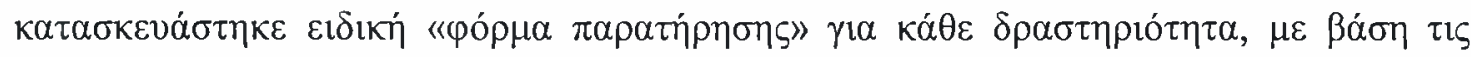

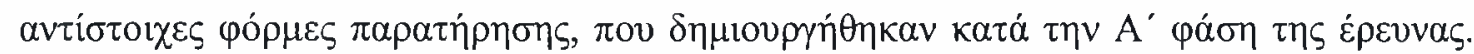

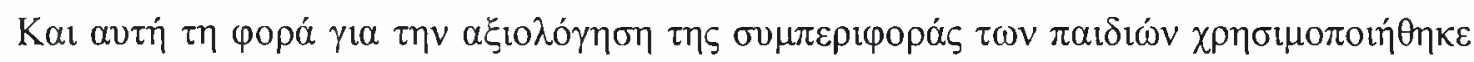

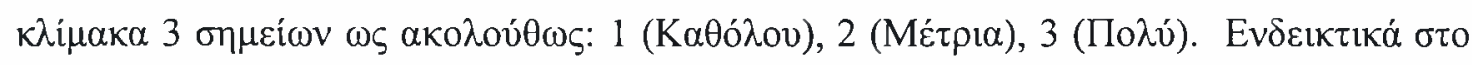

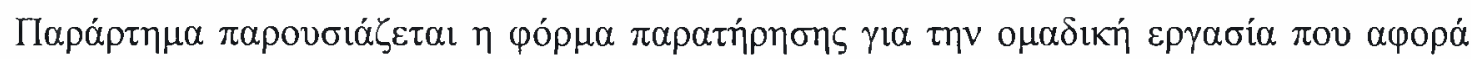

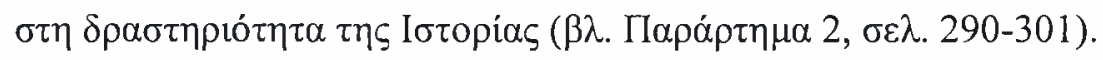

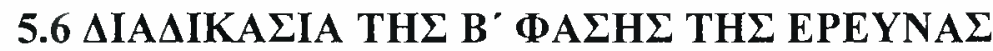

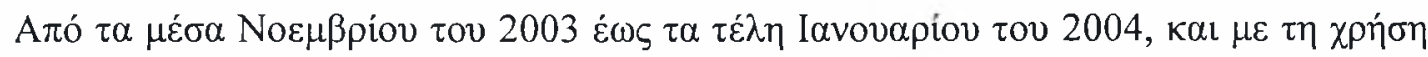

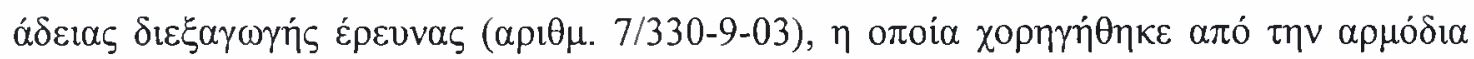

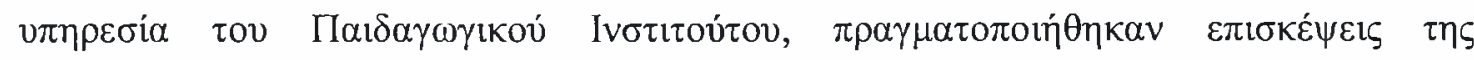

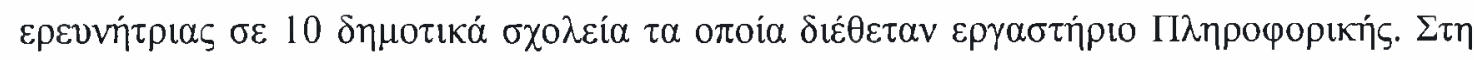

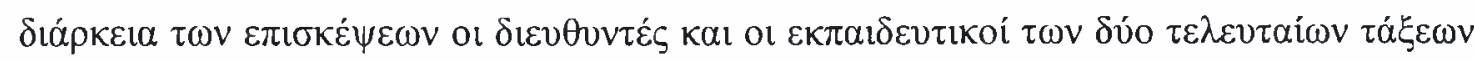

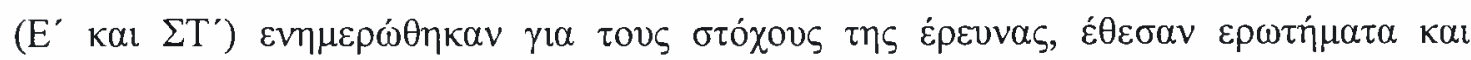

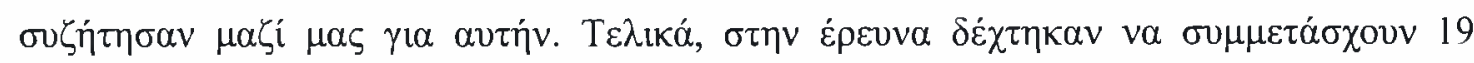

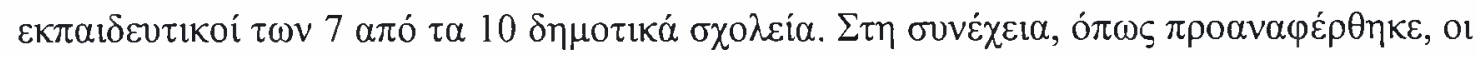

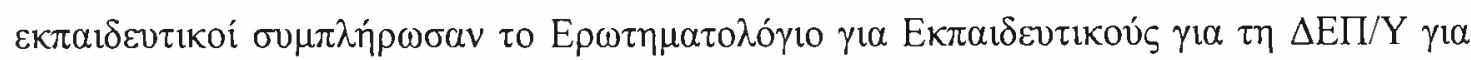

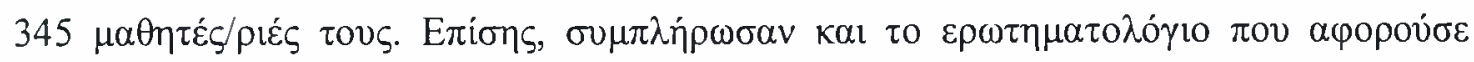

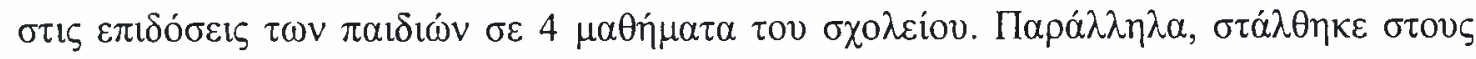




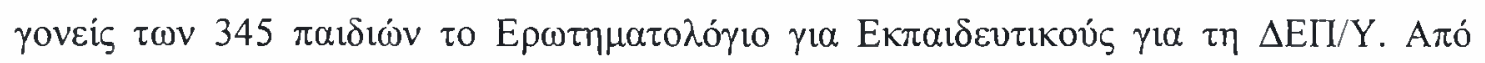

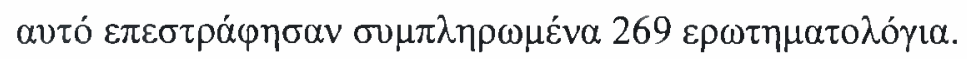

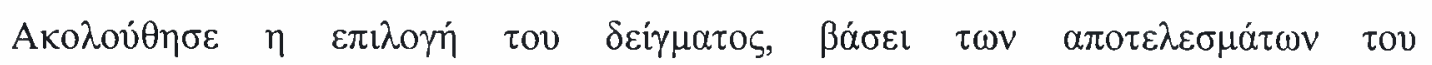

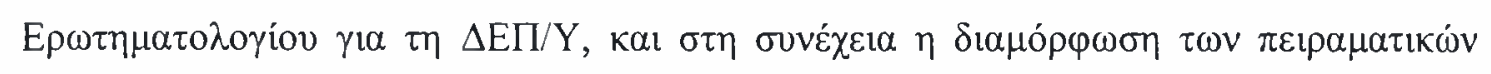
o $\mu \alpha ́ \delta \omega v \kappa \alpha \iota \tau \omega v$ o $\mu \alpha \dot{\delta} \delta \omega v \varepsilon \lambda \varepsilon \dot{\gamma \chi \chi 0}$.

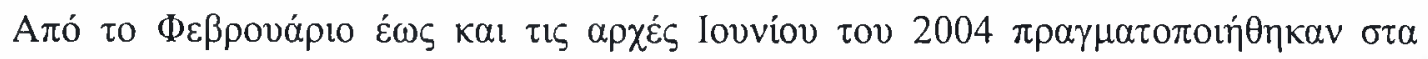

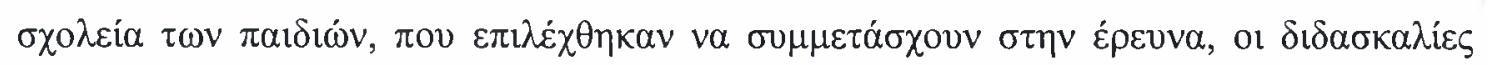

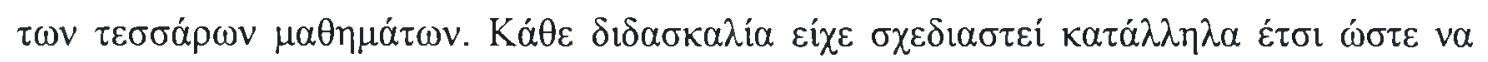

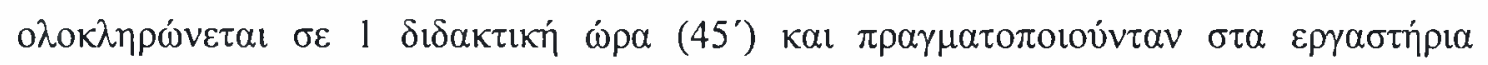

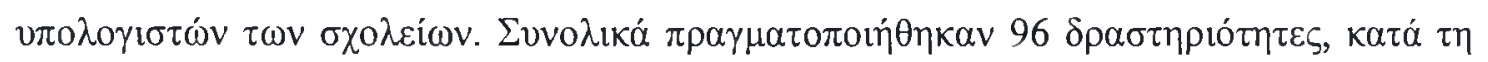

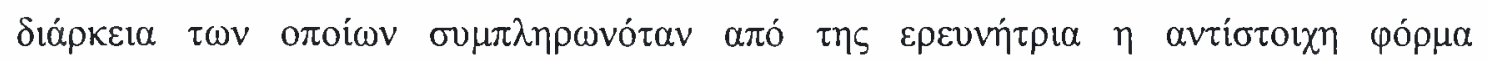
$\pi \alpha \rho \alpha \tau \dot{\rho} \eta \sigma \eta \zeta$.

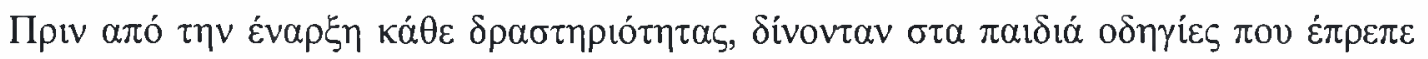

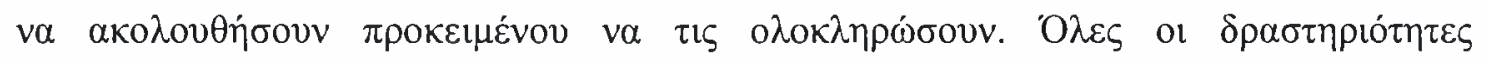

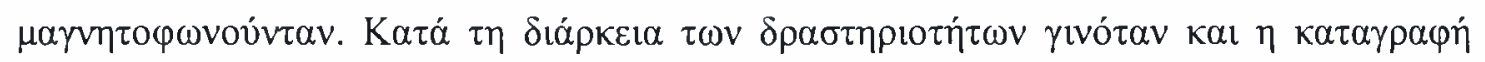

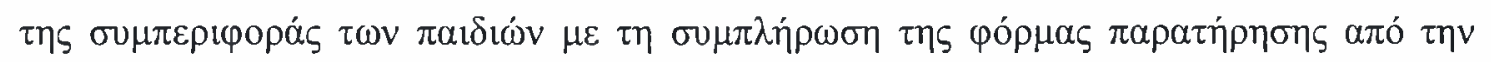

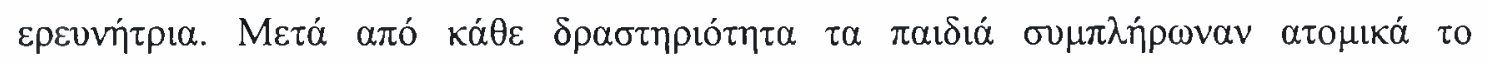

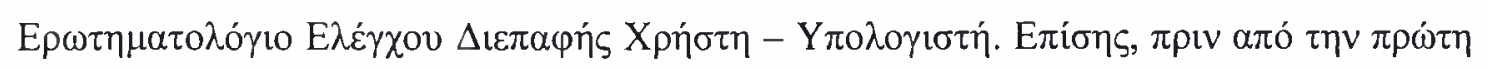

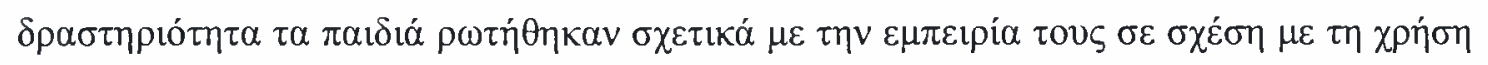

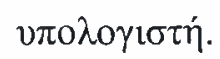

\subsection{EПE}

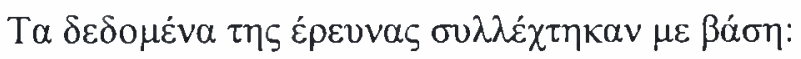

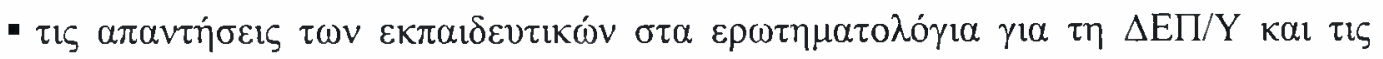

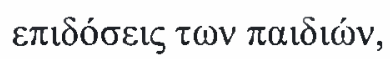

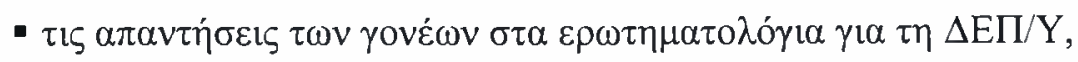

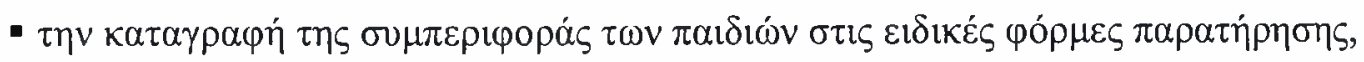

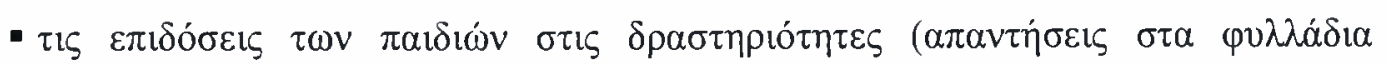

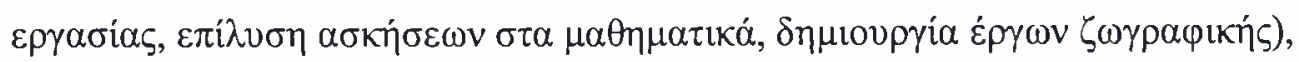




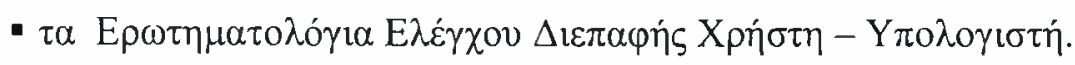

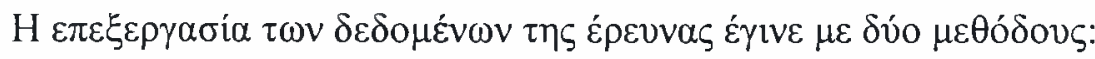

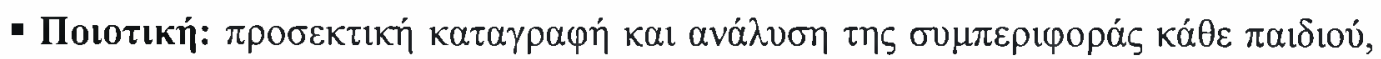

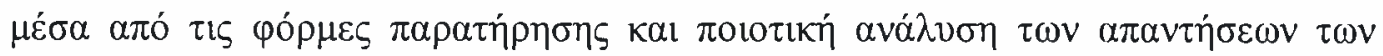

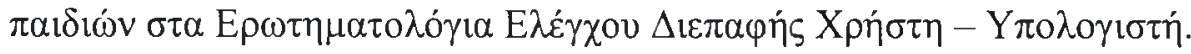

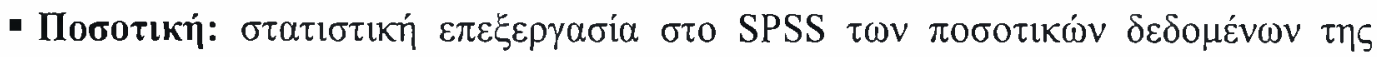

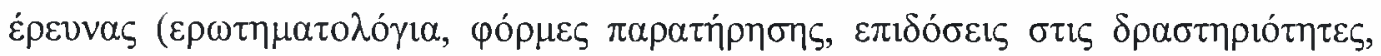

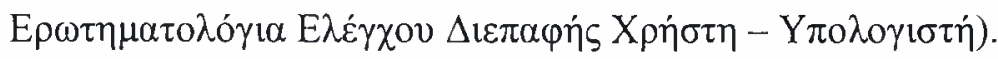

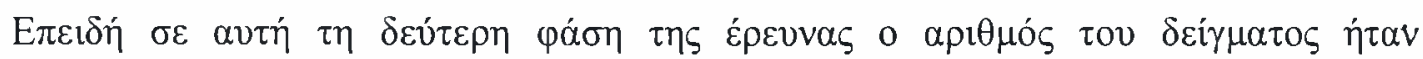

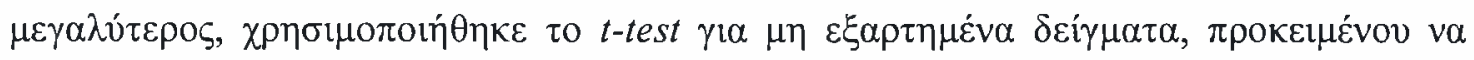

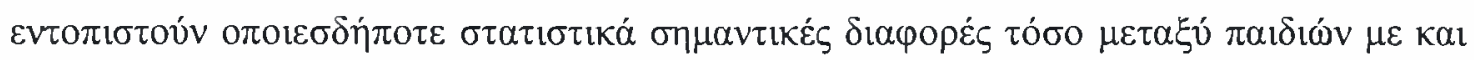

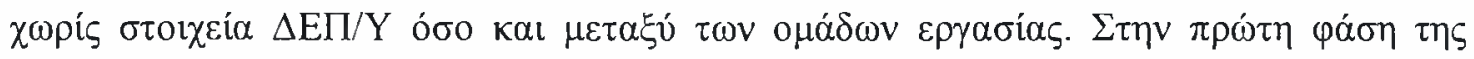

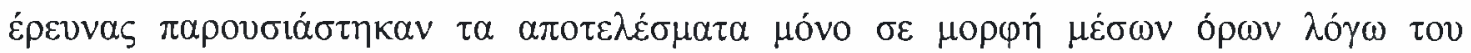

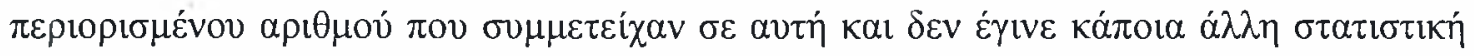
$\alpha v \alpha \dot{\lambda} v \sigma \eta$.

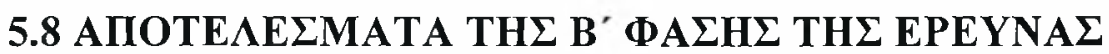

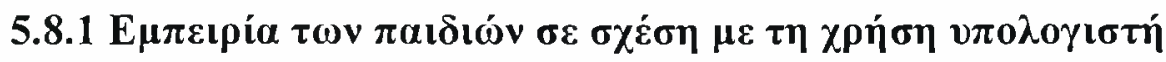

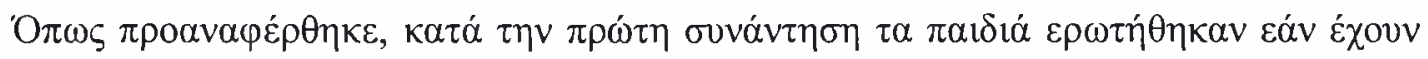

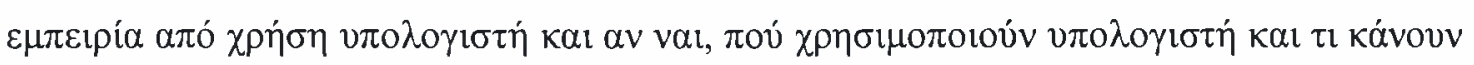

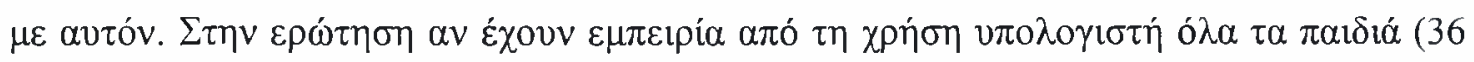

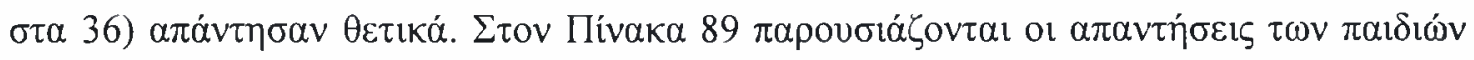

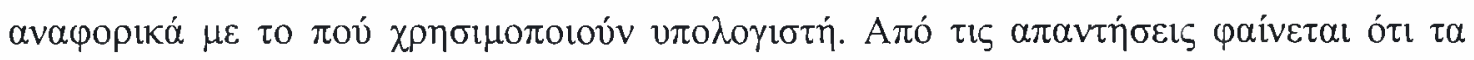

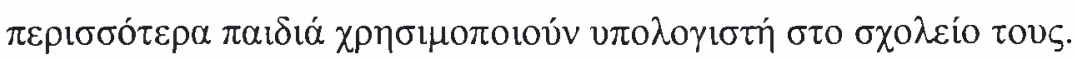




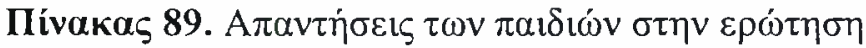

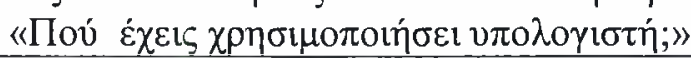

\begin{tabular}{|c|c|}
\hline$A \pi \alpha \nu \tau \dot{\sigma} \sigma \varepsilon l \varsigma$ & $\begin{array}{c}A \rho \imath \theta \mu o ́ \varsigma \pi \alpha \imath \delta t \omega v \\
N(=36)\end{array}$ \\
\hline 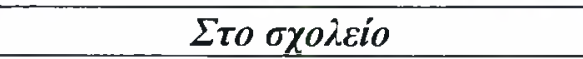 & 12 \\
\hline$\sum \tau 0 \sigma \pi i \tau l$ & 5 \\
\hline 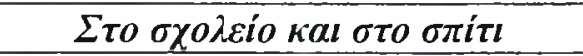 & 8 \\
\hline 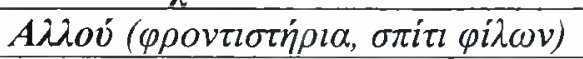 & 7 \\
\hline 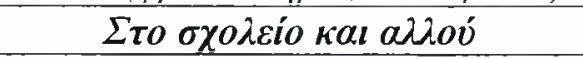 & 2 \\
\hline 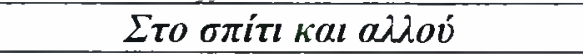 & 2 \\
\hline
\end{tabular}

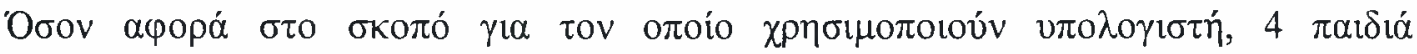

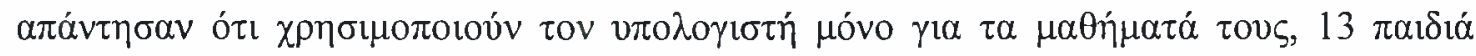

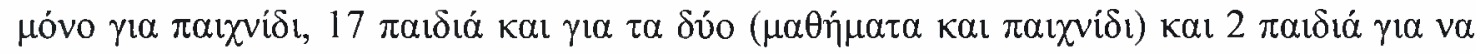

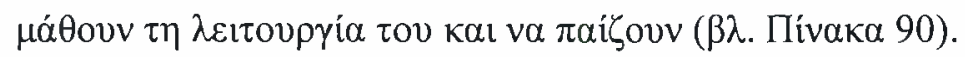

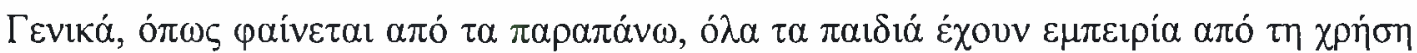

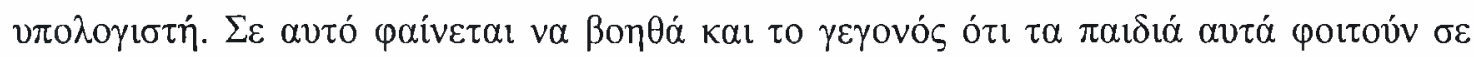

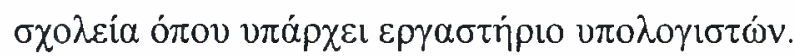

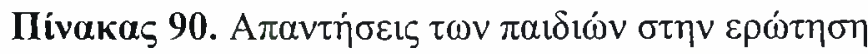

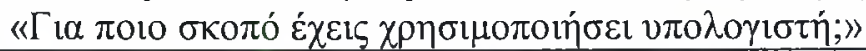

\begin{tabular}{|c|c|}
\hline$A \pi \alpha \nu \tau \eta ́ \sigma \varepsilon \iota \varsigma$ & 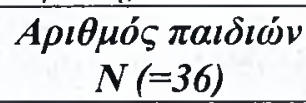 \\
\hline 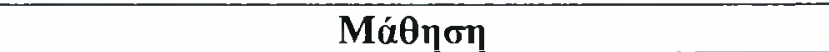 & 4 \\
\hline 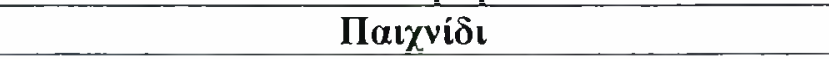 & 13 \\
\hline 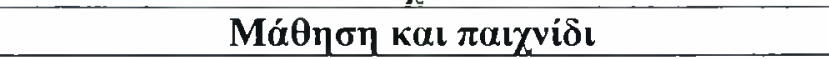 & 17 \\
\hline 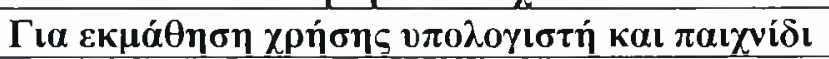 & 2 \\
\hline
\end{tabular}

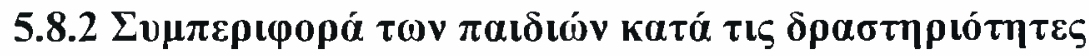

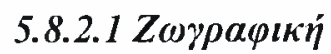

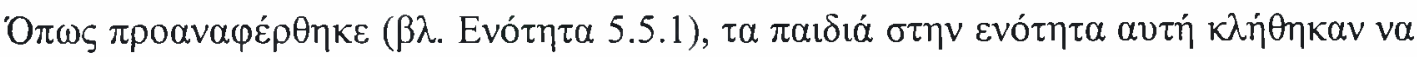

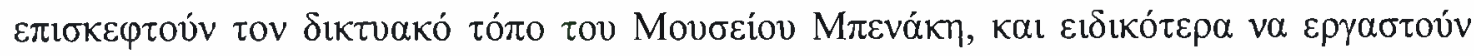

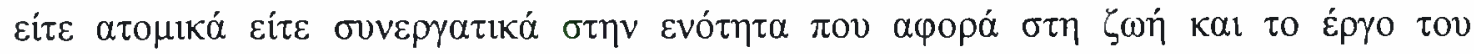

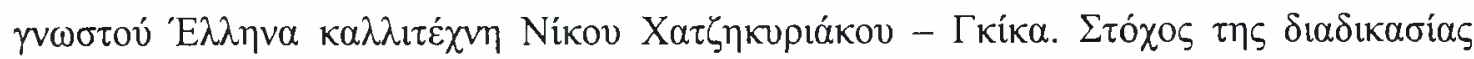




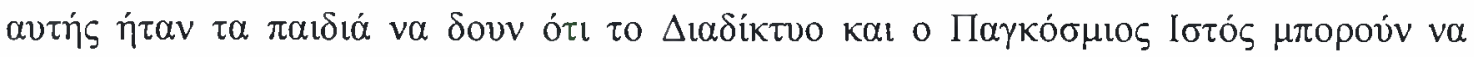

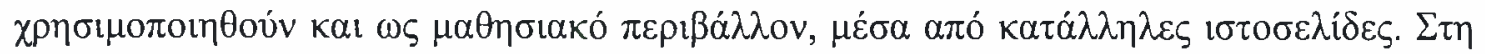

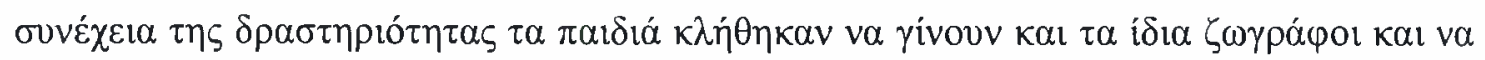

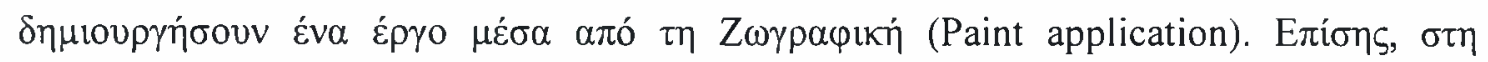

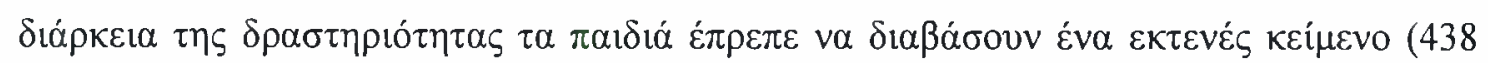

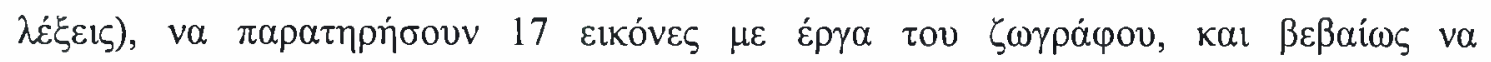

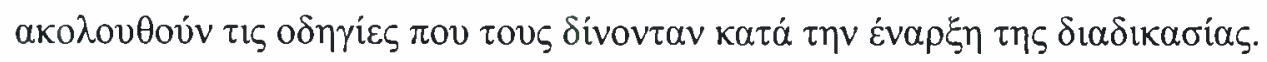

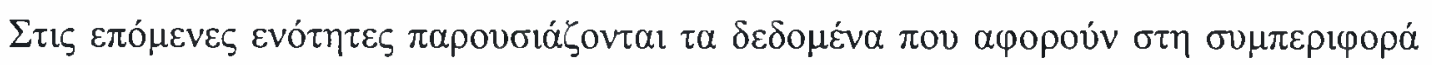

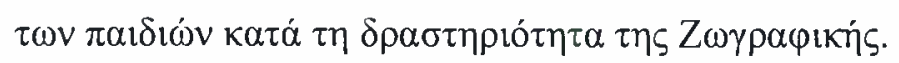

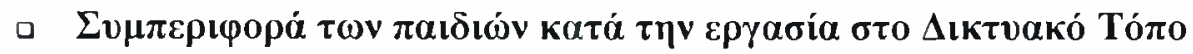

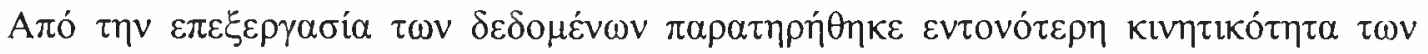

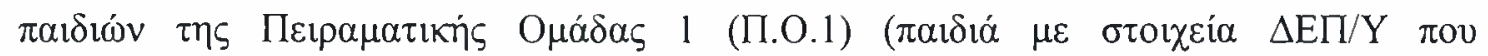

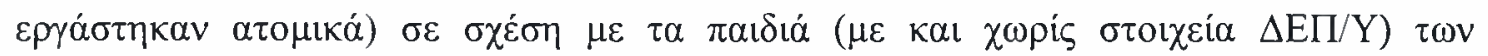

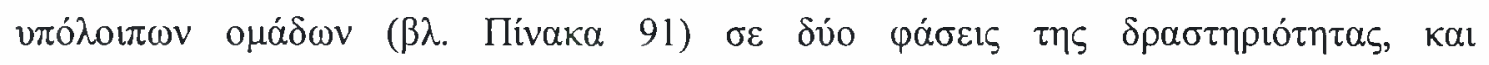

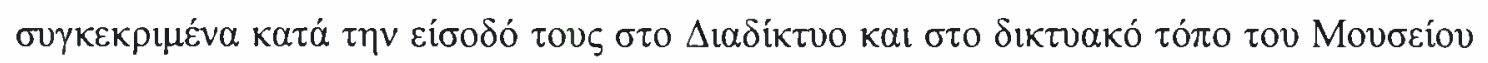

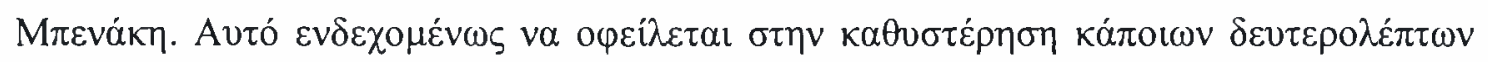

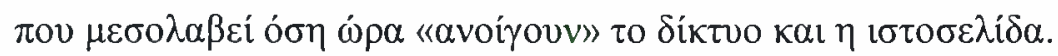

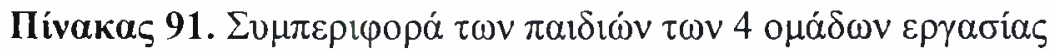

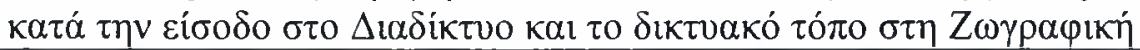

\begin{tabular}{|c|c|c|c|c|c|c|c|c|}
\hline \multirow{3}{*}{$\begin{array}{c}\Sigma \tau o \iota \chi \varepsilon i ́ \alpha \\
\sigma u \mu \pi \varepsilon \rho \iota \varphi о \rho a ́ \varsigma\end{array}$} & \multicolumn{8}{|c|}{ 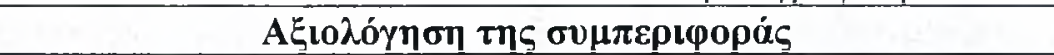 } \\
\hline & \multicolumn{2}{|c|}{$\begin{array}{l}\Pi .0 .1 \\
(N=7)\end{array}$} & \multicolumn{2}{|c|}{$\begin{array}{l}\text { П.0.2 } \\
(N=14)\end{array}$} & \multicolumn{2}{|c|}{$\begin{array}{l}\text { O.E.1 } \\
(N=5)\end{array}$} & \multicolumn{2}{|c|}{$\begin{array}{c}\text { O.E.2 } \\
(N=10)\end{array}$} \\
\hline & M.O. & T.A. & M.O. & T.A. & M.O. & T.A. & M.O. & T.A. \\
\hline 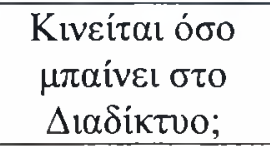 & 1,29 & 0,49 & 1,00 & 0,00 & 1,00 & 0,00 & 1,00 & 0,00 \\
\hline 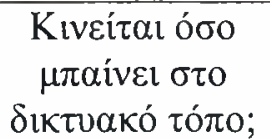 & 1,43 & 0,53 & 1,07 & 0,27 & 1,00 & 0,00 & 1,00 & 0,00 \\
\hline
\end{tabular}

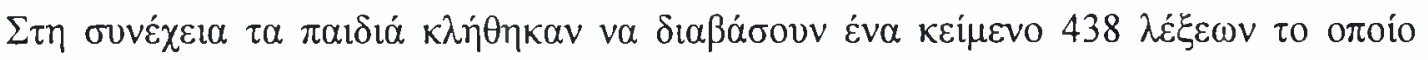

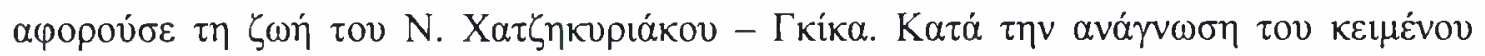




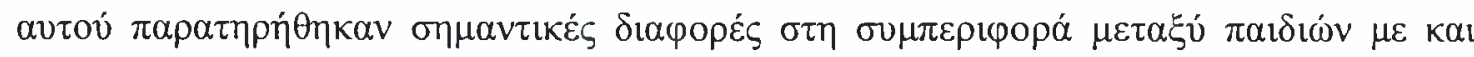

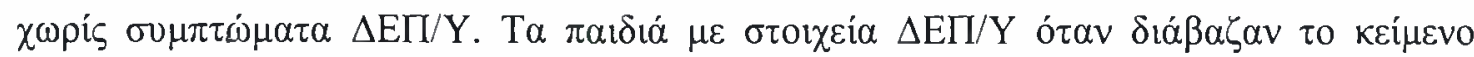

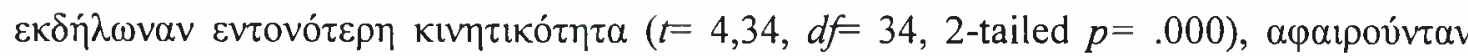

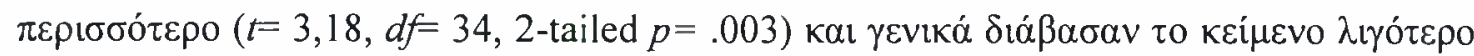

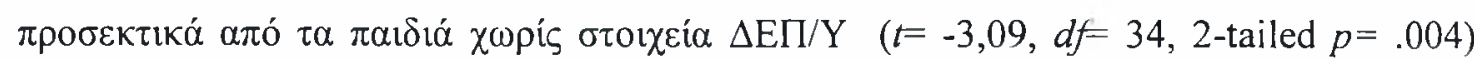

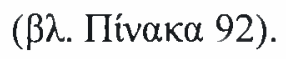

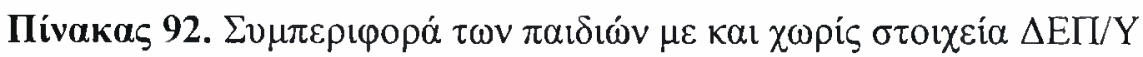

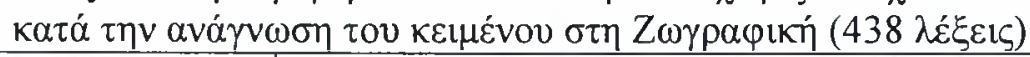

\begin{tabular}{|c|c|c|c|c|}
\hline \multirow{3}{*}{ 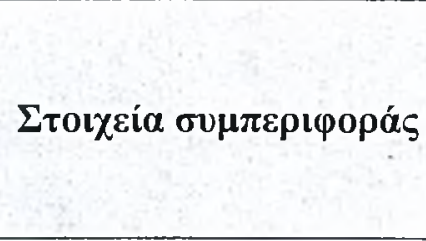 } & \multicolumn{4}{|c|}{ 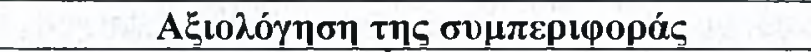 } \\
\hline & \multicolumn{2}{|c|}{$\begin{array}{c}\Pi \alpha \_\delta \text { i } \alpha \varepsilon \sigma \tau o \imath \chi \varepsilon i \alpha \\
\Delta E \Pi / Y \\
(14)\end{array}$} & \multicolumn{2}{|c|}{ 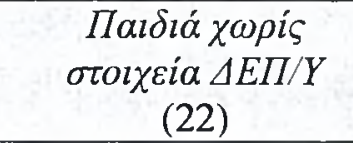 } \\
\hline & M.O. & T.A. & M.O. & T.A. \\
\hline 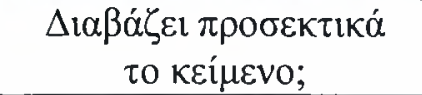 & 2,36 & 0,63 & 2,86 & 0,35 \\
\hline 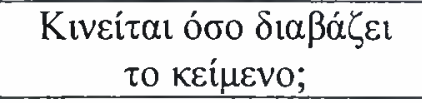 & 1,79 & 0,43 & 1,18 & 0,39 \\
\hline 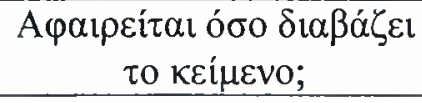 & 1,71 & 0,61 & 1,18 & 0,39 \\
\hline
\end{tabular}

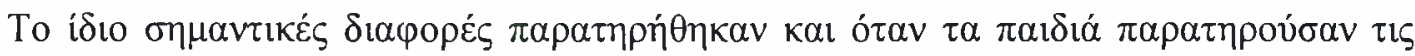

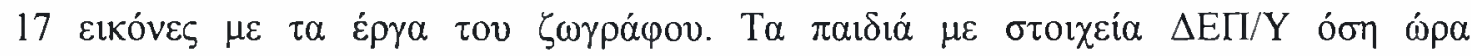

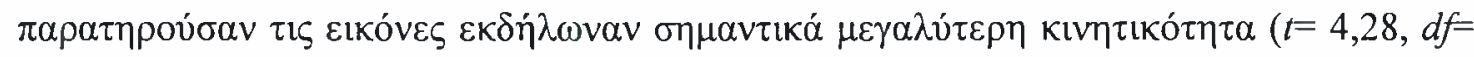

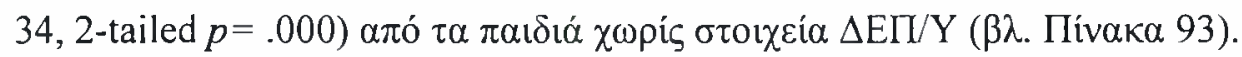

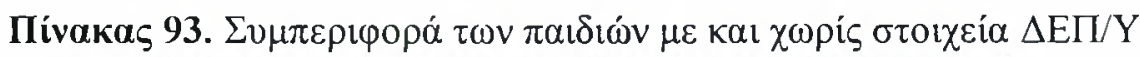

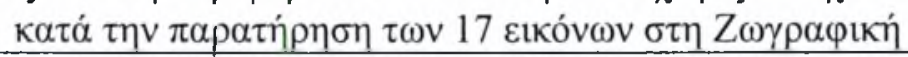

\begin{tabular}{|c|c|c|c|c|}
\hline \multirow{3}{*}{ 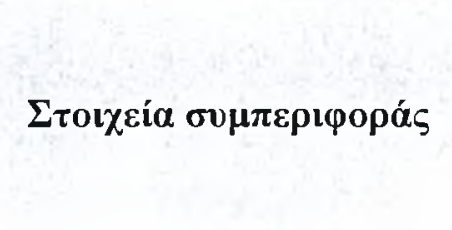 } & \multicolumn{4}{|c|}{ 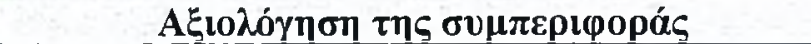 } \\
\hline & \multicolumn{2}{|c|}{ 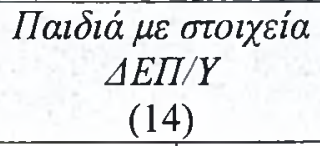 } & \multicolumn{2}{|c|}{ 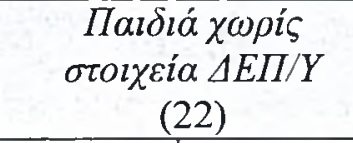 } \\
\hline & M.O. & T.A. & M.O. & T.A. \\
\hline 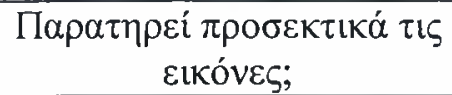 & 2,64 & 0,50 & 2,91 & 0,29 \\
\hline 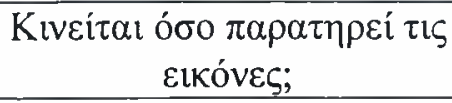 & 1,57 & 0,51 & 1,05 & 0,21 \\
\hline 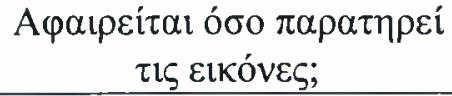 & 1,14 & 0,36 & 1,00 & 0,00 \\
\hline
\end{tabular}




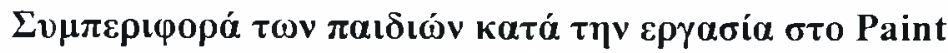

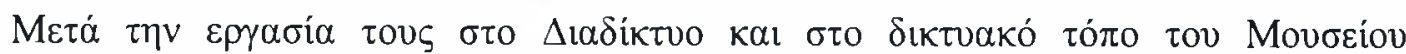

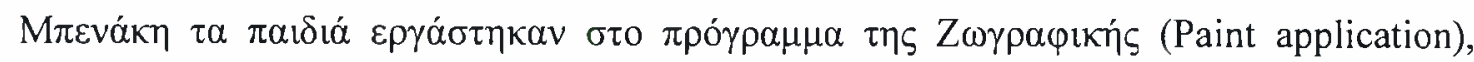

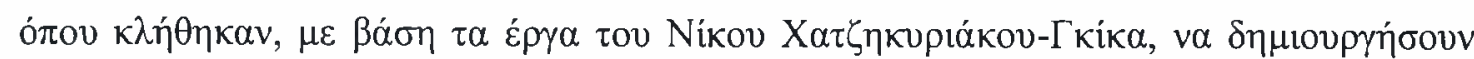

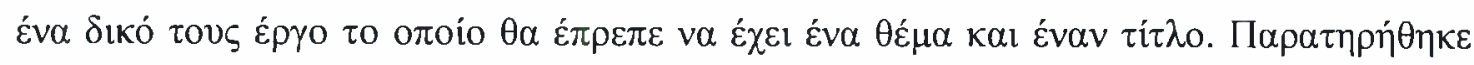

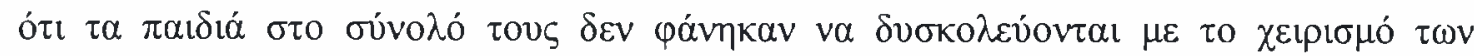

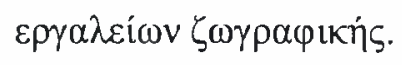

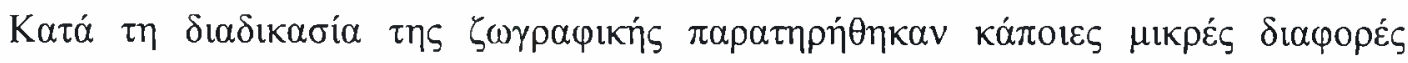

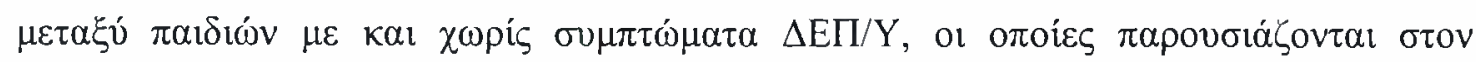
Пívaкa 94.

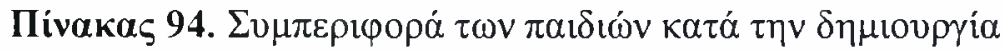

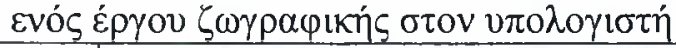

\begin{tabular}{|c|c|c|c|c|}
\hline \multirow{3}{*}{ 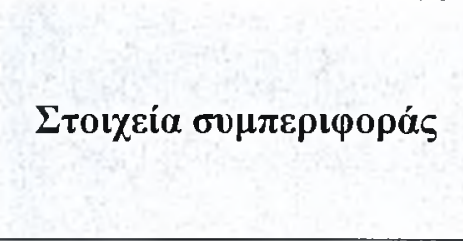 } & \multicolumn{4}{|c|}{ 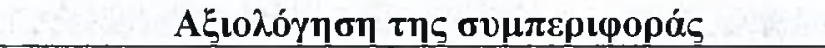 } \\
\hline & \multicolumn{2}{|c|}{ 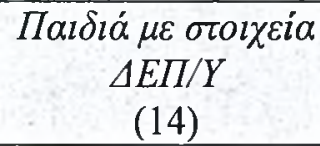 } & \multicolumn{2}{|c|}{ 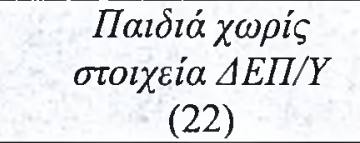 } \\
\hline & M.O. & T.A. & M.O. & T.A. \\
\hline 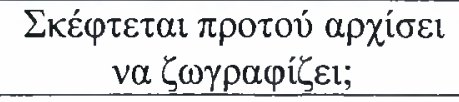 & 2,00 & 0,88 & 2,36 & 0,58 \\
\hline 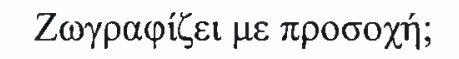 & 2,71 & 0,83 & 2,95 & 0,21 \\
\hline 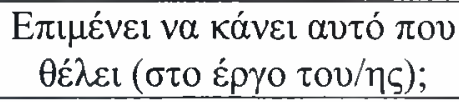 & 2,64 & 0,84 & 2,86 & 0,35 \\
\hline 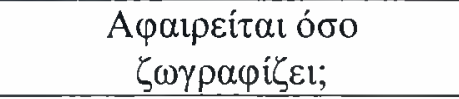 & 1,07 & 0,47 & 1,00 & 0,00 \\
\hline
\end{tabular}

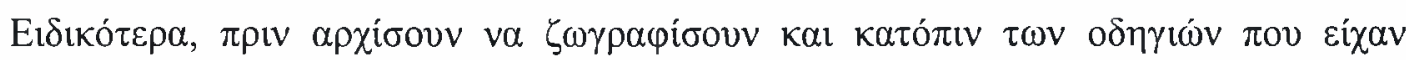

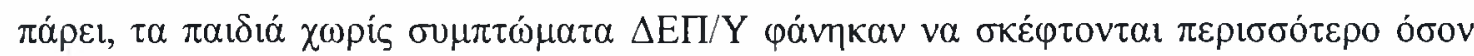

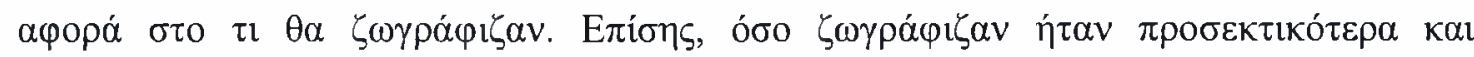

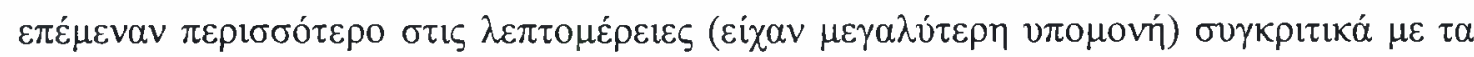
$\pi \alpha \imath \delta i \alpha \dot{\alpha} \mu \varepsilon \sigma \tau \mathrm{o} \chi \varepsilon \dot{i} \alpha \Delta \mathrm{E} \Pi / \mathrm{Y}$.

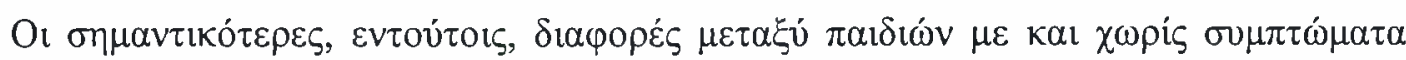
$\Delta \mathrm{E \Pi /Y} \mathrm{\pi \alpha \rho \alpha \tau \rho \eta ́ \theta \eta \kappa \alpha v} \mathrm{\sigma \varepsilon} \mathrm{\alpha v \tau \alpha ́} \mathrm{\pi ov} \mathrm{\varepsilon \rho \gamma \alpha ́ \zeta ov \tau \alpha v} \mathrm{\alpha v \alpha ́} \mathrm{\delta v́o} \mathrm{[П \varepsilon เ \rho \alpha \mu \alpha \tau i \kappa \eta ்} \mathrm{O \mu \alpha ́ \delta \alpha} 2$

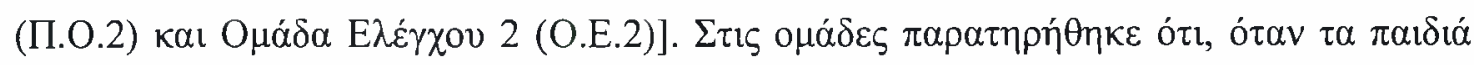




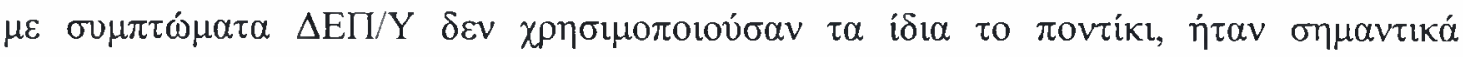

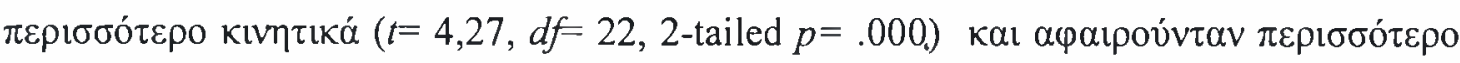

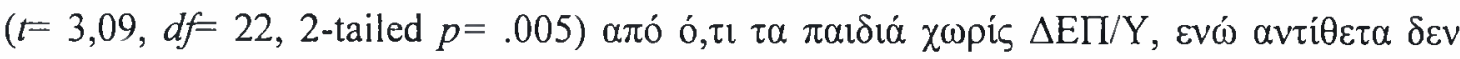

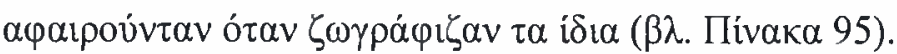

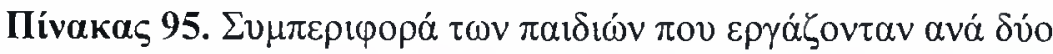

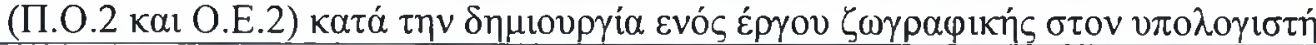

\begin{tabular}{|c|c|c|c|c|}
\hline \multirow{3}{*}{$\Sigma \tau о\llcorner\chi \varepsilon i ́ \alpha \sigma v \mu \pi \varepsilon \rho \iota \varphi \circ \rho \alpha \dot{~} \varsigma$} & \multicolumn{4}{|c|}{ 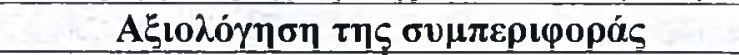 } \\
\hline & \multicolumn{2}{|c|}{ 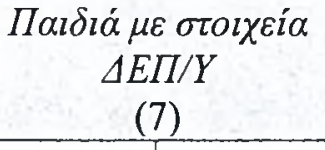 } & \multicolumn{2}{|c|}{$\begin{array}{c}\text { Пaldid } \chi \omega \rho i \varsigma \\
\sigma \tau o l \chi \varepsilon i \alpha \Delta E \Pi / Y \\
(17)\end{array}$} \\
\hline & M.O. & T.A. & M.O. & T.A. \\
\hline 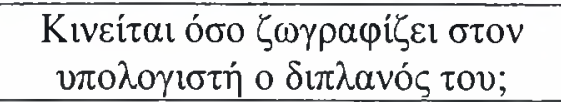 & 1,86 & 0,38 & 1,06 & 0,43 \\
\hline 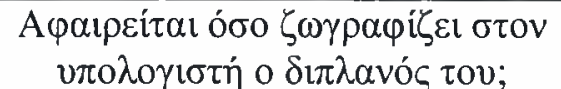 & 1,57 & 0,53 & 1,00 & 0,35 \\
\hline
\end{tabular}

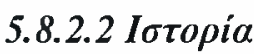

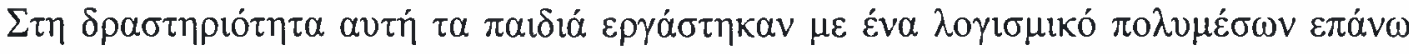

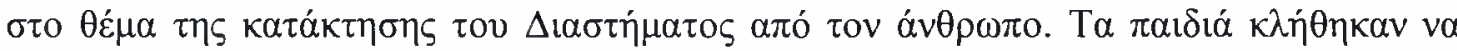

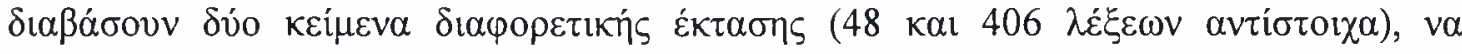

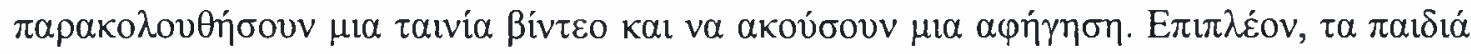

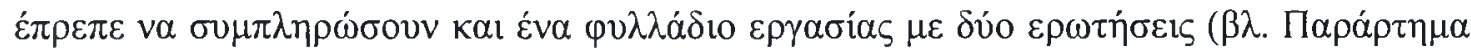

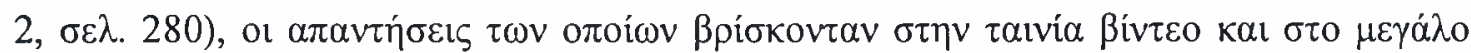

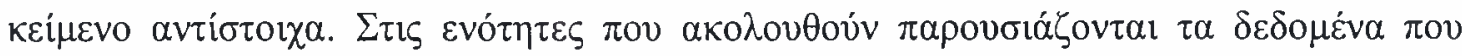

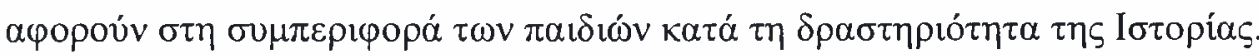

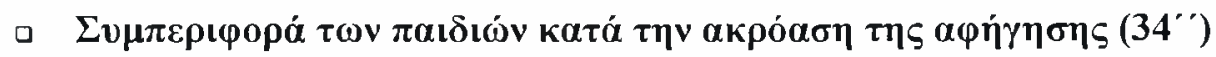

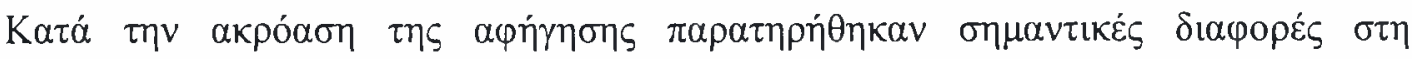

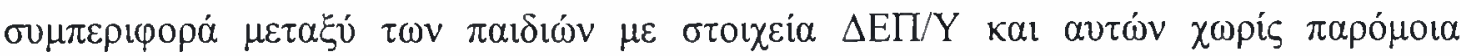

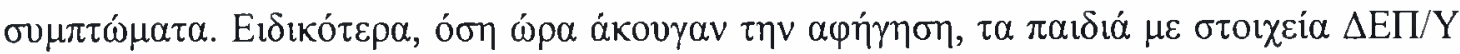

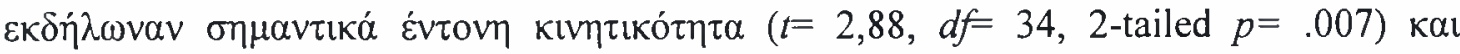




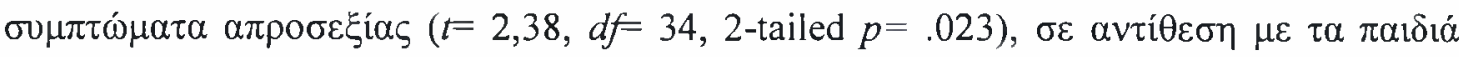

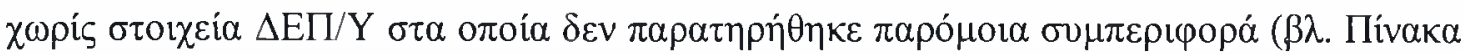

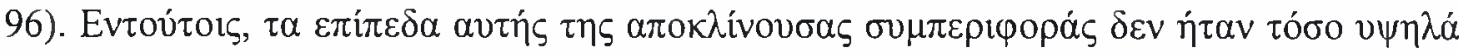
$\dot{\omega} \sigma \tau \varepsilon v \alpha \pi \alpha \rho \alpha \kappa \omega \lambda v ́ o v v \tau \eta \delta \rho \alpha \sigma \tau \eta \rho \operatorname{t} \tau \tau \alpha$.

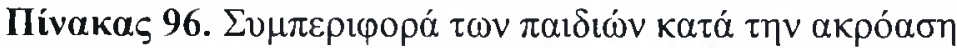

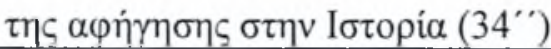

\begin{tabular}{|c|c|c|c|c|}
\hline \multirow{3}{*}{ 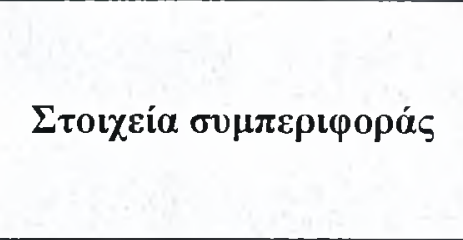 } & \multicolumn{4}{|c|}{ 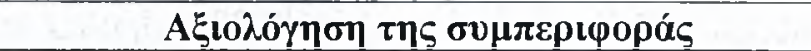 } \\
\hline & \multicolumn{2}{|c|}{ 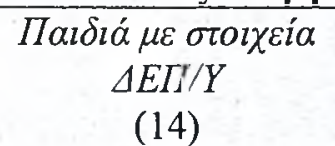 } & \multicolumn{2}{|c|}{ 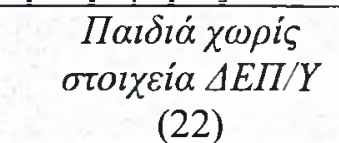 } \\
\hline & M.O. & T.A. & M.O. & T.A. \\
\hline 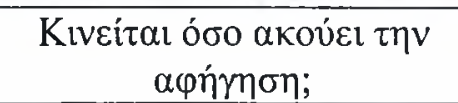 & 1,29 & 0,47 & 1,00 & 0,00 \\
\hline 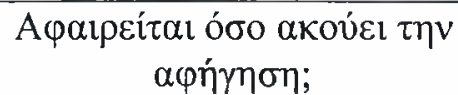 & 1,21 & 0,43 & 1,00 & 0,00 \\
\hline
\end{tabular}

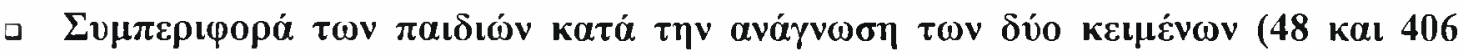
$\lambda \dot{\varepsilon} \xi \varepsilon \omega v \alpha v \tau i \sigma \tau o\llcorner\chi \alpha)$

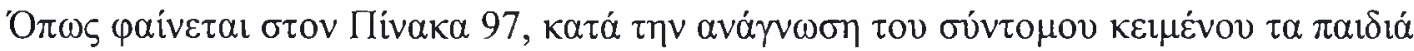

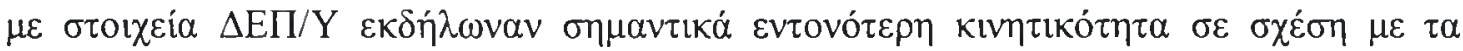

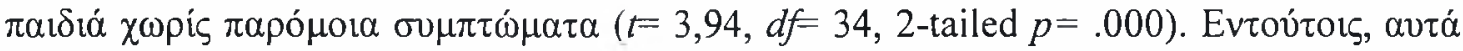

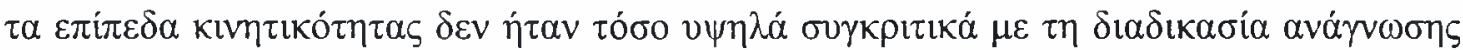

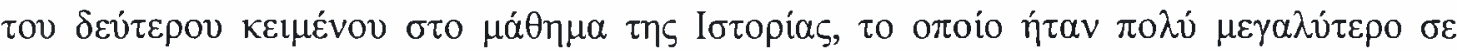

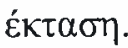

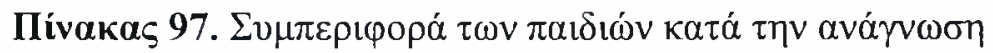

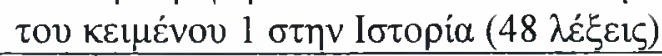

\begin{tabular}{|c|c|c|c|c|}
\hline \multirow{3}{*}{$\Sigma \tau o \iota \chi \varepsilon i ́ \alpha \sigma v \mu \pi \varepsilon \rho \iota \varphi \circ \rho \alpha \varsigma$} & \multicolumn{4}{|c|}{ 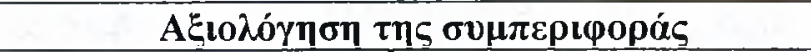 } \\
\hline & \multicolumn{2}{|c|}{ 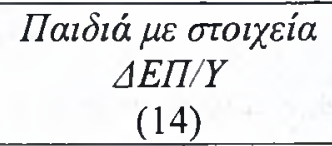 } & \multicolumn{2}{|c|}{ 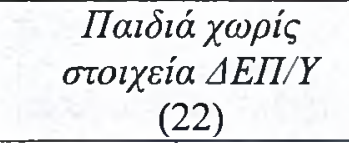 } \\
\hline & M.O. & T.A. & M.O. & T.A. \\
\hline 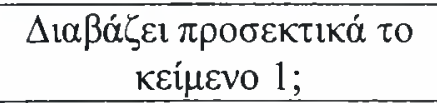 & 2,64 & 050 & 2,86 & 0,35 \\
\hline 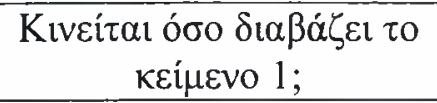 & 1,43 & 0,51 & 1,00 & 0,00 \\
\hline
\end{tabular}




\begin{tabular}{|c|c|c|c|c|}
\hline 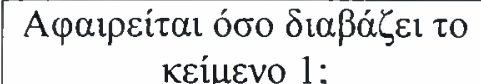 & 1,14 & 0,36 & 1,09 & 0,29 \\
\hline
\end{tabular}

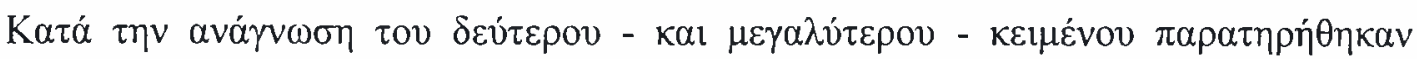

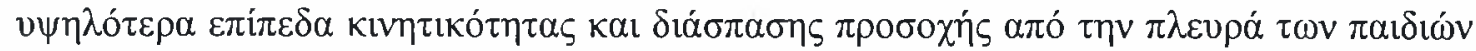

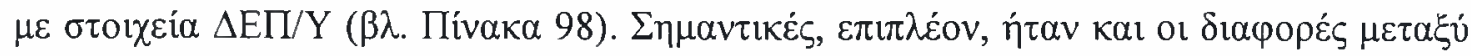

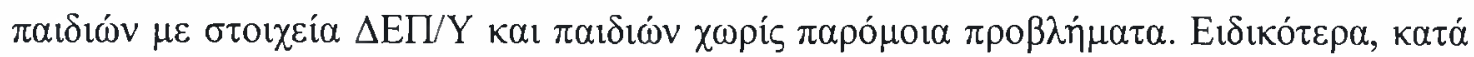

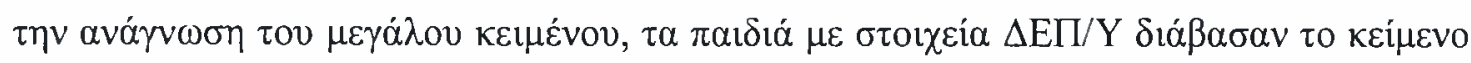

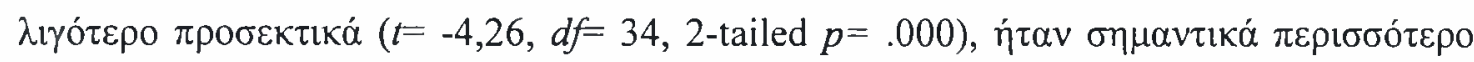

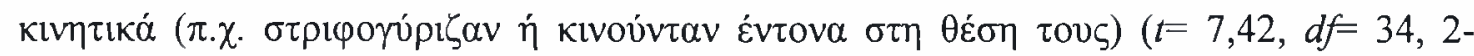

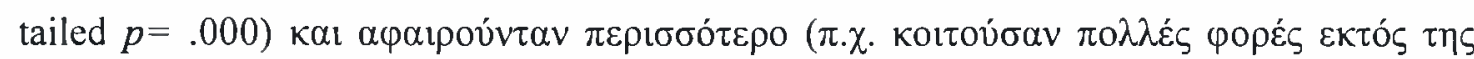

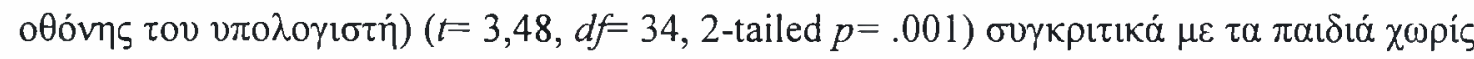

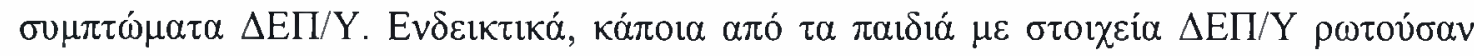

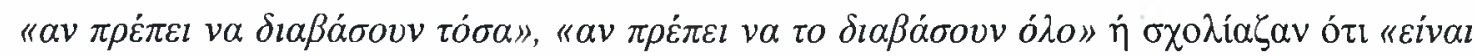
$\pi o \lambda \dot{u} \mu \varepsilon \gamma \alpha \dot{\lambda} o$ !».

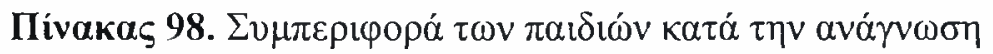

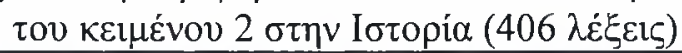

\begin{tabular}{|c|c|c|c|c|}
\hline \multirow{3}{*}{ 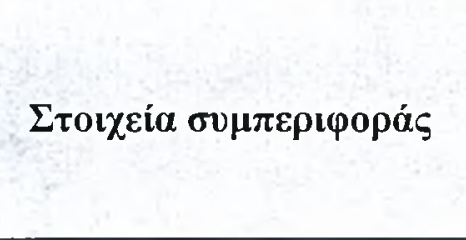 } & \multicolumn{4}{|c|}{ 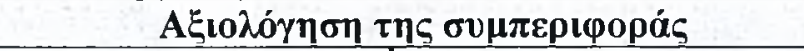 } \\
\hline & \multicolumn{2}{|c|}{ 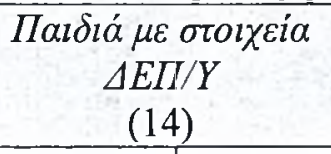 } & \multicolumn{2}{|c|}{ 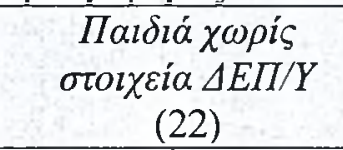 } \\
\hline & M.O. & T.A. & M.O. & T.A. \\
\hline 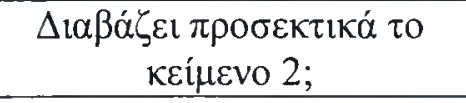 & 1,93 & 0,47 & 2,64 & 0,49 \\
\hline 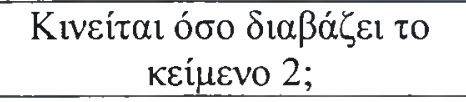 & 2,21 & 0,43 & 1,18 & 0,39 \\
\hline 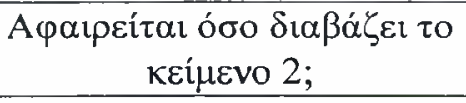 & 2,07 & 0,73 & 1,36 & 0,49 \\
\hline
\end{tabular}

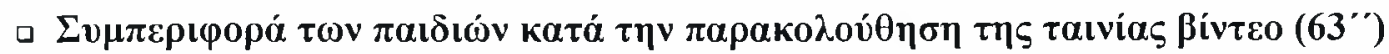

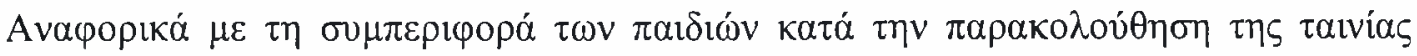

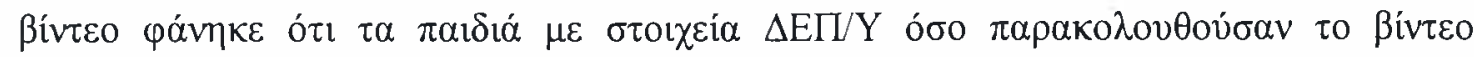

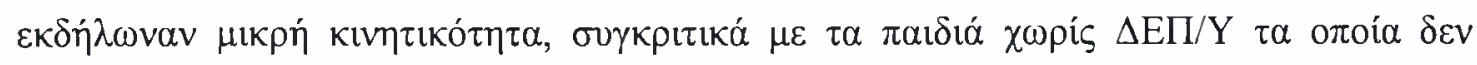




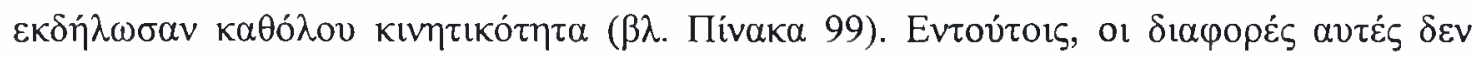

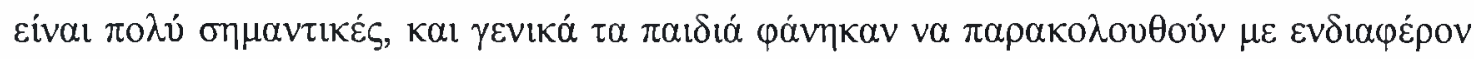

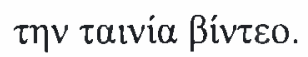

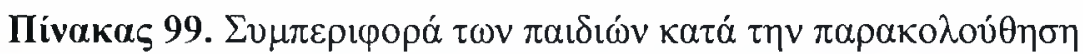

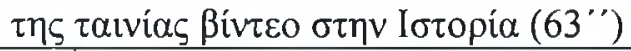

\begin{tabular}{|c|c|c|c|c|}
\hline \multirow{3}{*}{ 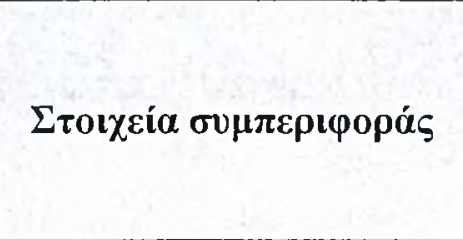 } & \multicolumn{4}{|c|}{ 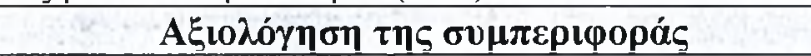 } \\
\hline & \multicolumn{2}{|c|}{ 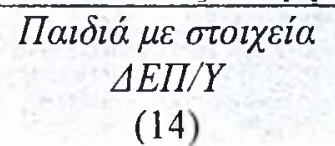 } & \multicolumn{2}{|c|}{ 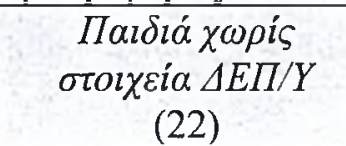 } \\
\hline & M.O. & T.A. & M.O. & T.A. \\
\hline 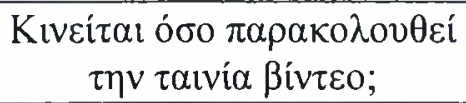 & 1,21 & 0,43 & 1,00 & 0,00 \\
\hline
\end{tabular}

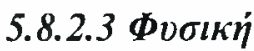

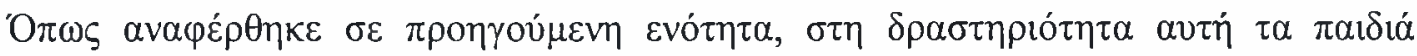

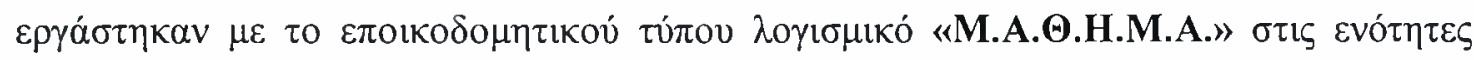

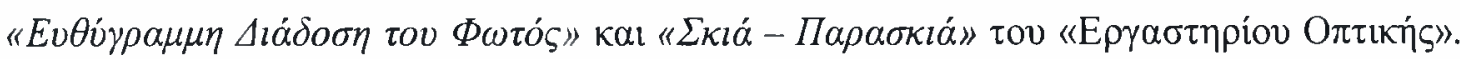

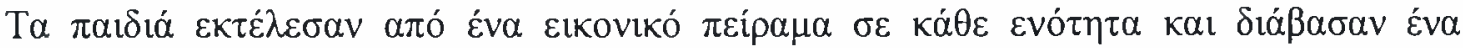

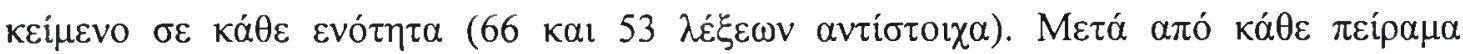

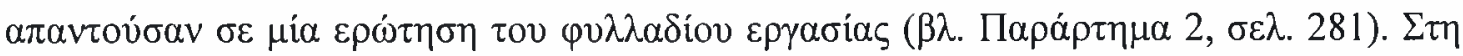

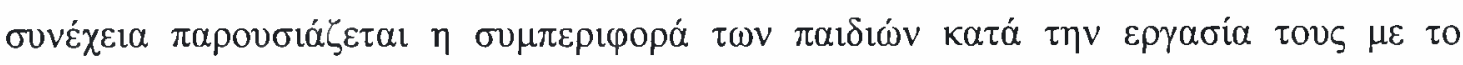

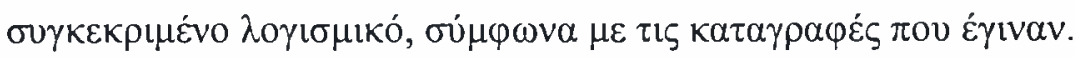

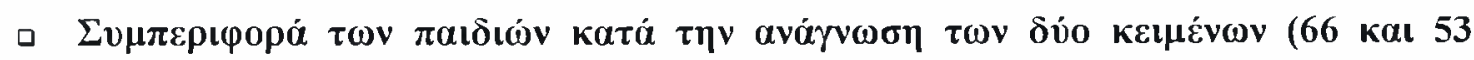

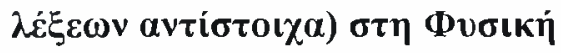

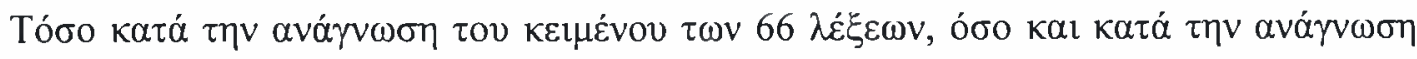

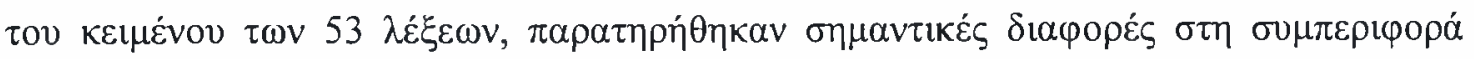

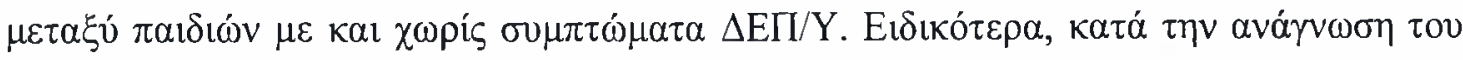

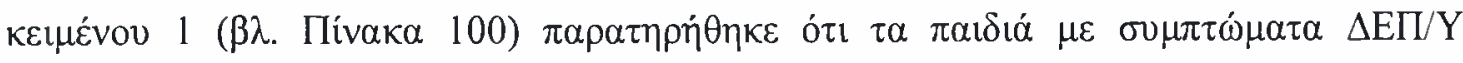

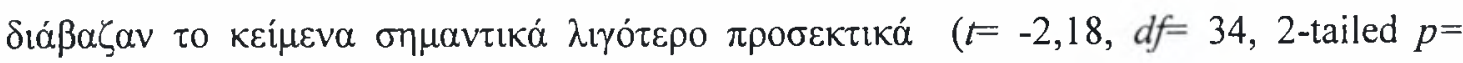




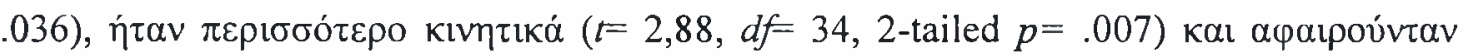

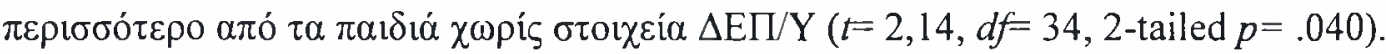

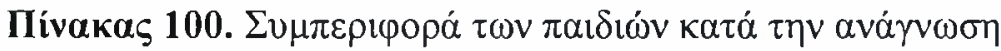

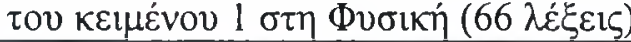

\begin{tabular}{|c|c|c|c|c|}
\hline \multirow{3}{*}{ 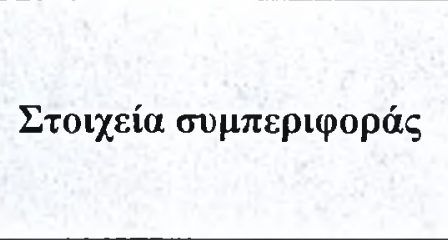 } & \multicolumn{4}{|c|}{ 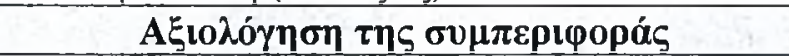 } \\
\hline & \multicolumn{2}{|c|}{ 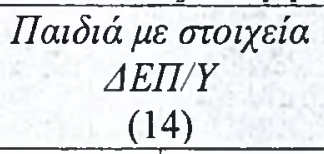 } & \multicolumn{2}{|c|}{ 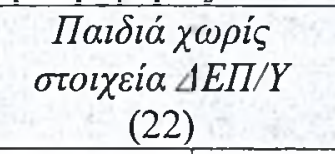 } \\
\hline & M.O. & T.A. & M.O. & T.A. \\
\hline 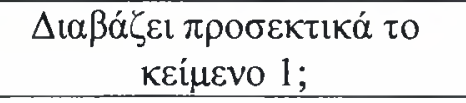 & 2,50 & 0,65 & 2,86 & 0,35 \\
\hline 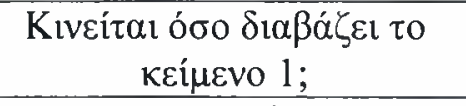 & 1,29 & 0,47 & 1,00 & 0,00 \\
\hline 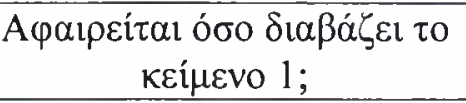 & 1,36 & 0,63 & 1,05 & 0,21 \\
\hline
\end{tabular}

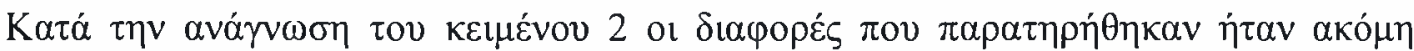

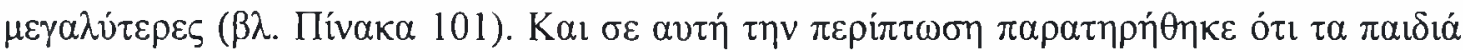

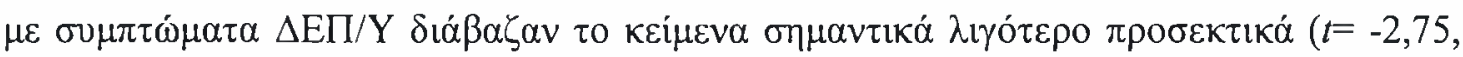

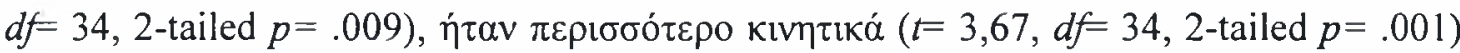

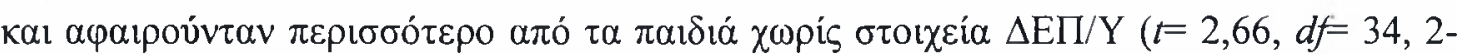
tailed $p=.012$ ).

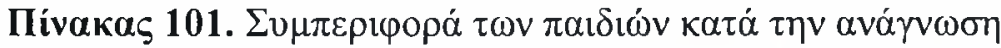

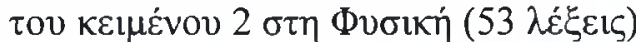

\begin{tabular}{|c|c|c|c|c|}
\hline \multirow{3}{*}{ 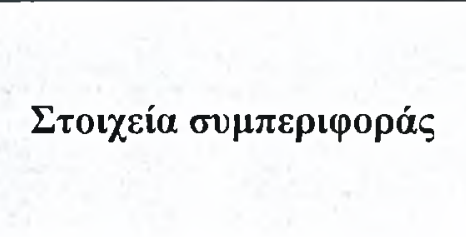 } & \multicolumn{4}{|c|}{ 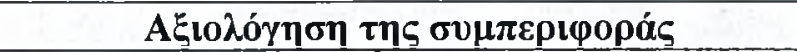 } \\
\hline & \multicolumn{2}{|c|}{ 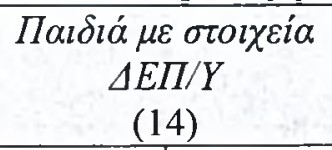 } & \multicolumn{2}{|c|}{ 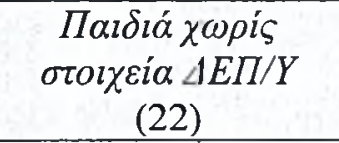 } \\
\hline & M.O. & T.A. & M.O. & T.A. \\
\hline 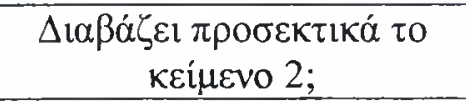 & 2,36 & 0,74 & 2,86 & 0,35 \\
\hline 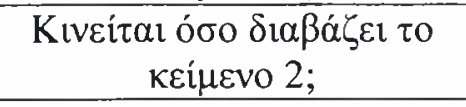 & 1,50 & 0,52 & 1,05 & 0,21 \\
\hline 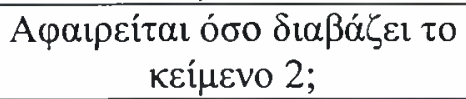 & 1,50 & 0,76 & 1,05 & 0,21 \\
\hline
\end{tabular}

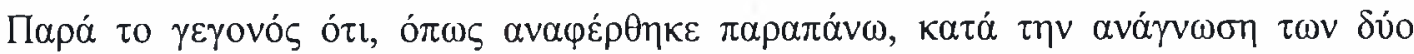

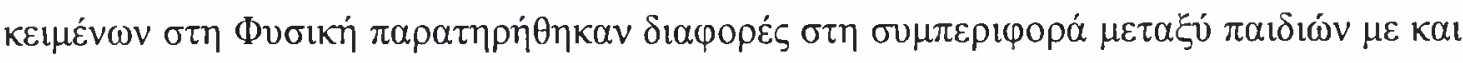




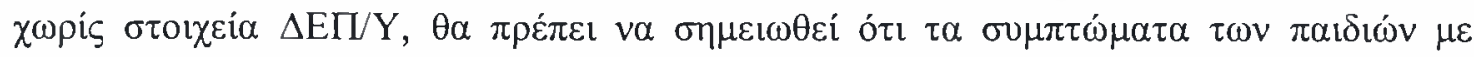

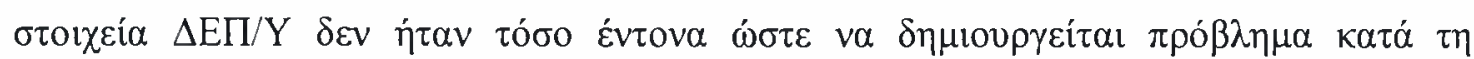

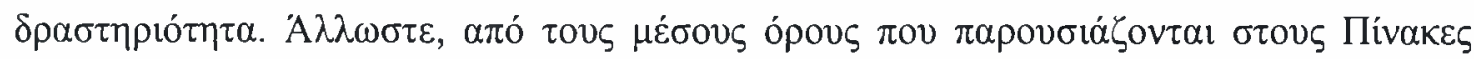

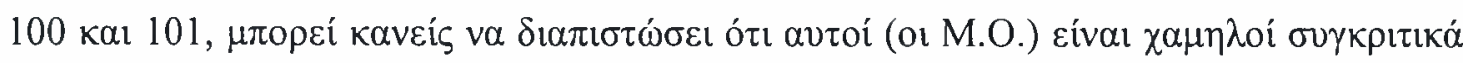

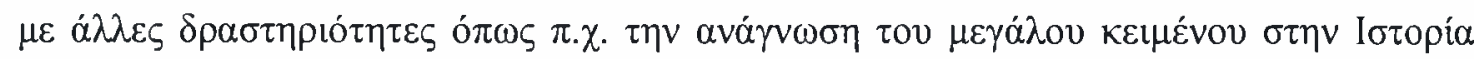
$(\beta \lambda$. Evó $\tau \tau \alpha$ 5.8.2.2).

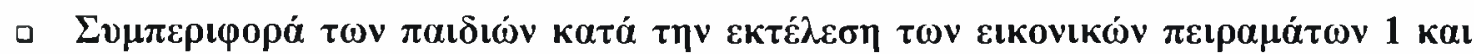

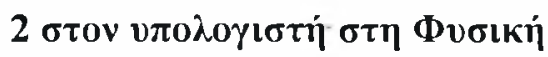

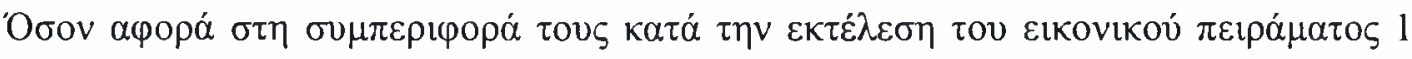

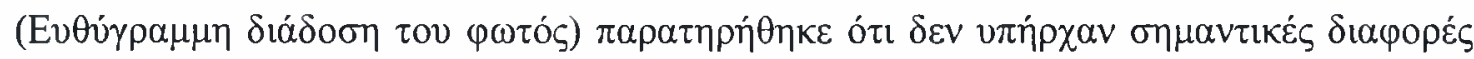

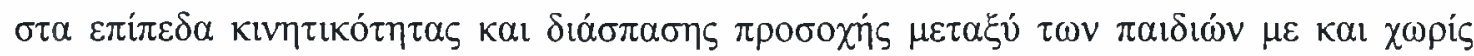

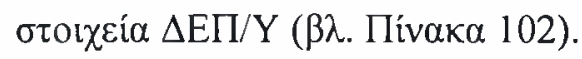

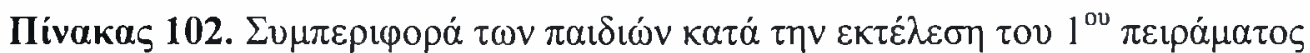

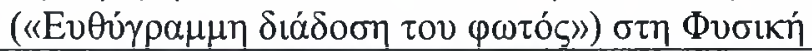

\begin{tabular}{|c|c|c|c|c|}
\hline \multirow{3}{*}{ 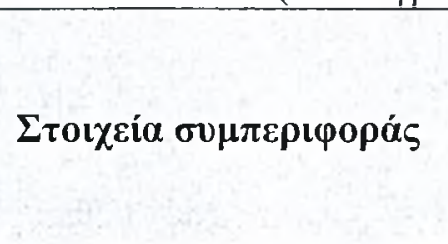 } & \multicolumn{4}{|c|}{ 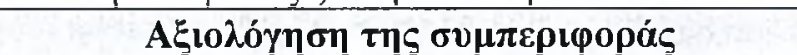 } \\
\hline & \multicolumn{2}{|c|}{ 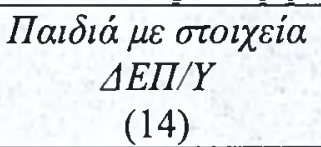 } & \multicolumn{2}{|c|}{ 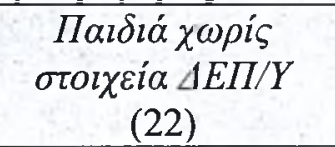 } \\
\hline & M.O. & T.A. & M.O. & T.A. \\
\hline 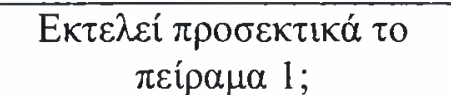 & 2,93 & 0,27 & 3,00 & 0,00 \\
\hline 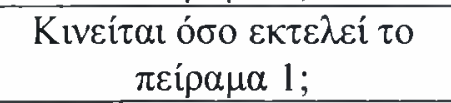 & 1,14 & 0,36 & 1,00 & 0,00 \\
\hline 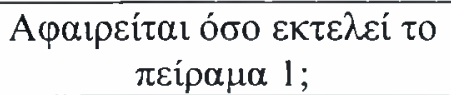 & 1,07 & 0,27 & 1,00 & 0,00 \\
\hline
\end{tabular}

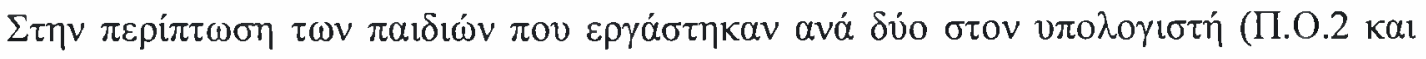

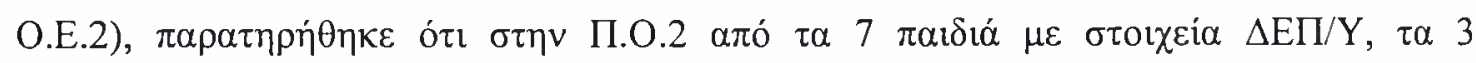

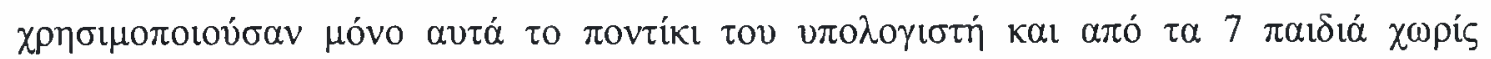

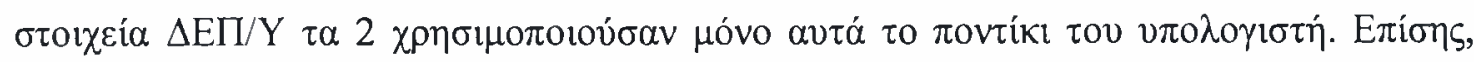

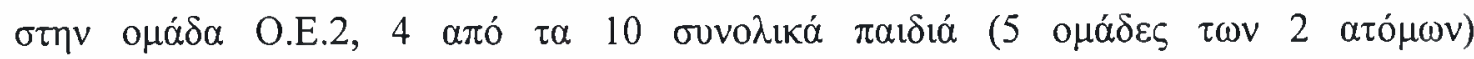

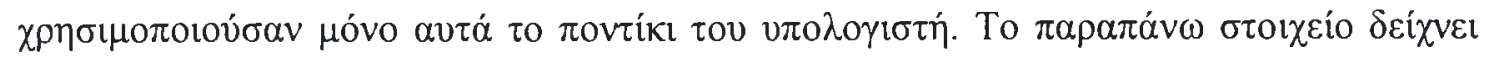




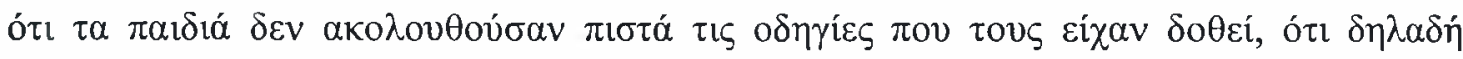

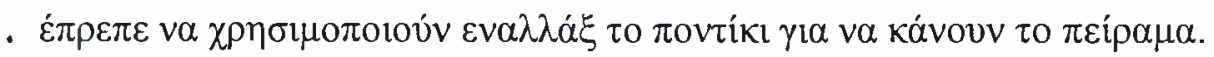

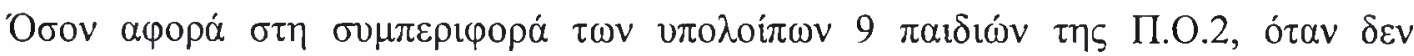

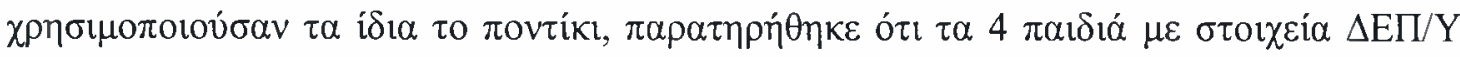

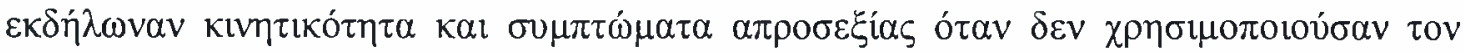

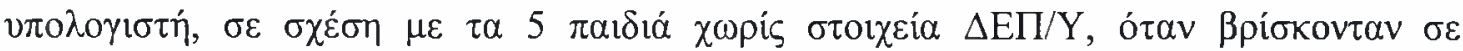

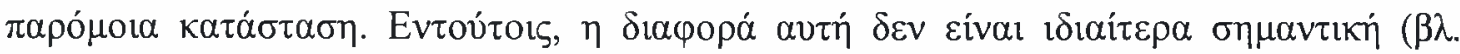

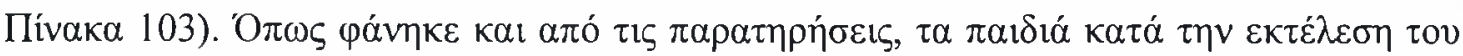

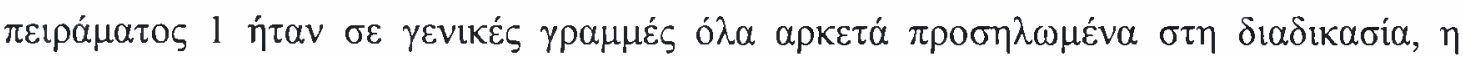

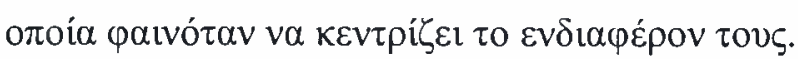

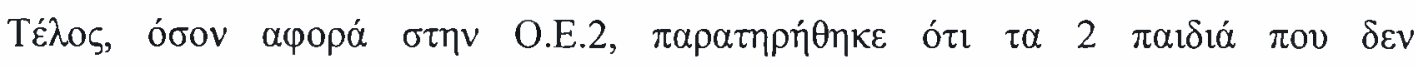

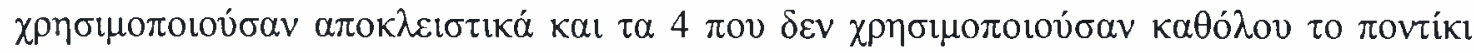

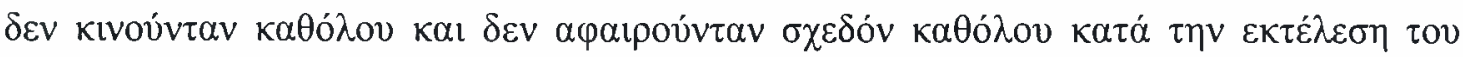

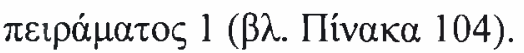

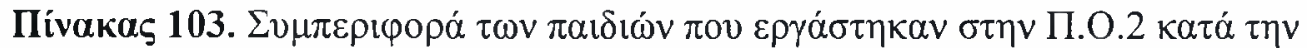

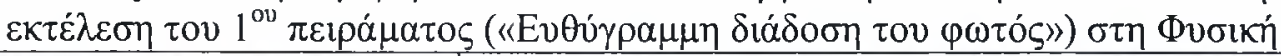

\begin{tabular}{|c|c|c|c|c|}
\hline \multirow{3}{*}{ 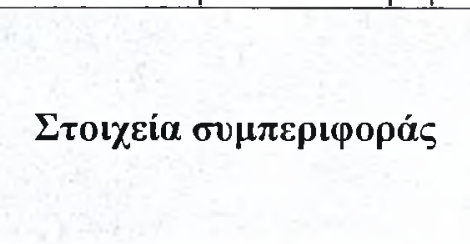 } & \multicolumn{4}{|c|}{ 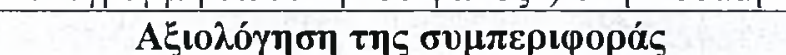 } \\
\hline & \multicolumn{2}{|c|}{ 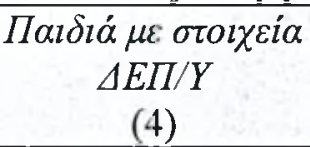 } & \multicolumn{2}{|c|}{ 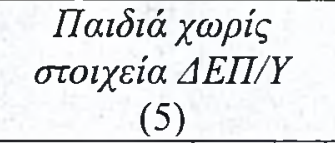 } \\
\hline & M.O. & T.A. & M.O. & T.A. \\
\hline 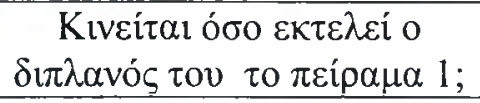 & 1,25 & 0,50 & 1,00 & 0,00 \\
\hline 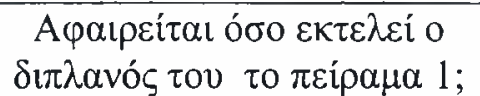 & 1,25 & 0,50 & 1,00 & 0,00 \\
\hline
\end{tabular}

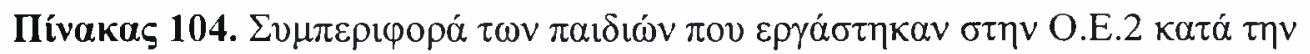

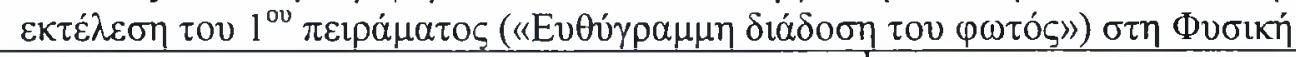

\begin{tabular}{|c|c|c|}
\hline \multirow{3}{*}{ 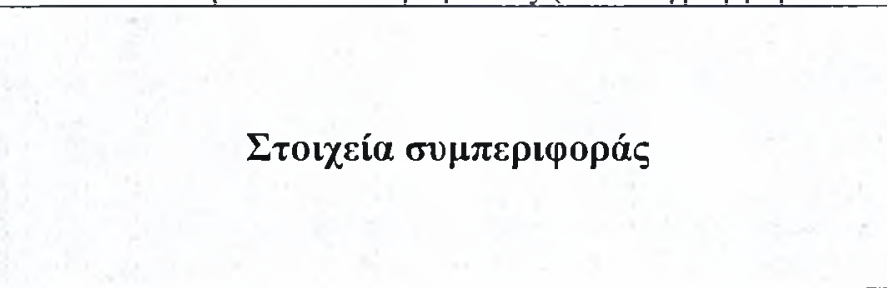 } & \multirow{2}{*}{\multicolumn{2}{|c|}{ 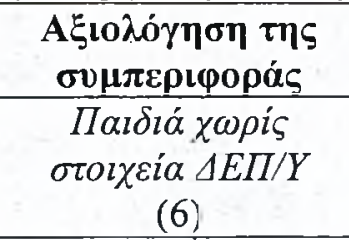 }} \\
\hline & & \\
\hline & M.O. & T.A. \\
\hline 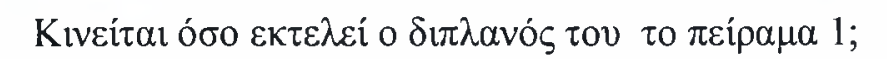 & 1,00 & 0,00 \\
\hline 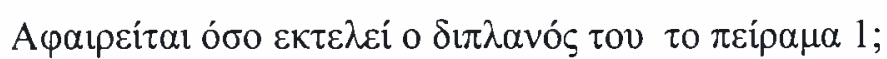 & 1,17 & 0,41 \\
\hline
\end{tabular}




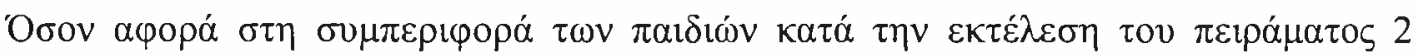

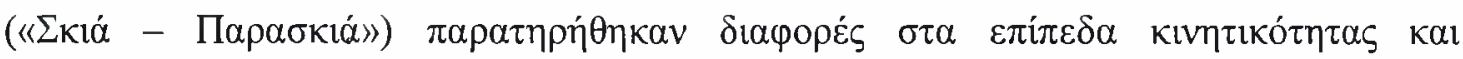

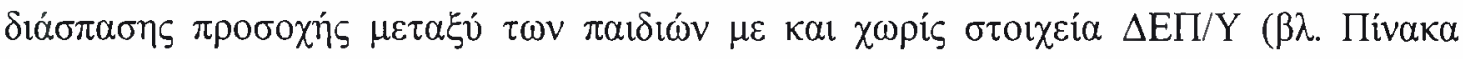

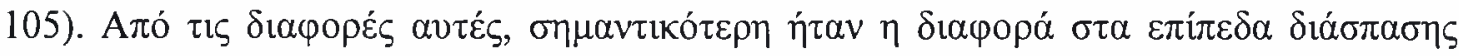

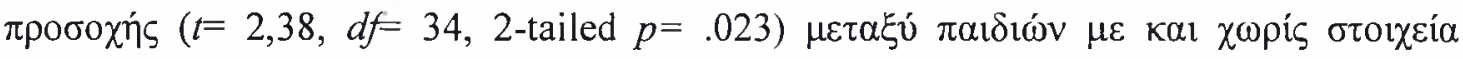
$\Delta \mathrm{E} \Pi / \mathrm{Y}$.

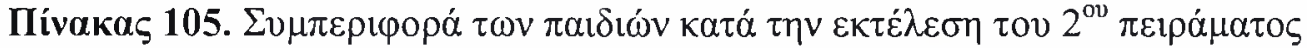

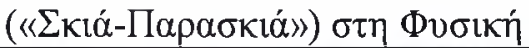

\begin{tabular}{|c|c|c|c|c|}
\hline \multirow{3}{*}{ 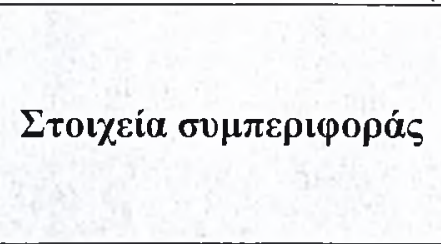 } & \multicolumn{4}{|c|}{ 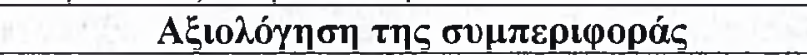 } \\
\hline & \multicolumn{2}{|c|}{ 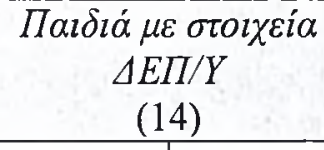 } & \multicolumn{2}{|c|}{ 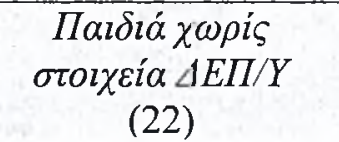 } \\
\hline & M.O. & T.A. & M.O. & T.A. \\
\hline 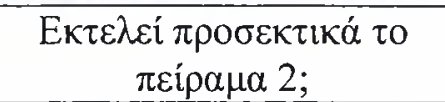 & 2,86 & 0,36 & 3,00 & 0,00 \\
\hline 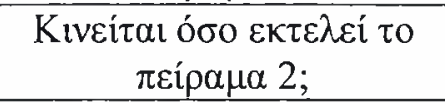 & 1,14 & 0,36 & 1,00 & 0,00 \\
\hline 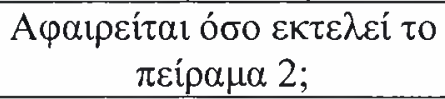 & 1,21 & 0,43 & 1,00 & 0,00 \\
\hline
\end{tabular}

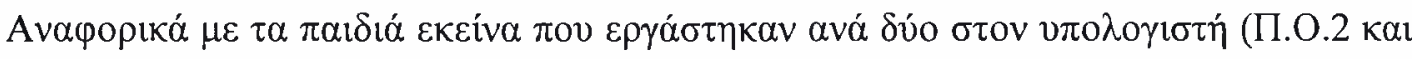

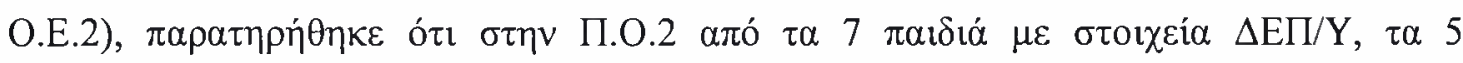

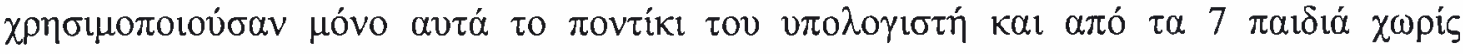

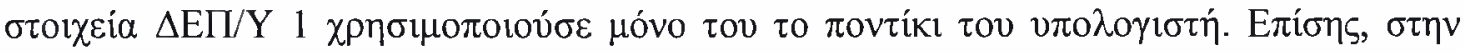

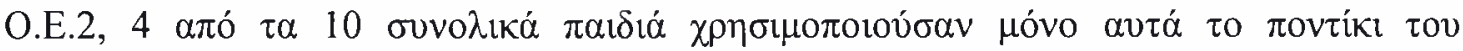

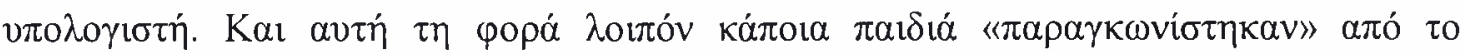

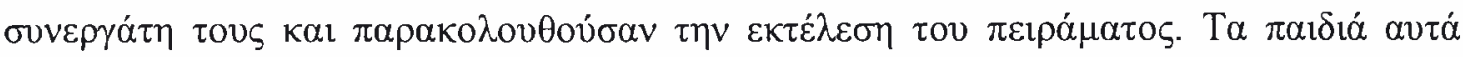

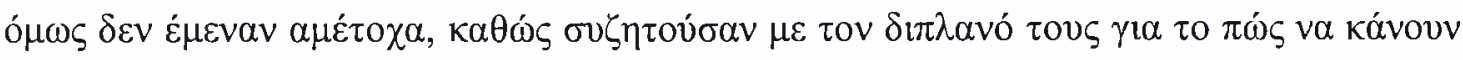
$\tau \alpha \pi \varepsilon i \rho \alpha ́ \alpha \alpha \tau \alpha$.

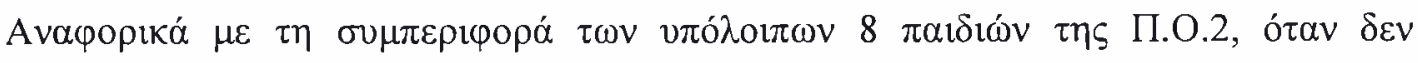

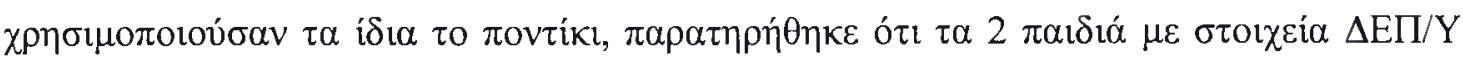

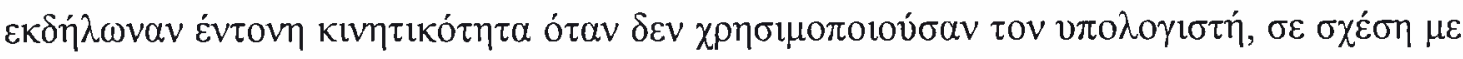

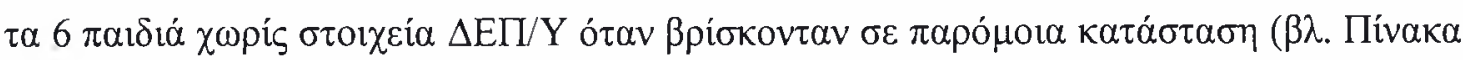

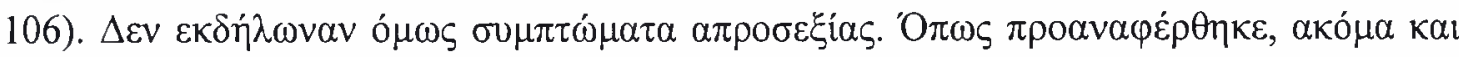

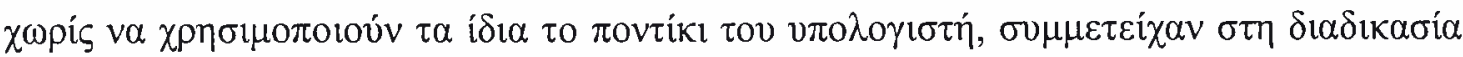




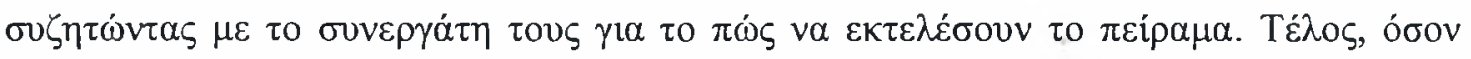

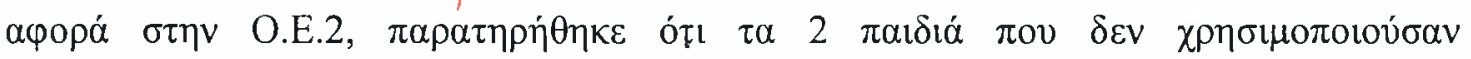

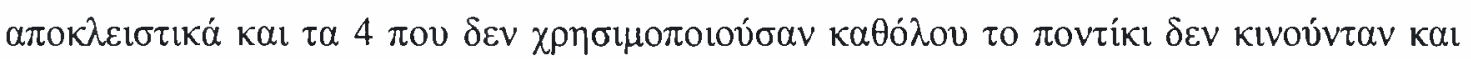

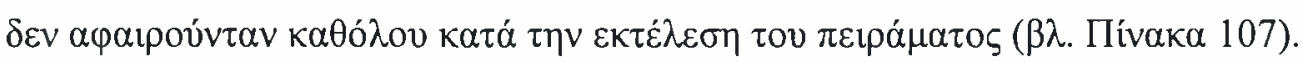

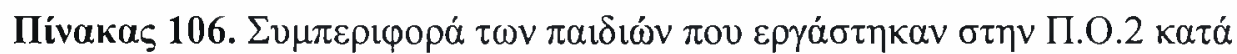

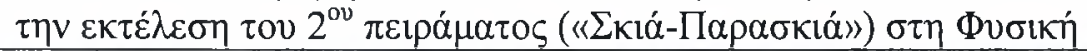

\begin{tabular}{|c|c|c|c|c|}
\hline \multirow{3}{*}{ 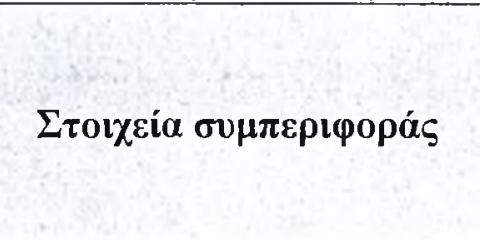 } & \multicolumn{4}{|c|}{ 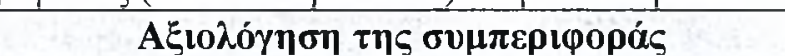 } \\
\hline & \multicolumn{2}{|c|}{$\begin{array}{c}\Pi \alpha \iota \delta \imath \alpha \dot{\alpha} \mu \varepsilon \sigma \tau o \iota \chi \varepsilon i \alpha \\
\Delta E \Pi / Y \\
(2)\end{array}$} & \multicolumn{2}{|c|}{ 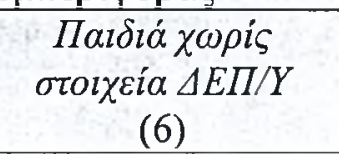 } \\
\hline & M.O. & T.A. & M.O. & T.A. \\
\hline 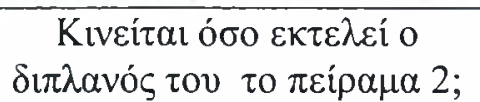 & 1,50 & 0,71 & 1,00 & 0,00 \\
\hline 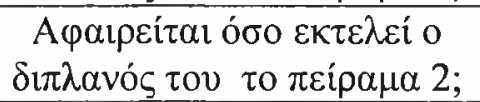 & 1,00 & 0,00 & 1,00 & 0,00 \\
\hline
\end{tabular}

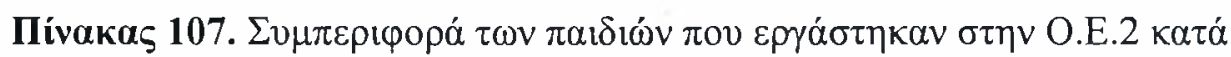

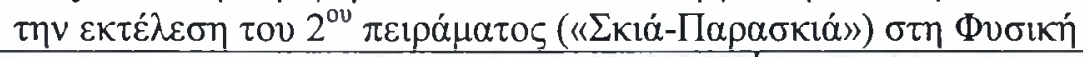

\begin{tabular}{|c|c|c|}
\hline \multirow{3}{*}{ 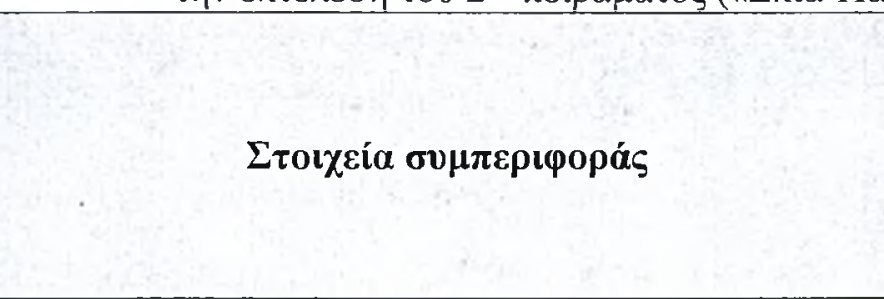 } & \multicolumn{2}{|c|}{ 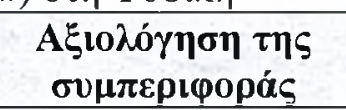 } \\
\hline & \multicolumn{2}{|c|}{ 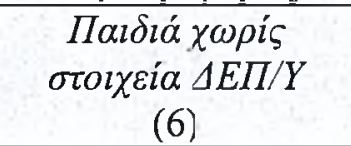 } \\
\hline & M.O. & T.A. \\
\hline 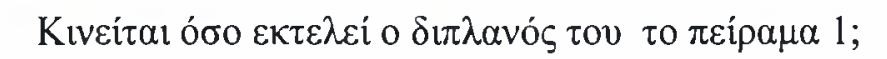 & 1,00 & 0,00 \\
\hline 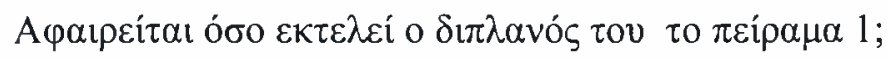 & 1,00 & 0,00 \\
\hline
\end{tabular}

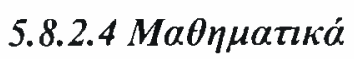

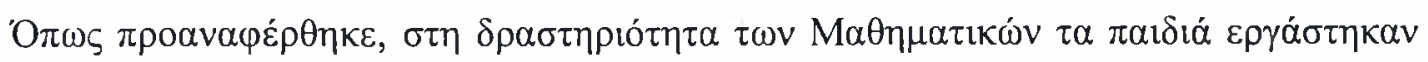

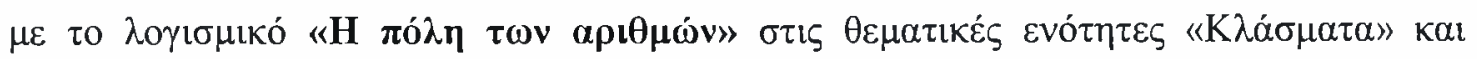

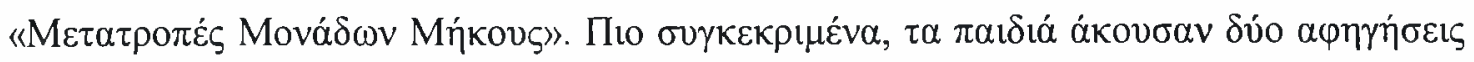

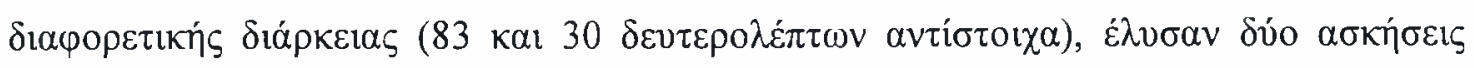

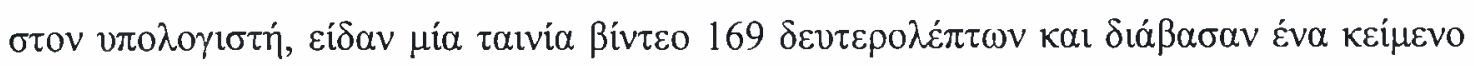

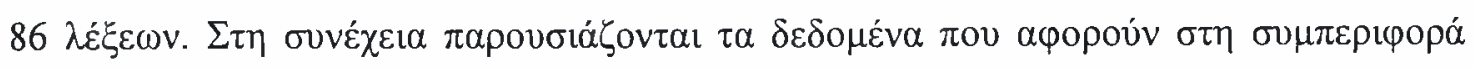




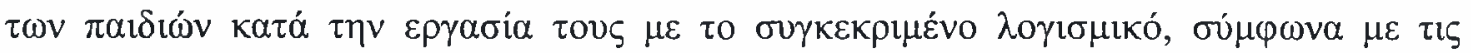

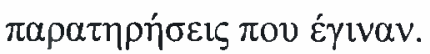

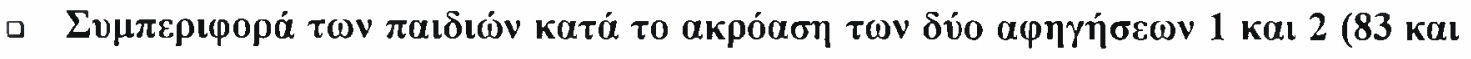

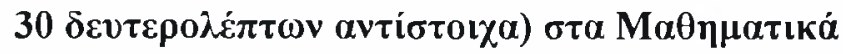

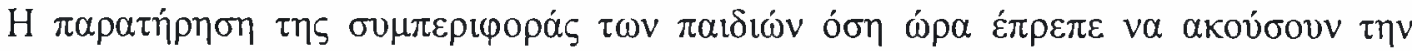

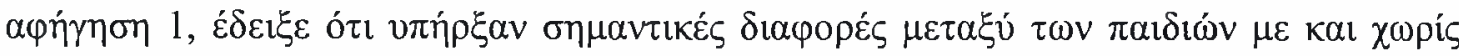

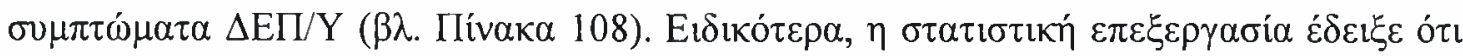

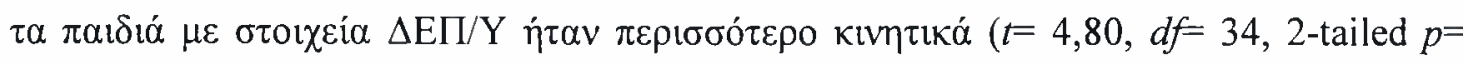

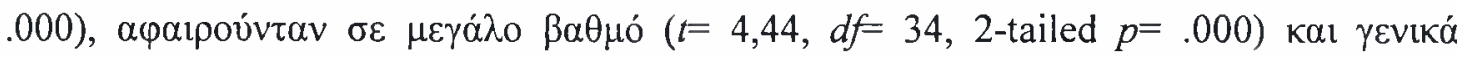

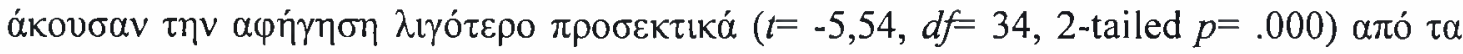

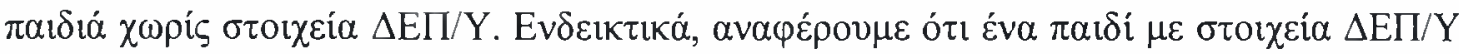

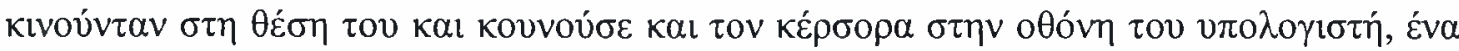

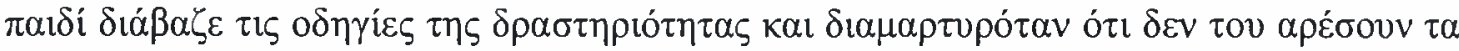

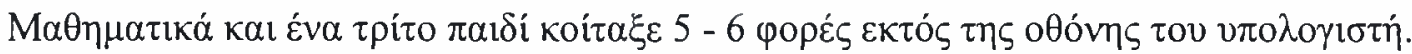

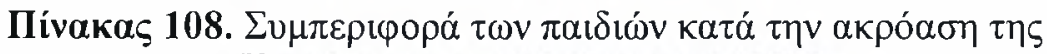

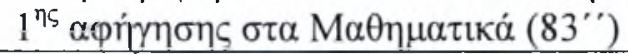

\begin{tabular}{|c|c|c|c|c|}
\hline \multirow{3}{*}{ 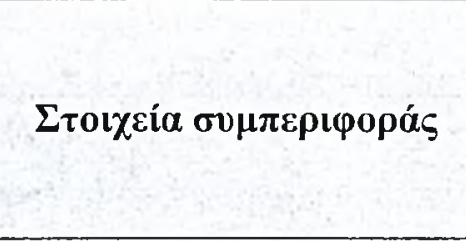 } & \multicolumn{4}{|c|}{ 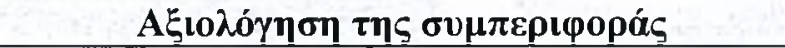 } \\
\hline & \multicolumn{2}{|c|}{ 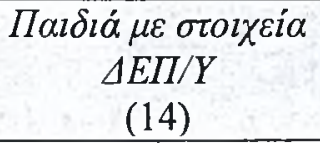 } & \multicolumn{2}{|c|}{ 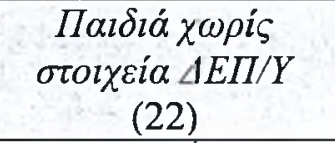 } \\
\hline & M.O. & T.A. & M.O. & T.A. \\
\hline 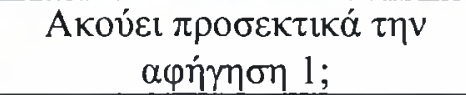 & 2,00 & 0,68 & 2,91 & 0,29 \\
\hline 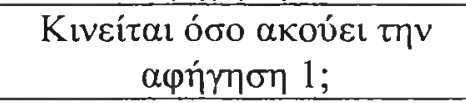 & 1,64 & 0,63 & 1,00 & 0,00 \\
\hline 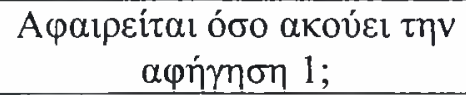 & 1,93 & 0,62 & 1,18 & 0,39 \\
\hline
\end{tabular}

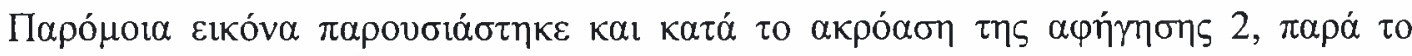

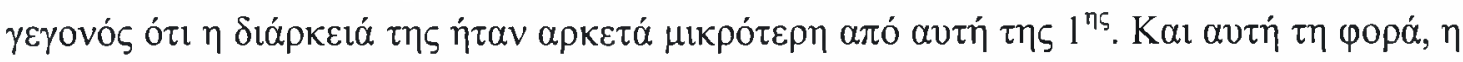

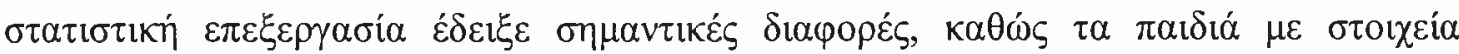

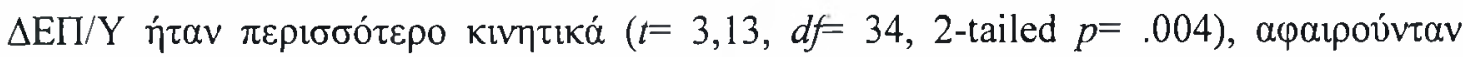

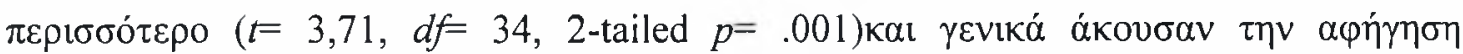




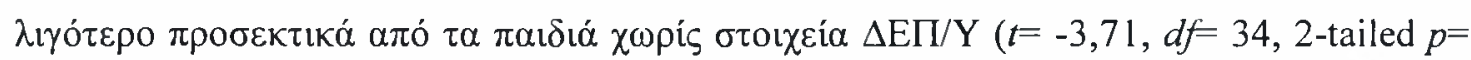

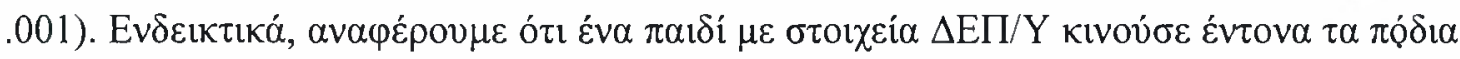

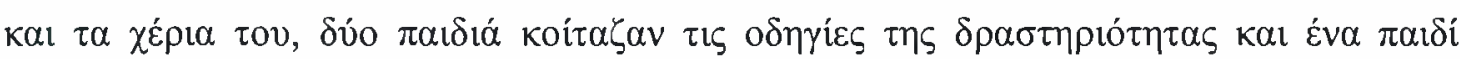

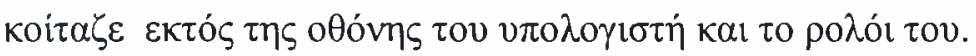

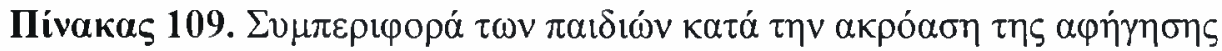

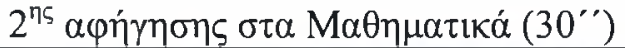

\begin{tabular}{|c|c|c|c|c|}
\hline \multirow{3}{*}{ 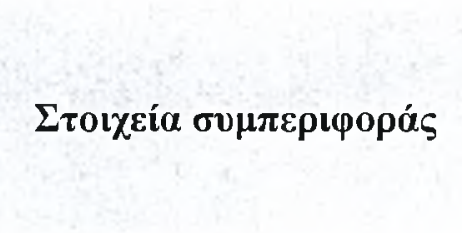 } & \multicolumn{4}{|c|}{ 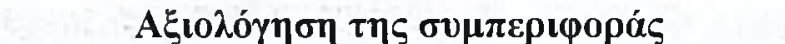 } \\
\hline & \multicolumn{2}{|c|}{ 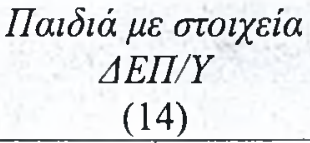 } & \multicolumn{2}{|c|}{ 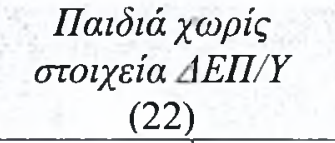 } \\
\hline & M.O. & T.A. & M.O. & T.A. \\
\hline 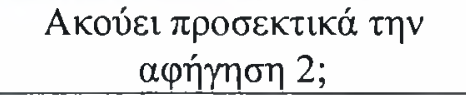 & 2,21 & 0,80 & 2,91 & 0,29 \\
\hline 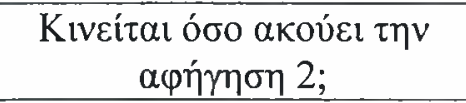 & 1,64 & 0,74 & 1,09 & 0,00 \\
\hline 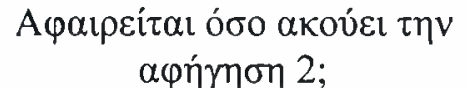 & 1,79 & 0,80 & 1,09 & 0,29 \\
\hline
\end{tabular}

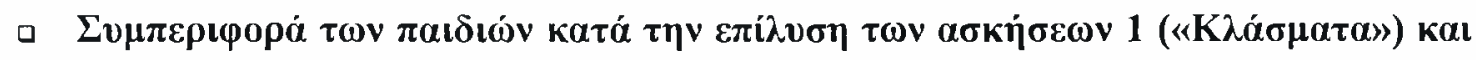

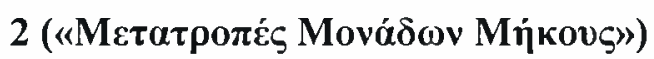

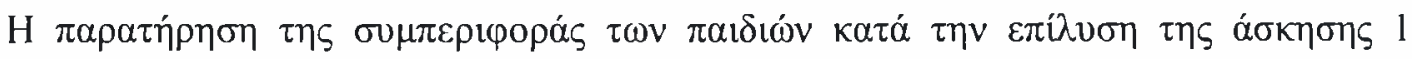

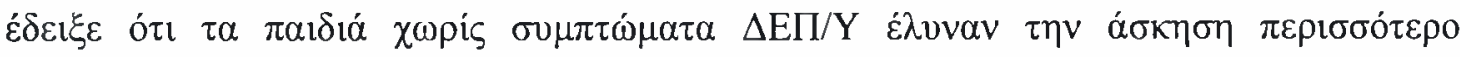

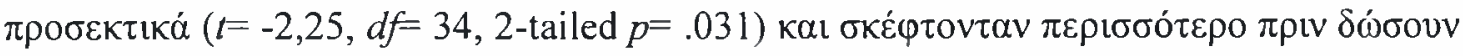

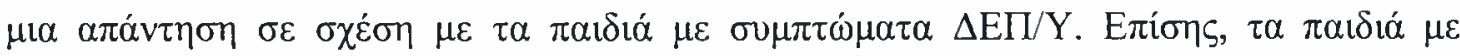

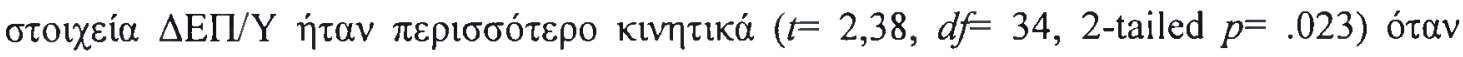

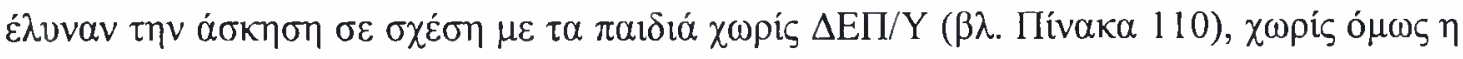

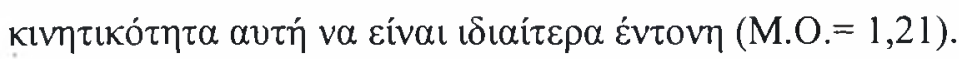

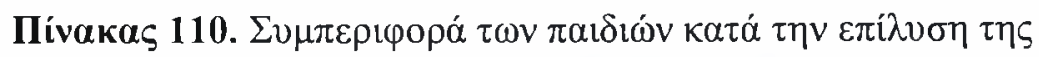

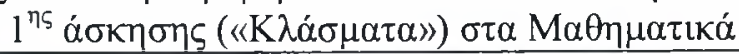

\begin{tabular}{|c|c|c|c|}
\hline \multirow{3}{*}{ 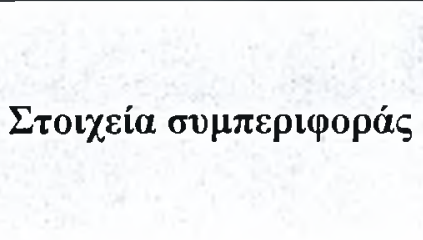 } & \multicolumn{3}{|c|}{ 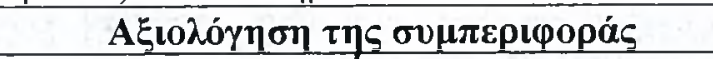 } \\
\hline & $\begin{array}{c}\Pi \alpha_{l} \delta l \alpha \dot{\alpha} \mu \varepsilon \sigma \tau o l \chi \varepsilon i \alpha \\
\Delta E \Pi / Y \\
(14)\end{array}$ & $\begin{array}{c}\Pi \alpha \\
\sigma \tau o t\end{array}$ & $\begin{array}{l}\rho i s \\
M / Y\end{array}$ \\
\hline & M.O. & M.O. & T.A. \\
\hline
\end{tabular}




\begin{tabular}{|c|c|c|c|c|}
\hline 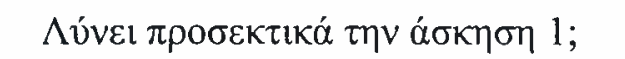 & 2,43 & 0,65 & 2,82 & 0,39 \\
\hline 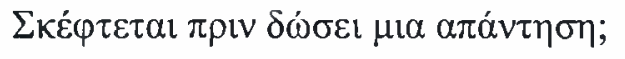 & 2,43 & 0,65 & 2,73 & 0,46 \\
\hline 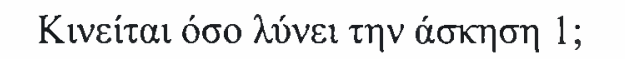 & 1,21 & 0,43 & 1,00 & 0,00 \\
\hline 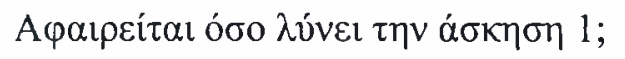 & 1,14 & 0,53 & 1,00 & 0,00 \\
\hline
\end{tabular}

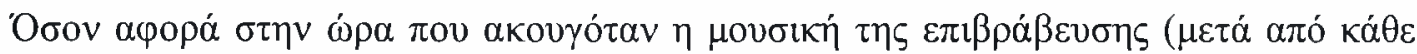

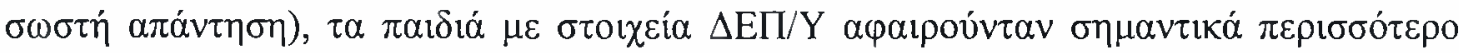

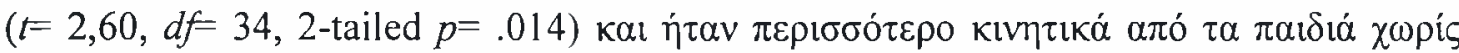

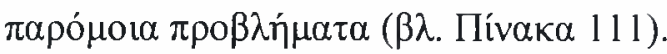

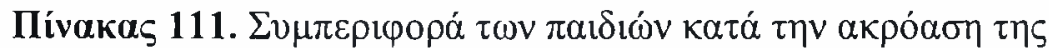

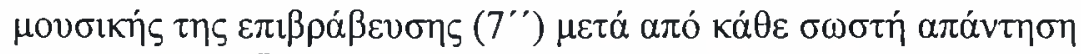

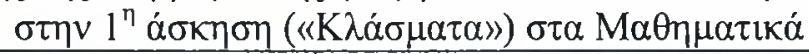

\begin{tabular}{|c|c|c|c|c|}
\hline \multirow{3}{*}{ 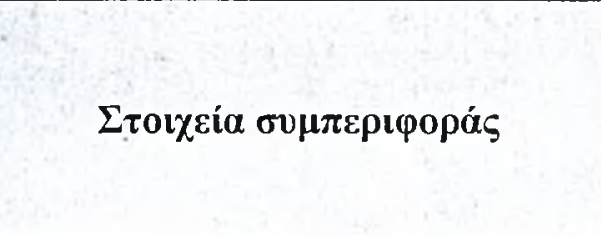 } & \multicolumn{4}{|c|}{ 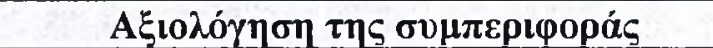 } \\
\hline & \multicolumn{2}{|c|}{ 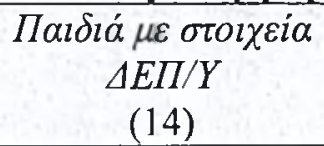 } & \multicolumn{2}{|c|}{ 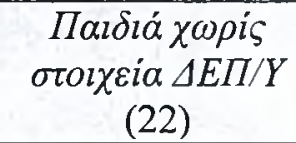 } \\
\hline & M.O. & T.A. & M.O. & T.A. \\
\hline 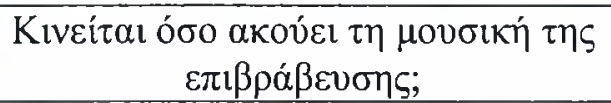 & 1,50 & 0,65 & 1,27 & 0,46 \\
\hline 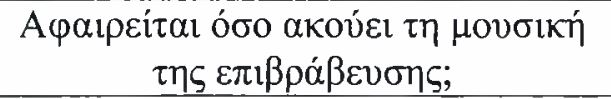 & 1,36 & 0,50 & 1,05 & 0,21 \\
\hline
\end{tabular}

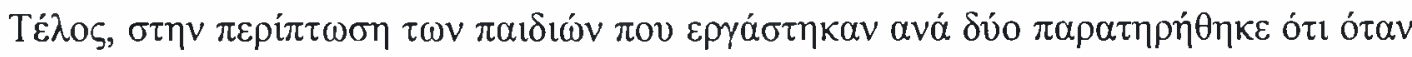

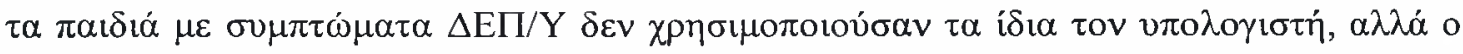

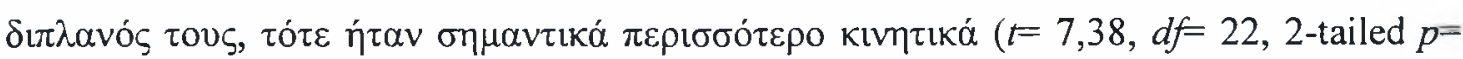

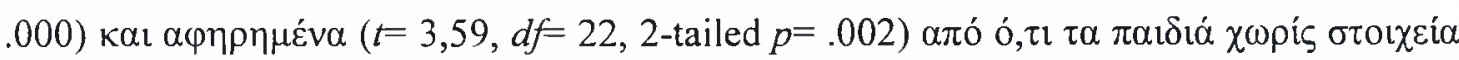

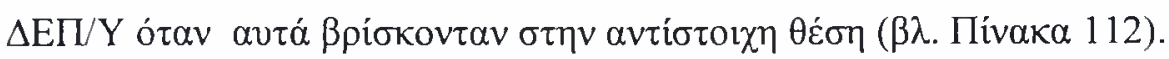




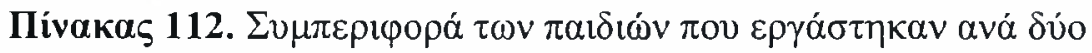

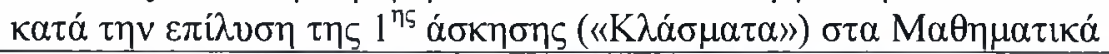

\begin{tabular}{|c|c|c|c|c|}
\hline \multirow{3}{*}{ 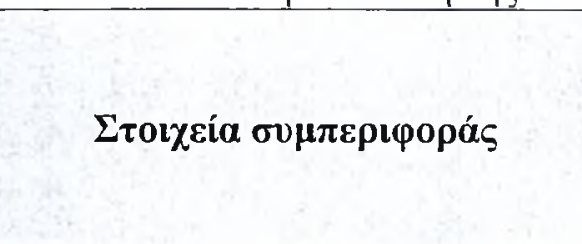 } & \multicolumn{4}{|c|}{ 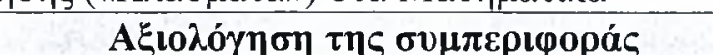 } \\
\hline & \multicolumn{2}{|c|}{ 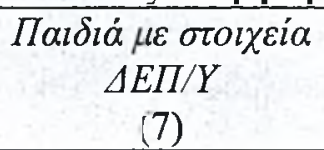 } & \multicolumn{2}{|c|}{ 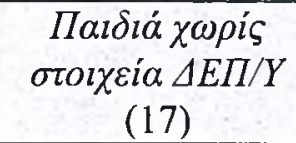 } \\
\hline & M.O. & T.A. & M.O. & T.A. \\
\hline 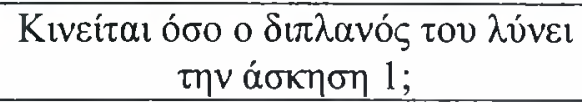 & 2,00 & 0,58 & 1,00 & 0,00 \\
\hline 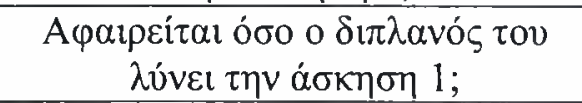 & 1,86 & 0,69 & 1,12 & 0,33 \\
\hline
\end{tabular}

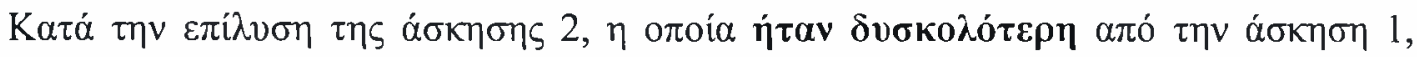

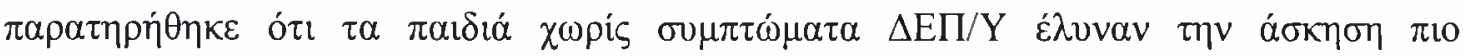

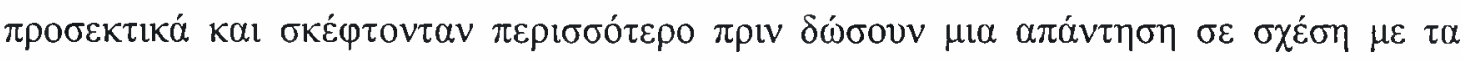

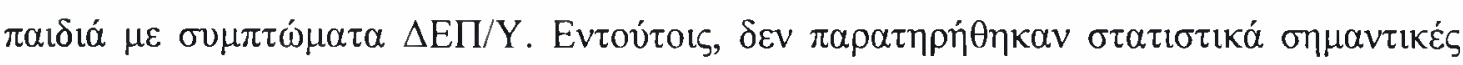

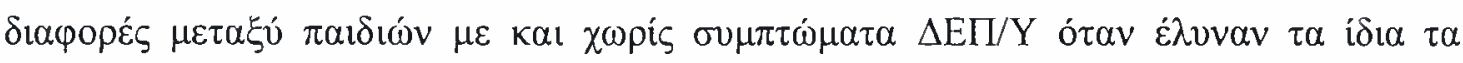

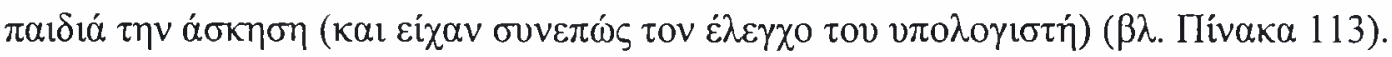

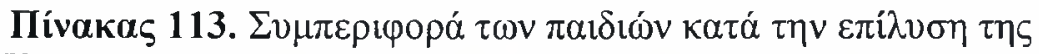

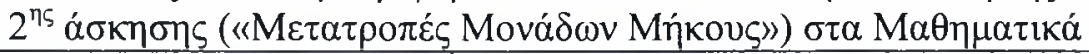

\begin{tabular}{|c|c|c|c|c|}
\hline \multirow{3}{*}{ 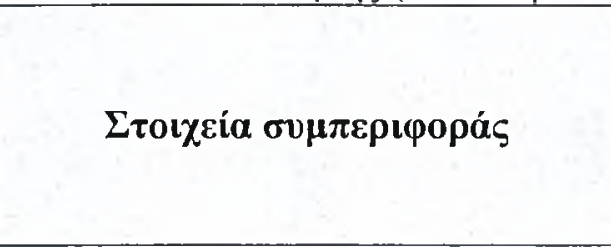 } & \multicolumn{4}{|c|}{ 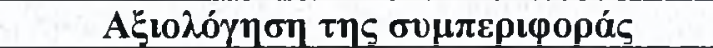 } \\
\hline & \multicolumn{2}{|c|}{ 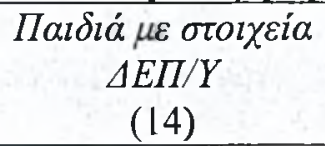 } & \multicolumn{2}{|c|}{ 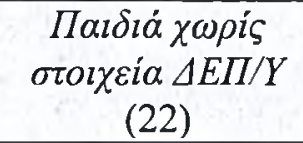 } \\
\hline & M.O. & T.A. & M.O. & T.A. \\
\hline 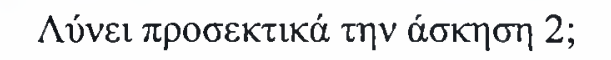 & 2,36 & 0,74 & 2,73 & 0,46 \\
\hline 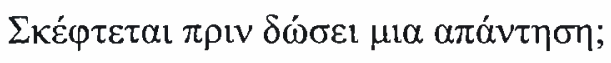 & 2,36 & 0,74 & 2,68 & 0,48 \\
\hline 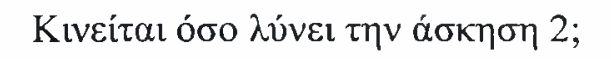 & 1,14 & 0,36 & 1,00 & 0,00 \\
\hline 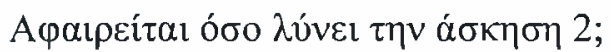 & 1,00 & 0,00 & 1,00 & 0,00 \\
\hline
\end{tabular}

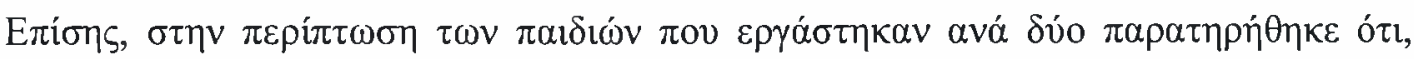

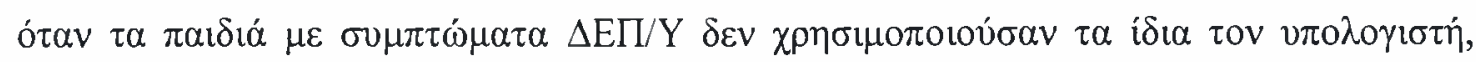

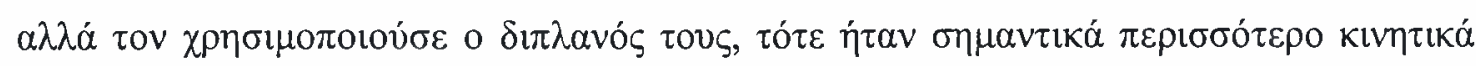

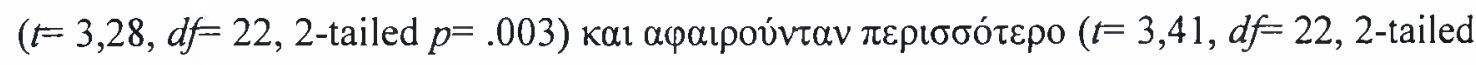




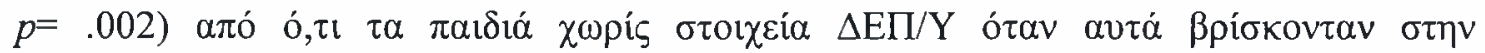

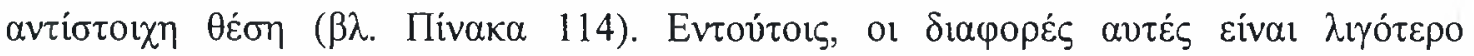

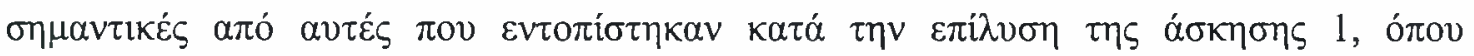

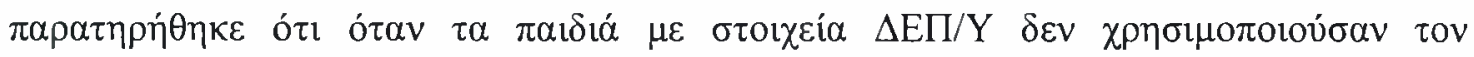

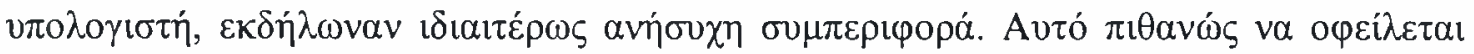

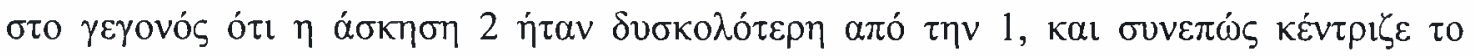

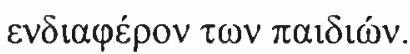

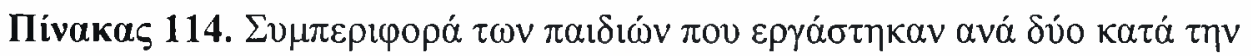

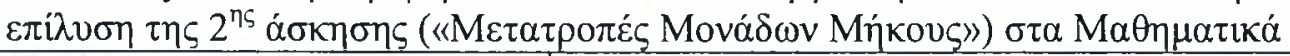

\begin{tabular}{|c|c|c|c|c|}
\hline \multirow{3}{*}{ 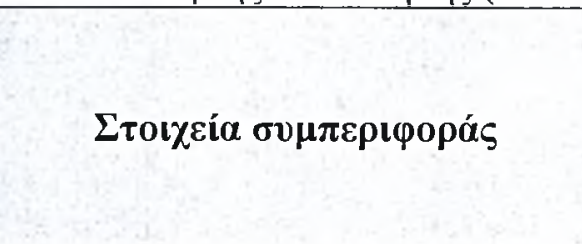 } & \multicolumn{4}{|c|}{ 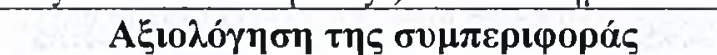 } \\
\hline & \multicolumn{2}{|c|}{ 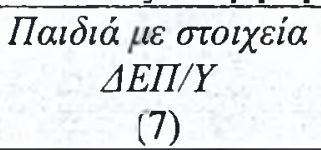 } & \multicolumn{2}{|c|}{ 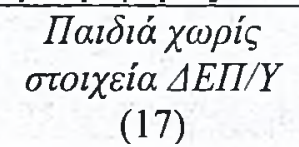 } \\
\hline & M.O. & T.A. & M.O. & T.A. \\
\hline 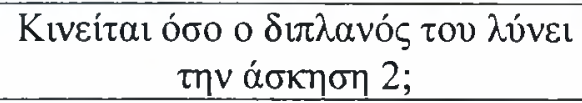 & 1,57 & 0,53 & 1,06 & 0,24 \\
\hline 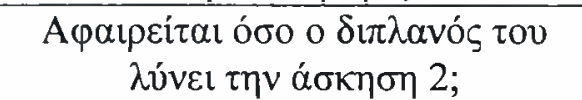 & 1,43 & 0,53 & 1,00 & 0,00 \\
\hline
\end{tabular}

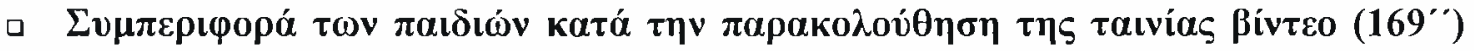 $\sigma \tau \alpha \mathrm{M \alpha \theta \eta \mu \alpha \tau \iota \kappa \alpha ́}$}

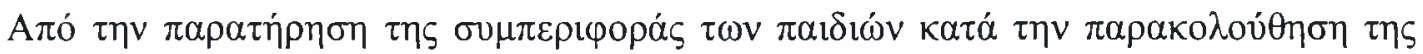

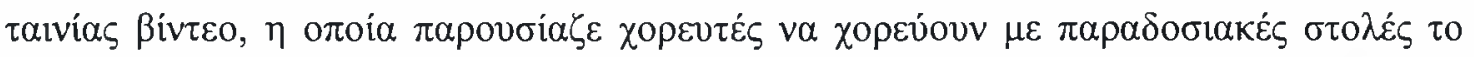

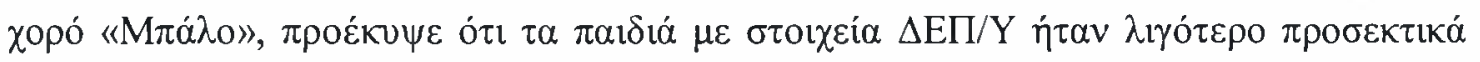

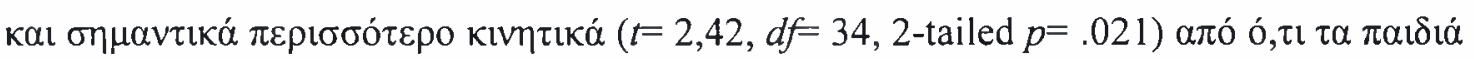

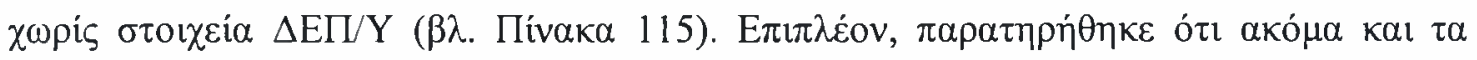

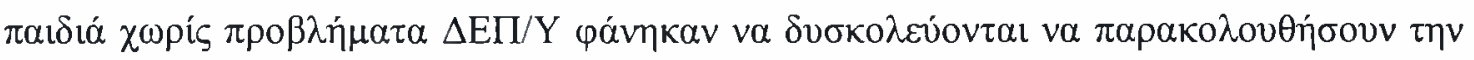

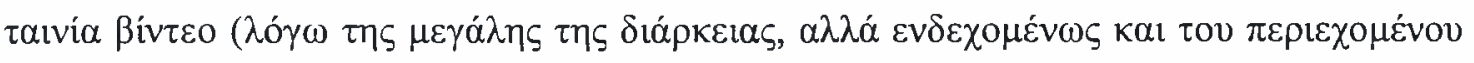
$\tau \eta \varsigma)$. 


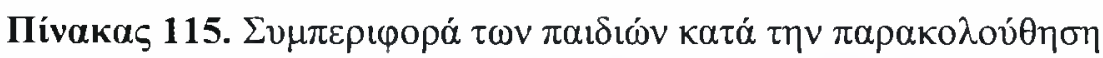

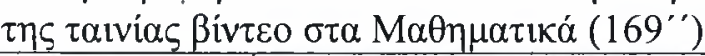

\begin{tabular}{|c|c|c|c|c|}
\hline \multirow{3}{*}{ 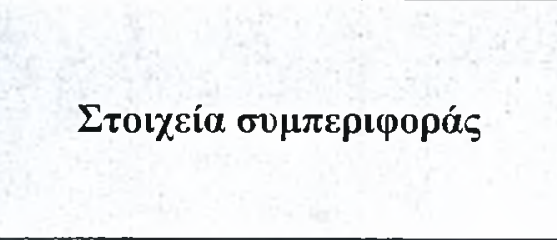 } & \multicolumn{4}{|c|}{ 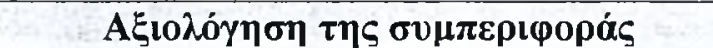 } \\
\hline & \multicolumn{2}{|c|}{ 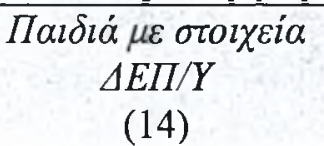 } & \multicolumn{2}{|c|}{ 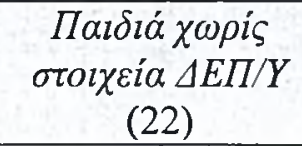 } \\
\hline & M.O. & T.A. & M.O. & T.A. \\
\hline 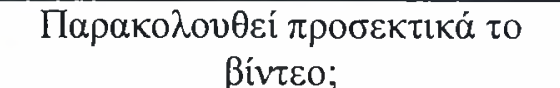 & 1,57 & 0,65 & 1,91 & 0,53 \\
\hline 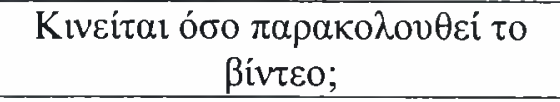 & 2,14 & 0,66 & 1,68 & 0,48 \\
\hline 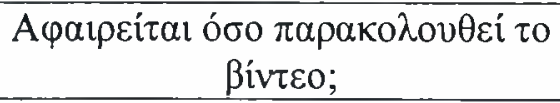 & 2,36 & 0,63 & 2,09 & 0,61 \\
\hline
\end{tabular}

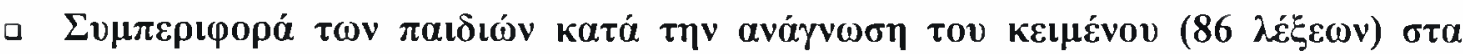

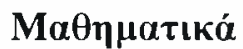

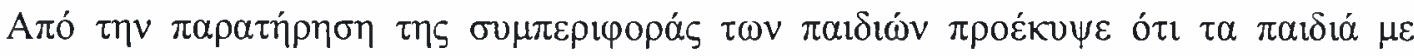

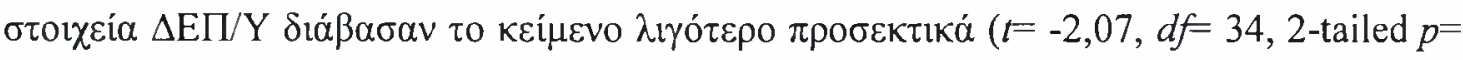

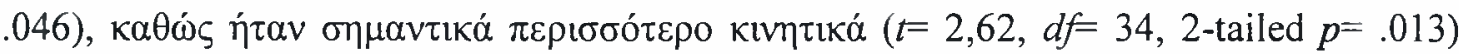

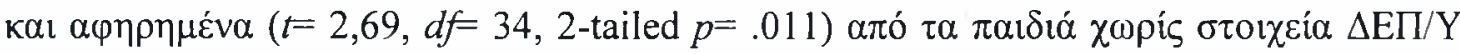

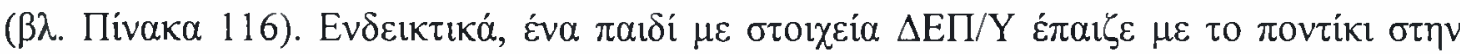

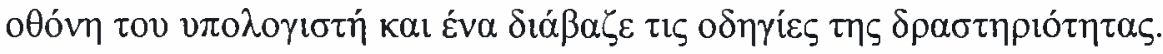

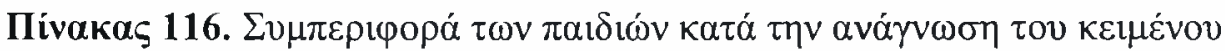

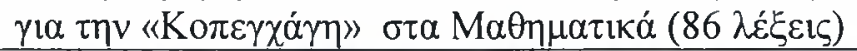

\begin{tabular}{|c|c|c|c|c|}
\hline \multirow{3}{*}{ 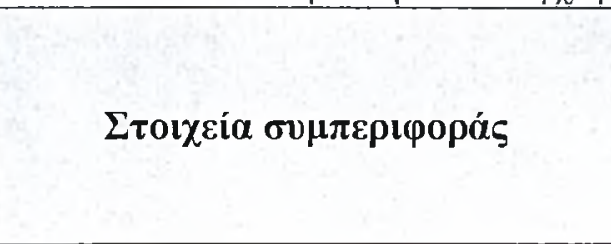 } & \multicolumn{4}{|c|}{ 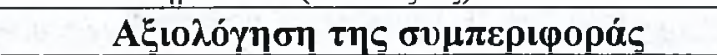 } \\
\hline & \multicolumn{2}{|c|}{ 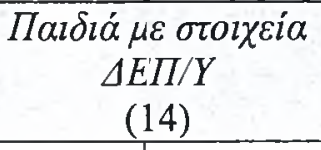 } & \multicolumn{2}{|c|}{ 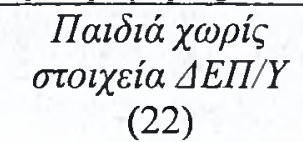 } \\
\hline & M.O. & T.A. & M.O. & T.A. \\
\hline 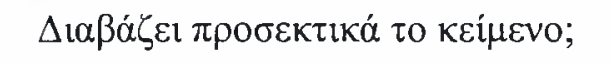 & 2,29 & 0,73 & 2,73 & 0,55 \\
\hline 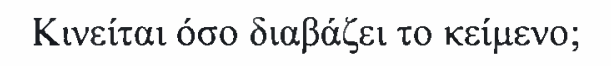 & 1,57 & 0,65 & 1,14 & 0,35 \\
\hline 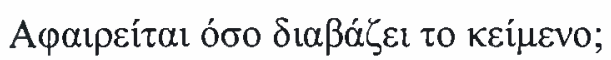 & 1,57 & 0,76 & 1,09 & 0,29 \\
\hline
\end{tabular}




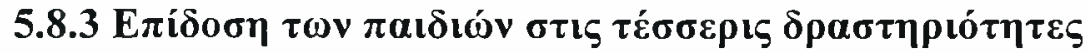

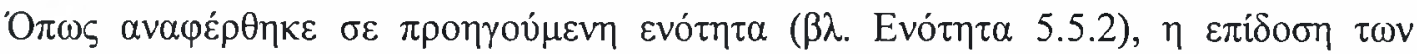

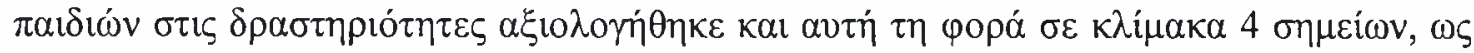

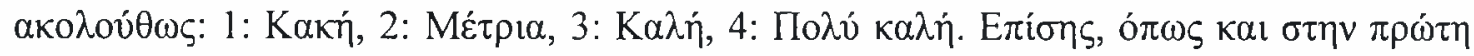

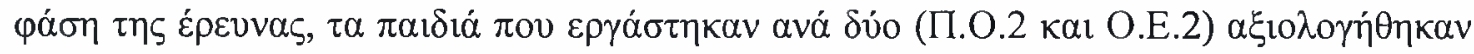

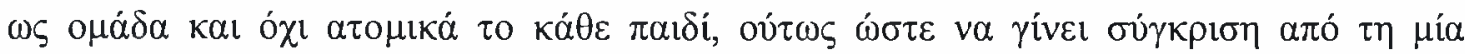

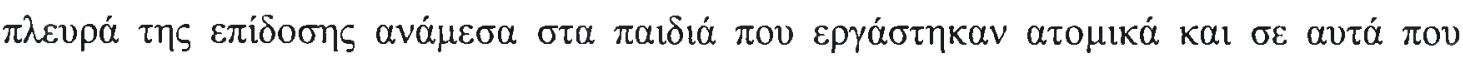

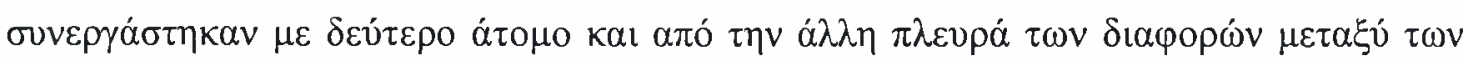

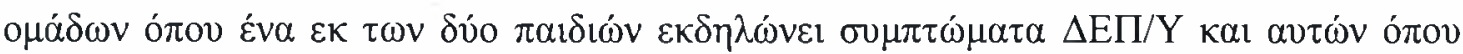

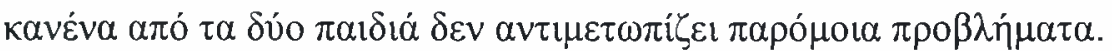

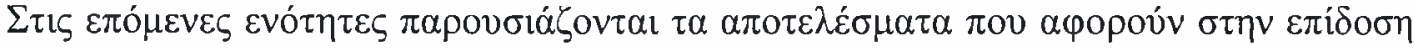

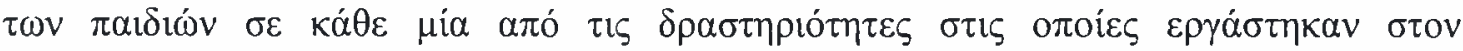

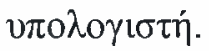

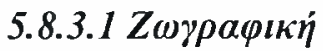

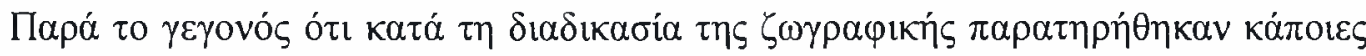

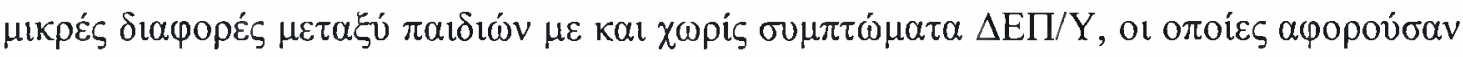

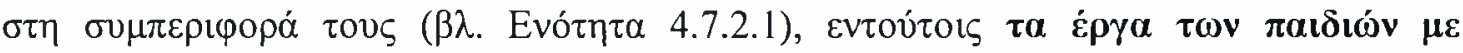

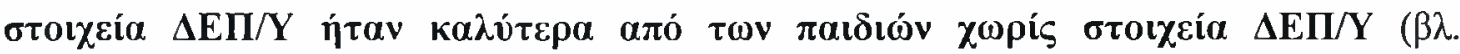

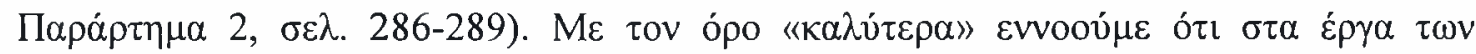

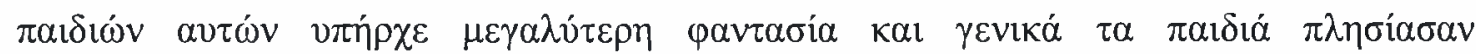

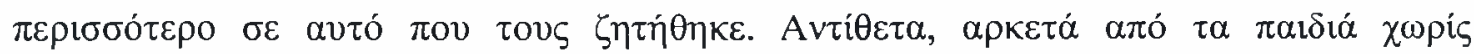

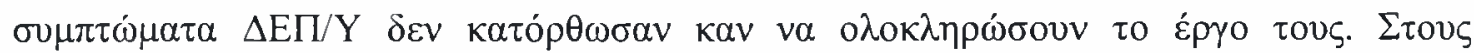

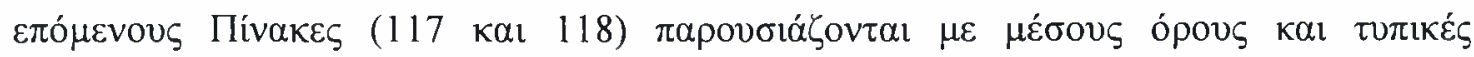

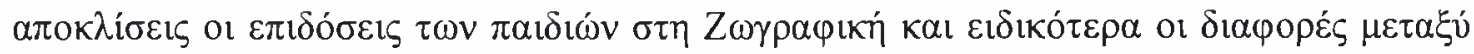

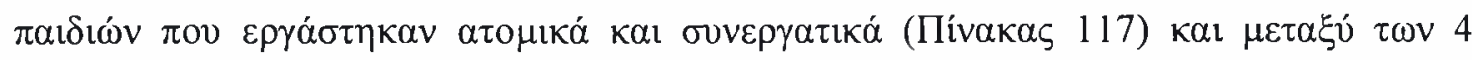

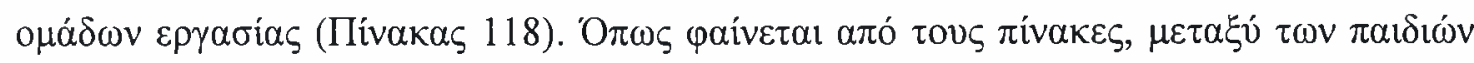

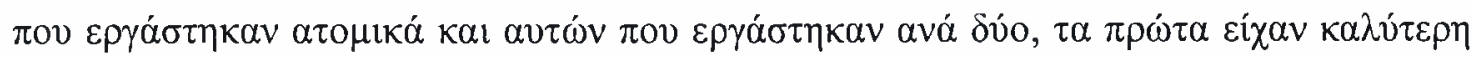

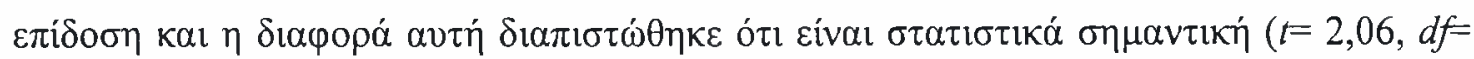




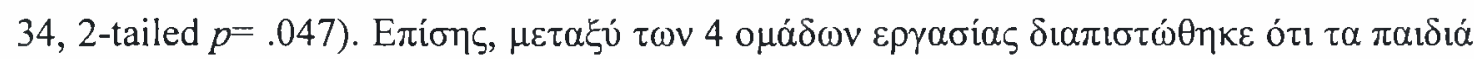

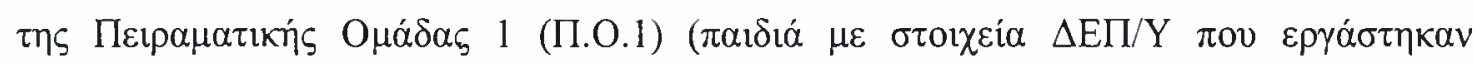

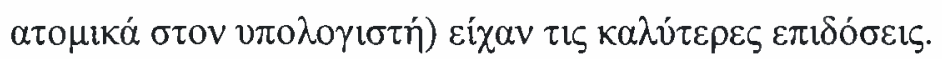

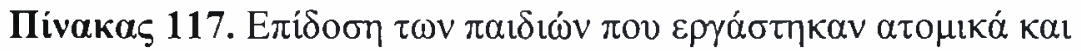

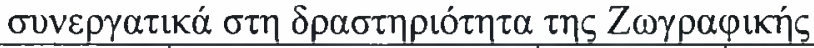

\begin{tabular}{|c|c|c|c|c|}
\hline & 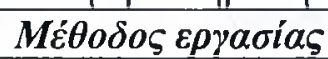 & $N(=36)$ & M.O. & T.A. \\
\hline \multirow{2}{*}{ 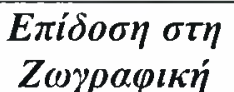 } & ATOMIKH & 12 & 2,67 & 0,65 \\
\hline & OMA $\triangle \mathrm{IKH}$ & 24 & 2,17 & 0,70 \\
\hline
\end{tabular}

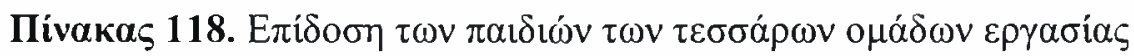

\begin{tabular}{|c|c|c|c|c|}
\hline & 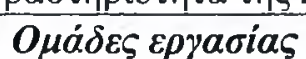 & $N(=36)$ & M.O. & T. $A$. \\
\hline \multirow{4}{*}{$\begin{array}{c}E \pi i \delta o \sigma \eta \sigma \tau \eta \\
Z \omega \gamma \rho \alpha \varphi \iota \kappa \eta\end{array}$} & П.0.1 & 7 & 2,86 & 0,69 \\
\hline & П.0.2 & 14 & 2,29 & 0,91 \\
\hline & O.E.1 & 5 & 2,40 & 0,55 \\
\hline & O.E.2 & 10 & 2,00 & 0,00 \\
\hline
\end{tabular}

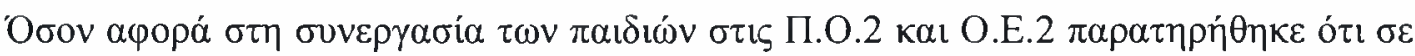

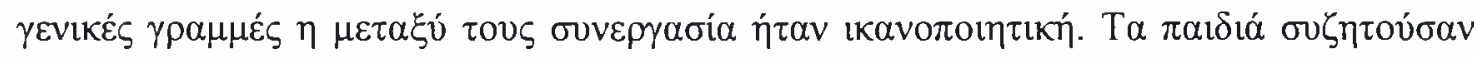

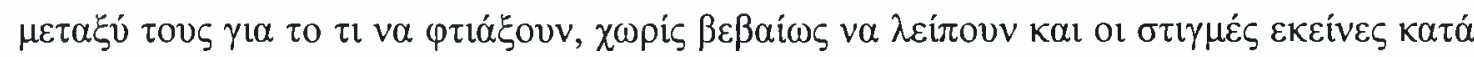

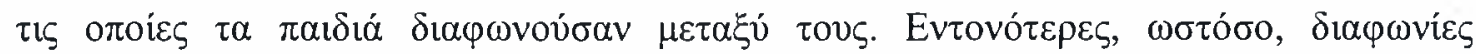

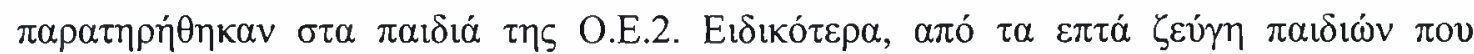

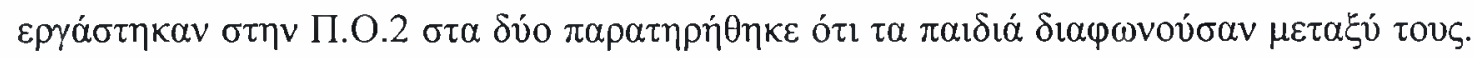

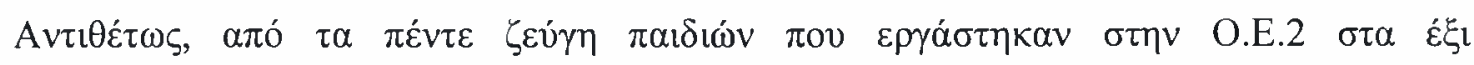

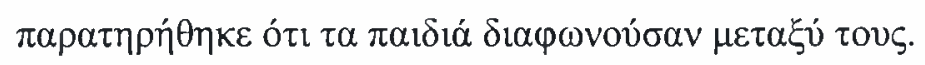

\subsubsection{Iбтopia}

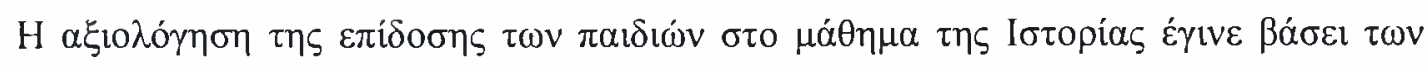

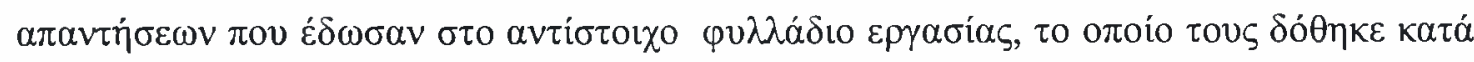

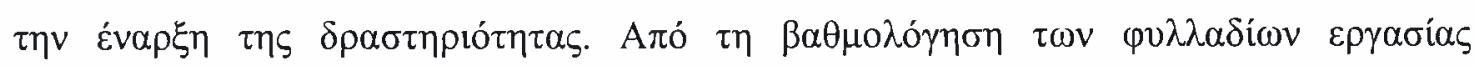

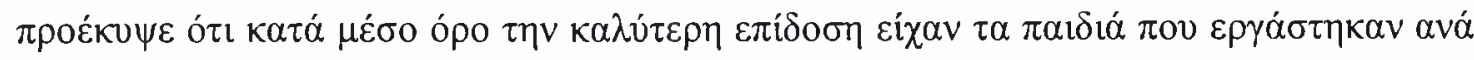

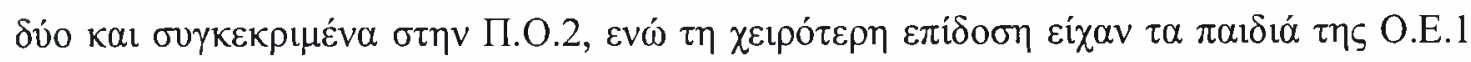

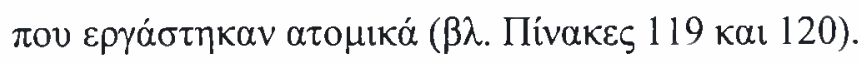




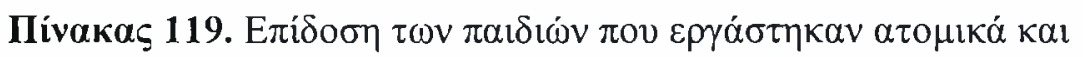

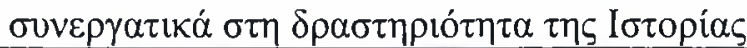

\begin{tabular}{|c|c|c|c|c|}
\hline & 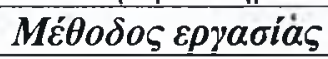 & $N(=36)$ & M.O. & T.A. \\
\hline \multirow{2}{*}{ 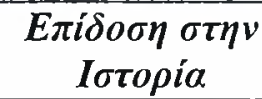 } & ATOMIKH & 12 & 2,83 & 1,03 \\
\hline & $\mathrm{OMA} \triangle \mathrm{IKH}$ & 24 & 3,42 & 0,78 \\
\hline
\end{tabular}

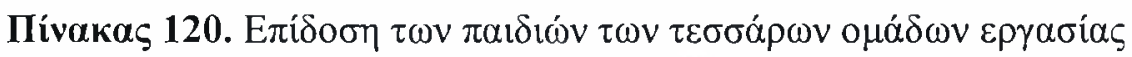
$\sigma \tau \eta \delta \rho \alpha \sigma \tau \eta \rho t o ́ \tau \eta \tau \alpha \tau \eta \zeta ~ l \sigma \tau o \rho i ́ \alpha s$

\begin{tabular}{|c|c|c|c|c|}
\hline & 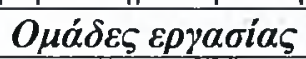 & $N(=36)$ & M.O. & T.A. \\
\hline \multirow{4}{*}{ 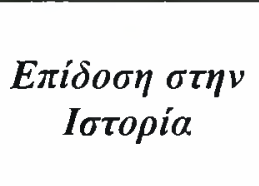 } & ПI.0.1 & 7 & 3,14 & 0,90 \\
\hline & П.0.2 & 14 & 3,57 & 0,76 \\
\hline & O.E.1 & 5 & 2,40 & 1,14 \\
\hline & O.E.2 & 10 & 3,20 & 0,79 \\
\hline
\end{tabular}

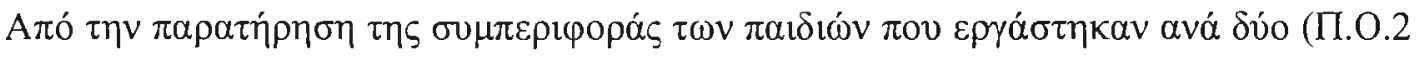

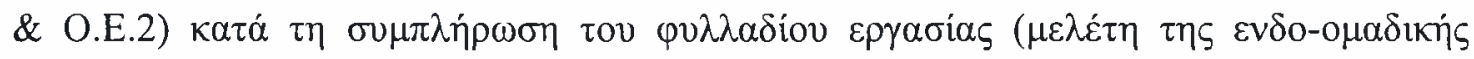

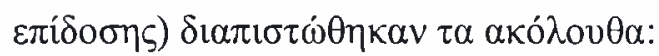

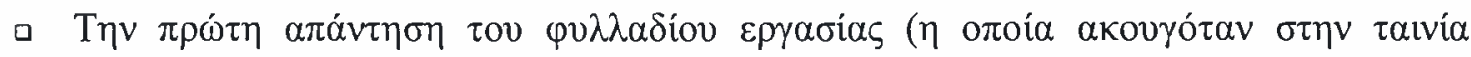

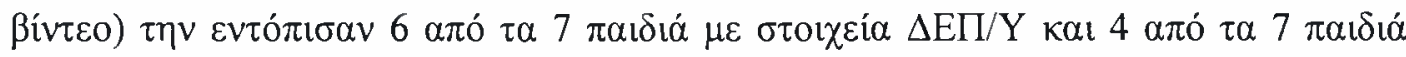

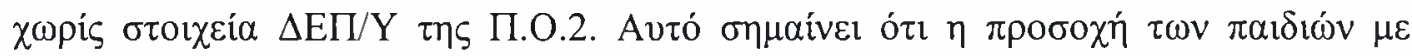

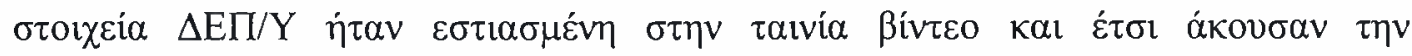

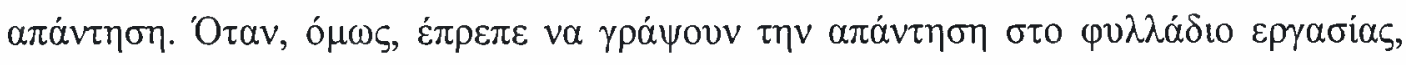

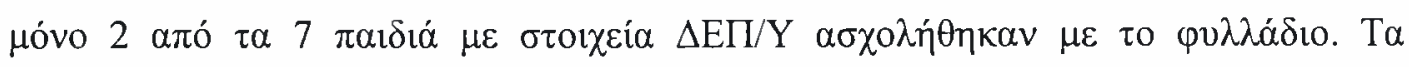

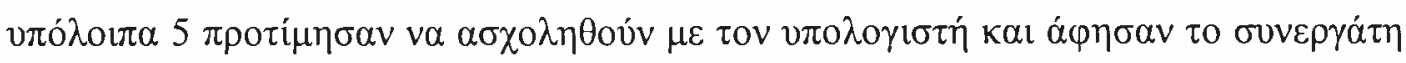

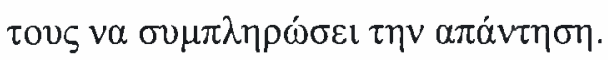

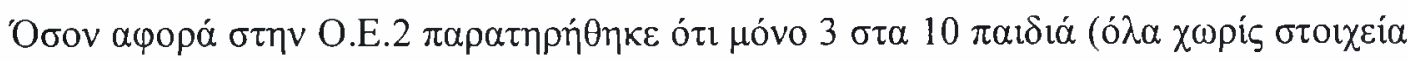

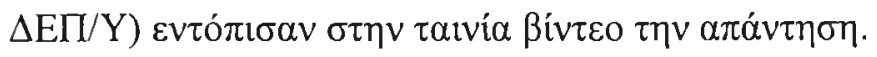

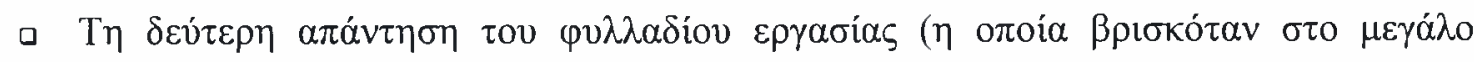

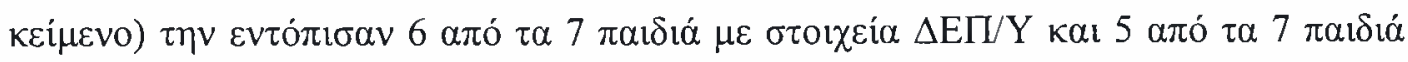

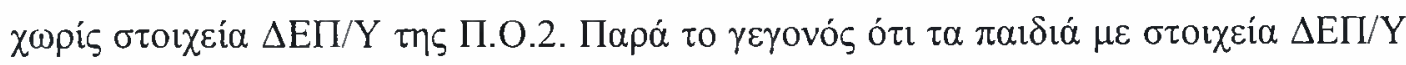

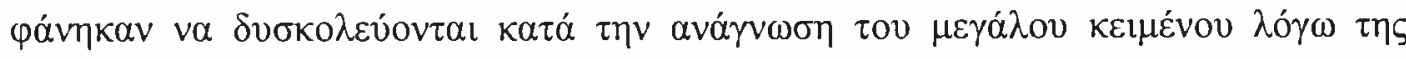

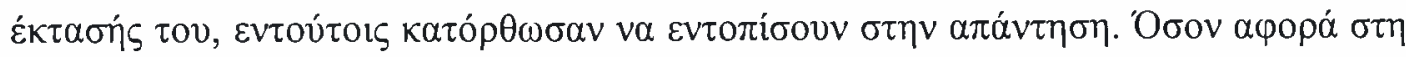

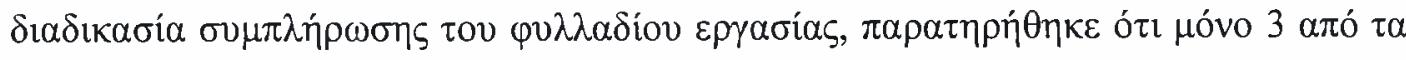

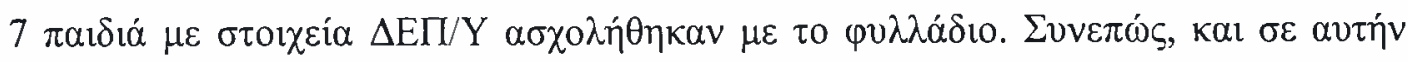




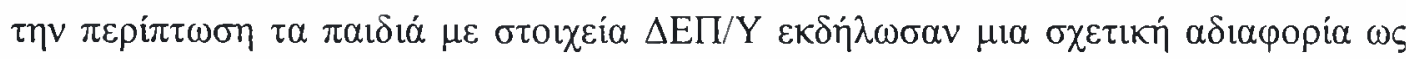

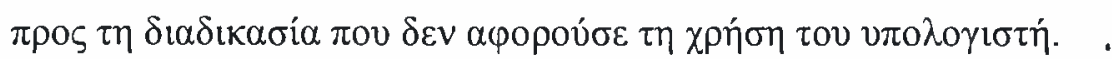

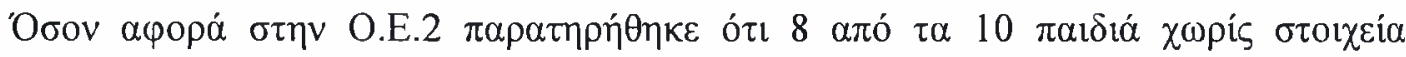

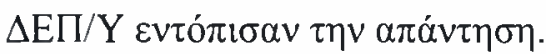

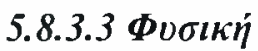

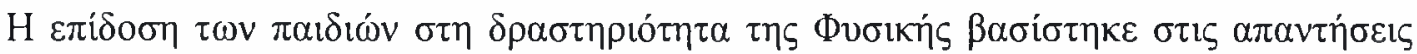

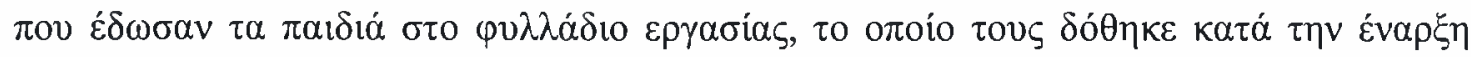

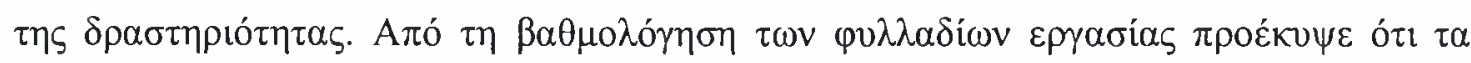

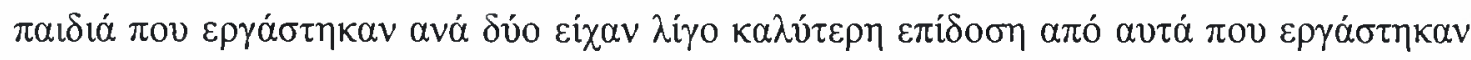

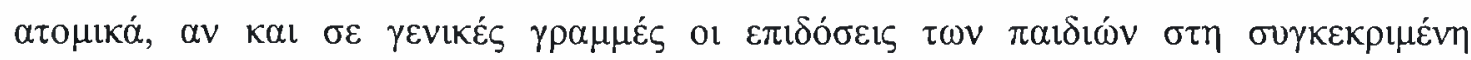

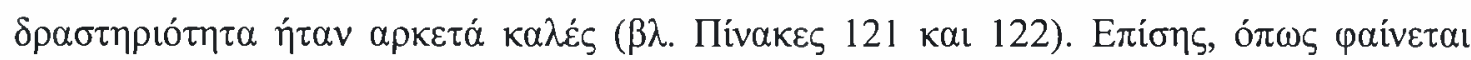

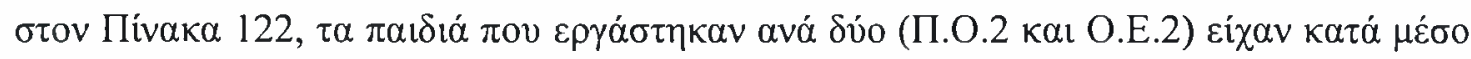

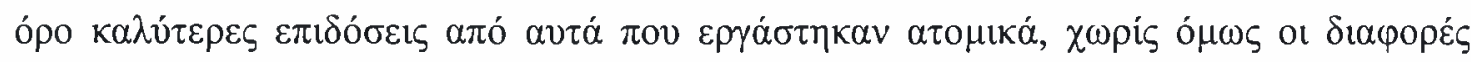

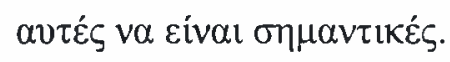

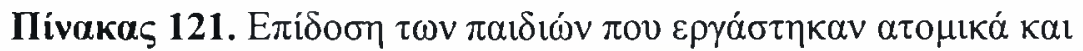

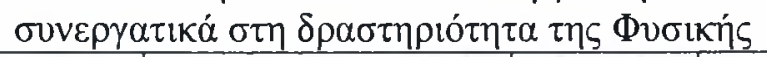

\begin{tabular}{|c|c|c|c|c|}
\hline & 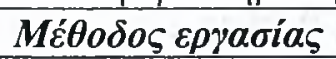 & $N(=36)$ & M.O. & T.A. \\
\hline \multirow{2}{*}{ 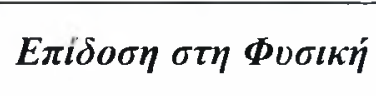 } & ATOMIKH & 12 & 3,25 & 0,75 \\
\hline & OMA $\triangle \mathrm{IKH}$ & 24 & 3,42 & 0,50 \\
\hline
\end{tabular}

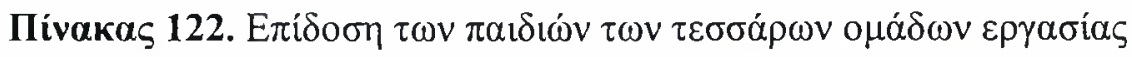

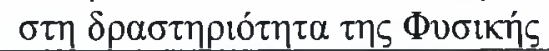

\begin{tabular}{|c|c|c|c|c|}
\hline & 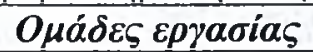 & $N(=36)$ & M.O. & T.A. \\
\hline \multirow{4}{*}{ 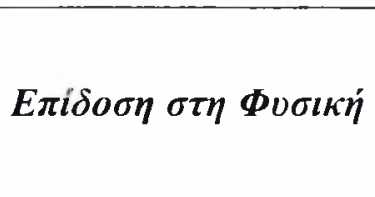 } & П.0.1 & 7 & 3,29 & 0,95 \\
\hline & П.0.2 & 14 & 3,43 & 0,51 \\
\hline & O.E.1 & 5 & 3,20 & 0,45 \\
\hline & O.E.2 & 10 & 3,40 & 0,52 \\
\hline
\end{tabular}

A

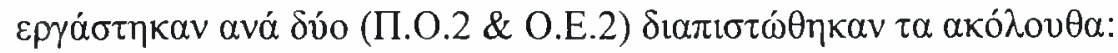

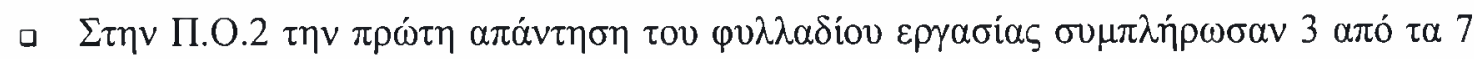

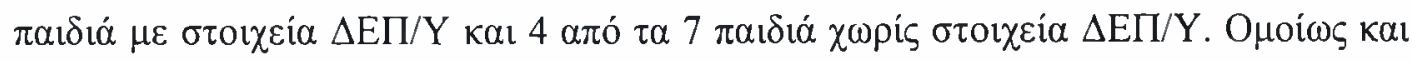




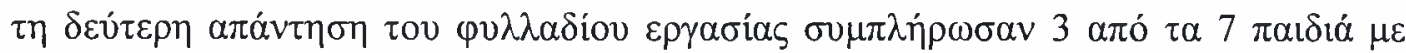

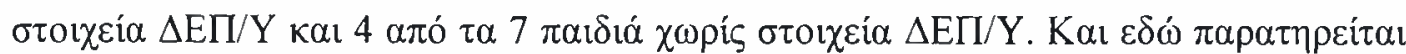

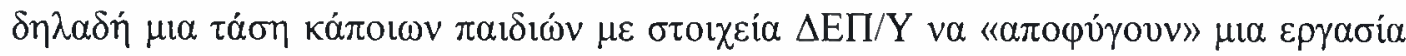

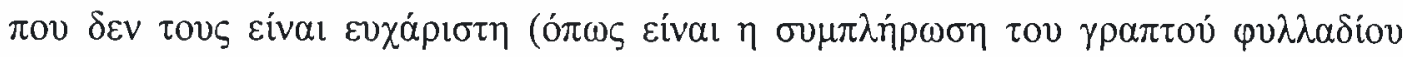
$\varepsilon \rho \gamma \alpha \sigma i \alpha \varsigma)$.

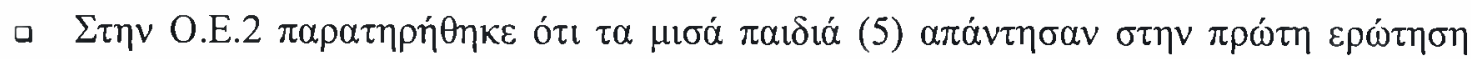

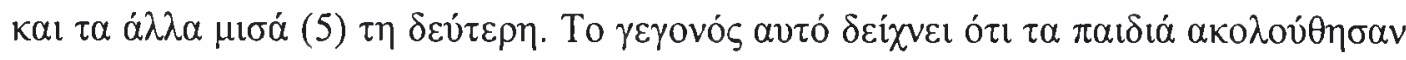

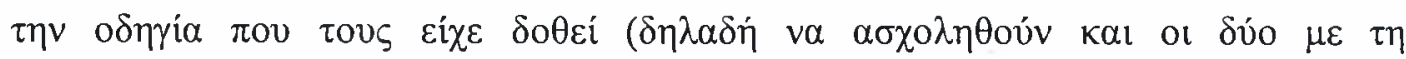

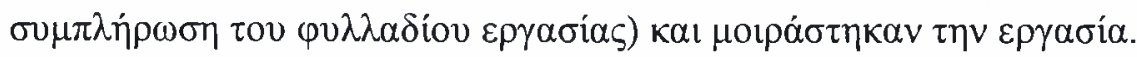

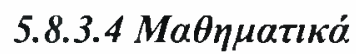

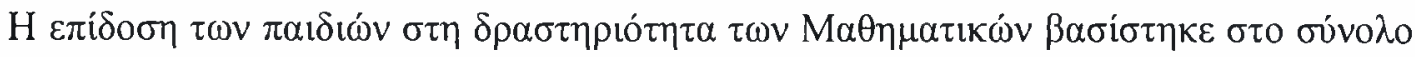

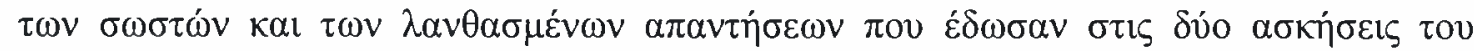

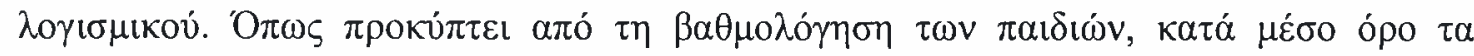

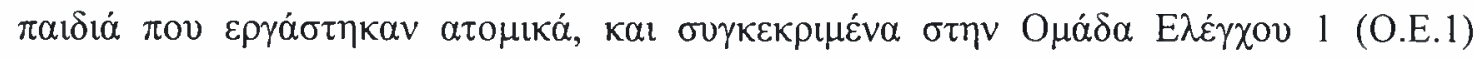

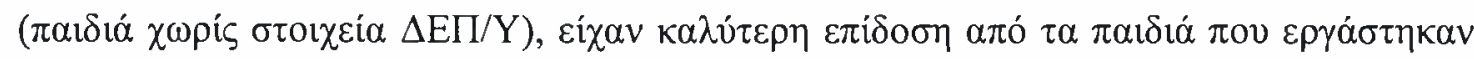

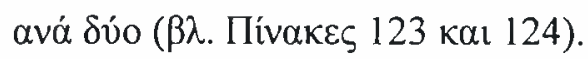

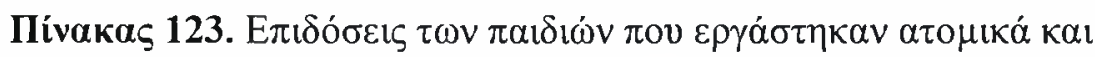

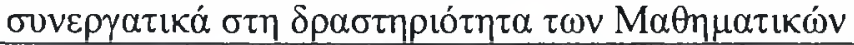

\begin{tabular}{|c|c|c|c|c|}
\hline & 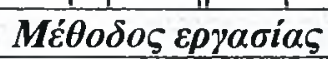 & $N(=36)$ & M.O. & T.A. \\
\hline \multirow{2}{*}{ 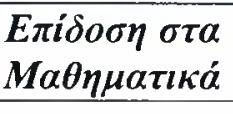 } & ATOMIKH & 12 & 2,75 & 0,75 \\
\hline & OMA $\triangle \mathrm{IKH}$ & 24 & 2,33 & 0,76 \\
\hline
\end{tabular}

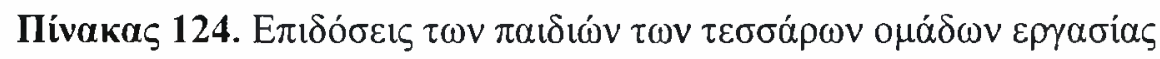

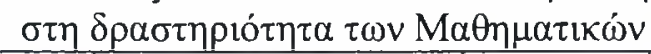

\begin{tabular}{|c|c|c|c|c|}
\hline & 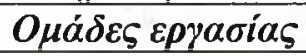 & $N(=36)$ & M.O. & T.A. \\
\hline \multirow{4}{*}{ 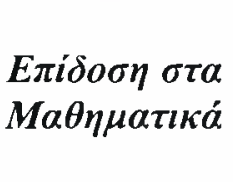 } & П.0.1 & 7 & 2,57 & 0,79 \\
\hline & II.0.2 & 14 & 2,29 & 0,47 \\
\hline & O.E.1 & 5 & 3,00 & 0,71 \\
\hline & O.E.2 & 10 & 2,40 & 1,07 \\
\hline
\end{tabular}

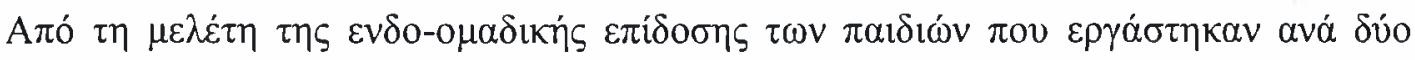

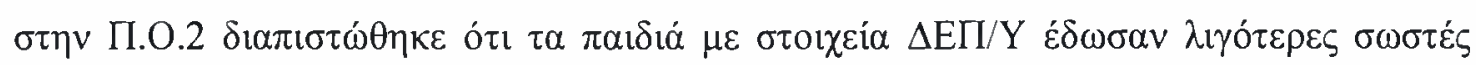




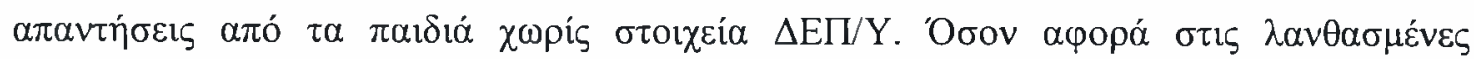

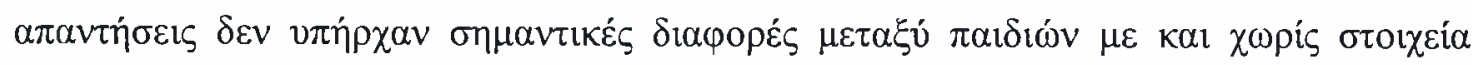

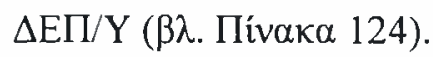

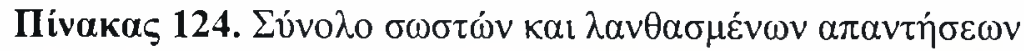

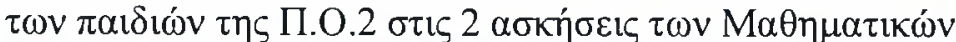

\begin{tabular}{|c|c|c|c|c|}
\hline & $\begin{array}{c}\text { Пaıdı́́ } \\
\mu \varepsilon \\
\Delta E \Pi / Y \\
\end{array}$ & $\begin{array}{c}N \\
(=14)\end{array}$ & 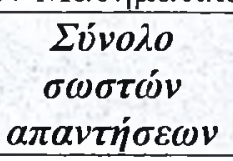 & 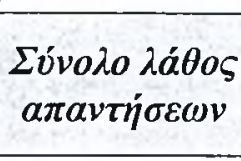 \\
\hline \multirow{2}{*}{ 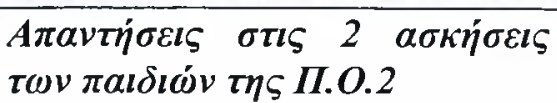 } & NAI & 7 & 42 & 45 \\
\hline & OXI & 7 & 58 & 47 \\
\hline
\end{tabular}

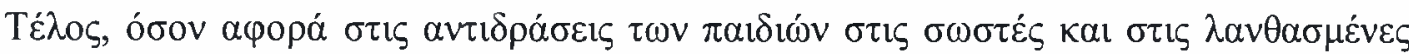

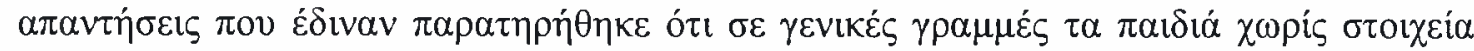

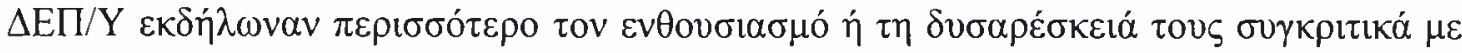

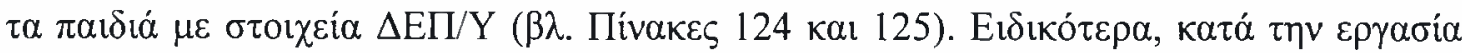

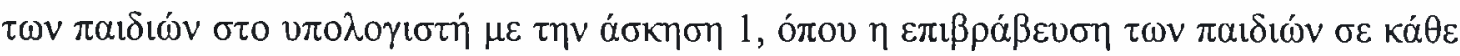

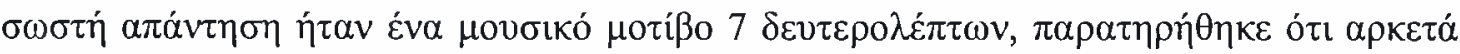

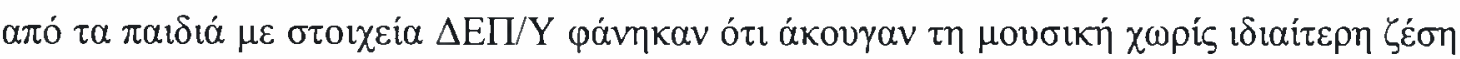

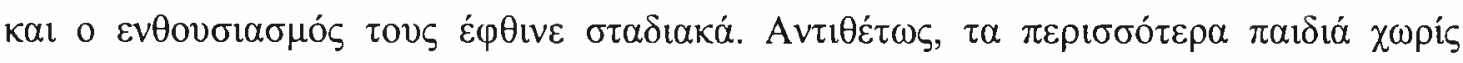

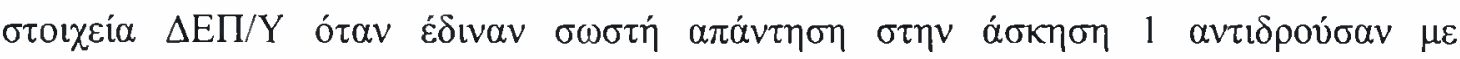

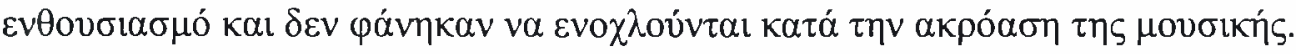

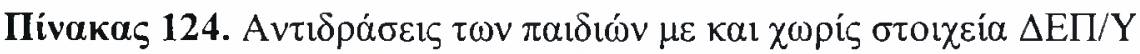

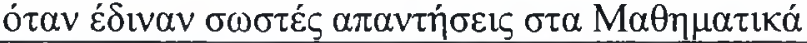

\begin{tabular}{|c|c|c|c|}
\hline & 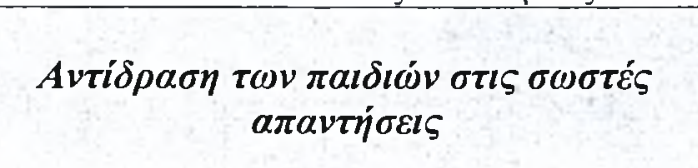 & $\begin{array}{c}\Pi a ı \delta ı \alpha ́ \mu \varepsilon \\
\Delta E \Pi / Y \\
(N=14)\end{array}$ & $\begin{array}{c}\text { Maısıá } \chi \omega \rho i ́ \varsigma \\
\Delta E \Pi / Y \\
(N=22)\end{array}$ \\
\hline \multirow{4}{*}{$A \sigma \kappa \eta \sigma \eta 1$} & 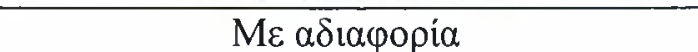 & 4 & 9 \\
\hline & 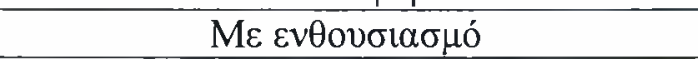 & 5 & 11 \\
\hline & 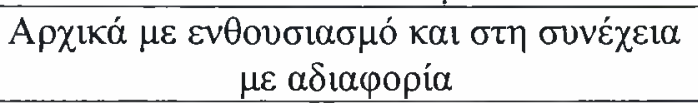 & 3 & 2 \\
\hline & 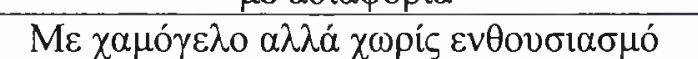 & 2 & \\
\hline
\end{tabular}




\begin{tabular}{|c|c|c|c|}
\hline \multirow{4}{*}{ 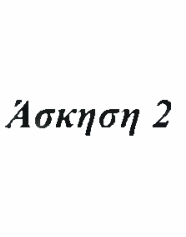 } & 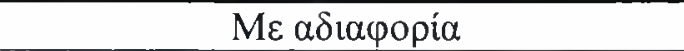 & 6 & 10 \\
\hline & 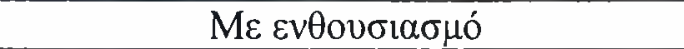 & 6 & 11 \\
\hline & 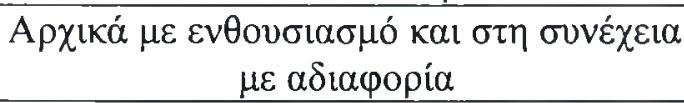 & 1 & 1 \\
\hline & 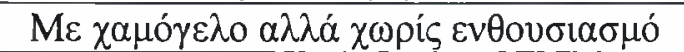 & 1 & \\
\hline
\end{tabular}

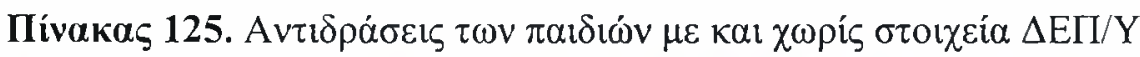

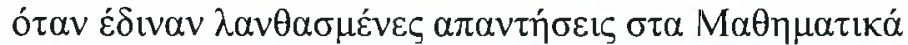

\begin{tabular}{|c|c|c|c|}
\hline & 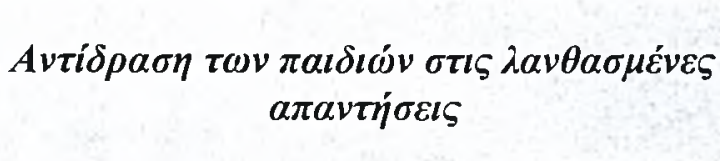 & $\begin{array}{c}\Pi \alpha \iota \delta \imath \alpha \dot{\alpha} \mu \varepsilon \\
\Delta E \Pi / Y \\
(N=14)\end{array}$ & 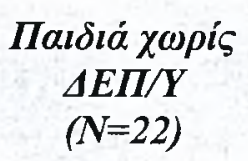 \\
\hline \multirow{4}{*}{$A \sigma \kappa \eta \sigma \eta 1$} & Me $\alpha \delta 1 \alpha \varphi \circ \rho i \alpha$ & 4 & 5 \\
\hline & $\mathrm{M \varepsilon} \delta v \sigma \alpha \rho \varepsilon ́ \sigma \kappa \varepsilon l \alpha$ & 6 & 7 \\
\hline & 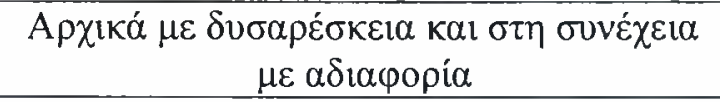 & 1 & 2 \\
\hline & $\Delta \varepsilon v \varepsilon \dot{\varepsilon} \delta \omega \sigma \alpha v \lambda \alpha \dot{\theta} \theta \circ \varsigma \alpha \pi \alpha v \tau \eta \dot{\sigma} \sigma \varepsilon \iota \zeta$ & 3 & 8 \\
\hline \multirow{4}{*}{ 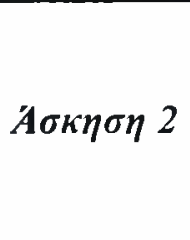 } & M $\varepsilon \alpha \delta 1 \alpha \varphi \circ \rho i \alpha$ & 4 & 7 \\
\hline & $\mathrm{M} \varepsilon \delta v \sigma \alpha \rho \varepsilon \dot{\sigma} \sigma \varepsilon \varepsilon \alpha$ & 9 & 14 \\
\hline & 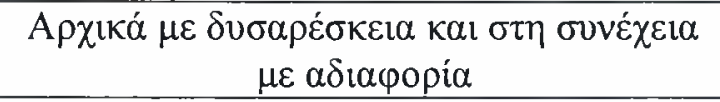 & 1 & \\
\hline & $\Delta \varepsilon v \varepsilon \dot{\varepsilon} \delta \omega \sigma \alpha \nu \lambda \alpha \dot{\alpha} \theta \circ \varsigma \alpha \pi \alpha v \tau \eta \dot{\sigma} \sigma \varepsilon l \varsigma$ & & 1 \\
\hline
\end{tabular}

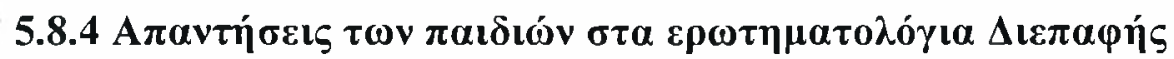

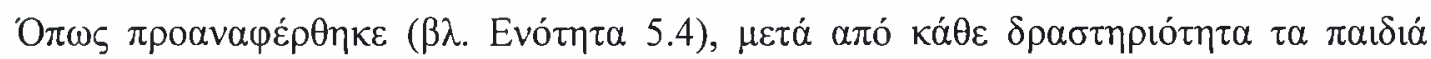

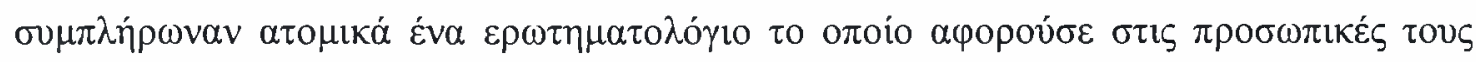

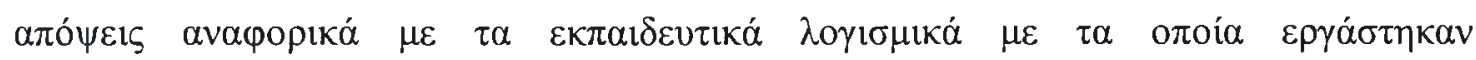

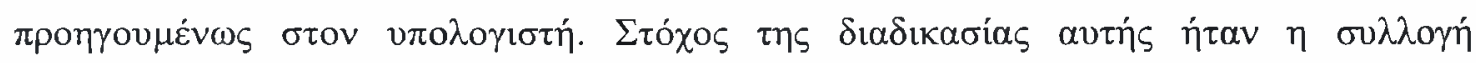

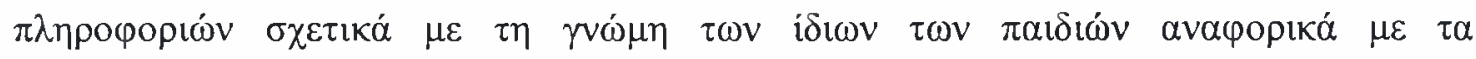

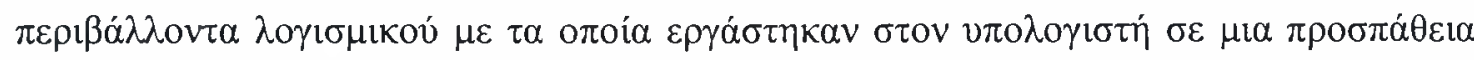

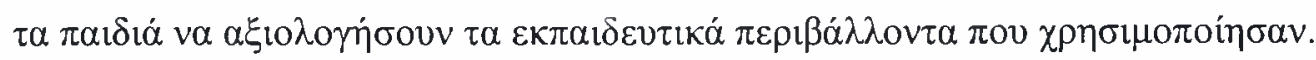

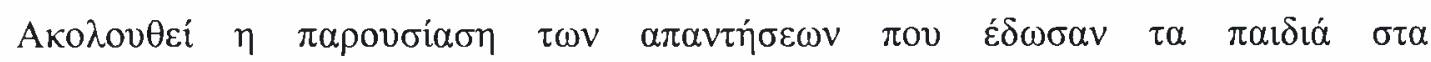
$\varepsilon \rho \omega \tau \eta \mu \alpha \tau o \lambda o ́ \gamma i \alpha \alpha v \tau \dot{\alpha}$. 


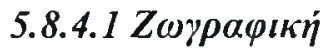

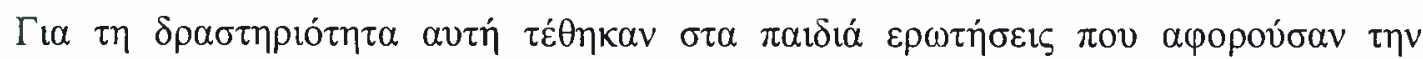

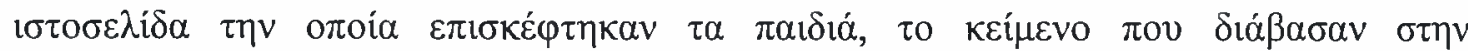

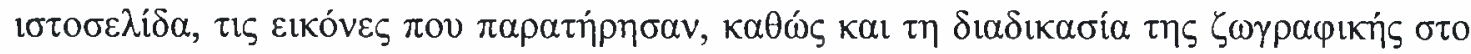
Paint.

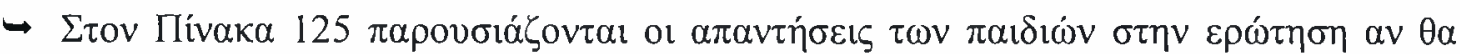

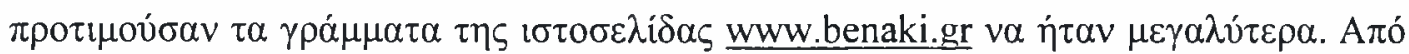

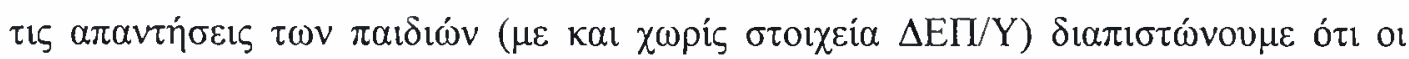

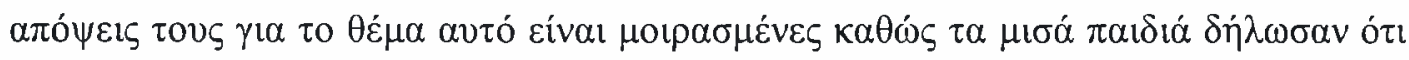

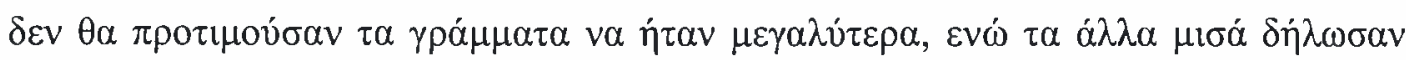

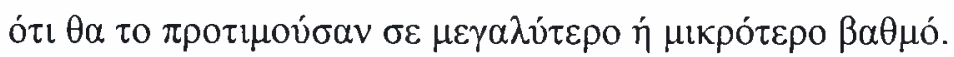

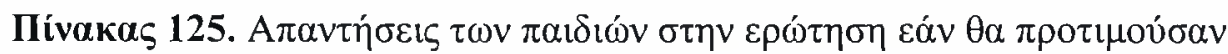

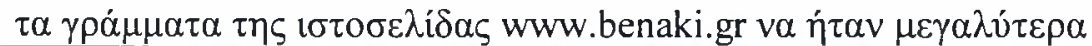

\begin{tabular}{|c|c|c|c|c|}
\hline \multirow{4}{*}{ 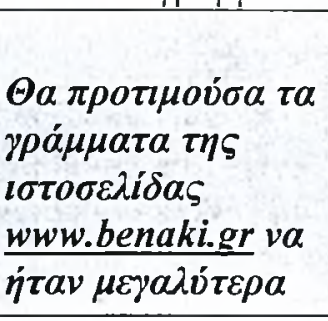 } & & 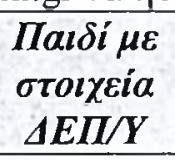 & 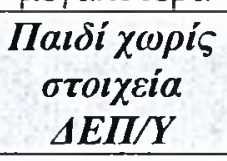 & Lúvoìo \\
\hline & $\Delta ı \alpha \varphi \omega \nu \omega ́$ & 7 & 11 & 18 \\
\hline & $\sum v \mu \varphi \omega v \dot{\omega} \lambda \dot{i} \gamma o$ & 4 & 9 & 13 \\
\hline & $\Sigma v \mu \varphi \omega v \dot{\omega} \alpha \pi \dot{\lambda} \lambda v \tau \alpha$ & 3 & 2 & 5 \\
\hline & Ev́voגo & 14 & 22 & 36 \\
\hline
\end{tabular}

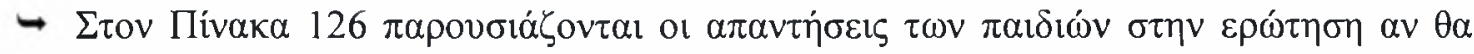

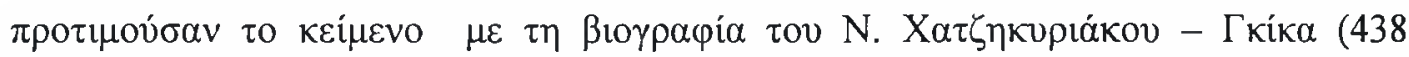

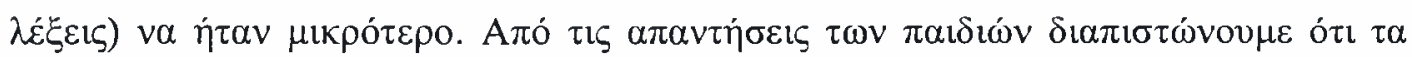

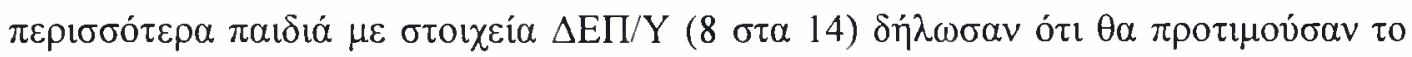

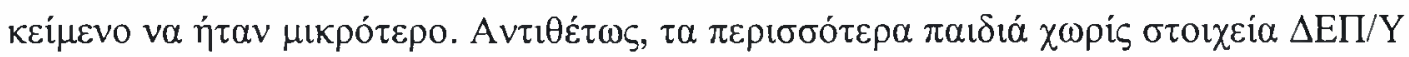

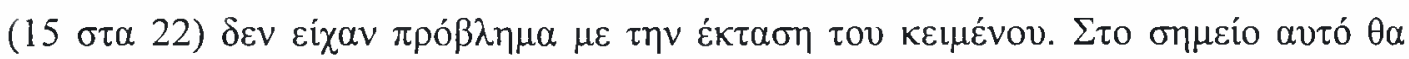

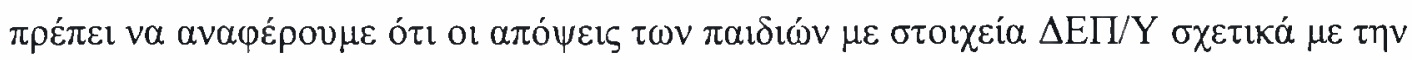

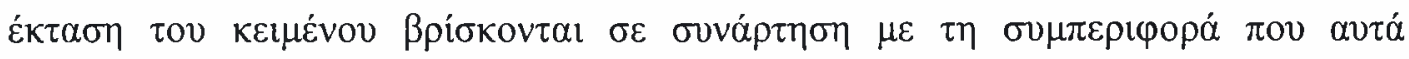

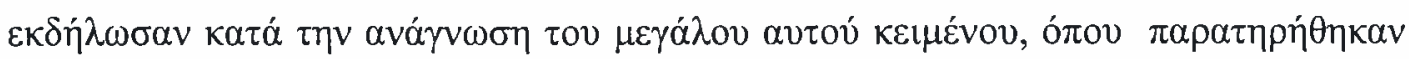




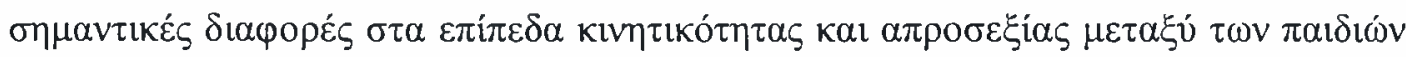

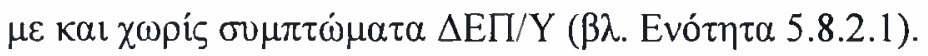

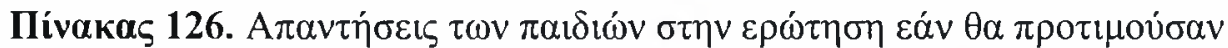

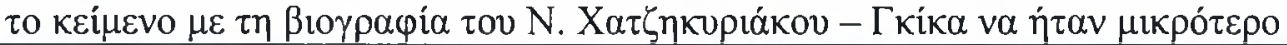

\begin{tabular}{|c|c|c|c|c|}
\hline \multirow{5}{*}{ 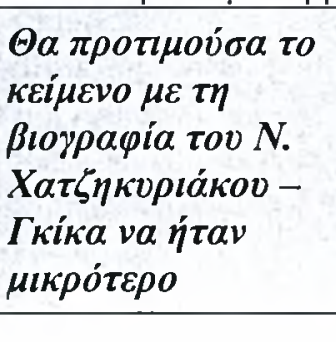 } & & 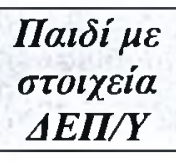 & 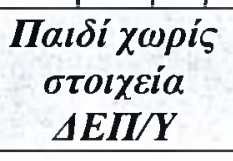 & Lúvodo \\
\hline & $\Delta \iota \alpha \varphi \omega v \omega ́$ & 6 & 15 & 21 \\
\hline & $\Sigma v \mu \varphi \omega v \dot{\omega} \lambda i \gamma_{0}$ & 4 & 7 & 11 \\
\hline & $\Sigma v \mu \varphi \omega v \omega^{\prime} \alpha \pi \dot{\alpha}^{\prime} \lambda v \tau \alpha$ & 4 & & 4 \\
\hline & Ev́voגo & 14 & 22 & 36 \\
\hline
\end{tabular}

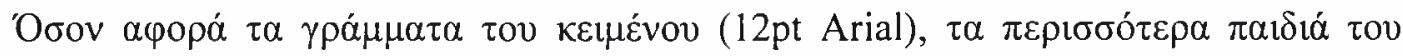

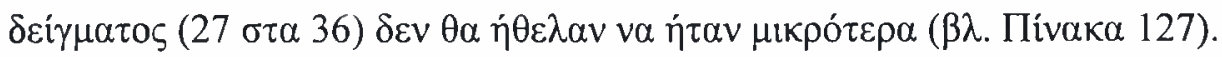

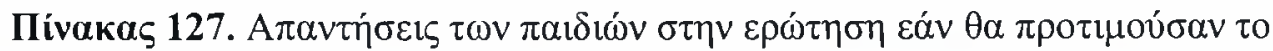

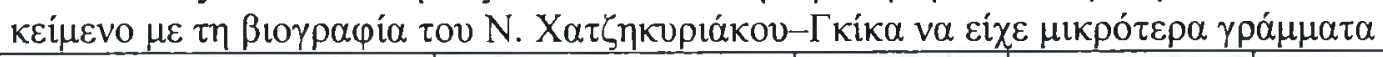

\begin{tabular}{|c|c|c|c|c|}
\hline \multirow{4}{*}{ 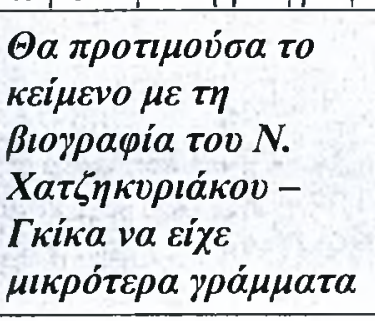 } & & 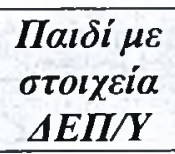 & 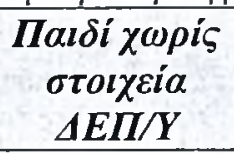 & इúvoגo \\
\hline & $\Delta \imath \alpha \varphi \omega \nu \omega ́$ & 9 & 18 & 27 \\
\hline & $\sum v \mu \varphi \omega v \dot{~ \lambda i ́ \gamma o}$ & 2 & 4 & 6 \\
\hline & $\Sigma v \mu \varphi \omega v \dot{\omega} \alpha \pi \dot{\lambda} \nu \tau \tau \alpha$ & 3 & & 3 \\
\hline & Eúvoגo & 14 & 22 & 36 \\
\hline
\end{tabular}

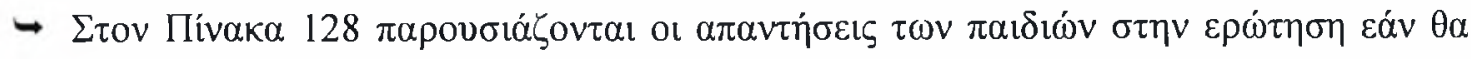

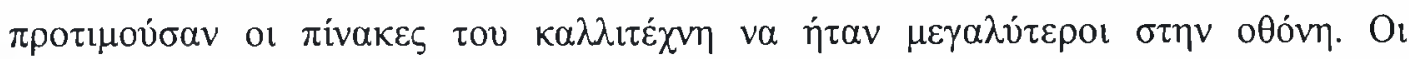

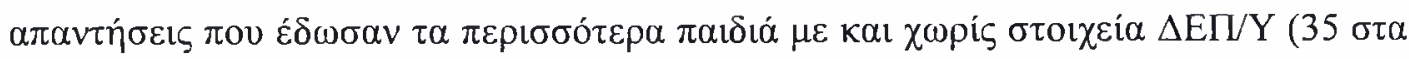

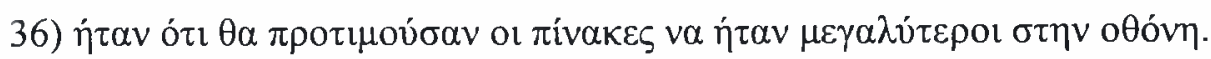

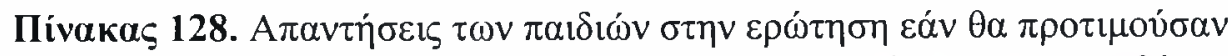

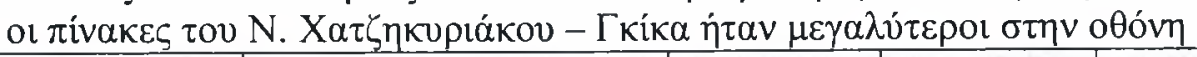

\begin{tabular}{|c|c|c|c|c|}
\hline \multirow{3}{*}{ 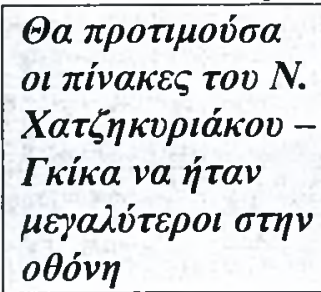 } & & $\begin{array}{c}\text { Паıьі } \mu \varepsilon \\
\sigma \tau o \iota \chi \varepsilon i ́ a \\
\Delta E \Pi / Y\end{array}$ & 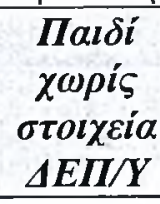 & $\Sigma v ́ v o \lambda o$ \\
\hline & $\Delta \iota \alpha \varphi \omega v \omega ́$ & 1 & & 1 \\
\hline & 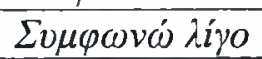 & 4 & 14 & 18 \\
\hline
\end{tabular}




\begin{tabular}{|c|c|c|c|c|}
\hline & $\Sigma v \mu \varphi \omega v \omega \dot{\alpha} \alpha \dot{\lambda} \dot{\prime} v \tau \alpha$ & 9 & 8 & 17 \\
\hline & 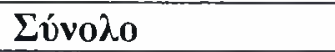 & 14 & 22 & 36 \\
\hline
\end{tabular}

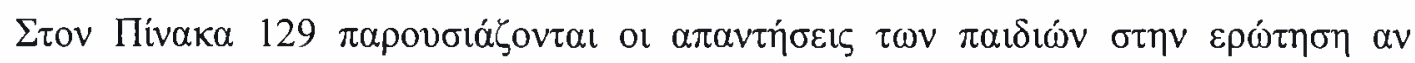

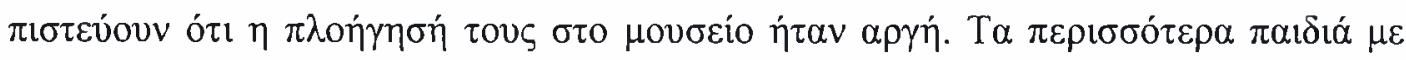

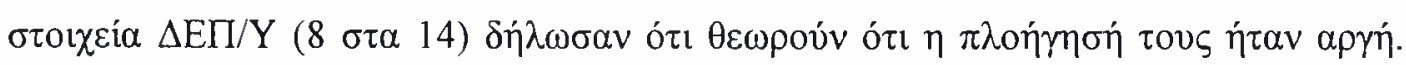

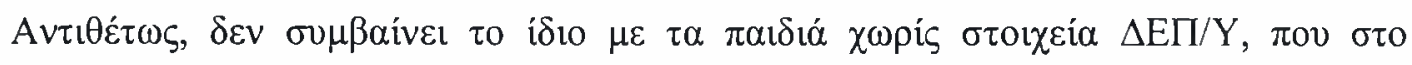

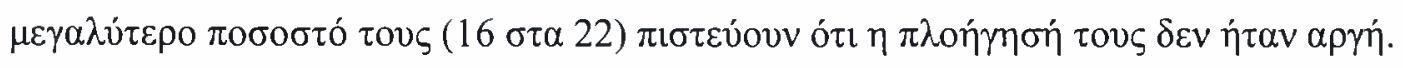

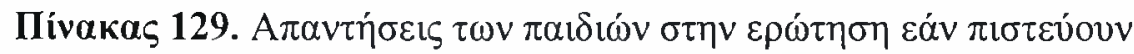

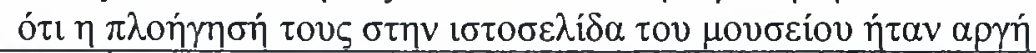

\begin{tabular}{|c|c|c|c|c|}
\hline \multirow{5}{*}{ 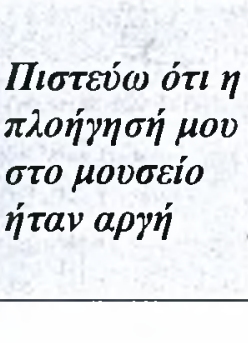 } & & 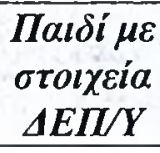 & 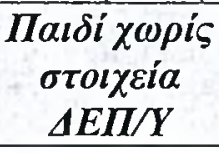 & 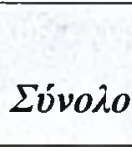 \\
\hline & $\Delta \imath \alpha \varphi \omega \nu \omega ́$ & 6 & 16 & 1 \\
\hline & $\Sigma v \mu \varphi \omega v \dot{\omega} \lambda i^{\prime} \gamma o$ & 5 & 6 & 18 \\
\hline & $\Sigma v \mu \varphi \omega v \dot{\omega} \alpha \pi \dot{\lambda} v \tau \tau \alpha$ & 3 & & 17 \\
\hline & 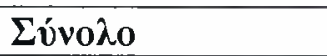 & 14 & 22 & 36 \\
\hline
\end{tabular}

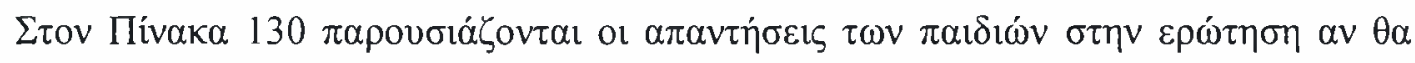

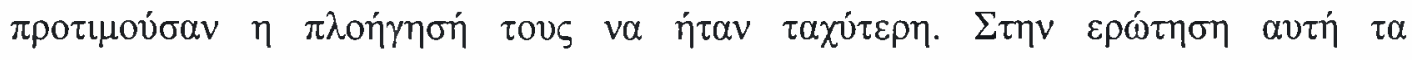

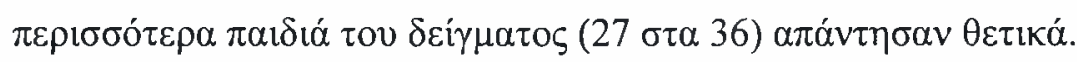

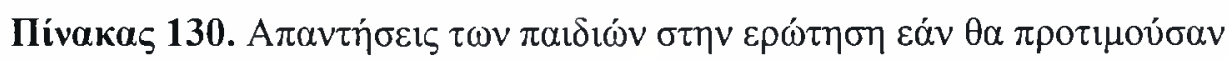

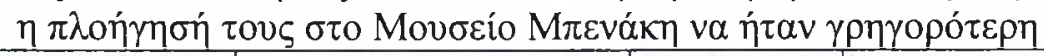

\begin{tabular}{|c|c|c|c|c|}
\hline \multirow{5}{*}{ 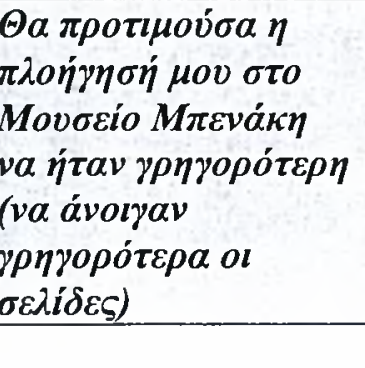 } & & $\begin{array}{c}\text { Пaıঠi } \mu \varepsilon \\
\sigma \tau o \iota \chi \varepsilon i ́ a \\
\Delta E \Pi / Y\end{array}$ & 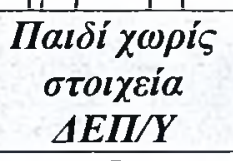 & Lúvoגo \\
\hline & $\Delta \imath \alpha \varphi \omega \nu \omega ́$ & 4 & 5 & 3 \\
\hline & $\Sigma v \mu \varphi \omega \nu \dot{~ \lambda i ́ \gamma o ~}$ & 4 & 10 & 14 \\
\hline & 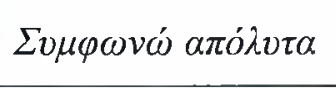 & 6 & 7 & 13 \\
\hline & 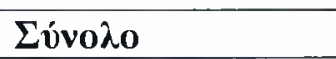 & 14 & 22 & 36 \\
\hline
\end{tabular}

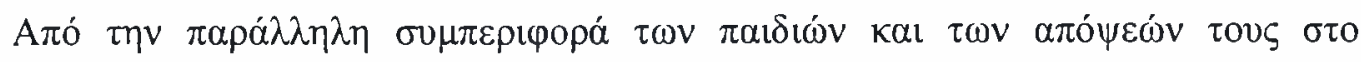

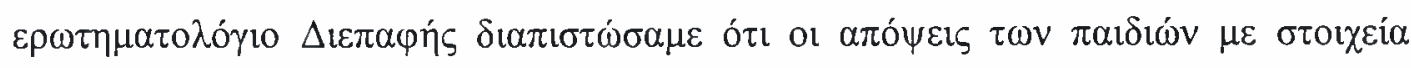

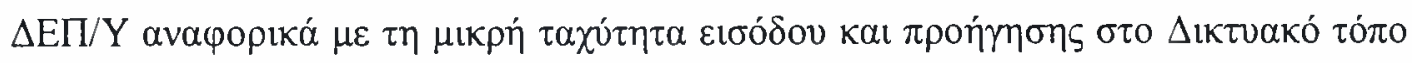




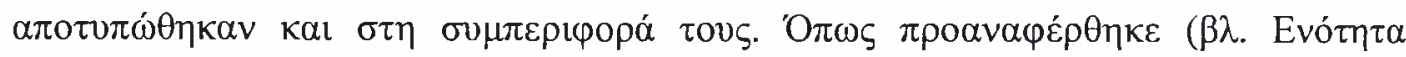

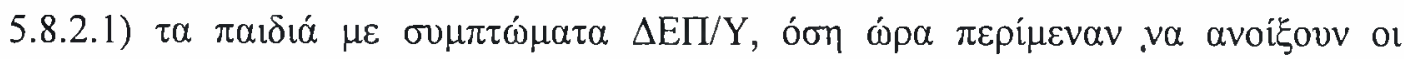

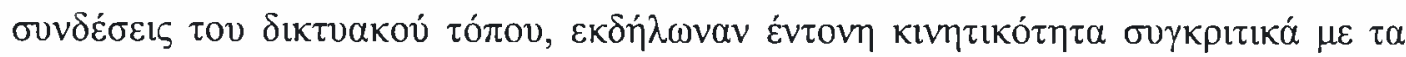

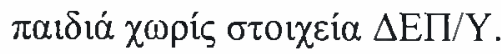

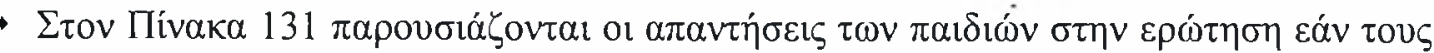

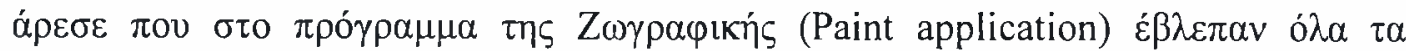

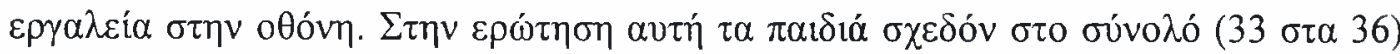

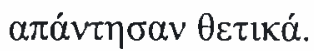

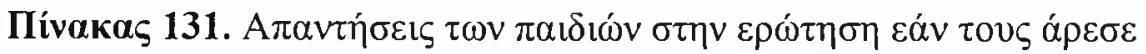

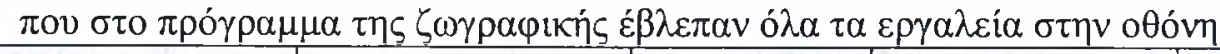

\begin{tabular}{|c|c|c|c|c|}
\hline \multirow{5}{*}{ 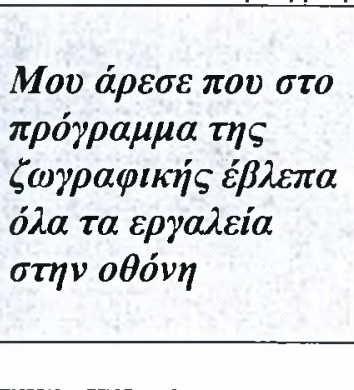 } & & 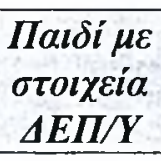 & 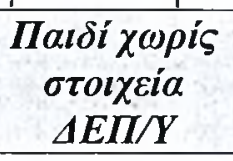 & इúvoגo \\
\hline & $\Delta \iota \alpha \varphi \omega v \omega ́$ & 1 & 2 & 3 \\
\hline & 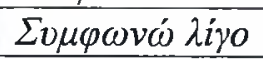 & 2 & 1 & 3 \\
\hline & $\begin{array}{l}\Sigma v \mu \varphi \omega v \dot{~} \\
\alpha \pi \dot{\lambda} \lambda v \tau \alpha\end{array}$ & 11 & 19 & 30 \\
\hline & 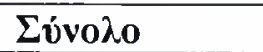 & 14 & 22 & 36 \\
\hline
\end{tabular}

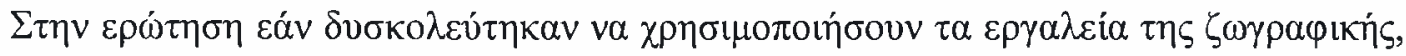

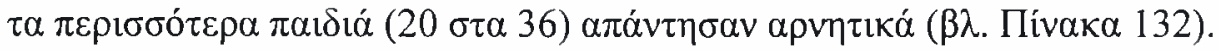

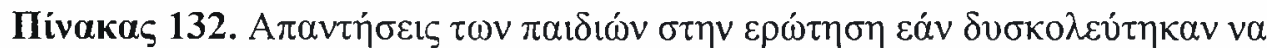

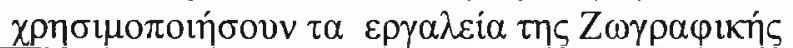

\begin{tabular}{|c|c|c|c|c|}
\hline \multirow{5}{*}{ 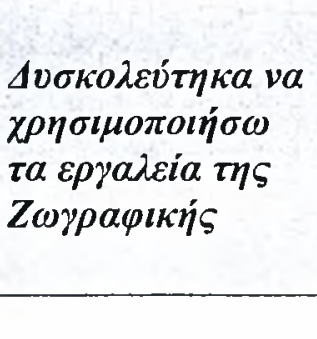 } & & $\begin{array}{c}\text { Пaıji } \mu \varepsilon \\
\sigma \tau o \iota \chi \varepsilon i \alpha \\
\Delta E \Pi / Y\end{array}$ & 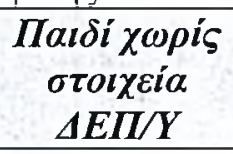 & इúvodo \\
\hline & $\Delta \imath \alpha \varphi \omega \nu \omega$ & 8 & 12 & 20 \\
\hline & 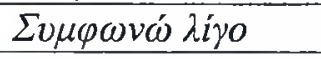 & 6 & 7 & 13 \\
\hline & $\Sigma v \mu \varphi \omega v \omega ́ \alpha \pi o ́ \lambda v \tau \alpha$ & & 3 & 3 \\
\hline & 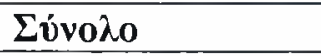 & 14 & 22 & 36 \\
\hline
\end{tabular}




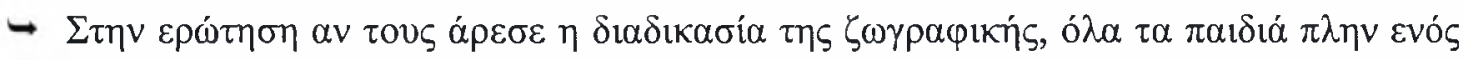

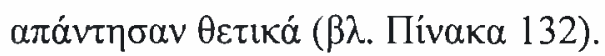

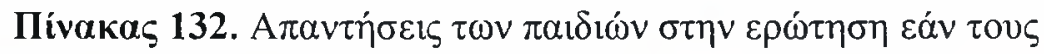

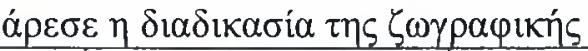

\begin{tabular}{|c|c|c|c|c|}
\hline \multirow{3}{*}{ 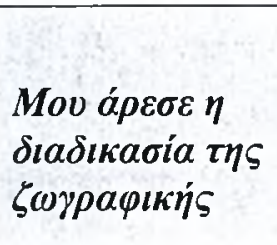 } & & 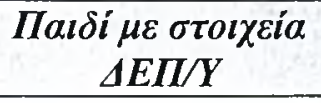 & 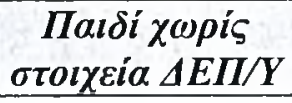 & $\Sigma u ́ v o \lambda o$ \\
\hline & $N \alpha l$ & 13 & 22 & 35 \\
\hline & $O \chi l$ & 1 & & 1 \\
\hline & Ev́voגo & 14 & 22 & 36 \\
\hline
\end{tabular}

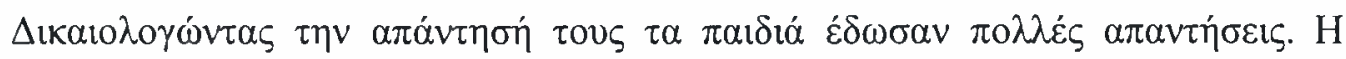

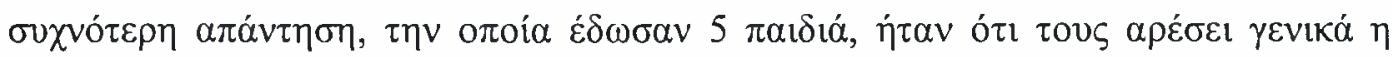

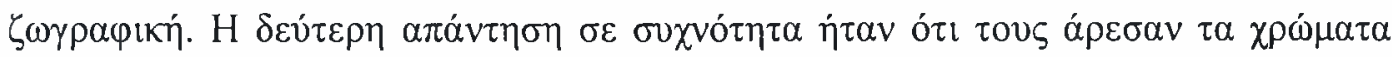

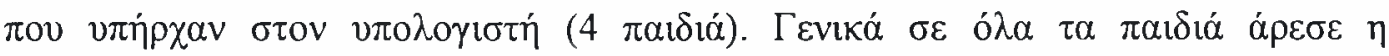

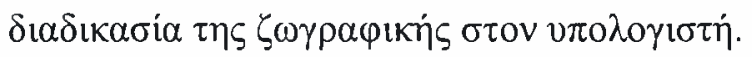

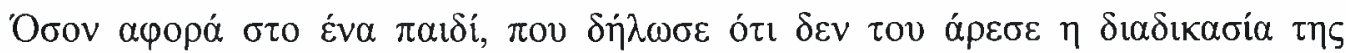

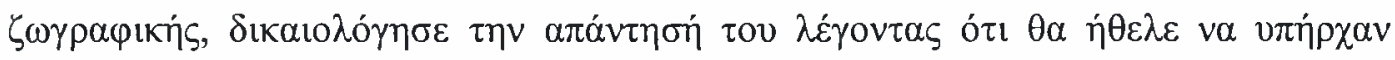

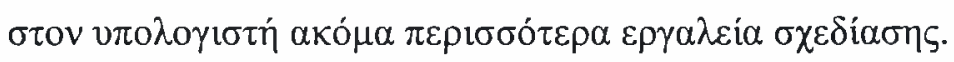

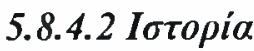

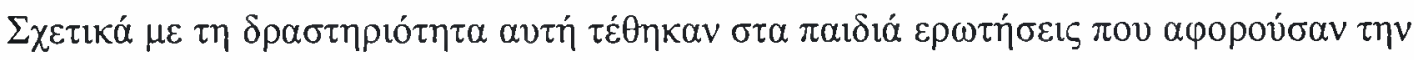

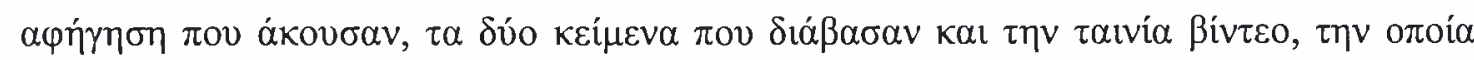
$\pi \alpha \rho \alpha \kappa \circ \lambda \circ \theta_{\eta} \sigma \alpha \nu$.

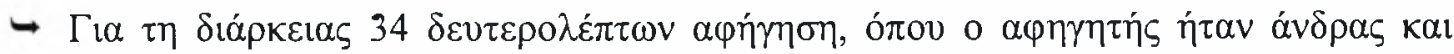

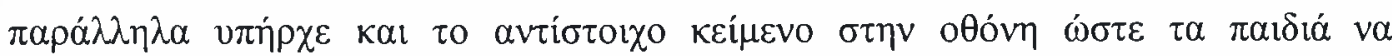

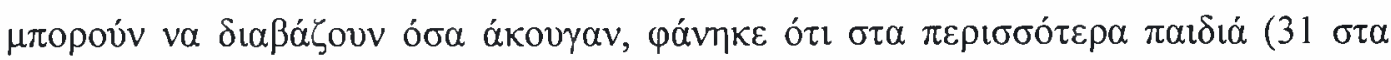

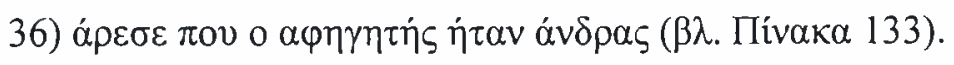




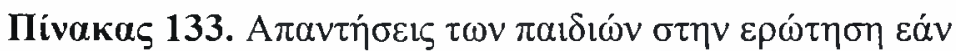

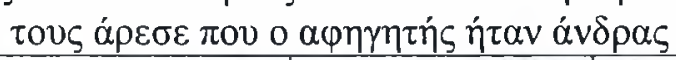

\begin{tabular}{|c|c|c|c|c|}
\hline \multirow{4}{*}{ 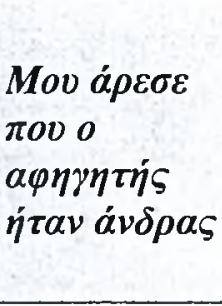 } & & 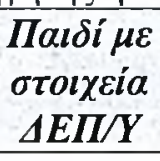 & 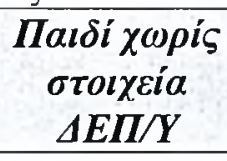 & $\Sigma u ́ v o \lambda o$ \\
\hline & $\Delta \imath \alpha \varphi \omega v \omega ́$ & 2 & 3 & 5 \\
\hline & 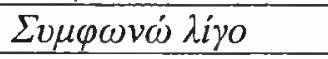 & 3 & 7 & 10 \\
\hline & $\Sigma v \mu \varphi \omega v \dot{\omega} \alpha \pi \delta ́ \lambda v \tau \alpha$ & 9 & 12 & 21 \\
\hline & Ev́voגo & 14 & 22 & 36 \\
\hline
\end{tabular}

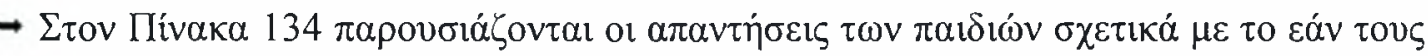

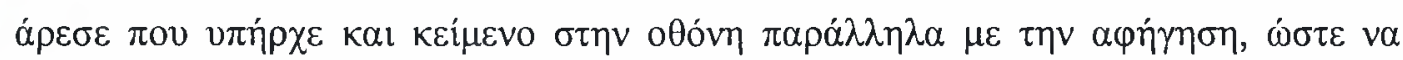

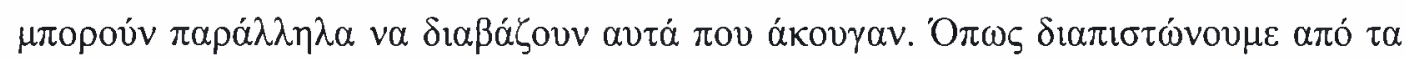

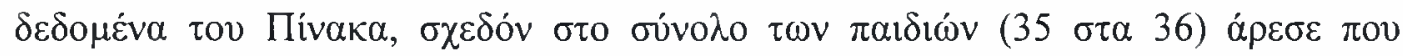

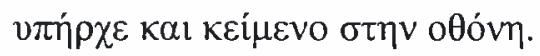

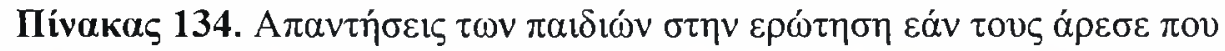

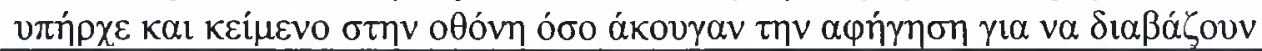

\begin{tabular}{|c|c|c|c|c|}
\hline \multirow{5}{*}{ 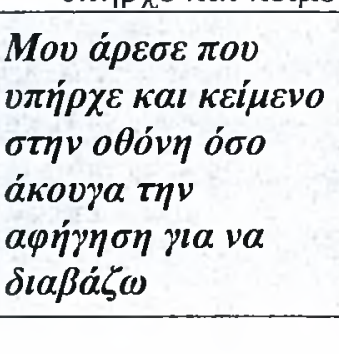 } & & 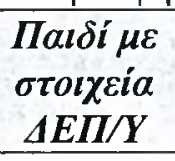 & 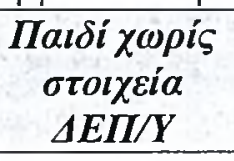 & इóvodo \\
\hline & $\Delta \imath \alpha \varphi \omega \nu \omega ́$ & & 1 & 1 \\
\hline & $\Sigma v \mu \varphi \omega \nu \dot{\omega} \lambda \dot{\gamma} \gamma o$ & 1 & 4 & 5 \\
\hline & $\Sigma v \mu \varphi \omega v \dot{\omega} \alpha \pi \dot{\lambda} \lambda v \tau \alpha$ & 13 & 17 & 30 \\
\hline & Ev́voגo & 14 & 22 & 36 \\
\hline
\end{tabular}

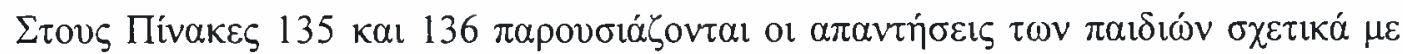

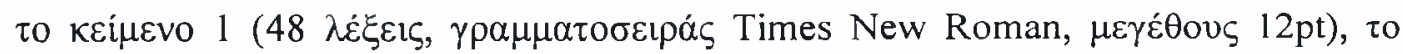

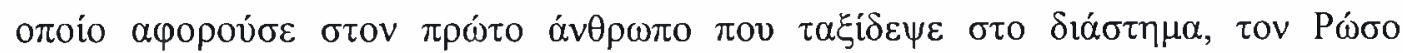

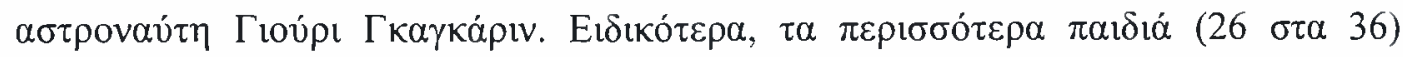

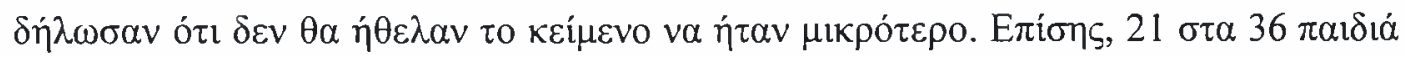

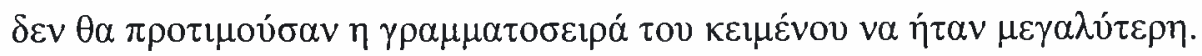




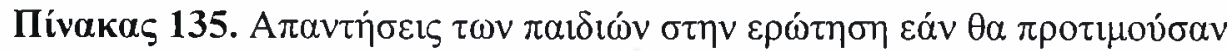

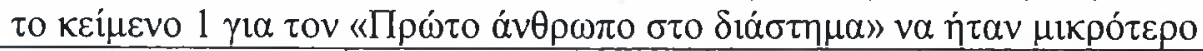

\begin{tabular}{|c|c|c|c|c|}
\hline \multirow{5}{*}{ 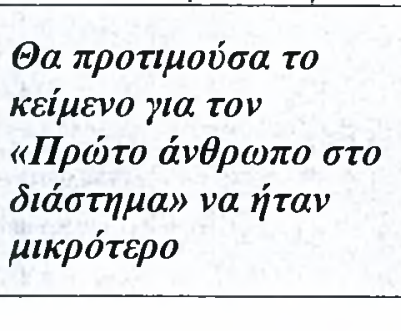 } & & $\begin{array}{c}\text { Пaı } \delta i \mu \varepsilon \\
\sigma \tau o \imath \chi \varepsilon i ́ a \\
\Delta E \Pi / Y\end{array}$ & 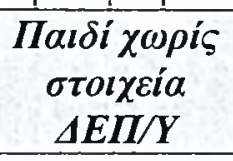 & $\Sigma v ́ v o \lambda o$ \\
\hline & $\Delta \iota \alpha \varphi \omega v \omega ́$ & 12 & 14 & 26 \\
\hline & 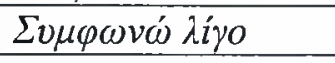 & 2 & 7 & 9 \\
\hline & $\Sigma v \mu \varphi \omega v \omega \dot{\alpha} \alpha \pi \dot{\lambda} \lambda v \tau \alpha$ & & 1 & 1 \\
\hline & Eúvoגo & 14 & 22 & 36 \\
\hline
\end{tabular}

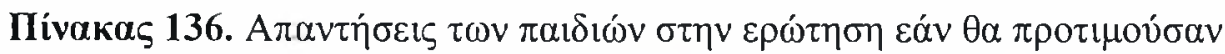

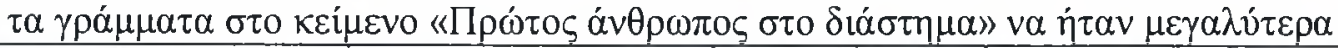

\begin{tabular}{|c|c|c|c|c|}
\hline \multirow{5}{*}{ 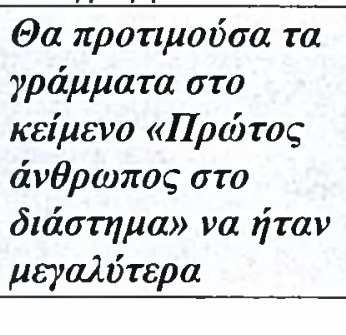 } & & 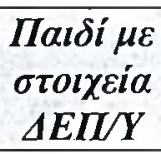 & 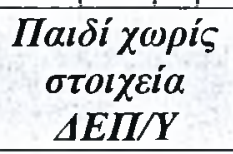 & 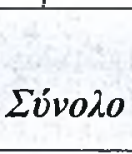 \\
\hline & $\Delta \iota \alpha \varphi \omega \nu \omega$ & 8 & 13 & 21 \\
\hline & 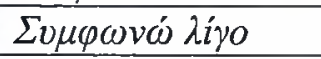 & 5 & 9 & 14 \\
\hline & $\sum v \mu \varphi \omega v \omega ́ \alpha \pi{ }^{\prime} \lambda v \tau \alpha$ & 1 & & 1 \\
\hline & 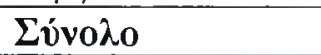 & 14 & 22 & 36 \\
\hline
\end{tabular}

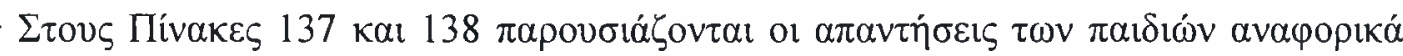

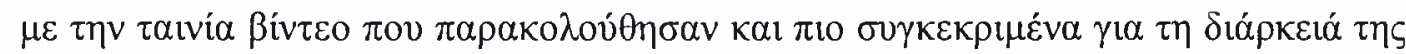

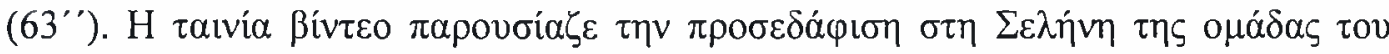

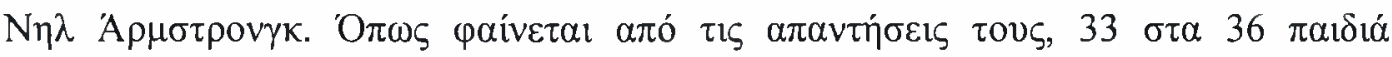

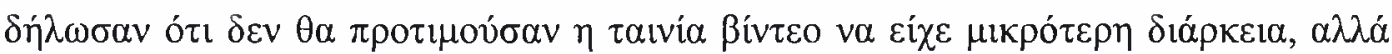

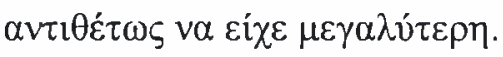

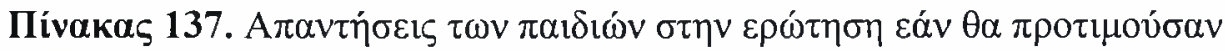

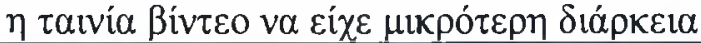

\begin{tabular}{|c|c|c|c|c|}
\hline \multirow{5}{*}{ 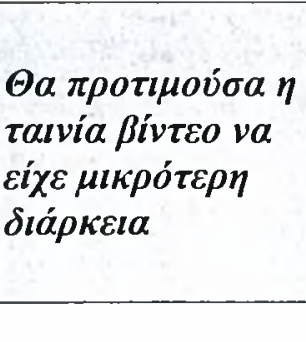 } & - & 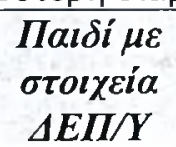 & $\begin{array}{c}\text { Пaısi } \chi \omega \rho i \varsigma \\
\sigma \tau o \imath \chi \varepsilon i ́ a \\
\Delta E \Pi / Y\end{array}$ & 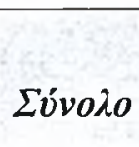 \\
\hline & $\Delta \imath \alpha \varphi \omega \nu \omega \dot{\omega}$ & 14 & 19 & 33 \\
\hline & 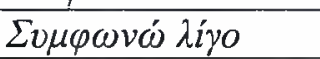 & & 2 & 2 \\
\hline & 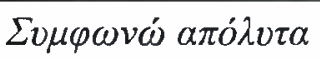 & & 1 & 1 \\
\hline & 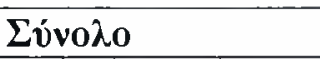 & 14 & 22 & 36 \\
\hline
\end{tabular}




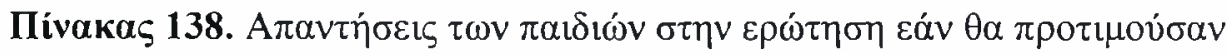

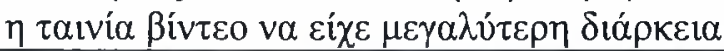

\begin{tabular}{|c|c|c|c|c|}
\hline \multirow{5}{*}{ 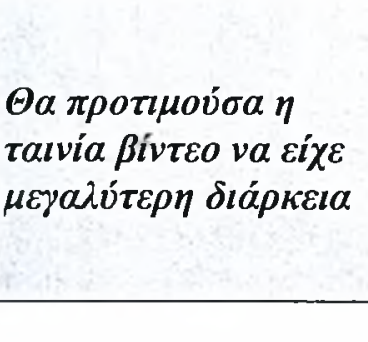 } & & $\begin{array}{c}\text { ПIaısí } \mu \varepsilon \\
\sigma \tau o \iota \chi \varepsilon i ́ a \\
\Delta E \Pi / Y\end{array}$ & 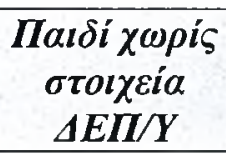 & $\begin{array}{c}\text { Ev́vod } \\
o\end{array}$ \\
\hline & $\Delta \iota \alpha \varphi \omega v \omega ́$ & 2 & 1 & 3 \\
\hline & 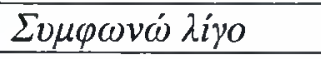 & 4 & 9 & 13 \\
\hline & $\Sigma v \mu \varphi \omega v \dot{\omega} \alpha \pi \dot{\lambda} \lambda v \tau \alpha$ & 8 & 12 & 20 \\
\hline & Eúvoגo & 14 & 22 & 36 \\
\hline
\end{tabular}

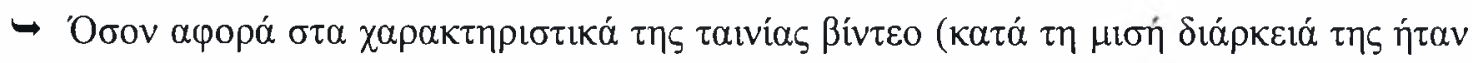

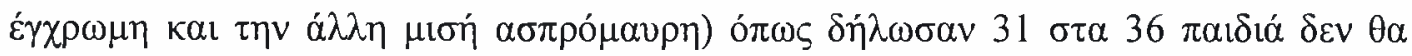

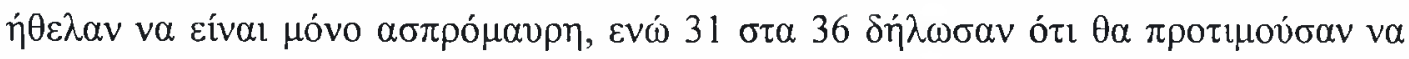

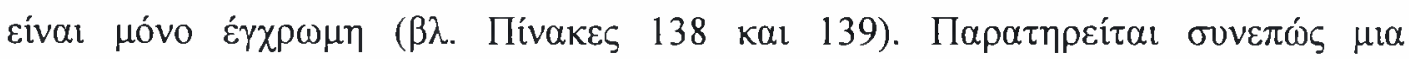

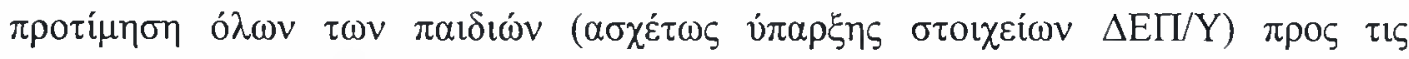

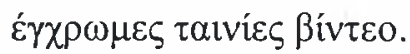

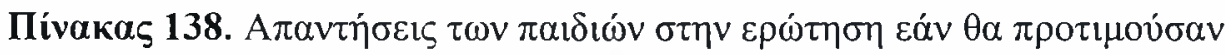

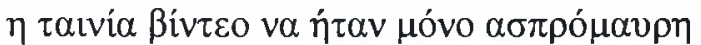

\begin{tabular}{|c|c|c|c|c|}
\hline \multirow{4}{*}{ 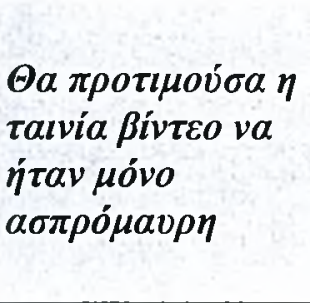 } & & 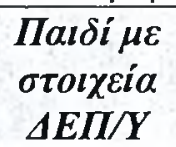 & 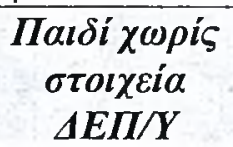 & Lv́voגo \\
\hline & $\Delta \iota \alpha \varphi \omega \nu \dot{~}$ & 12 & 19 & 31 \\
\hline & 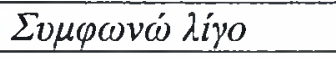 & 1 & 2 & 3 \\
\hline & $\Sigma v \mu \varphi \omega v \omega \dot{\omega} \alpha \pi \dot{\lambda} \nu \tau \tau$ & 1 & 1 & 2 \\
\hline & 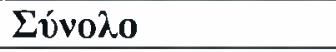 & 14 & 22 & 36 \\
\hline
\end{tabular}

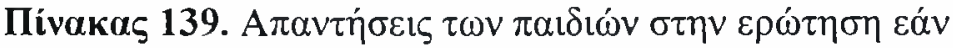

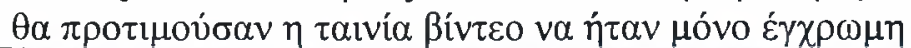

\begin{tabular}{|c|c|c|c|c|}
\hline \multirow{5}{*}{ 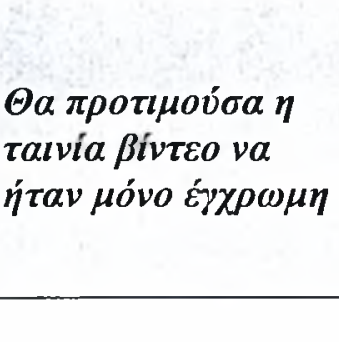 } & & 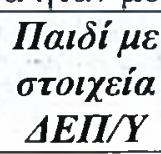 & 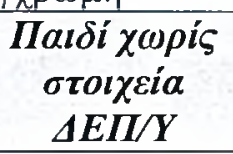 & $\Sigma v ́ v o \lambda o$ \\
\hline & $\Delta \iota \alpha \varphi \omega v \omega ́$ & 2 & 3 & 5 \\
\hline & $\Sigma v \mu \varphi \omega v \dot{\omega} \lambda i \gamma o$ & 5 & 5 & 10 \\
\hline & $\Sigma v \mu \varphi \omega v \dot{\alpha} \alpha \pi \dot{\lambda} \lambda v \tau \alpha$ & 7 & 14 & 21 \\
\hline & 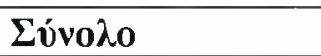 & 14 & 22 & 36 \\
\hline
\end{tabular}

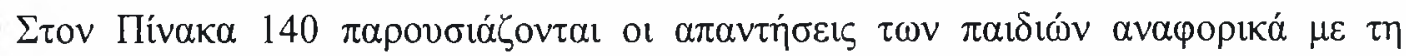

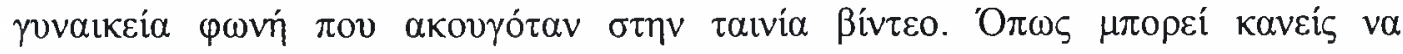




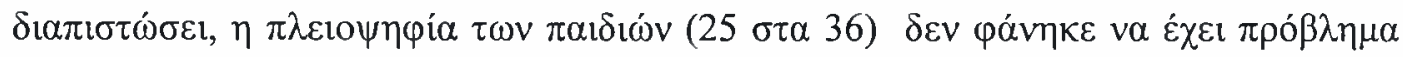

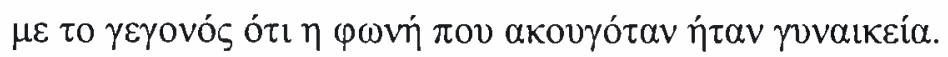

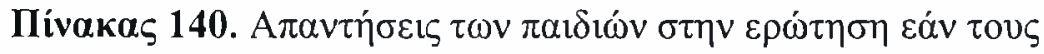

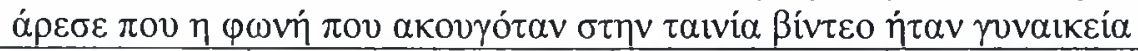

\begin{tabular}{|c|c|c|c|c|}
\hline \multirow{4}{*}{ 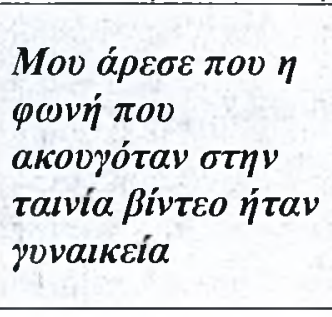 } & & $\begin{array}{c}\text { Iaısí } \mu \varepsilon \\
\sigma \tau o \iota \chi \varepsilon i ́ a \\
\Delta E \Pi / Y\end{array}$ & 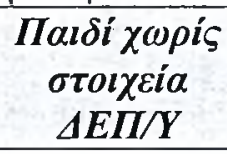 & $\Sigma v ́ v o \lambda o$ \\
\hline & $\Delta \imath \alpha \varphi \omega v \omega \dot{\omega}$ & 6 & 5 & 11 \\
\hline & 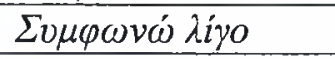 & 7 & 12 & 19 \\
\hline & $\Sigma v \mu \varphi \omega v \dot{~} \alpha \pi \dot{\lambda} \lambda v \tau \alpha$ & 1 & 5 & 6 \\
\hline & 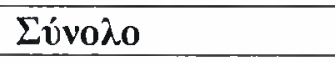 & 14 & 22 & 36 \\
\hline
\end{tabular}

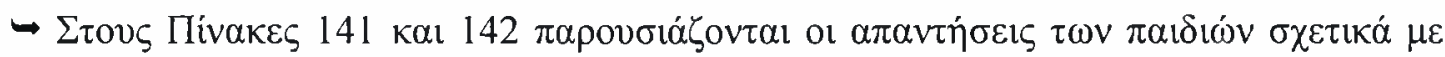

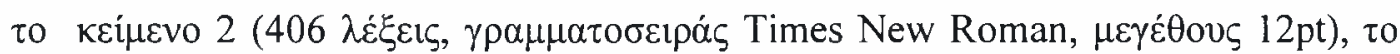

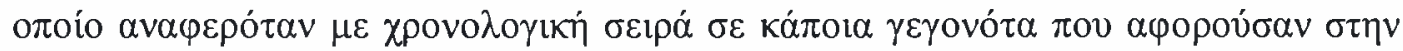

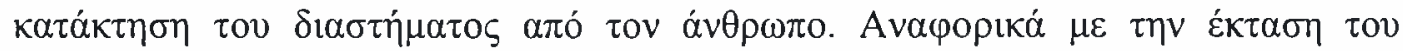

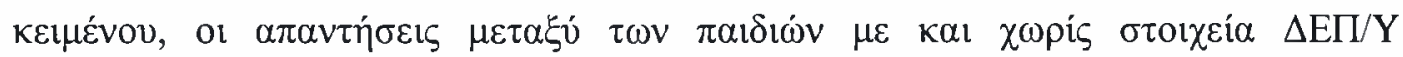

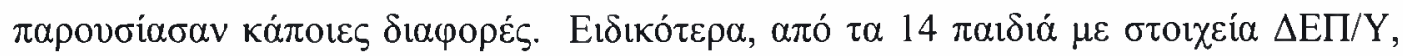

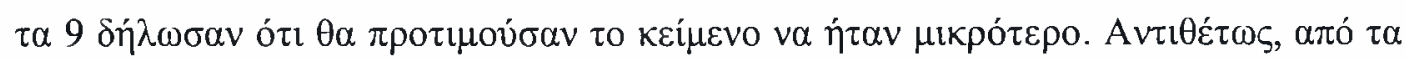

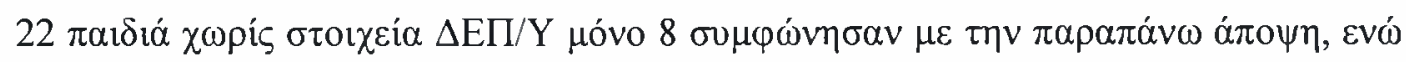

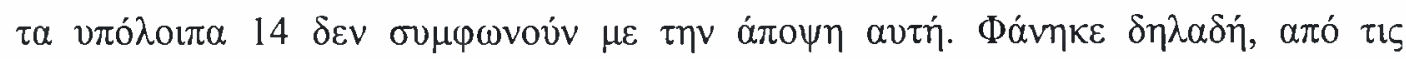

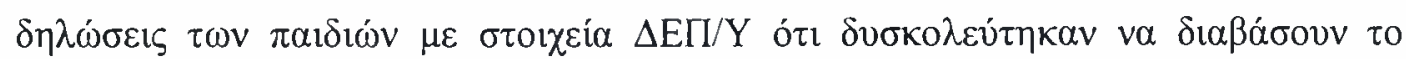

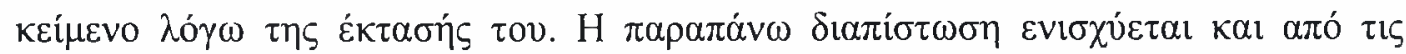

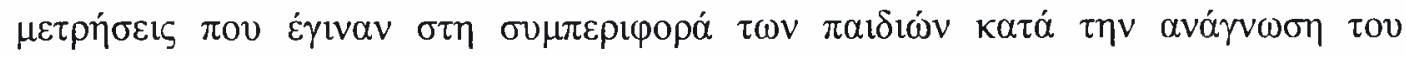

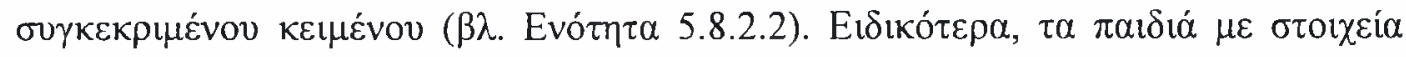

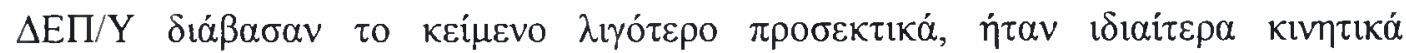

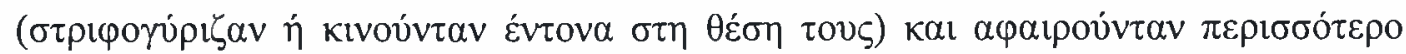

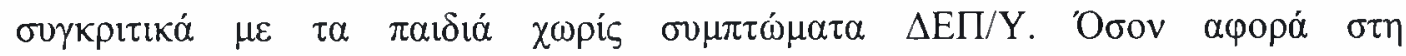

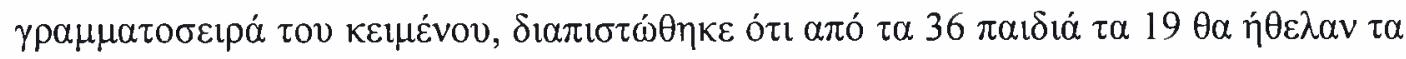

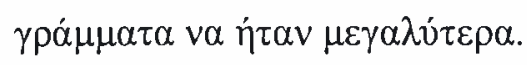




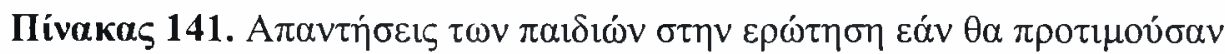

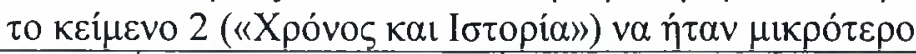

\begin{tabular}{|c|c|c|c|c|}
\hline \multirow{5}{*}{ 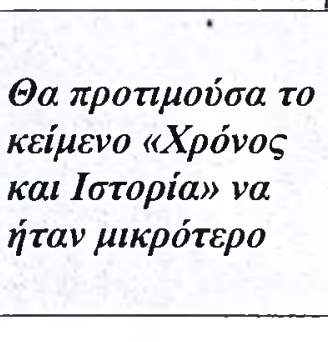 } & & 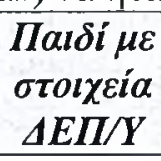 & $\begin{array}{c}\Pi \alpha \iota \delta i \chi \chi \omega \rho i \varsigma \\
\sigma \tau o \imath \chi \varepsilon i \alpha \\
\Delta E \Pi / Y\end{array}$ & 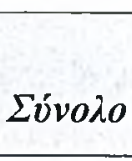 \\
\hline & $\Delta l \alpha \varphi \omega \nu \dot{\omega}$ & 5 & 14 & 19 \\
\hline & 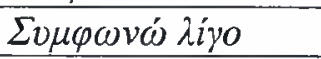 & 6 & 6 & 12 \\
\hline & 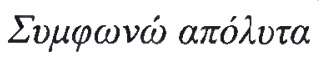 & 3 & 2 & 5 \\
\hline & Ev́voגo & 14 & 22 & 36 \\
\hline
\end{tabular}

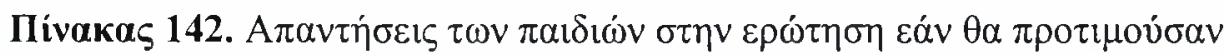

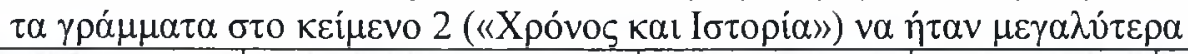

\begin{tabular}{|c|c|c|c|c|}
\hline \multirow{5}{*}{ 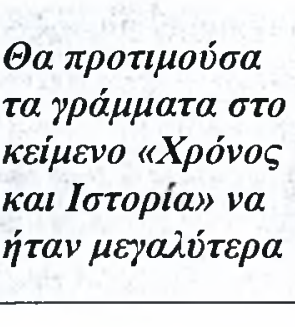 } & & 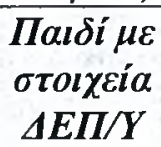 & 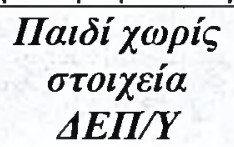 & Ev́voגo \\
\hline & $\Delta l \alpha \varphi \omega \nu \omega ́$ & 7 & 10 & 17 \\
\hline & 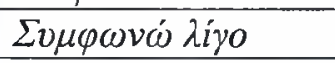 & 5 & 9 & 14 \\
\hline & 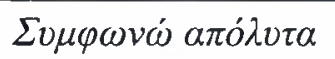 & 2 & 3 & 5 \\
\hline & Ev่voגo & 14 & 22 & 36 \\
\hline
\end{tabular}

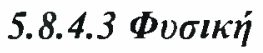

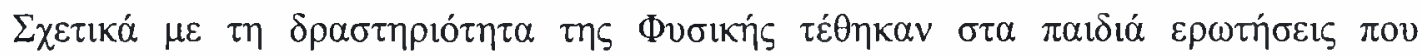
$\alpha \varphi$

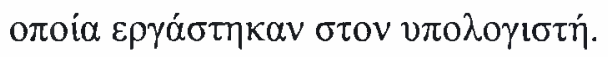

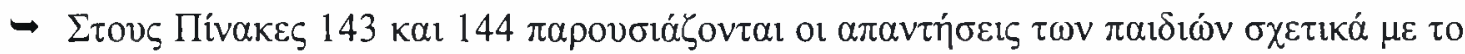

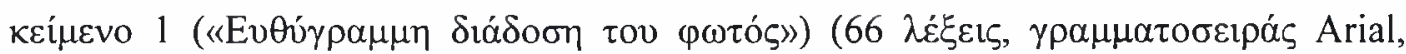

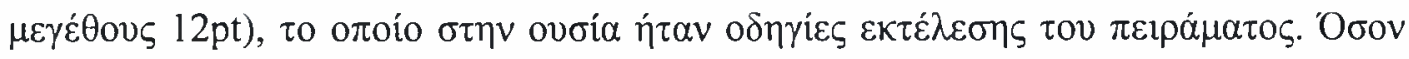

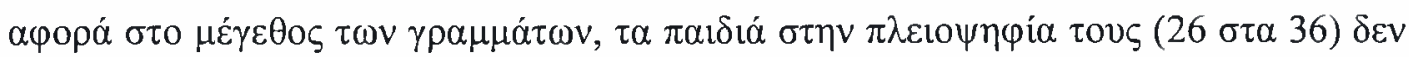

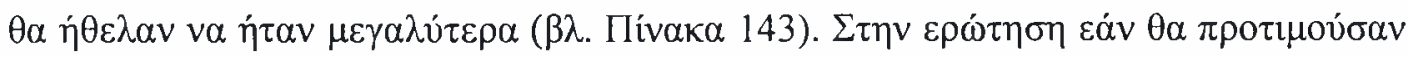

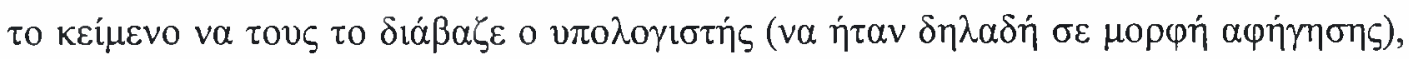

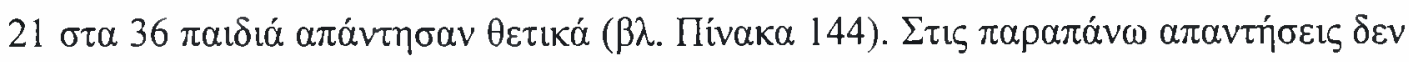

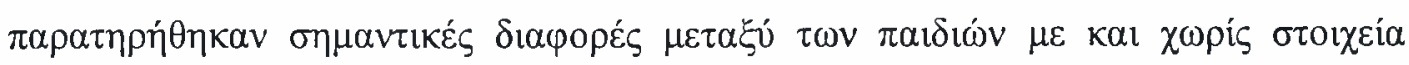
$\Delta \mathrm{E} \Pi / \mathrm{Y}$. 


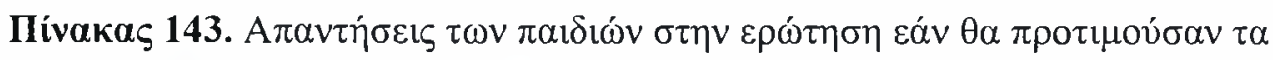

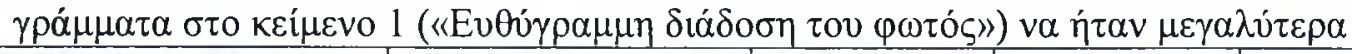

\begin{tabular}{|c|c|c|c|c|}
\hline \multirow{5}{*}{ 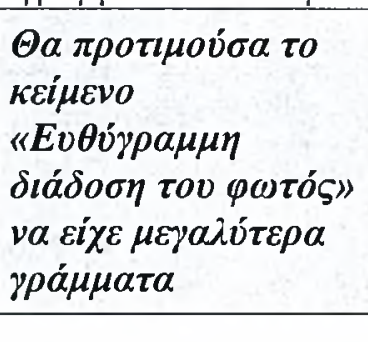 } & ${ }^{\circ}$ & $\begin{array}{c}\Pi a ı \delta i \mu \varepsilon \\
\sigma \tau o \imath \chi \varepsilon i ́ a \\
\Delta E \Pi \| Y\end{array}$ & 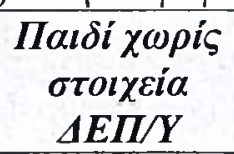 & 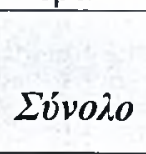 \\
\hline & $\Delta \iota \alpha \varphi \omega v \omega \dot{0}$ & 11 & 15 & 26 \\
\hline & 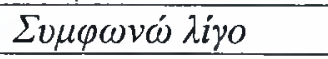 & 3 & 6 & 9 \\
\hline & $\Sigma v \mu \varphi \omega v \omega ́ \alpha \pi \delta ́ \lambda v \tau \alpha$ & & 1 & 1 \\
\hline & Ev́voגo & 14 & 22 & 36 \\
\hline
\end{tabular}

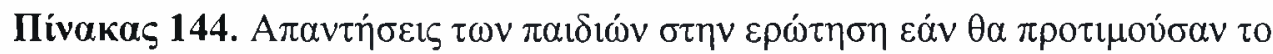

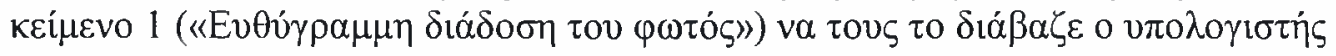

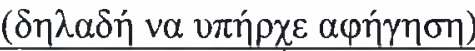

\begin{tabular}{|c|c|c|c|c|}
\hline \multirow{4}{*}{ 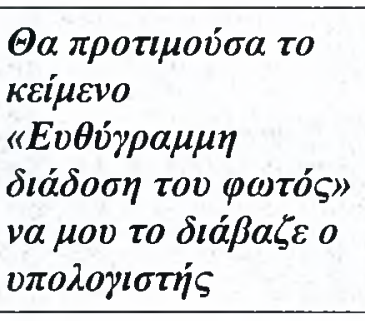 } & & $\begin{array}{c}\text { Паı } \delta i \mu \varepsilon \\
\sigma \tau o \iota \chi \varepsilon i \alpha \\
\Delta E \Pi / Y\end{array}$ & 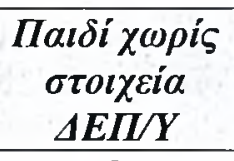 & Lúvoגo \\
\hline & $\Delta \imath \alpha \varphi \omega v \dot{\omega}$ & 6 & 9 & 15 \\
\hline & 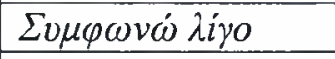 & 2 & 8 & 10 \\
\hline & $\Sigma v \mu \varphi \omega v \dot{\alpha} \alpha \pi \dot{\lambda} \lambda v \tau \alpha$ & 6 & 5 & 11 \\
\hline & Ev́voגo & 14 & 22 & 36 \\
\hline
\end{tabular}

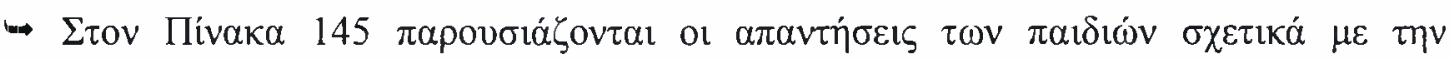

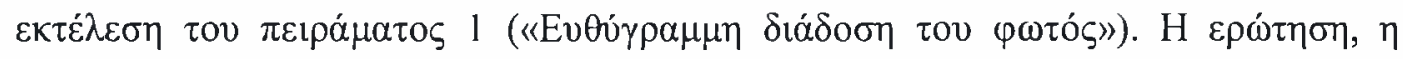

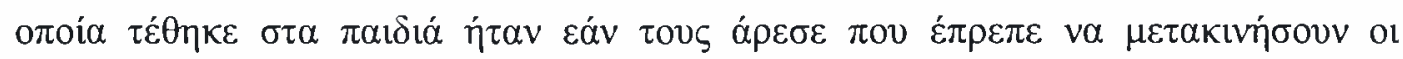

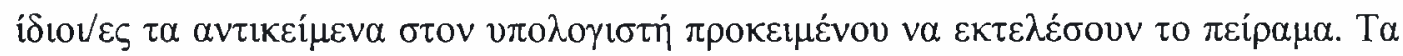

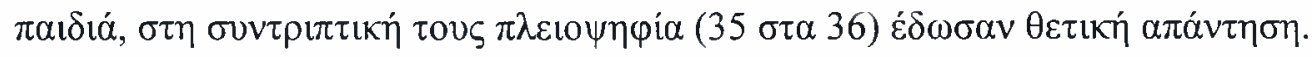

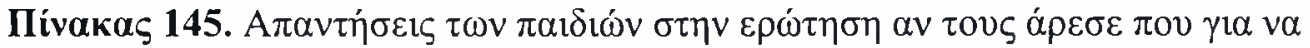

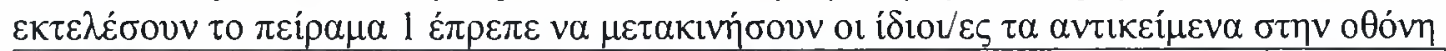

\begin{tabular}{|c|c|c|c|c|}
\hline \multirow{4}{*}{ 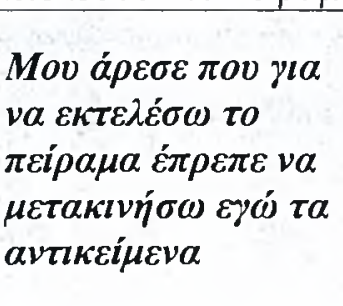 } & & $\begin{array}{c}\Pi a ı \delta i \mu \varepsilon \\
\sigma \tau o \iota \chi \varepsilon i ́ a \\
\Delta E \Pi / Y\end{array}$ & 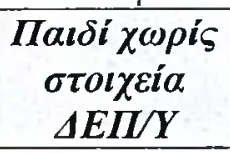 & 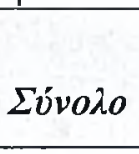 \\
\hline & $\Delta l \alpha \varphi \omega \nu \omega ́$ & & 1 & 1 \\
\hline & $\Sigma v \mu \varphi \omega v \dot{~} \lambda i \gamma_{0}$ & 2 & 2 & 4 \\
\hline & $\Sigma v \mu \varphi \omega v \omega ́ \alpha \pi \dot{\lambda} \lambda v \tau \alpha$ & 12 & 19 & 31 \\
\hline & 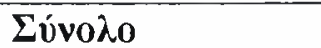 & 14 & 22 & 36 \\
\hline
\end{tabular}

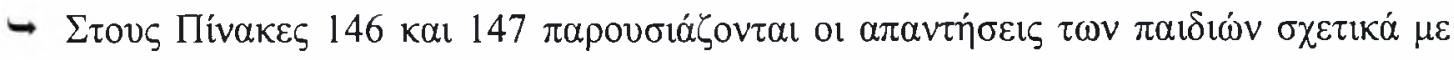

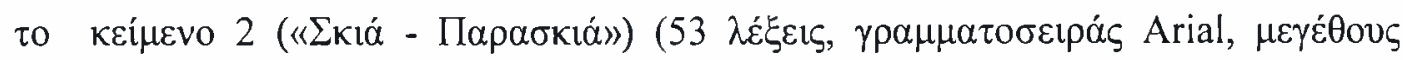




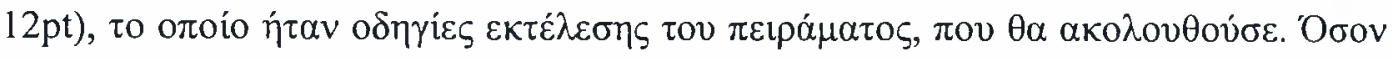

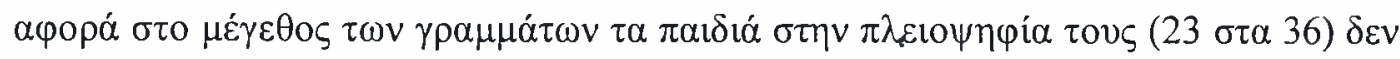

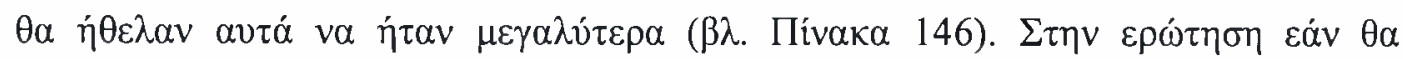

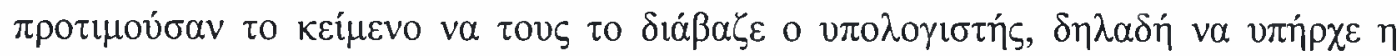

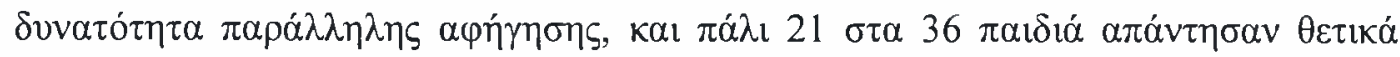

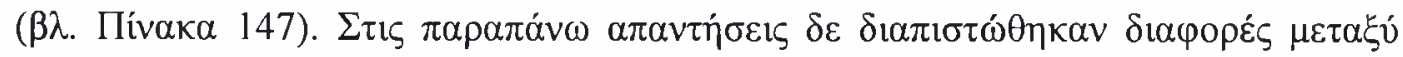

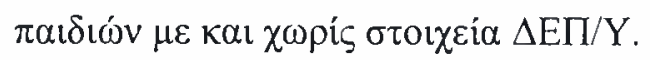

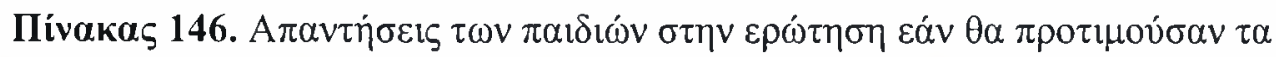

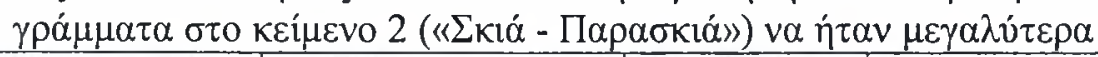

\begin{tabular}{|c|c|c|c|c|}
\hline \multirow{4}{*}{ 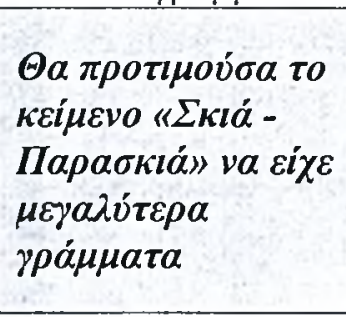 } & & $\begin{array}{l}\text { Пaı́i } \mu \varepsilon \\
\sigma \tau o \iota \chi \varepsilon i \alpha \\
\Delta E \Pi / Y\end{array}$ & 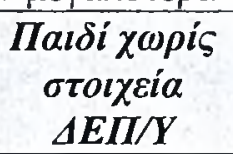 & $\Sigma v ́ v o \lambda o$ \\
\hline & $\Delta \iota \alpha \varphi \omega \nu \omega ́$ & 9 & 14 & 23 \\
\hline & 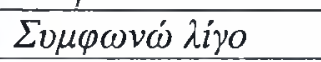 & 4 & 6 & 10 \\
\hline & $\Sigma v \mu \varphi \omega v \omega \dot{\alpha} \alpha \pi \dot{\lambda} v \tau \alpha$ & 1 & 2 & 3 \\
\hline & Súvoגo & 14 & 22 & 36 \\
\hline
\end{tabular}

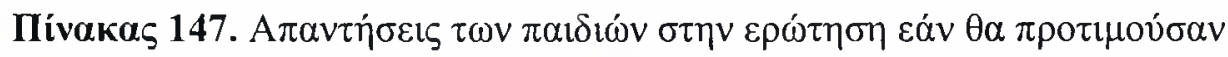

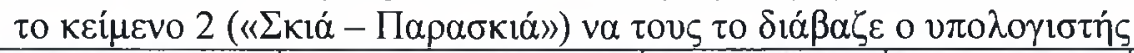

\begin{tabular}{|c|c|c|c|c|}
\hline \multirow{4}{*}{ 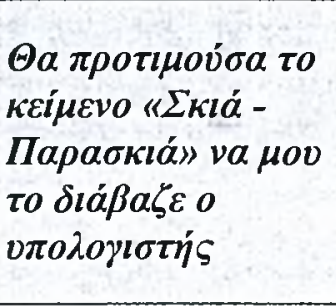 } & & 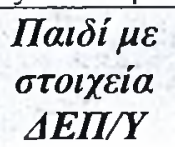 & 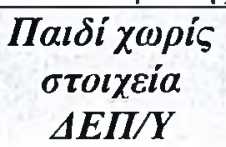 & $\Sigma v ́ v o \lambda o$ \\
\hline & $\Delta \iota \alpha \varphi \omega \nu \omega ́$ & 7 & 8 & 15 \\
\hline & 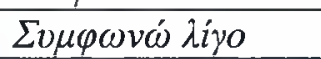 & 3 & 9 & 12 \\
\hline & $\Sigma v \mu \varphi \omega v \omega \dot{\alpha} \alpha \pi \dot{\lambda} \nu \tau \tau \alpha$ & 4 & 5 & 9 \\
\hline & 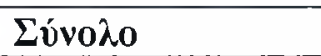 & 14 & 22 & 36 \\
\hline
\end{tabular}

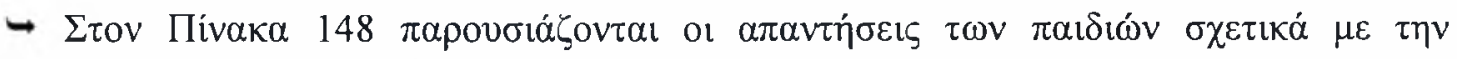

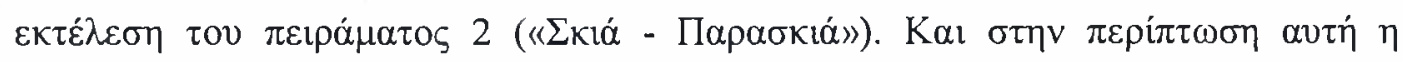

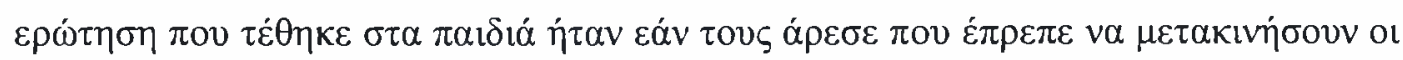

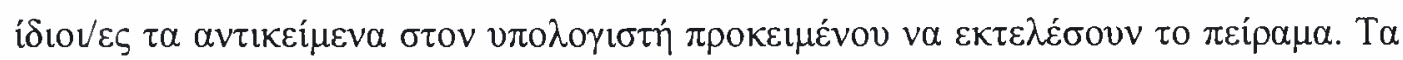

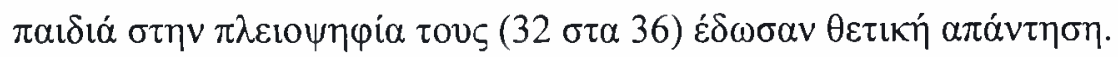




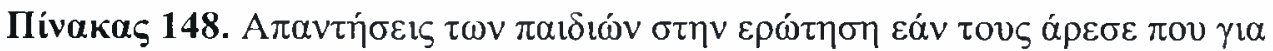

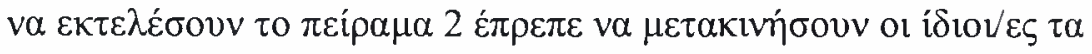
$\alpha v \tau \iota k \varepsilon i ́ \mu \varepsilon v \alpha \sigma \tau \eta$ oӨóvm

\begin{tabular}{|c|c|c|c|c|}
\hline \multirow{4}{*}{ 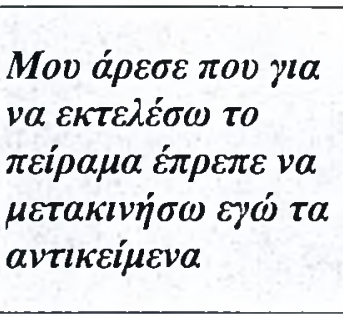 } & & 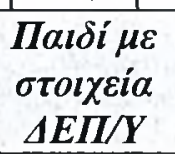 & $\begin{array}{c}\text { Паı } \delta i ́ \chi \omega \rho i ́ \varsigma \\
\sigma \tau o \imath \chi \varepsilon i ́ a \\
\Delta E \Pi / Y\end{array}$ & ¿v́voגo \\
\hline & $\Delta \iota \alpha \varphi \omega v \omega ́$ & 2 & 2 & 4 \\
\hline & $\sum v \mu \varphi \omega v \dot{~ \lambda i ́ \gamma o}$ & & 1 & 1 \\
\hline & $\Sigma v \mu \varphi \omega v \dot{s} \alpha \pi o ́ \lambda v \tau \alpha$ & 12 & 19 & 31 \\
\hline & Ev́voגo & 14 & 22 & 36 \\
\hline
\end{tabular}

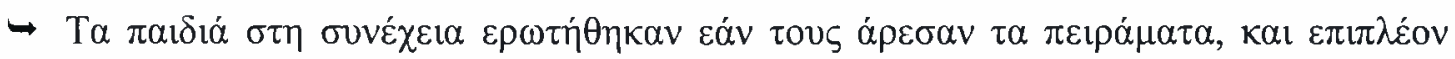

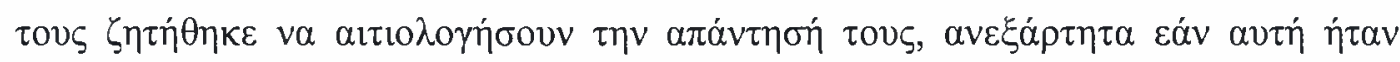

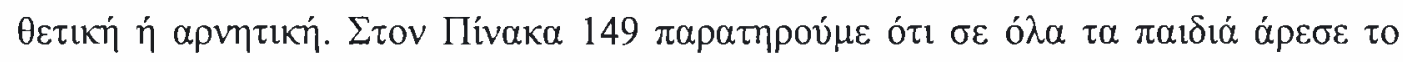

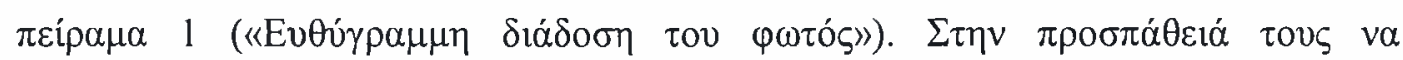

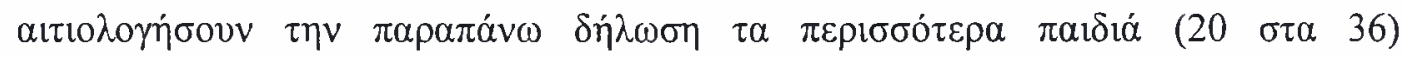

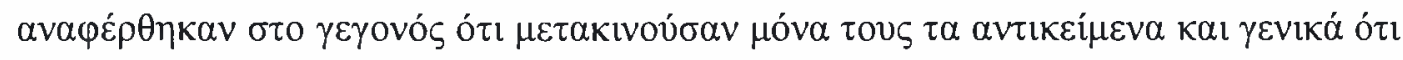

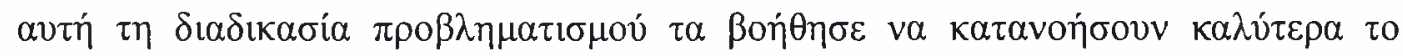

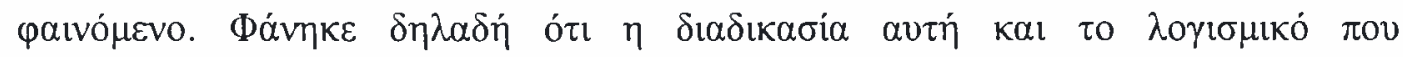

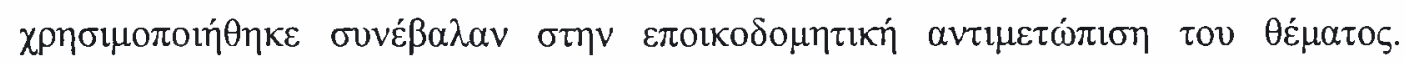

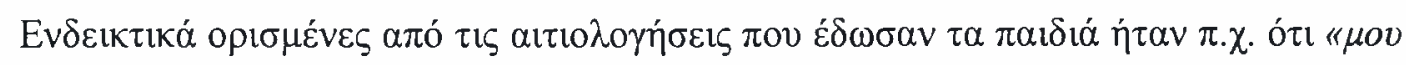

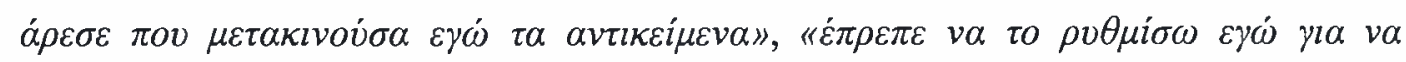

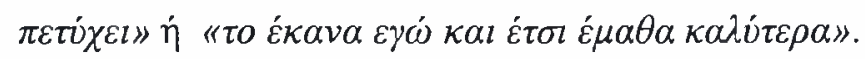

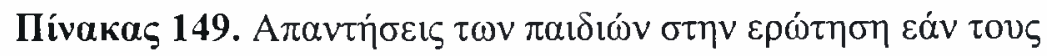

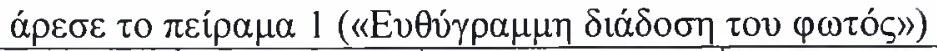

\begin{tabular}{|c|c|c|c|c|}
\hline \multirow{3}{*}{ 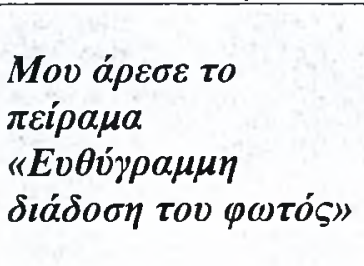 } & & 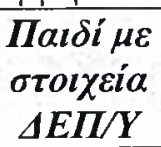 & 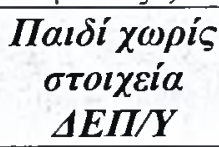 & ¿v́voגo \\
\hline & $N \alpha l$ & 14 & 22 & 36 \\
\hline & $O \chi_{l}$ & & & \\
\hline & Ev́voגo & 14 & 22 & 36 \\
\hline
\end{tabular}

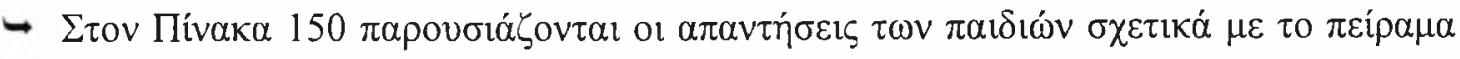

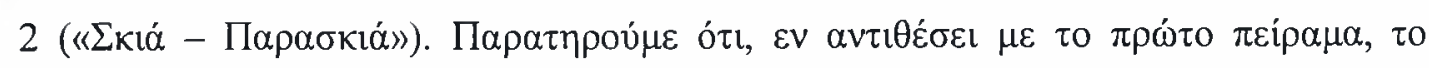

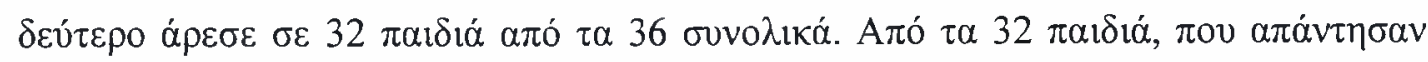




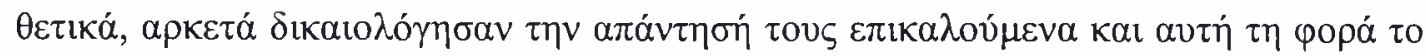

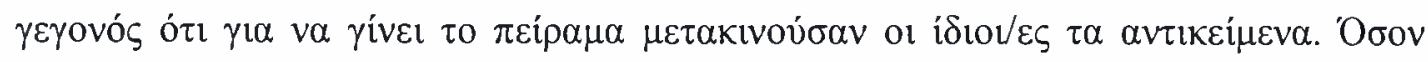

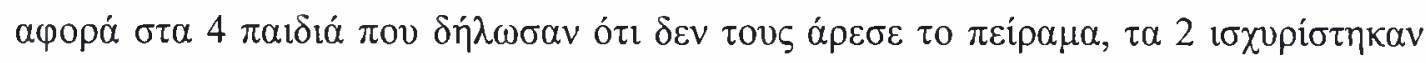

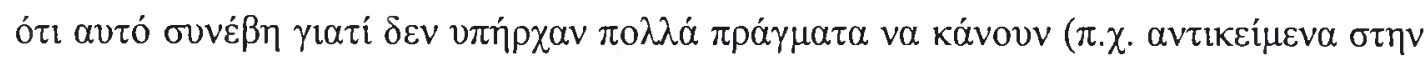

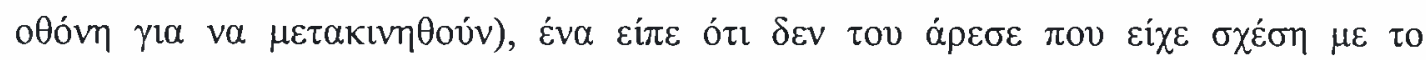

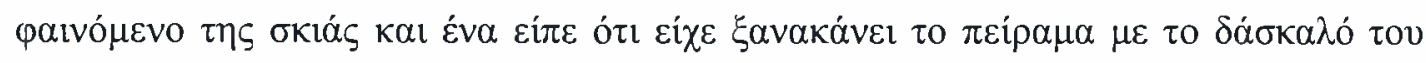
$\sigma \tau o \sigma \chi 0 \lambda \varepsilon i o(\mu \varepsilon \pi \varepsilon i \rho \alpha \mu \alpha \sigma \tau \eta \nu \tau \alpha \dot{\alpha} \xi \eta)$.

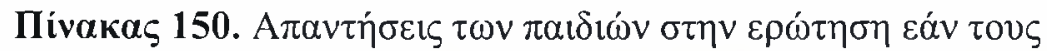

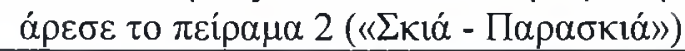

\begin{tabular}{|c|c|c|c|c|}
\hline \multirow{3}{*}{ 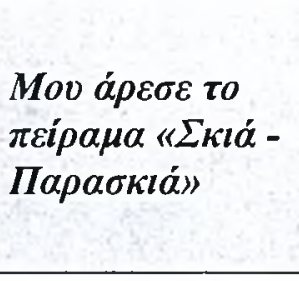 } & & 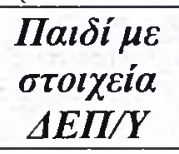 & 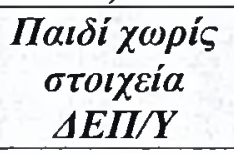 & $\sum v ́ v o \lambda o$ \\
\hline & $N \alpha l$ & 12 & 20 & 32 \\
\hline & $O \chi_{l}$ & 2 & 2 & 4 \\
\hline & 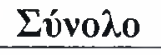 & 14 & 22 & 36 \\
\hline
\end{tabular}

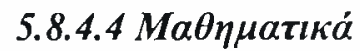

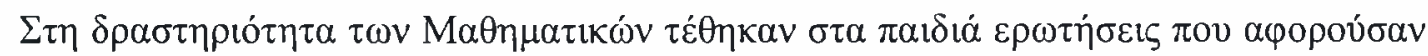

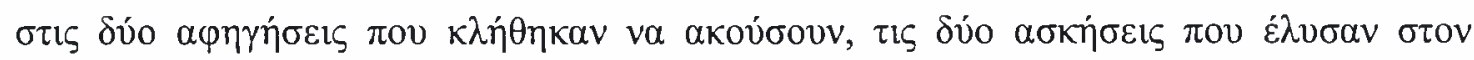

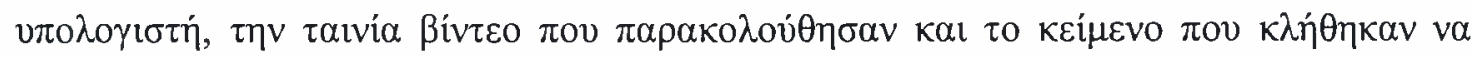
$\delta \imath \alpha \beta \dot{\sigma o v v . ~}$

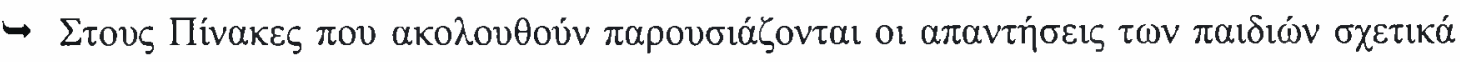

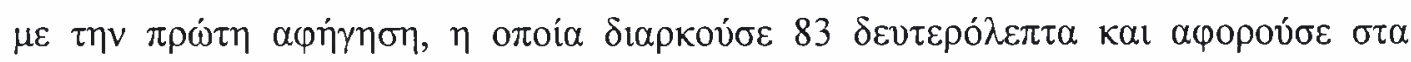

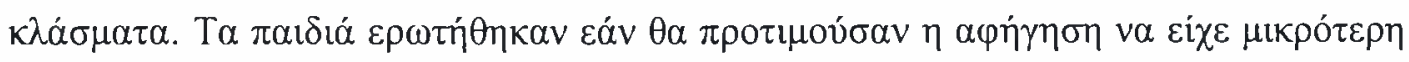

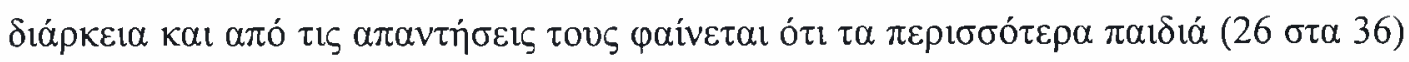

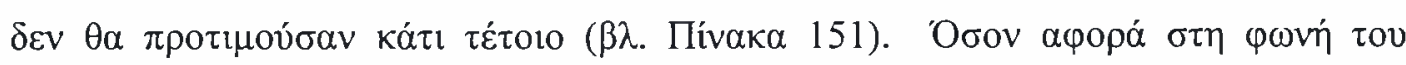

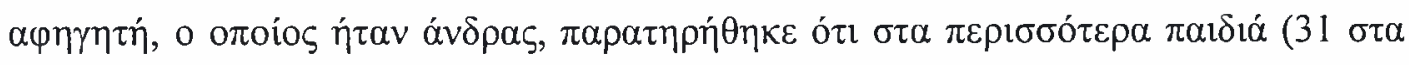

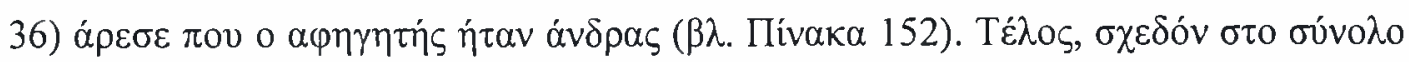

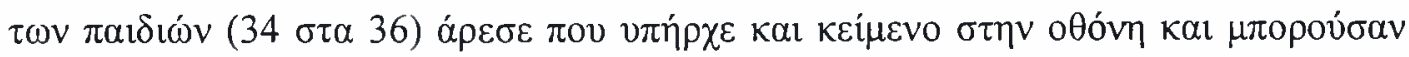

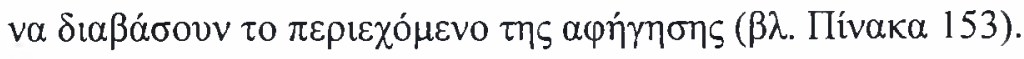




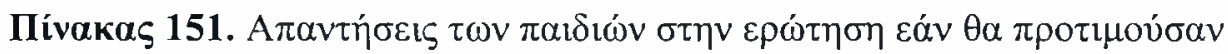

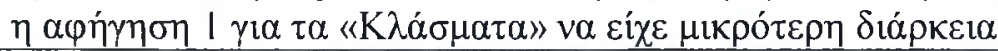

\begin{tabular}{|c|c|c|c|c|}
\hline \multirow{5}{*}{ 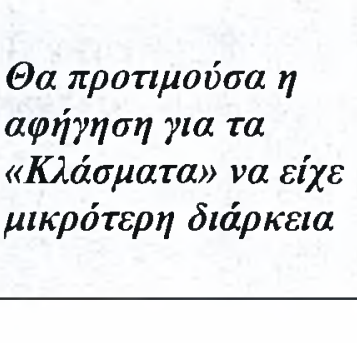 } & & 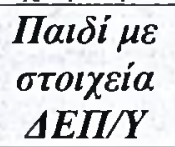 & 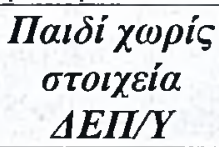 & 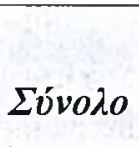 \\
\hline & $\Delta l \alpha \varphi \omega v \omega ́$ & 10 & 16 & 26 \\
\hline & $\Sigma v \mu \varphi \omega v \dot{\omega} \lambda i \gamma_{0}$ & 3 & 6 & 9 \\
\hline & 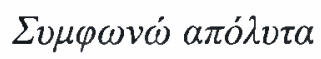 & 1 & & 1 \\
\hline & Ev́voגo & 14 & 22 & 36 \\
\hline
\end{tabular}

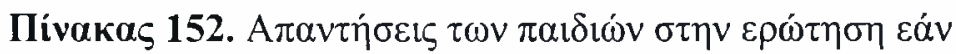

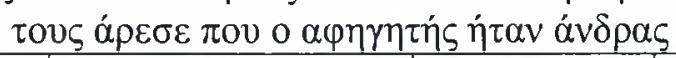

\begin{tabular}{|c|c|c|c|c|}
\hline \multirow{5}{*}{ 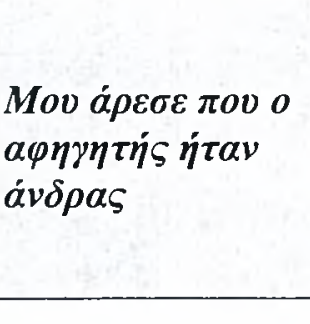 } & & 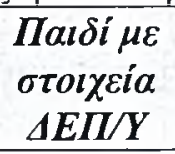 & 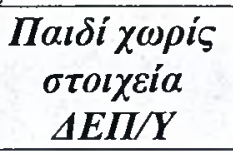 & $\Sigma v ́ v o \lambda o$ \\
\hline & $\Delta \iota \alpha \varphi \omega v \dot{~}$ & 2 & 3 & 5 \\
\hline & $\Sigma v \mu \varphi \omega v \omega \dot{\lambda} \lambda \dot{\imath} \gamma o$ & 2 & 6 & 8 \\
\hline & $\Sigma v \mu \varphi \omega v \dot{\omega} \alpha \pi \delta ́ \lambda v \tau \alpha$ & 10 & 13 & 23 \\
\hline & 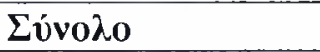 & 14 & 22 & 36 \\
\hline
\end{tabular}

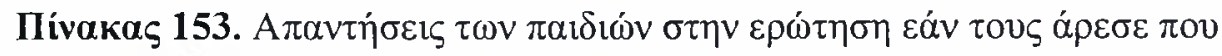

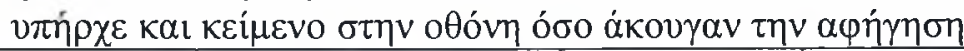

\begin{tabular}{|c|c|c|c|c|}
\hline \multirow{5}{*}{ 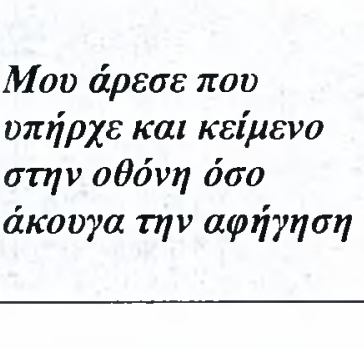 } & 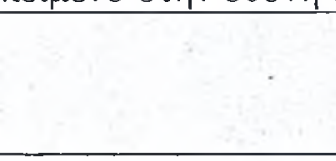 & $\begin{array}{c}\Pi \alpha \iota \delta i \mu \varepsilon \\
\sigma \tau o \iota \chi \varepsilon i \alpha \\
\Delta E \Pi / Y\end{array}$ & 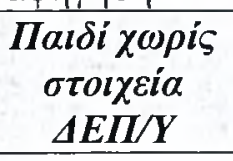 & 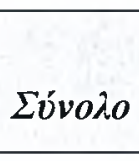 \\
\hline & $\Delta \imath \alpha \varphi \omega \nu \omega ́$ & & 2 & 2 \\
\hline & 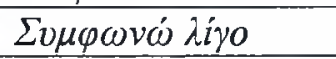 & 4 & 3 & 7 \\
\hline & $\Sigma v \mu \varphi \omega v \dot{\omega} \alpha \pi \delta ́ \lambda v \tau \alpha$ & 10 & 17 & 27 \\
\hline & 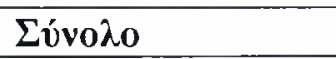 & 14 & 22 & 36 \\
\hline
\end{tabular}

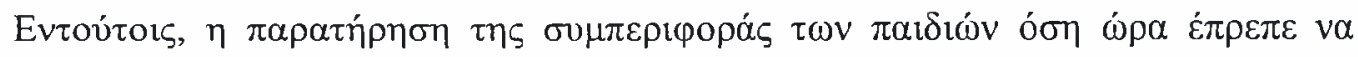

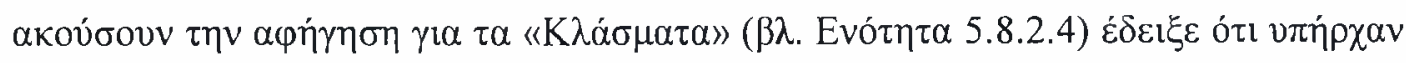

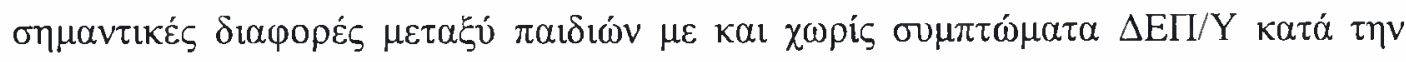

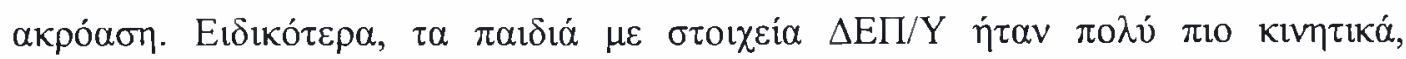

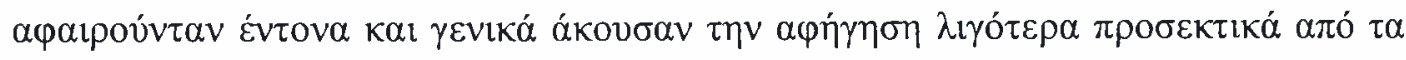

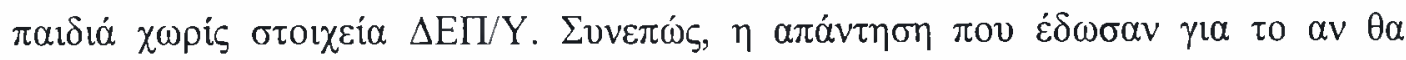

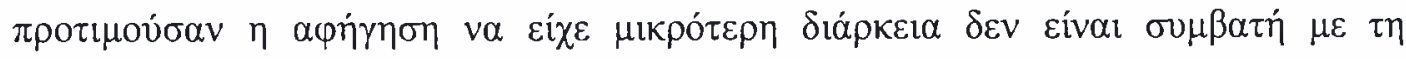

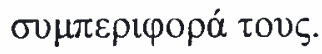




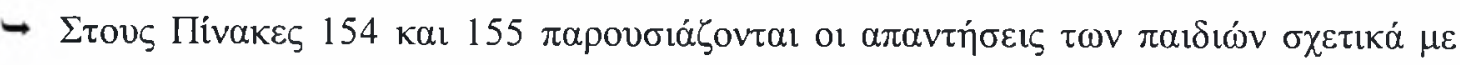

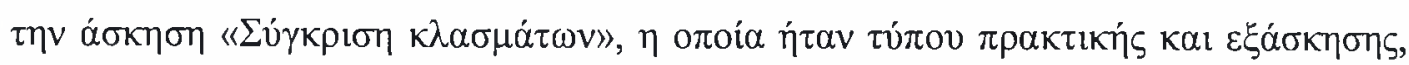

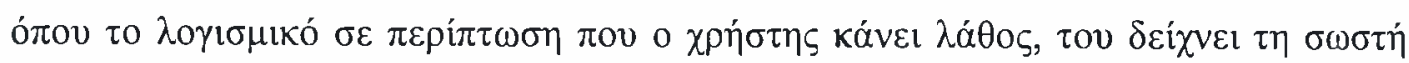

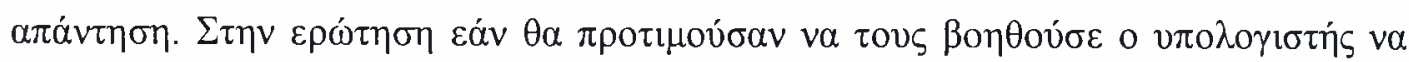

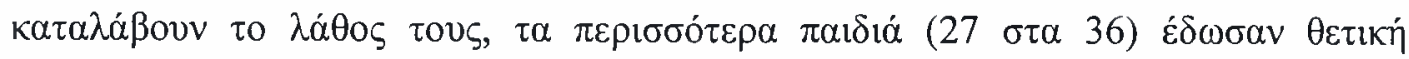

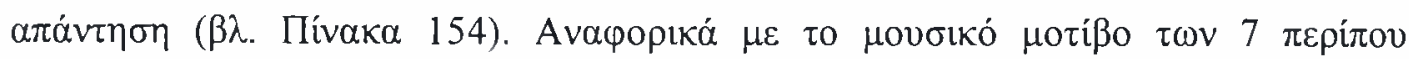

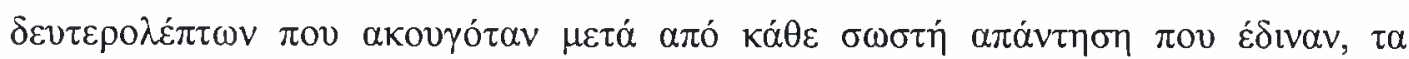

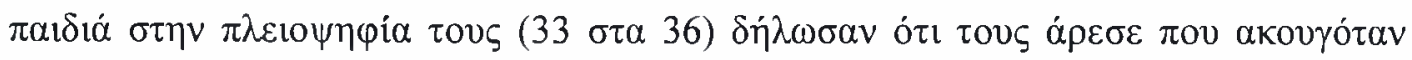

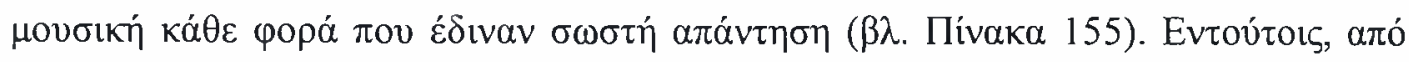

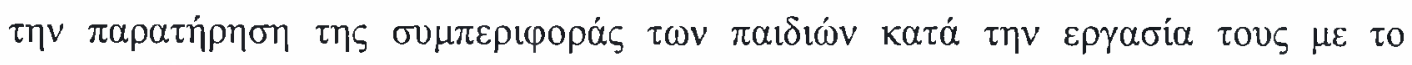

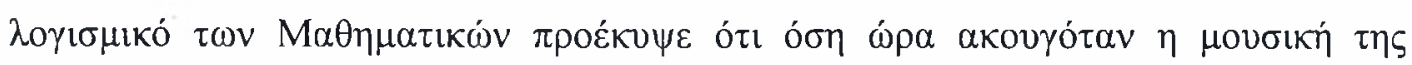

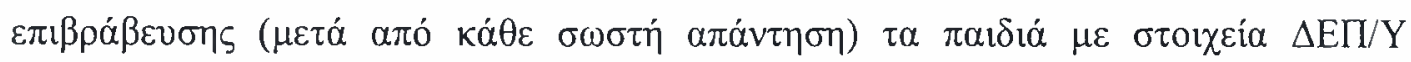

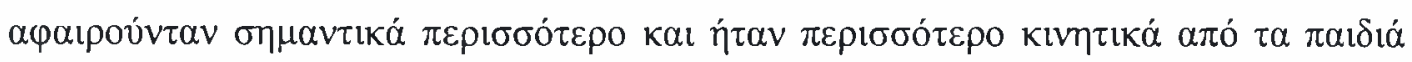

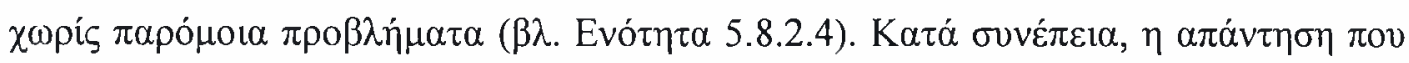

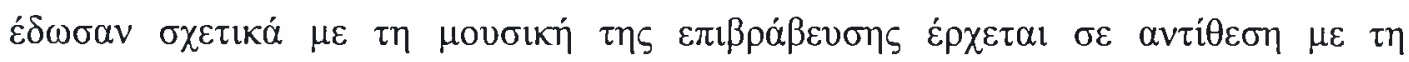

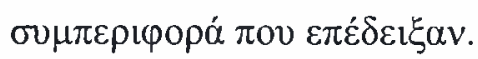

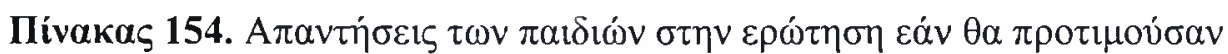

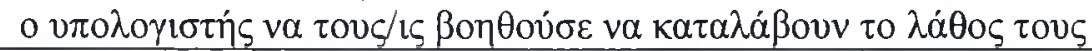

\begin{tabular}{|c|c|c|c|c|}
\hline \multirow{4}{*}{ 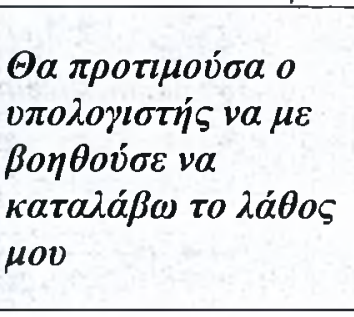 } & & 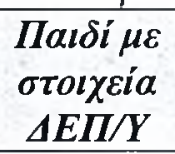 & 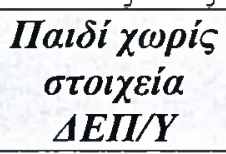 & 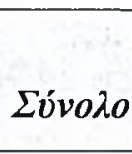 \\
\hline & $\Delta \iota \alpha \varphi \omega v \omega \dot{~}$ & 3 & 6 & 9 \\
\hline & 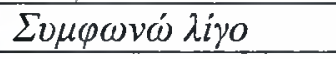 & 6 & 12 & 18 \\
\hline & $\Sigma v \mu \varphi \omega v \omega ́ \alpha \pi \dot{\lambda} v \tau \alpha$ & 5 & 4 & 9 \\
\hline & 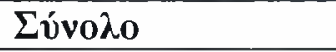 & 14 & 22 & 36 \\
\hline
\end{tabular}

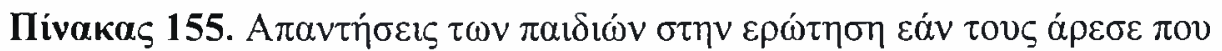

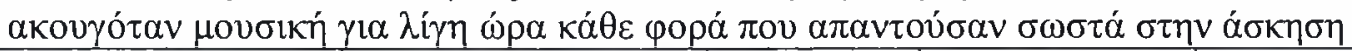

\begin{tabular}{|c|c|c|c|c|}
\hline \multirow{4}{*}{ 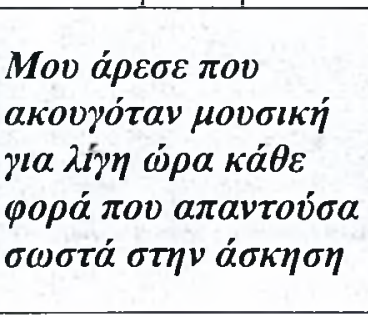 } & & 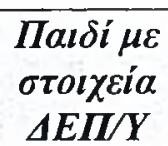 & 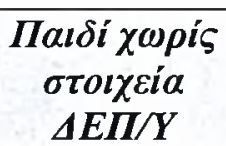 & $\Sigma v ́ v o \lambda o$ \\
\hline & $\Delta \iota \alpha \varphi \omega \nu \omega \dot{\omega}$ & 2 & 1 & 3 \\
\hline & 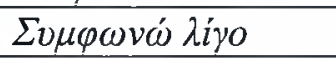 & & 5 & 5 \\
\hline & $\Sigma v \mu \varphi \omega v \omega ́ \alpha \pi \dot{\lambda} \lambda v \tau \alpha$ & 12 & 16 & 28 \\
\hline & 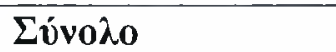 & 14 & 22 & 36 \\
\hline
\end{tabular}




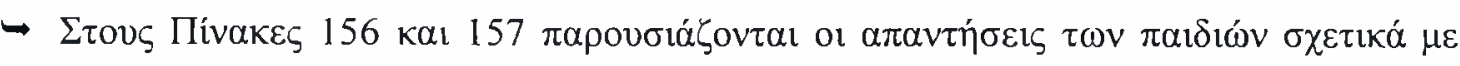

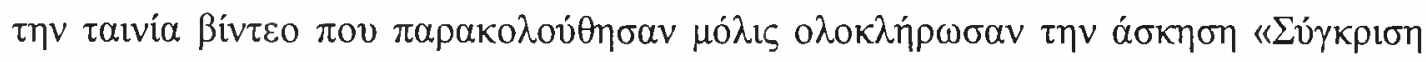

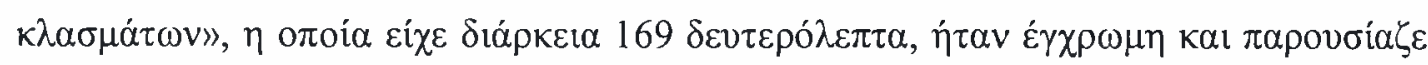

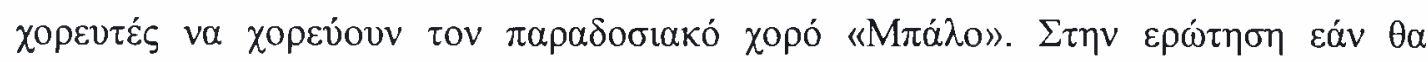

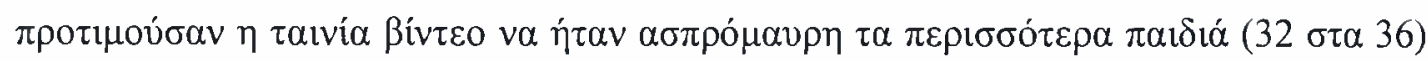

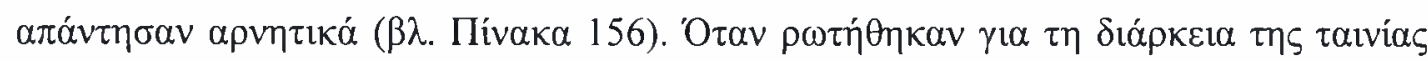

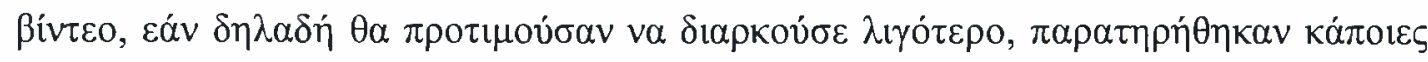

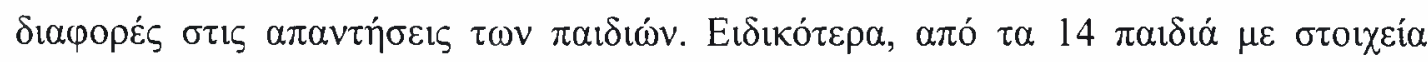

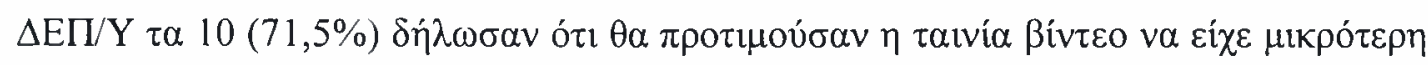

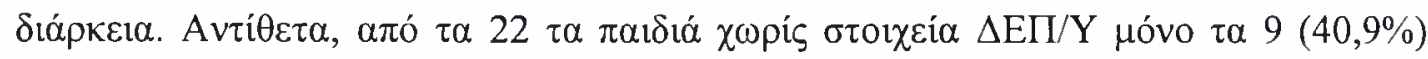

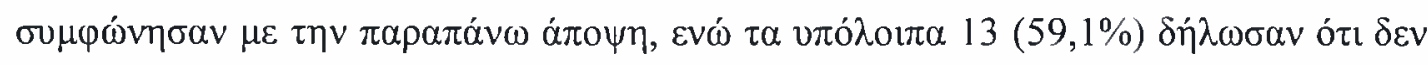

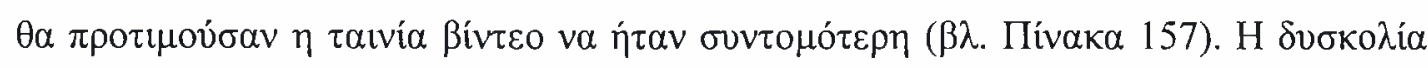

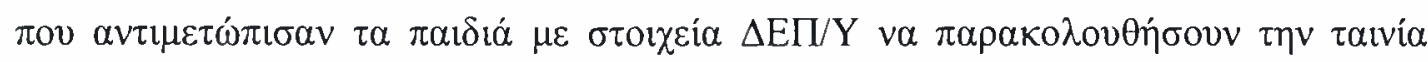

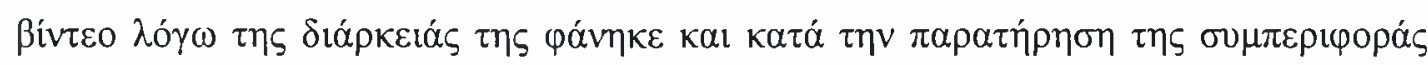

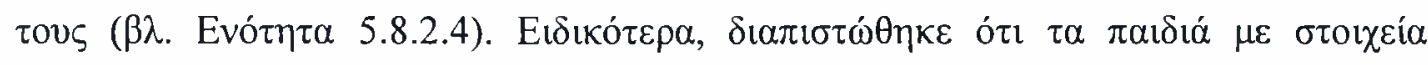

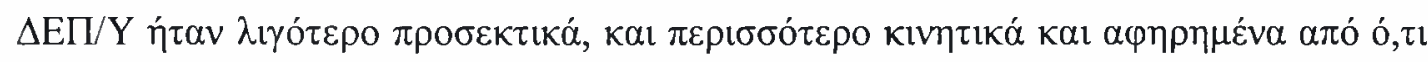

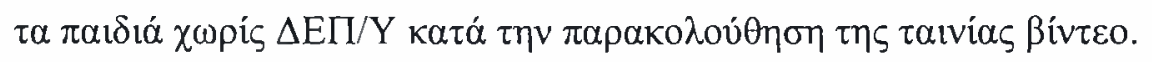

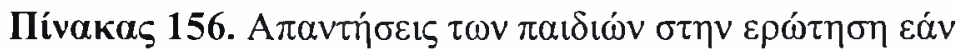

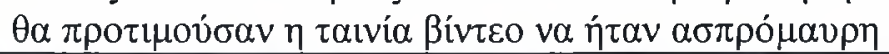

\begin{tabular}{|c|c|c|c|c|}
\hline \multirow{5}{*}{ 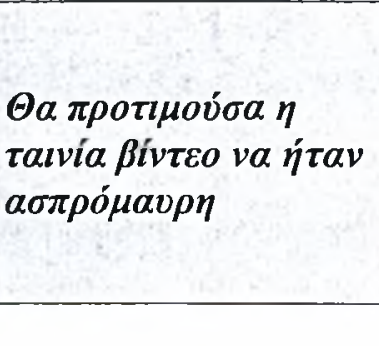 } & & $\begin{array}{c}\Pi a \imath \delta i \mu \varepsilon \\
\sigma \tau o \iota \chi \varepsilon i ́ a \\
\Delta E \Pi / Y\end{array}$ & 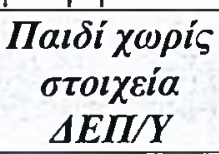 & 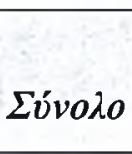 \\
\hline & $\Delta \imath \alpha \varphi \omega v \omega ́$ & 13 & 19 & 32 \\
\hline & $\Sigma v \mu \varphi \omega v \dot{\omega} \lambda i \gamma_{o}$ & & 3 & 3 \\
\hline & $\Sigma v \mu \varphi \omega v \dot{\omega} \alpha \pi \dot{\lambda} \lambda v \tau \alpha$ & 1 & & 1 \\
\hline & Ev́voגo & 14 & 22 & 36 \\
\hline
\end{tabular}

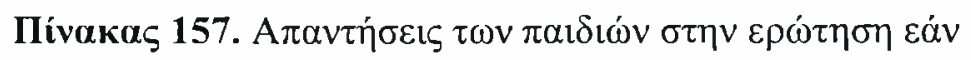

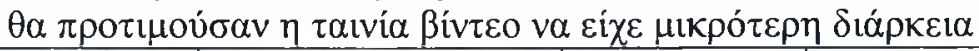

\begin{tabular}{|c|c|c|c|c|}
\hline \multirow{4}{*}{ 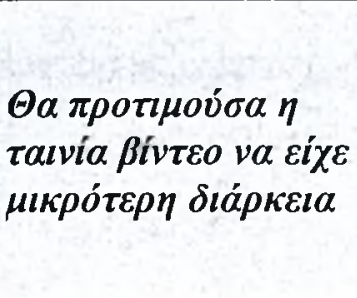 } & & $\begin{array}{c}\text { Паıьí } \mu \varepsilon \\
\sigma \tau o \imath \chi \varepsilon i ́ a \\
\Delta E \Pi / Y\end{array}$ & 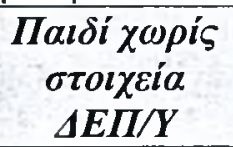 & 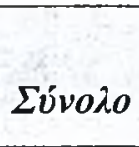 \\
\hline & $\Delta \imath \alpha \varphi \omega \nu \omega ́$ & 4 & 13 & 17 \\
\hline & $\sum v \mu \varphi \omega \nu \dot{\omega} \lambda i \gamma_{0}$ & 6 & 6 & 12 \\
\hline & $\Sigma v \mu \varphi \omega v \dot{\omega} \alpha \pi \delta ́ \lambda v \tau \alpha$ & 4 & 3 & 7 \\
\hline
\end{tabular}




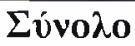
14 22 36

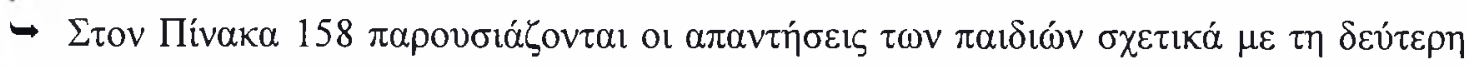

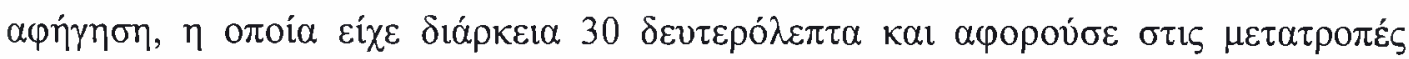

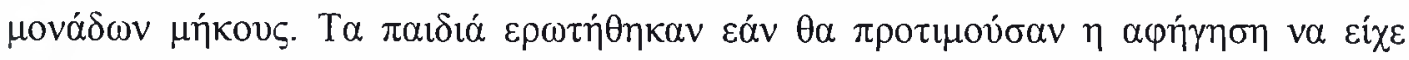

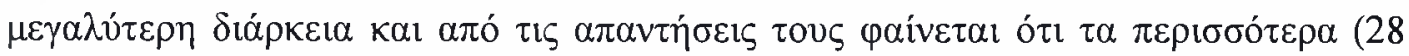

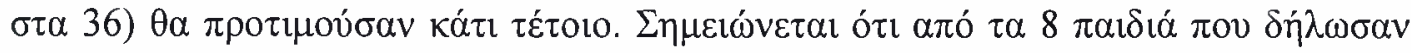

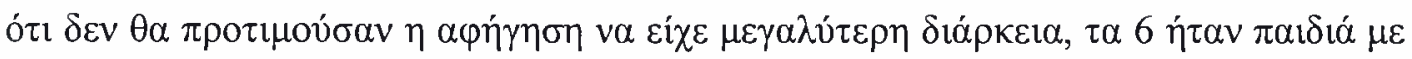

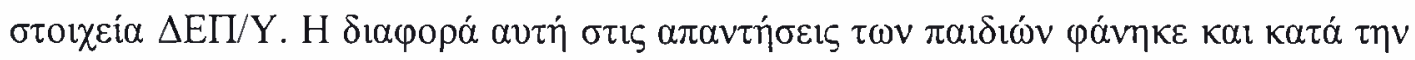

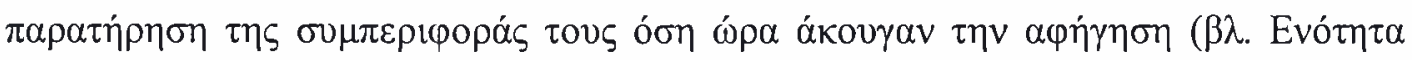

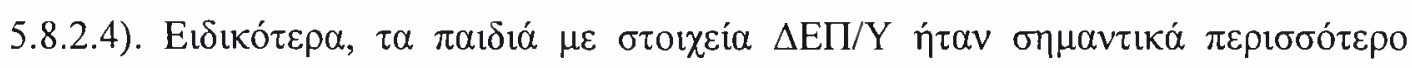

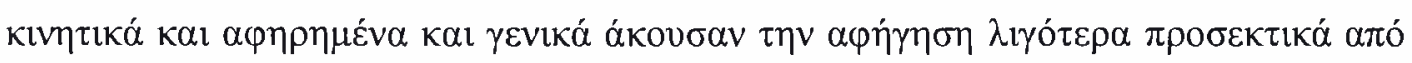

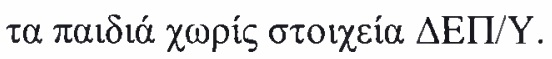

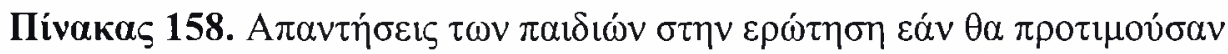

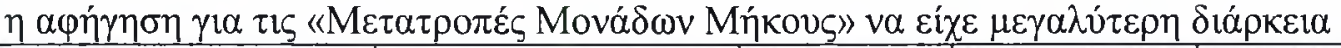

\begin{tabular}{|c|c|c|c|c|}
\hline \multirow{4}{*}{ 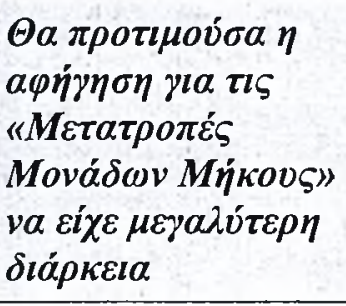 } & & 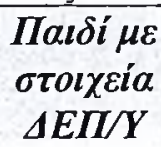 & 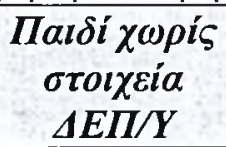 & 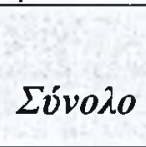 \\
\hline & $\Delta \iota \alpha \varphi \omega v \dot{\omega}$ & 6 & 2 & 8 \\
\hline & $\Sigma v \mu \varphi \omega v \omega^{\prime} \lambda i \gamma_{0}$ & 6 & 13 & 19 \\
\hline & $\Sigma v \mu \varphi \omega v \omega ́ \alpha \pi \delta ́ \lambda v \tau \alpha$ & 2 & 7 & 9 \\
\hline & Eúvoגo & 14 & 22 & 36 \\
\hline
\end{tabular}

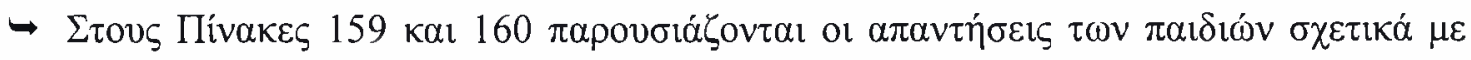

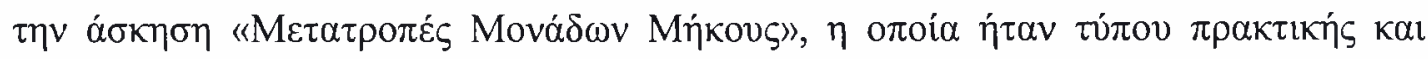

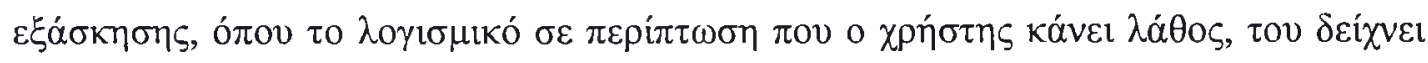

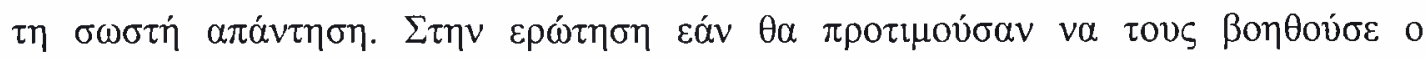

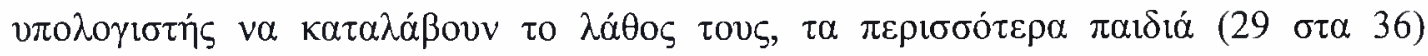

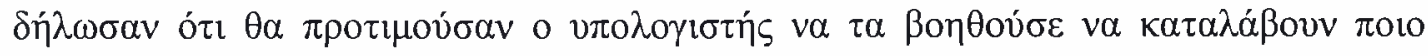

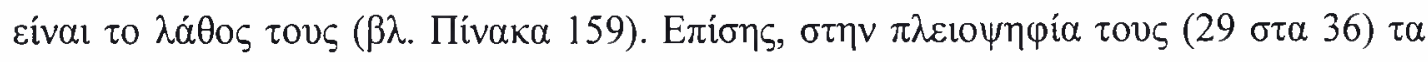

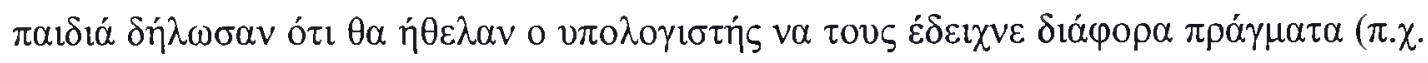

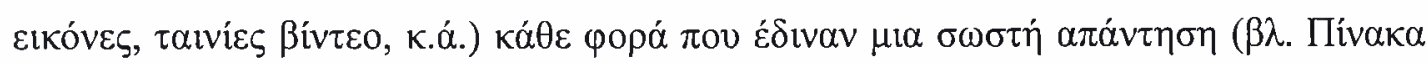

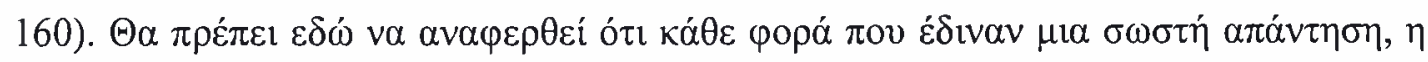




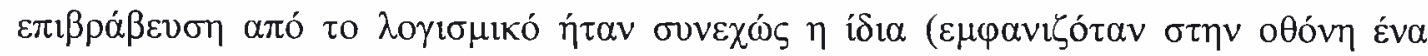
$\alpha \varepsilon \rho \circ \pi \lambda a ́ v o \pi$ ov $\alpha \pi \mathrm{a} \gamma \varepsilon(\omega v o ́ \tau \alpha v)$.

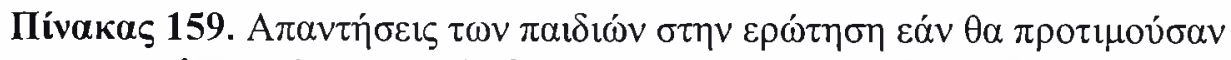

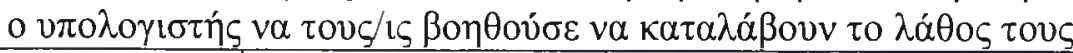

\begin{tabular}{|c|c|c|c|c|}
\hline \multirow{4}{*}{ 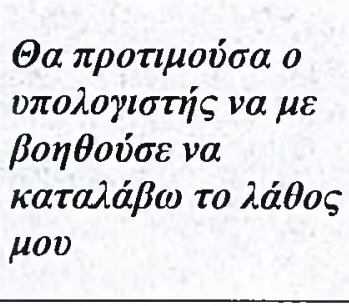 } & & 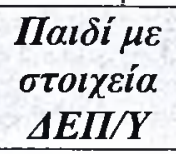 & 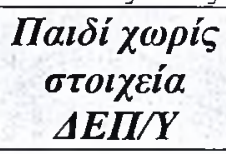 & 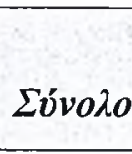 \\
\hline & $\Delta \imath \alpha \varphi \omega v \omega ́$ & 4 & 3 & 7 \\
\hline & $\Sigma v \mu \varphi \omega v \dot{~ \lambda i ́ \gamma o}$ & 5 & 12 & 17 \\
\hline & $\Sigma v \mu \varphi \omega v \omega \dot{\alpha} \alpha \pi \delta^{\prime} \nu \tau \tau \alpha$ & 5 & 7 & 12 \\
\hline & Ev́voגo & 14 & 22 & 36 \\
\hline
\end{tabular}

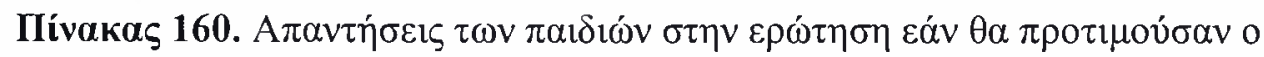

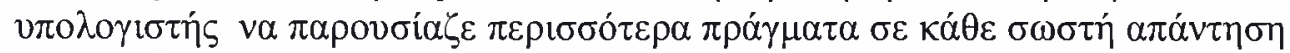

\begin{tabular}{|c|c|c|c|c|}
\hline \multirow{5}{*}{ 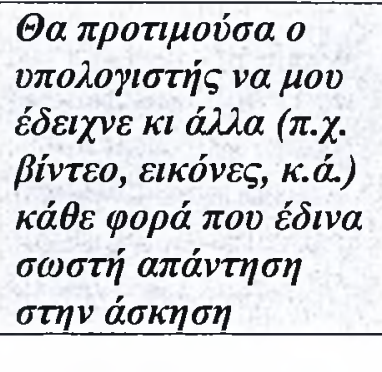 } & & 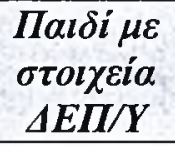 & 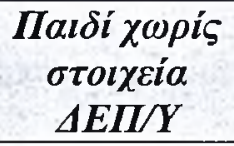 & $\Sigma v ́ v o \lambda o$ \\
\hline & $\Delta l \alpha \varphi \omega \nu \omega \dot{ }$ & 4 & 3 & 7 \\
\hline & $\Sigma v \mu \varphi \omega v \omega \dot{\omega} \lambda i \gamma o$ & 3 & 10 & 13 \\
\hline & $\Sigma v \mu \varphi \omega v \dot{\omega} \alpha \pi \delta ́ \lambda v \tau \alpha$ & 7 & 9 & 16 \\
\hline & Ev́voגo & 14 & 22 & 36 \\
\hline
\end{tabular}

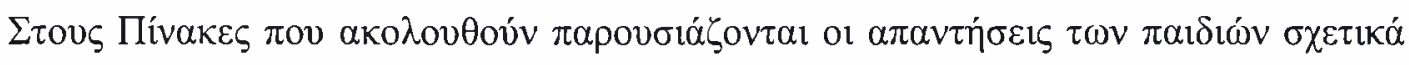

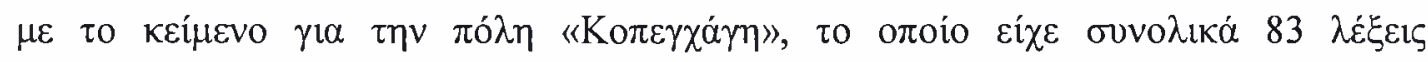

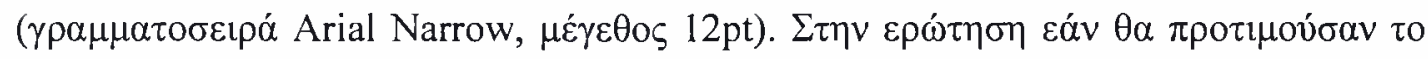

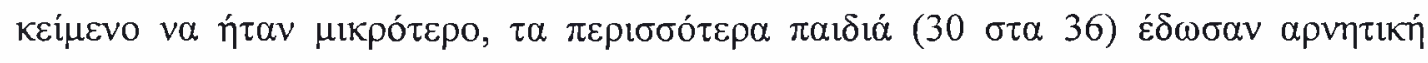

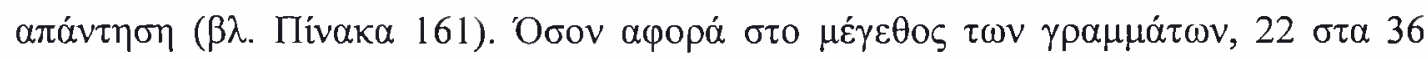

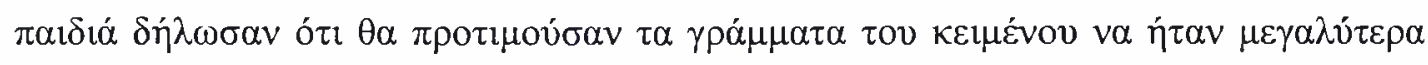

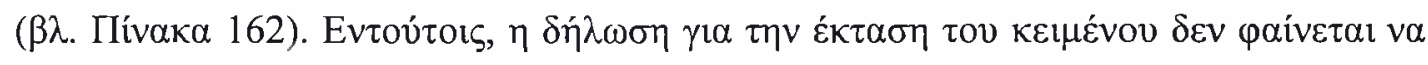

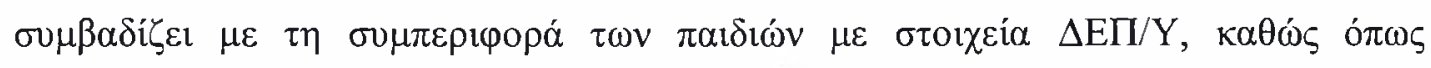

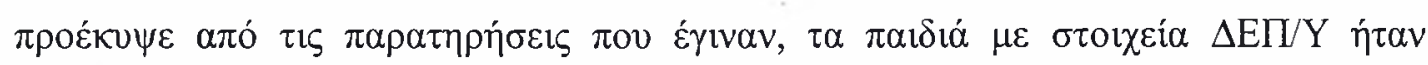

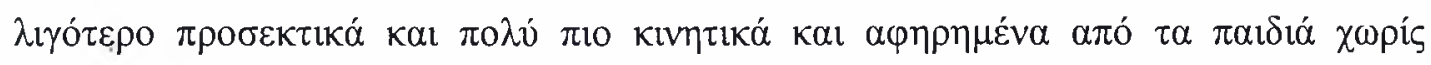

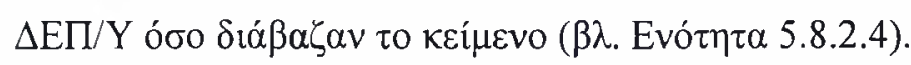




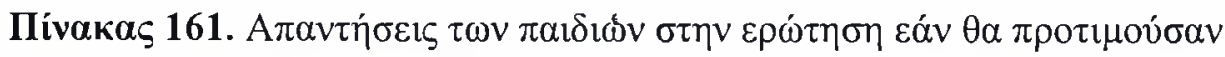

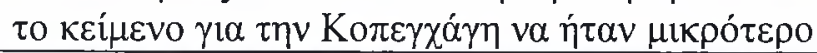

\begin{tabular}{|c|c|c|c|c|}
\hline \multirow{4}{*}{ 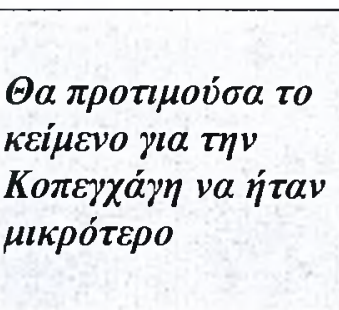 } & & 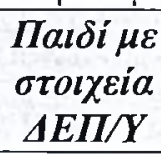 & 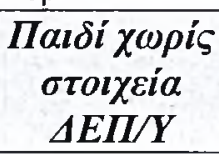 & $\Sigma v ́ v o \lambda o$ \\
\hline & $\Delta l \alpha \varphi \omega \nu \omega ́$ & 13 & 17 & 30 \\
\hline & 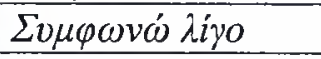 & 1 & 5 & 6 \\
\hline & $\Sigma v \mu \varphi \omega \nu \omega^{\prime} \alpha \pi \dot{\lambda} \lambda v \tau \alpha$ & & & \\
\hline & 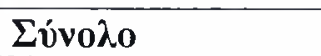 & 14 & 22 & 36 \\
\hline
\end{tabular}

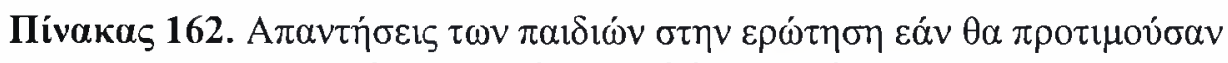

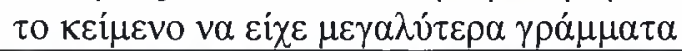

\begin{tabular}{|c|c|c|c|c|}
\hline \multirow{5}{*}{ 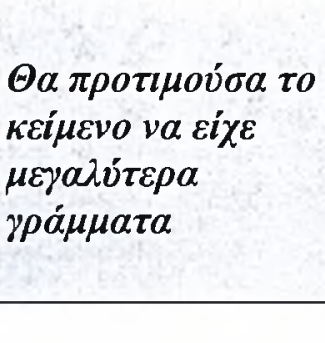 } & & 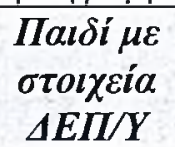 & $\begin{array}{c}\text { Maısi } \chi \omega \rho i \varsigma \\
\sigma \tau o \imath \chi \varepsilon i \alpha \\
\Delta E \Pi / Y\end{array}$ & $\Sigma v ́ v o \lambda o$ \\
\hline & $\Delta \imath \alpha \varphi \omega \nu \omega \dot{\omega}$ & 5 & 9 & 14 \\
\hline & 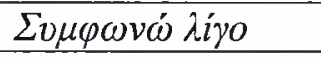 & 7 & 11 & 18 \\
\hline & 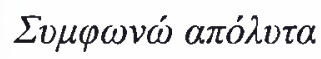 & 2 & 2 & 4 \\
\hline & Ev́voגo & 14 & 22 & 36 \\
\hline
\end{tabular}




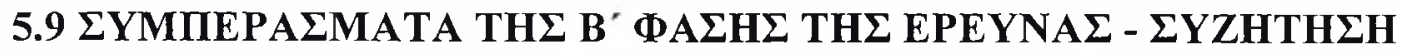

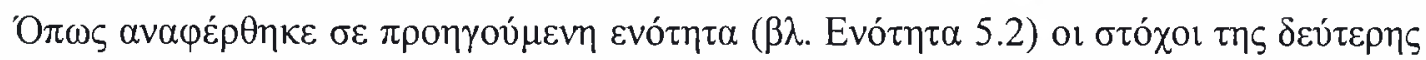

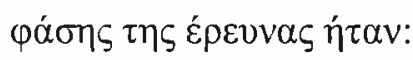

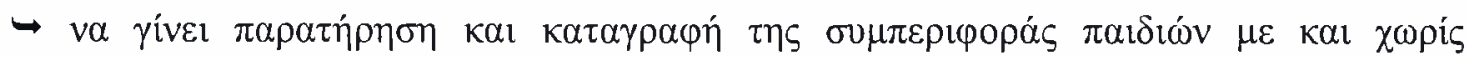

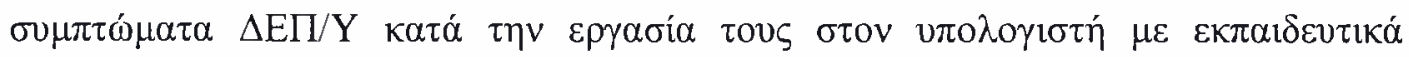

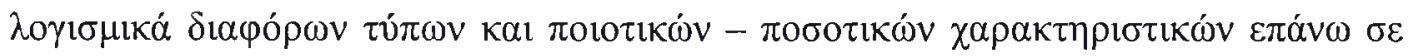

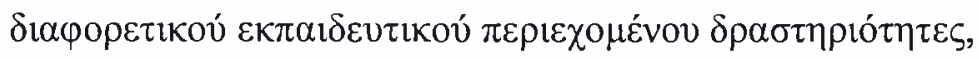

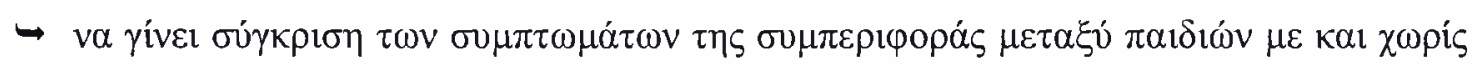

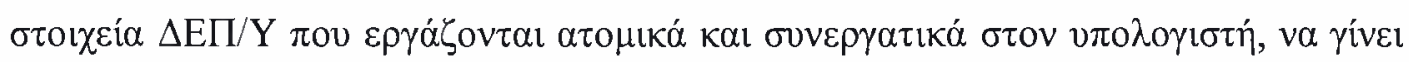

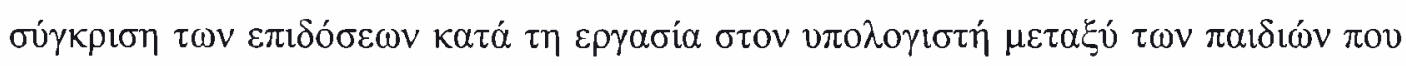

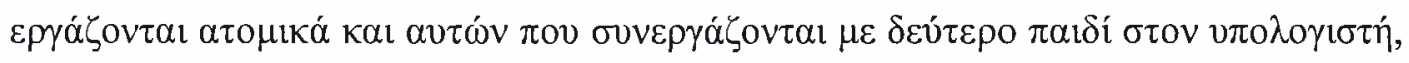
$\kappa \alpha l$,

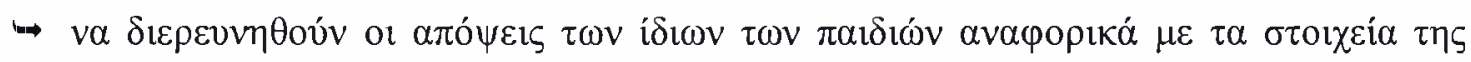

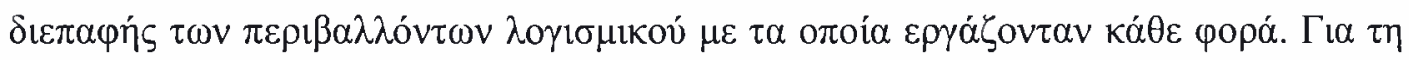

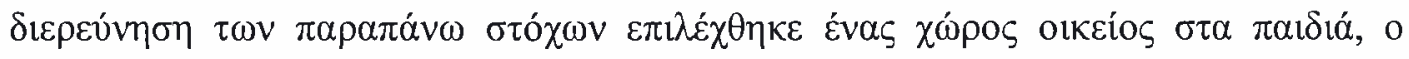

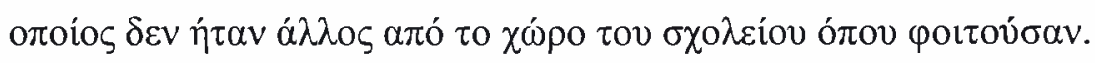

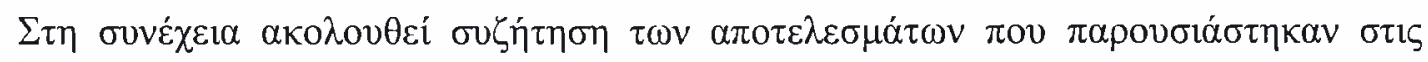

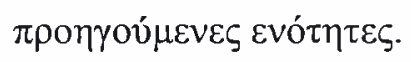

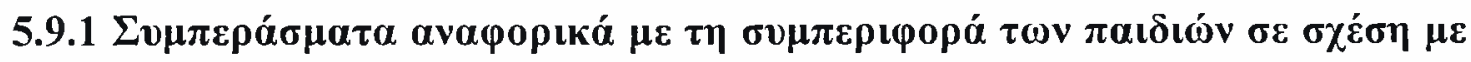

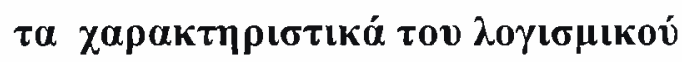

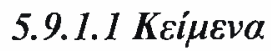

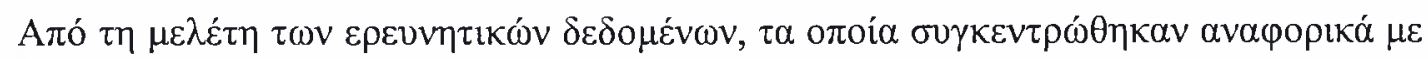

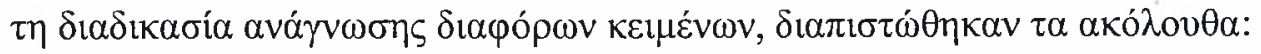

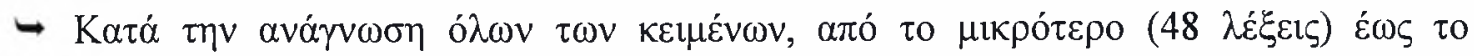

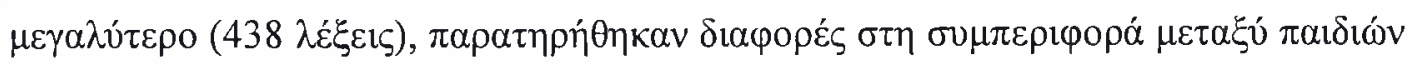

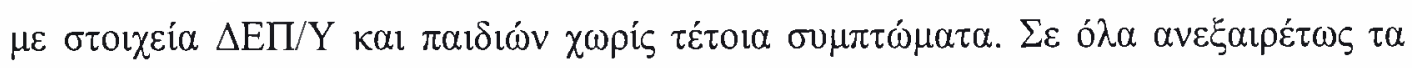




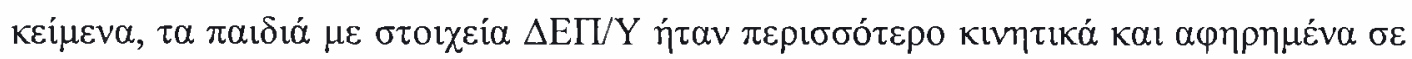

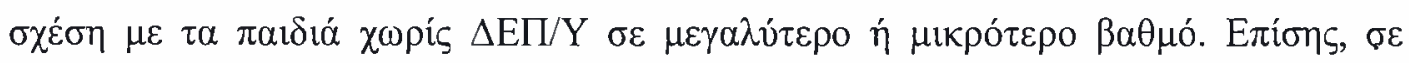

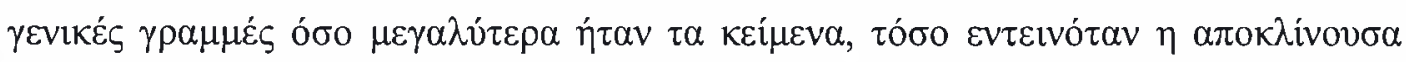

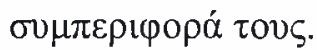

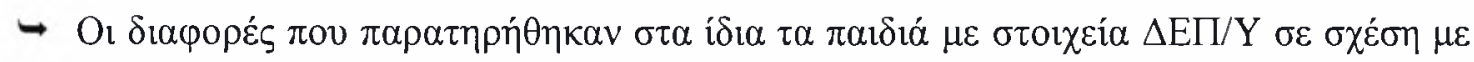

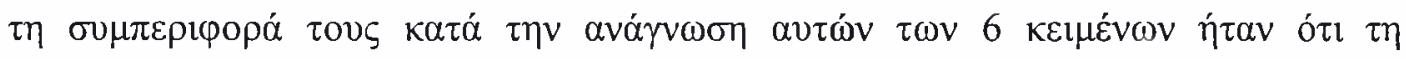

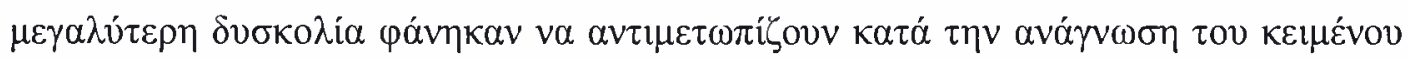

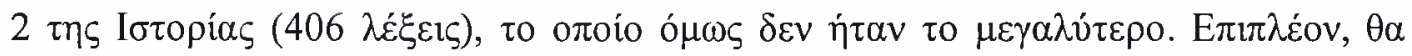

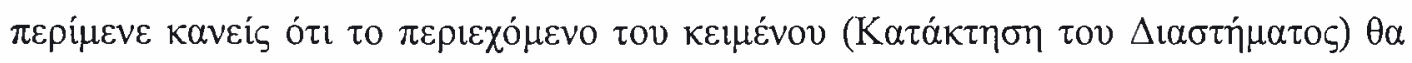

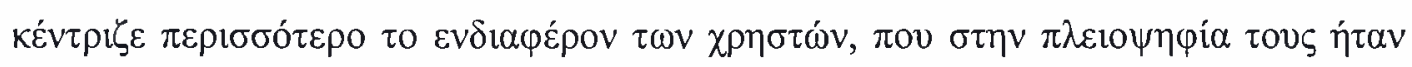

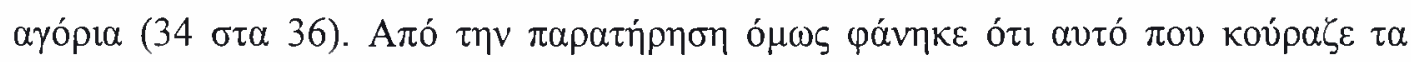

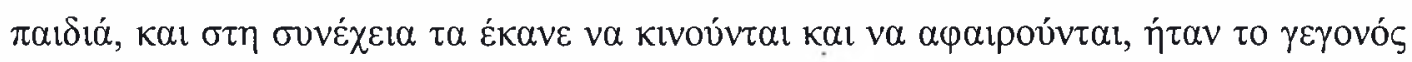

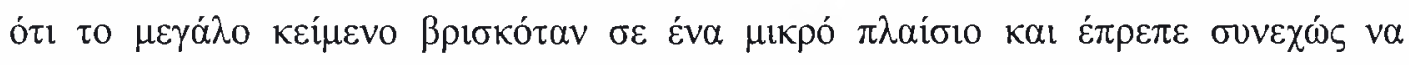

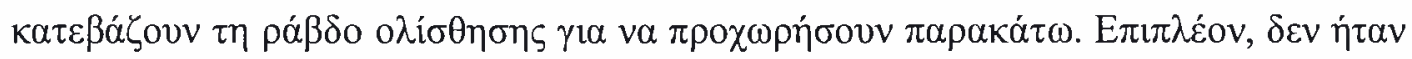

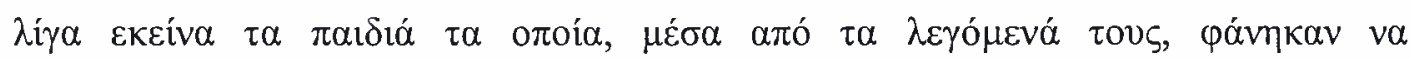

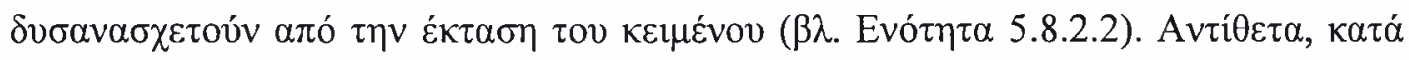

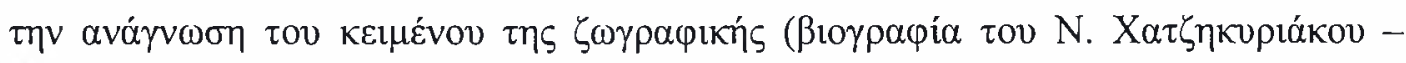

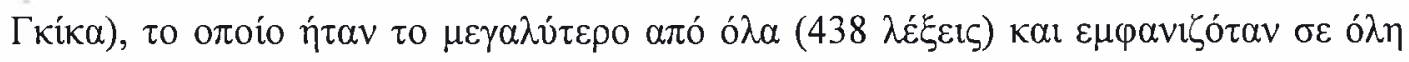

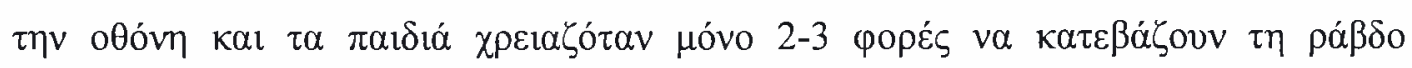

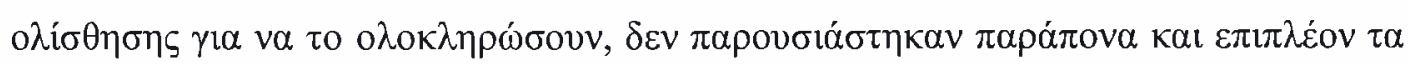

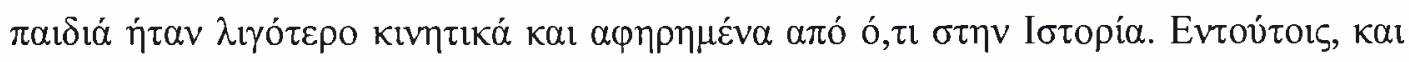

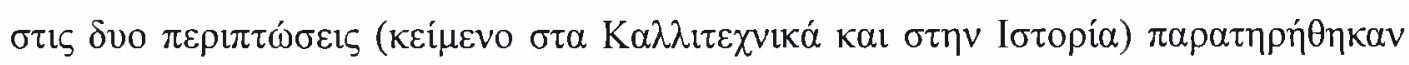

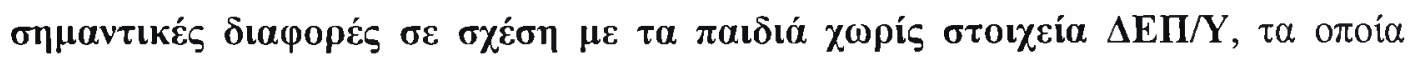

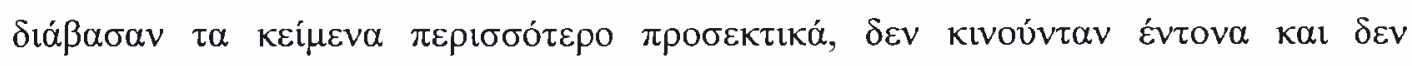

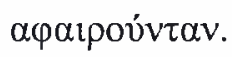

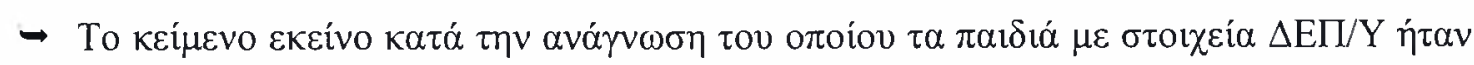

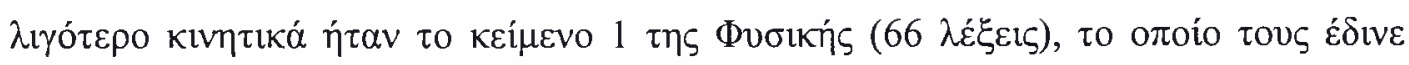

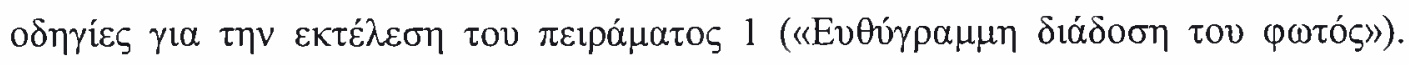

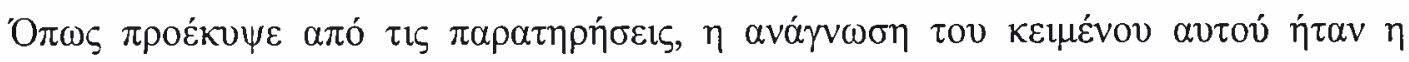

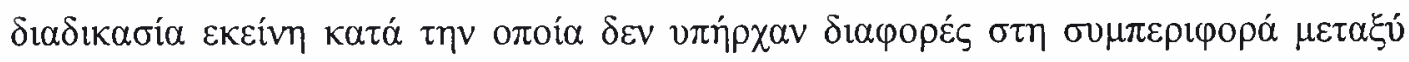

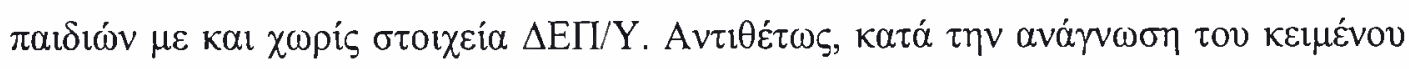




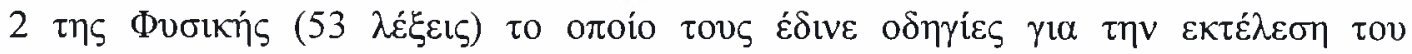

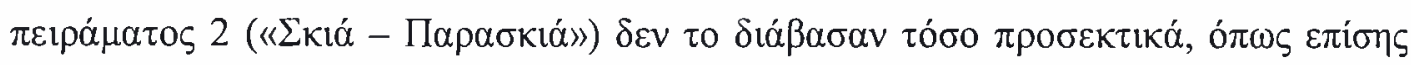

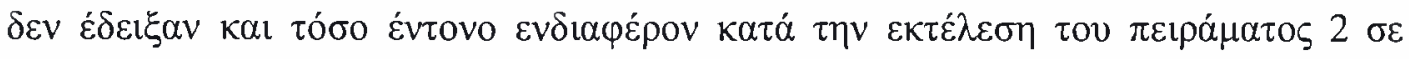

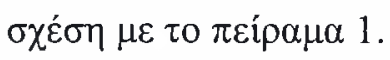

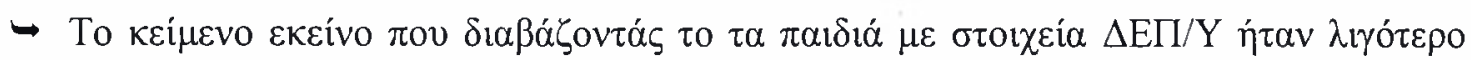

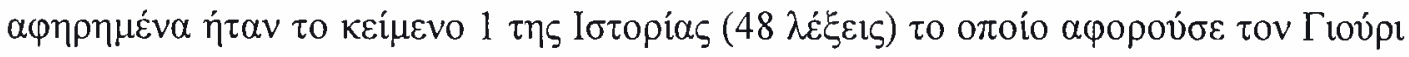

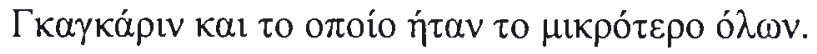

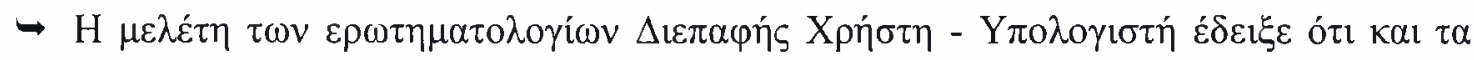

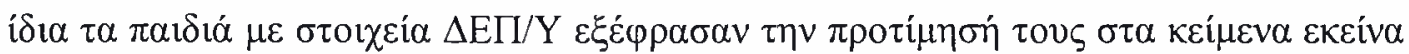

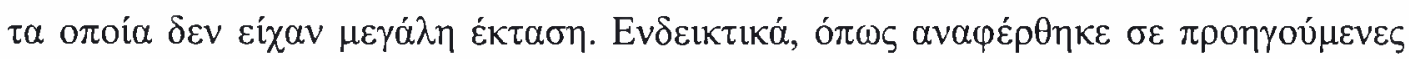

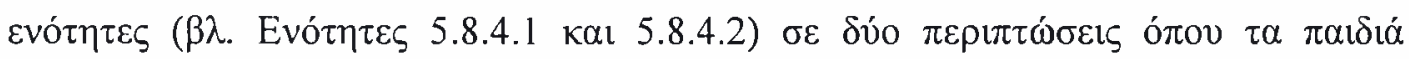

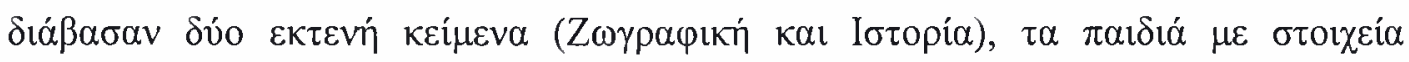

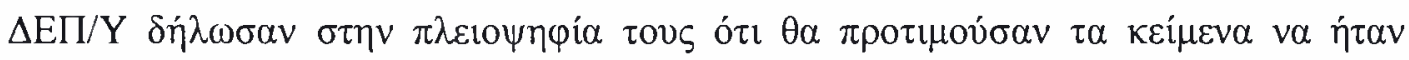

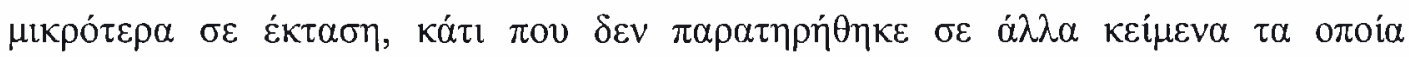

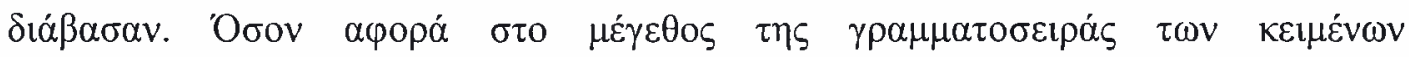

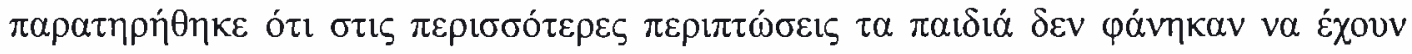

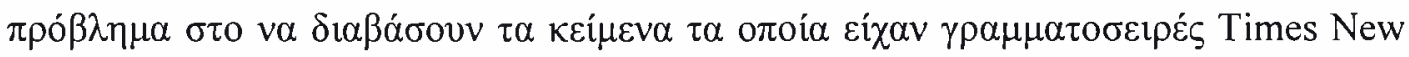

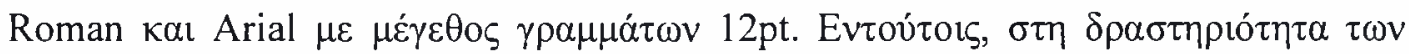

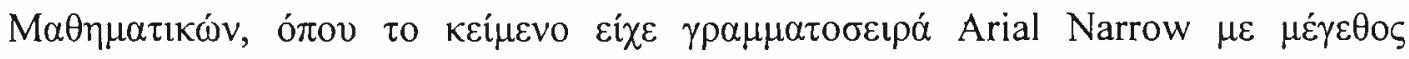

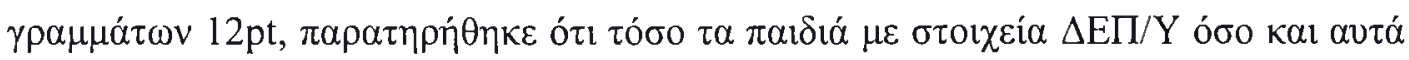

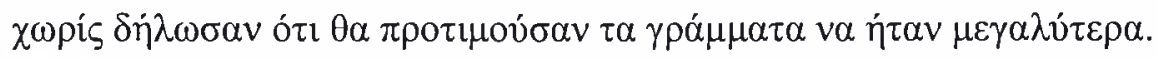

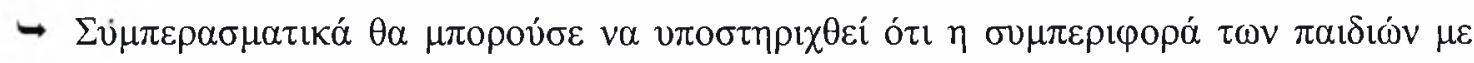

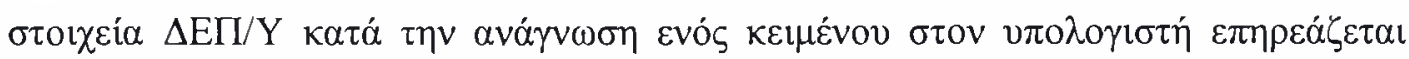
$\alpha \pi$ ó:

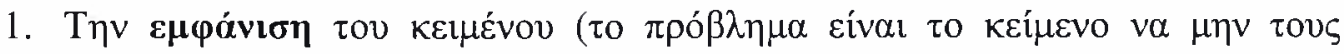

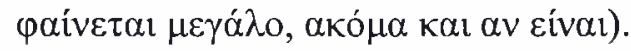

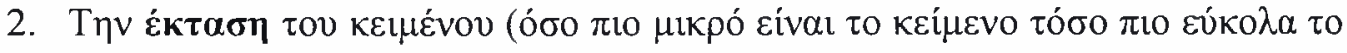

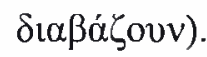

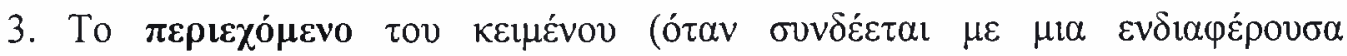

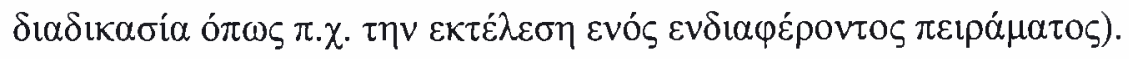




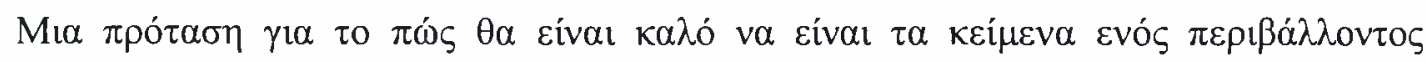

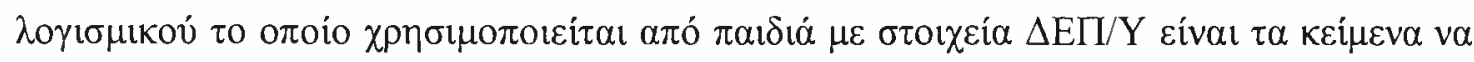

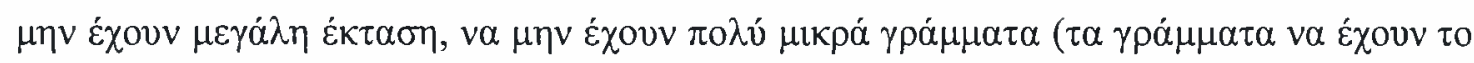

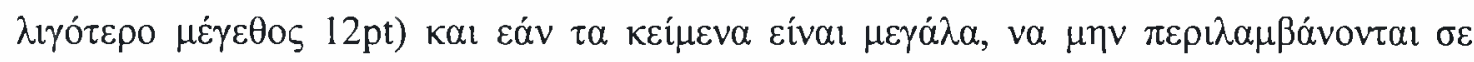

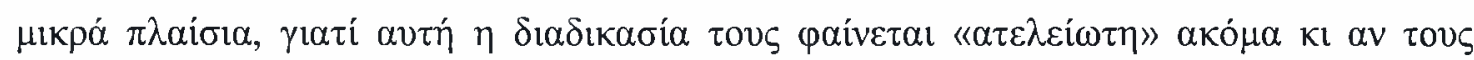

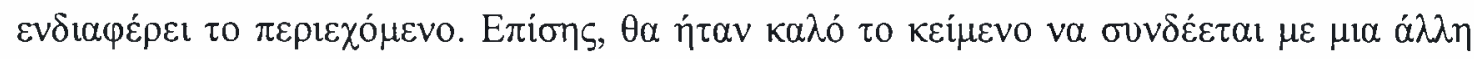

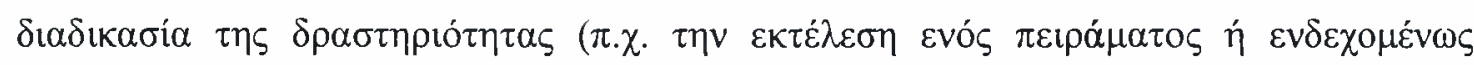

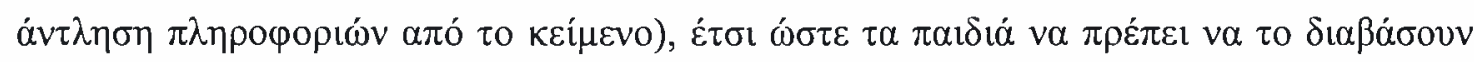

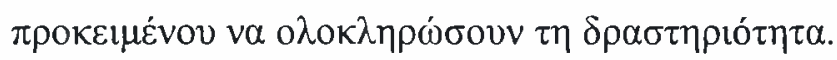

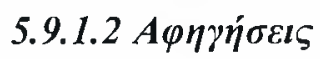

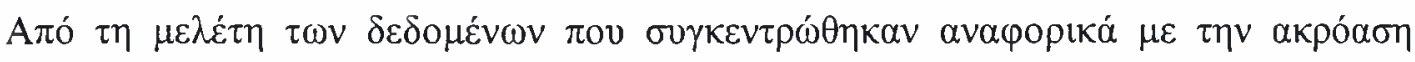

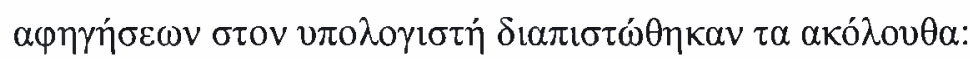

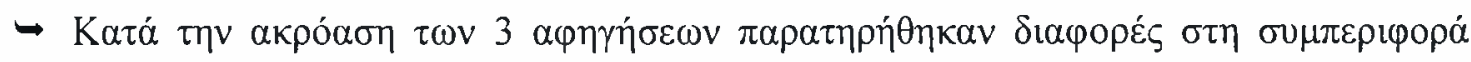

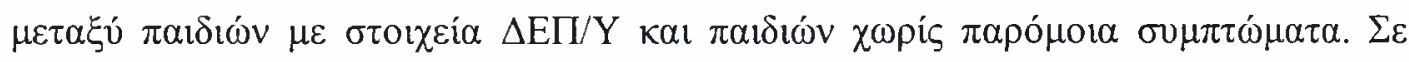

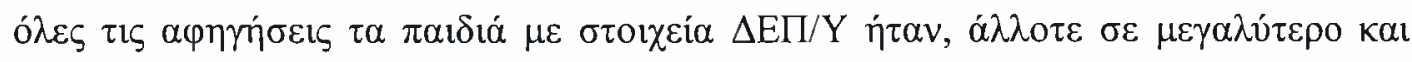

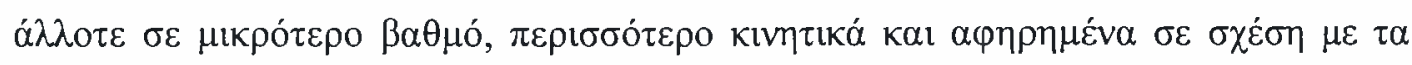

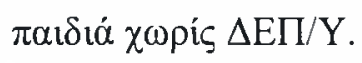

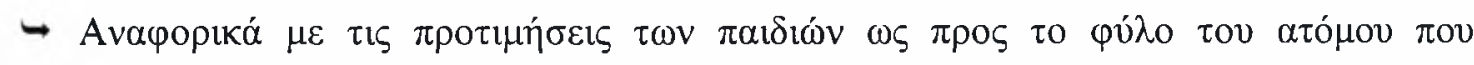

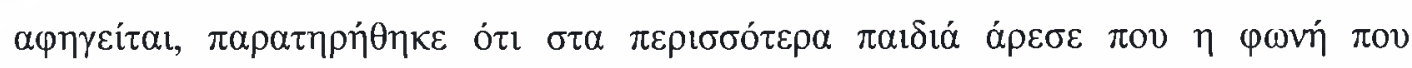

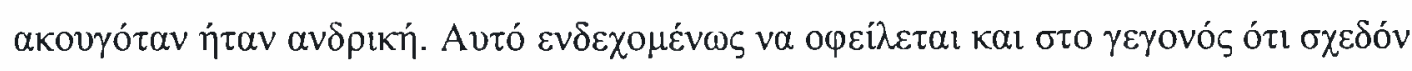

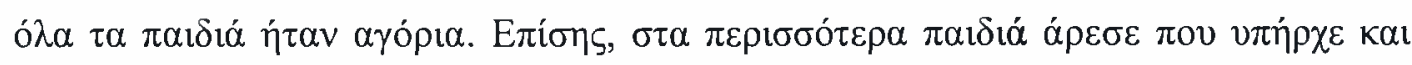

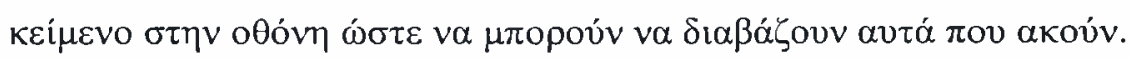

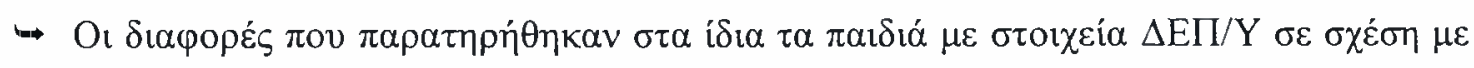

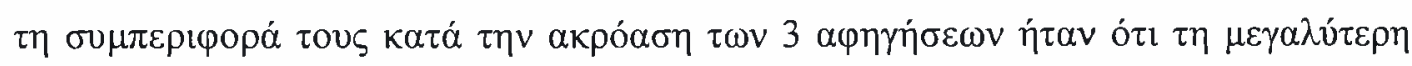

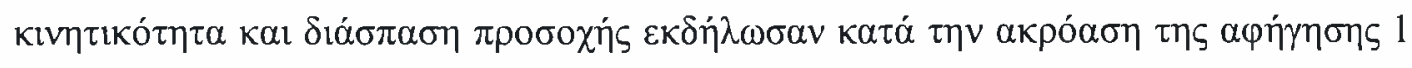

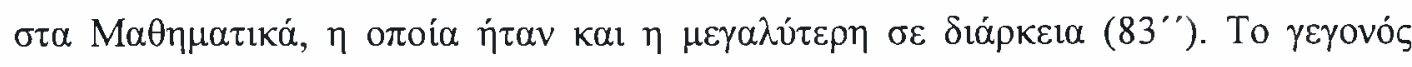

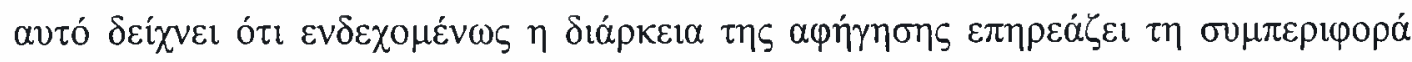

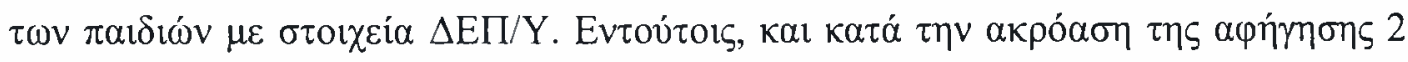

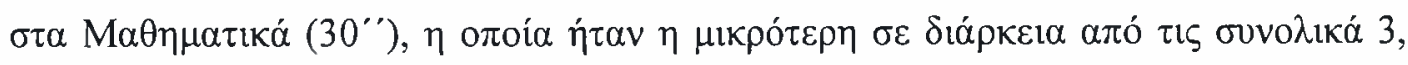




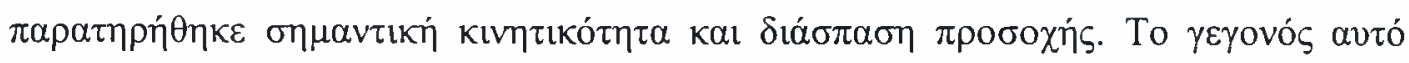

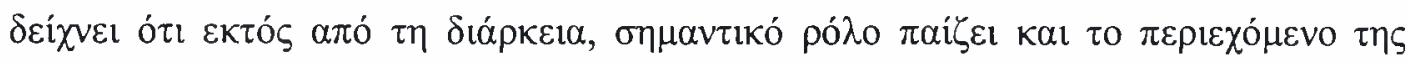

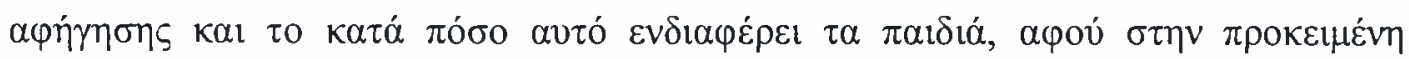

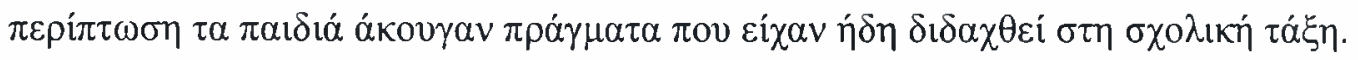

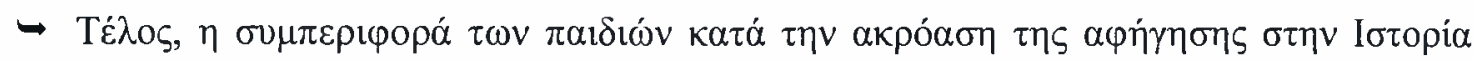

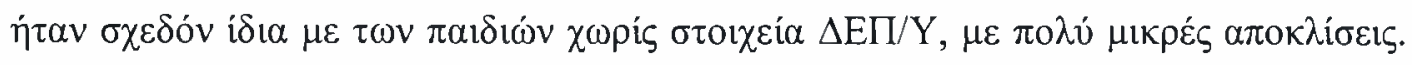

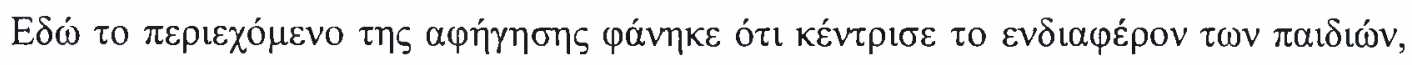

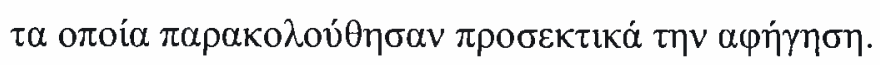

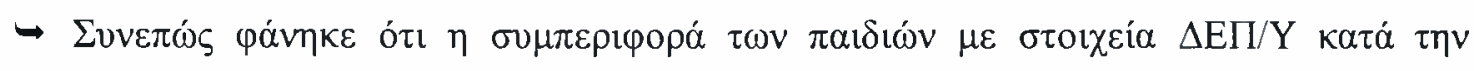

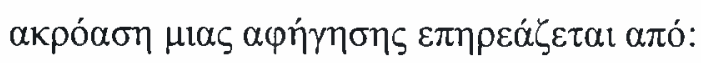

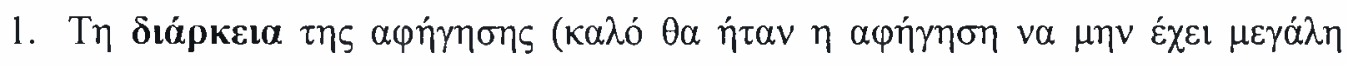

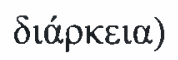

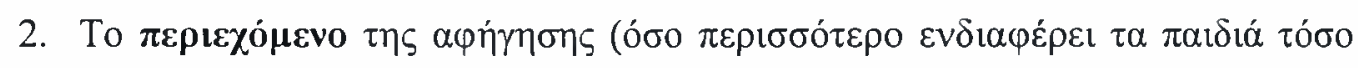
$\pi ı 0 \pi \rho о \sigma \varepsilon \kappa \tau \iota k \alpha ́$ cival).

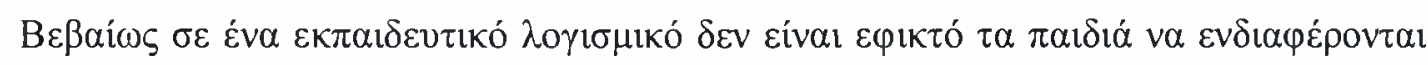

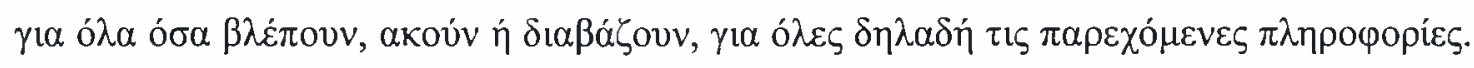

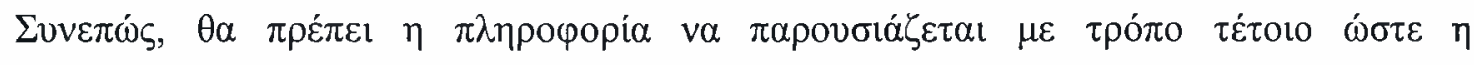

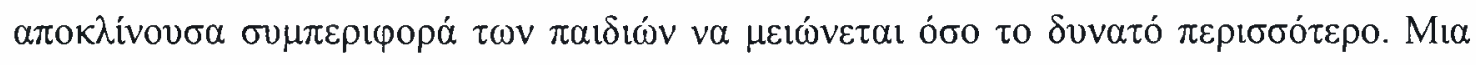

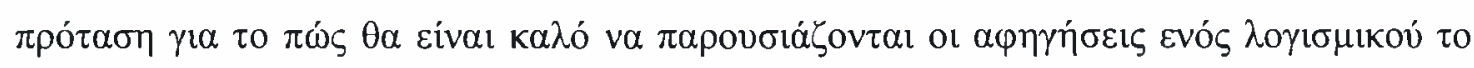

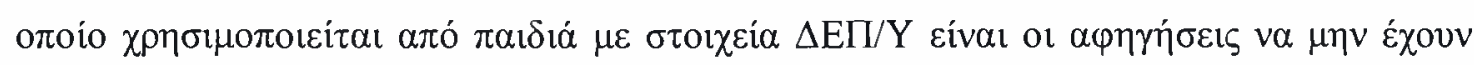

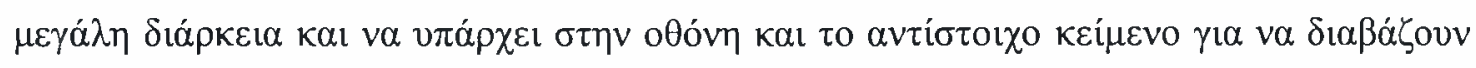

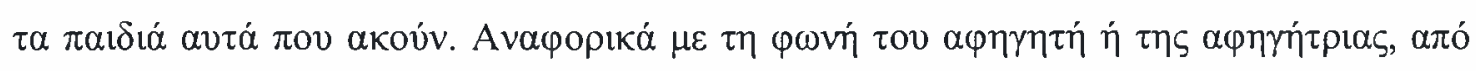

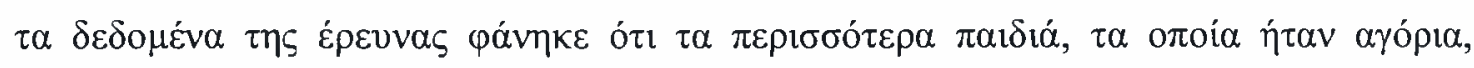

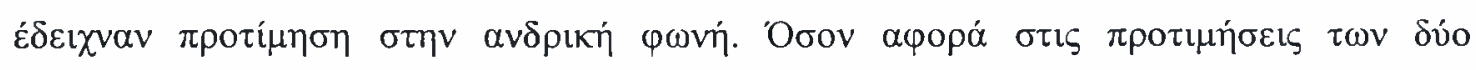

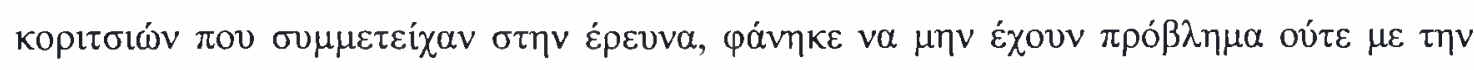

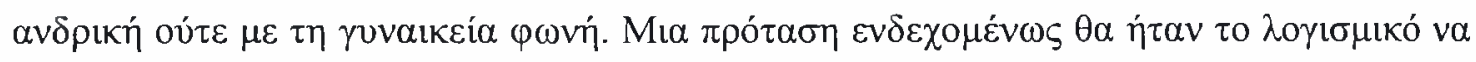

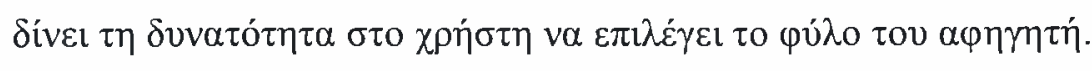




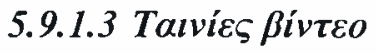

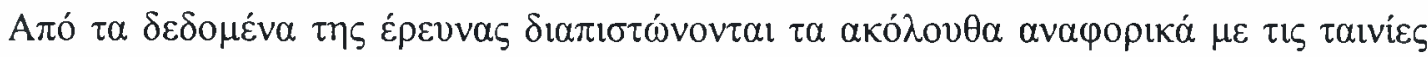

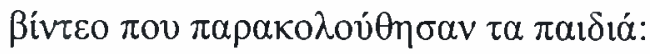

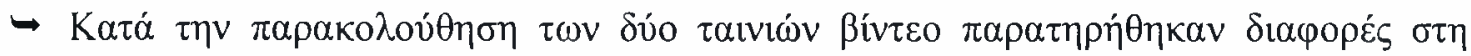

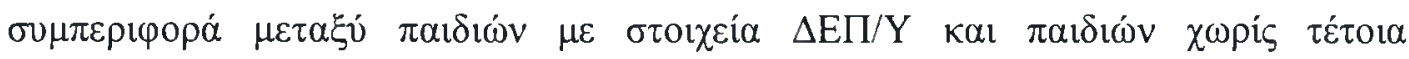

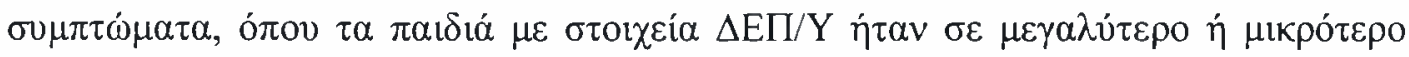

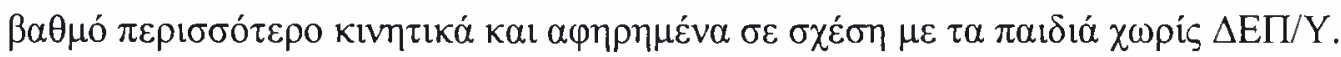

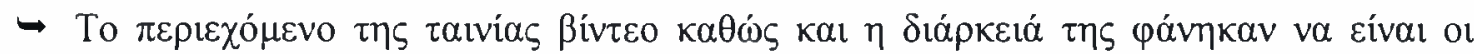

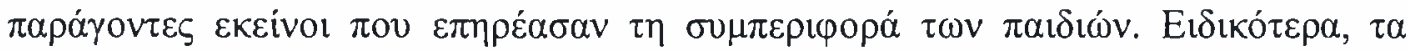

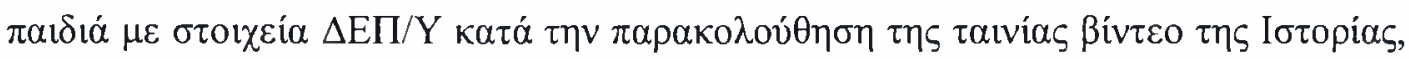

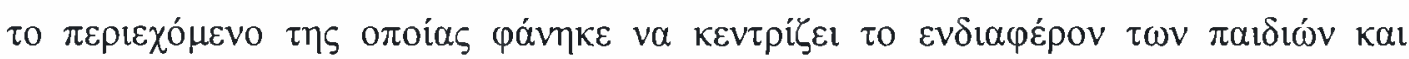

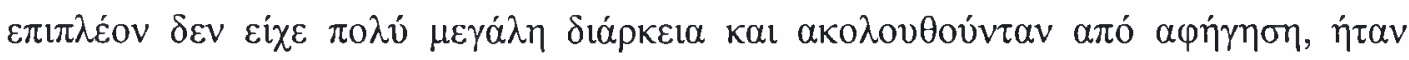

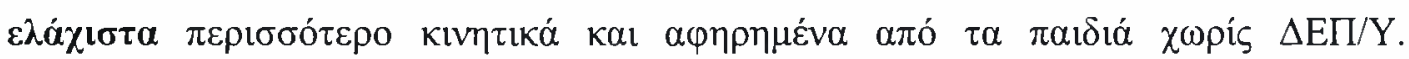

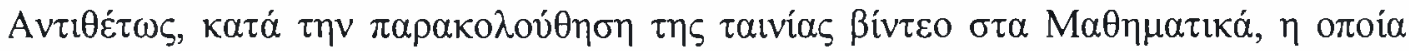

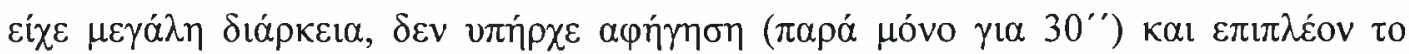

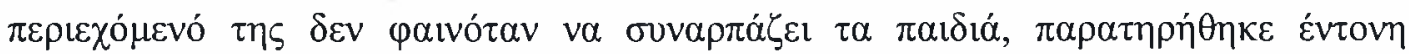

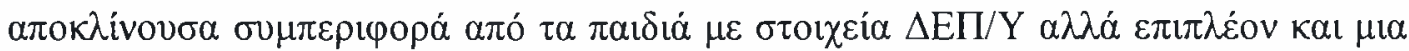

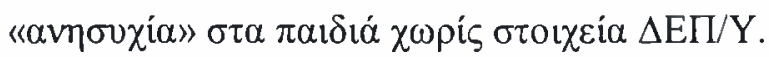

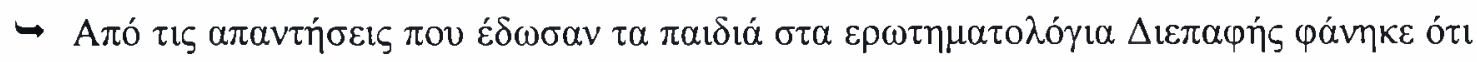

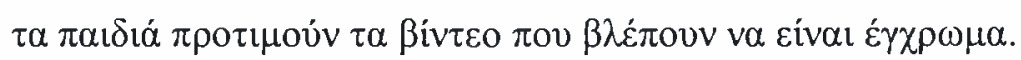

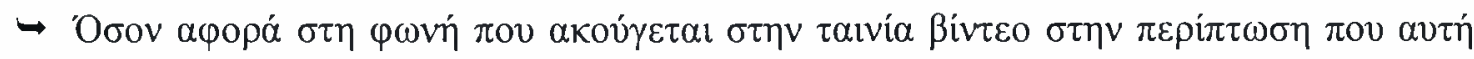

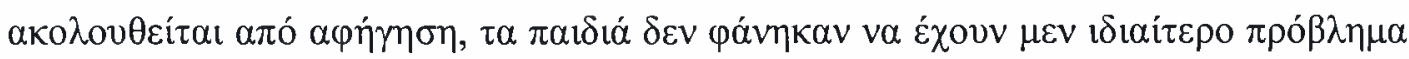

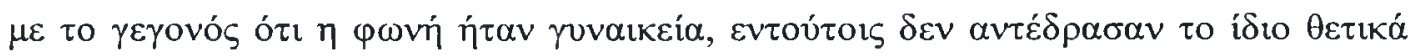

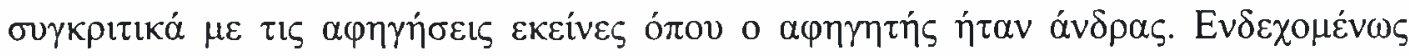

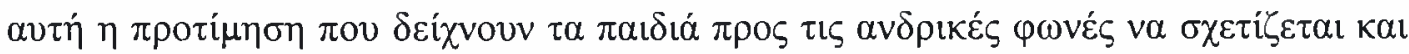

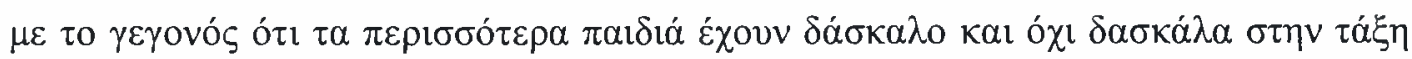

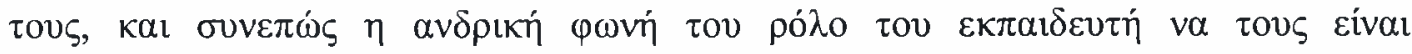

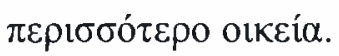

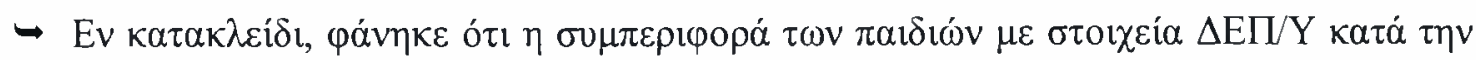

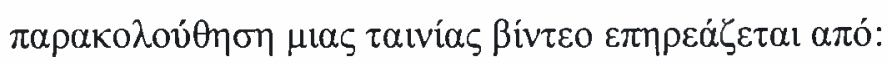




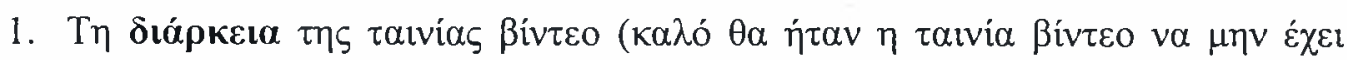

- $\mu \varepsilon \gamma \alpha \dot{\lambda} \eta \delta \delta \alpha ́ \rho \kappa \varepsilon 1 \alpha)$

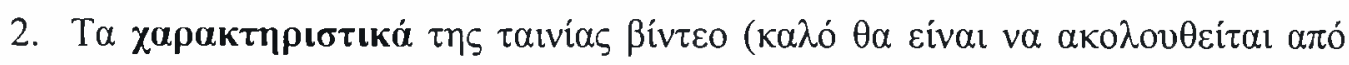

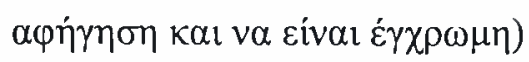

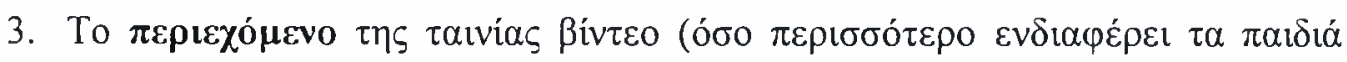

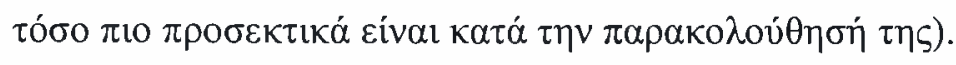

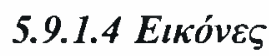

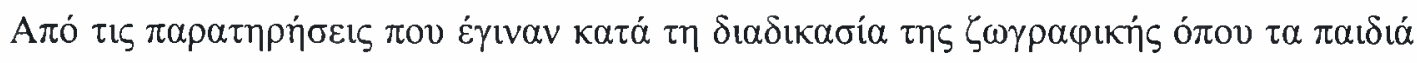

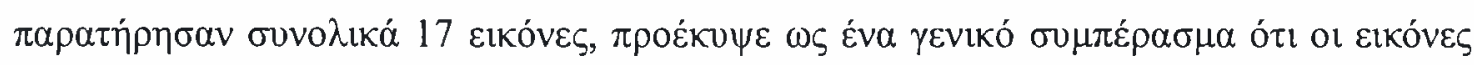

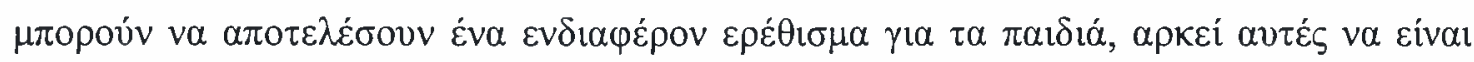

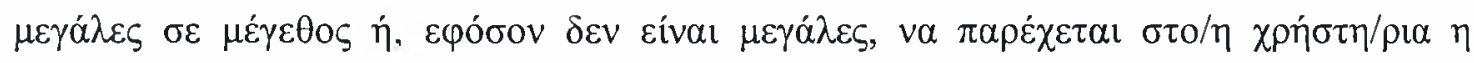

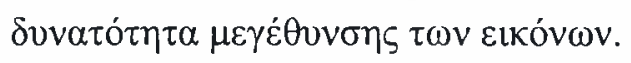

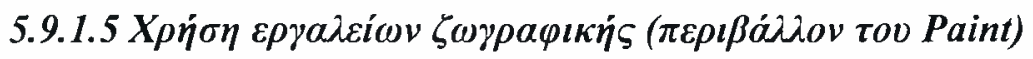

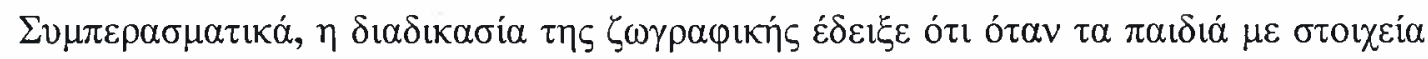

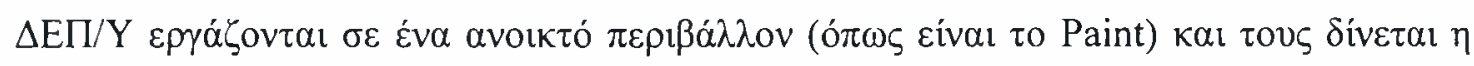

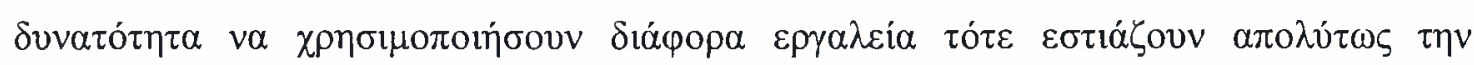

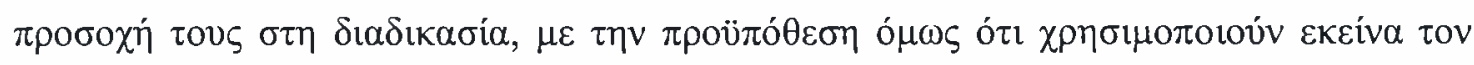

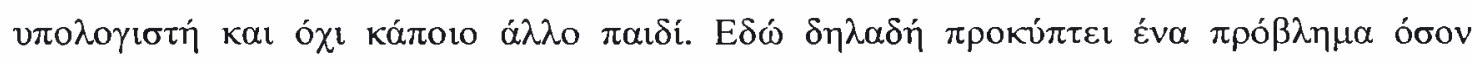

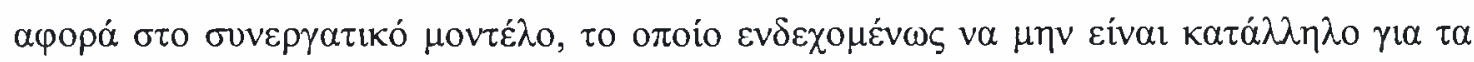

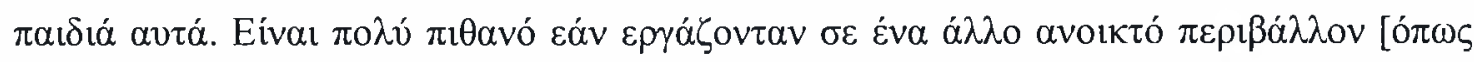

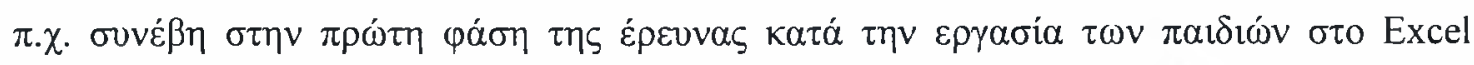

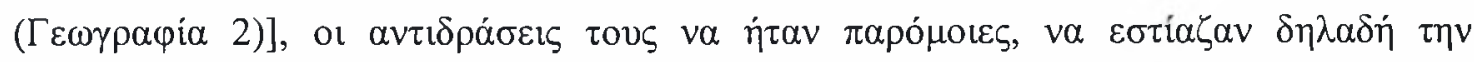

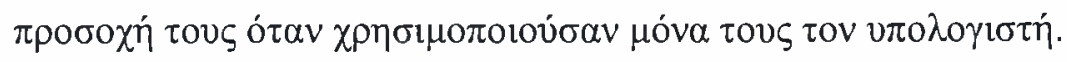




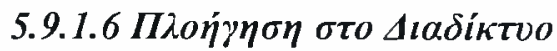

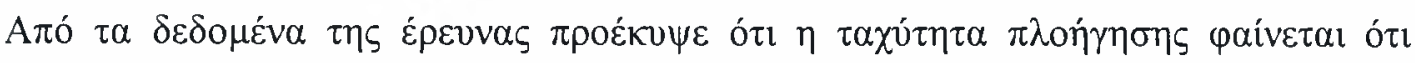

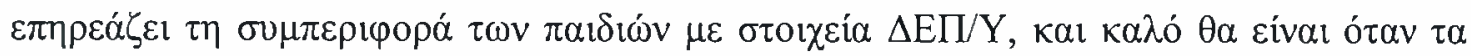

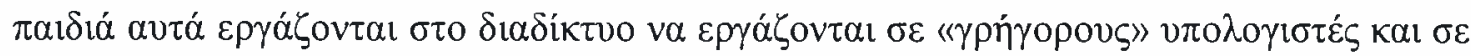

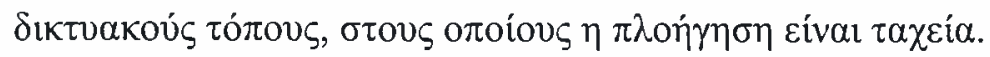

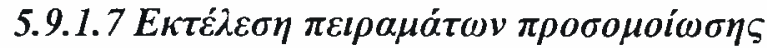

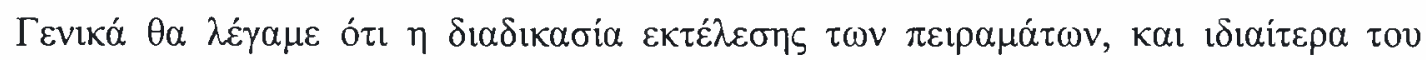

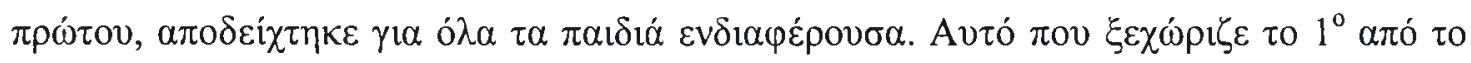

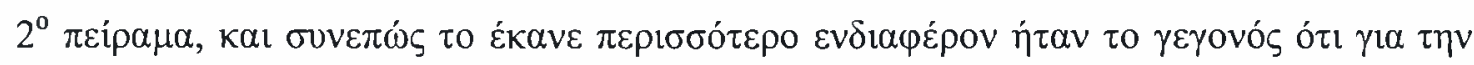

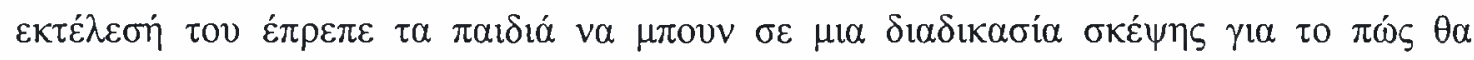

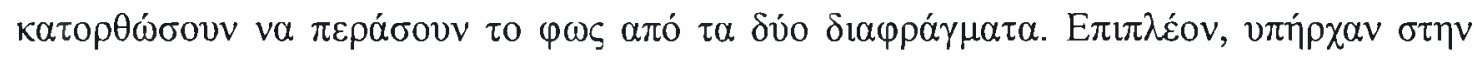

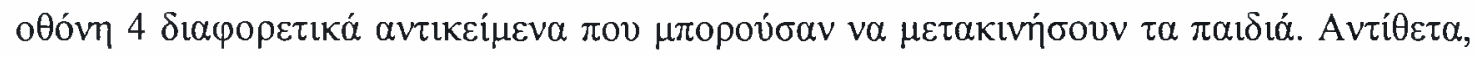

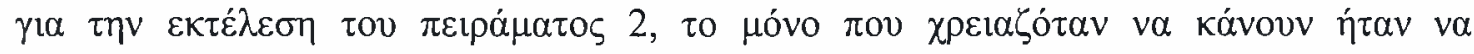

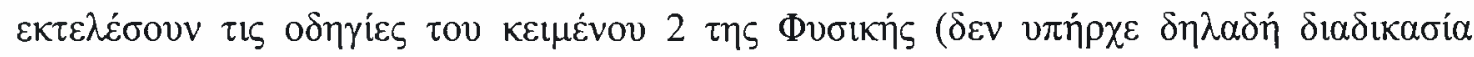

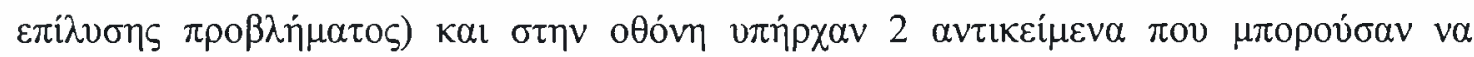

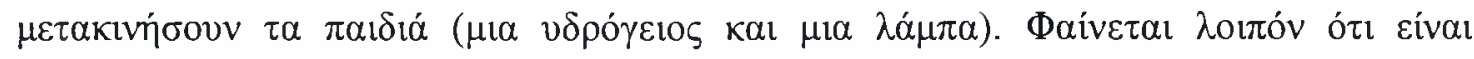

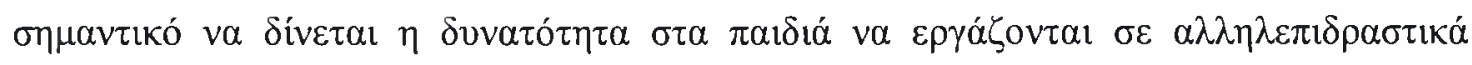

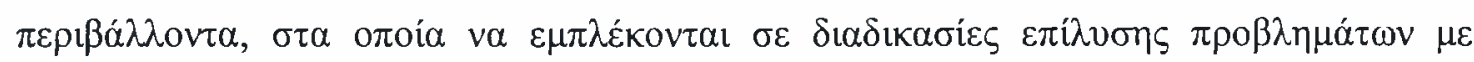

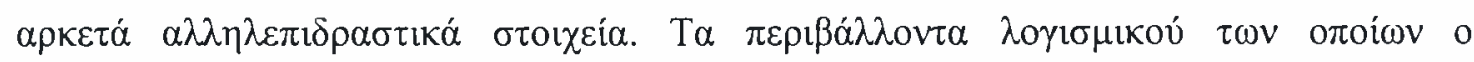

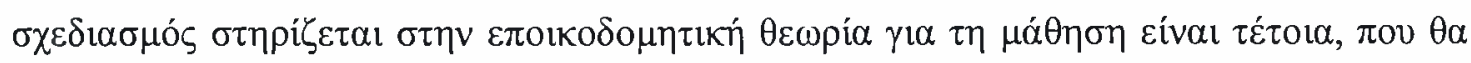

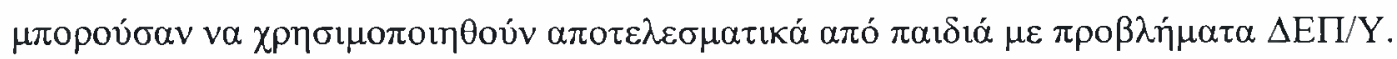

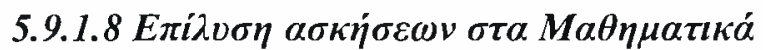

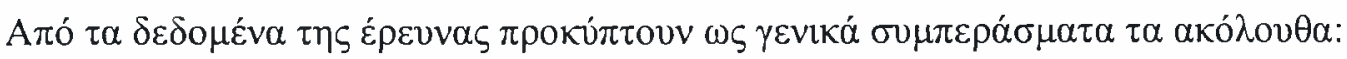

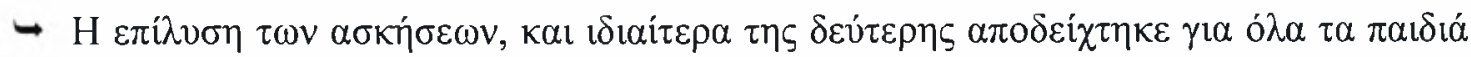

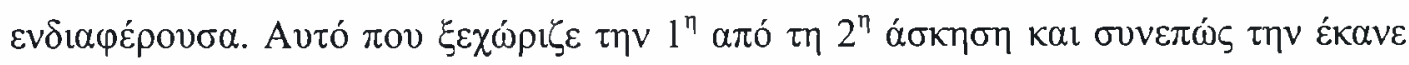

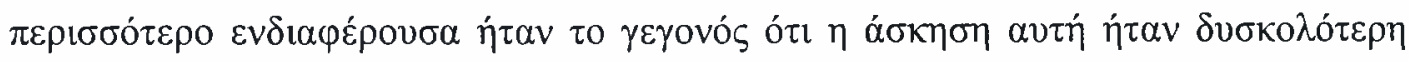




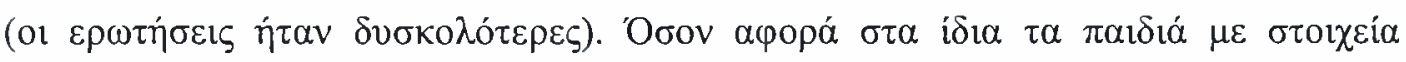

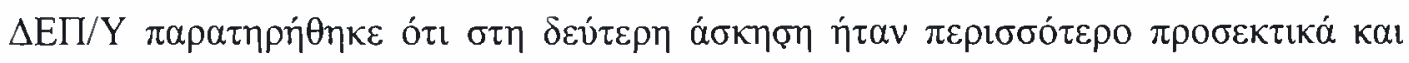

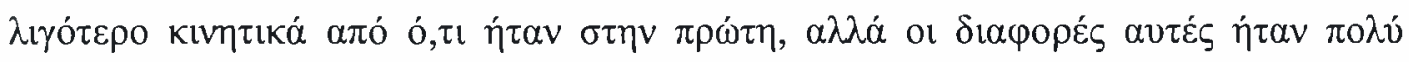

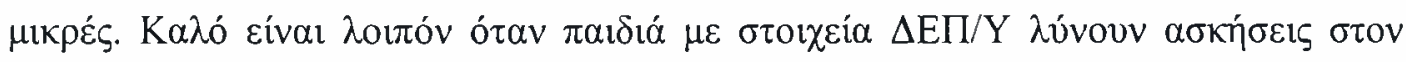

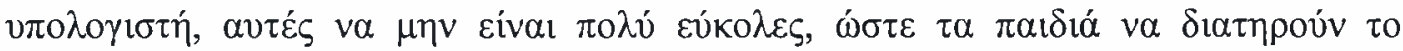

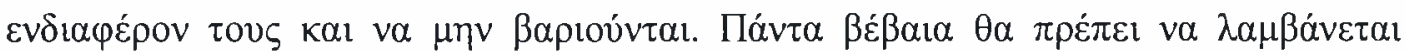

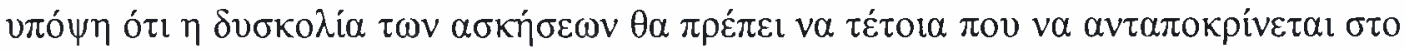
$\varepsilon \pi i \pi \varepsilon \delta$ o $\tau \omega \vee \pi \alpha ı \delta i \omega ́ v$.

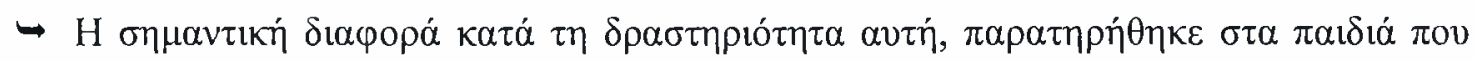

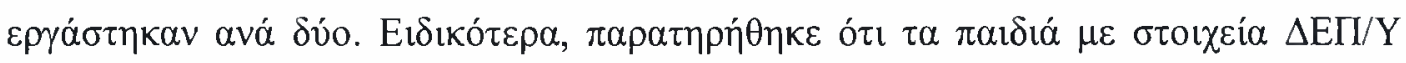

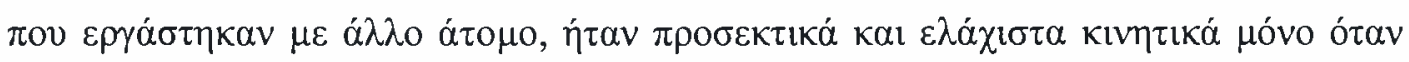

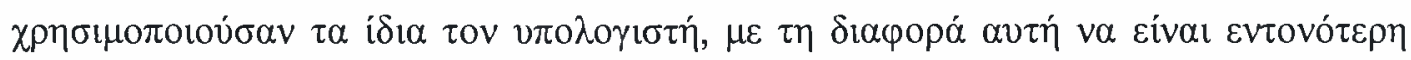

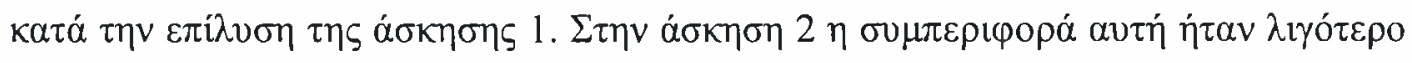

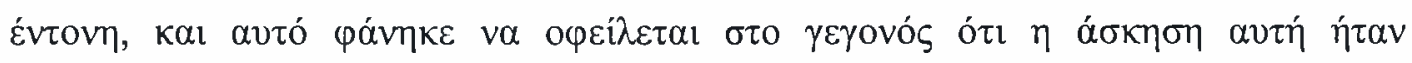

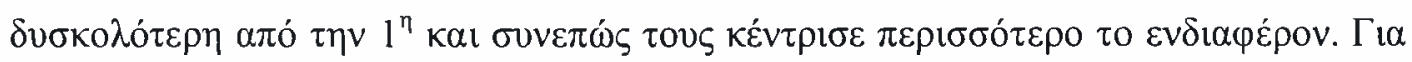

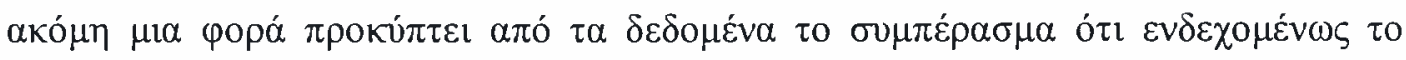

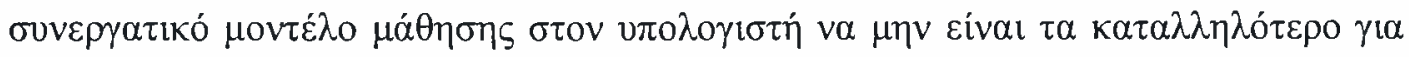

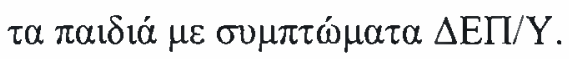

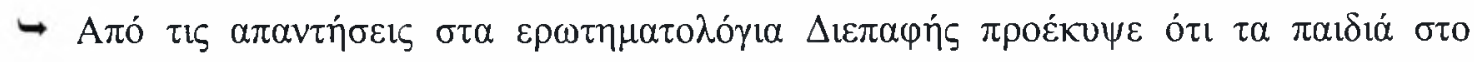

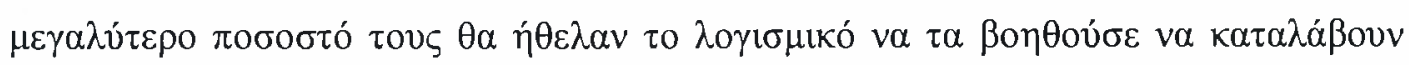

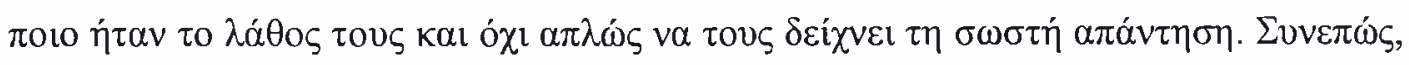

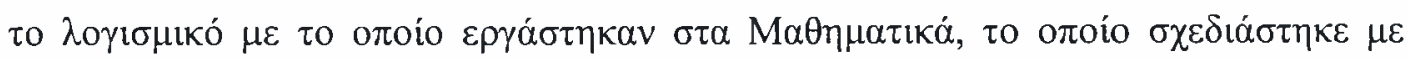

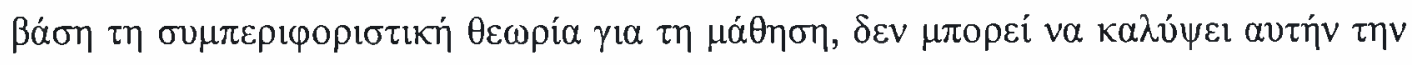

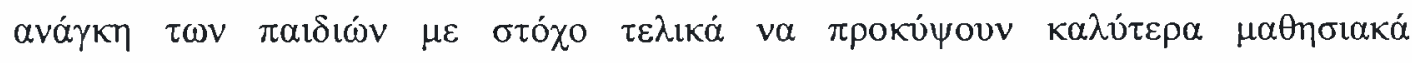
$\alpha \pi \circ \tau \varepsilon \lambda \dot{\varepsilon} \sigma \mu \alpha \tau \alpha$.

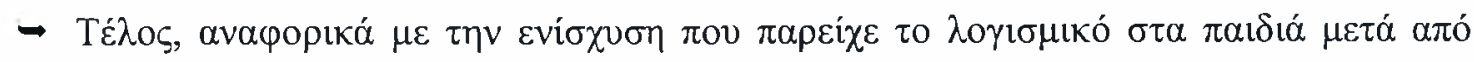

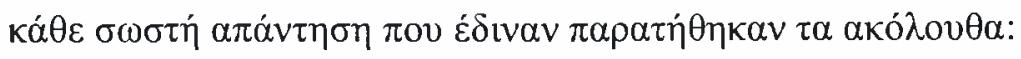

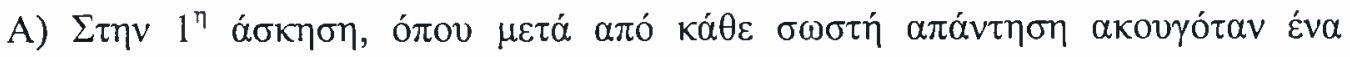

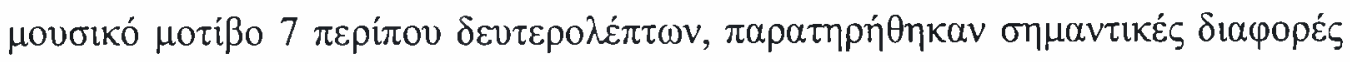

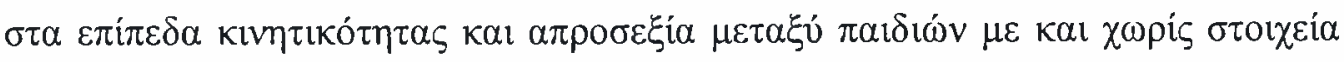
$\Delta \mathrm{E} \Pi / \mathrm{Y}$, 


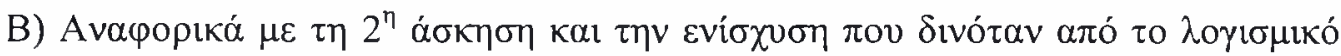

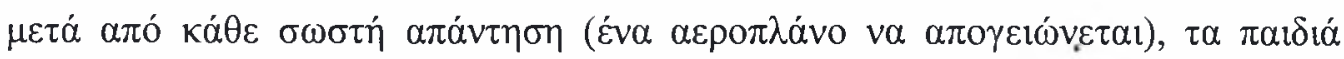

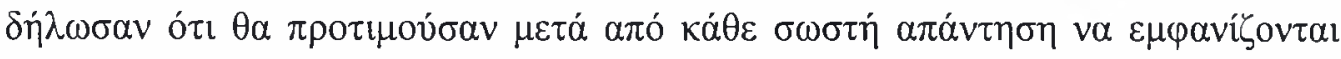

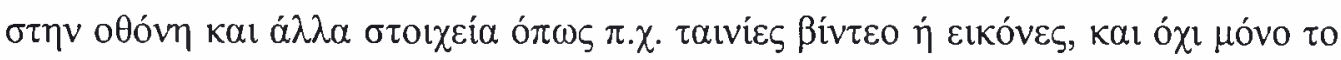
$\alpha \varepsilon \rho o \pi \lambda \alpha \dot{\alpha} v o$.

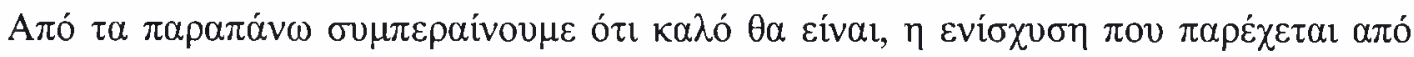

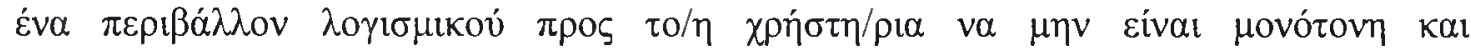

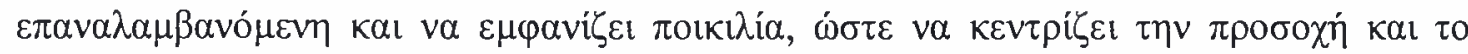

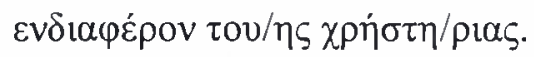

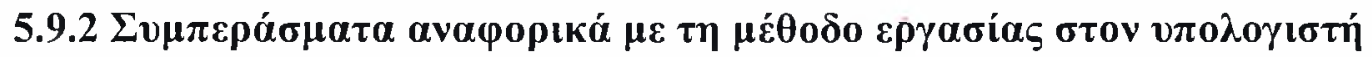

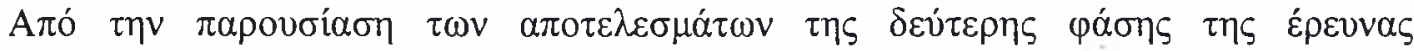

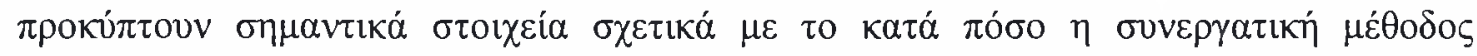

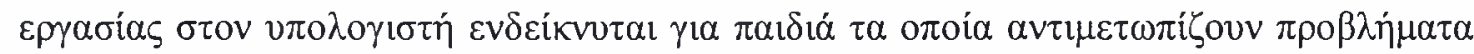

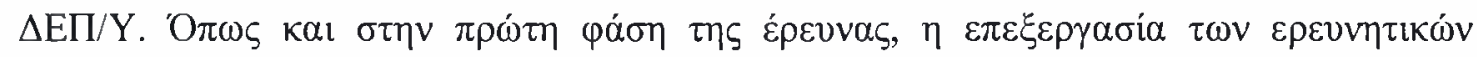

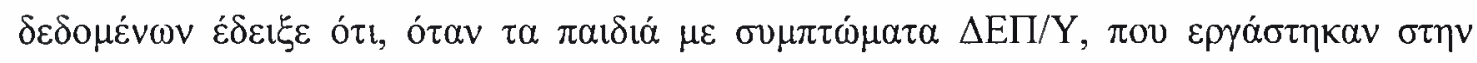

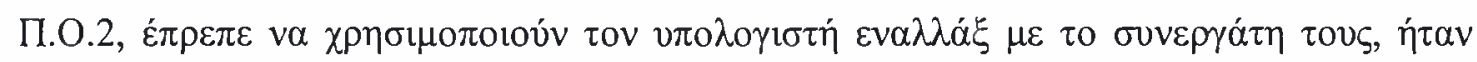

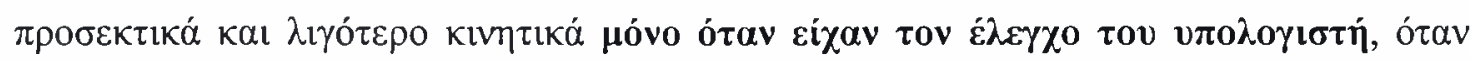
$\delta \eta \lambda \alpha \delta \dot{\eta} \chi \rho \eta \sigma \mu$ o

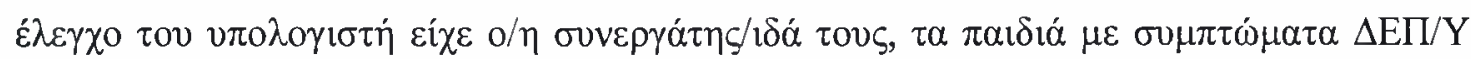

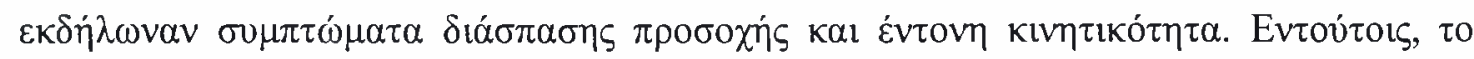

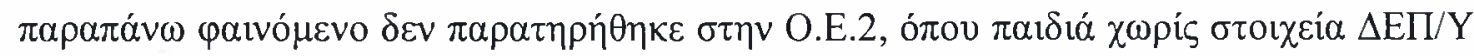

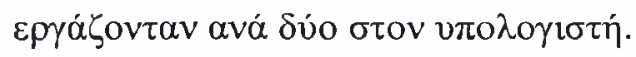

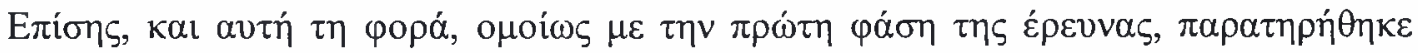

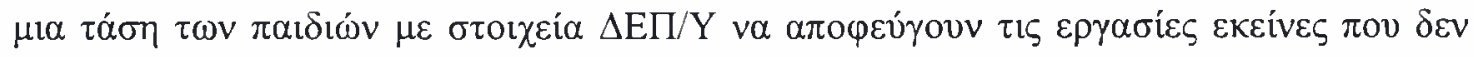

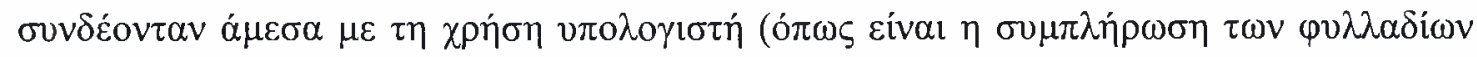

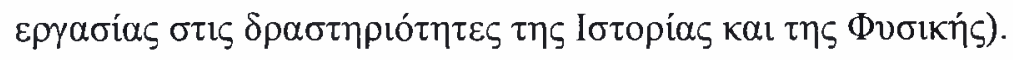

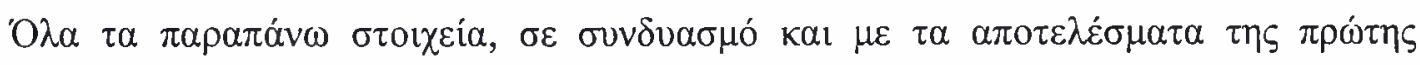

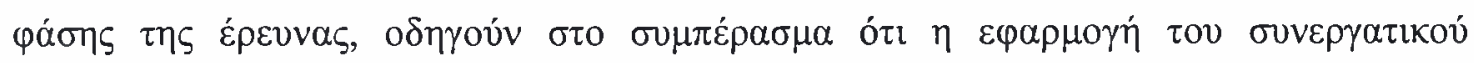

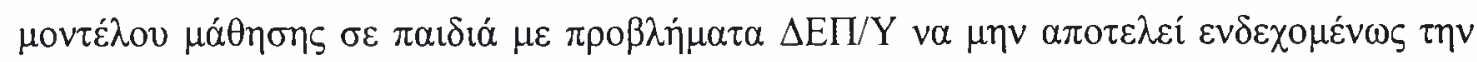




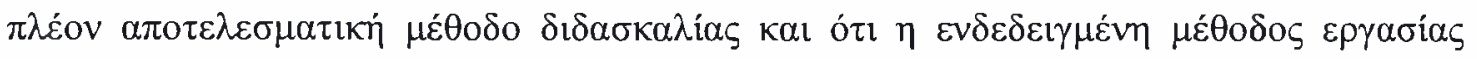

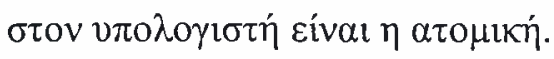

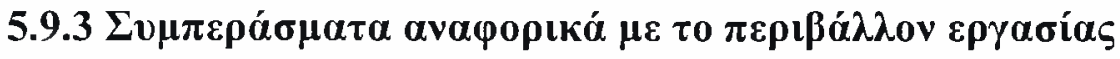

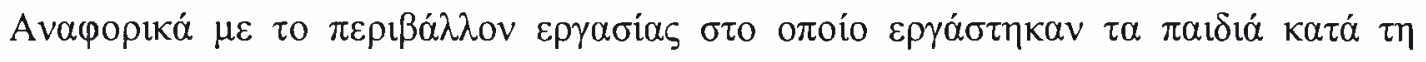

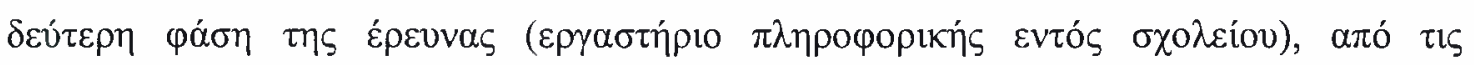

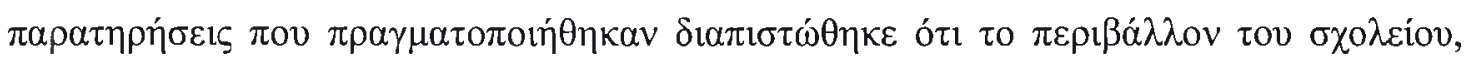

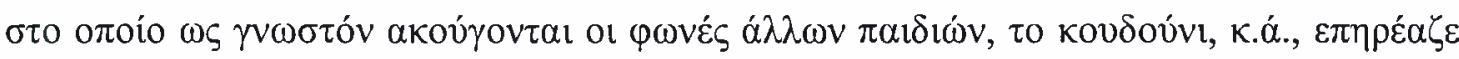

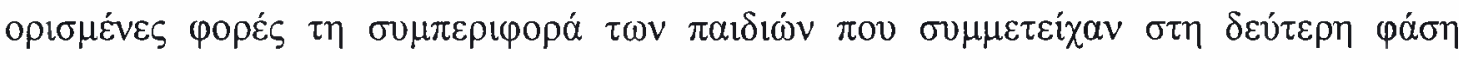

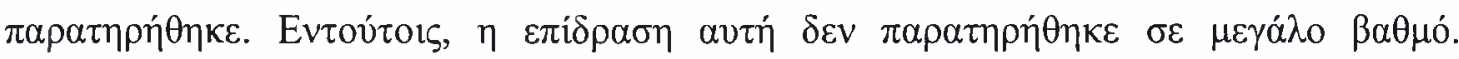

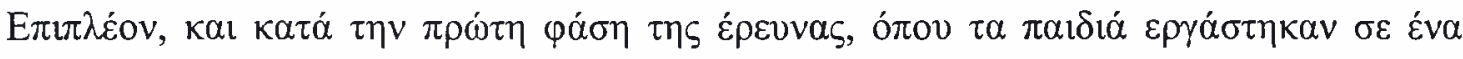

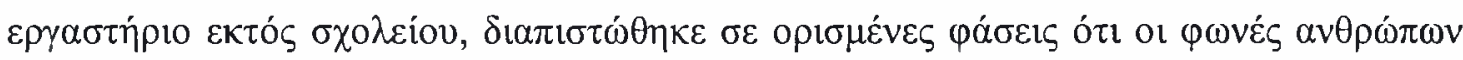

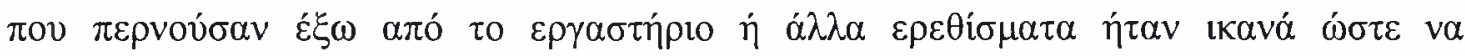

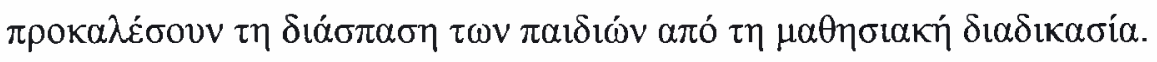

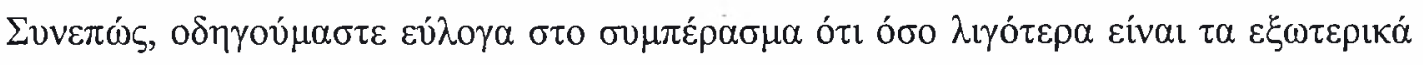

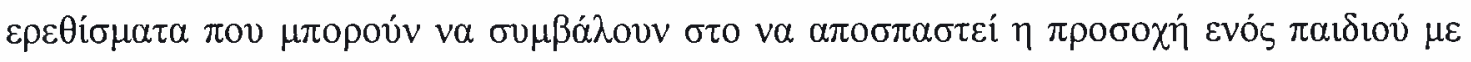

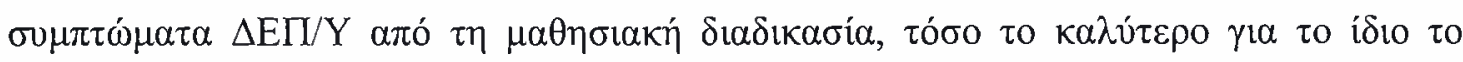
$\pi \alpha\llcorner\delta i ́$. 
$\underline{\underline{E K T O ~ K E} \Phi A \Lambda A I O}$

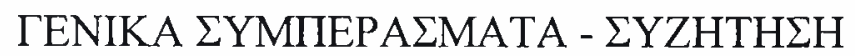

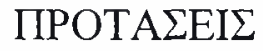

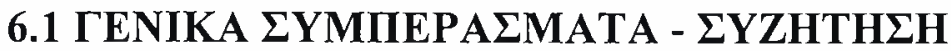

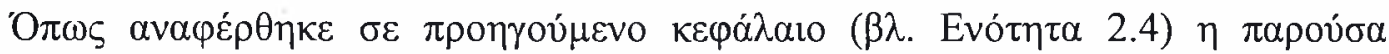

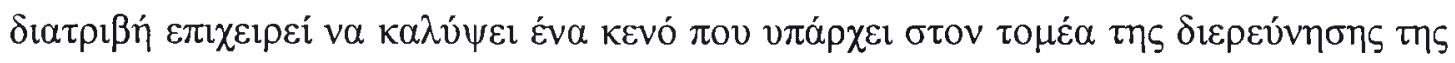

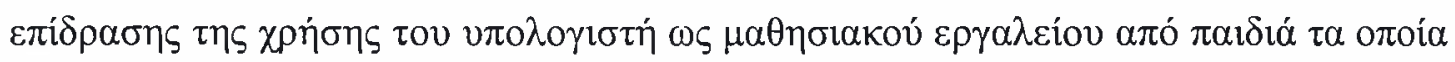

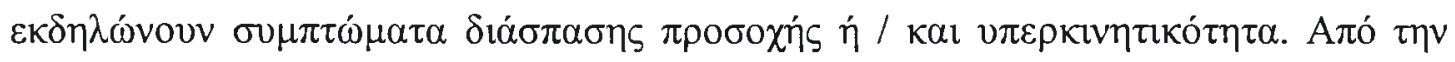

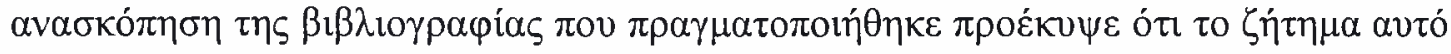

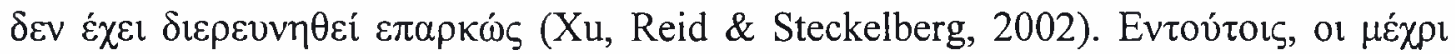

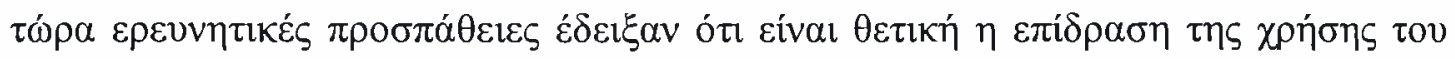

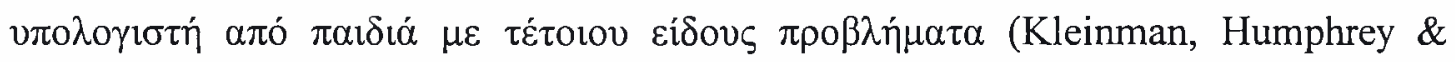
Lindsay 1981; Millman, 1984, Ford, Poe \& Cox, 1993, Elkind, Cohen \& Murray, 1993, Carey \& Sale, 1997, Farrace-Di Zinno, Douglas, Houghton, Lawrence, West \& Whiting, 2001, Hecker, Burns, Elkind, Elkind \& Katz, 2002).

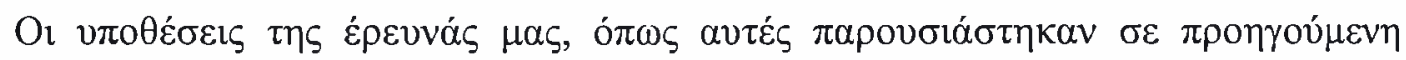

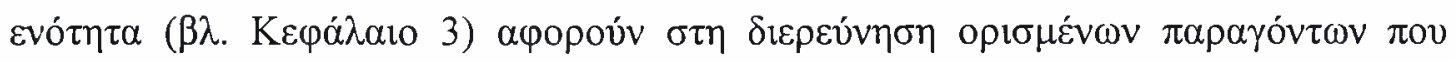

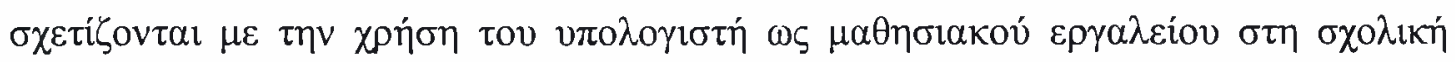

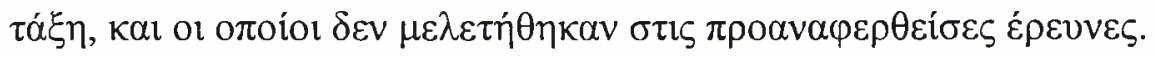

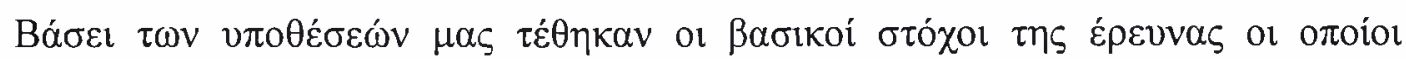

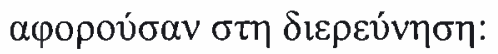

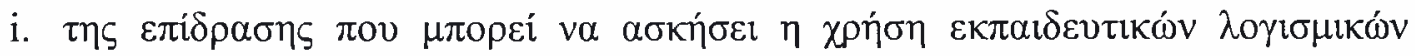

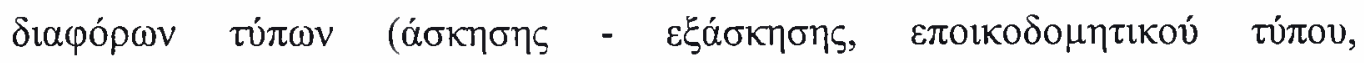

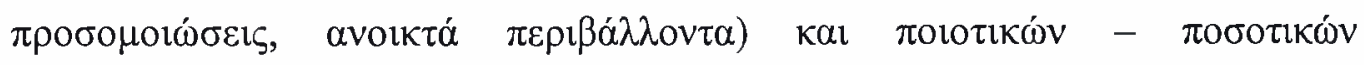

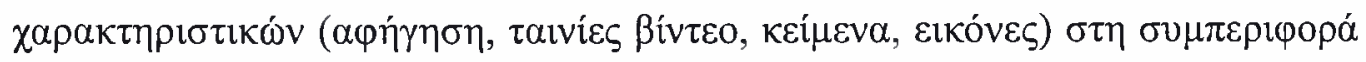

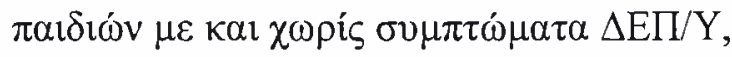




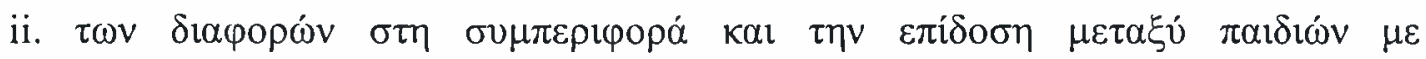

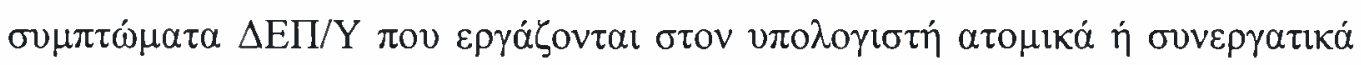

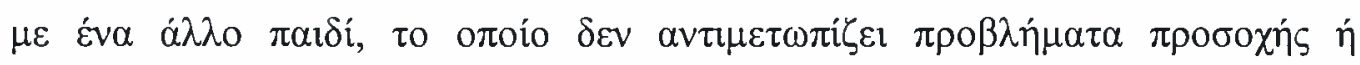

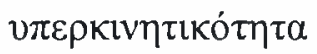

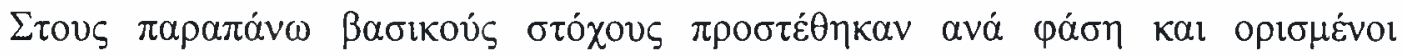

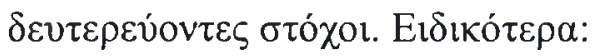

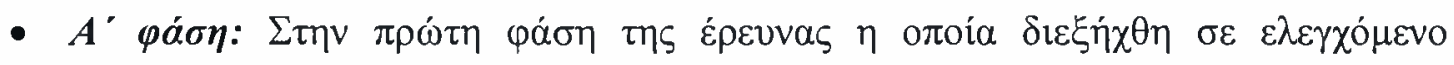

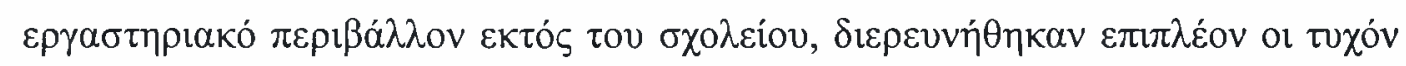

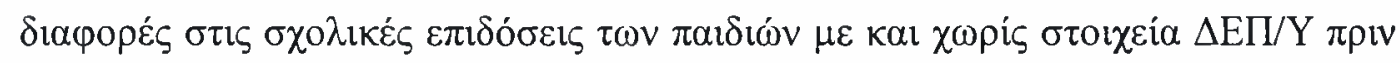

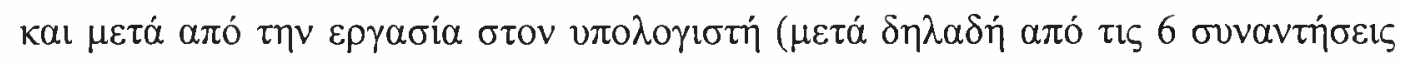

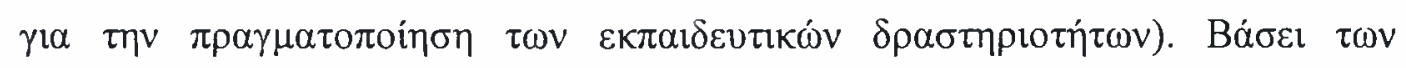

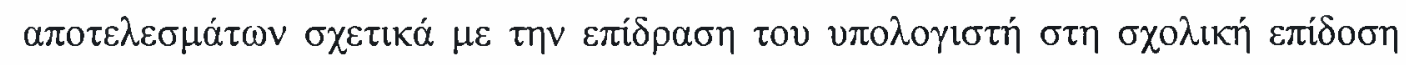

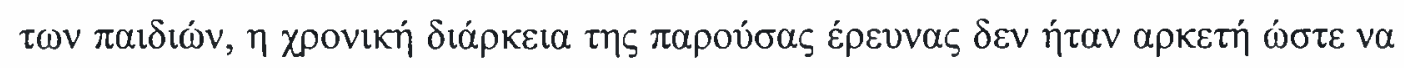

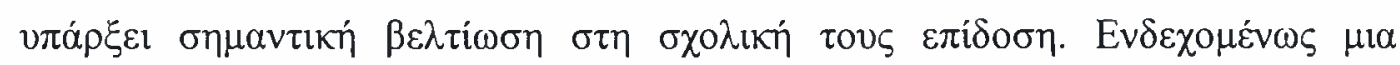

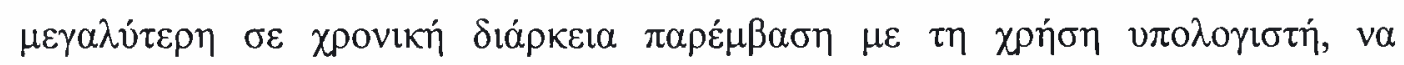

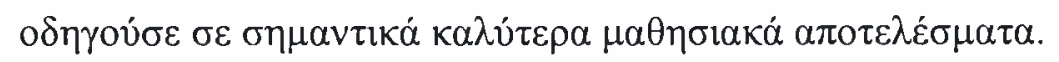

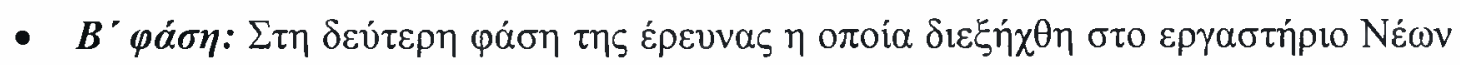

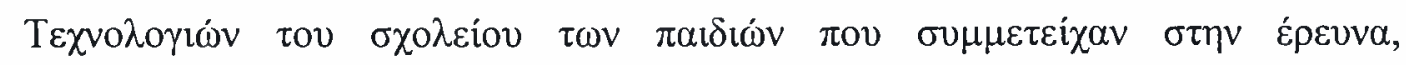

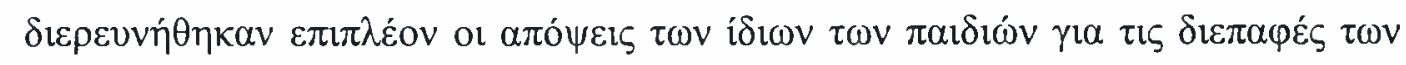

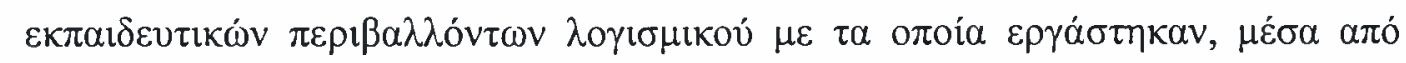

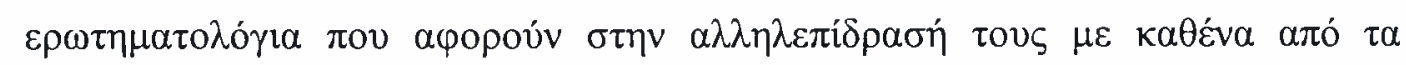

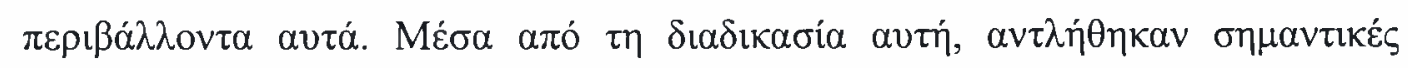

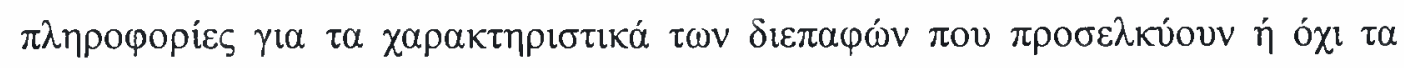

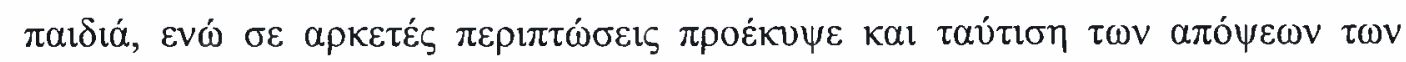

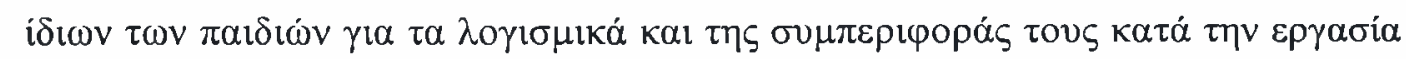

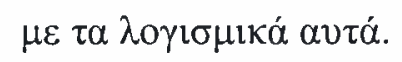

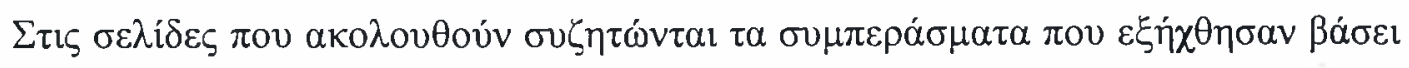

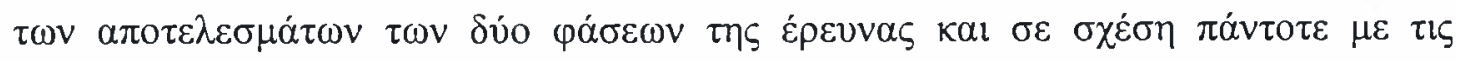

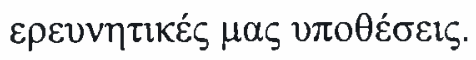




\subsection{1 Ү}

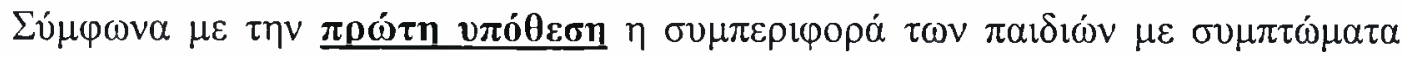

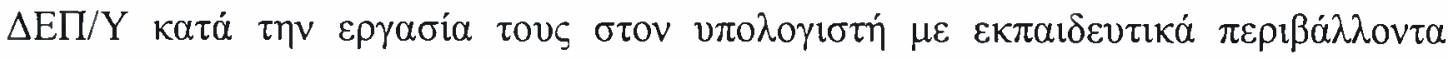

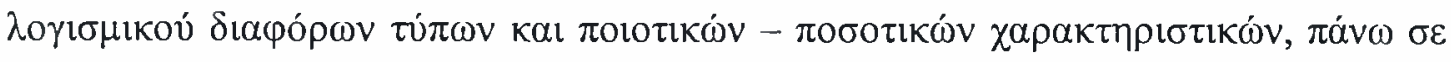

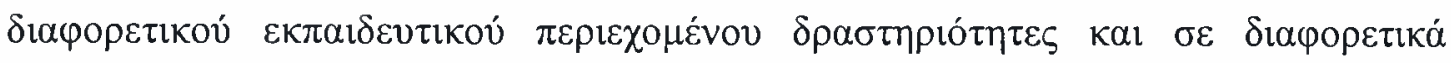

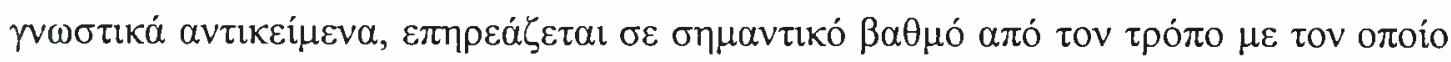

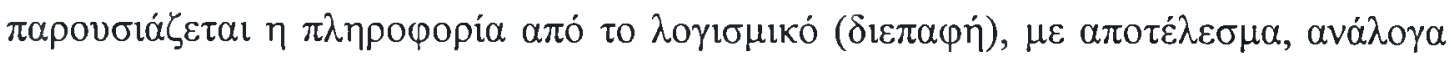

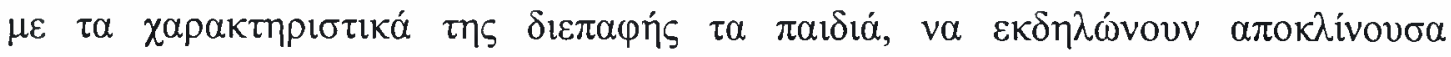

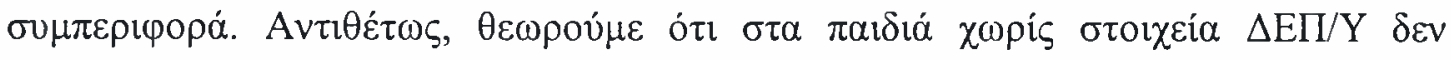

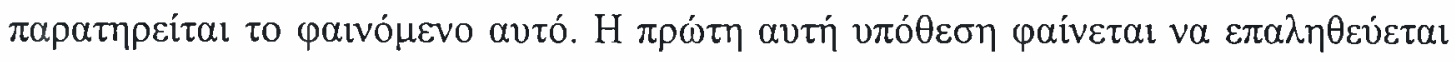

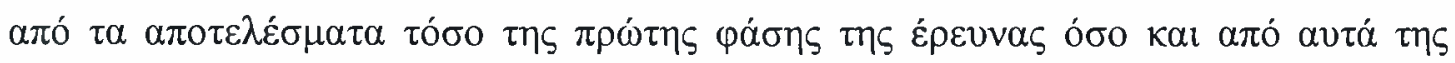

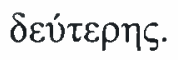

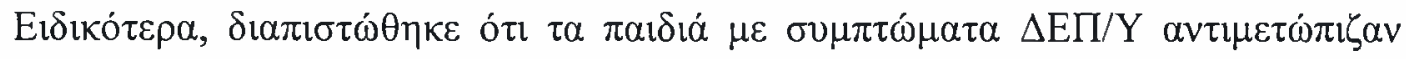

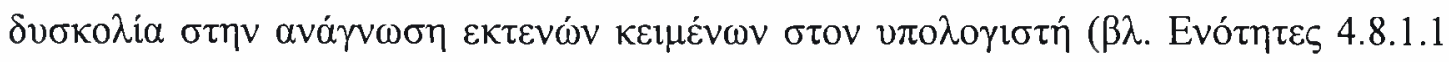

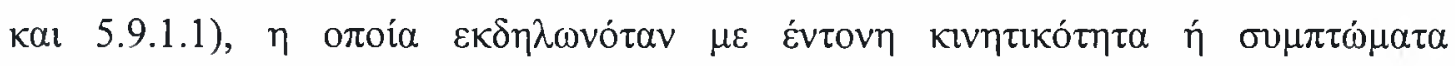

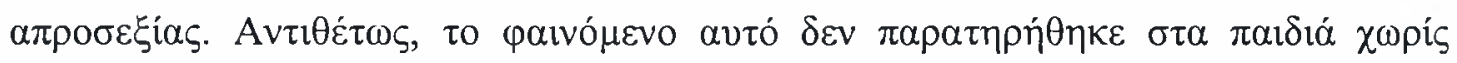

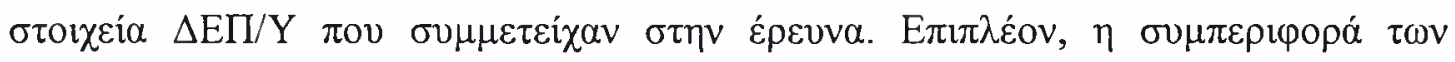

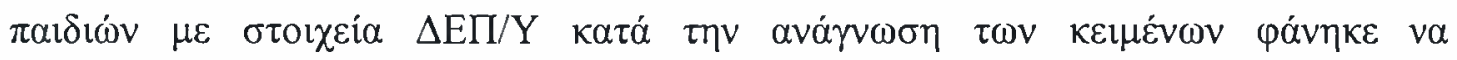

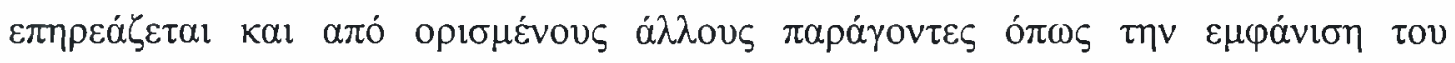

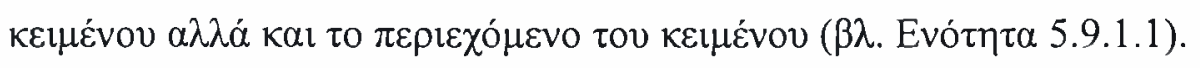

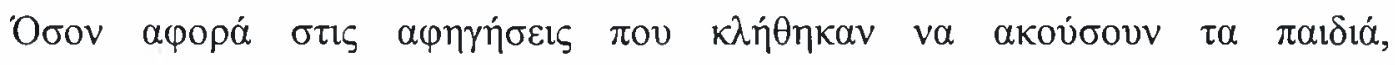

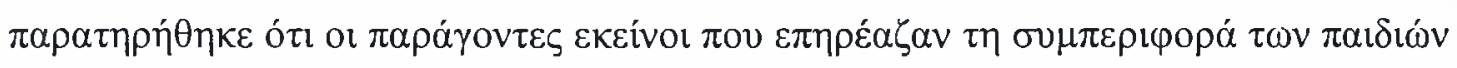

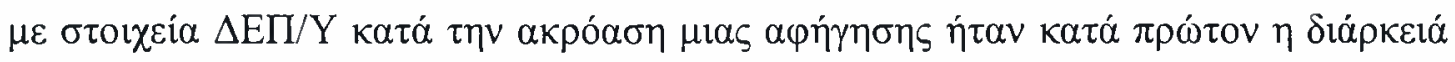

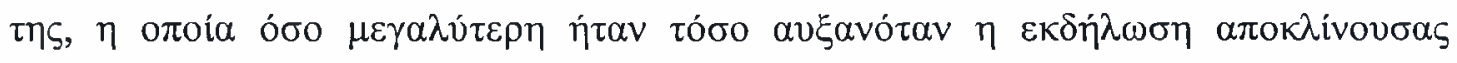

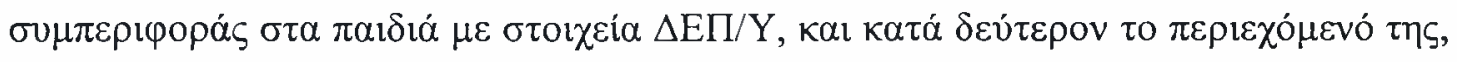

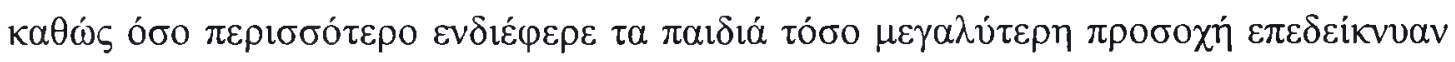

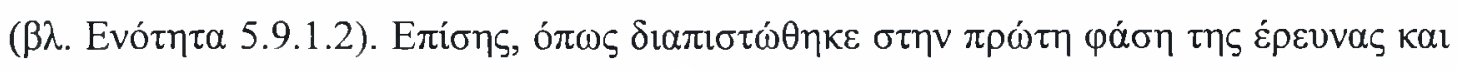

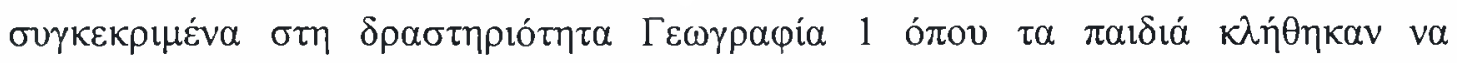

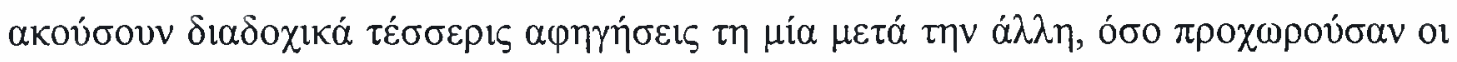

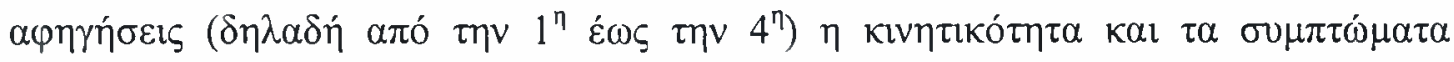

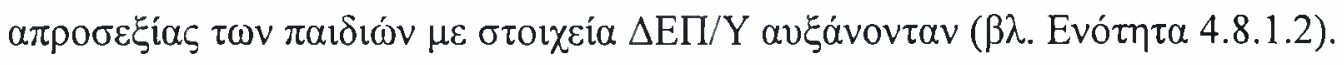




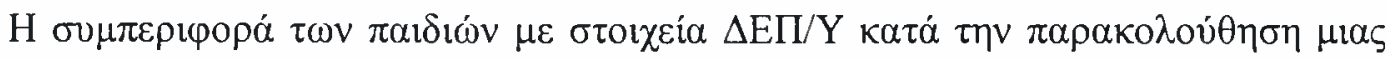

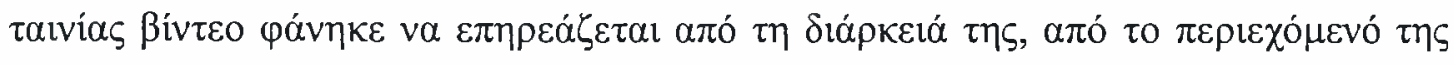

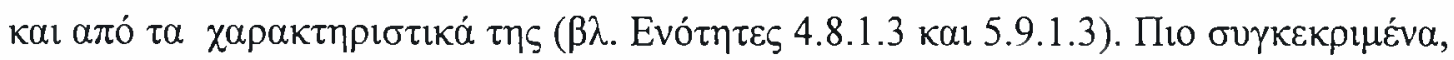

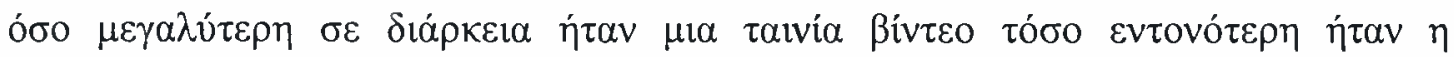

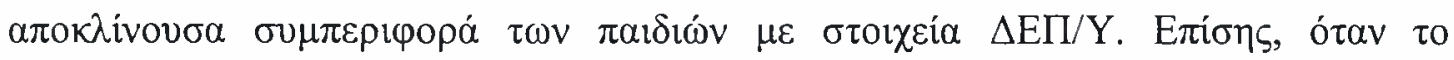

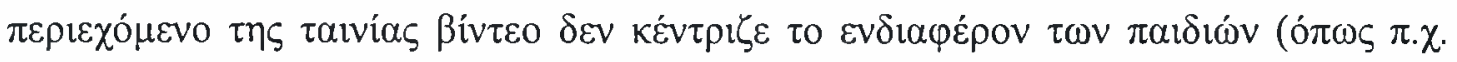

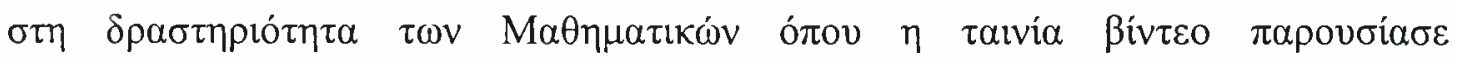

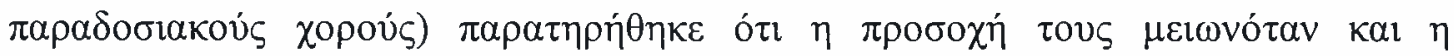

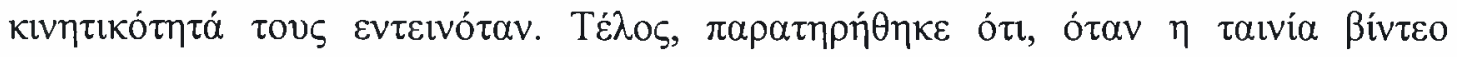

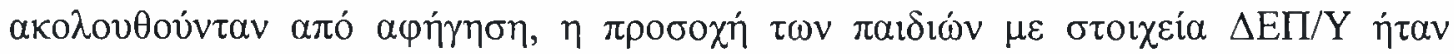

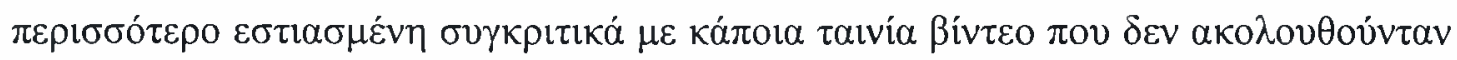
$\alpha \pi \delta ́ \alpha \varphi ́ \gamma \eta \sigma \eta$.

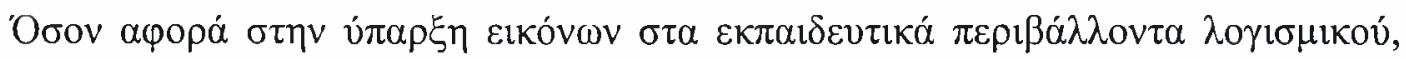

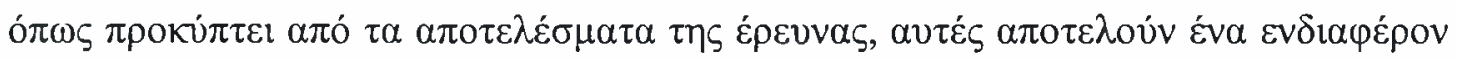

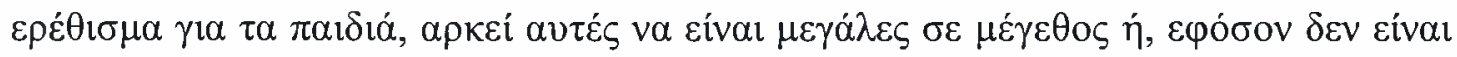

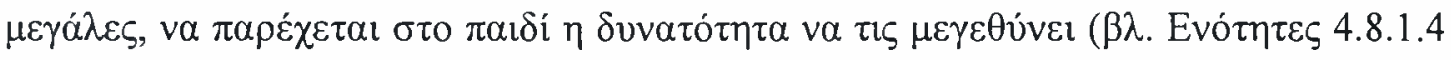
Kal 5.9.1.4).

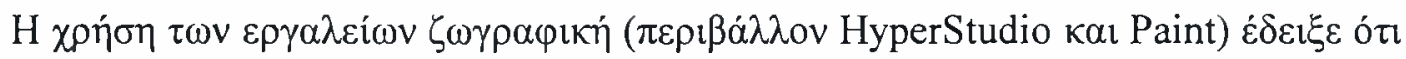

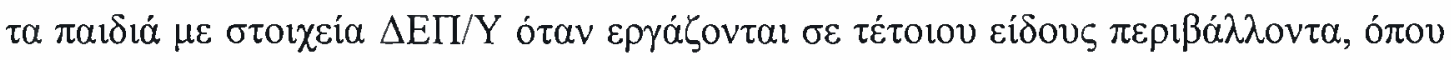

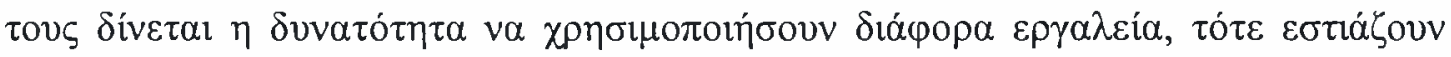

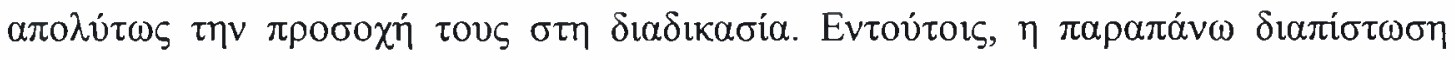

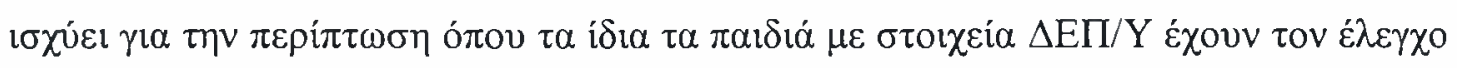

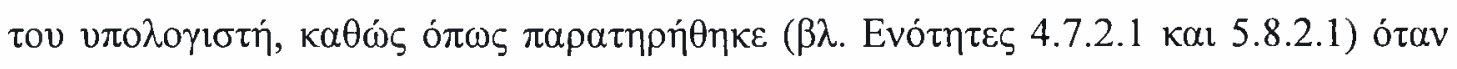

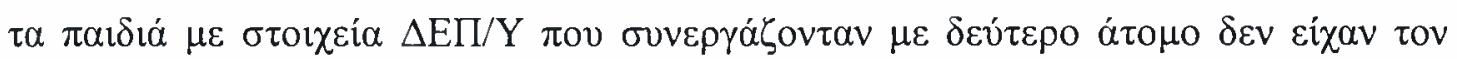

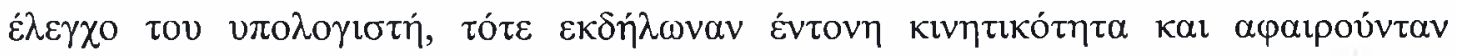

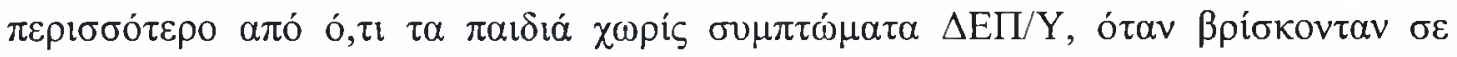
$\pi \alpha \rho o ́ \mu o l \alpha \theta \dot{\varepsilon} \sigma \eta$.

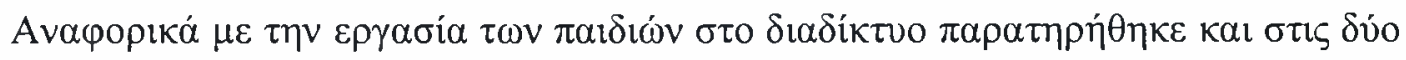

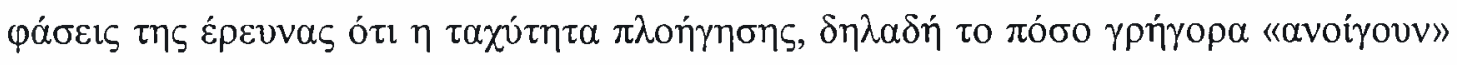

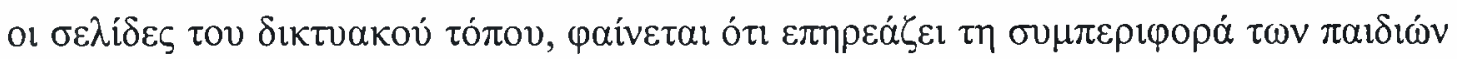

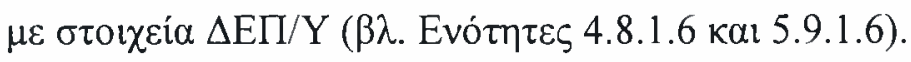

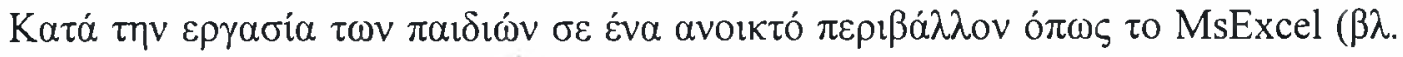

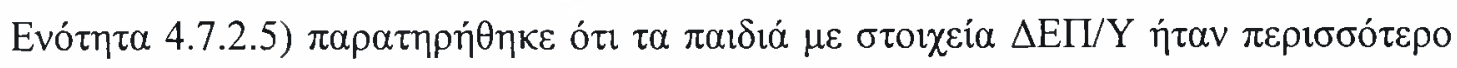

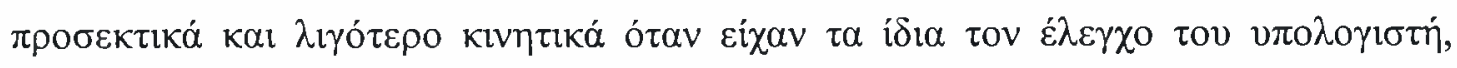




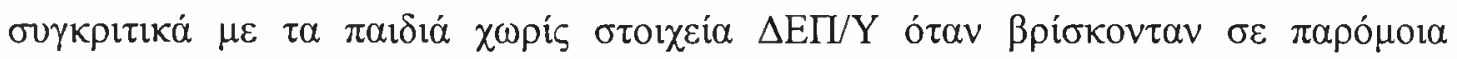
$\kappa \alpha \tau \alpha \dot{\sigma} \tau \alpha \sigma \eta$.

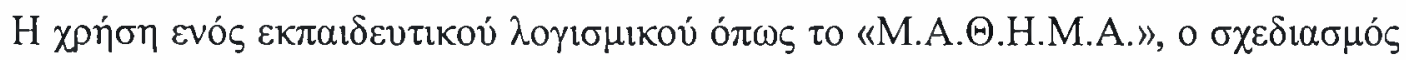

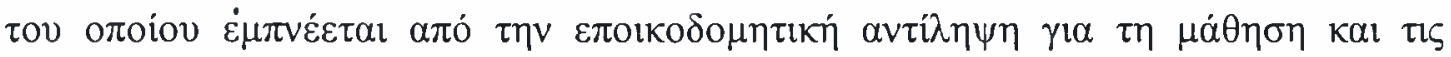

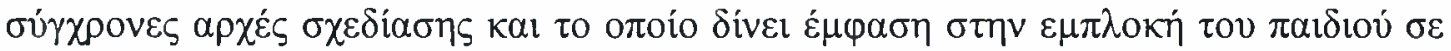

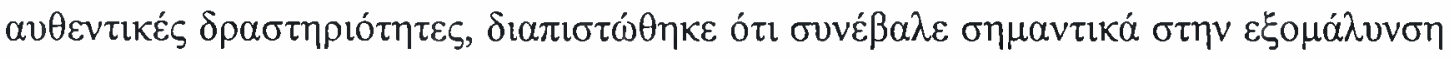

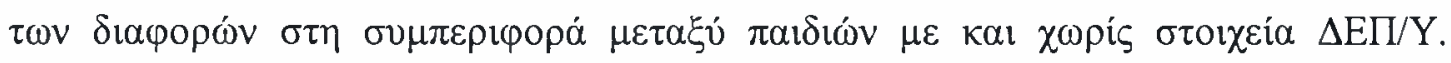

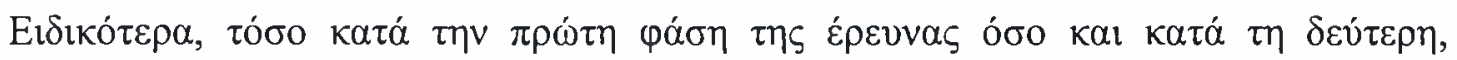

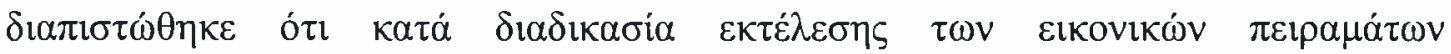

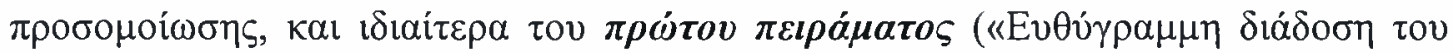


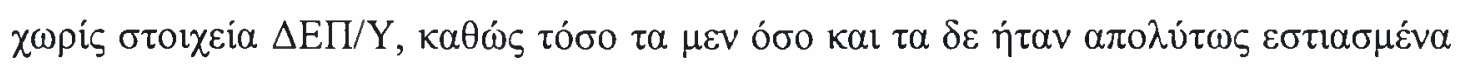

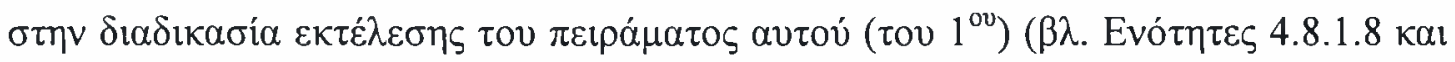

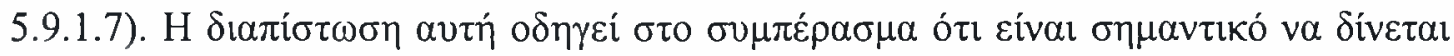

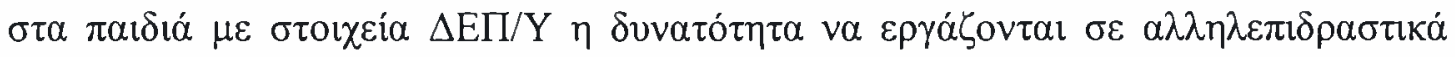

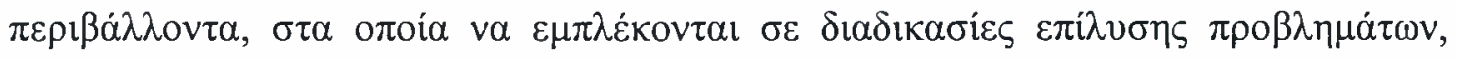

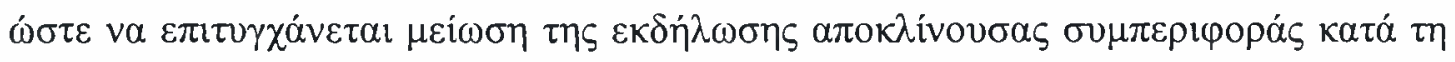
$\delta i \delta \alpha \sigma \kappa \alpha \lambda i \alpha$.

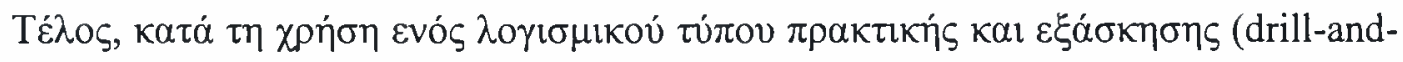

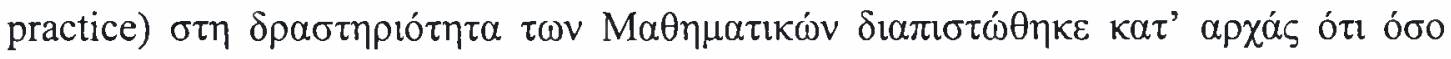

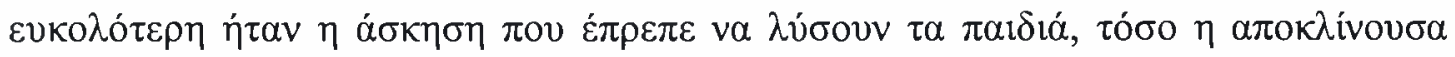

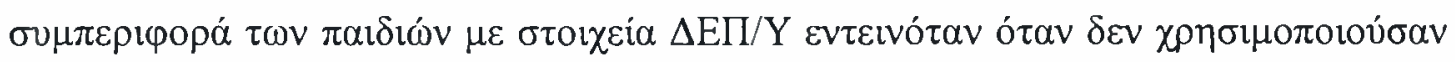

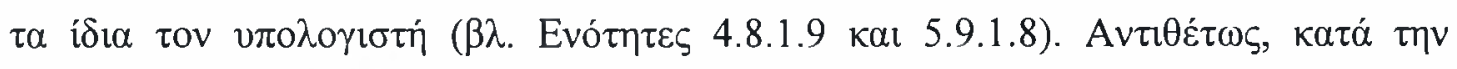

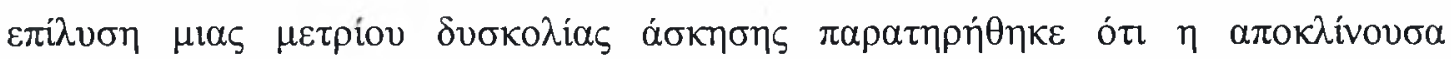

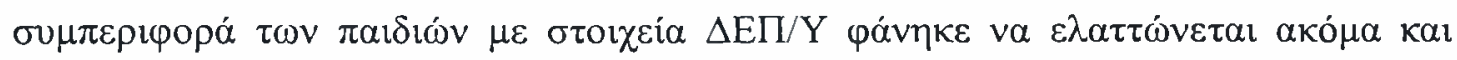

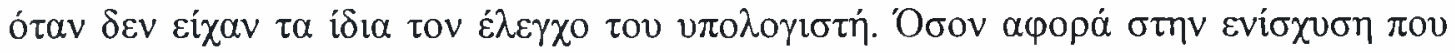

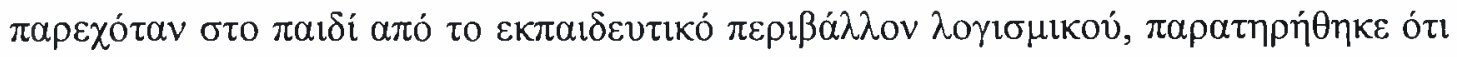

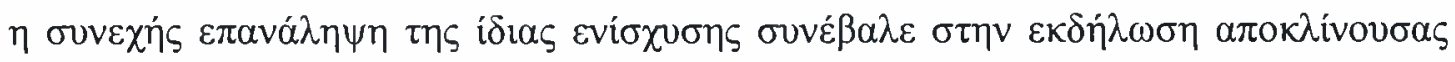

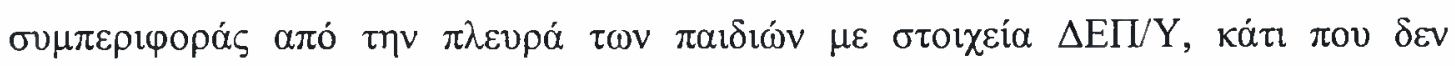

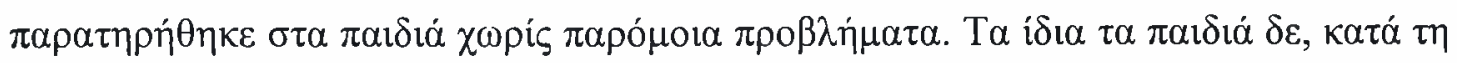

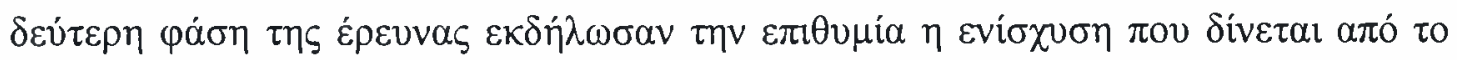

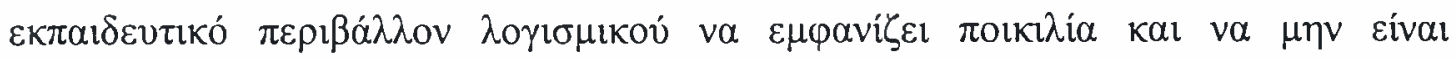

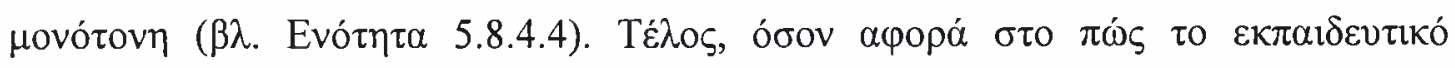

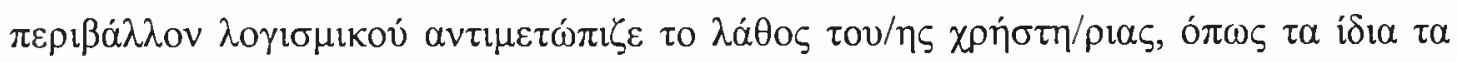




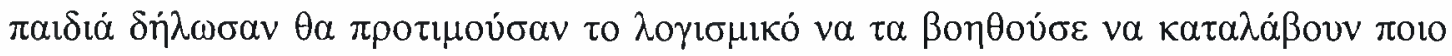

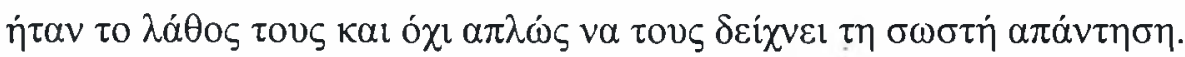

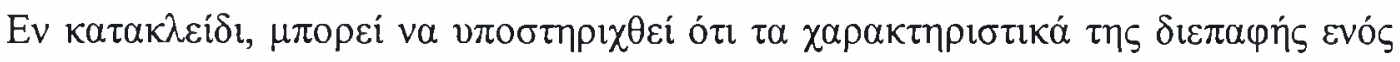

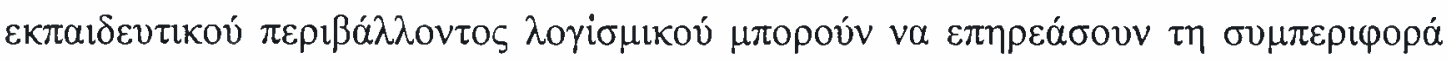

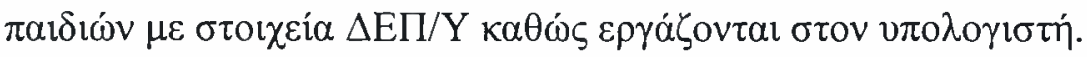

\subsection{2 Ү}

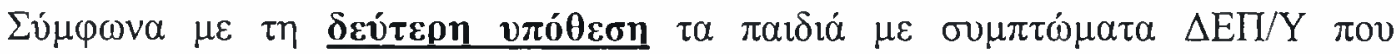

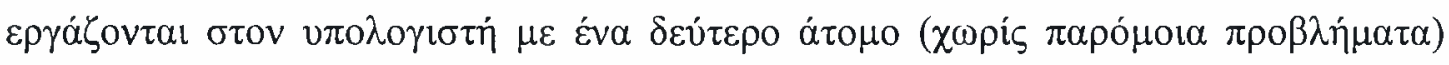

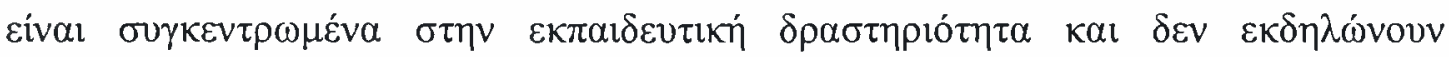

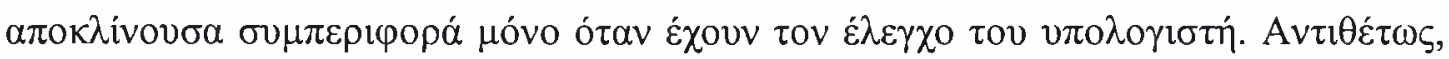

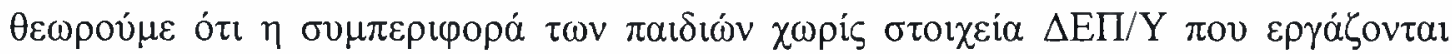

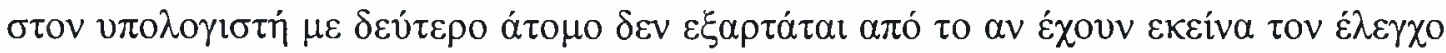

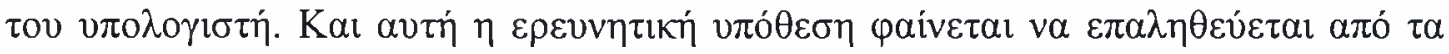

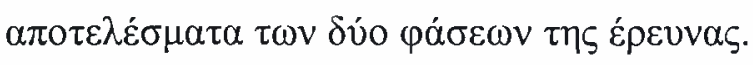

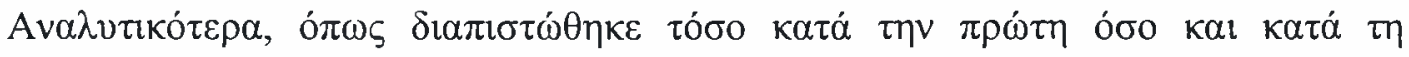

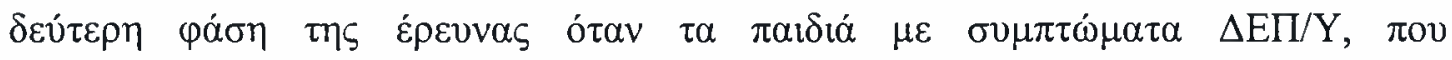

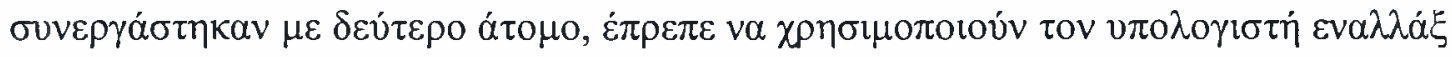

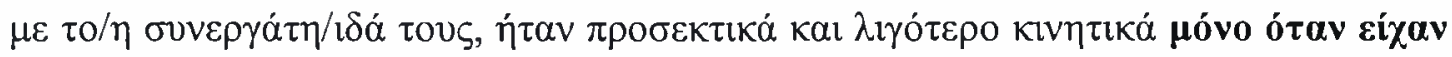

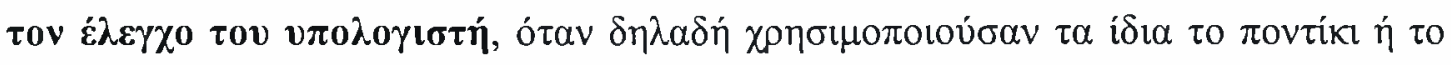

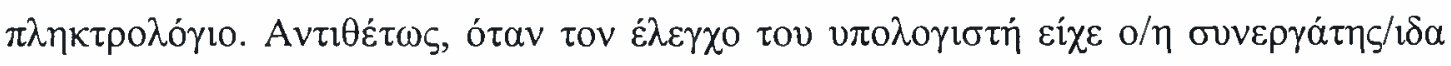

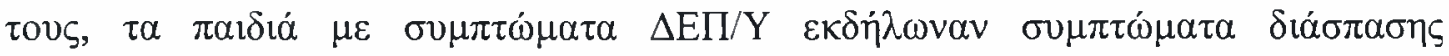

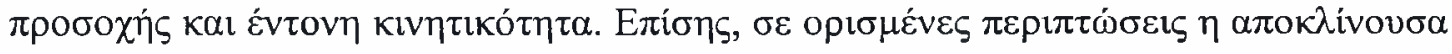

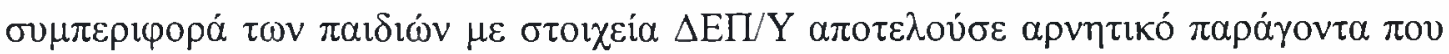

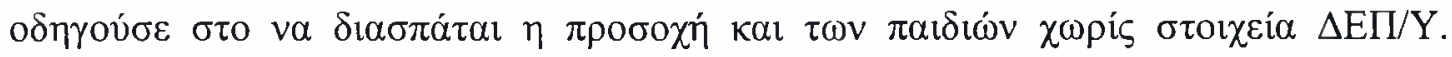

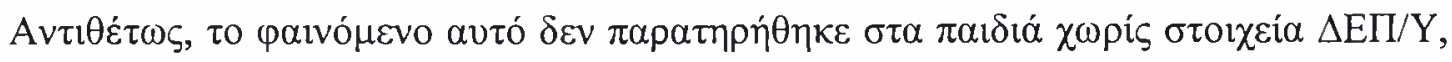

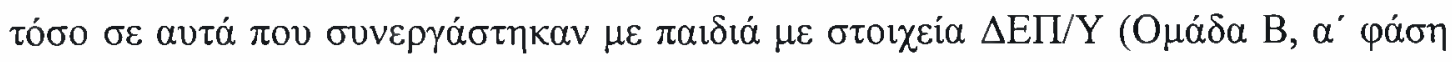

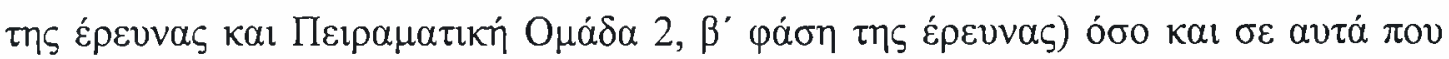

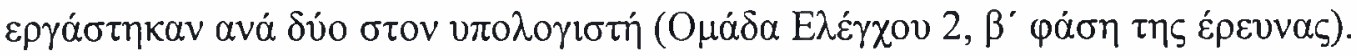

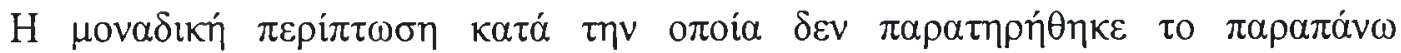

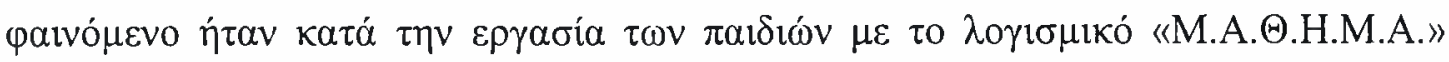




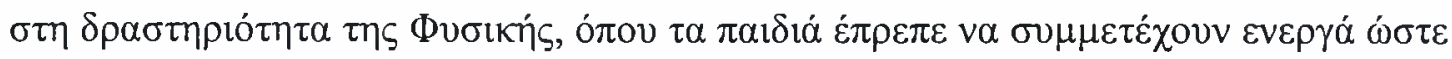

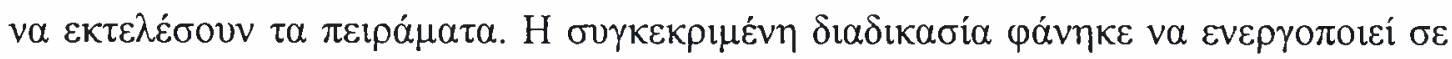

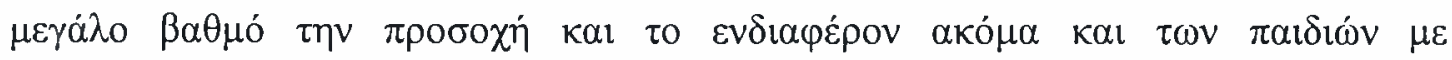

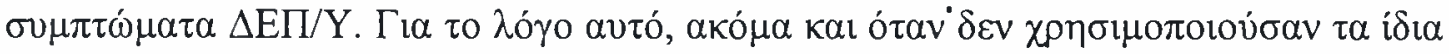

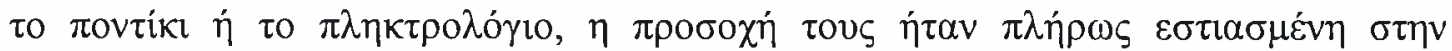

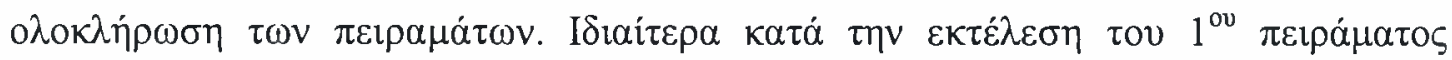

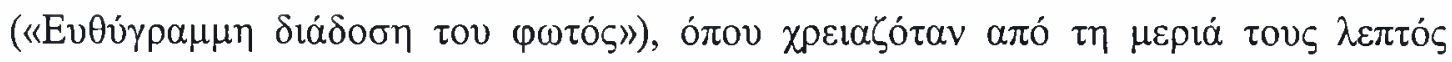

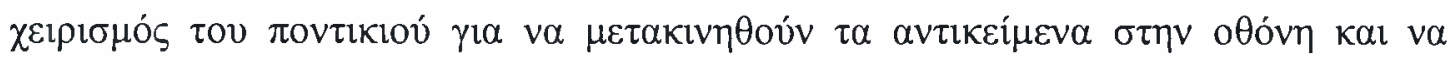

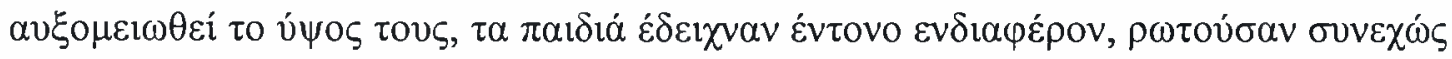

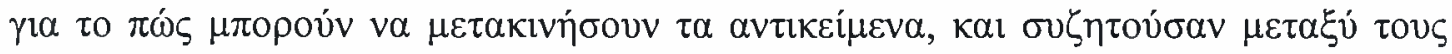

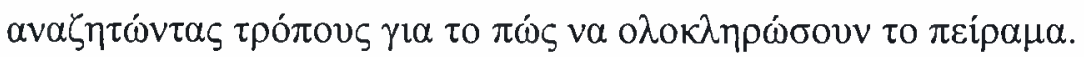

\subsubsection{Y}

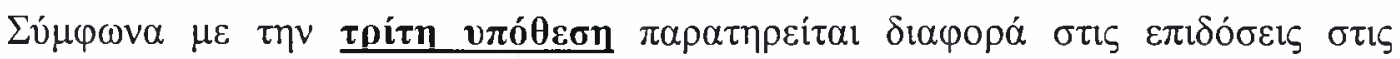

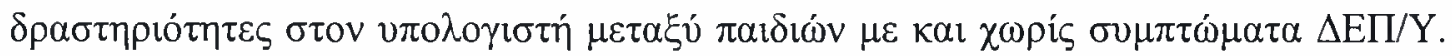

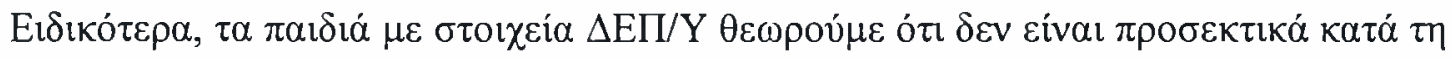

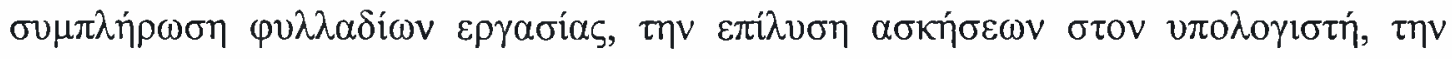

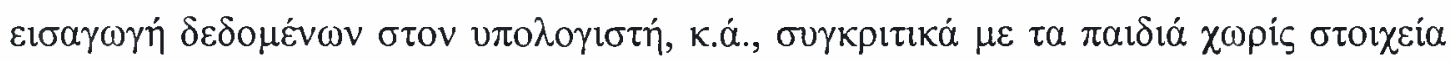

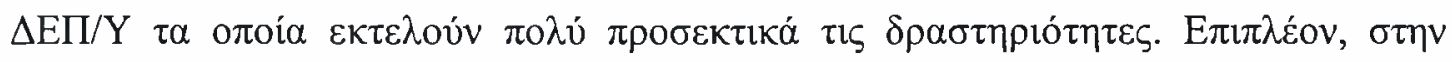

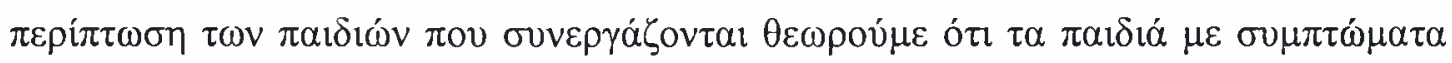
$\triangle \mathrm{E \Pi /Y} \mathrm{\beta \alpha \sigma i \zeta ov \tau \alpha l} \mathrm{\sigma \tau о/ \eta} \mathrm{\sigma uv \varepsilon \rho \gamma \alpha ́ \tau \eta /l \delta á} \mathrm{\tau ov \zeta} \mathrm{\kappa \alpha l} \mathrm{\alpha \pi o \varphi \varepsilon v́ \gamma ovv} \mathrm{v \alpha} \mathrm{\kappa \alpha ́vovv} \mathrm{\pi \rho \alpha ́ \gamma \mu \alpha \tau \alpha}$

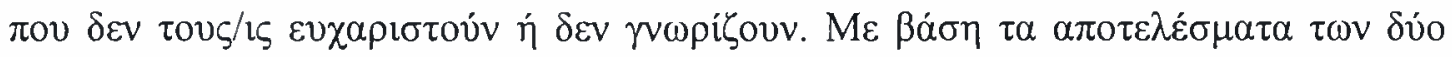

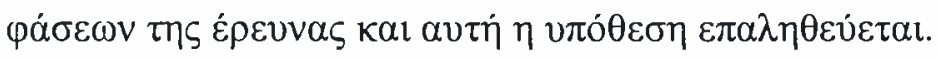

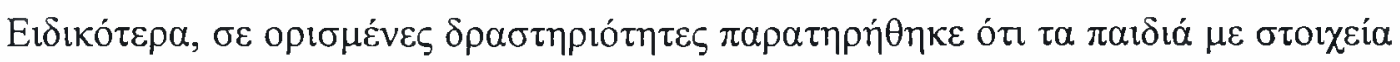

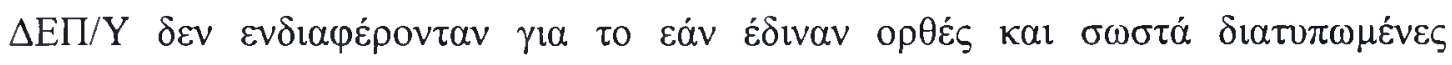

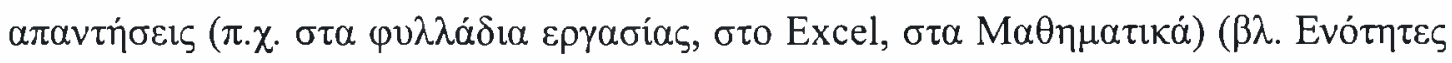

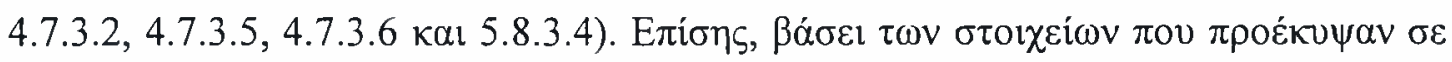

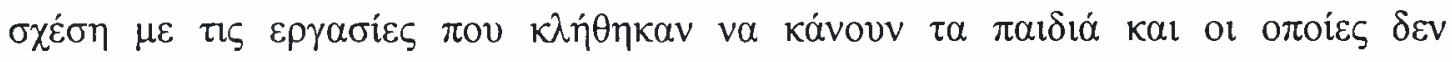

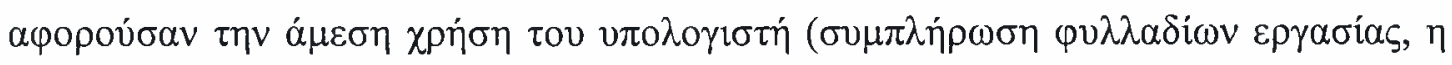

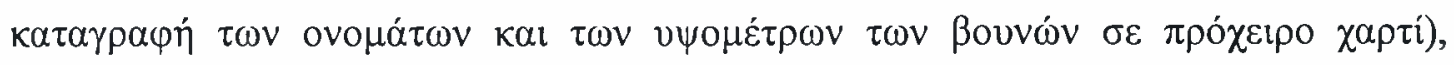

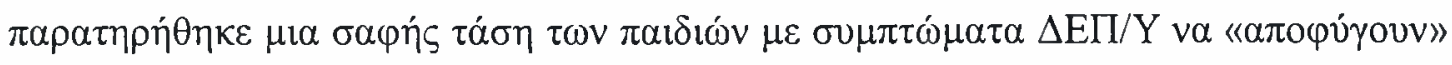




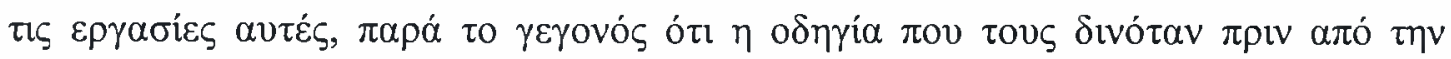

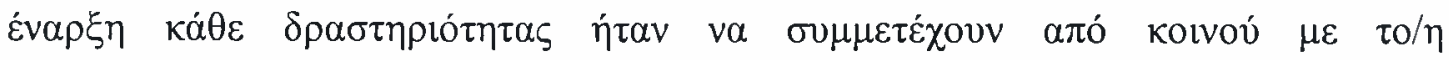

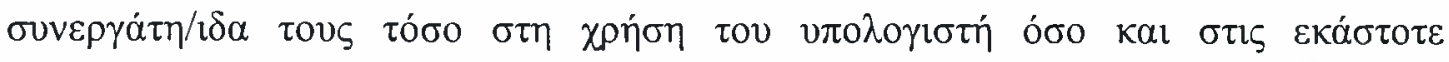

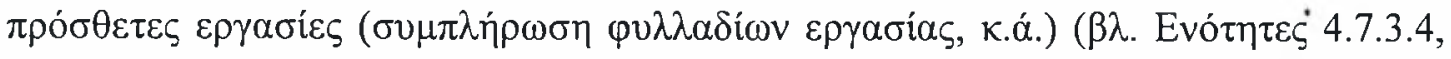
4.7.3.5, 5.8.3.2 каı 5.8.3.3).

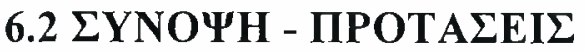

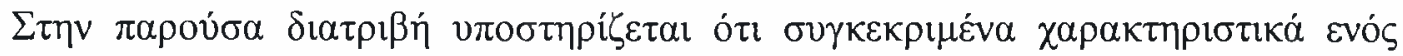

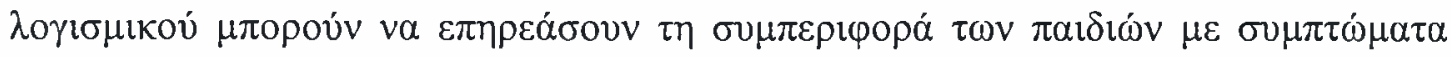

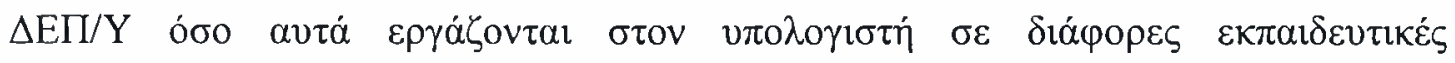

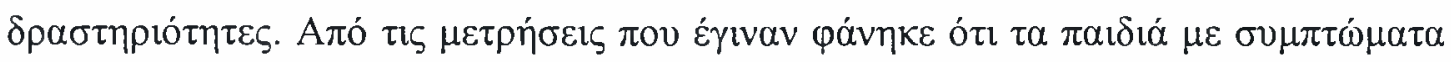

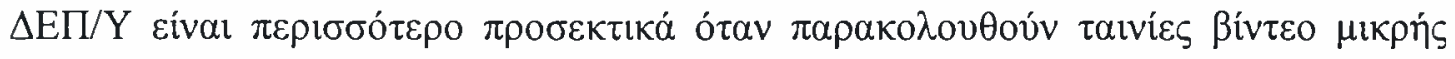

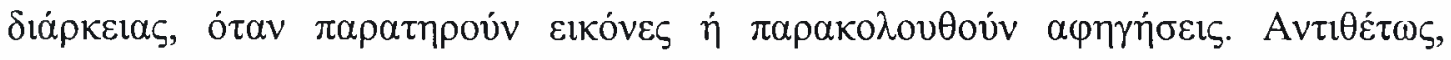

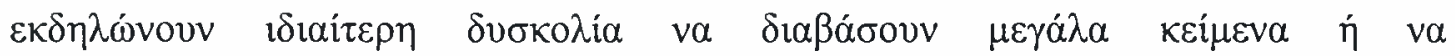

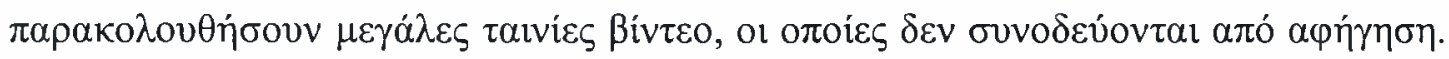

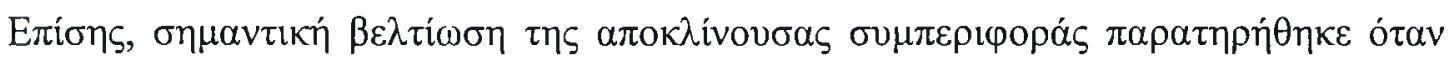

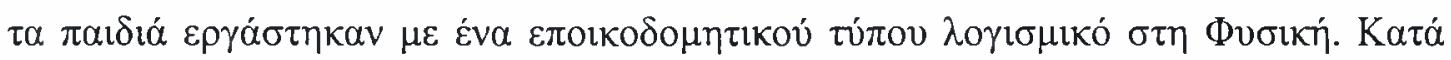

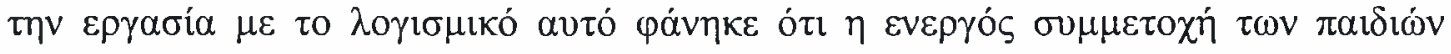

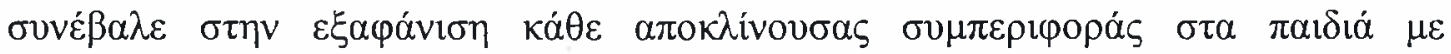

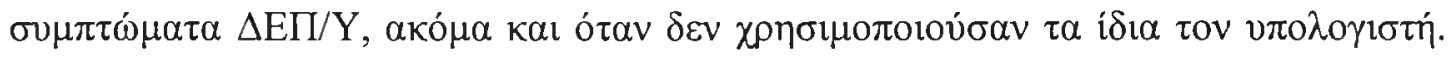

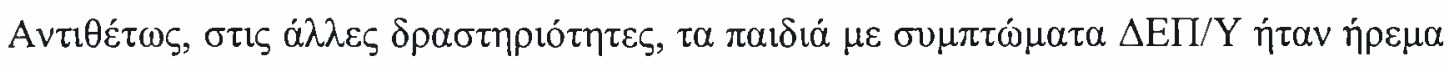

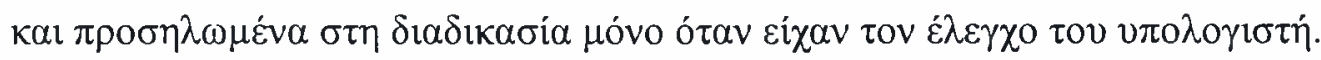

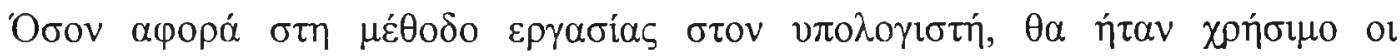

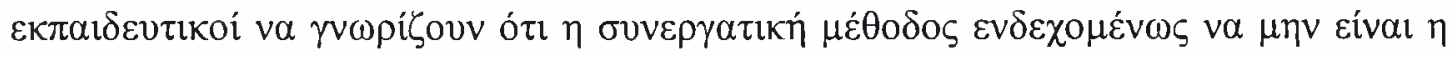

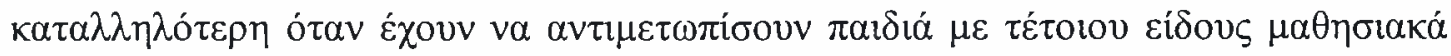

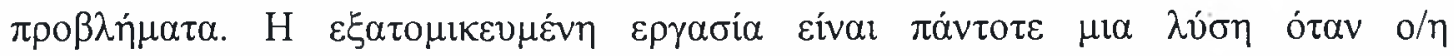

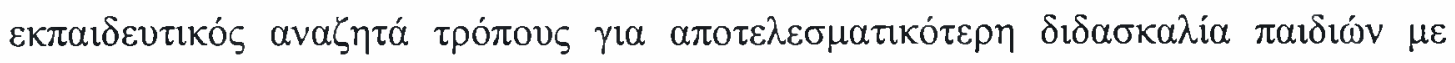

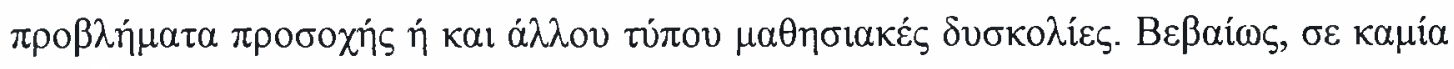

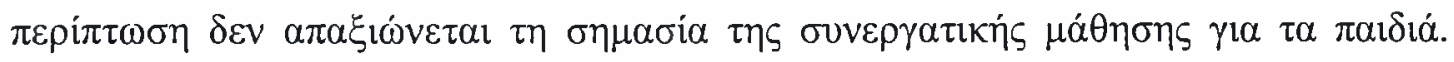

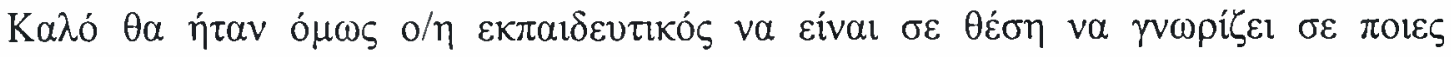

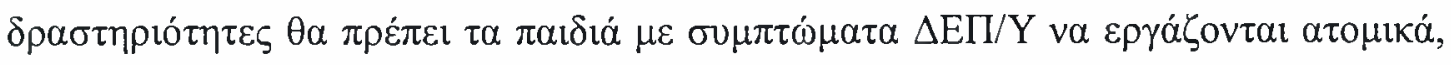




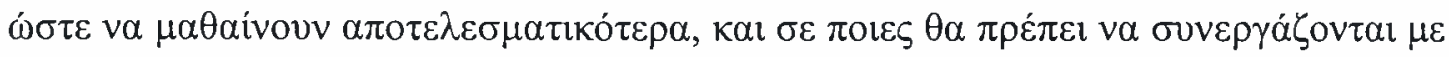

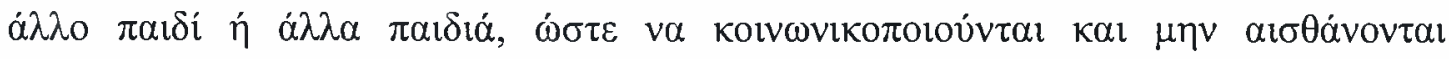
$\pi \alpha \rho \alpha \gamma \kappa \omega \vee v \sigma \mu \varepsilon \dot{v} \alpha$.

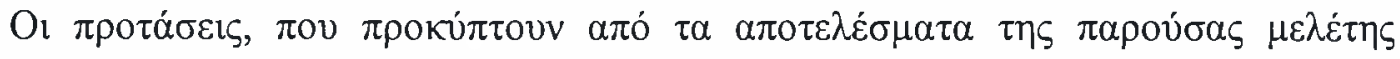

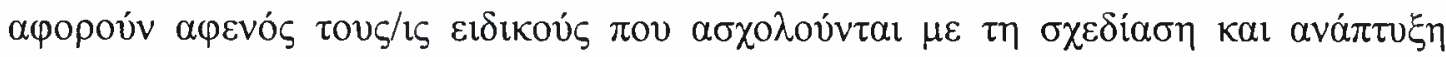

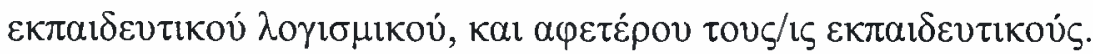

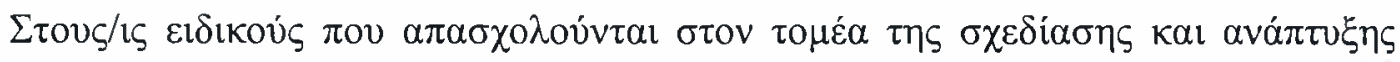

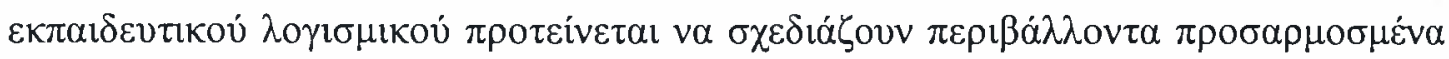

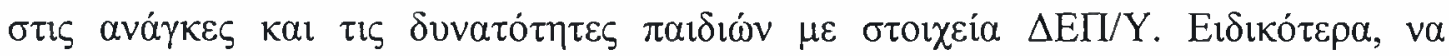

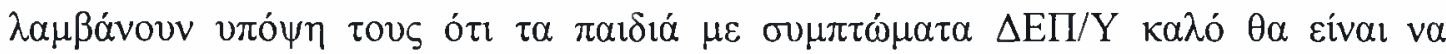

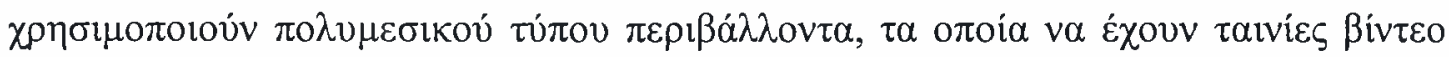

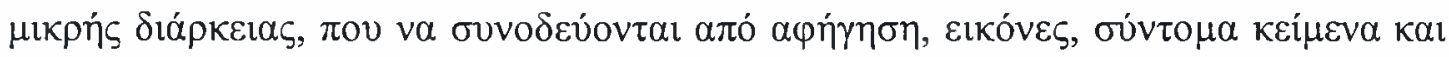

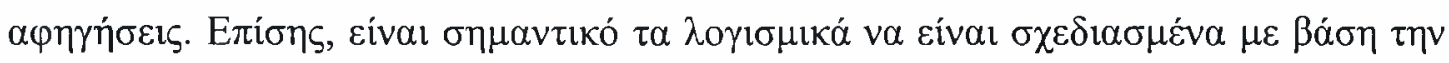

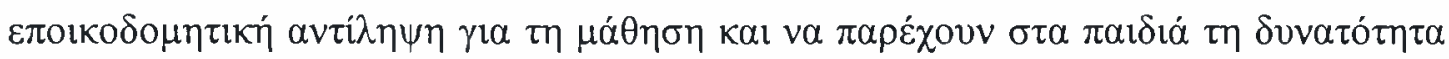

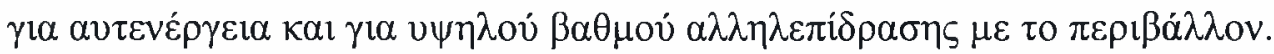

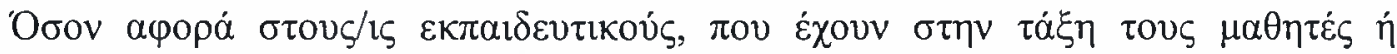

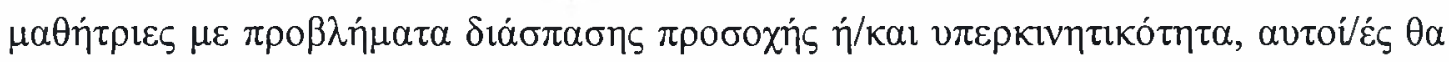

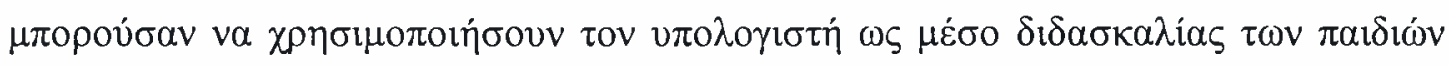

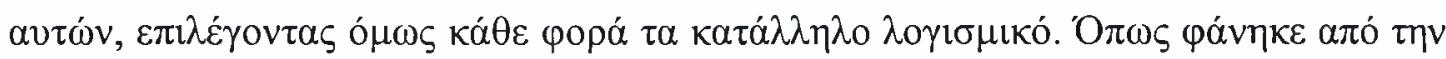

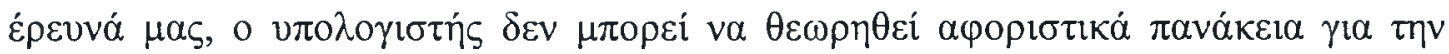

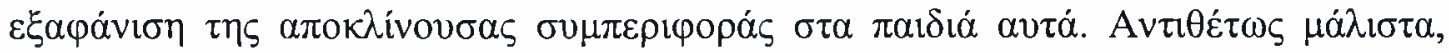

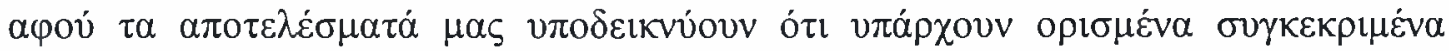

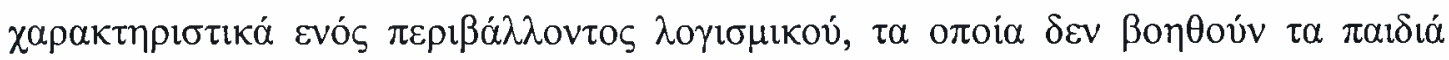

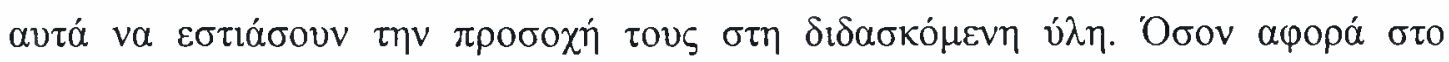

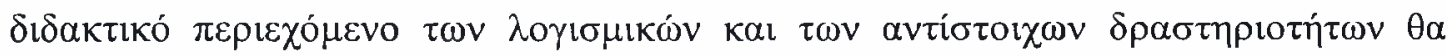

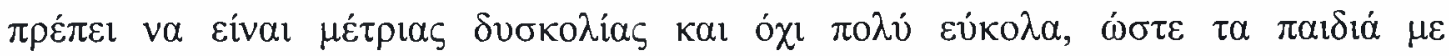

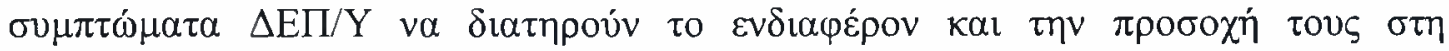

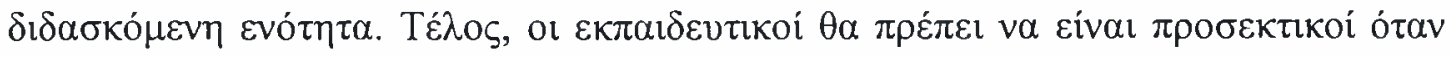

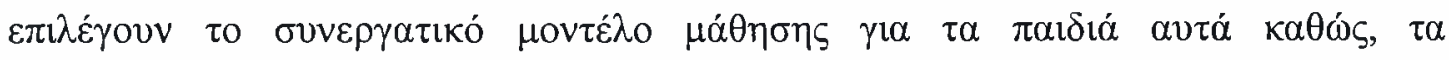

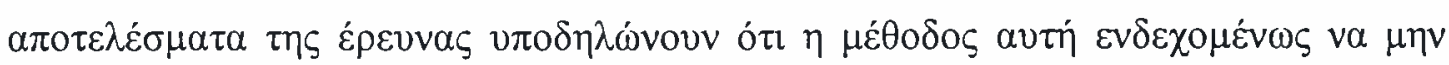

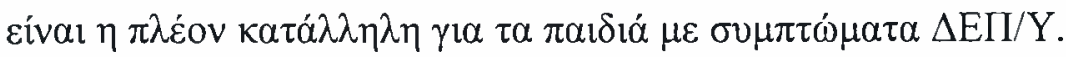

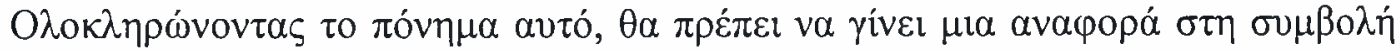

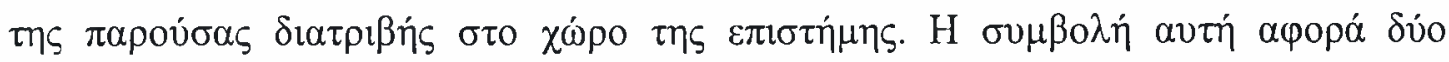

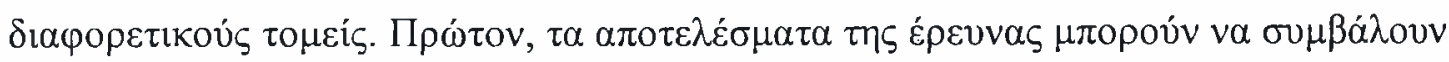




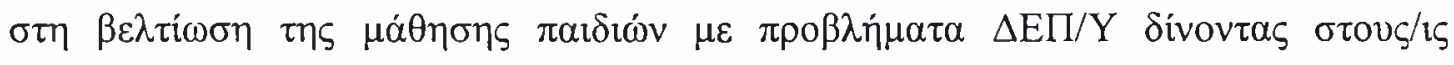

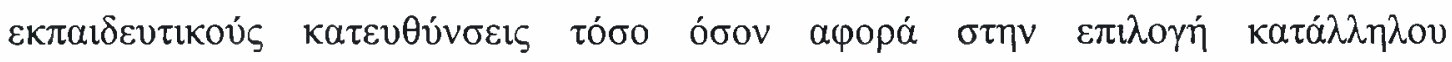

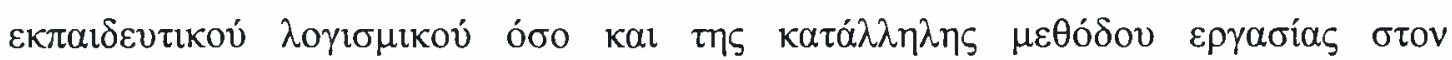

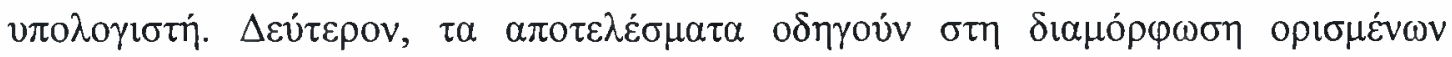

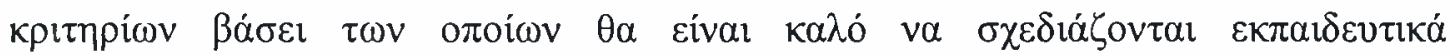

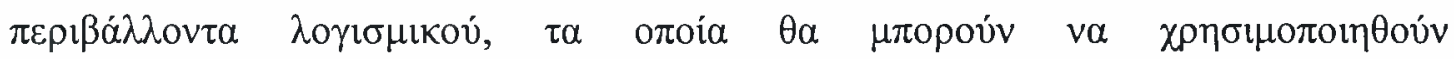

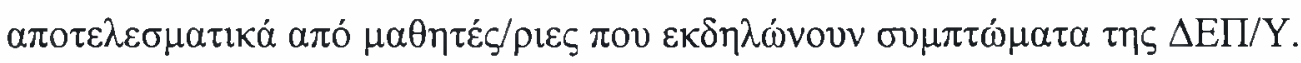




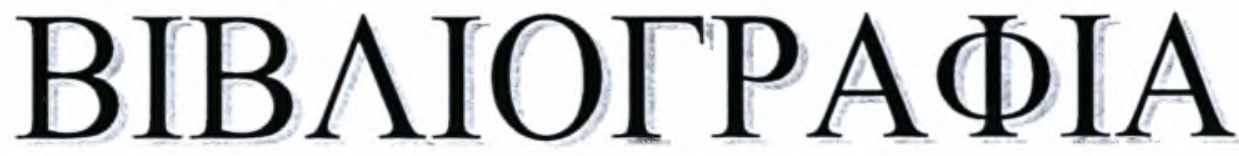




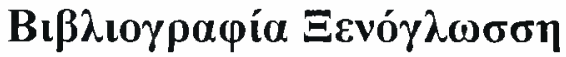

Abikoff, H. (1991). Cognitive Training in ADHD children: Less to it than meets the eye. Journal of Learning Disabilities, 24 (4), 205-209.

American Psychiatric Association (1968). Diagnostic and Statistical Manual of Mental Disorders ( $2^{\text {nd }}$ ed.). Washington, DC: Author.

American Psychiatric Association (1980). Diagnostic and Statistical Manual of Mental Disorders ( $3^{\text {rd }}$ ed.). Washington, DC: Author.

American Psychiatric Association (1987). Diagnostic and Statistical Manual of Mental Disorders ( $3^{\text {rd }}$ ed. - Revised). Washington, DC: Author.

American Psychiatric Association (1994). Diagnostic and Statistical Manual of Mental Disorders ( $4^{\text {th }}$ ed.). Washington, DC: Author.

Anderson-Inman, L. (Dec. 1990 - Jan. 1991). Enabling students with learning disabilities: Insights from research. The Computing Teacher, 18 (4), 26-29.

Barkley. R. A. (1995). Taking charge of ADHD. New York: The Guilford Press.

Barkley. R. A. (1998a, $2^{\text {nd }}$ edition). Attention-Deficit Hyperactivity Disorder: A handbook for diagnosis and treatment. New York: The Guilford Press.

Barkley, R. A. (1998b, September). Attention-deficit hyperactivity disorder. Scientific American, 44-49.

Bauder, D. \& Simmons, T. J. (2003). Universal Design for Learning: Accessing the curriculum through digitized text. In C. Stephanidis (Ed.) Universal Access in HCI. Inclusive Design in the Information Society, Proceedings of HCI International 2003, vol. 4, 1073-1077. Mahwah, NJ: Lawrence Erlbaum Associates.

Bender, R. \& Bender, W. (1996). Computer-Assisted Instruction for students at Risk for ADHD, Mild Disabilities, or Academic Problems. Massachusetts: Allyn and Bacon.

Berk, L. E. \& Potts, M. K. (1991). Development and functional significance of private speech among attention-deficit hyperactivity disordered and normal boys. Journal of Abnormal Child Psychology, 19, 357-377.

Bing, J., Swicegood, P., Delaney, E. \& Hallum, D (1993). Technology and whole language for students with learning disabilities. LD Forum, 19 (1), 6-12. 
Bitter, G. \& Pierson, M. (1999). Using Technology in the Classroom (4 $4^{\text {th }}$ edition). Boston: Allyn and Bacon.

Bradley, C. (1937). The behavior of children receiving benzedrine. American Journal of Psychiatry, 94, 577-585.

Brannan, K. (1984). Moving the writing process along. Learning, 13, 221.

Brim, S. A. \& Whitaker, D. (2000). Motivation and students with Attention Deficit Hyperactivity Disorder. Preventing school failure, 44 (2), 57-60.

Carey, D. \& Sale, P. (1997). Technology use benefits for learners with attention deficits. In J. Willis, J. D. Price, S. Mc Neil, B. Robin \& D. A. Willis (Eds.). Technology and Teacher Education Annual, 1997. Charlottesville, VA: Association for the Advancement of Computing in Education, 1037-1041.

Chelune, G. J., Ferguson, W., Koon, R. \& Dickey, T. O. (1986). Frontal lobe disinhibition in attention deficit disorder. Child Psychiatry and Human Psychology, 17, 73-80.

Cherkes - Julkowski, M. (1998). Learning disability, attention - deficit disorder, and language impairment as outcomes of prematurity: A longitudinal descriptive study. Journal of learning disabilities, 31(3), 294-306.

Chess, S. (1960). Diagnosis and treatment of the hyperactive child. New York State Journal of Medicine, 60, 2379-2385.

Collins, J., Hammond, M. \& Wellington, J. (1997). Teaching and learning with multimedia. London-New York: Routledge.

Conners, C. K. (1990). The Conners Rating Scales. North Tonawanda, NY: MultiHealth Systems.

Cook, E. H., Stein, M. A., Krasowski, M. D., Cox, N. J., Olkon, D. M., Kieffer, J. E. \& Leventhal, B. L. (1995). Association of attention-deficit disorder and the dopamine transporter gene. American Journal of Human Genetics, 56, 993-998.

Cunningham, C. E. \& Barkley, R. (1978). The role of academic failure in hyperactive behavior. Journal of Learning Disabilities, 11, 15-21.

Dailey, E. \& Rosemberg, M (1994). ADD, Computers \& Learning: Using Computers to Help Children with ADD Become Efficient Learners. Attention!, 1 (2), 8-16.

Davis, N., Desforges, C., Jessel, J., Somekh, B., Taylor, C. \& Vaughan, G. (1997). Can quality in learning be enhanced through the use of IT? In B. Somekh \& N. Davis (Eds.) Using Information Technology effectively in Teaching and Learning, 14-27. London - New York: Routledge. 
Dehler, C. \& Porras - Hernandez, L. H. (1998). Using Computer Mediated communication (CMC) to promote experiential learning in graduate studies. Educational Technology, 38 (3), 52-56.

Douglas, V. (1972). 'Stop, look and listen: The problem of sustained attention and impulse control in hyperactive and normal children. Canadian Journal of Behavioural Science, 4, 259-282.

Douglas, V. (1983). Attentional and cognitive problems. In M. Rutter (Ed.) Developmental neuropsychiatry, pp 280-329. New York: The Guilford Press.

Driver, R., \& Oldham, V. A. (1986), A constructivist approach to curriculum development in science. Studies in Science Education, 10, 37-60.

DuPaul, G., Power, T., Anastopoulos, A. \& Reid, R. (1998). ADHD Rating Scale-IV: Checklists, Norms and Clinical Interpretation. New York: The Guilford Press.

Elkind, J., Cohen, K. \& Murray, C. (1993). Using computer-based readers to improve reading comprehension of students with dyslexia. Annals of Dyslexia, 42, 238259.

Faraone, S.V. \& Biederman, J. (1998). Neurobiology of attention - deficit hyperactivity disorder. Biological Psychiatry, 44 (10), 951-958.

Farrace-Di Zinno, A.M., Douglas, G., Houghton, S., Lawrence, V., West, J., \& Whiting, K. (2001). Body movements of boys with Attention Deficit Hyperactivity Disorder (ADHD) during computer video game play. British Journal of Educational Technology, 32 (5), 607-618.

Feingold, B. (1973). Food additives and child development. Hospital Practice, 8, 11 $12,17-18,21$.

Feingold, B. (1975). Why your child is hyperactive. New York: Random House.

Fitzgerald, G. \& Koury, K. (1996). Empirical advances in technology assisted instruction for students with mild and moderate disabilities. Journal of Research on Computing in Education, 28 (4), 526-553.

Fitzgerald, G. \& Werner, J. (1996). The use of the computer to support cognitivebehavioral interventions for students with behavioral disorders. Journal of Computing in Childhood Education, 7 (3/4), 127-148.

Fitzgerald, G. (1996). Technology-Assisted instruction for students with learning disabilities. Preface to the Special Issue. Journal of Computing in Childhood Education, 7 (3/4), 123-125. 
Ford, M.J., Poe, V. \& Cox, J. (1993). Attending behaviors of ADHD children in Math and Reading using various types of software. Journal of Computing in Childhood Education, 4, 183-196.

Fowler, M. (1994). Attention-Deficit/Hyperactivity Disorder. NICHCY (National Information Center for Children and Youth with Disabilities) Briefing Paper, 116.

Frick, P. J., Kamphaus, R. W, Lahey, B. B., Loeber, R., Christ, M. A., Hart, E. L. \& Tannenbaum, L. E. (1991). Academic underachievement and disruptive behavior disorders. Journal of Consulting and Clinical Psychology, 59, pp. 289294.

Goldstein, S \& Goldstein M. (1992). Hyperactivity. Why won't my child pay attention? USA: John Wiley \& Sons, Inc.

Greve, J., Soriano, V. \& Weber, H. (2003). Providing ICT/IST professionals with access to resources in Special Education Needs (SEN) and vice versa - a European network approach. In C. Stephanidis (Ed.) Universal Access in HCI. Inclusive Design in the Information Society, Proceedings of HCI International 2003, vol. 4, 1103-1107. Mahwah, NJ: Lawrence Erlbaum Associates.

Hall, T. E., Hughes, C. A. \& Filbert, M. (2000). Computer assisted instruction in reading for students with learning disabilities: a research synthesis. Education and Treatment of Children, 23 (2), 173 - 193.

Hasselbring, T. (1982). Remediating spelling problems of learning-handicapped students through the use of microcomputers. Educational Technology, 31-32.

Hay, D., McStephen, M. \& Levy, F. (2001). The developmental genetics of ADHD. In F. Levy \& D. Hay (Eds.) Attention, genes and ADHD, 58-79. USA-Canada: Brunner - Routledge.

Hecker, L, Burns, L., Elkind, J., Elkind, K., \& Katz, L. (2002). Benefits of assistive reading software for students with attention disorders. Annals of Dyslexia, 52, 243-271.

Horton, S., Lovitt, T. \& Slocum, T. (1988). Teaching geography to high school students with academic deficits: Effects of a computerized map tutorial. Learning Disability Quarterly, 11, 371-379.

Johnson, D. \& Johnson, R. (1994). Learning together and alone: Cooperative, Competitive and Individualistic Learning. Boston: Allyn and Bacon.

Jonassen, D. (1996). Computers in the classroom. Prentice-Hall. New Jersey. 
Jones, K. U., Torgeson, J. K. \& Sexton, M. A. (1987). Using computer-guided practice to increase decoding fluency in learning disabled children: A study using the Hint and Hunt I program. Journal of Learning Disabilities, 20, 122128.

Kahn, E. \& Cohen, L. H. (1934). Organic driveness: A brainstorm syndrome and experience. New England Journal of Medicine, 210, 748-756.

Kakouros, E. \& Maniadaki, K. (1998). The effects of ADHD on children's academic achievement and behaviour. Oral presentation at the International Conference on Developmental / Intellectual Disabilities, March 1998, Larnaka, Cyprus.

Kearsley, G. \& Shneiderman, B. (1998). Engagement Theory: A framework for Technology-Based Teaching and Learning. Educational Technology, September-October, 20-23.

Keates, S., Clarkson, P. J., \& Robinson, P. (2002), Developing a practical inclusive interface approach. Interacting with computers, 14, 271-299.

Keough, B. K. (1971). Hyperactivity and learning disorders: review and speculation. Exceptional Children, 38, 101-109.

Kitz, W. \& Thorpe, H. W. (1995). A comparison of the effectiveness of videodisc and traditional algebra instruction for college-age students with learning disabilities. Remedial and Special Education, 16 (5), 295-306.

Kleiman, G., Humphrey, M. \& Lindsay, P. H. (1981). Microcomputers and hyperactive children. Creative Computing, 7 (3), 93-94.

LaHoste, G. J. et al. (1996). Dopamine D4 receptor gene polymorphism is associated with Attention Deficit Hyperactivity Disorder. Molecular Psychiatry, l (2), 121-124.

Larose, S., Gagnon, S., Ferland, C. \& Pepin, M. (1989). Psychology of computers: XIV. Cognitive rehabilitation through computers games. Perceptual and Motor Skills, 69, 851-858.

Lomangino, A., Nicholson, J. \& Sulzby, E. (1999). The influence of power relations and social goals on children's collaborative interactions while composing on computer. Early Childhood Research Quarterly, 14 (2), 197-228.

Long, G., Pence, H. \& Zielinsky, T. J. (1995). New tools vs. old methods: a description of the CHEMCOMF 93 discussion. Computers and Education, 24 (4), 259-269. 
Maccini, P., McNaughton, D. \& Ruhl, K. L. (1999). Algebra instruction for students with learning disabilities: implications from a research review. Learning Disability Quarterly, 22 (2), $113-126$.

Maccini, P., Gagnon, J. C. \& Hughes, C. A. (2002). Technology - based practices for secondary students with learning disabilities. Learning Disability Quarterly, 25, 247-261.

Male, M. (2003). Technology for inclusion. Meeting the special needs of all students. USA: Pearson Education Group Inc.

Marshall, P. (1989). Attention deficit disorder and allergy: A neurochemical model of the relation between the illness. Psychological Bulletin, 106, 434-446.

McArthur, C. A. \& Graham, S. (1987). Learning disabled students' composing under three methods of text production: Handwriting, word processing and dictation. Journal of Special Education, 20 (3), 22-42.

McArthur, C. A., Ferretti, R. P. \& Okolo, C. M. (2001). Technology applications for students with literacy problems: a critical review. The Elementary School Journal, 101 (3), 273-301.

McArthur, C. A., Haynes, J., Malouf, D. B. \& Harris, K (1987). Computer assisted instruction with learning disabled students: Achievement, engagement, and other factors related to achievement. Paper presented at the annual meeting of the American Educational Research Association, Washinghton, DC.

Metaxaki-Kossionides, C., Lialiou, S. \& Kouroupetroglou, G. (1999). Applying the object oriented design in combination with the hypertext mode for prototyping in different topics. Proceedings of ED-Media 1999-World Conference on Educational, Multimedia, Hypermedia \& Telecommunications, Seattle, Washington, USA, June 19-24 1999, 917-922.

Millman, P. G. (1984). The effects of computer-assisted instruction on attention deficits, achievement and attitudes of learning disabled children (Doctoral dissertation, University of Virginia, 1984). Dissertation Abstracts International, $45,3114 \mathrm{~A}$.

Mitsis, E., McKay, K., Schulz, K., Newcorn, J. \& Halperin, J. (2000). Parent-teacher concordance for DSM-IV Attention-Deficit/Hyperactivity Disorder in a clinicreferred sample. Journal of American Academy of Child \& Adolescent Psychiatry, 39 (3), 308-313. 
Navarro, J., Ruiz, G., Alcalde, C., Marchena, E. \& Aguilar, M. (2001). How to increase attention using a computer assisted teaching procedure. Paper presented at the International Conference on Education and Technology, Florida, May 2001.

Ostoits, J. (1999). Reading strategies for students with ADD and ADHD in the inclusive classroom. Preventing school failure, 43 (3), 129-32.

Page, M. (2002). Technology-Enriched Classrooms Effects on students of low socioeconomic status. Journal of Research on Technology in Education, 34, 389-409.

Passig, D. \& Levin, H. (2001). The interaction between gender, age, and multimedia interface design. Education and Information Technologies, 6 (4), 241-250.

Reif, S. F. (1993). How to reach and teach $A D D / A D H D$ children. Boston: Allyn \& Bacon.

Poole, B. J. (1997, $2^{\text {nd }}$ edition). Education for an Information Age: Teaching in the Computerized Classroom. USA: McGraw-Hill.

Preece, J., Rogers, Y. \& Sharp, E. (2002). Interaction design: beyond human computer interaction. USA: John Wiley \& sons Inc.

Reid, R. (1999). Attention Deficit Hyperactivity Disorder: Effective Methods for the classroom. Focus on Exceptional Children, 32 (4), 1-20.

Roth, S. F. \& Beck, I. L. (1987). Theoretical and instructional implications of the assessment of two microcomputer word recognition programs. Reading Research Quarterly, 22 (2), 197-218.

Seiger, M. (2001). Tips for helping ADHD students succeed. American Teacher, 85 (5), 23.

Shih - Chung, L. (1996). Evaluation of the user interface design for Chinese instructional multimedia programs. International Journal of Instructional Media, 23 (3), 219-127.

Shneiderman, B. (2000), Universal Usability: pushing human-computer interaction research to empower every citizen. Communications of the ACM, 43, 84-91.

Sieg, K. G., Gaffney, G. R., Preston, D. F. \& Hellings, J. A. (1995). SPECT brain imaging abnormalities in attention deficit hyperactivity disorder. Clinical Nuclear Medicine, 21 (1), 55-60.

Silverman, B. (1995). Computer Supported Collaborative Learning. Computers and Education, 25 (3), 81-91. 
Slate, S.E., Meyer, T., Burns, W. \& Montgomery, D. (1998). Computerized cognitive training for severely disturbed children with ADHD. Behaviour Modification, 22, 415-437.

Stephanidis, C. (ed.) (2003). Universal Access in HCI. Inclusive Design in the Information Society. Proceedings of HCI International 2003, vol. 4. Mahwah, NJ: Lawrence Erlbaum Associates.

Still, G. F. (1902). Some abnormal psychical conditions in children: Lectures I, II and III. Lancet, $i$, March 29: 1008-1012, April 19: 1077-1082, April 26: 1163-1168.

Wicks - Nelson, R. \& Israel, A. C. (1997). Behavioral disorders of childhood, third edition. New Jersey: Prentice-Hall.

Willer, A. (1984). Creative writing with computers: What do elementary students have to say? Computers, Reading and Language Arts, 2 (1), 39-42.

Xu, C., Reid, R., \& Steckelberg, A (2002), Technology applications for children with ADHD: Assessing the empirical support. Education and Treatment of Children, $25,224-248$.

Zametkin, A. et al. (1990). Cerebral glucose metabolism in adults with hyperactivity of childhood onset. New England Journal of Medicine, 323 (2), 1361-1366.

Zentall, S. (1993). Research on the Educational Implications of Attention Deficit Hyperactivity Disorder. Exceptional Children, 60 (2), 143-153. 


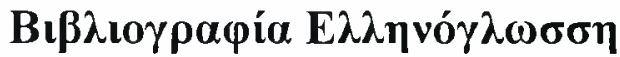

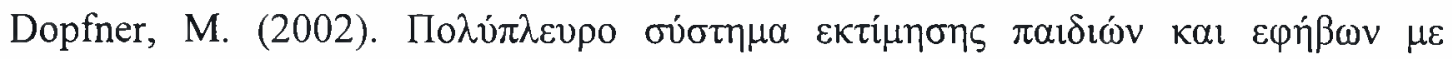

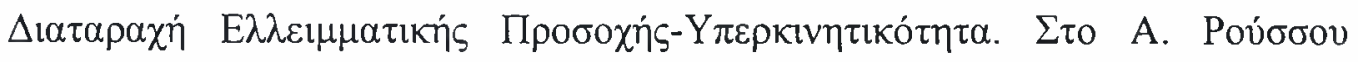

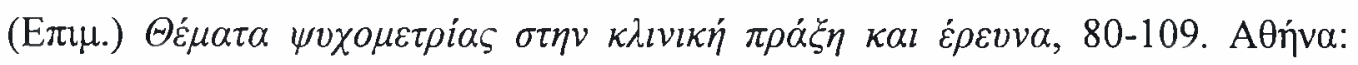

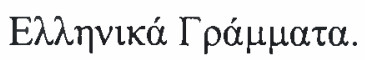

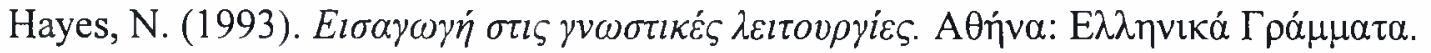

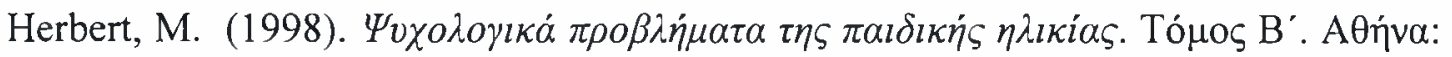

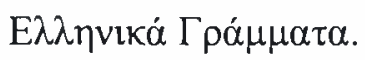

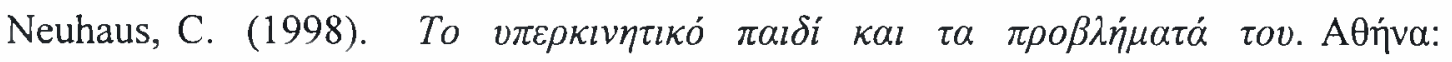

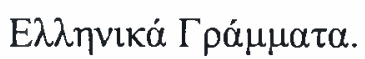

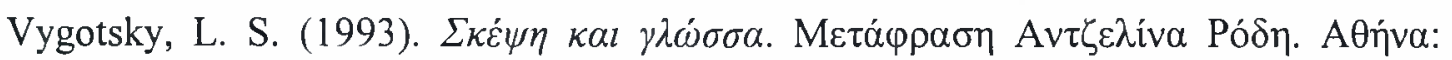

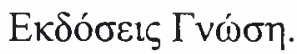

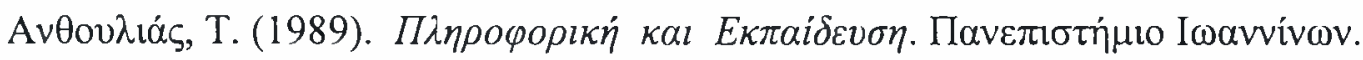

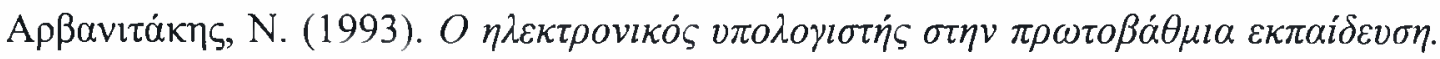

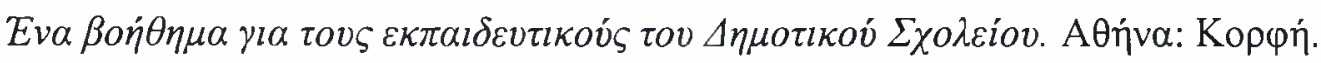

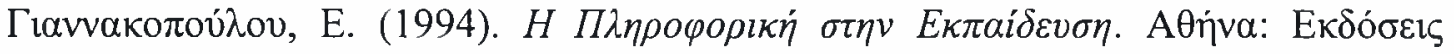

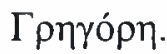

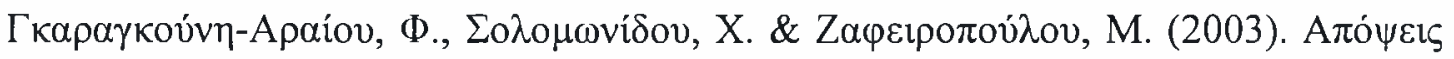

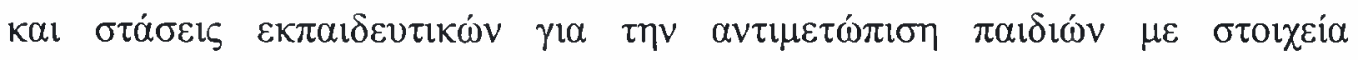

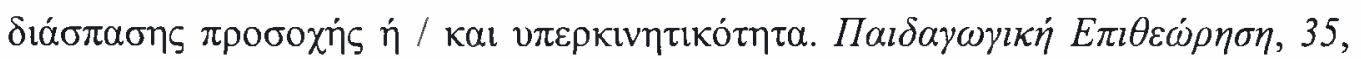
77-96.

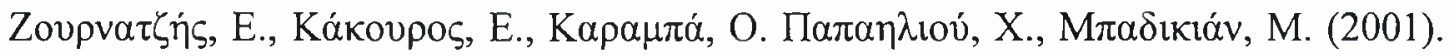

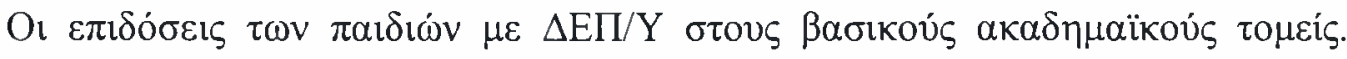

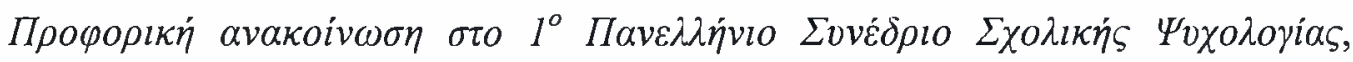

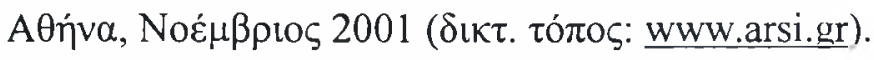

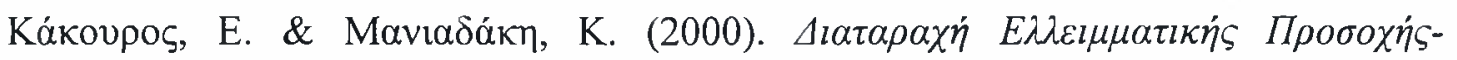

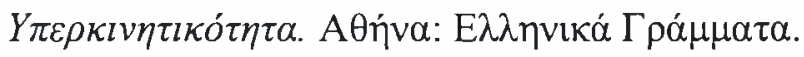

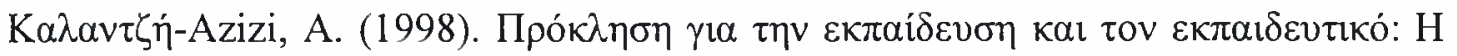

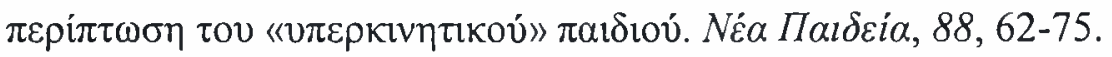

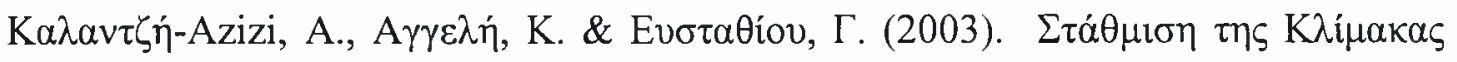

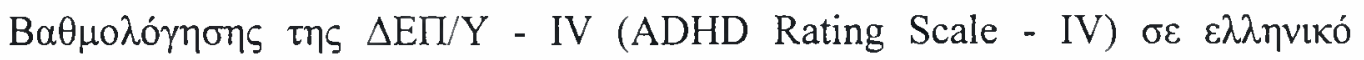




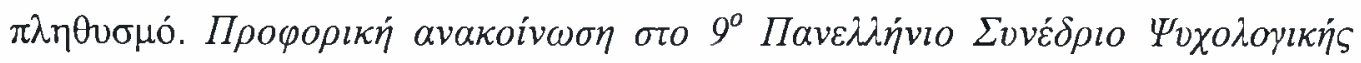

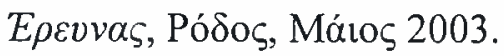

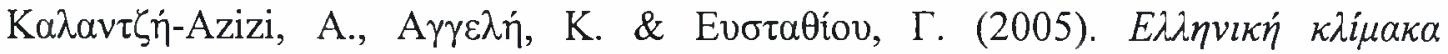

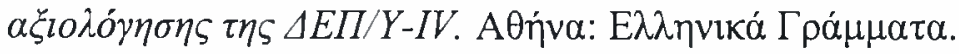

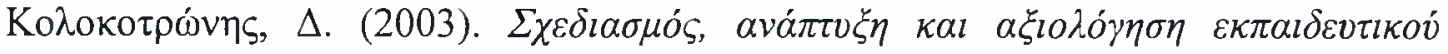

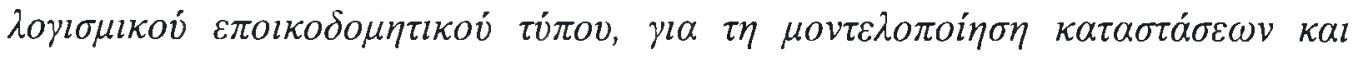

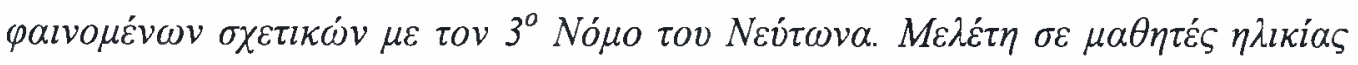

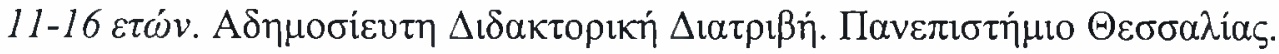

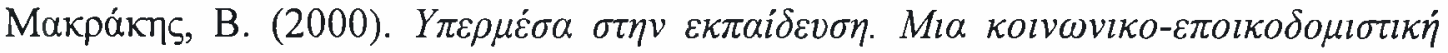

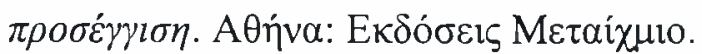

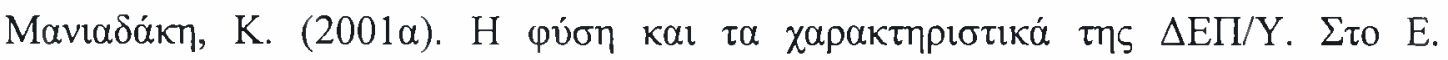

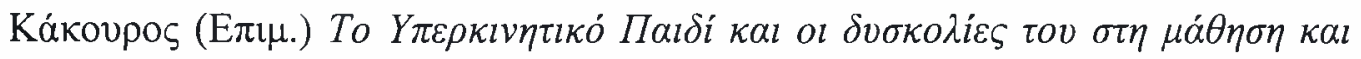

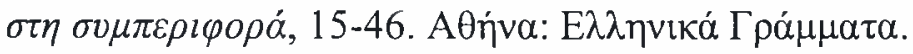

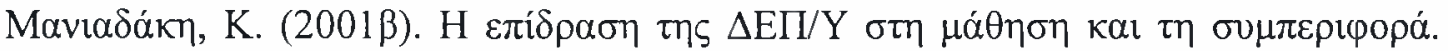

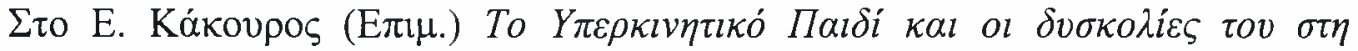

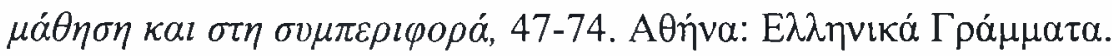

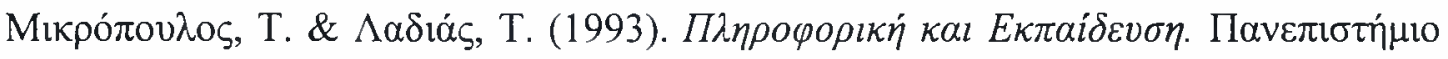
I $\omega \alpha v v i v \omega v$.

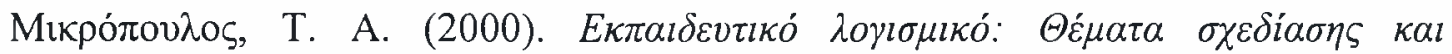

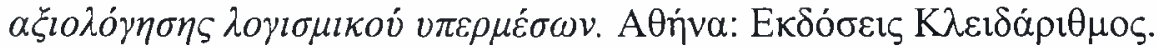

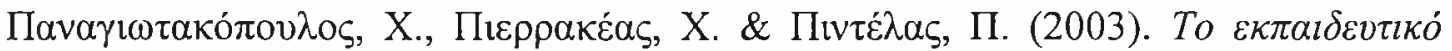

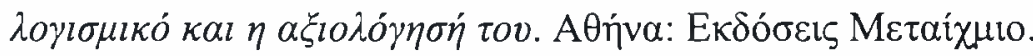

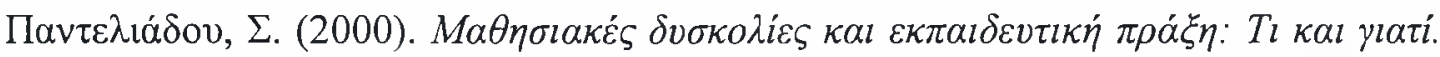
A $\theta \eta \dot{v \alpha: ~ E \lambda \lambda \eta v ı к \alpha ́ ~ Г \rho \alpha ́ \mu \mu \alpha \tau \alpha . ~}$

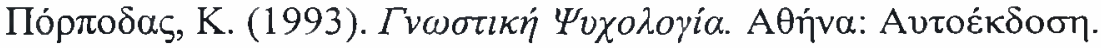

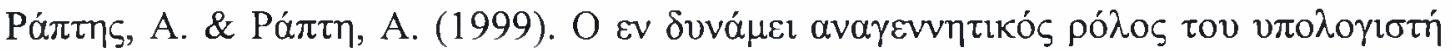

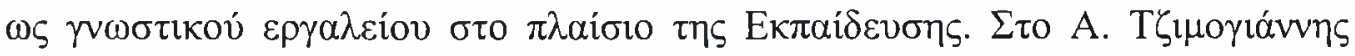

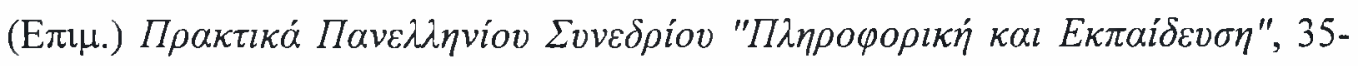
52, Iwávviva, 14-15 Maî́ov 1999.

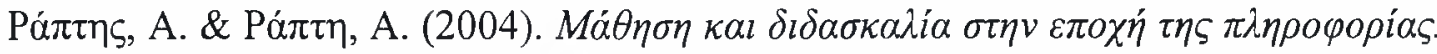

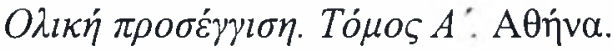

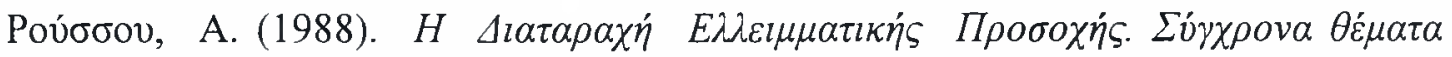

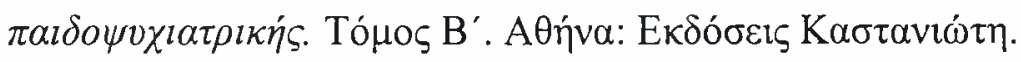




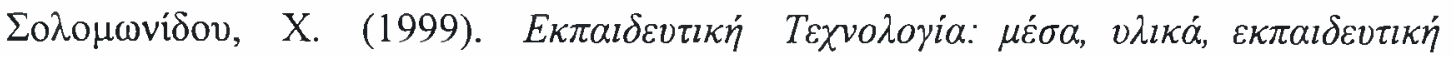

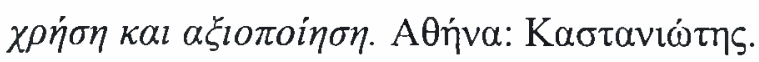

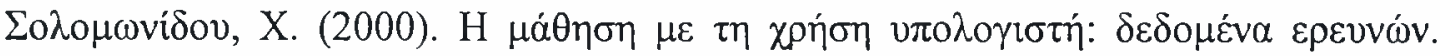
Themes in Education, 1 (1), 75-100.

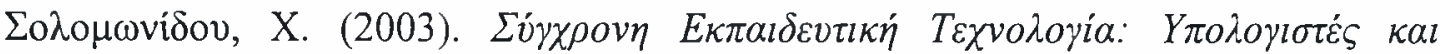

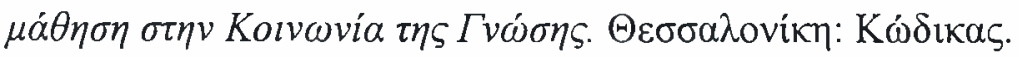

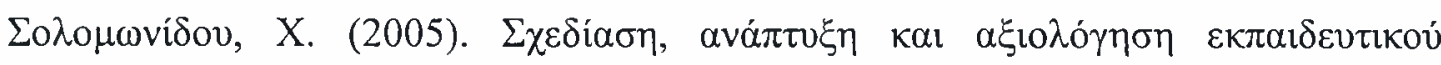

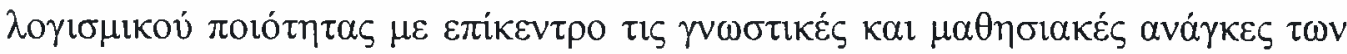

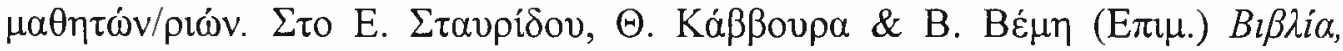

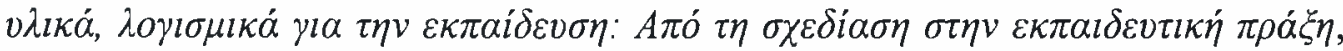
96-104, Bóגos.

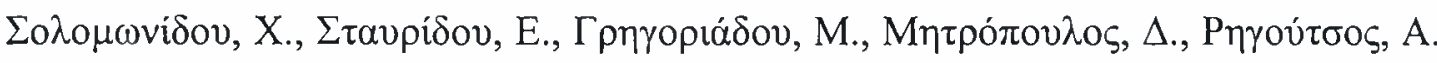

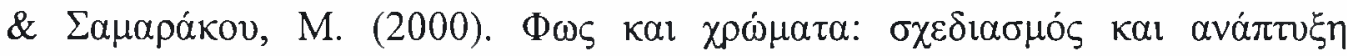

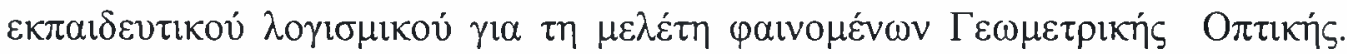

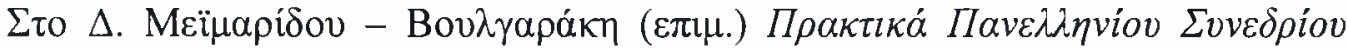

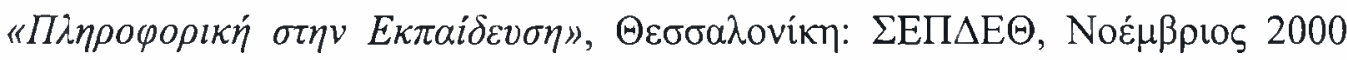

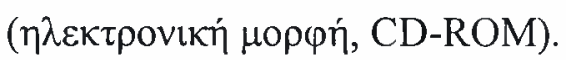

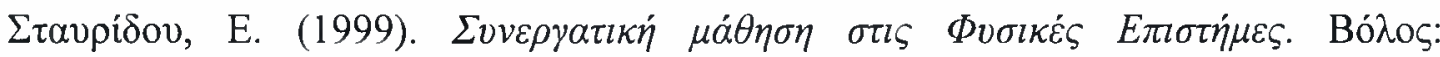

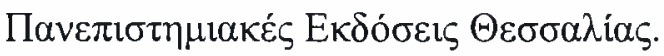

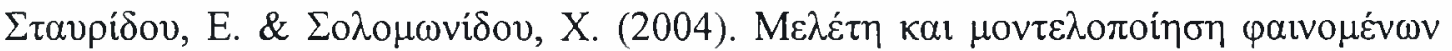

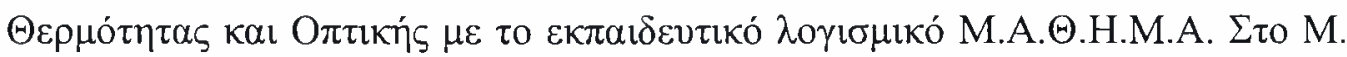

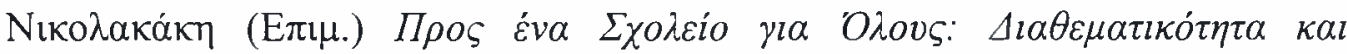

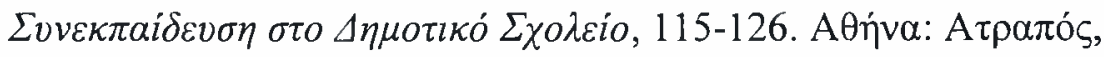

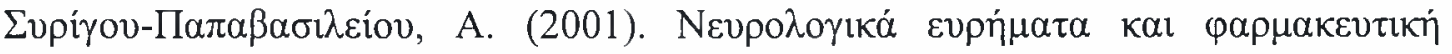

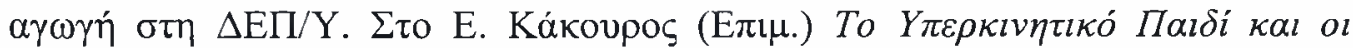

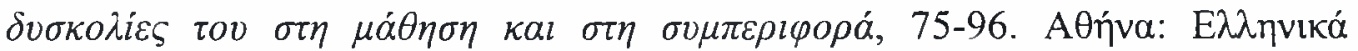
$\Gamma \rho \alpha \dot{\mu} \mu \alpha \tau \alpha$. 


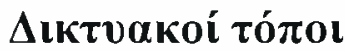

National Joint Committee on Learning Disabilities (NJCLD):

http://www.ldonline.org/ld indepth/general info/definitions.html

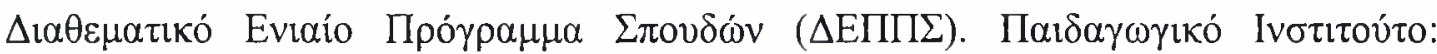
http://www.pi-school.gr

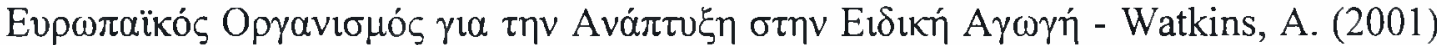

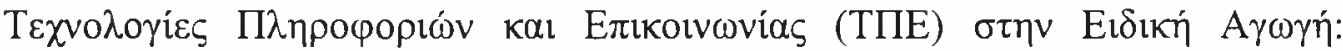
http://www.european-agency.org/ict sen db/index.html).

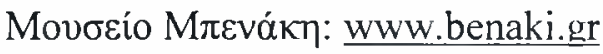

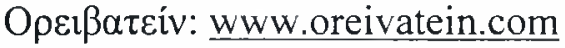




\section{Парápтnpa 1}

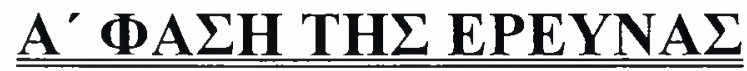




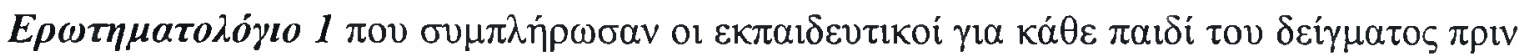

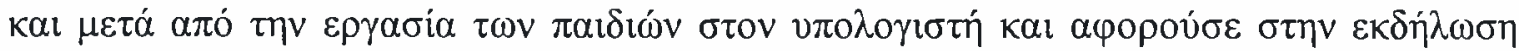

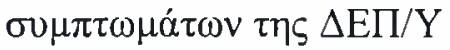

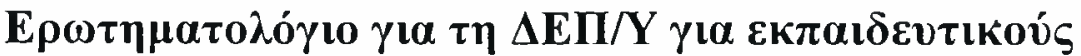

(DuPaul, Power, Anastopoulos, \& Reid, 1998)

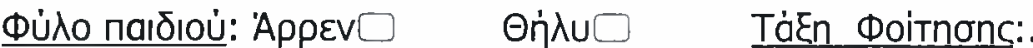

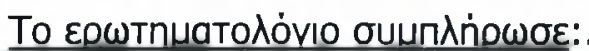

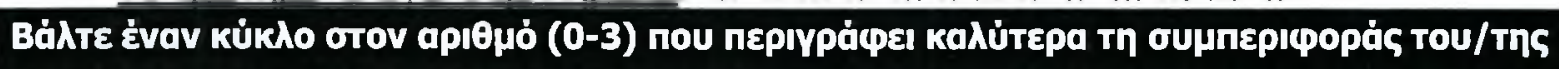

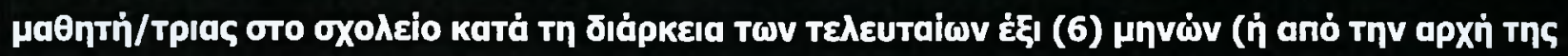
oxodikís xpoviác).

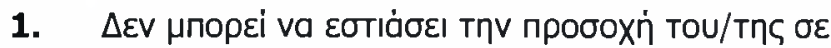

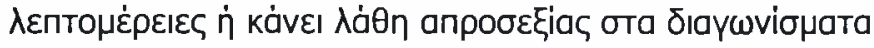

\begin{tabular}{|c|c|c|c|}
\hline 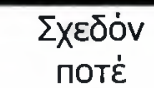 & Enávıa & 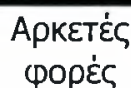 & $\begin{array}{l}\text { Пoגú } \\
\text { ouxvé }\end{array}$ \\
\hline
\end{tabular}

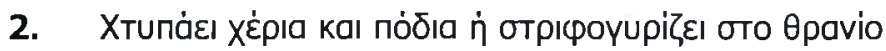

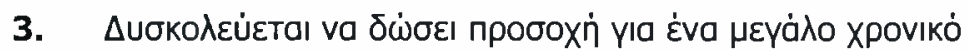

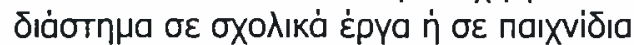

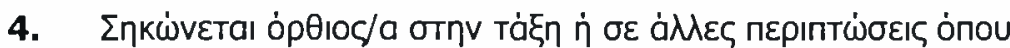

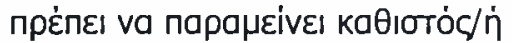

0

0

0

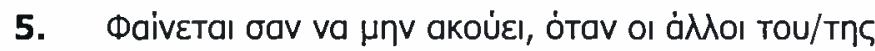

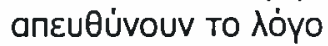

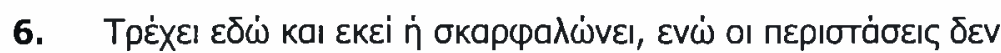

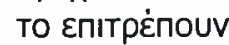

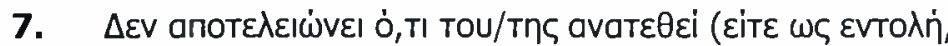

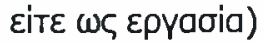

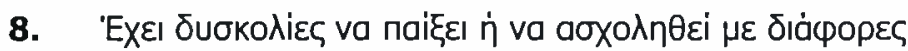

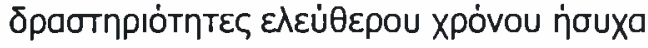

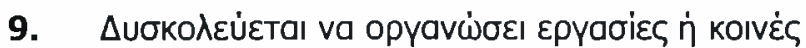
ठраотпріо́тптєৎ

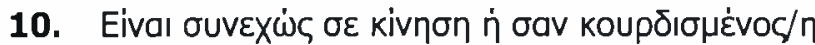

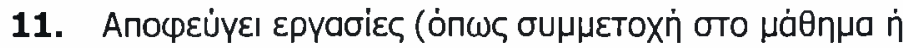

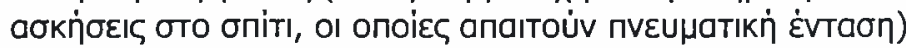

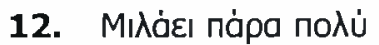

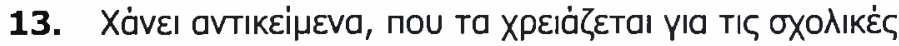

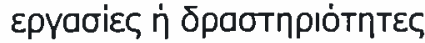

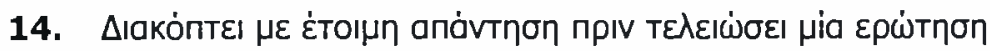

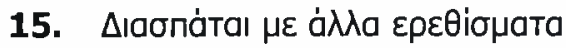

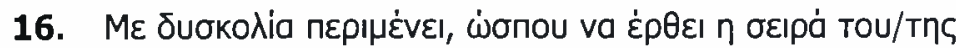

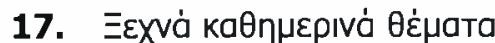

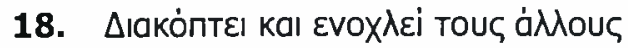

\begin{tabular}{|c|c|c|c|}
\hline 0 & 1 & 2 & 3 \\
\hline 0 & 1 & 2 & 3 \\
\hline 0 & 1 & 2 & 3 \\
\hline 0 & 1 & 2 & 3 \\
\hline 0 & 1 & 2 & 3 \\
\hline 0 & 1 & 2 & 3 \\
\hline 0 & 1 & 2 & 3 \\
\hline 0 & 1 & 2 & 3 \\
\hline 0 & 1 & 2 & 3 \\
\hline 0 & 1 & 2 & 3 \\
\hline 0 & 1 & 2 & 3 \\
\hline 0 & 1 & 2 & 3 \\
\hline 0 & 1 & 2 & 3 \\
\hline 0 & 1 & 2 & 3 \\
\hline 0 & 1 & 2 & 3 \\
\hline 0 & 1 & 2 & 3 \\
\hline 0 & 1 & 2 & 3 \\
\hline 0 & 1 & 2 & 3 \\
\hline
\end{tabular}




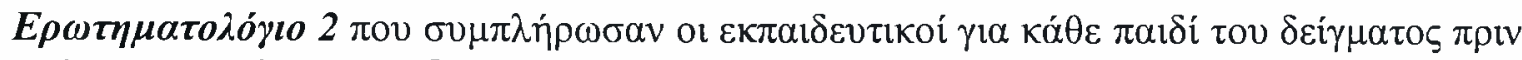

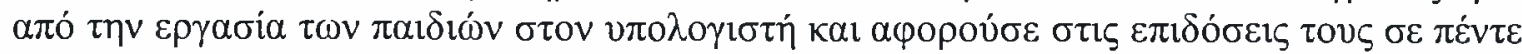

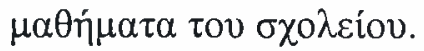

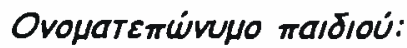

Tákn:

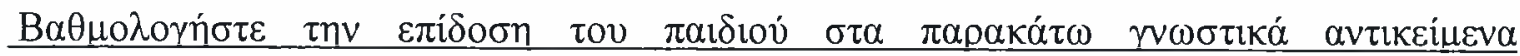

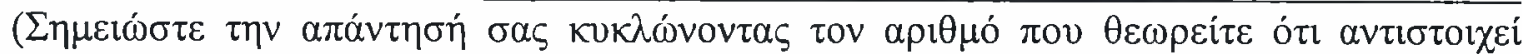

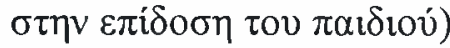

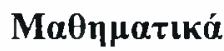

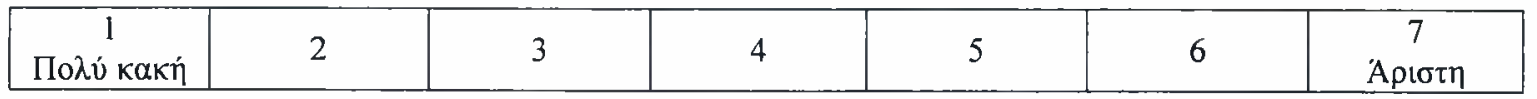

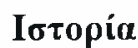

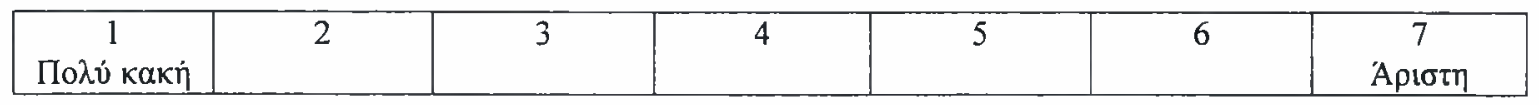

\section{Фขбเкท́}

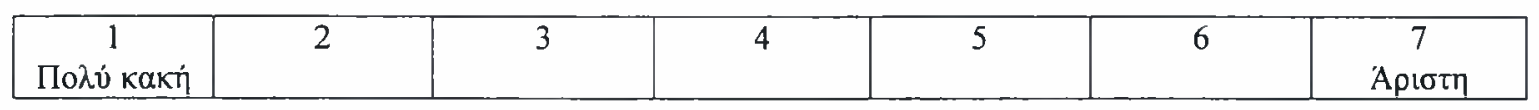

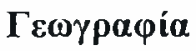

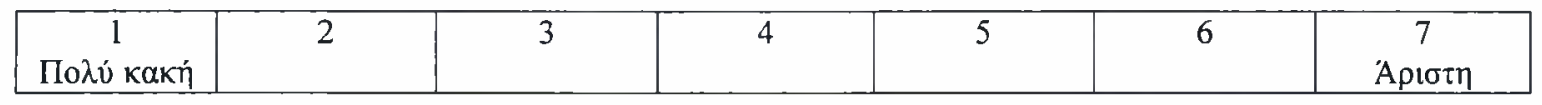

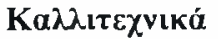

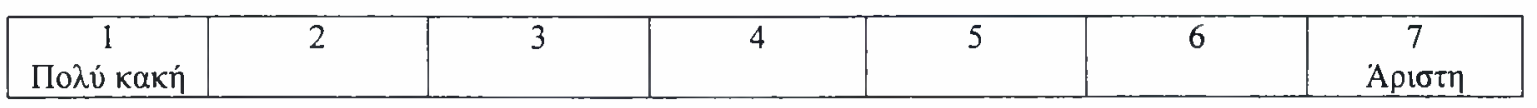




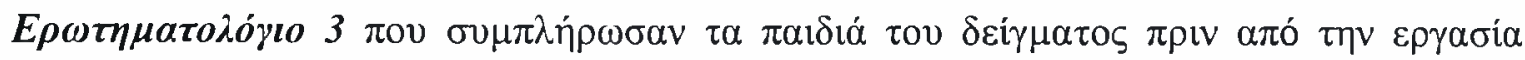

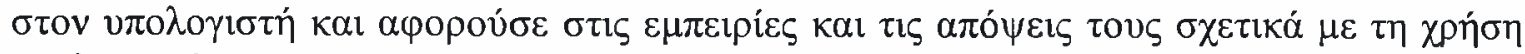

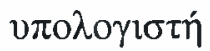

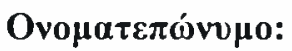

Tágฺ:

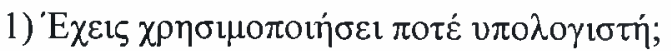

1. $\mathrm{N \alpha l}$

2. $\mathrm{O} \chi \mathrm{l}$

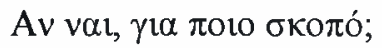

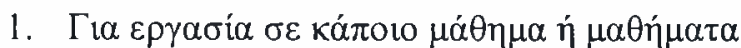

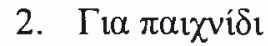

3. $A \lambda \lambda 0$

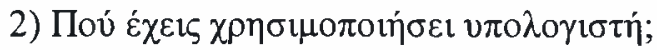

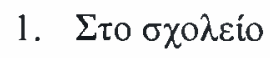

2. $\Sigma$ то $\sigma \pi i \tau 1$

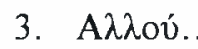

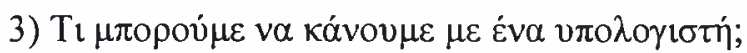

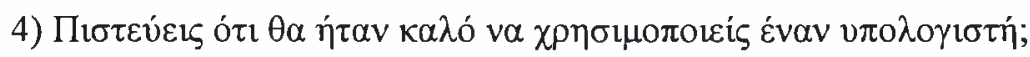

1. $\mathrm{Nar}$

2. $\mathrm{O} \chi \mathrm{l}$

Гıаті; 


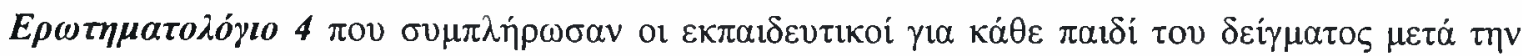

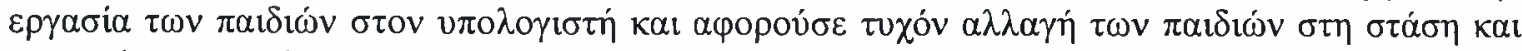

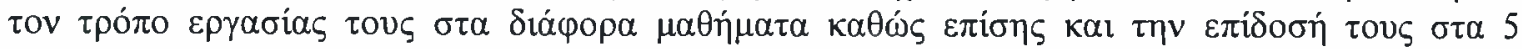

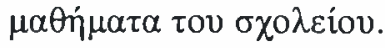

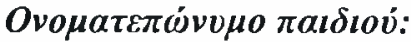

Ták $\xi$ :

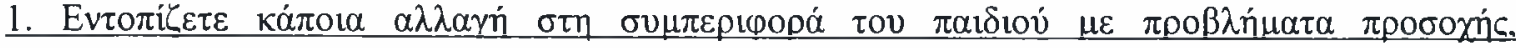

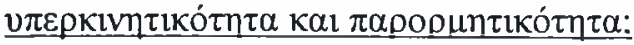

i. Nat

ii. $\mathrm{O} \chi \mathrm{l}$

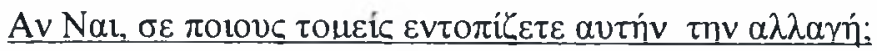

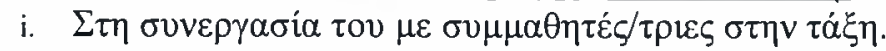

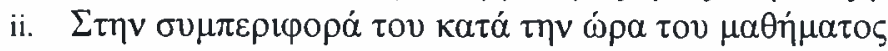

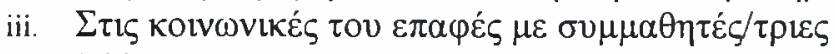

iv. A A $\lambda \lambda_{0}$.

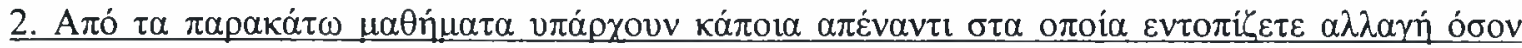

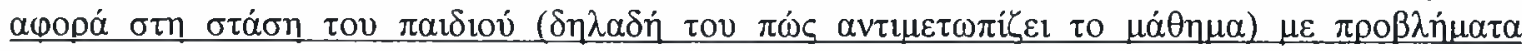

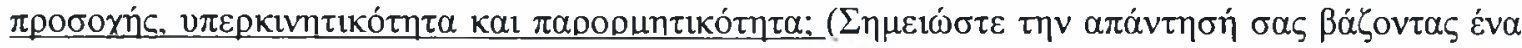

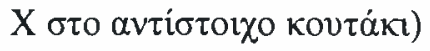

$\mathrm{M} \alpha \theta \eta \mu \alpha \tau \iota \kappa \alpha ́$

NAI $\square$ OXI

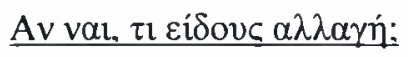

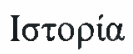

NAI $\square$ OXI

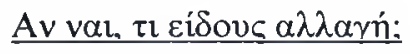

Фvбเки́

NAI $\square$ OXI

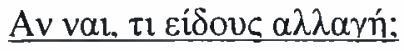


$\Gamma \varepsilon \omega \gamma \rho \alpha \varphi i \alpha$

NAI

OXI

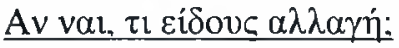

$\mathrm{K} \alpha \lambda \lambda \iota \tau \varepsilon \chi \nu \imath \kappa \alpha ́$

NAI

OXI

Avval. $\tau l \varepsilon i \delta$ ovc $\alpha \lambda \lambda \alpha \gamma \eta \dot{:}$

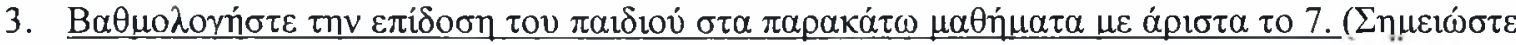

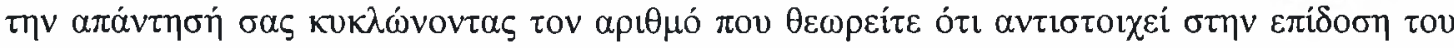
$\pi \propto\llcorner\delta\llcorner 0 \dot{)}$

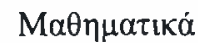

\begin{tabular}{|c|c|c|c|c|c|c|}
\hline 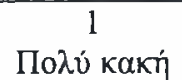 & 2 & 3 & 4 & 5 & 6 & 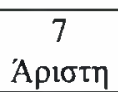 \\
\hline
\end{tabular}

\begin{tabular}{|c|c|c|c|c|c|c|}
\hline $\begin{array}{c}1 \\
\Pi о \lambda \dot{v} \kappa a k \eta ́\end{array}$ & 2 & 3 & 4 & 5 & 6 & $\begin{array}{c}7 \\
\text { Aptorm }\end{array}$ \\
\hline
\end{tabular}

\begin{tabular}{|c|c|c|c|c|c|c|}
\hline $\begin{array}{c}1 \\
\text { Подú } \kappa \alpha \kappa \eta ́\end{array}$ & 2 & 3 & 4 & 5 & 6 & 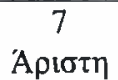 \\
\hline
\end{tabular}

$\Gamma \varepsilon \omega \gamma p \alpha \omega i \alpha$

\begin{tabular}{|c|l|l|l|l|l|c|}
\hline $\begin{array}{c}1 \\
\Pi о \lambda \dot{v} \kappa \alpha \kappa \eta ்\end{array}$ & 2 & 3 & 4 & 5 & 6 & 7 \\
Apı $\sigma \tau$ \\
\hline
\end{tabular}

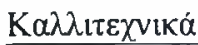

\begin{tabular}{|c|c|c|c|c|c|c|}
\hline $\begin{array}{c}1 \\
\Pi о \lambda u ́ \\
\kappa \alpha \kappa \eta ́\end{array}$ & 2 & 3 & 4 & 5 & 6 & $\begin{array}{c}7 \\
\text { Apı } \sigma \tau \eta\end{array}$ \\
\hline
\end{tabular}




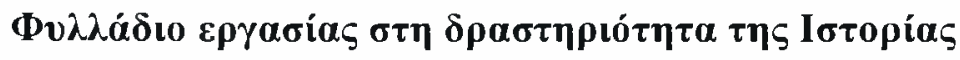

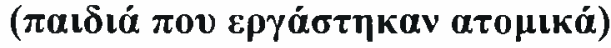

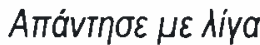

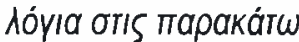

$\varepsilon \rho \omega r \eta ́ \sigma \varepsilon I \zeta \ldots$

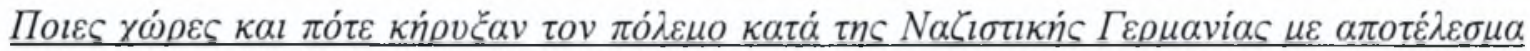

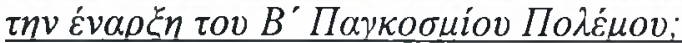

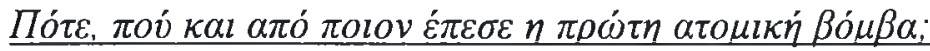

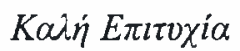




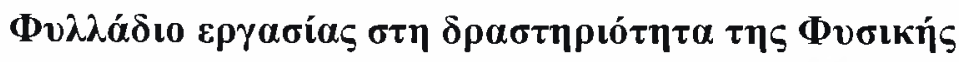

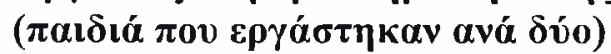

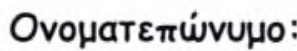

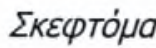

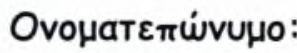

\section{$A^{\prime}$ Mépos}

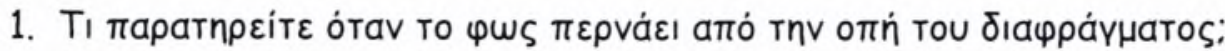

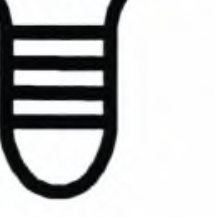

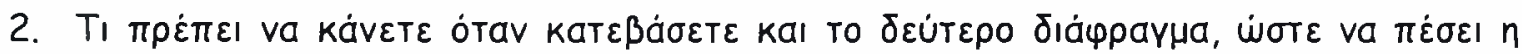

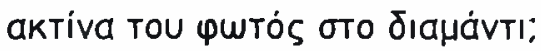

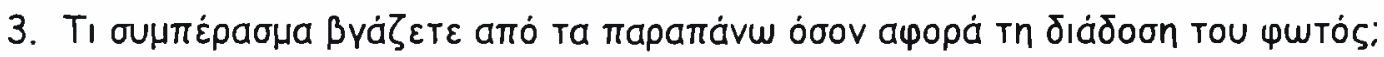


$B^{\prime}$ Mépos

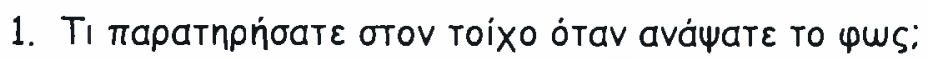

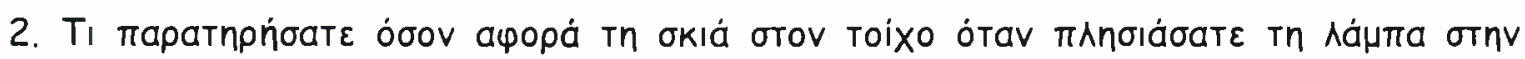

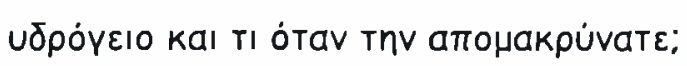

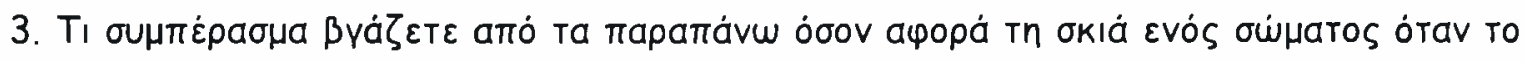
$\pi \wedge n \sigma ı \dot{a} \zeta \varepsilon ।$ To $\varphi \omega \varsigma:$ 


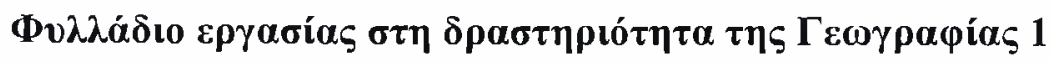

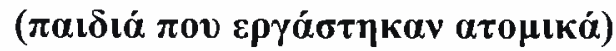

Amóvrnoє $\mu \varepsilon$ aUVTopía aT/S

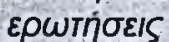

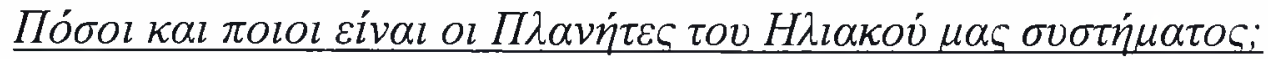

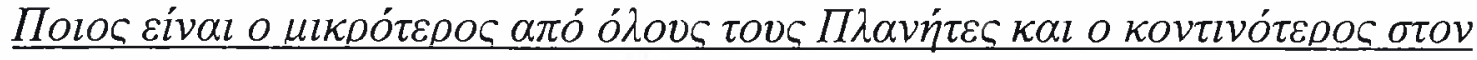

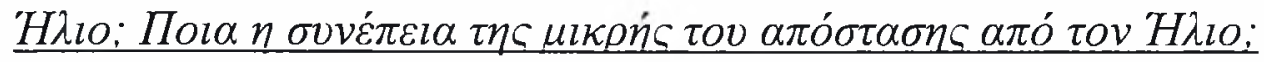

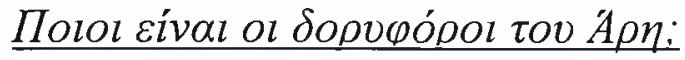

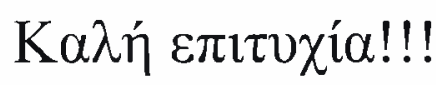




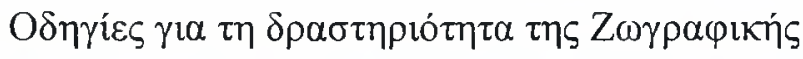

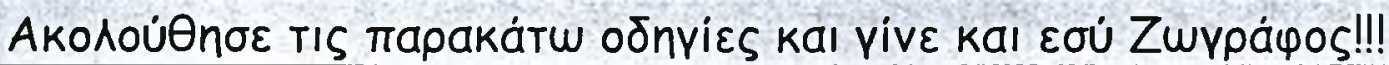

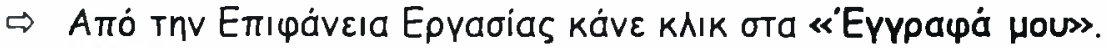

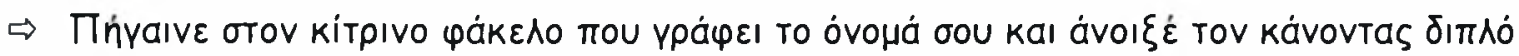
KAIK.

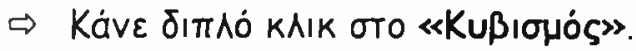

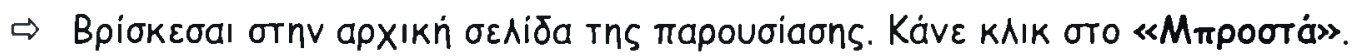

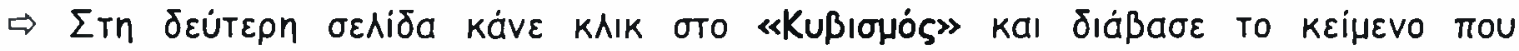

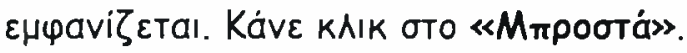

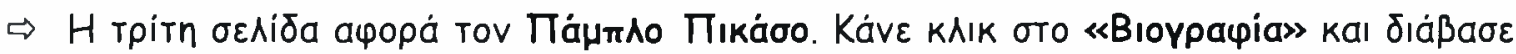

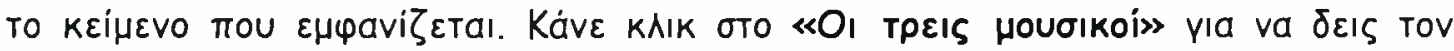

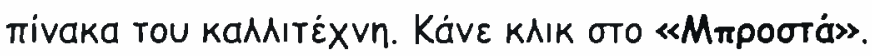

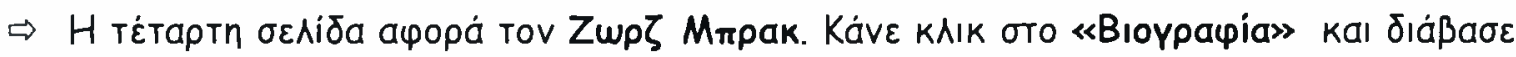

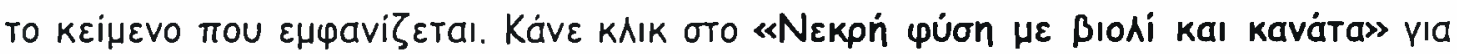

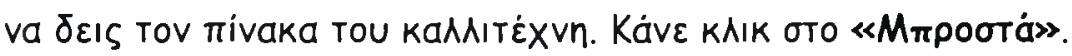

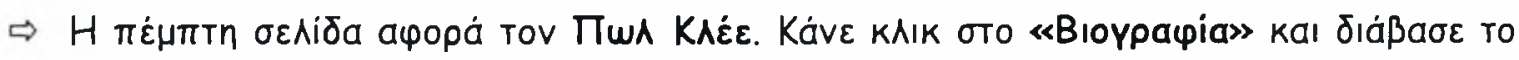

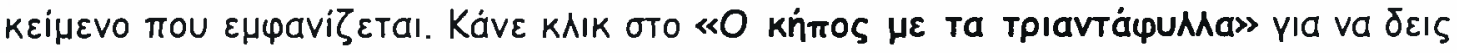

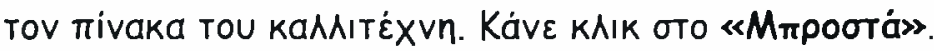

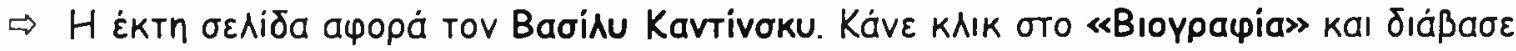

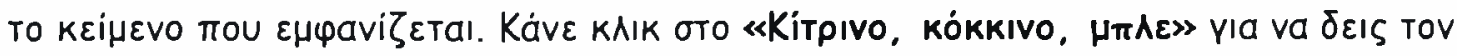

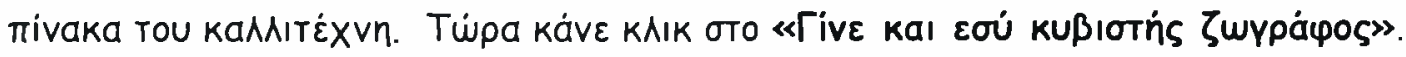

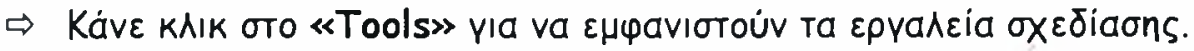

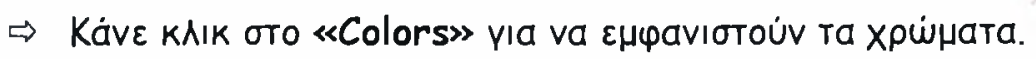

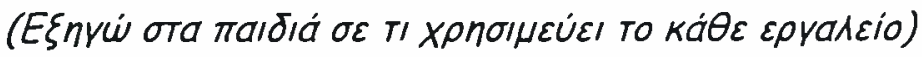

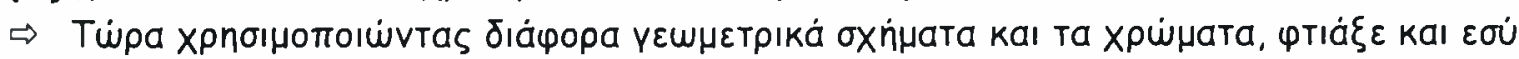

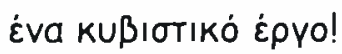




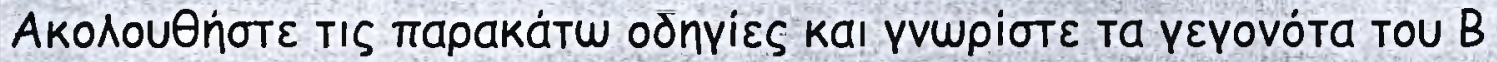

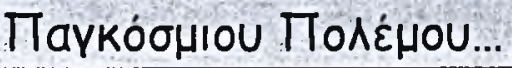

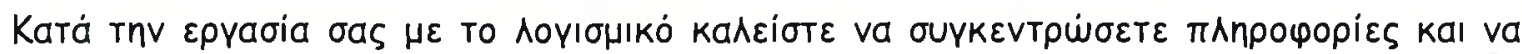

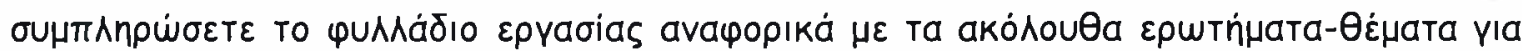
Ta:

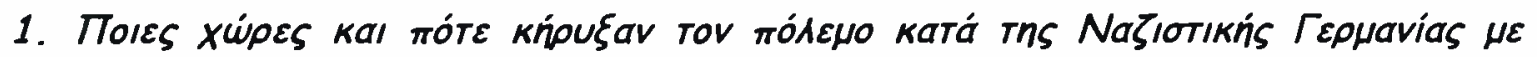

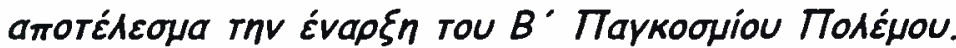

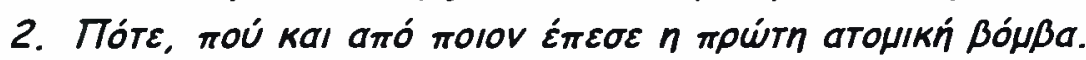

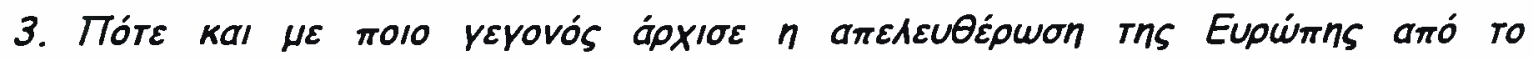

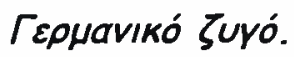

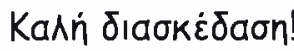

$\Rightarrow$ A

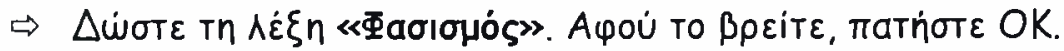

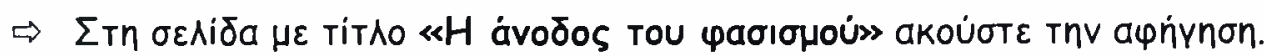

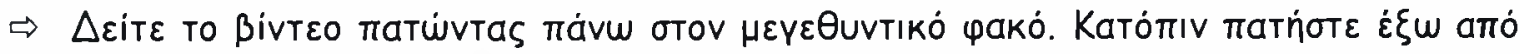
Thv عikóva.

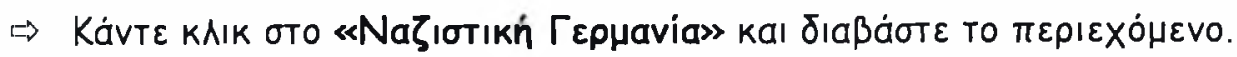

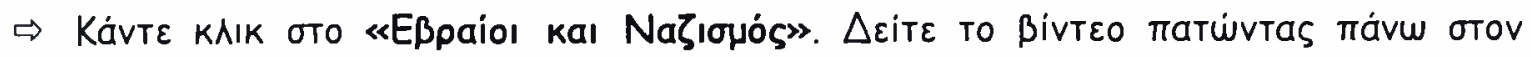

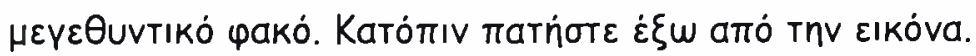

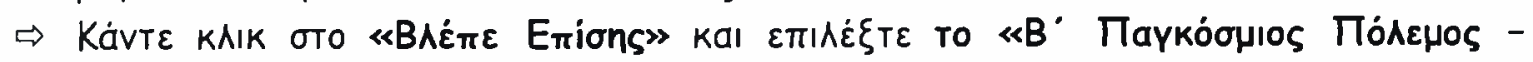
Eupúmn».

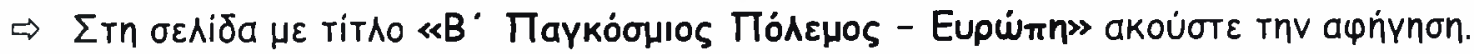

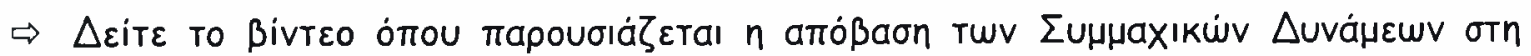

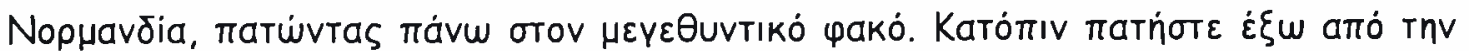
Eıkóva.

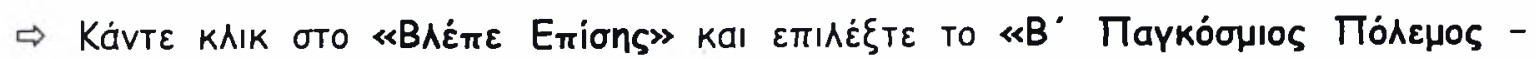
Eıрnvikós».

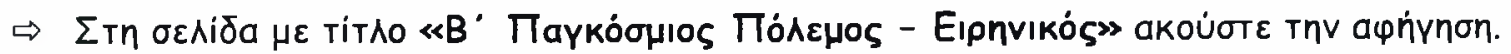

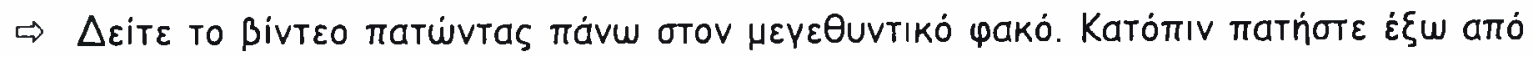
TnV EIKóva.

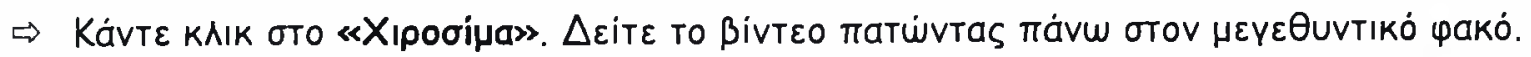

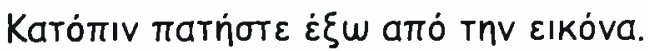

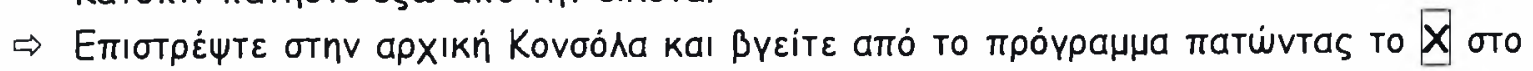

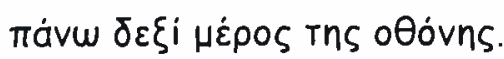




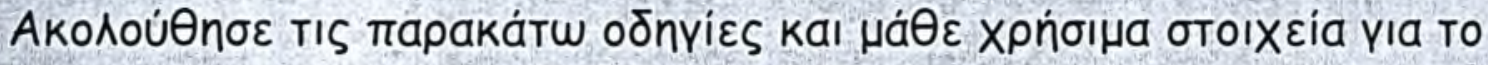
$\Phi \omega \zeta ! ! !$

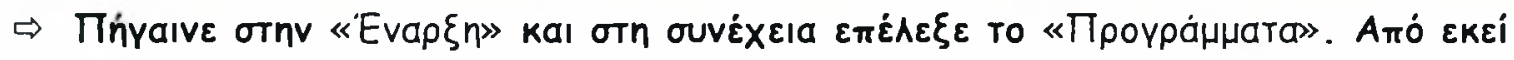
$\varepsilon \pi \varepsilon \wedge \varepsilon \xi \varepsilon$ Tо «MAOHMA».

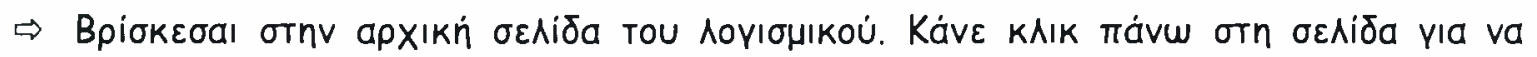

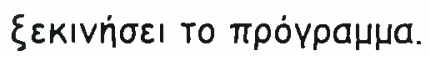

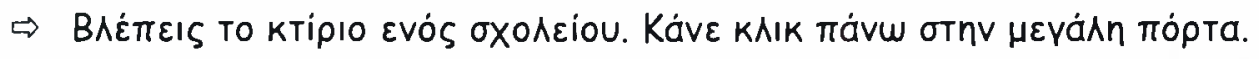

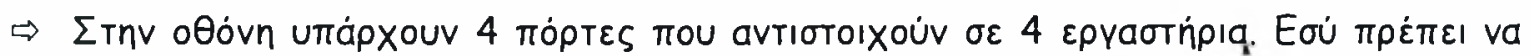

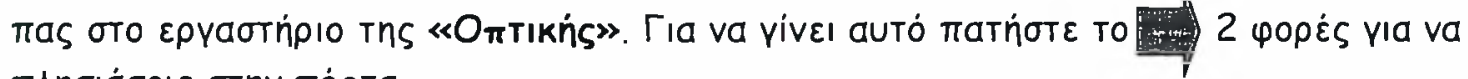

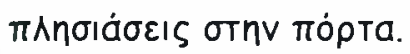

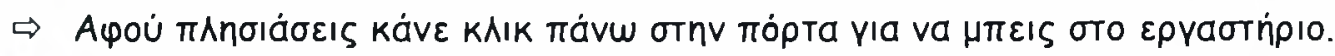

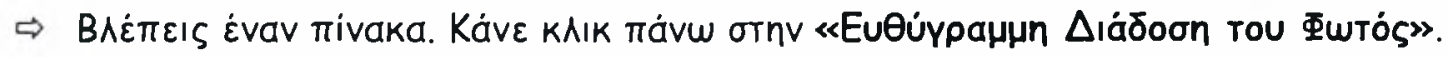

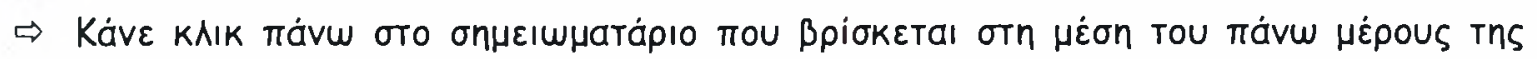

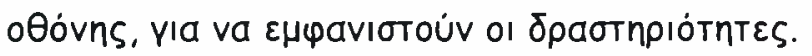

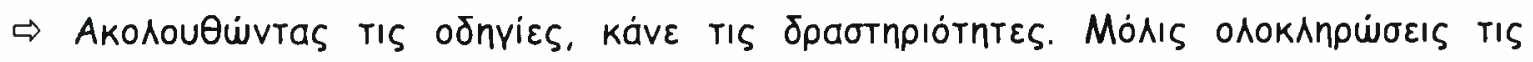

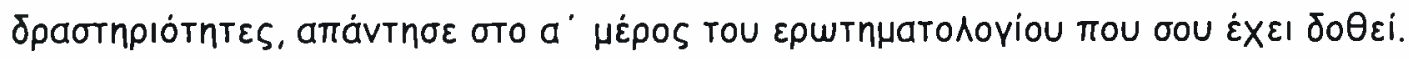

$\Rightarrow$ A

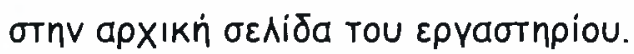

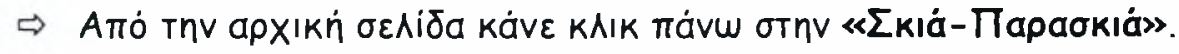

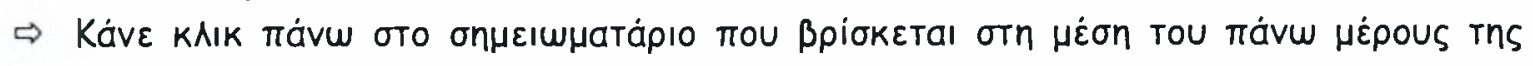

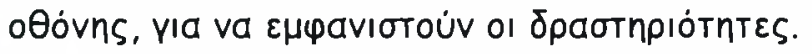

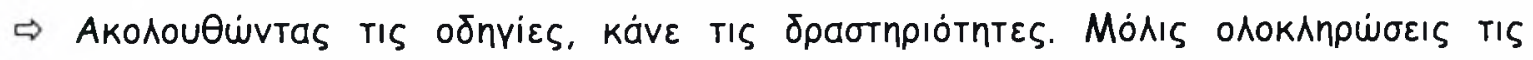

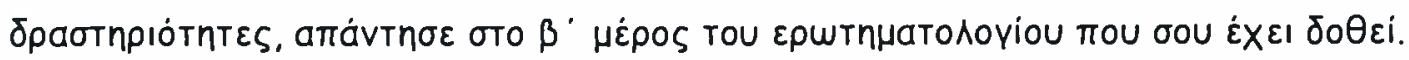

$\Rightarrow$ A

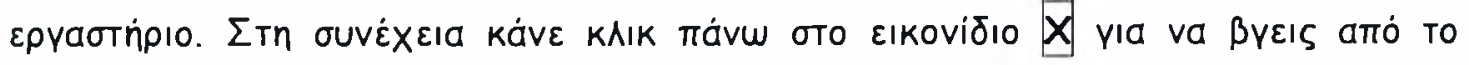
$\pi \rho \dot{v p a \mu \mu a . ~}$ 


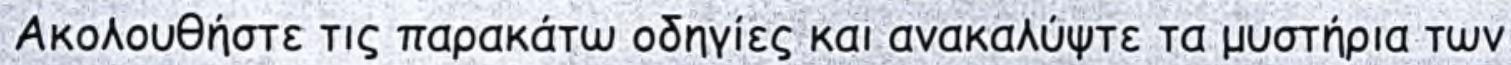
$\pi \wedge a v \eta T \dot{u} \vee ! ! !$

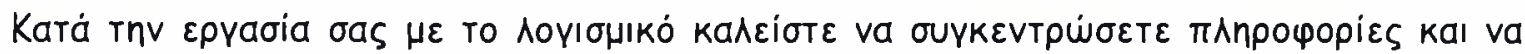

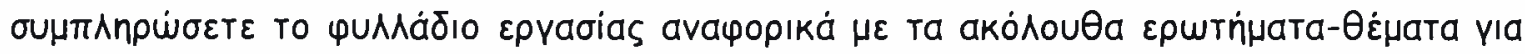
Ta:

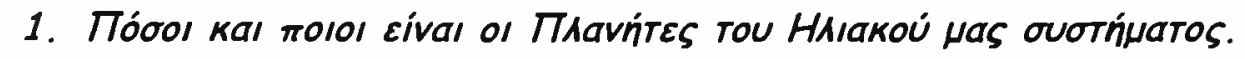

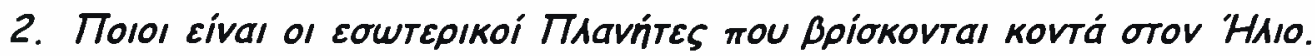

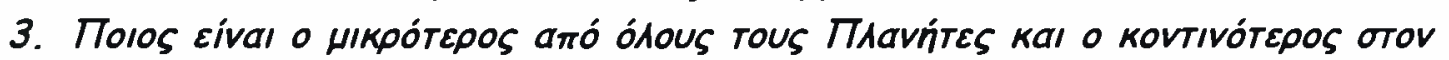

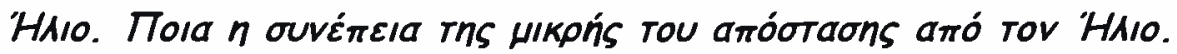

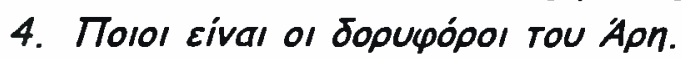

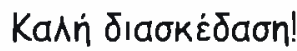

\section{$\Rightarrow$ Eıбаүшүń бто Про́үранца}

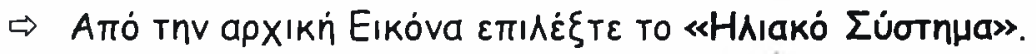

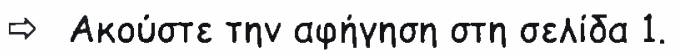

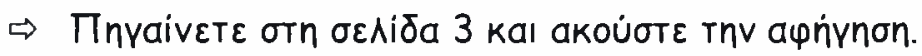

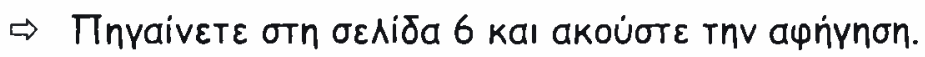

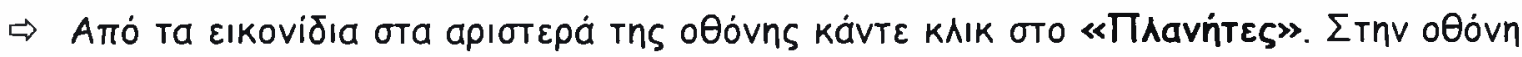

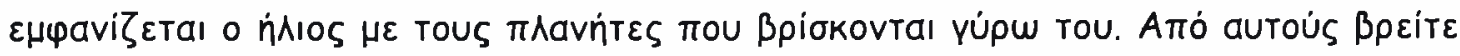

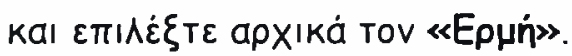

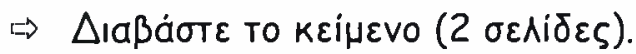

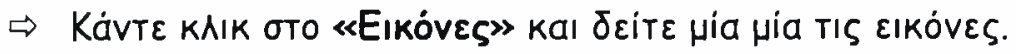

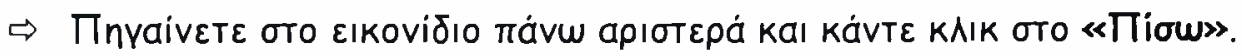

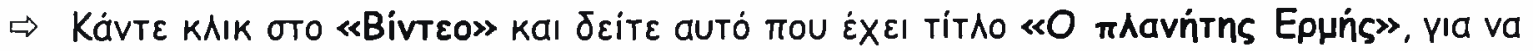

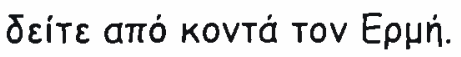

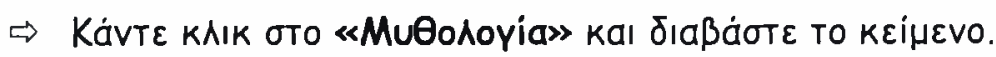

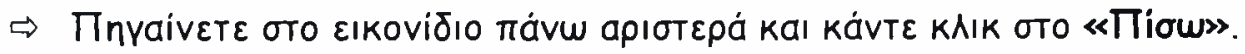

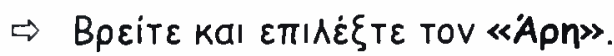

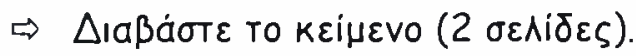

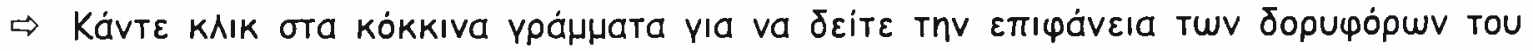
Apn.

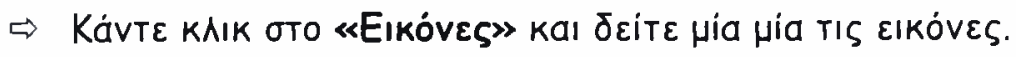

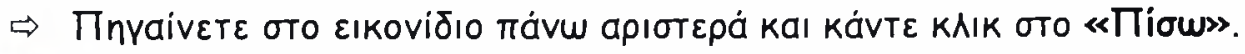

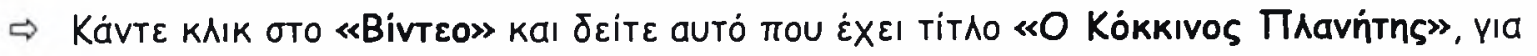

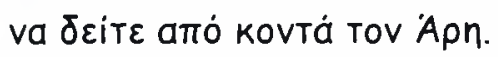

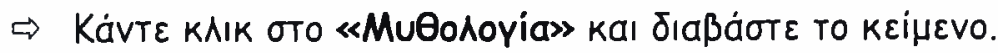

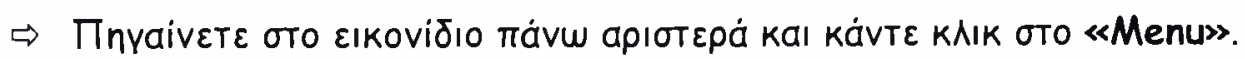

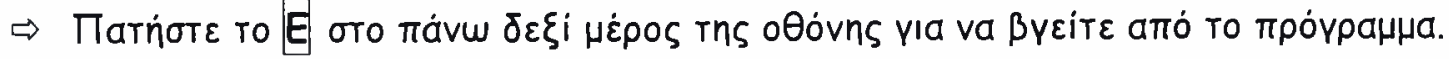




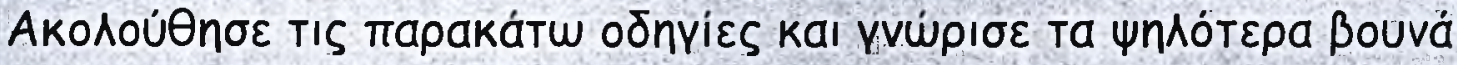 Ths Eᄊরádas!!!}

\section{$A^{\cdot}$ yáon}

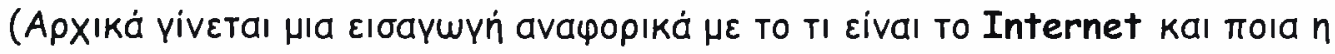
Xрпбінótntá тоu).

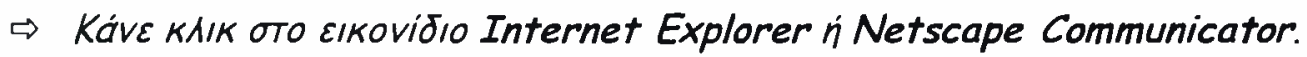

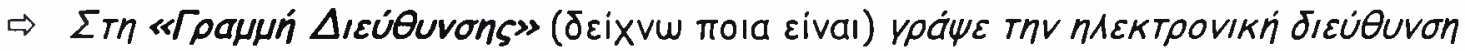
www.oreivatein.com

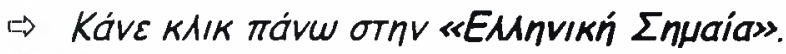

$\Rightarrow$ Káve KAIK oTo «Bouvó».

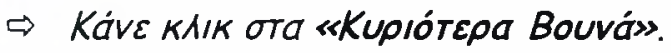

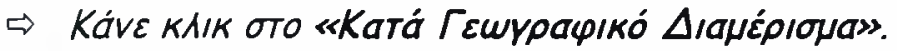

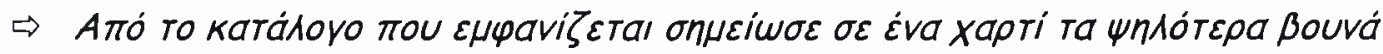

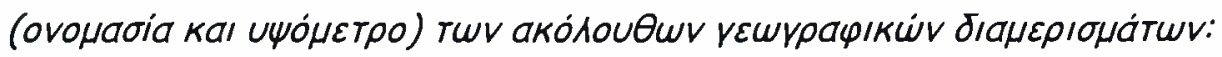

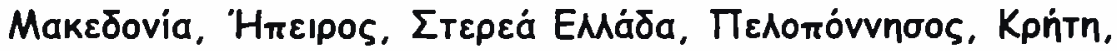

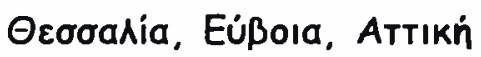

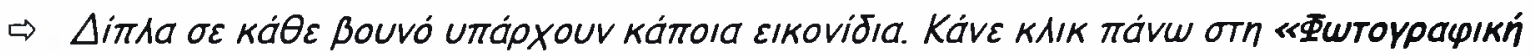

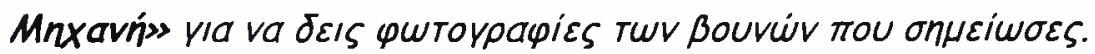

$\Rightarrow$ A

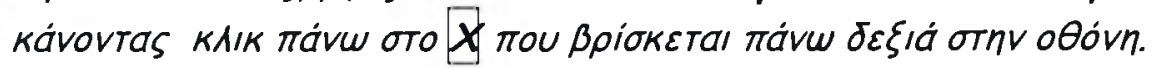

\section{B' yáon}

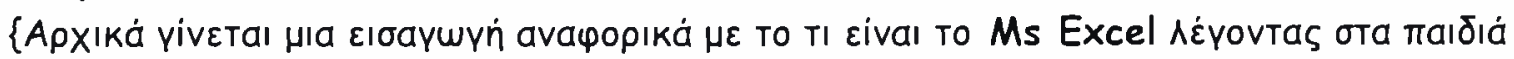

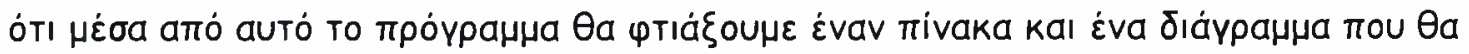

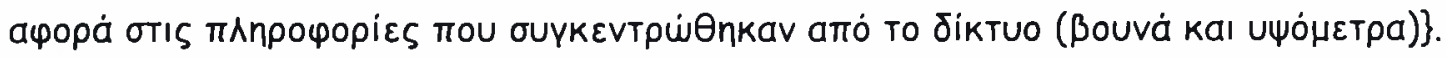

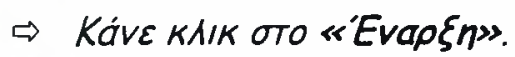

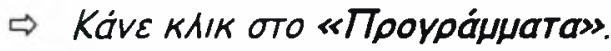

$\Rightarrow$ Káve KAik oto «Microsoft Excel».

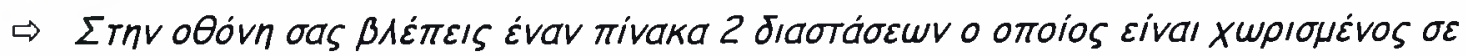

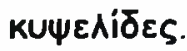

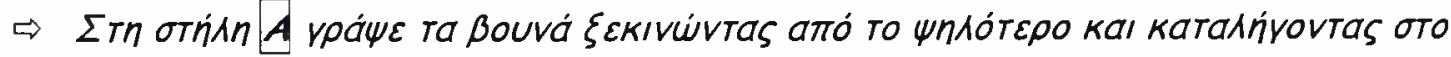

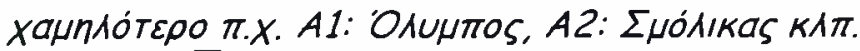

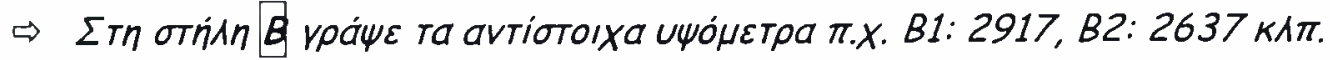

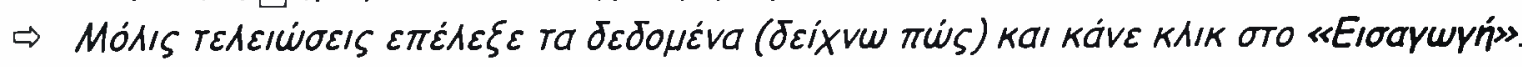

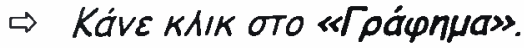

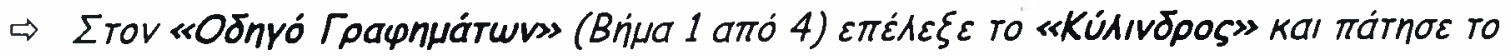
«Enópevo».

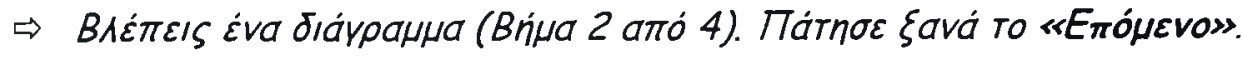




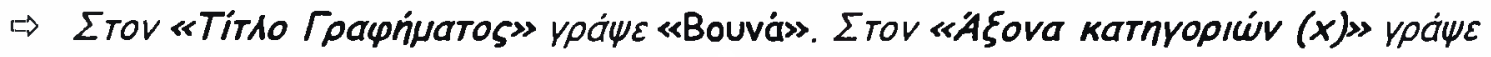

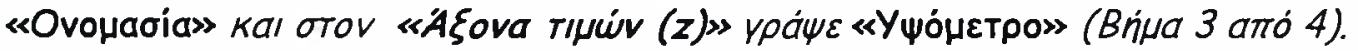

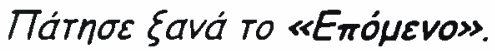

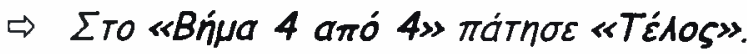

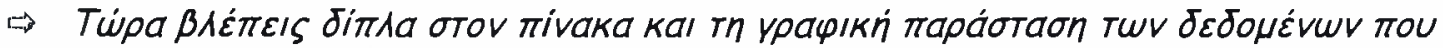
EIOńvaYes $\pi \rho \mathrm{V}$.

$\Rightarrow$ Káve KAIK OTO «ApXeio».

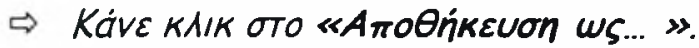

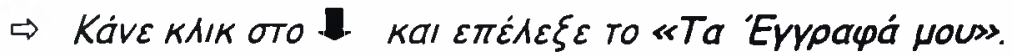

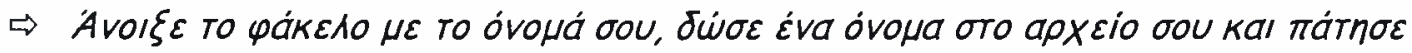

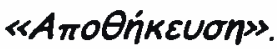

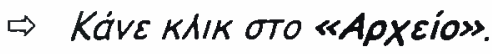

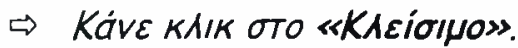

$\Rightarrow$ Káve Gavá kAIK oTo «Apxeio»s.

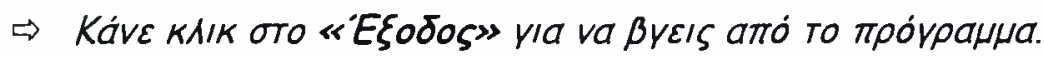




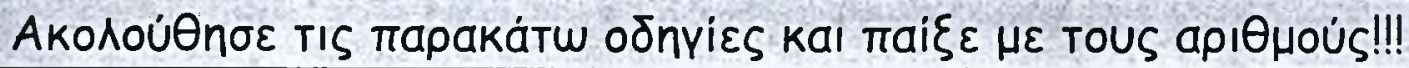

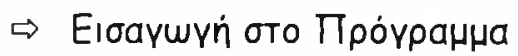

$A^{\circ}$ áoknon

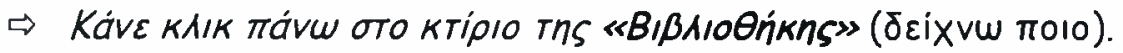

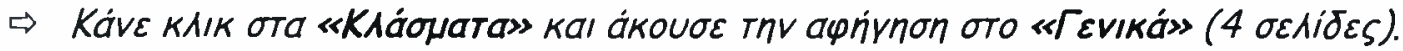

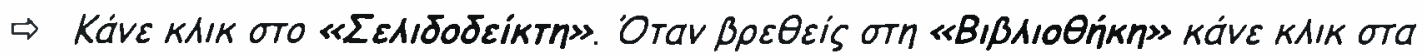
«Bńpara».

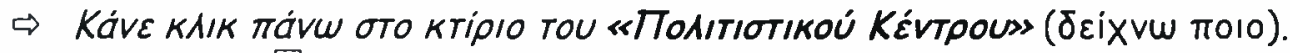

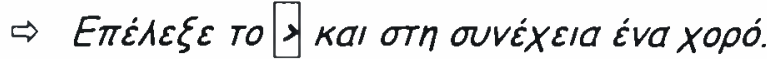

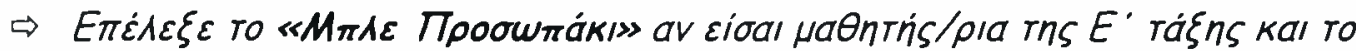

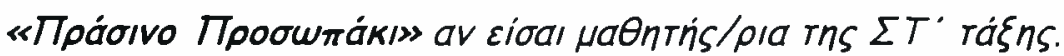

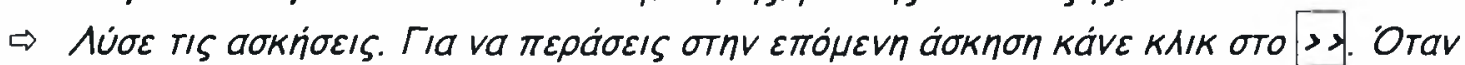

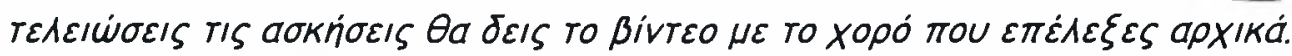

$\Rightarrow$ A

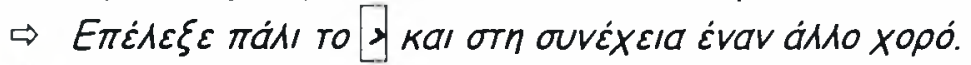

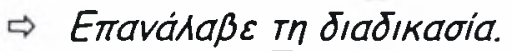

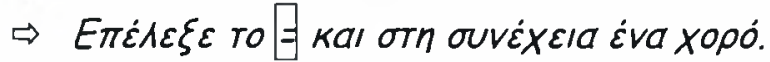

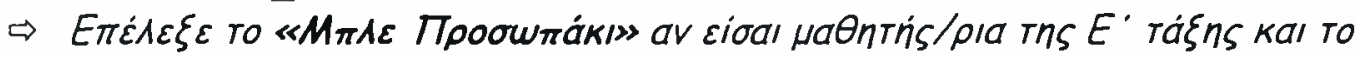

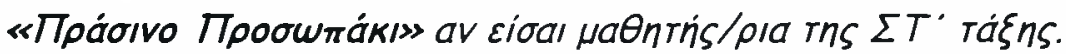

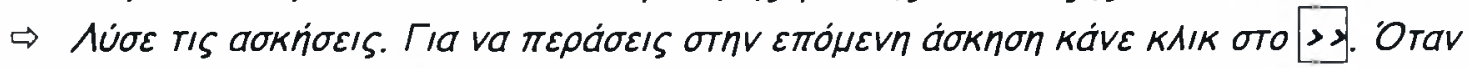

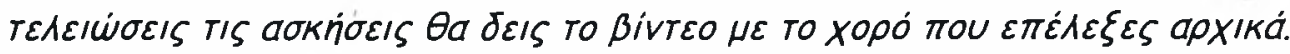

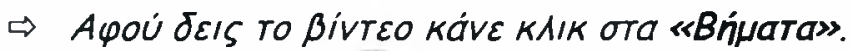

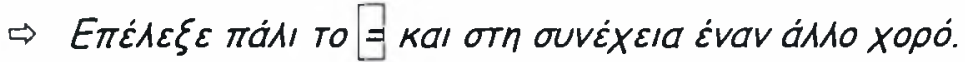

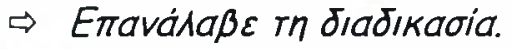

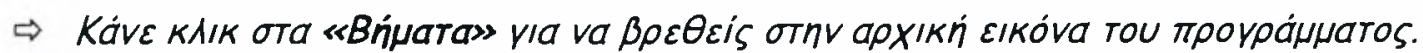

$\mathrm{B}^{\prime}$ áoknon

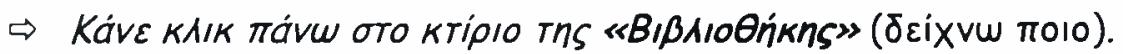

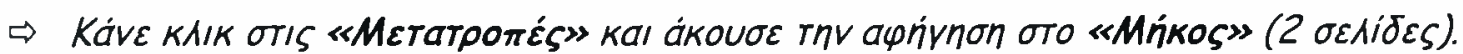

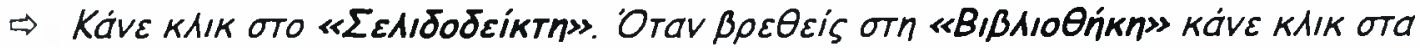
«Bńuata».

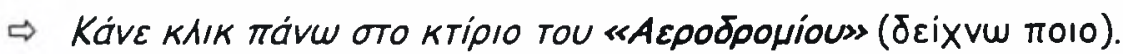

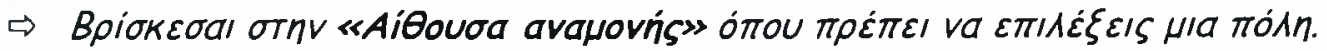

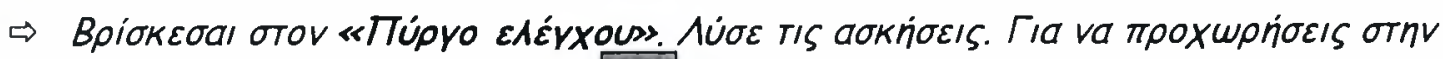

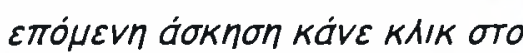

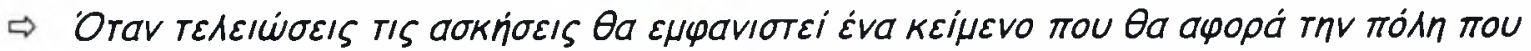

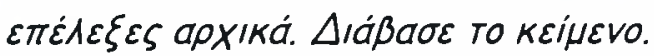

$\Rightarrow$ Káve KAIK oTa «Bńpata».

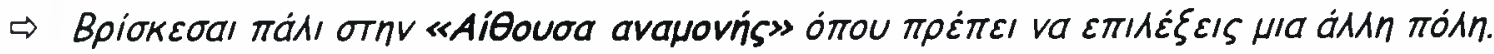

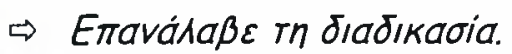

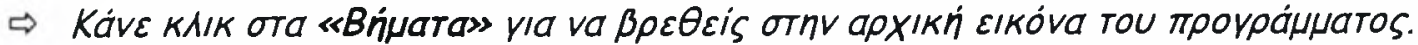

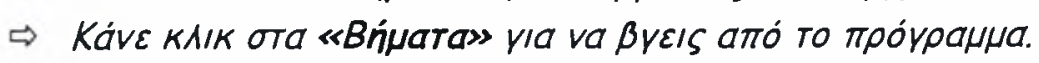




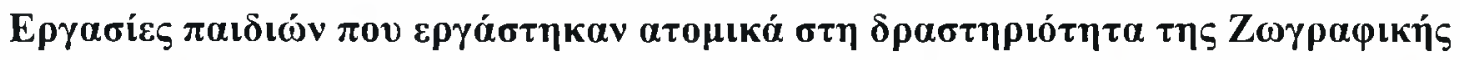

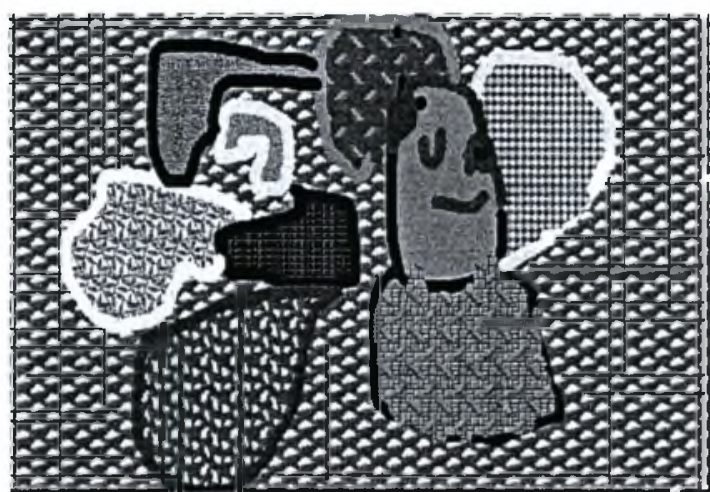

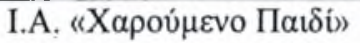

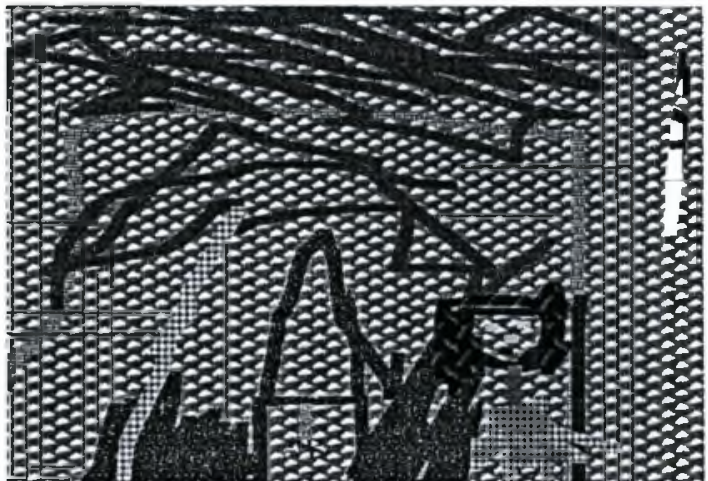

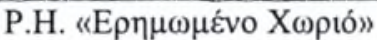
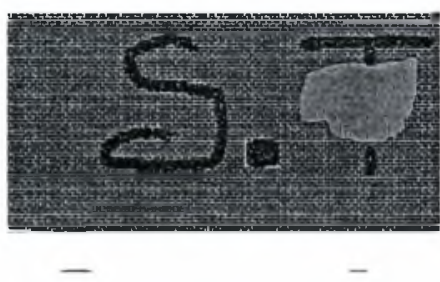

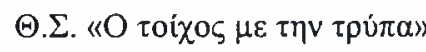

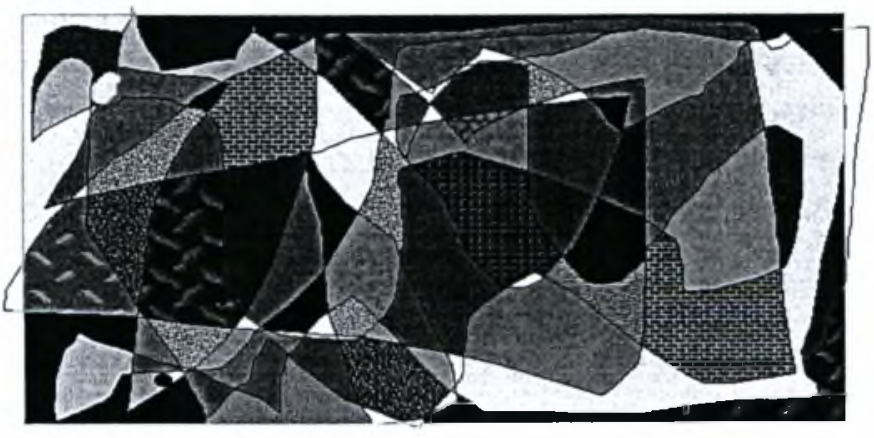

Т.П. «Н $\chi \omega ́ \rho \alpha \tau \omega \vee \chi \rho \omega \mu \alpha ́ \tau \omega v »$ 


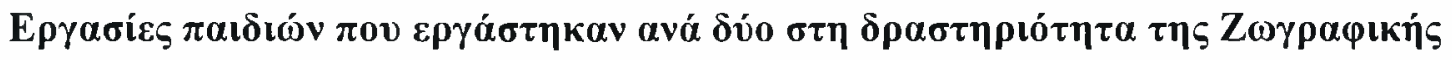

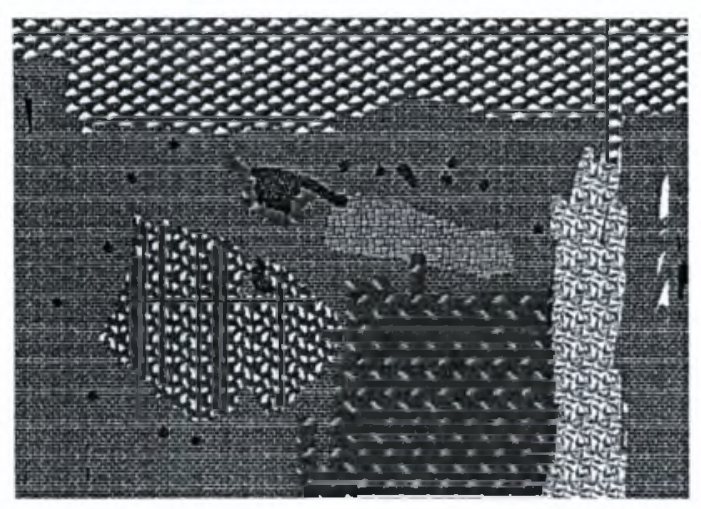

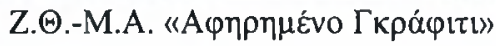

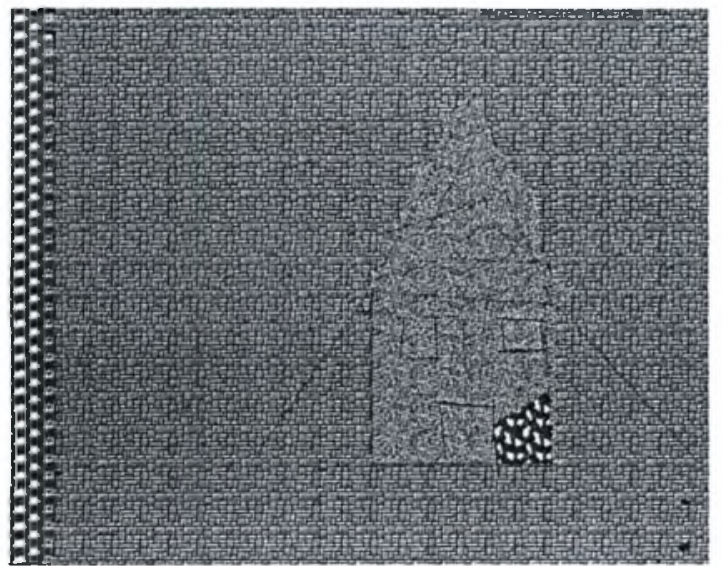

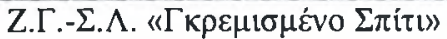

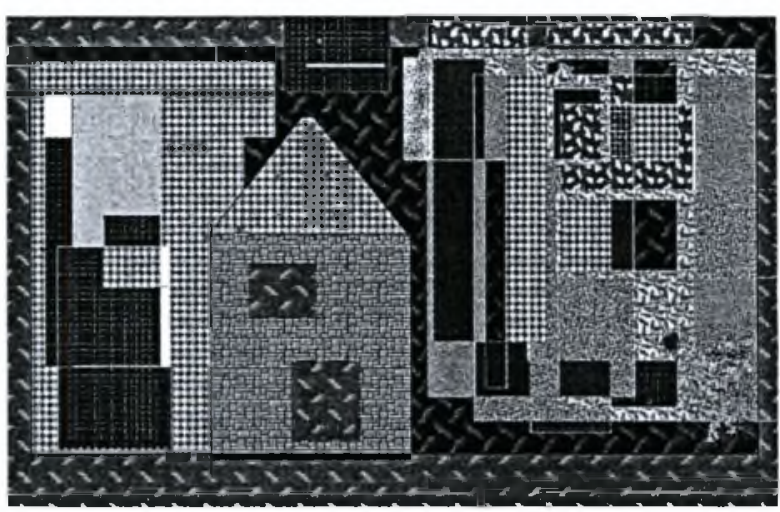

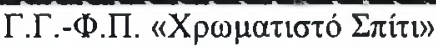

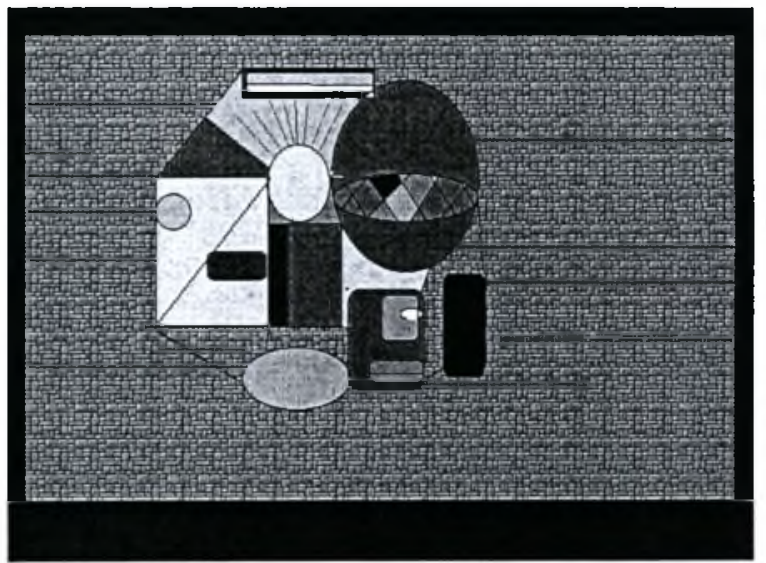

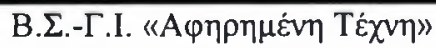




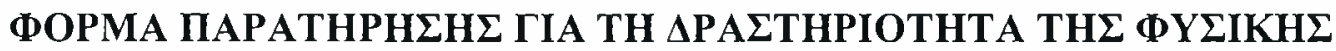

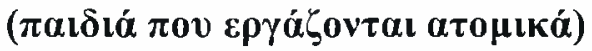

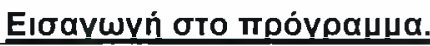

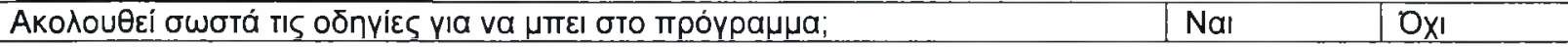

\begin{tabular}{|c|c|c|c|}
\hline 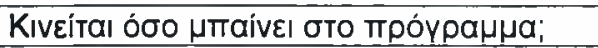 & 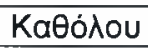 & MÉTpıа & Пoגú \\
\hline 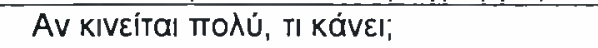 & & & \\
\hline 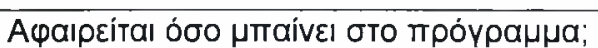 & 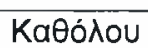 & Мغ́трıа & По入ú \\
\hline
\end{tabular}

\begin{tabular}{|c|c|c|}
\hline 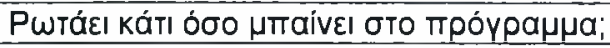 & $\mathrm{Nal}$ & $\overline{O X I}$ \\
\hline 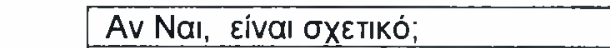 & $\mathrm{Nal}$ & OXI \\
\hline 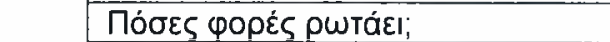 & & \\
\hline
\end{tabular}

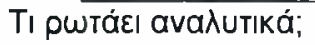

\begin{tabular}{|c|c|c|}
\hline 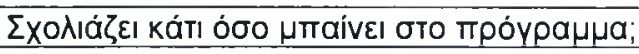 & Nal & OXI \\
\hline 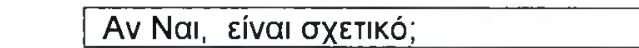 & $\mathrm{Nal}$ & OXI \\
\hline 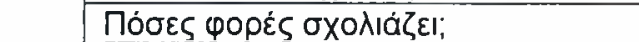 & & \\
\hline
\end{tabular}

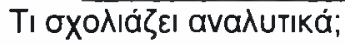

\begin{tabular}{|c|c|c|}
\hline 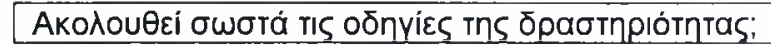 & Nal & OXI \\
\hline
\end{tabular}

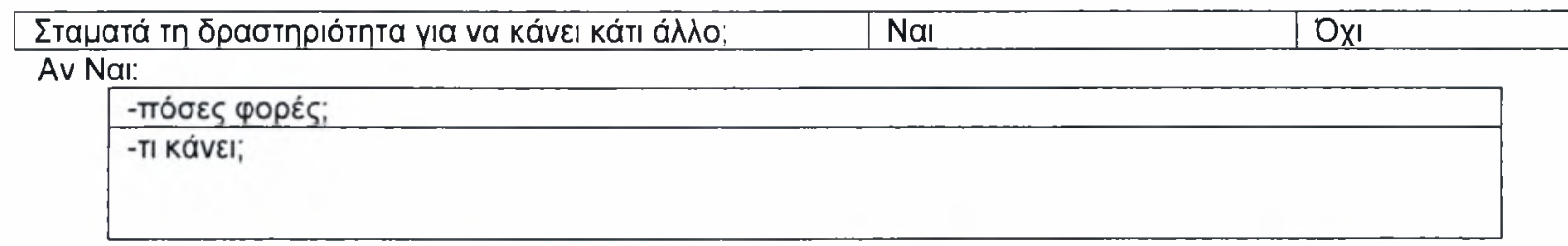

\begin{tabular}{|c|c|c|}
\hline 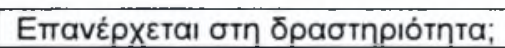 & $\mathrm{Nal}$ & OXI \\
\hline
\end{tabular}

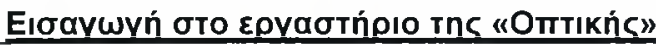

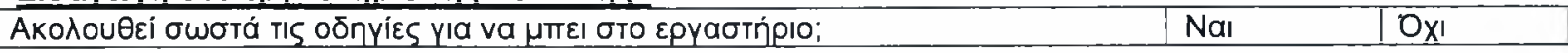

\begin{tabular}{|c|c|c|c|}
\hline 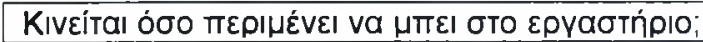 & KaӨó̀ou & Мغ́трıа & Поरें \\
\hline
\end{tabular}

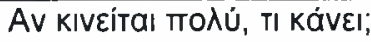

\begin{tabular}{|c|c|c|c|}
\hline 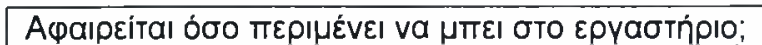 & 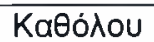 & Мغ́трıа & Пంגن́ \\
\hline
\end{tabular}

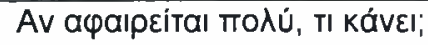

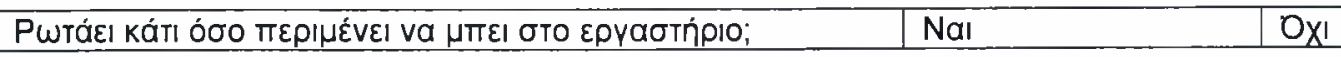




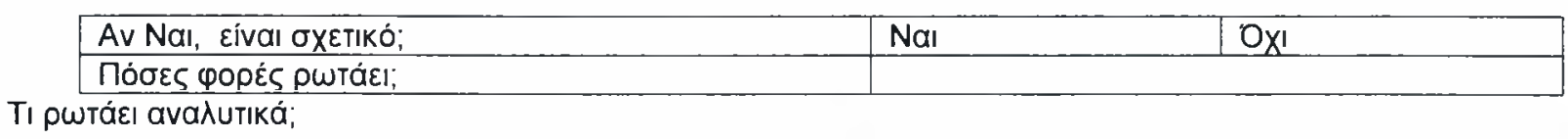

\begin{tabular}{|c|c|c|}
\hline 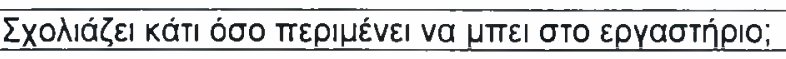 & $\mathrm{Nal}$ & OXI \\
\hline 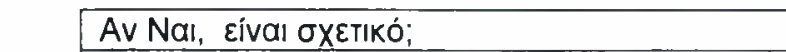 & $\mathrm{Nal}$ & OXI \\
\hline 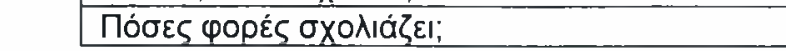 & & \\
\hline
\end{tabular}

\begin{tabular}{|c|c|c|}
\hline 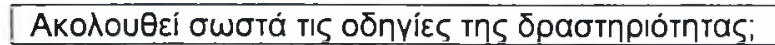 & $\mathrm{Nal}$ & OxI \\
\hline
\end{tabular}

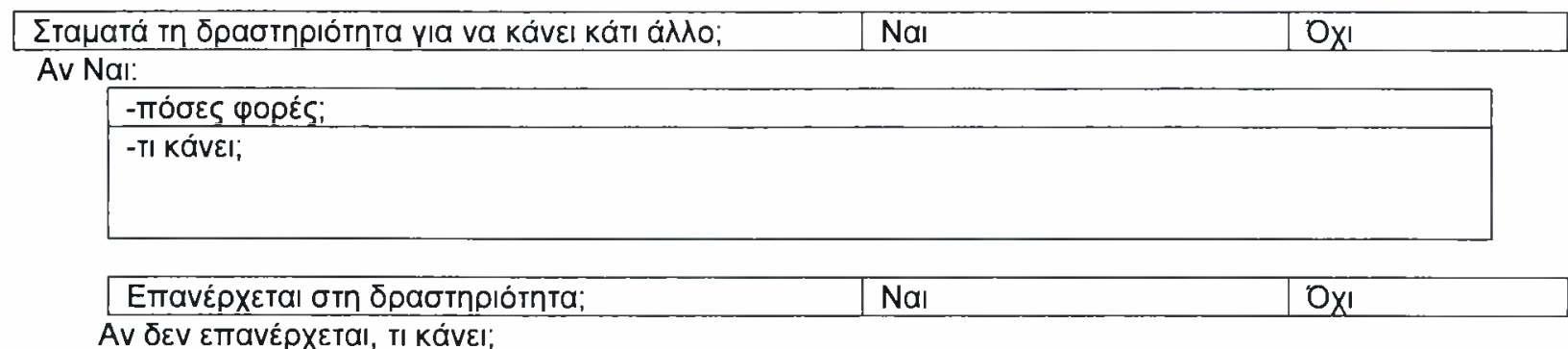

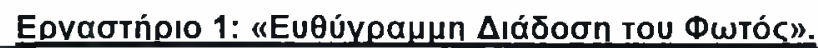

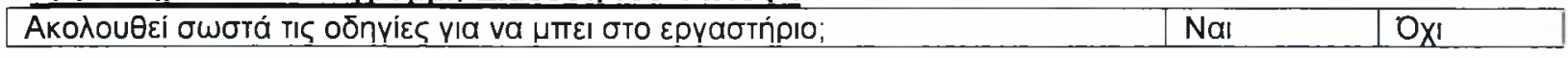

\begin{tabular}{|c|c|c|c|}
\hline 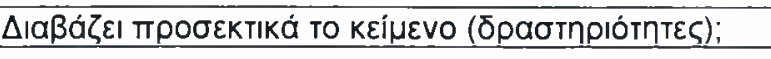 & Käó̀ou & Мغ́трıа & Пoגú \\
\hline 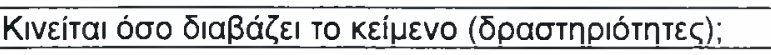 & Käódou & MÉtpıа & По入ú \\
\hline \multicolumn{4}{|l|}{ Av KIVEíTaı TOAÚ, TI KávEI; } \\
\hline 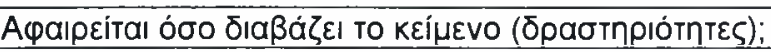 & Käó內ou & Мغ́тр|а & По入ú \\
\hline 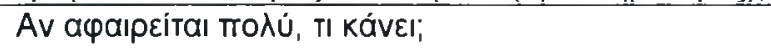 & & & \\
\hline
\end{tabular}

\begin{tabular}{|c|c|c|}
\hline 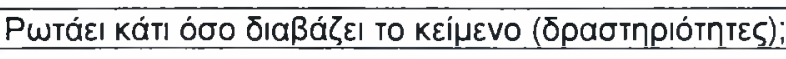 & Nal & OXI \\
\hline 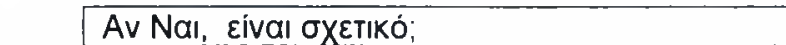 & $\mathrm{Nal}$ & $O \times 1$ \\
\hline 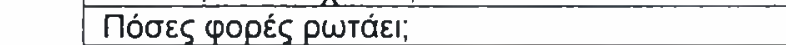 & & \\
\hline
\end{tabular}

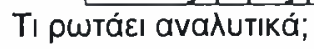

\begin{tabular}{|c|c|c|}
\hline 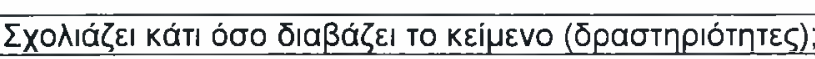 & $\mathrm{Nal}$ & OXI \\
\hline Av Nal, eival бXETIKó; & $\mathrm{Nal}$ & OXI \\
\hline 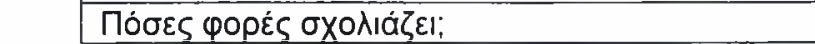 & & \\
\hline
\end{tabular}

\begin{tabular}{|c|c|c|}
\hline 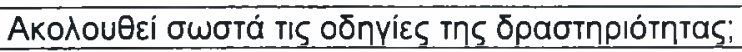 & $\mathrm{Nal}$ & $O \times 1$ \\
\hline Av OXI, TI Kável; & & \\
\hline 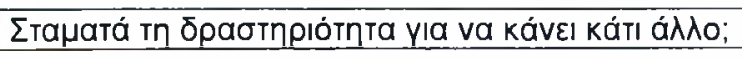 & $\mathrm{Nal}$ & $O x$ \\
\hline
\end{tabular}


Av Nal:

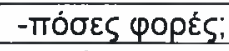

-TI Kável;

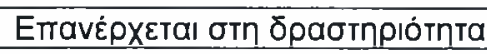

Naı

OXI

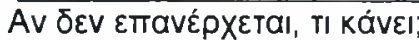

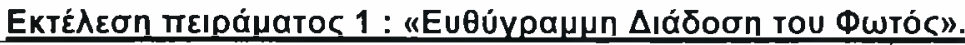

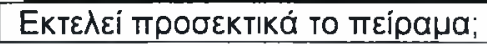

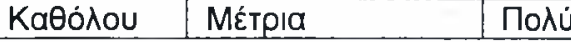

\begin{tabular}{|c|c|c|c|}
\hline 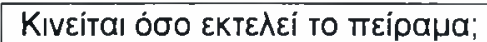 & KaӨónou & Мغ́трıа & חоर́ \\
\hline
\end{tabular}

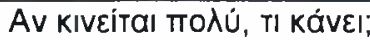

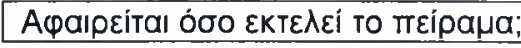

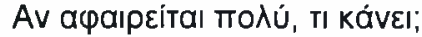

\begin{tabular}{|l|l|l|}
\hline Kąó̀ou & Métpıa & Пoגú \\
\hline
\end{tabular}

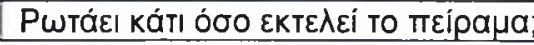

Nal $10 \times 1$

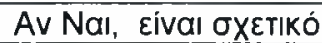

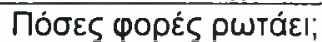

\begin{tabular}{l|l} 
Naı & OXı
\end{tabular}

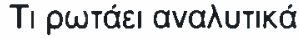

\begin{tabular}{|c|c|c|}
\hline 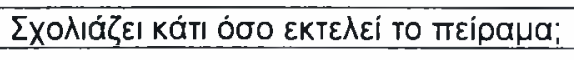 & Nal & OXI \\
\hline 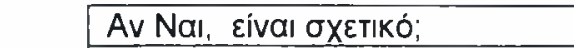 & Naı & OXI \\
\hline
\end{tabular}

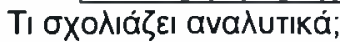

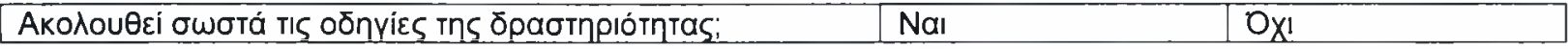

Av OXI, TI Kável;

\begin{tabular}{|c|c|c|}
\hline 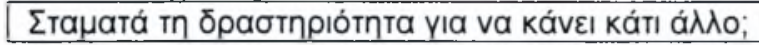 & Nal & OXI \\
\hline
\end{tabular}

Av Naı:

\begin{tabular}{|c|}
\hline 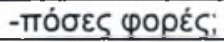 \\
\hline -TI kávعI; \\
\hline
\end{tabular}

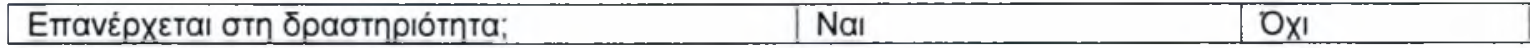

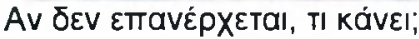

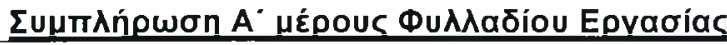

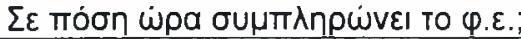

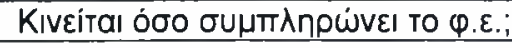

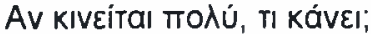

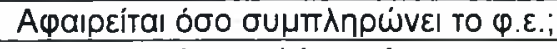

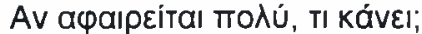

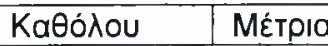

Поגú 


\begin{tabular}{|c|c|c|}
\hline 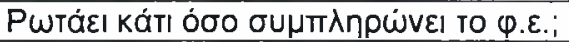 & Naı & $\mathrm{OXI}$ \\
\hline Av Naı, Eívaı бXETIKó; & Naı & OXI \\
\hline 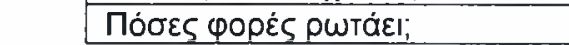 & & \\
\hline
\end{tabular}

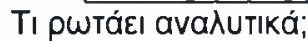

\begin{tabular}{|c|c|c|}
\hline 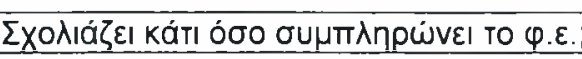 & $\mathrm{Nal}$ & OXI \\
\hline Av Nal, EÍvaı бXETIKó; & $\mathrm{Nal}$ & OxI \\
\hline 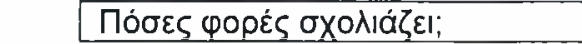 & & \\
\hline
\end{tabular}

\begin{tabular}{|c|c|c|}
\hline 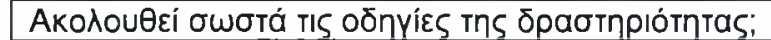 & Nal & $O \times 1$ \\
\hline
\end{tabular}

\begin{tabular}{|c|c|c|}
\hline 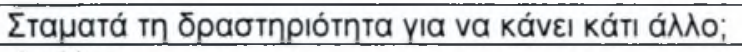 & Nal & $0 \times 1$ \\
\hline \multicolumn{3}{|l|}{ Av Nal: } \\
\hline 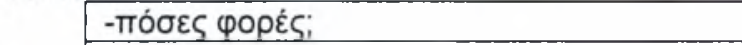 & & \\
\hline -TI KávEl; & & \\
\hline
\end{tabular}

\begin{tabular}{|c|c|c|}
\hline 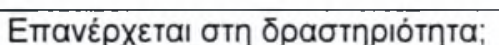 & Nal & $0 \times 1$ \\
\hline
\end{tabular}

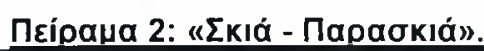

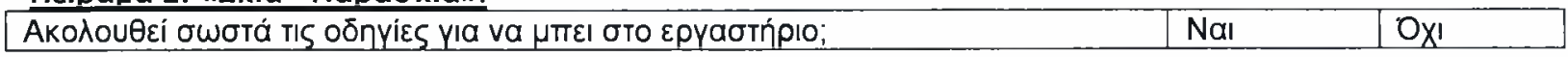

\begin{tabular}{|c|c|c|c|}
\hline 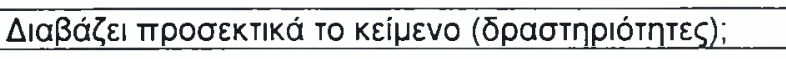 & 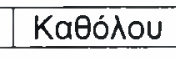 & Мغ́трıа & По入ú \\
\hline 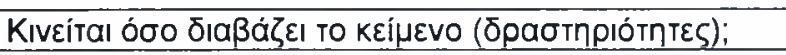 & KaGódou & Мغ́трıа & По入ú \\
\hline \multicolumn{4}{|l|}{ 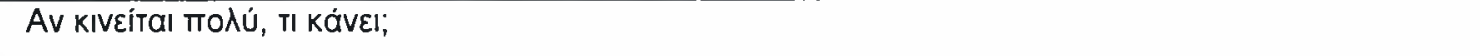 } \\
\hline 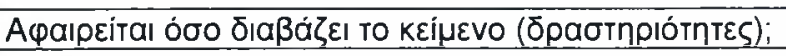 & KaӨóđou & MÉtpıd & По^ú \\
\hline 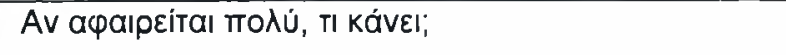 & & & \\
\hline
\end{tabular}

\begin{tabular}{|c|c|c|}
\hline P & $\mathrm{Nal}$ & OXI \\
\hline Av Nal, eíval бXETIKó; & $\mathrm{Nal}$ & OXI \\
\hline 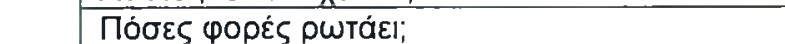 & & \\
\hline
\end{tabular}

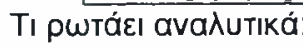

\begin{tabular}{|c|c|c|}
\hline 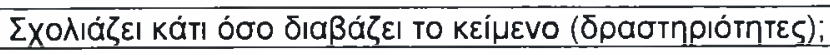 & $\mathrm{Nal}$ & OXI \\
\hline Av Nal, eival бXETIKó; & $\mathrm{Nal}$ & OXI \\
\hline 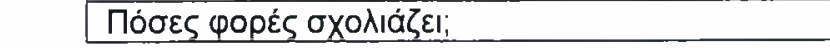 & & \\
\hline
\end{tabular}

\begin{tabular}{|c|c|c|}
\hline 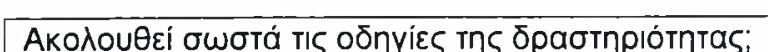 & $\mathrm{Nal}$ & OXI \\
\hline
\end{tabular}




\begin{tabular}{|c|c|c|}
\hline 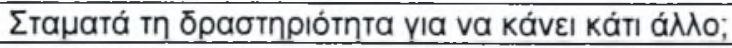 & $\mathrm{Nal}$ & Ox! \\
\hline \multicolumn{3}{|l|}{ Av Nal: } \\
\hline 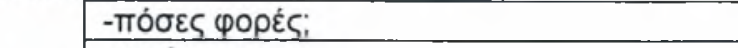 & & \\
\hline -TI kável; & & \\
\hline
\end{tabular}

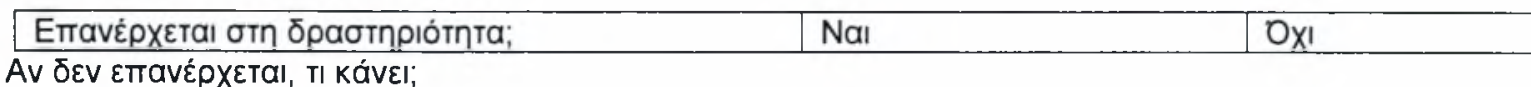

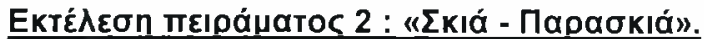

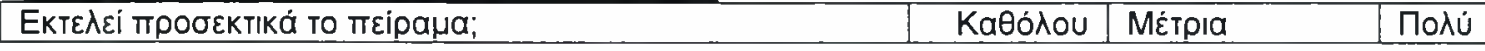

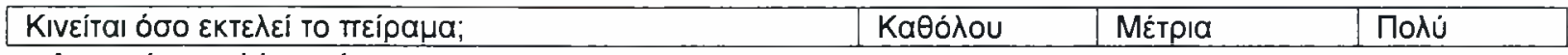

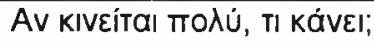

\begin{tabular}{|c|c|c|c|c|}
\hline \multirow{2}{*}{ 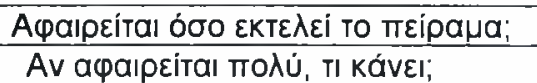 } & 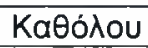 & \multicolumn{2}{|l|}{ Мغ̇трıа } & Пoגú \\
\hline & & & & \\
\hline 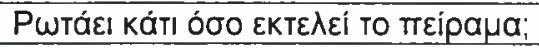 & Na! & & OXI & \\
\hline Av Nal, É́val oXETIKó; & $\mathrm{Nal}$ & & OXI & \\
\hline
\end{tabular}

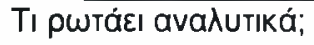

\begin{tabular}{|c|c|c|}
\hline 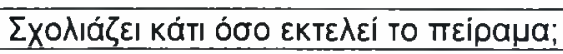 & $\mathrm{NaI}$ & OXI \\
\hline Av Nal, Eívaı бXETIKó; & $\mathrm{Nai}$ & OXI \\
\hline 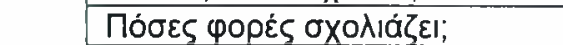 & & \\
\hline
\end{tabular}

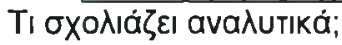

\begin{tabular}{|c|c|c|}
\hline 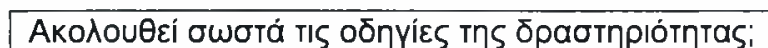 & Nal & OXI \\
\hline
\end{tabular}

\begin{tabular}{|c|c|c|}
\hline 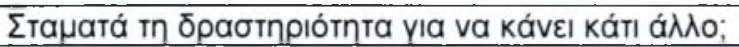 & Nal & $0 \times 1$ \\
\hline \multicolumn{3}{|l|}{ Av Nal: } \\
\hline \multicolumn{3}{|l|}{ 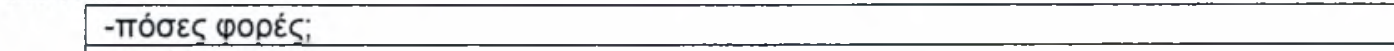 } \\
\hline \multicolumn{3}{|l|}{-TI KávEl; } \\
\hline 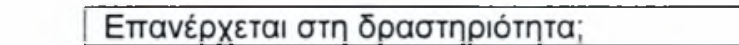 & Na! & OXI \\
\hline
\end{tabular}

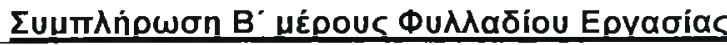

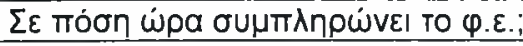

\begin{tabular}{|c|c|c|c|}
\hline 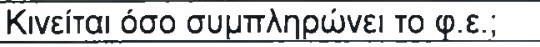 & 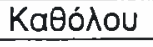 & Мغ́трıа & Поגú \\
\hline 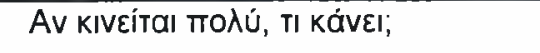 & & & \\
\hline 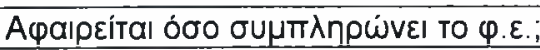 & KaӨó̀ou & Мغ́тріа & По入ú \\
\hline
\end{tabular}

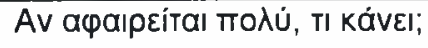




\begin{tabular}{|c|c|c|}
\hline 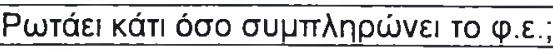 & $\mathrm{NaI}$ & $0 \times 1$ \\
\hline Av Nal, eíval $\sigma X \varepsilon T I K o ́ ;$ & $\mathrm{Nal}$ & $0 \times 1$ \\
\hline 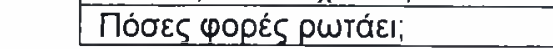 & & \\
\hline
\end{tabular}

\begin{tabular}{|c|c|c|}
\hline 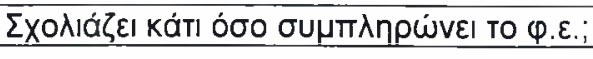 & $\mathrm{Nal}$ & $\mathrm{OXI}$ \\
\hline Av Nal, Eival $\sigma \times \varepsilon T I K o ́ ;$ & Naı & OXI \\
\hline 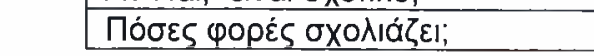 & & \\
\hline
\end{tabular}

\begin{tabular}{|c|c|c|}
\hline 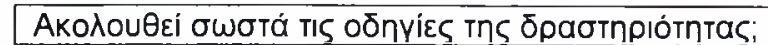 & $\mathrm{Nal}$ & $0 \times 1$ \\
\hline
\end{tabular}

\begin{tabular}{|c|c|c|}
\hline 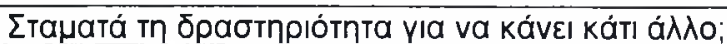 & $\mathrm{Nal}$ & OXI \\
\hline \multicolumn{3}{|l|}{ Av Nal: } \\
\hline 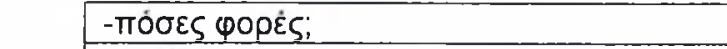 & & \\
\hline -TI KávEI; & & \\
\hline
\end{tabular}

\begin{tabular}{|c|c|c|}
\hline 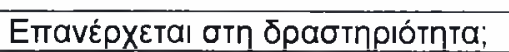 & $\mathrm{Nal}$ & $O \times 1$ \\
\hline
\end{tabular}

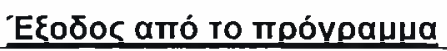

\begin{tabular}{|c|c|c|}
\hline 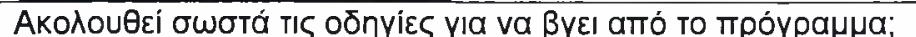 & Naı & Oxi \\
\hline
\end{tabular}

\begin{tabular}{|c|c|c|c|}
\hline 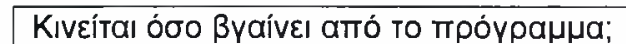 & 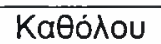 & MÉтpı & По入ú \\
\hline
\end{tabular}

\begin{tabular}{|c|c|c|c|}
\hline 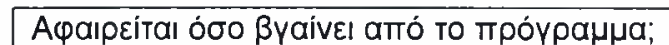 & Käó̀ou & Métpla & По入ú \\
\hline
\end{tabular}

\begin{tabular}{|c|c|c|}
\hline 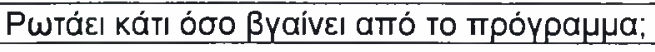 & Nal & OXI \\
\hline Av NaI, Eívaı бXETIKó; & Naı & OXI \\
\hline 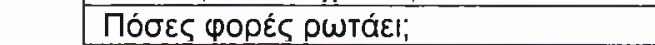 & & \\
\hline
\end{tabular}

\begin{tabular}{|c|c|c|}
\hline 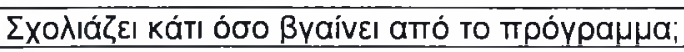 & $\mathrm{Nal}$ & OXI \\
\hline Av NaI, EÍvaI бXXETIKó; & $\mathrm{NaI}$ & $0 \times 1$ \\
\hline 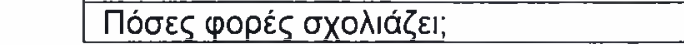 & & \\
\hline
\end{tabular}

\begin{tabular}{|c|c|c|}
\hline 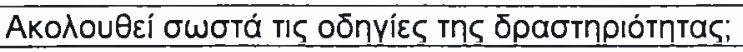 & $\mathrm{Nal}$ & $O \times 1$ \\
\hline Av OXI, TI KávEl; & & \\
\hline 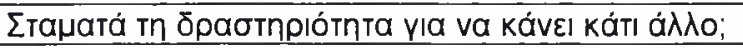 & $\mathrm{NaI}$ & $\mathrm{O} \times 1$ \\
\hline
\end{tabular}




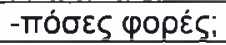

-TI Kável;

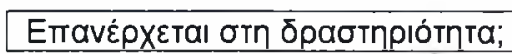

Nal

OXI

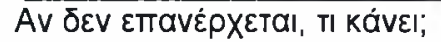




\section{Парápтnpa 2}

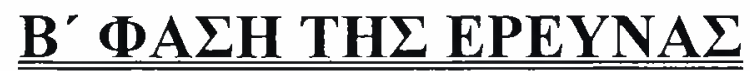




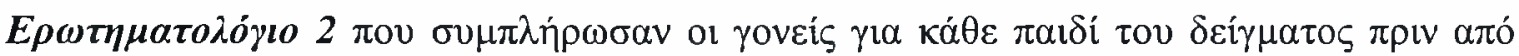

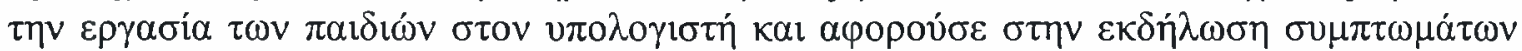
$\tau \eta \zeta \triangle \mathrm{E} \Pi / \mathrm{Y}$

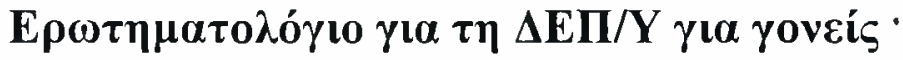

(DuPaul, Power, Anastopoulos, \& Reid, 1998)

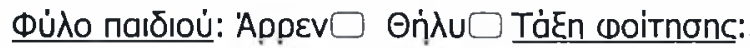

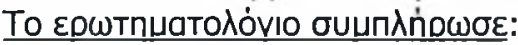

o natépac口

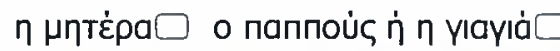

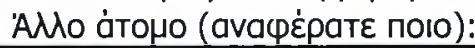

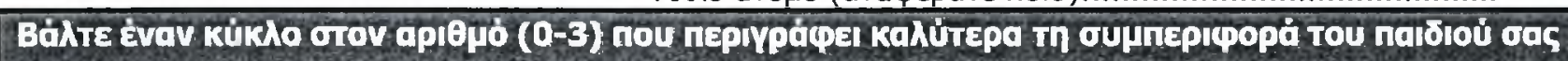

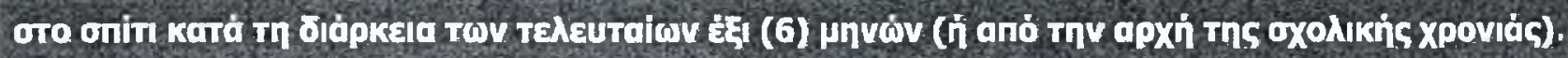

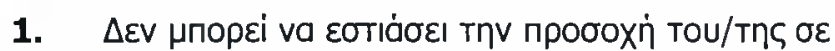

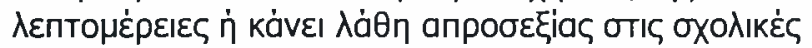

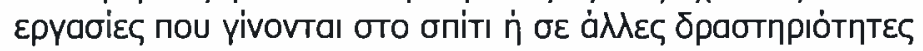

\begin{tabular}{|c|c|c|}
\hline $\begin{array}{c}\Sigma x \varepsilon \delta \dot{v} v \\
\text { потв́ }\end{array}$ & ¿návıa & 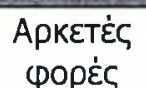 \\
\hline
\end{tabular}

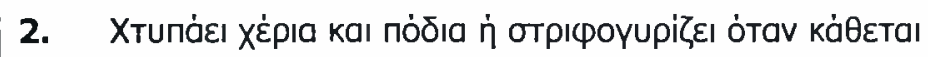

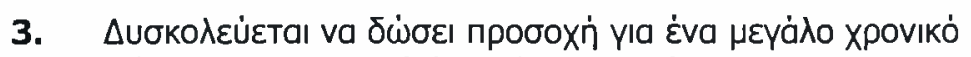

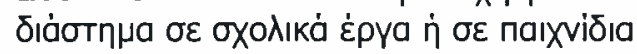

\begin{tabular}{|c|c|c|c|}
\hline 0 & 1 & 2 & 3 \\
\hline 0 & 1 & 2 & 3 \\
\hline 0 & 1 & 2 & 3 \\
\hline 0 & 1 & 2 & 3 \\
\hline 0 & 1 & 2 & 3 \\
\hline 0 & 1 & 2 & 3 \\
\hline 0 & 1 & 2 & 3 \\
\hline 0 & 1 & 2 & 3 \\
\hline 0 & 1 & 2 & 3 \\
\hline 0 & 1 & 2 & 3 \\
\hline 0 & 1 & 2 & 3 \\
\hline 0 & 1 & 2 & 3 \\
\hline 0 & 1 & 2 & 3 \\
\hline 0 & 1 & 2 & 3 \\
\hline 0 & 1 & 2 & 3 \\
\hline 0 & 1 & 2 & 3 \\
\hline 0 & 1 & 2 & 3 \\
\hline 0 & 1 & 2 & 3 \\
\hline
\end{tabular}

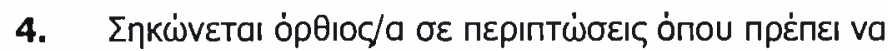

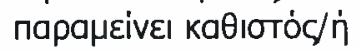

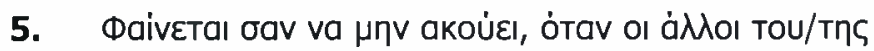

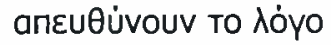

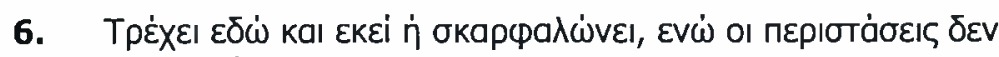
то घпітреंпоUV

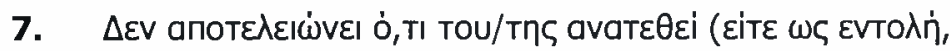

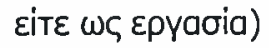

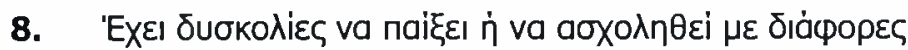

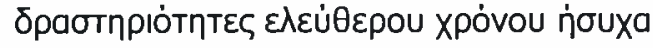

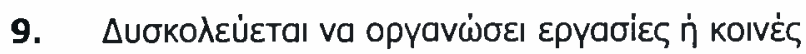
ठрабтпріо́тптєৎ

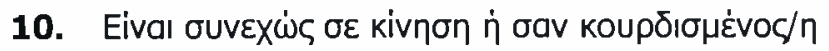

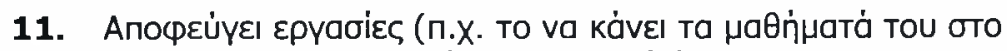

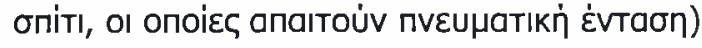

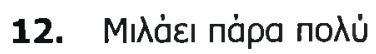

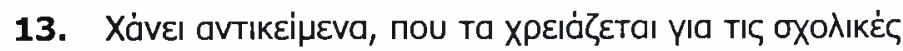

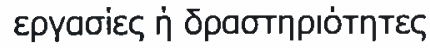

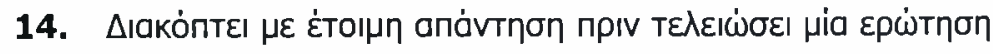

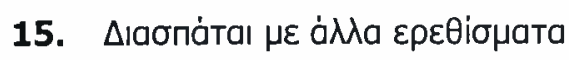

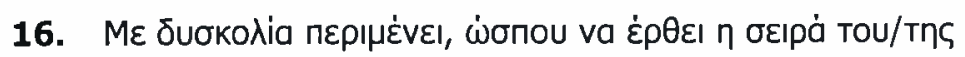

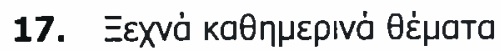

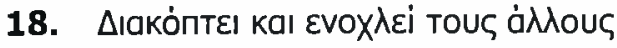




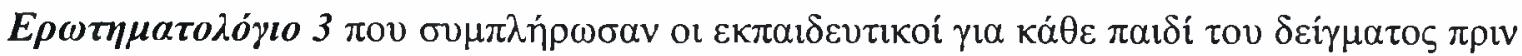

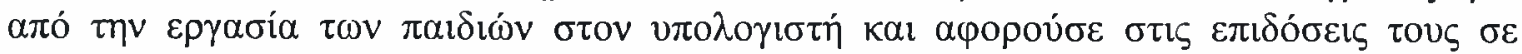

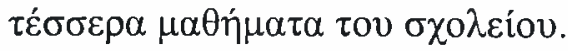

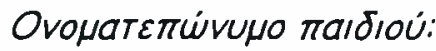

Táłn:

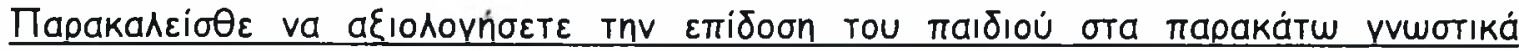

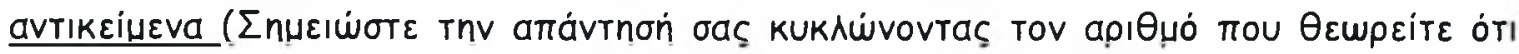

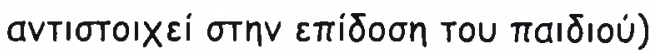

\section{MaӨnuatıká}

\begin{tabular}{|l|l|l|l|l|l|l|l|l|l|}
\hline 1 & 2 & 3 & 4 & 5 & 6 & 7 & 8 & 9 & 10 \\
\hline
\end{tabular}

\section{Iotopia}

\begin{tabular}{|l|l|l|l|l|l|l|l|l|l|}
\hline 1 & 2 & 3 & 4 & 5 & 6 & 7 & 8 & 9 & 10 \\
\hline
\end{tabular}

\section{ФUणıkń}

\begin{tabular}{|l|l|l|l|l|l|l|l|l|l|}
\hline 1 & 2 & 3 & 4 & 5 & 6 & 7 & 8 & 9 & 10 \\
\hline
\end{tabular}

\section{KaMııтeXviká}

\begin{tabular}{|l|l|l|l|l|l|l|l|l|l|}
\hline 1 & 2 & 3 & 4 & 5 & 6 & 7 & 8 & 9 & 10 \\
\hline
\end{tabular}




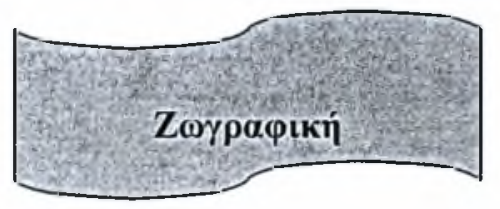

Ovo $\mu \alpha \tau \varepsilon \pi \dot{v} v \mu$ :

Tákn:

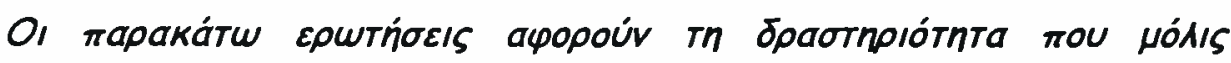

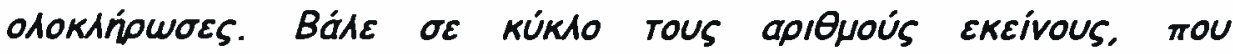

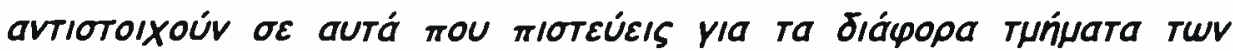

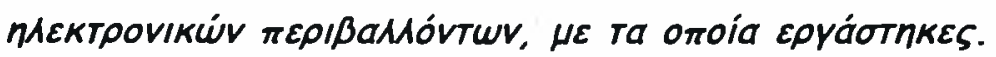

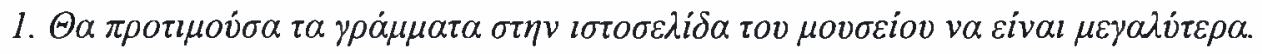

\begin{tabular}{|c|}
\hline$\Delta \imath \alpha \varphi \omega \vee \omega ́$ \\
\hline 1 \\
\hline
\end{tabular}

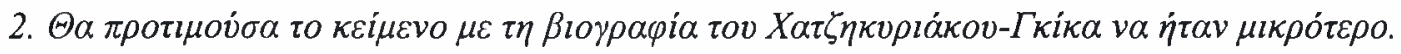

$\begin{array}{ccc}\Delta t \alpha \varphi \omega v \omega ́ & \Sigma v \mu \varphi \omega v \dot{\omega} \lambda i ́ r o & \sum v \mu \varphi \omega v \omega ́ \alpha \pi o ́ \lambda v \tau \alpha \\ 1 & 2 & 3\end{array}$

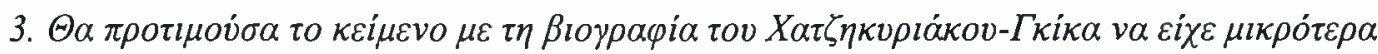
$\gamma \rho \alpha \dot{\mu} \mu \alpha \tau \alpha$.

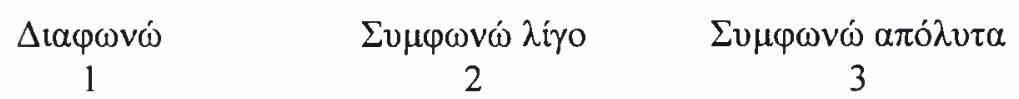

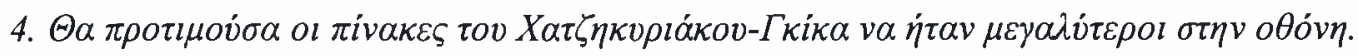

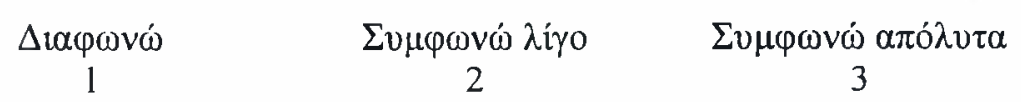

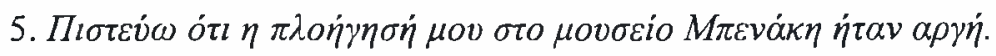

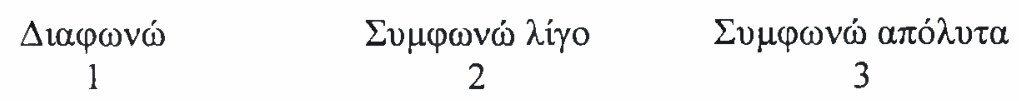

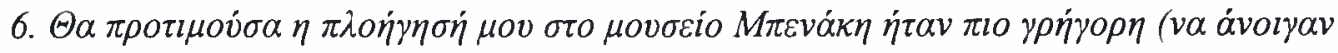

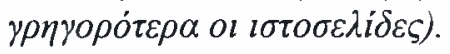

\begin{tabular}{|c|}
\hline$\Delta \imath \alpha \varphi \omega v \dot{~}$ \\
\hline 1 \\
\hline
\end{tabular}




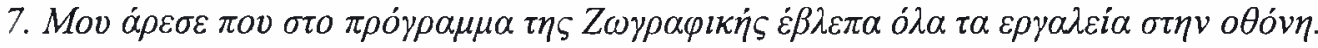

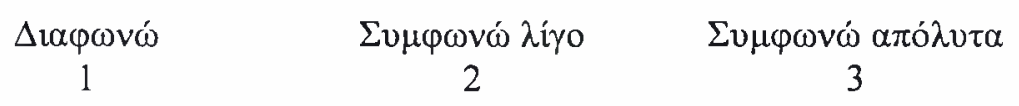

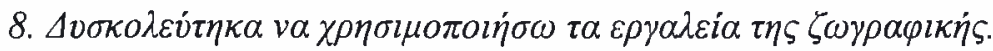

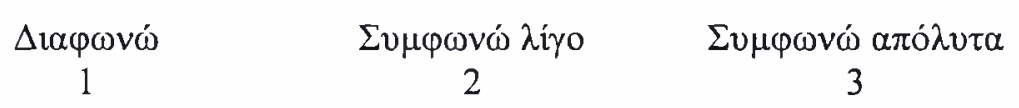

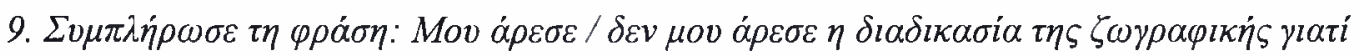




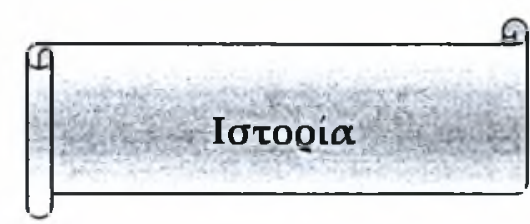

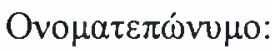

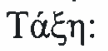

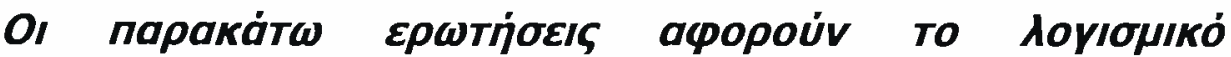

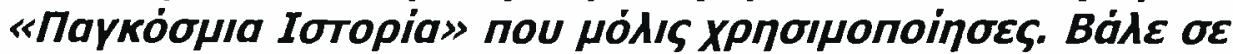

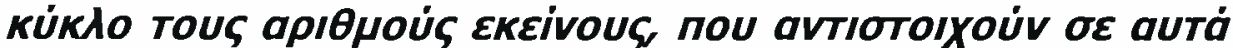

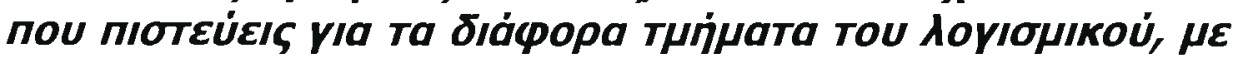

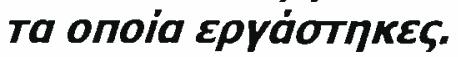

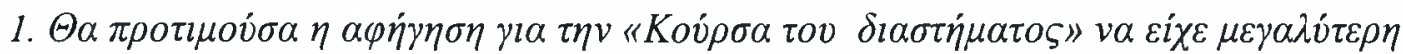
$\delta l \alpha ́ \rho \kappa \varepsilon l \alpha$.

\begin{tabular}{|c|c|c|}
\hline$\Delta \imath \alpha \varphi \omega \vee \omega ́$ & 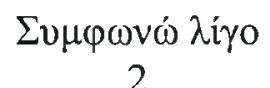 & $\Sigma v \mu \varphi \omega v \omega ́ \alpha \pi \delta ́ \lambda v \tau o$ \\
\hline
\end{tabular}

2. Mov $\alpha \rho \varepsilon \sigma \varepsilon \pi o v$ o $\alpha \varphi \eta \gamma \eta \tau \dot{\eta} \varsigma \dot{\eta} \tau \alpha \nu \alpha \dot{\alpha} \nu \delta \rho \varsigma$.
$\triangle\llcorner\alpha \varphi \omega \vee \omega ́$

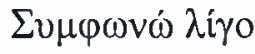
$\Sigma v \mu \varphi \omega \vee \omega ́ \alpha \pi o ́ \lambda v \tau \alpha$
1
2
3

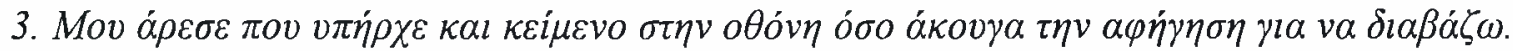

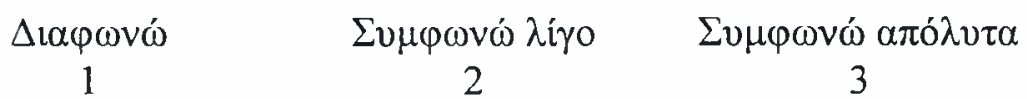

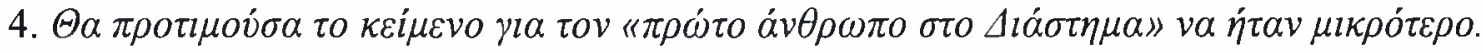

\begin{tabular}{|c|c|c|}
\hline$\Delta \iota \alpha \varphi \omega \vee \omega ́$ & $\begin{array}{c}\Sigma v \mu \varphi \omega v \omega ́ \\
2\end{array}$ & $\sum_{3}^{\Sigma v \mu \varphi \omega v \omega ́ ~ \alpha \pi o ́ \lambda v \tau \alpha}$ \\
\hline
\end{tabular}

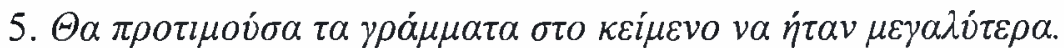

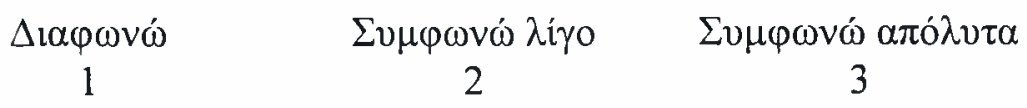

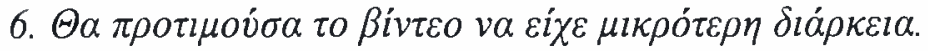

\begin{tabular}{|c|c|c|}
\hline 4 & 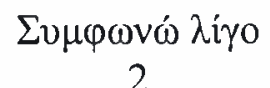 & $\Sigma v \mu \varphi \omega v \dot{~} \alpha \pi \delta ́ \lambda v \tau \alpha$ \\
\hline
\end{tabular}




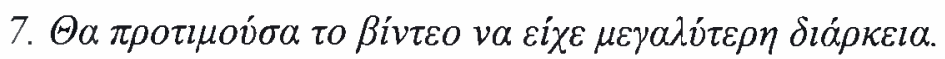

\begin{tabular}{|c|c|c|}
\hline$\Delta \iota \alpha \varphi \omega \vee \omega ́$ & 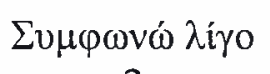 & $\Sigma v \mu \varphi \omega v \omega ́$ c \\
\hline 1 & 2 & 3 \\
\hline
\end{tabular}

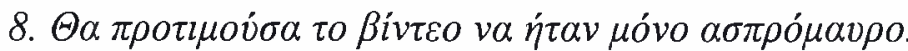

\begin{tabular}{|c|c|}
\hline$\Delta$ เ $\alpha \varphi \omega \vee \omega ́$ & 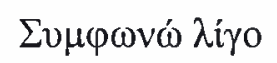 \\
\hline 1 & 2 \\
\hline
\end{tabular}

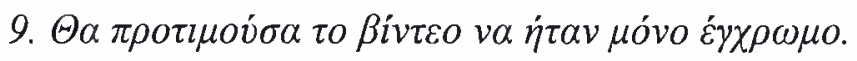

\begin{tabular}{|c|c|}
\hline$\Delta \mathrm{t} \alpha \varphi \omega \vee \omega ́$ & 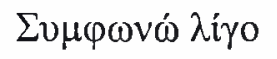 \\
\hline 1 & 2 \\
\hline
\end{tabular}

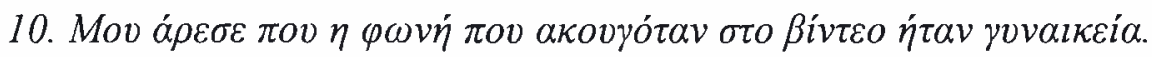

$\begin{array}{ccc}\Delta \iota \alpha \varphi \omega \vee \omega ́ & \sum v \mu \varphi \omega \vee \omega ́ \lambda i ́ \gamma o & \Sigma v \mu \varphi \omega \vee \omega ́ \alpha \pi o ́ \lambda v \tau \alpha \\ 1 & 2 & 3\end{array}$

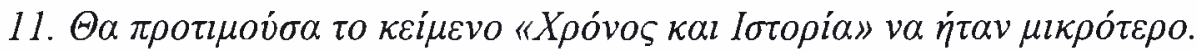

\begin{tabular}{|c|c|}
\hline$\Delta \iota \alpha \varphi \omega \vee \omega ́$ & $\Sigma v \mu \varphi \omega v \dot{\omega} \lambda \dot{i} \gamma o$ \\
\hline 1 & 2 \\
\hline
\end{tabular}

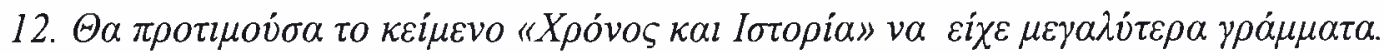

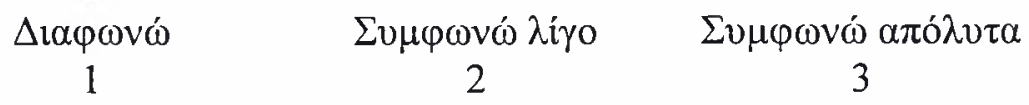




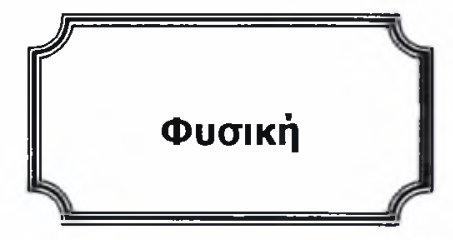

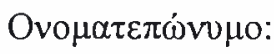

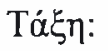

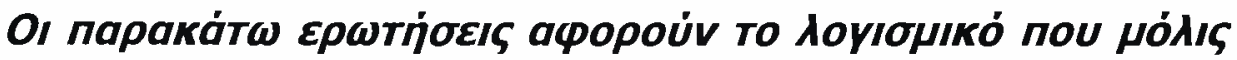

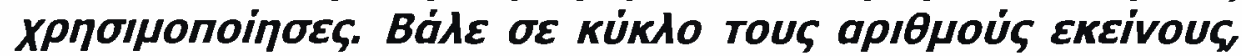

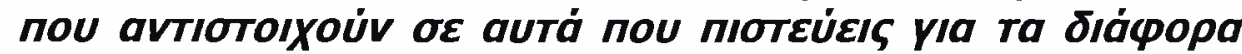

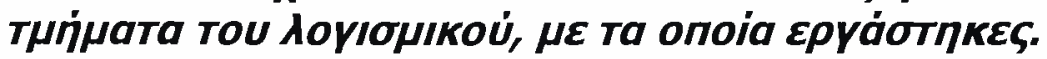

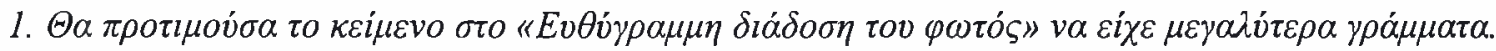

\begin{tabular}{|c|}
\hline$\Delta \iota \alpha \varphi \omega \vee \omega ́$ \\
\hline 1 \\
\hline
\end{tabular}

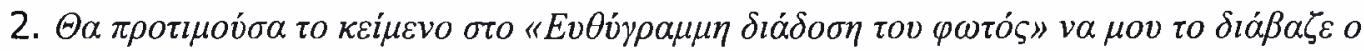

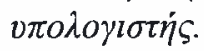

\begin{tabular}{|c|c|c|}
\hline$\Delta \iota \alpha \varphi \omega \vee \omega ́$ & 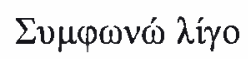 & $\Sigma v \mu \varphi \omega v \omega ́ \alpha \pi \operatorname{có}_{\lambda v \tau o}$ \\
\hline 1 & 2 & 3 \\
\hline
\end{tabular}

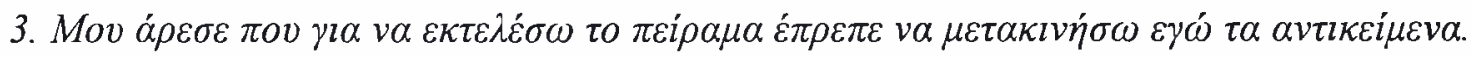

\begin{tabular}{|c|c|}
\hline$\Delta \iota \alpha \varphi \omega \vee \omega ́$ & $\lambda i \gamma o$ \\
\hline 1 & \\
\hline
\end{tabular}

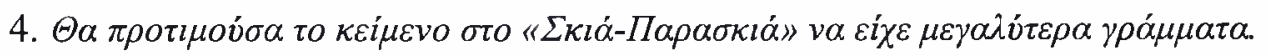

\begin{tabular}{|c|c|c|}
\hline$\Delta \imath \alpha \varphi \omega \vee \omega ́$ & 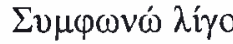 & 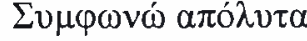 \\
\hline 1 & 2 & 3 \\
\hline
\end{tabular}

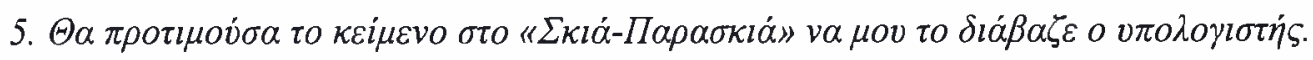

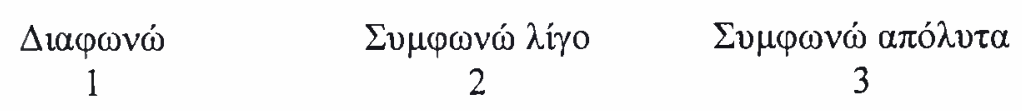




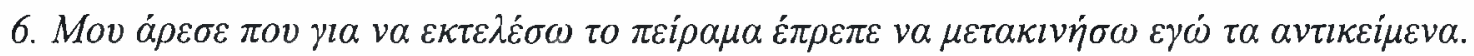

\begin{tabular}{|c|c|c|}
\hline$\Delta \imath \alpha \varphi \omega \vee \omega ́$ & $\Sigma v \mu \varphi \omega v \omega \dot{~} \lambda \dot{i} \gamma o$ & $\Sigma v \mu \varphi \omega v \omega \dot{\alpha} \alpha \pi \delta ́ \lambda v \tau \alpha$ \\
\hline 1 & 2 & 3 \\
\hline
\end{tabular}

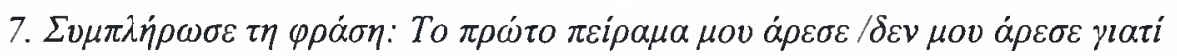

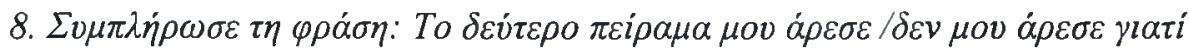




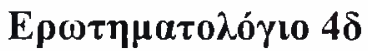

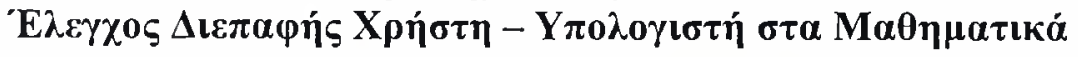

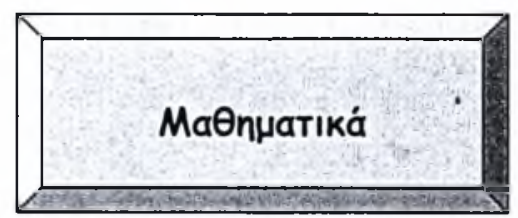

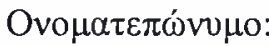

Táłฤ:

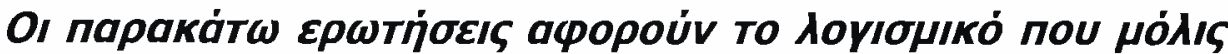

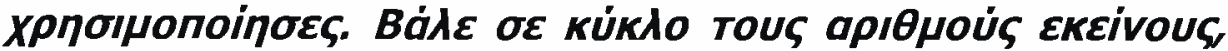

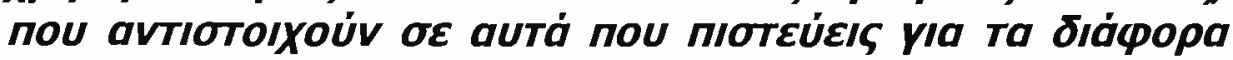

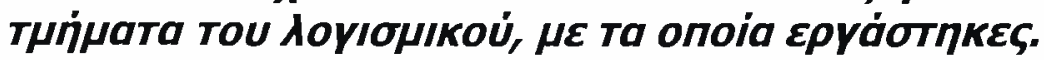

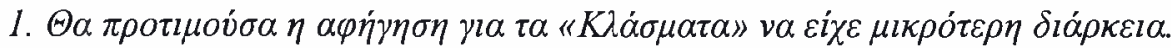

\begin{tabular}{|c|c|c|}
\hline$\Delta \iota \alpha \varphi \omega v \omega ́$ & 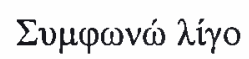 & $\Sigma v \mu \varphi \omega v \omega ́ \alpha \pi \delta ́ \lambda v \tau \alpha$ \\
\hline 1 & 2 & 3 \\
\hline
\end{tabular}

2. Mov $\dot{\alpha} \rho \varepsilon \sigma \varepsilon \pi o v o \alpha \varphi \eta \gamma \eta \tau \dot{\eta} \varsigma \dot{\eta} \tau \alpha v \dot{\alpha} v \delta \rho \alpha \varsigma$.

\begin{tabular}{|c|c|c|}
\hline$\Delta \iota \alpha \varphi \omega v \omega ́$ & $\Sigma v \mu \varphi \omega v \omega ́ \lambda i ́ \gamma o$ & 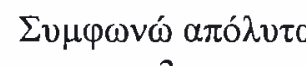 \\
\hline 1 & 2 & 3 \\
\hline
\end{tabular}

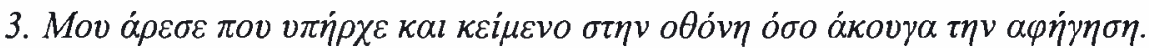

\begin{tabular}{|c|c|c|}
\hline$\Delta \mathrm{l} \alpha \varphi \omega \vee \omega ́$ & 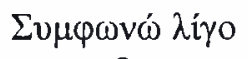 & $\Sigma v \mu \varphi \omega v \omega ́ \alpha \pi \delta ́ \lambda v \tau \sigma$ \\
\hline 1 & 2 & 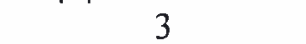 \\
\hline
\end{tabular}

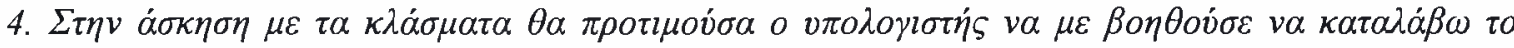
$\lambda \dot{\alpha} \theta \circ \varsigma \mu \nu$.

\begin{tabular}{|c|c|c|}
\hline$\Delta \operatorname{l} \alpha \varphi \omega \vee \omega ́$ & 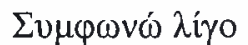 & $\Sigma v \mu \varphi \omega v \omega ́ \alpha \pi \delta ́ \lambda v \tau 0$ \\
\hline 1 & 2 & 3 \\
\hline
\end{tabular}

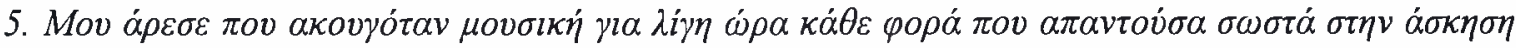
$\mu \varepsilon \tau \alpha \kappa \lambda \dot{\alpha} \sigma \mu \alpha \tau \alpha$.

\begin{tabular}{|c|c|c|}
\hline$\Delta \iota \alpha \varphi \omega \vee \omega ́$ & 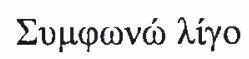 & $\Sigma v \mu \varphi \omega v \omega ́ \alpha \pi \delta ́ \lambda v \tau 0$ \\
\hline 1 & 2 & \\
\hline
\end{tabular}

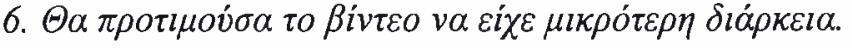

\begin{tabular}{|c|}
\hline 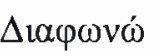 \\
\hline 1 \\
\hline
\end{tabular}

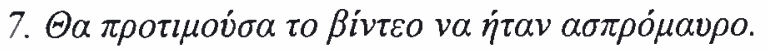

\begin{tabular}{|c|c|}
\hline$\Delta l \alpha \varphi \omega$ & 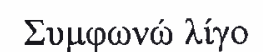 \\
\hline
\end{tabular}




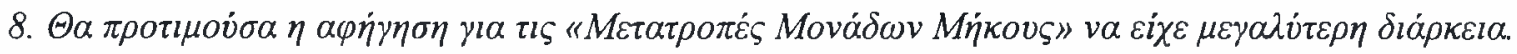

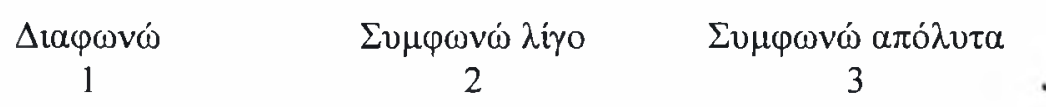

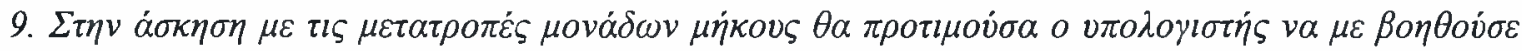

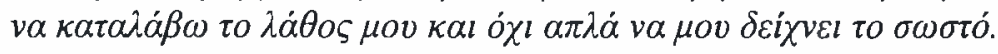

\begin{tabular}{|c|c|}
\hline$\Delta \alpha \omega \varphi \omega \omega \dot{~}$ & 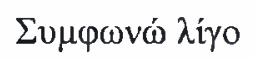 \\
\hline 1 & 2 \\
\hline
\end{tabular}

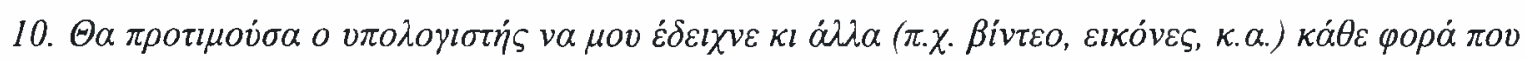
$\varepsilon \dot{\delta} \iota v \alpha \sigma \omega \sigma \tau \dot{\eta} \alpha \pi \dot{\alpha} v \tau \eta \sigma \eta \sigma \tau \eta v \dot{\alpha} \sigma \kappa \eta \sigma \eta \mu \varepsilon \tau \iota \varsigma \mu \varepsilon \tau \alpha \tau \rho o \pi \varepsilon \dot{\varepsilon} \mu o v \dot{\alpha} \delta \omega v \mu \dot{\eta} \kappa o v \varsigma$.

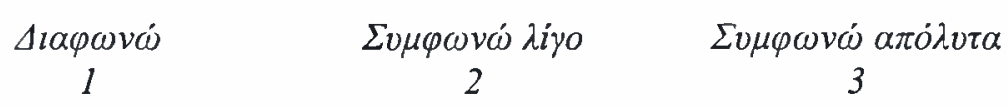

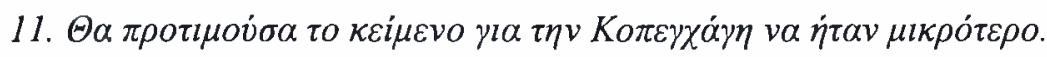

\begin{tabular}{|c|}
\hline$\Delta \mathrm{t} \alpha \varphi \omega \nu \omega \dot{~}$ \\
\hline l \\
\hline
\end{tabular}

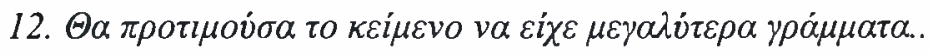

\begin{tabular}{|c|c|c|}
\hline$\Delta t \alpha \varphi \omega \vee \omega ́$ & $\Sigma v \mu \varphi \omega v \omega \dot{\lambda} \lambda \dot{i} о$ & $\Sigma v \mu \varphi \omega v \omega ́ \alpha \pi \delta \lambda^{\prime} v \tau \alpha$ \\
\hline 1 & 2 & 3 \\
\hline
\end{tabular}




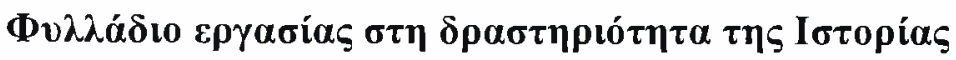

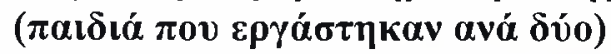

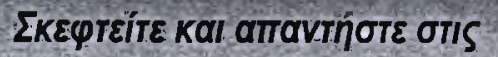

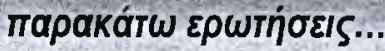

Ovo $\mu \alpha \tau \varepsilon \pi \omega ́ v v \mu$ o $\pi \alpha 1 \delta$ เov́:

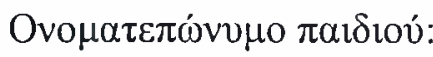

Tákฑ:

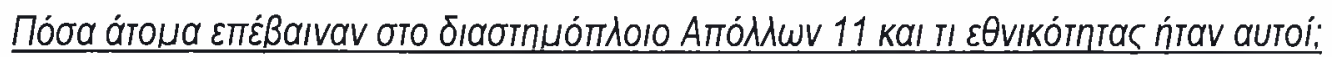

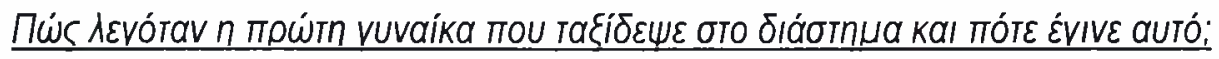

Kaגí Emituxía 


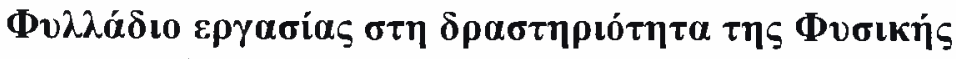

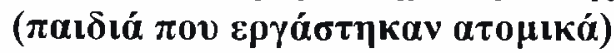

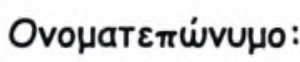

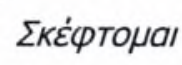

кaı anavтம்!!!

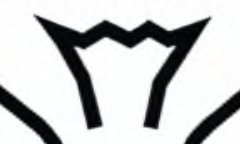

$\bar{\square}$

\section{$A^{\prime}$ Mépos}

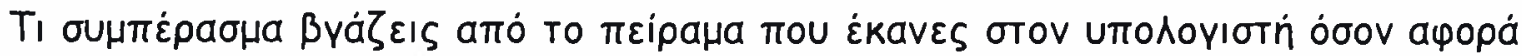

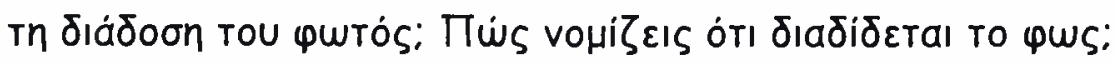

\section{B'Mépos}

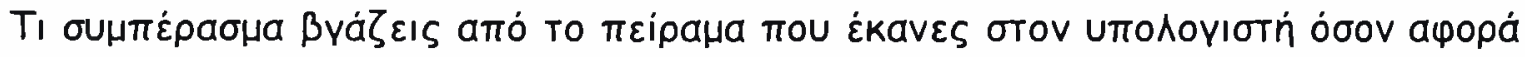

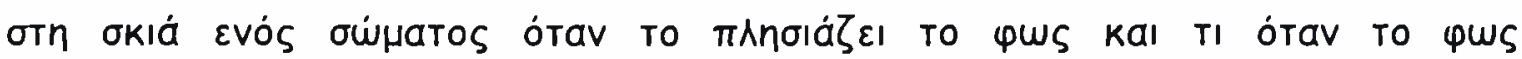

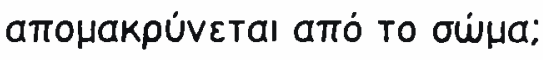




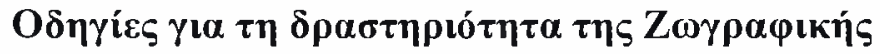

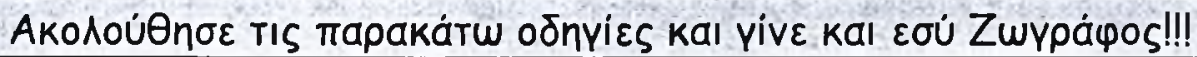

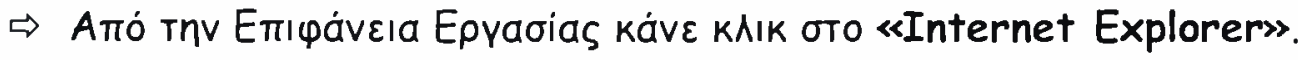

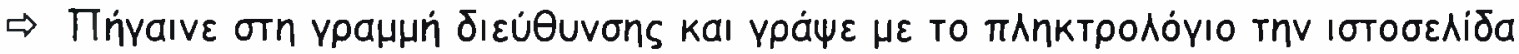

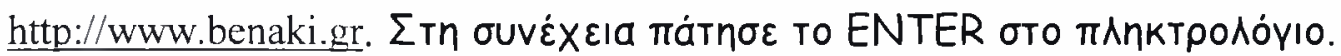

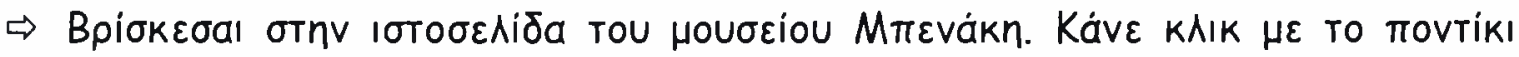

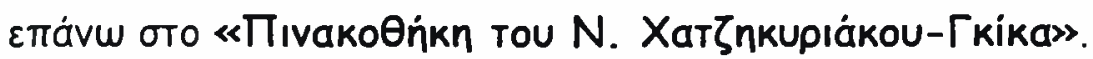

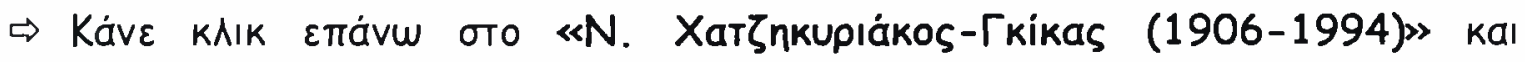

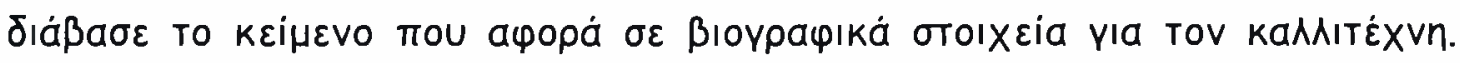

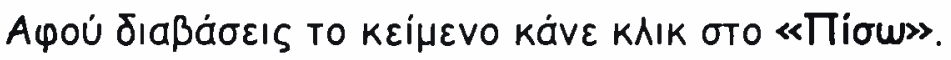

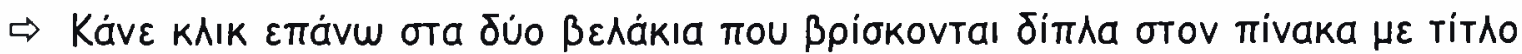

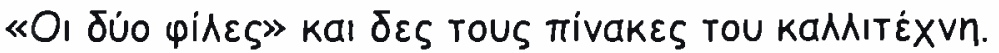

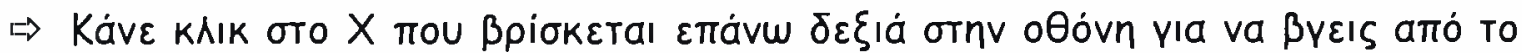
«Internet Explorer».

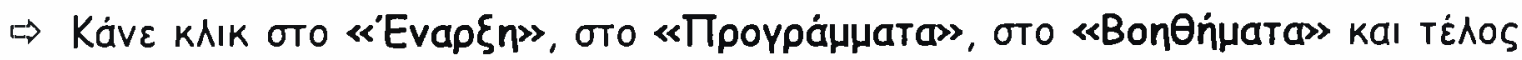

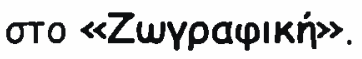

$\Rightarrow$ Tẃpa

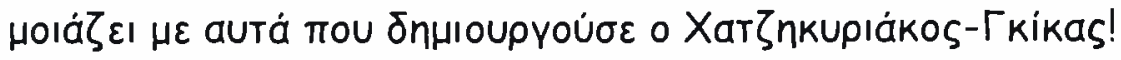




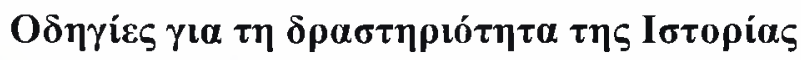

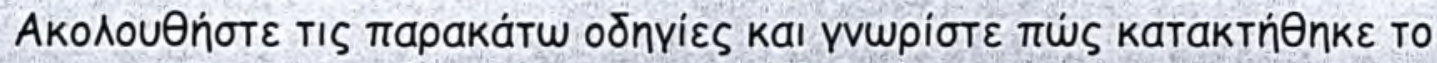

ঠıáotnua!!!

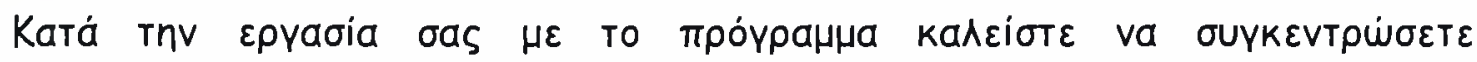

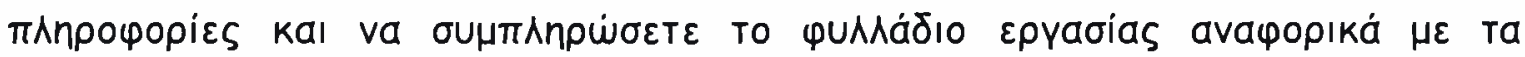

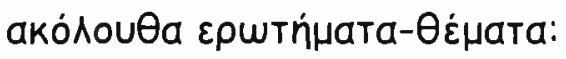

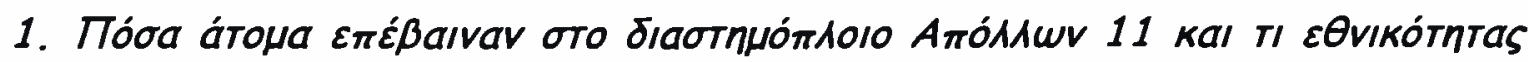
ńtav autoí.

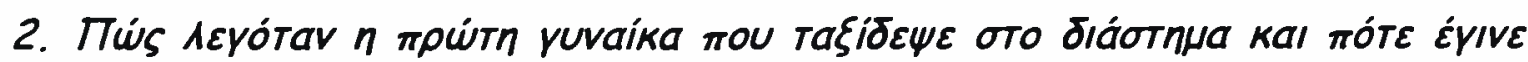
autó.

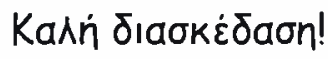

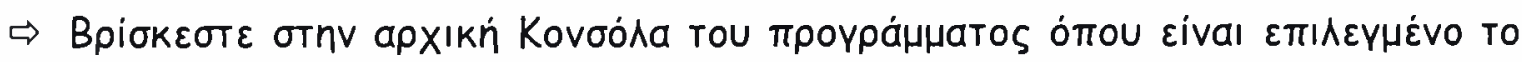

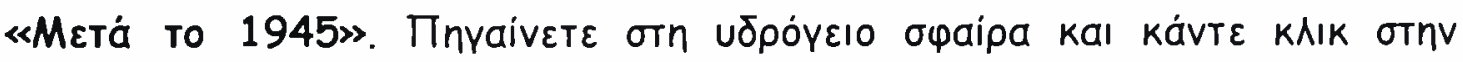
«Uอம்inn».

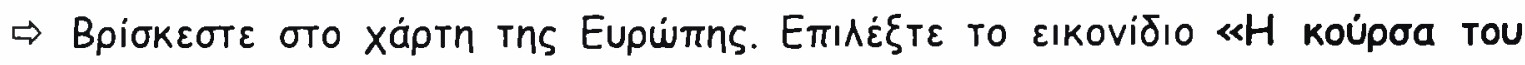

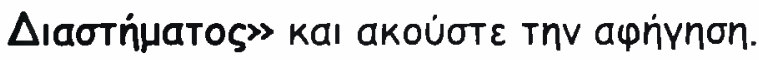

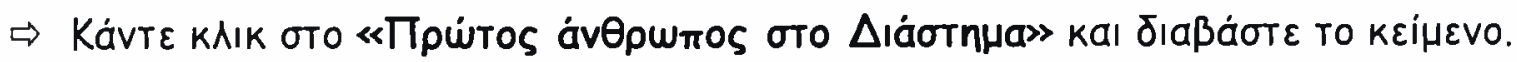

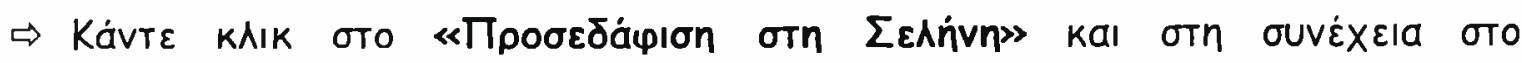

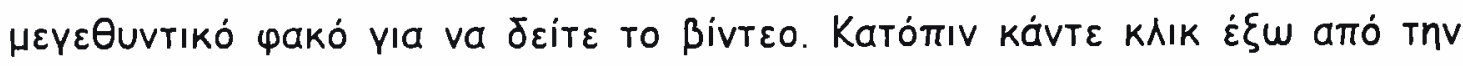
Elkóva.

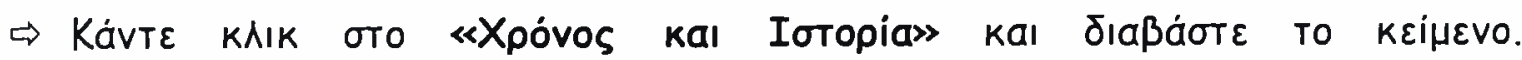

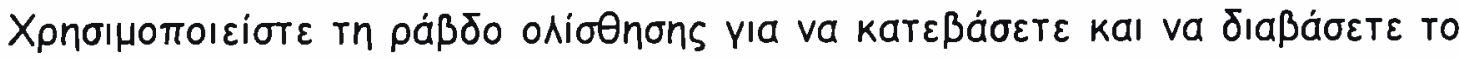
Keífevo.

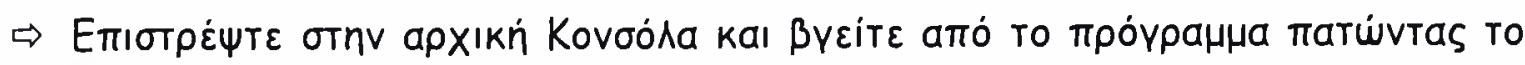

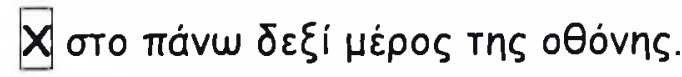




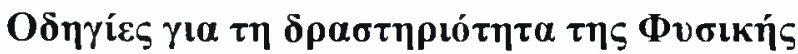

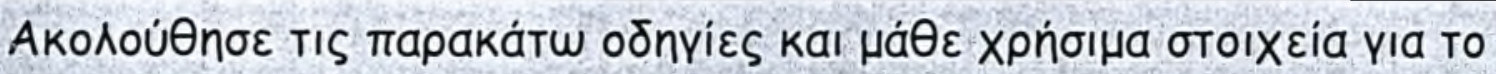
$\Phi \omega \varsigma ! ! !$

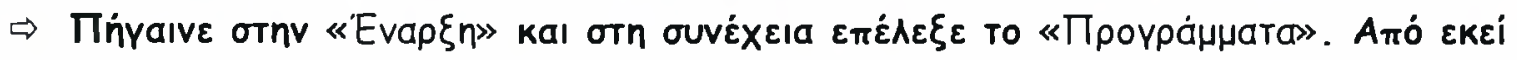
$\varepsilon \pi \varepsilon \dot{\wedge} \varepsilon \varepsilon \varepsilon$ то «MAOHMA».

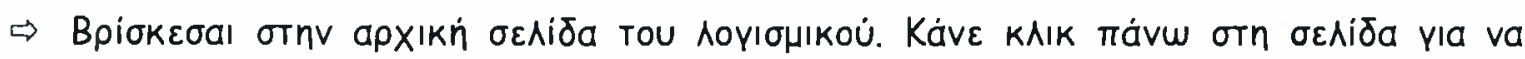

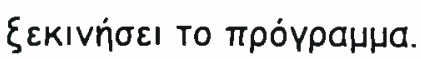

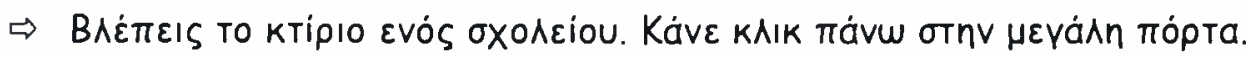

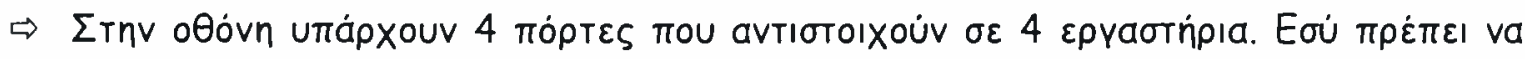

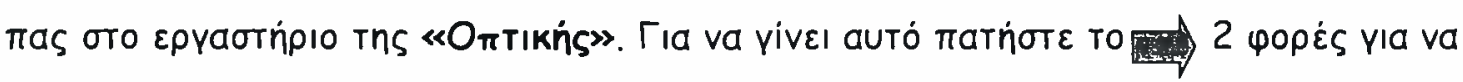
$\pi \wedge$

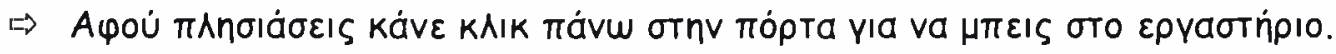

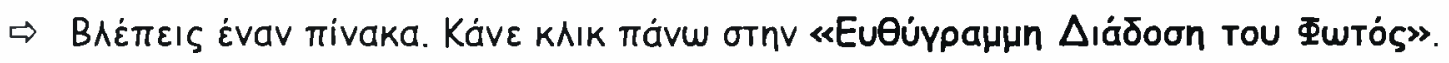

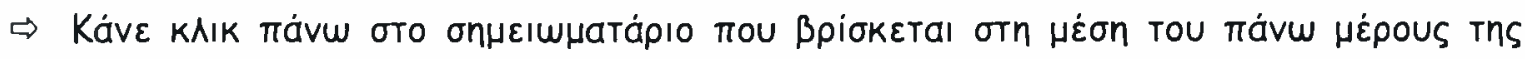

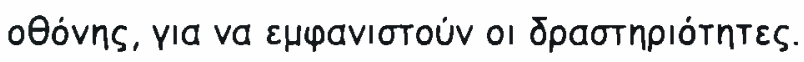

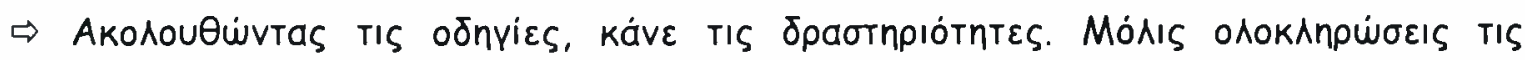

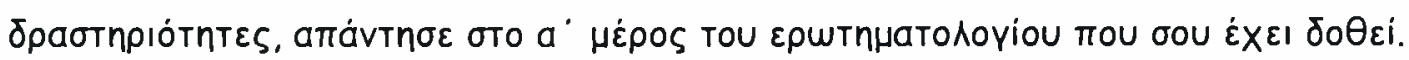

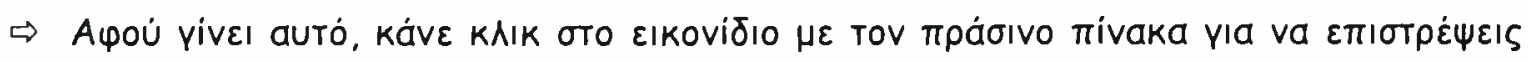

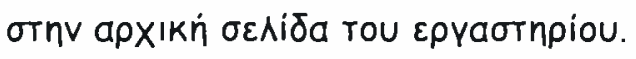

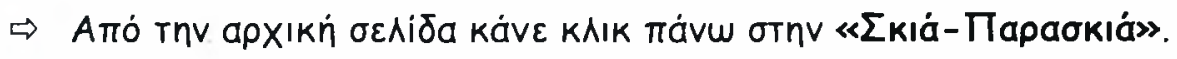

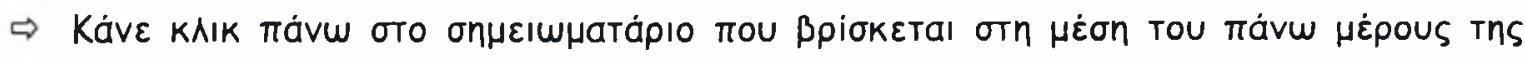

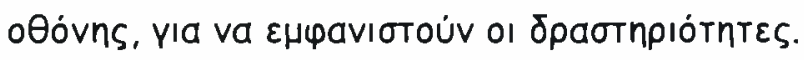

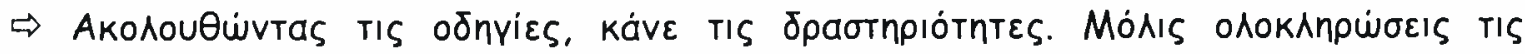

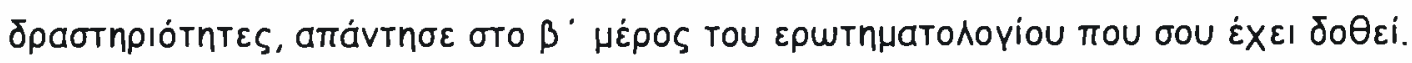

A

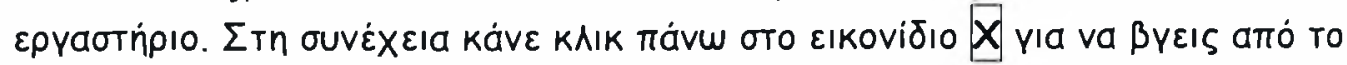
$\pi \rho \dot{\gamma} \rho а \mu \mu a$. 


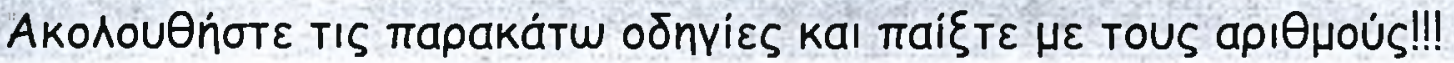

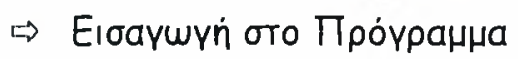

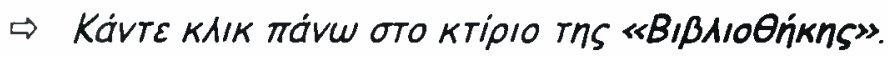

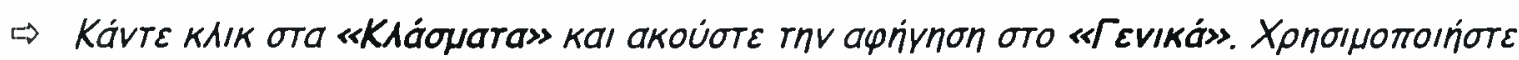

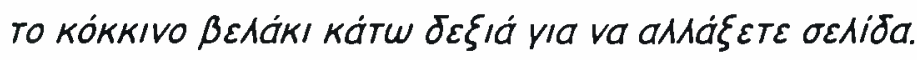

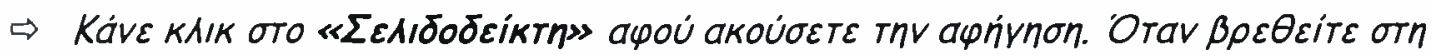

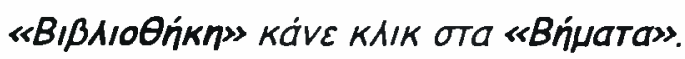

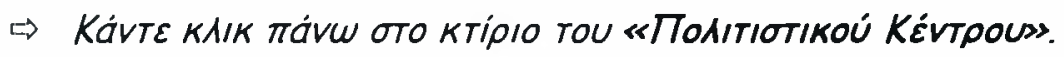

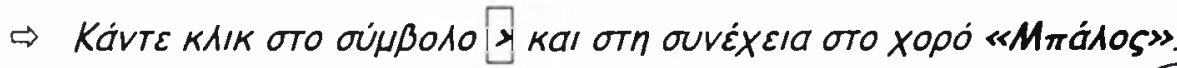

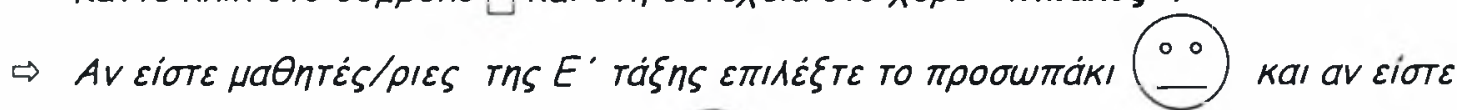

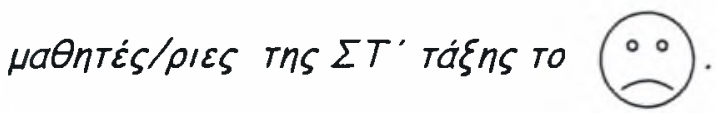

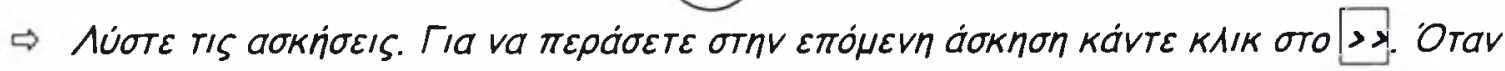

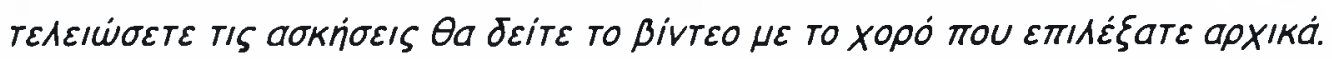

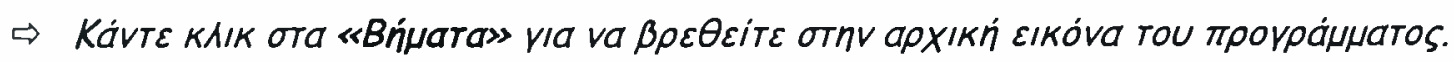

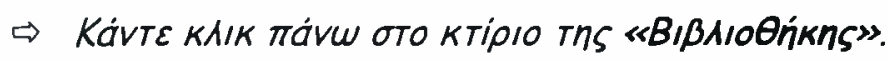

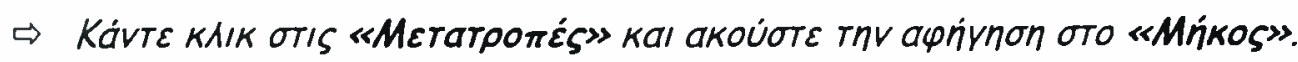

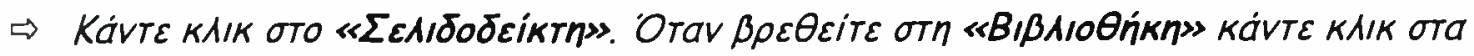
«Bńmata».

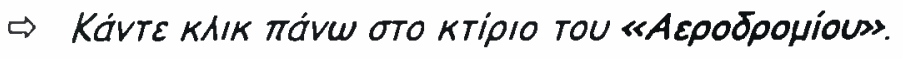

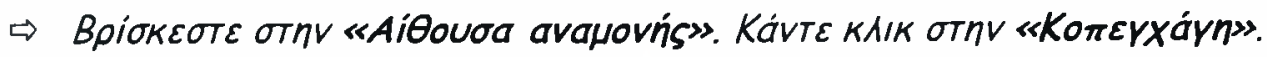

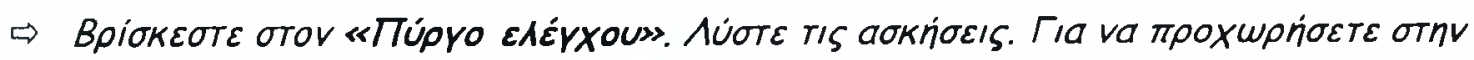

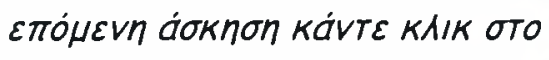

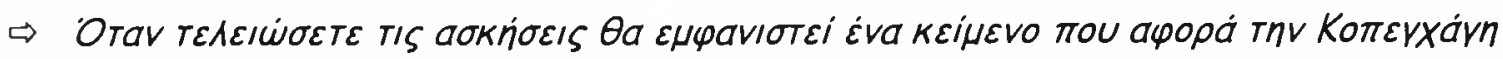

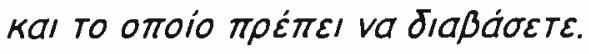

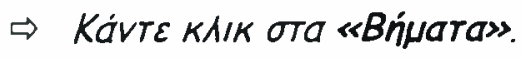

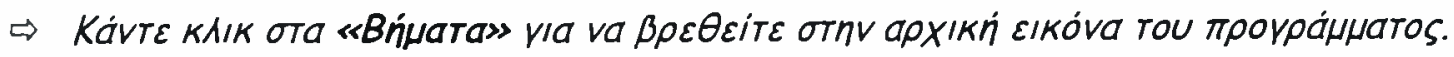

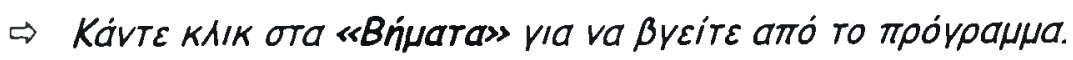




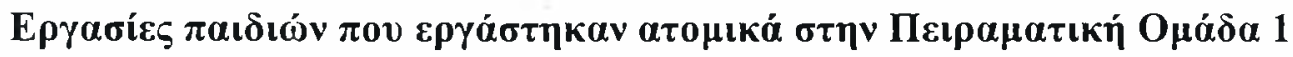

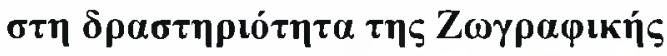






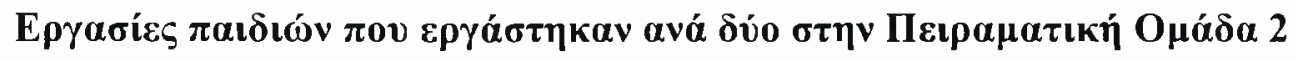

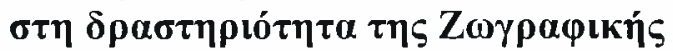

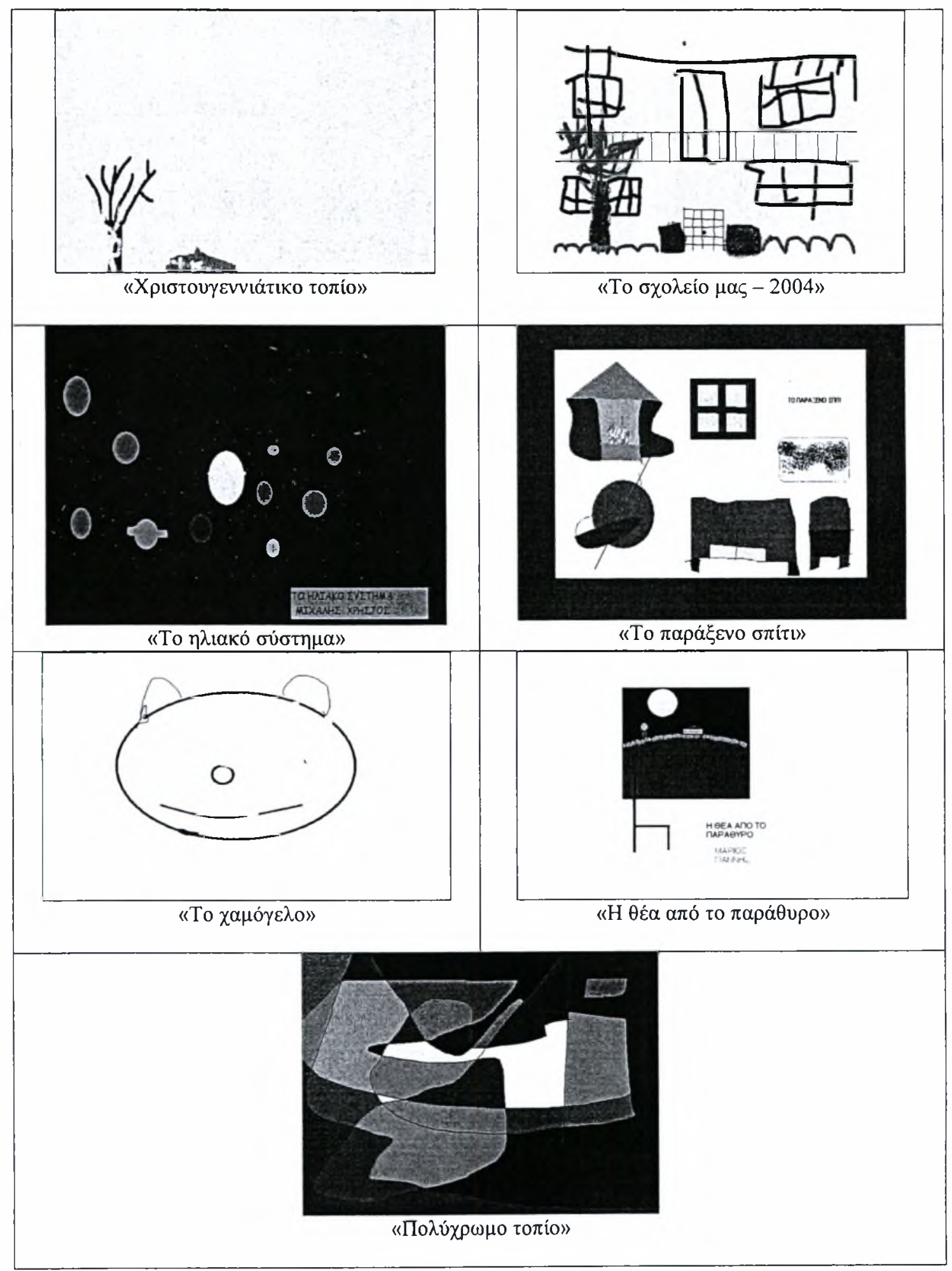




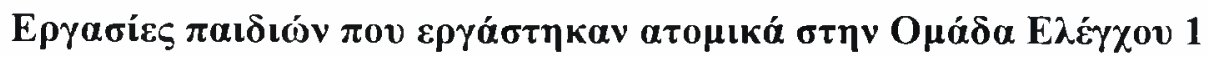
$\sigma \tau \eta \delta \rho \alpha \sigma \tau \rho\llcorner o ́ \tau \eta \tau \alpha \tau \eta \varsigma Z$ Z

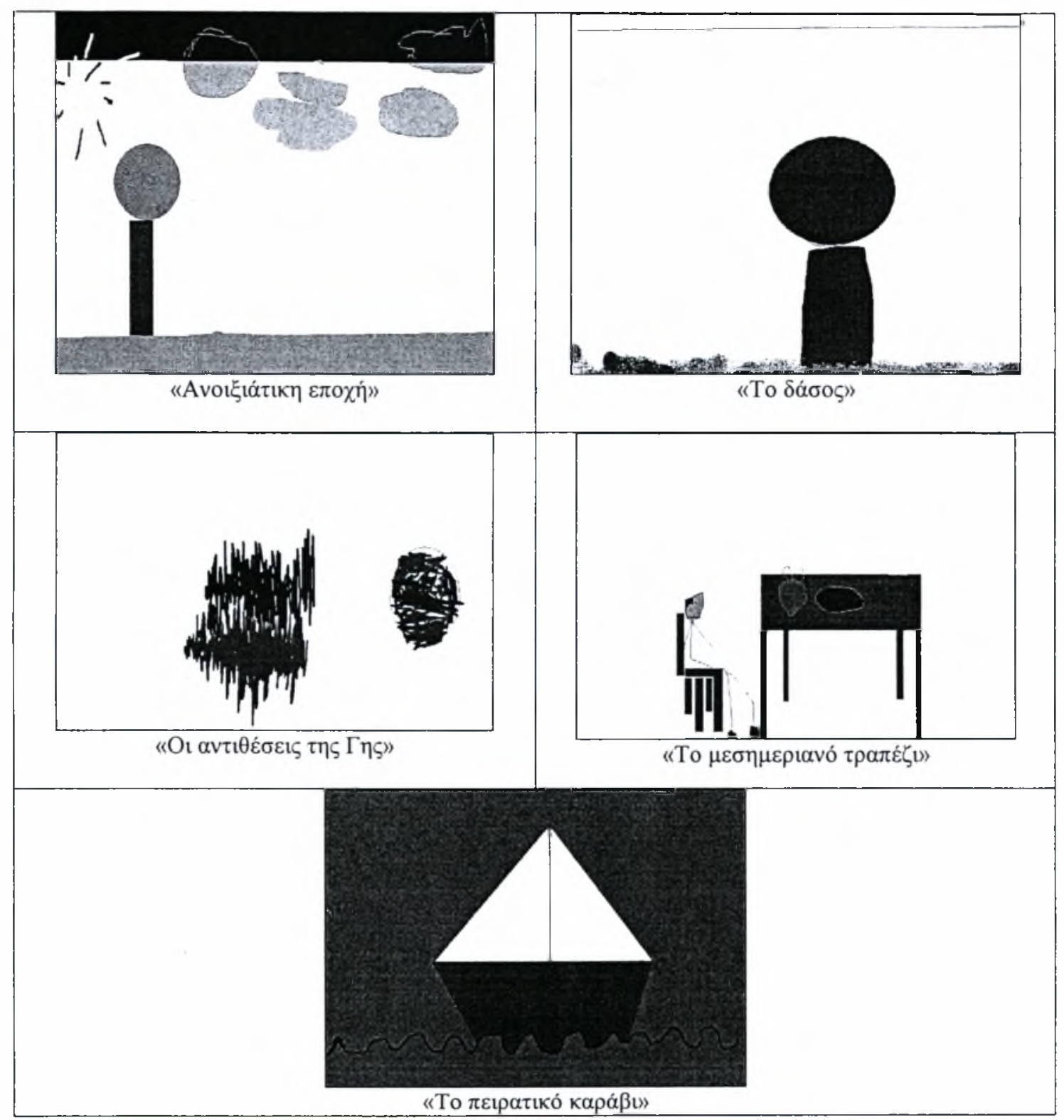




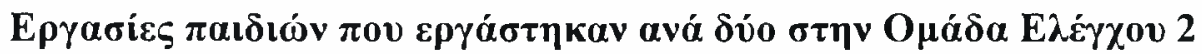

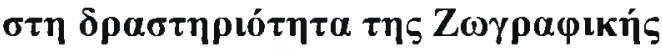

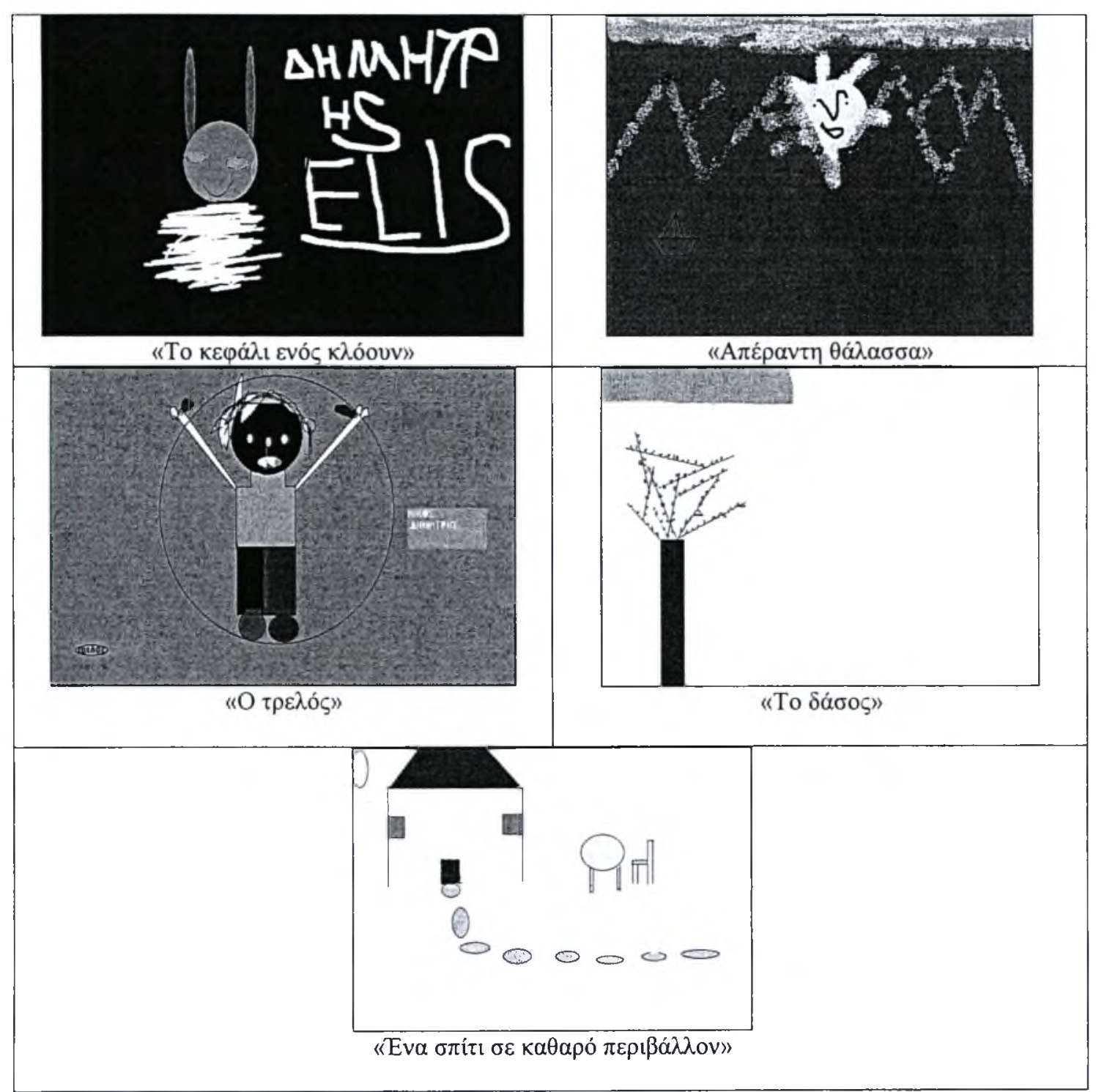




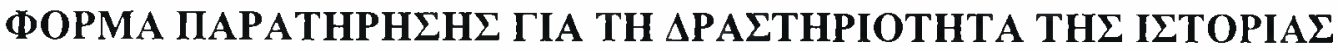

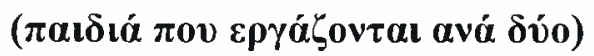

O $\mu \alpha \dot{\delta} \delta$ :

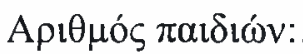

\section{Ạńynon 1.}

A

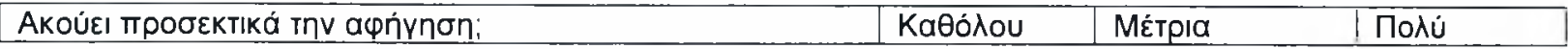

B

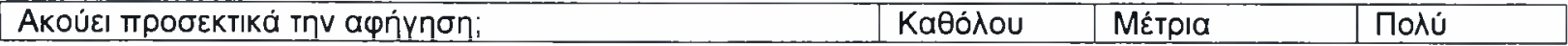

A

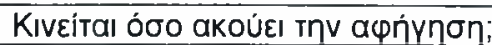
Käó̀ou

Méтpıа

Пoגú

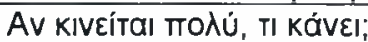

B

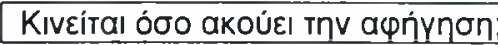

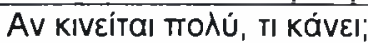

Käó̀ou

MÉтpıа

Пoגú

A

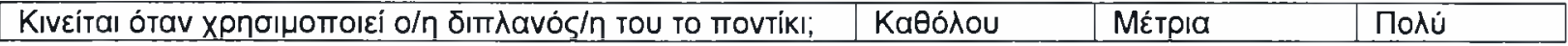

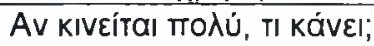

B

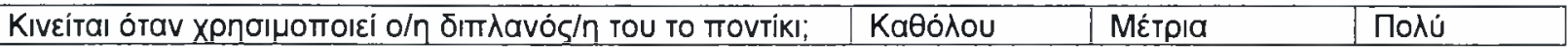

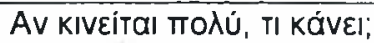

A

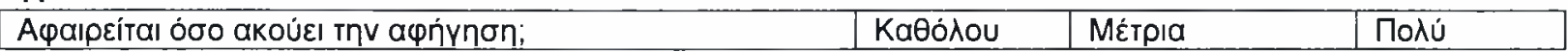

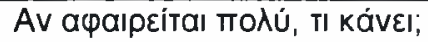

B

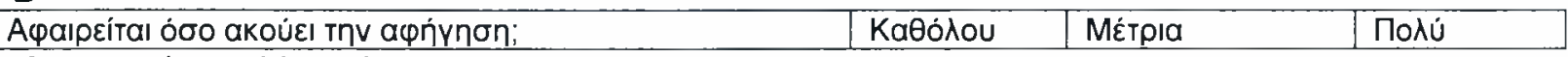

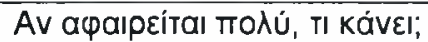

A

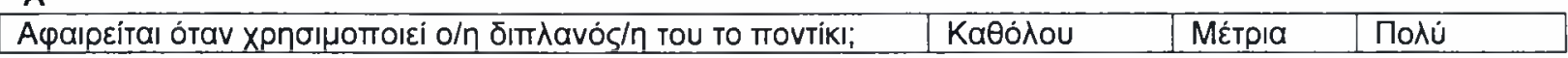

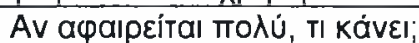

B

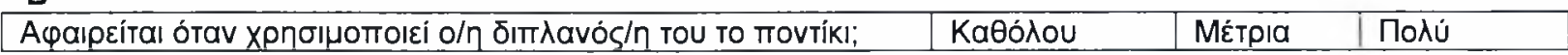

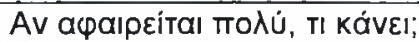

A

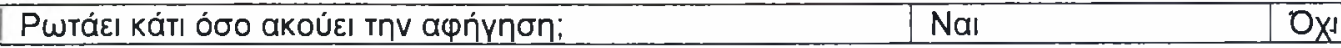

\begin{tabular}{|c|c|}
\hline Av Nal, Eivaı бXETIKÓ; & $\mathrm{Nal}$ \\
\hline
\end{tabular}

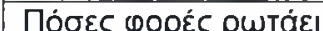

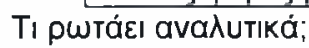

B

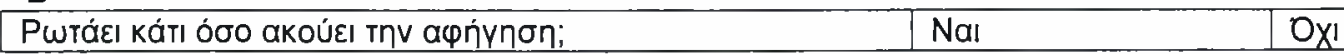

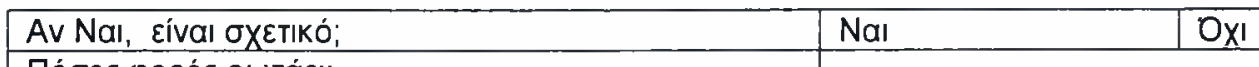

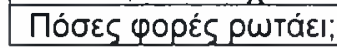

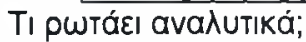

A

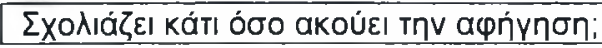

NaI

OXI 


\begin{tabular}{|c|c|c|}
\hline Av Nal, eivaı бXETIKó; & $\mathrm{Nal}$ & $O \times 1$ \\
\hline 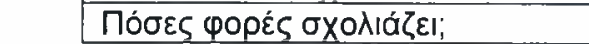 & & \\
\hline \multicolumn{3}{|l|}{ 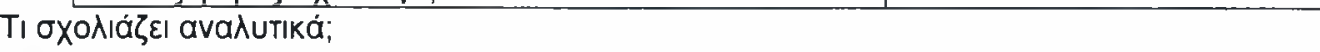 } \\
\hline \multicolumn{3}{|l|}{ B } \\
\hline 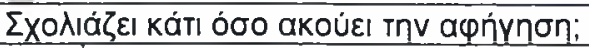 & $\mathrm{Nal}$ & OXI \\
\hline Av Naı, Eívaı бXETIKó; & $\mathrm{Nal}$ & OxI \\
\hline 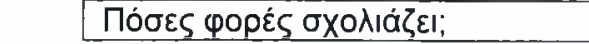 & & \\
\hline
\end{tabular}

A

\begin{tabular}{|c|c|c|}
\hline 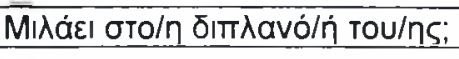 & Nal & $O \times 1$ \\
\hline 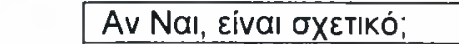 & Nal & OXI \\
\hline 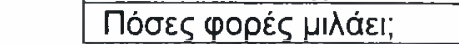 & & \\
\hline
\end{tabular}
T। $\lambda \varepsilon ́ \varepsilon \mid ;$

B

\begin{tabular}{|c|c|c|}
\hline 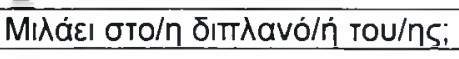 & Nal & OXI \\
\hline 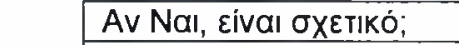 & $\mathrm{Nal}$ & OXI \\
\hline 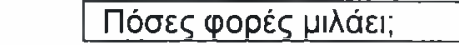 & & \\
\hline
\end{tabular}
T। $\lambda \dot{\varepsilon} \varepsilon I_{;}$

A

\begin{tabular}{|c|c|c|c|}
\hline 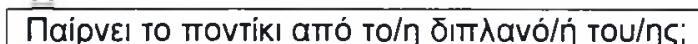 & Käódou & Мغ́трıа & Пoגú \\
\hline
\end{tabular}

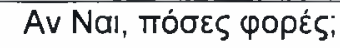

B

\begin{tabular}{|c|c|c|c|}
\hline 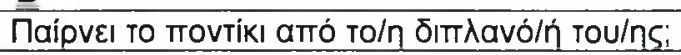 & Käódou & Мغ́тoı & Todó \\
\hline
\end{tabular}

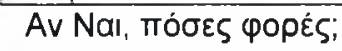

A

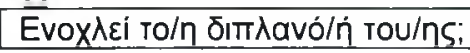
Av Nal, TI Kável;

\begin{tabular}{|l|l|}
\hline $\mathrm{NaI}$ & $\mathrm{OXI}$ \\
\hline
\end{tabular}

B

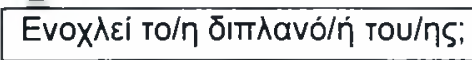

\begin{tabular}{l|l}
$\mathrm{Nal}$ & $\mathrm{OXI}$ \\
\hline
\end{tabular}

Av Nal, TI Kável;

A

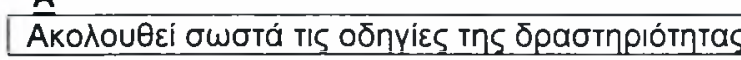
Av OXI, TI Kável;

NaI

OXI

B

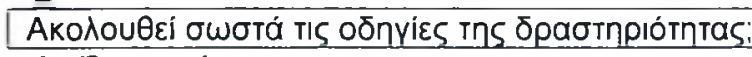

Av OXI, TI Kável;

Naı

Oxi

$\underline{A}$

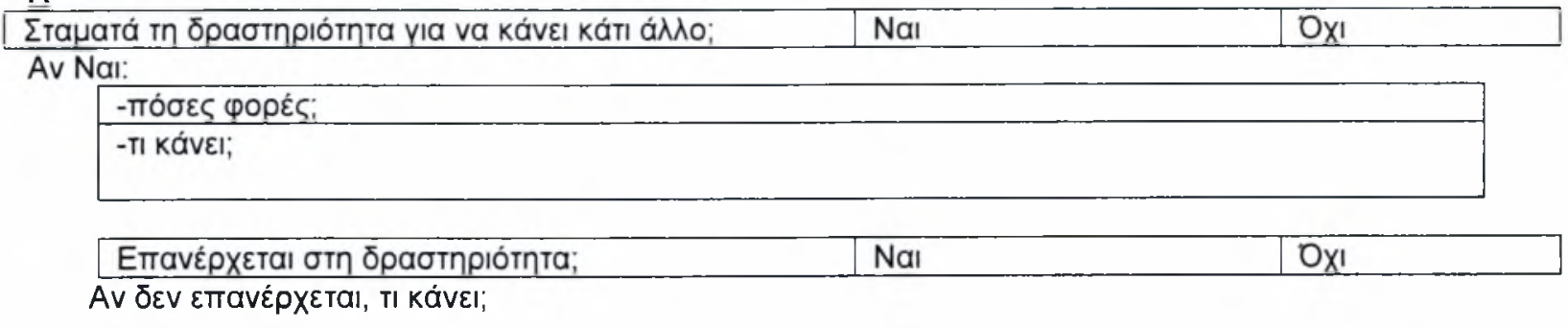

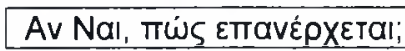

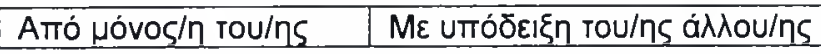




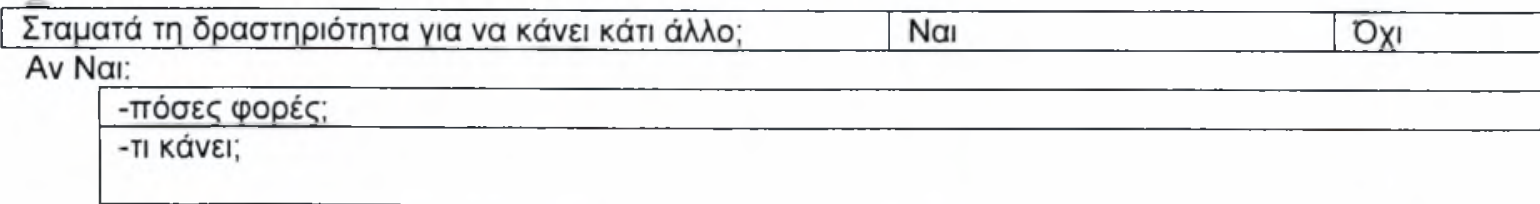

\begin{tabular}{|c|c|c|}
\hline 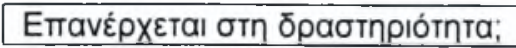 & $\mathrm{Nal}$ & OXI \\
\hline
\end{tabular}

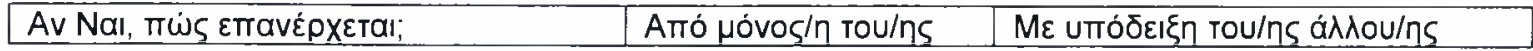

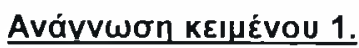

\section{A}

\begin{tabular}{|c|c|c|c|}
\hline 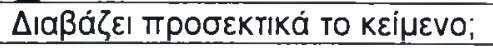 & KaӨóגou & 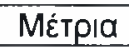 & Поגú \\
\hline
\end{tabular}

B

\begin{tabular}{|c|c|c|c|}
\hline$\triangle$ Іаßа́弓̨ & KaӨó^ou & Мغ́тріа & Поגú \\
\hline
\end{tabular}

A

\begin{tabular}{|c|c|c|c|}
\hline 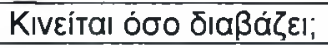 & 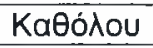 & Мغ̇трıа & Пoגú \\
\hline
\end{tabular}

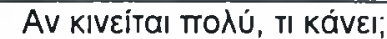

$\underline{B}$

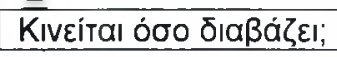

Käóরou

Мغ́тріа

Пoגú

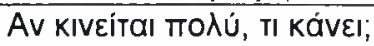

A

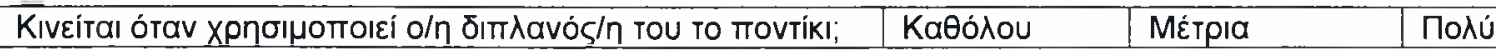

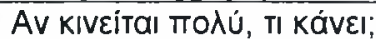

$\underline{\underline{B}}$

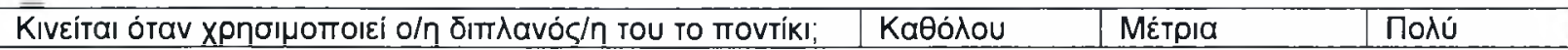

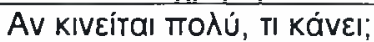

A

\begin{tabular}{|c|c|c|c|}
\hline 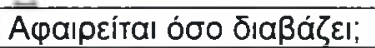 & KaӨó৯ou & Мغ́трıа & Пoגú \\
\hline \multicolumn{4}{|c|}{ 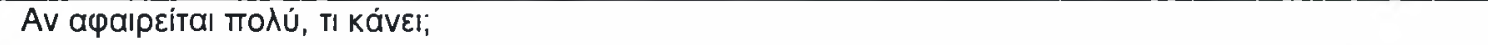 } \\
\hline \multicolumn{4}{|l|}{ B } \\
\hline 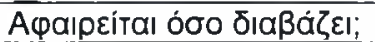 & Käó^ou & Мغ́трıа & Пoגú \\
\hline
\end{tabular}

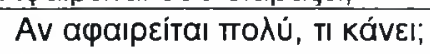

$\underline{\underline{A}}$

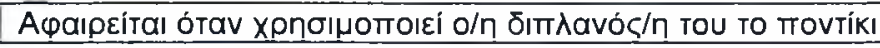

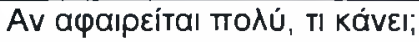

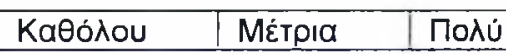

B

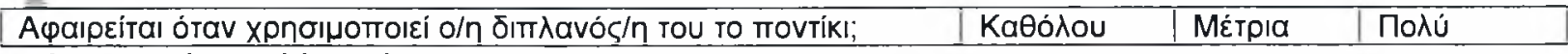

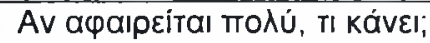

A

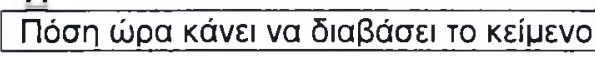

B

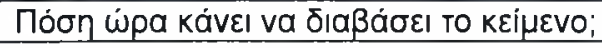

A

\begin{tabular}{|c|c|c|}
\hline 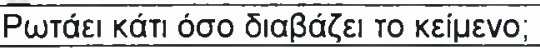 & Naı & OXI \\
\hline 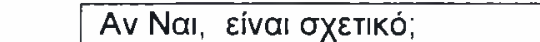 & Naı & OXI \\
\hline
\end{tabular}




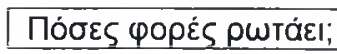

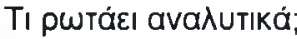

B

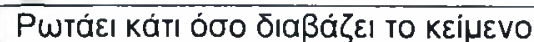

\begin{tabular}{l|l|} 
Nal & OXI \\
\hline
\end{tabular}

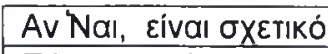

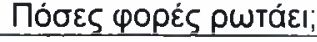

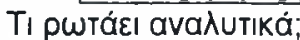

A

\begin{tabular}{|c|c|c|}
\hline 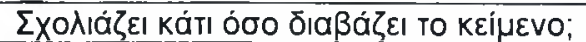 & Naı & OXI \\
\hline
\end{tabular}

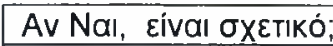

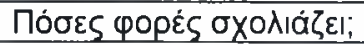

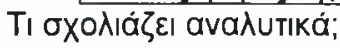

$B$

\begin{tabular}{|c|c|c|}
\hline 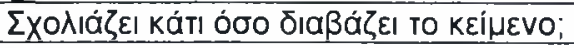 & Naı & OXI \\
\hline 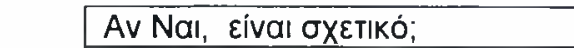 & Naı & OXI \\
\hline 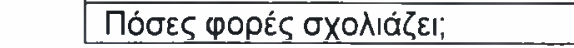 & & \\
\hline
\end{tabular}

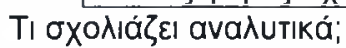

A

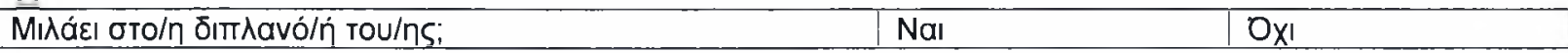

\begin{tabular}{|c|c|c|}
\hline Av Nal, Eívaı oXETıKó; & $\mathrm{Nal}$ & OXI \\
\hline 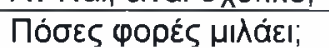 & & \\
\hline
\end{tabular}

T। $\wedge \dot{\varepsilon \varepsilon \mid}$

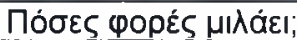

B

\begin{tabular}{|c|c|c|}
\hline 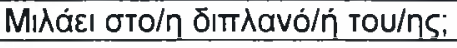 & Naı & OXI \\
\hline 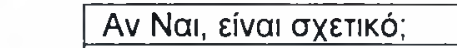 & Naı & OX! \\
\hline
\end{tabular}

T। $\lambda \varepsilon ́ \varepsilon \mid ;$

A

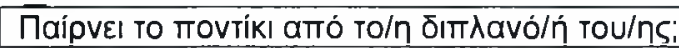

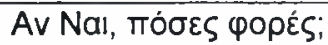

B

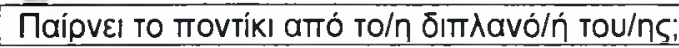

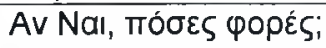

\begin{tabular}{|c|c|}
\hline KaӨóגou & Мغ́трıа \\
\hline
\end{tabular}

A

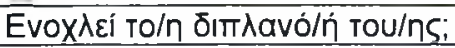

Av Naı, Tı Kável;

B

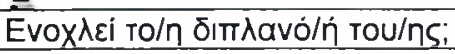

Av Naı, Tı Kável;

KaӨóגou

Мغ́трıа

Пoגú

A

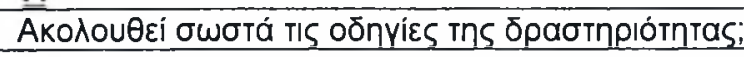

Av OXI, TI KáveI;

B

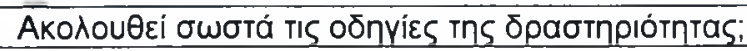

Av OXI, TI Kável;

\begin{tabular}{|l|l|} 
NaI & OXI \\
\hline
\end{tabular}

\begin{tabular}{|l|l|}
\hline NaI & OXI \\
\hline
\end{tabular}




\begin{tabular}{|c|c|c|}
\hline 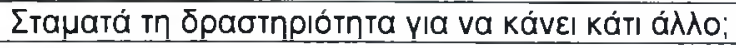 & Naı & $\mathrm{OX}$ \\
\hline \multicolumn{3}{|l|}{ Av Nal: } \\
\hline \multicolumn{3}{|l|}{ 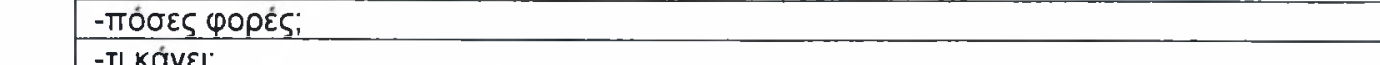 } \\
\hline \multicolumn{3}{|l|}{-TI Kável; } \\
\hline$\because$ & & \\
\hline 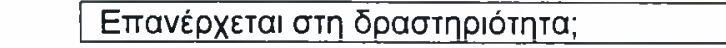 & Naı & OXI \\
\hline
\end{tabular}

B

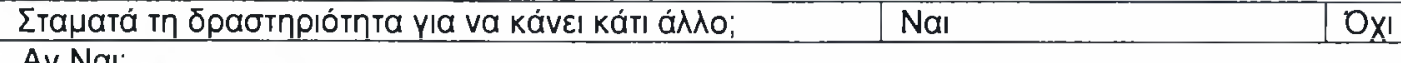

Av Nal:

-ாӧбES Форह்ร;

-TI Kável;

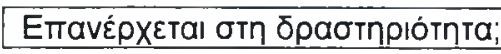

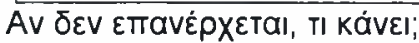

$\mathrm{Na}$

OXI

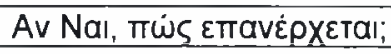

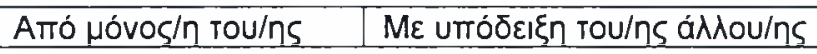

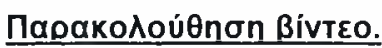

A

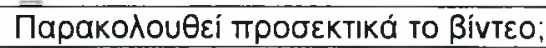

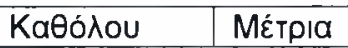

По^ú

B

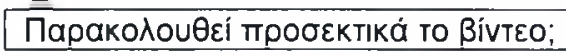

\begin{tabular}{|l|l|}
\hline Käódou & MÉTpıa \\
\hline
\end{tabular}

Пoגú

A

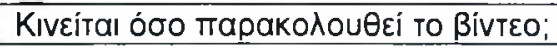

Av KIvEítaı Toגú, TI Kável;

KaӨónou

MÉTpıа

Поגن்

B

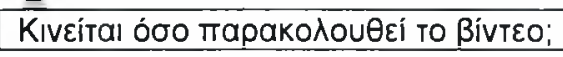

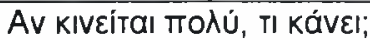

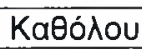

Мغ́трıа

Поגú

A

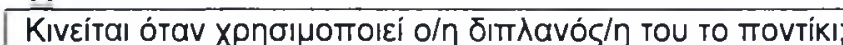

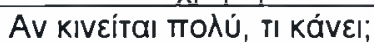

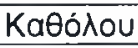

Mغ́трıа

Поגú

B

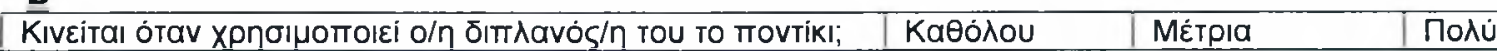

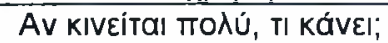

$\underline{A}$

A

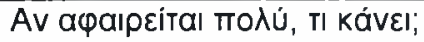

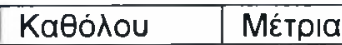

П○גú

B

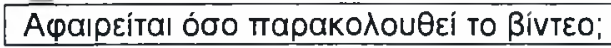

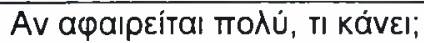

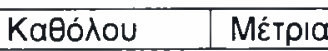

По入ú

A

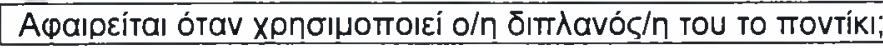

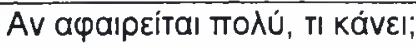

Kąó̀ou

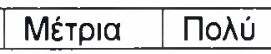

B

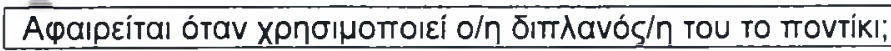

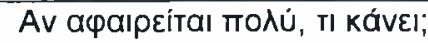

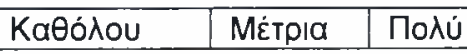




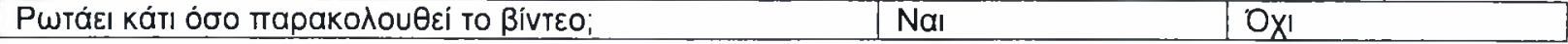

\begin{tabular}{|c|c|c|}
\hline Av Nal, eival бXETIKó; & $\mathrm{Nal}$ & OXI \\
\hline 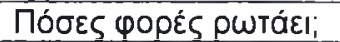 & & \\
\hline
\end{tabular}

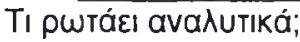

B

\begin{tabular}{|c|c|c|}
\hline 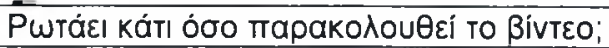 & $\mathrm{Na}$ & OXI \\
\hline 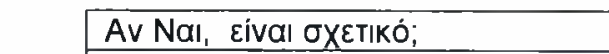 & $\mathrm{Naı}$ & OXI \\
\hline 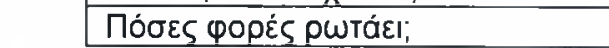 & & \\
\hline
\end{tabular}

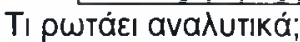

A

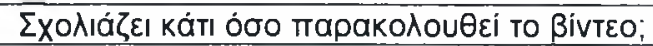

Nal $\quad$ OXI

Av Naı, عívaı бXعтıKó;

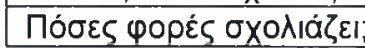

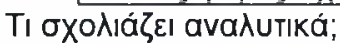

$\underline{\underline{B}}$

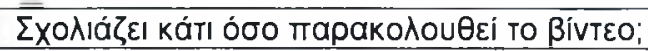

\begin{tabular}{|l|l|}
\hline NaI & OXI \\
\hline
\end{tabular}

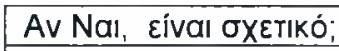

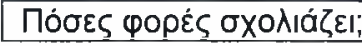

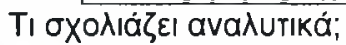

A

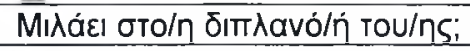
Naı
OXI

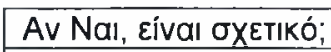

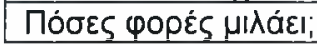

\begin{tabular}{|l|l|}
\hline Naı & OXI \\
\hline
\end{tabular}

Т। Аદ่દા;

B

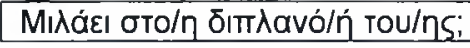

Nal $\quad$ OXI

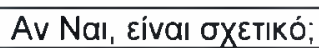

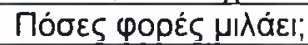

Nal $\quad$ OxI

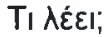

A

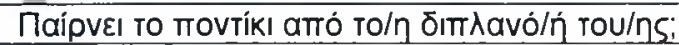

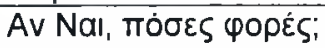

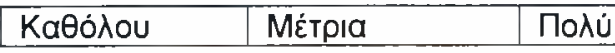

B

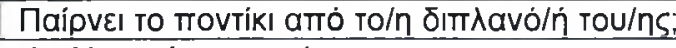

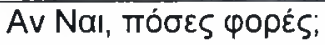

KaӨó̀ou

Méтрıа

Пoגú

A

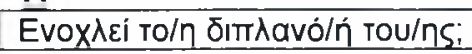

Av Naı, TI Kável;

NaI

OXI

B

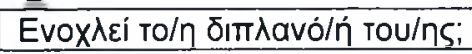

Av Naı, Tı Káveı;

Na!

OxI

A

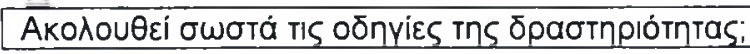

Av OXI, TI Kável;

Naı

OXI 
A

\begin{tabular}{|c|c|c|}
\hline 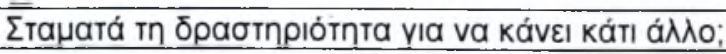 & $\mathrm{Nal}$ & OXI \\
\hline Av Nal: & & \\
\hline 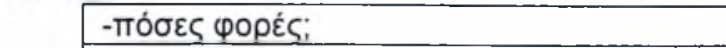 & & \\
\hline -TI Kável; & & \\
\hline 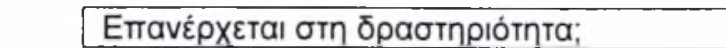 & Naı & OXI \\
\hline
\end{tabular}

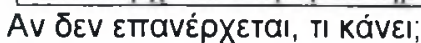

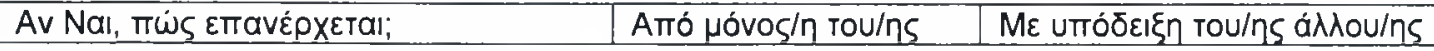

B

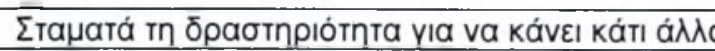
Av Nal:

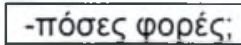

-TI Kávعl;

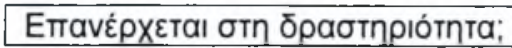

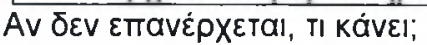

Naı

OXI

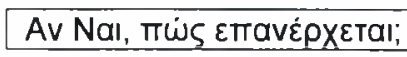

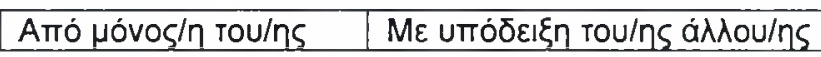

A

Bpiokel tnv $1^{\eta}$ aாávinơ rou $\varphi . \varepsilon$; Naı

OXI

$\underline{B}$

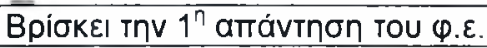

Naı

OXI

A

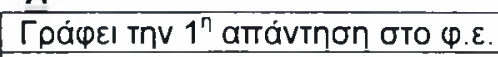

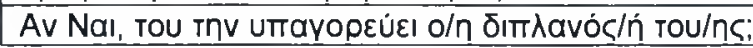

\begin{tabular}{|l|l} 
Nal _... OX! \\
\hline Nal
\end{tabular}

B

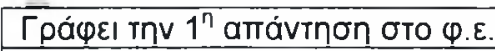

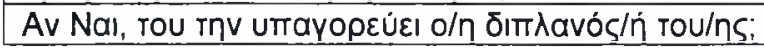

\begin{tabular}{|l|l|}
\hline Naı & OXı \\
\hline Naı & OX! \\
\hline
\end{tabular}

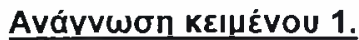

\section{A}

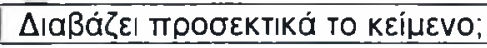

Kä́ó̀ou

Мغ́трıа

Поגن́

B

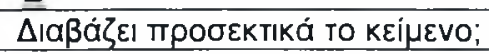

KaӨ́ó̀ou

Mغ́трıа

По入ú

A

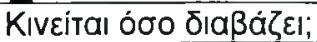

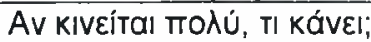

KaӨó^ou

Мغ́трıа

Поגú

B

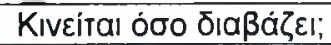

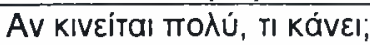

KaӨó̀ou

Мغ́тр।a

Поגن́

A

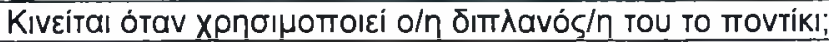

Kąó^ou

Mغ́трıа

По^ú 
Av kıveítaı moגú, Tı Kável;

B

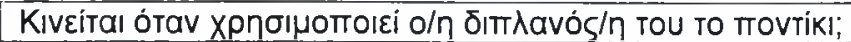

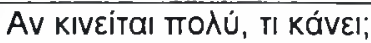

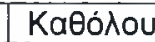

MÉTpIa

По^ú

A

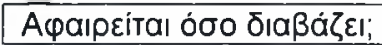

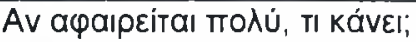

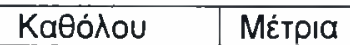

Поגú

B

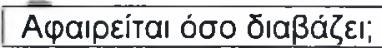

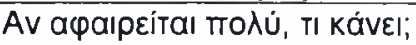
KaӨó̀ou

Мغ́трıа

Пoגú

A

\begin{tabular}{|c|c|c|c|}
\hline 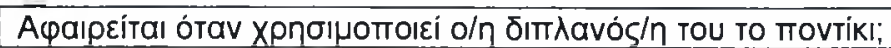 & 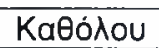 & Мغ́трıа & Пoגú \\
\hline
\end{tabular}

B

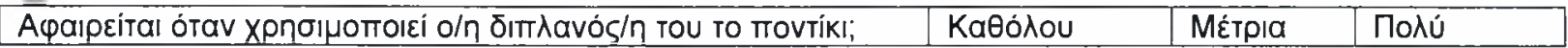

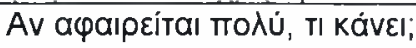

A

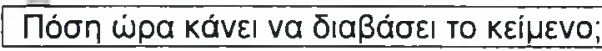

B

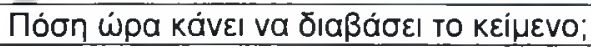

A

\begin{tabular}{|c|c|c|}
\hline 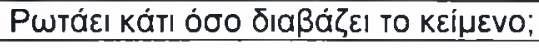 & $\mathrm{Na}$ & OXI \\
\hline 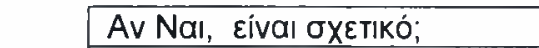 & $\mathrm{Nal}$ & Ox! \\
\hline
\end{tabular}

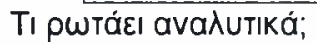

B

\begin{tabular}{|c|c|c|}
\hline 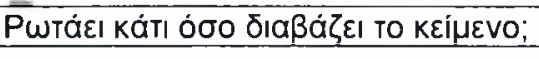 & Nal & OXI \\
\hline Av Nal, Eivaı oXETIKó; & $\mathrm{Nal}$ & $O X I$ \\
\hline
\end{tabular}

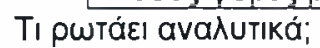

A

\begin{tabular}{|c|c|c|}
\hline 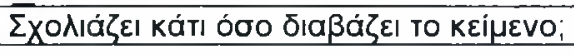 & Nal & OXI \\
\hline 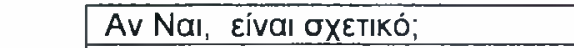 & Nal & $0 \times 1$ \\
\hline 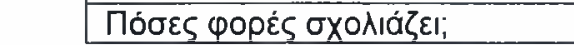 & & \\
\hline
\end{tabular}

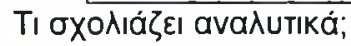

B

\begin{tabular}{|c|c|c|}
\hline 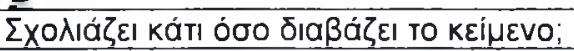 & Nal & $0 \times 1$ \\
\hline 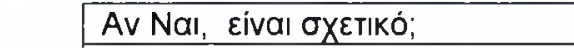 & Nal & OXI \\
\hline 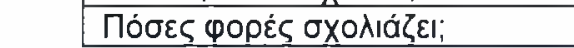 & & \\
\hline
\end{tabular}

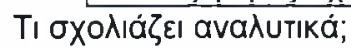

A

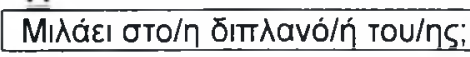

Naı

OXI

Av Naı, Eíval $\sigma \times \varepsilon T$ TIKó

Naı

OXI

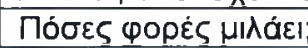

(


TІ $\lambda \varepsilon \varepsilon \mid ;$

B

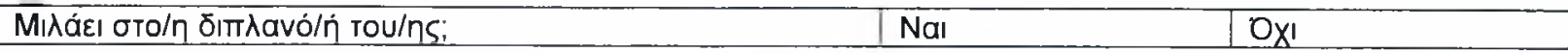

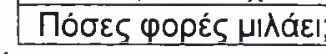

\begin{tabular}{|c|c|}
\hline 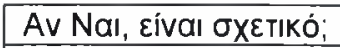 & $\mathrm{Nal}$ \\
\hline
\end{tabular}

A

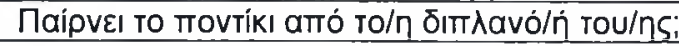

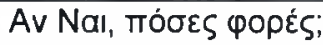

KąóAou

Мغ́трıа

По入ú

B

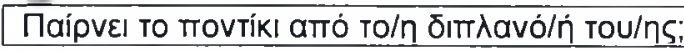

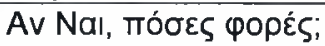

KaӨóরou

Mغ́rpıа

Поגú

A

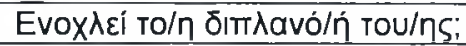

Av Naı, Tı Kável;

Naı

OXI

B

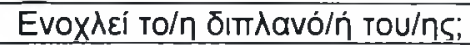

Av Naı, Tı Kável;

\begin{tabular}{|l|l|}
\hline Nal & OXI
\end{tabular}

A

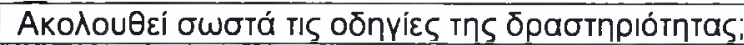

Av OXI, TI Kável;

\begin{tabular}{|l|l}
\hline $\mathrm{Nal}$ & $\mathrm{O} \times \mathrm{I}$ \\
\hline
\end{tabular}

B

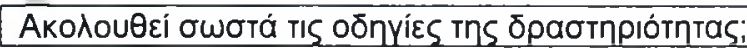

Av OXI, T। Kável;

\begin{tabular}{|l|l|}
\hline Naı & Ox! \\
\hline
\end{tabular}

A

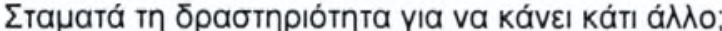

Av Nal:

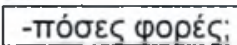

-TI KávعI;

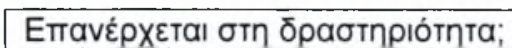

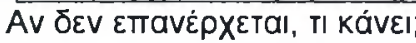

Naı

OxI

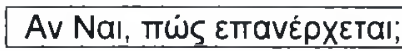

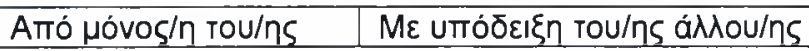

B

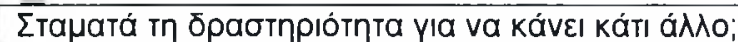

Av Nal:

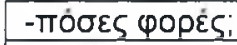

-TI Kável;

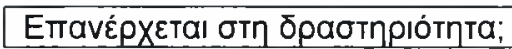

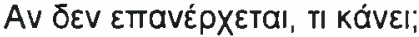

Naı

OXI

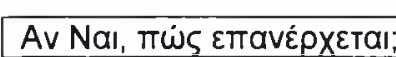

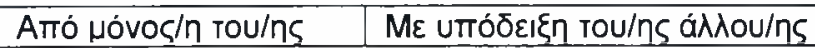

A

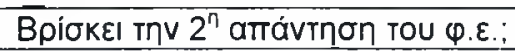
$\mathrm{Na} ı$

OxI 


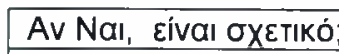

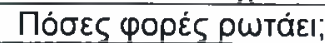

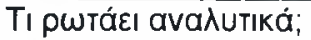

A

\begin{tabular}{|c|c|c|}
\hline 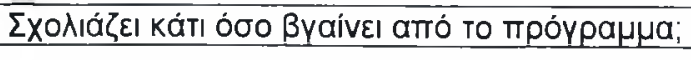 & Nai & OXI \\
\hline 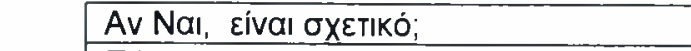 & Nal & OXI \\
\hline 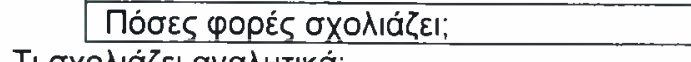 & & \\
\hline
\end{tabular}

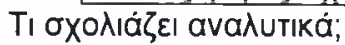

B

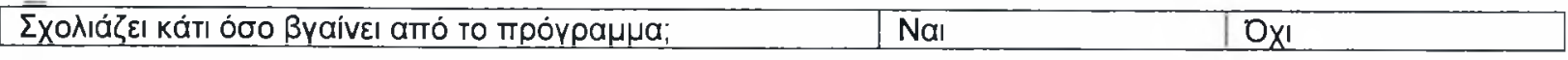

\begin{tabular}{|c|c|c|}
\hline Av Naı, zívaı бXETIKó; & $\mathrm{Nal}$ & OXI \\
\hline
\end{tabular}

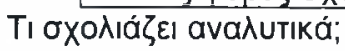

A

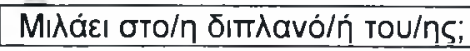

$\mathrm{Nal}$

|OxI

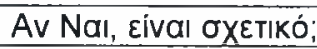

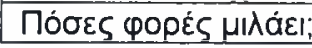

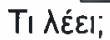

B

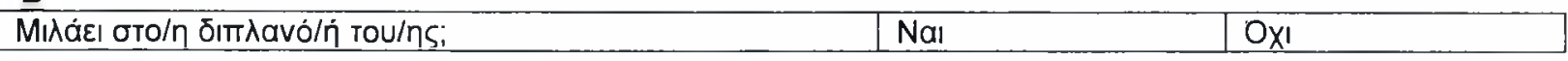

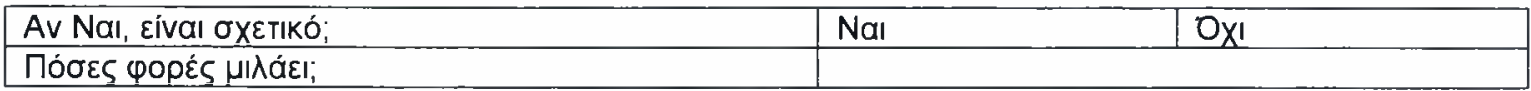
TІ $\lambda \dot{\varepsilon \varepsilon l ; ~}$

A

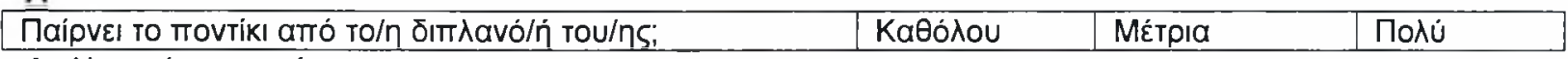

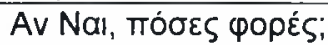

B

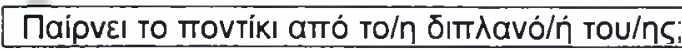

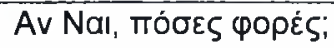

Käó̀ou

Mغ́трıа

Пoגú

A

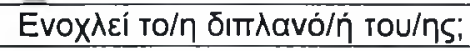

Av Naı, Tı Kável;

Naı $\quad$ OXI

B

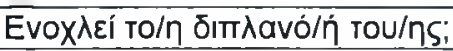

Av Naı, Tı Kável;

Nal

OXI

A

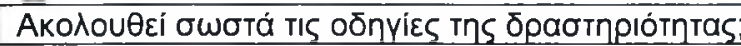

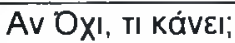

Naı

OXI

B

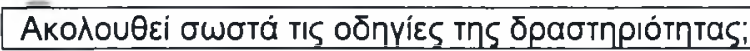

Av OXI, TI KáveI;

Nal

OxI

A

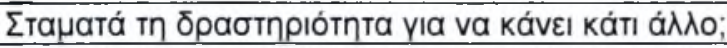

Av Nal:

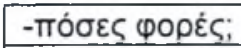

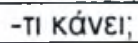


$+$

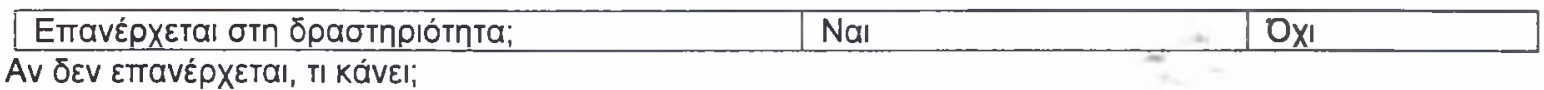

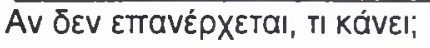

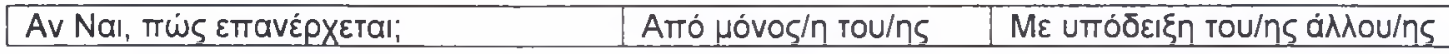

B

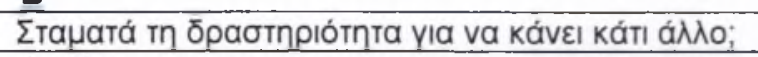

Av Nal:

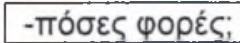

-TI KávEl;

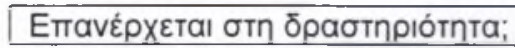

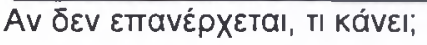

$\mathrm{Nal}$

OXI

$10 \times 1$

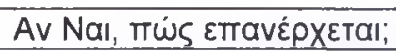

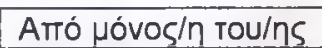

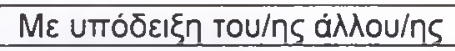




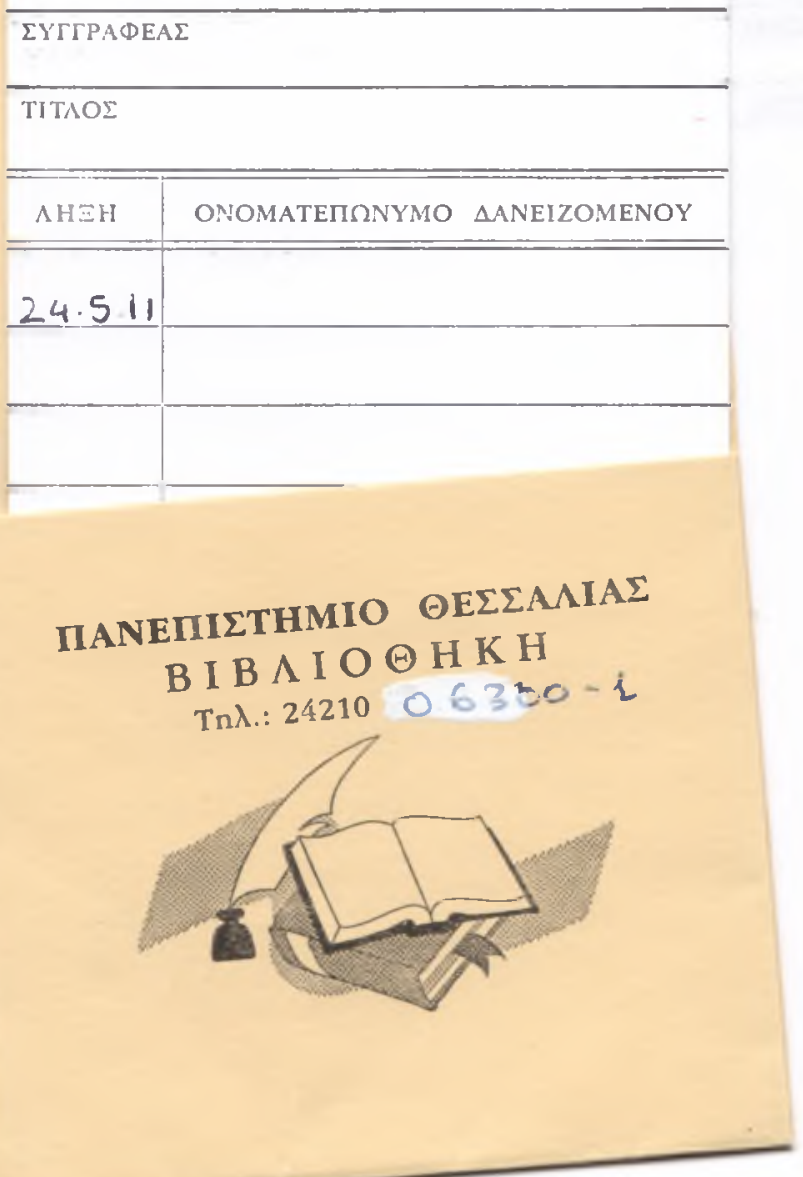


Institutional Repository - Library \& Information Centre - University of Thessaly 26/04/2023 14:08:38 EEST - 54.89.56.158 